http://kitaibelia.unideb.hu/

ISSN 2064-4507 (Online) • ISSN 1219-9672 (Print)

(C) 2016, Department of Botany, University of Debrecen, Hungary

22 (1): 55-59.; 2017

DOI: $10.17542 /$ kit.22.55

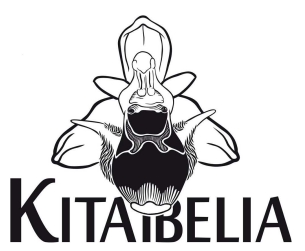

\title{
Az ELTE Füvészkert herbáriuma (BPU)
}

\author{
NóTÁRI Krisztina1* ${ }^{*}$, NAGY Timea ${ }^{2}$, LÖKI Viktor ${ }^{1}$, LJUBKA Tibor ${ }^{3}$, \\ MoLnÁR V. Attila ${ }^{1}$ \& TAKÁCS Attila ${ }^{1}$
}

(1) Debreceni Egyetem TTK Növénytani Tanszék, H-4032 Debrecen, Egyetem tér 1.; *notari.krisztina@gmail.com (2) Pannon Egyetem GK Növénytudományi és Biotechnológiai Tanszék, H-8360 Keszthely, Festetics u. 7.

(3) II. Rákóczi Ferenc Kárpátaljai Magyar Főiskola, Fodor István Kutatóközpont, UA-90202 Beregszász, Kossuth tér 6.

\section{The herbarium of the Botanical Garden of Eötvös Loránd University (BPU)}

Abstract - The paper introduces the herbarium of Eötvös Loránd University (BPU), currently stored at the Botanical Garden of the University, according to its state in the year 2013. The BPU herbarium consists of ca. 16000 specimens the relevant data of which have been organised into an OpenOffice Calc spreadsheet database. The specimens were collected in 16 European countries, mainly in the current territory of Hungary (80\%), Romania (12\%) and Slovakia (5\%). Other countries are represented with very few specimens. The vast majority (ca. 70\%) of the Hungarian specimens were collected in Pest, Veszprém, Borsod-Abaúj-Zemplén, Hajdú-Bihar and Szabolcs-Szatmár-Bereg counties. Although the gatherings cover a century and a half period of time, the most rapid growth of the collection took place from the 1930s to the 1960s. The most prolific collectors were Rezső Soó and his followers: Lajos Felföldy, Tibor Simon and Szaniszló Priszter. Felföldy and Simon enriched the collection through more than 60 years. Data on the native plants collected in the present-day Hungary are summarized in Electronic Appendix 1. (incl. taxon name, settlement, collecting year, collector, file name of documentary photograph).

Keywords: flora of Hungary, Lajos Felföldy, natural history collections, Szaniszló Priszter, Rezső Soó, Tibor Simon

Összefoglalás - Dolgozatunk az ELTE Füvészkert herbáriumát (BPU) mutatja be annak 2013-as állapota alapján. A herbárium kb. 16000 példányáról készült fényképekről a cédulákon szereplő adatokat OpenOffice Calc táblázatba rögzítettük. Összesen 16 európai országból származnak a lapok. Ezek közel 80\%-a magyarországi, 12\%-a romániai, 5\%-a szlovákiai. A hazai példányok közel 70\%-a Pest, Veszprém, Borsod-Abaúj-Zemplén, Hajdú-Bihar és Szabolcs-Szatmár-Bereg megyéből származik. Az 1850 és 2007 között keletkezett példányok több mint 70\%-át 1931 és 1960 között gyüjtötték. A példányok harmadát Soó Rezső gyűjtötte, de kiemelkedő Felföldy Lajos, Simon Tibor és Priszter Szaniszló hozzájárulása is. Felföldy Lajos és Simon Tibor több mint 60 éven át gyarapították a gyüjteményt. Az adatbázis magyarországi, nem kultivációból származó példányainak főbb adatait elektronikus mellékletben szabad felhasználásra közreadjuk, igény szerint részletesebb adatokat is közlünk.

Kulcsszavak: Felföldy Lajos, Magyarország flórája, Priszter Szaniszló, Simon Tibor, Soó Rezső, természettudományi gyűjtemények

\section{Bevezetés}

Az utóbbi években örvendetesen nőtt a herbáriumainkat bemutató közlemények száma, amelyek a több mint fél évszázada útjára indult Magyar herbáriumok sorozat (PRISZTER 1959) szellemiségét viszik tovább. Az Eötvös Loránd Tudományegyetem Botanikus Kertjének, azaz 
a Füvészkert herbáriumának (BPU) feldolgozásával ehhez a folyamathoz kívánunk csatlakozni. Munkánk célja a BPU gyưjtemény digitális archiválása és adatfeltárása volt. A feldolgozás módszere lényegében megegyezik korábbi munkáinkkal (TAKÁcS et al. 2014, 2015, E. VojTKó et al. 2014, NAGY et al. 2016): minden egyes herbáriumi lapról digitális felvétel készült, majd a fényképek fájlnevei folytonos sorszámot kaptak. A cédulákon olvasható információkat (fajnév, gyűjtő, ország, megye, település, pontos lelőhely, dátum) OpenOffice Calc táblázatban rögzítettük. Végül a példányokhoz sorszámot és taxonnevet rendeltünk KIRÁLY (2009) műve alapján. Az Új magyar füvészkönyv által alkalmazott nevektől csak akkor tértünk el, ha a korábban alkalmazott taxonnevek nem, vagy csak bizonytalanul egyeztethetők össze a jelenlegiekkel - jellemzően az adott csoport taxonómiai megítélésének változása miatt. Ilyen esetekben nemzetség szinten vagy agg. ill. s.l. értelemben rendeltünk nevet a példányokhoz. A közelmúltbeli taxonómiai kutatások eredményeit is igyekeztünk figyelembe venni, így például az Adonis hybrida G. Wolff ex Simonk. helyett az A. volgensis Steven ex DC. (SRAmKó et al. 2012), a Himantoglossum caprinum (M. Bieb.) Spreng. helyett a H. jankae Somlyay, Kreutz \& Óvári (MolNÁR V. et al. 2012) nevek alkalmazását tartottuk helyesnek.

Közleményünkben áttekintjük a BPU herbárium főbb tulajdonságait (példányszám, a példányok térbeli és időbeli eloszlása, gyűjtők stb.). A kutathatóság, áttekinthetőség megkönnyítése érdekében a gyűjtemény hazai anyagának fóbb adatait elektronikus mellékletben közreadjuk.

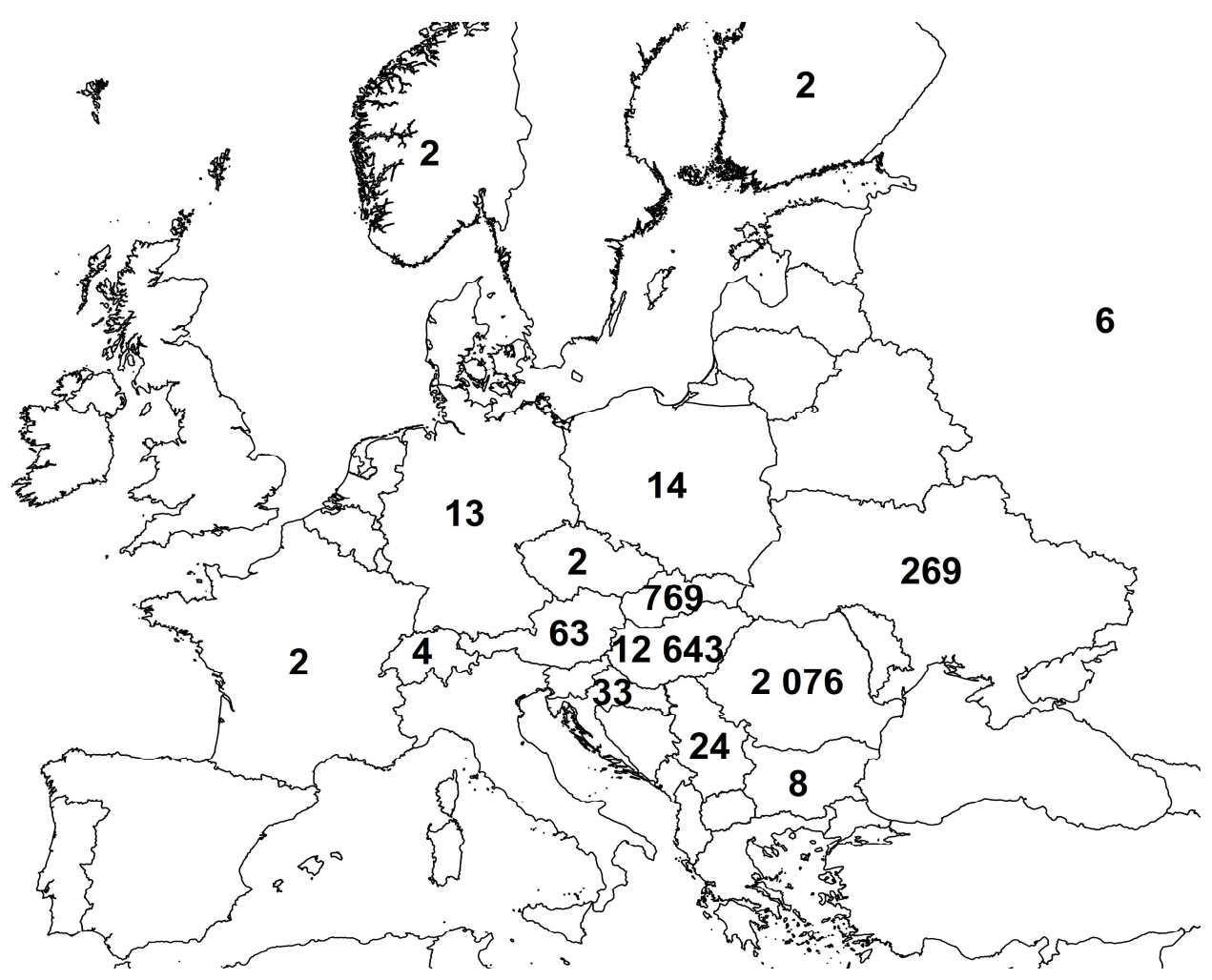

1. ábra. A BPU gyűjteményben őrzött példányok száma országonként

Fig. 1. The number of collected specimens per countries as represented in the BPU herbarium 


\section{A gyüjtemény bemutatása}

A Soó \& JÁvoRKA (1951) sorszámai alapján rendezett gyújtemény mintegy 16000 lapot számlál (az említett műben nem szereplő taxonok adott nemzetség vagy család végén alfabetikus sorrendben vannak elhelyezve). A gyűjteményt Soó Rezső herbáriumának ide került része alapozta meg (vö. Soó 1972). Ennek megfelelően az anyag térbeli és időbeli reprezentativitása, valamint a legaktívabb gyűjtők köre igen hasonló a Debreceni Egyetemen őrzött Soó-herbáriuméhoz (vö. TAKÁcs et al. 2014). A Szabolcs-Szatmár-Bereg, Hajdú-Bihar és Borsod-Abaúj-Zemplén megye területéről származó példányok viszonylag magas számát (2. ábra) jórészt a Soó-hagyaték magyarázza, ahogy az 1930-as, 1940-es és 1950-es évek magas példányszámait (3. ábra) is. Ugyanakkor a gyüjtemény „utóélete” a debreceniétől eltérő: Felföldy Lajos 2003-2005 között foglalkozott annak rendezésével (FELFöLDY 2005). Ez a Soó-gyűjtemény mellett felhalmozódott anyagok válogatását, határozását, revízióját, ragasztását, cédulázását és beosztását jelentette. A munka legnagyobb számban Priszter Szaniszló és Simon Tibor addig feldolgozatlan példányait érintette (FELFöLDY 2005), de több ezer saját lapot is minden bizonnyal ekkor épített be a gyüjteménybe. Veszprém megye, illetve az 1950-es évek magas példányszámához Felföldy Balaton környéki gyújtései nagymértékben hozzájárulnak.

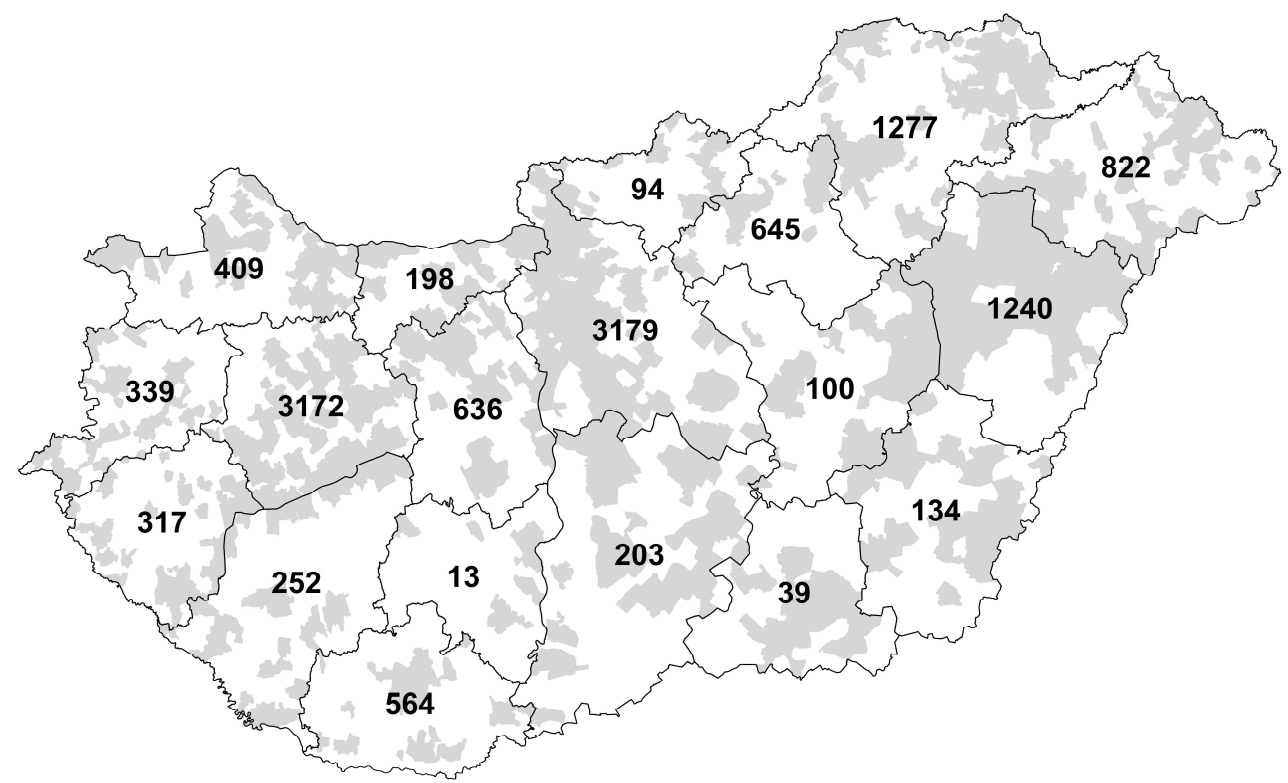

2. ábra. A BPU gyűjteményben őrzött hazai példányok száma megyénként. A szürke szín a példánnyal reprezentált települések közigazgatási területét jelöli

Fig. 2. The number of collected specimens per Hungarian counties as represented in the BPU herbarium. The territory of settlements represented by vouchers is indicated with grey

A lapok többsége tehát Soó és tanítványai, kollégái tevékenységéhez kapcsolódik, de - a debreceni gyűjteményhez hasonlóan - megtaláljuk többek között Polgár Sándor, Boros Ádám, Kárpáti Zoltán, Kováts Ferenc, Károlyi Árpád példányait (1. táblázat), ahogy növénytári duplumokat és a Degen-féle exsiccata lapjait is.

A példányok - nyilván Felföldy Lajos gondos válogatásának köszönhetően (FELFöLDY 2005) - szinte kivétel nélkül jól lokalizáltak. Magyarországon túl Európa 15 további 
országából találunk példányokat a gyűjteményben (1. ábra), de ezek nagytöbbsége is magyar gyűjtőktől származik. Valójában a 2000-et meghaladó számú erdélyi lapon kívül a gyűjteményben nem találunk jelentős mennyiségű külföldi anyagot.

A lapok több mint 90\%-a napra pontos dátummal feliratozott. A gyűjtemény legidősebb példányai az 1850-es (Theodor Kotschy néhány erdélyi lapja) és 1870-es (Feichtinger Sándor, Simonkai Lajos, Haynald Lajos gyưjtései) évekből származnak, míg az utolsók a 2000-es években keletkeztek (elsősorban Felföldy Lajos és Simon Tibor gyűjtései).

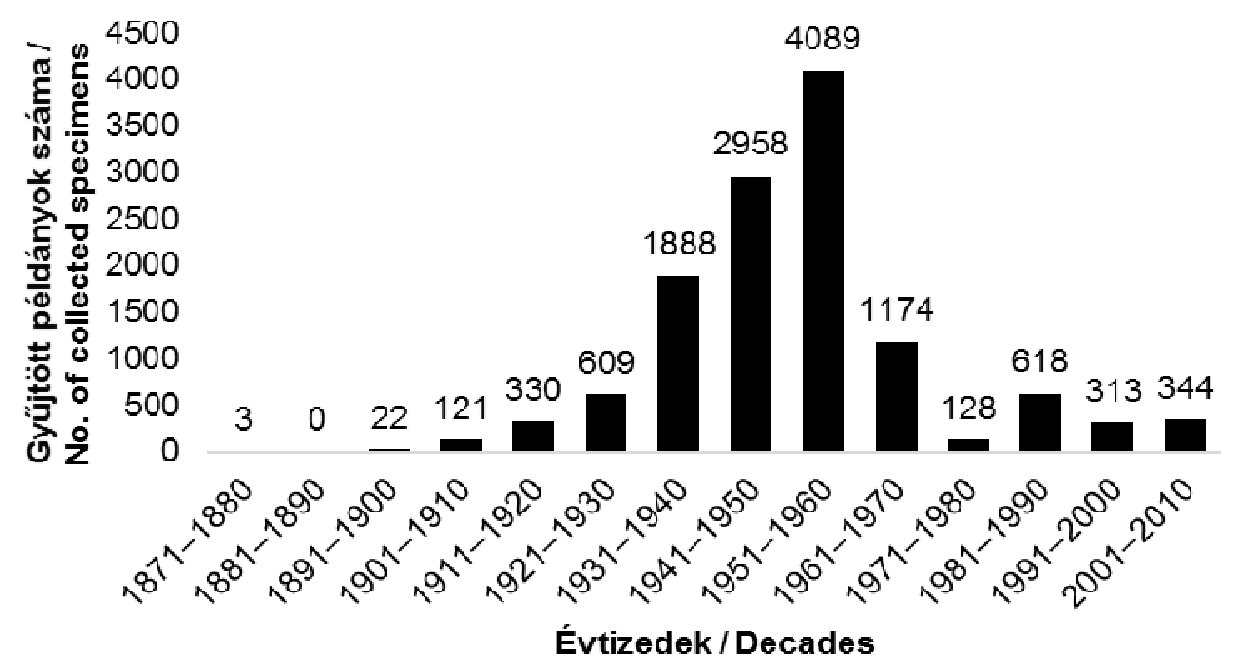

3. ábra. A Magyarországon gyűjtött példányok száma évtizedenként

Fig. 3. The number of collected specimens in Hungary per decades

A BPU gyűjteményben kijelölt típusanyagot nem ismerünk, de megtalálhatók számos taxon típus értékű originális anyagai, a teljesség igénye nélkül például Theodor Kotschy-tól a Rhododendron myrtifolium Schott \& Kotschy, Simonkai Lajostól a Quercus ×tabajdiana Simonk., Borbás Vincétől a Rumex pseudonatronatus Borbás és a Gypsophila ×digenea Borbás, Jávorka Sándortól a Minuartia graminifolia Jáv., Bihari Gyulától a Rumex ×hungaricus Bihari, Hulják Jánostól a Galium ×buekkense Hulják, Priszter Szaniszlótól az Amaranthus ×polgarianus Priszter \& Kárpáti, Polgár Sándortól az Ornithogalum ×degenianum Polgár és a Solanum probstianum Polgár, Kárpáti Zoltántól a Sorbus borosiana Kárpáti, a S. adami Kárpáti, a S. pseudobakonyensis Kárpáti, a S. eugenii-kelleri Kárpáti lapjai. Mivel az említett nevek egy részének státusza - különösen a nothotaxonoké - máig tisztázatlan, az itt őrzött példányoknak fontos szerepük lehet a jövő taxonómiai és nevezéktani kutatásaiban.

\section{Köszönetnyilvánítás}

Hálásak vagyunk Isépy Istvánnak, ifj. Papp Lászlónak és Orlóci Lászlónak, hogy a BPU gyưjtemény feldolgozását számunkra lehetővé tették. Isépy Istvánnak és ifj. Papp Lászlónak kéziratunkhoz fűzött hasznos észrevételeiket is köszönjük. A munkát az OTKA K108992 számú pályázata támogatta. 
1. táblázat. A BPU legjelentősebb gyűjtői

Table 1. The most prolific collectors of the BPU herbarium

\begin{tabular}{|c|c|c|c|c|c|c|}
\hline 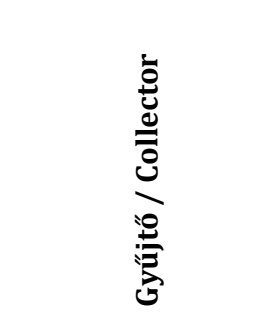 & 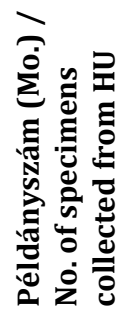 & 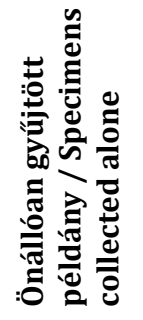 & 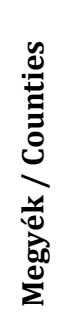 & 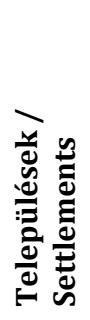 & 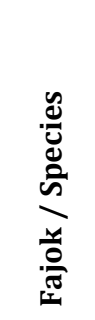 & 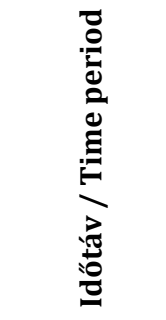 \\
\hline Soó Rezső & 4639 & 4378 & 18 & 225 & 1742 & 1921-1965 \\
\hline Felföldy Lajos & 3252 & 2765 & 18 & 113 & 1070 & 1936-2005 \\
\hline Simon Tibor & 1271 & 585 & 16 & 133 & 806 & 1943-2007 \\
\hline Priszter Szaniszló & 1014 & 917 & 18 & 128 & 477 & $1935-1980$ \\
\hline Borsos Olga & 578 & 217 & 15 & 52 & 398 & 1947-1995 \\
\hline Tóth László & 440 & 17 & 10 & 45 & 294 & 1950-1989 \\
\hline Jakucs Pál & 360 & 28 & 6 & 47 & 294 & 1920-1955 \\
\hline Jávorka Sándor & 243 & 21 & 9 & 30 & 194 & 1910-1959 \\
\hline Degen Árpád & 181 & 154 & 10 & 32 & 131 & $1900-1930$ \\
\hline Polgár Sándor & 176 & 175 & 8 & 49 & 137 & $1896-1940$ \\
\hline Boros Ádám & 166 & 166 & 18 & 103 & 115 & 1916-1957 \\
\hline Kárpáti Zoltán & 161 & 159 & 9 & 37 & 148 & 1930-1951 \\
\hline Isépy István & 140 & 140 & 11 & 37 & 131 & 1956-1964 \\
\hline Vozáry Elemér & 137 & 21 & 6 & 16 & 117 & 1933-1953 \\
\hline Pólya László & 134 & 40 & 7 & 19 & 116 & 1943-1949 \\
\hline Kováts Ferenc & 125 & 125 & 8 & 21 & 29 & 1925-1958 \\
\hline Kulcsár Gábor & 120 & 46 & 5 & 15 & 100 & 1920-1953 \\
\hline Károlyi Árpád & 109 & 109 & 6 & 37 & 95 & $1948-1956$ \\
\hline
\end{tabular}

\section{Irodalom}

E. VojtKó A., TAKÁcs A., Molnár V. A. \& VojTKó A. (2014): Herbarium database of the vascular collection of Eszterházy Károly College (EGR). - Kitaibelia 19: 339-348.

FELFöLDY L. (2005): A HERBARIUM HORTI BOTANICI UNIV. SCI. BUDAPESTIENSIS 2003-2005 évi rendezésének leírása. - Kézirat, ELTE Botanikus Kert, 5 pp.

Molnár V. A., Kreutz K. C., Óvari M., Sennikov A. N., Bateman R. M., Takács A., Somlyay L. \& Sramkó G. (2012): Himantoglossum jankae (Orchidaceae: Orchideae), a new name for a long-misnamed lizard orchid. - Phytotaxa 73: 8-12.

NAGY T., TAKÁCS A., \& BódIS J. (2016): Magyar herbáriumok 15. A keszthelyi Balatoni Múzeum herbáriuma (KBM). - Botanikai Közlemények 103: 213-226.

PRISZTER Sz. (1959): Magyar herbáriumok 1. A keszthelyi általános gimnázium herbáriuma. - Botanikai Közlemények 48: 109-113.

Soó R. \& JávorKA S. (1951): A magyar növényvilág kézikönyve I-II. - Akadémiai Kiadó, Budapest.

Soó R. (1972): Növénygyűjtéseim, 1917-1968. - Botanikai Közlemények 59: 211-214.

SRAmkó G., BARTha L., SAllainé KaPocsi J., PodAni J. \& MolnÁr V. A. (2012): A csorvási hérics filogenetikai helyzetének molekuláris és morfometriai vizsgálata. - Kitaibelia 17: 55.

TakÁcs A., Nagy T., Fekete R., Lovas-Kiss Á., Ljubka T., LöKi V., LisZTes-Szabó Zs. \& Molnár V. A. (2014): A Debreceni Egyetem Herbáriuma (DE) I.: A „Soó Rezső Herbárium”. - Kitaibelia 19: 142-155.

TAKÁcs A., SÜVeges K., LJubKa T., LöKI V., LisZTes-Szabó Zs. \& Molnár V. A. (2015): A Debreceni Egyetem Herbáriuma (DE) II.: A „Siroki Zoltán Herbárium”. - Kitaibelia 20: 15-22.

Beérkezett: 2017. 03. 12. • Elfogadva: 2017. 03. 20. 
NóTÁRI Krisztina, NAGY Timea, LöKI Viktor, LJUBKA Tibor, MoLNÁR V. Attila \& TAKÁcs Attila (2017):

Az ELTE Füvészkert herbáriuma (BPU) /

The herbarium of the Botanical Garden of Eötvös Loránd University (BPU) - Kitaibelia 22 (1): 55-59.

(DOI: 10.17542/kit.22.55)

1. Elektronikus melléklet - Részlet a BPU gyűjtemény adatbázisának attribútum-táblájából (Magyarországon gyűjtött, nem kultivált növények példányainak fő gyűjtési adatai).

Electronic Appendix 1. - Main attributes of the BPU specimens of non-cultivated plants collected in present-day Hungary

\section{Sorszámozás és nevezéktan / Numbers and nomenclature: KIRÁLY (2009)}

\begin{tabular}{|c|c|c|c|c|c|}
\hline $\begin{array}{l}\text { Sorszám / } \\
\text { Number }\end{array}$ & Taxon-név / Taxon-name & $\begin{array}{l}\text { Település / } \\
\text { Settlement }\end{array}$ & $\begin{array}{l}\text { Év / } \\
\text { Year }\end{array}$ & Gyűjtő / Collector & $\begin{array}{l}\text { Fájlnév / } \\
\text { File-name }\end{array}$ \\
\hline 1 & Huperzia selago (L.) Bernh. & Kishuta & 1952 & Vida Gábor & BPU-09234.JPG \\
\hline 1 & Huperzia selago (L.) Bernh. & Kishuta & 1955 & Simon Tibor & BPU-09235.JPG \\
\hline 1 & Huperzia selago (L.) Bernh. & Kishuta & 1963 & Isépy István & BPU-09236.JPG \\
\hline 1 & Huperzia selago (L.) Bernh. & Pálháza & 1952 & Simon Tibor & BPU-09233.JPG \\
\hline 2 & Diphasium issleri (Rouy) Holub & Miskolc & 1949 & Jávorka Sándor & BPU-09244.JPG \\
\hline 3 & Diphasium complanatum (L.) Rothm. & Bozsok & 1938 & Soó Rezső & BPU-09251.JPG \\
\hline 3 & Diphasium complanatum (L.) Rothm. & Kishuta & 1952 & Vida Gábor & BPU-09246.JPG \\
\hline 3 & Diphasium complanatum (L.) Rothm. & Kishuta & 1963 & Isépy István & BPU-09249.JPG \\
\hline 3 & Diphasium complanatum (L.) Rothm. & Kishuta & 1963 & Simon Tibor & BPU-09248.JPG \\
\hline 3 & Diphasium complanatum (L.) Rothm. & Nagyhuta & 1981 & $\begin{array}{l}\text { Simon Tibor, Simon- } \\
\text { Wolcsánszky E. }\end{array}$ & BPU-09243.JPG \\
\hline 3 & Diphasium complanatum (L.) Rothm. & Pálháza & 1949 & Simon Tibor, Jakucs Pál & BPU-09245.JPG \\
\hline 3 & Diphasium complanatum (L.) Rothm. & Pálháza & 1961 & Simon Tibor & BPU-09247.JPG \\
\hline 3 & Diphasium complanatum (L.) Rothm. & Regéc & 1981 & Simon Tibor & BPU-09250.JPG \\
\hline 5 & Lycopodium clavatum L. & Alsószölnök & 1955 & Vajda László & BPU-09264.JPG \\
\hline 5 & Lycopodium clavatum L. & Bakonybél & 1954 & Tallós Pál & BPU-09266.JPG \\
\hline 5 & Lycopodium clavatum L. & Kishuta & 1963 & Isépy István & BPU-09262.JPG \\
\hline 5 & Lycopodium clavatum L. & Nagyhuta & 1952 & Simon Tibor & BPU-09263.JPG \\
\hline 5 & Lycopodium clavatum L. & Szőce & 1955 & Soó Rezső & BPU-09265.JPG \\
\hline 5 & Lycopodium clavatum L. & Zalalövő & 1953 & Pócs Tamás & BPU-09267.JPG \\
\hline 6 & Lycopodium annotinum L. & Kishuta & 1961 & Simon Tibor & BPU-09274.JPG \\
\hline 6 & Lycopodium annotinum L. & Kishuta & 1963 & Isépy István & BPU-09275.JPG \\
\hline 7 & Selaginella helvetica (L.) Link & Felsőcsatár & 1957 & Pócs Tamás, Tallós Pál & BPU-09287.JPG \\
\hline 8 & Equisetum telmateia Ehrh. & Alsószölnök & 1955 & Soó Rezső & BPU-09319.JPG \\
\hline 8 & Equisetum telmateia Ehrh. & Bátorliget & 1931 & Soó Rezső & BPU-09318.JPG \\
\hline 8 & Equisetum telmateia Ehrh. & Bisse & 1965 & $\begin{array}{l}\text { Simon Tibor, Priszter } \\
\text { Szaniszló, Borhidi Attila }\end{array}$ & BPU-09314.JPG \\
\hline 8 & Equisetum telmateia Ehrh. & Hidegkút & 1957 & Felföldy Lajos & BPU-09322.JPG \\
\hline 8 & Equisetum telmateia Ehrh. & Hidegkút & 1959 & Felföldy Lajos & BPU-09321.JPG \\
\hline 8 & Equisetum telmateia Ehrh. & Komló & 1941 & Priszter Szaniszló & BPU-09315.JPG \\
\hline 8 & Equisetum telmateia Ehrh. & Mogyoróska & 1939 & Soó Rezső & BPU-09324.JPG \\
\hline 8 & Equisetum telmateia Ehrh. & Sopron & 1942 & Priszter Szaniszló & BPU-09316.JPG \\
\hline 8 & Equisetum telmateia Ehrh. & Sopron & 1960 & Borsos Olga, Simon Tibor & BPU-09317.JPG \\
\hline 8 & Equisetum telmateia Ehrh. & Telekes & 1955 & Soó Rezső & BPU-09320.JPG \\
\hline 8 & Equisetum telmateia Ehrh. & Uzsa & 1952 & Simon Tibor & BPU-09323.JPG \\
\hline 9 & Equisetum arvense L. & Balatonszőlős & 1956 & $\begin{array}{l}\text { Felföldy Lajos, Tóth } \\
\text { László }\end{array}$ & BPU-09309.JPG \\
\hline
\end{tabular}


1. Elektroniukus melléklet - Electronic Appendix 1.

\begin{tabular}{|c|c|c|c|c|c|}
\hline $\begin{array}{l}\text { Sorszám / } \\
\text { Number }\end{array}$ & Taxon-név / Taxon-name & $\begin{array}{l}\text { Település / } \\
\text { Settlement }\end{array}$ & $\begin{array}{l}\text { Év / } \\
\text { Year }\end{array}$ & Gyưjtő / Collector & $\begin{array}{l}\text { Fájlnév / } \\
\text { File-name }\end{array}$ \\
\hline 9 & Equisetum arvense L. & Bátorliget & 1951 & Simon Tibor & BPU-09308.JPG \\
\hline 9 & Equisetum arvense L. & Budapest & 1991 & Felföldy Lajos & BPU-09301.JPG \\
\hline 9 & Equisetum arvense L. & Budapest & 2004 & Felföldy Lajos & BPU-09300.JPG \\
\hline 9 & Equisetum arvense L. & Jósvafő & 1939 & Priszter Szaniszló & BPU-09303.JPG \\
\hline 9 & Equisetum arvense L. & Jósvafő & 1952 & Kulcsár Gábor & BPU-09302.JPG \\
\hline 9 & Equisetum arvense L. & Kapolcs & 1959 & $\begin{array}{l}\text { Jávorka Sándor, Felföldy } \\
\text { Lajos, Tóth László }\end{array}$ & BPU-09310.JPG \\
\hline 9 & Equisetum arvense L. & Pálháza & 1939 & Soó Rezső & BPU-09304.JPG \\
\hline 9 & Equisetum arvense L. & Szilvásvárad & 1953 & $\begin{array}{l}\text { Felföldy Lajos, Tóth } \\
\text { László }\end{array}$ & BPU-09307.JPG \\
\hline 9 & Equisetum arvense L. & Téglás & 1937 & Soó Rezső & BPU-09306.JPG \\
\hline 9 & Equisetum arvense L. & Uzsa & 1959 & $\begin{array}{l}\text { Jávorka Sándor, Felföldy } \\
\text { Lajos }\end{array}$ & BPU-09311.JPG \\
\hline 9 & Equisetum arvense L. & Zámoly & 1982 & Felföldy Lajos & BPU-09305.JPG \\
\hline 10 & Equisetum sylvaticum L. & Alsószölnök & 1955 & Soó Rezső & BPU-09299.JPG \\
\hline 10 & Equisetum sylvaticum L. & Pálháza & 1938 & Soó Rezső & BPU-09295.JPG \\
\hline 10 & Equisetum sylvaticum L. & Pálháza & 1952 & Vida Gábor & BPU-09296.JPG \\
\hline 10 & Equisetum sylvaticum L. & Pilismarót & 1924 & $\begin{array}{l}\text { Gyelnik Vilmos, } \\
\text { Trautmann Róbert }\end{array}$ & BPU-09297.JPG \\
\hline 10 & Equisetum sylvaticum L. & Sopron & 1960 & Borsos Olga, Simon Tibor & BPU-09298.JPG \\
\hline 11 & Equisetum variegatum Schleich. & Tatabánya & 1937 & Boros Ádám & BPU-09388.JPG \\
\hline 11 & Equisetum variegatum Schleich. & Telekes & 1955 & Soó Rezső & BPU-09389.JPG \\
\hline 12 & Equisetum fluviatile L. em. Ehrh. & Baktalórántháza & 1932 & Soó Rezső & BPU-09347.JPG \\
\hline 12 & Equisetum fluviatile L. em. Ehrh. & Gávavencsellő & 1990 & Felföldy Lajos & BPU-09346.JPG \\
\hline 12 & Equisetum fluviatile L. em. Ehrh. & Miskolc & 1923 & Kümmerle Jenő Béla & BPU-09342.JPG \\
\hline 12 & Equisetum fluviatile L. em. Ehrh. & Pálháza & 1938 & Soó Rezső & BPU-09343.JPG \\
\hline 12 & Equisetum fluviatile L. em. Ehrh. & Penészlek & 1932 & Soó Rezső & BPU-09348.JPG \\
\hline 12 & Equisetum fluviatile L. em. Ehrh. & Tarpa & 1949 & Soó Rezső & BPU-09344.JPG \\
\hline 12 & Equisetum fluviatile L. em. Ehrh. & Veresegyház & 1949 & Soó Rezső & BPU-09345.JPG \\
\hline 13 & Equisetum palustre L. & Badacsonytomaj & 1942 & Priszter Szaniszló & BPU-09338.JPG \\
\hline 13 & Equisetum palustre L. & Balatonfüred & 1946 & Felföldy Lajos & BPU-09339.JPG \\
\hline 13 & Equisetum palustre L. & Bisse & 1965 & $\begin{array}{l}\text { Simon Tibor, Priszter } \\
\text { Szaniszló, Borhidi Attila }\end{array}$ & BPU-09333.JPG \\
\hline 13 & Equisetum palustre L. & Budapest & 2004 & Felföldy Lajos & BPU-09331.JPG \\
\hline 13 & Equisetum palustre L. & Gáborján & - & Kovács & BPU-09334.JPG \\
\hline 13 & Equisetum palustre L. & Kányavár & 1949 & Károlyi Árpád & BPU-09340.JPG \\
\hline 13 & Equisetum palustre L. & Nagykanizsa & 1956 & Felföldy Lajos & BPU-09341.JPG \\
\hline 13 & Equisetum palustre L. & Nyírpilis & 1951 & Simon Tibor & BPU-09337.JPG \\
\hline 13 & Equisetum palustre L. & Pálháza & 1938 & Soó Rezső & BPU-09332.JPG \\
\hline 13 & Equisetum palustre L. & Piliscsaba & 1942 & Priszter Szaniszló & BPU-09336.JPG \\
\hline 13 & Equisetum palustre L. & Pilisszentiván & 1913 & $\begin{array}{l}\text { Filarszky Nándor, Jávorka } \\
\text { Sándor, Timkó György }\end{array}$ & BPU-09330.JPG \\
\hline 13 & Equisetum palustre L. & Szalonna & 1966 & Felföldy Lajos & BPU-09335.JPG \\
\hline 14 & Equisetum ramosissimum Desf. & Balatonakali & 1958 & Felföldy Lajos & BPU-09385.JPG \\
\hline 14 & Equisetum ramosissimum Desf. & Balatonszepezd & 1958 & Felföldy Lajos & BPU-09386.JPG \\
\hline 14 & Equisetum ramosissimum Desf. & Budapest & 1915 & Kümmerle Jenő Béla & BPU-09363.JPG \\
\hline 14 & Equisetum ramosissimum Desf. & Budapest & 1915 & Kümmerle Jenő Béla & BPU-09366.JPG \\
\hline 14 & Equisetum ramosissimum Desf. & Budapest & 1923 & Soó Rezső & BPU-09370.JPG \\
\hline 14 & Equisetum ramosissimum Desf. & Budapest & 1924 & Filarszky Nándor & BPU-09369.JPG \\
\hline 14 & Equisetum ramosissimum Desf. & Budapest & 1948 & Soó Rezső & BPU-09371.JPG \\
\hline
\end{tabular}


1. Elektroniukus melléklet - Electronic Appendix 1.

\begin{tabular}{|c|c|c|c|c|c|}
\hline $\begin{array}{l}\text { Sorszám / } \\
\text { Number }\end{array}$ & Taxon-név / Taxon-name & $\begin{array}{l}\text { Település / } \\
\text { Settlement }\end{array}$ & $\begin{array}{l}\text { Év / } \\
\text { Year }\end{array}$ & Gyüjtő / Collector & $\begin{array}{l}\text { Fájlnév / } \\
\text { File-name }\end{array}$ \\
\hline 14 & Equisetum ramosissimum Desf. & Budapest & 1982 & Felföldy Lajos & BPU-09372.JPG \\
\hline 14 & Equisetum ramosissimum Desf. & Budapest & 1982 & Felföldy Lajos & BPU-09377.JPG \\
\hline 14 & Equisetum ramosissimum Desf. & Budapest & 1989 & Felföldy Lajos & BPU-09374.JPG \\
\hline 14 & Equisetum ramosissimum Desf. & Budapest & 1991 & Felföldy Lajos & BPU-09373.JPG \\
\hline 14 & Equisetum ramosissimum Desf. & Budapest & 1991 & Felföldy Lajos & BPU-09375.JPG \\
\hline 14 & Equisetum ramosissimum Desf. & Budapest & 1993 & Felföldy Lajos & BPU-09376.JPG \\
\hline 14 & Equisetum ramosissimum Desf. & Budapest & 1993 & Felföldy Lajos & BPU-09378.JPG \\
\hline 14 & Equisetum ramosissimum Desf. & Debrecen & 1946 & Soó Rezső & BPU-09380.JPG \\
\hline 14 & Equisetum ramosissimum Desf. & Hortobágy & 1947 & Soó Rezső & BPU-09381.JPG \\
\hline 14 & Equisetum ramosissimum Desf. & Konyár & 1949 & Soó Rezső & BPU-09379.JPG \\
\hline 14 & Equisetum ramosissimum Desf. & Nagykanizsa & 1949 & Károlyi Árpád & BPU-09387.JPG \\
\hline 14 & Equisetum ramosissimum Desf. & Pannonhalma & 1925 & Kálovics Rezső & BPU-09365.JPG \\
\hline 14 & Equisetum ramosissimum Desf. & Pannonhalma & 1925 & Kálovics Rezső & BPU-09368.JPG \\
\hline 14 & Equisetum ramosissimum Desf. & Sárszentmihály & 1924 & $\begin{array}{l}\text { Filarszky Nándor, } \\
\text { Kümmerle Jenő Béla }\end{array}$ & BPU-09364.JPG \\
\hline 14 & Equisetum ramosissimum Desf. & Sárszentmihály & 1924 & $\begin{array}{l}\text { Filarszky Nándor, } \\
\text { Kümmerle Jenő Béla }\end{array}$ & BPU-09367.JPG \\
\hline 14 & Equisetum ramosissimum Desf. & Solymár & 1942 & Priszter Szaniszló & BPU-09383.JPG \\
\hline 14 & Equisetum ramosissimum Desf. & Solymár & 1992 & Felföldy Lajos & BPU-09382.JPG \\
\hline 14 & Equisetum ramosissimum Desf. & Szigetcsép & 1992 & Felföldy Lajos & BPU-09384.JPG \\
\hline 15 & Equisetum hyemale L. & Alsószölnök & 1955 & Soó Rezső & BPU-09354.JPG \\
\hline 15 & Equisetum hyemale L. & Ercsi & 1962 & Vida Gábor & BPU-09353.JPG \\
\hline 15 & Equisetum hyemale L. & Kemence & 1952 & Simon Tibor & BPU-09357.JPG \\
\hline 15 & Equisetum hyemale L. & Telkibánya & 1961 & Simon Tibor & BPU-09352.JPG \\
\hline 16 & Equisetum $\times$ moorei Newman & Balatonszemes & 1974 & Tóth László & BPU-09359.JPG \\
\hline 16 & Equisetum $\times$ moorei Newman & Bátorliget & 1932 & Soó Rezső & BPU-09360.JPG \\
\hline 16 & Equisetum $\times$ moorei Newman & Budapest & 1950 & Károlyi Árpád & BPU-09361.JPG \\
\hline 16 & Equisetum $\times$ moorei Newman & Debrecen & 1938 & Soó Rezső & BPU-09355.JPG \\
\hline 16 & Equisetum $\times$ moorei Newman & Göd & 1948 & Soó Rezső & BPU-09356.JPG \\
\hline 16 & Equisetum $\times$ moorei Newman & Szigetújfalu & 1943 & Kárpáti Zoltán & BPU-09358.JPG \\
\hline 17 & Ophioglossum vulgatum L. & Bátorliget & 1951 & Simon Tibor & BPU-00699.JPG \\
\hline 17 & Ophioglossum vulgatum L. & Miskolc & 1950 & Soó Rezső, Simon Tibor & BPU-00694.JPG \\
\hline 17 & Ophioglossum vulgatum L. & Nagyvisnyó & 1952 & Vida Gábor & BPU-00696.JPG \\
\hline 17 & Ophioglossum vulgatum L. & Nagyvisnyó & 1953 & Soó Rezső & BPU-00695.JPG \\
\hline 17 & Ophioglossum vulgatum L. & Piricse & 1932 & Soó Rezső & BPU-00700.JPG \\
\hline 17 & Ophioglossum vulgatum L. & Szentendre & 1947 & Priszter Szaniszló & BPU-00697.JPG \\
\hline 17 & Ophioglossum vulgatum L. & Szigetújfalu & 1943 & Kárpáti Zoltán & BPU-00698.JPG \\
\hline 17 & Ophioglossum vulgatum L. & Tarpa & 1949 & Soó Rezső & BPU-00692.JPG \\
\hline 18 & Botrychium lunaria (L.) Sw. & Miskolc & 1952 & Vida Gábor & BPU-00702.JPG \\
\hline 23 & Notholaena marantae (L.) Desv. & Hegymagas & 1940 & Priszter Szaniszló & BPU-00637.JPG \\
\hline 23 & Notholaena marantae (L.) Desv. & Kisapáti & 1920 & $\begin{array}{l}\text { Timkó György, Zsák } \\
\text { Zoltán, Szatala Ödön }\end{array}$ & BPU-00635.JPG \\
\hline 23 & Notholaena marantae (L.) Desv. & Kisapáti & 1929 & Soó Rezső & BPU-00636.JPG \\
\hline 23 & Notholaena marantae (L.) Desv. & Tapolca & 1941 & Kárpáti Zoltán & BPU-00638.JPG \\
\hline 23 & Notholaena marantae (L.) Desv. & Tapolca & 1949 & Károlyi Árpád & BPU-00639.JPG \\
\hline 23 & Pteris cretica L. & Eger & 1927 & Boros Ádám & BPU-00654.JPG \\
\hline 26 & Polypodium vulgare L. & Baktalórántháza & 1932 & Soó Rezső & BPU-00669.JPG \\
\hline 26 & Polypodium vulgare L. & Budapest & 1913 & Timkó György & BPU-00655.JPG \\
\hline 26 & Polypodium vulgare L. & Budapest & 1942 & Priszter Szaniszló & BPU-00657.JPG \\
\hline
\end{tabular}


1. Elektroniukus melléklet - Electronic Appendix 1.

\begin{tabular}{|c|c|c|c|c|c|}
\hline $\begin{array}{l}\text { Sorszám / } \\
\text { Number }\end{array}$ & Taxon-név / Taxon-name & $\begin{array}{l}\text { Település / } \\
\text { Settlement }\end{array}$ & $\begin{array}{l}\text { Év / } \\
\text { Year }\end{array}$ & Gyújtő / Collector & $\begin{array}{l}\text { Fájlnév / } \\
\text { File-name }\end{array}$ \\
\hline 26 & Polypodium vulgare L. & Dömös & 1948 & Soó Rezső & BPU-00660.JPG \\
\hline 26 & Polypodium vulgare L. & Erdőbénye & 1962 & Simon Tibor & BPU-00664.JPG \\
\hline 26 & Polypodium vulgare L. & Füzér & 1949 & Simon Tibor, Jakucs Pál & BPU-00658.JPG \\
\hline 26 & Polypodium vulgare L. & Gánt & 1949 & Soó Rezső & BPU-00666.JPG \\
\hline 26 & Polypodium vulgare L. & Kemence & 1949 & Pólya László & BPU-00661.JPG \\
\hline 26 & Polypodium vulgare L. & Szilvásvárad & 1949 & Koppányi Tibor & BPU-00659.JPG \\
\hline 26 & Polypodium vulgare L. & Téglás & 1937 & Soó Rezső & BPU-00667.JPG \\
\hline 26 & Polypodium vulgare L. & Tihany & 1958 & Felföldy Lajos & BPU-00663.JPG \\
\hline 26 & Polypodium vulgare L. & Vác & 1941 & Priszter Szaniszló & BPU-00662.JPG \\
\hline 26 & Polypodium vulgare L. & Vác & 1949 & $\begin{array}{l}\text { Soó Rezső, Simon Tibor, } \\
\text { Jakucs Pál }\end{array}$ & BPU-00668.JPG \\
\hline 28 & Pteridium aquilinum (L.) Kuhn & Bátorliget & 1950 & Simon Tibor & BPU-00649.JPG \\
\hline 28 & Pteridium aquilinum (L.) Kuhn & Budapest & 1952 & Vida Gábor & BPU-00642.JPG \\
\hline 28 & Pteridium aquilinum (L.) Kuhn & Budapest & 1952 & Vida Gábor & BPU-00643.JPG \\
\hline 28 & Pteridium aquilinum (L.) Kuhn & Csesznek & 1924 & Polgár Sándor & BPU-00651.JPG \\
\hline 28 & Pteridium aquilinum (L.) Kuhn & Dömös & 1918 & Boros Ádám & BPU-00648.JPG \\
\hline 28 & Pteridium aquilinum (L.) Kuhn & Nyíracsád & 1937 & Soó Rezső & BPU-00650.JPG \\
\hline 28 & Pteridium aquilinum (L.) Kuhn & Pécs & 1948 & Priszter Szaniszló & BPU-00645.JPG \\
\hline 28 & Pteridium aquilinum (L.) Kuhn & Piliscsaba & 1916 & $\begin{array}{l}\text { Filarszky Nándor, } \\
\text { Kümmerle Jenő Béla }\end{array}$ & BPU-00641.JPG \\
\hline 28 & Pteridium aquilinum (L.) Kuhn & Regéc & 1963 & Isépy István & BPU-00644.JPG \\
\hline 28 & Pteridium aquilinum (L.) Kuhn & Szentendre & 1942 & Priszter Szaniszló & BPU-00647.JPG \\
\hline 28 & Pteridium aquilinum (L.) Kuhn & Tarpa & 1949 & Soó Rezső & BPU-00646.JPG \\
\hline 28 & Pteridium aquilinum (L.) Kuhn & Uzsa & 1951 & Borsos Olga & BPU-00652.JPG \\
\hline 29 & Thelypteris palustris Schott & Balatonfüred & 1982 & $\begin{array}{l}\text { Felföldy Lajos, Tóth } \\
\text { László }\end{array}$ & BPU-09563.JPG \\
\hline 29 & Thelypteris palustris Schott & Bátorliget & 1950 & $\begin{array}{l}\text { Simon Tibor, Kárpáti } \\
\text { István }\end{array}$ & BPU-09559.JPG \\
\hline 29 & Thelypteris palustris Schott & Csaroda & 1951 & $\begin{array}{l}\text { Simon Tibor, Vozáry } \\
\text { Elemér }\end{array}$ & BPU-09549.JPG \\
\hline 29 & Thelypteris palustris Schott & Debrecen & 1931 & Soó Rezső & BPU-09554.JPG \\
\hline 29 & Thelypteris palustris Schott & Debrecen & 1953 & Kulcsár Gábor & BPU-15435.JPG \\
\hline 29 & Thelypteris palustris Schott & Debrecen & 1953 & Kulcsár Gábor & BPU-15436.JPG \\
\hline 29 & Thelypteris palustris Schott & Gárdony & 1980 & Felföldy Lajos & BPU-09552.JPG \\
\hline 29 & Thelypteris palustris Schott & Isaszeg & 1949 & Soó Rezső & BPU-09556.JPG \\
\hline 29 & Thelypteris palustris Schott & Kállósemjén & 1950 & Simon Tibor & BPU-09560.JPG \\
\hline 29 & Thelypteris palustris Schott & Kelemér & 1950 & Soó Rezső & BPU-09553.JPG \\
\hline 29 & Thelypteris palustris Schott & Lesenceistvánd & 1928 & Soó Rezső & BPU-09564.JPG \\
\hline 29 & Thelypteris palustris Schott & Nagykanizsa & 1949 & Károlyi Árpád & BPU-09567.JPG \\
\hline 29 & Thelypteris palustris Schott & Nyírábrány & 1987 & Felföldy Lajos & BPU-09555.JPG \\
\hline 29 & Thelypteris palustris Schott & Penészlek & 1932 & Soó Rezső & BPU-09561.JPG \\
\hline 29 & Thelypteris palustris Schott & Sümeg & 1952 & Vida Gábor & BPU-09565.JPG \\
\hline 29 & Thelypteris palustris Schott & Szenta & 1955 & Soó Rezső & BPU-09558.JPG \\
\hline 29 & Thelypteris palustris Schott & Szigetcsép & 1992 & Felföldy Lajos & BPU-09557.JPG \\
\hline 29 & Thelypteris palustris Schott & Tarpa & 1948 & $\begin{array}{l}\text { Pólya László, Simon } \\
\text { Tibor, Jakucs Pál }\end{array}$ & BPU-09550.JPG \\
\hline 29 & Thelypteris palustris Schott & Uzsa & 1952 & Simon Tibor & BPU-09566.JPG \\
\hline 29 & Thelypteris palustris Schott & Vasvár & 1949 & Károlyi Árpád & BPU-09562.JPG \\
\hline 29 & Thelypteris palustris Schott & Zsombó & 1947 & Timár Lajos & BPU-09551.JPG \\
\hline 30 & Oreopteris limbosperma (All.) Holub & Alsószölnök & 1955 & Soó Rezső & BPU-00230.JPG \\
\hline
\end{tabular}


1. Elektroniukus melléklet - Electronic Appendix 1.

\begin{tabular}{|c|c|c|c|c|c|}
\hline $\begin{array}{l}\text { Sorszám / } \\
\text { Number }\end{array}$ & Taxon-név / Taxon-name & $\begin{array}{l}\text { Település / } \\
\text { Settlement }\end{array}$ & $\begin{array}{l}\text { Év / } \\
\text { Year }\end{array}$ & Gyüjtő / Collector & $\begin{array}{l}\text { Fájlnév / } \\
\text { File-name }\end{array}$ \\
\hline 30 & Oreopteris limbosperma (All.) Holub & Szakonyfalu & 1955 & Soó Rezső & BPU-00232.JPG \\
\hline 30 & Oreopteris limbosperma (All.) Holub & Szőce & 1955 & Soó Rezső & BPU-00233.JPG \\
\hline 31 & Phegopteris connectilis (Michx.) Watt & Alsószölnök & 1955 & Soó Rezső & BPU-09517.JPG \\
\hline 31 & Phegopteris connectilis (Michx.) Watt & Erdőbénye & 1948 & Soó Rezső & BPU-09518.JPG \\
\hline 31 & Phegopteris connectilis (Michx.) Watt & Kemence & 1949 & Pólya László & BPU-09516.JPG \\
\hline 31 & Phegopteris connectilis (Michx.) Watt & Regéc & 1963 & Isépy István & BPU-09515.JPG \\
\hline 31 & Phegopteris connectilis (Michx.) Watt & Telkibánya & 1959 & Simon Tibor & BPU-09514.JPG \\
\hline 32 & Asplenium scolopendrium L. & Bódvaszilas & 1950 & Jakucs Pál, Kulcsár Gábor & BPU-15465.JPG \\
\hline 32 & Asplenium scolopendrium L. & Bódvaszilas & 1950 & Jakucs Pál, Kulcsár Gábor & BPU-15466.JPG \\
\hline 32 & Asplenium scolopendrium L. & Dömös & 1948 & Priszter Szaniszló & BPU-00537.JPG \\
\hline 32 & Asplenium scolopendrium L. & Dömös & 1948 & Soó Rezső & BPU-00536.JPG \\
\hline 32 & Asplenium scolopendrium L. & Gánt & 1946 & Boros Ádám & BPU-00538.JPG \\
\hline 32 & Asplenium scolopendrium L. & Gyula & 1935 & Soó Rezső & BPU-00530.JPG \\
\hline 32 & Asplenium scolopendrium L. & Lasztonya & 1948 & Károlyi Árpád & BPU-00540.JPG \\
\hline 32 & Asplenium scolopendrium L. & Miskolc & 1915 & Hulják János & BPU-00533.JPG \\
\hline 32 & Asplenium scolopendrium L. & Miskolc & 1949 & Priszter Szaniszló & BPU-00532.JPG \\
\hline 32 & Asplenium scolopendrium L. & Miskolc & 1949 & Priszter Szaniszló & BPU-00534.JPG \\
\hline 32 & Asplenium scolopendrium L. & Miskolc & 1950 & Priszter Szaniszló & BPU-00535.JPG \\
\hline 32 & Asplenium scolopendrium L. & Orfü & 1940 & Boros Ádám & BPU-00528.JPG \\
\hline 32 & Asplenium scolopendrium L. & Pécs & 1956 & Soó Rezső & BPU-00529.JPG \\
\hline 32 & Asplenium scolopendrium L. & Tibolddaróc & 1953 & Soó Rezső & BPU-00531.JPG \\
\hline 32 & Asplenium scolopendrium L. & Vác & 1942 & Priszter Szaniszló & BPU-00539.JPG \\
\hline 33 & Asplenium ceterach L. & Budapest & 1916 & Boros Ádám & BPU-00608.JPG \\
\hline 33 & Asplenium ceterach L. & Budapest & 1947 & Soó Rezső & BPU-00609.JPG \\
\hline 33 & Asplenium ceterach L. & Csákberény & 1949 & Priszter Szaniszló & BPU-00615.JPG \\
\hline 33 & Asplenium ceterach L. & Csókakő & 1948 & Priszter Szaniszló & BPU-00612.JPG \\
\hline 33 & Asplenium ceterach L. & Kisapáti & 1942 & Priszter Szaniszló & BPU-00613.JPG \\
\hline 33 & Asplenium ceterach L. & Tapolca & 1950 & Papp József & BPU-00614.JPG \\
\hline 33 & Asplenium ceterach L. & Villány & 1941 & Priszter Szaniszló & BPU-00610.JPG \\
\hline 33 & Asplenium ceterach L. & Villány & 1956 & Soó Rezső & BPU-00611.JPG \\
\hline 34 & Asplenium javorkeanum Vida & Csesznek & 1929 & Soó Rezső & BPU-00623.JPG \\
\hline 34 & Asplenium javorkeanum Vida & Csókakő & 1927 & Boros Ádám & BPU-00620.JPG \\
\hline 34 & Asplenium javorkeanum Vida & Mór & 1953 & Felföldy Lajos & BPU-00621.JPG \\
\hline 34 & Asplenium javorkeanum Vida & Nagyharsány & 1948 & Priszter Szaniszló & BPU-00618.JPG \\
\hline 34 & Asplenium javorkeanum Vida & Nagyharsány & 1961 & Simon Tibor & BPU-00619.JPG \\
\hline 34 & Asplenium javorkeanum Vida & Vác & 1949 & Simon Tibor, Jakucs Pál & BPU-00622.JPG \\
\hline 35 & Asplenium septentrionale (L.) Hoffm. & Dömös & 1948 & Soó Rezső & BPU-00544.JPG \\
\hline 35 & Asplenium septentrionale (L.) Hoffm. & Füzér & 1939 & Soó Rezső & BPU-00543.JPG \\
\hline 35 & Asplenium septentrionale (L.) Hoffm. & Nagymaros & 1948 & Priszter Szaniszló & BPU-00547.JPG \\
\hline 35 & Asplenium septentrionale (L.) Hoffm. & Sukoró & 1934 & Boros Ádám & BPU-00545.JPG \\
\hline 35 & Asplenium septentrionale (L.) Hoffm. & Szarvaskő & 1930 & Soó Rezső & BPU-00546.JPG \\
\hline 35 & Asplenium septentrionale (L.) Hoffm. & Tapolca & 1931 & Soó Rezső & BPU-00548.JPG \\
\hline 37 & Asplenium trichomanes L. & Bagamér & 1949 & Soó Rezső & BPU-00568.JPG \\
\hline 37 & Asplenium trichomanes L. & Budapest & 1915 & $\begin{array}{l}\text { Kümmerle Jenő Béla, } \\
\text { Timkó György }\end{array}$ & BPU-00564.JPG \\
\hline 37 & Asplenium trichomanes L. & Budapest & 1947 & Soó Rezső & BPU-00565.JPG \\
\hline 37 & Asplenium trichomanes L. & Csesznek & 1963 & Soó Rezső & BPU-00573.JPG \\
\hline 37 & Asplenium trichomanes L. & Kapolcs & 1959 & Felföldy Lajos & BPU-00572.JPG \\
\hline
\end{tabular}


1. Elektroniukus melléklet - Electronic Appendix 1.

\begin{tabular}{|c|c|c|c|c|c|}
\hline $\begin{array}{l}\text { Sorszám / } \\
\text { Number }\end{array}$ & Taxon-név / Taxon-name & $\begin{array}{l}\text { Település / } \\
\text { Settlement }\end{array}$ & $\begin{array}{l}\text { Év / } \\
\text { Year }\end{array}$ & Gyújtő / Collector & $\begin{array}{l}\text { Fájlnév / } \\
\text { File-name }\end{array}$ \\
\hline 37 & Asplenium trichomanes L. & Miskolc & 1937 & Soó Rezső & BPU-00570.JPG \\
\hline 37 & Asplenium trichomanes L. & Miskolc & 1952 & Borsos Olga & BPU-00571.JPG \\
\hline 37 & Asplenium trichomanes L. & Sátoraljaújhely & 1937 & Soó Rezső & BPU-00576.JPG \\
\hline 37 & Asplenium trichomanes L. & Szarvas & 1947 & Soó Rezső & BPU-00567.JPG \\
\hline 37 & Asplenium trichomanes L. & Tibolddaróc & 1953 & Soó Rezső & BPU-00569.JPG \\
\hline 37 & Asplenium trichomanes L. & Tihany & 1958 & Felföldy Lajos & BPU-00574.JPG \\
\hline 37 & Asplenium trichomanes L. & Vászoly & 1958 & Felföldy Lajos & BPU-00575.JPG \\
\hline 37 & Asplenium trichomanes L. & Villány & 1936 & Soó Rezső & BPU-00566.JPG \\
\hline 38 & Asplenium viride Huds. & Miskolc & 1952 & Vida Gábor & BPU-00551.JPG \\
\hline 38 & Asplenium viride Huds. & Nagykanizsa & 1948 & Károlyi Árpád & BPU-00552.JPG \\
\hline 38 & Asplenium viride Huds. & Nagyvisnyó & 1952 & $\begin{array}{l}\text { Simon Tibor, Vozáry } \\
\text { Elemér }\end{array}$ & BPU-00550.JPG \\
\hline 39 & Asplenium adiantum-nigrum L. & Uzsa & 1953 & $\begin{array}{l}\text { Felföldy Lajos, Tóth } \\
\text { László }\end{array}$ & BPU-00598.JPG \\
\hline 39 & Asplenium adiantum-nigrum L. & Visegrád & 1949 & Soó Rezső & BPU-00596.JPG \\
\hline 40 & Asplenium ruta-muraria L. & Bélapátfalva & 1948 & $\begin{array}{l}\text { Simon Tibor, Pólya } \\
\text { László, Jakucs Pál }\end{array}$ & BPU-00584.JPG \\
\hline 40 & Asplenium ruta-muraria L. & Bódvarákó & 1951 & $\begin{array}{l}\text { Soó Rezső, Jakucs Pál, Ér } \\
\text { Lajos }\end{array}$ & BPU-00580.JPG \\
\hline 40 & Asplenium ruta-muraria L. & Budapest & 1947 & Soó Rezső & BPU-00579.JPG \\
\hline 40 & Asplenium ruta-muraria L. & Csákberény & 1948 & Priszter Szaniszló & BPU-00586.JPG \\
\hline 40 & Asplenium ruta-muraria L. & Csákberény & 1948 & Priszter Szaniszló & BPU-00587.JPG \\
\hline 40 & Asplenium ruta-muraria L. & Csókakő & 1948 & Priszter Szaniszló & BPU-00588.JPG \\
\hline 40 & Asplenium ruta-muraria L. & Isztimér & 1949 & Soó Rezső & BPU-00589.JPG \\
\hline 40 & Asplenium ruta-muraria L. & Jósvafó & 1948 & $\begin{array}{l}\text { Simon Tibor, Pólya } \\
\text { László, Böszörményi } \\
\text { Zoltán }\end{array}$ & BPU-00581.JPG \\
\hline 40 & Asplenium ruta-muraria L. & Kőszeg & 1898 & Waisbecker Antal & BPU-00590.JPG \\
\hline 40 & Asplenium ruta-muraria L. & Nagyharsány & 1965 & $\begin{array}{l}\text { Simon Tibor, Priszter } \\
\text { Szaniszló, Borhidi Attila }\end{array}$ & BPU-00583.JPG \\
\hline 40 & Asplenium ruta-muraria L. & Őriszentpéter & 1955 & Soó Rezső & BPU-00591.JPG \\
\hline 40 & Asplenium ruta-muraria L. & Piliscsaba & 1916 & $\begin{array}{l}\text { Filarszky Nándor, } \\
\text { Kümmerle Jenő Béla }\end{array}$ & BPU-00578.JPG \\
\hline 40 & Asplenium ruta-muraria L. & Telkibánya & 1959 & Simon Tibor & BPU-00582.JPG \\
\hline 40 & Asplenium ruta-muraria L. & Tibolddaróc & 1953 & Soó Rezső & BPU-00585.JPG \\
\hline 40 & Asplenium ruta-muraria L. & Tihany & 1947 & Pólya László & BPU-00593.JPG \\
\hline 40 & Asplenium ruta-muraria L. & Tihany & 1958 & Felföldy Lajos & BPU-00592.JPG \\
\hline 40 & Asplenium ruta-muraria L. & Vác & 1923 & Soó Rezső & BPU-00595.JPG \\
\hline 42 & Athyrium filix-femina (L.) Roth & Bátorliget & 1950 & Simon Tibor & BPU-00369.JPG \\
\hline 42 & Athyrium filix-femina (L.) Roth & Bózsva & 1950 & Simon Tibor & BPU-00359.JPG \\
\hline 42 & Athyrium filix-femina (L.) Roth & Debrecen & 1947 & Soó Rezső & BPU-00367.JPG \\
\hline 42 & Athyrium filix-femina (L.) Roth & Dömös & 1948 & Soó Rezső & BPU-00366.JPG \\
\hline 42 & Athyrium filix-femina (L.) Roth & Erdőbénye & 1952 & Borsos Olga & BPU-00376.JPG \\
\hline 42 & Athyrium filix-femina (L.) Roth & Kemence & 1949 & Pólya László & BPU-00368.JPG \\
\hline 42 & Athyrium filix-femina (L.) Roth & Kishuta & 1963 & Isépy István & BPU-00358.JPG \\
\hline 42 & Athyrium filix-femina (L.) Roth & Kőszeg & 1932 & Soó Rezső & BPU-00373.JPG \\
\hline 42 & Athyrium filix-femina (L.) Roth & Miskolc & 1939 & Soó Rezső & BPU-00365.JPG \\
\hline 42 & Athyrium filix-femina (L.) Roth & Miskolc & 1950 & Soó Rezső & BPU-00364.JPG \\
\hline 42 & Athyrium filix-femina (L.) Roth & Nyíregyháza & 1933 & Soó Rezső & BPU-00370.JPG \\
\hline 42 & Athyrium filix-femina (L.) Roth & Pálháza & 1939 & Soó Rezső & BPU-00360.JPG \\
\hline 42 & Athyrium filix-femina (L.) Roth & Pécs & 1928 & Soó Rezső & BPU-00362.JPG \\
\hline
\end{tabular}


1. Elektroniukus melléklet - Electronic Appendix 1.

\begin{tabular}{|c|c|c|c|c|c|}
\hline $\begin{array}{l}\text { Sorszám / } \\
\text { Number }\end{array}$ & Taxon-név / Taxon-name & $\begin{array}{l}\text { Település / } \\
\text { Settlement }\end{array}$ & $\begin{array}{l}\text { Év / } \\
\text { Year }\end{array}$ & Gyűjtő / Collector & $\begin{array}{l}\text { Fájlnév / } \\
\text { File-name }\end{array}$ \\
\hline 42 & Athyrium filix-femina (L.) Roth & Regéc & 1955 & Simon Tibor & BPU-00361.JPG \\
\hline 42 & Athyrium filix-femina (L.) Roth & Szőce & 1955 & Soó Rezső & BPU-00374.JPG \\
\hline 42 & Athyrium filix-femina (L.) Roth & Tarpa & 1948 & $\begin{array}{l}\text { Pólya László, Simon } \\
\text { Tibor, Jakucs Pál }\end{array}$ & BPU-00363.JPG \\
\hline 42 & Athyrium filix-femina (L.) Roth & Tornyospálca & 1933 & Soó Rezső & BPU-00371.JPG \\
\hline 42 & Athyrium filix-femina (L.) Roth & Uzsa & 1953 & Borsos Olga & BPU-00375.JPG \\
\hline 43 & Cystopteris fragilis (L.) Bernh. & Badacsonytomaj & 1942 & Priszter Szaniszló & BPU-09465.JPG \\
\hline 43 & Cystopteris fragilis (L.) Bernh. & Baktalórántháza & 1934 & Máthé Imre & BPU-09464.JPG \\
\hline 43 & Cystopteris fragilis (L.) Bernh. & Balatonszőlős & 1959 & Felföldy Lajos & BPU-09466.JPG \\
\hline 43 & Cystopteris fragilis (L.) Bernh. & Budapest & 1948 & Kárpáti Zoltán & BPU-09450.JPG \\
\hline 43 & Cystopteris fragilis (L.) Bernh. & Csesznek & 1929 & Soó Rezső & BPU-09467.JPG \\
\hline 43 & Cystopteris fragilis (L.) Bernh. & Füzér & 1937 & Soó Rezső & BPU-09451.JPG \\
\hline 43 & Cystopteris fragilis (L.) Bernh. & Gyöngyös & 1953 & Soó Rezső & BPU-09457.JPG \\
\hline 43 & Cystopteris fragilis (L.) Bernh. & Gyöngyös & 1962 & Soó Rezső, Borsos Olga & BPU-09456.JPG \\
\hline 43 & Cystopteris fragilis (L.) Bernh. & Hajdúhadház & 1932 & Soó Rezső & BPU-09455.JPG \\
\hline 43 & Cystopteris fragilis (L.) Bernh. & Kapolcs & 1959 & $\begin{array}{l}\text { Jávorka Sándor, Felföldy } \\
\text { Lajos, Tóth László }\end{array}$ & BPU-09468.JPG \\
\hline 43 & Cystopteris fragilis (L.) Bernh. & Kemence & 1952 & Simon Tibor & BPU-09459.JPG \\
\hline 43 & Cystopteris fragilis (L.) Bernh. & Köveskál & 1959 & $\begin{array}{l}\text { Jávorka Sándor, Felföldy } \\
\text { Lajos, Tóth László }\end{array}$ & BPU-09469.JPG \\
\hline 43 & Cystopteris fragilis (L.) Bernh. & Miskolc & 1939 & Soó Rezső & BPU-09453.JPG \\
\hline 43 & Cystopteris fragilis (L.) Bernh. & Miskolc & 1950 & Soó Rezső & BPU-09454.JPG \\
\hline 43 & Cystopteris fragilis (L.) Bernh. & Nagycserkesz & 1933 & Soó Rezső & BPU-09463.JPG \\
\hline 43 & Cystopteris fragilis (L.) Bernh. & Pálháza & 1960 & Simon Tibor & BPU-09452.JPG \\
\hline 43 & Cystopteris fragilis (L.) Bernh. & Pilisszentlászló & 1918 & Trautmann Róbert & BPU-09460.JPG \\
\hline 43 & Cystopteris fragilis (L.) Bernh. & Somlóvásárhely & 1953 & $\begin{array}{l}\text { Kulcsár Gábor, Vozáry } \\
\text { Elemér }\end{array}$ & BPU-09470.JPG \\
\hline 43 & Cystopteris fragilis (L.) Bernh. & Szentendre & 1947 & Priszter Szaniszló & BPU-09461.JPG \\
\hline 43 & Cystopteris fragilis (L.) Bernh. & Szilvásvárad & 1953 & $\begin{array}{l}\text { Felföldy Lajos, Tóth } \\
\text { László }\end{array}$ & BPU-09458.JPG \\
\hline 43 & Cystopteris fragilis (L.) Bernh. & Tapolca & 1953 & Borsos Olga & BPU-09471.JPG \\
\hline 43 & Cystopteris fragilis (L.) Bernh. & Vászoly & 1958 & Felföldy Lajos & BPU-09472.JPG \\
\hline 43 & Cystopteris fragilis (L.) Bernh. & Vászoly & 1958 & Felföldy Lajos & BPU-09473.JPG \\
\hline 43 & Cystopteris fragilis (L.) Bernh. & Vászoly & 1958 & Felföldy Lajos & BPU-09474.JPG \\
\hline 43 & Cystopteris fragilis (L.) Bernh. & Visegrád & 1948 & Priszter Szaniszló & BPU-09462.JPG \\
\hline 44 & Woodsia ilvensis (L.) R. Br. & Füzér & 1938 & Soó Rezső & BPU-09445.JPG \\
\hline 44 & Woodsia ilvensis (L.) R. Br. & Füzér & 1947 & Boros Ádám & BPU-09446.JPG \\
\hline 44 & Woodsia ilvensis (L.) R. Br. & Füzér & 1952 & Vida Gábor & BPU-09447.JPG \\
\hline 44 & Woodsia ilvensis (L.) R. Br. & Füzér & 1960 & Simon Tibor & BPU-09448.JPG \\
\hline 44 & Woodsia ilvensis (L.) R. Br. & Gyöngyös & 1953 & Soó Rezső & BPU-09449.JPG \\
\hline 46 & $\begin{array}{l}\text { Gymnocarpium dryopteris (L.) } \\
\text { Newman }\end{array}$ & Füzér & 1938 & Soó Rezső & BPU-09523.JPG \\
\hline 46 & $\begin{array}{l}\text { Gymnocarpium dryopteris (L.) } \\
\text { Newman }\end{array}$ & Gyöngyös & 1953 & Soó Rezső & BPU-09528.JPG \\
\hline 46 & $\begin{array}{l}\text { Gymnocarpium dryopteris (L.) } \\
\text { Newman }\end{array}$ & Kemence & 1949 & Pólya László & BPU-09529.JPG \\
\hline 46 & $\begin{array}{l}\text { Gymnocarpium dryopteris (L.) } \\
\text { Newman }\end{array}$ & Miskolc & 1939 & Soó Rezső & BPU-09526.JPG \\
\hline 46 & $\begin{array}{l}\text { Gymnocarpium dryopteris (L.) } \\
\text { Newman }\end{array}$ & Miskolc & 1950 & Soó Rezső & BPU-09527.JPG \\
\hline 46 & $\begin{array}{l}\text { Gymnocarpium dryopteris (L.) } \\
\text { Newman }\end{array}$ & Pusztamiske & 1948 & Borsos Olga & BPU-09531.JPG \\
\hline
\end{tabular}


1. Elektroniukus melléklet - Electronic Appendix 1.

\begin{tabular}{|c|c|c|c|c|c|}
\hline $\begin{array}{l}\text { Sorszám / } \\
\text { Number }\end{array}$ & Taxon-név / Taxon-name & $\begin{array}{l}\text { Település / } \\
\text { Settlement }\end{array}$ & $\begin{array}{l}\text { Év / } \\
\text { Year }\end{array}$ & Gyújtő / Collector & $\begin{array}{l}\text { Fájlnév / } \\
\text { File-name }\end{array}$ \\
\hline 46 & $\begin{array}{l}\text { Gymnocarpium dryopteris (L.) } \\
\text { Newman }\end{array}$ & Tibolddaróc & 1953 & Soó Rezső & BPU-09525.JPG \\
\hline 46 & $\begin{array}{l}\text { Gymnocarpium dryopteris (L.) } \\
\text { Newman }\end{array}$ & Szőce & 1955 & Soó Rezső & BPU-09530.JPG \\
\hline 46 & $\begin{array}{l}\text { Gymnocarpium dryopteris (L.) } \\
\text { Newman }\end{array}$ & Telkibánya & 1959 & Simon Tibor & BPU-09524.JPG \\
\hline 47 & $\begin{array}{l}\text { Gymnocarpium robertianum (Hoffm.) } \\
\text { Newman }\end{array}$ & Bozsok & 1938 & Soó Rezső & BPU-09542.JPG \\
\hline 47 & $\begin{array}{l}\text { Gymnocarpium robertianum (Hoffm.) } \\
\text { Newman }\end{array}$ & Budapest & 1947 & Kárpáti Zoltán & BPU-09538.JPG \\
\hline 47 & $\begin{array}{l}\text { Gymnocarpium robertianum (Hoffm.) } \\
\text { Newman }\end{array}$ & Csesznek & 1929 & Soó Rezső & BPU-09541.JPG \\
\hline 47 & $\begin{array}{l}\text { Gymnocarpium robertianum (Hoffm.) } \\
\text { Newman }\end{array}$ & Miskolc & 1960 & Simon Tibor & BPU-09539.JPG \\
\hline 47 & $\begin{array}{l}\text { Gymnocarpium robertianum (Hoffm.) } \\
\text { Newman }\end{array}$ & Nagykanizsa & 1948 & Károlyi Árpád & BPU-09543.JPG \\
\hline 47 & $\begin{array}{l}\text { Gymnocarpium robertianum (Hoffm.) } \\
\text { Newman }\end{array}$ & Nagykanizsa & 1949 & Károlyi Árpád & BPU-09544.JPG \\
\hline 47 & $\begin{array}{l}\text { Gymnocarpium robertianum (Hoffm.) } \\
\text { Newman }\end{array}$ & Répáshuta & 1952 & Vida Gábor & BPU-09540.JPG \\
\hline 48 & Matteuccia struthiopteris (L.) Tod. & Pálháza & 1938 & Soó Rezső & BPU-09499.JPG \\
\hline 48 & Matteuccia struthiopteris (L.) Tod. & Pálháza & 1948 & Bánó L. & BPU-09500.JPG \\
\hline 48 & Matteuccia struthiopteris (L.) Tod. & Pálháza & 1952 & Vida Gábor & BPU-09501.JPG \\
\hline 48 & Matteuccia struthiopteris (L.) Tod. & Regéc & 1963 & Isépy István & BPU-09503.JPG \\
\hline 48 & Matteuccia struthiopteris (L.) Tod. & Sátoraljaújhely & 1954 & $\begin{array}{l}\text { Máthé Imre, Jeanplon } \\
\text { József, Kovács Margit }\end{array}$ & BPU-09502.JPG \\
\hline 50 & $\begin{array}{l}\text { Polystichum setiferum (Forssk.) } \\
\text { Woyn. }\end{array}$ & Mánfa & 1946 & Boros Ádám & BPU-00349.JPG \\
\hline 50 & $\begin{array}{l}\text { Polystichum setiferum (Forssk.) } \\
\text { Woyn. }\end{array}$ & Orfü & 1940 & Boros Ádám & BPU-00350.JPG \\
\hline 50 & $\begin{array}{l}\text { Polystichum setiferum (Forssk.) } \\
\text { Woyn. }\end{array}$ & Zákány & 1948 & Károlyi Árpád & BPU-00351.JPG \\
\hline 51 & Polystichum aculeatum (L.) Roth & Bakonybél & 1955 & Soó Rezső & BPU-00338.JPG \\
\hline 51 & Polystichum aculeatum (L.) Roth & Bátorliget & 1947 & Simon Tibor & BPU-00335.JPG \\
\hline 51 & Polystichum aculeatum (L.) Roth & Dömös & 1948 & Priszter Szaniszló & BPU-00332.JPG \\
\hline 51 & Polystichum aculeatum (L.) Roth & Dömös & 1948 & Soó Rezső & BPU-00331.JPG \\
\hline 51 & Polystichum aculeatum (L.) Roth & Kőszeg & 1933 & Visnya Aladár & BPU-00336.JPG \\
\hline 51 & Polystichum aculeatum (L.) Roth & Miskolc & 1950 & Soó Rezső & BPU-00330.JPG \\
\hline 51 & Polystichum aculeatum (L.) Roth & Pécs & 1936 & Soó Rezső & BPU-00329.JPG \\
\hline 51 & Polystichum aculeatum (L.) Roth & Sand & 1950 & Károlyi Árpád & BPU-00328.JPG \\
\hline 51 & Polystichum aculeatum (L.) Roth & Sátoraljaújhely & 1952 & $\begin{array}{l}\text { Kulcsár Gábor, Vozáry } \\
\text { Elemér }\end{array}$ & BPU-15547.JPG \\
\hline 51 & Polystichum aculeatum (L.) Roth & Szenna & 1957 & Soó Rezső & BPU-00334.JPG \\
\hline 51 & Polystichum aculeatum (L.) Roth & Szőce & 1955 & Soó Rezső & BPU-00337.JPG \\
\hline 51 & Polystichum aculeatum (L.) Roth & Tatabánya & 1949 & Priszter Szaniszló & BPU-00333.JPG \\
\hline 51 & Polystichum aculeatum (L.) Roth & Tormafölde & 1949 & Papp József & BPU-00339.JPG \\
\hline 52 & Polystichum braunii (Spenn.) Fée & Nagyhuta & 1952 & Vida Gábor & BPU-00318.JPG \\
\hline 52 & Polystichum braunii (Spenn.) Fée & Parád & 1931 & Boros Ádám & BPU-00319.JPG \\
\hline 53 & Dryopteris cristata (L.) A. Gray & Kelemér & 1928 & Zólyomi Bálint & BPU-00257.JPG \\
\hline 53 & Dryopteris cristata (L.) A. Gray & Kelemér & 1950 & Soó Rezső & BPU-00258.JPG \\
\hline 53 & Dryopteris cristata (L.) A. Gray & Uzsa & 1952 & Vida Gábor & BPU-00259.JPG \\
\hline 53 & Dryopteris cristata (L.) A. Gray & Uzsa & 1954 & Soó Rezső & BPU-00260.JPG \\
\hline 53 & Dryopteris cristata (L.) A. Gray & Uzsa & 1959 & Felföldy Lajos & BPU-00261.JPG \\
\hline 54 & Dryopteris affinis (Lowe) Fraser-Jenk. & Pálháza & 1952 & Simon Tibor & BPU-00255.JPG \\
\hline
\end{tabular}


1. Elektroniukus melléklet - Electronic Appendix 1.

\begin{tabular}{|c|c|c|c|c|c|}
\hline $\begin{array}{l}\text { Sorszám / } \\
\text { Number }\end{array}$ & Taxon-név / Taxon-name & $\begin{array}{l}\text { Település / } \\
\text { Settlement }\end{array}$ & $\begin{array}{l}\text { Év / } \\
\text { Year }\end{array}$ & Gyújtő / Collector & $\begin{array}{l}\text { Fájlnév / } \\
\text { File-name }\end{array}$ \\
\hline 55 & Dryopteris filix-mas (L.) Schott & Alsószölnök & 1955 & Vajda László & BPU-00248.JPG \\
\hline 55 & Dryopteris filix-mas (L.) Schott & Baktalórántháza & 1932 & Soó Rezső & BPU-00246.JPG \\
\hline 55 & Dryopteris filix-mas (L.) Schott & Bátorliget & 1950 & Simon Tibor & BPU-00245.JPG \\
\hline 55 & Dryopteris filix-mas (L.) Schott & Budapest & 1917 & Trautmann Róbert & BPU-00234.JPG \\
\hline 55 & Dryopteris filix-mas (L.) Schott & Csesznek & 1953 & $\begin{array}{l}\text { Kulcsár Gábor, Vozáry } \\
\text { Elemér }\end{array}$ & BPU-00251.JPG \\
\hline 55 & Dryopteris filix-mas (L.) Schott & Csesznek & 1953 & $\begin{array}{l}\text { Kulcsár Gábor, Vozáry } \\
\text { Elemér }\end{array}$ & BPU-00252.JPG \\
\hline 55 & Dryopteris filix-mas (L.) Schott & Gyöngyös & 1935 & Soó Rezső & BPU-00241.JPG \\
\hline 55 & Dryopteris filix-mas (L.) Schott & Hajdúhadház & 1932 & Soó Rezső & BPU-00240.JPG \\
\hline 55 & Dryopteris filix-mas (L.) Schott & Kőszeg & 1932 & Soó Rezső & BPU-00250.JPG \\
\hline 55 & Dryopteris filix-mas (L.) Schott & Miskolc & 1929 & Soó Rezső & BPU-00239.JPG \\
\hline 55 & Dryopteris filix-mas (L.) Schott & Nagybajom & 1957 & Soó Rezső & BPU-00242.JPG \\
\hline 55 & Dryopteris filix-mas (L.) Schott & Pálháza & 1952 & Simon Tibor & BPU-00235.JPG \\
\hline 55 & Dryopteris filix-mas (L.) Schott & Pálháza & 1952 & Simon Tibor & BPU-00236.JPG \\
\hline 55 & Dryopteris filix-mas (L.) Schott & Pálháza & 1952 & Simon Tibor & BPU-00237.JPG \\
\hline 55 & Dryopteris filix-mas (L.) Schott & Pécs & 1953 & Soó Rezső, Borsos Olga & BPU-00238.JPG \\
\hline 55 & Dryopteris filix-mas (L.) Schott & Sopron & 1948 & Priszter Szaniszló & BPU-00244.JPG \\
\hline 55 & Dryopteris filix-mas (L.) Schott & Szenna & 1957 & Soó Rezső & BPU-00243.JPG \\
\hline 55 & Dryopteris filix-mas (L.) Schott & Tornyospálca & 1933 & Soó Rezső & BPU-00247.JPG \\
\hline 56 & $\begin{array}{l}\text { Dryopteris carthusiana (Vill.) H.P. } \\
\text { Fuchs }\end{array}$ & Baktalórántháza & 1933 & Soó Rezső & BPU-00277.JPG \\
\hline 56 & $\begin{array}{l}\text { Dryopteris carthusiana (Vill.) H.P. } \\
\text { Fuchs }\end{array}$ & Dabas & 1964 & Soó Rezső & BPU-00271.JPG \\
\hline 56 & $\begin{array}{l}\text { Dryopteris carthusiana (Vill.) H.P. } \\
\text { Fuchs }\end{array}$ & Fenyőfő & 1955 & Soó Rezső & BPU-00279.JPG \\
\hline 56 & $\begin{array}{l}\text { Dryopteris carthusiana (Vill.) H.P. } \\
\text { Fuchs }\end{array}$ & Gelénes & 1951 & $\begin{array}{l}\text { Simon Tibor, Vozáry } \\
\text { Elemér }\end{array}$ & BPU-00267.JPG \\
\hline 56 & $\begin{array}{l}\text { Dryopteris carthusiana (Vill.) H.P. } \\
\text { Fuchs }\end{array}$ & Ócsa & 1955 & Soó Rezső & BPU-00270.JPG \\
\hline 56 & $\begin{array}{l}\text { Dryopteris carthusiana (Vill.) H.P. } \\
\text { Fuchs }\end{array}$ & Pálháza & 1938 & Soó Rezső & BPU-00264.JPG \\
\hline 56 & $\begin{array}{l}\text { Dryopteris carthusiana (Vill.) H.P. } \\
\text { Fuchs }\end{array}$ & Pécs & 1956 & Soó Rezső & BPU-00266.JPG \\
\hline 56 & $\begin{array}{l}\text { Dryopteris carthusiana (Vill.) H.P. } \\
\text { Fuchs }\end{array}$ & Porva & 1929 & Soó Rezső & BPU-00280.JPG \\
\hline 56 & $\begin{array}{l}\text { Dryopteris carthusiana (Vill.) H.P. } \\
\text { Fuchs }\end{array}$ & Somogyszob & 1957 & Soó Rezső & BPU-00274.JPG \\
\hline 56 & $\begin{array}{l}\text { Dryopteris carthusiana (Vill.) H.P. } \\
\text { Fuchs }\end{array}$ & Sopron & 1951 & Csapody István & BPU-00275.JPG \\
\hline 56 & $\begin{array}{l}\text { Dryopteris carthusiana (Vill.) H.P. } \\
\text { Fuchs }\end{array}$ & Sopron & 1960 & Borsos Olga, Simon Tibor & BPU-00276.JPG \\
\hline 56 & $\begin{array}{l}\text { Dryopteris carthusiana (Vill.) H.P. } \\
\text { Fuchs }\end{array}$ & Sümeg & 1952 & Vida Gábor & BPU-00281.JPG \\
\hline 56 & $\begin{array}{l}\text { Dryopteris carthusiana (Vill.) H.P. } \\
\text { Fuchs }\end{array}$ & Szenta & 1955 & Soó Rezső & BPU-00273.JPG \\
\hline 56 & $\begin{array}{l}\text { Dryopteris carthusiana (Vill.) H.P. } \\
\text { Fuchs }\end{array}$ & Szentendre & 1945 & Kárpáti Zoltán & BPU-00272.JPG \\
\hline 56 & $\begin{array}{l}\text { Dryopteris carthusiana (Vill.) H.P. } \\
\text { Fuchs }\end{array}$ & Szőce & 1955 & Soó Rezső & BPU-00278.JPG \\
\hline 56 & $\begin{array}{l}\text { Dryopteris carthusiana (Vill.) H.P. } \\
\text { Fuchs }\end{array}$ & Szögliget & 1951 & $\begin{array}{l}\text { Soó Rezső, Jakucs Pál, Ér } \\
\text { Lajos }\end{array}$ & BPU-00265.JPG \\
\hline 56 & $\begin{array}{l}\text { Dryopteris carthusiana (Vill.) H.P. } \\
\text { Fuchs }\end{array}$ & Téglás & 1937 & Soó Rezső & BPU-00269.JPG \\
\hline 56 & $\begin{array}{l}\text { Dryopteris carthusiana (Vill.) H.P. } \\
\text { Fuchs }\end{array}$ & Tiszakerecseny & 1987 & Felföldy Lajos & BPU-00268.JPG \\
\hline
\end{tabular}


1. Elektroniukus melléklet - Electronic Appendix 1.

\begin{tabular}{|c|c|c|c|c|c|}
\hline $\begin{array}{l}\text { Sorszám / } \\
\text { Number }\end{array}$ & Taxon-név / Taxon-name & $\begin{array}{l}\text { Település / } \\
\text { Settlement }\end{array}$ & $\begin{array}{l}\text { Év / } \\
\text { Year }\end{array}$ & Gyüjtő / Collector & $\begin{array}{l}\text { Fájlnév / } \\
\text { File-name }\end{array}$ \\
\hline 56 & $\begin{array}{l}\text { Dryopteris carthusiana (Vill.) H.P. } \\
\text { Fuchs }\end{array}$ & Uzsa & 1952 & Simon Tibor & BPU-00283.JPG \\
\hline 56 & $\begin{array}{l}\text { Dryopteris carthusiana (Vill.) H.P. } \\
\text { Fuchs }\end{array}$ & Uzsa & 1953 & Borsos Olga & BPU-00282.JPG \\
\hline 56 & $\begin{array}{l}\text { Dryopteris carthusiana (Vill.) H.P. } \\
\text { Fuchs }\end{array}$ & Uzsa & 1959 & $\begin{array}{l}\text { Jávorka Sándor, Felföldy } \\
\text { Lajos }\end{array}$ & BPU-00284.JPG \\
\hline 56 & $\begin{array}{l}\text { Dryopteris carthusiana (Vill.) H.P. } \\
\text { Fuchs }\end{array}$ & Bódvaszilas & 1951 & $\begin{array}{l}\text { Jakucs Pál, Kulcsár Gábor, } \\
\text { Ér Lajos }\end{array}$ & BPU-15479.JPG \\
\hline 57 & Dryopteris dilatata (Hoffm.) A. Gray & Fenyőfő & 1955 & Soó Rezső & BPU-00291.JPG \\
\hline 57 & Dryopteris dilatata (Hoffm.) A. Gray & Kám & 1956 & Jeanplong József & BPU-00290.JPG \\
\hline 57 & Dryopteris dilatata (Hoffm.) A. Gray & Lébény & 1932 & Zólyomi Bálint & BPU-00287.JPG \\
\hline 57 & Dryopteris dilatata (Hoffm.) A. Gray & Ócsa & 1955 & Soó Rezső & BPU-00288.JPG \\
\hline 57 & Dryopteris dilatata (Hoffm.) A. Gray & Sopron & 1942 & Priszter Szaniszló & BPU-00289.JPG \\
\hline 58 & $\begin{array}{l}\text { Dryopteris expansa (C. Presl) Fraser- } \\
\text { Jenk. }\end{array}$ & Bozsok & 1932 & Soó Rezső & BPU-00300.JPG \\
\hline 58 & $\begin{array}{l}\text { Dryopteris expansa (C. Presl) Fraser- } \\
\text { Jenk. }\end{array}$ & Hollóháza & 2007 & Simon Tibor & BPU-15507.JPG \\
\hline 58 & $\begin{array}{l}\text { Dryopteris expansa (C. Presl) Fraser- } \\
\text { Jenk. }\end{array}$ & Nagykanizsa & 1949 & Károlyi Árpád & BPU-00302.JPG \\
\hline 58 & $\begin{array}{l}\text { Dryopteris expansa (C. Presl) Fraser- } \\
\text { Jenk. }\end{array}$ & Oroszlány & 1937 & Boros Ádám & BPU-00299.JPG \\
\hline 58 & $\begin{array}{l}\text { Dryopteris expansa (C. Presl) Fraser- } \\
\text { Jenk. }\end{array}$ & Szepetnek & 1948 & Károlyi Árpád & BPU-00301.JPG \\
\hline $54 \times 55$ & Dryopteris $\times$ tavelii Rothm. & Somogyszob & 1957 & Soó Rezső & BPU-00307.JPG \\
\hline $54 \times 55$ & Dryopteris $\times$ tavelii Rothm. & Szőce & 1955 & Soó Rezső & BPU-00308.JPG \\
\hline $54 \times 55$ & Dryopteris $\times$ tavelii Rothm & Velem & 1932 & Soó Rezső & BPU-00309.JPG \\
\hline 59 & Blechnum spicant (L.) Roth & Alsószölnök & 1955 & Soó Rezső & BPU-00626.JPG \\
\hline 59 & Blechnum spicant (L.) Roth & Alsószölnök & 1955 & Vajda László & BPU-00627.JPG \\
\hline 60 & Marsilea quadrifolia L. & Hortobágy & 1947 & Simon Tibor & BPU-00675.JPG \\
\hline 60 & Marsilea quadrifolia L. & Hortobágy & 1947 & Soó Rezső & BPU-00673.JPG \\
\hline 60 & Marsilea quadrifolia L. & Hortobágy & 1947 & Soó Rezső & BPU-00674.JPG \\
\hline 60 & Marsilea quadrifolia L. & Sárospatak & 1939 & Soó Rezső & BPU-00678.JPG \\
\hline 60 & Marsilea quadrifolia L. & Szarvas & 1947 & Ubrizsy Gábor & BPU-00672.JPG \\
\hline 61 & Salvinia natans (L.) All. & Barbacs & 1946 & Boros Ádám & BPU-00687.JPG \\
\hline 61 & Salvinia natans (L.) All. & Csaroda & 1951 & $\begin{array}{l}\text { Simon Tibor, Vozáry } \\
\text { Elemér }\end{array}$ & BPU-00689.JPG \\
\hline 61 & Salvinia natans (L.) All. & Demecser & 1912 & $\begin{array}{l}\text { Kümmerle Jenő Béla, } \\
\text { Timkó György }\end{array}$ & BPU-00681.JPG \\
\hline 61 & Salvinia natans (L.) All. & Egyek & 1942 & Felföldy Lajos & BPU-00690.JPG \\
\hline 61 & Salvinia natans (L.) All. & Gelénes & 1951 & $\begin{array}{l}\text { Simon Tibor, Vozáry } \\
\text { Elemér }\end{array}$ & BPU-00682.JPG \\
\hline 61 & Salvinia natans (L.) All. & Göd & 1947 & Kárpáti Zoltán & BPU-00691.JPG \\
\hline 61 & Salvinia natans (L.) All. & Göd & 1948 & Soó Rezső & BPU-00685.JPG \\
\hline 61 & Salvinia natans (L.) All. & Kunmadaras & 1991 & Csányi Béla & BPU-00691.JPG \\
\hline 61 & Salvinia natans (L.) All. & Sárospatak & 1939 & Soó Rezső & BPU-00690.JPG \\
\hline 61 & Salvinia natans (L.) All. & Sükösd & 1914 & Greinich Ferenc & BPU-00681.JPG \\
\hline 61 & Salvinia natans (L.) All. & Sükösd & 1914 & Greinich Ferenc & BPU-00682.JPG \\
\hline 61 & Salvinia natans (L.) All. & Szamosszeg & 1951 & Simon Tibor & BPU-00689.JPG \\
\hline 61 & Salvinia natans (L.) All. & Szarvas & 1947 & Soó Rezső & BPU-00683.JPG \\
\hline 61 & Salvinia natans (L.) All. & Szeged & 1942 & Timár Lajos & BPU-00684.JPG \\
\hline 61 & Salvinia natans (L.) All. & Szenta & 1957 & Soó Rezső & BPU-00686.JPG \\
\hline 61 & Salvinia natans (L.) All. & Tiszafüred & 1953 & Borsos Olga & BPU-00689.JPG \\
\hline
\end{tabular}


1. Elektroniukus melléklet - Electronic Appendix 1.

\begin{tabular}{|c|c|c|c|c|c|}
\hline $\begin{array}{l}\text { Sorszám / } \\
\text { Number }\end{array}$ & Taxon-név / Taxon-name & $\begin{array}{l}\text { Település / } \\
\text { Settlement }\end{array}$ & $\begin{array}{l}\text { Év / } \\
\text { Year }\end{array}$ & Gyưjtő / Collector & $\begin{array}{l}\text { Fájlnév / } \\
\text { File-name }\end{array}$ \\
\hline 61 & Salvinia natans (L.) All. & Tiszafüred & 1963 & Isépy István & BPU-00688.JPG \\
\hline 61 & Salvinia natans (L.) All. & Zalkod & 1993 & Felföldy Lajos & BPU-00691.JPG \\
\hline-- & Adiantum capillus-veneris L. & Nagykanizsa & 1948 & Károlyi Árpád & BPU-00640.JPG \\
\hline 69 & Abies alba Mill. & Szigliget & 1962 & Soó Rezső & BPU-09416.JPG \\
\hline 74 & Larix decidua Mill. & Szakonyfalu & 1955 & Soó Rezső & BPU-09415.JPG \\
\hline 80 & Pinus sylvestris L. & Fenyőfő & 1955 & Soó Rezső & BPU-09403.JPG \\
\hline 80 & Pinus sylvestris L. & Kőszeg & 1941 & Priszter Szaniszló & BPU-09402.JPG \\
\hline 80 & Pinus sylvestris L. & Sopron & 1942 & Priszter Szaniszló & BPU-09399.JPG \\
\hline 80 & Pinus sylvestris L. & Sopron & 1947 & Priszter Szaniszló & BPU-09400.JPG \\
\hline 80 & Pinus sylvestris L. & Szentgotthárd & 1955 & Soó Rezső & BPU-09401.JPG \\
\hline 86 & Thuja orientalis L. & Budapest & 1947 & Priszter Szaniszló & BPU-09418.JPG \\
\hline 86 & Thuja orientalis L. & Miskolc & 1949 & Priszter Szaniszló & BPU-09419.JPG \\
\hline 89 & Juniperus communis L. & Bugac & 1952 & Vozáry Elemér & BPU-09421.JPG \\
\hline 89 & Juniperus communis L. & Nagyvisnyó & 1953 & Soó Rezső & BPU-09420.JPG \\
\hline 89 & Juniperus communis L. & Pomáz & 1948 & Priszter Szaniszló & BPU-09422.JPG \\
\hline 89 & Juniperus communis L. & Tatárszentgyörgy & 1950 & Soó Rezső & BPU-09423.JPG \\
\hline 89 & Juniperus communis L. & Tihany & 1928 & Soó Rezső & BPU-09424.JPG \\
\hline 92 & Taxus baccata L. & Szentgál & 1927 & Jávorka Sándor & BPU-09392.JPG \\
\hline 93 & Ephedra distachya L. & Budapest & 1942 & Priszter Szaniszló & BPU-09435.JPG \\
\hline 93 & Ephedra distachya L. & Budapest & 1947 & Soó Rezső & BPU-09436.JPG \\
\hline 93 & Ephedra distachya L. & Dabas & 1951 & Borsos Olga, Simon Tibor & BPU-09437.JPG \\
\hline 93 & Ephedra distachya L. & Dabas & 1959 & Simon Tibor & BPU-09438.JPG \\
\hline 93 & Ephedra distachya L. & Dunaharaszti & 1948 & Priszter Szaniszló & BPU-09440.JPG \\
\hline 93 & Ephedra distachya L. & Dunakeszi & 1940 & Kárpáti Zoltán & BPU-09439.JPG \\
\hline 93 & Ephedra distachya L. & Kunpeszér & 1947 & Papp József & BPU-09441.JPG \\
\hline 93 & Ephedra distachya L. & Szigetmonostor & 1947 & Papp József & BPU-09442.JPG \\
\hline 93 & Ephedra distachya L. & Szigetmonostor & 1947 & Priszter Szaniszló & BPU-09443.JPG \\
\hline 94 & Salix alba L. & Balatonszőlős & 1957 & $\begin{array}{l}\text { Felföldy Lajos, Kovács } \\
\text { Margit, Tóth László }\end{array}$ & BPU-09716.JPG \\
\hline 94 & Salix alba L. & Budapest & 1982 & Felföldy Lajos & BPU-09712.JPG \\
\hline 94 & Salix alba L. & Debrecen & 1947 & Soó Rezső & BPU-09714.JPG \\
\hline 94 & Salix alba L. & Gánt & 1949 & Soó Rezső & BPU-09713.JPG \\
\hline 94 & Salix alba L. & Hidegkút & 1957 & Felföldy Lajos & BPU-09717.JPG \\
\hline 94 & Salix alba L. & Tiszafüred & 1952 & Soó Rezső & BPU-09715.JPG \\
\hline 95 & Salix fragilis L. & Baktalórántháza & 1934 & Máthé Imre & BPU-09729.JPG \\
\hline 95 & Salix fragilis L. & Balatonakali & 1958 & Felföldy Lajos & BPU-09727.JPG \\
\hline 95 & Salix fragilis L. & Balatonszőlős & 1957 & $\begin{array}{l}\text { Felföldy Lajos, Kovács } \\
\text { Margit, Tóth László }\end{array}$ & BPU-09728.JPG \\
\hline 95 & Salix fragilis L. & Balatonszőlős & 1959 & Felföldy Lajos & BPU-09725.JPG \\
\hline 95 & Salix fragilis $\mathrm{L}$. & Debrecen & 1934 & Soó Rezső & BPU-09731.JPG \\
\hline 96 & Salix triandra L. & Aszófő & 1959 & Felföldy Lajos & BPU-09734.JPG \\
\hline 96 & Salix triandra L. & Budapest & 1982 & Felföldy Lajos & BPU-09732.JPG \\
\hline 96 & Salix triandra L. & Gyenesdiás & 1960 & Priszter Szaniszló & BPU-09735.JPG \\
\hline 96 & Salix triandra L. & Szolnok & 1937 & Soó Rezső & BPU-09733.JPG \\
\hline 97 & Salix pentandra L. & Bátorliget & 1932 & Soó Rezső & BPU-09720.JPG \\
\hline 97 & Salix pentandra $\mathrm{L}$. & Bátorliget & 1950 & $\begin{array}{l}\text { Simon Tibor, Kárpáti } \\
\text { István }\end{array}$ & BPU-09721.JPG \\
\hline 97 & Salix pentandra L. & Csaroda & 1952 & Soó Rezső & BPU-09718.JPG \\
\hline 97 & Salix pentandra L. & Csaroda & 1963 & Isépy István & BPU-09719.JPG \\
\hline
\end{tabular}


1. Elektroniukus melléklet - Electronic Appendix 1.

\begin{tabular}{|c|c|c|c|c|c|}
\hline $\begin{array}{l}\text { Sorszám / } \\
\text { Number }\end{array}$ & Taxon-név / Taxon-name & $\begin{array}{l}\text { Település / } \\
\text { Settlement }\end{array}$ & $\begin{array}{l}\text { Év / } \\
\text { Year }\end{array}$ & Gyüjtő / Collector & $\begin{array}{l}\text { Fájlnév / } \\
\text { File-name }\end{array}$ \\
\hline 98 & Salix rosmarinifolia L. & Balatonszőlős & 1956 & $\begin{array}{l}\text { Felföldy Lajos, Tóth } \\
\text { László }\end{array}$ & BPU-09751.JPG \\
\hline 98 & Salix rosmarinifolia $\mathrm{L}$. & Beregdaróc & 1951 & $\begin{array}{l}\text { Simon Tibor, Vozáry } \\
\text { Elemér }\end{array}$ & BPU-09747.JPG \\
\hline 98 & Salix rosmarinifolia L. & Budapest & 1923 & Soó Rezső & BPU-09746.JPG \\
\hline 98 & Salix rosmarinifolia L. & Csaroda & 1952 & Soó Rezső, Simon Tibor & BPU-09748.JPG \\
\hline 98 & Salix rosmarinifolia L. & Debrecen & 1934 & Soó Rezső & BPU-09750.JPG \\
\hline 98 & Salix rosmarinifolia L. & Miskolc & 1950 & Soó Rezső & BPU-09749.JPG \\
\hline 99 & Salix purpurea L. & Aszófő & 1959 & Felföldy Lajos & BPU-09738.JPG \\
\hline 99 & Salix purpurea L. & Balatonszepezd & 1958 & Felföldy Lajos & BPU-09740.JPG \\
\hline 99 & Salix purpurea L. & Balatonszőlős & 1957 & $\begin{array}{l}\text { Felföldy Lajos, Kovács } \\
\text { Margit, Tóth László }\end{array}$ & BPU-09741.JPG \\
\hline 99 & Salix purpurea L. & Budapest & 1991 & Felföldy Lajos & BPU-09737.JPG \\
\hline 99 & Salix purpurea L. & Tarpa & 1949 & Soó Rezső & BPU-09739.JPG \\
\hline 100 & Salix elaeagnos Scop. & Ásványráró & 1931 & Soó Rezső & BPU-09744.JPG \\
\hline 101 & Salix viminalis L. & Abádszalók & 1989 & $\begin{array}{l}\text { Felföldy Lajos, Hamar } \\
\text { József }\end{array}$ & BPU-09754.JPG \\
\hline 101 & Salix viminalis L. & Balatonszőlős & 1957 & $\begin{array}{l}\text { Felföldy Lajos, Kovács } \\
\text { Margit, Tóth László }\end{array}$ & BPU-09755.JPG \\
\hline 101 & Salix viminalis L. & Csesznek & 1929 & Soó Rezső & BPU-09756.JPG \\
\hline 101 & Salix viminalis L. & Tarpa & 1949 & Soó Rezső & BPU-09753.JPG \\
\hline 102 & Salix caprea L. & Balatonszőlős & 1959 & Felföldy Lajos & BPU-13446.JPG \\
\hline 102 & Salix caprea L. & Balatonszőlős & 1959 & Felföldy Lajos & BPU-13447.JPG \\
\hline 102 & Salix caprea L. & Bódvaszilas & 1951 & $\begin{array}{l}\text { Jakucs Pál, Kulcsár Gábor, } \\
\text { Ér Lajos }\end{array}$ & BPU-13451.JPG \\
\hline 102 & Salix caprea L. & Budapest & 2004 & Felföldy Lajos & BPU-11122.JPG \\
\hline 102 & Salix caprea L. & Budapest & 2004 & Felföldy Lajos & BPU-13452.JPG \\
\hline 102 & Salix caprea L. & Csesznek & 1929 & Soó Rezső & BPU-13445.JPG \\
\hline 102 & Salix caprea L. & Gyöngyös & 1964 & Soó Rezső & BPU-13448.JPG \\
\hline 102 & Salix caprea L. & Hidegkút & 1957 & Felföldy Lajos & BPU-13444.JPG \\
\hline 102 & Salix caprea L. & Miskolc & 1937 & Soó Rezső & BPU-13449.JPG \\
\hline 102 & Salix caprea L. & Miskolc & 1953 & Soó Rezső & BPU-13450.JPG \\
\hline 104 & Salix cinerea L. & Aszófő & 1959 & Felföldy Lajos & BPU-09771.JPG \\
\hline 104 & Salix cinerea L. & Balatonszepezd & 1958 & Felföldy Lajos & BPU-09769.JPG \\
\hline 104 & Salix cinerea L. & Balatonszepezd & 1958 & Felföldy Lajos & BPU-09770.JPG \\
\hline 104 & Salix cinerea L. & Balatonszőlős & 1957 & $\begin{array}{l}\text { Felföldy Lajos, Kovács } \\
\text { Margit, Tóth László }\end{array}$ & BPU-09768.JPG \\
\hline 104 & Salix cinerea L. & Bátorliget & 1934 & Soó Rezső & BPU-09767.JPG \\
\hline 104 & Salix cinerea L. & Bicske & 1991 & Felföldy Lajos & BPU-09760.JPG \\
\hline 104 & Salix cinerea L. & Budapest & 1982 & Felföldy Lajos & BPU-09757.JPG \\
\hline 104 & Salix cinerea L. & Csaroda & 1952 & $\begin{array}{l}\text { Simon Tibor, Vozáry } \\
\text { Elemér }\end{array}$ & BPU-13413.JPG \\
\hline 104 & Salix cinerea L. & Csaroda & 1952 & Soó Rezső & BPU-09759.JPG \\
\hline 104 & Salix cinerea L. & Csaroda & 1952 & Soó Rezső, Simon Tibor & BPU-09758.JPG \\
\hline 104 & Salix cinerea L. & Debrecen & 1932 & Soó Rezső & BPU-09766.JPG \\
\hline 104 & Salix cinerea L. & Kelemér & 1950 & Soó Rezső & BPU-09765.JPG \\
\hline 104 & Salix cinerea L. & Olaszliszka & 1993 & Felföldy Lajos & BPU-09772.JPG \\
\hline 104 & Salix cinerea L. & Pákozd & 1980 & Felföldy Lajos & BPU-09761.JPG \\
\hline 104 & Salix cinerea L. & Pákozd & 1980 & Felföldy Lajos & BPU-09762.JPG \\
\hline 104 & Salix cinerea L. & Pákozd & 1980 & Felföldy Lajos & BPU-09763.JPG \\
\hline 104 & Salix cinerea L. & Pákozd & 1980 & Felföldy Lajos & BPU-13420.JPG \\
\hline
\end{tabular}


1. Elektroniukus melléklet - Electronic Appendix 1.

\begin{tabular}{|c|c|c|c|c|c|}
\hline $\begin{array}{l}\text { Sorszám / } \\
\text { Number }\end{array}$ & Taxon-név / Taxon-name & $\begin{array}{l}\text { Település / } \\
\text { Settlement }\end{array}$ & $\begin{array}{l}\text { Év / } \\
\text { Year }\end{array}$ & Gyújtő / Collector & $\begin{array}{l}\text { Fájlnév / } \\
\text { File-name }\end{array}$ \\
\hline 104 & Salix cinerea L. & Zámoly & 1982 & Felföldy Lajos & BPU-09764.JPG \\
\hline 105 & Salix aurita L. & Baktalórántháza & 1932 & Soó Rezső & BPU-09776.JPG \\
\hline 105 & Salix aurita L. & Csaroda & 1963 & Isépy István & BPU-09774.JPG \\
\hline 105 & Salix aurita L. & Csaroda & - & $\begin{array}{l}\text { Simon Tibor, Vozáry } \\
\text { Elemér }\end{array}$ & BPU-09773.JPG \\
\hline 105 & Salix aurita L. & Kőszeg & 1932 & Soó Rezső & BPU-09777.JPG \\
\hline 105 & Salix aurita L. & Szakonyfalu & 1955 & Soó Rezső & BPU-09778.JPG \\
\hline 105 & Salix aurita L. & Szenta & 1957 & Soó Rezső & BPU-09775.JPG \\
\hline $94-105$ & Salix & Hidegkút & 1957 & Felföldy Lajos & BPU-13411.JPG \\
\hline $94-105$ & Salix & Pákozd & 1980 & Felföldy Lajos & BPU-13421.JPG \\
\hline $94 \times 95$ & Salix $\times$ rubens Schrank & Bátorliget & 1934 & Soó Rezső & BPU-09726.JPG \\
\hline $94 \times 95$ & Salix $\times$ ubens Schrank & Debrecen & 1931 & Soó Rezső & BPU-13419.JPG \\
\hline $94 \times 95$ & Salix $\times$ ubens Schrank & Miskolc & 1937 & Soó Rezső & BPU-13418.JPG \\
\hline $95 \times 96$ & Salix fragilis L. $\times$ Salix triandra L. & Baktalórántháza & 1933 & Soó Rezső & BPU-13402.JPG \\
\hline $95 \times 96$ & Salix fragilis L. $\times$ Salix triandra L. & Budapest & 2004 & Felföldy Lajos & BPU-13422.JPG \\
\hline $95 \times 97$ & Salix fragilis L. $\times$ Salix pentandra L. & Bátorliget & 1932 & Soó Rezső & BPU-13403.JPG \\
\hline $\begin{array}{c}96 \times 99 \times \\
101\end{array}$ & $\begin{array}{l}\text { Salix triandra L. } \times \text { Salix purpurea L. } \times \\
\text { Salix viminalis L. }\end{array}$ & Győr & 1940 & Polgár Sándor & BPU-13401.JPG \\
\hline $98 \times 101$ & $\begin{array}{l}\text { Salix rosmarinifolia L. } \times \text { Salix viminalis } \\
\text { L. }\end{array}$ & Debrecen & 1934 & Soó Rezső & BPU-13400.JPG \\
\hline $98 \times 104$ & $\begin{array}{l}\text { Salix rosmarinifolia L. } \times \text { Salix cinerea } \\
\text { L. }\end{array}$ & Budapest & 1925 & Jávorka Sándor & BPU-13406.JPG \\
\hline $104 \times 105$ & Salix $\times$ multinervis Döll & Baktalórántháza & 1932 & Soó Rezső & BPU-13410.JPG \\
\hline $104 \times 105$ & Salix $\times$ multinervis Döll & Csaroda & 1952 & Soó Rezső & BPU-13412.JPG \\
\hline $104 \times 105$ & Salix $\times$ multinervis Döll & Pálháza & 1938 & Soó Rezső & BPU-13417.JPG \\
\hline $104 \times 105$ & Salix $\times$ multinervis Döll & Pálháza & 1949 & Simon Tibor, Jakucs Pál & BPU-13416.JPG \\
\hline $104 \times 105$ & Salix $\times$ multinervis Döll & Regéc & 1952 & Simon Tibor & BPU-13414.JPG \\
\hline $104 \times 105$ & Salix $\times$ multinervis Döll & Szentgotthárd & 1955 & Soó Rezső & BPU-13409.JPG \\
\hline $104 \times 105$ & Salix $\times$ multinervis Döll & Szőce & 1955 & Soó Rezső & BPU-13408.JPG \\
\hline $104 \times 105$ & Salix $\times$ multinervis Döll & Telkibánya & 1952 & Simon Tibor & BPU-13415.JPG \\
\hline 106 & Populus alba L. & Budapest & 1922 & Soó Rezső & BPU-09700.JPG \\
\hline 106 & Populus alba L. & Debrecen & 1932 & Soó Rezső & BPU-09699.JPG \\
\hline 106 & Populus alba L. & Debrecen & 1945 & Felföldy Lajos & BPU-09698.JPG \\
\hline 107 & Populus tremula L. & Bátorliget & 1927 & Gombocz Endre & BPU-09695.JPG \\
\hline 107 & Populus tremula L. & Bátorliget & 1927 & Gombocz Endre & BPU-09696.JPG \\
\hline 107 & Populus tremula L. & Budapest & 1949 & Soó Rezső & BPU-09692.JPG \\
\hline 107 & Populus tremula L. & Budapest & 2004 & Felföldy Lajos & BPU-09691.JPG \\
\hline 107 & Populus tremula L. & Debrecen & 1931 & Soó Rezső & BPU-09693.JPG \\
\hline 107 & Populus tremula L. & Gyöngyös & 1964 & Soó Rezső & BPU-09694.JPG \\
\hline 109 & Populus nigra L. & Eszteregnye & 1949 & Károlyi Árpád & BPU-09702.JPG \\
\hline 109 & Populus nigra L. & Tarpa & 1949 & Soó Rezső & BPU-09701.JPG \\
\hline $106 \times 107$ & Populus $\times$ canescens Sm. & "Csepel-sziget" & 1923 & Gombocz Endre & BPU-09707.JPG \\
\hline $106 \times 107$ & Populus $\times$ canescens Sm. & "Csepel-sziget" & 1923 & Gombocz Endre & BPU-09708.JPG \\
\hline $106 \times 107$ & Populus $\times$ canescens $\mathrm{Sm}$. & Budapest & 1929 & Boros Ádám & BPU-09711.JPG \\
\hline $106 \times 107$ & Populus $\times$ canescens Sm. & Csévharaszt & 1959 & Simon Tibor, Borsos Olga & BPU-09703.JPG \\
\hline $106 \times 107$ & Populus $\times$ canescens Sm. & Monor & 1925 & Gombocz Endre & BPU-09709.JPG \\
\hline $106 \times 107$ & Populus $\times$ canescens $\mathrm{Sm}$. & Nagykáta & 1924 & Gombocz Endre & BPU-09706.JPG \\
\hline $106 \times 107$ & Populus $\times$ canescens Sm. & Pilisborosjenő & 1944 & Kárpáti Zoltán & BPU-09705.JPG \\
\hline $106 \times 107$ & Populus $\times$ canescens Sm. & Szigetcsép & - & Gombocz Endre & BPU-09710.JPG \\
\hline
\end{tabular}


1. Elektroniukus melléklet - Electronic Appendix 1.

\begin{tabular}{|c|c|c|c|c|c|}
\hline $\begin{array}{l}\text { Sorszám / } \\
\text { Number }\end{array}$ & Taxon-név / Taxon-name & $\begin{array}{l}\text { Település / } \\
\text { Settlement }\end{array}$ & $\begin{array}{l}\text { Év / } \\
\text { Year }\end{array}$ & Gyűjtő / Collector & $\begin{array}{l}\text { Fájlnév / } \\
\text { File-name }\end{array}$ \\
\hline 113 & Betula pendula Roth & Bátorliget & 1932 & Soó Rezső & BPU-09577.JPG \\
\hline 113 & Betula pendula Roth & Csévharaszt & 1959 & Simon Tibor, Borsos Olga & BPU-09575.JPG \\
\hline 113 & Betula pendula Roth & Dávod & 1955 & Soó Rezső & BPU-09576.JPG \\
\hline 113 & Betula pendula Roth & Debrecen & 1931 & Soó Rezső & BPU-09574.JPG \\
\hline 113 & Betula pendula Roth & Kecskemét & 1918 & Degen Árpád & BPU-09568.JPG \\
\hline 113 & Betula pendula Roth & Pákozd & 1980 & Felföldy Lajos & BPU-09571.JPG \\
\hline 113 & Betula pendula Roth & Pécs & 1953 & Soó Rezső, Borsos Olga & BPU-09570.JPG \\
\hline 113 & Betula pendula Roth & Rudabánya & 1936 & Soó Rezső & BPU-09573.JPG \\
\hline 113 & Betula pendula Roth & Uzsa & 1959 & $\begin{array}{l}\text { Jávorka Sándor, Felföldy } \\
\text { Lajos }\end{array}$ & BPU-09578.JPG \\
\hline 114 & Betula pubescens Ehrh. & Aggtelek & 1952 & Jakucs Pál & BPU-09580.JPG \\
\hline 114 & Betula pubescens Ehrh. & Bátorliget & 1934 & Soó Rezső & BPU-09585.JPG \\
\hline 114 & Betula pubescens Ehrh. & Bátorliget & 1950 & $\begin{array}{l}\text { Simon Tibor, Kárpáti } \\
\text { István }\end{array}$ & BPU-09584.JPG \\
\hline 114 & Betula pubescens Ehrh. & Kelemér & 1950 & Soó Rezső & BPU-09582.JPG \\
\hline 114 & Betula pubescens Ehrh. & Kelemér & 1950 & Soó Rezső & BPU-09583.JPG \\
\hline 114 & Betula pubescens Ehrh. & Szentgotthárd & 1955 & Soó Rezső & BPU-09586.JPG \\
\hline 116 & Alnus glutinosa (L.) Gaertn. & Bátorliget & 1934 & Soó Rezső & BPU-09605.JPG \\
\hline 116 & Alnus glutinosa (L.) Gaertn. & Bicske & 1991 & Felföldy Lajos & BPU-09601.JPG \\
\hline 116 & Alnus glutinosa (L.) Gaertn. & Csévharaszt & 1961 & Simon Tibor & BPU-09602.JPG \\
\hline 116 & Alnus glutinosa (L.) Gaertn. & Miskolc & 1934 & Soó Rezső & BPU-09600.JPG \\
\hline 116 & Alnus glutinosa (L.) Gaertn. & Nagybajom & 1955 & Soó Rezső & BPU-09604.JPG \\
\hline 116 & Alnus glutinosa (L.) Gaertn. & Révfülöp & 1956 & Felföldy Lajos & BPU-09607.JPG \\
\hline 116 & Alnus glutinosa (L.) Gaertn. & Révfülöp & 1956 & Felföldy Lajos & BPU-09608.JPG \\
\hline 116 & Alnus glutinosa (L.) Gaertn. & Tarpa & 1949 & Soó Rezső & BPU-09599.JPG \\
\hline 116 & Alnus glutinosa (L.) Gaertn. & Vashosszúfalu & 1951 & Felföldy Lajos & BPU-09606.JPG \\
\hline 116 & Alnus glutinosa (L.) Gaertn. & Visegrád & 1968 & Priszter Szaniszló & BPU-09603.JPG \\
\hline 117 & Alnus incana (L.) Moench & Alsószölnök & 1955 & Soó Rezső & BPU-09595.JPG \\
\hline 117 & Alnus incana (L.) Moench & Göd & 1948 & Soó Rezső & BPU-09594.JPG \\
\hline $115 \times 117$ & $\begin{array}{l}\text { Alnus incana (L.) Moench } \times \text { Alnus } \\
\text { viridis (Chaix) DC. }\end{array}$ & Alsószölnök & 1955 & Soó Rezső & BPU-09613.JPG \\
\hline $115 \times 117$ & $\begin{array}{l}\text { Alnus incana (L.) Moench } \times \text { Alnus } \\
\text { viridis (Chaix) DC. }\end{array}$ & Alsószölnök & 1955 & Soó Rezső & BPU-09614.JPG \\
\hline $116 \times 117$ & Alnus $\times$ hybrida A. Braun ex Rchb. & Alsószölnök & 1955 & Soó Rezső & BPU-09612.JPG \\
\hline $116 \times 117$ & Alnus $\times$ hybrida A. Braun ex Rchb. & Győr & 1901 & Polgár Sándor & BPU-09615.JPG \\
\hline 118 & Carpinus betulus L. & Budapest & 1922 & Soó Rezső & BPU-11136.JPG \\
\hline 118 & Carpinus betulus L. & Budapest & 1944 & Kárpáti Zoltán & BPU-11135.JPG \\
\hline 118 & Carpinus betulus L. & Budapest & 1947 & Soó Rezső & BPU-11128.JPG \\
\hline 118 & Carpinus betulus L. & Dömös & 1964 & Isépy István & BPU-11134.JPG \\
\hline 118 & Carpinus betulus L. & Gánt & 1943 & Kárpáti Zoltán & BPU-11133.JPG \\
\hline 118 & Carpinus betulus L. & Gyöngyös & 1952 & Soó Rezső & BPU-11130.JPG \\
\hline 118 & Carpinus betulus L. & Gyöngyös & 1964 & Soó Rezső & BPU-11131.JPG \\
\hline 118 & Carpinus betulus L. & Gyöngyös & 1964 & Soó Rezső & BPU-11132.JPG \\
\hline 118 & Carpinus betulus L. & Sátoraljaújhely & 1993 & Felföldy Lajos & BPU-11126.JPG \\
\hline 118 & Carpinus betulus L. & Somogyszob & 1957 & Soó Rezső & BPU-11127.JPG \\
\hline 118 & Carpinus betulus L. & Zebegény & 1947 & Soó Rezső & BPU-11129.JPG \\
\hline 119 & Carpinus orientalis Mill. & Csákvár & 1955 & Soó Rezső & BPU-11124.JPG \\
\hline 119 & Carpinus orientalis Mill. & Csákvár & 1955 & Soó Rezső & BPU-11125.JPG \\
\hline 123 & Fagus sylvatica L. & Beregdaróc & 1951 & Simon Tibor & BPU-09620.JPG \\
\hline
\end{tabular}


1. Elektroniukus melléklet - Electronic Appendix 1.

\begin{tabular}{|c|c|c|c|c|c|}
\hline $\begin{array}{l}\text { Sorszám / } \\
\text { Number }\end{array}$ & Taxon-név / Taxon-name & $\begin{array}{l}\text { Település / } \\
\text { Settlement }\end{array}$ & $\begin{array}{l}\text { Év / } \\
\text { Year }\end{array}$ & Gyűjtő / Collector & $\begin{array}{l}\text { Fájlnév / } \\
\text { File-name }\end{array}$ \\
\hline 123 & Fagus sylvatica L. & Budapest & 1953 & Borsos Olga & BPU-15412.JPG \\
\hline 123 & Fagus sylvatica L. & Budapest & 1982 & Felföldy Lajos & BPU-09616.JPG \\
\hline 123 & Fagus sylvatica L. & Pécs & 1936 & Soó Rezső & BPU-09619.JPG \\
\hline 123 & Fagus sylvatica L. & Pécs & 1956 & Soó Rezső & BPU-09618.JPG \\
\hline 123 & Fagus sylvatica L. & Pusztafalu & 1962 & Simon Tibor & BPU-09617.JPG \\
\hline 124 & Castanea sativa Mill. & Bódvarákó & 1951 & Jakucs Pál, Ér Lajos & BPU-09624.JPG \\
\hline 124 & Castanea sativa Mill. & Kőszeg & 1943 & Soó Rezső & BPU-09622.JPG \\
\hline 124 & Castanea sativa Mill. & Kőszeg & 1963 & Soó Rezső, Borsos Olga & BPU-09626.JPG \\
\hline 124 & Castanea sativa Mill. & Nagybajom & 1957 & Soó Rezső & BPU-09625.JPG \\
\hline 124 & Castanea sativa Mill. & Pécs & 1953 & Soó Rezső, Borsos Olga & BPU-09627.JPG \\
\hline 125 & Quercus cerris L. & Budapest & 1964 & Soó Rezső & BPU-09638.JPG \\
\hline 125 & Quercus cerris L. & Budapest & 2005 & Felföldy Lajos & BPU-09628.JPG \\
\hline 125 & Quercus cerris L. & Csesznek & 1929 & Soó Rezső & BPU-09630.JPG \\
\hline 125 & Quercus cerris L. & Fót & 1960 & Soó Rezső & BPU-09634.JPG \\
\hline 125 & Quercus cerris L. & Gánt & 1947 & Vajda László & BPU-09632.JPG \\
\hline 125 & Quercus cerris L. & Hajdúnánás & - & Igmándy József & BPU-09631.JPG \\
\hline 125 & Quercus cerris L. & Hidegkút & 1958 & Felföldy Lajos & BPU-09640.JPG \\
\hline 125 & Quercus cerris L. & Isztimér & 1949 & Soó Rezső & BPU-09639.JPG \\
\hline 125 & Quercus cerris L. & Kaszó & 1957 & Soó Rezső & BPU-09629.JPG \\
\hline 125 & Quercus cerris L. & Kerecsend & 1957 & Soó Rezső & BPU-09637.JPG \\
\hline 125 & Quercus cerris L. & Lesenceistvánd & 1952 & Borsos Olga & BPU-09635.JPG \\
\hline 125 & Quercus cerris L. & Tibolddaróc & 1953 & Soó Rezső & BPU-09636.JPG \\
\hline 125 & Quercus cerris L. & Tihany & 1946 & Udvardy Miklós & BPU-09633.JPG \\
\hline 128 & Quercus robur L. & Debrecen & 1934 & Soó Rezső & BPU-09680.JPG \\
\hline 128 & Quercus robur L. & Egyek & 1932 & Soó Rezső & BPU-09677.JPG \\
\hline 128 & Quercus robur L. & Magyarlukafa & 1957 & Soó Rezső & BPU-09678.JPG \\
\hline 129 & Quercus dalechampii Ten. & Gyöngyös & 1962 & Soó Rezső, Borsos Olga & BPU-09666.JPG \\
\hline 129 & Quercus dalechampii Ten. & Kovácsvágás & 1962 & Simon Tibor & BPU-09659.JPG \\
\hline 129 & Quercus dalechampii Ten. & Kőszeg & 1963 & Soó Rezső, Borsos Olga & BPU-09672.JPG \\
\hline 129 & Quercus dalechampii Ten. & Pusztafalu & 1962 & Simon Tibor & BPU-09658.JPG \\
\hline 129 & Quercus petraea agg. & Badacsonytördemic & 1953 & $\begin{array}{l}\text { Felföldy Lajos, Tóth } \\
\text { László }\end{array}$ & BPU-09673.JPG \\
\hline 129 & Quercus petraea agg. & Budaörs & 1961 & Borsos Olga, Simon Tibor & BPU-09679.JPG \\
\hline 129 & Quercus petraea agg. & Gyöngyös & 1962 & Soó Rezső, Borsos Olga & BPU-09662.JPG \\
\hline 129 & Quercus petraea agg. & Gyöngyös & 1962 & Soó Rezső, Borsos Olga & BPU-09663.JPG \\
\hline 129 & Quercus petraea agg. & Gyöngyös & 1962 & Soó Rezső, Borsos Olga & BPU-09664.JPG \\
\hline 129 & Quercus petraea agg. & Gyöngyös & 1962 & Soó Rezső, Borsos Olga & BPU-09665.JPG \\
\hline 129 & Quercus petraea agg. & Kerecsend & 1957 & Soó Rezső & BPU-09683.JPG \\
\hline 129 & Quercus petraea agg. & Kishuta & 1962 & Simon Tibor & BPU-09657.JPG \\
\hline 129 & Quercus petraea agg. & Pomáz & 1948 & Koppányi Tibor & BPU-09667.JPG \\
\hline 129 & Quercus petraea agg. & Szenna & 1957 & Soó Rezső & BPU-09671.JPG \\
\hline 129 & Quercus petraea agg. & Vác & 1949 & $\begin{array}{l}\text { Soó Rezső, Simon Tibor, } \\
\text { Jakucs Pál }\end{array}$ & BPU-09668.JPG \\
\hline 129 & Quercus petraea agg. & Visegrád & 1964 & Soó Rezső, Borsos Olga & BPU-09669.JPG \\
\hline 129 & Quercus petraea agg. & Visegrád & 1964 & Soó Rezső, Borsos Olga & BPU-09670.JPG \\
\hline 129 & Quercus polycarpa Schur & Kovácsvágás & 1962 & Simon Tibor & BPU-09660.JPG \\
\hline 130 & Quercus frainetto Ten. & Magyarlukafa & 1957 & Soó Rezső & BPU-09641.JPG \\
\hline 131 & Quercus pubescens agg. & Arka & 1961 & Simon Tibor & BPU-09646.JPG \\
\hline
\end{tabular}


1. Elektroniukus melléklet - Electronic Appendix 1.

\begin{tabular}{|c|c|c|c|c|c|}
\hline $\begin{array}{l}\text { Sorszám / } \\
\text { Number }\end{array}$ & Taxon-név / Taxon-name & $\begin{array}{l}\text { Település / } \\
\text { Settlement }\end{array}$ & $\begin{array}{l}\text { Év / } \\
\text { Year }\end{array}$ & Gyưjtő / Collector & $\begin{array}{l}\text { Fájlnév / } \\
\text { File-name }\end{array}$ \\
\hline 131 & Quercus pubescens agg. & Arka & 1961 & Simon Tibor & BPU-09647.JPG \\
\hline 131 & Quercus pubescens agg. & Budapest & 1947 & Soó Rezső & BPU-09644.JPG \\
\hline 131 & Quercus pubescens agg. & Budapest & 1953 & Soó Rezső & BPU-09645.JPG \\
\hline 131 & Quercus pubescens agg. & Budapest & 1958 & Simon Tibor & BPU-09642.JPG \\
\hline 131 & Quercus pubescens agg. & Budapest & 1958 & Soó Rezső & BPU-09643.JPG \\
\hline 131 & Quercus pubescens agg. & Csévharaszt & 1959 & Simon Tibor, Borsos Olga & BPU-09651.JPG \\
\hline 131 & Quercus pubescens agg. & Csévharaszt & 1961 & Simon Tibor & BPU-09652.JPG \\
\hline 131 & Quercus pubescens agg. & Kerecsend & 1957 & Soó Rezső & BPU-09649.JPG \\
\hline 131 & Quercus pubescens agg. & Kerecsend & 1957 & Soó Rezső & BPU-09650.JPG \\
\hline 131 & Quercus pubescens agg. & Kerecsend & 1957 & Soó Rezső & BPU-09681.JPG \\
\hline 131 & Quercus pubescens agg. & Kerecsend & 1957 & Soó Rezső & BPU-09684.JPG \\
\hline 131 & Quercus pubescens agg. & Pécs & 1925 & Soó Rezső & BPU-09648.JPG \\
\hline 131 & Quercus pubescens agg. & Tokaj & 1947 & Soó Rezső & BPU-09653.JPG \\
\hline 131 & Quercus virgiliana Ten. & Érd & 2007 & Simon Tibor & BPU-15502.JPG \\
\hline 131 & Quercus virgiliana Ten. & Kerecsend & 1962 & Simon Tibor & BPU-09655.JPG \\
\hline 131 & Quercus virgiliana Ten. & Miskolc & 1937 & Soó Rezső & BPU-09656.JPG \\
\hline 131 & Quercus virgiliana Ten. & Nagyharsány & 1955 & $\begin{array}{l}\text { Simon Tibor, Priszter } \\
\text { Szaniszló, Borhidi Attila }\end{array}$ & BPU-09654.JPG \\
\hline 131 & Quercus virgiliana Ten. & Nagymaros & 2007 & Simon Tibor & BPU-15501.JPG \\
\hline $128 \times 129$ & $\begin{array}{l}\text { Quercus robur L. } \times \text { Quercus petraea } \\
\text { (Matt.) Liebl. }\end{array}$ & Bodajk & 1955 & Soó Rezső & BPU-09685.JPG \\
\hline $128 \times 129$ & $\begin{array}{l}\text { Quercus robur L. × Quercus petraea } \\
\text { (Matt.) Liebl. }\end{array}$ & Bodajk & 1955 & Soó Rezső & BPU-09686.JPG \\
\hline $129 \times 130$ & $\begin{array}{l}\text { Quercus frainetto Ten. × Quercus } \\
\text { petraea (Matt.) Liebl. }\end{array}$ & Gyöngyös & - & Priszter Szaniszló & BPU-09682.JPG \\
\hline 132 & Ulmus laevis Pall. & Bátorliget & 1934 & Soó Rezső & BPU-11159.JPG \\
\hline 132 & Ulmus laevis Pall. & Hévíz & 1951 & Felföldy Lajos & BPU-11157.JPG \\
\hline 132 & Ulmus laevis Pall. & Mór & 1953 & Felföldy Lajos & BPU-11160.JPG \\
\hline 132 & Ulmus laevis Pall. & Nyíregyháza & 1935 & Soó Rezső & BPU-11158.JPG \\
\hline 132 & Ulmus laevis Pall. & Sárospatak & 1939 & Soó Rezső & BPU-11156.JPG \\
\hline 133 & Ulmus glabra Huds. & Bátorliget & 1953 & Simon Tibor & BPU-11140.JPG \\
\hline 133 & Ulmus glabra Huds. & Budapest & 2004 & Felföldy Lajos & BPU-11146.JPG \\
\hline 133 & Ulmus glabra Huds. & Debrecen & 1931 & Soó Rezső & BPU-11141.JPG \\
\hline 133 & Ulmus glabra Huds. & Gánt & 1948 & Soó Rezső & BPU-11142.JPG \\
\hline 133 & Ulmus glabra Huds. & Kisharsány & 1961 & Simon Tibor & BPU-11145.JPG \\
\hline 133 & Ulmus glabra Huds. & Miskolc & 1950 & Soó Rezső & BPU-11143.JPG \\
\hline 133 & Ulmus glabra Huds. & Miskolc & 1953 & Soó Rezső & BPU-11144.JPG \\
\hline 134 & Ulmus minor Mill. & Balatonszőlős & 1957 & Felföldy Lajos & BPU-11149.JPG \\
\hline 134 & Ulmus minor Mill. & Debrecen & 1947 & Borsos Olga & BPU-11153.JPG \\
\hline 134 & Ulmus minor Mill. & Debrecen & 1947 & Soó Rezső & BPU-11154.JPG \\
\hline 134 & Ulmus minor Mill. & Hidegkút & 1958 & Felföldy Lajos & BPU-11148.JPG \\
\hline 134 & Ulmus minor Mill. & Mór & 1953 & Felföldy Lajos & BPU-11147.JPG \\
\hline 134 & Ulmus minor Mill. & Nagykovácsi & 1948 & Soó Rezső & BPU-15426.JPG \\
\hline 134 & Ulmus minor Mill. & Nagykovácsi & 1949 & Soó Rezső & BPU-11152.JPG \\
\hline 134 & Ulmus minor Mill. & Pécsvárad & 1936 & Soó Rezső & BPU-11155.JPG \\
\hline 134 & Ulmus minor Mill. & Tihany & 1957 & $\begin{array}{l}\text { Felföldy Lajos, Tóth } \\
\text { László }\end{array}$ & BPU-11150.JPG \\
\hline 134 & Ulmus minor Mill. & Tihany & 1958 & Felföldy Lajos & BPU-11151.JPG \\
\hline 143 & Humulus lupulus L. & Budapest & 2004 & Felföldy Lajos & BPU-11192.JPG \\
\hline 143 & Humulus lupulus L. & Debrecen & 1949 & Soó Rezső & BPU-11191.JPG \\
\hline
\end{tabular}


1. Elektroniukus melléklet - Electronic Appendix 1.

\begin{tabular}{|c|c|c|c|c|c|}
\hline $\begin{array}{l}\text { Sorszám / } \\
\text { Number }\end{array}$ & Taxon-név / Taxon-name & $\begin{array}{l}\text { Település / } \\
\text { Settlement }\end{array}$ & $\begin{array}{l}\text { Év / } \\
\text { Year }\end{array}$ & Gyüjtő / Collector & $\begin{array}{l}\text { Fájlnév / } \\
\text { File-name }\end{array}$ \\
\hline 143 & Humulus lupulus L. & Tokaj & 1949 & Soó Rezső & BPU-11190.JPG \\
\hline 144 & Humulus scandens (Lour.) Merr. & Budapest & 1947 & Papp József & BPU-11189.JPG \\
\hline 145 & Cannabis sativa L. & Bugac & 1964 & Soó Rezső & BPU-11188.JPG \\
\hline 145 & Cannabis sativa L. & Kiskunhalas & 1964 & Soó Rezső & BPU-11186.JPG \\
\hline 145 & Cannabis sativa L. & Kiskunmajsa & 1964 & Soó Rezső & BPU-11185.JPG \\
\hline 145 & Cannabis sativa L. & Szár & 1960 & Priszter Szaniszló & BPU-11184.JPG \\
\hline 146 & Urtica urens L. & Debrecen & 1947 & Soó Rezső & BPU-11183.JPG \\
\hline 147 & Urtica dioica L. & Debrecen & 1931 & Soó Rezső & BPU-11181.JPG \\
\hline 147 & Urtica dioica L. & Kimle & 1962 & Borsos Olga & BPU-11177.JPG \\
\hline 147 & Urtica dioica L. & Nyírpilis & 1948 & $\begin{array}{l}\text { Simon Tibor, Jakucs Pál, } \\
\text { Borsos Olga }\end{array}$ & BPU-11178.JPG \\
\hline 147 & Urtica dioica L. & Veresegyház & 1949 & Soó Rezső & BPU-11180.JPG \\
\hline 147 & Urtica dioica L. & Veresegyház & 1949 & Soó Rezső & BPU-11182.JPG \\
\hline 148 & Urtica kioviensis Rogow. & Bátorliget & 1932 & Zólyomi Bálint & BPU-11170.JPG \\
\hline 148 & Urtica kioviensis Rogow. & Kecskemét & 1953 & Soó Rezső & BPU-11174.JPG \\
\hline 148 & Urtica kioviensis Rogow. & Ócsa & 1950 & Soó Rezső & BPU-11172.JPG \\
\hline 148 & Urtica kioviensis Rogow. & Szigetcsép & 1992 & Felföldy Lajos & BPU-11171.JPG \\
\hline 148 & Urtica kioviensis Rogow. & Tákos & 1949 & Soó Rezső & BPU-11173.JPG \\
\hline 149 & Parietaria officinalis L. & Budapest & 2004 & Felföldy Lajos & BPU-11167.JPG \\
\hline 149 & Parietaria officinalis L. & Miskolc & 1952 & Borsos Olga & BPU-11166.JPG \\
\hline 149 & Parietaria officinalis L. & Szentendre & 1947 & Soó Rezső & BPU-11164.JPG \\
\hline 149 & Parietaria officinalis L. & Szigliget & 1962 & Soó Rezső & BPU-11162.JPG \\
\hline 149 & Parietaria officinalis L. & Szilvásvárad & 1953 & $\begin{array}{l}\text { Felföldy Lajos, Tóth } \\
\text { László }\end{array}$ & BPU-11165.JPG \\
\hline 149 & Parietaria officinalis L. & Tihany & 1947 & Pólya László & BPU-11163.JPG \\
\hline 151 & Thesium ramosum Hayne & Aszófő & 1959 & Felföldy Lajos & BPU-11016.JPG \\
\hline 151 & Thesium ramosum Hayne & Budapest & 1923 & Soó Rezső & BPU-11023.JPG \\
\hline 151 & Thesium ramosum Hayne & Csarnóta & 1968 & Priszter Szaniszló & BPU-11022.JPG \\
\hline 151 & Thesium ramosum Hayne & Egyek & 1947 & Soó Rezső & BPU-11020.JPG \\
\hline 151 & Thesium ramosum Hayne & Kerecsend & 1957 & Soó Rezső & BPU-11019.JPG \\
\hline 151 & Thesium ramosum Hayne & Sopron & 1960 & Borsos Olga, Simon Tibor & BPU-11018.JPG \\
\hline 151 & Thesium ramosum Hayne & Szalonna & 1966 & Felföldy Lajos & BPU-11021.JPG \\
\hline 151 & Thesium ramosum Hayne & Tihany & 1928 & Soó Rezső & BPU-11012.JPG \\
\hline 151 & Thesium ramosum Hayne & Tihany & 1956 & Felföldy Lajos & BPU-11017.JPG \\
\hline 151 & Thesium ramosum Hayne & Tihany & 1959 & Felföldy Lajos & BPU-11011.JPG \\
\hline 151 & Thesium ramosum Hayne & Tihany & 1959 & Felföldy Lajos & BPU-11015.JPG \\
\hline 151 & Thesium ramosum Hayne & Uzsa & 1959 & $\begin{array}{l}\text { Jávorka Sándor, Felföldy } \\
\text { Lajos }\end{array}$ & BPU-11013.JPG \\
\hline 152 & Thesium dollineri Murb. & Balmazújváros & 1932 & Soó Rezső & BPU-11010.JPG \\
\hline 153 & Thesium linophyllon L. & Balatonszőlős & 1958 & Felföldy Lajos & BPU-10998.JPG \\
\hline 153 & Thesium linophyllon L. & Bátorliget & 1934 & Soó Rezső & BPU-11008.JPG \\
\hline 153 & Thesium linophyllon L. & Bodajk & 1961 & Borsos Olga, Simon Tibor & BPU-11002.JPG \\
\hline 153 & Thesium linophyllon L. & Bozsok & 1938 & Soó Rezső & BPU-11009.JPG \\
\hline 153 & Thesium linophyllon L. & Budaörs & 1961 & Borsos Olga, Simon Tibor & BPU-11005.JPG \\
\hline 153 & Thesium linophyllon L. & Budapest & 1982 & Felföldy Lajos & BPU-11001.JPG \\
\hline 153 & Thesium linophyllon L. & Budapest & 2004 & Felföldy Lajos & BPU-11000.JPG \\
\hline 153 & Thesium linophyllon L. & Budapest & 2005 & Felföldy Lajos & BPU-10999.JPG \\
\hline 153 & Thesium linophyllon L. & Gyöngyös & 1961 & Soó Rezső & BPU-11003.JPG \\
\hline 153 & Thesium linophyllon L. & Lábatlan & 1953 & Felföldy Lajos & BPU-11004.JPG \\
\hline
\end{tabular}


1. Elektroniukus melléklet - Electronic Appendix 1.

\begin{tabular}{|c|c|c|c|c|c|}
\hline $\begin{array}{l}\text { Sorszám / } \\
\text { Number }\end{array}$ & Taxon-név / Taxon-name & $\begin{array}{l}\text { Település / } \\
\text { Settlement }\end{array}$ & $\begin{array}{l}\text { Év / } \\
\text { Year }\end{array}$ & Gyűjtő / Collector & $\begin{array}{l}\text { Fájlnév / } \\
\text { File-name }\end{array}$ \\
\hline 153 & Thesium linophyllon L. & Ócsa & 1953 & Soó Rezső, Borsos Olga & BPU-11006.JPG \\
\hline 153 & Thesium linophyllon L. & Vác & 1923 & Soó Rezső & BPU-11007.JPG \\
\hline 155 & Loranthus europaeus Jacq. & Debrecen & 1947 & Soó Rezső & BPU-10994.JPG \\
\hline 155 & Loranthus europaeus Jacq. & Nagykanizsa & 1950 & Károlyi Árpád & BPU-10995.JPG \\
\hline 156 & Viscum album L. & Bátorliget & 1934 & Soó Rezső & BPU-10996.JPG \\
\hline 156 & Viscum album L. & Csákvár & 1948 & Soó Rezső & BPU-10997.JPG \\
\hline 157 & Asarum europaeum L. & Bakonybél & 1955 & Soó Rezső & BPU-01109.JPG \\
\hline 157 & Asarum europaeum L. & Bakonybél & 1955 & Soó Rezső & BPU-01110.JPG \\
\hline 157 & Asarum europaeum L. & Balatonszőlős & 1959 & Felföldy Lajos & BPU-01111.JPG \\
\hline 157 & Asarum europaeum L. & Bátorliget & 1950 & $\begin{array}{l}\text { Simon Tibor, Kárpáti } \\
\text { István }\end{array}$ & BPU-01108.JPG \\
\hline 157 & Asarum europaeum L. & Beregdaróc & 1951 & $\begin{array}{l}\text { Simon Tibor, Jakucs Pál, } \\
\text { Kulcsár Gábor }\end{array}$ & BPU-01105.JPG \\
\hline 157 & Asarum europaeum L. & Csesznek & 1953 & $\begin{array}{l}\text { Kulcsár Gábor, Vozáry } \\
\text { Elemér }\end{array}$ & BPU-01113.JPG \\
\hline 157 & Asarum europaeum L. & Dömös & 1948 & Soó Rezső & BPU-01106.JPG \\
\hline 157 & Asarum europaeum L. & Gánt & 1949 & Soó Rezső & BPU-01107.JPG \\
\hline 157 & Asarum europaeum L. & Háromhuta & 1955 & Simon Tibor & BPU-01103.JPG \\
\hline 157 & Asarum europaeum L. & Pécs & 1953 & Soó Rezső, Borsos Olga & BPU-01104.JPG \\
\hline 157 & Asarum europaeum L. & Regéc & 1939 & Soó Rezső & BPU-01102.JPG \\
\hline 157 & Asarum europaeum L. & Vászoly & 1958 & Felföldy Lajos & BPU-01112.JPG \\
\hline 158 & Aristolochia clematitis L. & Budapest & 1982 & Felföldy Lajos & BPU-01114.JPG \\
\hline 158 & Aristolochia clematitis L. & Egyek & 1933 & Soó Rezső & BPU-01116.JPG \\
\hline 158 & Aristolochia clematitis L. & Szeged & 1965 & Priszter Szaniszló & BPU-01115.JPG \\
\hline 159 & Persicaria amphibia (L.) Delarbre & Bélapátfalva & 1953 & Simon Tibor & BPU-11298.JPG \\
\hline 159 & Persicaria amphibia (L.) Delarbre & Budaörs & 1991 & Felföldy Lajos & BPU-11293.JPG \\
\hline 159 & Persicaria amphibia (L.) Delarbre & Budapest & 1990 & Felföldy Lajos & BPU-11299.JPG \\
\hline 159 & Persicaria amphibia (L.) Delarbre & Csobánka & 1992 & Felföldy Lajos & BPU-11292.JPG \\
\hline 159 & Persicaria amphibia (L.) Delarbre & Debrecen & 1951 & Soó Rezső & BPU-11296.JPG \\
\hline 159 & Persicaria amphibia (L.) Delarbre & Debrecen & 1952 & Kulcsár Gábor & BPU-11295.JPG \\
\hline 159 & Persicaria amphibia (L.) Delarbre & Hortobágy & 1947 & Soó Rezső & BPU-11294.JPG \\
\hline 159 & Persicaria amphibia (L.) Delarbre & Nagybajom & 1957 & Soó Rezső & BPU-11291.JPG \\
\hline 159 & Persicaria amphibia (L.) Delarbre & Nyírpilis & 1932 & Soó Rezső & BPU-11290.JPG \\
\hline 160 & Persicaria bistorta (L.) Samp. & Büttös & 1952 & Jakucs Pál & BPU-11300.JPG \\
\hline 160 & Persicaria bistorta (L.) Samp. & Szőce & 1955 & Soó Rezső & BPU-11302.JPG \\
\hline 161 & Persicaria lapathifolia (L.) Delarbre & Budapest & 1989 & Felföldy Lajos & BPU-11282.JPG \\
\hline 161 & Persicaria lapathifolia (L.) Delarbre & Debrecen & 1946 & Soó Rezső & BPU-11284.JPG \\
\hline 161 & Persicaria lapathifolia (L.) Delarbre & Hajdúbagos & 1933 & Soó Rezső & BPU-11286.JPG \\
\hline 161 & Persicaria lapathifolia (L.) Delarbre & Hortobágy & 1947 & Soó Rezső & BPU-11287.JPG \\
\hline 161 & Persicaria lapathifolia (L.) Delarbre & Nyírmada & 1933 & Soó Rezső & BPU-11283.JPG \\
\hline 161 & Persicaria lapathifolia (L.) Delarbre & Tihany & 1947 & Pólya László & BPU-11285.JPG \\
\hline 161 & Persicaria lapathifolia (L.) Delarbre & Vácrátót & 1949 & Soó Rezső & BPU-11288.JPG \\
\hline 162 & Persicaria maculosa Gray & Balatonakali & 1950 & Felföldy Lajos & BPU-11280.JPG \\
\hline 162 & Persicaria maculosa Gray & Hortobágy & 1934 & Soó Rezső & BPU-11281.JPG \\
\hline 162 & Persicaria maculosa Gray & Hosszúpályi & 1933 & Soó Rezső & BPU-11277.JPG \\
\hline 162 & Persicaria maculosa Gray & Révfülöp & 1956 & $\begin{array}{l}\text { Felföldy Lajos, Tóth } \\
\text { László }\end{array}$ & BPU-11278.JPG \\
\hline 162 & Persicaria maculosa Gray & Vonyarcvashegy & 1958 & Tóth László & BPU-11279.JPG \\
\hline 163 & Persicaria orientalis (L.) Gray & Debrecen & 1934 & Juhász Lajos & BPU-11200.JPG \\
\hline
\end{tabular}


1. Elektroniukus melléklet - Electronic Appendix 1.

\begin{tabular}{|c|c|c|c|c|c|}
\hline $\begin{array}{l}\text { Sorszám / } \\
\text { Number }\end{array}$ & Taxon-név / Taxon-name & $\begin{array}{l}\text { Település / } \\
\text { Settlement }\end{array}$ & $\begin{array}{l}\text { Év / } \\
\text { Year }\end{array}$ & Gyűjtő / Collector & $\begin{array}{l}\text { Fájlnév / } \\
\text { File-name }\end{array}$ \\
\hline 164 & Persicaria hydropiper (L.) Delarbre & Dömös & 1948 & Soó Rezső & BPU-11263.JPG \\
\hline 164 & Persicaria hydropiper (L.) Delarbre & Gyomaendrőd & 1947 & Soó Rezső & BPU-11264.JPG \\
\hline 165 & Persicaria dubia (Stein) Fourr. & Balatonszőlős & 1957 & $\begin{array}{l}\text { Felföldy Lajos, Kovács } \\
\text { Margit, Tóth László }\end{array}$ & BPU-11267.JPG \\
\hline 165 & Persicaria dubia (Stein) Fourr. & Budapest & 1990 & Felföldy Lajos & BPU-11272.JPG \\
\hline 165 & Persicaria dubia (Stein) Fourr. & Miskolc & 1939 & Soó Rezső & BPU-11270.JPG \\
\hline 165 & Persicaria dubia (Stein) Fourr. & Monoszló & 1982 & Felföldy Lajos & BPU-11266.JPG \\
\hline 165 & Persicaria dubia (Stein) Fourr. & Ócsa & 1950 & Soó Rezső & BPU-11269.JPG \\
\hline 165 & Persicaria dubia (Stein) Fourr. & Szarvas & 1991 & Felföldy Lajos & BPU-11271.JPG \\
\hline 165 & Persicaria dubia (Stein) Fourr. & Szigetmonostor & 1964 & Isépy István & BPU-11268.JPG \\
\hline 165 & Persicaria dubia (Stein) Fourr. & Vonyarcvashegy & 1958 & Tóth László & BPU-11265.JPG \\
\hline 166 & Persicaria minor (Huds.) Opiz & Debrecen & 1933 & Soó Rezső & BPU-11276.JPG \\
\hline 166 & Persicaria minor (Huds.) Opiz & Debrecen & 1945 & Felföldy Lajos & BPU-15485.JPG \\
\hline 166 & Persicaria minor (Huds.) Opiz & Dömös & 1948 & Soó Rezső & BPU-11273.JPG \\
\hline 166 & Persicaria minor (Huds.) Opiz & Nagykanizsa & 1956 & Felföldy Lajos & BPU-11274.JPG \\
\hline 166 & Persicaria minor (Huds.) Opiz & Ócsa & 1950 & Soó Rezső & BPU-11275.JPG \\
\hline 167 & Polygonum arenarium Waldst. et Kit. & Budapest & 1948 & Soó Rezső & BPU-11259.JPG \\
\hline 167 & Polygonum arenarium Waldst. et Kit. & Budapest & - & Woloszczak & BPU-11261.JPG \\
\hline 167 & Polygonum arenarium Waldst. et Kit. & Bugac & 1964 & Soó Rezső & BPU-11260.JPG \\
\hline 167 & Polygonum arenarium Waldst. et Kit. & Debrecen & 1946 & Soó Rezső & BPU-11258.JPG \\
\hline 167 & Polygonum arenarium Waldst. et Kit. & Nagybajom & 1957 & Soó Rezső & BPU-11262.JPG \\
\hline 167 & Polygonum arenarium Waldst. et Kit. & Nagykanizsa & 1948 & Károlyi Árpád & BPU-15486.JPG \\
\hline 168 & Polygonum bellardii All. & Budapest & 1932 & Pénzes Antal & BPU-11254.JPG \\
\hline 168 & Polygonum bellardii All. & Budapest & 1982 & Felföldy Lajos & BPU-11257.JPG \\
\hline 168 & Polygonum bellardii All. & Egyek & 1933 & Soó Rezső & BPU-11256.JPG \\
\hline 168 & Polygonum bellardii All. & Egyek & 1949 & Pénzes Antal & BPU-11255.JPG \\
\hline 168 & Polygonum bellardii All. & Hajdúhadház & 1947 & Simon Tibor & BPU-11252.JPG \\
\hline 169 & Polygonum graminifolium Wierzb. & Egyek & 1949 & Pénzes Antal & BPU-11255.JPG \\
\hline 169 & Polygonum graminifolium Wierzb. & Szigetújfalu & 1949 & Vajda László & BPU-11251.JPG \\
\hline 170 & Polygonum arenastrum Boreau & Szeged & 1950 & Timár Lajos & BPU-11244.JPG \\
\hline 171 & Polygonum rurivagum Jord. & Debrecen & 1945 & Felföldy Lajos & BPU-11214.JPG \\
\hline $170-172$ & Polygonum aviculare agg. & Budaörs & 1991 & Felföldy Lajos & BPU-11238.JPG \\
\hline $170-172$ & Polygonum aviculare agg. & Budapest & 1982 & Felföldy Lajos & BPU-11209.JPG \\
\hline $170-172$ & Polygonum aviculare agg. & Budapest & 1982 & Felföldy Lajos & BPU-11237.JPG \\
\hline $170-172$ & Polygonum aviculare agg. & Budapest & 1982 & Felföldy Lajos & BPU-11240.JPG \\
\hline $170-172$ & Polygonum aviculare agg. & Budapest & 1989 & Felföldy Lajos & BPU-11235.JPG \\
\hline $170-172$ & Polygonum aviculare agg. & Budapest & 1991 & Felföldy Lajos & BPU-11239.JPG \\
\hline $170-172$ & Polygonum aviculare agg. & Budapest & 1992 & Felföldy Lajos & BPU-11245.JPG \\
\hline $170-172$ & Polygonum aviculare agg. & Budapest & 2004 & Felföldy Lajos & BPU-11249.JPG \\
\hline $170-172$ & Polygonum aviculare agg. & Budapest & 2004 & Felföldy Lajos & BPU-11250.JPG \\
\hline $170-172$ & Polygonum aviculare agg. & Debrecen & 1945 & Csedreky Olga & BPU-11208.JPG \\
\hline $170-172$ & Polygonum aviculare agg. & Debrecen & 1945 & Csedreky Olga & BPU-11213.JPG \\
\hline $170-172$ & Polygonum aviculare agg. & Debrecen & 1945 & Csedreky Olga & BPU-11222.JPG \\
\hline $170-172$ & Polygonum aviculare agg. & Debrecen & 1945 & Felföldy Lajos & BPU-11210.JPG \\
\hline $170-172$ & Polygonum aviculare agg. & Debrecen & 1945 & Felföldy Lajos & BPU-11211.JPG \\
\hline $170-172$ & Polygonum aviculare agg. & Debrecen & 1945 & Felföldy Lajos & BPU-11212.JPG \\
\hline $170-172$ & Polygonum aviculare agg. & Debrecen & 1945 & Felföldy Lajos & BPU-11215.JPG \\
\hline $170-172$ & Polygonum aviculare agg. & Debrecen & 1945 & Felföldy Lajos & BPU-11216.JPG \\
\hline
\end{tabular}


1. Elektroniukus melléklet - Electronic Appendix 1.

\begin{tabular}{|c|c|c|c|c|c|}
\hline $\begin{array}{l}\text { Sorszám / } \\
\text { Number }\end{array}$ & Taxon-név / Taxon-name & $\begin{array}{l}\text { Település / } \\
\text { Settlement }\end{array}$ & $\begin{array}{l}\text { Év / } \\
\text { Year }\end{array}$ & Gyújtő / Collector & $\begin{array}{l}\text { Fájlnév / } \\
\text { File-name }\end{array}$ \\
\hline $170-172$ & Polygonum aviculare agg. & Debrecen & 1945 & Felföldy Lajos & BPU-11217.JPG \\
\hline $170-172$ & Polygonum aviculare agg. & Debrecen & 1945 & Felföldy Lajos & BPU-11218.JPG \\
\hline $170-172$ & Polygonum aviculare agg. & Debrecen & 1945 & Felföldy Lajos & BPU-11219.JPG \\
\hline $170-172$ & Polygonum aviculare agg. & Debrecen & 1945 & Felföldy Lajos & BPU-11220.JPG \\
\hline $170-172$ & Polygonum aviculare agg. & Debrecen & 1945 & Felföldy Lajos & BPU-11221.JPG \\
\hline $170-172$ & Polygonum aviculare agg. & Debrecen & 1945 & Felföldy Lajos & BPU-11223.JPG \\
\hline $170-172$ & Polygonum aviculare agg. & Debrecen & 1945 & Felföldy Lajos & BPU-11224.JPG \\
\hline $170-172$ & Polygonum aviculare agg. & Debrecen & 1945 & Felföldy Lajos & BPU-11225.JPG \\
\hline $170-172$ & Polygonum aviculare agg. & Debrecen & 1945 & Felföldy Lajos & BPU-11226.JPG \\
\hline $170-172$ & Polygonum aviculare agg. & Debrecen & 1945 & Felföldy Lajos & BPU-11227.JPG \\
\hline $170-172$ & Polygonum aviculare agg. & Debrecen & 1945 & Felföldy Lajos & BPU-11228.JPG \\
\hline $170-172$ & Polygonum aviculare agg. & Debrecen & 1945 & Felföldy Lajos & BPU-11229.JPG \\
\hline $170-172$ & Polygonum aviculare agg. & Debrecen & 1945 & Felföldy Lajos & BPU-11230.JPG \\
\hline $170-172$ & Polygonum aviculare agg. & Debrecen & 1945 & Felföldy Lajos & BPU-11241.JPG \\
\hline $170-172$ & Polygonum aviculare agg. & Egyek & 1933 & Soó Rezső & BPU-11232.JPG \\
\hline $170-172$ & Polygonum aviculare agg. & Egyek & 1947 & Soó Rezső & BPU-11231.JPG \\
\hline $170-172$ & Polygonum aviculare agg. & Gánt & 1980 & Felföldy Lajos & BPU-11246.JPG \\
\hline $170-172$ & Polygonum aviculare agg. & Hortobágy & 1947 & Soó Rezső & BPU-11242.JPG \\
\hline $170-172$ & Polygonum aviculare agg. & Szár & 1948 & Soó Rezső & BPU-11243.JPG \\
\hline $170-172$ & Polygonum aviculare agg. & Szentendre & 1963 & Soó Rezső & BPU-11234.JPG \\
\hline $170-172$ & Polygonum aviculare agg. & Tiborszállás & 1948 & Simon Tibor, Jakucs Pál & BPU-11207.JPG \\
\hline $170-172$ & Polygonum aviculare agg. & Vásárosnamény & 1933 & Soó Rezső & BPU-11233.JPG \\
\hline $170-172$ & Polygonum aviculare agg. & Zámoly & 1982 & Felföldy Lajos & BPU-11247.JPG \\
\hline $170-172$ & Polygonum aviculare agg. & Zámoly & 1982 & Felföldy Lajos & BPU-11248.JPG \\
\hline 173 & Fallopia japonica (Houtt.) Ronse Decr. & Gyöngyös & 1953 & Soó Rezső & BPU-11198.JPG \\
\hline 173 & Fallopia japonica (Houtt.) Ronse Decr. & Nagyharsány & 1960 & Simon Tibor & BPU-11199.JPG \\
\hline 178 & Fallopia dumetorum (L.) Holub & Nyíradony & 1932 & Soó Rezső & BPU-11202.JPG \\
\hline 178 & Fallopia dumetorum (L.) Holub & Szentendre & 1963 & Soó Rezső & BPU-11203.JPG \\
\hline 179 & Fallopia convolvulus (L.) A. Löve & Budapest & 1991 & Felföldy Lajos & BPU-11205.JPG \\
\hline 179 & Fallopia convolvulus (L.) A. Löve & Budapest & 2005 & Felföldy Lajos & BPU-11206.JPG \\
\hline 179 & Fallopia convolvulus (L.) A. Löve & Hajdúbagos & 1933 & Soó Rezső & BPU-11204.JPG \\
\hline 180 & Fagopyrum esculentum Moench & Egyek & 1950 & $\begin{array}{l}\text { Simon Tibor, Borsos Olga, } \\
\text { Kulcsár Gábor }\end{array}$ & BPU-11193.JPG \\
\hline 185 & Rumex acetosella L. & Baktalórántháza & 1932 & Soó Rezső & BPU-11339.JPG \\
\hline 185 & Rumex acetosella L. & Budapest & 1982 & Felföldy Lajos & BPU-11345.JPG \\
\hline 185 & Rumex acetosella L. & Csévharaszt & 1936 & Polgár Sándor & BPU-11342.JPG \\
\hline 185 & Rumex acetosella L. & Csévharaszt & 1959 & Simon Tibor, Borsos Olga & BPU-11341.JPG \\
\hline 185 & Rumex acetosella L. & Debrecen & 1930 & Soó Rezső & BPU-11344.JPG \\
\hline 185 & Rumex acetosella L. & Gyöngyös & 1965 & Soó Rezső & BPU-11343.JPG \\
\hline 185 & Rumex acetosella L. & Szenta & 1957 & Soó Rezső & BPU-11340.JPG \\
\hline 187 & Rumex acetosa L. & "Cuha-völgy" & 1928 & Polgár Sándor & BPU-11334.JPG \\
\hline 187 & Rumex acetosa L. & Bakonybél & 1955 & Soó Rezső & BPU-11337.JPG \\
\hline 187 & Rumex acetosa L. & Hidegkút & 1958 & Felföldy Lajos & BPU-11333.JPG \\
\hline 187 & Rumex acetosa L. & Hosszúpályi & 1933 & Soó Rezső & BPU-11335.JPG \\
\hline 187 & Rumex acetosa L. & Uzsa & 1959 & $\begin{array}{l}\text { Jávorka Sándor, Felföldy } \\
\text { Lajos }\end{array}$ & BPU-11332.JPG \\
\hline 188 & Rumex thyrsiflorus Fingerh. & Tiszavasvári & 1949 & Igmándy József & BPU-11336.JPG \\
\hline 188 & Rumex thyrsiflorus Fingerh. & Zebegény & 1947 & Soó Rezső & BPU-11338.JPG \\
\hline
\end{tabular}


1. Elektroniukus melléklet - Electronic Appendix 1.

\begin{tabular}{|c|c|c|c|c|c|}
\hline $\begin{array}{l}\text { Sorszám / } \\
\text { Number }\end{array}$ & Taxon-név / Taxon-name & $\begin{array}{l}\text { Település / } \\
\text { Settlement }\end{array}$ & $\begin{array}{l}\text { Év / } \\
\text { Year }\end{array}$ & Gyűjtő / Collector & $\begin{array}{l}\text { Fájlnév / } \\
\text { File-name }\end{array}$ \\
\hline 191 & Rumex pseudonatronatus Borbás & Bélmegyer & - & Borbás Vince & BPU-11351.JPG \\
\hline 192 & Rumex maritimus L. & Bátorliget & 1932 & Soó Rezső & BPU-11396.JPG \\
\hline 193 & Rumex palustris Sm. & Balatonakali & 1950 & Felföldy Lajos & BPU-11389.JPG \\
\hline 193 & Rumex palustris Sm. & Balatonszepezd & 1958 & Felföldy Lajos & BPU-11388.JPG \\
\hline 193 & Rumex palustris Sm. & Budaörs & 1991 & Felföldy Lajos & BPU-11391.JPG \\
\hline 193 & Rumex palustris Sm. & Csaroda & 1951 & $\begin{array}{l}\text { Simon Tibor, Vozáry } \\
\text { Elemér }\end{array}$ & BPU-11395.JPG \\
\hline 193 & Rumex palustris Sm. & Debrecen & 1989 & Felföldy Lajos & BPU-11393.JPG \\
\hline 193 & Rumex palustris Sm. & Gárdony & 1980 & Felföldy Lajos & BPU-11394.JPG \\
\hline 193 & Rumex palustris Sm. & Hortobágy & 1947 & Soó Rezső & BPU-11392.JPG \\
\hline 193 & Rumex palustris Sm. & Szenta & 1957 & Soó Rezső & BPU-11390.JPG \\
\hline 196 & Rumex obtusifolius L. & Bagamér & 1938 & Soó Rezső & BPU-11372.JPG \\
\hline 196 & Rumex obtusifolius L. & Debrecen & 1933 & Soó Rezső & BPU-11370.JPG \\
\hline 196 & Rumex obtusifolius L. & Gyöngyös & 1952 & Soó Rezső & BPU-11369.JPG \\
\hline 196 & Rumex obtusifolius L. & Mátraszentimre & 1935 & Soó Rezső & BPU-11368.JPG \\
\hline 196 & Rumex obtusifolius L. & Miskolc & 1950 & Soó Rezső & BPU-11371.JPG \\
\hline 196 & Rumex obtusifolius L. & Tihany & 1958 & Felföldy Lajos & BPU-11365.JPG \\
\hline 196 & Rumex obtusifolius L. & Vácrátót & 1949 & Soó Rezső & BPU-11367.JPG \\
\hline 196 & Rumex obtusifolius L. & Visegrád & 1968 & Priszter Szaniszló & BPU-11366.JPG \\
\hline 197 & Rumex confertus Willd. & Gyöngyös & 1923 & Soó Rezső & BPU-11374.JPG \\
\hline 197 & Rumex confertus Willd. & Miskolc & 1938 & Soó Rezső & BPU-11376.JPG \\
\hline 197 & Rumex confertus Willd. & Szolnok & 1937 & Soó Rezső & BPU-11375.JPG \\
\hline 198 & Rumex conglomeratus Murray & Balatonszepezd & 1958 & Felföldy Lajos & BPU-11384.JPG \\
\hline 198 & Rumex conglomeratus Murray & Balatonszőlős & 1957 & $\begin{array}{l}\text { Felföldy Lajos, Kovács } \\
\text { Margit, Tóth László }\end{array}$ & BPU-11385.JPG \\
\hline 198 & Rumex conglomeratus Murray & Debrecen & 1933 & Soó Rezső & BPU-11382.JPG \\
\hline 198 & Rumex conglomeratus Murray & Fadd & - & Haynald Lajos & BPU-11373.JPG \\
\hline 198 & Rumex conglomeratus Murray & Hegykő & 1960 & Tóth László & BPU-11386.JPG \\
\hline 198 & Rumex conglomeratus Murray & Miskolc & 1937 & Soó Rezső & BPU-11387.JPG \\
\hline 198 & Rumex conglomeratus Murray & Örvényes & 1958 & Felföldy Lajos & BPU-11383.JPG \\
\hline 199 & Rumex sanguineus L. & Debrecen & 1931 & Soó Rezső & BPU-11380.JPG \\
\hline 199 & Rumex sanguineus L. & Fadd & - & Haynald Lajos & BPU-11378.JPG \\
\hline 199 & Rumex sanguineus L. & Gyöngyös & 1961 & Soó Rezső & BPU-11377.JPG \\
\hline 199 & Rumex sanguineus L. & Tákos & 1949 & Soó Rezső & BPU-11381.JPG \\
\hline 199 & Rumex sanguineus L. & Villány & 1935 & Soó Rezső & BPU-11379.JPG \\
\hline 200 & Rumex hydrolapathum Huds. & Balatonfüred & 1942 & Felföldy Lajos & BPU-11357.JPG \\
\hline 200 & Rumex hydrolapathum Huds. & Balatonszemes & 1974 & Tóth László & BPU-11358.JPG \\
\hline 200 & Rumex hydrolapathum Huds. & Debrecen & 1934 & Soó Rezső & BPU-11356.JPG \\
\hline 200 & Rumex hydrolapathum Huds. & Isaszeg & 1949 & Soó Rezső & BPU-11359.JPG \\
\hline 201 & Rumex stenophyllus Ledeb. & Budaörs & 1991 & Felföldy Lajos & BPU-11362.JPG \\
\hline 201 & Rumex stenophyllus Ledeb. & Debrecen & 1942 & Felföldy Lajos & BPU-11361.JPG \\
\hline 201 & Rumex stenophyllus Ledeb. & Püspökladány & 1935 & Soó Rezső & BPU-11360.JPG \\
\hline 201 & Rumex stenophyllus Ledeb. & Vésztő & - & Borbás Vince & BPU-11363.JPG \\
\hline 202 & Rumex crispus L. & Bélmegyer & 1935 & Soó Rezső & BPU-11349.JPG \\
\hline 202 & Rumex crispus L. & Debrecen & 1934 & Soó Rezső & BPU-11353.JPG \\
\hline 202 & Rumex crispus L. & Gelénes & 1951 & $\begin{array}{l}\text { Simon Tibor, Vozáry } \\
\text { Elemér }\end{array}$ & BPU-11352.JPG \\
\hline 202 & Rumex crispus L. & Tihany & 1958 & Felföldy Lajos & BPU-11355.JPG \\
\hline 202 & Rumex crispus L. & Tihany & 1959 & Felföldy Lajos & BPU-11354.JPG \\
\hline
\end{tabular}


1. Elektroniukus melléklet - Electronic Appendix 1.

\begin{tabular}{|c|c|c|c|c|c|}
\hline $\begin{array}{l}\text { Sorszám / } \\
\text { Number }\end{array}$ & Taxon-név / Taxon-name & $\begin{array}{l}\text { Település / } \\
\text { Settlement }\end{array}$ & $\begin{array}{l}\text { Év / } \\
\text { Year }\end{array}$ & Gyüjtő / Collector & $\begin{array}{l}\text { Fájlnév / } \\
\text { File-name }\end{array}$ \\
\hline 202 & Rumex crispus L. & Vésztő & 1935 & Soó Rezső & BPU-11350.JPG \\
\hline 203 & Rumex patientia L. & Hortobágy & 1947 & Soó Rezső & BPU-11348.JPG \\
\hline 203 & Rumex patientia L. & Jósvafő & 1951 & Soó Rezső, Jakucs Pál & BPU-11347.JPG \\
\hline 204 & Rumex kerneri Borbás & Győr & 1928 & Polgár Sándor & BPU-11346.JPG \\
\hline $191 \times 201$ & $\begin{array}{l}\text { Rumex pseudonatronatus Borbás } \times \\
\text { Rumex stenophyllus Ledeb. }\end{array}$ & Miskolc & 1914 & Budai József & BPU-11314.JPG \\
\hline $196 \times 197$ & $\begin{array}{l}\text { Rumex obtusifolius L. } \times \text { Rumex } \\
\text { confertus Willd. }\end{array}$ & Miskolc & 1920 & Bihari Gyula & BPU-11316.JPG \\
\hline $196 \times 202$ & $\begin{array}{l}\text { Rumex } \times \text { pratensis Mert. Et W.D.J. } \\
\text { Koch }\end{array}$ & Győr & 1915 & Polgár Sándor & BPU-11320.JPG \\
\hline $196 \times 203$ & $\begin{array}{l}\text { Rumex obtusifolius L. } \times \text { Rumex } \\
\text { patientia L. }\end{array}$ & Gyöngyössolymos & 1920 & Bihari Gyula & BPU-11319.JPG \\
\hline $197 \times 200$ & $\begin{array}{l}\text { Rumex confertus Willd. } \times \text { Rumex } \\
\text { hydrolapathum Huds. }\end{array}$ & Mezőcsát & 1920 & Bihari Gyula & BPU-11317.JPG \\
\hline $197 \times 202$ & $\begin{array}{l}\text { Rumex confertus Willd. } \times \text { Rumex } \\
\text { crispus L. }\end{array}$ & Abádszalók & 1930 & Bihari Gyula & BPU-11315.JPG \\
\hline $198 \times 202$ & $\begin{array}{l}\text { Rumex conglomeratus Murray } \times \\
\text { Rumex crispus L. }\end{array}$ & Budapest & 1915 & Degen Árpád & BPU-11318.JPG \\
\hline 205 & Polycnemum heuffelii Láng & Bagamér & 1947 & Soó Rezső & BPU-10323.JPG \\
\hline 205 & Polycnemum heuffelii Láng & Kaposvár & 1948 & Károlyi Árpád & BPU-10322.JPG \\
\hline 206 & Polycnemum majus A. Braun & Dörgicse & 1957 & Felföldy Lajos & BPU-10330.JPG \\
\hline 206 & Polycnemum majus A. Braun & Hajdúszoboszló & 1915 & Polgár Sándor & BPU-10328.JPG \\
\hline 206 & Polycnemum majus A. Braun & Tihany & 1954 & Felföldy Lajos & BPU-10329.JPG \\
\hline 207 & Polycnemum arvense L. & Budapest & 1948 & Soó Rezső & BPU-10327.JPG \\
\hline 207 & Polycnemum arvense L. & Egyek & 1932 & Soó Rezső & BPU-10325.JPG \\
\hline 207 & Polycnemum arvense L. & Pálháza & 1952 & Simon Tibor & BPU-10325.JPG \\
\hline 207 & Polycnemum arvense L. & Pomáz & 1948 & Vajda László & BPU-10326.JPG \\
\hline 208 & Polycnemum verrucosum Láng & Dorog & 1876 & Haynald Lajos & BPU-10324.JPG \\
\hline 209 & Beta vulgaris L. & Debrecen & 1951 & Soó Rezső & BPU-10332.JPG \\
\hline 210 & Chenopodium aristatum L. & Örkény & 1964 & Soó Rezső & BPU-10335.JPG \\
\hline 210 & Chenopodium aristatum L. & Szigethalom & 1947 & Papp József & BPU-10336.JPG \\
\hline 210 & Chenopodium aristatum L. & Vácrátót & 1950 & Soó Rezső & BPU-10333.JPG \\
\hline 210 & Chenopodium aristatum L. & Vácrátót & 1950 & Soó Rezső & BPU-10334.JPG \\
\hline 211 & Chenopodium ambrosioides L. & Budapest & 1947 & Soó Rezső & BPU-10337.JPG \\
\hline 211 & Chenopodium ambrosioides L. & Paks & 1961 & Simon Tibor & BPU-10339.JPG \\
\hline 211 & Chenopodium ambrosioides L. & Paks & 1966 & Vöröss László Zs. & BPU-10340.JPG \\
\hline 211 & Chenopodium ambrosioides L. & Paks & 1966 & Vöröss László Zs. & BPU-10341.JPG \\
\hline 211 & Chenopodium ambrosioides L. & Visegrád & 1968 & Priszter Szaniszló & BPU-10338.JPG \\
\hline 212 & Chenopodium pumilio R. Br. & Gárdony & 1960 & Priszter Szaniszló & BPU-10434.JPG \\
\hline 213 & Chenopodium botrys L. & Budapest & 1947 & Soó Rezső & BPU-10344.JPG \\
\hline 214 & Chenopodium schraderianum Schult. & Bátorliget & 1932 & Soó Rezső & BPU-10342.JPG \\
\hline 216 & Chenopodium bonus-henricus L. & Csesznek & 1932 & Polgár Sándor & BPU-10348.JPG \\
\hline 216 & Chenopodium bonus-henricus L. & Miskolc & 1950 & Soó Rezső & BPU-10345.JPG \\
\hline 216 & Chenopodium bonus-henricus L. & Nagyvisnyó & 1953 & Soó Rezső & BPU-10346.JPG \\
\hline 216 & Chenopodium bonus-henricus L. & Szilvásvárad & 1953 & $\begin{array}{l}\text { Felföldy Lajos, Tóth } \\
\text { László }\end{array}$ & BPU-10347.JPG \\
\hline 217 & Chenopodium glaucum L. & Budapest & 1990 & Felföldy Lajos & BPU-10381.JPG \\
\hline 217 & Chenopodium glaucum $\mathrm{L}$. & Debrecen & 1932 & Soó Rezső & BPU-10379.JPG \\
\hline 217 & Chenopodium glaucum $\mathrm{L}$. & Debrecen & 1946 & Soó Rezső & BPU-10378.JPG \\
\hline 217 & Chenopodium glaucum L. & Hajdúszoboszló & 1937 & Soó Rezső & BPU-10377.JPG \\
\hline 217 & Chenopodium glaucum $\mathrm{L}$. & Kiskunhalas & 1964 & Soó Rezső & BPU-10376.JPG \\
\hline
\end{tabular}


1. Elektroniukus melléklet - Electronic Appendix 1.

\begin{tabular}{|c|c|c|c|c|c|}
\hline $\begin{array}{l}\text { Sorszám / } \\
\text { Number }\end{array}$ & Taxon-név / Taxon-name & $\begin{array}{l}\text { Település / } \\
\text { Settlement }\end{array}$ & $\begin{array}{l}\text { Év / } \\
\text { Year }\end{array}$ & Gyűjtő / Collector & $\begin{array}{l}\text { Fájlnév / } \\
\text { File-name }\end{array}$ \\
\hline 217 & Chenopodium glaucum L. & Kiskunmajsa & 1964 & Soó Rezső & BPU-10372.JPG \\
\hline 217 & Chenopodium glaucum L. & Kiskunmajsa & 1964 & Soó Rezső & BPU-10380.JPG \\
\hline 217 & Chenopodium glaucum L. & Tihany & 1950 & Felföldy Lajos & BPU-10373.JPG \\
\hline 217 & Chenopodium glaucum L. & Vácrátót & 1953 & Felföldy Lajos & BPU-10374.JPG \\
\hline 217 & Chenopodium glaucum L. & Vácrátót & 1953 & Felföldy Lajos & BPU-10375.JPG \\
\hline 218 & Chenopodium rubrum $\mathrm{L}$. & Debrecen & 1943 & Felföldy Lajos & BPU-10362.JPG \\
\hline 218 & Chenopodium rubrum L. & Debrecen & 1946 & Soó Rezső & BPU-10364.JPG \\
\hline 218 & Chenopodium rubrum L. & Kiskunmajsa & 1964 & Soó Rezső & BPU-10361.JPG \\
\hline 218 & Chenopodium rubrum L. & Nyíregyháza & 1933 & Soó Rezső & BPU-10367.JPG \\
\hline 218 & Chenopodium rubrum L. & Tiszafüred & 1952 & Soó Rezső & BPU-10363.JPG \\
\hline 218 & Chenopodium rubrum L. & Tiszafüred & 1952 & Soó Rezső & BPU-15542.JPG \\
\hline 219 & $\begin{array}{l}\text { Chenopodium chenopodioides (L.) } \\
\text { Aellen }\end{array}$ & Hortobágy & 1947 & Soó Rezső & BPU-10368.JPG \\
\hline 219 & $\begin{array}{l}\text { Chenopodium chenopodioides (L.) } \\
\text { Aellen }\end{array}$ & Hortobágy & 1947 & Soó Rezső & BPU-10370.JPG \\
\hline 219 & $\begin{array}{l}\text { Chenopodium chenopodioides (L.) } \\
\text { Aellen }\end{array}$ & Kiskunmajsa & 1964 & Soó Rezső & BPU-10366.JPG \\
\hline 219 & $\begin{array}{l}\text { Chenopodium chenopodioides (L.) } \\
\text { Aellen }\end{array}$ & Tihany & 1946 & Felföldy Lajos & BPU-10365.JPG \\
\hline 220 & Chenopodium vulvaria $\mathrm{L}$. & Tarpa & 1949 & Soó Rezső & BPU-10357.JPG \\
\hline 220 & Chenopodium vulvaria L. & Tihany & 1959 & Felföldy Lajos & BPU-10358.JPG \\
\hline 221 & Chenopodium polyspermum L. & Dömös & 1948 & Soó Rezső & BPU-10351.JPG \\
\hline 221 & Chenopodium polyspermum L. & Egyek & 1932 & Soó Rezső & BPU-10353.JPG \\
\hline 221 & Chenopodium polyspermum L. & Egyek & 1947 & Soó Rezső & BPU-10352.JPG \\
\hline 221 & Chenopodium polyspermum L. & Kiskunhalas & 1964 & Soó Rezső & BPU-10354.JPG \\
\hline 221 & Chenopodium polyspermum L. & Szenna & 1957 & Soó Rezső & BPU-10355.JPG \\
\hline 222 & Chenopodium hybridum L. & Debrecen & 1932 & Soó Rezső & BPU-10360.JPG \\
\hline 222 & Chenopodium hybridum L. & Füzér & 1949 & Simon Tibor, Jakucs Pál & BPU-10359.JPG \\
\hline 223 & Chenopodium murale L. & Tarpa & 1949 & Soó Rezső & BPU-10430.JPG \\
\hline 223 & Chenopodium murale L. & Vác & 1949 & $\begin{array}{l}\text { Soó Rezső, Simon Tibor, } \\
\text { Jakucs Pál }\end{array}$ & BPU-10429.JPG \\
\hline 224 & Chenopodium urbicum L. & Balatonszőlős & 1957 & Felföldy Lajos & BPU-10427.JPG \\
\hline 224 & Chenopodium urbicum L. & Debrecen & 1942 & Felföldy Lajos & BPU-10428.JPG \\
\hline 224 & Chenopodium urbicum L. & Hortobágy & 1947 & Soó Rezső & BPU-10422.JPG \\
\hline 224 & Chenopodium urbicum L. & Hortobágy & 1947 & Soó Rezső & BPU-10425.JPG \\
\hline 224 & Chenopodium urbicum L. & Kaszó & 1957 & Soó Rezső & BPU-10426.JPG \\
\hline 225 & Chenopodium ficifolium Sm. & Győr & 1914 & Polgár Sándor & BPU-10382.JPG \\
\hline 226 & Chenopodium opulifolium Schrad. & Győr & 1909 & Polgár Sándor & BPU-10383.JPG \\
\hline 226 & Chenopodium opulifolium Schrad. & Nyúl & 1913 & Polgár Sándor & BPU-10384.JPG \\
\hline 228 & Chenopodium strictum Roth & Budapest & 1948 & Soó Rezső & BPU-10387.JPG \\
\hline 228 & Chenopodium strictum Roth & Budapest & 1949 & Soó Rezső & BPU-10385.JPG \\
\hline 228 & Chenopodium strictum Roth & Debrecen & 1932 & Soó Rezső & BPU-10386.JPG \\
\hline 229 & Chenopodium album L. & Budapest & 1948 & Soó Rezső & BPU-10391.JPG \\
\hline 229 & Chenopodium album L. & Budapest & 2004 & Felföldy Lajos & BPU-10400.JPG \\
\hline 229 & Chenopodium album L. & Csesznek & 1963 & Soó Rezső & BPU-10401.JPG \\
\hline 229 & Chenopodium album L. & Csesznek & 1963 & Soó Rezső & BPU-10406.JPG \\
\hline 229 & Chenopodium album L. & Csorna & 1925 & Polgár Sándor & BPU-10419.JPG \\
\hline 229 & Chenopodium album L. & Debrecen & 1934 & Soó Rezső & BPU-10394.JPG \\
\hline 229 & Chenopodium album L. & Debrecen & 1942 & Felföldy Lajos & BPU-10398.JPG \\
\hline 229 & Chenopodium album L. & Debrecen & 1945 & Felföldy Lajos & BPU-10404.JPG \\
\hline
\end{tabular}


1. Elektroniukus melléklet - Electronic Appendix 1.

\begin{tabular}{|c|c|c|c|c|c|}
\hline $\begin{array}{l}\text { Sorszám / } \\
\text { Number }\end{array}$ & Taxon-név / Taxon-name & $\begin{array}{l}\text { Település / } \\
\text { Settlement }\end{array}$ & $\begin{array}{l}\text { Év / } \\
\text { Year }\end{array}$ & Gyűjtő / Collector & $\begin{array}{l}\text { Fájlnév / } \\
\text { File-name }\end{array}$ \\
\hline 229 & Chenopodium album L. & Debrecen & 1945 & Felföldy Lajos & BPU-10405.JPG \\
\hline 229 & Chenopodium album L. & Debrecen & 1945 & Felföldy Lajos & BPU-10421.JPG \\
\hline 229 & Chenopodium album L. & Egyek & 1942 & Felföldy Lajos & BPU-10402.JPG \\
\hline 229 & Chenopodium album L. & Gelénes & 1951 & $\begin{array}{l}\text { Simon Tibor, Vozáry } \\
\text { Elemér }\end{array}$ & BPU-10409.JPG \\
\hline 229 & Chenopodium album L. & Gelénes & 1951 & $\begin{array}{l}\text { Simon Tibor, Vozáry } \\
\text { Elemér }\end{array}$ & BPU-10410.JPG \\
\hline 229 & Chenopodium album L. & Győr & 1915 & Polgár Sándor & BPU-10415.JPG \\
\hline 229 & Chenopodium album L. & Győr & 1915 & Polgár Sándor & BPU-10416.JPG \\
\hline 229 & Chenopodium album L. & Győr & 1916 & Polgár Sándor & BPU-10399.JPG \\
\hline 229 & Chenopodium album L. & Győr & 1918 & Polgár Sándor & BPU-10392.JPG \\
\hline 229 & Chenopodium album L. & Győr & 1919 & Polgár Sándor & BPU-10414.JPG \\
\hline 229 & Chenopodium album L. & Győr & 1924 & Polgár Sándor & BPU-10393.JPG \\
\hline 229 & Chenopodium album L. & Győr & 1924 & Polgár Sándor & BPU-10407.JPG \\
\hline 229 & Chenopodium album L. & Győr & 1924 & Polgár Sándor & BPU-10418.JPG \\
\hline 229 & Chenopodium album L. & Győr & 1925 & Polgár Sándor & BPU-10417.JPG \\
\hline 229 & Chenopodium album L. & Győr & 1927 & Polgár Sándor & BPU-10413.JPG \\
\hline 229 & Chenopodium album L. & Győr & 1928 & Polgár Sándor & BPU-10412.JPG \\
\hline 229 & Chenopodium album L. & Győr & 1933 & Polgár Sándor & BPU-10390.JPG \\
\hline 229 & Chenopodium album L. & Hortobágy & 1931 & Soó Rezső & BPU-10395.JPG \\
\hline 229 & Chenopodium album L. & Nyíregyháza & 1933 & Soó Rezső & BPU-10397.JPG \\
\hline 229 & Chenopodium album L. & Nyíregyháza & 1933 & Soó Rezső & BPU-10403.JPG \\
\hline 229 & Chenopodium album L. & Révfülöp & 1956 & $\begin{array}{l}\text { Felföldy Lajos, Tóth } \\
\text { László }\end{array}$ & BPU-10408.JPG \\
\hline 229 & Chenopodium album L. & Sárospatak & 1949 & Simon Tibor & BPU-10388.JPG \\
\hline 229 & Chenopodium album L. & Sárospatak & 1949 & Simon Tibor & BPU-10420.JPG \\
\hline 229 & Chenopodium album L. & Szarvas & 1947 & Soó Rezső & BPU-10389.JPG \\
\hline 229 & Chenopodium album L. & Vácrátót & 1949 & Soó Rezső & BPU-10396.JPG \\
\hline 229 & Chenopodium album L. & Vácrátót & 1952 & Felföldy Lajos & BPU-10411.JPG \\
\hline--- & Chenopodium carnosulum Moq. & Visegrád & 1948 & Priszter Szaniszló & BPU-10356.JPG \\
\hline--- & $\begin{array}{l}\text { Chenopodium leptophyllum (Nutt. ex } \\
\text { Moq.) B.D.Jacks. }\end{array}$ & Győr & 1928 & Polgár Sándor & BPU-10431.JPG \\
\hline--- & Chenopodium multifidum L. & Görgeteg & 1964 & Almádi László & BPU-10432.JPG \\
\hline--- & Chenopodium multifidum L. & Görgeteg & 1964 & Almádi László & BPU-10433.JPG \\
\hline 232 & Atriplex tatarica L. & Budapest & 1948 & Soó Rezső & BPU-10446.JPG \\
\hline 232 & Atriplex tatarica L. & Debrecen & 1932 & Soó Rezső & BPU-10444.JPG \\
\hline 232 & Atriplex tatarica L. & Egyek & 1947 & Soó Rezső & BPU-10447.JPG \\
\hline 232 & Atriplex tatarica L. & Kiskunmajsa & 1964 & Soó Rezső & BPU-10445.JPG \\
\hline 232 & Atriplex tatarica L. & Konyár & 1933 & Soó Rezső & BPU-10448.JPG \\
\hline 233 & Atriplex rosea L. & Tihany & 1928 & Soó Rezső & BPU-10449.JPG \\
\hline 234 & Atriplex littoralis L. & Bugyi & 1950 & Soó Rezső & BPU-10440.JPG \\
\hline 234 & Atriplex littoralis L. & Egyek & 1960 & Simon Tibor & BPU-10443.JPG \\
\hline 234 & Atriplex littoralis L. & Nyíregyháza & 1933 & Soó Rezső & BPU-10439.JPG \\
\hline 234 & Atriplex littoralis L. & Szenna & 1957 & Soó Rezső & BPU-10442.JPG \\
\hline 235 & Atriplex oblongifolia Waldst. et Kit. & Budapest & 1948 & Soó Rezső & BPU-10458.JPG \\
\hline 235 & Atriplex oblongifolia Waldst. et Kit. & Budapest & 1982 & Felföldy Lajos & BPU-10459.JPG \\
\hline 235 & Atriplex oblongifolia Waldst. et Kit. & Debrecen & 1938 & Soó Rezső & BPU-10461.JPG \\
\hline 235 & Atriplex oblongifolia Waldst. et Kit. & Tihany & 1958 & $\begin{array}{l}\text { Felföldy Lajos, Tóth } \\
\text { László }\end{array}$ & BPU-10460.JPG \\
\hline 235 & Atriplex oblongifolia Waldst. et Kit. & Tiszafüred & 1952 & Soó Rezső & BPU-15539.JPG \\
\hline
\end{tabular}


1. Elektroniukus melléklet - Electronic Appendix 1.

\begin{tabular}{|c|c|c|c|c|c|}
\hline $\begin{array}{l}\text { Sorszám / } \\
\text { Number }\end{array}$ & Taxon-név / Taxon-name & $\begin{array}{l}\text { Település / } \\
\text { Settlement }\end{array}$ & $\begin{array}{l}\text { Év / } \\
\text { Year }\end{array}$ & Gyújtő / Collector & $\begin{array}{l}\text { Fájlnév / } \\
\text { File-name }\end{array}$ \\
\hline 236 & Atriplex patula L. & Balmazújváros & 1932 & Soó Rezső & BPU-10462.JPG \\
\hline 236 & Atriplex patula L. & Egyek & 1932 & Soó Rezső & BPU-10463.JPG \\
\hline 236 & Atriplex patula L. & Hortobágy & 1931 & Soó Rezső & BPU-10464.JPG \\
\hline 237 & Atriplex prostrata Boucher & Debrecen & 1932 & Soó Rezső & BPU-10452.JPG \\
\hline 237 & Atriplex prostrata Boucher & Fertőhomok & 1960 & Tóth László & BPU-10456.JPG \\
\hline 237 & Atriplex prostrata Boucher & Hegykő & 1960 & Tóth László & BPU-10455.JPG \\
\hline 237 & Atriplex prostrata Boucher & Hortobágy & 1947 & Soó Rezső & BPU-10450.JPG \\
\hline 237 & Atriplex prostrata Boucher & Hortobágy & 1947 & Soó Rezső & BPU-10451.JPG \\
\hline 237 & Atriplex prostrata Boucher & Hortobágy & 1947 & Soó Rezső & BPU-10453.JPG \\
\hline 237 & Atriplex prostrata Boucher & Konyár & 1933 & Soó Rezső & BPU-10454.JPG \\
\hline 237 & Atriplex prostrata Boucher & Tihany & 1956 & $\begin{array}{l}\text { Felföldy Lajos, Tóth } \\
\text { László }\end{array}$ & BPU-10457.JPG \\
\hline 238 & Atriplex sagittata Borkh. & Gyomaendrőd & 1947 & Soó Rezső & BPU-10437.JPG \\
\hline 238 & Atriplex sagittata Borkh. & Sáránd & 1933 & Soó Rezső & BPU-10438.JPG \\
\hline 238 & Atriplex sagittata Borkh. & Tiszafüred & 1952 & Soó Rezső & BPU-15540.JPG \\
\hline 239 & Atriplex hortensis L. & Debrecen & 1947 & Pólya László & BPU-10436.JPG \\
\hline 240 & $\begin{array}{l}\text { Krascheninnikovia ceratoides (L.) } \\
\text { Gueldenst. }\end{array}$ & Sárbogárd & 1922 & Boros Ádám & BPU-10465.JPG \\
\hline 241 & Camphorosma annua Pall. & Bugyi & 1950 & Soó Rezső & BPU-10466.JPG \\
\hline 241 & Camphorosma annua Pall. & Kiskunmajsa & 1964 & Soó Rezső & BPU-10467.JPG \\
\hline 242 & Kochia scoparia (L.) Schrad. & Budapest & 1982 & Felföldy Lajos & BPU-10470.JPG \\
\hline 242 & Kochia scoparia (L.) Schrad. & Csarnóta & 1968 & Priszter Szaniszló & BPU-10471.JPG \\
\hline 242 & Kochia scoparia (L.) Schrad. & Debrecen & 1938 & Máthé Imre & BPU-10473.JPG \\
\hline 242 & Kochia scoparia (L.) Schrad. & Nagyharsány & 1968 & Priszter Szaniszló & BPU-10472.JPG \\
\hline 243 & Kochia prostrata (L.) Schrad. & Bugac & 1964 & Soó Rezső & BPU-10476.JPG \\
\hline 243 & Kochia prostrata (L.) Schrad. & Hortobágy & 1947 & Soó Rezső & BPU-10474.JPG \\
\hline 243 & Kochia prostrata (L.) Schrad. & Tokaj & 1949 & Soó Rezső & BPU-10475.JPG \\
\hline 244 & Kochia laniflora (S.G. Gmel.) Borbás & Bugac & 1952 & Vozáry Elemér & BPU-10480.JPG \\
\hline 244 & Kochia laniflora (S.G. Gmel.) Borbás & Bugac & 1964 & Soó Rezső & BPU-10477.JPG \\
\hline 244 & Kochia laniflora (S.G. Gmel.) Borbás & Bugac & 1964 & Soó Rezső & BPU-10478.JPG \\
\hline 244 & Kochia laniflora (S.G. Gmel.) Borbás & Dunakeszi & 1948 & Soó Rezső & BPU-10479.JPG \\
\hline 245 & Bassia sedoides (Pall.) Asch. & Gyomaendrőd & 1947 & Soó Rezső & BPU-10468.JPG \\
\hline 245 & Bassia sedoides (Pall.) Asch. & Hortobágy & 1947 & Soó Rezső & BPU-10469.JPG \\
\hline 246 & Corispermum nitidum Kit. & Budapest & 1948 & Soó Rezső & BPU-10483.JPG \\
\hline 246 & Corispermum nitidum Kit. & Győr & 1908 & Polgár Sándor & BPU-10481.JPG \\
\hline 246 & Corispermum nitidum Kit. & Veresegyház & 1950 & Soó Rezső & BPU-10482.JPG \\
\hline 248 & Corispermum canescens Kit. & Budapest & 1913 & Degen Árpád & BPU-10486.JPG \\
\hline 248 & Corispermum canescens Kit. & Tatárszentgyörgy & 1950 & Soó Rezső & BPU-10487.JPG \\
\hline 248 & Corispermum canescens Kit. & Vácrátót & 1949 & Soó Rezső & BPU-10484.JPG \\
\hline 248 & Corispermum canescens Kit. & Vácrátót & 1950 & Soó Rezső & BPU-10485.JPG \\
\hline 250 & Salicornia prostrata Pall. & Budapest & 1903 & Kocsis István & BPU-10489.JPG \\
\hline 251 & Suaeda pannonica (Beck) Graebn. & Kiskunmajsa & 1964 & Soó Rezső & BPU-10496.JPG \\
\hline 251 & Suaeda pannonica (Beck) Graebn. & Kunadacs & 1964 & Soó Rezső & BPU-10495.JPG \\
\hline 251 & Suaeda pannonica (Beck) Graebn. & Nyíregyháza & 1933 & Soó Rezső & BPU-10492.JPG \\
\hline 251 & Suaeda pannonica (Beck) Graebn. & Sárkeresztúr & 1935 & Boros Ádám & BPU-10497.JPG \\
\hline 254 & Salsola soda L. & Egyek & 1933 & Soó Rezső & BPU-10499.JPG \\
\hline 254 & Salsola soda L. & Szabadszállás & 1918 & Andrasovszky József & BPU-10500.JPG \\
\hline 255 & Salsola kali L. & Debrecen & 1930 & Soó Rezső & BPU-10498.JPG \\
\hline
\end{tabular}


1. Elektroniukus melléklet - Electronic Appendix 1.

\begin{tabular}{|c|c|c|c|c|c|}
\hline $\begin{array}{l}\text { Sorszám / } \\
\text { Number }\end{array}$ & Taxon-név / Taxon-name & $\begin{array}{l}\text { Település / } \\
\text { Settlement }\end{array}$ & $\begin{array}{l}\text { Év / } \\
\text { Year }\end{array}$ & Gyűjtő / Collector & $\begin{array}{l}\text { Fájlnév / } \\
\text { File-name }\end{array}$ \\
\hline 260 & Amaranthus retroflexus L. & Balmazújváros & 1932 & Soó Rezső & BPU-10522.JPG \\
\hline 260 & Amaranthus retroflexus L. & Bodajk & 1948 & Kárpáti Zoltán & BPU-10521.JPG \\
\hline 260 & Amaranthus retroflexus L. & Budapest & 1915 & Lyka Károly & BPU-10506.JPG \\
\hline 260 & Amaranthus retroflexus L. & Budapest & 1945 & Priszter Szaniszló & BPU-10509.JPG \\
\hline 260 & Amaranthus retroflexus L. & Budapest & 1945 & Priszter Szaniszló & BPU-10510.JPG \\
\hline 260 & Amaranthus retroflexus L. & Budapest & 1946 & Priszter Szaniszló & BPU-10501.JPG \\
\hline 260 & Amaranthus retroflexus L. & Budapest & 1946 & Priszter Szaniszló & BPU-10512.JPG \\
\hline 260 & Amaranthus retroflexus L. & Budapest & 1946 & Priszter Szaniszló & BPU-10513.JPG \\
\hline 260 & Amaranthus retroflexus L. & Budapest & 1946 & Priszter Szaniszló & BPU-10514.JPG \\
\hline 260 & Amaranthus retroflexus L. & Budapest & 1946 & Priszter Szaniszló & BPU-10515.JPG \\
\hline 260 & Amaranthus retroflexus L. & Budapest & 1946 & Priszter Szaniszló & BPU-10516.JPG \\
\hline 260 & Amaranthus retroflexus L. & Budapest & 1947 & Priszter Szaniszló & BPU-10502.JPG \\
\hline 260 & Amaranthus retroflexus L. & Budapest & 1947 & Priszter Szaniszló & BPU-10503.JPG \\
\hline 260 & Amaranthus retroflexus L. & Budapest & 1947 & Priszter Szaniszló & BPU-10504.JPG \\
\hline 260 & Amaranthus retroflexus L. & Budapest & 1947 & Priszter Szaniszló & BPU-10508.JPG \\
\hline 260 & Amaranthus retroflexus L. & Budapest & 1947 & Priszter Szaniszló & BPU-10518.JPG \\
\hline 260 & Amaranthus retroflexus L. & Budapest & 1947 & Priszter Szaniszló & BPU-10519.JPG \\
\hline 260 & Amaranthus retroflexus L. & Budapest & 1947 & Priszter Szaniszló & BPU-10520.JPG \\
\hline 260 & Amaranthus retroflexus L. & Budapest & 1947 & Soó Rezső & BPU-10511.JPG \\
\hline 260 & Amaranthus retroflexus L. & Budapest & 1948 & Kárpáti Zoltán & BPU-10507.JPG \\
\hline 260 & Amaranthus retroflexus L. & Budapest & 1948 & Soó Rezső & BPU-10505.JPG \\
\hline 260 & Amaranthus retroflexus L. & Budapest & $?$ & Priszter Szaniszló & BPU-10517.JPG \\
\hline 260 & Amaranthus retroflexus L. & Debrecen & 1931 & Soó Rezső & BPU-10524.JPG \\
\hline 260 & Amaranthus retroflexus L. & Hortobágy & 1947 & Soó Rezső & BPU-10523.JPG \\
\hline 260 & Amaranthus retroflexus L. & Szolnok & 1968 & Priszter Szaniszló & BPU-10525.JPG \\
\hline 261 & Amaranthus patulus Bertol. & Budapest & 1947 & Priszter Szaniszló & BPU-10544.JPG \\
\hline 261 & Amaranthus patulus Bertol. & Budapest & 1947 & Priszter Szaniszló & BPU-10545.JPG \\
\hline 261 & Amaranthus patulus Bertol. & Budapest & 1947 & Priszter Szaniszló & BPU-10546.JPG \\
\hline 261 & Amaranthus patulus Bertol. & Budapest & 1948 & Soó Rezső & BPU-10547.JPG \\
\hline 262 & Amaranthus powellii S. Watson & "Csepel-sziget" & 1947 & Priszter Szaniszló & BPU-10526.JPG \\
\hline 262 & Amaranthus powellii S. Watson & Budapest & 1946 & Priszter Szaniszló & BPU-10527.JPG \\
\hline 262 & Amaranthus powellii S. Watson & Budapest & 1946 & Priszter Szaniszló & BPU-10528.JPG \\
\hline 262 & Amaranthus powellii S. Watson & Budapest & 1947 & Priszter Szaniszló & BPU-10529.JPG \\
\hline 262 & Amaranthus powellii S. Watson & Budapest & 1948 & Soó Rezső & BPU-10531.JPG \\
\hline 262 & Amaranthus powellii S. Watson & Budapest & 1949 & Priszter Szaniszló & BPU-10530.JPG \\
\hline 262 & Amaranthus powellii S. Watson & Bugac & 1964 & Soó Rezső & BPU-10532.JPG \\
\hline 262 & Amaranthus powellii S. Watson & Debrecen & 1948 & Soó Rezső & BPU-10536.JPG \\
\hline 262 & Amaranthus powellii S. Watson & Dömös & 1948 & Soó Rezső & BPU-10534.JPG \\
\hline 262 & Amaranthus powellii S. Watson & Esztergom & 1947 & Priszter Szaniszló & BPU-10533.JPG \\
\hline 262 & Amaranthus powellii S. Watson & Gyöngyös & 1955 & Soó Rezső & BPU-10537.JPG \\
\hline 262 & Amaranthus powellii S. Watson & Ócsa & 1965 & Priszter Szaniszló & BPU-10539.JPG \\
\hline 262 & Amaranthus powellii S. Watson & Sopron & 1947 & Priszter Szaniszló & BPU-10541.JPG \\
\hline 262 & Amaranthus powellii S. Watson & Sopron & 1947 & Priszter Szaniszló & BPU-10542.JPG \\
\hline 262 & Amaranthus powellii S. Watson & Tárkány & 1932 & Polgár Sándor & BPU-10538.JPG \\
\hline 262 & Amaranthus powellii S. Watson & Visegrád & 1968 & Priszter Szaniszló & BPU-10540.JPG \\
\hline 262 & Amaranthus powellii S. Watson & Zámoly & 1982 & Felföldy Lajos & BPU-10535.JPG \\
\hline 263 & Amaranthus viridis L. & Budapest & 1948 & Priszter Szaniszló & BPU-14852.JPG \\
\hline
\end{tabular}


1. Elektroniukus melléklet - Electronic Appendix 1.

\begin{tabular}{|c|c|c|c|c|c|}
\hline $\begin{array}{l}\text { Sorszám / } \\
\text { Number }\end{array}$ & Taxon-név / Taxon-name & $\begin{array}{l}\text { Település / } \\
\text { Settlement }\end{array}$ & $\begin{array}{l}\text { Év / } \\
\text { Year }\end{array}$ & Gyújtő / Collector & $\begin{array}{l}\text { Fájlnév / } \\
\text { File-name }\end{array}$ \\
\hline 263 & Amaranthus viridis L. & Győr & 1927 & Polgár Sándor & BPU-14851.JPG \\
\hline 264 & Amaranthus blitum L. & Budapest & 1947 & Priszter Szaniszló & BPU-14920.JPG \\
\hline 264 & Amaranthus blitum L. & Debrecen & 1942 & Felföldy Lajos & BPU-14918.JPG \\
\hline 264 & Amaranthus blitum L. & Hortobágy & 1947 & Soó Rezső & BPU-14918.JPG \\
\hline 264 & Amaranthus blitum L. & Kishuta & 1952 & Simon Tibor & BPU-14919.JPG \\
\hline 264 & Amaranthus blitum L. & Nagykanizsa & 1950 & Károlyi Árpád & BPU-10575.JPG \\
\hline 264 & Amaranthus blitum L. & Nagykanizsa & 1950 & Károlyi Árpád & BPU-14915.JPG \\
\hline 264 & Amaranthus blitum L. & Szolnok & 1947 & Timár Lajos & BPU-14917.JPG \\
\hline 264 & Amaranthus blitum L. & Tihany & 1942 & Felföldy Lajos & BPU-14916.JPG \\
\hline 265 & Amaranthus deflexus L. & Budapest & 1946 & Priszter Szaniszló & BPU-14910.JPG \\
\hline 265 & Amaranthus deflexus L. & Budapest & 1946 & Priszter Szaniszló & BPU-14912.JPG \\
\hline 265 & Amaranthus deflexus L. & Budapest & 1946 & Priszter Szaniszló & BPU-14913.JPG \\
\hline 265 & Amaranthus deflexus L. & Budapest & 1947 & Soó Rezső & BPU-14909.JPG \\
\hline 265 & Amaranthus deflexus L. & Budapest & 1948 & Priszter Szaniszló & BPU-14914.JPG \\
\hline 265 & Amaranthus deflexus L. & Budapest & 1949 & Priszter Szaniszló & BPU-14911.JPG \\
\hline 265 & Amaranthus deflexus L. & Debrecen & 1948 & Soó Rezső & BPU-14908.JPG \\
\hline 265 & Amaranthus deflexus L. & Kaszó & 1957 & Soó Rezső & BPU-14907.JPG \\
\hline 265 & Amaranthus deflexus L. & Nagykanizsa & 1948 & Károlyi Árpád & BPU-14906.JPG \\
\hline 266 & Amaranthus blitoides S. Watson & Budapest & 1947 & Priszter Szaniszló & BPU-10558.JPG \\
\hline 266 & Amaranthus blitoides S. Watson & Budapest & 1947 & Priszter Szaniszló & BPU-10560.JPG \\
\hline 266 & Amaranthus blitoides S. Watson & Budapest & 1947 & Soó Rezső & BPU-10564.JPG \\
\hline 266 & Amaranthus blitoides S. Watson & Budapest & 1948 & Priszter Szaniszló & BPU-10559.JPG \\
\hline 266 & Amaranthus blitoides S. Watson & Budapest & 1948 & Priszter Szaniszló & BPU-10561.JPG \\
\hline 266 & Amaranthus blitoides S. Watson & Budapest & 1948 & Soó Rezső & BPU-10562.JPG \\
\hline 266 & Amaranthus blitoides S. Watson & Budapest & 1948 & Soó Rezső & BPU-10563.JPG \\
\hline 266 & Amaranthus blitoides S. Watson & Bugac & 1964 & Soó Rezső & BPU-10565.JPG \\
\hline 266 & Amaranthus blitoides S. Watson & Zámoly & 1982 & Felföldy Lajos & BPU-10566.JPG \\
\hline 267 & $\begin{array}{l}\text { Amaranthus crispus (Lesp. et } \\
\text { Thévenau) N. Terracc. }\end{array}$ & Budapest & 1946 & Priszter Szaniszló & BPU-14904.JPG \\
\hline 267 & $\begin{array}{l}\text { Amaranthus crispus (Lesp. et } \\
\text { Thévenau) N. Terracc. }\end{array}$ & Budapest & 1947 & Soó Rezső & BPU-14903.JPG \\
\hline 267 & $\begin{array}{l}\text { Amaranthus crispus (Lesp. et } \\
\text { Thévenau) N. Terracc. }\end{array}$ & Budapest & 1948 & Priszter Szaniszló & BPU-14905.JPG \\
\hline 267 & $\begin{array}{l}\text { Amaranthus crispus (Lesp. et } \\
\text { Thévenau) N. Terracc. }\end{array}$ & Bükkszék & 1949 & Károlyi Árpád & BPU-14899.JPG \\
\hline 267 & $\begin{array}{l}\text { Amaranthus crispus (Lesp. et } \\
\text { Thévenau) N. Terracc. }\end{array}$ & Debrecen & 1915 & Polgár Sándor & BPU-14901.JPG \\
\hline 267 & $\begin{array}{l}\text { Amaranthus crispus (Lesp. et } \\
\text { Thévenau) N. Terracc. }\end{array}$ & Egyek & 1947 & Soó Rezső & BPU-14900.JPG \\
\hline 267 & $\begin{array}{l}\text { Amaranthus crispus (Lesp. et } \\
\text { Thévenau) N. Terracc. }\end{array}$ & Szeged & 1957 & Timár Lajos & BPU-14902.JPG \\
\hline 267 & $\begin{array}{l}\text { Amaranthus crispus (Lesp. et } \\
\text { Thévenau) N. Terracc. }\end{array}$ & Tokaj & 1946 & Priszter Szaniszló & BPU-14898.JPG \\
\hline 269 & Amaranthus albus L. & Budapest & 1946 & Priszter Szaniszló & BPU-10549.JPG \\
\hline 269 & Amaranthus albus L. & Budapest & 1947 & Priszter Szaniszló & BPU-10552.JPG \\
\hline 269 & Amaranthus albus L. & Budapest & 1947 & Soó Rezső & BPU-10550.JPG \\
\hline 269 & Amaranthus albus L. & Budapest & 1956 & Priszter Szaniszló & BPU-10551.JPG \\
\hline 269 & Amaranthus albus L. & Bugyi & 1917 & Magyar Ant. & BPU-10556.JPG \\
\hline 269 & Amaranthus albus L. & Debrecen & 1931 & Soó Rezső & BPU-10553.JPG \\
\hline 269 & Amaranthus albus L. & Debrecen & 1953 & Kulcsár Gábor & BPU-15456.JPG \\
\hline 269 & Amaranthus albus L. & Egyek & 1947 & Soó Rezső & BPU-10554.JPG \\
\hline
\end{tabular}


1. Elektroniukus melléklet - Electronic Appendix 1.

\begin{tabular}{|c|c|c|c|c|c|}
\hline $\begin{array}{l}\text { Sorszám / } \\
\text { Number }\end{array}$ & Taxon-név / Taxon-name & $\begin{array}{l}\text { Település / } \\
\text { Settlement }\end{array}$ & $\begin{array}{l}\text { Év / } \\
\text { Year }\end{array}$ & Gyújtő / Collector & $\begin{array}{l}\text { Fájlnév / } \\
\text { File-name }\end{array}$ \\
\hline 269 & Amaranthus albus L. & Nagymaros & 1950 & Simon Tibor, Borsos Olga & BPU-10555.JPG \\
\hline 269 & Amaranthus albus L. & Szenna & 1957 & Soó Rezső & BPU-10557.JPG \\
\hline 270 & Amaranthus graecizans L. & Balatonalmádi & 1963 & Soó Rezső & BPU-10573.JPG \\
\hline 270 & Amaranthus graecizans L. & Balatongyörök & 1949 & Priszter Szaniszló & BPU-10574.JPG \\
\hline 270 & Amaranthus graecizans L. & Budapest & 1947 & Priszter Szaniszló & BPU-10567.JPG \\
\hline 270 & Amaranthus graecizans L. & Budapest & 1947 & Priszter Szaniszló & BPU-10568.JPG \\
\hline 270 & Amaranthus graecizans L. & Budapest & 1948 & Soó Rezső & BPU-10569.JPG \\
\hline 270 & Amaranthus graecizans L. & Dömös & 1947 & Priszter Szaniszló & BPU-10570.JPG \\
\hline 270 & Amaranthus graecizans L. & Esztergom & 1948 & Priszter Szaniszló & BPU-10571.JPG \\
\hline 270 & Amaranthus graecizans L. & Győr & 1913 & Polgár Sándor & BPU-10572.JPG \\
\hline $260-261$ & $\begin{array}{l}\text { Amaranthus retroflexus L. - } \\
\text { Amaranthus patulus Bertol. }\end{array}$ & Budapest & 1947 & Priszter Szaniszló & BPU-14892.JPG \\
\hline $261-262$ & $\begin{array}{l}\text { Amaranthus patulus Bertol. - } \\
\text { Amaranthus powellii S. Watson }\end{array}$ & Budapest & 1946 & Priszter Szaniszló & BPU-14895.JPG \\
\hline $261-262$ & $\begin{array}{l}\text { Amaranthus patulus Bertol. - } \\
\text { Amaranthus powellii S. Watson }\end{array}$ & Budapest & 1947 & Priszter Szaniszló & BPU-14894.JPG \\
\hline $261-262$ & $\begin{array}{l}\text { Amaranthus patulus Bertol. - } \\
\text { Amaranthus powellii S. Watson }\end{array}$ & Dömös & 1947 & Priszter Szaniszló & BPU-14893.JPG \\
\hline $260 \times 261$ & $\begin{array}{l}\text { Amaranthus retroflexus L. } \times \\
\text { Amaranthus patulus Bertol. }\end{array}$ & Budapest & 1946 & Priszter Szaniszló & BPU-14869.JPG \\
\hline $260 \times 261$ & $\begin{array}{l}\text { Amaranthus retroflexus L. } \times \\
\text { Amaranthus patulus Bertol. }\end{array}$ & Budapest & 1947 & Priszter Szaniszló & BPU-14864.JPG \\
\hline $260 \times 261$ & $\begin{array}{l}\text { Amaranthus retroflexus L. } \times \\
\text { Amaranthus patulus Bertol. }\end{array}$ & Budapest & 1947 & Priszter Szaniszló & BPU-14867.JPG \\
\hline $260 \times 261$ & $\begin{array}{l}\text { Amaranthus retroflexus L. } \times \\
\text { Amaranthus patulus Bertol. }\end{array}$ & Budapest & 1947 & Priszter Szaniszló & BPU-14868.JPG \\
\hline $260 \times 261$ & $\begin{array}{l}\text { Amaranthus retroflexus L. } \times \\
\text { Amaranthus patulus Bertol. }\end{array}$ & Budapest & 1947 & $\begin{array}{l}\text { Priszter Szaniszló, Kárpáti } \\
\text { Zoltán }\end{array}$ & BPU-14866.JPG \\
\hline $260 \times 261$ & $\begin{array}{l}\text { Amaranthus retroflexus L. } \times \\
\text { Amaranthus patulus Bertol. }\end{array}$ & Budapest & 1948 & Priszter Szaniszló & BPU-14865.JPG \\
\hline $260 \times 262$ & $\begin{array}{l}\text { Amaranthus retroflexus L. } \times \\
\text { Amaranthus powellii S. Watson }\end{array}$ & Bodajk & 1948 & Kárpáti Zoltán & BPU-14860.JPG \\
\hline $260 \times 262$ & $\begin{array}{l}\text { Amaranthus retroflexus L. } \times \\
\text { Amaranthus powellii S. Watson }\end{array}$ & Budapest & 1949 & Priszter Szaniszló & BPU-14863.JPG \\
\hline $260 \times 262$ & $\begin{array}{l}\text { Amaranthus retroflexus L. } \times \\
\text { Amaranthus powellii S. Watson }\end{array}$ & Dömös & 1948 & Soó Rezső & BPU-14861.JPG \\
\hline $260 \times 262$ & $\begin{array}{l}\text { Amaranthus retroflexus L. } \times \\
\text { Amaranthus powellii S. Watson }\end{array}$ & Győr & 1926 & Polgár Sándor & BPU-14846.JPG \\
\hline $260 \times 262$ & $\begin{array}{l}\text { Amaranthus retroflexus L. } \times \\
\text { Amaranthus powellii S. Watson }\end{array}$ & Sopron & 1942 & Kárpáti Zoltán & BPU-14896.JPG \\
\hline $260 \times 262$ & $\begin{array}{l}\text { Amaranthus retroflexus L. } \times \\
\text { Amaranthus powellii S. Watson }\end{array}$ & Sopron & 1947 & Kárpáti Zoltán & BPU-14858.JPG \\
\hline $260 \times 262$ & $\begin{array}{l}\text { Amaranthus retroflexus L. } \times \\
\text { Amaranthus powellii S. Watson }\end{array}$ & Sopron & 1947 & Priszter Szaniszló & BPU-14897.JPG \\
\hline $260 \times 262$ & $\begin{array}{l}\text { Amaranthus retroflexus L. } \times \\
\text { Amaranthus powellii S. Watson }\end{array}$ & Szeged & 1949 & Timár Lajos & BPU-14862.JPG \\
\hline $260 \times 262$ & $\begin{array}{l}\text { Amaranthus retroflexus L. } \times \\
\text { Amaranthus powellii S. Watson }\end{array}$ & Tököl & 1947 & Priszter Szaniszló & BPU-14859.JPG \\
\hline $265 \times 267$ & $\begin{array}{l}\text { Amaranthus deflexus L. } \times \text { Amaranthus } \\
\text { crispus (Lesp. et Thévenau) N. } \\
\text { Terracc. }\end{array}$ & Budapest & 1946 & $\begin{array}{l}\text { Kárpáti Zoltán, Priszter } \\
\text { Szaniszló }\end{array}$ & BPU-14887.JPG \\
\hline $265 \times 267$ & $\begin{array}{l}\text { Amaranthus deflexus L. } \times \text { Amaranthus } \\
\text { crispus (Lesp. et Thévenau) N. } \\
\text { Terracc. }\end{array}$ & Budapest & 1947 & Kárpáti Zoltán & BPU-14854.JPG \\
\hline $265 \times 267$ & $\begin{array}{l}\text { Amaranthus deflexus L. } \times \text { Amaranthus } \\
\text { crispus (Lesp. et Thévenau) N. } \\
\text { Terracc. }\end{array}$ & Budapest & 1947 & Kárpáti Zoltán & BPU-14855.JPG \\
\hline
\end{tabular}


1. Elektroniukus melléklet - Electronic Appendix 1.

\begin{tabular}{|c|c|c|c|c|c|}
\hline $\begin{array}{l}\text { Sorszám / } \\
\text { Number }\end{array}$ & Taxon-név / Taxon-name & $\begin{array}{l}\text { Település / } \\
\text { Settlement }\end{array}$ & $\begin{array}{l}\text { Év / } \\
\text { Year }\end{array}$ & Gyưjtő / Collector & $\begin{array}{l}\text { Fájlnév / } \\
\text { File-name }\end{array}$ \\
\hline $265 \times 267$ & $\begin{array}{l}\text { Amaranthus deflexus L. } \times \text { Amaranthus } \\
\text { crispus (Lesp. et Thévenau) N. } \\
\text { Terracc. }\end{array}$ & Budapest & 1947 & Kárpáti Zoltán & BPU-14856.JPG \\
\hline $265 \times 267$ & $\begin{array}{l}\text { Amaranthus deflexus L. } \times \text { Amaranthus } \\
\text { crispus (Lesp. et Thévenau) N. } \\
\text { Terracc. }\end{array}$ & Budapest & 1947 & Priszter Szaniszló & BPU-14857.JPG \\
\hline $265 \times 267$ & $\begin{array}{l}\text { Amaranthus deflexus L. } \times \text { Amaranthus } \\
\text { crispus (Lesp. et Thévenau) N. } \\
\text { Terracc. }\end{array}$ & Budapest & 1947 & Priszter Szaniszló & BPU-14888.JPG \\
\hline $265 \times 267$ & $\begin{array}{l}\text { Amaranthus deflexus L. } \times \text { Amaranthus } \\
\text { crispus (Lesp. et Thévenau) N. } \\
\text { Terracc. }\end{array}$ & Budapest & 1947 & Priszter Szaniszló & BPU-14889.JPG \\
\hline $265 \times 267$ & $\begin{array}{l}\text { Amaranthus deflexus L. } \times \text { Amaranthus } \\
\text { crispus (Lesp. et Thévenau) N. } \\
\text { Terracc. }\end{array}$ & Budapest & 1947 & Priszter Szaniszló & BPU-14890.JPG \\
\hline $265 \times 267$ & $\begin{array}{l}\text { Amaranthus deflexus L. } \times \text { Amaranthus } \\
\text { crispus (Lesp. et Thévenau) N. } \\
\text { Terracc. }\end{array}$ & Budapest & 1947 & Priszter Szaniszló & BPU-14891.JPG \\
\hline $265 \times 267$ & $\begin{array}{l}\text { Amaranthus deflexus L. } \times \text { Amaranthus } \\
\text { crispus (Lesp. et Thévenau) N. } \\
\text { Terracc. }\end{array}$ & Budapest & 1947 & Soó Rezső & BPU-14886.JPG \\
\hline $265 \times 267$ & $\begin{array}{l}\text { Amaranthus deflexus L. } \times \text { Amaranthus } \\
\text { crispus (Lesp. et Thévenau) N. } \\
\text { Terracc. }\end{array}$ & Budapest & 1948 & Kárpáti Zoltán & BPU-14884.JPG \\
\hline $265 \times 267$ & $\begin{array}{l}\text { Amaranthus deflexus L. } \times \text { Amaranthus } \\
\text { crispus (Lesp. et Thévenau) N. } \\
\text { Terracc. }\end{array}$ & Cegléd & 1947 & Boros Ádám & BPU-14885.JPG \\
\hline $266 \times 269$ & $\begin{array}{l}\text { Amaranthus blitoides S. Watson } \times \\
\text { Amaranthus albus L. }\end{array}$ & Budapest & 1946 & Priszter Szaniszló & BPU-14871.JPG \\
\hline $266 \times 269$ & $\begin{array}{l}\text { Amaranthus blitoides S. Watson } \times \\
\text { Amaranthus albus L. }\end{array}$ & Budapest & 1946 & Priszter Szaniszló & BPU-14876.JPG \\
\hline $266 \times 269$ & $\begin{array}{l}\text { Amaranthus blitoides S. Watson } \times \\
\text { Amaranthus albus L. }\end{array}$ & Budapest & 1946 & Priszter Szaniszló & BPU-15425.JPG \\
\hline $266 \times 269$ & $\begin{array}{l}\text { Amaranthus blitoides S. Watson } \times \\
\text { Amaranthus albus L. }\end{array}$ & Budapest & 1947 & Priszter Szaniszló & BPU-14870.JPG \\
\hline $266 \times 269$ & $\begin{array}{l}\text { Amaranthus blitoides S. Watson } \times \\
\text { Amaranthus albus L. }\end{array}$ & Budapest & 1947 & Priszter Szaniszló & BPU-14872.JPG \\
\hline $266 \times 269$ & $\begin{array}{l}\text { Amaranthus blitoides S. Watson } \times \\
\text { Amaranthus albus L. }\end{array}$ & Budapest & 1947 & Priszter Szaniszló & BPU-14873.JPG \\
\hline $266 \times 269$ & $\begin{array}{l}\text { Amaranthus blitoides S. Watson } \times \\
\text { Amaranthus albus L. }\end{array}$ & Budapest & 1947 & Priszter Szaniszló & BPU-14874.JPG \\
\hline $266 \times 269$ & $\begin{array}{l}\text { Amaranthus blitoides S. Watson } \times \\
\text { Amaranthus albus L. }\end{array}$ & Budapest & 1947 & Priszter Szaniszló & BPU-14875.JPG \\
\hline $266 \times 269$ & $\begin{array}{l}\text { Amaranthus blitoides S. Watson } \times \\
\text { Amaranthus albus L. }\end{array}$ & Budapest & 1947 & Priszter Szaniszló & BPU-14877.JPG \\
\hline $266 \times 269$ & $\begin{array}{l}\text { Amaranthus blitoides S. Watson } \times \\
\text { Amaranthus albus L. }\end{array}$ & Budapest & 1947 & Priszter Szaniszló & BPU-14878.JPG \\
\hline $266 \times 269$ & $\begin{array}{l}\text { Amaranthus blitoides S. Watson } \times \\
\text { Amaranthus albus L. }\end{array}$ & Budapest & 1947 & Priszter Szaniszló & BPU-14879.JPG \\
\hline $266 \times 269$ & $\begin{array}{l}\text { Amaranthus blitoides S. Watson } \times \\
\text { Amaranthus albus L. }\end{array}$ & Budapest & 1947 & Priszter Szaniszló & BPU-14880.JPG \\
\hline $266 \times 269$ & $\begin{array}{l}\text { Amaranthus blitoides S. Watson } \times \\
\text { Amaranthus albus L. }\end{array}$ & Budapest & 1948 & Soó Rezső & BPU-14881.JPG \\
\hline $266 \times 269$ & $\begin{array}{l}\text { Amaranthus blitoides S. Watson } \times \\
\text { Amaranthus albus L. }\end{array}$ & Budapest & 1948 & Soó Rezső & BPU-14882.JPG \\
\hline $266 \times 269$ & $\begin{array}{l}\text { Amaranthus blitoides S. Watson } \times \\
\text { Amaranthus albus L. }\end{array}$ & Bugac & 1964 & Soó Rezső & BPU-14883.JPG \\
\hline--- & Amaranthus hypochondriacus L. & Győr & 1915 & Polgár Sándor & BPU-10543.JPG \\
\hline--- & Amaranthus paniculatus L. & Debrecen & 1947 & Soó Rezső & BPU-10548.JPG \\
\hline--- & Amaranthus quitensis & Győr & 1913 & Polgár Sándor & BPU-14849.JPG \\
\hline
\end{tabular}


1. Elektroniukus melléklet - Electronic Appendix 1.

\begin{tabular}{|c|c|c|c|c|c|}
\hline $\begin{array}{l}\text { Sorszám / } \\
\text { Number }\end{array}$ & Taxon-név / Taxon-name & $\begin{array}{l}\text { Település / } \\
\text { Settlement }\end{array}$ & $\begin{array}{l}\text { Év / } \\
\text { Year }\end{array}$ & Gyưjtő / Collector & $\begin{array}{l}\text { Fájlnév / } \\
\text { File-name }\end{array}$ \\
\hline--- & Amaranthus quitensis & Győr & 1913 & Polgár Sándor & BPU-14850.JPG \\
\hline--- & Amaranthus quitensis & Győr & 1915 & Polgár Sándor & BPU-14848.JPG \\
\hline--- & Amaranthus quitensis & Győr & 1918 & Polgár Sándor & BPU-14847.JPG \\
\hline--- & Amaranthus quitensis & Győr & 1926 & Polgár Sándor & BPU-14845.JPG \\
\hline 272 & $\begin{array}{l}\text { Oxybaphus nyctagineus (Michx.) } \\
\text { Sweet }\end{array}$ & Budapest & 1947 & Soó Rezső & BPU-11034.JPG \\
\hline 272 & $\begin{array}{l}\text { Oxybaphus nyctagineus (Michx.) } \\
\text { Sweet }\end{array}$ & Budapest & 1982 & Felföldy Lajos & BPU-11033.JPG \\
\hline 273 & Phytolacca americana L. & Nyíregyháza & 1937 & Soó Rezső & BPU-11035.JPG \\
\hline 274 & Phytolacca esculenta van Houtte & Budapest & 1964 & Priszter Szaniszló & BPU-11036.JPG \\
\hline 276 & Portulaca oleracea L. & Debrecen & 1953 & Kulcsár Gábor & BPU-11038.JPG \\
\hline 278 & Montia fontana L. & Görgeteg & 1923 & Boros Ádám & BPU-11037.JPG \\
\hline 280 & Arenaria procera Spreng. & Kisgyőr & 1913 & Hulják János & BPU-14977.JPG \\
\hline 280 & Arenaria procera Spreng. & Szin & 1951 & Jakucs Pál, Ér Lajos & BPU-14979.JPG \\
\hline 281 & Arenaria serpyllifolia L. & Budaörs & 1982 & Felföldy Lajos & BPU-14970.JPG \\
\hline 281 & Arenaria serpyllifolia L. & Budapest & 1991 & Felföldy Lajos & BPU-14975.JPG \\
\hline 281 & Arenaria serpyllifolia L. & Budapest & 1991 & Felföldy Lajos & BPU-14976.JPG \\
\hline 281 & Arenaria serpyllifolia L. & Hajdúnánás & 1935 & Soó Rezső & BPU-14972.JPG \\
\hline 281 & Arenaria serpyllifolia L. & Hortobágy & 1930 & Soó Rezső & BPU-14971.JPG \\
\hline 281 & Arenaria serpyllifolia L. & Kapolcs & 1959 & $\begin{array}{l}\text { Jávorka Sándor, Felföldy } \\
\text { Lajos, Tóth László }\end{array}$ & BPU-14968.JPG \\
\hline 281 & Arenaria serpyllifolia L. & Nagyharsány & 1965 & $\begin{array}{l}\text { Simon Tibor, Priszter } \\
\text { Szaniszló }\end{array}$ & BPU-14974.JPG \\
\hline 281 & Arenaria serpyllifolia L. & Pécs & 1953 & Soó Rezső, Borsos Olga & BPU-14973.JPG \\
\hline 281 & Arenaria serpyllifolia L. & Tihany & 1956 & Felföldy Lajos & BPU-14967.JPG \\
\hline 281 & Arenaria serpyllifolia L. & Tihany & 1959 & Felföldy Lajos & BPU-14964.JPG \\
\hline 281 & Arenaria serpyllifolia L. & Tihany & 1959 & Felföldy Lajos & BPU-14965.JPG \\
\hline 281 & Arenaria serpyllifolia L. & Tihany & 1959 & Felföldy Lajos & BPU-14966.JPG \\
\hline 281 & Arenaria serpyllifolia L. & Uzsa & 1959 & $\begin{array}{l}\text { Jávorka Sándor, Felföldy } \\
\text { Lajos }\end{array}$ & BPU-14962.JPG \\
\hline 281 & Arenaria serpyllifolia L. & Uzsa & 1959 & $\begin{array}{l}\text { Jávorka Sándor, Felföldy } \\
\text { Lajos }\end{array}$ & BPU-14963.JPG \\
\hline 281 & Arenaria serpyllifolia L. & Vácrátót & 1953 & Felföldy Lajos & BPU-14969.JPG \\
\hline 282 & Arenaria leptoclados (Rchb.) Guss. & Budapest & 2004 & Felföldy Lajos & BPU-14961.JPG \\
\hline 282 & Arenaria leptoclados (Rchb.) Guss. & Lábatlan & 1953 & Felföldy Lajos & BPU-14960.JPG \\
\hline 283 & Moehringia trinervia (L.) Clairv. & "Cuha-völgy" & 1954 & Soó Rezső & BPU-14950.JPG \\
\hline 283 & Moehringia trinervia (L.) Clairv. & Debrecen & 1934 & Soó Rezső & BPU-14953.JPG \\
\hline 283 & Moehringia trinervia (L.) Clairv. & Kapolcs & 1959 & $\begin{array}{l}\text { Jávorka Sándor, Felföldy } \\
\text { Lajos, Tóth László }\end{array}$ & BPU-14949.JPG \\
\hline 283 & Moehringia trinervia (L.) Clairv. & Kemence & 1952 & Simon Tibor & BPU-14951.JPG \\
\hline 283 & Moehringia trinervia (L.) Clairv. & Lábatlan & 1953 & Felföldy Lajos & BPU-14952.JPG \\
\hline 283 & Moehringia trinervia (L.) Clairv. & Miskolc & 1937 & Soó Rezső & BPU-14954.JPG \\
\hline 283 & Moehringia trinervia (L.) Clairv. & Uzsa & 1959 & $\begin{array}{l}\text { Jávorka Sándor, Felföldy } \\
\text { Lajos }\end{array}$ & BPU-14948.JPG \\
\hline 284 & Moehringia muscosa L. & Bakonybél & 1955 & Soó Rezső & BPU-14943.JPG \\
\hline 284 & Moehringia muscosa L. & Csesznek & 1963 & Soó Rezső & BPU-14942.JPG \\
\hline 284 & Moehringia muscosa L. & Garadna & 1955 & Tóth László & BPU-14945.JPG \\
\hline 284 & Moehringia muscosa L. & Isztimér & 1949 & Soó Rezső & BPU-14944.JPG \\
\hline 284 & Moehringia muscosa L. & Miskolc & 1937 & Soó Rezső & BPU-14947.JPG \\
\hline 284 & Moehringia muscosa L. & Miskolc & 1950 & Soó Rezső & BPU-14946.JPG \\
\hline 285 & Minuartia setacea (Thuill.) Hayek & Budapest & 1982 & Felföldy Lajos & BPU-15018.JPG \\
\hline
\end{tabular}


1. Elektroniukus melléklet - Electronic Appendix 1.

\begin{tabular}{|c|c|c|c|c|c|}
\hline $\begin{array}{l}\text { Sorszám / } \\
\text { Number }\end{array}$ & Taxon-név / Taxon-name & $\begin{array}{l}\text { Település / } \\
\text { Settlement }\end{array}$ & $\begin{array}{l}\text { Év / } \\
\text { Year }\end{array}$ & Gyüjtő / Collector & $\begin{array}{l}\text { Fájlnév / } \\
\text { File-name }\end{array}$ \\
\hline 285 & Minuartia setacea (Thuill.) Hayek & Bugac & 1954 & Soó Rezső & BPU-15015.JPG \\
\hline 285 & Minuartia setacea (Thuill.) Hayek & Csákvár & 1955 & Soó Rezső & BPU-15016.JPG \\
\hline 285 & Minuartia setacea (Thuill.) Hayek & Csákvár & 1955 & Soó Rezső & BPU-15017.JPG \\
\hline 285 & Minuartia setacea (Thuill.) Hayek & Domony & 1992 & Felföldy Lajos & BPU-15014.JPG \\
\hline 285 & Minuartia setacea (Thuill.) Hayek & Fót & 1960 & Soó Rezső & BPU-15012.JPG \\
\hline 285 & Minuartia setacea (Thuill.) Hayek & Gánt & 1948 & Soó Rezső & BPU-15013.JPG \\
\hline 285 & Minuartia setacea (Thuill.) Hayek & Uzsa & 1959 & $\begin{array}{l}\text { Jávorka Sándor, Felföldy } \\
\text { Lajos }\end{array}$ & BPU-15011.JPG \\
\hline 286 & Minuartia fastigiata (Sm.) Rchb. & Bódvarákó & 1951 & $\begin{array}{l}\text { Soó Rezső, Jakucs Pál, Ér } \\
\text { Lajos }\end{array}$ & BPU-15023.JPG \\
\hline 286 & Minuartia fastigiata (Sm.) Rchb. & Tihany & 1928 & Soó Rezső & BPU-15024.JPG \\
\hline 286 & Minuartia fastigiata (Sm.) Rchb. & Tihany & 1947 & Pólya László & BPU-15025.JPG \\
\hline 286 & Minuartia fastigiata (Sm.) Rchb. & Tihany & 1949 & Simon Tibor & BPU-15028.JPG \\
\hline 286 & Minuartia fastigiata (Sm.) Rchb. & Tihany & 1958 & Felföldy Lajos & BPU-15022.JPG \\
\hline 286 & Minuartia fastigiata (Sm.) Rchb. & Tihany & 1959 & Felföldy Lajos & BPU-15026.JPG \\
\hline 286 & Minuartia fastigiata (Sm.) Rchb. & Tihany & 1959 & Felföldy Lajos & BPU-15027.JPG \\
\hline 287 & Minuartia glomerata (M. Bieb.) Degen & Budaörs & 1947 & Soó Rezső & BPU-15021.JPG \\
\hline 287 & Minuartia glomerata (M. Bieb.) Degen & Budapest & 1923 & Soó Rezső & BPU-15019.JPG \\
\hline 287 & Minuartia glomerata (M. Bieb.) Degen & Villány & 1956 & Soó Rezső & BPU-15020.JPG \\
\hline 288 & $\begin{array}{l}\text { Minuartia viscosa (Schreb.) Schinz et } \\
\text { Thell. }\end{array}$ & Debrecen & 1938 & Soó Rezső & BPU-15030.JPG \\
\hline 288 & $\begin{array}{l}\text { Minuartia viscosa (Schreb.) Schinz et } \\
\text { Thell. }\end{array}$ & Tököl & 1918 & Degen Árpád & BPU-15029.JPG \\
\hline 289 & Minuartia glaucina Dvořáková & Budapest & 1982 & Felföldy Lajos & BPU-15008.JPG \\
\hline 289 & Minuartia glaucina Dvořáková & Csomád & 1953 & Felföldy Lajos & BPU-14999.JPG \\
\hline 289 & Minuartia glaucina Dvořáková & Gyöngyös & 1953 & Soó Rezső & BPU-15003.JPG \\
\hline 289 & Minuartia glaucina Dvořáková & Isztimér & 1955 & Soó Rezső & BPU-15004.JPG \\
\hline 289 & Minuartia glaucina Dvořáková & Nagyharsány & 1936 & Soó Rezső & BPU-15005.JPG \\
\hline 289 & Minuartia glaucina Dvořáková & Nagyharsány & 1956 & Soó Rezső & BPU-15006.JPG \\
\hline 289 & Minuartia glaucina Dvořáková & Nagyharsány & 1965 & $\begin{array}{l}\text { Simon Tibor, Priszter } \\
\text { Szaniszló }\end{array}$ & BPU-15002.JPG \\
\hline 289 & Minuartia glaucina Dvořáková & Vácrátót & 1952 & Felföldy Lajos & BPU-14998.JPG \\
\hline 290 & Minuartia frutescens (Kit.) Tuzson & Boldogkőváralja & 1939 & Soó Rezső & BPU-14994.JPG \\
\hline 290 & Minuartia frutescens (Kit.) Tuzson & Füzér & 1949 & Simon Tibor, Jakucs Pál & BPU-14995.JPG \\
\hline 290 & Minuartia frutescens (Kit.) Tuzson & Szarvaskő & 1928 & Zólyomi Bálint & BPU-14992.JPG \\
\hline 291 & Stellaria nemorum L. & Devecser & 1957 & Tallós Pál & BPU-15203.JPG \\
\hline 294 & Stellaria media (L.) Vill. & Balatonszőlős & 1959 & Felföldy Lajos & BPU-15197.JPG \\
\hline 294 & Stellaria media (L.) Vill. & Budapest & 1982 & Felföldy Lajos & BPU-15198.JPG \\
\hline 294 & Stellaria media (L.) Vill. & Debrecen & 1936 & Soó Rezső & BPU-15199.JPG \\
\hline 294 & Stellaria media (L.) Vill. & Nyíregyháza & 1910 & Radó E. & BPU-15200.JPG \\
\hline 294 & Stellaria media (L.) Vill. & Tihany & 1958 & Felföldy Lajos & BPU-15196.JPG \\
\hline 294 & Stellaria media (L.) Vill. & Tihany & 1958 & Felföldy Lajos & BPU-15201.JPG \\
\hline 294 & Stellaria media (L.) Vill. & Tihany & 1958 & Felföldy Lajos & BPU-15202.JPG \\
\hline 295 & Stellaria holostea L. & Biatorbágy & 1992 & Felföldy Lajos & BPU-15192.JPG \\
\hline 295 & Stellaria holostea L. & Budapest & 1923 & Soó Rezső & BPU-15195.JPG \\
\hline 295 & Stellaria holostea L. & Debrecen & 1949 & Soó Rezső & BPU-15194.JPG \\
\hline 295 & Stellaria holostea L. & Lábatlan & 1953 & Felföldy Lajos & BPU-15193.JPG \\
\hline 295 & Stellaria holostea L. & Sátoraljaújhely & 1952 & $\begin{array}{l}\text { Kulcsár Gábor, Vozáry } \\
\text { Elemér }\end{array}$ & BPU-15537.JPG \\
\hline 295 & Stellaria holostea L. & Visegrád & 1964 & Soó Rezső, Borsos Olga & BPU-15191.JPG \\
\hline
\end{tabular}


1. Elektroniukus melléklet - Electronic Appendix 1.

\begin{tabular}{|c|c|c|c|c|c|}
\hline $\begin{array}{l}\text { Sorszám / } \\
\text { Number }\end{array}$ & Taxon-név / Taxon-name & $\begin{array}{l}\text { Település / } \\
\text { Settlement }\end{array}$ & $\begin{array}{l}\text { Év / } \\
\text { Year }\end{array}$ & Gyüjtő / Collector & $\begin{array}{l}\text { Fájlnév / } \\
\text { File-name }\end{array}$ \\
\hline 296 & Stellaria alsine Grimm & Háromhuta & 1960 & Simon Tibor & BPU-15190.JPG \\
\hline 296 & Stellaria alsine Grimm & Szőce & 1955 & Soó Rezső & BPU-15188.JPG \\
\hline 297 & Stellaria graminea L. & Budapest & 1982 & Felföldy Lajos & BPU-15186.JPG \\
\hline 297 & Stellaria graminea L. & Budapest & 1991 & Felföldy Lajos & BPU-15185.JPG \\
\hline 297 & Stellaria graminea L. & Budapest & 2005 & Felföldy Lajos & BPU-15187.JPG \\
\hline 297 & Stellaria graminea $\mathrm{L}$. & Egyek & 1935 & Soó Rezső & BPU-15184.JPG \\
\hline 297 & Stellaria graminea L. & Gelénes & 1953 & $\begin{array}{l}\text { Simon Tibor, Vozáry } \\
\text { Elemér }\end{array}$ & BPU-15487.JPG \\
\hline 297 & Stellaria graminea L. & Nagykovácsi & 2005 & Felföldy Lajos & BPU-15183.JPG \\
\hline 297 & Stellaria graminea L. & Nyíregyháza & 1933 & Soó Rezső & BPU-15182.JPG \\
\hline 297 & Stellaria graminea L. & Uzsa & 1959 & $\begin{array}{l}\text { Jávorka Sándor, Felföldy } \\
\text { Lajos }\end{array}$ & BPU-15181.JPG \\
\hline 298 & Stellaria palustris Retz. & Gelénes & 1951 & $\begin{array}{l}\text { Simon Tibor, Borsos Olga, } \\
\text { Jakucs Pál }\end{array}$ & BPU-15180.JPG \\
\hline 298 & Stellaria palustris Retz. & Gelénes & 1951 & $\begin{array}{l}\text { Simon Tibor, Vozáry } \\
\text { Elemér }\end{array}$ & BPU-15176.JPG \\
\hline 298 & Stellaria palustris Retz. & Gelénes & 1951 & $\begin{array}{l}\text { Simon Tibor, Vozáry } \\
\text { Elemér }\end{array}$ & BPU-15178.JPG \\
\hline 298 & Stellaria palustris Retz. & Nyírpilis & 1934 & Soó Rezső & BPU-15179.JPG \\
\hline 298 & Stellaria palustris Retz. & Tákos & 1949 & Soó Rezső & BPU-15177.JPG \\
\hline 299 & Holosteum umbellatum L. & Budapest & 1982 & Felföldy Lajos & BPU-15055.JPG \\
\hline 299 & Holosteum umbellatum L. & Debrecen & 1935 & Soó Rezső & BPU-15059.JPG \\
\hline 299 & Holosteum umbellatum L. & Hencida & 1927 & Kovács I. & BPU-15058.JPG \\
\hline 299 & Holosteum umbellatum L. & Tihany & 1958 & Felföldy Lajos & BPU-15057.JPG \\
\hline 299 & Holosteum umbellatum L. & Vácrátót & 1953 & Felföldy Lajos & BPU-15056.JPG \\
\hline 300 & Cerastium dubium (Bastard) Guépin & Beregdaróc & 1951 & Simon Tibor & BPU-15092.JPG \\
\hline 300 & Cerastium dubium (Bastard) Guépin & Hortobágy & 1934 & Soó Rezső & BPU-15089.JPG \\
\hline 300 & Cerastium dubium (Bastard) Guépin & Zámoly & 1982 & Felföldy Lajos & BPU-15090.JPG \\
\hline 300 & Cerastium dubium (Bastard) Guépin & Zámoly & 1982 & Felföldy Lajos & BPU-15091.JPG \\
\hline 301 & Cerastium glomeratum Thuill. & Budapest & 1991 & Felföldy Lajos & BPU-15174.JPG \\
\hline 301 & Cerastium glomeratum Thuill. & Uzsa & 1959 & $\begin{array}{l}\text { Jávorka Sándor, Felföldy } \\
\text { Lajos }\end{array}$ & BPU-15173.JPG \\
\hline 302 & Cerastium tenoreanum Ser. & Budapest & 2004 & Felföldy Lajos & BPU-15169.JPG \\
\hline 303 & Cerastium brachypetalum Desp. & Balatonszőlős & 1959 & Felföldy Lajos & BPU-15165.JPG \\
\hline 303 & Cerastium brachypetalum Desp. & Budapest & 2004 & Felföldy Lajos & BPU-15170.JPG \\
\hline 303 & Cerastium brachypetalum Desp. & Budapest & 2004 & Felföldy Lajos & BPU-15171.JPG \\
\hline 303 & Cerastium brachypetalum Desp. & Budapest & 2004 & Felföldy Lajos & BPU-15172.JPG \\
\hline 303 & Cerastium brachypetalum Desp. & Hajdúhadház & 1933 & Soó Rezső & BPU-15166.JPG \\
\hline 303 & Cerastium brachypetalum Desp. & Lábatlan & 1953 & Felföldy Lajos & BPU-15168.JPG \\
\hline 303 & Cerastium brachypetalum Desp. & Mór & 1953 & Felföldy Lajos & BPU-15167.JPG \\
\hline 303 & Cerastium brachypetalum Desp. & Tihany & 1946 & Felföldy Lajos & BPU-15164.JPG \\
\hline 303 & Cerastium brachypetalum Desp. & Tihany & 1956 & Felföldy Lajos & BPU-15162.JPG \\
\hline 303 & Cerastium brachypetalum Desp. & Tihany & 1958 & Felföldy Lajos & BPU-15161.JPG \\
\hline 303 & Cerastium brachypetalum Desp. & Tihany & 1958 & Felföldy Lajos & BPU-15163.JPG \\
\hline 303 & Cerastium brachypetalum Desp. & Tihany & 1959 & Felföldy Lajos & BPU-15160.JPG \\
\hline 303 & Cerastium brachypetalum Desp. & Uzsa & 1959 & $\begin{array}{l}\text { Jávorka Sándor, Felföldy } \\
\text { Lajos }\end{array}$ & BPU-15159.JPG \\
\hline 304 & $\begin{array}{l}\text { Cerastium subtetrandrum (Lange) } \\
\text { Murb. }\end{array}$ & Tihany & 1958 & Felföldy Lajos & BPU-15134.JPG \\
\hline 304 & $\begin{array}{l}\text { Cerastium subtetrandrum (Lange) } \\
\text { Murb. }\end{array}$ & Zámoly & 1982 & Felföldy Lajos & BPU-15135.JPG \\
\hline
\end{tabular}


1. Elektroniukus melléklet - Electronic Appendix 1.

\begin{tabular}{|c|c|c|c|c|c|}
\hline $\begin{array}{l}\text { Sorszám / } \\
\text { Number }\end{array}$ & Taxon-név / Taxon-name & $\begin{array}{l}\text { Település / } \\
\text { Settlement }\end{array}$ & $\begin{array}{l}\text { Év / } \\
\text { Year }\end{array}$ & Gyűjtő / Collector & $\begin{array}{l}\text { Fájlnév / } \\
\text { File-name }\end{array}$ \\
\hline 305 & Cerastium semidecandrum L. & Bagamér & 1949 & Soó Rezső & BPU-15128.JPG \\
\hline 305 & Cerastium semidecandrum L. & Budaörs & 1982 & Felföldy Lajos & BPU-15127.JPG \\
\hline 305 & Cerastium semidecandrum L. & Budapest & 1982 & Felföldy Lajos & BPU-15133.JPG \\
\hline 305 & Cerastium semidecandrum L. & Budapest & 2004 & Felföldy Lajos & BPU-15132.JPG \\
\hline 305 & Cerastium semidecandrum L. & Debrecen & 1934 & Soó Rezső & BPU-15129.JPG \\
\hline 305 & Cerastium semidecandrum L. & Gödöllő & 1991 & Felföldy Lajos & BPU-15126.JPG \\
\hline 305 & Cerastium semidecandrum L. & Nyíracsád & 1932 & Máthé Imre & BPU-15124.JPG \\
\hline 305 & Cerastium semidecandrum L. & Nyíradony & 1951 & Soó Rezső & BPU-15123.JPG \\
\hline 305 & Cerastium semidecandrum L. & Tass & 1982 & Felföldy Lajos & BPU-15130.JPG \\
\hline 305 & Cerastium semidecandrum L. & Tass & 1982 & Felföldy Lajos & BPU-15131.JPG \\
\hline 305 & Cerastium semidecandrum L. & Tihany & 1958 & Felföldy Lajos & BPU-15116.JPG \\
\hline 305 & Cerastium semidecandrum L. & Tihany & 1958 & Felföldy Lajos & BPU-15117.JPG \\
\hline 305 & Cerastium semidecandrum L. & Tihany & 1958 & Felföldy Lajos & BPU-15118.JPG \\
\hline 305 & Cerastium semidecandrum L. & Tihany & 1958 & Felföldy Lajos & BPU-15119.JPG \\
\hline 305 & Cerastium semidecandrum L. & Tihany & 1958 & Felföldy Lajos & BPU-15120.JPG \\
\hline 305 & Cerastium semidecandrum L. & Tihany & 1958 & Felföldy Lajos & BPU-15121.JPG \\
\hline 305 & Cerastium semidecandrum L. & Tihany & 1958 & Felföldy Lajos & BPU-15122.JPG \\
\hline 305 & Cerastium semidecandrum L. & Vácrátót & 1953 & Felföldy Lajos & BPU-15125.JPG \\
\hline $306-307$ & Cerastium pumilum agg. & Balatonszőlős & 1959 & Felföldy Lajos & BPU-15100.JPG \\
\hline $306-307$ & Cerastium pumilum agg. & Balmazújváros & 1932 & Soó Rezső & BPU-15104.JPG \\
\hline $306-307$ & Cerastium pumilum agg. & Budaörs & 1982 & Felföldy Lajos & BPU-15103.JPG \\
\hline $306-307$ & Cerastium pumilum agg. & Budapest & 1982 & Felföldy Lajos & BPU-15109.JPG \\
\hline $306-307$ & Cerastium pumilum agg. & Budapest & 1982 & Felföldy Lajos & BPU-15110.JPG \\
\hline $306-307$ & Cerastium pumilum agg. & Budapest & 1991 & Felföldy Lajos & BPU-15111.JPG \\
\hline $306-307$ & Cerastium pumilum agg. & Budapest & 2004 & Felföldy Lajos & BPU-15112.JPG \\
\hline $306-307$ & Cerastium pumilum agg. & Budapest & 2004 & Felföldy Lajos & BPU-15114.JPG \\
\hline $306-307$ & Cerastium pumilum agg. & Budapest & 2004 & Felföldy Lajos & BPU-15115.JPG \\
\hline $306-307$ & Cerastium pumilum agg. & Budapest & 2005 & Felföldy Lajos & BPU-15113.JPG \\
\hline $306-307$ & Cerastium pumilum agg. & Csákberény & 1982 & Felföldy Lajos & BPU-15108.JPG \\
\hline $306-307$ & Cerastium pumilum agg. & Nagycserkesz & 1933 & Soó Rezső & BPU-15101.JPG \\
\hline $306-307$ & Cerastium pumilum agg. & Nagykovácsi & 1949 & Soó Rezső & BPU-15102.JPG \\
\hline $306-307$ & Cerastium pumilum agg. & Pátka & 1987 & Felföldy Lajos & BPU-15107.JPG \\
\hline $306-307$ & Cerastium pumilum agg. & Tihany & 1942 & Felföldy Lajos & BPU-15094.JPG \\
\hline $306-307$ & Cerastium pumilum agg. & Tihany & 1958 & Felföldy Lajos & BPU-15093.JPG \\
\hline $306-307$ & Cerastium pumilum agg. & Tihany & 1958 & Felföldy Lajos & BPU-15095.JPG \\
\hline $306-307$ & Cerastium pumilum agg. & Tihany & 1958 & Felföldy Lajos & BPU-15096.JPG \\
\hline $306-307$ & Cerastium pumilum agg. & Tihany & 1958 & Felföldy Lajos & BPU-15097.JPG \\
\hline $306-307$ & Cerastium pumilum agg. & Tihany & 1958 & Felföldy Lajos & BPU-15098.JPG \\
\hline $306-307$ & Cerastium pumilum agg. & Tihany & 1958 & Felföldy Lajos & BPU-15099.JPG \\
\hline $306-307$ & Cerastium pumilum agg. & Zámoly & 1982 & Felföldy Lajos & BPU-15105.JPG \\
\hline $306-307$ & Cerastium pumilum agg. & Zámoly & 1982 & Felföldy Lajos & BPU-15106.JPG \\
\hline 308 & Cerastium sylvaticum Waldst. et Kit. & Alsószölnök & 1955 & Soó Rezső & BPU-15083.JPG \\
\hline 308 & Cerastium sylvaticum Waldst. et Kit. & Bakonybél & 1955 & Soó Rezső & BPU-15081.JPG \\
\hline 308 & Cerastium sylvaticum Waldst. et Kit. & Bakonybél & 1955 & Soó Rezső & BPU-15082.JPG \\
\hline 308 & Cerastium sylvaticum Waldst. et Kit. & Gánt & 1953 & Vajda László & BPU-15085.JPG \\
\hline 308 & Cerastium sylvaticum Waldst. et Kit. & Pécs & 1936 & Soó Rezső & BPU-15087.JPG \\
\hline 308 & Cerastium sylvaticum Waldst. et Kit. & Regéc & 1960 & Simon Tibor & BPU-15088.JPG \\
\hline
\end{tabular}


1. Elektroniukus melléklet - Electronic Appendix 1.

\begin{tabular}{|c|c|c|c|c|c|}
\hline $\begin{array}{l}\text { Sorszám / } \\
\text { Number }\end{array}$ & Taxon-név / Taxon-name & $\begin{array}{l}\text { Település / } \\
\text { Settlement }\end{array}$ & $\begin{array}{l}\text { Év / } \\
\text { Year }\end{array}$ & Gyújtő / Collector & $\begin{array}{l}\text { Fájlnév / } \\
\text { File-name }\end{array}$ \\
\hline 308 & Cerastium sylvaticum Waldst. et Kit. & Szenna & 1957 & Soó Rezső & BPU-15084.JPG \\
\hline 308 & Cerastium sylvaticum Waldst. et Kit. & Tarpa & 1949 & Soó Rezső & BPU-15086.JPG \\
\hline 308 & Cerastium sylvaticum Waldst. et Kit. & Uzsa & 1950 & Felföldy Lajos & BPU-15080.JPG \\
\hline $309-310$ & Cerastium fontanum agg. & Bagamér & 1933 & Soó Rezső & BPU-15148.JPG \\
\hline $309-310$ & Cerastium fontanum agg. & Balatonberény & 1958 & Tóth László & BPU-15145.JPG \\
\hline $309-310$ & Cerastium fontanum agg. & Balatonszőlős & 1956 & $\begin{array}{l}\text { Felföldy Lajos, Tóth } \\
\text { László }\end{array}$ & BPU-15144.JPG \\
\hline $309-310$ & Cerastium fontanum agg. & Bicske & 1991 & Felföldy Lajos & BPU-15152.JPG \\
\hline $309-310$ & Cerastium fontanum agg. & Budapest & 1982 & Felföldy Lajos & BPU-15155.JPG \\
\hline $309-310$ & Cerastium fontanum agg. & Budapest & 1991 & Felföldy Lajos & BPU-15154.JPG \\
\hline $309-310$ & Cerastium fontanum agg. & Budapest & 2005 & Felföldy Lajos & BPU-15156.JPG \\
\hline $309-310$ & Cerastium fontanum agg. & Debrecen & 1989 & Felföldy Lajos & BPU-15149.JPG \\
\hline $309-310$ & Cerastium fontanum agg. & Hidegkút & 1958 & Felföldy Lajos & BPU-15143.JPG \\
\hline $309-310$ & Cerastium fontanum agg. & Monoszló & 1982 & Felföldy Lajos & BPU-15142.JPG \\
\hline $309-310$ & Cerastium fontanum agg. & Mór & 1953 & Felföldy Lajos & BPU-15151.JPG \\
\hline $309-310$ & Cerastium fontanum agg. & Tarpa & 1948 & Simon Tibor, Jakucs Pál & BPU-15153.JPG \\
\hline $309-310$ & Cerastium fontanum agg. & Tihany & 1959 & Felföldy Lajos & BPU-15140.JPG \\
\hline $309-310$ & Cerastium fontanum agg. & Tihany & 1959 & Felföldy Lajos & BPU-15141.JPG \\
\hline $309-310$ & Cerastium fontanum agg. & Uzsa & 1950 & Felföldy Lajos & BPU-15139.JPG \\
\hline $309-310$ & Cerastium fontanum agg. & Vácrátót & 1952 & Felföldy Lajos & BPU-15146.JPG \\
\hline $309-310$ & Cerastium fontanum agg. & Vácrátót & 1953 & Felföldy Lajos & BPU-15147.JPG \\
\hline $309-310$ & Cerastium fontanum agg. & Zámoly & 1982 & Felföldy Lajos & BPU-15150.JPG \\
\hline 311 & Cerastium arvense L. & Bélapátfalva & 1948 & $\begin{array}{l}\text { Simon Tibor, Pólya } \\
\text { László, Jakucs Pál }\end{array}$ & BPU-15078.JPG \\
\hline 311 & Cerastium arvense L. & Fertőrákos & 1938 & Soó Rezső & BPU-15077.JPG \\
\hline 311 & Cerastium arvense L. & Pocsaj & 1949 & Soó Rezső & BPU-15079.JPG \\
\hline 312 & Cerastium tomentosum L. & Mátraszentimre & 1961 & Soó Rezső & BPU-15060.JPG \\
\hline 313 & Moenchia mantica (L.) Bartl. & Bisse & 1965 & $\begin{array}{l}\text { Simon Tibor, Priszter } \\
\text { Szaniszló, Borhidi Attila }\end{array}$ & BPU-15054.JPG \\
\hline 313 & Moenchia mantica (L.) Bartl. & Gánt & 1953 & Vajda László & BPU-15052.JPG \\
\hline 313 & Moenchia mantica (L.) Bartl. & Kapolcs & 1959 & $\begin{array}{l}\text { Jávorka Sándor, Felföldy } \\
\text { Lajos, Tóth László }\end{array}$ & BPU-15051.JPG \\
\hline 313 & Moenchia mantica (L.) Bartl. & Pécs & 1953 & Soó Rezső, Borsos Olga & BPU-15053.JPG \\
\hline 313 & Moenchia mantica (L.) Bartl. & Uzsa & 1950 & Felföldy Lajos & BPU-15050.JPG \\
\hline 313 & Moenchia mantica (L.) Bartl. & Uzsa & 1959 & $\begin{array}{l}\text { Jávorka Sándor, Felföldy } \\
\text { Lajos }\end{array}$ & BPU-15048.JPG \\
\hline 313 & Moenchia mantica (L.) Bartl. & Uzsa & 1959 & $\begin{array}{l}\text { Jávorka Sándor, Felföldy } \\
\text { Lajos }\end{array}$ & BPU-15049.JPG \\
\hline 314 & Myosoton aquaticum (L.) Moench & Budaörs & 1991 & Felföldy Lajos & BPU-15208.JPG \\
\hline 314 & Myosoton aquaticum (L.) Moench & Budapest & 1991 & Felföldy Lajos & BPU-15209.JPG \\
\hline 314 & Myosoton aquaticum (L.) Moench & Budapest & 2005 & Felföldy Lajos & BPU-15210.JPG \\
\hline 314 & Myosoton aquaticum (L.) Moench & Sárospatak & 1938 & Soó Rezső & BPU-15207.JPG \\
\hline 315 & Sagina procumbens $\mathrm{L}$. & Abaliget & 1938 & Soó Rezső & BPU-15047.JPG \\
\hline 315 & Sagina procumbens L. & Abaliget & 1938 & Soó Rezső & BPU-15047.JPG \\
\hline 315 & Sagina procumbens L. & Dömös & 1948 & Soó Rezső & BPU-15047.JPG \\
\hline 315 & Sagina procumbens L. & Dömös & 1961 & Simon Tibor & BPU-15047.JPG \\
\hline 315 & Sagina procumbens L. & Dörgicse & 1957 & Felföldy Lajos & BPU-15045.JPG \\
\hline 315 & Sagina procumbens L. & Gibárt & 1961 & Simon Tibor & BPU-15047.JPG \\
\hline 315 & Sagina procumbens $\mathrm{L}$. & Hajdúbagos & 1933 & Soó Rezső & BPU-15046.JPG \\
\hline 315 & Sagina procumbens L. & Háromhuta & 1960 & Simon Tibor & BPU-15044.JPG \\
\hline
\end{tabular}


1. Elektroniukus melléklet - Electronic Appendix 1.

\begin{tabular}{|c|c|c|c|c|c|}
\hline $\begin{array}{l}\text { Sorszám / } \\
\text { Number }\end{array}$ & Taxon-név / Taxon-name & $\begin{array}{l}\text { Település / } \\
\text { Settlement }\end{array}$ & $\begin{array}{l}\text { Év / } \\
\text { Year }\end{array}$ & Gyújtő / Collector & $\begin{array}{l}\text { Fájlnév / } \\
\text { File-name }\end{array}$ \\
\hline 316 & Sagina apetala Ard. & Balatonhenye & 1959 & $\begin{array}{l}\text { Jávorka Sándor, Felföldy } \\
\text { Lajos, Tóth László }\end{array}$ & BPU-15041.JPG \\
\hline 316 & Sagina apetala Ard. & Balatonhenye & 1959 & $\begin{array}{l}\text { Jávorka Sándor, Felföldy } \\
\text { Lajos, Tóth László }\end{array}$ & BPU-15042.JPG \\
\hline 316 & Sagina apetala Ard. & Kapolcs & 1959 & $\begin{array}{l}\text { Jávorka Sándor, Felföldy } \\
\text { Lajos, Tóth László }\end{array}$ & BPU-15040.JPG \\
\hline 316 & Sagina apetala Ard. & Monostorapáti & 1959 & $\begin{array}{l}\text { Jávorka Sándor, Felföldy } \\
\text { Lajos, Tóth László }\end{array}$ & BPU-15039.JPG \\
\hline 316 & Sagina apetala Ard. & Nagykanizsa & 1952 & Károlyi Árpád & BPU-15038.JPG \\
\hline 317 & Sagina nodosa (L.) Fenzl & Sávoly & 1923 & Boros Ádám & BPU-15037.JPG \\
\hline 318 & Sagina subulata (Sw.) C. Presl & Debrecen & 1953 & Dobos L. & BPU-15035.JPG \\
\hline 318 & Sagina subulata (Sw.) C. Presl & Győr & 1912 & Polgár Sándor & BPU-15034.JPG \\
\hline 318 & Sagina subulata (Sw.) C. Presl & Iharosberény & 1948 & Károlyi Árpád & BPU-15036.JPG \\
\hline 318 & Sagina subulata (Sw.) C. Presl & Révfülöp & 1926 & Jávorka Sándor & BPU-15034.JPG \\
\hline 319 & Sagina sabuletorum (Gay) Lange & Nagykanizsa & 1949 & Károlyi Árpád & BPU-15032.JPG \\
\hline 320 & Scleranthus perennis L. & Nagyvisnyó & 1953 & Soó Rezső & BPU-10305.JPG \\
\hline 322 & Scleranthus polycarpos L. & Budapest & 2004 & Felföldy Lajos & BPU-10309.JPG \\
\hline 322 & Scleranthus polycarpos L. & Uzsa & 1959 & $\begin{array}{l}\text { Jávorka Sándor, Felföldy } \\
\text { Lajos }\end{array}$ & BPU-10310.JPG \\
\hline 323 & Scleranthus annuus L. & Badacsonytomaj & 1982 & Felföldy Lajos & BPU-10312.JPG \\
\hline 323 & Scleranthus annuus L. & Debrecen & 1933 & Soó Rezső & BPU-10311.JPG \\
\hline 323 & Scleranthus annuus L. & Egyek & 1950 & $\begin{array}{l}\text { Simon Tibor, Borsos Olga, } \\
\text { Kulcsár Gábor }\end{array}$ & BPU-10317.JPG \\
\hline 323 & Scleranthus annuus L. & Gyöngyös & 1963 & Soó Rezső & BPU-10316.JPG \\
\hline 323 & Scleranthus annuus L. & Molnaszecsőd & 1955 & Soó Rezső & BPU-10314.JPG \\
\hline 323 & Scleranthus annuus L. & Nyíradony & 1951 & Simon Tibor & BPU-10311.JPG \\
\hline 323 & Scleranthus annuus L. & Tihany & 1958 & Felföldy Lajos & BPU-10315.JPG \\
\hline 323 & Scleranthus annuus L. & Uzsa & 1959 & $\begin{array}{l}\text { Jávorka Sándor, Felföldy } \\
\text { Lajos }\end{array}$ & BPU-10313.JPG \\
\hline 324 & $\begin{array}{l}\text { Paronychia cephalotes (M. Bieb.) } \\
\text { Besser }\end{array}$ & Bodajk & 1961 & Borsos Olga, Simon Tibor & BPU-10293.JPG \\
\hline 324 & $\begin{array}{l}\text { Paronychia cephalotes (M. Bieb.) } \\
\text { Besser }\end{array}$ & Budaörs & 1947 & Soó Rezső & BPU-10289.JPG \\
\hline 324 & $\begin{array}{l}\text { Paronychia cephalotes (M. Bieb.) } \\
\text { Besser }\end{array}$ & Csákvár & 1955 & Soó Rezső & BPU-10290.JPG \\
\hline 324 & $\begin{array}{l}\text { Paronychia cephalotes (M. Bieb.) } \\
\text { Besser }\end{array}$ & Gánt & 1948 & Soó Rezső & BPU-10291.JPG \\
\hline 324 & $\begin{array}{l}\text { Paronychia cephalotes (M. Bieb.) } \\
\text { Besser }\end{array}$ & Uzsa & 1959 & $\begin{array}{l}\text { Jávorka Sándor, Felföldy } \\
\text { Lajos }\end{array}$ & BPU-10294.JPG \\
\hline 325 & Herniaria glabra L. & Gyöngyös & 1964 & Soó Rezső & BPU-10295.JPG \\
\hline 325 & Herniaria glabra L. & Kővágószőlős & 1938 & Soó Rezső & BPU-10297.JPG \\
\hline 326 & Herniaria hirsuta L. & Dávod & 1955 & Soó Rezső & BPU-10298.JPG \\
\hline 326 & Herniaria hirsuta L. & Kőszeg & 1894 & Waisbecker Antal & BPU-10299.JPG \\
\hline 326 & Herniaria hirsuta L. & Szőce & 1955 & Soó Rezső & BPU-10298.JPG \\
\hline 327 & Herniaria incana Lam. & Debrecen & 1946 & Soó Rezső & BPU-10300.JPG \\
\hline 327 & Herniaria incana Lam. & Tihany & 1950 & Felföldy Lajos & BPU-10303.JPG \\
\hline 327 & Herniaria incana Lam. & Tihany & 1954 & Felföldy Lajos & BPU-10302.JPG \\
\hline 327 & Herniaria incana Lam. & Tihany & 1959 & Felföldy Lajos & BPU-10304.JPG \\
\hline 327 & Herniaria incana Lam. & Vác & 1923 & Soó Rezső & BPU-10301.JPG \\
\hline 329 & Spergula arvensis L. & Bódvarákó & 1951 & $\begin{array}{l}\text { Soó Rezső, Jakucs Pál, Ér } \\
\text { Lajos }\end{array}$ & BPU-14934.JPG \\
\hline 330 & Spergula pentandra L. & Debrecen & 1935 & Soó Rezső & BPU-14931.JPG \\
\hline 330 & Spergula pentandra L. & Nyíregyháza & 1938 & Soó Rezső & BPU-14932.JPG \\
\hline
\end{tabular}


1. Elektroniukus melléklet - Electronic Appendix 1.

\begin{tabular}{|c|c|c|c|c|c|}
\hline $\begin{array}{l}\text { Sorszám / } \\
\text { Number }\end{array}$ & Taxon-név / Taxon-name & $\begin{array}{l}\text { Település / } \\
\text { Settlement }\end{array}$ & $\begin{array}{l}\text { Év / } \\
\text { Year }\end{array}$ & Gyűjtő / Collector & $\begin{array}{l}\text { Fájlnév / } \\
\text { File-name }\end{array}$ \\
\hline 331 & Spergularia maritima (All.) Chiov. & Budapest & 1908 & Lyka Károly & BPU-14925.JPG \\
\hline 331 & Spergularia maritima (All.) Chiov. & Kiskunmajsa & 1954 & Soó Rezső & BPU-14928.JPG \\
\hline 331 & Spergularia maritima (All.) Chiov. & Kunmadaras & 1991 & Csányi Béla & BPU-14929.JPG \\
\hline 331 & Spergularia maritima (All.) Chiov. & Pákozd & 1980 & Felföldy Lajos & BPU-14930.JPG \\
\hline 331 & Spergularia maritima (All.) Chiov. & Püspökladány & 1935 & Soó Rezső & BPU-14927.JPG \\
\hline 332 & $\begin{array}{l}\text { Spergularia rubra (L.) J. Presl et C. } \\
\text { Presl }\end{array}$ & Baja & 1968 & Priszter Szaniszló & BPU-14923.JPG \\
\hline 332 & $\begin{array}{l}\text { Spergularia rubra (L.) J. Presl et C. } \\
\text { Presl }\end{array}$ & Egyek & 1947 & Soó Rezső & BPU-14921.JPG \\
\hline 332 & $\begin{array}{l}\text { Spergularia rubra (L.) J. Presl et C. } \\
\text { Presl }\end{array}$ & Egyek & 1950 & $\begin{array}{l}\text { Simon Tibor, Borsos Olga, } \\
\text { Kulcsár Gábor }\end{array}$ & BPU-10286.JPG \\
\hline 332 & $\begin{array}{l}\text { Spergularia rubra (L.) J. Presl et C. } \\
\text { Presl }\end{array}$ & Pécs & 1938 & Soó Rezső & BPU-14922.JPG \\
\hline 332 & $\begin{array}{l}\text { Spergularia rubra (L.) J. Presl et C. } \\
\text { Presl }\end{array}$ & Pusztafalu & 1939 & Soó Rezső & BPU-14924.JPG \\
\hline 333 & Spergularia salina J. Presl et C. Presl & Konyár & 1933 & Soó Rezső & BPU-10288.JPG \\
\hline 334 & Lychnis flos-cuculi L. & Balatonszőlős & 1957 & Felföldy Lajos & BPU-15395.JPG \\
\hline 334 & Lychnis flos-cuculi L. & Felsőtelekes & 2005 & Gulyás Pál & BPU-15396.JPG \\
\hline 334 & Lychnis flos-cuculi L. & Hajdúbagos & 1935 & Soó Rezső & BPU-15397.JPG \\
\hline 334 & Lychnis flos-cuculi L. & Örvényes & 1959 & Felföldy Lajos & BPU-15394.JPG \\
\hline 334 & Lychnis flos-cuculi L. & Tákos & 1948 & $\begin{array}{l}\text { Pólya László, Simon } \\
\text { Tibor, Jakucs Pál }\end{array}$ & BPU-15398.JPG \\
\hline 335 & Lychnis coronaria (L.) Desr. & Csévharaszt & 1959 & Simon Tibor, Borsos Olga & BPU-15403.JPG \\
\hline 335 & Lychnis coronaria (L.) Desr. & Gyöngyös & 1961 & Soó Rezső & BPU-15400.JPG \\
\hline 335 & Lychnis coronaria (L.) Desr. & Kerecsend & 1957 & Soó Rezső & BPU-15402.JPG \\
\hline 335 & Lychnis coronaria (L.) Desr. & Somoskőújfalu & 1936 & Soó Rezső & BPU-15401.JPG \\
\hline 335 & Lychnis coronaria (L.) Desr. & Szentendre & 1925 & Soó Rezső & BPU-15399.JPG \\
\hline 336 & Lychnis viscaria L. & Gyöngyös & 1961 & Soó Rezső & BPU-11044.JPG \\
\hline 336 & Lychnis viscaria $L$. & Gyöngyös & 1965 & Soó Rezső & BPU-11046.JPG \\
\hline 336 & Lychnis viscaria L. & Hajdúbagos & 1933 & Soó Rezső & BPU-11045.JPG \\
\hline 336 & Lychnis viscaria L. & Köveskál & 1959 & $\begin{array}{l}\text { Jávorka Sándor, Felföldy } \\
\text { Lajos, Tóth László }\end{array}$ & BPU-11043.JPG \\
\hline 336 & Lychnis viscaria L. & Miskolc & 1935 & Soó Rezső & BPU-11047.JPG \\
\hline 336 & Lychnis viscaria L. & Uzsa & 1950 & Felföldy Lajos & BPU-11050.JPG \\
\hline 336 & Lychnis viscaria L. & Uzsa & 1959 & $\begin{array}{l}\text { Jávorka Sándor, Felföldy } \\
\text { Lajos }\end{array}$ & BPU-11042.JPG \\
\hline 337 & Agrostemma githago L. & Debrecen & 1946 & Soó Rezső & BPU-11039.JPG \\
\hline 337 & Agrostemma githago L. & Felsőtelekes & 2005 & Gulyás Pál & BPU-11040.JPG \\
\hline 337 & Agrostemma githago L. & Nagyvisnyó & 1948 & $\begin{array}{l}\text { Simon Tibor, Pólya } \\
\text { László, Jakucs Pál }\end{array}$ & BPU-11041.JPG \\
\hline 338 & Silene vulgaris (Moench) Garcke & Balatongyörök & 1961 & Felföldy Lajos & BPU-11057.JPG \\
\hline 338 & Silene vulgaris (Moench) Garcke & Budapest & 2004 & Felföldy Lajos & BPU-11051.JPG \\
\hline 338 & Silene vulgaris (Moench) Garcke & Budapest & 2005 & Felföldy Lajos & BPU-11052.JPG \\
\hline 338 & Silene vulgaris (Moench) Garcke & Debrecen & 1934 & Soó Rezső & BPU-11053.JPG \\
\hline 338 & Silene vulgaris (Moench) Garcke & Gödöllő & 1949 & Soó Rezső & BPU-11055.JPG \\
\hline 338 & Silene vulgaris (Moench) Garcke & Recsk & 1922 & Soó Rezső & BPU-11054.JPG \\
\hline 338 & Silene vulgaris (Moench) Garcke & Vácrátót & 1949 & Soó Rezső & BPU-11056.JPG \\
\hline 339 & Silene otites (L.) Wibel & Aggtelek & 1951 & Soó Rezső & BPU-11065.JPG \\
\hline 339 & Silene otites (L.) Wibel & Balmazújváros & 1937 & Soó Rezső & BPU-11069.JPG \\
\hline 339 & Silene otites (L.) Wibel & Budaörs & 1947 & Soó Rezső & BPU-11071.JPG \\
\hline 339 & Silene otites (L.) Wibel & Budapest & 1947 & Soó Rezső & BPU-11064.JPG \\
\hline
\end{tabular}


1. Elektroniukus melléklet - Electronic Appendix 1.

\begin{tabular}{|c|c|c|c|c|c|}
\hline $\begin{array}{l}\text { Sorszám / } \\
\text { Number }\end{array}$ & Taxon-név / Taxon-name & $\begin{array}{l}\text { Település / } \\
\text { Settlement }\end{array}$ & $\begin{array}{l}\text { Év / } \\
\text { Year }\end{array}$ & Gyújtő / Collector & $\begin{array}{l}\text { Fájlnév / } \\
\text { File-name }\end{array}$ \\
\hline 339 & Silene otites (L.) Wibel & Budapest & 1982 & Felföldy Lajos & BPU-11063.JPG \\
\hline 339 & Silene otites (L.) Wibel & Csákvár & 1955 & Soó Rezső & BPU-11068.JPG \\
\hline 339 & Silene otites (L.) Wibel & Debrecen & 1933 & Soó Rezső & BPU-11070.JPG \\
\hline 339 & Silene otites (L.) Wibel & Miskolc & 1936 & Soó Rezső & BPU-11067.JPG \\
\hline 339 & Silene otites (L.) Wibel & Nagyharsány & 1967 & Priszter Szaniszló & BPU-11066.JPG \\
\hline 340 & Silene borysthenica (Gruner) Walters & Bakonyszentlászló & 1963 & Soó Rezső & BPU-11072.JPG \\
\hline 341 & Silene alba (Mill.) E.H.L. Krause & Budapest & 1982 & Felföldy Lajos & BPU-15379.JPG \\
\hline 341 & Silene alba (Mill.) E.H.L. Krause & Nyíracsád & 1949 & Soó Rezső & BPU-15380.JPG \\
\hline 341 & Silene alba (Mill.) E.H.L. Krause & Szilvásvárad & 1953 & $\begin{array}{l}\text { Felföldy Lajos, Tóth } \\
\text { László }\end{array}$ & BPU-15381.JPG \\
\hline 341 & Silene alba (Mill.) E.H.L. Krause & Tihany & 1958 & Felföldy Lajos & BPU-15382.JPG \\
\hline 342 & Silene dioica (L.) Clairv. & Bakonybél & 1955 & Soó Rezső & BPU-15372.JPG \\
\hline 342 & Silene dioica (L.) Clairv. & Csesznek & 1963 & Soó Rezső & BPU-15373.JPG \\
\hline 342 & Silene dioica (L.) Clairv. & Pécs & 1936 & Soó Rezső & BPU-15374.JPG \\
\hline 342 & Silene dioica (L.) Clairv. & Pécs & 1956 & Soó Rezső & BPU-15375.JPG \\
\hline 343 & Silene conica L. & Csévharaszt & 1959 & Simon Tibor, Borsos Olga & BPU-11074.JPG \\
\hline 343 & Silene conica L. & Dabas & 1959 & Simon Tibor & BPU-11075.JPG \\
\hline 343 & Silene conica L. & Debrecen & 1938 & Soó Rezső & BPU-11073.JPG \\
\hline 343 & Silene conica L. & Ócsa & 1953 & Soó Rezső, Borsos Olga & BPU-11076.JPG \\
\hline 343 & Silene conica L. & Pilisszentiván & 1953 & Soó Rezső & BPU-11077.JPG \\
\hline 343 & Silene conica L. & Vácrátót & 1953 & Felföldy Lajos & BPU-11078.JPG \\
\hline 346 & Silene dichotoma Ehrh. & Balatonszőlős & 1956 & $\begin{array}{l}\text { Felföldy Lajos, Tóth } \\
\text { László }\end{array}$ & BPU-11082.JPG \\
\hline 346 & Silene dichotoma Ehrh. & Dorog & 1965 & Priszter Szaniszló & BPU-11079.JPG \\
\hline 346 & Silene dichotoma Ehrh. & Pilisszentkereszt & 1955 & Soó Rezső & BPU-11081.JPG \\
\hline 346 & Silene dichotoma Ehrh. & Szár & 1960 & Priszter Szaniszló & BPU-11080.JPG \\
\hline 348 & Silene bupleuroides L. & Budapest & 1947 & Soó Rezső & BPU-11089.JPG \\
\hline 348 & Silene bupleuroides L. & Budapest & 1982 & Felföldy Lajos & BPU-11090.JPG \\
\hline 349 & Silene multiflora (Waldst. et Kit.) Pers. & Bagamér & 1933 & Soó Rezső & BPU-11087.JPG \\
\hline 349 & Silene multiflora (Waldst. et Kit.) Pers. & Debrecen & 1951 & Kulcsár Gábor & BPU-11086.JPG \\
\hline 349 & Silene multiflora (Waldst. et Kit.) Pers. & Gárdony & 1970 & Priszter Szaniszló & BPU-11085.JPG \\
\hline 349 & Silene multiflora (Waldst. et Kit.) Pers. & Ócsa & 1953 & Soó Rezső, Borsos Olga & BPU-11088.JPG \\
\hline 350 & Silene viscosa (L.) Pers. & Debrecen & 1947 & Soó Rezső & BPU-15383.JPG \\
\hline 350 & Silene viscosa (L.) Pers. & Szigetmonostor & 1947 & Soó Rezső & BPU-15384.JPG \\
\hline 351 & Silene nemoralis Waldst. et Kit. & Gyöngyös & 1951 & Jakucs Pál, Ér Lajos & BPU-11091.JPG \\
\hline 351 & Silene nemoralis Waldst. et Kit. & Gyöngyös & 1953 & Soó Rezső & BPU-11092.JPG \\
\hline 351 & Silene nemoralis Waldst. et Kit. & Miskolc & 1953 & Soó Rezső & BPU-11093.JPG \\
\hline 351 & Silene nemoralis Waldst. et Kit. & Nagyharsány & 1967 & Priszter Szaniszló & BPU-11095.JPG \\
\hline 351 & Silene nemoralis Waldst. et Kit. & Pákozd & 1956 & Borhidi Attila & BPU-15370.JPG \\
\hline 351 & Silene nemoralis Waldst. et Kit. & Pákozd & 1956 & Simon Tibor & BPU-15371.JPG \\
\hline 351 & Silene nemoralis Waldst. et Kit. & Szögliget & 1951 & Jakucs Pál, Ér Lajos & BPU-11094.JPG \\
\hline 352 & Silene noctiflora L. & Budapest & 2005 & Felföldy Lajos & BPU-15387.JPG \\
\hline 352 & Silene noctiflora L. & Örvényes & 1958 & Felföldy Lajos & BPU-15385.JPG \\
\hline 353 & Silene nutans L. & Bakonybél & 1955 & Soó Rezső & BPU-11105.JPG \\
\hline 353 & Silene nutans L. & Budapest & 2004 & Felföldy Lajos & BPU-11100.JPG \\
\hline 353 & Silene nutans L. & Dabas & 1959 & Soó Rezső & BPU-11103.JPG \\
\hline 353 & Silene nutans L. & Isztimér & 1949 & Soó Rezső & BPU-11102.JPG \\
\hline 353 & Silene nutans L. & Lábatlan & 1953 & Felföldy Lajos & BPU-11101.JPG \\
\hline
\end{tabular}


1. Elektroniukus melléklet - Electronic Appendix 1.

\begin{tabular}{|c|c|c|c|c|c|}
\hline $\begin{array}{l}\text { Sorszám / } \\
\text { Number }\end{array}$ & Taxon-név / Taxon-name & $\begin{array}{l}\text { Település / } \\
\text { Settlement }\end{array}$ & $\begin{array}{l}\text { Év / } \\
\text { Year }\end{array}$ & Gyűjtő / Collector & $\begin{array}{l}\text { Fájlnév / } \\
\text { File-name }\end{array}$ \\
\hline 353 & Silene nutans L. & Ófehértó & 1934 & Máthé Imre & BPU-11104.JPG \\
\hline 353 & Silene nutans L. & Tihany & 1958 & Felföldy Lajos & BPU-11106.JPG \\
\hline 354 & Silene viridiflora $\mathrm{L}$. & Bátorliget & 1947 & Simon Tibor & BPU-11098.JPG \\
\hline 354 & Silene viridiflora L. & Miskolc & 1929 & Soó Rezső & BPU-11099.JPG \\
\hline 354 & Silene viridiflora L. & Uzsa & 1925 & Polgár Sándor & BPU-11097.JPG \\
\hline 355 & Cucubalus baccifer L. & Debrecen & 1989 & Felföldy Lajos & BPU-15362.JPG \\
\hline 355 & Cucubalus baccifer L. & Miskolc & 1929 & Soó Rezső & BPU-15364.JPG \\
\hline 355 & Cucubalus baccifer L. & Tornyospálca & 1933 & Soó Rezső & BPU-15361.JPG \\
\hline 355 & Cucubalus baccifer L. & Vashosszúfalu & 1951 & Felföldy Lajos & BPU-15360.JPG \\
\hline 355 & Cucubalus baccifer L. & Zámoly & 1982 & Felföldy Lajos & BPU-15363.JPG \\
\hline 356 & Gypsophila muralis L. & Bátorliget & 1948 & $\begin{array}{l}\text { Simon Tibor, Jakucs Pál, } \\
\text { Borsos Olga }\end{array}$ & BPU-15357.JPG \\
\hline 356 & Gypsophila muralis L. & Karcag & 1926 & Oláh J. & BPU-15356.JPG \\
\hline 356 & Gypsophila muralis L. & Kishuta & 1952 & Simon Tibor & BPU-15358.JPG \\
\hline 358 & Gypsophila perfoliata L. & Győr & 1919 & Polgár Sándor & BPU-15342.JPG \\
\hline 358 & Gypsophila perfoliata L. & Győr & 1924 & Polgár Sándor & BPU-15341.JPG \\
\hline 359 & Gypsophila arenaria Waldst. et Kit. & Budapest & 1948 & Soó Rezső & BPU-15355.JPG \\
\hline 359 & Gypsophila arenaria Waldst. et Kit. & Fenyőfö & 1929 & Soó Rezső & BPU-15352.JPG \\
\hline 359 & Gypsophila arenaria Waldst. et Kit. & Fenyőfő & 1955 & Soó Rezső & BPU-15353.JPG \\
\hline 359 & Gypsophila arenaria Waldst. et Kit. & Pilisszentiván & 1948 & Kárpáti Zoltán & BPU-15354.JPG \\
\hline 360 & Gypsophila paniculata L. & Baktalórántháza & 1933 & Soó Rezső & BPU-15347.JPG \\
\hline 360 & Gypsophila paniculata L. & Budapest & 1989 & Felföldy Lajos & BPU-15351.JPG \\
\hline 360 & Gypsophila paniculata L. & Budapest & 1992 & Felföldy Lajos & BPU-15350.JPG \\
\hline 360 & Gypsophila paniculata L. & Göd & 1948 & Soó Rezső & BPU-15348.JPG \\
\hline 360 & Gypsophila paniculata L. & Szigetmonostor & 1947 & Soó Rezső & BPU-15349.JPG \\
\hline $359 \times 360$ & $\begin{array}{l}\text { Gypsophila × digenea Borbás (G. } \\
\text { arenaria Waldst. Et Kit. × G. paniculata } \\
\text { L.) }\end{array}$ & Budapest & - & Borbás Vince & BPU-15340.JPG \\
\hline 362 & Vaccaria hispanica (Mill.) Rauschert & Debrecen & 1933 & Soó Rezső & BPU-15331.JPG \\
\hline 362 & Vaccaria hispanica (Mill.) Rauschert & Eger & 1953 & Simon Tibor & BPU-15330.JPG \\
\hline 362 & Vaccaria hispanica (Mill.) Rauschert & Nagyharsány & 1967 & Priszter Szaniszló & BPU-15332.JPG \\
\hline 363 & Petrorhagia saxifraga (L.) Link & Tapolca & 1928 & Soó Rezső & BPU-15339.JPG \\
\hline 363 & Petrorhagia saxifraga (L.) Link & Tihany & 1959 & Felföldy Lajos & BPU-15337.JPG \\
\hline 363 & Petrorhagia saxifraga (L.) Link & Tihany & 1959 & Felföldy Lajos & BPU-15338.JPG \\
\hline 364 & $\begin{array}{l}\text { Petrorhagia prolifera (L.) P.W. Ball et } \\
\text { Heywood }\end{array}$ & Debrecen & 1932 & Máthé Imre & BPU-15335.JPG \\
\hline 364 & $\begin{array}{l}\text { Petrorhagia prolifera (L.) P.W. Ball et } \\
\text { Heywood }\end{array}$ & Pécs & 1969 & Priszter Szaniszló & BPU-15336.JPG \\
\hline 364 & $\begin{array}{l}\text { Petrorhagia prolifera (L.) P.W. Ball et } \\
\text { Heywood }\end{array}$ & Tihany & 1942 & Felföldy Lajos & BPU-15334.JPG \\
\hline 364 & $\begin{array}{l}\text { Petrorhagia prolifera (L.) P.W. Ball et } \\
\text { Heywood }\end{array}$ & Tihany & 1959 & Felföldy Lajos & BPU-15333.JPG \\
\hline 366 & Dianthus superbus L. & Bátorliget & 1950 & $\begin{array}{l}\text { Simon Tibor, Kárpáti } \\
\text { István }\end{array}$ & BPU-15326.JPG \\
\hline 366 & Dianthus superbus L. & Göd & 1948 & Soó Rezső & BPU-15327.JPG \\
\hline 366 & Dianthus superbus L. & Ócsa & 1951 & Pócs Tamás & BPU-15328.JPG \\
\hline 366 & Dianthus superbus L. & Ócsa & 1953 & Soó Rezső, Borsos Olga & BPU-15329.JPG \\
\hline 366 & Dianthus superbus L. & Szentgotthárd & 1955 & Soó Rezső & BPU-15325.JPG \\
\hline 367 & Dianthus plumarius L. & Bélapátfalva & 1932 & Soó Rezső & BPU-15310.JPG \\
\hline 367 & Dianthus plumarius L. & Bódvarákó & 1951 & $\begin{array}{l}\text { Soó Rezső, Jakucs Pál, Ér } \\
\text { Lajos }\end{array}$ & BPU-15312.JPG \\
\hline
\end{tabular}


1. Elektroniukus melléklet - Electronic Appendix 1.

\begin{tabular}{|c|c|c|c|c|c|}
\hline $\begin{array}{l}\text { Sorszám / } \\
\text { Number }\end{array}$ & Taxon-név / Taxon-name & $\begin{array}{l}\text { Település / } \\
\text { Settlement }\end{array}$ & $\begin{array}{l}\text { Év / } \\
\text { Year }\end{array}$ & Gyújtő / Collector & $\begin{array}{l}\text { Fájlnév / } \\
\text { File-name }\end{array}$ \\
\hline 369 & Dianthus serotinus Waldst. et Kit. & Bakonyszentlászló & 1963 & Soó Rezső & BPU-15323.JPG \\
\hline 369 & Dianthus serotinus Waldst. et Kit. & Bátorliget & 1932 & Soó Rezső & BPU-15315.JPG \\
\hline 369 & Dianthus serotinus Waldst. et Kit. & Budapest & 1948 & Soó Rezső & BPU-15316.JPG \\
\hline 369 & Dianthus serotinus Waldst. et Kit. & Bugac & 1964 & Soó Rezső & BPU-15313.JPG \\
\hline 369 & Dianthus serotinus Waldst. et Kit. & Bugac & 1964 & Soó Rezső & BPU-15317.JPG \\
\hline 369 & Dianthus serotinus Waldst. et Kit. & Csákvár & 1955 & Soó Rezső & BPU-15314.JPG \\
\hline 369 & Dianthus serotinus Waldst. et Kit. & Gyenesdiás & 1948 & Károlyi Árpád & BPU-15321.JPG \\
\hline 369 & Dianthus serotinus Waldst. et Kit. & Isztimér & 1949 & Soó Rezső & BPU-15320.JPG \\
\hline 369 & Dianthus serotinus Waldst. et Kit. & Nagybajom & 1957 & Soó Rezső & BPU-15322.JPG \\
\hline 369 & Dianthus serotinus Waldst. et Kit. & Pilisszentiván & 1932 & Soó Rezső & BPU-15319.JPG \\
\hline 369 & Dianthus serotinus Waldst. et Kit. & Pilisszentiván & 1953 & Soó Rezső & BPU-15318.JPG \\
\hline 369 & Dianthus serotinus Waldst. et Kit. & Szentendre & 1952 & Vozáry Elemér & BPU-15407.JPG \\
\hline 370 & Dianthus deltoides L. & Alsószölnök & 1955 & Soó Rezső & BPU-15307.JPG \\
\hline 370 & Dianthus deltoides L. & Mátraszentimre & 1965 & Priszter Szaniszló & BPU-15308.JPG \\
\hline 370 & Dianthus deltoides L. & Nagyvisnyó & 1955 & Soó Rezső & BPU-15309.JPG \\
\hline 370 & Dianthus deltoides L. & Uzsa & 1953 & $\begin{array}{l}\text { Felföldy Lajos, Tóth } \\
\text { László }\end{array}$ & BPU-15306.JPG \\
\hline 370 & Dianthus deltoides L. & Uzsa & 1959 & $\begin{array}{l}\text { Jávorka Sándor, Felföldy } \\
\text { Lajos }\end{array}$ & BPU-15305.JPG \\
\hline 371 & Dianthus diutinus Kit. & Bugac & 1952 & Vozáry Elemér & BPU-15303.JPG \\
\hline 371 & Dianthus diutinus Kit. & Csévharaszt & 1959 & Simon Tibor, Borsos Olga & BPU-15302.JPG \\
\hline 371 & Dianthus diutinus Kit. & Kiskunhalas & 1964 & Soó Rezső & BPU-15301.JPG \\
\hline 372 & Dianthus carthusianorum L. & Beregdaróc & 1951 & Simon Tibor & BPU-15273.JPG \\
\hline 372 & Dianthus carthusianorum L. & Füzér & 1938 & Soó Rezső & BPU-15270.JPG \\
\hline 372 & Dianthus carthusianorum L. & Perkupa & 1940 & $\begin{array}{l}\text { Ubrizsy Gábor, Felföldy } \\
\text { Lajos, Szűcs Lajos }\end{array}$ & BPU-15268.JPG \\
\hline 372 & Dianthus carthusianorum L. & Tarpa & 1949 & Soó Rezső & BPU-15278.JPG \\
\hline 373 & Dianthus pontederae A. Kern. & "Szentendrei-sziget" & 1953 & Borsos Olga & BPU-15289.JPG \\
\hline 373 & Dianthus pontederae A. Kern. & Abaújszántó & 1938 & Soó Rezső & BPU-15285.JPG \\
\hline 373 & Dianthus pontederae A. Kern. & Bélapátfalva & 1948 & $\begin{array}{l}\text { Simon Tibor, Pólya } \\
\text { László, Jakucs Pál }\end{array}$ & BPU-15294.JPG \\
\hline 373 & Dianthus pontederae A. Kern. & Budapest & 1982 & Felföldy Lajos & BPU-15298.JPG \\
\hline 373 & Dianthus pontederae A. Kern. & Budapest & 1991 & Felföldy Lajos & BPU-15299.JPG \\
\hline 373 & Dianthus pontederae A. Kern. & Budapest & 2005 & Felföldy Lajos & BPU-15300.JPG \\
\hline 373 & Dianthus pontederae A. Kern. & Bugac & 1963 & Isépy István & BPU-15297.JPG \\
\hline 373 & Dianthus pontederae A. Kern. & Debrecen & 1953 & Kulcsár Gábor & BPU-15455.JPG \\
\hline 373 & Dianthus pontederae A. Kern. & Hajdúbagos & 1933 & Soó Rezső & BPU-15295.JPG \\
\hline 373 & Dianthus pontederae A. Kern. & Hidegkút & 1958 & Felföldy Lajos & BPU-15288.JPG \\
\hline 373 & Dianthus pontederae A. Kern. & Isztimér & 1949 & Soó Rezső & BPU-15292.JPG \\
\hline 373 & Dianthus pontederae A. Kern. & Nagybajom & 1957 & Soó Rezső & BPU-15286.JPG \\
\hline 373 & Dianthus pontederae A. Kern. & Nagyharsány & 1967 & Priszter Szaniszló & BPU-15296.JPG \\
\hline 373 & Dianthus pontederae A. Kern. & Nagykovácsi & 1963 & Isépy István & BPU-15290.JPG \\
\hline 373 & Dianthus pontederae A. Kern. & Nagyvisnyó & 1936 & Soó Rezső & BPU-15293.JPG \\
\hline 373 & Dianthus pontederae A. Kern. & Pákozd & 1944 & Papp József & BPU-15291.JPG \\
\hline 373 & Dianthus pontederae A. Kern. & Tihany & 1959 & Felföldy Lajos & BPU-15287.JPG \\
\hline 374 & Dianthus giganteiformis Borbás & Bisse & 1965 & $\begin{array}{l}\text { Simon Tibor, Priszter } \\
\text { Szaniszló, Borhidi Attila }\end{array}$ & BPU-15284.JPG \\
\hline 374 & Dianthus giganteiformis Borbás & Kaszó & 1957 & Soó Rezső & BPU-15259.JPG \\
\hline 374 & Dianthus giganteiformis Borbás & Kisharsány & 1961 & Simon Tibor & BPU-15261.JPG \\
\hline 374 & Dianthus giganteiformis Borbás & Mór & 1959 & Priszter Szaniszló & BPU-15279.JPG \\
\hline
\end{tabular}


1. Elektroniukus melléklet - Electronic Appendix 1.

\begin{tabular}{|c|c|c|c|c|c|}
\hline $\begin{array}{l}\text { Sorszám / } \\
\text { Number }\end{array}$ & Taxon-név / Taxon-name & $\begin{array}{l}\text { Település / } \\
\text { Settlement }\end{array}$ & $\begin{array}{l}\text { Év / } \\
\text { Year }\end{array}$ & Gyújtő / Collector & $\begin{array}{l}\text { Fájlnév / } \\
\text { File-name }\end{array}$ \\
\hline 374 & Dianthus giganteiformis Borbás & Nagyharsány & 1956 & Soó Rezső & BPU-15282.JPG \\
\hline 374 & Dianthus giganteiformis Borbás & Nagyharsány & 1965 & $\begin{array}{l}\text { Simon Tibor, Priszter } \\
\text { Szaniszló }\end{array}$ & BPU-15281.JPG \\
\hline 374 & Dianthus giganteiformis Borbás & Nagyharsány & 1967 & Priszter Szaniszló & BPU-15280.JPG \\
\hline 374 & Dianthus giganteiformis Borbás & Nagykanizsa & 1949 & Károlyi Árpád & BPU-15257.JPG \\
\hline 374 & Dianthus giganteiformis Borbás & Somogyszob & 1923 & Boros Ádám & BPU-15260.JPG \\
\hline 374 & Dianthus giganteiformis Borbás & Szenta & 1957 & Soó Rezső & BPU-15258.JPG \\
\hline 374 & Dianthus giganteiformis Borbás & Villány & 1934 & Kárpáti Zoltán & BPU-15283.JPG \\
\hline 375 & Dianthus armeria L. & Egyek & 1953 & Borsos Olga & BPU-15264.JPG \\
\hline 375 & Dianthus armeria L. & Gyöngyös & 1961 & Soó Rezső & BPU-15263.JPG \\
\hline 375 & Dianthus armeria L. & Hosszúpályi & 1933 & Soó Rezső & BPU-15262.JPG \\
\hline 375 & Dianthus armeria L. & Szilvásvárad & 1953 & $\begin{array}{l}\text { Felföldy Lajos, Tóth } \\
\text { László }\end{array}$ & BPU-15265.JPG \\
\hline 376 & Dianthus collinus Waldst. et Kit. & Debrecen & 1931 & Soó Rezső & BPU-15252.JPG \\
\hline 376 & Dianthus collinus Waldst. et Kit. & Erdőbénye & 1948 & Soó Rezső & BPU-15247.JPG \\
\hline 376 & Dianthus collinus Waldst. et Kit. & Gyöngyös & 1963 & Soó Rezső & BPU-15249.JPG \\
\hline 376 & Dianthus collinus Waldst. et Kit. & Háromhuta & 1949 & Simon Tibor, Jakucs Pál & BPU-15246.JPG \\
\hline 376 & Dianthus collinus Waldst. et Kit. & Mátraszentimre & 1965 & Priszter Szaniszló & BPU-15250.JPG \\
\hline 376 & Dianthus collinus Waldst. et Kit. & Mátraszentimre & 1965 & Priszter Szaniszló & BPU-15251.JPG \\
\hline 376 & Dianthus collinus Waldst. et Kit. & Nagyhuta & 1952 & Simon Tibor & BPU-15254.JPG \\
\hline 376 & Dianthus collinus Waldst. et Kit. & Telkibánya & 1952 & Simon Tibor & BPU-15253.JPG \\
\hline 376 & Dianthus collinus Waldst. et Kit. & Tokaj & 1949 & Soó Rezső & BPU-15244.JPG \\
\hline 376 & Dianthus collinus Waldst. et Kit. & Tokaj & 1949 & Soó Rezső & BPU-15245.JPG \\
\hline 376 & Dianthus collinus Waldst. et Kit. & Zebegény & 1947 & Soó Rezső & BPU-15248.JPG \\
\hline 377 & Dianthus barbatus L. & Nagybajom & 1955 & Soó Rezső & BPU-15255.JPG \\
\hline $370 \times 375$ & $\begin{array}{l}\text { Dianthus deltoides L. } \times \text { Dianthus } \\
\text { armeria L. }\end{array}$ & Pusztamiske & 1948 & Borsos Olga & BPU-15213.JPG \\
\hline 379 & Nymphaea rubra Roxb. & Hévíz & 1952 & Simon Tibor & BPU-01075.JPG \\
\hline 379 & Nymphaea rubra Roxb. & Hévíz & 1961 & Felföldy Lajos & BPU-01078.JPG \\
\hline 381 & Nymphaea alba L. & Csaroda & 1948 & $\begin{array}{l}\text { Pólya László, Simon } \\
\text { Tibor, Jakucs Pál }\end{array}$ & BPU-01074.JPG \\
\hline 381 & Nymphaea alba L. & Kállósemjén & 1932 & Soó Rezső & BPU-01072.JPG \\
\hline 381 & Nymphaea alba L. & Murarátka & 1948 & Károlyi Árpád & BPU-01071.JPG \\
\hline 381 & Nymphaea alba L. & Tiszafüred & 1954 & Dobos L. & BPU-01073.JPG \\
\hline 382 & Nuphar lutea (L.) Sm. & Balatongyörök & 1956 & $\begin{array}{l}\text { Felföldy Lajos, Tóth } \\
\text { László }\end{array}$ & BPU-01080.JPG \\
\hline 382 & Nuphar lutea (L.) Sm. & Csaroda & 1948 & $\begin{array}{l}\text { Pólya László, Simon } \\
\text { Tibor, Jakucs Pál }\end{array}$ & BPU-01081.JPG \\
\hline 382 & Nuphar lutea (L.) Sm. & Vonyarcvashegy & 1958 & Felföldy Lajos & BPU-01082.JPG \\
\hline 383 & Cabomba caroliniana A. Gray & Miskolc & 1963 & Isépy István & BPU-01079.JPG \\
\hline 384 & Ceratophyllum submersum L. & Budapest & 1982 & Felföldy Lajos & BPU-01085.JPG \\
\hline 384 & Ceratophyllum submersum L. & Budapest & 1989 & Felföldy Lajos & BPU-01083.JPG \\
\hline 384 & Ceratophyllum submersum L. & Budapest & 1992 & Felföldy Lajos & BPU-01084.JPG \\
\hline 384 & Ceratophyllum submersum L. & Debrecen & 1937 & Soó Rezső & BPU-01086.JPG \\
\hline 384 & Ceratophyllum submersum L. & Érd & 1980 & Csányi Béla & BPU-01087.JPG \\
\hline 384 & Ceratophyllum submersum L. & Kunmadaras & 1991 & Csányi Béla & BPU-01088.JPG \\
\hline 384 & Ceratophyllum submersum L. & Paloznak & 1956 & $\begin{array}{l}\text { Felföldy Lajos, Tóth } \\
\text { László }\end{array}$ & BPU-01089.JPG \\
\hline 384 & Ceratophyllum submersum L. & Szentbékkálla & 1959 & $\begin{array}{l}\text { Jávorka Sándor, Felföldy } \\
\text { Lajos, Tóth László }\end{array}$ & BPU-01090.JPG \\
\hline 384 & Ceratophyllum submersum L. & Uzsa & 1952 & Simon Tibor & BPU-01091.JPG \\
\hline
\end{tabular}


1. Elektroniukus melléklet - Electronic Appendix 1.

\begin{tabular}{|c|c|c|c|c|c|}
\hline $\begin{array}{l}\text { Sorszám / } \\
\text { Number }\end{array}$ & Taxon-név / Taxon-name & $\begin{array}{l}\text { Település / } \\
\text { Settlement }\end{array}$ & $\begin{array}{l}\text { Év / } \\
\text { Year }\end{array}$ & Gyújtő / Collector & $\begin{array}{l}\text { Fájlnév / } \\
\text { File-name }\end{array}$ \\
\hline 385 & Ceratophyllum demersum L. & Budapest & 1982 & Felföldy Lajos & BPU-01092.JPG \\
\hline 385 & Ceratophyllum demersum L. & Budapest & 1989 & Felföldy Lajos & BPU-01093.JPG \\
\hline 385 & Ceratophyllum demersum L. & Gávavencsellő & 1990 & Felföldy Lajos & BPU-01097.JPG \\
\hline 385 & Ceratophyllum demersum L. & Gávavencsellő & 1990 & Felföldy Lajos & BPU-01098.JPG \\
\hline 385 & Ceratophyllum demersum L. & Gávavencsellő & 1990 & Felföldy Lajos & BPU-01099.JPG \\
\hline 385 & Ceratophyllum demersum L. & Hortobágy & 1947 & Soó Rezső & BPU-01095.JPG \\
\hline 385 & Ceratophyllum demersum L. & Szarvas & 1991 & Felföldy Lajos & BPU-01094.JPG \\
\hline 385 & Ceratophyllum demersum L. & Tiszafüred & 1987 & Felföldy Lajos & BPU-01100.JPG \\
\hline 385 & Ceratophyllum demersum L. & Tiszafüred & 1990 & Felföldy Lajos & BPU-01101.JPG \\
\hline 385 & Ceratophyllum demersum L. & Veresegyház & 1949 & Soó Rezső & BPU-01096.JPG \\
\hline 385 & Ceratophyllum demersum L. & Veresegyház & 1949 & Soó Rezső & BPU-15561.JPG \\
\hline 386 & $\begin{array}{l}\text { Helleborus purpurascens Waldst. et } \\
\text { Kit. }\end{array}$ & Miskolc & 1937 & Soó Rezső & BPU-00423.JPG \\
\hline 387 & Helleborus dumetorum Waldst. et Kit. & Budapest & 1923 & Miltényi & BPU-00414.JPG \\
\hline 387 & Helleborus dumetorum Waldst. et Kit. & Gánt & 1932 & Lengyel Géza & BPU-00416.JPG \\
\hline 387 & Helleborus dumetorum Waldst. et Kit. & Gánt & 1949 & Soó Rezső & BPU-00417.JPG \\
\hline 387 & Helleborus dumetorum Waldst. et Kit. & Nadap & 1922 & Soó Rezső & BPU-00415.JPG \\
\hline 388 & Helleborus odorus Waldst. et Kit. & Bisse & 1965 & Priszter Szaniszló & BPU-00418.JPG \\
\hline 388 & Helleborus odorus Waldst. et Kit. & Kisharsány & 1961 & Simon Tibor & BPU-00422.JPG \\
\hline 388 & Helleborus odorus Waldst. et Kit. & Pécs & 1967 & Priszter Szaniszló & BPU-00421.JPG \\
\hline 388 & Helleborus odorus Waldst. et Kit. & Villány & 1935 & Soó Rezső & BPU-00419.JPG \\
\hline 388 & Helleborus odorus Waldst. et Kit. & Villány & 1956 & Soó Rezső & BPU-00420.JPG \\
\hline 389 & Eranthis hyemalis (L.) Salisb. & Ács & 1953 & Soó Rezső & BPU-00426.JPG \\
\hline 389 & Eranthis hyemalis (L.) Salisb. & Aszófő & 1933 & Entz Géza & BPU-00427.JPG \\
\hline 389 & Eranthis hyemalis (L.) Salisb. & Aszófő & 1965 & Priszter Szaniszló & BPU-00428.JPG \\
\hline 389 & Eranthis hyemalis (L.) Salisb. & Budapest & 1922 & Soó Rezső & BPU-00425.JPG \\
\hline 389 & Eranthis hyemalis (L.) Salisb. & Budapest & 1922 & Soó Rezső & BPU-00425.JPG \\
\hline 391 & Nigella arvensis L. & Vácrátót & 1953 & Felföldy Lajos & BPU-00429.JPG \\
\hline 393 & Trollius europaeus L. & Bátorliget & 1950 & Simon Tibor & BPU-00405.JPG \\
\hline 393 & Trollius europaeus L. & Pápa & 1952 & Tallós Pál & BPU-00406.JPG \\
\hline 393 & Trollius europaeus L. & Szakonyfalu & 1955 & Soó Rezső & BPU-00404.JPG \\
\hline 394 & Isopyrum thalictroides L. & Bátorliget & 1934 & Soó Rezső & BPU-00433.JPG \\
\hline 394 & Isopyrum thalictroides L. & Beregdaróc & 1962 & Simon Tibor & BPU-00434.JPG \\
\hline 394 & Isopyrum thalictroides L. & Budapest & 1943 & Kárpáti Zoltán & BPU-00430.JPG \\
\hline 394 & Isopyrum thalictroides L. & Gyöngyös & 1964 & Soó Rezső & BPU-00431.JPG \\
\hline 394 & Isopyrum thalictroides L. & Sopron & 1951 & Csapody István & BPU-00432.JPG \\
\hline 395 & Actaea spicata L. & Baktalórántháza & 1933 & Soó Rezső & BPU-00442.JPG \\
\hline 395 & Actaea spicata L. & Bátorliget & 1950 & $\begin{array}{l}\text { Simon Tibor, Kárpáti } \\
\text { István }\end{array}$ & BPU-00443.JPG \\
\hline 395 & Actaea spicata L. & Csesznek & 1929 & Soó Rezső & BPU-00445.JPG \\
\hline 395 & Actaea spicata L. & Gyöngyös & 1951 & Jakucs Pál, Ér Lajos & BPU-00441.JPG \\
\hline 395 & Actaea spicata L. & Ivánc & 1955 & Soó Rezső & BPU-00444.JPG \\
\hline 395 & Actaea spicata L. & Miskolc & 1950 & Soó Rezső & BPU-00439.JPG \\
\hline 395 & Actaea spicata L. & Miskolc & 1952 & Borsos Olga & BPU-00437.JPG \\
\hline 395 & Actaea spicata L. & Miskolc & 1953 & Soó Rezső & BPU-00438.JPG \\
\hline 395 & Actaea spicata L. & Nagyvisnyó & 1953 & Soó Rezső & BPU-00440.JPG \\
\hline 395 & Actaea spicata L. & Pécs & 1953 & Soó Rezső, Borsos Olga & BPU-00436.JPG \\
\hline 397 & Caltha palustris L. & Aszófő & 1959 & Felföldy Lajos & BPU-00389.JPG \\
\hline
\end{tabular}


1. Elektroniukus melléklet - Electronic Appendix 1.

\begin{tabular}{|c|c|c|c|c|c|}
\hline $\begin{array}{l}\text { Sorszám / } \\
\text { Number }\end{array}$ & Taxon-név / Taxon-name & $\begin{array}{l}\text { Település / } \\
\text { Settlement }\end{array}$ & $\begin{array}{l}\text { Év / } \\
\text { Year }\end{array}$ & Gyűjtő / Collector & $\begin{array}{l}\text { Fájlnév / } \\
\text { File-name }\end{array}$ \\
\hline 397 & Caltha palustris L. & Beregdaróc & 1962 & Simon Tibor & BPU-00388.JPG \\
\hline 397 & Caltha palustris L. & Debrecen & 1937 & Soó Rezső & BPU-00385.JPG \\
\hline 397 & Caltha palustris L. & Debrecen & 1947 & Simon Tibor & BPU-00386.JPG \\
\hline 397 & Caltha palustris L. & Gyöngyös & 1964 & Soó Rezső & BPU-00394.JPG \\
\hline 397 & Caltha palustris L. & Nagyvisnyó & 1953 & Soó Rezső & BPU-00393.JPG \\
\hline 397 & Caltha palustris L. & Nagyvisnyó & 1954 & Simon Tibor & BPU-00398.JPG \\
\hline 397 & Caltha palustris L. & Pusztamiske & 1948 & Borsos Olga & BPU-00390.JPG \\
\hline 397 & Caltha palustris L. & Sárospatak & 1939 & Soó Rezső & BPU-00401.JPG \\
\hline 397 & Caltha palustris L. & Sopron & 1947 & Kárpáti Zoltán & BPU-00397.JPG \\
\hline 397 & Caltha palustris L. & Szilvásvárad & 1953 & $\begin{array}{l}\text { Felföldy Lajos, Tóth } \\
\text { László }\end{array}$ & BPU-00387.JPG \\
\hline 397 & Caltha palustris L. & Tihany & 1928 & Soó Rezső & BPU-00391.JPG \\
\hline 397 & Caltha palustris L. & Tihany & 1928 & Soó Rezső & BPU-00391.JPG \\
\hline 398 & Aconitum anthora L. & Bélapátfalva & 1930 & Soó Rezső & BPU-00475.JPG \\
\hline 398 & Aconitum anthora L. & Bózsva & 1950 & Simon Tibor & BPU-00478.JPG \\
\hline 398 & Aconitum anthora L. & Füzér & 1939 & Soó Rezső & BPU-00473.JPG \\
\hline 398 & Aconitum anthora L. & Gyöngyös & 1952 & Soó Rezső & BPU-00476.JPG \\
\hline 398 & Aconitum anthora L. & Miskolc & 1952 & Borsos Olga & BPU-00474.JPG \\
\hline 399 & Aconitum vulparia Rchb. & Bisse & 1965 & $\begin{array}{l}\text { Simon Tibor, Priszter } \\
\text { Szaniszló, Borhidi Attila }\end{array}$ & BPU-00485.JPG \\
\hline 399 & Aconitum vulparia Rchb. & Budapest & 1947 & Soó Rezső & BPU-00484.JPG \\
\hline 399 & Aconitum vulparia Rchb. & Csesznek & 1963 & Soó Rezső & BPU-00493.JPG \\
\hline 399 & Aconitum vulparia Rchb. & Gyöngyös & 1951 & Jakucs Pál, Ér Lajos & BPU-00487.JPG \\
\hline 399 & Aconitum vulparia Rchb. & Gyöngyös & 1953 & Soó Rezső & BPU-00490.JPG \\
\hline 399 & Aconitum vulparia Rchb. & Gyöngyös & 1962 & Soó Rezső, Borsos Olga & BPU-00488.JPG \\
\hline 399 & Aconitum vulparia Rchb. & Gyöngyös & 1963 & Soó Rezső & BPU-00489.JPG \\
\hline 399 & Aconitum vulparia Rchb. & Kemence & 1952 & Simon Tibor & BPU-00491.JPG \\
\hline 399 & Aconitum vulparia Rchb. & Szalonna & 1948 & $\begin{array}{l}\text { Simon Tibor, Borsos Olga, } \\
\text { Jakucs Pál }\end{array}$ & BPU-00486.JPG \\
\hline 399 & Aconitum vulparia Rchb. & Szenna & 1957 & Soó Rezső & BPU-00492.JPG \\
\hline 400 & Aconitum variegatum L. & Bózsva & 1955 & Vajda László & BPU-00479.JPG \\
\hline 400 & Aconitum variegatum L. & Miskolc & 1952 & Borsos Olga & BPU-00482.JPG \\
\hline 400 & Aconitum variegatum $\mathrm{L}$. & Nagyvisnyó & 1953 & Soó Rezső & BPU-00481.JPG \\
\hline 400 & Aconitum variegatum L. & Telkibánya & 1952 & Simon Tibor & BPU-00480.JPG \\
\hline 401 & Aconitum moldavicum Hacq. & Kishuta & 1950 & Soó Rezső & BPU-00505.JPG \\
\hline 401 & Aconitum moldavicum Hacq. & Miskolc & 1930 & Soó Rezső & BPU-00502.JPG \\
\hline 403 & Consolida regalis Gray & Budapest & 1993 & Felföldy Lajos & BPU-00460.JPG \\
\hline 403 & Consolida regalis Gray & Gödöllő & 1922 & Soó Rezső & BPU-00463.JPG \\
\hline 403 & Consolida regalis Gray & Hortobágy & 1947 & Soó Rezső & BPU-00462.JPG \\
\hline 403 & Consolida regalis Gray & Keszthely & 1961 & Laki István & BPU-00464.JPG \\
\hline 403 & Consolida regalis Gray & Nagyvisnyó & 1948 & $\begin{array}{l}\text { Simon Tibor, Pólya } \\
\text { László, Jakucs Pál }\end{array}$ & BPU-00461.JPG \\
\hline 404 & $\begin{array}{l}\text { Consolida orientalis (J. Gay) } \\
\text { Schrödinger }\end{array}$ & Debrecen & 1946 & Soó Rezső & BPU-00465.JPG \\
\hline 404 & $\begin{array}{l}\text { Consolida orientalis (J. Gay) } \\
\text { Schrödinger }\end{array}$ & Kimle & 1962 & Borsos Olga & BPU-00467.JPG \\
\hline 404 & $\begin{array}{l}\text { Consolida orientalis (J. Gay) } \\
\text { Schrödinger }\end{array}$ & Mosonmagyaróvár & 1962 & Borsos Olga & BPU-00468.JPG \\
\hline 404 & $\begin{array}{l}\text { Consolida orientalis (J. Gay) } \\
\text { Schrödinger }\end{array}$ & Nagyvisnyó & 1953 & Soó Rezső & BPU-00466.JPG \\
\hline 406 & Aquilegia vulgaris $\mathrm{L}$. & Bozsok & 1938 & Soó Rezső & BPU-00453.JPG \\
\hline
\end{tabular}


1. Elektroniukus melléklet - Electronic Appendix 1.

\begin{tabular}{|c|c|c|c|c|c|}
\hline $\begin{array}{l}\text { Sorszám / } \\
\text { Number }\end{array}$ & Taxon-név / Taxon-name & $\begin{array}{l}\text { Település / } \\
\text { Settlement }\end{array}$ & $\begin{array}{l}\text { Év / } \\
\text { Year }\end{array}$ & Gyújtő / Collector & $\begin{array}{l}\text { Fájlnév / } \\
\text { File-name }\end{array}$ \\
\hline 406 & Aquilegia vulgaris L. & Gyöngyös & 1961 & Soó Rezső & BPU-00450.JPG \\
\hline 406 & Aquilegia vulgaris L. & Pilisszentiván & 1943 & Kárpáti Zoltán & BPU-00452.JPG \\
\hline 408 & Anemone ranunculoides L. & Bakonypölöske & 1962 & Simon Tibor, Borsos Olga & BPU-02127.JPG \\
\hline 408 & Anemone ranunculoides L. & Balatonszőlős & 1958 & $\begin{array}{l}\text { Felföldy Lajos, Tóth } \\
\text { László }\end{array}$ & BPU-02130.JPG \\
\hline 408 & Anemone ranunculoides L. & Balatonszőlős & 1959 & Felföldy Lajos & BPU-02128.JPG \\
\hline 408 & Anemone ranunculoides L. & Beregdaróc & 1962 & Simon Tibor & BPU-02121.JPG \\
\hline 408 & Anemone ranunculoides L. & Debrecen & 1934 & Soó Rezső & BPU-02123.JPG \\
\hline 408 & Anemone ranunculoides L. & Gánt & 1949 & Soó Rezső & BPU-02122.JPG \\
\hline 408 & Anemone ranunculoides L. & Gyöngyös & 1964 & Soó Rezső & BPU-02124.JPG \\
\hline 408 & Anemone ranunculoides L. & Háromhuta & 1938 & Soó Rezső & BPU-02132.JPG \\
\hline 408 & Anemone ranunculoides L. & Jósvafő & 1952 & Kulcsár Gábor & BPU-02120.JPG \\
\hline 408 & Anemone ranunculoides L. & Mosonmagyaróvár & 1965 & Borsos Olga & BPU-02125.JPG \\
\hline 408 & Anemone ranunculoides L. & Sopron & 1960 & Borsos Olga, Simon Tibor & BPU-02126.JPG \\
\hline 408 & Anemone ranunculoides L. & Tihany & 1957 & $\begin{array}{l}\text { Felföldy Lajos, Tóth } \\
\text { László }\end{array}$ & BPU-02129.JPG \\
\hline 408 & Anemone ranunculoides L. & Tihany & 1958 & $\begin{array}{l}\text { Felföldy Lajos, Tóth } \\
\text { László }\end{array}$ & BPU-02131.JPG \\
\hline 409 & Anemone sylvestris L. & Debrecen & 1933 & Soó Rezső & BPU-02134.JPG \\
\hline 409 & Anemone sylvestris L. & Fenyőfő & 1955 & Soó Rezső & BPU-02136.JPG \\
\hline 409 & Anemone sylvestris L. & Fenyőfő & 1955 & Soó Rezső & BPU-02137.JPG \\
\hline 409 & Anemone sylvestris L. & Nadap & 1922 & Soó Rezső & BPU-02133.JPG \\
\hline 409 & Anemone sylvestris L. & Szentendre & 1963 & Soó Rezső & BPU-02135.JPG \\
\hline 409 & Anemone sylvestris L. & Szin & 1951 & Jakucs Pál, Ér Lajos & BPU-15481.JPG \\
\hline 409 & Anemone sylvestris L. & Tihany & 1956 & Farkas Tiborné & BPU-02138.JPG \\
\hline 411 & Anemone nemorosa L. & Beregdaróc & 1952 & Kulcsár Gábor & BPU-02119.JPG \\
\hline 411 & Anemone nemorosa L. & Háromhuta & 1938 & Soó Rezső & BPU-02118.JPG \\
\hline 411 & Anemone nemorosa L. & Szakonyfalu & 1955 & Soó Rezső & BPU-02117.JPG \\
\hline 412 & Hepatica nobilis Schreb. & Balatonszőlős & 1959 & Farkas Tiborné & BPU-02150.JPG \\
\hline 412 & Hepatica nobilis Schreb. & Balatonszőlős & 1959 & Felföldy Lajos & BPU-02151.JPG \\
\hline 412 & Hepatica nobilis Schreb. & Budapest & 1956 & Isépy István & BPU-02146.JPG \\
\hline 412 & Hepatica nobilis Schreb. & Csobánka & 1947 & Kárpáti Zoltán & BPU-02147.JPG \\
\hline 412 & Hepatica nobilis Schreb. & Hidegkút & 1959 & Felföldy Lajos & BPU-02152.JPG \\
\hline 412 & Hepatica nobilis Schreb. & Hidegkút & 1959 & Felföldy Lajos & BPU-02153.JPG \\
\hline 412 & Hepatica nobilis Schreb. & Nagybajom & 1955 & Soó Rezső & BPU-02148.JPG \\
\hline 412 & Hepatica nobilis Schreb. & Nagykanizsa & 1949 & Károlyi Árpád & BPU-02149.JPG \\
\hline 412 & Hepatica nobilis Schreb. & Szentbékkálla & 1957 & Felföldy Lajos & BPU-02154.JPG \\
\hline 413 & Pulsatilla patens (L.) Mill. & Bagamér & 1932 & Soó Rezső & BPU-02075.JPG \\
\hline 413 & Pulsatilla patens (L.) Mill. & Bátorliget & 1960 & $\begin{array}{l}\text { Simon Tibor, } \\
\text { Zimmermann W. }\end{array}$ & BPU-02076.JPG \\
\hline 414 & Pulsatilla grandis Wender. & "Szentendrei-sziget" & 1939 & Zsolt János & BPU-02094.JPG \\
\hline 414 & Pulsatilla grandis Wender. & Balatonszőlős & 1959 & Felföldy Lajos & BPU-02095.JPG \\
\hline 414 & Pulsatilla grandis Wender. & Balatonudvari & 1958 & Entz Béla & BPU-02097.JPG \\
\hline 414 & Pulsatilla grandis Wender. & Balatonudvari & 1964 & Entz Béla & BPU-02096.JPG \\
\hline 414 & Pulsatilla grandis Wender. & Bódvarákó & 1953 & Jakucs Pál & BPU-02084.JPG \\
\hline 414 & Pulsatilla grandis Wender. & Bódvaszilas & 1951 & $\begin{array}{l}\text { Jakucs Pál, Kulcsár Gábor, } \\
\text { Ér Lajos }\end{array}$ & BPU-02085.JPG \\
\hline 414 & Pulsatilla grandis Wender. & Fenyőfő & 1955 & Soó Rezső & BPU-02098.JPG \\
\hline 414 & Pulsatilla grandis Wender. & Gyenesdiás & 1965 & Priszter Szaniszló & BPU-02100.JPG \\
\hline 414 & Pulsatilla grandis Wender. & Gyöngyös & 1964 & Soó Rezső & BPU-02092.JPG \\
\hline
\end{tabular}


1. Elektroniukus melléklet - Electronic Appendix 1.

\begin{tabular}{|c|c|c|c|c|c|}
\hline $\begin{array}{l}\text { Sorszám / } \\
\text { Number }\end{array}$ & Taxon-név / Taxon-name & $\begin{array}{l}\text { Település / } \\
\text { Settlement }\end{array}$ & $\begin{array}{l}\text { Év / } \\
\text { Year }\end{array}$ & Gyújtő / Collector & $\begin{array}{l}\text { Fájlnév / } \\
\text { File-name }\end{array}$ \\
\hline 414 & Pulsatilla grandis Wender. & Hidegkút & 1959 & Felföldy Lajos & BPU-02099.JPG \\
\hline 414 & Pulsatilla grandis Wender. & Jósvafő & 1949 & Jakucs Pál & BPU-02086.JPG \\
\hline 414 & Pulsatilla grandis Wender. & Jósvafő & 1951 & Jakucs Pál & BPU-02090.JPG \\
\hline 414 & Pulsatilla grandis Wender. & Jósvafő & 1951 & $\begin{array}{l}\text { Jakucs Pál, Kulcsár Gábor, } \\
\text { Ér Lajos }\end{array}$ & BPU-02089.JPG \\
\hline 414 & Pulsatilla grandis Wender. & Jósvafó & 1951 & $\begin{array}{l}\text { Soó Rezső, Jakucs Pál, Ér } \\
\text { Lajos }\end{array}$ & BPU-02088.JPG \\
\hline 414 & Pulsatilla grandis Wender. & Nagykovácsi & 1949 & Soó Rezső & BPU-02093.JPG \\
\hline 414 & Pulsatilla grandis Wender. & Nagykovácsi & 1949 & Soó Rezső & BPU-15560.JPG \\
\hline 414 & Pulsatilla grandis Wender. & Tornanádaska & 1953 & Jakucs Pál & BPU-02091.JPG \\
\hline 415 & Pulsatilla flavescens (Hazsl.) Borbás & Bátorliget & 1932 & Soó Rezső & BPU-02170.JPG \\
\hline 415 & Pulsatilla flavescens (Hazsl.) Borbás & Hosszúpályi & 1933 & Soó Rezső & BPU-02169.JPG \\
\hline 416 & Pulsatilla nigricans Störck & Ács & 1921 & Polgár Sándor & BPU-02104.JPG \\
\hline 416 & Pulsatilla nigricans Störck & Balatonfüred & 1959 & Felföldy Lajos & BPU-02107.JPG \\
\hline 416 & Pulsatilla nigricans Störck & Balatonszőlős & 1958 & Felföldy Lajos & BPU-02108.JPG \\
\hline 416 & Pulsatilla nigricans Störck & Balatonszőlős & 1959 & Felföldy Lajos & BPU-02109.JPG \\
\hline 416 & Pulsatilla nigricans Störck & Budapest & 1989 & Felföldy Lajos & BPU-02101.JPG \\
\hline 416 & Pulsatilla nigricans Störck & Budapest & 1991 & Felföldy Lajos & BPU-02101.JPG \\
\hline 416 & Pulsatilla nigricans Störck & Esztergom & 1943 & Kárpáti Zoltán & BPU-02102.JPG \\
\hline 416 & Pulsatilla nigricans Störck & Gánt & 1949 & Soó Rezső & BPU-02103.JPG \\
\hline 416 & Pulsatilla nigricans Störck & Gyenesdiás & 1965 & Priszter Szaniszló & BPU-02116.JPG \\
\hline 416 & Pulsatilla nigricans Störck & Hidegkút & 1959 & Felföldy Lajos & BPU-02110.JPG \\
\hline 416 & Pulsatilla nigricans Störck & Hidegkút & 1959 & Felföldy Lajos & BPU-02111.JPG \\
\hline 416 & Pulsatilla nigricans Störck & Nagykovácsi & 1949 & Soó Rezső & BPU-02105.JPG \\
\hline 416 & Pulsatilla nigricans Störck & Noszlop & 1962 & Simon Tibor, Borsos Olga & BPU-02112.JPG \\
\hline 416 & Pulsatilla nigricans Störck & Pécsely & 1959 & Felföldy Lajos & BPU-02113.JPG \\
\hline 416 & Pulsatilla nigricans Störck & Pusztavacs & 1959 & $\begin{array}{l}\text { Simon Tibor, } \\
\text { Zimmermann W. }\end{array}$ & BPU-02106.JPG \\
\hline 416 & Pulsatilla nigricans Störck & Tihany & 1958 & Felföldy Lajos & BPU-02114.JPG \\
\hline 416 & Pulsatilla nigricans Störck & Tihany & 1958 & Felföldy Lajos & BPU-02115.JPG \\
\hline 417 & Pulsatilla zimmermannii Soó & Boldogkőváralja & 1960 & Simon Tibor & BPU-02163.JPG \\
\hline 417 & Pulsatilla zimmermannii Soó & Kisgyőr & 1925 & Boros Ádám & BPU-02165.JPG \\
\hline 417 & Pulsatilla zimmermannii Soó & Sárospatak & 1938 & Hargitai Zoltán & BPU-02168.JPG \\
\hline 417 & Pulsatilla zimmermannii Soó & Sárospatak & 1939 & Soó Rezső & BPU-02167.JPG \\
\hline 417 & Pulsatilla zimmermannii Soó & Sárospatak & 1949 & Simon Tibor, Jakucs Pál & BPU-02166.JPG \\
\hline 417 & Pulsatilla zimmermannii Soó & Telkibánya & 1955 & Simon Tibor & BPU-02164.JPG \\
\hline $414 \times 415$ & $\begin{array}{l}\text { Pulsatilla grandis Wender. × Pulsatilla } \\
\text { flavescens (Hazsl.) Borbás }\end{array}$ & Debrecen & 1932 & Soó Rezső & BPU-02155.JPG \\
\hline $414 \times 416$ & $\begin{array}{l}\text { Pulsatilla grandis Wender. } \times \text { Pulsatilla } \\
\text { nigricans Störck }\end{array}$ & Esztergom & 1935 & Kárpáti Zoltán & BPU-02156.JPG \\
\hline--- & Pulsatilla slavica (G.Reuss) G.Reuss & Jósvafő & 1949 & Jakucs Pál & BPU-02087.JPG \\
\hline 418 & Clematis integrifolia L. & Miskolc & 1936 & Soó Rezső & BPU-02176.JPG \\
\hline 418 & Clematis integrifolia L. & Tiszafüred & 1932 & Soó Rezső & BPU-02175.JPG \\
\hline 422 & Clematis vitalba L. & Baktalórántháza & 1933 & Soó Rezső & BPU-02185.JPG \\
\hline 422 & Clematis vitalba L. & Budapest & 2004 & Felföldy Lajos & BPU-02184.JPG \\
\hline 422 & Clematis vitalba L. & Budapest & 2005 & Felföldy Lajos & BPU-02183.JPG \\
\hline 422 & Clematis vitalba L. & Tihany & 1947 & Pólya László & BPU-02186.JPG \\
\hline 422 & Clematis vitalba L. & Tokaj & 1949 & Soó Rezső & BPU-02187.JPG \\
\hline 423 & Clematis recta L. & Debrecen & 1935 & Soó Rezső & BPU-02190.JPG \\
\hline 423 & Clematis recta L. & Miskolc & 1938 & Soó Rezső & BPU-02189.JPG \\
\hline
\end{tabular}


1. Elektroniukus melléklet - Electronic Appendix 1.

\begin{tabular}{|c|c|c|c|c|c|}
\hline $\begin{array}{l}\text { Sorszám / } \\
\text { Number }\end{array}$ & Taxon-név / Taxon-name & $\begin{array}{l}\text { Település / } \\
\text { Settlement }\end{array}$ & $\begin{array}{l}\text { Év / } \\
\text { Year }\end{array}$ & Gyújtő / Collector & $\begin{array}{l}\text { Fájlnév / } \\
\text { File-name }\end{array}$ \\
\hline 423 & Clematis recta L. & Nagybajom & 1957 & Soó Rezső & BPU-02193.JPG \\
\hline 423 & Clematis recta L. & Nagyvisnyó & 1953 & Soó Rezső & BPU-02188.JPG \\
\hline 423 & Clematis recta $\mathrm{L}$. & Pomáz & 1963 & Simon Tibor & BPU-02192.JPG \\
\hline 423 & Clematis recta L. & Szigetmonostor & 1947 & Soó Rezső & BPU-02191.JPG \\
\hline--- & Clematis flammula L. & Nagykanizsa & 1948 & Károlyi Árpád & BPU-02195.JPG \\
\hline 424 & Adonis vernalis L. & Balatonfüred & 1959 & Felföldy Lajos & BPU-01050.JPG \\
\hline 424 & Adonis vernalis L. & Balatonszőlős & 1958 & Felföldy Lajos & BPU-01051.JPG \\
\hline 424 & Adonis vernalis L. & Balatonszőlős & 1959 & Felföldy Lajos & BPU-01052.JPG \\
\hline 424 & Adonis vernalis L. & Bátorliget & 1934 & Soó Rezső & BPU-01048.JPG \\
\hline 424 & Adonis vernalis L. & Budapest & 1953 & Soó Rezső & BPU-01039.JPG \\
\hline 424 & Adonis vernalis L. & Budapest & 1990 & Felföldy Lajos & BPU-01040.JPG \\
\hline 424 & Adonis vernalis L. & Budapest & 2005 & Felföldy Lajos & BPU-01038.JPG \\
\hline 424 & Adonis vernalis L. & Gánt & 1949 & Soó Rezső & BPU-01043.JPG \\
\hline 424 & Adonis vernalis L. & Gyenesdiás & 1965 & Priszter Szaniszló & BPU-01056.JPG \\
\hline 424 & Adonis vernalis L. & Győr & 1959 & $\begin{array}{l}\text { Simon Tibor, Borhidi } \\
\text { Attila }\end{array}$ & BPU-01044.JPG \\
\hline 424 & Adonis vernalis L. & Hidegkút & 1959 & Felföldy Lajos & BPU-01053.JPG \\
\hline 424 & Adonis vernalis L. & Lábatlan & 1953 & Felföldy Lajos & BPU-01045.JPG \\
\hline 424 & Adonis vernalis L. & Mór & 1953 & Felföldy Lajos & BPU-01042.JPG \\
\hline 424 & Adonis vernalis L. & Nagykovácsi & 1949 & Soó Rezső & BPU-01046.JPG \\
\hline 424 & Adonis vernalis L. & Nyíradony & 1951 & Simon Tibor & BPU-01049.JPG \\
\hline 424 & Adonis vernalis L. & Pécs & 1953 & Soó Rezső, Borsos Olga & BPU-01041.JPG \\
\hline 424 & Adonis vernalis L. & Pécsely & 1959 & Felföldy Lajos & BPU-01054.JPG \\
\hline 424 & Adonis vernalis L. & Tihany & 1958 & Felföldy Lajos & BPU-01055.JPG \\
\hline 424 & Adonis vernalis L. & Zebegény & 1962 & Simon Tibor & BPU-01047.JPG \\
\hline 425 & Adonis volgensis Steven ex DC. & Csorvás & 1968 & Priszter Szaniszló & BPU-01059.JPG \\
\hline 426 & Adonis flammea Jacq. & Aszófő & 1959 & Felföldy Lajos & BPU-01065.JPG \\
\hline 426 & Adonis flammea Jacq. & Budapest & 1991 & Felföldy Lajos & BPU-01062.JPG \\
\hline 426 & Adonis flammea Jacq. & Budapest & 2004 & Felföldy Lajos & BPU-01061.JPG \\
\hline 426 & Adonis flammea Jacq. & Egyek & 1932 & Soó Rezső & BPU-01063.JPG \\
\hline 426 & Adonis flammea Jacq. & Pécsely & 1950 & Felföldy Lajos & BPU-01066.JPG \\
\hline 426 & Adonis flammea Jacq. & Üröm & 1992 & Felföldy Lajos & BPU-01064.JPG \\
\hline 428 & Ranunculus circinatus Sibth. & Balatonakali & 1956 & $\begin{array}{l}\text { Felföldy Lajos, Tóth } \\
\text { László }\end{array}$ & BPU-02208.JPG \\
\hline 428 & Ranunculus circinatus Sibth. & Budapest & 1916 & Degen Árpád & BPU-02205.JPG \\
\hline 428 & Ranunculus circinatus Sibth. & Paloznak & 1956 & $\begin{array}{l}\text { Felföldy Lajos, Tóth } \\
\text { László }\end{array}$ & BPU-02207.JPG \\
\hline 428 & Ranunculus circinatus Sibth. & Szolnok & 1937 & Soó Rezső & BPU-02206.JPG \\
\hline 430 & Ranunculus aquatilis L. & Budapest & 1917 & Degen Árpád & BPU-02234.JPG \\
\hline 430 & Ranunculus aquatilis L. & Debrecen & 1930 & Soó Rezső & BPU-02227.JPG \\
\hline 430 & Ranunculus aquatilis L. & Debrecen & 1932 & Soó Rezső & BPU-02229.JPG \\
\hline 430 & Ranunculus aquatilis L. & Debrecen & 1933 & Soó Rezső & BPU-02227.JPG \\
\hline 430 & Ranunculus aquatilis L. & Hajdúnánás & 1937 & Ujvárosi Miklós & BPU-02233.JPG \\
\hline 430 & Ranunculus aquatilis L. & Sarkadkeresztúr & 1938 & Boros Ádám & BPU-02230.JPG \\
\hline 431 & Ranunculus peltatus Schrank & Budapest & 1919 & Degen Árpád & BPU-02223.JPG \\
\hline 431 & Ranunculus peltatus Schrank & Egyek & 1947 & Soó Rezső & BPU-02231.JPG \\
\hline 431 & Ranunculus peltatus Schrank & Hencida & 1926 & Kovács I. & BPU-02228.JPG \\
\hline 431 & Ranunculus peltatus Schrank & Püspökladány & 1900 & Degen Árpád & BPU-02226.JPG \\
\hline 431 & Ranunculus peltatus Schrank & Sárszentágota & 1952 & Boros Ádám & BPU-02224.JPG \\
\hline
\end{tabular}


1. Elektroniukus melléklet - Electronic Appendix 1.

\begin{tabular}{|c|c|c|c|c|c|}
\hline $\begin{array}{l}\text { Sorszám / } \\
\text { Number }\end{array}$ & Taxon-név / Taxon-name & $\begin{array}{l}\text { Település / } \\
\text { Settlement }\end{array}$ & $\begin{array}{l}\text { Év / } \\
\text { Year }\end{array}$ & Gyűjtő / Collector & $\begin{array}{c}\text { Fájlnév / } \\
\text { File-name }\end{array}$ \\
\hline 432 & Ranunculus baudotii Godr. & Gelénes & 1951 & $\begin{array}{l}\text { Simon Tibor, Vozáry } \\
\text { Elemér }\end{array}$ & BPU-02225.JPG \\
\hline 432 & Ranunculus baudotii Godr. & Győr & 1919 & Polgár Sándor & BPU-02221.JPG \\
\hline 432 & Ranunculus baudotii Godr. & Szalonna & 1966 & Felföldy Lajos & BPU-02222.JPG \\
\hline 433 & Ranunculus rionii Lagger & Alcsútdoboz & 1992 & Felföldy Lajos & BPU-02215.JPG \\
\hline 434 & Ranunculus trichophyllus Chaix & Bicske & 1992 & Felföldy Lajos & BPU-02216.JPG \\
\hline 434 & Ranunculus trichophyllus Chaix & Budapest & 1915 & Degen Árpád & BPU-02209.JPG \\
\hline 434 & Ranunculus trichophyllus Chaix & Budapest & 1915 & Degen Árpád & BPU-02210.JPG \\
\hline 434 & Ranunculus trichophyllus Chaix & Hajdúbagos & 1934 & Soó Rezső & BPU-02213.JPG \\
\hline 434 & Ranunculus trichophyllus Chaix & Kiskunmajsa & 1964 & Soó Rezső & BPU-02211.JPG \\
\hline 434 & Ranunculus trichophyllus Chaix & Nagykovácsi & 1925 & Degen Árpád & BPU-02219.JPG \\
\hline 434 & Ranunculus trichophyllus Chaix & Ócsa & 1955 & Soó Rezső & BPU-02218.JPG \\
\hline 434 & Ranunculus trichophyllus Chaix & Tarpa & 1951 & Simon Tibor, Jakucs Pál & BPU-02212.JPG \\
\hline 434 & Ranunculus trichophyllus Chaix & Tiszafüred & 1990 & Felföldy Lajos & BPU-02220.JPG \\
\hline 434 & Ranunculus trichophyllus Chaix & Tiszaújváros & 1980 & Felföldy Lajos & BPU-02214.JPG \\
\hline 434 & Ranunculus trichophyllus Chaix & Zámoly & 1982 & Felföldy Lajos & BPU-02217.JPG \\
\hline 435 & Ranunculus ficaria L. & Bátorliget & 1934 & Soó Rezső & BPU-02242.JPG \\
\hline 435 & Ranunculus ficaria L. & Egyek & 1948 & Soó Rezső & BPU-02240.JPG \\
\hline 435 & Ranunculus ficaria L. & Gánt & 1949 & Soó Rezső & BPU-02238.JPG \\
\hline 435 & Ranunculus ficaria L. & Gyöngyös & 1964 & Soó Rezső & BPU-02241.JPG \\
\hline 435 & Ranunculus ficaria L. & Győr & 1913 & Polgár Sándor & BPU-02239.JPG \\
\hline 435 & Ranunculus ficaria L. & Kesztölc & 1943 & Kárpáti Zoltán & BPU-02237.JPG \\
\hline 436 & Ranunculus pedatus Waldst. et Kit. & Budapest & 1946 & Papp József & BPU-02245.JPG \\
\hline 436 & Ranunculus pedatus Waldst. et Kit. & Budapest & 2005 & Felföldy Lajos & BPU-02244.JPG \\
\hline 436 & Ranunculus pedatus Waldst. et Kit. & Debrecen & 1947 & Soó Rezső & BPU-02247.JPG \\
\hline 436 & Ranunculus pedatus Waldst. et Kit. & Tass & 1982 & Felföldy Lajos & BPU-02246.JPG \\
\hline 437 & Ranunculus illyricus L. & Budapest & 1951 & Pócs Tamás & BPU-02248.JPG \\
\hline 437 & Ranunculus illyricus L. & Gyöngyös & 1965 & Soó Rezső & BPU-02250.JPG \\
\hline 437 & Ranunculus illyricus L. & Nagyharsány & 1936 & Soó Rezső & BPU-02251.JPG \\
\hline 437 & Ranunculus illyricus L. & Tihany & 1956 & Felföldy Lajos & BPU-02249.JPG \\
\hline 439 & Ranunculus lateriflorus DC. & Egyek & 1947 & Soó Rezső & BPU-02254.JPG \\
\hline 439 & Ranunculus lateriflorus DC. & Elek & 1923 & Boros Ádám & BPU-02252.JPG \\
\hline 439 & Ranunculus lateriflorus DC. & Gyöngyös & 1923 & Soó Rezső & BPU-02253.JPG \\
\hline 439 & Ranunculus lateriflorus DC. & Monostorapáti & 1959 & $\begin{array}{l}\text { Jávorka Sándor, Felföldy } \\
\text { Lajos, Tóth László }\end{array}$ & BPU-02255.JPG \\
\hline 440 & Ranunculus polyphyllus Waldst. et Kit. & Egyek & 1948 & Soó Rezső & BPU-02257.JPG \\
\hline 440 & Ranunculus polyphyllus Waldst. et Kit. & Gyöngyös & 1923 & Soó Rezső & BPU-02256.JPG \\
\hline 440 & Ranunculus polyphyllus Waldst. et Kit. & Tarpa & 1948 & $\begin{array}{l}\text { Pólya László, Simon } \\
\text { Tibor, Jakucs Pál }\end{array}$ & BPU-02258.JPG \\
\hline 441 & Ranunculus cymbalaria Pursh & Debrecen & 1955 & Soó Rezső & BPU-00976.JPG \\
\hline 442 & Ranunculus flammula L. & Alsószölnök & 1955 & Soó Rezső & BPU-02262.JPG \\
\hline 442 & Ranunculus flammula $\mathrm{L}$. & Debrecen & 1945 & Soó Rezső & BPU-02265.JPG \\
\hline 442 & Ranunculus flammula L. & Debrecen & 1951 & Soó Rezső & BPU-02259.JPG \\
\hline 442 & Ranunculus flammula L. & Kőszeg & 1932 & Soó Rezső & BPU-02263.JPG \\
\hline 442 & Ranunculus flammula L. & Túrricse & 1953 & Simon Tibor & BPU-02264.JPG \\
\hline 443 & Ranunculus lingua $\mathrm{L}$. & Beregdaróc & 1951 & $\begin{array}{l}\text { Simon Tibor, Vozáry } \\
\text { Elemér }\end{array}$ & BPU-02266.JPG \\
\hline 443 & Ranunculus lingua L. & Debrecen & 1949 & Soó Rezső & BPU-02268.JPG \\
\hline 443 & Ranunculus lingua L. & Debrecen & 1949 & Soó Rezső & BPU-15544.JPG \\
\hline
\end{tabular}


1. Elektroniukus melléklet - Electronic Appendix 1.

\begin{tabular}{|c|c|c|c|c|c|}
\hline $\begin{array}{l}\text { Sorszám / } \\
\text { Number }\end{array}$ & Taxon-név / Taxon-name & $\begin{array}{l}\text { Település / } \\
\text { Settlement }\end{array}$ & $\begin{array}{l}\text { Év / } \\
\text { Year }\end{array}$ & Gyújtő / Collector & $\begin{array}{l}\text { Fájlnév / } \\
\text { File-name }\end{array}$ \\
\hline 443 & Ranunculus lingua L. & Győr & 1907 & Polgár Sándor & BPU-02267.JPG \\
\hline 443 & Ranunculus lingua L. & Ócsa & 1953 & Soó Rezső, Borsos Olga & BPU-02269.JPG \\
\hline 443 & Ranunculus lingua L. & Ócsa & 1953 & Soó Rezső, Borsos Olga & BPU-02270.JPG \\
\hline 444 & Ranunculus sceleratus L. & Budapest & 1993 & Felföldy Lajos & BPU-02273.JPG \\
\hline 444 & Ranunculus sceleratus L. & Budapest & 2005 & Felföldy Lajos & BPU-02272.JPG \\
\hline 444 & Ranunculus sceleratus L. & Pákozd & 1980 & Felföldy Lajos & BPU-02274.JPG \\
\hline 444 & Ranunculus sceleratus L. & Pátka & 1987 & Felföldy Lajos & BPU-02275.JPG \\
\hline 444 & Ranunculus sceleratus L. & Tihany & 1946 & Felföldy Lajos & BPU-02278.JPG \\
\hline 444 & Ranunculus sceleratus L. & Tihany & 1959 & Felföldy Lajos & BPU-02277.JPG \\
\hline 444 & Ranunculus sceleratus L. & Újfehértó & 1936 & Soó Rezső & BPU-02276.JPG \\
\hline 445 & Ranunculus bulbosus L. & Hidegkút & 1958 & Felföldy Lajos & BPU-02281.JPG \\
\hline 445 & Ranunculus bulbosus L. & Pécs & 1956 & Soó Rezső & BPU-02284.JPG \\
\hline 445 & Ranunculus bulbosus L. & Tarpa & 1949 & Soó Rezső & BPU-02283.JPG \\
\hline 445 & Ranunculus bulbosus L. & Tihany & 1942 & Felföldy Lajos & BPU-02282.JPG \\
\hline 445 & Ranunculus bulbosus L. & Tihany & 1956 & Felföldy Lajos & BPU-02280.JPG \\
\hline 445 & Ranunculus bulbosus L. & Uzsa & 1959 & $\begin{array}{l}\text { Jávorka Sándor, Felföldy } \\
\text { Lajos }\end{array}$ & BPU-02279.JPG \\
\hline 446 & Ranunculus sardous Crantz & Aszófő & 1957 & $\begin{array}{l}\text { Felföldy Lajos, Kovács } \\
\text { Margit, Tóth László }\end{array}$ & BPU-02288.JPG \\
\hline 446 & Ranunculus sardous Crantz & Hortobágy & 1947 & Soó Rezső & BPU-02286.JPG \\
\hline 446 & Ranunculus sardous Crantz & Mór & 1942 & Felföldy Lajos & BPU-02285.JPG \\
\hline 446 & Ranunculus sardous Crantz & Vácrátót & 1953 & Felföldy Lajos & BPU-02287.JPG \\
\hline 447 & Ranunculus repens L. & Debrecen & 1931 & Soó Rezső & BPU-02291.JPG \\
\hline 447 & Ranunculus repens L. & Örvényes & 1959 & Felföldy Lajos & BPU-02292.JPG \\
\hline 447 & Ranunculus repens L. & Pátka & 1987 & Felföldy Lajos & BPU-02290.JPG \\
\hline 448 & Ranunculus arvensis L. & Budapest & 1899 & Polgár Sándor & BPU-02294.JPG \\
\hline 448 & Ranunculus arvensis L. & Keszthely & 1961 & Szabó Gyula & BPU-02296.JPG \\
\hline 448 & Ranunculus arvensis L. & Vásárosnamény & 1951 & $\begin{array}{l}\text { Simon Tibor, Jakucs Pál, } \\
\text { Kulcsár Gábor }\end{array}$ & BPU-02295.JPG \\
\hline 450 & Ranunculus auricomus agg. & $\begin{array}{l}\text { Badacsonytomaj, } \\
\text { Badacsonytördemic }\end{array}$ & 1928 & Soó Rezső & BPU-00917.JPG \\
\hline 450 & Ranunculus auricomus agg. & Bakonyoszlop & 1906 & Polgár Sándor & BPU-00863.JPG \\
\hline 450 & Ranunculus auricomus agg. & Balatonszőlős & 1951 & Felföldy Lajos & BPU-00861.JPG \\
\hline 450 & Ranunculus auricomus agg. & Balatonszőlős & 1951 & Felföldy Lajos & BPU-00895.JPG \\
\hline 450 & Ranunculus auricomus agg. & Bátonyterenye & 1928 & Boros Ádám & BPU-00934.JPG \\
\hline 450 & Ranunculus auricomus agg. & Bátorliget & 1922 & Boros Ádám & BPU-00852.JPG \\
\hline 450 & Ranunculus auricomus agg. & Bátorliget & 1934 & Soó Rezső & BPU-00868.JPG \\
\hline 450 & Ranunculus auricomus agg. & Bátorliget & 1935 & Soó Rezső & BPU-00887.JPG \\
\hline 450 & Ranunculus auricomus agg. & Bátorliget & 1935 & Soó Rezső & BPU-00846.JPG \\
\hline 450 & Ranunculus auricomus agg. & Beregdaróc & 1953 & $\begin{array}{l}\text { Simon Tibor, Helmeczy } \\
\text { Balázs }\end{array}$ & BPU-00898.JPG \\
\hline 450 & Ranunculus auricomus agg. & Budapest & 1918 & Trautmann Róbert & BPU-00853.JPG \\
\hline 450 & Ranunculus auricomus agg. & Budapest & 1918 & Trautmann Róbert & BPU-00858.JPG \\
\hline 450 & Ranunculus auricomus agg. & Budapest & 1918 & Trautmann Róbert & BPU-00935.JPG \\
\hline 450 & Ranunculus auricomus agg. & Budapest & 1919 & Boros Ádám & BPU-00854.JPG \\
\hline 450 & Ranunculus auricomus agg. & Budapest & 1919 & Boros Ádám & BPU-00856.JPG \\
\hline 450 & Ranunculus auricomus agg. & Budapest & 1919 & Schiller, Sigismundi & BPU-00855.JPG \\
\hline 450 & Ranunculus auricomus agg. & Budapest & 1934 & Bán Elek & BPU-00937.JPG \\
\hline 450 & Ranunculus auricomus agg. & Budapest & 1949 & Simon Tibor & BPU-00899.JPG \\
\hline 450 & Ranunculus auricomus agg. & Budapest & 1949 & Simon Tibor & BPU-00936.JPG \\
\hline
\end{tabular}


1. Elektroniukus melléklet - Electronic Appendix 1.

\begin{tabular}{|c|c|c|c|c|c|}
\hline $\begin{array}{l}\text { Sorszám / } \\
\text { Number }\end{array}$ & Taxon-név / Taxon-name & $\begin{array}{l}\text { Település / } \\
\text { Settlement }\end{array}$ & $\begin{array}{l}\text { Év / } \\
\text { Year }\end{array}$ & Gyưjtő / Collector & $\begin{array}{l}\text { Fájlnév / } \\
\text { File-name }\end{array}$ \\
\hline 450 & Ranunculus auricomus agg. & Budapest & 1982 & Felföldy Lajos & BPU-00890.JPG \\
\hline 450 & Ranunculus auricomus agg. & Budapest & 1982 & Felföldy Lajos & BPU-00891.JPG \\
\hline 450 & Ranunculus auricomus agg. & Budapest & 2004 & Felföldy Lajos & BPU-00841.JPG \\
\hline 450 & Ranunculus auricomus agg. & "Cuha-völgy" & 1954 & Soó Rezső & BPU-00885.JPG \\
\hline 450 & Ranunculus auricomus agg. & Csesznek & 1929 & Soó Rezső & BPU-00882.JPG \\
\hline 450 & Ranunculus auricomus agg. & Debrecen & 1931 & Soó Rezső & BPU-00909.JPG \\
\hline 450 & Ranunculus auricomus agg. & Debrecen & 1933 & Soó Rezső & BPU-00908.JPG \\
\hline 450 & Ranunculus auricomus agg. & Debrecen & 1948 & Soó Rezső & BPU-00883.JPG \\
\hline 450 & Ranunculus auricomus agg. & Doboz & 1935 & Máthé Imre & BPU-00896.JPG \\
\hline 450 & Ranunculus auricomus agg. & Dömös & 1965 & Borsos Olga & BPU-00877.JPG \\
\hline 450 & Ranunculus auricomus agg. & Écs & 1921 & Polgár Sándor & BPU-00921.JPG \\
\hline 450 & Ranunculus auricomus agg. & Egyek & 1932 & Soó Rezső & BPU-00918.JPG \\
\hline 450 & Ranunculus auricomus agg. & Egyek & 1947 & Soó Rezső & BPU-00911.JPG \\
\hline 450 & Ranunculus auricomus agg. & Esztergom & 1928 & Boros Ádám & BPU-00938.JPG \\
\hline 450 & Ranunculus auricomus agg. & Felsőtárkány & 1931 & Boros Ádám & BPU-00941.JPG \\
\hline 450 & Ranunculus auricomus agg. & Gönc & 1952 & Boros Ádám & BPU-00849.JPG \\
\hline 450 & Ranunculus auricomus agg. & Gyöngyös & 1961 & Soó Rezső & BPU-00903.JPG \\
\hline 450 & Ranunculus auricomus agg. & Gyöngyös & 1964 & Soó Rezső & BPU-00884.JPG \\
\hline 450 & Ranunculus auricomus agg. & Gyöngyös & 1964 & Soó Rezső & BPU-00919.JPG \\
\hline 450 & Ranunculus auricomus agg. & Hajdúbagos & 1948 & Soó Rezső & BPU-00904.JPG \\
\hline 450 & Ranunculus auricomus agg. & Hajdúbagos & 1948 & Soó Rezső & BPU-00916.JPG \\
\hline 450 & Ranunculus auricomus agg. & Hajdúnánás & 1933 & Igmándy József & BPU-00920.JPG \\
\hline 450 & Ranunculus auricomus agg. & Háromhuta & 1960 & Priszter Szaniszló & BPU-00874.JPG \\
\hline 450 & Ranunculus auricomus agg. & Hencida & 1927 & Kovács L. & BPU-00932.JPG \\
\hline 450 & Ranunculus auricomus agg. & Hencida & 1948 & Boros Ádám & BPU-00930.JPG \\
\hline 450 & Ranunculus auricomus agg. & Hont & 1950 & Boros Ádám & BPU-00940.JPG \\
\hline 450 & Ranunculus auricomus agg. & Jósvafő & 1953 & Boros Ádám & BPU-00872.JPG \\
\hline 450 & Ranunculus auricomus agg. & Jósvafő & 1953 & Borsos Olga & BPU-00857.JPG \\
\hline 450 & Ranunculus auricomus agg. & Kapolcs & 1959 & $\begin{array}{l}\text { Jávorka Sándor, Felföldy } \\
\text { Lajos, Tóth László }\end{array}$ & BPU-00892.JPG \\
\hline 450 & Ranunculus auricomus agg. & Kesztölc & 1943 & Kárpáti Zoltán & BPU-00924.JPG \\
\hline 450 & Ranunculus auricomus agg. & Lábatlan & 1953 & Felföldy Lajos & BPU-00878.JPG \\
\hline 450 & Ranunculus auricomus agg. & Lábatlan & 1953 & Felföldy Lajos & BPU-00879.JPG \\
\hline 450 & Ranunculus auricomus agg. & Lenti & 1949 & Károlyi Árpád & BPU-00906.JPG \\
\hline 450 & Ranunculus auricomus agg. & Mosonmagyaróvár & 1965 & Borsos Olga & BPU-00889.JPG \\
\hline 450 & Ranunculus auricomus agg. & Nagykanizsa & 1948 & Károlyi Árpád & BPU-00914.JPG \\
\hline 450 & Ranunculus auricomus agg. & Nagykovácsi & 1949 & Soó Rezső & BPU-00905.JPG \\
\hline 450 & Ranunculus auricomus agg. & Nyíradony & 1931 & Soó Rezső & BPU-00927.JPG \\
\hline 450 & Ranunculus auricomus agg. & Nyírlugos & 1939 & Felföldy Lajos & BPU-00925.JPG \\
\hline 450 & Ranunculus auricomus agg. & Nyírpilis & 1942 & Máthé Imre & BPU-00931.JPG \\
\hline 450 & Ranunculus auricomus agg. & Pálháza & 1938 & Soó Rezső & BPU-00847.JPG \\
\hline 450 & Ranunculus auricomus agg. & Pilisszentiván & 1913 & $\begin{array}{l}\text { Filarszky Nándor, } \\
\text { Kümmerle Jenő Béla }\end{array}$ & BPU-00902.JPG \\
\hline 450 & Ranunculus auricomus agg. & Pilisszentiván & 1913 & $\begin{array}{l}\text { Filarszky Nándor, } \\
\text { Kümmerle Jenő Béla }\end{array}$ & BPU-00939.JPG \\
\hline 450 & Ranunculus auricomus agg. & Pilisszentkereszt & 1919 & Boros Ádám & BPU-00910.JPG \\
\hline 450 & Ranunculus auricomus agg. & Pilisszentkereszt & 1964 & Simon Tibor & BPU-00875.JPG \\
\hline 450 & Ranunculus auricomus agg. & Pilisszentkereszt & 1964 & Simon Tibor & BPU-00876.JPG \\
\hline 450 & Ranunculus auricomus agg. & Sárospatak & 1938 & Soó Rezső & BPU-00871.JPG \\
\hline
\end{tabular}


1. Elektroniukus melléklet - Electronic Appendix 1.

\begin{tabular}{|c|c|c|c|c|c|}
\hline $\begin{array}{l}\text { Sorszám / } \\
\text { Number }\end{array}$ & Taxon-név / Taxon-name & $\begin{array}{l}\text { Település / } \\
\text { Settlement }\end{array}$ & $\begin{array}{l}\text { Év / } \\
\text { Year }\end{array}$ & Gyújtő / Collector & $\begin{array}{l}\text { Fájlnév / } \\
\text { File-name }\end{array}$ \\
\hline 450 & Ranunculus auricomus agg. & Sátoraljaújhely & 1938 & Soó Rezső & BPU-00881.JPG \\
\hline 450 & Ranunculus auricomus agg. & Sopron & 1960 & Borsos Olga, Simon Tibor & BPU-00880.JPG \\
\hline 450 & Ranunculus auricomus agg. & Szamosszeg & 1948 & $\begin{array}{l}\text { Simon Tibor, Pólya } \\
\text { László, Jakucs Pál }\end{array}$ & BPU-00894.JPG \\
\hline 450 & Ranunculus auricomus agg. & Szarvas & 1949 & Soó Rezső & BPU-00933.JPG \\
\hline 450 & Ranunculus auricomus agg. & Tákos & 1948 & $\begin{array}{l}\text { Pólya László, Simon } \\
\text { Tibor, Jakucs Pál }\end{array}$ & BPU-00897.JPG \\
\hline 450 & Ranunculus auricomus agg. & Tarpa & 1951 & Simon Tibor, Jakucs Pál & BPU-00893.JPG \\
\hline 450 & Ranunculus auricomus agg. & Uzsa & 1951 & Felföldy Lajos & BPU-00900.JPG \\
\hline 450 & Ranunculus auricomus agg. & Zirc & 1925 & Polgár Sándor & BPU-00915.JPG \\
\hline 451 & Ranunculus polyanthemos L. & Balatonfüred & 1959 & Priszter Szaniszló & BPU-00969.JPG \\
\hline 451 & Ranunculus polyanthemos L. & Budapest & 2005 & Felföldy Lajos & BPU-00961.JPG \\
\hline 451 & Ranunculus polyanthemos L. & Budapest & 2005 & Felföldy Lajos & BPU-00962.JPG \\
\hline 451 & Ranunculus polyanthemos L. & Bugyi & 1959 & Soó Rezső & BPU-00968.JPG \\
\hline 451 & Ranunculus polyanthemos L. & Debrecen & 1931 & Soó Rezső & BPU-00966.JPG \\
\hline 451 & Ranunculus polyanthemos L. & Felsőtelekes & 2005 & Gulyás Pál & BPU-00963.JPG \\
\hline 451 & Ranunculus polyanthemos L. & Gyöngyös & 1965 & Soó Rezső & BPU-00967.JPG \\
\hline 451 & Ranunculus polyanthemos L. & Győr & 1918 & Polgár Sándor & BPU-00965.JPG \\
\hline 451 & Ranunculus polyanthemos L. & Szalonna & 1966 & Felföldy Lajos & BPU-00964.JPG \\
\hline 451 & Ranunculus polyanthemos L. & Tihany & 1958 & Felföldy Lajos & BPU-00970.JPG \\
\hline 451 & Ranunculus polyanthemos L. & Tihany & 1958 & Felföldy Lajos & BPU-00971.JPG \\
\hline 451 & Ranunculus polyanthemos L. & Tihany & 1958 & Felföldy Lajos & BPU-00972.JPG \\
\hline 454 & Ranunculus lanuginosus L. & Csesznek & 1929 & Soó Rezső & BPU-00946.JPG \\
\hline 454 & Ranunculus lanuginosus L. & Hercegkút & 1955 & Simon Tibor & BPU-00948.JPG \\
\hline 454 & Ranunculus lanuginosus L. & Kapolcs & 1959 & $\begin{array}{l}\text { Jávorka Sándor, Felföldy } \\
\text { Lajos, Tóth László }\end{array}$ & BPU-00947.JPG \\
\hline 454 & Ranunculus lanuginosus L. & Nagyvisnyó & 1953 & Soó Rezső & BPU-00944.JPG \\
\hline 454 & Ranunculus lanuginosus L. & Pécs & 1953 & Soó Rezső, Borsos Olga & BPU-00943.JPG \\
\hline 454 & Ranunculus lanuginosus L. & Pécs & 1956 & Soó Rezső & BPU-00942.JPG \\
\hline 454 & Ranunculus lanuginosus L. & Sopron & 1960 & Borsos Olga, Simon Tibor & BPU-00945.JPG \\
\hline 455 & Ranunculus acris L. & Háromhuta & 1960 & Priszter Szaniszló & BPU-00950.JPG \\
\hline 455 & Ranunculus acris L. & Örvényes & 1959 & Felföldy Lajos & BPU-00956.JPG \\
\hline 455 & Ranunculus acris L. & Somogyszob & 1957 & Soó Rezső & BPU-00955.JPG \\
\hline 455 & Ranunculus acris L. & Szalonna & 1966 & Felföldy Lajos & BPU-00954.JPG \\
\hline 455 & Ranunculus acris L. & Tarpa & 1948 & Simon Tibor, Jakucs Pál & BPU-00952.JPG \\
\hline 455 & Ranunculus acris L. & Tarpa & 1948 & Simon Tibor, Jakucs Pál & BPU-00953.JPG \\
\hline 455 & Ranunculus acris L. & Tihany & 1946 & Felföldy Lajos & BPU-00957.JPG \\
\hline 455 & Ranunculus acris L. & Tihany & 1959 & Felföldy Lajos & BPU-00958.JPG \\
\hline 455 & Ranunculus acris L. & Villány & 1962 & Priszter Szaniszló & BPU-00951.JPG \\
\hline 457 & $\begin{array}{l}\text { Ceratocephala testiculata (Crantz) } \\
\text { Roth }\end{array}$ & Abaújszántó & 1938 & Soó Rezső & BPU-02202.JPG \\
\hline 457 & $\begin{array}{l}\text { Ceratocephala testiculata (Crantz) } \\
\text { Roth }\end{array}$ & Budapest & 1912 & $\begin{array}{l}\text { Moesz Gusztáv, Szurák } \\
\text { János }\end{array}$ & BPU-02204.JPG \\
\hline 457 & $\begin{array}{l}\text { Ceratocephala testiculata (Crantz) } \\
\text { Roth }\end{array}$ & Budapest & 1947 & Papp József & BPU-02201.JPG \\
\hline 457 & $\begin{array}{l}\text { Ceratocephala testiculata (Crantz) } \\
\text { Roth }\end{array}$ & Budapest & 1949 & Soó Rezső & BPU-02203.JPG \\
\hline 459 & Myosurus minimus L. & Balmazújváros & 1932 & Soó Rezső & BPU-02200.JPG \\
\hline 459 & Myosurus minimus L. & Budapest & 1949 & Soó Rezső & BPU-02198.JPG \\
\hline 459 & Myosurus minimus L. & Budapest & 1949 & Soó Rezső & BPU-15562.JPG \\
\hline
\end{tabular}


1. Elektroniukus melléklet - Electronic Appendix 1.

\begin{tabular}{|c|c|c|c|c|c|}
\hline $\begin{array}{l}\text { Sorszám / } \\
\text { Number }\end{array}$ & Taxon-név / Taxon-name & $\begin{array}{l}\text { Település / } \\
\text { Settlement }\end{array}$ & $\begin{array}{l}\text { Év / } \\
\text { Year }\end{array}$ & Gyújtő / Collector & $\begin{array}{l}\text { Fájlnév / } \\
\text { File-name }\end{array}$ \\
\hline 459 & Myosurus minimus L. & Csaroda & 1951 & $\begin{array}{l}\text { Simon Tibor, Jakucs Pál, } \\
\text { Kulcsár Gábor }\end{array}$ & BPU-02197.JPG \\
\hline 459 & Myosurus minimus L. & Gyöngyös & 1923 & Soó Rezső & BPU-02199.JPG \\
\hline 459 & Myosurus minimus L. & Vásárosnamény & 1951 & Simon Tibor & BPU-02196.JPG \\
\hline 460 & Thalictrum aquilegiifolium L. & Balatonszőlős & 1951 & Felföldy Lajos & BPU-01001.JPG \\
\hline 460 & Thalictrum aquilegiifolium $\mathrm{L}$. & Debrecen & 1935 & Soó Rezső & BPU-01002.JPG \\
\hline 461 & Thalictrum foetidum L. & Bélapátfalva & 1950 & $\begin{array}{l}\text { Simon Tibor, Jakucs Pál, } \\
\text { Kárpáti István }\end{array}$ & BPU-01007.JPG \\
\hline 461 & Thalictrum foetidum $\mathrm{L}$. & Bélapátfalva & 1953 & Simon Tibor & BPU-01006.JPG \\
\hline 461 & Thalictrum foetidum L. & Bódvarákó & 1911 & Thaisz Lajos & BPU-01008.JPG \\
\hline 462 & Thalictrum minus L. & Bátorliget & 1950 & $\begin{array}{l}\text { Simon Tibor, Kárpáti } \\
\text { István }\end{array}$ & BPU-01020.JPG \\
\hline 462 & Thalictrum minus L. & Bélapátfalva & 1948 & $\begin{array}{l}\text { Simon Tibor, Pólya } \\
\text { László, Jakucs Pál }\end{array}$ & BPU-01012.JPG \\
\hline 462 & Thalictrum minus L. & Debrecen & 1933 & Soó Rezső & BPU-01014.JPG \\
\hline 462 & Thalictrum minus L. & Debrecen & 1933 & Soó Rezső & BPU-01015.JPG \\
\hline 462 & Thalictrum minus L. & Debrecen & 1935 & Soó Rezső & BPU-01017.JPG \\
\hline 462 & Thalictrum minus L. & Debrecen & 1935 & Soó Rezső & BPU-01018.JPG \\
\hline 462 & Thalictrum minus L. & Jósvafó & 1951 & $\begin{array}{l}\text { Soó Rezső, Jakucs Pál, Ér } \\
\text { Lajos }\end{array}$ & BPU-01019.JPG \\
\hline 462 & Thalictrum minus L. & Kőszeg & 1932 & Soó Rezső & BPU-01016.JPG \\
\hline 462 & Thalictrum minus L. & Pilisszentiván & 1914 & Degen Árpád & BPU-01013.JPG \\
\hline 462 & Thalictrum minus L. & Pilisszentiván & 1953 & Soó Rezső & BPU-01011.JPG \\
\hline 462 & Thalictrum minus L. & Ravazd & 1936 & Polgár Sándor & BPU-01022.JPG \\
\hline 462 & Thalictrum minus L. & Telki & 1947 & Soó Rezső & BPU-01010.JPG \\
\hline 464 & Thalictrum simplex L. & Aszófő & 1956 & $\begin{array}{l}\text { Felföldy Lajos, Tóth } \\
\text { László }\end{array}$ & BPU-01024.JPG \\
\hline 464 & Thalictrum simplex L. & Budapest & 1946 & Kárpáti Zoltán & BPU-01037.JPG \\
\hline 465 & Thalictrum flavum L. & Győr & 1905 & Polgár Sándor & BPU-01028.JPG \\
\hline 465 & Thalictrum flavum L. & Nagykanizsa & 1948 & Károlyi Árpád & BPU-01027.JPG \\
\hline 465 & Thalictrum flavum L. & Tiszacsege & 1951 & Borsos Olga, Simon Tibor & BPU-01025.JPG \\
\hline 465 & Thalictrum flavum L. & Tiszafüred & 1953 & Borsos Olga & BPU-01026.JPG \\
\hline 466 & Thalictrum lucidum L. & Bátorliget & 1948 & Simon Tibor, Jakucs Pál & BPU-01036.JPG \\
\hline 466 & Thalictrum lucidum L. & Debrecen & 1932 & Soó Rezső & BPU-01030.JPG \\
\hline 466 & Thalictrum lucidum L. & Magyarlukafa & 1957 & Soó Rezső & BPU-01034.JPG \\
\hline 466 & Thalictrum lucidum L. & Magyarlukafa & 1957 & Soó Rezső & BPU-01035.JPG \\
\hline 466 & Thalictrum lucidum L. & Miskolc & 1937 & Soó Rezső & BPU-01029.JPG \\
\hline 466 & Thalictrum lucidum L. & Nyírpilis & 1932 & Soó Rezső & BPU-01031.JPG \\
\hline 466 & Thalictrum lucidum L. & Ócsa & 1953 & Soó Rezső, Borsos Olga & BPU-01032.JPG \\
\hline 466 & Thalictrum lucidum L. & Vámosszabadi & 1931 & Soó Rezső & BPU-01033.JPG \\
\hline 467 & Paeonia officinalis L. & Pécsvárad & 1936 & Soó Rezső & BPU-00384.JPG \\
\hline 470 & Berberis vulgaris L. & Csévharaszt & 1959 & $\begin{array}{l}\text { Simon Tibor, Horánszky } \\
\text { András }\end{array}$ & BPU-01068.JPG \\
\hline 470 & Berberis vulgaris L. & Dabas & 1965 & Priszter Szaniszló & BPU-01069.JPG \\
\hline 470 & Berberis vulgaris L. & Sopron & 1960 & Borsos Olga, Simon Tibor & BPU-01070.JPG \\
\hline 477 & Papaver argemone L. & Nagykanizsa & 1948 & Károlyi Árpád & BPU-06462.JPG \\
\hline 478 & Papaver hybridum L. & Békéscsaba & 1923 & Soó Rezső & BPU-06461.JPG \\
\hline 479 & Papaver rhoeas L. & Debrecen & 1938 & Felföldy Lajos & BPU-06487.JPG \\
\hline 479 & Papaver rhoeas L. & Debrecen & 1945 & Felföldy Lajos & BPU-06466.JPG \\
\hline 479 & Papaver rhoeas L. & Debrecen & 1945 & Felföldy Lajos & BPU-06476.JPG \\
\hline
\end{tabular}


1. Elektroniukus melléklet - Electronic Appendix 1.

\begin{tabular}{|c|c|c|c|c|c|}
\hline $\begin{array}{l}\text { Sorszám / } \\
\text { Number }\end{array}$ & Taxon-név / Taxon-name & $\begin{array}{l}\text { Település / } \\
\text { Settlement }\end{array}$ & $\begin{array}{l}\text { Év / } \\
\text { Year }\end{array}$ & Gyüjtő / Collector & $\begin{array}{l}\text { Fájlnév / } \\
\text { File-name }\end{array}$ \\
\hline 479 & Papaver rhoeas L. & Debrecen & 1945 & Felföldy Lajos & BPU-06477.JPG \\
\hline 479 & Papaver rhoeas L. & Debrecen & 1945 & Felföldy Lajos & BPU-06480.JPG \\
\hline 479 & Papaver rhoeas L. & Debrecen & 1945 & Felföldy Lajos & BPU-06484.JPG \\
\hline 479 & Papaver rhoeas L. & Kapolcs & 1959 & $\begin{array}{l}\text { Jávorka Sándor, Felföldy } \\
\text { Lajos, Tóth László }\end{array}$ & BPU-06474.JPG \\
\hline 479 & Papaver rhoeas L. & Tihany & 1946 & Felföldy Lajos & BPU-06467.JPG \\
\hline 479 & Papaver rhoeas L. & Tihany & 1946 & Felföldy Lajos & BPU-06468.JPG \\
\hline 479 & Papaver rhoeas L. & Tihany & 1946 & Felföldy Lajos & BPU-06469.JPG \\
\hline 479 & Papaver rhoeas L. & Tihany & 1946 & Felföldy Lajos & BPU-06475.JPG \\
\hline 479 & Papaver rhoeas L. & Tihany & 1946 & Felföldy Lajos & BPU-06478.JPG \\
\hline 479 & Papaver rhoeas L. & Tihany & 1946 & Felföldy Lajos & BPU-06479.JPG \\
\hline 479 & Papaver rhoeas L. & Tihany & 1946 & Felföldy Lajos & BPU-06481.JPG \\
\hline 479 & Papaver rhoeas L. & Tihany & 1946 & Felföldy Lajos & BPU-06482.JPG \\
\hline 479 & Papaver rhoeas L. & Tihany & 1946 & Felföldy Lajos & BPU-06483.JPG \\
\hline 479 & Papaver rhoeas L. & Tihany & 1946 & Felföldy Lajos & BPU-06485.JPG \\
\hline 479 & Papaver rhoeas L. & Tihany & 1946 & Felföldy Lajos & BPU-06486.JPG \\
\hline 479 & Papaver rhoeas L. & Tihany & 1946 & Felföldy Lajos & BPU-06488.JPG \\
\hline 479 & Papaver rhoeas L. & Tihany & 1959 & Felföldy Lajos & BPU-06473.JPG \\
\hline 480 & Papaver dubium L. & Debrecen & 1943 & Máthé Imre & BPU-06458.JPG \\
\hline 480 & Papaver dubium L. & Debrecen & 1945 & Felföldy Lajos & BPU-06457.JPG \\
\hline 480 & Papaver dubium L. & Debrecen & 1947 & Soó Rezső & BPU-06460.JPG \\
\hline 480 & Papaver dubium L. & Pécs & 1953 & Soó Rezső, Borsos Olga & BPU-06459.JPG \\
\hline 482 & Glaucium corniculatum (L.) Rudolph & Budapest & 1947 & Soó Rezső & BPU-06465.JPG \\
\hline 482 & Glaucium corniculatum (L.) Rudolph & Hajdúszoboszló & 1937 & Soó Rezső & BPU-06464.JPG \\
\hline 482 & Glaucium corniculatum (L.) Rudolph & Tihany & 1950 & Felföldy Lajos & BPU-06463.JPG \\
\hline---- & Macleaya cordata (Willd.) R.Br. & Budapest & 1948 & Soó Rezső & BPU-06456.JPG \\
\hline 485 & Corydalis cava $\mathrm{L}$. & Aszófő & 1964 & Priszter Szaniszló & BPU-06500.JPG \\
\hline 485 & Corydalis cava L. & Balatonszőlős & 1959 & Felföldy Lajos & BPU-06501.JPG \\
\hline 485 & Corydalis cava L. & Balatonszőlős & 1959 & Felföldy Lajos & BPU-06502.JPG \\
\hline 485 & Corydalis cava L. & Beregdaróc & 1962 & Simon Tibor & BPU-06491.JPG \\
\hline 485 & Corydalis cava L. & Budapest & 1961 & Isépy István & BPU-06489.JPG \\
\hline 485 & Corydalis cava L. & Debrecen & 1932 & Soó Rezső & BPU-06495.JPG \\
\hline 485 & Corydalis cava L. & Gánt & 1949 & Soó Rezső & BPU-06492.JPG \\
\hline 485 & Corydalis cava L. & Gánt & 1949 & Soó Rezső & BPU-06493.JPG \\
\hline 485 & Corydalis cava L. & Gánt & 1949 & Soó Rezső & BPU-06494.JPG \\
\hline 485 & Corydalis cava L. & Gyöngyös & 1964 & Soó Rezső & BPU-06496.JPG \\
\hline 485 & Corydalis cava L. & Háromhuta & 1939 & Balázs Ferenc & BPU-06508.JPG \\
\hline 485 & Corydalis cava L. & Mosonmagyaróvár & 1965 & Borsos Olga & BPU-06497.JPG \\
\hline 485 & Corydalis cava L. & Panyola & 1948 & $\begin{array}{l}\text { Simon Tibor, Pólya } \\
\text { László, Jakucs Pál }\end{array}$ & BPU-06499.JPG \\
\hline 485 & Corydalis cava L. & Pécs & 1953 & Soó Rezső, Borsos Olga & BPU-06490.JPG \\
\hline 485 & Corydalis cava L. & Pécsely & 1959 & Felföldy Lajos & BPU-06503.JPG \\
\hline 485 & Corydalis cava L. & Sátoraljaújhely & 1952 & $\begin{array}{l}\text { Kulcsár Gábor, Vozáry } \\
\text { Elemér }\end{array}$ & BPU-06509.JPG \\
\hline 485 & Corydalis cava L. & Sopron & 1960 & Borsos Olga, Simon Tibor & BPU-06498.JPG \\
\hline 485 & Corydalis cava L. & Tihany & 1958 & Felföldy Lajos & BPU-06504.JPG \\
\hline 485 & Corydalis cava $\mathrm{L}$. & Tihany & 1958 & Felföldy Lajos & BPU-06505.JPG \\
\hline 485 & Corydalis cava L. & Tihany & 1958 & Felföldy Lajos & BPU-06506.JPG \\
\hline 485 & Corydalis cava $\mathrm{L}$. & Tihany & 1958 & Felföldy Lajos & BPU-06507.JPG \\
\hline
\end{tabular}


1. Elektroniukus melléklet - Electronic Appendix 1.

\begin{tabular}{|c|c|c|c|c|c|}
\hline $\begin{array}{l}\text { Sorszám / } \\
\text { Number }\end{array}$ & Taxon-név / Taxon-name & $\begin{array}{l}\text { Település / } \\
\text { Settlement }\end{array}$ & $\begin{array}{l}\text { Év / } \\
\text { Year }\end{array}$ & Gyújtő / Collector & $\begin{array}{l}\text { Fájlnév / } \\
\text { File-name }\end{array}$ \\
\hline 486 & Corydalis intermedia Link & Gánt & 1949 & Soó Rezső & BPU-06510.JPG \\
\hline 486 & Corydalis intermedia Link & Gánt & 1949 & Soó Rezső & BPU-06512.JPG \\
\hline 486 & Corydalis intermedia Link & Gánt & 1949 & Soó Rezső & BPU-15463.JPG \\
\hline 486 & Corydalis intermedia Link & Pilisszántó & 1943 & Kárpáti Zoltán & BPU-06511.JPG \\
\hline 487 & Corydalis pumila Rchb. & Aszófő & 1964 & Priszter Szaniszló & BPU-06525.JPG \\
\hline 487 & Corydalis pumila Rchb. & Balatonalmádi & 1929 & Soó Rezső & BPU-06534.JPG \\
\hline 487 & Corydalis pumila Rchb. & Balatonszőlős & 1959 & Felföldy Lajos & BPU-06526.JPG \\
\hline 487 & Corydalis pumila Rchb. & Balatonszőlős & 1959 & Felföldy Lajos & BPU-06527.JPG \\
\hline 487 & Corydalis pumila Rchb. & Gánt & 1949 & Soó Rezső & BPU-06522.JPG \\
\hline 487 & Corydalis pumila Rchb. & Gánt & 1949 & Soó Rezső & BPU-06523.JPG \\
\hline 487 & Corydalis pumila Rchb. & Pécsely & 1959 & Felföldy Lajos & BPU-06528.JPG \\
\hline 487 & Corydalis pumila Rchb. & Pilisszántó & 1943 & Kárpáti Zoltán & BPU-06524.JPG \\
\hline 487 & Corydalis pumila Rchb. & Tihany & 1951 & Felföldy Lajos & BPU-06530.JPG \\
\hline 487 & Corydalis pumila Rchb. & Tihany & 1958 & Felföldy Lajos & BPU-06529.JPG \\
\hline 487 & Corydalis pumila Rchb. & Tihany & 1958 & Felföldy Lajos & BPU-06531.JPG \\
\hline 487 & Corydalis pumila Rchb. & Tihany & 1958 & Felföldy Lajos & BPU-06532.JPG \\
\hline 487 & Corydalis pumila Rchb. & Tihany & 1958 & Felföldy Lajos & BPU-06533.JPG \\
\hline 488 & Corydalis solida L. & Budapest & 1961 & Isépy István & BPU-06513.JPG \\
\hline 488 & Corydalis solida L. & Budapest & 1992 & Felföldy Lajos & BPU-06514.JPG \\
\hline 488 & Corydalis solida L. & Gyöngyös & 1964 & Soó Rezső & BPU-06516.JPG \\
\hline 488 & Corydalis solida L. & Jósvafő & 1952 & Kulcsár Gábor & BPU-06515.JPG \\
\hline 488 & Corydalis solida L. & Nagykovácsi & 1949 & Soó Rezső & BPU-06517.JPG \\
\hline 488 & Corydalis solida L. & Nagykovácsi & 1949 & Soó Rezső & BPU-06518.JPG \\
\hline 488 & Corydalis solida L. & Nagykovácsi & 1949 & Soó Rezső & BPU-06519.JPG \\
\hline $485 \times 488$ & Corydalis cava L. $\times$ Corydalis solida L. & Kehidakustány & 1946 & Ujvárosi Miklós & BPU-06537.JPG \\
\hline 491 & Fumaria officinalis L. & Gyöngyös & 1923 & Soó Rezső & BPU-06542.JPG \\
\hline 491 & Fumaria officinalis L. & Nagyharsány & 1965 & Priszter Szaniszló & BPU-06539.JPG \\
\hline 491 & Fumaria officinalis L. & Pécs & 1953 & Soó Rezső, Borsos Olga & BPU-06540.JPG \\
\hline 491 & Fumaria officinalis L. & Pécs & 1953 & Soó Rezső, Borsos Olga & BPU-06541.JPG \\
\hline 491 & Fumaria officinalis L. & Tihany & 1956 & Felföldy Lajos & BPU-06543.JPG \\
\hline 492 & Fumaria schleicheri Soy.-Will. & Bisse & 1965 & Priszter Szaniszló & BPU-06546.JPG \\
\hline 492 & Fumaria schleicheri Soy.-Will. & Budapest & 1961 & Isépy István & BPU-06544.JPG \\
\hline 492 & Fumaria schleicheri Soy.-Will. & Budapest & 1991 & Felföldy Lajos & BPU-06545.JPG \\
\hline 492 & Fumaria schleicheri Soy.-Will. & Debrecen & 1989 & Felföldy Lajos & BPU-06548.JPG \\
\hline 492 & Fumaria schleicheri Soy.-Will. & Egyek & 1950 & $\begin{array}{l}\text { Simon Tibor, Borsos Olga, } \\
\text { Kulcsár Gábor }\end{array}$ & BPU-06549.JPG \\
\hline 492 & Fumaria schleicheri Soy.-Will. & Nagyharsány & 1965 & $\begin{array}{l}\text { Simon Tibor, Priszter } \\
\text { Szaniszló }\end{array}$ & BPU-06547.JPG \\
\hline 492 & Fumaria schleicheri Soy.-Will. & Salgótarján & 1936 & Soó Rezső & BPU-06550.JPG \\
\hline 492 & Fumaria schleicheri Soy.-Will. & Tihany & 1928 & Soó Rezső & BPU-06554.JPG \\
\hline 492 & Fumaria schleicheri Soy.-Will. & Tihany & 1928 & Soó Rezső & BPU-06554.JPG \\
\hline 492 & Fumaria schleicheri Soy.-Will. & Tiszabercel & 1990 & Felföldy Lajos & BPU-06551.JPG \\
\hline 492 & Fumaria schleicheri Soy.-Will. & Újfehértó & 1936 & Soó Rezső & BPU-06552.JPG \\
\hline 493 & Fumaria vaillantii Loisel. & Budapest & 1993 & Felföldy Lajos & BPU-06556.JPG \\
\hline 493 & Fumaria vaillantii Loisel. & Debrecen & 1936 & Soó Rezső & BPU-06557.JPG \\
\hline 493 & Fumaria vaillantii Loisel. & Monostorapáti & 1959 & $\begin{array}{l}\text { Jávorka Sándor, Felföldy } \\
\text { Lajos, Tóth László }\end{array}$ & BPU-06560.JPG \\
\hline 493 & Fumaria vaillantii Loisel. & Örvényes & 1959 & Felföldy Lajos & BPU-06561.JPG \\
\hline 493 & Fumaria vaillantii Loisel. & Szigetmonostor & 1947 & Soó Rezső & BPU-06558.JPG \\
\hline
\end{tabular}


1. Elektroniukus melléklet - Electronic Appendix 1.

\begin{tabular}{|c|c|c|c|c|c|}
\hline $\begin{array}{l}\text { Sorszám / } \\
\text { Number }\end{array}$ & Taxon-név / Taxon-name & $\begin{array}{l}\text { Település / } \\
\text { Settlement }\end{array}$ & $\begin{array}{l}\text { Év / } \\
\text { Year }\end{array}$ & Gyújtő / Collector & $\begin{array}{l}\text { Fájlnév / } \\
\text { File-name }\end{array}$ \\
\hline 493 & Fumaria vaillantii Loisel. & Tihany & 1958 & Felföldy Lajos & BPU-06562.JPG \\
\hline 493 & Fumaria vaillantii Loisel. & Vácrátót & 1953 & Felföldy Lajos & BPU-06559.JPG \\
\hline 494 & Fumaria parviflora Lam. & Tihany & 1958 & Felföldy Lajos & BPU-06555.JPG \\
\hline 494 & Fumaria parviflora Lam. & Vasboldogasszony & 1951 & Károlyi Árpád & BPU-06553.JPG \\
\hline 495 & Sisymbrium officinale (L.) Scop. & Nagycserkesz & 1933 & Soó Rezső & BPU-07143.JPG \\
\hline 496 & Sisymbrium strictissimum L. & Budapest & 1947 & Soó Rezső & BPU-07149.JPG \\
\hline 496 & Sisymbrium strictissimum L. & Budapest & 2005 & Felföldy Lajos & BPU-07150.JPG \\
\hline 496 & Sisymbrium strictissimum L. & Szigetmonostor & 1947 & Soó Rezső & BPU-07151.JPG \\
\hline 497 & Sisymbrium altissimum L. & Debrecen & 1946 & Soó Rezső & BPU-07160.JPG \\
\hline 497 & Sisymbrium altissimum L. & Ócsa & 1923 & Soó Rezső & BPU-07159.JPG \\
\hline 498 & Sisymbrium orientale L. & Balatongyörök & 1961 & Felföldy Lajos & BPU-07161.JPG \\
\hline 498 & Sisymbrium orientale L. & Pécs & 1953 & Soó Rezső, Borsos Olga & BPU-07163.JPG \\
\hline 498 & Sisymbrium orientale L. & Szigetmonostor & 1947 & Soó Rezső & BPU-07162.JPG \\
\hline 498 & Sisymbrium orientale L. & Tihany & 1928 & Soó Rezső & BPU-07164.JPG \\
\hline 499 & $\begin{array}{l}\text { Sisymbrium polymorphum (Murray) } \\
\text { Roth }\end{array}$ & Abaújszántó & 1938 & Soó Rezső & BPU-07148.JPG \\
\hline 500 & Sisymbrium loeselii L. & Budaörs & 1991 & Felföldy Lajos & BPU-07157.JPG \\
\hline 500 & Sisymbrium loeselii L. & Budapest & 1947 & Soó Rezső & BPU-07152.JPG \\
\hline 500 & Sisymbrium loeselii L. & Budapest & 1982 & Felföldy Lajos & BPU-07154.JPG \\
\hline 500 & Sisymbrium loeselii L. & Budapest & 1982 & Felföldy Lajos & BPU-07155.JPG \\
\hline 500 & Sisymbrium loeselii L. & Budapest & 1982 & Felföldy Lajos & BPU-07156.JPG \\
\hline 500 & Sisymbrium loeselii L. & Budapest & 1991 & Felföldy Lajos & BPU-07153.JPG \\
\hline 501 & Descurainia sophia (L.) Webb & Bátorliget & 1932 & Soó Rezső & BPU-07147.JPG \\
\hline 501 & Descurainia sophia (L.) Webb & Gárdony & 1963 & Isépy István & BPU-07144.JPG \\
\hline 501 & Descurainia sophia (L.) Webb & Gárdony & 1963 & Isépy István & BPU-07145.JPG \\
\hline 501 & Descurainia sophia (L.) Webb & Vácrátót & 1953 & Felföldy Lajos & BPU-07146.JPG \\
\hline 502 & $\begin{array}{l}\text { Alliaria petiolata (M. Bieb.) Cavara et } \\
\text { Grande }\end{array}$ & Budapest & 1961 & Isépy István & BPU-07140.JPG \\
\hline 502 & $\begin{array}{l}\text { Alliaria petiolata (M. Bieb.) Cavara et } \\
\text { Grande }\end{array}$ & Debrecen & 1934 & Soó Rezső & BPU-07141.JPG \\
\hline 502 & $\begin{array}{l}\text { Alliaria petiolata (M. Bieb.) Cavara et } \\
\text { Grande }\end{array}$ & Vászoly & 1955 & $\begin{array}{l}\text { Felföldy Lajos, Tóth } \\
\text { László }\end{array}$ & BPU-07142.JPG \\
\hline 503 & Arabidopsis thaliana (L.) Heynh. & Balatonszőlős & 1959 & Felföldy Lajos & BPU-07166.JPG \\
\hline 503 & Arabidopsis thaliana (L.) Heynh. & Farmos & 1923 & Soó Rezső & BPU-07165.JPG \\
\hline 504 & Myagrum perfoliatum L. & Budapest & 1922 & Soó Rezső & BPU-06724.JPG \\
\hline 504 & Myagrum perfoliatum L. & Hajdúbagos & 1935 & Soó Rezső & BPU-06723.JPG \\
\hline 504 & Myagrum perfoliatum L. & Tihany & 1961 & $\begin{array}{l}\text { Pankov H., Felföldy Lajos, } \\
\text { Sólymosy G. }\end{array}$ & BPU-06722.JPG \\
\hline 505 & Isatis tinctoria $\mathrm{L}$. & Bugyi & 1959 & Soó Rezső & BPU-06648.JPG \\
\hline 505 & Isatis tinctoria $\mathrm{L}$. & Debrecen & 1932 & Soó Rezső & BPU-06647.JPG \\
\hline 506 & Bunias orientalis L. & Budaörs & 1947 & Soó Rezső & BPU-06729.JPG \\
\hline 506 & Bunias orientalis L. & Budapest & 1991 & Felföldy Lajos & BPU-06727.JPG \\
\hline 506 & Bunias orientalis L. & Budapest & 2005 & Felföldy Lajos & BPU-06728.JPG \\
\hline 506 & Bunias orientalis L. & Nyíracsád & 1935 & Soó Rezső & BPU-06730.JPG \\
\hline 508 & Erysimum cheiranthoides L. & Budapest & 1923 & Soó Rezső & BPU-07106.JPG \\
\hline 508 & Erysimum cheiranthoides L. & Lakitelek & 1991 & Felföldy Lajos & BPU-07104.JPG \\
\hline 508 & Erysimum cheiranthoides L. & Sárospatak & 1938 & Soó Rezső & BPU-07107.JPG \\
\hline 508 & Erysimum cheiranthoides L. & Tiszacsege & 1951 & Borsos Olga, Simon Tibor & BPU-07105.JPG \\
\hline 509 & Erysimum repandum L. & Hajdúnánás & 1935 & Soó Rezső & BPU-07108.JPG \\
\hline 510 & Erysimum diffusum Ehrh. & Budapest & 2005 & Felföldy Lajos & BPU-07113.JPG \\
\hline
\end{tabular}


1. Elektroniukus melléklet - Electronic Appendix 1.

\begin{tabular}{|c|c|c|c|c|c|}
\hline $\begin{array}{l}\text { Sorszám / } \\
\text { Number }\end{array}$ & Taxon-név / Taxon-name & $\begin{array}{l}\text { Település / } \\
\text { Settlement }\end{array}$ & $\begin{array}{l}\text { Év / } \\
\text { Year }\end{array}$ & Gyújtő / Collector & $\begin{array}{l}\text { Fájlnév / } \\
\text { File-name }\end{array}$ \\
\hline 510 & Erysimum diffusum Ehrh. & Nagycserkesz & 1933 & Soó Rezső & BPU-07110.JPG \\
\hline 510 & Erysimum diffusum Ehrh. & Szigetmonostor & 1947 & Soó Rezső & BPU-07112.JPG \\
\hline 510 & Erysimum diffusum Ehrh. & Tihany & 1949 & Simon Tibor & BPU-07109.JPG \\
\hline 510 & Erysimum diffusum Ehrh. & Vácrátót & 1953 & Felföldy Lajos & BPU-07111.JPG \\
\hline 513 & Erysimum witmannii Zaw. & Tállya & 1927 & Hulják János & BPU-07117.JPG \\
\hline 513 & Erysimum witmannii Zaw. & Vác & 1923 & Soó Rezső & BPU-07116.JPG \\
\hline 514 & Erysimum odoratum Ehrh. & Budapest & 1947 & Soó Rezső & BPU-07126.JPG \\
\hline 514 & Erysimum odoratum Ehrh. & Budapest & 1982 & Felföldy Lajos & BPU-07121.JPG \\
\hline 514 & Erysimum odoratum Ehrh. & Miskolc & 1939 & Soó Rezső & BPU-07125.JPG \\
\hline 514 & Erysimum odoratum Ehrh. & Nagyvisnyó & 1953 & Soó Rezső & BPU-07127.JPG \\
\hline 514 & Erysimum odoratum Ehrh. & Pécs & 1935 & Soó Rezső & BPU-07124.JPG \\
\hline 514 & Erysimum odoratum Ehrh. & Uzsa & 1959 & $\begin{array}{l}\text { Jávorka Sándor, Felföldy } \\
\text { Lajos }\end{array}$ & BPU-07120.JPG \\
\hline 514 & Erysimum odoratum Ehrh. & Várpalota & 1955 & Soó Rezső & BPU-07123.JPG \\
\hline 515 & Syrenia cana (Piller et Mitterp.) Neilr. & Budapest & 1923 & Soó Rezső & BPU-07139.JPG \\
\hline 515 & Syrenia cana (Piller et Mitterp.) Neilr. & Bugac & 1964 & Soó Rezső & BPU-07136.JPG \\
\hline 515 & Syrenia cana (Piller et Mitterp.) Neilr. & Bugac & 1964 & Soó Rezső & BPU-07137.JPG \\
\hline 515 & Syrenia cana (Piller et Mitterp.) Neilr. & Csévharaszt & 1959 & Simon Tibor, Borsos Olga & BPU-07135.JPG \\
\hline 515 & Syrenia cana (Piller et Mitterp.) Neilr. & Veresegyház & 1949 & Soó Rezső & BPU-15488.JPG \\
\hline 515 & Syrenia cana (Piller et Mitterp.) Neilr. & Veresegyház & 1950 & Soó Rezső & BPU-07138.JPG \\
\hline 516 & Hesperis tristis L. & Budapest & 1953 & Soó Rezső & BPU-07083.JPG \\
\hline 516 & Hesperis tristis L. & Egyek & 1935 & Soó Rezső & BPU-07085.JPG \\
\hline 516 & Hesperis tristis L. & Győr & 1959 & $\begin{array}{l}\text { Simon Tibor, Borhidi } \\
\text { Attila }\end{array}$ & BPU-07081.JPG \\
\hline 516 & Hesperis tristis L. & Nagyharsány & 1956 & Soó Rezső & BPU-07086.JPG \\
\hline 516 & Hesperis tristis L. & Pécs & 1953 & Soó Rezső, Borsos Olga & BPU-07084.JPG \\
\hline 516 & Hesperis tristis L. & Tihany & 1956 & Felföldy Lajos & BPU-07079.JPG \\
\hline 516 & Hesperis tristis L. & Tihany & 1958 & Felföldy Lajos & BPU-07080.JPG \\
\hline 516 & Hesperis tristis L. & Tihany & 1958 & Kozma Erika & BPU-07078.JPG \\
\hline 516 & Hesperis tristis L. & Tokaj & 1933 & Soó Rezső & BPU-07082.JPG \\
\hline 517 & Hesperis matronalis L. & "Pilis" & 1932 & Soó Rezső & BPU-07093.JPG \\
\hline 517 & Hesperis matronalis L. & Budaörs & 1961 & Borsos Olga, Simon Tibor & BPU-07087.JPG \\
\hline 517 & Hesperis matronalis L. & Gyöngyös & 1953 & Soó Rezső & BPU-07090.JPG \\
\hline 517 & Hesperis matronalis L. & Miskolc & 1930 & Soó Rezső & BPU-07089.JPG \\
\hline 517 & Hesperis matronalis L. & Miskolc & 1948 & $\begin{array}{l}\text { Simon Tibor, Borsos Olga, } \\
\text { Jakucs Pál }\end{array}$ & BPU-07092.JPG \\
\hline 517 & Hesperis matronalis L. & Miskolc & 1953 & Soó Rezső & BPU-07088.JPG \\
\hline 517 & Hesperis matronalis L. & Pilisszentkereszt & 1955 & Soó Rezső & BPU-07091.JPG \\
\hline 517 & Hesperis matronalis L. & Szilvásvárad & 1912 & Hulják János & BPU-07099.JPG \\
\hline 517 & Hesperis matronalis L. & Szilvásvárad & 1948 & $\begin{array}{l}\text { Simon Tibor, Pólya } \\
\text { László, Jakucs Pál }\end{array}$ & BPU-07098.JPG \\
\hline 518 & Hesperis sylvestris Crantz & Gyula & 1935 & Soó Rezső & BPU-07102.JPG \\
\hline 518 & Hesperis sylvestris Crantz & Tákos & 1951 & Simon Tibor & BPU-07100.JPG \\
\hline 519 & Malcolmia africana (L.) R. Br. & Budapest & 1909 & Kocsis István & BPU-07103.JPG \\
\hline 523 & Euclidium syriacum (L.) R. Br. & Balatonudvari & 1959 & Felföldy Lajos & BPU-06735.JPG \\
\hline 523 & Euclidium syriacum (L.) R. Br. & Gárdony & 1966 & Priszter Szaniszló & BPU-06732.JPG \\
\hline 523 & Euclidium syriacum (L.) R. Br. & Hajdúnánás & 1935 & Soó Rezső & BPU-06733.JPG \\
\hline 523 & Euclidium syriacum (L.) R. Br. & Nagykáta & 1933 & Boros Ádám & BPU-06734.JPG \\
\hline 523 & Euclidium syriacum (L.) R. Br. & Tihany & 1961 & Felföldy Lajos & BPU-06736.JPG \\
\hline
\end{tabular}


1. Elektroniukus melléklet - Electronic Appendix 1.

\begin{tabular}{|c|c|c|c|c|c|}
\hline $\begin{array}{l}\text { Sorszám / } \\
\text { Number }\end{array}$ & Taxon-név / Taxon-name & $\begin{array}{l}\text { Település / } \\
\text { Settlement }\end{array}$ & $\begin{array}{l}\text { Év / } \\
\text { Year }\end{array}$ & Gyűjtő / Collector & $\begin{array}{l}\text { Fájlnév / } \\
\text { File-name }\end{array}$ \\
\hline 525 & Barbarea stricta Andrz. & Tákos & 1948 & $\begin{array}{l}\text { Pólya László, Simon } \\
\text { Tibor, Jakucs Pál }\end{array}$ & BPU-06938.JPG \\
\hline 526 & Barbarea vulgaris R. Br. & Csákberény & 1982 & Felföldy Lajos & BPU-06940.JPG \\
\hline 526 & Barbarea vulgaris R. Br. & Debrecen & 1935 & Soó Rezső & BPU-06941.JPG \\
\hline 526 & Barbarea vulgaris R. Br. & Háromhuta & 1955 & Simon Tibor & BPU-06942.JPG \\
\hline 526 & Barbarea vulgaris R. Br. & Vásárosnamény & 1951 & Simon Tibor & BPU-06939.JPG \\
\hline 527 & Rapistrum perenne (L.) All. & Budaörs & 1947 & Soó Rezső & BPU-06608.JPG \\
\hline 527 & Rapistrum perenne (L.) All. & Budapest & 1982 & Felföldy Lajos & BPU-06604.JPG \\
\hline 527 & Rapistrum perenne (L.) All. & Budapest & 1982 & Felföldy Lajos & BPU-06605.JPG \\
\hline 527 & Rapistrum perenne (L.) All. & Hajdúszoboszló & 1937 & Soó Rezső & BPU-06607.JPG \\
\hline 527 & Rapistrum perenne (L.) All. & Pilisszentkereszt & 1955 & Soó Rezső & BPU-06606.JPG \\
\hline 529 & Nasturtium officinale R. Br. & Kishuta & 1952 & Simon Tibor & BPU-07030.JPG \\
\hline 529 & Nasturtium officinale R. Br. & Lesencetomaj & 1952 & Simon Tibor & BPU-07032.JPG \\
\hline 529 & Nasturtium officinale R. Br. & Pethőhenye & 1953 & Pócs Tamás & BPU-07034.JPG \\
\hline 529 & Nasturtium officinale R. Br. & Tapolca & 1928 & Soó Rezső & BPU-07033.JPG \\
\hline 529 & Nasturtium officinale R. Br. & Tata & 1932 & Jávorka Sándor & BPU-07031.JPG \\
\hline 530 & $\begin{array}{l}\text { Armoracia rusticana G. Gaertn., B. Mey. } \\
\text { et Scherb. }\end{array}$ & Konyár & 1949 & Soó Rezső & BPU-06862.JPG \\
\hline 530 & $\begin{array}{l}\text { Armoracia rusticana G. Gaertn., B. Mey. } \\
\text { et Scherb. }\end{array}$ & Konyár & 1949 & Soó Rezső & BPU-15459.JPG \\
\hline 531 & $\begin{array}{l}\text { Armoracia macrocarpa (Waldst. et } \\
\text { Kit.) Kit. ex Baumg. }\end{array}$ & Egyek & 1932 & Soó Rezső & BPU-06861.JPG \\
\hline 532 & Cardamine impatiens L. & Balatonszőlős & 1958 & Felföldy Lajos & BPU-06866.JPG \\
\hline 532 & Cardamine impatiens L. & Isztimér & 1949 & Soó Rezső & BPU-06870.JPG \\
\hline 532 & Cardamine impatiens L. & Miskolc & 1948 & $\begin{array}{l}\text { Simon Tibor, Borsos Olga, } \\
\text { Jakucs Pál }\end{array}$ & BPU-06865.JPG \\
\hline 532 & Cardamine impatiens L. & Nagyvisnyó & 1953 & Soó Rezső & BPU-06868.JPG \\
\hline 532 & Cardamine impatiens L. & Nyíradony & 1951 & Simon Tibor & BPU-06867.JPG \\
\hline 532 & Cardamine impatiens L. & Pécs & 1953 & Soó Rezső, Borsos Olga & BPU-06869.JPG \\
\hline 535 & Cardamine enneaphyllos (L.) Crantz & Budapest & 1961 & Isépy István & BPU-06915.JPG \\
\hline 535 & Cardamine enneaphyllos (L.) Crantz & Gánt & 1949 & Soó Rezső & BPU-06918.JPG \\
\hline 535 & Cardamine enneaphyllos (L.) Crantz & Isztimér & 1949 & Soó Rezső & BPU-15470.JPG \\
\hline 535 & Cardamine enneaphyllos (L.) Crantz & Lasztonya & 1948 & Károlyi Árpád & BPU-15458.JPG \\
\hline 535 & Cardamine enneaphyllos (L.) Crantz & Pécs & 1953 & Soó Rezső, Borsos Olga & BPU-06917.JPG \\
\hline 535 & Cardamine enneaphyllos (L.) Crantz & Pécs & 1956 & Soó Rezső & BPU-06916.JPG \\
\hline 536 & Cardamine glanduligera O. Schwarz & Háromhuta & 1939 & Balázs Ferenc & BPU-06922.JPG \\
\hline 536 & Cardamine glanduligera O. Schwarz & Szögliget & 1954 & Jakucs Pál & BPU-06924.JPG \\
\hline 537 & Cardamine bulbifera (L.) Crantz & "Cuha-völgy" & 1954 & Soó Rezső & BPU-06936.JPG \\
\hline 537 & Cardamine bulbifera (L.) Crantz & Bátorliget & 1935 & Soó Rezső & BPU-06934.JPG \\
\hline 537 & Cardamine bulbifera (L.) Crantz & Budapest & 1953 & Soó Rezső & BPU-15415.JPG \\
\hline 537 & Cardamine bulbifera (L.) Crantz & Budapest & 1961 & Isépy István & BPU-06926.JPG \\
\hline 537 & Cardamine bulbifera (L.) Crantz & Budapest & 1964 & Soó Rezső & BPU-06927.JPG \\
\hline 537 & Cardamine bulbifera (L.) Crantz & Csesznek & 1929 & Soó Rezső & BPU-06935.JPG \\
\hline 537 & Cardamine bulbifera (L.) Crantz & Lábatlan & 1953 & Felföldy Lajos & BPU-06929.JPG \\
\hline 537 & Cardamine bulbifera (L.) Crantz & Mosonmagyaróvár & 1965 & Borsos Olga & BPU-06930.JPG \\
\hline 537 & Cardamine bulbifera (L.) Crantz & Nagykovácsi & 1963 & Isépy István & BPU-06931.JPG \\
\hline 537 & Cardamine bulbifera (L.) Crantz & Nagymaros & 1962 & Simon Tibor & BPU-06932.JPG \\
\hline 537 & Cardamine bulbifera (L.) Crantz & Pécs & 1953 & Soó Rezső, Borsos Olga & BPU-06928.JPG \\
\hline 537 & Cardamine bulbifera (L.) Crantz & Sopron & 1960 & Borsos Olga, Simon Tibor & BPU-06933.JPG \\
\hline 538 & Cardamine parviflora L. & Barbacs & 1932 & Zólyomi Bálint & BPU-06874.JPG \\
\hline
\end{tabular}


1. Elektroniukus melléklet - Electronic Appendix 1.

\begin{tabular}{|c|c|c|c|c|c|}
\hline $\begin{array}{l}\text { Sorszám / } \\
\text { Number }\end{array}$ & Taxon-név / Taxon-name & $\begin{array}{l}\text { Település / } \\
\text { Settlement }\end{array}$ & $\begin{array}{l}\text { Év / } \\
\text { Year }\end{array}$ & Gyüjtő / Collector & $\begin{array}{l}\text { Fájlnév / } \\
\text { File-name }\end{array}$ \\
\hline 539 & Cardamine hirsuta L. & Bátorliget & 1942 & Máthé Imre & BPU-06873.JPG \\
\hline 539 & Cardamine hirsuta L. & Bisse & 1965 & $\begin{array}{l}\text { Simon Tibor, Priszter } \\
\text { Szaniszló, Borhidi Attila }\end{array}$ & BPU-06871.JPG \\
\hline 539 & Cardamine hirsuta L. & Siklós & 1969 & Priszter Szaniszló & BPU-06872.JPG \\
\hline 539 & Cardamine hirsuta L. & Uzsa & 1950 & Felföldy Lajos & BPU-06878.JPG \\
\hline 541 & Cardamine amara L. & Bakonybél & 1955 & Soó Rezső & BPU-06889.JPG \\
\hline 541 & Cardamine amara L. & Csesznek & 1929 & Soó Rezső & BPU-06892.JPG \\
\hline 541 & Cardamine amara L. & Kisbodak & 1991 & Simon Tibor & BPU-06881.JPG \\
\hline 541 & Cardamine amara L. & Miskolc & 1925 & Zólyomi Bálint & BPU-06886.JPG \\
\hline 541 & Cardamine amara L. & Miskolc & 1955 & Tóth László & BPU-06885.JPG \\
\hline 541 & Cardamine amara L. & Nagykanizsa & 1949 & Károlyi Árpád & BPU-06883.JPG \\
\hline 541 & Cardamine amara L. & Örvényes & 1959 & Felföldy Lajos & BPU-06879.JPG \\
\hline 541 & Cardamine amara L. & Örvényes & 1959 & Felföldy Lajos & BPU-06880.JPG \\
\hline 541 & Cardamine amara L. & Pécs & 1956 & Soó Rezső & BPU-06888.JPG \\
\hline 542 & Cardamine pratensis L. & Balmazújváros & 1932 & Soó Rezső & BPU-06898.JPG \\
\hline 542 & Cardamine pratensis L. & Bozsok & 1938 & Soó Rezső & BPU-06901.JPG \\
\hline 542 & Cardamine pratensis L. & Debrecen & 1947 & Soó Rezső & BPU-06896.JPG \\
\hline 542 & Cardamine pratensis L. & Felsőtelekes & 2005 & Gulyás Pál & BPU-06895.JPG \\
\hline 542 & Cardamine pratensis L. & Gyöngyös & 1964 & Soó Rezső & BPU-06897.JPG \\
\hline 542 & Cardamine pratensis L. & Kőszeg & 1938 & Soó Rezső & BPU-06902.JPG \\
\hline 542 & Cardamine pratensis L. & Lenti & 1948 & Károlyi Árpád & BPU-06903.JPG \\
\hline 542 & Cardamine pratensis L. & Sióagárd & 1965 & $\begin{array}{l}\text { Priszter Szaniszló, Simon } \\
\text { Tibor }\end{array}$ & BPU-06900.JPG \\
\hline 542 & Cardamine pratensis L. & Sopron & 1960 & Borsos Olga, Simon Tibor & BPU-06899.JPG \\
\hline 542 & Cardamine pratensis L. & Tákos & 1948 & $\begin{array}{l}\text { Pólya László, Simon } \\
\text { Tibor, Jakucs Pál }\end{array}$ & BPU-06894.JPG \\
\hline 544 & Cardaminopsis petraea (L.) Hiitonen & Gyenesdiás & 1928 & Soó Rezső & BPU-06961.JPG \\
\hline 545 & Cardaminopsis arenosa (L.) Hayek & Budapest & 1961 & Isépy István & BPU-06946.JPG \\
\hline 545 & Cardaminopsis arenosa (L.) Hayek & Budapest & 2004 & Felföldy Lajos & BPU-06945.JPG \\
\hline 545 & Cardaminopsis arenosa (L.) Hayek & Budapest & 2005 & Felföldy Lajos & BPU-06943.JPG \\
\hline 545 & Cardaminopsis arenosa (L.) Hayek & Budapest & 2005 & Felföldy Lajos & BPU-06944.JPG \\
\hline 545 & Cardaminopsis arenosa (L.) Hayek & Dömös & 1948 & Soó Rezső & BPU-06948.JPG \\
\hline 545 & Cardaminopsis arenosa (L.) Hayek & Füzér & 1937 & Soó Rezső & BPU-06957.JPG \\
\hline 545 & Cardaminopsis arenosa (L.) Hayek & Gánt & 1949 & Soó Rezső & BPU-06949.JPG \\
\hline 545 & Cardaminopsis arenosa (L.) Hayek & Gyöngyös & 1953 & Soó Rezső & BPU-06950.JPG \\
\hline 545 & Cardaminopsis arenosa (L.) Hayek & Hidegkút & 1959 & Felföldy Lajos & BPU-06954.JPG \\
\hline 545 & Cardaminopsis arenosa (L.) Hayek & Miskolc & 1949 & Jakucs Pál & BPU-06947.JPG \\
\hline 545 & Cardaminopsis arenosa (L.) Hayek & Nagykovácsi & 1949 & Soó Rezső & BPU-06951.JPG \\
\hline 545 & Cardaminopsis arenosa (L.) Hayek & Tihany & 1958 & Felföldy Lajos & BPU-06955.JPG \\
\hline 545 & Cardaminopsis arenosa (L.) Hayek & Vácrátót & 1950 & Soó Rezső & BPU-06952.JPG \\
\hline 545 & Cardaminopsis arenosa (L.) Hayek & Vászoly & 1955 & $\begin{array}{l}\text { Felföldy Lajos, Tóth } \\
\text { László }\end{array}$ & BPU-06956.JPG \\
\hline 545 & Cardaminopsis arenosa (L.) Hayek & Visegrád & 1964 & Soó Rezső, Borsos Olga & BPU-06953.JPG \\
\hline 546 & Arabis turrita L. & Budapest & 1964 & Soó Rezső & BPU-06991.JPG \\
\hline 546 & Arabis turrita L. & Gánt & 1949 & Soó Rezső & BPU-06996.JPG \\
\hline 546 & Arabis turrita L. & Miskolc & 1930 & Soó Rezső & BPU-06993.JPG \\
\hline 546 & Arabis turrita L. & Miskolc & 1950 & Soó Rezső & BPU-06995.JPG \\
\hline 546 & Arabis turrita L. & Miskolc & 1953 & Soó Rezső & BPU-06994.JPG \\
\hline 546 & Arabis turrita L. & Nagykovácsi & 1963 & Isépy István & BPU-06997.JPG \\
\hline
\end{tabular}


1. Elektroniukus melléklet - Electronic Appendix 1.

\begin{tabular}{|c|c|c|c|c|c|}
\hline $\begin{array}{l}\text { Sorszám / } \\
\text { Number }\end{array}$ & Taxon-név / Taxon-name & $\begin{array}{l}\text { Település / } \\
\text { Settlement }\end{array}$ & $\begin{array}{l}\text { Év / } \\
\text { Year }\end{array}$ & Gyújtő / Collector & $\begin{array}{l}\text { Fájlnév / } \\
\text { File-name }\end{array}$ \\
\hline 546 & Arabis turrita L. & Pécs & 1935 & Soó Rezső & BPU-06992.JPG \\
\hline 546 & Arabis turrita L. & Tihany & 1958 & Felföldy Lajos & BPU-06999.JPG \\
\hline 546 & Arabis turrita L. & Visegrád & 1964 & Soó Rezső, Borsos Olga & BPU-06998.JPG \\
\hline 547 & Arabis glabra (L.) Bernh. & Bagamér & 1932 & Soó Rezső & BPU-07028.JPG \\
\hline 547 & Arabis glabra (L.) Bernh. & Dömös & 1952 & Soó Rezső & BPU-07027.JPG \\
\hline 547 & Arabis glabra (L.) Bernh. & Szigetmonostor & 1947 & Soó Rezső & BPU-07029.JPG \\
\hline 547 & Arabis glabra (L.) Bernh. & Tihany & 1956 & Felföldy Lajos & BPU-07025.JPG \\
\hline 547 & Arabis glabra (L.) Bernh. & Tihany & 1958 & Felföldy Lajos & BPU-07026.JPG \\
\hline 547 & Arabis glabra (L.) Bernh. & Uzsa & 1959 & $\begin{array}{l}\text { Jávorka Sándor, Felföldy } \\
\text { Lajos }\end{array}$ & BPU-07024.JPG \\
\hline 548 & Arabis alpina $\mathrm{L}$. & Bélapátfalva & 1932 & Soó Rezső & BPU-06989.JPG \\
\hline 548 & Arabis alpina L. & Nagyvisnyó & 1953 & Vozáry Elemér & BPU-06982.JPG \\
\hline 548 & Arabis alpina L. & Tibolddaróc & 1953 & Soó Rezső & BPU-06990.JPG \\
\hline 549 & Arabis recta Vill. & Balatonszőlős & 1959 & Felföldy Lajos & BPU-07003.JPG \\
\hline 549 & Arabis recta Vill. & Budaörs & 1982 & Felföldy Lajos & BPU-07002.JPG \\
\hline 549 & Arabis recta Vill. & Budapest & 1982 & Felföldy Lajos & BPU-07001.JPG \\
\hline 549 & Arabis recta Vill. & Dabas & 1959 & Simon Tibor & BPU-07009.JPG \\
\hline 549 & Arabis recta Vill. & Győr & 1959 & $\begin{array}{l}\text { Simon Tibor, Borhidi } \\
\text { Attila }\end{array}$ & BPU-07008.JPG \\
\hline 549 & Arabis recta Vill. & Mór & 1953 & Felföldy Lajos & BPU-07006.JPG \\
\hline 549 & Arabis recta Vill. & Nadap & 1922 & Soó Rezső & BPU-07010.JPG \\
\hline 549 & Arabis recta Vill. & Örvényes & 1959 & Felföldy Lajos & BPU-07004.JPG \\
\hline 549 & Arabis recta Vill. & Pécsely & 1959 & Felföldy Lajos & BPU-07005.JPG \\
\hline 549 & Arabis recta Vill. & Tihany & 1958 & Felföldy Lajos & BPU-07007.JPG \\
\hline 551 & Arabis sagittata (Bertol.) DC. & Csákberény & 1982 & Felföldy Lajos & BPU-07014.JPG \\
\hline 552 & Arabis hirsuta (L.) Scop. & Budapest & 1947 & Soó Rezső & BPU-07011.JPG \\
\hline 552 & Arabis hirsuta (L.) Scop. & Debrecen & 1933 & Soó Rezső & BPU-07015.JPG \\
\hline 552 & Arabis hirsuta (L.) Scop. & Háromhuta & 1955 & Simon Tibor & BPU-07020.JPG \\
\hline 552 & Arabis hirsuta (L.) Scop. & Isztimér & 1955 & Soó Rezső & BPU-07019.JPG \\
\hline 552 & Arabis hirsuta (L.) Scop. & Lábatlan & 1953 & Felföldy Lajos & BPU-07013.JPG \\
\hline 552 & Arabis hirsuta (L.) Scop. & Ócsa & 1953 & Soó Rezső, Borsos Olga & BPU-07016.JPG \\
\hline 552 & Arabis hirsuta (L.) Scop. & Pécs & 1935 & Soó Rezső & BPU-07012.JPG \\
\hline 552 & Arabis hirsuta (L.) Scop. & Tihany & 1959 & Felföldy Lajos & BPU-07017.JPG \\
\hline 552 & Arabis hirsuta (L.) Scop. & Uzsa & 1959 & $\begin{array}{l}\text { Jávorka Sándor, Felföldy } \\
\text { Lajos }\end{array}$ & BPU-07018.JPG \\
\hline 553 & Lunaria rediviva L. & Bakonybél & 1955 & Soó Rezső & BPU-06747.JPG \\
\hline 553 & Lunaria rediviva L. & Csesznek & 1933 & $\begin{array}{l}\text { Kulcsár Gábor, Vozáry } \\
\text { Elemér }\end{array}$ & BPU-06740.JPG \\
\hline 553 & Lunaria rediviva L. & Isztimér & 1959 & Simon Tibor & BPU-06737.JPG \\
\hline 553 & Lunaria rediviva L. & Isztimér & 1959 & Simon Tibor & BPU-06746.JPG \\
\hline 553 & Lunaria rediviva L. & Mátraszentimre & 1961 & Soó Rezső & BPU-06743.JPG \\
\hline 553 & Lunaria rediviva L. & Miskolc & 1948 & $\begin{array}{l}\text { Simon Tibor, Borsos Olga, } \\
\text { Jakucs Pál }\end{array}$ & BPU-06738.JPG \\
\hline 553 & Lunaria rediviva L. & Pécs & 1956 & Soó Rezső & BPU-06744.JPG \\
\hline 553 & Lunaria rediviva L. & Tibolddaróc & 1953 & Soó Rezső & BPU-06742.JPG \\
\hline 555 & Peltaria alliacea Jacq. & Vasvár & 1950 & Károlyi Árpád & BPU-06751.JPG \\
\hline 555 & Peltaria alliacea Jacq. & Vasvár & 1950 & Károlyi Árpád & BPU-06752.JPG \\
\hline 556 & Alyssum alyssoides L. & Aszófő & 1959 & Felföldy Lajos & BPU-06775.JPG \\
\hline 556 & Alyssum alyssoides L. & Budapest & 1982 & Felföldy Lajos & BPU-06770.JPG \\
\hline 556 & Alyssum alyssoides L. & Budapest & 2004 & Felföldy Lajos & BPU-06769.JPG \\
\hline
\end{tabular}


1. Elektroniukus melléklet - Electronic Appendix 1.

\begin{tabular}{|c|c|c|c|c|c|}
\hline $\begin{array}{l}\text { Sorszám / } \\
\text { Number }\end{array}$ & Taxon-név / Taxon-name & $\begin{array}{l}\text { Település / } \\
\text { Settlement }\end{array}$ & $\begin{array}{l}\text { Év / } \\
\text { Year }\end{array}$ & Gyújtő / Collector & $\begin{array}{l}\text { Fájlnév / } \\
\text { File-name }\end{array}$ \\
\hline 556 & Alyssum alyssoides L. & Csévharaszt & 1959 & Simon Tibor, Borsos Olga & BPU-06773.JPG \\
\hline 556 & Alyssum alyssoides L. & Nagyharsány & 1965 & Priszter Szaniszló & BPU-06771.JPG \\
\hline 556 & Alyssum alyssoides L. & Nagykovácsi & 1932 & Soó Rezső & BPU-06774.JPG \\
\hline 556 & Alyssum alyssoides L. & Pécs & 1953 & Soó Rezső, Borsos Olga & BPU-06772.JPG \\
\hline 556 & Alyssum alyssoides L. & Pusztamiske & 1948 & Borsos Olga & BPU-06780.JPG \\
\hline 556 & Alyssum alyssoides L. & Tihany & 1951 & Felföldy Lajos & BPU-06776.JPG \\
\hline 556 & Alyssum alyssoides L. & Tihany & 1958 & Felföldy Lajos & BPU-06777.JPG \\
\hline 556 & Alyssum alyssoides L. & Tihany & 1958 & Felföldy Lajos & BPU-06778.JPG \\
\hline 556 & Alyssum alyssoides L. & Tihany & 1958 & Felföldy Lajos & BPU-06779.JPG \\
\hline 557 & Alyssum desertorum Stapf & Győr & 1898 & Polgár Sándor & BPU-06781.JPG \\
\hline 557 & Alyssum desertorum Stapf & Szigetmonostor & 1947 & Soó Rezső & BPU-06782.JPG \\
\hline 557 & Alyssum desertorum Stapf & Tihany & 1958 & Felföldy Lajos & BPU-06784.JPG \\
\hline 557 & Alyssum desertorum Stapf & Vácrátót & 1953 & Felföldy Lajos & BPU-06783.JPG \\
\hline 558 & Alyssum montanum L. & Abaújszántó & 1948 & Soó Rezső & BPU-06757.JPG \\
\hline 558 & Alyssum montanum L. & Ács & 1959 & $\begin{array}{l}\text { Simon Tibor, Borhidi } \\
\text { Attila }\end{array}$ & BPU-06759.JPG \\
\hline 558 & Alyssum montanum L. & Bőny & 1927 & Polgár Sándor & BPU-06760.JPG \\
\hline 558 & Alyssum montanum L. & Budaörs & 1982 & Felföldy Lajos & BPU-06762.JPG \\
\hline 558 & Alyssum montanum L. & Budapest & 1922 & Soó Rezső & BPU-06755.JPG \\
\hline 558 & Alyssum montanum L. & Budapest & 1923 & Soó Rezső & BPU-06756.JPG \\
\hline 558 & Alyssum montanum L. & Budapest & 1982 & Felföldy Lajos & BPU-06754.JPG \\
\hline 558 & Alyssum montanum L. & Csévharaszt & 1959 & Simon Tibor, Borsos Olga & BPU-06763.JPG \\
\hline 558 & Alyssum montanum L. & Debrecen & 1947 & Soó Rezső & BPU-06761.JPG \\
\hline 558 & Alyssum montanum L. & Gánt & 1949 & Soó Rezső & BPU-06758.JPG \\
\hline 558 & Alyssum montanum L. & Gyenesdiás & 1957 & Entz Béla, Szabó E. & BPU-06766.JPG \\
\hline 558 & Alyssum montanum L. & Gyenesdiás & 1965 & Priszter Szaniszló & BPU-06767.JPG \\
\hline 558 & Alyssum montanum L. & Szigetmonostor & 1947 & Soó Rezső & BPU-06764.JPG \\
\hline 558 & Alyssum montanum L. & Visegrád & 1964 & Soó Rezső, Borsos Olga & BPU-06765.JPG \\
\hline 559 & Alyssum tortuosum Willd. & Csévharaszt & 1959 & $\begin{array}{l}\text { Simon Tibor, Horánszky } \\
\text { András }\end{array}$ & BPU-06787.JPG \\
\hline 559 & Alyssum tortuosum Willd. & Dabas & 1951 & Borsos Olga, Simon Tibor & BPU-06785.JPG \\
\hline 559 & Alyssum tortuosum Willd. & Kunadacs & 1964 & Soó Rezső & BPU-06790.JPG \\
\hline 559 & Alyssum tortuosum Willd. & Nagykovácsi & 1949 & Soó Rezső & BPU-06791.JPG \\
\hline 559 & Alyssum tortuosum Willd. & Pilisszentiván & 1953 & Soó Rezső & BPU-06789.JPG \\
\hline 559 & Alyssum tortuosum Willd. & Veresegyház & 1949 & Soó Rezső & BPU-06788.JPG \\
\hline 560 & Berteroa incana (L.) DC. & Budapest & 2004 & Felföldy Lajos & BPU-06804.JPG \\
\hline 560 & Berteroa incana (L.) DC. & Keszthely & 1961 & Váczy-Hübschl Ildikó & BPU-06809.JPG \\
\hline 560 & Berteroa incana (L.) DC. & Keszthely & 1963 & Pavlovszky Béla & BPU-06810.JPG \\
\hline 560 & Berteroa incana (L.) DC. & Nyíracsád & 1949 & Soó Rezső & BPU-06807.JPG \\
\hline 560 & Berteroa incana (L.) DC. & Sopron & 1969 & Priszter Szaniszló & BPU-06806.JPG \\
\hline 560 & Berteroa incana (L.) DC. & Tihany & 1961 & $\begin{array}{l}\text { Pankov H., Felföldy Lajos, } \\
\text { Sólymosy G. }\end{array}$ & BPU-06808.JPG \\
\hline 560 & Berteroa incana (L.) DC. & Vokány & 1963 & Magyar István & BPU-06805.JPG \\
\hline 561 & Aurinia saxatilis (L.) Desv. & Badacsonytomaj & 1928 & Soó Rezső & BPU-06799.JPG \\
\hline 561 & Aurinia saxatilis (L.) Desv. & Füzér & 1938 & Soó Rezső & BPU-06798.JPG \\
\hline 561 & Aurinia saxatilis (L.) Desv. & Kisapáti & 1930 & Lengyel Géza & BPU-06793.JPG \\
\hline 561 & Aurinia saxatilis (L.) Desv. & Tapolca & 1953 & Borsos Olga & BPU-06795.JPG \\
\hline 562 & Rorippa austriaca (Crantz) Besser & Egyek & 1947 & Soó Rezső & BPU-07039.JPG \\
\hline 562 & Rorippa austriaca (Crantz) Besser & Pákozd & 1980 & Felföldy Lajos & BPU-07038.JPG \\
\hline
\end{tabular}


1. Elektroniukus melléklet - Electronic Appendix 1.

\begin{tabular}{|c|c|c|c|c|c|}
\hline $\begin{array}{l}\text { Sorszám / } \\
\text { Number }\end{array}$ & Taxon-név / Taxon-name & $\begin{array}{l}\text { Település / } \\
\text { Settlement }\end{array}$ & $\begin{array}{l}\text { Év / } \\
\text { Year }\end{array}$ & Gyújtő / Collector & $\begin{array}{l}\text { Fájlnév / } \\
\text { File-name }\end{array}$ \\
\hline 562 & Rorippa austriaca (Crantz) Besser & Tarpa & 1949 & Soó Rezső & BPU-07040.JPG \\
\hline 563 & $\begin{array}{l}\text { Rorippa } \times \text { armoracioides (Tausch) } \\
\text { Fuss }\end{array}$ & Bükkszentkereszt & 1937 & Soó Rezső & BPU-07043.JPG \\
\hline 563 & $\begin{array}{l}\text { Rorippa } \times \text { armoracioides (Tausch) } \\
\text { Fuss }\end{array}$ & Egyek & 1947 & Soó Rezső & BPU-07041.JPG \\
\hline 563 & $\begin{array}{l}\text { Rorippa } \times \text { armoracioides (Tausch) } \\
\text { Fuss }\end{array}$ & Győr & 1936 & Polgár Sándor & BPU-07042.JPG \\
\hline 563 & $\begin{array}{l}\text { Rorippa } \times \text { armoracioides (Tausch) } \\
\text { Fuss }\end{array}$ & Tarpa & 1949 & Soó Rezső & BPU-07044.JPG \\
\hline 564 & Rorippa amphibia (L.) Besser & Debrecen & 1933 & Soó Rezső & BPU-07050.JPG \\
\hline 564 & Rorippa amphibia (L.) Besser & Gárdony & 1963 & Isépy István & BPU-07046.JPG \\
\hline 564 & Rorippa amphibia (L.) Besser & Gárdony & 1980 & Felföldy Lajos & BPU-07047.JPG \\
\hline 564 & Rorippa amphibia (L.) Besser & Hajdúsámson & 1932 & Soó Rezső & BPU-07051.JPG \\
\hline 564 & Rorippa amphibia (L.) Besser & Piricse & 1932 & Soó Rezső & BPU-07053.JPG \\
\hline 564 & Rorippa amphibia (L.) Besser & Tarpa & 1948 & $\begin{array}{l}\text { Pólya László, Simon } \\
\text { Tibor, Jakucs Pál }\end{array}$ & BPU-07045.JPG \\
\hline 564 & Rorippa amphibia (L.) Besser & Zamárdi & 1957 & Entz Béla & BPU-07052.JPG \\
\hline 564 & Rorippa amphibia (L.) Besser & Zámoly & 1982 & Felföldy Lajos & BPU-07048.JPG \\
\hline 565 & Rorippa palustris (L.) Besser & Jánd & 1951 & Simon Tibor & BPU-07036.JPG \\
\hline 565 & Rorippa palustris (L.) Besser & Pogányszentpéter & 1948 & Károlyi Árpád & BPU-07035.JPG \\
\hline 565 & Rorippa palustris (L.) Besser & Tiszacsege & 1951 & Borsos Olga, Simon Tibor & BPU-07037.JPG \\
\hline 566 & Rorippa $\times$ anceps (Wahlenb.) Rchb. & Budapest & 1982 & Felföldy Lajos & BPU-07062.JPG \\
\hline 566 & Rorippa $\times$ anceps (Wahlenb.) Rchb. & Debrecen & 1939 & Soó Rezső & BPU-07060.JPG \\
\hline 566 & Rorippa × anceps (Wahlenb.) Rchb. & Hajdúsámson & 1933 & Soó Rezső & BPU-07061.JPG \\
\hline 566 & Rorippa $\times$ anceps (Wahlenb.) Rchb. & Szalonna & 1944 & Felföldy Lajos & BPU-07057.JPG \\
\hline 567 & Rorippa sylvestris (L.) Besser & Akasztó & 1961 & Borsos Olga, Simon Tibor & BPU-07068.JPG \\
\hline 567 & Rorippa sylvestris (L.) Besser & Aranyosapáti & 1933 & Soó Rezső & BPU-07056.JPG \\
\hline 567 & Rorippa sylvestris (L.) Besser & Beregdaróc & 1951 & Simon Tibor, Jakucs Pál & BPU-07066.JPG \\
\hline 567 & Rorippa sylvestris (L.) Besser & Budapest & 1990 & Felföldy Lajos & BPU-07058.JPG \\
\hline 567 & Rorippa sylvestris (L.) Besser & Egyek & 1947 & Soó Rezső & BPU-07069.JPG \\
\hline 567 & Rorippa sylvestris (L.) Besser & Győr & 1915 & Polgár Sándor & BPU-07054.JPG \\
\hline 567 & Rorippa sylvestris (L.) Besser & Konyár & 1949 & Soó Rezső & BPU-07055.JPG \\
\hline 567 & Rorippa sylvestris (L.) Besser & Konyár & 1949 & Soó Rezső & BPU-15461.JPG \\
\hline 567 & Rorippa sylvestris (L.) Besser & Nagykanizsa & 1956 & Felföldy Lajos & BPU-07059.JPG \\
\hline 567 & Rorippa sylvestris (L.) Besser & Nyírpilis & 1932 & Soó Rezső & BPU-07065.JPG \\
\hline 567 & Rorippa sylvestris (L.) Besser & Szarvas & 1949 & Soó Rezső & BPU-07064.JPG \\
\hline 567 & Rorippa sylvestris (L.) Besser & Szarvas & 1949 & Soó Rezső & BPU-15460.JPG \\
\hline 567 & Rorippa sylvestris (L.) Besser & Szigetmonostor & 1964 & Isépy István & BPU-07063.JPG \\
\hline 567 & Rorippa sylvestris (L.) Besser & Tihany & 1959 & Felföldy Lajos & BPU-07067.JPG \\
\hline 567 & Rorippa sylvestris (L.) Besser & Vácrátót & 1953 & Felföldy Lajos & BPU-07072.JPG \\
\hline $562 \times 564$ & Rorippa $\times$ hungarica Borbás & Debrecen & - & Máthé Imre & BPU-07075.JPG \\
\hline $562 \times 564$ & Rorippa $\times$ hungarica Borbás & Hortobágy & 1931 & Soó Rezső & BPU-07049.JPG \\
\hline $562 \times 564$ & Rorippa $\times$ hungarica Borbás & Tihany & 1942 & Felföldy Lajos & BPU-07074.JPG \\
\hline $564 \times 567$ & $\begin{array}{l}\text { Rorippa amphibia (L.) Besser } \times \\
\text { Rorippa sylvestris (L.) Besser }\end{array}$ & Debrecen & 1933 & Soó Rezső & BPU-07073.JPG \\
\hline 568 & Draba lasiocarpa Rochel & Budapest & 1958 & Isépy István & BPU-06811.JPG \\
\hline 568 & Draba lasiocarpa Rochel & Gánt & 1949 & Soó Rezső & BPU-06813.JPG \\
\hline 568 & Draba lasiocarpa Rochel & Gyenesdiás & 1965 & Priszter Szaniszló & BPU-06814.JPG \\
\hline 568 & Draba lasiocarpa Rochel & Miskolc & 1939 & Soó Rezső & BPU-06812.JPG \\
\hline 569 & Draba nemorosa L. & Gödöllő & 1991 & Felföldy Lajos & BPU-06818.JPG \\
\hline
\end{tabular}


1. Elektroniukus melléklet - Electronic Appendix 1.

\begin{tabular}{|c|c|c|c|c|c|}
\hline $\begin{array}{l}\text { Sorszám / } \\
\text { Number }\end{array}$ & Taxon-név / Taxon-name & $\begin{array}{l}\text { Település / } \\
\text { Settlement }\end{array}$ & $\begin{array}{l}\text { Év / } \\
\text { Year }\end{array}$ & Gyújtő / Collector & $\begin{array}{l}\text { Fájlnév / } \\
\text { File-name }\end{array}$ \\
\hline 569 & Draba nemorosa L. & Gödöllő & 1991 & Felföldy Lajos & BPU-06819.JPG \\
\hline 569 & Draba nemorosa L. & Hajdúhadház & 1933 & Soó Rezső & BPU-06817.JPG \\
\hline 570 & Draba muralis L. & Fenyőfő & 1955 & Soó Rezső & BPU-06822.JPG \\
\hline 570 & Draba muralis L. & Tihany & 1928 & Soó Rezső & BPU-06821.JPG \\
\hline $571-573$ & Erophila verna agg. & Badacsonytomaj & 1982 & Felföldy Lajos & BPU-06835.JPG \\
\hline $571-573$ & Erophila verna agg. & Balatonszőlős & 1959 & Felföldy Lajos & BPU-06834.JPG \\
\hline $571-573$ & Erophila verna agg. & Budapest & 1922 & Soó Rezső & BPU-06858.JPG \\
\hline $571-573$ & Erophila verna agg. & Budapest & 1991 & Felföldy Lajos & BPU-06830.JPG \\
\hline $571-573$ & Erophila verna agg. & Budapest & 2004 & Felföldy Lajos & BPU-06854.JPG \\
\hline $571-573$ & Erophila verna agg. & Budapest & 2005 & Felföldy Lajos & BPU-06852.JPG \\
\hline $571-573$ & Erophila verna agg. & Budapest & 2005 & Felföldy Lajos & BPU-06853.JPG \\
\hline $571-573$ & Erophila verna agg. & Debrecen & 1931 & Soó Rezső & BPU-06844.JPG \\
\hline $571-573$ & Erophila verna agg. & Debrecen & 1934 & Soó Rezső & BPU-06846.JPG \\
\hline $571-573$ & Erophila verna agg. & Farmos & 1923 & Soó Rezső & BPU-06841.JPG \\
\hline $571-573$ & Erophila verna agg. & Gyenesdiás & 1965 & Priszter Szaniszló & BPU-06851.JPG \\
\hline $571-573$ & Erophila verna agg. & Hajdúböszörmény & 1934 & Soó Rezső & BPU-06843.JPG \\
\hline $571-573$ & Erophila verna agg. & Hajdúsámson & 1935 & Soó Rezső & BPU-06845.JPG \\
\hline $571-573$ & Erophila verna agg. & Hidegkút & 1959 & Felföldy Lajos & BPU-06832.JPG \\
\hline $571-573$ & Erophila verna agg. & Nagyharsány & 1965 & Priszter Szaniszló & BPU-06848.JPG \\
\hline $571-573$ & Erophila verna agg. & Nagykovácsi & 1949 & Soó Rezső & BPU-06842.JPG \\
\hline $571-573$ & Erophila verna agg. & Pécsely & 1951 & Felföldy Lajos & BPU-06847.JPG \\
\hline $571-573$ & Erophila verna agg. & Tákos & 1953 & $\begin{array}{l}\text { Simon Tibor, Helmeczy } \\
\text { Balázs }\end{array}$ & BPU-06840.JPG \\
\hline $571-573$ & Erophila verna agg. & Tass & 1982 & Felföldy Lajos & BPU-06833.JPG \\
\hline $571-573$ & Erophila verna agg. & Tihany & 1958 & Felföldy Lajos & BPU-06836.JPG \\
\hline $571-573$ & Erophila verna agg. & Tihany & 1958 & Felföldy Lajos & BPU-06836.JPG \\
\hline $571-573$ & Erophila verna agg. & Tihany & 1958 & Felföldy Lajos & BPU-06837.JPG \\
\hline $571-573$ & Erophila verna agg. & Tihany & 1958 & Felföldy Lajos & BPU-06850.JPG \\
\hline $571-573$ & Erophila verna agg. & Tihany & 1958 & Felföldy Lajos & BPU-06855.JPG \\
\hline $571-573$ & Erophila verna agg. & Tihany & 1958 & Felföldy Lajos & BPU-06856.JPG \\
\hline $571-573$ & Erophila verna agg. & Tihany & 1958 & Felföldy Lajos & BPU-06857.JPG \\
\hline $571-573$ & Erophila verna agg. & Tihany & 1958 & Felföldy Lajos & BPU-06857.JPG \\
\hline $571-573$ & Erophila verna agg. & Üröm & 1991 & Felföldy Lajos & BPU-06831.JPG \\
\hline $571-573$ & Erophila verna agg. & Vácrátót & 1953 & Felföldy Lajos & BPU-06849.JPG \\
\hline 574 & Camelina microcarpa Andrz. ex DC. & Budapest & 1991 & Felföldy Lajos & BPU-07175.JPG \\
\hline 574 & Camelina microcarpa Andrz. ex DC. & Gödöllő & 1922 & Soó Rezső & BPU-07172.JPG \\
\hline 574 & Camelina microcarpa Andrz. ex DC. & Hajdúsámson & 1932 & Soó Rezső & BPU-07173.JPG \\
\hline 575 & Camelina rumelica Velen. & Győr & 1911 & Polgár Sándor & BPU-07171.JPG \\
\hline 576 & Camelina alyssum (Mill.) Thell. & Budapest & 1982 & Felföldy Lajos & BPU-07167.JPG \\
\hline 576 & Camelina alyssum (Mill.) Thell. & Hatvan & 1903 & Degen Árpád & BPU-07169.JPG \\
\hline 577 & Camelina sativa (L.) Crantz & Eplény & 1930 & Polgár Sándor & BPU-07170.JPG \\
\hline 579 & Capsella bursa-pastoris (L.) Medik. & Balatonszőlős & 1959 & Felföldy Lajos & BPU-06706.JPG \\
\hline 579 & Capsella bursa-pastoris (L.) Medik. & Budakeszi & 1958 & Isépy István & BPU-06704.JPG \\
\hline 579 & Capsella bursa-pastoris (L.) Medik. & Debrecen & 1934 & Soó Rezső & BPU-06696.JPG \\
\hline 579 & Capsella bursa-pastoris (L.) Medik. & Debrecen & 1935 & Soó Rezső & BPU-06702.JPG \\
\hline 579 & Capsella bursa-pastoris (L.) Medik. & Debrecen & 1939 & Felföldy Lajos & BPU-06697.JPG \\
\hline 579 & Capsella bursa-pastoris (L.) Medik. & Debrecen & 1945 & Felföldy Lajos & BPU-06698.JPG \\
\hline 579 & Capsella bursa-pastoris (L.) Medik. & Debrecen & 1945 & Felföldy Lajos & BPU-06699.JPG \\
\hline
\end{tabular}


1. Elektroniukus melléklet - Electronic Appendix 1.

\begin{tabular}{|c|c|c|c|c|c|}
\hline $\begin{array}{l}\text { Sorszám / } \\
\text { Number }\end{array}$ & Taxon-név / Taxon-name & $\begin{array}{l}\text { Település / } \\
\text { Settlement }\end{array}$ & $\begin{array}{l}\text { Év / } \\
\text { Year }\end{array}$ & Gyüjtő / Collector & $\begin{array}{l}\text { Fájlnév / } \\
\text { File-name }\end{array}$ \\
\hline 579 & Capsella bursa-pastoris (L.) Medik. & Debrecen & 1945 & Felföldy Lajos & BPU-06700.JPG \\
\hline 579 & Capsella bursa-pastoris (L.) Medik. & Debrecen & 1945 & Felföldy Lajos & BPU-06701.JPG \\
\hline 579 & Capsella bursa-pastoris (L.) Medik. & Hajdúszoboszló & 1937 & Fäller & BPU-06703.JPG \\
\hline 579 & Capsella bursa-pastoris (L.) Medik. & Keszthely & 1959 & Göcsei Mária & BPU-06707.JPG \\
\hline 579 & Capsella bursa-pastoris (L.) Medik. & Keszthely & 1959 & Horváth István & BPU-06708.JPG \\
\hline 579 & Capsella bursa-pastoris (L.) Medik. & Pat & 1959 & Darab Csaba & BPU-06709.JPG \\
\hline 579 & Capsella bursa-pastoris (L.) Medik. & Vácrátót & 1953 & Felföldy Lajos & BPU-06705.JPG \\
\hline 581 & Hornungia petraea (L.) Rchb. & Aszófő & 1928 & Soó Rezső & BPU-06714.JPG \\
\hline 581 & Hornungia petraea (L.) Rchb. & Balatonszőlős & 1959 & Felföldy Lajos & BPU-06713.JPG \\
\hline 581 & Hornungia petraea (L.) Rchb. & Budapest & 1982 & Felföldy Lajos & BPU-06710.JPG \\
\hline 581 & Hornungia petraea (L.) Rchb. & Gyenesdiás & 1965 & Priszter Szaniszló & BPU-06715.JPG \\
\hline 581 & Hornungia petraea (L.) Rchb. & Pilisszentiván & 1949 & Soó Rezső & BPU-06711.JPG \\
\hline 581 & Hornungia petraea (L.) Rchb. & Pilisszentiván & 1949 & Soó Rezső & BPU-15548.JPG \\
\hline 581 & Hornungia petraea (L.) Rchb. & Üröm & 1991 & Felföldy Lajos & BPU-06712.JPG \\
\hline 583 & Thlaspi perfoliatum L. & Aszófő & 1965 & Priszter Szaniszló & BPU-06681.JPG \\
\hline 583 & Thlaspi perfoliatum L. & Budapest & 1982 & Felföldy Lajos & BPU-06676.JPG \\
\hline 583 & Thlaspi perfoliatum L. & Budapest & 1991 & Felföldy Lajos & BPU-06677.JPG \\
\hline 583 & Thlaspi perfoliatum L. & Budapest & 2005 & Felföldy Lajos & BPU-06675.JPG \\
\hline 583 & Thlaspi perfoliatum L. & Mosonmagyaróvár & 1965 & Borsos Olga & BPU-06679.JPG \\
\hline 583 & Thlaspi perfoliatum L. & Nagyharsány & 1965 & $\begin{array}{l}\text { Simon Tibor, Priszter } \\
\text { Szaniszló, Borhidi Attila }\end{array}$ & BPU-06678.JPG \\
\hline 583 & Thlaspi perfoliatum L. & Tihany & 1946 & Felföldy Lajos & BPU-06684.JPG \\
\hline 583 & Thlaspi perfoliatum L. & Tihany & 1958 & Felföldy Lajos & BPU-06682.JPG \\
\hline 583 & Thlaspi perfoliatum L. & Tihany & 1958 & Felföldy Lajos & BPU-06683.JPG \\
\hline 583 & Thlaspi perfoliatum L. & Tihany & 1958 & Felföldy Lajos & BPU-06685.JPG \\
\hline 583 & Thlaspi perfoliatum L. & Vácrátót & 1953 & Felföldy Lajos & BPU-06680.JPG \\
\hline 584 & Thlaspi alliaceum L. & Szegilong & 1936 & Kiss Árpád & BPU-06686.JPG \\
\hline 585 & Thlaspi arvense L. & Monostorapáti & 1959 & $\begin{array}{l}\text { Jávorka Sándor, Felföldy } \\
\text { Lajos, Tóth László }\end{array}$ & BPU-06672.JPG \\
\hline 585 & Thlaspi arvense L. & Nógrád & 1922 & Soó Rezső & BPU-06673.JPG \\
\hline 585 & Thlaspi arvense L. & Tiszavasvári & 1949 & Igmándy József & BPU-06674.JPG \\
\hline 587 & Thlaspi montanum L. & Solymár & 1932 & Jávorka Sándor & BPU-06688.JPG \\
\hline 589 & Thlaspi jankae A. Kern. & Budapest & 1949 & Soó Rezső & BPU-15462.JPG \\
\hline 589 & Thlaspi jankae A. Kern. & Gyöngyös & 1933 & Soó Rezső & BPU-06691.JPG \\
\hline 591 & Aethionema saxatile (L.) R. Br. & Balatonfüred & 1928 & Soó Rezső & BPU-06668.JPG \\
\hline 591 & Aethionema saxatile (L.) R. Br. & Csákvár & 1955 & Soó Rezső & BPU-06667.JPG \\
\hline 591 & Aethionema saxatile (L.) R. Br. & Isztimér & 1955 & Soó Rezső & BPU-06670.JPG \\
\hline 591 & Aethionema saxatile (L.) R. Br. & Isztimér & 1955 & Soó Rezső & BPU-06671.JPG \\
\hline 591 & Aethionema saxatile (L.) R. Br. & Tihany & 1958 & Felföldy Lajos & BPU-06669.JPG \\
\hline 593 & Biscutella laevigata L. & Budapest & 1945 & Boros Ádám & BPU-06649.JPG \\
\hline 593 & Biscutella laevigata L. & Budapest & 1947 & Boros Ádám & BPU-06650.JPG \\
\hline 593 & Biscutella laevigata L. & Gánt & 1949 & Soó Rezső & BPU-06652.JPG \\
\hline 593 & Biscutella laevigata L. & Gyenesdiás & 1957 & Entz Béla, Szabó E. & BPU-06655.JPG \\
\hline 593 & Biscutella laevigata L. & Hajmáskér & 1940 & Boros Ádám & BPU-06656.JPG \\
\hline 593 & Biscutella laevigata $\mathrm{L}$. & Nagykovácsi & 1932 & Soó Rezső & BPU-06653.JPG \\
\hline 593 & Biscutella laevigata L. & Pilisszentiván & 1953 & Soó Rezső & BPU-06654.JPG \\
\hline 593 & Biscutella laevigata L. & Uzsa & 1959 & $\begin{array}{l}\text { Jávorka Sándor, Felföldy } \\
\text { Lajos }\end{array}$ & BPU-06657.JPG \\
\hline 593 & Biscutella laevigata L. & Várpalota & 1955 & Boros Ádám & BPU-06651.JPG \\
\hline
\end{tabular}


1. Elektroniukus melléklet - Electronic Appendix 1.

\begin{tabular}{|c|c|c|c|c|c|}
\hline $\begin{array}{l}\text { Sorszám / } \\
\text { Number }\end{array}$ & Taxon-név / Taxon-name & $\begin{array}{l}\text { Település / } \\
\text { Settlement }\end{array}$ & $\begin{array}{l}\text { Év / } \\
\text { Year }\end{array}$ & Gyújtő / Collector & $\begin{array}{l}\text { Fájlnév / } \\
\text { File-name }\end{array}$ \\
\hline 594 & $\begin{array}{l}\text { Lepidium cartilagineum (J.C. Mayer) } \\
\text { Thell. }\end{array}$ & Akasztó & 1961 & Borsos Olga, Simon Tibor & BPU-06628.JPG \\
\hline 594 & $\begin{array}{l}\text { Lepidium cartilagineum (J.C. Mayer) } \\
\text { Thell. }\end{array}$ & Akasztó & 1963 & Isépy István & BPU-06629.JPG \\
\hline 594 & $\begin{array}{l}\text { Lepidium cartilagineum (J.C. Mayer) } \\
\text { Thell. }\end{array}$ & Bugyi & 1950 & Soó Rezső & BPU-06631.JPG \\
\hline 594 & $\begin{array}{l}\text { Lepidium cartilagineum (J.C. Mayer) } \\
\text { Thell. }\end{array}$ & Farmos & 1923 & Soó Rezső & BPU-06630.JPG \\
\hline 594 & $\begin{array}{l}\text { Lepidium cartilagineum (J.C. Mayer) } \\
\text { Thell. }\end{array}$ & Kiskunmajsa & 1964 & Soó Rezső & BPU-06632.JPG \\
\hline 595 & Lepidium perfoliatum L. & Akasztó & 1963 & Isépy István & BPU-06612.JPG \\
\hline 595 & Lepidium perfoliatum L. & Kunszentmiklós & 1925 & Boros Ádám & BPU-06613.JPG \\
\hline 595 & Lepidium perfoliatum L. & Örvényes & 1959 & Felföldy Lajos & BPU-06615.JPG \\
\hline 595 & Lepidium perfoliatum L. & Szarvas & 1949 & Soó Rezső & BPU-06614.JPG \\
\hline 596 & Lepidium campestre (L.) R. Br. & Balatonszőlős & 1958 & Felföldy Lajos & BPU-06620.JPG \\
\hline 596 & Lepidium campestre (L.) R. Br. & Budapest & 2004 & Felföldy Lajos & BPU-06616.JPG \\
\hline 596 & Lepidium campestre (L.) R. Br. & Debrecen & 1931 & Erendics E. & BPU-06619.JPG \\
\hline 596 & Lepidium campestre (L.) R. Br. & Mór & 1953 & Felföldy Lajos & BPU-06618.JPG \\
\hline 596 & Lepidium campestre (L.) R. Br. & Pocsaj & 1949 & Soó Rezső & BPU-06617.JPG \\
\hline 596 & Lepidium campestre (L.) R. Br. & Tihany & 1956 & Felföldy Lajos & BPU-06623.JPG \\
\hline 596 & Lepidium campestre (L.) R. Br. & Tihany & 1958 & Felföldy Lajos & BPU-06622.JPG \\
\hline 596 & Lepidium campestre (L.) R. Br. & Tihany & 1959 & Felföldy Lajos & BPU-06621.JPG \\
\hline 598 & Lepidium graminifolium L. & Szántód & 1928 & Soó Rezső & BPU-06635.JPG \\
\hline 599 & Lepidium virginicum L. & Zákány & 1948 & Károlyi Árpád & BPU-06636.JPG \\
\hline 600 & Lepidium ruderale $\mathrm{L}$. & Budapest & 1982 & Felföldy Lajos & BPU-06633.JPG \\
\hline 600 & Lepidium ruderale $\mathrm{L}$. & Egyek & 1938 & Soó Rezső & BPU-06634.JPG \\
\hline 601 & Lepidium densiflorum Schrad. & Bátorliget & 1953 & Soó Rezső & BPU-06643.JPG \\
\hline 601 & Lepidium densiflorum Schrad. & Budapest & 1947 & Soó Rezső & BPU-06637.JPG \\
\hline 601 & Lepidium densiflorum Schrad. & Budapest & 1968 & Priszter Szaniszló & BPU-06638.JPG \\
\hline 601 & Lepidium densiflorum Schrad. & Debrecen & 1939 & Ubrizsy Gábor & BPU-06640.JPG \\
\hline 601 & Lepidium densiflorum Schrad. & Debrecen & 1946 & Soó Rezső & BPU-06641.JPG \\
\hline 601 & Lepidium densiflorum Schrad. & Konyár & 1949 & Soó Rezső & BPU-06639.JPG \\
\hline 601 & Lepidium densiflorum Schrad. & Mosonmagyaróvár & 1962 & Borsos Olga & BPU-06642.JPG \\
\hline--- & Lepidium bonariense L. & Győr & 1927 & Polgár Sándor & BPU-06644.JPG \\
\hline 602 & Cardaria draba (L.) Desv. & Besenyszög & 1969 & Priszter Szaniszló & BPU-06626.JPG \\
\hline 602 & Cardaria draba (L.) Desv. & Budapest & 1947 & Soó Rezső & BPU-06624.JPG \\
\hline 602 & Cardaria draba (L.) Desv. & Tihany & 1946 & Felföldy Lajos & BPU-06627.JPG \\
\hline 602 & Cardaria draba (L.) Desv. & Zebegény & 1963 & Isépy István & BPU-06625.JPG \\
\hline 603 & Coronopus squamatus (Forssk.) Asch. & Besenyszög & 1969 & Priszter Szaniszló & BPU-06645.JPG \\
\hline 605 & Conringia orientalis (L.) Dumort. & Győr & 1916 & Polgár Sándor & BPU-06610.JPG \\
\hline 605 & Conringia orientalis (L.) Dumort. & Örvényes & 1950 & Felföldy Lajos & BPU-06609.JPG \\
\hline 606 & Conringia austriaca (Jacq.) Sweet & Budapest & 1923 & Soó Rezső & BPU-06611.JPG \\
\hline 608 & Diplotaxis muralis (L.) DC. & Budapest & 1921 & Soó Rezső & BPU-06588.JPG \\
\hline 609 & Diplotaxis tenuifolia (L.) DC. & Balatonalmádi & 1963 & Soó Rezső & BPU-06593.JPG \\
\hline 609 & Diplotaxis tenuifolia (L.) DC. & Boldogkőváralja & 1943 & Siroki Zoltán & BPU-06591.JPG \\
\hline 609 & Diplotaxis tenuifolia (L.) DC. & Budapest & 1982 & Felföldy Lajos & BPU-06590.JPG \\
\hline 609 & Diplotaxis tenuifolia (L.) DC. & Budapest & 1989 & Felföldy Lajos & BPU-06589.JPG \\
\hline 609 & Diplotaxis tenuifolia (L.) DC. & Debrecen & 1933 & Soó Rezső & BPU-06592.JPG \\
\hline 610 & Brassica elongata Ehrh. & Budaörs & 1982 & Felföldy Lajos & BPU-06567.JPG \\
\hline 610 & Brassica elongata Ehrh. & Budapest & 1912 & Degen Árpád & BPU-06563.JPG \\
\hline
\end{tabular}


1. Elektroniukus melléklet - Electronic Appendix 1.

\begin{tabular}{|c|c|c|c|c|c|}
\hline $\begin{array}{l}\text { Sorszám / } \\
\text { Number }\end{array}$ & Taxon-név / Taxon-name & $\begin{array}{l}\text { Település / } \\
\text { Settlement }\end{array}$ & $\begin{array}{l}\text { Év / } \\
\text { Year }\end{array}$ & Gyújtő / Collector & $\begin{array}{l}\text { Fájlnév / } \\
\text { File-name }\end{array}$ \\
\hline 610 & Brassica elongata Ehrh. & Budapest & 1947 & Soó Rezső & BPU-06566.JPG \\
\hline 610 & Brassica elongata Ehrh. & Budapest & 1982 & Felföldy Lajos & BPU-06565.JPG \\
\hline 610 & Brassica elongata Ehrh. & Budapest & 1991 & Felföldy Lajos & BPU-06564.JPG \\
\hline 610 & Brassica elongata Ehrh. & Törökbálint & 2004 & Simon Tibor & BPU-06568.JPG \\
\hline 611 & Brassica nigra (L.) W.D.J. Koch & Nagykanizsa & 1948 & Károlyi Árpád & BPU-06570.JPG \\
\hline 611 & Brassica nigra (L.) W.D.J. Koch & Szamosszeg & 1948 & Simon Tibor, Jakucs Pál & BPU-06571.JPG \\
\hline 612 & Brassica $\times$ juncea (L.) Czern. & Győr & 1930 & Polgár Sándor & BPU-06574.JPG \\
\hline 614 & Brassica rapa L. & Tarpa & 1949 & Soó Rezső & BPU-06573.JPG \\
\hline 615 & Brassica $\times$ napus L. & Győr & 1919 & Polgár Sándor & BPU-06575.JPG \\
\hline--- & Brassica incana Ten. & Győr & 1940 & Polgár Sándor & BPU-06583.JPG \\
\hline 616 & Sinapis arvensis L. & Budapest & 2005 & Felföldy Lajos & BPU-06584.JPG \\
\hline 616 & Sinapis arvensis L. & Tihany & 1928 & Soó Rezső & BPU-06585.JPG \\
\hline 617 & Sinapis alba L. & Vác & 1923 & Soó Rezső & BPU-06586.JPG \\
\hline 618 & Eruca vesicaria (L.) Cav. & Nagykanizsa & 1950 & Károlyi Árpád & BPU-06587.JPG \\
\hline 619 & $\begin{array}{l}\text { Erucastrum gallicum (Willd.) O.E. } \\
\text { Schulz }\end{array}$ & Keszthely & 1961 & Pálfalvi Ferenc & BPU-06579.JPG \\
\hline 619 & $\begin{array}{l}\text { Erucastrum gallicum (Willd.) O.E. } \\
\text { Schulz }\end{array}$ & Kimle & 1962 & Borsos Olga & BPU-06581.JPG \\
\hline 619 & $\begin{array}{l}\text { Erucastrum gallicum (Willd.) O.E. } \\
\text { Schulz }\end{array}$ & Mosonmagyaróvár & 1962 & Borsos Olga & BPU-06578.JPG \\
\hline 619 & $\begin{array}{l}\text { Erucastrum gallicum (Willd.) O.E. } \\
\text { Schulz }\end{array}$ & Nagyszentjános & 1929 & Polgár Sándor & BPU-06582.JPG \\
\hline 619 & $\begin{array}{l}\text { Erucastrum gallicum (Willd.) O.E. } \\
\text { Schulz }\end{array}$ & Pécs & 1961 & Somlyai József & BPU-06577.JPG \\
\hline 620 & $\begin{array}{l}\text { Erucastrum nasturtiifolium (Poir.) O.E. } \\
\text { Schulz }\end{array}$ & Budapest & 1947 & Vajda László & BPU-06580.JPG \\
\hline 621 & Crambe tataria Sebeók & Balatonkenese & 1951 & Soó Rezső & BPU-06603.JPG \\
\hline 622 & Calepina irregularis (Asso) Thell. & Bisse & 1965 & $\begin{array}{l}\text { Simon Tibor, Priszter } \\
\text { Szaniszló, Borhidi Attila }\end{array}$ & BPU-06598.JPG \\
\hline 622 & Calepina irregularis (Asso) Thell. & Budapest & 1991 & Felföldy Lajos & BPU-06596.JPG \\
\hline 622 & Calepina irregularis (Asso) Thell. & Kaba & 1942 & Felföldy Lajos & BPU-06597.JPG \\
\hline 622 & Calepina irregularis (Asso) Thell. & Nagyharsány & 1965 & $\begin{array}{l}\text { Simon Tibor, Priszter } \\
\text { Szaniszló, Borhidi Attila }\end{array}$ & BPU-06599.JPG \\
\hline 622 & Calepina irregularis (Asso) Thell. & Pécs & 1953 & Soó Rezső, Borsos Olga & BPU-06600.JPG \\
\hline 622 & Calepina irregularis (Asso) Thell. & Tihany & 1928 & Soó Rezső & BPU-06601.JPG \\
\hline 623 & Raphanus raphanistrum L. & Nyíregyháza & 1947 & Soó Rezső & BPU-06595.JPG \\
\hline 623 & Raphanus raphanistrum L. & Pilisszentkereszt & 1955 & Soó Rezső & BPU-06594.JPG \\
\hline---- & Matthiola lunata DC. & Budapest & 1911 & Kocsis István & BPU-07076.JPG \\
\hline 625 & Reseda luteola L. & Balatonszepezd & 1958 & Felföldy Lajos & BPU-07178.JPG \\
\hline 625 & Reseda luteola L. & Bükkszentkereszt & 1938 & Soó Rezső & BPU-07177.JPG \\
\hline 626 & Reseda lutea L. & Dabas & 1963 & Isépy István & BPU-07182.JPG \\
\hline 626 & Reseda lutea L. & Debrecen & 1931 & Soó Rezső & BPU-07180.JPG \\
\hline 626 & Reseda lutea L. & Debrecen & 1934 & Soó Rezső & BPU-07181.JPG \\
\hline 626 & Reseda lutea L. & Győr & 1919 & Polgár Sándor & BPU-07179.JPG \\
\hline 626 & Reseda lutea L. & Tihany & 1958 & Felföldy Lajos & BPU-07183.JPG \\
\hline 627 & Reseda inodora Rchb. & Budapest & 1947 & Soó Rezső & BPU-07184.JPG \\
\hline 627 & Reseda inodora Rchb. & Sukoró & 1949 & Vajda László & BPU-07185.JPG \\
\hline 628 & Reseda phyteuma L. & Balatonfüred & 1928 & Soó Rezső & BPU-07190.JPG \\
\hline 628 & Reseda phyteuma L. & Budapest & 1982 & Felföldy Lajos & BPU-07186.JPG \\
\hline 628 & Reseda phyteuma L. & Nagyharsány & 1965 & $\begin{array}{l}\text { Simon Tibor, Priszter } \\
\text { Szaniszló, Borhidi Attila }\end{array}$ & BPU-07188.JPG \\
\hline
\end{tabular}


1. Elektroniukus melléklet - Electronic Appendix 1.

\begin{tabular}{|c|c|c|c|c|c|}
\hline $\begin{array}{l}\text { Sorszám / } \\
\text { Number }\end{array}$ & Taxon-név / Taxon-name & $\begin{array}{l}\text { Település / } \\
\text { Settlement }\end{array}$ & $\begin{array}{l}\text { Év / } \\
\text { Year }\end{array}$ & Gyűjtő / Collector & $\begin{array}{l}\text { Fájlnév / } \\
\text { File-name }\end{array}$ \\
\hline 628 & Reseda phyteuma L. & Tihany & 1957 & $\begin{array}{l}\text { Felföldy Lajos, Tóth } \\
\text { László }\end{array}$ & BPU-07187.JPG \\
\hline 628 & Reseda phyteuma L. & Tihany & 1958 & Felföldy Lajos & BPU-07189.JPG \\
\hline 629 & Aldrovanda vesiculosa L. & Szenta & 1930 & Boros Ádám & BPU-07192.JPG \\
\hline 629 & Aldrovanda vesiculosa L. & Szenta & 1957 & Soó Rezső & BPU-07191.JPG \\
\hline 629 & Aldrovanda vesiculosa L. & Szenta & 1980 & Priszter Szaniszló & BPU-07191.JPG \\
\hline 630 & Drosera rotundifolia L. & Lesenceistvánd & 1928 & Soó Rezső & BPU-07195.JPG \\
\hline 630 & Drosera rotundifolia L. & Szentgotthárd & 1955 & Soó Rezső & BPU-07193.JPG \\
\hline 630 & Drosera rotundifolia L. & Szőce & 1955 & Soó Rezső & BPU-07194.JPG \\
\hline 631 & Drosera anglica Huds. & Uzsa & 1951 & Kovács Béla & BPU-07201.JPG \\
\hline 631 & Drosera anglica Huds. & Uzsa & 1951 & Kovács Béla & BPU-07202.JPG \\
\hline 633 & Sempervivum marmoreum Griseb. & Abaújszántó & 1948 & Soó Rezső & BPU-01842.JPG \\
\hline 633 & Sempervivum marmoreum Griseb. & Bélapátfalva & 1974 & Priszter Szaniszló & BPU-01844.JPG \\
\hline 633 & Sempervivum marmoreum Griseb. & Dömös & 1948 & Soó Rezső & BPU-01843.JPG \\
\hline 633 & Sempervivum marmoreum Griseb. & Vác & 1949 & $\begin{array}{l}\text { Soó Rezső, Simon Tibor, } \\
\text { Jakucs Pál }\end{array}$ & BPU-01845.JPG \\
\hline 634 & Jovibarba globifera (L.) J. Parn. & Bélapátfalva & 1974 & Priszter Szaniszló & BPU-01848.JPG \\
\hline 634 & Jovibarba globifera (L.) J. Parn. & Gánt & 1948 & Soó Rezső & BPU-01847.JPG \\
\hline 634 & Jovibarba globifera (L.) J. Parn. & Miskolc & 1937 & Soó Rezső & BPU-01846.JPG \\
\hline 634 & Jovibarba globifera (L.) J. Parn. & Vác & 1949 & $\begin{array}{l}\text { Soó Rezső, Simon Tibor, } \\
\text { Jakucs Pál }\end{array}$ & BPU-15559.JPG \\
\hline 634 & Jovibarba globifera (L.) J. Parn. & Veszprém & 1931 & Soó Rezső & BPU-01849.JPG \\
\hline 635 & Hylotelephium telephium (L.) H. Ohba & Egyek & 1932 & Soó Rezső & BPU-01804.JPG \\
\hline 637 & Sedum caespitosum (Cav.) DC. & Makó & 1905 & Thaisz Lajos & BPU-01801.JPG \\
\hline 637 & Sedum caespitosum (Cav.) DC. & Nadap & 1933 & Boros Ádám & BPU-01802.JPG \\
\hline 638 & Sedum hispanicum Jusl. & Miskolc & 1929 & Soó Rezső & BPU-01807.JPG \\
\hline 638 & Sedum hispanicum Jusl. & Szarvaskő & 1948 & $\begin{array}{l}\text { Simon Tibor, Pólya } \\
\text { László, Jakucs Pál }\end{array}$ & BPU-01808.JPG \\
\hline 639 & Sedum album L. & Gánt & 1948 & Soó Rezső & BPU-01814.JPG \\
\hline 639 & Sedum album L. & Gyöngyös & 1963 & Soó Rezső & BPU-01813.JPG \\
\hline 639 & Sedum album L. & Nagyvisnyó & 1948 & $\begin{array}{l}\text { Simon Tibor, Pólya } \\
\text { László, Jakucs Pál }\end{array}$ & BPU-01811.JPG \\
\hline 639 & Sedum album L. & Uppony & 1950 & Soó Rezső, Simon Tibor & BPU-01815.JPG \\
\hline 640 & Sedum rupestre L. & Pécs & 1925 & Soó Rezső & BPU-01816.JPG \\
\hline 641 & Sedum urvillei DC. & Budapest & 1923 & Soó Rezső & BPU-01821.JPG \\
\hline 641 & Sedum urvillei DC. & Gyöngyös & 1923 & Soó Rezső & BPU-01822.JPG \\
\hline 642 & Sedum sexangulare L. & Debrecen & 1933 & Soó Rezső & BPU-01823.JPG \\
\hline 642 & Sedum sexangulare L. & Pilisszentiván & 1969 & Priszter Szaniszló & BPU-01824.JPG \\
\hline 642 & Sedum sexangulare L. & Vác & 1923 & Soó Rezső & BPU-01825.JPG \\
\hline 643 & Sedum acre L. & Nagyharsány & 1936 & Soó Rezső & BPU-01818.JPG \\
\hline 643 & Sedum acre L. & Püspökladány & 1935 & Soó Rezső & BPU-01819.JPG \\
\hline 643 & Sedum acre L. & Siklós & 1967 & Priszter Szaniszló & BPU-01817.JPG \\
\hline 644 & Saxifraga paniculata Mill. & Bélapátfalva & 1948 & $\begin{array}{l}\text { Simon Tibor, Pólya } \\
\text { László, Jakucs Pál }\end{array}$ & BPU-01862.JPG \\
\hline 644 & Saxifraga paniculata Mill. & Füzér & 1937 & Soó Rezső & BPU-01860.JPG \\
\hline 644 & Saxifraga paniculata Mill. & Füzér & 1960 & Simon Tibor & BPU-01861.JPG \\
\hline 644 & Saxifraga paniculata Mill. & Gyöngyös & 1953 & Soó Rezső & BPU-01863.JPG \\
\hline 644 & Saxifraga paniculata Mill. & Szarvaskő & 1930 & Soó Rezső & BPU-01864.JPG \\
\hline 644 & Saxifraga paniculata Mill. & Vác & 1949 & $\begin{array}{l}\text { Soó Rezső, Simon Tibor, } \\
\text { Jakucs Pál }\end{array}$ & BPU-01865.JPG \\
\hline
\end{tabular}


1. Elektroniukus melléklet - Electronic Appendix 1.

\begin{tabular}{|c|c|c|c|c|c|}
\hline $\begin{array}{l}\text { Sorszám / } \\
\text { Number }\end{array}$ & Taxon-név / Taxon-name & $\begin{array}{l}\text { Település / } \\
\text { Settlement }\end{array}$ & $\begin{array}{l}\text { Év / } \\
\text { Year }\end{array}$ & Gyűjtő / Collector & $\begin{array}{l}\text { Fájlnév / } \\
\text { File-name }\end{array}$ \\
\hline 645 & Saxifraga bulbifera L. & Debrecen & 1930 & Soó Rezső & BPU-01879.JPG \\
\hline 645 & Saxifraga bulbifera L. & Egyek & 1948 & Soó Rezső & BPU-01881.JPG \\
\hline 645 & Saxifraga bulbifera L. & Hidegkút & 1958 & Felföldy Lajos & BPU-01875.JPG \\
\hline 645 & Saxifraga bulbifera L. & Miskolc & 1939 & Soó Rezső & BPU-01880.JPG \\
\hline 645 & Saxifraga bulbifera L. & Nyíradony & 1951 & Simon Tibor & BPU-01877.JPG \\
\hline 645 & Saxifraga bulbifera L. & Sátoraljaújhely & 1952 & $\begin{array}{l}\text { Kulcsár Gábor, Vozáry } \\
\text { Elemér }\end{array}$ & BPU-15474.JPG \\
\hline 645 & Saxifraga bulbifera L. & Somlóvásárhely & 1953 & $\begin{array}{l}\text { Kulcsár Gábor, Vozáry } \\
\text { Elemér }\end{array}$ & BPU-01876.JPG \\
\hline 647 & Saxifraga tridactylites L. & Bagamér & 1933 & Soó Rezső & BPU-01886.JPG \\
\hline 647 & Saxifraga tridactylites L. & Balatonszőlős & 1959 & Felföldy Lajos & BPU-01885.JPG \\
\hline 647 & Saxifraga tridactylites L. & Bódvaszilas & 1951 & $\begin{array}{l}\text { Jakucs Pál, Kulcsár Gábor, } \\
\text { Ér Lajos }\end{array}$ & BPU-01884.JPG \\
\hline 647 & Saxifraga tridactylites L. & Budapest & 1991 & Felföldy Lajos & BPU-01882.JPG \\
\hline 647 & Saxifraga tridactylites L. & Budapest & 1991 & Felföldy Lajos & BPU-01883.JPG \\
\hline 647 & Saxifraga tridactylites L. & Csákberény & 1982 & Felföldy Lajos & BPU-01888.JPG \\
\hline 647 & Saxifraga tridactylites L. & Debrecen & 1947 & Soó Rezső & BPU-01885.JPG \\
\hline 647 & Saxifraga tridactylites L. & Mór & 1953 & Felföldy Lajos & BPU-01889.JPG \\
\hline 647 & Saxifraga tridactylites L. & Tihany & 1958 & Felföldy Lajos & BPU-01891.JPG \\
\hline 647 & Saxifraga tridactylites L. & Uzsa & 1951 & Felföldy Lajos & BPU-01892.JPG \\
\hline 647 & Saxifraga tridactylites L. & Üröm & 1991 & Felföldy Lajos & BPU-01887.JPG \\
\hline 647 & Saxifraga tridactylites L. & Zámoly & 1982 & Felföldy Lajos & BPU-01890.JPG \\
\hline 650 & Chrysosplenium alternifolium L. & Gyöngyös & 1954 & Soó Rezső & BPU-02382.JPG \\
\hline 650 & Chrysosplenium alternifolium L. & Isztimér & 1959 & Simon Tibor & BPU-02386.JPG \\
\hline 650 & Chrysosplenium alternifolium L. & Miskolc & 1937 & Soó Rezső & BPU-02384.JPG \\
\hline 650 & Chrysosplenium alternifolium L. & Pécs & 1936 & Soó Rezső & BPU-02385.JPG \\
\hline 652 & Deutzia scabra Thunb. & Gyöngyös & 1962 & Soó Rezső, Borsos Olga & BPU-02396.JPG \\
\hline 653 & Parnassia palustris L. & Lesencetomaj & 1964 & Isépy István & BPU-02392.JPG \\
\hline 653 & Parnassia palustris L. & Pálháza & 1949 & Simon Tibor, Jakucs Pál & BPU-15571.JPG \\
\hline 653 & Parnassia palustris L. & Vácrátót & 1950 & Soó Rezső & BPU-02390.JPG \\
\hline 653 & Parnassia palustris L. & Veresegyház & 1949 & Soó Rezső & BPU-02391.JPG \\
\hline 654 & Ribes uva-crispa L. & Gyöngyös & 1952 & Soó Rezső & BPU-02401.JPG \\
\hline 654 & Ribes uva-crispa L. & Miskolc & 1929 & Soó Rezső & BPU-02399.JPG \\
\hline 654 & Ribes uva-crispa L. & Nagyvisnyó & 1953 & Soó Rezső & BPU-02400.JPG \\
\hline 654 & Ribes uva-crispa L. & Pécs & 1956 & Soó Rezső & BPU-02403.JPG \\
\hline 654 & Ribes uva-crispa L. & Szentendre & 1963 & Soó Rezső & BPU-02402.JPG \\
\hline 654 & Ribes uva-crispa L. & Szilvásvárad & 1953 & $\begin{array}{l}\text { Felföldy Lajos, Tóth } \\
\text { László }\end{array}$ & BPU-02404.JPG \\
\hline 657 & Ribes alpinum L. & Nagyvisnyó & 1953 & Soó Rezső & BPU-02408.JPG \\
\hline 657 & Ribes alpinum L. & Telkibánya & 1948 & Borsos Olga & BPU-02411.JPG \\
\hline 660 & Ribes rubrum agg. & Debrecen & 1949 & Soó Rezső & BPU-02416.JPG \\
\hline 660 & Ribes rubrum agg. & Putnok & 1951 & Soó Rezső & BPU-02415.JPG \\
\hline 663 & Spiraea media Schmidt & Bélapátfalva & 1949 & $\begin{array}{l}\text { Simon Tibor, Pólya } \\
\text { László, Jakucs Pál }\end{array}$ & BPU-01125.JPG \\
\hline 663 & Spiraea media Schmidt & Bódvarákó & 1951 & $\begin{array}{l}\text { Soó Rezső, Jakucs Pál, Ér } \\
\text { Lajos }\end{array}$ & BPU-01123.JPG \\
\hline 663 & Spiraea media Schmidt & Dömös & 1952 & Soó Rezső & BPU-01119.JPG \\
\hline 663 & Spiraea media Schmidt & Gyöngyös & 1965 & Soó Rezső & BPU-01122.JPG \\
\hline 663 & Spiraea media Schmidt & Pécs & 1935 & Soó Rezső & BPU-01121.JPG \\
\hline 663 & Spiraea media Schmidt & Pécs & 1953 & Soó Rezső, Borsos Olga & BPU-01120.JPG \\
\hline
\end{tabular}


1. Elektroniukus melléklet - Electronic Appendix 1.

\begin{tabular}{|c|c|c|c|c|c|}
\hline $\begin{array}{l}\text { Sorszám / } \\
\text { Number }\end{array}$ & Taxon-név / Taxon-name & $\begin{array}{l}\text { Település / } \\
\text { Settlement }\end{array}$ & $\begin{array}{l}\text { Év / } \\
\text { Year }\end{array}$ & Gyújtő / Collector & $\begin{array}{l}\text { Fájlnév / } \\
\text { File-name }\end{array}$ \\
\hline 663 & Spiraea media Schmidt & Tállya & 1938 & Soó Rezső & BPU-01124.JPG \\
\hline 663 & Spiraea media Schmidt & Vác & 1949 & $\begin{array}{l}\text { Soó Rezső, Simon Tibor, } \\
\text { Jakucs Pál }\end{array}$ & BPU-01118.JPG \\
\hline 666 & Aruncus dioicus (Walter) Fernald & Csesznek & 1963 & Soó Rezső & BPU-01135.JPG \\
\hline 666 & Aruncus dioicus (Walter) Fernald & Ivánc & 1955 & Soó Rezső & BPU-01133.JPG \\
\hline 666 & Aruncus dioicus (Walter) Fernald & Kishuta & 1950 & Soó Rezső & BPU-01131.JPG \\
\hline 666 & Aruncus dioicus (Walter) Fernald & Kishuta & 1963 & Isépy István & BPU-01132.JPG \\
\hline 666 & Aruncus dioicus (Walter) Fernald & Kőszeg & 1932 & Soó Rezső & BPU-01134.JPG \\
\hline 667 & Filipendula ulmaria (L.) Maxim. & Bátorliget & 1932 & Soó Rezső & BPU-01610.JPG \\
\hline 667 & Filipendula ulmaria (L.) Maxim. & Bátorliget & 1950 & $\begin{array}{l}\text { Simon Tibor, Kárpáti } \\
\text { István }\end{array}$ & BPU-01611.JPG \\
\hline 667 & Filipendula ulmaria (L.) Maxim. & Budapest & 1990 & Felföldy Lajos & BPU-01607.JPG \\
\hline 667 & Filipendula ulmaria (L.) Maxim. & Domony & 1992 & Felföldy Lajos & BPU-01608.JPG \\
\hline 667 & Filipendula ulmaria (L.) Maxim. & Kemence & 1952 & Simon Tibor & BPU-01609.JPG \\
\hline 667 & Filipendula ulmaria (L.) Maxim. & Nagykanizsa & 1948 & Károlyi Árpád & BPU-01614.JPG \\
\hline 667 & Filipendula ulmaria (L.) Maxim. & Uzsa & 1952 & Simon Tibor & BPU-01612.JPG \\
\hline 667 & Filipendula ulmaria (L.) Maxim. & Zalagyömörő & 1957 & Felföldy Lajos & BPU-01613.JPG \\
\hline 668 & Filipendula vulgaris Moench & Balatonszőlős & 1957 & Felföldy Lajos & BPU-01620.JPG \\
\hline 668 & Filipendula vulgaris Moench & Budapest & 1982 & Felföldy Lajos & BPU-01617.JPG \\
\hline 668 & Filipendula vulgaris Moench & Budapest & 2005 & Felföldy Lajos & BPU-01616.JPG \\
\hline 668 & Filipendula vulgaris Moench & Diósjenő & 1949 & Pólya László & BPU-01622.JPG \\
\hline 668 & Filipendula vulgaris Moench & Felsőtelekes & 2005 & Gulyás Pál & BPU-01618.JPG \\
\hline 668 & Filipendula vulgaris Moench & Szigetmonostor & 1947 & Soó Rezső & BPU-01619.JPG \\
\hline 668 & Filipendula vulgaris Moench & Uzsa & 1959 & $\begin{array}{l}\text { Jávorka Sándor, Felföldy } \\
\text { Lajos }\end{array}$ & BPU-01621.JPG \\
\hline 670 & Rubus idaeus L. & Bózsva & 1950 & Simon Tibor & BPU-01353.JPG \\
\hline 670 & Rubus idaeus L. & Dömös & 1948 & Soó Rezső & BPU-01354.JPG \\
\hline 672 & Rubus fruticosus agg. & Bakonyszentlászló & 1930 & Polgár Sándor & BPU-01373.JPG \\
\hline 672 & Rubus fruticosus agg. & Bakonyszentlászló & 1930 & Polgár Sándor & BPU-01394.JPG \\
\hline 672 & Rubus fruticosus agg. & Barabás & 1948 & Simon Tibor, Jakucs Pál & BPU-01363.JPG \\
\hline 672 & Rubus fruticosus agg. & Budapest & 1947 & Soó Rezső & BPU-01366.JPG \\
\hline 672 & Rubus fruticosus agg. & Csaroda & 1951 & Simon Tibor & BPU-01388.JPG \\
\hline 672 & Rubus fruticosus agg. & Jósvafő & 1951 & $\begin{array}{l}\text { Soó Rezső, Jakucs Pál, Ér } \\
\text { Lajos }\end{array}$ & BPU-01367.JPG \\
\hline 672 & Rubus fruticosus agg. & Kőszeg & 1892 & Waisbecker Antal & BPU-01384.JPG \\
\hline 672 & Rubus fruticosus agg. & Kőszeg & 1892 & Waisbecker Antal & BPU-01391.JPG \\
\hline 672 & Rubus fruticosus agg. & Kőszeg & 1893 & Waisbecker Antal & BPU-01378.JPG \\
\hline 672 & Rubus fruticosus agg. & Kőszeg & 1896 & Waisbecker Antal & BPU-01382.JPG \\
\hline 672 & Rubus fruticosus agg. & Mátraszentimre & 1961 & Soó Rezső & BPU-01392.JPG \\
\hline 672 & Rubus fruticosus agg. & Miskolc & 1912 & Budai József & BPU-01390.JPG \\
\hline 672 & Rubus fruticosus agg. & Miskolc & 1950 & Soó Rezső & BPU-01364.JPG \\
\hline 672 & Rubus fruticosus agg. & Pécs & 1925 & Soó Rezső & BPU-01365.JPG \\
\hline 672 & Rubus fruticosus agg. & Pilisszentiván & 1917 & Degen Árpád & BPU-01374.JPG \\
\hline 672 & Rubus fruticosus agg. & Pilisszentiván & 1925 & Degen Árpád & BPU-01372.JPG \\
\hline 672 & Rubus fruticosus agg. & Pilisszentlászló & 1917 & Degen Árpád & BPU-01393.JPG \\
\hline 672 & Rubus fruticosus agg. & Pusztamiske & 1948 & Borsos Olga & BPU-01376.JPG \\
\hline 672 & Rubus fruticosus agg. & Ravazd & 1909 & Polgár Sándor & BPU-01375.JPG \\
\hline 672 & Rubus fruticosus agg. & Ravazd & 1909 & Polgár Sándor & BPU-01389.JPG \\
\hline 672 & Rubus fruticosus agg. & Szakonyfalu & 1955 & Soó Rezső & BPU-01377.JPG \\
\hline
\end{tabular}


1. Elektroniukus melléklet - Electronic Appendix 1.

\begin{tabular}{|c|c|c|c|c|c|}
\hline $\begin{array}{l}\text { Sorszám / } \\
\text { Number }\end{array}$ & Taxon-név / Taxon-name & $\begin{array}{l}\text { Település / } \\
\text { Settlement }\end{array}$ & $\begin{array}{l}\text { Év / } \\
\text { Year }\end{array}$ & Gyüjtő / Collector & $\begin{array}{l}\text { Fájlnév / } \\
\text { File-name }\end{array}$ \\
\hline 672 & Rubus fruticosus agg. & Szenna & 1957 & Soó Rezső & BPU-01383.JPG \\
\hline 672 & Rubus fruticosus agg. & Szenna & 1957 & Soó Rezső & BPU-01385.JPG \\
\hline 673 & Rubus caesius L. & Diósjenő & 1952 & Simon Tibor & BPU-01369.JPG \\
\hline 673 & Rubus caesius L. & Hajdúbagos & 1933 & Soó Rezső & BPU-01358.JPG \\
\hline 673 & Rubus caesius L. & Hortobágy & 1947 & Soó Rezső & BPU-01360.JPG \\
\hline 673 & Rubus caesius L. & Miskolc & 1911 & Budai József & BPU-01371.JPG \\
\hline 673 & Rubus caesius L. & Pusztamiske & 1948 & Borsos Olga & BPU-01362.JPG \\
\hline 673 & Rubus caesius L. & Zebegény & 1947 & Soó Rezső & BPU-01370.JPG \\
\hline 677 & Rosa arvensis Huds. & Pécs & 1925 & Soó Rezső & BPU-09152.JPG \\
\hline 678 & Rosa spinosissima L. & Abaújszántó & 1948 & Soó Rezső & BPU-09155.JPG \\
\hline 678 & Rosa spinosissima L. & Bélapátfalva & 1950 & $\begin{array}{l}\text { Simon Tibor, Jakucs Pál, } \\
\text { Kárpáti István }\end{array}$ & BPU-09154.JPG \\
\hline 678 & Rosa spinosissima L. & Budapest & 1925 & Soó Rezső & BPU-09156.JPG \\
\hline 678 & Rosa spinosissima L. & Nagyharsány & 1956 & Soó Rezső & BPU-09153.JPG \\
\hline 681 & Rosa pendulina L. & Bélapátfalva & 1932 & Soó Rezső & BPU-09160.JPG \\
\hline 681 & Rosa pendulina L. & Gyöngyös & 1923 & Soó Rezső & BPU-09157.JPG \\
\hline 681 & Rosa pendulina L. & Gyöngyös & 1953 & Soó Rezső & BPU-09158.JPG \\
\hline 681 & Rosa pendulina L. & Mátraszentimre & 1961 & Soó Rezső & BPU-09151.JPG \\
\hline 681 & Rosa pendulina L. & Miskolc & 1953 & Soó Rezső & BPU-09163.JPG \\
\hline 681 & Rosa pendulina L. & Telkibánya & 1950 & Soó Rezső & BPU-09159.JPG \\
\hline 681 & Rosa pendulina L. & Telkibánya & 1950 & Soó Rezső & BPU-09161.JPG \\
\hline 685 & Rosa gallica L. & Balatonszőlős & 1957 & Felföldy Lajos & BPU-09169.JPG \\
\hline 685 & Rosa gallica L. & Budaörs & 1947 & Soó Rezső & BPU-09168.JPG \\
\hline 685 & Rosa gallica L. & Budapest & 2005 & Felföldy Lajos & BPU-09138.JPG \\
\hline 685 & Rosa gallica L. & Hajdúdorog & 1935 & Ujvárosi Miklós & BPU-09139.JPG \\
\hline 685 & Rosa gallica L. & Isztimér & 1949 & Soó Rezső & BPU-09167.JPG \\
\hline 685 & Rosa gallica L. & Jósvafő & 1951 & $\begin{array}{l}\text { Soó Rezső, Jakucs Pál, Ér } \\
\text { Lajos }\end{array}$ & BPU-09165.JPG \\
\hline 685 & Rosa gallica L. & Pécs & 1935 & Soó Rezső & BPU-09166.JPG \\
\hline 685 & Rosa gallica L. & Tokaj & 1937 & Soó Rezső & BPU-09140.JPG \\
\hline $686-694$ & Rosa rubiginosa agg. & Csesznek & 1929 & Soó Rezső & BPU-09175.JPG \\
\hline $686-694$ & Rosa rubiginosa agg. & Dunaszeg & 1921 & Polgár Sándor & BPU-09148.JPG \\
\hline $686-694$ & Rosa rubiginosa agg. & Erdőbénye & 1938 & Hulják János & BPU-09137.JPG \\
\hline $686-694$ & Rosa rubiginosa agg. & Lókút & 1932 & Polgár Sándor & BPU-09142.JPG \\
\hline $686-694$ & Rosa rubiginosa agg. & Monostorapáti & 1959 & $\begin{array}{l}\text { Jávorka Sándor, Felföldy } \\
\text { Lajos, Tóth László }\end{array}$ & BPU-09173.JPG \\
\hline $686-694$ & Rosa rubiginosa agg. & Pannonhalma & 1918 & Polgár Sándor & BPU-09172.JPG \\
\hline $686-694$ & Rosa rubiginosa agg. & Pilisszentiván & 1953 & Soó Rezső & BPU-09144.JPG \\
\hline $686-694$ & Rosa rubiginosa agg. & Pilisszentiván & 1953 & Soó Rezső & BPU-09145.JPG \\
\hline $686-694$ & Rosa rubiginosa agg. & Tihany & 1928 & Soó Rezső & BPU-09150.JPG \\
\hline $686-694$ & Rosa rubiginosa agg. & Tihany & 1959 & Felföldy Lajos & BPU-09146.JPG \\
\hline $686-694$ & Rosa rubiginosa agg. & Tihany & 1959 & Felföldy Lajos & BPU-09147.JPG \\
\hline $686-694$ & Rosa rubiginosa agg. & Tihany & 1959 & Felföldy Lajos & BPU-09174.JPG \\
\hline 695 & Rosa jundzillii Besser & Eplény & 1955 & Soó Rezső & BPU-09171.JPG \\
\hline 695 & Rosa jundzillii Besser & Pomáz & 1920 & Degen Árpád & BPU-09132.JPG \\
\hline 695 & Rosa jundzillii Besser & Szentbékkálla & 1959 & $\begin{array}{l}\text { Jávorka Sándor, Felföldy } \\
\text { Lajos, Tóth László }\end{array}$ & BPU-09141.JPG \\
\hline 695 & Rosa jundzillii Besser & Tállya & 1936 & Hulják János & BPU-09170.JPG \\
\hline 697 & Rosa villosa L. & Szentendre & 1943 & Kárpáti Zoltán & BPU-09133.JPG \\
\hline
\end{tabular}


1. Elektroniukus melléklet - Electronic Appendix 1.

\begin{tabular}{|c|c|c|c|c|c|}
\hline $\begin{array}{l}\text { Sorszám / } \\
\text { Number }\end{array}$ & Taxon-név / Taxon-name & $\begin{array}{l}\text { Település / } \\
\text { Settlement }\end{array}$ & $\begin{array}{l}\text { Év / } \\
\text { Year }\end{array}$ & Gyújtő / Collector & $\begin{array}{l}\text { Fájlnév / } \\
\text { File-name }\end{array}$ \\
\hline $700-707$ & Rosa canina agg. & Aszófő & 1957 & Felföldy Lajos & BPU-09177.JPG \\
\hline $700-707$ & Rosa canina agg. & Balatonszőlős & 1957 & Felföldy Lajos & BPU-09200.JPG \\
\hline $700-707$ & Rosa canina agg. & Balatonszőlős & 1957 & Felföldy Lajos & BPU-09201.JPG \\
\hline $700-707$ & Rosa canina agg. & Balatonszőlős & 1957 & Felföldy Lajos & BPU-09211.JPG \\
\hline 700-707 & Rosa canina agg. & Balatonszőlős & 1957 & Felföldy Lajos & BPU-09212.JPG \\
\hline 700-707 & Rosa canina agg. & Balatonszőlős & 1957 & Felföldy Lajos & BPU-09213.JPG \\
\hline $700-707$ & Rosa canina agg. & Barabás & 1951 & Simon Tibor & BPU-09192.JPG \\
\hline $700-707$ & Rosa canina agg. & Bélapátfalva & 1932 & Soó Rezső & BPU-09183.JPG \\
\hline 700-707 & Rosa canina agg. & Budapest & 1922 & Soó Rezső & BPU-09187.JPG \\
\hline $700-707$ & Rosa canina agg. & Budapest & 1922 & Soó Rezső & BPU-09206.JPG \\
\hline $700-707$ & Rosa canina agg. & Budapest & 1947 & Soó Rezső & BPU-09191.JPG \\
\hline $700-707$ & Rosa canina agg. & Budapest & 1952 & Soó Rezső & BPU-15444.JPG \\
\hline $700-707$ & Rosa canina agg. & Csaroda & 1951 & Simon Tibor & BPU-09134.JPG \\
\hline $700-707$ & Rosa canina agg. & Csesznek & 1936 & Polgár Sándor & BPU-09130.JPG \\
\hline $700-707$ & Rosa canina agg. & Egyek & 1947 & Soó Rezső & BPU-09180.JPG \\
\hline $700-707$ & Rosa canina agg. & Egyek & 1947 & Soó Rezső & BPU-09218.JPG \\
\hline $700-707$ & Rosa canina agg. & Gyöngyös & 1923 & Soó Rezső & BPU-09190.JPG \\
\hline 700-707 & Rosa canina agg. & Gyula & 1935 & Soó Rezső & BPU-09217.JPG \\
\hline $700-707$ & Rosa canina agg. & Hejce & 2007 & Simon Tibor & BPU-15508.JPG \\
\hline 700-707 & Rosa canina agg. & Hortobágy & 1947 & Soó Rezső & BPU-09186.JPG \\
\hline 700-707 & Rosa canina agg. & Hortobágy & 1947 & Soó Rezső & BPU-09209.JPG \\
\hline $700-707$ & Rosa canina agg. & Jósvafő & 1951 & $\begin{array}{l}\text { Soó Rezső, Jakucs Pál, Ér } \\
\text { Lajos }\end{array}$ & BPU-09182.JPG \\
\hline $700-707$ & Rosa canina agg. & Monostorapáti & 1959 & $\begin{array}{l}\text { Jávorka Sándor, Felföldy } \\
\text { Lajos, Tóth László }\end{array}$ & BPU-09202.JPG \\
\hline $700-707$ & Rosa canina agg. & Nagyharsány & 1936 & Soó Rezső & BPU-09207.JPG \\
\hline $700-707$ & Rosa canina agg. & Nagyharsány & 1936 & Soó Rezső & BPU-09208.JPG \\
\hline 700-707 & Rosa canina agg. & Nagykovácsi & 1932 & Soó Rezső & BPU-09189.JPG \\
\hline 700-707 & Rosa canina agg. & Nagykovácsi & 1932 & Soó Rezső & BPU-09210.JPG \\
\hline 700-707 & Rosa canina agg. & Nagyvisnyó & 1953 & Soó Rezső & BPU-09164.JPG \\
\hline $700-707$ & Rosa canina agg. & Pomáz & 1920 & Degen Árpád & BPU-09135.JPG \\
\hline $700-707$ & Rosa canina agg. & Pusztamiske & 1948 & Borsos Olga & BPU-09219.JPG \\
\hline 700-707 & Rosa canina agg. & Salgótarján & 1936 & Soó Rezső & BPU-09197.JPG \\
\hline $700-707$ & Rosa canina agg. & Solymár & 1923 & Degen Árpád & BPU-09136.JPG \\
\hline 700-707 & Rosa canina agg. & Solymár & 1923 & Degen Árpád & BPU-09188.JPG \\
\hline 700-707 & Rosa canina agg. & Szarvas & 1949 & Soó Rezső & BPU-09184.JPG \\
\hline $700-707$ & Rosa canina agg. & Szigetmonostor & 1947 & Soó Rezső & BPU-09185.JPG \\
\hline $700-707$ & Rosa canina agg. & Tákos & 1948 & Simon Tibor, Jakucs Pál & BPU-09179.JPG \\
\hline 700-707 & Rosa canina agg. & Tákos & 1948 & Simon Tibor, Jakucs Pál & BPU-09195.JPG \\
\hline 700-707 & Rosa canina agg. & Tarcal & 1933 & Soó Rezső & BPU-09215.JPG \\
\hline $700-707$ & Rosa canina agg. & Tarpa & 1948 & $\begin{array}{l}\text { Pólya László, Simon } \\
\text { Tibor, Jakucs Pál }\end{array}$ & BPU-09131.JPG \\
\hline 700-707 & Rosa canina agg. & Tarpa & 1949 & Soó Rezső & BPU-09193.JPG \\
\hline $700-707$ & Rosa canina agg. & Tarpa & 1949 & Soó Rezső & BPU-09194.JPG \\
\hline 700-707 & Rosa canina agg. & Tarpa & 1949 & Soó Rezső & BPU-09196.JPG \\
\hline 700-707 & Rosa canina agg. & Tihany & 1959 & Felföldy Lajos & BPU-09203.JPG \\
\hline 700-707 & Rosa canina agg. & Tihany & 1959 & Felföldy Lajos & BPU-09204.JPG \\
\hline $700-707$ & Rosa canina agg. & Tihany & 1959 & Felföldy Lajos & BPU-09205.JPG \\
\hline 700-707 & Rosa canina agg. & Tihany & 1959 & Felföldy Lajos & BPU-09214.JPG \\
\hline
\end{tabular}


1. Elektroniukus melléklet - Electronic Appendix 1.

\begin{tabular}{|c|c|c|c|c|c|}
\hline $\begin{array}{l}\text { Sorszám / } \\
\text { Number }\end{array}$ & Taxon-név / Taxon-name & $\begin{array}{l}\text { Település / } \\
\text { Settlement }\end{array}$ & $\begin{array}{l}\text { Év / } \\
\text { Year }\end{array}$ & Gyújtő / Collector & $\begin{array}{l}\text { Fájlnév / } \\
\text { File-name }\end{array}$ \\
\hline $700-707$ & Rosa canina agg. & Tokaj & 1937 & Soó Rezső & BPU-09181.JPG \\
\hline $700-707$ & Rosa canina agg. & Tokaj & 1937 & Soó Rezső & BPU-09216.JPG \\
\hline $700-707$ & Rosa canina agg. & Törökbálint & 1926 & Degen Árpád & BPU-09178.JPG \\
\hline $700-707$ & Rosa canina agg. & Vámosatya & 1949 & Soó Rezső & BPU-09198.JPG \\
\hline $700-707$ & Rosa canina agg. & Visegrád & 1932 & Soó Rezső & BPU-09199.JPG \\
\hline 708 & Agrimonia eupatoria L. & Budapest & 1991 & Felföldy Lajos & BPU-01722.JPG \\
\hline 708 & Agrimonia eupatoria L. & Budapest & 2004 & Felföldy Lajos & BPU-01720.JPG \\
\hline 708 & Agrimonia eupatoria L. & Budapest & 2004 & Felföldy Lajos & BPU-01721.JPG \\
\hline 708 & Agrimonia eupatoria L. & Csomád & 1953 & Felföldy Lajos & BPU-01725.JPG \\
\hline 708 & Agrimonia eupatoria L. & Magyarlukafa & 1957 & Soó Rezső & BPU-01723.JPG \\
\hline 708 & Agrimonia eupatoria L. & Solymár & 1992 & Felföldy Lajos & BPU-01726.JPG \\
\hline 708 & Agrimonia eupatoria L. & Szilvásvárad & 1953 & $\begin{array}{l}\text { Felföldy Lajos, Tóth } \\
\text { László }\end{array}$ & BPU-01724.JPG \\
\hline 709 & Agrimonia procera Wallr. & Isztimér & 1932 & Boros Ádám & BPU-01727.JPG \\
\hline 710 & Aremonia agrimonioides (L.) DC. & Szenna & 1957 & Soó Rezső & BPU-01729.JPG \\
\hline 711 & Sanguisorba officinalis L. & Balatongyörök & 1961 & Felföldy Lajos & BPU-01738.JPG \\
\hline 711 & Sanguisorba officinalis L. & Debrecen & 1932 & Máthé Imre & BPU-01732.JPG \\
\hline 711 & Sanguisorba officinalis L. & Kőszeg & 1963 & Soó Rezső, Borsos Olga & BPU-01735.JPG \\
\hline 711 & Sanguisorba officinalis L. & Örvényes & 1958 & Felföldy Lajos & BPU-01736.JPG \\
\hline 711 & Sanguisorba officinalis L. & Pálháza & 1949 & Simon Tibor, Jakucs Pál & BPU-01730.JPG \\
\hline 711 & Sanguisorba officinalis L. & Pálháza & 1963 & Isépy István & BPU-01731.JPG \\
\hline 711 & Sanguisorba officinalis L. & Somogyszob & 1957 & Soó Rezső & BPU-01734.JPG \\
\hline 711 & Sanguisorba officinalis L. & Tatárszentgyörgy & 1950 & Soó Rezső & BPU-01733.JPG \\
\hline 711 & Sanguisorba officinalis L. & Zalagyömörő & 1957 & Felföldy Lajos & BPU-01737.JPG \\
\hline 712 & Sanguisorba minor Scop. & Abaújszántó & 1938 & Soó Rezső & BPU-01744.JPG \\
\hline 712 & Sanguisorba minor Scop. & Balatonszőlős & 1958 & Felföldy Lajos & BPU-01749.JPG \\
\hline 712 & Sanguisorba minor Scop. & Budapest & 1922 & Soó Rezső & BPU-01742.JPG \\
\hline 712 & Sanguisorba minor Scop. & Budapest & 1964 & Soó Rezső & BPU-01743.JPG \\
\hline 712 & Sanguisorba minor Scop. & Budapest & 2004 & Felföldy Lajos & BPU-01740.JPG \\
\hline 712 & Sanguisorba minor Scop. & Budapest & 2005 & Felföldy Lajos & BPU-01741.JPG \\
\hline 712 & Sanguisorba minor Scop. & Konyár & 1949 & Soó Rezső & BPU-01746.JPG \\
\hline 712 & Sanguisorba minor Scop. & Pécs & 1953 & Soó Rezső, Borsos Olga & BPU-01745.JPG \\
\hline 712 & Sanguisorba minor Scop. & Szigetmonostor & 1947 & Soó Rezső & BPU-01747.JPG \\
\hline 712 & Sanguisorba minor Scop. & Uzsa & 1950 & Felföldy Lajos & BPU-01750.JPG \\
\hline 712 & Sanguisorba minor Scop. & Vác & 1947 & Kárpáti Zoltán & BPU-01748.JPG \\
\hline 714 & Geum urbanum L. & Mátraszentimre & 1963 & Isépy István & BPU-01584.JPG \\
\hline 714 & Geum urbanum L. & Miskolc & 1950 & Soó Rezső & BPU-01583.JPG \\
\hline 714 & Geum urbanum L. & Sáránd & - & anonim & BPU-01582.JPG \\
\hline 716 & Waldsteinia geoides Willd. & Gyöngyös & 1964 & Soó Rezső & BPU-01577.JPG \\
\hline 716 & Waldsteinia geoides Willd. & Pécs & 1935 & Soó Rezső & BPU-01576.JPG \\
\hline 716 & Waldsteinia geoides Willd. & Sátoraljaújhely & 1952 & $\begin{array}{l}\text { Kulcsár Gábor, Vozáry } \\
\text { Elemér }\end{array}$ & BPU-15545.JPG \\
\hline 716 & Waldsteinia geoides Willd. & Szögliget & 1951 & $\begin{array}{l}\text { Jakucs Pál, Kulcsár Gábor, } \\
\text { Ér Lajos }\end{array}$ & BPU-15483.JPG \\
\hline 716 & Waldsteinia geoides Willd. & Vác & 1947 & Papp József & BPU-01579.JPG \\
\hline 718 & Potentilla palustris (L.) Scop. & Bátorliget & 1950 & $\begin{array}{l}\text { Simon Tibor, Kárpáti } \\
\text { István }\end{array}$ & BPU-01408.JPG \\
\hline 718 & Potentilla palustris (L.) Scop. & Csaroda & 1951 & $\begin{array}{l}\text { Simon Tibor, Vozáry } \\
\text { Elemér }\end{array}$ & BPU-01410.JPG \\
\hline
\end{tabular}


1. Elektroniukus melléklet - Electronic Appendix 1.

\begin{tabular}{|c|c|c|c|c|c|}
\hline $\begin{array}{l}\text { Sorszám / } \\
\text { Number }\end{array}$ & Taxon-név / Taxon-name & $\begin{array}{l}\text { Település / } \\
\text { Settlement }\end{array}$ & $\begin{array}{l}\text { Év / } \\
\text { Year }\end{array}$ & Gyűjtő / Collector & $\begin{array}{l}\text { Fájlnév / } \\
\text { File-name }\end{array}$ \\
\hline 718 & Potentilla palustris (L.) Scop. & Csaroda & 1951 & $\begin{array}{l}\text { Simon Tibor, Vozáry } \\
\text { Elemér }\end{array}$ & BPU-01411.JPG \\
\hline 718 & Potentilla palustris (L.) Scop. & Csaroda & 1952 & Soó Rezső & BPU-01413.JPG \\
\hline 718 & Potentilla palustris (L.) Scop. & Nyírpilis & 1951 & Simon Tibor & BPU-01409.JPG \\
\hline 718 & Potentilla palustris (L.) Scop. & Szőce & 1955 & Soó Rezső & BPU-01414.JPG \\
\hline 719 & Potentilla rupestris L. & Gánt & 1935 & Boros Ádám & BPU-01406.JPG \\
\hline 719 & Potentilla rupestris L. & Pécs & 1938 & Soó Rezső & BPU-01407.JPG \\
\hline 719 & Potentilla rupestris L. & Szőce & 1955 & Soó Rezső & BPU-01405.JPG \\
\hline 719 & Potentilla rupestris L. & Uzsa & 1959 & $\begin{array}{l}\text { Jávorka Sándor, Felföldy } \\
\text { Lajos }\end{array}$ & BPU-01404.JPG \\
\hline 720 & Potentilla alba L. & Bátorliget & 1953 & Simon Tibor & BPU-01432.JPG \\
\hline 720 & Potentilla alba L. & Budapest & 1949 & Simon Tibor & BPU-01427.JPG \\
\hline 720 & Potentilla alba L. & Gyöngyös & 1964 & Soó Rezső & BPU-01429.JPG \\
\hline 720 & Potentilla alba L. & Hidegkút & 1959 & Felföldy Lajos & BPU-01433.JPG \\
\hline 720 & Potentilla alba L. & Pécs & 1953 & Soó Rezső, Borsos Olga & BPU-01428.JPG \\
\hline 720 & Potentilla alba L. & Sopron & 1960 & Borsos Olga, Simon Tibor & BPU-01431.JPG \\
\hline 720 & Potentilla alba L. & Vác & 1947 & Kárpáti Zoltán & BPU-01430.JPG \\
\hline 721 & Potentilla micrantha Ramond ex DC. & Gyöngyös & 1964 & Soó Rezső & BPU-01435.JPG \\
\hline 721 & Potentilla micrantha Ramond ex DC. & Háromhuta & 1960 & Simon Tibor & BPU-01437.JPG \\
\hline 721 & Potentilla micrantha Ramond ex DC. & Pécs & 1953 & Soó Rezső, Borsos Olga & BPU-01434.JPG \\
\hline 721 & Potentilla micrantha Ramond ex DC. & Pomáz & 1920 & Degen Árpád & BPU-01436.JPG \\
\hline 722 & Potentilla anserina L. & Debrecen & 1934 & Soó Rezső & BPU-01445.JPG \\
\hline 723 & Potentilla supina L. & Hortobágy & 1947 & Soó Rezső & BPU-01442.JPG \\
\hline 723 & Potentilla supina L. & Konyár & 1949 & Soó Rezső & BPU-01443.JPG \\
\hline 724 & Potentilla erecta (L.) Rausch & Aszófő & 1959 & Felföldy Lajos & BPU-01446.JPG \\
\hline 724 & Potentilla erecta (L.) Rausch & Balatongyörök & 1961 & Felföldy Lajos & BPU-01447.JPG \\
\hline 724 & Potentilla erecta (L.) Rausch & Bátorliget & 1932 & Soó Rezső & BPU-01448.JPG \\
\hline 724 & Potentilla erecta (L.) Rausch & Beregdaróc & 1951 & $\begin{array}{l}\text { Simon Tibor, Vozáry } \\
\text { Elemér }\end{array}$ & BPU-01450.JPG \\
\hline 724 & Potentilla erecta (L.) Rausch & Lesenceistvánd & 1952 & Borsos Olga & BPU-01451.JPG \\
\hline 724 & Potentilla erecta (L.) Rausch & Ócsa & 1950 & Soó Rezső & BPU-01449.JPG \\
\hline 725 & Potentilla reptans L. & Balatonszepezd & 1958 & $\begin{array}{l}\text { Felföldy Lajos, Tóth } \\
\text { László }\end{array}$ & BPU-01454.JPG \\
\hline 725 & Potentilla reptans L. & Budapest & 2005 & Felföldy Lajos & BPU-01453.JPG \\
\hline 726 & Potentilla collina Wibel & Abaújszántó & 1938 & Soó Rezső & BPU-01461.JPG \\
\hline 726 & Potentilla collina Wibel & Debrecen & 1931 & Soó Rezső & BPU-01462.JPG \\
\hline 726 & Potentilla collina Wibel & Hajdúszoboszló & 1938 & Fäller & BPU-01463.JPG \\
\hline 726 & Potentilla collina Wibel & Háromhuta & 1960 & Simon Tibor & BPU-01464.JPG \\
\hline 726 & Potentilla collina Wibel & Háromhuta & 1960 & Simon Tibor & BPU-01465.JPG \\
\hline 726 & Potentilla leucopolitana P.J. Müll. & Nagycserkesz & 1933 & Soó Rezső & BPU-01459.JPG \\
\hline 726 & Potentilla leucopolitana P.J. Müll. & Pannonhalma & 1910 & Polgár Sándor & BPU-01466.JPG \\
\hline 726 & Potentilla leucopolitana P.J. Müll. & Pécsvárad & 1936 & Soó Rezső & BPU-01458.JPG \\
\hline 726 & Potentilla leucopolitana P.J. Müll. & Tihany & 1946 & Felföldy Lajos & BPU-01460.JPG \\
\hline 728 & Potentilla argentea L. & Aszófő & 1931 & Soó Rezső & BPU-01572.JPG \\
\hline 728 & Potentilla argentea L. & Bakonyszentlászló & 1963 & Soó Rezső & BPU-01482.JPG \\
\hline 728 & Potentilla argentea L. & Balatonfüred & 1928 & Soó Rezső & BPU-01501.JPG \\
\hline 728 & Potentilla argentea L. & Bátonyterenye & 1937 & Máthé Imre & BPU-01499.JPG \\
\hline 728 & Potentilla argentea L. & Bélmegyer & 1935 & Soó Rezső & BPU-01488.JPG \\
\hline 728 & Potentilla argentea L. & Budapest & 1952 & Soó Rezső & BPU-01472.JPG \\
\hline
\end{tabular}


1. Elektroniukus melléklet - Electronic Appendix 1.

\begin{tabular}{|c|c|c|c|c|c|}
\hline $\begin{array}{l}\text { Sorszám / } \\
\text { Number }\end{array}$ & Taxon-név / Taxon-name & $\begin{array}{l}\text { Település / } \\
\text { Settlement }\end{array}$ & $\begin{array}{l}\text { Év / } \\
\text { Year }\end{array}$ & Gyűjtő / Collector & $\begin{array}{l}\text { Fájlnév / } \\
\text { File-name }\end{array}$ \\
\hline 728 & Potentilla argentea L. & Budapest & 2004 & Felföldy Lajos & BPU-01484.JPG \\
\hline 728 & Potentilla argentea L. & Csarnóta & 1968 & Priszter Szaniszló & BPU-01473.JPG \\
\hline 728 & Potentilla argentea L. & Debrecen & 1931 & Soó Rezső & BPU-01477.JPG \\
\hline 728 & Potentilla argentea L. & Debrecen & 1938 & Soó Rezső & BPU-01494.JPG \\
\hline 728 & Potentilla argentea L. & Füzér & 1938 & Soó Rezső & BPU-01485.JPG \\
\hline 728 & Potentilla argentea L. & Geszt & 1947 & Soó Rezső & BPU-01474.JPG \\
\hline 728 & Potentilla argentea L. & Gödöllő & 1922 & Soó Rezső & BPU-01480.JPG \\
\hline 728 & Potentilla argentea L. & Gulács & 1993 & Felföldy Lajos & BPU-01489.JPG \\
\hline 728 & Potentilla argentea L. & Gyöngyös & 1961 & Soó Rezső & BPU-01498.JPG \\
\hline 728 & Potentilla argentea L. & Gyula & 1935 & Soó Rezső & BPU-01487.JPG \\
\hline 728 & Potentilla argentea L. & Hajdúbagos & 1933 & Soó Rezső & BPU-01491.JPG \\
\hline 728 & Potentilla argentea L. & Hajdúhadház & 1933 & Soó Rezső & BPU-01478.JPG \\
\hline 728 & Potentilla argentea L. & Hajdúsámson & 1933 & Soó Rezső & BPU-01495.JPG \\
\hline 728 & Potentilla argentea L. & Hortobágy & 1930 & Soó Rezső & BPU-01479.JPG \\
\hline 728 & Potentilla argentea L. & Hortobágy & 1947 & Soó Rezső & BPU-01496.JPG \\
\hline 728 & Potentilla argentea L. & Mezőcsát & 1934 & Soó Rezső & BPU-01492.JPG \\
\hline 728 & Potentilla argentea L. & Nyíracsád & 1932 & Máthé Imre & BPU-01481.JPG \\
\hline 728 & Potentilla argentea L. & Pusztamiske & 1948 & Borsos Olga & BPU-01502.JPG \\
\hline 728 & Potentilla argentea L. & Salgótarján & 1936 & Soó Rezső & BPU-01500.JPG \\
\hline 728 & Potentilla argentea L. & Sátoraljaújhely & 1937 & Soó Rezső & BPU-01483.JPG \\
\hline 728 & Potentilla argentea L. & Szalonna & 1966 & Felföldy Lajos & BPU-01493.JPG \\
\hline 728 & Potentilla argentea L. & Tarpa & 1949 & Soó Rezső & BPU-01490.JPG \\
\hline 728 & Potentilla argentea L. & Téglás & 1948 & Soó Rezső & BPU-01497.JPG \\
\hline 728 & Potentilla argentea L. & Villány & 1935 & Soó Rezső & BPU-01486.JPG \\
\hline 728 & Potentilla argentea L. & Villány & 1935 & Soó Rezső & BPU-01486.JPG \\
\hline 730 & Potentilla recta L. & Budapest & 1947 & Soó Rezső & BPU-01545.JPG \\
\hline 730 & Potentilla recta L. & Budapest & 1982 & Felföldy Lajos & BPU-01544.JPG \\
\hline 730 & Potentilla recta L. & Budapest & 1991 & Felföldy Lajos & BPU-01543.JPG \\
\hline 730 & Potentilla recta L. & Budapest & 1992 & Felföldy Lajos & BPU-01546.JPG \\
\hline 730 & Potentilla recta L. & Budapest & 2004 & Felföldy Lajos & BPU-01542.JPG \\
\hline 730 & Potentilla recta L. & Bükkszentkereszt & 1937 & Soó Rezső & BPU-01550.JPG \\
\hline 730 & Potentilla recta L. & Csopak & - & Entz Béla & BPU-01533.JPG \\
\hline 730 & Potentilla recta L. & Debrecen & 1933 & Soó Rezső & BPU-01551.JPG \\
\hline 730 & Potentilla recta L. & Debrecen & 1948 & Simon Tibor & BPU-01552.JPG \\
\hline 730 & Potentilla recta L. & Fertőrákos & 1937 & Soó Rezső & BPU-01531.JPG \\
\hline 730 & Potentilla recta L. & Gyöngyös & 1953 & Soó Rezső & BPU-01553.JPG \\
\hline 730 & Potentilla recta L. & Jósvafő & 1951 & Soó Rezső & BPU-01547.JPG \\
\hline 730 & Potentilla recta L. & Mátraszentimre & 1965 & Priszter Szaniszló & BPU-01554.JPG \\
\hline 730 & Potentilla recta L. & Mosonmagyaróvár & 1962 & Borsos Olga & BPU-01556.JPG \\
\hline 730 & Potentilla recta L. & Nagydém & 1918 & Polgár Sándor & BPU-01534.JPG \\
\hline 730 & Potentilla recta L. & Nagyharsány & 1967 & Borhidi Attila & BPU-01548.JPG \\
\hline 730 & Potentilla recta L. & Nyíracsád & 1931 & Soó Rezső & BPU-01532.JPG \\
\hline 730 & Potentilla recta L. & Pécs & 1925 & Soó Rezső & BPU-01549.JPG \\
\hline 730 & Potentilla recta L. & Pilisszentiván & 1953 & Soó Rezső & BPU-01529.JPG \\
\hline 730 & Potentilla recta L. & Pusztamiske & 1948 & Borsos Olga & BPU-01538.JPG \\
\hline 730 & Potentilla recta L. & Sárospatak & 1932 & Soó Rezső & BPU-01539.JPG \\
\hline 730 & Potentilla recta $\mathrm{L}$. & Szilvásvárad & 1953 & $\begin{array}{l}\text { Felföldy Lajos, Tóth } \\
\text { László }\end{array}$ & BPU-01555.JPG \\
\hline
\end{tabular}


1. Elektroniukus melléklet - Electronic Appendix 1.

\begin{tabular}{|c|c|c|c|c|c|}
\hline $\begin{array}{l}\text { Sorszám / } \\
\text { Number }\end{array}$ & Taxon-név / Taxon-name & $\begin{array}{l}\text { Település / } \\
\text { Settlement }\end{array}$ & $\begin{array}{l}\text { Év / } \\
\text { Year }\end{array}$ & Gyüjtő / Collector & $\begin{array}{l}\text { Fájlnév / } \\
\text { File-name }\end{array}$ \\
\hline 730 & Potentilla recta L. & Tihany & 1928 & Soó Rezső & BPU-01536.JPG \\
\hline 730 & Potentilla recta L. & Tihany & 1959 & Felföldy Lajos & BPU-01537.JPG \\
\hline 730 & Potentilla recta $\mathrm{L}$. & Tihany & 1961 & $\begin{array}{l}\text { Pankov H., Felföldy Lajos, } \\
\text { Sólymosy G. }\end{array}$ & BPU-01535.JPG \\
\hline 730 & Potentilla recta L. & Tokaj & 1947 & Soó Rezső & BPU-01540.JPG \\
\hline 730 & Potentilla recta L. & Visegrád & 1949 & Soó Rezső & BPU-01530.JPG \\
\hline 731 & Potentilla heptaphylla L. & Budapest & 1949 & Simon Tibor & BPU-01518.JPG \\
\hline 731 & Potentilla heptaphylla L. & Budapest & 2004 & Felföldy Lajos & BPU-01517.JPG \\
\hline 731 & Potentilla heptaphylla L. & Budapest & 2005 & Felföldy Lajos & BPU-01519.JPG \\
\hline 731 & Potentilla heptaphylla L. & Gánt & 1949 & Soó Rezső & BPU-01524.JPG \\
\hline 731 & Potentilla heptaphylla L. & Hidegkút & 1959 & Felföldy Lajos & BPU-01527.JPG \\
\hline 731 & Potentilla heptaphylla L. & Miskolc & 1937 & Soó Rezső & BPU-01523.JPG \\
\hline 731 & Potentilla heptaphylla L. & Mosonmagyaróvár & 1965 & Borsos Olga & BPU-01525.JPG \\
\hline 731 & Potentilla heptaphylla L. & Nyíradony & 1951 & Simon Tibor & BPU-01526.JPG \\
\hline 731 & Potentilla heptaphylla L. & Pécs & 1936 & Soó Rezső & BPU-01522.JPG \\
\hline 731 & Potentilla heptaphylla L. & Pécs & 1953 & Soó Rezső, Borsos Olga & BPU-01521.JPG \\
\hline 731 & Potentilla heptaphylla L. & Szin & 1953 & Vajda László & BPU-01520.JPG \\
\hline 732 & Potentilla patula Waldst. et Kit. & Bódvarákó & 1951 & Soó Rezső & BPU-01467.JPG \\
\hline 732 & Potentilla patula Waldst. et Kit. & Kőszeg & 1932 & Soó Rezső & BPU-01471.JPG \\
\hline 732 & Potentilla patula Waldst. et Kit. & Miskolc & 1929 & Soó Rezső & BPU-01468.JPG \\
\hline 732 & Potentilla patula Waldst. et Kit. & Miskolc & 1935 & Soó Rezső & BPU-01516.JPG \\
\hline 732 & Potentilla patula Waldst. et Kit. & Szurdokpüspöki & 1937 & Máthé Imre & BPU-01470.JPG \\
\hline 734 & Potentilla arenaria Borkh. & Alsóörs & 1982 & Felföldy Lajos & BPU-01511.JPG \\
\hline 734 & Potentilla arenaria Borkh. & Budapest & 1922 & Soó Rezső & BPU-01503.JPG \\
\hline 734 & Potentilla arenaria Borkh. & Budapest & 1949 & Simon Tibor & BPU-01506.JPG \\
\hline 734 & Potentilla arenaria Borkh. & Gyenesdiás & 1965 & Priszter Szaniszló & BPU-01513.JPG \\
\hline 734 & Potentilla arenaria Borkh. & Gyöngyös & 1964 & Soó Rezső & BPU-01505.JPG \\
\hline 734 & Potentilla arenaria Borkh. & Jósvafő & 1952 & Kulcsár Gábor & BPU-01504.JPG \\
\hline 734 & Potentilla arenaria Borkh. & Nyíracsád & 1949 & Soó Rezső & BPU-01509.JPG \\
\hline 734 & Potentilla arenaria Borkh. & Nyíradony & 1951 & Simon Tibor & BPU-01510.JPG \\
\hline 734 & Potentilla arenaria Borkh. & Pilisborosjenő & 1913 & Degen Árpád & BPU-01507.JPG \\
\hline 734 & Potentilla arenaria Borkh. & Uzsa & 1952 & Simon Tibor & BPU-01512.JPG \\
\hline 734 & Potentilla arenaria Borkh. & Vácrátót & 1953 & Felföldy Lajos & BPU-01508.JPG \\
\hline $734 \times 735$ & $\begin{array}{l}\text { Potentilla arenaria Borkh. } \times \text { Potentilla } \\
\text { pusilla Host }\end{array}$ & Kőszeg & 1896 & Waisbecker Antal & BPU-01574.JPG \\
\hline 736 & Fragaria viridis Duchesne & Szarvas & 1949 & Soó Rezső & BPU-01420.JPG \\
\hline 736 & Fragaria viridis Duchesne & Tihany & 1957 & $\begin{array}{l}\text { Felföldy Lajos, Tóth } \\
\text { László }\end{array}$ & BPU-01417.JPG \\
\hline 736 & Fragaria viridis Duchesne & Uzsa & 1950 & Felföldy Lajos & BPU-01418.JPG \\
\hline 736 & Fragaria viridis Duchesne & Zebegény & 1962 & Simon Tibor & BPU-01419.JPG \\
\hline 737 & Fragaria moschata Duchesne & Balatonszőlős & 1958 & Felföldy Lajos & BPU-01403.JPG \\
\hline 737 & Fragaria moschata Duchesne & Budapest & 1947 & Soó Rezső & BPU-01400.JPG \\
\hline 737 & Fragaria moschata Duchesne & Budapest & 1964 & Soó Rezső & BPU-01395.JPG \\
\hline 737 & Fragaria moschata Duchesne & Debrecen & 1934 & Soó Rezső & BPU-01399.JPG \\
\hline 737 & Fragaria moschata Duchesne & Pécs & 1953 & Soó Rezső, Borsos Olga & BPU-01397.JPG \\
\hline 737 & Fragaria moschata Duchesne & Pécs & 1956 & Soó Rezső & BPU-01396.JPG \\
\hline 737 & Fragaria moschata Duchesne & Pécsvárad & 1936 & Soó Rezső & BPU-01398.JPG \\
\hline 737 & Fragaria moschata Duchesne & Sopron & 1960 & Borsos Olga, Simon Tibor & BPU-01402.JPG \\
\hline 737 & Fragaria moschata Duchesne & Zebegény & 1962 & Simon Tibor & BPU-01401.JPG \\
\hline
\end{tabular}


1. Elektroniukus melléklet - Electronic Appendix 1.

\begin{tabular}{|c|c|c|c|c|c|}
\hline $\begin{array}{l}\text { Sorszám / } \\
\text { Number }\end{array}$ & Taxon-név / Taxon-name & $\begin{array}{l}\text { Település / } \\
\text { Settlement }\end{array}$ & $\begin{array}{l}\text { Év / } \\
\text { Year }\end{array}$ & Gyűjtő / Collector & $\begin{array}{l}\text { Fájlnév / } \\
\text { File-name }\end{array}$ \\
\hline 738 & Fragaria vesca L. & Debrecen & 1937 & Soó Rezső & BPU-01422.JPG \\
\hline 738 & Fragaria vesca L. & Gyula & - & Soó Rezső & BPU-01426.JPG \\
\hline 738 & Fragaria vesca $L$. & Hidegkút & 1958 & Felföldy Lajos & BPU-01424.JPG \\
\hline 738 & Fragaria vesca L. & Komárom & 1959 & $\begin{array}{l}\text { Simon Tibor, Borhidi } \\
\text { Attila }\end{array}$ & BPU-01423.JPG \\
\hline 738 & Fragaria vesca L. & Uzsa & 1959 & $\begin{array}{l}\text { Jávorka Sándor, Felföldy } \\
\text { Lajos }\end{array}$ & BPU-01421.JPG \\
\hline $736 \times 737$ & $\begin{array}{l}\text { Fragaria viridis Duchesne } \times \text { Fragaria } \\
\text { moschata Duchesne }\end{array}$ & Budapest & 1943 & Kárpáti Zoltán & BPU-01416.JPG \\
\hline 739 & Alchemilla glabra Neygenf. & Sopron & 1937 & Soó Rezső & BPU-01657.JPG \\
\hline 740 & Alchemilla xanthochlora Rothm. & Szakonyfalu & 1955 & Soó Rezső & BPU-01692.JPG \\
\hline 741 & Alchemilla subcrenata Buser & Háromhuta & 1960 & Simon Tibor & BPU-01627.JPG \\
\hline 743 & Alchemilla glaucescens Wallr. & Füzér & 1938 & Soó Rezső & BPU-01644.JPG \\
\hline 745 & Alchemilla monticola Opiz & Miskolc & 1905 & Hulják János & BPU-01641.JPG \\
\hline 747 & Alchemilla acutiloba Opiz & Háromhuta & 1960 & Simon Tibor & BPU-01626.JPG \\
\hline 748 & Alchemilla micans Buser & Pálháza & 1952 & Simon Tibor & BPU-01624.JPG \\
\hline 739-748 & Alchemilla & Bélapátfalva & 1948 & $\begin{array}{l}\text { Simon Tibor, Pólya } \\
\text { László, Jakucs Pál }\end{array}$ & BPU-01683.JPG \\
\hline 739-748 & Alchemilla & Mályinka & 1925 & Hulják János & BPU-01697.JPG \\
\hline 739-748 & Alchemilla & Miskolc & 1911 & Hulják János & BPU-01713.JPG \\
\hline 739-748 & Alchemilla & Miskolc & 1922 & Boros Ádám & BPU-01643.JPG \\
\hline 739-748 & Alchemilla & Miskolc & 1927 & Hulják János & BPU-01708.JPG \\
\hline 739-748 & Alchemilla & Miskolc & 1929 & Soó Rezső & BPU-01681.JPG \\
\hline 739-748 & Alchemilla & Miskolc & 1953 & Soó Rezső & BPU-01687.JPG \\
\hline 739-748 & Alchemilla & Pálháza & 1938 & Soó Rezső & BPU-01677.JPG \\
\hline 739-748 & Alchemilla & Pálháza & 1952 & Simon Tibor & BPU-01623.JPG \\
\hline 739-748 & Alchemilla & Regéc & 1952 & Simon Tibor & BPU-01625.JPG \\
\hline 739-748 & Alchemilla & Sopron & 1960 & Borsos Olga, Simon Tibor & BPU-01661.JPG \\
\hline 739-748 & Alchemilla & Szilvásvárad & 1953 & $\begin{array}{l}\text { Felföldy Lajos, Tóth } \\
\text { László }\end{array}$ & BPU-01693.JPG \\
\hline 749 & Aphanes arvensis L. & Balatonhenye & 1959 & $\begin{array}{l}\text { Jávorka Sándor, Felföldy } \\
\text { Lajos, Tóth László }\end{array}$ & BPU-01630.JPG \\
\hline 715 & Aphanes arvensis L. & Gelénes & 1953 & $\begin{array}{l}\text { Simon Tibor, Vozáry } \\
\text { Elemér }\end{array}$ & BPU-01629.JPG \\
\hline 749 & Aphanes arvensis L. & Monostorapáti & 1959 & $\begin{array}{l}\text { Jávorka Sándor, Felföldy } \\
\text { Lajos, Tóth László }\end{array}$ & BPU-01631.JPG \\
\hline 714 & Aphanes arvensis L. & Nagykanizsa & 1948 & Károlyi Árpád & BPU-01628.JPG \\
\hline 751 & Cydonia oblonga Mill. & Sárospatak & 1939 & Soó Rezső & BPU-01138.JPG \\
\hline 754 & Pyrus pyraster (L.) Burgsd. & Budapest & 1964 & Priszter Szaniszló & BPU-01157.JPG \\
\hline 754 & Pyrus pyraster (L.) Burgsd. & Sáránd & 1932 & Soó Rezső & BPU-01159.JPG \\
\hline 758 & Malus sylvestris (L.) Mill. & Debrecen & 1949 & Soó Rezső & BPU-01161.JPG \\
\hline 759 & Malus dasyphylla Borkh. & Visegrád & 1964 & Soó Rezső, Borsos Olga & BPU-01162.JPG \\
\hline 761 & Sorbus domestica L. & Balatongyörök & 1949 & Priszter Szaniszló & BPU-01173.JPG \\
\hline 761 & Sorbus domestica L. & Balatonszőlős & 1958 & Felföldy Lajos & BPU-01172.JPG \\
\hline 761 & Sorbus domestica L. & Budaörs & 1949 & Priszter Szaniszló & BPU-01169.JPG \\
\hline 761 & Sorbus domestica L. & Budapest & 1948 & Priszter Szaniszló & BPU-01164.JPG \\
\hline 761 & Sorbus domestica L. & Budapest & 1949 & Priszter Szaniszló & BPU-01163.JPG \\
\hline 761 & Sorbus domestica L. & Budapest & 1949 & Priszter Szaniszló & BPU-01165.JPG \\
\hline 761 & Sorbus domestica L. & Dömös & 1948 & Priszter Szaniszló & BPU-01168.JPG \\
\hline 761 & Sorbus domestica L. & Erdőbénye & 1948 & Soó Rezső & BPU-01174.JPG \\
\hline 761 & Sorbus domestica L. & Miskolc & 1939 & Soó Rezső & BPU-01167.JPG \\
\hline
\end{tabular}


1. Elektroniukus melléklet - Electronic Appendix 1.

\begin{tabular}{|c|c|c|c|c|c|}
\hline $\begin{array}{l}\text { Sorszám / } \\
\text { Number }\end{array}$ & Taxon-név / Taxon-name & $\begin{array}{l}\text { Település / } \\
\text { Settlement }\end{array}$ & $\begin{array}{l}\text { Év / } \\
\text { Year }\end{array}$ & Gyújtő / Collector & $\begin{array}{l}\text { Fájlnév / } \\
\text { File-name }\end{array}$ \\
\hline 761 & Sorbus domestica L. & Nagykovácsi & 1949 & Priszter Szaniszló & BPU-01170.JPG \\
\hline 761 & Sorbus domestica L. & Pécs & 1935 & Soó Rezső & BPU-01166.JPG \\
\hline 761 & Sorbus domestica L. & Tahitótfalu & 1950 & Priszter Szaniszló & BPU-01171.JPG \\
\hline 762 & Sorbus aucuparia L. & Miskolc & 1937 & Soó Rezső & BPU-01176.JPG \\
\hline 762 & Sorbus aucuparia L. & Telkibánya & 1950 & Soó Rezső & BPU-01175.JPG \\
\hline 763 & Sorbus aria (L.) Crantz & Budapest & 1948 & Soó Rezső & BPU-01269.JPG \\
\hline 763 & Sorbus aria (L.) Crantz & Budapest & 1958 & Simon Tibor & BPU-01260.JPG \\
\hline 763 & Sorbus aria (L.) Crantz & Csákberény & 1948 & Papp József & BPU-01342.JPG \\
\hline 763 & Sorbus aria (L.) Crantz & Csákberény & 1948 & Priszter Szaniszló & BPU-01244.JPG \\
\hline 763 & Sorbus aria (L.) Crantz & Csákberény & 1948 & Priszter Szaniszló & BPU-01245.JPG \\
\hline 763 & Sorbus aria (L.) Crantz & Csókakő & 1948 & Priszter Szaniszló & BPU-01243.JPG \\
\hline 763 & Sorbus aria (L.) Crantz & Gánt & 1926 & Boros Ádám & BPU-01313.JPG \\
\hline 763 & Sorbus aria (L.) Crantz & Gánt & 1948 & Priszter Szaniszló & BPU-01242.JPG \\
\hline 763 & Sorbus aria (L.) Crantz & Gánt & 1948 & Priszter Szaniszló & BPU-01246.JPG \\
\hline 763 & Sorbus aria (L.) Crantz & Gánt & 1948 & Soó Rezső & BPU-01256.JPG \\
\hline 763 & Sorbus aria (L.) Crantz & Gyöngyös & 1952 & Soó Rezső & BPU-01249.JPG \\
\hline 763 & Sorbus aria (L.) Crantz & Miskolc & 1937 & Soó Rezső & BPU-01250.JPG \\
\hline 763 & Sorbus aria (L.) Crantz & Miskolc & 1949 & Priszter Szaniszló & BPU-01252.JPG \\
\hline 763 & Sorbus aria (L.) Crantz & Miskolc & 1950 & Soó Rezső & BPU-01251.JPG \\
\hline 763 & Sorbus aria (L.) Crantz & Miskolc & 1950 & Soó Rezső & BPU-01262.JPG \\
\hline 763 & Sorbus aria (L.) Crantz & Sopron & 1947 & Priszter Szaniszló & BPU-01247.JPG \\
\hline 763 & Sorbus aria (L.) Crantz & Szilvásvárad & 1953 & $\begin{array}{l}\text { Felföldy Lajos, Tóth } \\
\text { László }\end{array}$ & BPU-01258.JPG \\
\hline 763 & Sorbus aria (L.) Crantz & Tibolddaróc & 1953 & Soó Rezső & BPU-01253.JPG \\
\hline 764 & Sorbus graeca (Spach) Kotschy & Budapest & 1946 & Priszter Szaniszló & BPU-01261.JPG \\
\hline 764 & Sorbus graeca (Spach) Kotschy & Budapest & 1948 & Priszter Szaniszló & BPU-01259.JPG \\
\hline 764 & Sorbus graeca (Spach) Kotschy & Csákberény & 1948 & Priszter Szaniszló & BPU-01254.JPG \\
\hline 764 & Sorbus graeca (Spach) Kotschy & Csákvár & 1965 & Simon Tibor & BPU-01255.JPG \\
\hline 765 & Sorbus pannonica Kárpáti & Bélapátfalva & 1948 & Simon Tibor & BPU-01310.JPG \\
\hline 765 & Sorbus pannonica Kárpáti & Budapest & 1948 & Priszter Szaniszló & BPU-01308.JPG \\
\hline 765 & Sorbus pannonica Kárpáti & Budapest & 1950 & Priszter Szaniszló & BPU-01309.JPG \\
\hline 765 & Sorbus pannonica Kárpáti & Csákvár & 1948 & Priszter Szaniszló & BPU-01311.JPG \\
\hline 765 & Sorbus pannonica Kárpáti & Csókakő & 1948 & Priszter Szaniszló & BPU-01312.JPG \\
\hline 765 & Sorbus pannonica Kárpáti & Gánt & 1948 & Priszter Szaniszló & BPU-01314.JPG \\
\hline 765 & Sorbus pannonica Kárpáti & Gánt & 1948 & Priszter Szaniszló & BPU-01315.JPG \\
\hline 768 & Sorbus danubialis (Jáv.) Kárpáti & Balatongyörök & 1949 & Priszter Szaniszló & BPU-01279.JPG \\
\hline 768 & Sorbus danubialis (Jáv.) Kárpáti & Bélapátfalva & 1932 & Soó Rezső & BPU-01257.JPG \\
\hline 768 & Sorbus danubialis (Jáv.) Kárpáti & Budaörs & 1950 & Priszter Szaniszló & BPU-01276.JPG \\
\hline 768 & Sorbus danubialis (Jáv.) Kárpáti & Budaörs & 1950 & Priszter Szaniszló & BPU-01277.JPG \\
\hline 768 & Sorbus danubialis (Jáv.) Kárpáti & Budapest & 1943 & Kárpáti Zoltán & BPU-01265.JPG \\
\hline 768 & Sorbus danubialis (Jáv.) Kárpáti & Budapest & 1947 & Priszter Szaniszló & BPU-01267.JPG \\
\hline 768 & Sorbus danubialis (Jáv.) Kárpáti & Budapest & 1948 & Priszter Szaniszló & BPU-01264.JPG \\
\hline 768 & Sorbus danubialis (Jáv.) Kárpáti & Budapest & 1948 & Priszter Szaniszló & BPU-01266.JPG \\
\hline 768 & Sorbus danubialis (Jáv.) Kárpáti & Budapest & 1948 & Priszter Szaniszló & BPU-01268.JPG \\
\hline 768 & Sorbus danubialis (Jáv.) Kárpáti & Budapest & 1948 & Priszter Szaniszló & BPU-01270.JPG \\
\hline 768 & Sorbus danubialis (Jáv.) Kárpáti & Csákberény & 1948 & Priszter Szaniszló & BPU-01274.JPG \\
\hline 768 & Sorbus danubialis (Jáv.) Kárpáti & Csákberény & 1948 & Priszter Szaniszló & BPU-01275.JPG \\
\hline 768 & Sorbus danubialis (Jáv.) Kárpáti & Dömös & 1948 & Priszter Szaniszló & BPU-01273.JPG \\
\hline
\end{tabular}


1. Elektroniukus melléklet - Electronic Appendix 1.

\begin{tabular}{|c|c|c|c|c|c|}
\hline $\begin{array}{l}\text { Sorszám / } \\
\text { Number }\end{array}$ & Taxon-név / Taxon-name & $\begin{array}{l}\text { Település / } \\
\text { Settlement }\end{array}$ & $\begin{array}{l}\text { Év / } \\
\text { Year }\end{array}$ & Gyújtő / Collector & $\begin{array}{l}\text { Fájlnév / } \\
\text { File-name }\end{array}$ \\
\hline 768 & Sorbus danubialis (Jáv.) Kárpáti & Dömös & 1948 & Soó Rezső & BPU-01272.JPG \\
\hline 768 & Sorbus danubialis (Jáv.) Kárpáti & Gyöngyös & 1952 & Soó Rezső & BPU-01263.JPG \\
\hline 768 & Sorbus danubialis (Jáv.) Kárpáti & Kisgyőr & 1938 & Soó Rezső & BPU-01271.JPG \\
\hline 768 & Sorbus danubialis (Jáv.) Kárpáti & Pomáz & 1950 & Priszter Szaniszló & BPU-01278.JPG \\
\hline 770 & Sorbus subdanubialis (Soó) Kárpáti & Csákberény & 1948 & Priszter Szaniszló & BPU-01341.JPG \\
\hline 772 & Sorbus torminalis (L.) Crantz & Balatongyörök & 1949 & Priszter Szaniszló & BPU-01240.JPG \\
\hline 772 & Sorbus torminalis (L.) Crantz & Budaörs & 1949 & Priszter Szaniszló & BPU-01222.JPG \\
\hline 772 & Sorbus torminalis (L.) Crantz & Budaörs & 1949 & Priszter Szaniszló & BPU-01224.JPG \\
\hline 772 & Sorbus torminalis (L.) Crantz & Budaörs & 1949 & Priszter Szaniszló & BPU-01225.JPG \\
\hline 772 & Sorbus torminalis (L.) Crantz & Budaörs & 1950 & Priszter Szaniszló & BPU-01223.JPG \\
\hline 772 & Sorbus torminalis (L.) Crantz & Budaörs & 1950 & Priszter Szaniszló & BPU-01227.JPG \\
\hline 772 & Sorbus torminalis (L.) Crantz & Budaörs & 1950 & Priszter Szaniszló & BPU-01228.JPG \\
\hline 772 & Sorbus torminalis (L.) Crantz & Budapest & 1915 & Andrasovszky József & BPU-01179.JPG \\
\hline 772 & Sorbus torminalis (L.) Crantz & Budapest & 1947 & Priszter Szaniszló & BPU-01182.JPG \\
\hline 772 & Sorbus torminalis (L.) Crantz & Budapest & 1948 & Papp József & BPU-01208.JPG \\
\hline 772 & Sorbus torminalis (L.) Crantz & Budapest & 1948 & Priszter Szaniszló & BPU-01183.JPG \\
\hline 772 & Sorbus torminalis (L.) Crantz & Budapest & 1948 & Priszter Szaniszló & BPU-01186.JPG \\
\hline 772 & Sorbus torminalis (L.) Crantz & Budapest & 1948 & Priszter Szaniszló & BPU-01188.JPG \\
\hline 772 & Sorbus torminalis (L.) Crantz & Budapest & 1948 & Priszter Szaniszló & BPU-01189.JPG \\
\hline 772 & Sorbus torminalis (L.) Crantz & Budapest & 1948 & Priszter Szaniszló & BPU-01190.JPG \\
\hline 772 & Sorbus torminalis (L.) Crantz & Budapest & 1948 & Priszter Szaniszló & BPU-01191.JPG \\
\hline 772 & Sorbus torminalis (L.) Crantz & Budapest & 1948 & Priszter Szaniszló & BPU-01193.JPG \\
\hline 772 & Sorbus torminalis (L.) Crantz & Budapest & 1948 & Priszter Szaniszló & BPU-01194.JPG \\
\hline 772 & Sorbus torminalis (L.) Crantz & Budapest & 1948 & Priszter Szaniszló & BPU-01195.JPG \\
\hline 772 & Sorbus torminalis (L.) Crantz & Budapest & 1948 & Priszter Szaniszló & BPU-01203.JPG \\
\hline 772 & Sorbus torminalis (L.) Crantz & Budapest & 1948 & Priszter Szaniszló & BPU-01205.JPG \\
\hline 772 & Sorbus torminalis (L.) Crantz & Budapest & 1948 & Priszter Szaniszló & BPU-01207.JPG \\
\hline 772 & Sorbus torminalis (L.) Crantz & Budapest & 1948 & Soó Rezső & BPU-01200.JPG \\
\hline 772 & Sorbus torminalis (L.) Crantz & Budapest & 1949 & Priszter Szaniszló & BPU-01180.JPG \\
\hline 772 & Sorbus torminalis (L.) Crantz & Budapest & 1949 & Priszter Szaniszló & BPU-01181.JPG \\
\hline 772 & Sorbus torminalis (L.) Crantz & Budapest & 1949 & Priszter Szaniszló & BPU-01229.JPG \\
\hline 772 & Sorbus torminalis (L.) Crantz & Budapest & 1950 & Priszter Szaniszló & BPU-01185.JPG \\
\hline 772 & Sorbus torminalis (L.) Crantz & Budapest & 1950 & Priszter Szaniszló & BPU-01187.JPG \\
\hline 772 & Sorbus torminalis (L.) Crantz & Budapest & 1950 & Priszter Szaniszló & BPU-01192.JPG \\
\hline 772 & Sorbus torminalis (L.) Crantz & Budapest & 1950 & Priszter Szaniszló & BPU-01196.JPG \\
\hline 772 & Sorbus torminalis (L.) Crantz & Budapest & 1950 & Priszter Szaniszló & BPU-01197.JPG \\
\hline 772 & Sorbus torminalis (L.) Crantz & Budapest & 1950 & Priszter Szaniszló & BPU-01198.JPG \\
\hline 772 & Sorbus torminalis (L.) Crantz & Budapest & 1950 & Priszter Szaniszló & BPU-01199.JPG \\
\hline 772 & Sorbus torminalis (L.) Crantz & Budapest & 1950 & Priszter Szaniszló & BPU-01202.JPG \\
\hline 772 & Sorbus torminalis (L.) Crantz & Budapest & 1950 & Priszter Szaniszló & BPU-01204.JPG \\
\hline 772 & Sorbus torminalis (L.) Crantz & Budapest & 1950 & Priszter Szaniszló & BPU-01206.JPG \\
\hline 772 & Sorbus torminalis (L.) Crantz & Budapest & 1950 & Priszter Szaniszló & BPU-01226.JPG \\
\hline 772 & Sorbus torminalis (L.) Crantz & Budapest & 1950 & Priszter Szaniszló & BPU-01232.JPG \\
\hline 772 & Sorbus torminalis (L.) Crantz & Budapest & 1950 & Priszter Szaniszló & BPU-01233.JPG \\
\hline 772 & Sorbus torminalis (L.) Crantz & Budapest & 1951 & Priszter Szaniszló & BPU-01201.JPG \\
\hline 772 & Sorbus torminalis (L.) Crantz & Budapest & 1958 & Simon Tibor & BPU-01184.JPG \\
\hline 772 & Sorbus torminalis (L.) Crantz & Csákberény & 1946 & Priszter Szaniszló & BPU-01211.JPG \\
\hline
\end{tabular}


1. Elektroniukus melléklet - Electronic Appendix 1.

\begin{tabular}{|c|c|c|c|c|c|}
\hline $\begin{array}{l}\text { Sorszám / } \\
\text { Number }\end{array}$ & Taxon-név / Taxon-name & $\begin{array}{l}\text { Település / } \\
\text { Settlement }\end{array}$ & $\begin{array}{l}\text { Év / } \\
\text { Year }\end{array}$ & Gyújtő / Collector & $\begin{array}{l}\text { Fájlnév / } \\
\text { File-name }\end{array}$ \\
\hline 772 & Sorbus torminalis (L.) Crantz & Csákberény & 1948 & Priszter Szaniszló & BPU-01210.JPG \\
\hline 772 & Sorbus torminalis (L.) Crantz & Csákberény & 1948 & Priszter Szaniszló & BPU-01212.JPG \\
\hline 772 & Sorbus torminalis (L.) Crantz & Csákvár & 1948 & Priszter Szaniszló & BPU-01213.JPG \\
\hline 772 & Sorbus torminalis (L.) Crantz & Csákvár & 1948 & Priszter Szaniszló & BPU-01214.JPG \\
\hline 772 & Sorbus torminalis (L.) Crantz & Csókakő & 1948 & Papp József & BPU-01217.JPG \\
\hline 772 & Sorbus torminalis (L.) Crantz & Csókakő & 1948 & Priszter Szaniszló & BPU-01215.JPG \\
\hline 772 & Sorbus torminalis (L.) Crantz & Csókakő & 1948 & Priszter Szaniszló & BPU-01216.JPG \\
\hline 772 & Sorbus torminalis (L.) Crantz & Gánt & 1948 & Priszter Szaniszló & BPU-01218.JPG \\
\hline 772 & Sorbus torminalis (L.) Crantz & Gánt & 1948 & Priszter Szaniszló & BPU-01219.JPG \\
\hline 772 & Sorbus torminalis (L.) Crantz & Gánt & 1948 & Priszter Szaniszló & BPU-01220.JPG \\
\hline 772 & Sorbus torminalis (L.) Crantz & Gyenesdiás & 1950 & Priszter Szaniszló & BPU-01241.JPG \\
\hline 772 & Sorbus torminalis (L.) Crantz & Gyöngyös & 1965 & Soó Rezső & BPU-01238.JPG \\
\hline 772 & Sorbus torminalis (L.) Crantz & Isztimér & 1949 & Soó Rezső & BPU-01221.JPG \\
\hline 772 & Sorbus torminalis (L.) Crantz & Miskolc & 1939 & Soó Rezső & BPU-01237.JPG \\
\hline 772 & Sorbus torminalis (L.) Crantz & Nagykovácsi & 1949 & Priszter Szaniszló & BPU-01230.JPG \\
\hline 772 & Sorbus torminalis (L.) Crantz & Nagykovácsi & 1949 & Priszter Szaniszló & BPU-01234.JPG \\
\hline 772 & Sorbus torminalis (L.) Crantz & Nagykovácsi & 1950 & Priszter Szaniszló & BPU-01231.JPG \\
\hline 772 & Sorbus torminalis (L.) Crantz & Nagymaros & 1950 & Priszter Szaniszló & BPU-01235.JPG \\
\hline 772 & Sorbus torminalis (L.) Crantz & Regéc & 1960 & Simon Tibor & BPU-01236.JPG \\
\hline 772 & Sorbus torminalis (L.) Crantz & Tihany & 1956 & Felföldy Lajos & BPU-01239.JPG \\
\hline 772 & Sorbus torminalis (L.) Crantz & Vác & 1949 & $\begin{array}{l}\text { Soó Rezső, Simon Tibor, } \\
\text { Jakucs Pál }\end{array}$ & BPU-15535.JPG \\
\hline 775 & Sorbus zolyomii (Soó) Kárpáti & Miskolc & 1953 & Soó Rezső & BPU-01350.JPG \\
\hline 776 & Sorbus javorkae (Soó) Kárpáti & Fony & 1961 & Simon Tibor & BPU-01306.JPG \\
\hline 780 & Sorbus borosiana Kárpáti & Gánt & 1943 & Kárpáti Zoltán & BPU-01286.JPG \\
\hline 780 & Sorbus borosiana Kárpáti & Gánt & 1948 & Priszter Szaniszló & BPU-01287.JPG \\
\hline 780 & Sorbus borosiana Kárpáti & Gánt & 1948 & Priszter Szaniszló & BPU-01288.JPG \\
\hline 781 & Sorbus degenii Jáv. & Csákberény & 1935 & Boros Ádám & BPU-01290.JPG \\
\hline 781 & Sorbus degenii Jáv. & Csákberény & 1948 & Priszter Szaniszló & BPU-01291.JPG \\
\hline 781 & Sorbus degenii Jáv. & Csákberény & 1948 & Priszter Szaniszló & BPU-01292.JPG \\
\hline 781 & Sorbus degenii Jáv. & Csákberény & 1948 & Priszter Szaniszló & BPU-01293.JPG \\
\hline 781 & Sorbus degenii Jáv. & Csókakő & 1948 & Priszter Szaniszló & BPU-01294.JPG \\
\hline 781 & Sorbus degenii Jáv. & Csókakő & 1948 & Priszter Szaniszló & BPU-01295.JPG \\
\hline 781 & Sorbus degenii Jáv. & Gánt & 1948 & Priszter Szaniszló & BPU-01296.JPG \\
\hline 781 & Sorbus degenii Jáv. & Gánt & 1948 & Priszter Szaniszló & BPU-01297.JPG \\
\hline 781 & Sorbus degenii Jáv. & Gánt & 1948 & Priszter Szaniszló & BPU-01298.JPG \\
\hline 782 & Sorbus decipientiformis Kárpáti & Balatongyörök & 1949 & Priszter Szaniszló & BPU-01289.JPG \\
\hline 783 & Sorbus pseudolatifolia Boros & Csákberény & 1948 & Priszter Szaniszló & BPU-01317.JPG \\
\hline 783 & Sorbus pseudolatifolia Boros & Csákberény & 1948 & Priszter Szaniszló & BPU-01319.JPG \\
\hline 783 & Sorbus pseudolatifolia Boros & Gánt & 1943 & Kárpáti Zoltán & BPU-01318.JPG \\
\hline 788 & Sorbus gayeriana Kárpáti & Balatongyörök & 1949 & Priszter Szaniszló & BPU-01301.JPG \\
\hline 788 & Sorbus gayeriana Kárpáti & Balatongyörök & 1949 & Priszter Szaniszló & BPU-01302.JPG \\
\hline 788 & Sorbus gayeriana Kárpáti & Gyenesdiás & 1949 & Priszter Szaniszló & BPU-01300.JPG \\
\hline 788 & Sorbus gayeriana Kárpáti & Rezi & 1953 & Borsos Olga & BPU-01303.JPG \\
\hline 789 & Sorbus karpatii Boros & Csákberény & 1948 & Priszter Szaniszló & BPU-01307.JPG \\
\hline 790 & Sorbus barthae Kárpáti & Isztimér & 1949 & Soó Rezső & BPU-01284.JPG \\
\hline 791 & Sorbus pseudovertesensis Boros & Csákberény & 1948 & Kárpáti Zoltán & BPU-01324.JPG \\
\hline 791 & Sorbus pseudovertesensis Boros & Csákberény & 1948 & Priszter Szaniszló & BPU-01321.JPG \\
\hline
\end{tabular}


1. Elektroniukus melléklet - Electronic Appendix 1.

\begin{tabular}{|c|c|c|c|c|c|}
\hline $\begin{array}{l}\text { Sorszám / } \\
\text { Number }\end{array}$ & Taxon-név / Taxon-name & $\begin{array}{l}\text { Település / } \\
\text { Settlement }\end{array}$ & $\begin{array}{l}\text { Év / } \\
\text { Year }\end{array}$ & Gyújtő / Collector & $\begin{array}{l}\text { Fájlnév / } \\
\text { File-name }\end{array}$ \\
\hline 791 & Sorbus pseudovertesensis Boros & Csákberény & 1948 & Priszter Szaniszló & BPU-01322.JPG \\
\hline 791 & Sorbus pseudovertesensis Boros & Csákberény & 1948 & Priszter Szaniszló & BPU-01323.JPG \\
\hline 791 & Sorbus pseudovertesensis Boros & Csákberény & 1948 & Priszter Szaniszló & BPU-01325.JPG \\
\hline 791 & Sorbus pseudovertesensis Boros & Csákberény & 1948 & Priszter Szaniszló & BPU-01326.JPG \\
\hline 791 & Sorbus pseudovertesensis Boros & Csókakő & 1948 & Priszter Szaniszló & BPU-01327.JPG \\
\hline 791 & Sorbus pseudovertesensis Boros & Gánt & 1948 & Priszter Szaniszló & BPU-01328.JPG \\
\hline 794 & Sorbus redliana Kárpáti & Isztimér & 1932 & Polgár Sándor & BPU-01330.JPG \\
\hline 794 & Sorbus redliana Kárpáti & Isztimér & 1949 & Soó Rezső & BPU-01329.JPG \\
\hline 798 & Sorbus vertesensis Boros & Csákberény & 1948 & Kárpáti Zoltán & BPU-01344.JPG \\
\hline 798 & Sorbus vertesensis Boros & Csákberény & 1948 & Priszter Szaniszló & BPU-01343.JPG \\
\hline 798 & Sorbus vertesensis Boros & Csákberény & 1948 & Priszter Szaniszló & BPU-01345.JPG \\
\hline 798 & Sorbus vertesensis Boros & Csákberény & 1948 & Priszter Szaniszló & BPU-01346.JPG \\
\hline 798 & Sorbus vertesensis Boros & Csákberény & 1948 & Priszter Szaniszló & BPU-01347.JPG \\
\hline 798 & Sorbus vertesensis Boros & Csókakő & 1948 & Priszter Szaniszló & BPU-01348.JPG \\
\hline 798 & Sorbus vertesensis Boros & Gánt & 1948 & Priszter Szaniszló & BPU-01349.JPG \\
\hline 799 & Sorbus pseudosemiincisa Boros & Csákberény & 1948 & Priszter Szaniszló & BPU-01320.JPG \\
\hline 800 & Sorbus simonkaiana Kárpáti & Csákberény & 1948 & Priszter Szaniszló & BPU-01339.JPG \\
\hline 800 & Sorbus simonkaiana Kárpáti & Gánt & 1948 & Priszter Szaniszló & BPU-01340.JPG \\
\hline 801 & Sorbus semiincisa Borbás & "Pilis" & 1932 & Soó Rezső & BPU-01337.JPG \\
\hline 801 & Sorbus semiincisa Borbás & Budaörs & 1948 & Priszter Szaniszló & BPU-01333.JPG \\
\hline 801 & Sorbus semiincisa Borbás & Budaörs & 1950 & Priszter Szaniszló & BPU-01334.JPG \\
\hline 801 & Sorbus semiincisa Borbás & Budapest & 1948 & Priszter Szaniszló & BPU-01331.JPG \\
\hline 801 & Sorbus semiincisa Borbás & Budapest & 1950 & Priszter Szaniszló & BPU-01332.JPG \\
\hline 801 & Sorbus semiincisa Borbás & Nagykovácsi & 1934 & Gyelnik Vilmos & BPU-01335.JPG \\
\hline 801 & Sorbus semiincisa Borbás & Nagykovácsi & 1949 & Priszter Szaniszló & BPU-01336.JPG \\
\hline 803 & Sorbus adami Kárpáti & Csókakő & 1948 & Priszter Szaniszló & BPU-01280.JPG \\
\hline 803 & Sorbus adami Kárpáti & Gánt & 1943 & Kárpáti Zoltán & BPU-01281.JPG \\
\hline 803 & Sorbus adami Kárpáti & Gánt & 1948 & Priszter Szaniszló & BPU-01282.JPG \\
\hline 803 & Sorbus adami Kárpáti & Gánt & 1948 & Priszter Szaniszló & BPU-01283.JPG \\
\hline 804 & Sorbus pseudobakonyensis Kárpáti & Gánt & 1943 & Kárpáti Zoltán & BPU-01316.JPG \\
\hline 804 & Sorbus pseudobakonyensis Kárpáti & Gánt & 1948 & Kárpáti Zoltán & BPU-15536.JPG \\
\hline 806 & Sorbus eugenii-kelleri Kárpáti & Gánt & 1943 & Kárpáti Zoltán & BPU-01299.JPG \\
\hline $763-807$ & Sorbus & Szár & 1944 & Papp József & BPU-01304.JPG \\
\hline 808 & Amelanchier ovalis Medik. & Isztimér & 1949 & Soó Rezső & BPU-01352.JPG \\
\hline 809 & Cotoneaster tomentosus (Aiton) Lindl. & Isztimér & 1949 & Soó Rezső & BPU-01139.JPG \\
\hline 809 & Cotoneaster tomentosus (Aiton) Lindl. & Várpalota & 1955 & Soó Rezső & BPU-01140.JPG \\
\hline 809 & Cotoneaster tomentosus (Aiton) Lindl. & Várpalota & 1955 & Soó Rezső & BPU-01143.JPG \\
\hline 810 & Cotoneaster niger (Thunb.) Fr. & Bódvarákó & 1951 & $\begin{array}{l}\text { Soó Rezső, Jakucs Pál, Ér } \\
\text { Lajos }\end{array}$ & BPU-01155.JPG \\
\hline 810 & Cotoneaster niger (Thunb.) Fr. & Gánt & 1943 & Kárpáti Zoltán & BPU-01141.JPG \\
\hline 810 & Cotoneaster niger (Thunb.) Fr. & Tihany & 1938 & Soó Rezső & BPU-01154.JPG \\
\hline 810 & Cotoneaster niger (Thunb.) Fr. & Tihany & 1959 & Felföldy Lajos & BPU-01156.JPG \\
\hline 811 & Cotoneaster integerrimus Medik. & Bélapátfalva & 1930 & Soó Rezső & BPU-01144.JPG \\
\hline 811 & Cotoneaster integerrimus Medik. & Bozsok & 1938 & Soó Rezső & BPU-01149.JPG \\
\hline 811 & Cotoneaster integerrimus Medik. & Dömös & 1952 & Soó Rezső & BPU-01147.JPG \\
\hline 811 & Cotoneaster integerrimus Medik. & Dömös & 1952 & Soó Rezső & BPU-01148.JPG \\
\hline 811 & Cotoneaster integerrimus Medik. & Gyöngyös & 1953 & Soó Rezső & BPU-01146.JPG \\
\hline 811 & Cotoneaster integerrimus Medik. & Gyöngyös & 1963 & Soó Rezső & BPU-01145.JPG \\
\hline
\end{tabular}


1. Elektroniukus melléklet - Electronic Appendix 1.

\begin{tabular}{|c|c|c|c|c|c|}
\hline $\begin{array}{l}\text { Sorszám / } \\
\text { Number }\end{array}$ & Taxon-név / Taxon-name & $\begin{array}{l}\text { Település / } \\
\text { Settlement }\end{array}$ & $\begin{array}{l}\text { Év / } \\
\text { Year }\end{array}$ & Gyüjtő / Collector & $\begin{array}{l}\text { Fájlnév / } \\
\text { File-name }\end{array}$ \\
\hline 813 & Crataegus nigra Waldst. et Kit. & Szigetújfalu & 1943 & Kárpáti Zoltán & BPU-09117.JPG \\
\hline 814 & Crataegus laevigata (Poir.) DC. & Budapest & 1947 & Soó Rezső & BPU-09119.JPG \\
\hline 814 & Crataegus laevigata (Poir.) DC. & Isztimér & 1949 & Soó Rezső & BPU-09118.JPG \\
\hline 815 & Crataegus monogyna Jacq. & Budapest & 1947 & Soó Rezső & BPU-09129.JPG \\
\hline 815 & Crataegus monogyna Jacq. & Gánt & 1948 & Soó Rezső & BPU-09120.JPG \\
\hline 815 & Crataegus monogyna Jacq. & Magyarlukafa & 1957 & Soó Rezső & BPU-09121.JPG \\
\hline 815 & Crataegus monogyna Jacq. & Törökbálint & 1991 & Felföldy Lajos & BPU-09126.JPG \\
\hline $\begin{array}{l}814 \times \\
816.2\end{array}$ & $\begin{array}{l}\text { Crataegus laevigata (Poir.) DC. } \times \\
\text { Crataegus calycina subsp. curvisepala } \\
\text { (Lindm.) Franco }\end{array}$ & Beregdaróc & 1951 & Simon Tibor & BPU-09124.JPG \\
\hline $\begin{array}{l}814 \times \\
816.2\end{array}$ & $\begin{array}{l}\text { Crataegus laevigata (Poir.) DC. } \times \\
\text { Crataegus calycina subsp. curvisepala } \\
\text { (Lindm.) Franco }\end{array}$ & Beregdaróc & 1951 & Simon Tibor, Jakucs Pál & BPU-09123.JPG \\
\hline $\begin{array}{l}815 \times \\
816.2\end{array}$ & $\begin{array}{l}\text { Crataegus monogyna Jacq. } \times \text { Crataegus } \\
\text { calycina subsp. curvisepala (Lindm.) } \\
\text { Franco }\end{array}$ & Fót & 1960 & Soó Rezső & BPU-09122.JPG \\
\hline 817 & Padus avium Mill. & Mosonmagyaróvár & 1965 & Borsos Olga & BPU-01757.JPG \\
\hline 817 & Padus avium Mill. & Pápa & 1953 & Tallós Pál & BPU-01751.JPG \\
\hline 817 & Padus avium Mill. & Sárospatak & 1939 & Soó Rezső & BPU-01752.JPG \\
\hline 819 & Cerasus mahaleb (L.) Mill. & Budaörs & 1982 & Felföldy Lajos & BPU-01762.JPG \\
\hline 819 & Cerasus mahaleb (L.) Mill. & Budapest & 1947 & Soó Rezső & BPU-01758.JPG \\
\hline 819 & Cerasus mahaleb (L.) Mill. & Budapest & 1991 & Felföldy Lajos & BPU-01759.JPG \\
\hline 819 & Cerasus mahaleb (L.) Mill. & Budapest & 2005 & Felföldy Lajos & BPU-01760.JPG \\
\hline 819 & Cerasus mahaleb (L.) Mill. & Csákvár & 1955 & Soó Rezső & BPU-01761.JPG \\
\hline 819 & Cerasus mahaleb (L.) Mill. & Tihany & 1956 & Felföldy Lajos & BPU-01763.JPG \\
\hline 820 & Cerasus avium (L.) Moench & Bélmegyer & 1935 & Máthé Imre & BPU-01777.JPG \\
\hline 820 & Cerasus avium (L.) Moench & Budapest & 1949 & Soó Rezső & BPU-01779.JPG \\
\hline 820 & Cerasus avium (L.) Moench & Debrecen & 1932 & Soó Rezső & BPU-01780.JPG \\
\hline 820 & Cerasus avium (L.) Moench & Szár & 1949 & Soó Rezső & BPU-01781.JPG \\
\hline 821 & Cerasus fruticosa (Pall.) Woronow & Abaújszántó & 1948 & Soó Rezső & BPU-01784.JPG \\
\hline 821 & Cerasus fruticosa (Pall.) Woronow & Arka & 1961 & Simon Tibor & BPU-01785.JPG \\
\hline 821 & Cerasus fruticosa (Pall.) Woronow & Budapest & 1943 & Kárpáti Zoltán & BPU-01783.JPG \\
\hline 821 & Cerasus fruticosa (Pall.) Woronow & Budapest & 1982 & Felföldy Lajos & BPU-01782.JPG \\
\hline 821 & Cerasus fruticosa (Pall.) Woronow & Csarnóta & 1965 & Simon Tibor & BPU-01786.JPG \\
\hline 821 & Cerasus fruticosa (Pall.) Woronow & Egyek & 1935 & Soó Rezső & BPU-01787.JPG \\
\hline 821 & Cerasus fruticosa (Pall.) Woronow & Kerecsend & 1957 & Soó Rezső & BPU-01789.JPG \\
\hline 821 & Cerasus fruticosa (Pall.) Woronow & Nadap & 1922 & Soó Rezső & BPU-01788.JPG \\
\hline 821 & Cerasus fruticosa (Pall.) Woronow & Tarcal & 1951 & Soó Rezső & BPU-01791.JPG \\
\hline 821 & Cerasus fruticosa (Pall.) Woronow & Tihany & 1959 & Felföldy Lajos & BPU-01790.JPG \\
\hline 819-822 & Cerasus & Tarcal & 1951 & Soó Rezső & BPU-01800.JPG \\
\hline $820 \times 821$ & $\begin{array}{l}\text { Cerasus avium (L.) Moench } \times \text { Cerasus } \\
\text { fruticosa (Pall.) Woronow }\end{array}$ & Budapest & 1944 & Kárpáti Zoltán & BPU-01795.JPG \\
\hline $820 \times 821$ & $\begin{array}{l}\text { Cerasus avium (L.) Moench } \times \text { Cerasus } \\
\text { fruticosa (Pall.) Woronow }\end{array}$ & Budapest & 1947 & Soó Rezső & BPU-01796.JPG \\
\hline $820 \times 821$ & $\begin{array}{l}\text { Cerasus avium (L.) Moench } \times \text { Cerasus } \\
\text { fruticosa (Pall.) Woronow }\end{array}$ & Csákvár & 1948 & Soó Rezső & BPU-01797.JPG \\
\hline $820 \times 821$ & $\begin{array}{l}\text { Cerasus avium (L.) Moench } \times \text { Cerasus } \\
\text { fruticosa (Pall.) Woronow }\end{array}$ & Kerecsend & 1957 & Soó Rezső & BPU-01798.JPG \\
\hline $821 \times 822$ & Prunus $\times$ eminens Beck & Felpéc & 1909 & Polgár Sándor & BPU-01799.JPG \\
\hline 824 & Amygdalus nana L. & Abaújszántó & 1948 & Soó Rezső & BPU-01766.JPG \\
\hline 824 & Amygdalus nana L. & Abaújszántó & 1961 & Simon Tibor & BPU-01767.JPG \\
\hline
\end{tabular}


1. Elektroniukus melléklet - Electronic Appendix 1.

\begin{tabular}{|c|c|c|c|c|c|}
\hline $\begin{array}{l}\text { Sorszám / } \\
\text { Number }\end{array}$ & Taxon-név / Taxon-name & $\begin{array}{l}\text { Település / } \\
\text { Settlement }\end{array}$ & $\begin{array}{l}\text { Év / } \\
\text { Year }\end{array}$ & Gyújtő / Collector & $\begin{array}{l}\text { Fájlnév / } \\
\text { File-name }\end{array}$ \\
\hline 824 & Amygdalus nana L. & Egyek & 1938 & Soó Rezső & BPU-01769.JPG \\
\hline 824 & Amygdalus nana L. & Kerecsend & 1957 & Soó Rezső & BPU-01770.JPG \\
\hline 824 & Amygdalus nana L. & Kerecsend & 1957 & Soó Rezső & BPU-01771.JPG \\
\hline 824 & Amygdalus nana L. & Nagyharsány & 1967 & Priszter Szaniszló & BPU-01768.JPG \\
\hline 824 & Amygdalus nana L. & Tarcal & 1951 & Soó Rezső & BPU-01772.JPG \\
\hline 827 & Prunus spinosa L. & Egyek & 1933 & Máthé Imre & BPU-01776.JPG \\
\hline 827 & Prunus spinosa L. & Gyöngyös & 1964 & Soó Rezső & BPU-01774.JPG \\
\hline 827 & Prunus spinosa L. & Muraszemenye & 1948 & Károlyi Árpád & BPU-01775.JPG \\
\hline 834 & Laburnum anagyroides Medik. & Budapest & 2004 & Felföldy Lajos & BPU-00756.JPG \\
\hline 834 & Laburnum anagyroides Medik. & Pécs & 1953 & Soó Rezső, Borsos Olga & BPU-00757.JPG \\
\hline 834 & Laburnum anagyroides Medik. & Pécs & 1956 & Soó Rezső & BPU-00758.JPG \\
\hline 835 & Lembotropis nigricans (L.) Griseb. & Bátorliget & 1932 & Soó Rezső & BPU-00774.JPG \\
\hline 835 & Lembotropis nigricans (L.) Griseb. & Budaörs & 1982 & Felföldy Lajos & BPU-00772.JPG \\
\hline 835 & Lembotropis nigricans (L.) Griseb. & Budapest & 1952 & Soó Rezső & BPU-00771.JPG \\
\hline 835 & Lembotropis nigricans (L.) Griseb. & Solymár & 2005 & Felföldy Lajos & BPU-00773.JPG \\
\hline 836 & $\begin{array}{l}\text { Sarothamnus scoparius (L.) Wimm. ex } \\
\text { W.D.J. Koch }\end{array}$ & Kaszó & 1957 & Soó Rezső & BPU-00762.JPG \\
\hline 836 & $\begin{array}{l}\text { Sarothamnus scoparius (L.) Wimm. ex } \\
\text { W.D.J. Koch }\end{array}$ & Nagybajom & 1957 & Soó Rezső & BPU-00763.JPG \\
\hline 836 & $\begin{array}{l}\text { Sarothamnus scoparius (L.) Wimm. ex } \\
\text { W.D.J. Koch }\end{array}$ & Téglás & 1937 & Soó Rezső & BPU-00761.JPG \\
\hline 836 & $\begin{array}{l}\text { Sarothamnus scoparius (L.) Wimm. ex } \\
\text { W.D.J. Koch }\end{array}$ & Uzsa & 1951 & Borsos Olga & BPU-00764.JPG \\
\hline 836 & $\begin{array}{l}\text { Sarothamnus scoparius (L.) Wimm. ex } \\
\text { W.D.J. Koch }\end{array}$ & Uzsa & 1959 & $\begin{array}{l}\text { Jávorka Sándor, Felföldy } \\
\text { Lajos }\end{array}$ & BPU-00765.JPG \\
\hline 837 & $\begin{array}{l}\text { Corothamnus procumbens (Waldst. et } \\
\text { Kit.) C. Presl }\end{array}$ & Bélapátfalva & 1932 & Soó Rezső & BPU-00766.JPG \\
\hline 837 & $\begin{array}{l}\text { Corothamnus procumbens (Waldst. et } \\
\text { Kit.) C. Presl }\end{array}$ & Gyöngyös & 1923 & Soó Rezső & BPU-00768.JPG \\
\hline 837 & $\begin{array}{l}\text { Corothamnus procumbens (Waldst. et } \\
\text { Kit.) C. Presl }\end{array}$ & Gyöngyös & 1965 & Soó Rezső & BPU-00769.JPG \\
\hline 837 & $\begin{array}{l}\text { Corothamnus procumbens (Waldst. et } \\
\text { Kit.) C. Presl }\end{array}$ & Jósvafő & 1953 & Vajda László & BPU-00767.JPG \\
\hline 838 & Chamaecytisus supinus (L.) Link & Balatonszőlős & 1958 & Felföldy Lajos & BPU-00790.JPG \\
\hline 838 & Chamaecytisus supinus (L.) Link & Budakeszi & 1947 & Soó Rezső & BPU-00786.JPG \\
\hline 838 & Chamaecytisus supinus (L.) Link & Budapest & 1961 & Soó Rezső & BPU-00781.JPG \\
\hline 838 & Chamaecytisus supinus (L.) Link & Csákvár & 1948 & Soó Rezső & BPU-00784.JPG \\
\hline 838 & Chamaecytisus supinus (L.) Link & Dörgicse & 1957 & Felföldy Lajos & BPU-00779.JPG \\
\hline 838 & Chamaecytisus supinus (L.) Link & Isztimér & 1949 & Soó Rezső & BPU-00785.JPG \\
\hline 838 & Chamaecytisus supinus (L.) Link & Kőszeg & 1963 & Soó Rezső, Borsos Olga & BPU-00777.JPG \\
\hline 838 & Chamaecytisus supinus (L.) Link & Magyarlukafa & 1957 & Soó Rezső & BPU-00796.JPG \\
\hline 838 & Chamaecytisus supinus (L.) Link & Monostorapáti & 1959 & $\begin{array}{l}\text { Jávorka Sándor, Felföldy } \\
\text { Lajos, Tóth László }\end{array}$ & BPU-00791.JPG \\
\hline 838 & Chamaecytisus supinus (L.) Link & Nagybajom & 1955 & Soó Rezső & BPU-00789.JPG \\
\hline 838 & Chamaecytisus supinus (L.) Link & Pécs & 1936 & Soó Rezső & BPU-00783.JPG \\
\hline 838 & Chamaecytisus supinus (L.) Link & Pécs & 1953 & Soó Rezső, Borsos Olga & BPU-00775.JPG \\
\hline 838 & Chamaecytisus supinus (L.) Link & Siklós & 1967 & Priszter Szaniszló & BPU-00782.JPG \\
\hline 838 & Chamaecytisus supinus (L.) Link & Solymár & 2005 & Felföldy Lajos & BPU-00787.JPG \\
\hline 838 & Chamaecytisus supinus (L.) Link & Solymár & 2005 & Felföldy Lajos & BPU-00788.JPG \\
\hline 838 & Chamaecytisus supinus (L.) Link & Szakonyfalu & 1955 & Soó Rezső & BPU-00778.JPG \\
\hline 838 & Chamaecytisus supinus (L.) Link & Tihany & 1959 & Felföldy Lajos & BPU-00792.JPG \\
\hline 838 & Chamaecytisus supinus (L.) Link & Tihany & 1959 & Felföldy Lajos & BPU-00793.JPG \\
\hline
\end{tabular}


1. Elektroniukus melléklet - Electronic Appendix 1.

\begin{tabular}{|c|c|c|c|c|c|}
\hline $\begin{array}{l}\text { Sorszám / } \\
\text { Number }\end{array}$ & Taxon-név / Taxon-name & $\begin{array}{l}\text { Település / } \\
\text { Settlement }\end{array}$ & $\begin{array}{l}\text { Év / } \\
\text { Year }\end{array}$ & Gyűjtő / Collector & $\begin{array}{l}\text { Fájlnév / } \\
\text { File-name }\end{array}$ \\
\hline 838 & Chamaecytisus supinus (L.) Link & Uzsa & 1952 & Borsos Olga & BPU-00794.JPG \\
\hline 839 & $\begin{array}{l}\text { Chamaecytisus virescens (Kováts ex } \\
\text { Neilr.) Dostál }\end{array}$ & Budapest & 1982 & Felföldy Lajos & BPU-00807.JPG \\
\hline 839 & $\begin{array}{l}\text { Chamaecytisus virescens (Kováts ex } \\
\text { Neilr.) Dostál }\end{array}$ & Budapest & 1982 & Felföldy Lajos & BPU-00808.JPG \\
\hline 839 & $\begin{array}{l}\text { Chamaecytisus virescens (Kováts ex } \\
\text { Neilr.) Dostál }\end{array}$ & Kunágota & 1957 & Boros Ádám & BPU-00803.JPG \\
\hline 840 & $\begin{array}{l}\text { Chamaecytisus rochelii (Wierzb.) } \\
\text { Rothm. }\end{array}$ & Debrecen & 1917 & Rapaics Rajmund & BPU-00797.JPG \\
\hline 840 & $\begin{array}{l}\text { Chamaecytisus rochelii (Wierzb.) } \\
\text { Rothm. }\end{array}$ & Debrecen & 1933 & Soó Rezső & BPU-00798.JPG \\
\hline 840 & $\begin{array}{l}\text { Chamaecytisus rochelii (Wierzb.) } \\
\text { Rothm. }\end{array}$ & Tokaj & 1936 & Hulják János & BPU-01900.JPG \\
\hline 841 & Chamaecytisus albus (Hacq.) Rothm. & Abaújszántó & 1948 & Soó Rezső & BPU-00799.JPG \\
\hline 841 & Chamaecytisus albus (Hacq.) Rothm. & Boldogkőváralja & 1952 & Boros Ádám & BPU-00802.JPG \\
\hline 841 & Chamaecytisus albus (Hacq.) Rothm. & Jósvafő & 1951 & Soó Rezső, Jakucs Pál & BPU-00801.JPG \\
\hline 841 & Chamaecytisus albus (Hacq.) Rothm. & Miskolc & 1934 & Soó Rezső & BPU-00800.JPG \\
\hline 841 & Chamaecytisus albus (Hacq.) Rothm. & Miskolc & 1936 & Hulják János & BPU-00804.JPG \\
\hline 842 & Chamaecytisus austriacus (L.) Link & Budaörs & 1967 & $\begin{array}{l}\text { Priszter Szaniszló, } \\
\text { Borhidi Attila }\end{array}$ & BPU-00813.JPG \\
\hline 842 & Chamaecytisus austriacus (L.) Link & Budaörs & 1982 & Felföldy Lajos & BPU-00814.JPG \\
\hline 842 & Chamaecytisus austriacus (L.) Link & Budapest & 1923 & Soó Rezső & BPU-00815.JPG \\
\hline 842 & Chamaecytisus austriacus (L.) Link & Budapest & 1947 & Boros Ádám & BPU-00805.JPG \\
\hline 842 & Chamaecytisus austriacus (L.) Link & Budapest & 1991 & Felföldy Lajos & BPU-00806.JPG \\
\hline 842 & Chamaecytisus austriacus (L.) Link & Csákvár & 1955 & Soó Rezső & BPU-00811.JPG \\
\hline 842 & Chamaecytisus austriacus (L.) Link & Csarnóta & 1965 & Simon Tibor & BPU-00809.JPG \\
\hline 842 & Chamaecytisus austriacus (L.) Link & Lábatlan & 1953 & Felföldy Lajos & BPU-00812.JPG \\
\hline 842 & Chamaecytisus austriacus (L.) Link & Nagyharsány & 1961 & Simon Tibor & BPU-00810.JPG \\
\hline 842 & Chamaecytisus austriacus (L.) Link & Tihany & 1947 & Pólya László & BPU-00816.JPG \\
\hline 842 & Chamaecytisus austriacus (L.) Link & Tihany & 1959 & Felföldy Lajos & BPU-00817.JPG \\
\hline 842 & Chamaecytisus austriacus (L.) Link & Tihany & 1959 & Felföldy Lajos & BPU-00818.JPG \\
\hline 842 & Chamaecytisus austriacus (L.) Link & Tihany & 1959 & Felföldy Lajos & BPU-00819.JPG \\
\hline 842 & Chamaecytisus austriacus (L.) Link & Tököl & 1917 & Degen Árpád & BPU-00821.JPG \\
\hline 844 & $\begin{array}{l}\text { Chamaecytisus ratisbonensis } \\
\text { (Schaeff.) Rothm. }\end{array}$ & Bagamér & 1933 & Máthé Imre & BPU-00827.JPG \\
\hline 844 & $\begin{array}{l}\text { Chamaecytisus ratisbonensis } \\
\text { (Schaeff.) Rothm. }\end{array}$ & Balatonfüred & 1959 & Felföldy Lajos & BPU-00837.JPG \\
\hline 844 & $\begin{array}{l}\text { Chamaecytisus ratisbonensis } \\
\text { (Schaeff.) Rothm. }\end{array}$ & Balatonfüred & 1959 & Felföldy Lajos & BPU-00838.JPG \\
\hline 844 & $\begin{array}{l}\text { Chamaecytisus ratisbonensis } \\
\text { (Schaeff.) Rothm. }\end{array}$ & Balatonszőlős & 1958 & Felföldy Lajos & BPU-00831.JPG \\
\hline 844 & $\begin{array}{l}\text { Chamaecytisus ratisbonensis } \\
\text { (Schaeff.) Rothm. }\end{array}$ & Balatonszőlős & 1959 & Felföldy Lajos & BPU-00836.JPG \\
\hline 844 & $\begin{array}{l}\text { Chamaecytisus ratisbonensis } \\
\text { (Schaeff.) Rothm. }\end{array}$ & Bátorliget & 1934 & Soó Rezső & BPU-00830.JPG \\
\hline 844 & $\begin{array}{l}\text { Chamaecytisus ratisbonensis } \\
\text { (Schaeff.) Rothm. }\end{array}$ & Dabas & 1959 & Simon Tibor & BPU-00828.JPG \\
\hline 844 & $\begin{array}{l}\text { Chamaecytisus ratisbonensis } \\
\text { (Schaeff.) Rothm. }\end{array}$ & Debrecen & 1912 & Rapaics Rajmund & BPU-00826.JPG \\
\hline 844 & $\begin{array}{l}\text { Chamaecytisus ratisbonensis } \\
\text { (Schaeff.) Rothm. }\end{array}$ & Fenyőfő & 1955 & Soó Rezső & BPU-00832.JPG \\
\hline 844 & $\begin{array}{l}\text { Chamaecytisus ratisbonensis } \\
\text { (Schaeff.) Rothm. }\end{array}$ & Fenyőfő & 1955 & Soó Rezső & BPU-00833.JPG \\
\hline 844 & $\begin{array}{l}\text { Chamaecytisus ratisbonensis } \\
\text { (Schaeff.) Rothm. }\end{array}$ & Gánt & 1949 & Soó Rezső & BPU-00824.JPG \\
\hline
\end{tabular}


1. Elektroniukus melléklet - Electronic Appendix 1.

\begin{tabular}{|c|c|c|c|c|c|}
\hline $\begin{array}{l}\text { Sorszám / } \\
\text { Number }\end{array}$ & Taxon-név / Taxon-name & $\begin{array}{l}\text { Település / } \\
\text { Settlement }\end{array}$ & $\begin{array}{l}\text { Év / } \\
\text { Year }\end{array}$ & Gyújtő / Collector & $\begin{array}{l}\text { Fájlnév / } \\
\text { File-name }\end{array}$ \\
\hline 844 & $\begin{array}{l}\text { Chamaecytisus ratisbonensis } \\
\text { (Schaeff.) Rothm. }\end{array}$ & Győr & 1916 & Polgár Sándor & BPU-00825.JPG \\
\hline 844 & $\begin{array}{l}\text { Chamaecytisus ratisbonensis } \\
\text { (Schaeff.) Rothm. }\end{array}$ & Háromhuta & 1960 & Simon Tibor & BPU-00835.JPG \\
\hline 844 & $\begin{array}{l}\text { Chamaecytisus ratisbonensis } \\
\text { (Schaeff.) Rothm. }\end{array}$ & Miskolc & 1938 & Soó Rezső & BPU-00823.JPG \\
\hline 844 & $\begin{array}{l}\text { Chamaecytisus ratisbonensis } \\
\text { (Schaeff.) Rothm. }\end{array}$ & Nagybajom & 1957 & Soó Rezső & BPU-00829.JPG \\
\hline 844 & $\begin{array}{l}\text { Chamaecytisus ratisbonensis } \\
\text { (Schaeff.) Rothm. }\end{array}$ & Uzsa & 1959 & $\begin{array}{l}\text { Jávorka Sándor, Felföldy } \\
\text { Lajos }\end{array}$ & BPU-00834.JPG \\
\hline 845 & $\begin{array}{l}\text { Chamaecytisus ciliatus (Wahlenb.) } \\
\text { Rothm. }\end{array}$ & Bélapátfalva & 1950 & $\begin{array}{l}\text { Simon Tibor, Jakucs Pál, } \\
\text { Kárpáti István }\end{array}$ & BPU-00840.JPG \\
\hline 846 & $\begin{array}{l}\text { Chamaecytisus triflorus (Lam.) } \\
\text { Skalická }\end{array}$ & Budapest & 1953 & Soó Rezső & BPU-15413.JPG \\
\hline 846 & $\begin{array}{l}\text { Chamaecytisus triflorus (Lam.) } \\
\text { Skalická }\end{array}$ & Budapest & 1982 & Felföldy Lajos & BPU-01897.JPG \\
\hline 846 & $\begin{array}{l}\text { Chamaecytisus triflorus (Lam.) } \\
\text { Skalická }\end{array}$ & Gyöngyös & 1964 & Soó Rezső & BPU-01893.JPG \\
\hline 846 & $\begin{array}{l}\text { Chamaecytisus triflorus (Lam.) } \\
\text { Skalická }\end{array}$ & Pilisszentiván & 1913 & Filarszky Nándor & BPU-01894.JPG \\
\hline 846 & $\begin{array}{l}\text { Chamaecytisus triflorus (Lam.) } \\
\text { Skalická }\end{array}$ & Visegrád & 1964 & Soó Rezső, Borsos Olga & BPU-01895.JPG \\
\hline 847 & Genistella sagittalis (L.) Gams & Kőszeg & 1932 & Soó Rezső & BPU-00721.JPG \\
\hline 847 & Genistella sagittalis (L.) Gams & Szakonyfalu & 1955 & Soó Rezső & BPU-00723.JPG \\
\hline 847 & Genistella sagittalis (L.) Gams & Szentgotthárd & 1955 & Soó Rezső & BPU-00722.JPG \\
\hline 848 & Genista germanica L. & Beregdaróc & 1951 & Simon Tibor & BPU-00711.JPG \\
\hline 848 & Genista germanica L. & Gyöngyös & 1961 & Soó Rezső & BPU-00716.JPG \\
\hline 848 & Genista germanica L. & Pécs & 1925 & Soó Rezső & BPU-00715.JPG \\
\hline 848 & Genista germanica L. & Pécs & 1953 & Soó Rezső, Borsos Olga & BPU-00712.JPG \\
\hline 848 & Genista germanica L. & Pusztafalu & 1939 & Soó Rezső & BPU-00713.JPG \\
\hline 848 & Genista germanica L. & Sárospatak & 1949 & Simon Tibor, Jakucs Pál & BPU-00719.JPG \\
\hline 848 & Genista germanica L. & Sátoraljaújhely & 1952 & $\begin{array}{l}\text { Kulcsár Gábor, Vozáry } \\
\text { Elemér }\end{array}$ & BPU-00720.JPG \\
\hline 848 & Genista germanica L. & Somoskőújfalu & 1937 & Balázs Ferenc & BPU-00717.JPG \\
\hline 848 & Genista germanica L. & Sopron & 1937 & Soó Rezső & BPU-00718.JPG \\
\hline 848 & Genista germanica L. & Telkibánya & 1955 & Simon Tibor & BPU-00714.JPG \\
\hline 849 & Genista pilosa L. & Biatorbágy & 1992 & Felföldy Lajos & BPU-00727.JPG \\
\hline 849 & Genista pilosa L. & Gyöngyös & 1953 & Soó Rezső & BPU-00726.JPG \\
\hline 849 & Genista pilosa L. & Miskolc & 1955 & Tóth László & BPU-00725.JPG \\
\hline 849 & Genista pilosa L. & Pécs & 1953 & Soó Rezső, Borsos Olga & BPU-00724.JPG \\
\hline 849 & Genista pilosa L. & Sárospatak & 1938 & Hargitai Zoltán & BPU-00730.JPG \\
\hline 849 & Genista pilosa L. & Sopron & 1937 & Soó Rezső & BPU-00728.JPG \\
\hline 849 & Genista pilosa L. & Sopron & 1960 & Borsos Olga, Simon Tibor & BPU-00729.JPG \\
\hline 850 & Genista ovata Waldst. et Kit. & Cserkút & 1938 & Soó Rezső & BPU-00732.JPG \\
\hline 850 & Genista ovata Waldst. et Kit. & Szentgotthárd & 1955 & Soó Rezső & BPU-00733.JPG \\
\hline 850 & Genista ovata Waldst. et Kit. & Szentgotthárd & 1955 & Soó Rezső & BPU-00734.JPG \\
\hline 851 & Genista tinctoria L. & Abaújszántó & 1938 & Soó Rezső & BPU-00739.JPG \\
\hline 851 & Genista tinctoria L. & Budapest & 1982 & Felföldy Lajos & BPU-00742.JPG \\
\hline 851 & Genista tinctoria L. & Budapest & 1992 & Felföldy Lajos & BPU-00751.JPG \\
\hline 851 & Genista tinctoria L. & Budapest & 2004 & Felföldy Lajos & BPU-00740.JPG \\
\hline 851 & Genista tinctoria L. & Budapest & 2004 & Felföldy Lajos & BPU-00741.JPG \\
\hline 851 & Genista tinctoria L. & Dabas & 1959 & Soó Rezső & BPU-00737.JPG \\
\hline
\end{tabular}


1. Elektroniukus melléklet - Electronic Appendix 1.

\begin{tabular}{|c|c|c|c|c|c|}
\hline $\begin{array}{l}\text { Sorszám / } \\
\text { Number }\end{array}$ & Taxon-név / Taxon-name & $\begin{array}{l}\text { Település / } \\
\text { Settlement }\end{array}$ & $\begin{array}{l}\text { Év / } \\
\text { Year }\end{array}$ & Gyűjtő / Collector & $\begin{array}{l}\text { Fájlnév / } \\
\text { File-name }\end{array}$ \\
\hline 851 & Genista tinctoria L. & Debrecen & 1933 & Soó Rezső & BPU-00745.JPG \\
\hline 851 & Genista tinctoria L. & Magyarlukafa & 1957 & Soó Rezső & BPU-00752.JPG \\
\hline 851 & Genista tinctoria L. & Nagyvisnyó & 1948 & $\begin{array}{l}\text { Simon Tibor, Pólya } \\
\text { László, Jakucs Pál }\end{array}$ & BPU-00744.JPG \\
\hline 851 & Genista tinctoria L. & Nagyvisnyó & 1953 & Soó Rezső & BPU-00738.JPG \\
\hline 851 & Genista tinctoria L. & Pálháza & 1949 & Simon Tibor, Jakucs Pál & BPU-00743.JPG \\
\hline 851 & Genista tinctoria L. & Solymár & 2005 & Felföldy Lajos & BPU-00750.JPG \\
\hline 851 & Genista tinctoria L. & Somoskőújfalu & 1937 & Balázs Ferenc & BPU-00749.JPG \\
\hline 851 & Genista tinctoria L. & Szilvásvárad & 1953 & $\begin{array}{l}\text { Felföldy Lajos, Tóth } \\
\text { László }\end{array}$ & BPU-00747.JPG \\
\hline 851 & Genista tinctoria L. & Tihany & 1956 & Felföldy Lajos & BPU-00746.JPG \\
\hline 852 & Lupinus luteus L. & Debrecen & 1947 & Soó Rezső & BPU-00710.JPG \\
\hline 853 & Lupinus polyphyllus Lindl. & Szilvásvárad & 1952 & Borsos Olga & BPU-00708.JPG \\
\hline 853 & Lupinus polyphyllus Lindl. & Szilvásvárad & 1953 & Soó Rezső & BPU-00707.JPG \\
\hline 860 & Galega officinalis L. & Dömös & 1948 & Soó Rezső & BPU-02419.JPG \\
\hline 861 & Colutea arborescens L. & Budapest & 1947 & Soó Rezső & BPU-02425.JPG \\
\hline 861 & Colutea arborescens L. & Csákvár & 1955 & Soó Rezső & BPU-02423.JPG \\
\hline 861 & Colutea arborescens L. & Nagyharsány & 1956 & Soó Rezső & BPU-02426.JPG \\
\hline 861 & Colutea arborescens L. & Nagyharsány & 1961 & Simon Tibor & BPU-02422.JPG \\
\hline 861 & Colutea arborescens L. & Pécs & 1925 & Soó Rezső & BPU-02421.JPG \\
\hline 861 & Colutea arborescens L. & Szilvásvárad & 1953 & Soó Rezső & BPU-02424.JPG \\
\hline 861 & Colutea arborescens L. & Tihany & 1959 & Felföldy Lajos & BPU-02427.JPG \\
\hline 865 & Astragalus contortuplicatus L. & Tiszaroff & 1938 & Soó Rezső & BPU-02428.JPG \\
\hline 866 & Astragalus exscapus L. & Göd & 1948 & Soó Rezső & BPU-02431.JPG \\
\hline 866 & Astragalus exscapus L. & Göd & 1959 & Simon Tibor & BPU-02430.JPG \\
\hline 866 & Astragalus exscapus L. & Győr & 1959 & $\begin{array}{l}\text { Simon Tibor, Borhidi } \\
\text { Attila }\end{array}$ & BPU-02429.JPG \\
\hline 868 & Astragalus sulcatus L. & Fertőrákos & 1932 & Soó Rezső & BPU-02432.JPG \\
\hline 869 & Astragalus glycyphyllos L. & Abaújalpár & 1948 & Soó Rezső & BPU-02435.JPG \\
\hline 869 & Astragalus glycyphyllos L. & Baktalórántháza & 1932 & Soó Rezső & BPU-02436.JPG \\
\hline 869 & Astragalus glycyphyllos L. & Tihany & 1959 & Felföldy Lajos & BPU-02437.JPG \\
\hline 870 & Astragalus cicer L. & Budapest & 1982 & Felföldy Lajos & BPU-02439.JPG \\
\hline 870 & Astragalus cicer L. & Budapest & 1991 & Felföldy Lajos & BPU-02438.JPG \\
\hline 870 & Astragalus cicer L. & Gárdony & 1982 & Felföldy Lajos & BPU-02441.JPG \\
\hline 870 & Astragalus cicer L. & Hajdúsámson & 1933 & Soó Rezső & BPU-02442.JPG \\
\hline 870 & Astragalus cicer L. & Pécs & 1925 & Soó Rezső & BPU-02440.JPG \\
\hline 871 & Astragalus asper Wulfen & Akasztó & 1961 & Borsos Olga, Simon Tibor & BPU-02454.JPG \\
\hline 871 & Astragalus asper Wulfen & Akasztó & 1964 & Simon Tibor & BPU-02455.JPG \\
\hline 871 & Astragalus asper Wulfen & Dabas & 1951 & Simon Tibor, Borsos Olga & BPU-02457.JPG \\
\hline 871 & Astragalus asper Wulfen & Gárdony & 1982 & Felföldy Lajos & BPU-02456.JPG \\
\hline 872 & Astragalus austriacus Jacq. & Akasztó & 1964 & Simon Tibor & BPU-02443.JPG \\
\hline 872 & Astragalus austriacus Jacq. & Budaörs & 1947 & Soó Rezső & BPU-02447.JPG \\
\hline 872 & Astragalus austriacus Jacq. & Fertőrákos & 1937 & Soó Rezső & BPU-02448.JPG \\
\hline 872 & Astragalus austriacus Jacq. & Hajdúnánás & 1936 & Ujvárosi Miklós & BPU-02446.JPG \\
\hline 872 & Astragalus austriacus Jacq. & Kunadacs & 1964 & Soó Rezső & BPU-02444.JPG \\
\hline 872 & Astragalus austriacus Jacq. & Nagyharsány & 1965 & $\begin{array}{l}\text { Simon Tibor, Priszter } \\
\text { Szaniszló, Borhidi Attila }\end{array}$ & BPU-02445.JPG \\
\hline 872 & Astragalus austriacus Jacq. & Tarcal & 1933 & Soó Rezső & BPU-02452.JPG \\
\hline 872 & Astragalus austriacus Jacq. & Tihany & 1928 & Soó Rezső & BPU-02449.JPG \\
\hline
\end{tabular}


1. Elektroniukus melléklet - Electronic Appendix 1.

\begin{tabular}{|c|c|c|c|c|c|}
\hline $\begin{array}{l}\text { Sorszám / } \\
\text { Number }\end{array}$ & Taxon-név / Taxon-name & $\begin{array}{l}\text { Település / } \\
\text { Settlement }\end{array}$ & $\begin{array}{l}\text { Év / } \\
\text { Year }\end{array}$ & Gyújtő / Collector & $\begin{array}{l}\text { Fájlnév / } \\
\text { File-name }\end{array}$ \\
\hline 872 & Astragalus austriacus Jacq. & Tihany & 1959 & Felföldy Lajos & BPU-02450.JPG \\
\hline 872 & Astragalus austriacus Jacq. & Tihany & 1959 & Felföldy Lajos & BPU-02451.JPG \\
\hline 873 & Astragalus vesicarius L. & Jósvafő & 1953 & Jakucs Pál & BPU-02461.JPG \\
\hline 873 & Astragalus vesicarius L. & Telki & 1947 & Soó Rezső & BPU-02460.JPG \\
\hline 874 & Astragalus onobrychis L. & Budapest & 1982 & Felföldy Lajos & BPU-02464.JPG \\
\hline 874 & Astragalus onobrychis L. & Budapest & 1991 & Felföldy Lajos & BPU-02463.JPG \\
\hline 874 & Astragalus onobrychis L. & Budapest & 2004 & Felföldy Lajos & BPU-02462.JPG \\
\hline 874 & Astragalus onobrychis L. & Mosonmagyaróvár & 1962 & Borsos Olga & BPU-02466.JPG \\
\hline 874 & Astragalus onobrychis L. & Pécs & 1935 & Soó Rezső & BPU-02465.JPG \\
\hline 874 & Astragalus onobrychis L. & Szigetmonostor & 1947 & Soó Rezső & BPU-02467.JPG \\
\hline 874 & Astragalus onobrychis L. & Tihany & 1956 & Felföldy Lajos & BPU-02471.JPG \\
\hline 874 & Astragalus onobrychis L. & Tihany & 1959 & Felföldy Lajos & BPU-02472.JPG \\
\hline 874 & Astragalus onobrychis L. & Tihany & 1959 & Felföldy Lajos & BPU-02473.JPG \\
\hline 874 & Astragalus onobrychis L. & Tihany & 1961 & $\begin{array}{l}\text { Pankov H., Felföldy Lajos, } \\
\text { Sólymosy G. }\end{array}$ & BPU-02468.JPG \\
\hline 874 & Astragalus onobrychis L. & Tihany & 1968 & Priszter Szaniszló & BPU-02469.JPG \\
\hline 874 & Astragalus onobrychis L. & Tihany & 1968 & Priszter Szaniszló & BPU-02470.JPG \\
\hline 875 & Astragalus varius S.G. Gmel. & Csévharaszt & 1959 & Simon Tibor, Borsos Olga & BPU-02476.JPG \\
\hline 875 & Astragalus varius S.G. Gmel. & Kiskunfélegyháza & 1923 & Soó Rezső & BPU-02477.JPG \\
\hline 875 & Astragalus varius S.G. Gmel. & Szigetszentmiklós & 1948 & Kárpáti Zoltán & BPU-02475.JPG \\
\hline 876 & Oxytropis pilosa (L.) DC. & Budapest & 1923 & Soó Rezső & BPU-02483.JPG \\
\hline 877 & Glycyrrhiza echinata L. & Tiszafüred & 1952 & Soó Rezső & BPU-02491.JPG \\
\hline 877 & Glycyrrhiza echinata L. & Tiszafüred & 1952 & Soó Rezső & BPU-15541.JPG \\
\hline 879 & Amorpha fruticosa L. & Bugac & 1964 & Soó Rezső & BPU-02418.JPG \\
\hline 887 & Vicia hirsuta (L.) Gray & Bisse & 1965 & $\begin{array}{l}\text { Simon Tibor, Priszter } \\
\text { Szaniszló }\end{array}$ & BPU-02732.JPG \\
\hline 887 & Vicia hirsuta (L.) Gray & Budapest & 1991 & Felföldy Lajos & BPU-02729.JPG \\
\hline 887 & Vicia hirsuta (L.) Gray & Budapest & 1991 & Felföldy Lajos & BPU-02730.JPG \\
\hline 887 & Vicia hirsuta (L.) Gray & Budapest & 1993 & Felföldy Lajos & BPU-02731.JPG \\
\hline 887 & Vicia hirsuta (L.) Gray & Lábatlan & 1953 & Felföldy Lajos & BPU-02734.JPG \\
\hline 887 & Vicia hirsuta (L.) Gray & Nagyharsány & 1968 & Priszter Szaniszló & BPU-02733.JPG \\
\hline 887 & Vicia hirsuta (L.) Gray & Nyíracsád & 1935 & Soó Rezső & BPU-02735.JPG \\
\hline 887 & Vicia hirsuta (L.) Gray & Tihany & 1958 & Felföldy Lajos & BPU-02736.JPG \\
\hline 887 & Vicia hirsuta (L.) Gray & Tokaj & 1933 & Soó Rezső & BPU-02737.JPG \\
\hline 888 & Vicia tetrasperma (L.) Schreb. & Badacsonytomaj & 1985 & Felföldy Lajos & BPU-02741.JPG \\
\hline 888 & Vicia tetrasperma (L.) Schreb. & Hencida & 1927 & Kovács L. & BPU-02738.JPG \\
\hline 888 & Vicia tetrasperma (L.) Schreb. & Kapolcs & 1959 & $\begin{array}{l}\text { Jávorka Sándor, Felföldy } \\
\text { Lajos, Tóth László }\end{array}$ & BPU-02742.JPG \\
\hline 888 & Vicia tetrasperma (L.) Schreb. & Lábatlan & 1953 & Felföldy Lajos & BPU-02740.JPG \\
\hline 888 & Vicia tetrasperma (L.) Schreb. & Rudabánya & 1936 & Soó Rezső & BPU-02739.JPG \\
\hline 888 & Vicia tetrasperma (L.) Schreb. & Uzsa & 1959 & $\begin{array}{l}\text { Jávorka Sándor, Felföldy } \\
\text { Lajos }\end{array}$ & BPU-02743.JPG \\
\hline 891 & Vicia pisiformis L. & Debrecen & 1951 & Kulcsár Gábor & BPU-02748.JPG \\
\hline 891 & Vicia pisiformis L. & Fót & 1960 & Soó Rezső & BPU-02753.JPG \\
\hline 891 & Vicia pisiformis L. & Kapolcs & 1959 & $\begin{array}{l}\text { Jávorka Sándor, Felföldy } \\
\text { Lajos, Tóth László }\end{array}$ & BPU-02752.JPG \\
\hline 891 & Vicia pisiformis L. & Miskolc & 1951 & Soó Rezső & BPU-02750.JPG \\
\hline 891 & Vicia pisiformis L. & Nagykovácsi & 1947 & Soó Rezső & BPU-02754.JPG \\
\hline 891 & Vicia pisiformis L. & Nyíregyháza & 1933 & Soó Rezső & BPU-02755.JPG \\
\hline 891 & Vicia pisiformis L. & Szilvásvárad & 1953 & Soó Rezső & BPU-02751.JPG \\
\hline
\end{tabular}


1. Elektroniukus melléklet - Electronic Appendix 1.

\begin{tabular}{|c|c|c|c|c|c|}
\hline $\begin{array}{l}\text { Sorszám / } \\
\text { Number }\end{array}$ & Taxon-név / Taxon-name & $\begin{array}{l}\text { Település / } \\
\text { Settlement }\end{array}$ & $\begin{array}{l}\text { Év / } \\
\text { Year }\end{array}$ & Gyújtő / Collector & $\begin{array}{l}\text { Fájlnév / } \\
\text { File-name }\end{array}$ \\
\hline 891 & Vicia pisiformis L. & Tarcal & 1947 & Soó Rezső & BPU-02749.JPG \\
\hline 892 & Vicia dumetorum $\mathrm{L}$. & Bélapátfalva & 1948 & $\begin{array}{l}\text { Simon Tibor, Pólya } \\
\text { László, Jakucs Pál }\end{array}$ & BPU-02758.JPG \\
\hline 892 & Vicia dumetorum L. & Csesznek & 1929 & Soó Rezső & BPU-02761.JPG \\
\hline 892 & Vicia dumetorum L. & Gyöngyös & 1952 & Soó Rezső & BPU-02756.JPG \\
\hline 892 & Vicia dumetorum L. & Gyöngyös & 1953 & Soó Rezső & BPU-02760.JPG \\
\hline 892 & Vicia dumetorum L. & Lesenceistvánd & 1952 & Borsos Olga & BPU-02757.JPG \\
\hline 892 & Vicia dumetorum L. & Miskolc & 1950 & Soó Rezső & BPU-02759.JPG \\
\hline 893 & Vicia sparsiflora Ten. & Budapest & 1947 & Soó Rezső & BPU-02762.JPG \\
\hline 893 & Vicia sparsiflora Ten. & Budapest & 1990 & Felföldy Lajos & BPU-02766.JPG \\
\hline 893 & Vicia sparsiflora Ten. & Háromhuta & 1910 & Hulják János & BPU-02763.JPG \\
\hline 893 & Vicia sparsiflora Ten. & Pomáz & 1940 & Kárpáti Zoltán & BPU-02764.JPG \\
\hline 893 & Vicia sparsiflora Ten. & Sokorópátka & 1913 & Polgár Sándor & BPU-02765.JPG \\
\hline 894 & Vicia cassubica L. & Gyöngyös & 1961 & Soó Rezső & BPU-02767.JPG \\
\hline 894 & Vicia cassubica L. & Hajdúböszörmény & 1933 & Soó Rezső & BPU-02771.JPG \\
\hline 894 & Vicia cassubica L. & Nagyvisnyó & 1953 & Soó Rezső & BPU-02770.JPG \\
\hline 894 & Vicia cassubica L. & Rudabánya & 1936 & Soó Rezső & BPU-02769.JPG \\
\hline 894 & Vicia cassubica L. & Tarpa & 1949 & Soó Rezső & BPU-02772.JPG \\
\hline 894 & Vicia cassubica L. & Uzsa & 1952 & Simon Tibor & BPU-02768.JPG \\
\hline 895 & Vicia biennis L. & Ópusztaszer & 1947 & Timár Lajos & BPU-02773.JPG \\
\hline 895 & Vicia biennis L. & Szolnok & 1968 & Priszter Szaniszló & BPU-02774.JPG \\
\hline 896 & Vicia villosa Roth & Bicske & 1991 & Felföldy Lajos & BPU-02803.JPG \\
\hline 896 & Vicia villosa Roth & Budapest & 1982 & Felföldy Lajos & BPU-02799.JPG \\
\hline 896 & Vicia villosa Roth & Budapest & 1983 & Felföldy Lajos & BPU-02800.JPG \\
\hline 896 & Vicia villosa Roth & Budapest & 1991 & Felföldy Lajos & BPU-02797.JPG \\
\hline 896 & Vicia villosa Roth & Budapest & 1991 & Felföldy Lajos & BPU-02798.JPG \\
\hline 896 & Vicia villosa Roth & Budapest & 2005 & Felföldy Lajos & BPU-02801.JPG \\
\hline 896 & Vicia villosa Roth & Csévharaszt & 1959 & Simon Tibor, Borsos Olga & BPU-02807.JPG \\
\hline 896 & Vicia villosa Roth & Debrecen & 1939 & Soó Rezső & BPU-02805.JPG \\
\hline 896 & Vicia villosa Roth & Győr & 1924 & Polgár Sándor & BPU-02810.JPG \\
\hline 896 & Vicia villosa Roth & Hortobágy & 1931 & Soó Rezső & BPU-02806.JPG \\
\hline 896 & Vicia villosa Roth & Pomáz & 1926 & Lengyel Géza & BPU-02808.JPG \\
\hline 896 & Vicia villosa Roth & Tarpa & 1949 & Soó Rezső & BPU-02802.JPG \\
\hline 896 & Vicia villosa Roth & Tihany & 1959 & Felföldy Lajos & BPU-02809.JPG \\
\hline 896 & Vicia villosa Roth & Zámoly & 1982 & Felföldy Lajos & BPU-02804.JPG \\
\hline 897 & Vicia tenuifolia Roth & Abaújszántó & 1939 & Soó Rezső & BPU-02793.JPG \\
\hline 897 & Vicia tenuifolia Roth & Budapest & 1952 & Baksay Leona & BPU-02788.JPG \\
\hline 897 & Vicia tenuifolia Roth & Budapest & 1953 & Vajda László & BPU-02787.JPG \\
\hline 897 & Vicia tenuifolia Roth & Pécs & 1953 & Soó Rezső, Borsos Olga & BPU-02791.JPG \\
\hline 897 & Vicia tenuifolia Roth & Szarvas & 1949 & Soó Rezső & BPU-02795.JPG \\
\hline 897 & Vicia tenuifolia Roth & Tihany & 1959 & Felföldy Lajos & BPU-02796.JPG \\
\hline 898 & Vicia cracca L. & Budapest & 1947 & Soó Rezső & BPU-02790.JPG \\
\hline 898 & Vicia cracca L. & Budapest & 1992 & Felföldy Lajos & BPU-02777.JPG \\
\hline 898 & Vicia cracca L. & Budapest & 1993 & Felföldy Lajos & BPU-02775.JPG \\
\hline 898 & Vicia cracca L. & Budapest & 2005 & Felföldy Lajos & BPU-02776.JPG \\
\hline 898 & Vicia cracca L. & Csévharaszt & 1953 & Baksay Leona & BPU-02781.JPG \\
\hline 898 & Vicia cracca L. & Debrecen & 1951 & Soó Rezső & BPU-02789.JPG \\
\hline 898 & Vicia cracca L. & Gyöngyös & 1961 & Soó Rezső & BPU-02792.JPG \\
\hline
\end{tabular}


1. Elektroniukus melléklet - Electronic Appendix 1.

\begin{tabular}{|c|c|c|c|c|c|}
\hline $\begin{array}{l}\text { Sorszám / } \\
\text { Number }\end{array}$ & Taxon-név / Taxon-name & $\begin{array}{l}\text { Település / } \\
\text { Settlement }\end{array}$ & $\begin{array}{l}\text { Év / } \\
\text { Year }\end{array}$ & Gyüjtő / Collector & $\begin{array}{l}\text { Fájlnév / } \\
\text { File-name }\end{array}$ \\
\hline 898 & Vicia cracca L. & Hosszúpályi & 1933 & Juhász Lajos & BPU-02778.JPG \\
\hline 898 & Vicia cracca L. & Lábatlan & 1953 & Felföldy Lajos & BPU-02779.JPG \\
\hline 898 & Vicia cracca L. & Nyíregyháza & 1933 & Soó Rezső & BPU-02782.JPG \\
\hline 898 & Vicia cracca L. & Salgótarján & 1936 & Soó Rezső & BPU-02780.JPG \\
\hline 899 & Vicia oroboides Wulfen & Szakonyfalu & 1955 & Soó Rezső & BPU-02812.JPG \\
\hline 899 & Vicia oroboides Wulfen & Szenna & 1957 & Soó Rezső & BPU-02811.JPG \\
\hline 900 & Vicia lathyroides L. & Debrecen & 1933 & Soó Rezső & BPU-02816.JPG \\
\hline 900 & Vicia lathyroides L. & Lábatlan & 1953 & Felföldy Lajos & BPU-02814.JPG \\
\hline 900 & Vicia lathyroides L. & Tihany & 1958 & Felföldy Lajos & BPU-02815.JPG \\
\hline 900 & Vicia lathyroides L. & Vácrátót & 1953 & Felföldy Lajos & BPU-02813.JPG \\
\hline 901 & Vicia pannonica Crantz & Budapest & 1991 & Felföldy Lajos & BPU-02825.JPG \\
\hline 901 & Vicia pannonica Crantz & Pécs & 1925 & Soó Rezső & BPU-02824.JPG \\
\hline 901 & Vicia pannonica Crantz & Tállya & 1938 & Soó Rezső & BPU-02823.JPG \\
\hline 901.2 & $\begin{array}{l}\text { Vicia pannonica Crantz subsp. striata } \\
\text { (M. Bieb.) Nyman }\end{array}$ & Egyek & 1947 & Simon Tibor & BPU-02821.JPG \\
\hline 901.2 & $\begin{array}{l}\text { Vicia pannonica Crantz subsp. striata } \\
\text { (M. Bieb.) Nyman }\end{array}$ & Miskolc & 1929 & Soó Rezső & BPU-02820.JPG \\
\hline 901.2 & $\begin{array}{l}\text { Vicia pannonica Crantz subsp. striata } \\
\text { (M. Bieb.) Nyman }\end{array}$ & Szarvas & 1949 & Soó Rezső & BPU-02822.JPG \\
\hline 903 & Vicia grandiflora Scop. & Badacsonytomaj & 1984 & Felföldy Lajos & BPU-02838.JPG \\
\hline 903 & Vicia grandiflora Scop. & Budapest & 1904 & Kocsis István & BPU-02833.JPG \\
\hline 903 & Vicia grandiflora Scop. & Budapest & 1991 & Felföldy Lajos & BPU-02832.JPG \\
\hline 903 & Vicia grandiflora Scop. & Budapest & 1991 & Felföldy Lajos & BPU-02834.JPG \\
\hline 903 & Vicia grandiflora Scop. & Bükkszentkereszt & 1938 & Soó Rezső & BPU-02836.JPG \\
\hline 903 & Vicia grandiflora Scop. & Debrecen & 1930 & Soó Rezső & BPU-02837.JPG \\
\hline 903 & Vicia grandiflora Scop. & Hajdúbagos & 1935 & Soó Rezső & BPU-02835.JPG \\
\hline 903 & Vicia grandiflora Scop. & Sárospatak & 1951 & Csávás Imre & BPU-02841.JPG \\
\hline 903 & Vicia grandiflora Scop. & Tihany & 1959 & Felföldy Lajos & BPU-02839.JPG \\
\hline 903 & Vicia grandiflora Scop. & Uzsa & 1959 & $\begin{array}{l}\text { Jávorka Sándor, Felföldy } \\
\text { Lajos }\end{array}$ & BPU-02840.JPG \\
\hline 904 & Vicia narbonensis L. & Bélmegyer & 1935 & Soó Rezső & BPU-02818.JPG \\
\hline 904 & Vicia narbonensis L. & Egyek & 1933 & Soó Rezső & BPU-02819.JPG \\
\hline 904 & Vicia narbonensis L. & Nagyharsány & 1956 & Soó Rezső & BPU-02817.JPG \\
\hline 905 & Vicia sepium L. & Debrecen & 1931 & Soó Rezső & BPU-02826.JPG \\
\hline 905 & Vicia sepium L. & Hidegkút & 1958 & Felföldy Lajos & BPU-02828.JPG \\
\hline 905 & Vicia sepium L. & Kapolcs & 1959 & $\begin{array}{l}\text { Jávorka Sándor, Felföldy } \\
\text { Lajos, Tóth László }\end{array}$ & BPU-02830.JPG \\
\hline 905 & Vicia sepium L. & Miskolc & 1948 & Simon Tibor & BPU-02827.JPG \\
\hline 905 & Vicia sepium L. & Tihany & 1958 & Felföldy Lajos & BPU-02829.JPG \\
\hline 907 & Vicia sativa L. & Bátorliget & 1948 & $\begin{array}{l}\text { Simon Tibor, Jakucs Pál, } \\
\text { Böszörményi Zoltán }\end{array}$ & BPU-02850.JPG \\
\hline 907 & Vicia sativa L. & Felsőtelekes & 2005 & Gulyás Pál & BPU-02849.JPG \\
\hline 908 & Vicia angustifolia L. & Budapest & 2004 & Felföldy Lajos & BPU-02844.JPG \\
\hline 908 & Vicia angustifolia L. & Csévharaszt & 1959 & Simon Tibor, Borsos Olga & BPU-02846.JPG \\
\hline 908 & Vicia angustifolia L. & Debrecen & 1933 & Juhász Lajos & BPU-02845.JPG \\
\hline 908 & Vicia angustifolia L. & Uzsa & 1959 & $\begin{array}{l}\text { Jávorka Sándor, Felföldy } \\
\text { Lajos }\end{array}$ & BPU-02847.JPG \\
\hline 909 & Lens culinaris Medik. & Vászoly & 1950 & Felföldy Lajos & BPU-02851.JPG \\
\hline 911 & Lathyrus nissolia L. & Gyula & 1935 & Soó Rezső & BPU-02858.JPG \\
\hline 911 & Lathyrus nissolia L. & Pécs & 1936 & Soó Rezső & BPU-02856.JPG \\
\hline
\end{tabular}


1. Elektroniukus melléklet - Electronic Appendix 1.

\begin{tabular}{|c|c|c|c|c|c|}
\hline $\begin{array}{l}\text { Sorszám / } \\
\text { Number }\end{array}$ & Taxon-név / Taxon-name & $\begin{array}{l}\text { Település / } \\
\text { Settlement }\end{array}$ & $\begin{array}{l}\text { Év / } \\
\text { Year }\end{array}$ & Gyújtő / Collector & $\begin{array}{l}\text { Fájlnév / } \\
\text { File-name }\end{array}$ \\
\hline 911 & Lathyrus nissolia L. & Pécs & 1953 & Soó Rezső, Borsos Olga & BPU-02857.JPG \\
\hline 911 & Lathyrus nissolia L. & Pomáz & 1910 & Kocsis István & BPU-02859.JPG \\
\hline 911 & Lathyrus nissolia L. & Siklós & 1967 & Priszter Szaniszló & BPU-02855.JPG \\
\hline 912 & Lathyrus pratensis L. & Bátorliget & 1932 & Soó Rezső & BPU-02892.JPG \\
\hline 912 & Lathyrus pratensis L. & Budapest & 2005 & Felföldy Lajos & BPU-02890.JPG \\
\hline 912 & Lathyrus pratensis L. & Kőszeg & 1963 & Soó Rezső, Borsos Olga & BPU-02893.JPG \\
\hline 912 & Lathyrus pratensis L. & Tiszafüred & 1953 & Borsos Olga & BPU-02891.JPG \\
\hline 913 & Lathyrus sphaericus Retz. & Nagyharsány & 1956 & Soó Rezső & BPU-02867.JPG \\
\hline 913 & Lathyrus sphaericus Retz. & Pilisszentkereszt & 1932 & Soó Rezső & BPU-02866.JPG \\
\hline 913 & Lathyrus sphaericus Retz. & Tokaj & 1933 & Soó Rezső & BPU-02868.JPG \\
\hline 914 & Lathyrus tuberosus L. & Budapest & 1982 & Felföldy Lajos & BPU-02885.JPG \\
\hline 914 & Lathyrus tuberosus L. & Budapest & 1991 & Felföldy Lajos & BPU-02883.JPG \\
\hline 914 & Lathyrus tuberosus L. & Budapest & 2004 & Felföldy Lajos & BPU-02882.JPG \\
\hline 914 & Lathyrus tuberosus L. & Budapest & 2005 & Felföldy Lajos & BPU-02884.JPG \\
\hline 914 & Lathyrus tuberosus L. & Budapest & 2005 & Felföldy Lajos & BPU-02886.JPG \\
\hline 914 & Lathyrus tuberosus L. & Debrecen & 1931 & Soó Rezső & BPU-02887.JPG \\
\hline 914 & Lathyrus tuberosus L. & Mátraszentimre & 1961 & Soó Rezső & BPU-02888.JPG \\
\hline 914 & Lathyrus tuberosus L. & Tihany & 1947 & Pólya László & BPU-02889.JPG \\
\hline 916 & Lathyrus hirsutus L. & Balatonszepezd & 1958 & Felföldy Lajos & BPU-02864.JPG \\
\hline 916 & Lathyrus hirsutus L. & Nagyharsány & 1961 & Simon Tibor & BPU-02863.JPG \\
\hline 916 & Lathyrus hirsutus L. & Parád & 1939 & Igmándy József & BPU-02860.JPG \\
\hline 916 & Lathyrus hirsutus L. & Püspökladány & 1935 & Soó Rezső & BPU-02861.JPG \\
\hline 916 & Lathyrus hirsutus L. & Rózsafa & 1961 & Békali Mihály & BPU-02862.JPG \\
\hline 919 & Lathyrus palustris L. & Dabas & 1951 & Borsos Olga, Simon Tibor & BPU-02877.JPG \\
\hline 919 & Lathyrus palustris L. & Debrecen & 1931 & Soó Rezső & BPU-02874.JPG \\
\hline 919 & Lathyrus palustris L. & Debrecen & 1951 & Soó Rezső, Simon Tibor & BPU-02873.JPG \\
\hline 919 & Lathyrus palustris L. & Debrecen & 1987 & Felföldy Lajos & BPU-02875.JPG \\
\hline 919 & Lathyrus palustris L. & Debrecen & 1987 & Felföldy Lajos & BPU-02876.JPG \\
\hline 919 & Lathyrus palustris L. & Gelénes & 1951 & $\begin{array}{l}\text { Simon Tibor, Vozáry } \\
\text { Elemér }\end{array}$ & BPU-02871.JPG \\
\hline 919 & Lathyrus palustris L. & Töltéstava & 1933 & Polgár Sándor & BPU-02872.JPG \\
\hline 920 & Lathyrus pisiformis L. & Gánt & 1953 & Vajda László & BPU-02879.JPG \\
\hline 920 & Lathyrus pisiformis L. & Tállya & 1938 & Soó Rezső & BPU-02880.JPG \\
\hline 921 & Lathyrus sylvestris L. & Bisse & 1965 & $\begin{array}{l}\text { Simon Tibor, Priszter } \\
\text { Szaniszló }\end{array}$ & BPU-02902.JPG \\
\hline 921 & Lathyrus sylvestris L. & Csesznek & 1929 & Soó Rezső & BPU-02899.JPG \\
\hline 921 & Lathyrus sylvestris L. & Diósjenő & 1952 & Simon Tibor & BPU-02903.JPG \\
\hline 921 & Lathyrus sylvestris L. & Egyek & 1947 & Soó Rezső & BPU-02898.JPG \\
\hline 921 & Lathyrus sylvestris L. & Hencida & 1939 & Kovács & BPU-02904.JPG \\
\hline 922 & Lathyrus latifolius L. & Budaörs & 1982 & Felföldy Lajos & BPU-02910.JPG \\
\hline 922 & Lathyrus latifolius L. & Budapest & 1982 & Felföldy Lajos & BPU-02908.JPG \\
\hline 922 & Lathyrus latifolius L. & Budapest & 1991 & Felföldy Lajos & BPU-02909.JPG \\
\hline 922 & Lathyrus latifolius L. & Gyöngyös & 1961 & Soó Rezső & BPU-02906.JPG \\
\hline 922 & Lathyrus latifolius L. & Lábatlan & 1953 & Felföldy Lajos & BPU-02912.JPG \\
\hline 922 & Lathyrus latifolius L. & Miskolc & 1937 & Soó Rezső & BPU-02900.JPG \\
\hline 922 & Lathyrus latifolius L. & Nagyharsány & 1961 & Simon Tibor & BPU-02907.JPG \\
\hline 922 & Lathyrus latifolius L. & Szigliget & 1962 & Soó Rezső & BPU-02905.JPG \\
\hline 923 & Lathyrus linifolius (Reichard) Bässler & Kőszeg & 1932 & Soó Rezső & BPU-02924.JPG \\
\hline
\end{tabular}


1. Elektroniukus melléklet - Electronic Appendix 1.

\begin{tabular}{|c|c|c|c|c|c|}
\hline $\begin{array}{l}\text { Sorszám / } \\
\text { Number }\end{array}$ & Taxon-név / Taxon-name & $\begin{array}{l}\text { Település / } \\
\text { Settlement }\end{array}$ & $\begin{array}{l}\text { Év / } \\
\text { Year }\end{array}$ & Gyüjtő / Collector & $\begin{array}{l}\text { Fájlnév / } \\
\text { File-name }\end{array}$ \\
\hline 924 & $\begin{array}{l}\text { Lathyrus transsylvanicus (Spreng.) } \\
\text { Fritsch }\end{array}$ & Miskolc & 1929 & Soó Rezső & BPU-02925.JPG \\
\hline 925 & Lathyrus pallescens (M. Bieb.) K. Koch & Budapest & 1948 & Kárpáti Zoltán & BPU-02913.JPG \\
\hline 926 & Lathyrus lacteus (M. Bieb.) Wissjul. & Budapest & 1989 & Felföldy Lajos & BPU-02916.JPG \\
\hline 926 & Lathyrus lacteus (M. Bieb.) Wissjul. & Budapest & 2005 & Felföldy Lajos & BPU-02917.JPG \\
\hline 926 & Lathyrus lacteus (M. Bieb.) Wissjul. & Gánt & 1949 & Soó Rezső & BPU-02919.JPG \\
\hline 926 & Lathyrus lacteus (M. Bieb.) Wissjul. & Hidegkút & 1958 & Felföldy Lajos & BPU-02920.JPG \\
\hline 926 & Lathyrus lacteus (M. Bieb.) Wissjul. & Pécs & 1953 & Soó Rezső, Borsos Olga & BPU-02918.JPG \\
\hline 926 & Lathyrus lacteus (M. Bieb.) Wissjul. & Tokaj & 1933 & Soó Rezső & BPU-02921.JPG \\
\hline 926 & Lathyrus lacteus (M. Bieb.) Wissjul. & Tolcsva & 1962 & Simon Tibor & BPU-02922.JPG \\
\hline 927 & Lathyrus pannonicus (Jacq.) Garcke & Pápakovácsi & 1952 & Tallós Istvánné & BPU-02914.JPG \\
\hline 927 & Lathyrus pannonicus (Jacq.) Garcke & Tapolca & 1949 & Vajda László & BPU-02915.JPG \\
\hline 928 & Lathyrus niger (L.) Bernh. & Budapest & 1964 & Soó Rezső & BPU-02927.JPG \\
\hline 928 & Lathyrus niger (L.) Bernh. & Budapest & 1982 & Felföldy Lajos & BPU-02928.JPG \\
\hline 928 & Lathyrus niger (L.) Bernh. & Gyöngyös & 1961 & Soó Rezső & BPU-02932.JPG \\
\hline 928 & Lathyrus niger (L.) Bernh. & Hajdúbagos & 1937 & Soó Rezső & BPU-02930.JPG \\
\hline 928 & Lathyrus niger (L.) Bernh. & Isztimér & 1949 & Soó Rezső & BPU-02931.JPG \\
\hline 928 & Lathyrus niger (L.) Bernh. & Kapolcs & 1959 & $\begin{array}{l}\text { Jávorka Sándor, Felföldy } \\
\text { Lajos, Tóth László }\end{array}$ & BPU-02933.JPG \\
\hline 928 & Lathyrus niger (L.) Bernh. & Tállya & 1961 & Simon Tibor & BPU-02934.JPG \\
\hline 928 & Lathyrus niger (L.) Bernh. & Tarpa & 1949 & $\begin{array}{l}\text { Soó Rezső, Simon Tibor, } \\
\text { Jakucs Pál }\end{array}$ & BPU-02929.JPG \\
\hline 929 & Lathyrus vernus (L.) Bernh. & Balatonszőlős & 1959 & Felföldy Lajos & BPU-02949.JPG \\
\hline 929 & Lathyrus vernus (L.) Bernh. & Bátorliget & 1938 & Soó Rezső & BPU-02947.JPG \\
\hline 929 & Lathyrus vernus (L.) Bernh. & Beregdaróc & 1951 & $\begin{array}{l}\text { Simon Tibor, Jakucs Pál, } \\
\text { Kulcsár Gábor }\end{array}$ & BPU-02941.JPG \\
\hline 929 & Lathyrus vernus (L.) Bernh. & Bisse & 1965 & Priszter Szaniszló & BPU-02944.JPG \\
\hline 929 & Lathyrus vernus (L.) Bernh. & Csesznek & 1953 & $\begin{array}{l}\text { Kulcsár Gábor, Vozáry } \\
\text { Elemér }\end{array}$ & BPU-02950.JPG \\
\hline 929 & Lathyrus vernus (L.) Bernh. & Csesznek & 1953 & $\begin{array}{l}\text { Kulcsár Gábor, Vozáry } \\
\text { Elemér }\end{array}$ & BPU-02954.JPG \\
\hline 929 & Lathyrus vernus (L.) Bernh. & Gánt & 1949 & Soó Rezső & BPU-02938.JPG \\
\hline 929 & Lathyrus vernus (L.) Bernh. & Gyöngyös & 1961 & Soó Rezső & BPU-02937.JPG \\
\hline 929 & Lathyrus vernus (L.) Bernh. & Gyöngyös & 1964 & Soó Rezső & BPU-02936.JPG \\
\hline 929 & Lathyrus vernus (L.) Bernh. & Hidegkút & 1958 & Felföldy Lajos & BPU-02952.JPG \\
\hline 929 & Lathyrus vernus (L.) Bernh. & Hidegkút & 1959 & Felföldy Lajos & BPU-02951.JPG \\
\hline 929 & Lathyrus vernus (L.) Bernh. & Ivánc & 1955 & Soó Rezső & BPU-02948.JPG \\
\hline 929 & Lathyrus vernus (L.) Bernh. & Kapolcs & 1959 & $\begin{array}{l}\text { Jávorka Sándor, Felföldy } \\
\text { Lajos, Tóth László }\end{array}$ & BPU-02953.JPG \\
\hline 929 & Lathyrus vernus (L.) Bernh. & Lábatlan & 1953 & Felföldy Lajos & BPU-02945.JPG \\
\hline 929 & Lathyrus vernus (L.) Bernh. & Miskolc & 1937 & Soó Rezső & BPU-02939.JPG \\
\hline 929 & Lathyrus vernus (L.) Bernh. & Miskolc & 1937 & Soó Rezső & BPU-02940.JPG \\
\hline 929 & Lathyrus vernus (L.) Bernh. & Pécs & 1953 & Soó Rezső, Borsos Olga & BPU-02943.JPG \\
\hline 929 & Lathyrus vernus (L.) Bernh. & Pécs & 1967 & Priszter Szaniszló & BPU-02942.JPG \\
\hline 929 & Lathyrus vernus (L.) Bernh. & Visegrád & 1964 & Soó Rezső, Borsos Olga & BPU-02946.JPG \\
\hline 930 & Lathyrus venetus (Mill.) Wohlf. & Bakonybél & 1954 & Tallós Pál & BPU-02962.JPG \\
\hline 930 & Lathyrus venetus (Mill.) Wohlf. & Eszteregnye & 1949 & Károlyi Árpád & BPU-02961.JPG \\
\hline 930 & Lathyrus venetus (Mill.) Wohlf. & Kapolcs & 1959 & $\begin{array}{l}\text { Jávorka Sándor, Felföldy } \\
\text { Lajos, Tóth László }\end{array}$ & BPU-02959.JPG \\
\hline 930 & Lathyrus venetus (Mill.) Wohlf. & Pécs & 1936 & Soó Rezső & BPU-02960.JPG \\
\hline 930 & Lathyrus venetus (Mill.) Wohlf. & Szenna & 1957 & Soó Rezső & BPU-02963.JPG \\
\hline
\end{tabular}


1. Elektroniukus melléklet - Electronic Appendix 1.

\begin{tabular}{|c|c|c|c|c|c|}
\hline $\begin{array}{l}\text { Sorszám / } \\
\text { Number }\end{array}$ & Taxon-név / Taxon-name & $\begin{array}{l}\text { Település / } \\
\text { Settlement }\end{array}$ & $\begin{array}{l}\text { Év / } \\
\text { Year }\end{array}$ & Gyűjtő / Collector & $\begin{array}{l}\text { Fájlnév / } \\
\text { File-name }\end{array}$ \\
\hline 931 & Pisum elatius Steven & Bátorliget & 1948 & $\begin{array}{l}\text { Simon Tibor, Jakucs Pál, } \\
\text { Borsos Olga }\end{array}$ & BPU-02979.JPG \\
\hline 931 & Pisum elatius Steven & Gyöngyös & 1900 & Degen Árpád & BPU-02980.JPG \\
\hline 931 & Pisum elatius Steven & Nagyharsány & 1961 & Simon Tibor & BPU-02978.JPG \\
\hline 932 & Pisum sativum L. & Nagyvisnyó & 1953 & Soó Rezső & BPU-02982.JPG \\
\hline 932 & Pisum sativum L. & Váncsod & 1929 & Kovács I. & BPU-02981.JPG \\
\hline 933 & Ononis pusilla L. & Budaörs & 1960 & Priszter Szaniszló & BPU-01903.JPG \\
\hline 933 & Ononis pusilla L. & Budapest & 1947 & Soó Rezső & BPU-01901.JPG \\
\hline 933 & Ononis pusilla L. & Budapest & 1982 & Felföldy Lajos & BPU-01902.JPG \\
\hline 933 & Ononis pusilla L. & Gyenesdiás & 1928 & Soó Rezső & BPU-01905.JPG \\
\hline 933 & Ononis pusilla L. & Tihany & 1961 & $\begin{array}{l}\text { Pankov H., Felföldy Lajos, } \\
\text { Sólymosy G. }\end{array}$ & BPU-01904.JPG \\
\hline 934 & Ononis spinosa L. & Balatonakarattya & 1958 & Rády P. Blanka & BPU-01909.JPG \\
\hline 934 & Ononis spinosa L. & Hajdúnánás & 1935 & Ujvárosi Miklós & BPU-01908.JPG \\
\hline 934 & Ononis spinosa L. & Kunszentmiklós & 1913 & Degen Árpád & BPU-01906.JPG \\
\hline 934 & Ononis spinosa L. & Ócsa & 1953 & Soó Rezső, Borsos Olga & BPU-01907.JPG \\
\hline 934 & Ononis spinosa L. & Zalagyömörő & 1957 & Felföldy Lajos & BPU-01910.JPG \\
\hline 934 & Ononis spinosa L. & Zánka & 1958 & Felföldy Lajos & BPU-01911.JPG \\
\hline 935 & Ononis arvensis L. & Debrecen & 1933 & Soó Rezső & BPU-01916.JPG \\
\hline 935 & Ononis arvensis L. & Hajdúsámson & 1933 & Soó Rezső & BPU-01915.JPG \\
\hline 935 & Ononis arvensis L. & Tarpa & 1948 & Simon Tibor, Jakucs Pál & BPU-01914.JPG \\
\hline 935 & Ononis arvensis L. & Tarpa & 1948 & Simon Tibor, Jakucs Pál & BPU-01919.JPG \\
\hline 935 & Ononis arvensis L. & Vácrátót & 1949 & Soó Rezső & BPU-01913.JPG \\
\hline 936 & Ononis spinosiformis Simonk. & Csaroda & 1951 & $\begin{array}{l}\text { Simon Tibor, Vozáry } \\
\text { Elemér }\end{array}$ & BPU-01920.JPG \\
\hline 936 & Ononis spinosiformis Simonk. & Hajdúnánás & 1935 & Ujvárosi Miklós & BPU-01917.JPG \\
\hline 937 & Melilotus albus Desr. & Nyíregyháza & 1933 & Soó Rezső & BPU-01993.JPG \\
\hline 938 & $\begin{array}{l}\text { Melilotus dentatus (Waldst. et Kit.) } \\
\text { Pers. }\end{array}$ & Balatonkenese & 1982 & Felföldy Lajos & BPU-01984.JPG \\
\hline 938 & $\begin{array}{l}\text { Melilotus dentatus (Waldst. et Kit.) } \\
\text { Pers. }\end{array}$ & Budapest & 1923 & Soó Rezső & BPU-01982.JPG \\
\hline 938 & $\begin{array}{l}\text { Melilotus dentatus (Waldst. et Kit.) } \\
\text { Pers. }\end{array}$ & Budapest & 1993 & Felföldy Lajos & BPU-01983.JPG \\
\hline 938 & $\begin{array}{l}\text { Melilotus dentatus (Waldst. et Kit.) } \\
\text { Pers. }\end{array}$ & Tihany & 1959 & Felföldy Lajos & BPU-01985.JPG \\
\hline 939 & Melilotus altissimus Thuill. & Badacsonytomaj & 1933 & Jávorka Sándor & BPU-01986.JPG \\
\hline 939 & Melilotus altissimus Thuill. & Balatongyörök & 1961 & Felföldy Lajos & BPU-01987.JPG \\
\hline 939 & Melilotus altissimus Thuill. & Budaörs & 1946 & Vajda László & BPU-01988.JPG \\
\hline 940 & Melilotus officinalis (L.) Pall. & Budapest & 2005 & Felföldy Lajos & BPU-01989.JPG \\
\hline 940 & Melilotus officinalis (L.) Pall. & Debrecen & 1937 & Soó Rezső & BPU-01991.JPG \\
\hline 940 & Melilotus officinalis (L.) Pall. & Felsőtelekes & 2005 & Gulyás Pál & BPU-01990.JPG \\
\hline 941 & Trigonella caerulea (L.) Ser. & Budapest & 1923 & Soó Rezső & BPU-01923.JPG \\
\hline 942 & Trigonella procumbens (Besser) Rchb. & Hajdúnánás & 1936 & Ujvárosi Miklós & BPU-01922.JPG \\
\hline 942 & Trigonella procumbens (Besser) Rchb. & Kunpeszér & 1912 & Degen Árpád & BPU-01921.JPG \\
\hline 944 & Trigonella gladiata Steven & Nagyharsány & 1948 & Priszter Szaniszló & BPU-01929.JPG \\
\hline 944 & Trigonella gladiata Steven & Nagyharsány & 1956 & Soó Rezső & BPU-01930.JPG \\
\hline 945 & Medicago sativa L. & Budapest & 1982 & Felföldy Lajos & BPU-01938.JPG \\
\hline 945 & Medicago sativa L. & Budapest & 1982 & Felföldy Lajos & BPU-01939.JPG \\
\hline 945 & Medicago sativa L. & Budapest & 1982 & Felföldy Lajos & BPU-01940.JPG \\
\hline 946 & Medicago $\times$ varia Martyn & Balatonszepezd & 1958 & Felföldy Lajos & BPU-01951.JPG \\
\hline
\end{tabular}


1. Elektroniukus melléklet - Electronic Appendix 1.

\begin{tabular}{|c|c|c|c|c|c|}
\hline $\begin{array}{l}\text { Sorszám / } \\
\text { Number }\end{array}$ & Taxon-név / Taxon-name & $\begin{array}{l}\text { Település / } \\
\text { Settlement }\end{array}$ & $\begin{array}{l}\text { Év / } \\
\text { Year }\end{array}$ & Gyüjtő / Collector & $\begin{array}{l}\text { Fájlnév / } \\
\text { File-name }\end{array}$ \\
\hline 946 & Medicago $\times$ varia Martyn & Budapest & 1947 & Soó Rezső & BPU-01952.JPG \\
\hline 946 & Medicago × varia Martyn & Budapest & 1982 & Felföldy Lajos & BPU-01955.JPG \\
\hline 946 & Medicago $\times$ varia Martyn & Budapest & 1982 & Felföldy Lajos & BPU-01956.JPG \\
\hline 946 & Medicago $\times$ varia Martyn & Budapest & 1982 & Felföldy Lajos & BPU-01958.JPG \\
\hline 946 & Medicago $\times$ varia Martyn & Budapest & 1982 & Felföldy Lajos & BPU-01959.JPG \\
\hline 946 & Medicago $\times$ varia Martyn & Gyöngyös & 1953 & Soó Rezső & BPU-01954.JPG \\
\hline 946 & Medicago $\times$ varia Martyn & Sáránd & 1934 & Soó Rezső & BPU-01953.JPG \\
\hline 946 & Medicago $\times$ varia Martyn & Tarpa & 1949 & Soó Rezső & BPU-01957.JPG \\
\hline 947 & Medicago falcata L. & Budapest & 1982 & Felföldy Lajos & BPU-01942.JPG \\
\hline 947 & Medicago falcata L. & Budapest & 2004 & Felföldy Lajos & BPU-01941.JPG \\
\hline 947 & Medicago falcata L. & Csákvár & 1965 & Simon Tibor & BPU-01943.JPG \\
\hline 947 & Medicago falcata L. & Fót & 1960 & Soó Rezső & BPU-01946.JPG \\
\hline 947 & Medicago falcata L. & Győr & 1938 & Polgár Sándor & BPU-01944.JPG \\
\hline 947 & Medicago falcata L. & Hajdúszoboszló & 1937 & Soó Rezső & BPU-01945.JPG \\
\hline 947 & Medicago falcata L. & Kemence & 1952 & Simon Tibor & BPU-01947.JPG \\
\hline 947 & Medicago falcata L. & Pécsely & 1950 & Felföldy Lajos & BPU-01949.JPG \\
\hline 947 & Medicago falcata L. & Zebegény & 1947 & Soó Rezső & BPU-01948.JPG \\
\hline 948 & Medicago lupulina L. & Balatongyörök & 1961 & Felföldy Lajos & BPU-01937.JPG \\
\hline 948 & Medicago lupulina L. & Budapest & 2005 & Felföldy Lajos & BPU-01931.JPG \\
\hline 948 & Medicago lupulina L. & Budapest & 2005 & Felföldy Lajos & BPU-01932.JPG \\
\hline 948 & Medicago lupulina L. & Debrecen & 1937 & Soó Rezső & BPU-01933.JPG \\
\hline 948 & Medicago lupulina L. & Diósjenő & 1952 & Simon Tibor & BPU-01934.JPG \\
\hline 948 & Medicago lupulina L. & Karcag & 1937 & Oláh J. & BPU-01936.JPG \\
\hline 948 & Medicago lupulina L. & Pilisszentiván & 1969 & Priszter Szaniszló & BPU-01935.JPG \\
\hline 949 & Medicago monspeliaca (L.) Trautv. & Dabas & 1959 & Simon Tibor & BPU-01926.JPG \\
\hline 949 & Medicago monspeliaca (L.) Trautv. & Farmos & 1923 & Soó Rezső & BPU-01926.JPG \\
\hline 949 & Medicago monspeliaca (L.) Trautv. & Nagyharsány & 1965 & $\begin{array}{l}\text { Simon Tibor, Priszter } \\
\text { Szaniszló, Borhidi Attila }\end{array}$ & BPU-01924.JPG \\
\hline 949 & Medicago monspeliaca (L.) Trautv. & Nagyharsány & 1967 & Priszter Szaniszló & BPU-01925.JPG \\
\hline 949 & Medicago monspeliaca (L.) Trautv. & Pilisszentiván & 1953 & Soó Rezső & BPU-01927.JPG \\
\hline 949 & Medicago monspeliaca (L.) Trautv. & Tihany & 1959 & Felföldy Lajos & BPU-01928.JPG \\
\hline 950 & Medicago prostrata Jacq. & Aszófő & 1950 & Felföldy Lajos & BPU-01963.JPG \\
\hline 950 & Medicago prostrata Jacq. & Budapest & 1947 & Soó Rezső & BPU-01960.JPG \\
\hline 950 & Medicago prostrata Jacq. & Füzér & 1937 & Soó Rezső & BPU-01961.JPG \\
\hline 950 & Medicago prostrata Jacq. & Füzér & 1949 & Simon Tibor, Jakucs Pál & BPU-01962.JPG \\
\hline 950 & Medicago prostrata Jacq. & Tihany & 1928 & Soó Rezső & BPU-01964.JPG \\
\hline 952 & Medicago rigidula (L.) All. & Szögliget & 1951 & Jakucs Pál, Ér Lajos & BPU-01967.JPG \\
\hline 952 & Medicago rigidula (L.) All. & Tihany & 1956 & $\begin{array}{l}\text { Felföldy Lajos, Tóth } \\
\text { László }\end{array}$ & BPU-01966.JPG \\
\hline 952 & Medicago rigidula (L.) All. & Verőce & 1923 & Boros Ádám & BPU-01965.JPG \\
\hline 953 & Medicago minima (L.) L. & Debrecen & 1930 & Soó Rezső & BPU-01973.JPG \\
\hline 953 & Medicago minima (L.) L. & Debrecen & 1946 & Soó Rezső & BPU-01974.JPG \\
\hline 953 & Medicago minima (L.) L. & Fenyőfő & 1955 & Soó Rezső & BPU-01978.JPG \\
\hline 953 & Medicago minima (L.) L. & Győr & 1914 & Polgár Sándor & BPU-01972.JPG \\
\hline 953 & Medicago minima (L.) L. & Győr & 1933 & Polgár Sándor & BPU-01971.JPG \\
\hline 953 & Medicago minima (L.) L. & Nagyharsány & 1965 & $\begin{array}{l}\text { Simon Tibor, Priszter } \\
\text { Szaniszló, Borhidi Attila }\end{array}$ & BPU-01969.JPG \\
\hline 953 & Medicago minima (L.) L. & Paks & 1971 & Priszter Szaniszló & BPU-01977.JPG \\
\hline 953 & Medicago minima (L.) L. & Pécs & 1953 & Soó Rezső, Borsos Olga & BPU-01970.JPG \\
\hline
\end{tabular}


1. Elektroniukus melléklet - Electronic Appendix 1.

\begin{tabular}{|c|c|c|c|c|c|}
\hline $\begin{array}{l}\text { Sorszám / } \\
\text { Number }\end{array}$ & Taxon-név / Taxon-name & $\begin{array}{l}\text { Település / } \\
\text { Settlement }\end{array}$ & $\begin{array}{l}\text { Év / } \\
\text { Year }\end{array}$ & Gyüjtő / Collector & $\begin{array}{l}\text { Fájlnév / } \\
\text { File-name }\end{array}$ \\
\hline 953 & Medicago minima (L.) L. & Pilisszentiván & 1953 & Soó Rezső & BPU-01975.JPG \\
\hline 953 & Medicago minima (L.) L. & Tihany & 1942 & Felföldy Lajos & BPU-01980.JPG \\
\hline 953 & Medicago minima (L.) L. & Tihany & 1956 & $\begin{array}{l}\text { Felföldy Lajos, Tóth } \\
\text { László }\end{array}$ & BPU-01979.JPG \\
\hline 953 & Medicago minima (L.) L. & Tihany & 1958 & Felföldy Lajos & BPU-01980.JPG \\
\hline 953 & Medicago minima (L.) L. & Tihany & 1959 & Felföldy Lajos & BPU-01981.JPG \\
\hline 953 & Medicago minima (L.) L. & Vácrátót & 1953 & Felföldy Lajos & BPU-01976.JPG \\
\hline 956 & Trifolium subterraneum L. & Hódmezővásárhely & 1952 & Timár Lajos & BPU-01994.JPG \\
\hline 958 & Trifolium hybridum L. & Budapest & 2005 & Felföldy Lajos & BPU-02528.JPG \\
\hline 958 & Trifolium hybridum L. & Miskolc & 1936 & Soó Rezső & BPU-02529.JPG \\
\hline 959 & Trifolium micranthum Viv. & Kétegyháza & 1923 & Soó Rezső & BPU-02020.JPG \\
\hline 960 & Trifolium patens Schreb. & Balatongyörök & 1961 & Felföldy Lajos & BPU-02029.JPG \\
\hline 960 & Trifolium patens Schreb. & Balatonszőlős & 1957 & $\begin{array}{l}\text { Felföldy Lajos, Kovács } \\
\text { Margit, Tóth László }\end{array}$ & BPU-02026.JPG \\
\hline 960 & Trifolium patens Schreb. & Hévíz & 1961 & Felföldy Lajos & BPU-02030.JPG \\
\hline 960 & Trifolium patens Schreb. & Lesenceistvánd & 1952 & Simon Tibor & BPU-02027.JPG \\
\hline 960 & Trifolium patens Schreb. & Somogyszob & 1957 & Soó Rezső & BPU-02023.JPG \\
\hline 960 & Trifolium patens Schreb. & Szentgotthárd & 1955 & Soó Rezső & BPU-02024.JPG \\
\hline 960 & Trifolium patens Schreb. & Szentgotthárd & 1955 & Soó Rezső & BPU-02025.JPG \\
\hline 960 & Trifolium patens Schreb. & Tihany & 1956 & Felföldy Lajos & BPU-02028.JPG \\
\hline 960 & Trifolium patens Schreb. & Vácrátót & 1950 & Soó Rezső & BPU-02021.JPG \\
\hline 960 & Trifolium patens Schreb. & Vácrátót & 1953 & Felföldy Lajos & BPU-02022.JPG \\
\hline 961 & Trifolium aureum Pollich & Bátorliget & 1948 & $\begin{array}{l}\text { Simon Tibor, Jakucs Pál, } \\
\text { Borsos Olga }\end{array}$ & BPU-02036.JPG \\
\hline 961 & Trifolium aureum Pollich & Beregdaróc & 1951 & $\begin{array}{l}\text { Simon Tibor, Vozáry } \\
\text { Elemér }\end{array}$ & BPU-02032.JPG \\
\hline 961 & Trifolium aureum Pollich & Füzér & 1949 & Simon Tibor, Jakucs Pál & BPU-02031.JPG \\
\hline 961 & Trifolium aureum Pollich & Mátraszentimre & 1961 & Soó Rezső & BPU-02034.JPG \\
\hline 961 & Trifolium aureum Pollich & Solymár & 2005 & Felföldy Lajos & BPU-02035.JPG \\
\hline 961 & Trifolium aureum Pollich & Szilvásvárad & 1952 & Borsos Olga & BPU-02033.JPG \\
\hline 961 & Trifolium aureum Pollich & Tokaj & 1949 & Soó Rezső & BPU-02037.JPG \\
\hline 962 & Trifolium dubium Sibth. & Bodajk & 1934 & Boros Ádám & BPU-02012.JPG \\
\hline 962 & Trifolium dubium Sibth. & Budapest & 1991 & Felföldy Lajos & BPU-02019.JPG \\
\hline 962 & Trifolium dubium Sibth. & Debrecen & 1934 & Soó Rezső & BPU-02015.JPG \\
\hline 962 & Trifolium dubium Sibth. & Kapolcs & 1959 & $\begin{array}{l}\text { Jávorka Sándor, Felföldy } \\
\text { Lajos, Tóth László }\end{array}$ & BPU-02017.JPG \\
\hline 962 & Trifolium dubium Sibth. & Örvényes & 1959 & Felföldy Lajos & BPU-02014.JPG \\
\hline 962 & Trifolium dubium Sibth. & Örvényes & 1959 & Felföldy Lajos & BPU-02016.JPG \\
\hline 962 & Trifolium dubium Sibth. & Uzsa & 1959 & $\begin{array}{l}\text { Jávorka Sándor, Felföldy } \\
\text { Lajos }\end{array}$ & BPU-02018.JPG \\
\hline 962 & Trifolium dubium Sibth. & Vácrátót & 1953 & Felföldy Lajos & BPU-02013.JPG \\
\hline 963 & Trifolium campestre Schreb. & Aszófő & 1959 & Felföldy Lajos & BPU-02054.JPG \\
\hline 963 & Trifolium campestre Schreb. & Badacsonytomaj & 1984 & Felföldy Lajos & BPU-02055.JPG \\
\hline 963 & Trifolium campestre Schreb. & Balatongyörök & 1961 & Felföldy Lajos & BPU-02060.JPG \\
\hline 963 & Trifolium campestre Schreb. & Budapest & 1991 & Felföldy Lajos & BPU-02040.JPG \\
\hline 963 & Trifolium campestre Schreb. & Budapest & 2004 & Felföldy Lajos & BPU-02039.JPG \\
\hline 963 & Trifolium campestre Schreb. & Debrecen & 1930 & Pátkay Imre & BPU-02047.JPG \\
\hline 963 & Trifolium campestre Schreb. & Debrecen & 1947 & Simon Tibor & BPU-02048.JPG \\
\hline 963 & Trifolium campestre Schreb. & Debrecen & 1989 & Felföldy Lajos & BPU-02049.JPG \\
\hline 963 & Trifolium campestre Schreb. & Debrecen & 1989 & Felföldy Lajos & BPU-02050.JPG \\
\hline
\end{tabular}


1. Elektroniukus melléklet - Electronic Appendix 1.

\begin{tabular}{|c|c|c|c|c|c|}
\hline $\begin{array}{l}\text { Sorszám / } \\
\text { Number }\end{array}$ & Taxon-név / Taxon-name & $\begin{array}{l}\text { Település / } \\
\text { Settlement }\end{array}$ & $\begin{array}{l}\text { Év / } \\
\text { Year }\end{array}$ & Gyüjtő / Collector & $\begin{array}{l}\text { Fájlnév / } \\
\text { File-name }\end{array}$ \\
\hline 963 & Trifolium campestre Schreb. & Farmos & 1923 & Soó Rezső & BPU-02052.JPG \\
\hline 963 & Trifolium campestre Schreb. & Gárdony & 1982 & Felföldy Lajos & BPU-02043.JPG \\
\hline 963 & Trifolium campestre Schreb. & Gárdony & 1982 & Felföldy Lajos & BPU-02044.JPG \\
\hline 963 & Trifolium campestre Schreb. & Nagyharsány & 1965 & $\begin{array}{l}\text { Simon Tibor, Priszter } \\
\text { Szaniszló }\end{array}$ & BPU-02041.JPG \\
\hline 963 & Trifolium campestre Schreb. & Nagyharsány & 1968 & Priszter Szaniszló & BPU-02042.JPG \\
\hline 963 & Trifolium campestre Schreb. & Püspökladány & 1935 & Soó Rezső & BPU-02051.JPG \\
\hline 963 & Trifolium campestre Schreb. & Tihany & 1942 & Felföldy Lajos & BPU-02056.JPG \\
\hline 963 & Trifolium campestre Schreb. & Tihany & 1959 & Felföldy Lajos & BPU-02057.JPG \\
\hline 963 & Trifolium campestre Schreb. & Tihany & 1959 & Felföldy Lajos & BPU-02058.JPG \\
\hline 963 & Trifolium campestre Schreb. & Uzsa & 1959 & Felföldy Lajos & BPU-02059.JPG \\
\hline 963 & Trifolium campestre Schreb. & Vácrátót & 1953 & Felföldy Lajos & BPU-02053.JPG \\
\hline 963 & Trifolium campestre Schreb. & Zámoly & 1982 & Felföldy Lajos & BPU-02045.JPG \\
\hline 963 & Trifolium campestre Schreb. & Zámoly & 1982 & Felföldy Lajos & BPU-02046.JPG \\
\hline 964 & Trifolium strictum L. & Hortobágy & 1931 & Soó Rezső & BPU-02063.JPG \\
\hline 964 & Trifolium strictum L. & Kétegyháza & 1923 & Soó Rezső & BPU-02062.JPG \\
\hline 965 & Trifolium repens L. & Budaörs & 1962 & Priszter Szaniszló & BPU-02532.JPG \\
\hline 965 & Trifolium repens L. & Hortobágy & 1947 & Soó Rezső & BPU-02531.JPG \\
\hline 965 & Trifolium repens L. & Tihany & 1946 & Felföldy Lajos & BPU-02533.JPG \\
\hline 966 & Trifolium angulatum Waldst. et Kit. & Balmazújváros & 1930 & Soó Rezső & BPU-02073.JPG \\
\hline 966 & Trifolium angulatum Waldst. et Kit. & Besenyszög & 1969 & Priszter Szaniszló & BPU-02074.JPG \\
\hline 966 & Trifolium angulatum Waldst. et Kit. & Ecsegfalva & 1969 & Priszter Szaniszló & BPU-02071.JPG \\
\hline 966 & Trifolium angulatum Waldst. et Kit. & Kétegyháza & 1923 & Soó Rezső & BPU-02072.JPG \\
\hline 967 & Trifolium montanum L. & Bagamér & 1932 & Soó Rezső & BPU-02008.JPG \\
\hline 967 & Trifolium montanum L. & Budapest & 1982 & Felföldy Lajos & BPU-02004.JPG \\
\hline 967 & Trifolium montanum L. & Budapest & 2004 & Felföldy Lajos & BPU-02002.JPG \\
\hline 967 & Trifolium montanum L. & Budapest & 2005 & Felföldy Lajos & BPU-02003.JPG \\
\hline 967 & Trifolium montanum L. & Felsőtelekes & 2005 & Gulyás Pál & BPU-02005.JPG \\
\hline 967 & Trifolium montanum L. & Hortobágy & 1947 & Soó Rezső & BPU-02007.JPG \\
\hline 967 & Trifolium montanum L. & Lábatlan & 1953 & Felföldy Lajos & BPU-02006.JPG \\
\hline 967 & Trifolium montanum L. & Tihany & 1928 & Soó Rezső & BPU-02010.JPG \\
\hline 967 & Trifolium montanum L. & Tihany & 1959 & Felföldy Lajos & BPU-02011.JPG \\
\hline 967 & Trifolium montanum L. & Zebegény & 1963 & Isépy István & BPU-02009.JPG \\
\hline 968 & Trifolium retusum L. & Debrecen & 1945 & Felföldy Lajos & BPU-02069.JPG \\
\hline 968 & Trifolium retusum L. & Ecsegfalva & 1969 & Priszter Szaniszló & BPU-02064.JPG \\
\hline 968 & Trifolium retusum L. & Egyek & 1950 & $\begin{array}{l}\text { Simon Tibor, Borsos Olga, } \\
\text { Kulcsár Gábor }\end{array}$ & BPU-02065.JPG \\
\hline 968 & Trifolium retusum L. & Egyek & 1950 & $\begin{array}{l}\text { Simon Tibor, Borsos Olga, } \\
\text { Kulcsár Gábor }\end{array}$ & BPU-02066.JPG \\
\hline 968 & Trifolium retusum L. & Hortobágy & 1931 & Soó Rezső & BPU-02070.JPG \\
\hline 968 & Trifolium retusum L. & Kétegyháza & 1923 & Soó Rezső & BPU-02068.JPG \\
\hline 968 & Trifolium retusum $\mathrm{L}$. & Köveskál & 1959 & $\begin{array}{l}\text { Jávorka Sándor, Felföldy } \\
\text { Lajos, Tóth László }\end{array}$ & BPU-02067.JPG \\
\hline 969 & Trifolium vesiculosum Savi & Debrecen & 1950 & Siroki Zoltán & BPU-01999.JPG \\
\hline 970 & Trifolium fragiferum L. & Bugyi & 1950 & Soó Rezső & BPU-01996.JPG \\
\hline 970 & Trifolium fragiferum L. & Nyíregyháza & 1933 & Soó Rezső & BPU-01997.JPG \\
\hline 970 & Trifolium fragiferum $\mathrm{L}$. & Tihany & 1961 & $\begin{array}{l}\text { Pankov H., Felföldy Lajos, } \\
\text { Sólymosy G. }\end{array}$ & BPU-01998.JPG \\
\hline 971 & Trifolium resupinatum L. & Budapest & 1914 & Degen Árpád & BPU-02001.JPG \\
\hline 972 & Trifolium rubens L. & Kerecsend & 1957 & Soó Rezső & BPU-02537.JPG \\
\hline
\end{tabular}


1. Elektroniukus melléklet - Electronic Appendix 1.

\begin{tabular}{|c|c|c|c|c|c|}
\hline $\begin{array}{l}\text { Sorszám / } \\
\text { Number }\end{array}$ & Taxon-név / Taxon-name & $\begin{array}{l}\text { Település / } \\
\text { Settlement }\end{array}$ & $\begin{array}{l}\text { Év / } \\
\text { Year }\end{array}$ & Gyújtő / Collector & $\begin{array}{l}\text { Fájlnév / } \\
\text { File-name }\end{array}$ \\
\hline 972 & Trifolium rubens L. & Kisgyőr & 1938 & Soó Rezső & BPU-02536.JPG \\
\hline 972 & Trifolium rubens L. & Kőszeg & 1932 & Soó Rezső & BPU-02539.JPG \\
\hline 972 & Trifolium rubens L. & Pécs & 1925 & Soó Rezső & BPU-02538.JPG \\
\hline 972 & Trifolium rubens L. & Tarpa & 1949 & Soó Rezső & BPU-02540.JPG \\
\hline 973 & Trifolium alpestre L. & Budaörs & 1947 & Soó Rezső & BPU-02546.JPG \\
\hline 973 & Trifolium alpestre L. & Budapest & 2004 & Felföldy Lajos & BPU-02541.JPG \\
\hline 973 & Trifolium alpestre L. & Debrecen & 1933 & Soó Rezső & BPU-02542.JPG \\
\hline 973 & Trifolium alpestre L. & Debrecen & 1933 & Soó Rezső & BPU-02543.JPG \\
\hline 973 & Trifolium alpestre L. & Gyöngyös & 1961 & Soó Rezső & BPU-02544.JPG \\
\hline 973 & Trifolium alpestre L. & Lábatlan & 1953 & Felföldy Lajos & BPU-02545.JPG \\
\hline 973 & Trifolium alpestre L. & Monostorapáti & 1959 & $\begin{array}{l}\text { Jávorka Sándor, Felföldy } \\
\text { Lajos, Tóth László }\end{array}$ & BPU-02547.JPG \\
\hline 973 & Trifolium alpestre L. & Uzsa & 1959 & $\begin{array}{l}\text { Jávorka Sándor, Felföldy } \\
\text { Lajos }\end{array}$ & BPU-02548.JPG \\
\hline 975 & Trifolium ochroleucon Huds. & Balatonszőlős & 1956 & $\begin{array}{l}\text { Felföldy Lajos, Tóth } \\
\text { László }\end{array}$ & BPU-02554.JPG \\
\hline 975 & Trifolium ochroleucon Huds. & Egyek & 1932 & Soó Rezső & BPU-02550.JPG \\
\hline 975 & Trifolium ochroleucon Huds. & Gyöngyös & 1961 & Soó Rezső & BPU-02549.JPG \\
\hline 975 & Trifolium ochroleucon Huds. & Kelemér & 1950 & Soó Rezső & BPU-02552.JPG \\
\hline 975 & Trifolium ochroleucon Huds. & Tarpa & 1949 & Soó Rezső & BPU-02551.JPG \\
\hline 976 & Trifolium pannonicum Jacq. & Bátorliget & 1948 & $\begin{array}{l}\text { Simon Tibor, Jakucs Pál, } \\
\text { Borsos Olga }\end{array}$ & BPU-02560.JPG \\
\hline 976 & Trifolium pannonicum Jacq. & Gyöngyös & 1953 & Soó Rezső & BPU-02558.JPG \\
\hline 976 & Trifolium pannonicum Jacq. & Gyöngyös & 1962 & Soó Rezső, Borsos Olga & BPU-02556.JPG \\
\hline 976 & Trifolium pannonicum Jacq. & Tokaj & 1949 & Soó Rezső & BPU-02555.JPG \\
\hline 976 & Trifolium pannonicum Jacq. & Zebegény & 1947 & Soó Rezső & BPU-02557.JPG \\
\hline 977 & Trifolium medium L. & Diósjenő & 1952 & Simon Tibor & BPU-02566.JPG \\
\hline 977 & Trifolium medium L. & Gyöngyös & 1953 & Soó Rezső & BPU-02565.JPG \\
\hline 977 & Trifolium medium L. & Gyöngyös & 1961 & Soó Rezső & BPU-02563.JPG \\
\hline 977 & Trifolium medium L. & Jósvafó & 1951 & $\begin{array}{l}\text { Soó Rezső, Jakucs Pál, Ér } \\
\text { Lajos }\end{array}$ & BPU-02562.JPG \\
\hline 977 & Trifolium medium L. & Miskolc & 1937 & Soó Rezső & BPU-02564.JPG \\
\hline 977 & Trifolium medium L. & Nyíracsád & 1949 & Soó Rezső & BPU-02561.JPG \\
\hline 978 & Trifolium pratense L. & Hévíz & 1961 & Felföldy Lajos & BPU-02570.JPG \\
\hline 978 & Trifolium pratense L. & Örvényes & 1959 & Felföldy Lajos & BPU-02569.JPG \\
\hline 978 & Trifolium pratense L. & Szegvár & 1920 & Thaisz Lajos & BPU-02567.JPG \\
\hline 978 & Trifolium pratense L. & Újfehértó & 1936 & Soó Rezső & BPU-02568.JPG \\
\hline 980 & Trifolium striatum L. & Ecsegfalva & 1969 & Priszter Szaniszló & BPU-02574.JPG \\
\hline 980 & Trifolium striatum L. & Hajdúbagos & 1933 & Soó Rezső & BPU-02575.JPG \\
\hline 980 & Trifolium striatum L. & Hortobágy & 1916 & Rapaics Rajmund & BPU-02573.JPG \\
\hline 980 & Trifolium striatum L. & Köveskál & 1959 & $\begin{array}{l}\text { Jávorka Sándor, Felföldy } \\
\text { Lajos, Tóth László }\end{array}$ & BPU-02576.JPG \\
\hline 980 & Trifolium striatum L. & Monostorapáti & 1959 & $\begin{array}{l}\text { Jávorka Sándor, Felföldy } \\
\text { Lajos, Tóth László }\end{array}$ & BPU-02577.JPG \\
\hline 980 & Trifolium striatum L. & Tállya & 1938 & Soó Rezső & BPU-02579.JPG \\
\hline 980 & Trifolium striatum L. & Tihany & 1968 & Priszter Szaniszló & BPU-02578.JPG \\
\hline 981 & Trifolium diffusum Ehrh. & Debrecen & 1949 & Soó Rezső & BPU-02580.JPG \\
\hline 981 & Trifolium diffusum Ehrh. & Hosszúpályi & 1933 & Soó Rezső & BPU-02581.JPG \\
\hline 982 & Trifolium incarnatum L. & Kapolcs & 1959 & $\begin{array}{l}\text { Jávorka Sándor, Felföldy } \\
\text { Lajos, Tóth László }\end{array}$ & BPU-02583.JPG \\
\hline 982 & Trifolium incarnatum L. & Kétegyháza & 1923 & Soó Rezső & BPU-02584.JPG \\
\hline
\end{tabular}


1. Elektroniukus melléklet - Electronic Appendix 1.

\begin{tabular}{|c|c|c|c|c|c|}
\hline $\begin{array}{l}\text { Sorszám / } \\
\text { Number }\end{array}$ & Taxon-név / Taxon-name & $\begin{array}{l}\text { Település / } \\
\text { Settlement }\end{array}$ & $\begin{array}{l}\text { Év / } \\
\text { Year }\end{array}$ & Gyújtő / Collector & $\begin{array}{l}\text { Fájlnév / } \\
\text { File-name }\end{array}$ \\
\hline 982 & Trifolium incarnatum L. & Nyírbátor & 1932 & Soó Rezső & BPU-02582.JPG \\
\hline 983 & Trifolium arvense L. & Budapest & 2004 & Felföldy Lajos & BPU-02585.JPG \\
\hline 983 & Trifolium arvense L. & Debrecen & 1937 & Soó Rezső & BPU-02588.JPG \\
\hline 983 & Trifolium arvense L. & Nagyharsány & 1968 & Priszter Szaniszló & BPU-02586.JPG \\
\hline 983 & Trifolium arvense L. & Pécs & 1938 & Soó Rezső & BPU-02587.JPG \\
\hline 983 & Trifolium arvense L. & Tihany & 1928 & Soó Rezső & BPU-02589.JPG \\
\hline 984 & Dorycnium herbaceum Vill. & Budapest & 1991 & Felföldy Lajos & BPU-02623.JPG \\
\hline 984 & Dorycnium herbaceum Vill. & Diósjenő & 1952 & Simon Tibor & BPU-02629.JPG \\
\hline 984 & Dorycnium herbaceum Vill. & Eger & 1953 & Simon Tibor & BPU-02628.JPG \\
\hline 984 & Dorycnium herbaceum Vill. & Egyek & 1932 & Soó Rezső & BPU-02625.JPG \\
\hline 984 & Dorycnium herbaceum Vill. & Gyöngyös & 1961 & Soó Rezső & BPU-02624.JPG \\
\hline 984 & Dorycnium herbaceum Vill. & Nagyharsány & 1961 & Simon Tibor & BPU-02627.JPG \\
\hline 984 & Dorycnium herbaceum Vill. & Pécs & 1935 & Soó Rezső & BPU-02626.JPG \\
\hline 985 & Dorycnium germanicum (Gremli) Rikli & Budapest & 1947 & Soó Rezső & BPU-02619.JPG \\
\hline 985 & Dorycnium germanicum (Gremli) Rikli & Budapest & 1982 & Felföldy Lajos & BPU-02618.JPG \\
\hline 985 & Dorycnium germanicum (Gremli) Rikli & Budapest & 2004 & Felföldy Lajos & BPU-02617.JPG \\
\hline 985 & Dorycnium germanicum (Gremli) Rikli & Kőszeg & 1932 & Soó Rezső & BPU-02621.JPG \\
\hline 985 & Dorycnium germanicum (Gremli) Rikli & Nagyvisnyó & 1948 & $\begin{array}{l}\text { Simon Tibor, Pólya } \\
\text { László, Jakucs Pál }\end{array}$ & BPU-02620.JPG \\
\hline 985 & Dorycnium germanicum (Gremli) Rikli & Uzsa & 1959 & $\begin{array}{l}\text { Jávorka Sándor, Felföldy } \\
\text { Lajos }\end{array}$ & BPU-02622.JPG \\
\hline 986 & Lotus angustissimus L. & Gáborján & 1934 & Kovács L. & BPU-02639.JPG \\
\hline 986 & Lotus angustissimus L. & Hortobágy & 1953 & Dobos L. & BPU-02638.JPG \\
\hline 988 & Lotus tenuis Waldst. et Kit. & Berzence & 1923 & Boros Ádám & BPU-02723.JPG \\
\hline 988 & Lotus tenuis Waldst. et Kit. & Bicske & 1991 & Felföldy Lajos & BPU-02715.JPG \\
\hline 988 & Lotus tenuis Waldst. et Kit. & Gelénes & 1951 & $\begin{array}{l}\text { Simon Tibor, Vozáry } \\
\text { Elemér }\end{array}$ & BPU-02722.JPG \\
\hline 988 & Lotus tenuis Waldst. et Kit. & Hévíz & 1927 & Boros Ádám & BPU-02724.JPG \\
\hline 988 & Lotus tenuis Waldst. et Kit. & Hortobágy & 1931 & Máthé Imre & BPU-02720.JPG \\
\hline 988 & Lotus tenuis Waldst. et Kit. & Nyíregyháza & 1948 & Soó Rezső & BPU-02717.JPG \\
\hline 988 & Lotus tenuis Waldst. et Kit. & Tatabánya & 1937 & Boros Ádám & BPU-02716.JPG \\
\hline 988 & Lotus tenuis Waldst. et Kit. & Telekes & 1955 & Soó Rezső & BPU-02719.JPG \\
\hline 988 & Lotus tenuis Waldst. et Kit. & Tihany & 1954 & Felföldy Lajos & BPU-02725.JPG \\
\hline 988 & Lotus tenuis Waldst. et Kit. & Uzsa & 1952 & Simon Tibor & BPU-02721.JPG \\
\hline 988 & Lotus tenuis Waldst. et Kit. & Vácrátót & 1949 & Soó Rezső & BPU-02718.JPG \\
\hline 989 & Lotus borbasii Ujhelyi & Nyergesújfalu & 1946 & Boros Ádám & BPU-02728.JPG \\
\hline 990 & Lotus corniculatus L. & Akasztó & 1961 & Borsos Olga, Simon Tibor & BPU-02682.JPG \\
\hline 990 & Lotus corniculatus L. & Bajánsenye & 1956 & Gondola István & BPU-02665.JPG \\
\hline 990 & Lotus corniculatus L. & Bajánsenye & 1956 & Gondola István & BPU-02666.JPG \\
\hline 990 & Lotus corniculatus L. & Balatonszőlős & 1957 & Felföldy Lajos & BPU-02652.JPG \\
\hline 990 & Lotus corniculatus L. & Bánk & 1956 & Gondola István & BPU-02645.JPG \\
\hline 990 & Lotus corniculatus L. & Bánk & 1956 & Gondola István & BPU-02657.JPG \\
\hline 990 & Lotus corniculatus L. & Bánk & 1956 & Gondola István & BPU-02681.JPG \\
\hline 990 & Lotus corniculatus L. & Bánk & 1956 & Gondola István & BPU-02702.JPG \\
\hline 990 & Lotus corniculatus L. & Békéscsaba & 2004 & Kóra Judit & BPU-02726.JPG \\
\hline 990 & Lotus corniculatus L. & Bodajk & 1961 & Gondola István & BPU-02679.JPG \\
\hline 990 & Lotus corniculatus L. & Budaörs & 1947 & Soó Rezső & BPU-02683.JPG \\
\hline 990 & Lotus corniculatus L. & Budapest & 1917 & Boros Ádám & BPU-02676.JPG \\
\hline 990 & Lotus corniculatus L. & Budapest & 1930 & Lengyel Géza & BPU-02694.JPG \\
\hline
\end{tabular}


1. Elektroniukus melléklet - Electronic Appendix 1.

\begin{tabular}{|c|c|c|c|c|c|}
\hline $\begin{array}{l}\text { Sorszám / } \\
\text { Number }\end{array}$ & Taxon-név / Taxon-name & $\begin{array}{l}\text { Település / } \\
\text { Settlement }\end{array}$ & $\begin{array}{l}\text { Év / } \\
\text { Year }\end{array}$ & Gyűjtő / Collector & $\begin{array}{l}\text { Fájlnév / } \\
\text { File-name }\end{array}$ \\
\hline 990 & Lotus corniculatus L. & Budapest & 1956 & Gondola István & BPU-02695.JPG \\
\hline 990 & Lotus corniculatus L. & Budapest & 1957 & Gondola István & BPU-02641.JPG \\
\hline 990 & Lotus corniculatus L. & Budapest & 1982 & Felföldy Lajos & BPU-02640.JPG \\
\hline 990 & Lotus corniculatus L. & Budapest & 1982 & Felföldy Lajos & BPU-02675.JPG \\
\hline 990 & Lotus corniculatus L. & Budapest & 2004 & Felföldy Lajos & BPU-02673.JPG \\
\hline 990 & Lotus corniculatus L. & Budapest & - & Gondola István & BPU-02674.JPG \\
\hline 990 & Lotus corniculatus L. & Csákberény & 1966 & Borhidi Attila & BPU-02680.JPG \\
\hline 990 & Lotus corniculatus L. & Csákvár & 1965 & Simon Tibor & BPU-02700.JPG \\
\hline 990 & Lotus corniculatus L. & Csévharaszt & 1959 & Simon Tibor, Borsos Olga & BPU-02660.JPG \\
\hline 990 & Lotus corniculatus L. & Dabas & 1961 & Borsos Olga, Simon Tibor & BPU-02647.JPG \\
\hline 990 & Lotus corniculatus L. & Dabas & 1961 & Borsos Olga, Simon Tibor & BPU-02661.JPG \\
\hline 990 & Lotus corniculatus L. & Dabas & 1966 & Priszter Szaniszló & BPU-02648.JPG \\
\hline 990 & Lotus corniculatus L. & Debrecen & 1956 & Gondola István & BPU-02655.JPG \\
\hline 990 & Lotus corniculatus L. & Diósjenő & 1956 & Gondola István & BPU-02646.JPG \\
\hline 990 & Lotus corniculatus L. & Diósjenő & 1956 & Gondola István & BPU-02658.JPG \\
\hline 990 & Lotus corniculatus L. & Gödöllő & 1956 & Gondola István & BPU-02684.JPG \\
\hline 990 & Lotus corniculatus L. & Gödöllő & 1956 & Gondola István & BPU-02704.JPG \\
\hline 990 & Lotus corniculatus L. & Gyenesdiás & 1950 & Boros Ádám & BPU-02714.JPG \\
\hline 990 & Lotus corniculatus L. & Gyöngyös & 1962 & Soó Rezső, Borsos Olga & BPU-02644.JPG \\
\hline 990 & Lotus corniculatus L. & Hévízgyörk & 1957 & Gondola István & BPU-02643.JPG \\
\hline 990 & Lotus corniculatus L. & Hidegkút & 1958 & Felföldy Lajos & BPU-02671.JPG \\
\hline 990 & Lotus corniculatus L. & Hosszúpereszteg & 1955 & Soó Rezső & BPU-02667.JPG \\
\hline 990 & Lotus corniculatus L. & Isztimér & 1955 & Soó Rezső & BPU-02691.JPG \\
\hline 990 & Lotus corniculatus L. & Komló & 1956 & Gondola István & BPU-02642.JPG \\
\hline 990 & Lotus corniculatus L. & Körmend & 1956 & Gondola István & BPU-02650.JPG \\
\hline 990 & Lotus corniculatus L. & Körmend & 1956 & Gondola István & BPU-02668.JPG \\
\hline 990 & Lotus corniculatus L. & Kőszeg & 1963 & Soó Rezső, Borsos Olga & BPU-02649.JPG \\
\hline 990 & Lotus corniculatus L. & Lábatlan & 1953 & Felföldy Lajos & BPU-02678.JPG \\
\hline 990 & Lotus corniculatus L. & Ludányhalászi & 1956 & Gondola István & BPU-02659.JPG \\
\hline 990 & Lotus corniculatus L. & Mikepércs & 1947 & Simon Tibor & BPU-02656.JPG \\
\hline 990 & Lotus corniculatus L. & Monor & 1956 & Gondola István & BPU-02662.JPG \\
\hline 990 & Lotus corniculatus L. & Monor & 1956 & Gondola István & BPU-02663.JPG \\
\hline 990 & Lotus corniculatus L. & Monor & 1956 & Gondola István & BPU-02705.JPG \\
\hline 990 & Lotus corniculatus L. & Nógrád & 1956 & Gondola István & BPU-02703.JPG \\
\hline 990 & Lotus corniculatus L. & Őriszentpéter & 1956 & Gondola István & BPU-02669.JPG \\
\hline 990 & Lotus corniculatus L. & Örvényes & 1959 & Felföldy Lajos & BPU-02711.JPG \\
\hline 990 & Lotus corniculatus L. & Pécsely & 1951 & Felföldy Lajos & BPU-02710.JPG \\
\hline 990 & Lotus corniculatus L. & Pilisszentiván & 1961 & Isépy István & BPU-02685.JPG \\
\hline 990 & Lotus corniculatus L. & Pomáz & 1957 & Gondola István & BPU-02664.JPG \\
\hline 990 & Lotus corniculatus L. & Pomáz & 1957 & Gondola István & BPU-02686.JPG \\
\hline 990 & Lotus corniculatus L. & Pomáz & 1957 & Gondola István & BPU-02687.JPG \\
\hline 990 & Lotus corniculatus L. & Pomáz & 1957 & Gondola István & BPU-02688.JPG \\
\hline 990 & Lotus corniculatus L. & Pomáz & 1957 & Gondola István & BPU-02706.JPG \\
\hline 990 & Lotus corniculatus L. & Pomáz & 1957 & Gondola István & BPU-02707.JPG \\
\hline 990 & Lotus corniculatus L. & Püspökladány & 1935 & Soó Rezső & BPU-02701.JPG \\
\hline 990 & Lotus corniculatus L. & Sopron & 1960 & Borsos Olga, Simon Tibor & BPU-02690.JPG \\
\hline 990 & Lotus corniculatus L. & Szalafő & 1956 & Gondola István & BPU-02651.JPG \\
\hline
\end{tabular}


1. Elektroniukus melléklet - Electronic Appendix 1.

\begin{tabular}{|c|c|c|c|c|c|}
\hline $\begin{array}{l}\text { Sorszám / } \\
\text { Number }\end{array}$ & Taxon-név / Taxon-name & $\begin{array}{l}\text { Település / } \\
\text { Settlement }\end{array}$ & $\begin{array}{l}\text { Év / } \\
\text { Year }\end{array}$ & Gyüjtő / Collector & $\begin{array}{l}\text { Fájlnév / } \\
\text { File-name }\end{array}$ \\
\hline 990 & Lotus corniculatus L. & Szalafő & 1956 & Gondola István & BPU-02670.JPG \\
\hline 990 & Lotus corniculatus L. & Szalafő & 1956 & Gondola István & BPU-02709.JPG \\
\hline 990 & Lotus corniculatus L. & Szentgyörgyvölgy & 1955 & Boros Ádám & BPU-02653.JPG \\
\hline 990 & Lotus corniculatus L. & Szigetcsép & 1917 & Boros Ádám & BPU-02689.JPG \\
\hline 990 & Lotus corniculatus L. & Tihany & 1956 & Felföldy Lajos & BPU-02692.JPG \\
\hline 990 & Lotus corniculatus L. & Tihany & 1958 & Felföldy Lajos & BPU-02693.JPG \\
\hline 990 & Lotus corniculatus L. & Tihany & 1959 & Felföldy Lajos & BPU-02712.JPG \\
\hline 990 & Lotus corniculatus L. & Tihany & 1959 & Felföldy Lajos & BPU-02713.JPG \\
\hline 990 & Lotus corniculatus L. & Tiszakeszi & 1956 & Gondola István & BPU-02654.JPG \\
\hline 990 & Lotus corniculatus L. & Tiszakeszi & 1956 & Gondola István & BPU-02677.JPG \\
\hline 990 & Lotus corniculatus L. & Tiszakeszi & 1956 & Gondola István & BPU-02696.JPG \\
\hline 990 & Lotus corniculatus L. & Tiszakeszi & 1956 & Gondola István & BPU-02697.JPG \\
\hline 990 & Lotus corniculatus L. & Tiszakeszi & 1956 & Gondola István & BPU-02698.JPG \\
\hline 990 & Lotus corniculatus L. & Tiszakeszi & 1956 & Gondola István & BPU-02699.JPG \\
\hline 990 & Lotus corniculatus L. & Üröm & 1919 & Boros Ádám & BPU-02708.JPG \\
\hline 991 & Tetragonolobus maritimus (L.) Roth & Budapest & 2005 & Felföldy Lajos & BPU-02630.JPG \\
\hline 991 & Tetragonolobus maritimus (L.) Roth & Konyár & 1949 & Soó Rezső & BPU-02632.JPG \\
\hline 991 & Tetragonolobus maritimus (L.) Roth & Kunszentmiklós & 1968 & Priszter Szaniszló & BPU-02631.JPG \\
\hline 991 & Tetragonolobus maritimus (L.) Roth & Örvényes & 1959 & Felföldy Lajos & BPU-02633.JPG \\
\hline 991 & Tetragonolobus maritimus (L.) Roth & Örvényes & 1959 & Felföldy Lajos & BPU-02634.JPG \\
\hline 991 & Tetragonolobus maritimus (L.) Roth & Tihany & 1947 & Pólya László & BPU-02635.JPG \\
\hline 991 & Tetragonolobus maritimus (L.) Roth & Tihany & 1959 & Felföldy Lajos & BPU-02636.JPG \\
\hline 992 & Securigera varia (L.) Lassen & Bódvarákó & 1951 & Jakucs Pál, Ér Lajos & BPU-02495.JPG \\
\hline 992 & Securigera varia (L.) Lassen & Konyár & 1949 & Soó Rezső & BPU-02496.JPG \\
\hline 993 & Securigera elegans (Pančić) Lassen & Pálháza & 1949 & Vajda László & BPU-02497.JPG \\
\hline 993 & Securigera elegans (Pančić) Lassen & Pálháza & 1952 & Borsos Olga & BPU-02498.JPG \\
\hline 994 & Anthyllis vulneraria L. & Bátorliget & 1950 & $\begin{array}{l}\text { Simon Tibor, Kárpáti } \\
\text { István }\end{array}$ & BPU-02601.JPG \\
\hline 994 & Anthyllis vulneraria L. & Bélapátfalva & 1948 & $\begin{array}{l}\text { Simon Tibor, Pólya } \\
\text { László, Jakucs Pál }\end{array}$ & BPU-02599.JPG \\
\hline 994 & Anthyllis vulneraria L. & Budaörs & 1961 & Borsos Olga, Simon Tibor & BPU-02600.JPG \\
\hline 994 & Anthyllis vulneraria L. & Budapest & 1982 & Felföldy Lajos & BPU-02593.JPG \\
\hline 994 & Anthyllis vulneraria L. & Dorog & 1912 & Degen Árpád & BPU-02606.JPG \\
\hline 994 & Anthyllis vulneraria L. & Felsőtelekes & 2005 & Gulyás Pál & BPU-02596.JPG \\
\hline 994 & Anthyllis vulneraria L. & Hidegkút & 1958 & Felföldy Lajos & BPU-02602.JPG \\
\hline 994 & Anthyllis vulneraria L. & Isztimér & 1949 & Soó Rezső & BPU-02598.JPG \\
\hline 994 & Anthyllis vulneraria L. & Isztimér & 1949 & Soó Rezső & BPU-15427.JPG \\
\hline 994 & Anthyllis vulneraria L. & Isztimér & 1955 & Soó Rezső & BPU-02604.JPG \\
\hline 994 & Anthyllis vulneraria L. & Mátraszentimre & 1961 & Soó Rezső & BPU-02607.JPG \\
\hline 994 & Anthyllis vulneraria L. & Mátraszentimre & 1961 & Soó Rezső & BPU-02609.JPG \\
\hline 994 & Anthyllis vulneraria L. & Miskolc & 1930 & Soó Rezső & BPU-02597.JPG \\
\hline 994 & Anthyllis vulneraria L. & Nagyharsány & 1956 & Soó Rezső & BPU-02594.JPG \\
\hline 994 & Anthyllis vulneraria L. & Nagyharsány & 1961 & Simon Tibor & BPU-02595.JPG \\
\hline 994 & Anthyllis vulneraria L. & Rudabánya & 1936 & Soó Rezső & BPU-02608.JPG \\
\hline 994 & Anthyllis vulneraria L. & Tihany & 1956 & Felföldy Lajos & BPU-02603.JPG \\
\hline 994.3 & $\begin{array}{l}\text { Anthyllis vulneraria L. subsp. alpestris } \\
\text { (Kit.) Asch. et Graebn. }\end{array}$ & Csákvár & 1955 & Soó Rezső & BPU-02610.JPG \\
\hline 994.3 & $\begin{array}{l}\text { Anthyllis vulneraria L. subsp. alpestris } \\
\text { (Kit.) Asch. et Graebn. }\end{array}$ & Gánt & 1968 & Priszter Szaniszló & BPU-02611.JPG \\
\hline
\end{tabular}


1. Elektroniukus melléklet - Electronic Appendix 1.

\begin{tabular}{|c|c|c|c|c|c|}
\hline $\begin{array}{l}\text { Sorszám / } \\
\text { Number }\end{array}$ & Taxon-név / Taxon-name & $\begin{array}{l}\text { Település / } \\
\text { Settlement }\end{array}$ & $\begin{array}{l}\text { Év / } \\
\text { Year }\end{array}$ & Gyüjtő / Collector & $\begin{array}{l}\text { Fájlnév / } \\
\text { File-name }\end{array}$ \\
\hline 996 & Coronilla coronata L. & Budapest & 1952 & Soó Rezső & BPU-02500.JPG \\
\hline 996 & Coronilla coronata L. & Budapest & 1982 & Felföldy Lajos & BPU-02501.JPG \\
\hline 996 & Coronilla coronata L. & Pécs & 1953 & Soó Rezső, Borsos Olga & BPU-02499.JPG \\
\hline 996 & Coronilla coronata L. & Uzsa & 1959 & $\begin{array}{l}\text { Felföldy Lajos, Jávorka } \\
\text { Sándor }\end{array}$ & BPU-02502.JPG \\
\hline 997 & Coronilla vaginalis Lam. & Bodajk & 1955 & Soó Rezső & BPU-02509.JPG \\
\hline 997 & Coronilla vaginalis Lam. & Csákvár & 1955 & Soó Rezső & BPU-02510.JPG \\
\hline 997 & Coronilla vaginalis Lam. & Isztimér & 1932 & Boros Ádám & BPU-02503.JPG \\
\hline 997 & Coronilla vaginalis Lam. & Pilisszentiván & 1935 & Soó Rezső & BPU-02507.JPG \\
\hline 997 & Coronilla vaginalis Lam. & Pilisszentiván & 1953 & Soó Rezső & BPU-02508.JPG \\
\hline 998 & Hippocrepis emerus (L.) Lassen & Hidegkút & 1958 & Felföldy Lajos & BPU-02493.JPG \\
\hline 998 & Hippocrepis emerus (L.) Lassen & Tihany & 1958 & Felföldy Lajos & BPU-02492.JPG \\
\hline 998 & Hippocrepis emerus (L.) Lassen & Vászoly & 1950 & Felföldy Lajos & BPU-02494.JPG \\
\hline 999 & Hippocrepis comosa L. & Budapest & 1947 & Soó Rezső & BPU-02512.JPG \\
\hline 999 & Hippocrepis comosa L. & Tihany & 1946 & Felföldy Lajos & BPU-02513.JPG \\
\hline 999 & Hippocrepis comosa L. & Uzsa & 1959 & $\begin{array}{l}\text { Jávorka Sándor, Felföldy } \\
\text { Lajos }\end{array}$ & BPU-02514.JPG \\
\hline 1000 & Onobrychis arenaria (Kit.) DC. & Budapest & 1912 & Degen Árpád & BPU-02519.JPG \\
\hline 1000 & Onobrychis arenaria (Kit.) DC. & Fót & 1960 & Soó Rezső & BPU-02521.JPG \\
\hline 1000 & Onobrychis arenaria (Kit.) DC. & Tokaj & 1937 & Soó Rezső & BPU-02520.JPG \\
\hline 1000 & Onobrychis arenaria (Kit.) DC. & Vác & 1923 & Soó Rezső & BPU-02522.JPG \\
\hline 1001 & Onobrychis viciifolia Scop. & Konyár & 1949 & Soó Rezső & BPU-02515.JPG \\
\hline 1001 & Onobrychis viciifolia Scop. & Tihany & 1947 & Pólya László & BPU-02516.JPG \\
\hline 1002 & Oxalis acetosella L. & Csesznek & 1929 & Soó Rezső & BPU-04525.JPG \\
\hline 1002 & Oxalis acetosella L. & Gyöngyös & 1964 & Soó Rezső & BPU-04526.JPG \\
\hline 1002 & Oxalis acetosella L. & Miskolc & 1938 & Soó Rezső & BPU-04523.JPG \\
\hline 1002 & Oxalis acetosella L. & Sopron & 1960 & Borsos Olga, Simon Tibor & BPU-04524.JPG \\
\hline 1003 & Oxalis stricta L. & Dávod & 1955 & Soó Rezső & BPU-04531.JPG \\
\hline 1003 & Oxalis stricta L. & Debrecen & 1933 & Soó Rezső & BPU-04529.JPG \\
\hline 1003 & Oxalis stricta L. & Miskolc & 1950 & Soó Rezső & BPU-04528.JPG \\
\hline 1003 & Oxalis stricta L. & Pálháza & 1938 & Soó Rezső & BPU-04527.JPG \\
\hline 1003 & Oxalis stricta L. & Sárvár & 1965 & Tallós Pál & BPU-04532.JPG \\
\hline 1003 & Oxalis stricta L. & Szigliget & 1962 & Soó Rezső & BPU-04533.JPG \\
\hline 1003 & Oxalis stricta L. & Tarpa & 1949 & Soó Rezső & BPU-15567.JPG \\
\hline 1003 & Oxalis stricta L. & Vácrátót & 1949 & Soó Rezső & BPU-04530.JPG \\
\hline 1004 & Oxalis corniculata L. & Budapest & 1947 & Papp József & BPU-04534.JPG \\
\hline 1004 & Oxalis corniculata L. & Budapest & 1982 & Felföldy Lajos & BPU-04535.JPG \\
\hline 1004 & Oxalis corniculata L. & Debrecen & 1935 & Soó Rezső & BPU-04537.JPG \\
\hline 1004 & Oxalis corniculata L. & Kalocsa & 1964 & Soó Rezső & BPU-04536.JPG \\
\hline 1004 & Oxalis corniculata L. & Keszthely & 1965 & Priszter Szaniszló & BPU-04541.JPG \\
\hline 1004 & Oxalis corniculata L. & Sopron & 1951 & Tallós Pál & BPU-04538.JPG \\
\hline 1004 & Oxalis corniculata L. & Szőce & 1969 & Priszter Szaniszló & BPU-04539.JPG \\
\hline 1004 & Oxalis corniculata L. & Ugod & 1956 & Tallós Pál & BPU-04540.JPG \\
\hline 1007 & Geranium robertianum L. & Budapest & 1961 & Isépy István & BPU-04552.JPG \\
\hline 1007 & Geranium robertianum L. & Budapest & 1982 & Felföldy Lajos & BPU-04554.JPG \\
\hline 1007 & Geranium robertianum L. & Budapest & 2004 & Felföldy Lajos & BPU-04553.JPG \\
\hline 1007 & Geranium robertianum L. & Tihany & 1946 & Felföldy Lajos & BPU-04556.JPG \\
\hline 1007 & Geranium robertianum L. & Uzsa & 1959 & $\begin{array}{l}\text { Jávorka Sándor, Felföldy } \\
\text { Lajos }\end{array}$ & BPU-04557.JPG \\
\hline
\end{tabular}


1. Elektroniukus melléklet - Electronic Appendix 1.

\begin{tabular}{|c|c|c|c|c|c|}
\hline $\begin{array}{l}\text { Sorszám / } \\
\text { Number }\end{array}$ & Taxon-név / Taxon-name & $\begin{array}{l}\text { Település / } \\
\text { Settlement }\end{array}$ & $\begin{array}{l}\text { Év / } \\
\text { Year }\end{array}$ & Gyújtő / Collector & $\begin{array}{l}\text { Fájlnév / } \\
\text { File-name }\end{array}$ \\
\hline 1008 & Geranium phaeum L. & "Cuha-völgy" & 1954 & Soó Rezső & BPU-04550.JPG \\
\hline 1008 & Geranium phaeum L. & Bisse & 1965 & $\begin{array}{l}\text { Simon Tibor, Priszter } \\
\text { Szaniszló, Borhidi Attila }\end{array}$ & BPU-04543.JPG \\
\hline 1008 & Geranium phaeum L. & Bodajk & 1955 & Soó Rezső & BPU-04548.JPG \\
\hline 1008 & Geranium phaeum L. & Debrecen & 1939 & Soó Rezső & BPU-04549.JPG \\
\hline 1008 & Geranium phaeum L. & Kőszeg & 1963 & Soó Rezső, Borsos Olga & BPU-04607.JPG \\
\hline 1008 & Geranium phaeum L. & Nagyvisnyó & 1953 & Soó Rezső & BPU-04546.JPG \\
\hline 1008 & Geranium phaeum L. & Pécs & 1936 & Soó Rezső & BPU-04545.JPG \\
\hline 1008 & Geranium phaeum L. & Pécs & 1956 & Soó Rezső & BPU-04544.JPG \\
\hline 1008 & Geranium phaeum L. & Sima & 1961 & Simon Tibor & BPU-04542.JPG \\
\hline 1008 & Geranium phaeum L. & Tokaj & 1933 & Soó Rezső & BPU-04547.JPG \\
\hline 1009 & Geranium sanguineum L. & Balatonszőlős & 1958 & Felföldy Lajos & BPU-04616.JPG \\
\hline 1009 & Geranium sanguineum L. & Bátorliget & 1948 & $\begin{array}{l}\text { Simon Tibor, Jakucs Pál, } \\
\text { Böszörményi Zoltán }\end{array}$ & BPU-04615.JPG \\
\hline 1009 & Geranium sanguineum L. & Budapest & 1947 & Soó Rezső & BPU-04613.JPG \\
\hline 1009 & Geranium sanguineum L. & Budapest & 1953 & Soó Rezső & BPU-15454.JPG \\
\hline 1009 & Geranium sanguineum L. & Budapest & 1961 & Isépy István & BPU-04612.JPG \\
\hline 1009 & Geranium sanguineum L. & Budapest & 2004 & Felföldy Lajos & BPU-04611.JPG \\
\hline 1009 & Geranium sanguineum L. & Lábatlan & 1953 & Felföldy Lajos & BPU-04614.JPG \\
\hline 1010 & Geranium palustre E. Torner & Bózsva & 1950 & Simon Tibor & BPU-04603.JPG \\
\hline 1010 & Geranium palustre E. Torner & Nagyvisnyó & 1950 & Soó Rezső & BPU-04605.JPG \\
\hline 1010 & Geranium palustre E. Torner & Nagyvisnyó & 1953 & Soó Rezső & BPU-04606.JPG \\
\hline 1010 & Geranium palustre E. Torner & Szőce & 1959 & Priszter Szaniszló & BPU-04608.JPG \\
\hline 1010 & Geranium palustre E. Torner & Szögliget & 1950 & Jakucs Pál, Kulcsár Gábor & BPU-04604.JPG \\
\hline 1011 & Geranium pratense L. & Bükkszentkereszt & 1963 & Isépy István & BPU-04624.JPG \\
\hline 1011 & Geranium pratense L. & Pápa & 1950 & Tallós Pál & BPU-04625.JPG \\
\hline 1013 & Geranium lucidum L. & Bélapátfalva & 1932 & Soó Rezső, Máthé Ime & BPU-04560.JPG \\
\hline 1013 & Geranium lucidum L. & Bélapátfalva & 1953 & Simon Tibor & BPU-04562.JPG \\
\hline 1013 & Geranium lucidum L. & Budapest & 1947 & Soó Rezső & BPU-04558.JPG \\
\hline 1013 & Geranium lucidum L. & Lábatlan & 1953 & Felföldy Lajos & BPU-04563.JPG \\
\hline 1013 & Geranium lucidum L. & Tihany & 1947 & Pólya László & BPU-04559.JPG \\
\hline 1013 & Geranium lucidum L. & Vászoly & 1955 & $\begin{array}{l}\text { Felföldy Lajos, Tóth } \\
\text { László }\end{array}$ & BPU-04564.JPG \\
\hline 1014 & Geranium columbinum L. & Bisse & 1965 & $\begin{array}{l}\text { Simon Tibor, Priszter } \\
\text { Szaniszló, Borhidi Attila }\end{array}$ & BPU-04575.JPG \\
\hline 1014 & Geranium columbinum L. & Hajdúsámson & 1933 & Soó Rezső & BPU-04576.JPG \\
\hline 1014 & Geranium columbinum L. & Kemence & 1952 & Simon Tibor & BPU-04574.JPG \\
\hline 1014 & Geranium columbinum L. & Lábatlan & 1953 & Felföldy Lajos & BPU-04578.JPG \\
\hline 1014 & Geranium columbinum L. & Szarvaskő & 1930 & Soó Rezső & BPU-04571.JPG \\
\hline 1014 & Geranium columbinum L. & Tihany & 1956 & Felföldy Lajos & BPU-04577.JPG \\
\hline 1014 & Geranium columbinum L. & Tihany & 1956 & $\begin{array}{l}\text { Felföldy Lajos, Tóth } \\
\text { László }\end{array}$ & BPU-04573.JPG \\
\hline 1014 & Geranium columbinum L. & Uzsa & 1950 & Felföldy Lajos & BPU-04579.JPG \\
\hline 1014 & Geranium columbinum L. & Uzsa & 1959 & $\begin{array}{l}\text { Jávorka Sándor, Felföldy } \\
\text { Lajos }\end{array}$ & BPU-04572.JPG \\
\hline 1015 & Geranium dissectum Jusl. & Uzsa & 1959 & $\begin{array}{l}\text { Jávorka Sándor, Felföldy } \\
\text { Lajos }\end{array}$ & BPU-04588.JPG \\
\hline 1017 & Geranium sibiricum L. & Sopron & 1947 & Kárpáti Zoltán & BPU-04600.JPG \\
\hline 1018 & Geranium divaricatum Ehrh. & Debrecen & 1931 & Soó Rezső & BPU-04566.JPG \\
\hline 1019 & Geranium pyrenaicum Burm. f. & Ásványráró & 1928 & Polgár Sándor & BPU-04599.JPG \\
\hline
\end{tabular}


1. Elektroniukus melléklet - Electronic Appendix 1.

\begin{tabular}{|c|c|c|c|c|c|}
\hline $\begin{array}{l}\text { Sorszám / } \\
\text { Number }\end{array}$ & Taxon-név / Taxon-name & $\begin{array}{l}\text { Település / } \\
\text { Settlement }\end{array}$ & $\begin{array}{l}\text { Év / } \\
\text { Year }\end{array}$ & Gyűjtő / Collector & $\begin{array}{l}\text { Fájlnév / } \\
\text { File-name }\end{array}$ \\
\hline 1020 & Geranium molle L. & Felpéc & 1934 & Polgár Sándor & BPU-04568.JPG \\
\hline 1020 & Geranium molle L. & Nagyharsány & 1936 & Soó Rezső & BPU-04570.JPG \\
\hline 1020 & Geranium molle L. & Tihany & 1958 & Felföldy Lajos & BPU-04567.JPG \\
\hline 1020 & Geranium molle L. & Tihany & 1959 & Felföldy Lajos & BPU-04569.JPG \\
\hline 1021 & Geranium pusillum Burm. f. & Budapest & 1991 & Felföldy Lajos & BPU-04591.JPG \\
\hline 1021 & Geranium pusillum Burm. f. & Debrecen & 1945 & Felföldy Lajos & BPU-04593.JPG \\
\hline 1021 & Geranium pusillum Burm. f. & Hajdúnánás & 1935 & Soó Rezső & BPU-04592.JPG \\
\hline 1021 & Geranium pusillum Burm. f. & Sopron & 1969 & Priszter Szaniszló & BPU-04594.JPG \\
\hline 1021 & Geranium pusillum Burm. f. & Tihany & 1958 & Felföldy Lajos & BPU-04595.JPG \\
\hline 1021 & Geranium pusillum Burm. f. & Tihany & 1959 & Felföldy Lajos & BPU-04596.JPG \\
\hline 1021 & Geranium pusillum Burm. f. & Vászoly & 1958 & Felföldy Lajos & BPU-04597.JPG \\
\hline 1022 & Geranium rotundifolium L. & Lábatlan & 1953 & Felföldy Lajos & BPU-04587.JPG \\
\hline 1022 & Geranium rotundifolium L. & Mór & 1953 & Felföldy Lajos & BPU-04586.JPG \\
\hline 1022 & Geranium rotundifolium L. & Nagyharsány & 1965 & $\begin{array}{l}\text { Simon Tibor, Priszter } \\
\text { Szaniszló }\end{array}$ & BPU-04584.JPG \\
\hline 1022 & Geranium rotundifolium L. & Nagyharsány & 1965 & $\begin{array}{l}\text { Simon Tibor, Priszter } \\
\text { Szaniszló, Borhidi Attila }\end{array}$ & BPU-04583.JPG \\
\hline 1022 & Geranium rotundifolium L. & Nagyvisnyó & 1948 & $\begin{array}{l}\text { Simon Tibor, Pólya } \\
\text { László, Jakucs Pál }\end{array}$ & BPU-04580.JPG \\
\hline 1022 & Geranium rotundifolium L. & Szögliget & 1951 & Soó Rezső, Jakucs Pál & BPU-04590.JPG \\
\hline 1022 & Geranium rotundifolium L. & Tihany & 1928 & Soó Rezső & BPU-04581.JPG \\
\hline 1022 & Geranium rotundifolium L. & Tihany & 1956 & Felföldy Lajos & BPU-04585.JPG \\
\hline 1022 & Geranium rotundifolium L. & Tihany & 1959 & Felföldy Lajos & BPU-04582.JPG \\
\hline 1023 & Erodium cicutarium (L.) L'Hér. & Balatonszőlős & 1958 & Felföldy Lajos & BPU-04633.JPG \\
\hline 1023 & Erodium cicutarium (L.) L’Hér. & Budapest & 1922 & Soó Rezső & BPU-04628.JPG \\
\hline 1023 & Erodium cicutarium (L.) L’Hér. & Budapest & 1961 & Isépy István & BPU-04627.JPG \\
\hline 1023 & Erodium cicutarium (L.) L’Hér. & Gárdony & 1963 & Isépy István & BPU-04630.JPG \\
\hline 1023 & Erodium cicutarium (L.) L’Hér. & Győr & 1937 & Polgár Sándor & BPU-04631.JPG \\
\hline 1023 & Erodium cicutarium (L.) L’Hér. & Pécs & 1953 & Soó Rezső, Borsos Olga & BPU-04629.JPG \\
\hline 1023 & Erodium cicutarium (L.) L’Hér. & Püspökladány & 1991 & Gulyás Pál & BPU-04632.JPG \\
\hline 1024 & Erodium hoefftianum C.A. Mey. & Budapest & 1948 & Soó Rezső & BPU-04636.JPG \\
\hline 1025 & Erodium ciconium (Jusl.) L’Hér. & Budapest & 1932 & Pénzes Antal & BPU-04635.JPG \\
\hline 1025 & Erodium ciconium (Jusl.) L’Hér. & Tihany & 1946 & Felföldy Lajos & BPU-04634.JPG \\
\hline 1026 & Tribulus terrestris L. & Budapest & 1948 & Soó Rezső & BPU-04639.JPG \\
\hline 1026 & Tribulus terrestris L. & Bugac & 1964 & Soó Rezső & BPU-04641.JPG \\
\hline 1026 & Tribulus terrestris $\mathrm{L}$. & Debrecen & 1935 & Soó Rezső & BPU-04638.JPG \\
\hline 1026 & Tribulus terrestris L. & Győr & 1927 & Polgár Sándor & BPU-04637.JPG \\
\hline 1026 & Tribulus terrestris L. & Tihany & 1959 & Felföldy Lajos & BPU-04640.JPG \\
\hline 1027 & Linum catharticum L. & Balatongyörök & 1961 & Felföldy Lajos & BPU-04481.JPG \\
\hline 1027 & Linum catharticum L. & Balatonszőlős & 1956 & $\begin{array}{l}\text { Felföldy Lajos, Tóth } \\
\text { László }\end{array}$ & BPU-04477.JPG \\
\hline 1027 & Linum catharticum L. & Debrecen & 1932 & Soó Rezső & BPU-04475.JPG \\
\hline 1027 & Linum catharticum L. & Füzér & 1938 & Soó Rezső & BPU-04474.JPG \\
\hline 1027 & Linum catharticum L. & Hévíz & 1961 & Felföldy Lajos & BPU-04482.JPG \\
\hline 1027 & Linum catharticum L. & Révfülöp & 1956 & $\begin{array}{l}\text { Felföldy Lajos, Tóth } \\
\text { László }\end{array}$ & BPU-04478.JPG \\
\hline 1027 & Linum catharticum L. & Szilvásvárad & 1953 & $\begin{array}{l}\text { Felföldy Lajos, Tóth } \\
\text { László }\end{array}$ & BPU-04476.JPG \\
\hline 1027 & Linum catharticum L. & Uzsa & 1950 & Felföldy Lajos & BPU-04479.JPG \\
\hline
\end{tabular}


1. Elektroniukus melléklet - Electronic Appendix 1.

\begin{tabular}{|c|c|c|c|c|c|}
\hline $\begin{array}{l}\text { Sorszám / } \\
\text { Number }\end{array}$ & Taxon-név / Taxon-name & $\begin{array}{l}\text { Település / } \\
\text { Settlement }\end{array}$ & $\begin{array}{l}\text { Év / } \\
\text { Year }\end{array}$ & Gyüjtő / Collector & $\begin{array}{l}\text { Fájlnév / } \\
\text { File-name }\end{array}$ \\
\hline 1027 & Linum catharticum L. & Uzsa & 1959 & $\begin{array}{l}\text { Jávorka Sándor, Felföldy } \\
\text { Lajos }\end{array}$ & BPU-04480.JPG \\
\hline 1028 & Linum trigynum L. & Pogányszentpéter & 1948 & Károlyi Árpád & BPU-04495.JPG \\
\hline 1029 & Linum dolomiticum Borbás & Pilisszentiván & 1932 & Soó Rezső & BPU-04485.JPG \\
\hline 1030 & Linum flavum $\mathrm{L}$. & Eger & 1953 & Simon Tibor & BPU-04486.JPG \\
\hline 1030 & Linum flavum L. & Mátraszentimre & 1961 & Soó Rezső & BPU-04487.JPG \\
\hline 1030 & Linum flavum $\mathrm{L}$. & Mátraszentimre & 1963 & Isépy István & BPU-04488.JPG \\
\hline 1030 & Linum flavum L. & Szentendre & 1952 & Vozáry Elemér & BPU-04489.JPG \\
\hline 1030 & Linum flavum L. & Tállya & 1961 & Simon Tibor & BPU-04490.JPG \\
\hline 1030 & Linum flavum L. & Tállya & 1962 & Isépy István & BPU-04491.JPG \\
\hline 1030 & Linum flavum $\mathrm{L}$. & Tokaj & 1947 & Soó Rezső & BPU-04492.JPG \\
\hline 1031 & Linum tenuifolium L. & Budaörs & 1982 & Felföldy Lajos & BPU-04504.JPG \\
\hline 1031 & Linum tenuifolium L. & Budapest & 2004 & Felföldy Lajos & BPU-04502.JPG \\
\hline 1031 & Linum tenuifolium L. & Fót & 1960 & Soó Rezső & BPU-04505.JPG \\
\hline 1031 & Linum tenuifolium L. & Pécs & 1925 & Soó Rezső & BPU-04503.JPG \\
\hline 1031 & Linum tenuifolium L. & Tihany & 1954 & Felföldy Lajos & BPU-04507.JPG \\
\hline 1031 & Linum tenuifolium L. & Tihany & 1959 & Felföldy Lajos & BPU-04506.JPG \\
\hline 1031 & Linum tenuifolium L. & Tihany & 1959 & Felföldy Lajos & BPU-04508.JPG \\
\hline 1032 & Linum hirsutum L. & Abaújszántó & 1948 & Soó Rezső & BPU-04496.JPG \\
\hline 1032 & Linum hirsutum L. & Kiskunfélegyháza & 1923 & Soó Rezső & BPU-04497.JPG \\
\hline 1032 & Linum hirsutum L. & Salgótarján & 1940 & $\begin{array}{l}\text { Ubrizsy Gábor, Felföldy } \\
\text { Lajos, Szűcs Lajos }\end{array}$ & BPU-04499.JPG \\
\hline 1032 & Linum hirsutum L. & Villány & 1971 & Priszter Szaniszló & BPU-04498.JPG \\
\hline 1034 & Linum austriacum L. & "Szentendrei-sziget" & 1953 & Borsos Olga & BPU-04516.JPG \\
\hline 1034 & Linum austriacum L. & Eger & 1930 & Soó Rezső & BPU-04514.JPG \\
\hline 1034 & Linum austriacum L. & Hajdúnánás & 1935 & Soó Rezső & BPU-04513.JPG \\
\hline 1034 & Linum austriacum L. & Pécs & 1936 & Soó Rezső & BPU-04510.JPG \\
\hline 1034 & Linum austriacum L. & Pécs & 1953 & Soó Rezső, Borsos Olga & BPU-04511.JPG \\
\hline 1034 & Linum austriacum L. & Szigetmonostor & 1947 & Soó Rezső & BPU-04515.JPG \\
\hline 1034 & Linum austriacum L. & Zámoly & 1982 & Felföldy Lajos & BPU-04512.JPG \\
\hline 1035 & Linum perenne L. & Dabas & 1959 & Soó Rezső & BPU-04518.JPG \\
\hline 1036 & Radiola linoides Roth & Darány & 1923 & Boros Ádám & BPU-04472.JPG \\
\hline 1036 & Radiola linoides Roth & Szőce & 1955 & Soó Rezső & BPU-04473.JPG \\
\hline 1037 & Mercurialis annua L. & Balatonalmádi & 1963 & Soó Rezső & BPU-04645.JPG \\
\hline 1037 & Mercurialis annua L. & Balatongyörök & 1961 & Felföldy Lajos & BPU-04644.JPG \\
\hline 1037 & Mercurialis annua L. & Dömös & 1948 & Soó Rezső & BPU-04642.JPG \\
\hline 1037 & Mercurialis annua L. & Eger & 1951 & Simon Tibor & BPU-04643.JPG \\
\hline 1038 & Mercurialis perennis L. & Bátorliget & 1934 & Soó Rezső & BPU-04653.JPG \\
\hline 1038 & Mercurialis perennis L. & Gánt & 1949 & Soó Rezső & BPU-04646.JPG \\
\hline 1038 & Mercurialis perennis L. & Gyöngyös & 1964 & Soó Rezső & BPU-04652.JPG \\
\hline 1038 & Mercurialis perennis L. & Tihany & 1956 & Felföldy Lajos & BPU-04647.JPG \\
\hline 1038 & Mercurialis perennis L. & Tihany & 1956 & Felföldy Lajos & BPU-04648.JPG \\
\hline 1038 & Mercurialis perennis L. & Uzsa & 1959 & $\begin{array}{l}\text { Jávorka Sándor, Felföldy } \\
\text { Lajos }\end{array}$ & BPU-04654.JPG \\
\hline 1038 & Mercurialis perennis L. & Zebegény & 1962 & Simon Tibor & BPU-04649.JPG \\
\hline 1039 & Mercurialis ovata Sternb. et Hoppe & Hidegkút & 1959 & Felföldy Lajos & BPU-04655.JPG \\
\hline 1039 & Mercurialis ovata Sternb. et Hoppe & Telki & 1947 & Soó Rezső & BPU-04662.JPG \\
\hline 1039 & Mercurialis ovata Sternb. et Hoppe & Tihany & 1928 & Soó Rezső & BPU-04656.JPG \\
\hline 1039 & Mercurialis ovata Sternb. et Hoppe & Tihany & 1956 & Felföldy Lajos & BPU-04658.JPG \\
\hline
\end{tabular}


1. Elektroniukus melléklet - Electronic Appendix 1.

\begin{tabular}{|c|c|c|c|c|c|}
\hline $\begin{array}{l}\text { Sorszám / } \\
\text { Number }\end{array}$ & Taxon-név / Taxon-name & $\begin{array}{l}\text { Település / } \\
\text { Settlement }\end{array}$ & $\begin{array}{l}\text { Év / } \\
\text { Year }\end{array}$ & Gyűjtő / Collector & $\begin{array}{l}\text { Fájlnév / } \\
\text { File-name }\end{array}$ \\
\hline 1039 & Mercurialis ovata Sternb. et Hoppe & Tihany & 1956 & Felföldy Lajos & BPU-04659.JPG \\
\hline 1039 & Mercurialis ovata Sternb. et Hoppe & Tihany & 1956 & Felföldy Lajos & BPU-04660.JPG \\
\hline 1039 & Mercurialis ovata Sternb. et Hoppe & Tihany & 1957 & Felföldy Lajos & BPU-04657.JPG \\
\hline $\begin{array}{c}1038 \times \\
1039\end{array}$ & $\begin{array}{l}\text { Mercurialis perennis L. } \times \text { Mercurialis } \\
\text { ovata Sternb. et Hoppe }\end{array}$ & Pécsely & 1950 & Felföldy Lajos & BPU-04663.JPG \\
\hline 1042 & Euphorbia maculata L. & Balatonőszöd & 1949 & Károlyi Árpád & BPU-04664.JPG \\
\hline 1042 & Euphorbia maculata L. & Budapest & 1946 & Kárpáti Zoltán & BPU-04665.JPG \\
\hline 1044 & Euphorbia helioscopia L. & Csaroda & 1951 & $\begin{array}{l}\text { Simon Tibor, Vozáry } \\
\text { Elemér }\end{array}$ & BPU-04701.JPG \\
\hline 1044 & Euphorbia helioscopia L. & Pécs & 1953 & Soó Rezső, Borsos Olga & BPU-04702.JPG \\
\hline 1044 & Euphorbia helioscopia L. & Tihany & 1946 & Felföldy Lajos & BPU-04703.JPG \\
\hline 1044 & Euphorbia helioscopia L. & Tihany & 1958 & Felföldy Lajos & BPU-04700.JPG \\
\hline 1044 & Euphorbia helioscopia L. & Tokaj & 1949 & Soó Rezső & BPU-04698.JPG \\
\hline 1044 & Euphorbia helioscopia L. & Vácrátót & 1952 & Felföldy Lajos & BPU-04699.JPG \\
\hline 1045 & Euphorbia platyphyllos L. & Balatonakali & 1950 & Felföldy Lajos & BPU-04670.JPG \\
\hline 1045 & Euphorbia platyphyllos L. & Balatonakali & 1950 & Felföldy Lajos & BPU-04673.JPG \\
\hline 1045 & Euphorbia platyphyllos L. & Füzérkomlós & 1937 & Soó Rezső & BPU-04669.JPG \\
\hline 1045 & Euphorbia platyphyllos L. & Geszt & 1947 & Soó Rezső & BPU-04672.JPG \\
\hline 1046 & Euphorbia stricta L. & Doboz & 1935 & Soó Rezső & BPU-04674.JPG \\
\hline 1047 & Euphorbia villosa Waldst. et Kit. & Beregdaróc & 1951 & Simon Tibor & BPU-04707.JPG \\
\hline 1047 & Euphorbia villosa Waldst. et Kit. & Dabas & 1959 & Soó Rezső & BPU-04709.JPG \\
\hline 1047 & Euphorbia villosa Waldst. et Kit. & Debrecen & 1931 & Soó Rezső & BPU-04706.JPG \\
\hline 1047 & Euphorbia villosa Waldst. et Kit. & Debrecen & 1935 & Soó Rezső & BPU-04704.JPG \\
\hline 1047 & Euphorbia villosa Waldst. et Kit. & Hajdúhadház & 1933 & Soó Rezső & BPU-04705.JPG \\
\hline 1047 & Euphorbia villosa Waldst. et Kit. & Miskolc & 1929 & Soó Rezső & BPU-04708.JPG \\
\hline 1047 & Euphorbia villosa Waldst. et Kit. & Nagyvisnyó & 1953 & Soó Rezső & BPU-04711.JPG \\
\hline 1047 & Euphorbia villosa Waldst. et Kit. & Ócsa & 1953 & Soó Rezső, Borsos Olga & BPU-04710.JPG \\
\hline 1047 & Euphorbia villosa Waldst. et Kit. & Szalonna & 1966 & Felföldy Lajos & BPU-04712.JPG \\
\hline 1048 & Euphorbia seguieriana Neck. & Bátorliget & 1947 & Simon Tibor & BPU-04721.JPG \\
\hline 1048 & Euphorbia seguieriana Neck. & Budaörs & 1947 & Soó Rezső & BPU-04716.JPG \\
\hline 1048 & Euphorbia seguieriana Neck. & Csákvár & 1955 & Soó Rezső & BPU-04713.JPG \\
\hline 1048 & Euphorbia seguieriana Neck. & Csákvár & 1955 & Soó Rezső & BPU-04714.JPG \\
\hline 1048 & Euphorbia seguieriana Neck. & Diósjenő & 1952 & Simon Tibor & BPU-04717.JPG \\
\hline 1048 & Euphorbia seguieriana Neck. & Gánt & 1949 & Soó Rezső & BPU-04715.JPG \\
\hline 1048 & Euphorbia seguieriana Neck. & Isztimér & 1955 & Soó Rezső & BPU-04723.JPG \\
\hline 1048 & Euphorbia seguieriana Neck. & Lábatlan & 1953 & Feichtinger Sándor & BPU-04804.JPG \\
\hline 1048 & Euphorbia seguieriana Neck. & Ócsa & 1923 & Soó Rezső & BPU-04718.JPG \\
\hline 1048 & Euphorbia seguieriana Neck. & Pilisszentiván & 1953 & Soó Rezső & BPU-04719.JPG \\
\hline 1048 & Euphorbia seguieriana Neck. & Szigetmonostor & 1963 & Isépy István & BPU-04720.JPG \\
\hline 1048 & Euphorbia seguieriana Neck. & Tihany & 1947 & Pólya László & BPU-04722.JPG \\
\hline 1048 & Euphorbia seguieriana Neck. & Uzsa & 1953 & $\begin{array}{l}\text { Felföldy Lajos, Tóth } \\
\text { László }\end{array}$ & BPU-04724.JPG \\
\hline 1048 & Euphorbia seguieriana Neck. & Uzsa & 1959 & $\begin{array}{l}\text { Jávorka Sándor, Felföldy } \\
\text { Lajos }\end{array}$ & BPU-04725.JPG \\
\hline 1049 & Euphorbia glareosa Pall. & Budapest & 1947 & Soó Rezső & BPU-04726.JPG \\
\hline 1049 & Euphorbia glareosa Pall. & Budapest & 1952 & Soó Rezső & BPU-15406.JPG \\
\hline 1049 & Euphorbia glareosa Pall. & Budapest & 1982 & Felföldy Lajos & BPU-04728.JPG \\
\hline 1049 & Euphorbia glareosa Pall. & Budapest & 2005 & Felföldy Lajos & BPU-04727.JPG \\
\hline 1049 & Euphorbia glareosa Pall. & Lábatlan & 1953 & Felföldy Lajos & BPU-04729.JPG \\
\hline
\end{tabular}


1. Elektroniukus melléklet - Electronic Appendix 1.

\begin{tabular}{|c|c|c|c|c|c|}
\hline $\begin{array}{l}\text { Sorszám / } \\
\text { Number }\end{array}$ & Taxon-név / Taxon-name & $\begin{array}{l}\text { Település / } \\
\text { Settlement }\end{array}$ & $\begin{array}{l}\text { Év / } \\
\text { Year }\end{array}$ & Gyújtő / Collector & $\begin{array}{l}\text { Fájlnév / } \\
\text { File-name }\end{array}$ \\
\hline 1050 & Euphorbia palustris L. & Debrecen & 1930 & Soó Rezső & BPU-04666.JPG \\
\hline 1050 & Euphorbia palustris L. & Jánossomorja & 1926 & Polgár Sándor & BPU-04667.JPG \\
\hline 1050 & Euphorbia palustris L. & Tihany & 1949 & Simon Tibor & BPU-04671.JPG \\
\hline 1051 & Euphorbia carpatica Woł. & Tarpa & 1949 & Soó Rezső & BPU-04668.JPG \\
\hline 1052 & Euphorbia epithymoides L. & Budapest & 1949 & Soó Rezső & BPU-04676.JPG \\
\hline 1052 & Euphorbia epithymoides L. & Budapest & 1952 & Soó Rezső & BPU-15421.JPG \\
\hline 1052 & Euphorbia epithymoides L. & Cserépfalu & 1954 & Jakucs Pál & BPU-04679.JPG \\
\hline 1052 & Euphorbia epithymoides L. & Gyöngyös & 1965 & Soó Rezső & BPU-04682.JPG \\
\hline 1052 & Euphorbia epithymoides L. & Lábatlan & 1953 & Felföldy Lajos & BPU-04681.JPG \\
\hline 1052 & Euphorbia epithymoides L. & Miskolc & 1939 & Soó Rezső & BPU-04680.JPG \\
\hline 1052 & Euphorbia epithymoides L. & Nagykovácsi & 1963 & Isépy István & BPU-04683.JPG \\
\hline 1052 & Euphorbia epithymoides L. & Pécs & 1953 & Soó Rezső, Borsos Olga & BPU-04677.JPG \\
\hline 1052 & Euphorbia epithymoides L. & Tihany & 1956 & Felföldy Lajos & BPU-04685.JPG \\
\hline 1052 & Euphorbia epithymoides L. & Villány & 1935 & Soó Rezső & BPU-04678.JPG \\
\hline 1052 & Euphorbia epithymoides L. & Visegrád & 1964 & Soó Rezső, Borsos Olga & BPU-04684.JPG \\
\hline 1053 & Euphorbia verrucosa L. & Sopron & 1938 & Soó Rezső & BPU-04688.JPG \\
\hline 1054 & Euphorbia angulata Jacq. & Bakonybél & 1955 & Soó Rezső & BPU-04696.JPG \\
\hline 1054 & Euphorbia angulata Jacq. & Balatonszőlős & 1958 & Felföldy Lajos & BPU-04693.JPG \\
\hline 1054 & Euphorbia angulata Jacq. & Hidegkút & 1958 & Felföldy Lajos & BPU-04694.JPG \\
\hline 1054 & Euphorbia angulata Jacq. & Hidegkút & 1958 & Felföldy Lajos & BPU-04695.JPG \\
\hline 1054 & Euphorbia angulata Jacq. & Sopron & 1938 & Soó Rezső & BPU-04692.JPG \\
\hline 1054 & Euphorbia angulata Jacq. & Sopron & 1960 & Borsos Olga, Simon Tibor & BPU-04697.JPG \\
\hline 1055 & Euphorbia dulcis L. & Alsószölnök & 1955 & Soó Rezső & BPU-04690.JPG \\
\hline 1055 & Euphorbia dulcis L. & Nagykanizsa & 1948 & Károlyi Árpád & BPU-04691.JPG \\
\hline 1055 & Euphorbia dulcis L. & Szenna & 1957 & Soó Rezső & BPU-04689.JPG \\
\hline 1057 & Euphorbia amygdaloides L. & Bátorliget & 1934 & Soó Rezső & BPU-04783.JPG \\
\hline 1057 & Euphorbia amygdaloides L. & Beregdaróc & 1951 & $\begin{array}{l}\text { Simon Tibor, Jakucs Pál, } \\
\text { Kulcsár Gábor }\end{array}$ & BPU-04780.JPG \\
\hline 1057 & Euphorbia amygdaloides L. & Budapest & 1961 & Isépy István & BPU-04776.JPG \\
\hline 1057 & Euphorbia amygdaloides L. & Budapest & 1961 & Isépy István & BPU-04778.JPG \\
\hline 1057 & Euphorbia amygdaloides L. & Budapest & 1992 & Felföldy Lajos & BPU-04777.JPG \\
\hline 1057 & Euphorbia amygdaloides L. & Csesznek & 1953 & $\begin{array}{l}\text { Kulcsár Gábor, Vozáry } \\
\text { Elemér }\end{array}$ & BPU-04786.JPG \\
\hline 1057 & Euphorbia amygdaloides L. & Füzér & 1936 & Soó Rezső & BPU-04787.JPG \\
\hline 1057 & Euphorbia amygdaloides L. & Gánt & 1949 & Soó Rezső & BPU-04781.JPG \\
\hline 1057 & Euphorbia amygdaloides L. & Gyöngyös & 1964 & Soó Rezső & BPU-04782.JPG \\
\hline 1057 & Euphorbia amygdaloides L. & Hidegkút & 1959 & Felföldy Lajos & BPU-04784.JPG \\
\hline 1057 & Euphorbia amygdaloides L. & Uzsa & 1959 & $\begin{array}{l}\text { Jávorka Sándor, Felföldy } \\
\text { Lajos }\end{array}$ & BPU-04785.JPG \\
\hline 1058 & Euphorbia salicifolia Host & Budapest & 1982 & Felföldy Lajos & BPU-04730.JPG \\
\hline 1058 & Euphorbia salicifolia Host & Egyek & 1933 & Soó Rezső & BPU-04732.JPG \\
\hline 1058 & Euphorbia salicifolia Host & Szokolya & 1922 & Soó Rezső & BPU-04731.JPG \\
\hline 1060 & Euphorbia cyparissias L. & Balatonszőlős & 1958 & Felföldy Lajos & BPU-04741.JPG \\
\hline 1060 & Euphorbia cyparissias L. & Debrecen & 1943 & Felföldy Lajos & BPU-04737.JPG \\
\hline 1060 & Euphorbia cyparissias L. & Felsőtelekes & 2005 & Gulyás Pál & BPU-04735.JPG \\
\hline 1060 & Euphorbia cyparissias L. & Hidegkút & 1958 & Felföldy Lajos & BPU-04742.JPG \\
\hline 1060 & Euphorbia cyparissias L. & Nagyharsány & 1965 & $\begin{array}{l}\text { Simon Tibor, Priszter } \\
\text { Szaniszló }\end{array}$ & BPU-04733.JPG \\
\hline 1060 & Euphorbia cyparissias L. & Nagyharsány & 1965 & $\begin{array}{l}\text { Simon Tibor, Priszter } \\
\text { Szaniszló }\end{array}$ & BPU-04734.JPG \\
\hline
\end{tabular}


1. Elektroniukus melléklet - Electronic Appendix 1.

\begin{tabular}{|c|c|c|c|c|c|}
\hline $\begin{array}{l}\text { Sorszám / } \\
\text { Number }\end{array}$ & Taxon-név / Taxon-name & $\begin{array}{l}\text { Település / } \\
\text { Settlement }\end{array}$ & $\begin{array}{l}\text { Év / } \\
\text { Year }\end{array}$ & Gyűjtő / Collector & $\begin{array}{l}\text { Fájlnév / } \\
\text { File-name }\end{array}$ \\
\hline 1060 & Euphorbia cyparissias L. & Nagyvisnyó & 1953 & Vozáry Elemér & BPU-04736.JPG \\
\hline 1060 & Euphorbia cyparissias L. & Nyíradony & 1951 & Simon Tibor & BPU-04740.JPG \\
\hline 1060 & Euphorbia cyparissias L. & Szigetmonostor & 1947 & Soó Rezső & BPU-04738.JPG \\
\hline 1060 & Euphorbia cyparissias L. & Tihany & 1958 & $\begin{array}{l}\text { Felföldy Lajos, Tóth } \\
\text { László }\end{array}$ & BPU-04743.JPG \\
\hline 1060 & Euphorbia cyparissias L. & Vácrátót & 1953 & Felföldy Lajos & BPU-04739.JPG \\
\hline 1061 & Euphorbia esula L. & Aszófő & 1959 & Felföldy Lajos & BPU-04754.JPG \\
\hline 1061 & Euphorbia esula L. & Badacsonytomaj & 1928 & Soó Rezső & BPU-04755.JPG \\
\hline 1061 & Euphorbia esula L. & Baktalórántháza & 1934 & Soó Rezső & BPU-04752.JPG \\
\hline 1061 & Euphorbia esula L. & Balmazújváros & 1932 & Soó Rezső & BPU-04750.JPG \\
\hline 1061 & Euphorbia esula L. & Budapest & 1989 & Felföldy Lajos & BPU-04746.JPG \\
\hline 1061 & Euphorbia esula L. & Budapest & 1991 & Felföldy Lajos & BPU-04747.JPG \\
\hline 1061 & Euphorbia esula L. & Budapest & 2005 & Felföldy Lajos & BPU-04745.JPG \\
\hline 1061 & Euphorbia esula L. & Gárdony & 1963 & Isépy István & BPU-04749.JPG \\
\hline 1061 & Euphorbia esula L. & Gelénes & 1951 & $\begin{array}{l}\text { Simon Tibor, Vozáry } \\
\text { Elemér }\end{array}$ & BPU-04748.JPG \\
\hline 1061 & Euphorbia esula L. & Somlóvásárhely & 1953 & $\begin{array}{l}\text { Kulcsár Gábor, Vozáry } \\
\text { Elemér }\end{array}$ & BPU-04756.JPG \\
\hline 1061 & Euphorbia esula L. & Téglás & 1937 & Soó Rezső & BPU-04751.JPG \\
\hline 1061 & Euphorbia esula L. & Tihany & 1928 & Soó Rezső & BPU-04757.JPG \\
\hline 1062 & Euphorbia virgata Waldst. et Kit. & Balatonfüred & 1928 & Soó Rezső & BPU-04770.JPG \\
\hline 1062 & Euphorbia virgata Waldst. et Kit. & Bicske & 1992 & Felföldy Lajos & BPU-04765.JPG \\
\hline 1062 & Euphorbia virgata Waldst. et Kit. & Budaörs & 1982 & Felföldy Lajos & BPU-04767.JPG \\
\hline 1062 & Euphorbia virgata Waldst. et Kit. & Budapest & 1982 & Felföldy Lajos & BPU-04760.JPG \\
\hline 1062 & Euphorbia virgata Waldst. et Kit. & Budapest & 1982 & Felföldy Lajos & BPU-04763.JPG \\
\hline 1062 & Euphorbia virgata Waldst. et Kit. & Budapest & 1989 & Felföldy Lajos & BPU-04762.JPG \\
\hline 1062 & Euphorbia virgata Waldst. et Kit. & Budapest & 2004 & Felföldy Lajos & BPU-04759.JPG \\
\hline 1062 & Euphorbia virgata Waldst. et Kit. & Budapest & 2005 & Felföldy Lajos & BPU-04761.JPG \\
\hline 1062 & Euphorbia virgata Waldst. et Kit. & Győröcske & 1939 & Ubrizsy Gábor & BPU-04769.JPG \\
\hline 1062 & Euphorbia virgata Waldst. et Kit. & Hencida & 1927 & Kovács I. & BPU-04764.JPG \\
\hline 1062 & Euphorbia virgata Waldst. et Kit. & Püspökladány & 1935 & Soó Rezső & BPU-04766.JPG \\
\hline 1062 & Euphorbia virgata Waldst. et Kit. & Zánka & 1958 & Felföldy Lajos & BPU-04771.JPG \\
\hline 1062 & Euphorbia virgata Waldst. et Kit. & Zebegény & 1966 & Priszter Szaniszló & BPU-04768.JPG \\
\hline 1063 & Euphorbia lucida Waldst. et Kit. & Debrecen & 1931 & Soó Rezső & BPU-04773.JPG \\
\hline 1063 & Euphorbia lucida Waldst. et Kit. & Hajdúnánás & 1935 & Ujvárosi Miklós & BPU-04775.JPG \\
\hline 1063 & Euphorbia lucida Waldst. et Kit. & Szolnok & 1937 & Soó Rezső & BPU-04772.JPG \\
\hline 1063 & Euphorbia lucida Waldst. et Kit. & Tarpa & 1949 & Soó Rezső & BPU-04774.JPG \\
\hline 1064 & Euphorbia peplus L. & Budapest & 1991 & Felföldy Lajos & BPU-04796.JPG \\
\hline 1064 & Euphorbia peplus L. & Budapest & 2005 & Felföldy Lajos & BPU-04795.JPG \\
\hline 1065 & Euphorbia exigua L. & Budapest & 2004 & Felföldy Lajos & BPU-04789.JPG \\
\hline 1065 & Euphorbia exigua L. & Dabronc & 1960 & Németh Ferenc & BPU-04793.JPG \\
\hline 1065 & Euphorbia exigua L. & Dörgicse & 1957 & Felföldy Lajos & BPU-04794.JPG \\
\hline 1065 & Euphorbia exigua L. & Hajdúnánás & 1937 & Ujvárosi Miklós & BPU-04791.JPG \\
\hline 1065 & Euphorbia exigua L. & Jósvafő & 1951 & Soó Rezső & BPU-04790.JPG \\
\hline 1065 & Euphorbia exigua L. & Vácrátót & 1952 & Felföldy Lajos & BPU-04792.JPG \\
\hline 1066 & Euphorbia segetalis L. & Dorog & 1871 & Feichtinger Sándor & BPU-04802.JPG \\
\hline 1067 & Euphorbia taurinensis All. & Budaörs & 1982 & Felföldy Lajos & BPU-04801.JPG \\
\hline 1067 & Euphorbia taurinensis All. & Budapest & 1944 & Kárpáti Zoltán & BPU-04800.JPG \\
\hline 1068 & Euphorbia falcata L. & Kisharsány & 1961 & Simon Tibor & BPU-04798.JPG \\
\hline
\end{tabular}


1. Elektroniukus melléklet - Electronic Appendix 1.

\begin{tabular}{|c|c|c|c|c|c|}
\hline $\begin{array}{l}\text { Sorszám / } \\
\text { Number }\end{array}$ & Taxon-név / Taxon-name & $\begin{array}{l}\text { Település / } \\
\text { Settlement }\end{array}$ & $\begin{array}{l}\text { Év / } \\
\text { Year }\end{array}$ & Gyújtő / Collector & $\begin{array}{l}\text { Fájlnév / } \\
\text { File-name }\end{array}$ \\
\hline 1068 & Euphorbia falcata L. & Vácrátót & 1952 & Felföldy Lajos & BPU-04797.JPG \\
\hline $\begin{array}{c}1048 \times \\
1049\end{array}$ & $\begin{array}{l}\text { Euphorbia seguieriana Neck. } \times \\
\text { Euphorbia glareosa Pall. }\end{array}$ & Budapest & 1947 & Soó Rezső & BPU-04807.JPG \\
\hline $\begin{array}{c}1048 \times \\
1049\end{array}$ & $\begin{array}{l}\text { Euphorbia seguieriana Neck. × } \\
\text { Euphorbia glareosa Pall. }\end{array}$ & Diósjenő & 1952 & Simon Tibor & BPU-04805.JPG \\
\hline $\begin{array}{c}1048 \times \\
1049\end{array}$ & $\begin{array}{l}\text { Euphorbia seguieriana Neck. } \times \\
\text { Euphorbia glareosa Pall. }\end{array}$ & Pécsely & 1950 & Feichtinger Sándor & BPU-04806.JPG \\
\hline $\begin{array}{c}1050 \times \\
1063\end{array}$ & $\begin{array}{l}\text { Euphorbia palustris L. } \times \text { Euphorbia } \\
\text { lucida Waldst. et Kit. }\end{array}$ & Tarpa & 1949 & Soó Rezső & BPU-04812.JPG \\
\hline $\begin{array}{c}1050 \times \\
1063\end{array}$ & $\begin{array}{l}\text { Euphorbia palustris L. × Euphorbia } \\
\text { lucida Waldst. et Kit. }\end{array}$ & Tiszafüred & 1952 & Soó Rezső & BPU-04813.JPG \\
\hline $\begin{array}{c}1050 \times \\
1063\end{array}$ & $\begin{array}{l}\text { Euphorbia palustris L. } \times \text { Euphorbia } \\
\text { lucida Waldst. et Kit. }\end{array}$ & Tiszafüred & 1952 & Soó Rezső & BPU-15550.JPG \\
\hline $\begin{array}{c}1058 \times \\
1060\end{array}$ & $\begin{array}{l}\text { Euphorbia salicifolia Host } \times \text { Euphorbia } \\
\text { cyparissias L. }\end{array}$ & Egyek & 1938 & Soó Rezső & BPU-04811.JPG \\
\hline $\begin{array}{c}1058 \times \\
1061\end{array}$ & $\begin{array}{l}\text { Euphorbia salicifolia Host } \times \text { Euphorbia } \\
\text { esula L. }\end{array}$ & Kállósemjén & 1948 & Simon Tibor & BPU-04810.JPG \\
\hline $\begin{array}{c}1058 \times \\
1062\end{array}$ & $\begin{array}{l}\text { Euphorbia salicifolia Host } \times \text { Euphorbia } \\
\text { virgata Waldst. et Kit. }\end{array}$ & Debrecen & 1947 & Soó Rezső & BPU-04809.JPG \\
\hline $\begin{array}{c}1060 \times \\
1062\end{array}$ & $\begin{array}{l}\text { Euphorbia cyparissias L. × Euphorbia } \\
\text { virgata Waldst. et Kit. }\end{array}$ & Békéscsaba & 1923 & Soó Rezső & BPU-04808.JPG \\
\hline 1070 & Dictamnus albus L. & Bagamér & 1932 & Soó Rezső & BPU-03183.JPG \\
\hline 1070 & Dictamnus albus L. & Budapest & 1947 & Soó Rezső & BPU-03186.JPG \\
\hline 1070 & Dictamnus albus L. & Budapest & 1952 & Soó Rezső & BPU-15447.JPG \\
\hline 1070 & Dictamnus albus L. & Budapest & 1953 & Soó Rezső & BPU-15410.JPG \\
\hline 1070 & Dictamnus albus L. & Gyöngyös & 1961 & Soó Rezső & BPU-03180.JPG \\
\hline 1070 & Dictamnus albus L. & Kerecsend & 1957 & Soó Rezső & BPU-03187.JPG \\
\hline 1070 & Dictamnus albus L. & Monostorapáti & 1959 & $\begin{array}{l}\text { Jávorka Sándor, Felföldy } \\
\text { Lajos, Tóth László }\end{array}$ & BPU-03181.JPG \\
\hline 1070 & Dictamnus albus L. & Nagyharsány & 1956 & Soó Rezső & BPU-03185.JPG \\
\hline 1070 & Dictamnus albus L. & Pécs & 1953 & Soó Rezső, Borsos Olga & BPU-03182.JPG \\
\hline 1070 & Dictamnus albus L. & Somlóvásárhely & 1953 & $\begin{array}{l}\text { Kulcsár Gábor, Vozáry } \\
\text { Elemér }\end{array}$ & BPU-03179.JPG \\
\hline 1071 & Ptelea trifoliata L. & "Szentendrei-sziget" & 1964 & Isépy István & BPU-03190.JPG \\
\hline 1074 & Polygala major Jacq. & Budapest & 1982 & Felföldy Lajos & BPU-03193.JPG \\
\hline 1074 & Polygala major Jacq. & Háromhuta & 1955 & Simon Tibor & BPU-03194.JPG \\
\hline 1074 & Polygala major Jacq. & Miskolc & 1938 & Soó Rezső & BPU-03192.JPG \\
\hline 1074 & Polygala major Jacq. & Perkupa & 1949 & $\begin{array}{l}\text { Ubrizsy Gábor, Felföldy } \\
\text { Lajos, Szű́cs Lajos }\end{array}$ & BPU-03191.JPG \\
\hline 1075 & Polygala vulgaris L. & Alsószölnök & 1955 & Soó Rezső & BPU-03228.JPG \\
\hline 1075 & Polygala vulgaris L. & Bélapátfalva & 1948 & $\begin{array}{l}\text { Simon Tibor, Pólya } \\
\text { László, Jakucs Pál }\end{array}$ & BPU-03227.JPG \\
\hline 1075 & Polygala vulgaris L. & Beregdaróc & 1951 & Simon Tibor & BPU-03226.JPG \\
\hline 1075 & Polygala vulgaris L. & Komlóska & 1955 & Simon Tibor & BPU-03236.JPG \\
\hline 1075 & Polygala vulgaris L. & Komlóska & 1955 & Simon Tibor & BPU-03237.JPG \\
\hline 1075 & Polygala vulgaris L. & Kőszeg & 1938 & Soó Rezső & BPU-03229.JPG \\
\hline 1075 & Polygala vulgaris L. & Regéc & 1952 & Simon Tibor & BPU-03231.JPG \\
\hline 1075 & Polygala vulgaris L. & Telkibánya & 1952 & Simon Tibor & BPU-03225.JPG \\
\hline 1075 & Polygala vulgaris L. & Uzsa & 1959 & $\begin{array}{l}\text { Jávorka Sándor, Felföldy } \\
\text { Lajos }\end{array}$ & BPU-03230.JPG \\
\hline 1076 & Polygala comosa Schkuhr & Balatonakali & 1958 & Felföldy Lajos & BPU-03215.JPG \\
\hline 1076 & Polygala comosa Schkuhr & Balatonszőlős & 1958 & Felföldy Lajos & BPU-03216.JPG \\
\hline 1076 & Polygala comosa Schkuhr & Beregdaróc & 1951 & $\begin{array}{l}\text { Simon Tibor, Vozáry } \\
\text { Elemér }\end{array}$ & BPU-03198.JPG \\
\hline
\end{tabular}


1. Elektroniukus melléklet - Electronic Appendix 1.

\begin{tabular}{|c|c|c|c|c|c|}
\hline $\begin{array}{l}\text { Sorszám / } \\
\text { Number }\end{array}$ & Taxon-név / Taxon-name & $\begin{array}{l}\text { Település / } \\
\text { Settlement }\end{array}$ & $\begin{array}{l}\text { Év / } \\
\text { Year }\end{array}$ & Gyưjtő / Collector & $\begin{array}{l}\text { Fájlnév / } \\
\text { File-name }\end{array}$ \\
\hline 1076 & Polygala comosa Schkuhr & Budapest & 1947 & Pap S. & BPU-03211.JPG \\
\hline 1076 & Polygala comosa Schkuhr & Budapest & 2005 & Felföldy Lajos & BPU-03195.JPG \\
\hline 1076 & Polygala comosa Schkuhr & Bugyi & 1959 & Soó Rezső & BPU-03209.JPG \\
\hline 1076 & Polygala comosa Schkuhr & Csákberény & 1982 & Felföldy Lajos & BPU-03203.JPG \\
\hline 1076 & Polygala comosa Schkuhr & Dabas & 1966 & Priszter Szaniszló & BPU-03210.JPG \\
\hline 1076 & Polygala comosa Schkuhr & Debrecen & 1952 & Kulcsár Gábor & BPU-03204.JPG \\
\hline 1076 & Polygala comosa Schkuhr & Gelénes & 1951 & $\begin{array}{l}\text { Simon Tibor, Vozáry } \\
\text { Elemér }\end{array}$ & BPU-03199.JPG \\
\hline 1076 & Polygala comosa Schkuhr & Gyöngyös & 1965 & Soó Rezső & BPU-03206.JPG \\
\hline 1076 & Polygala comosa Schkuhr & Háromhuta & 1955 & Simon Tibor & BPU-03222.JPG \\
\hline 1076 & Polygala comosa Schkuhr & Kapolcs & 1959 & $\begin{array}{l}\text { Jávorka Sándor, Felföldy } \\
\text { Lajos, Tóth László }\end{array}$ & BPU-03217.JPG \\
\hline 1076 & Polygala comosa Schkuhr & Kecskemét & 1914 & Degen Árpád & BPU-03196.JPG \\
\hline 1076 & Polygala comosa Schkuhr & Kimle & 1962 & Borsos Olga & BPU-03208.JPG \\
\hline 1076 & Polygala comosa Schkuhr & Komárom & 1959 & $\begin{array}{l}\text { Simon Tibor, Borhidi } \\
\text { Attila }\end{array}$ & BPU-03207.JPG \\
\hline 1076 & Polygala comosa Schkuhr & Komlóska & 1955 & Simon Tibor & BPU-03221.JPG \\
\hline 1076 & Polygala comosa Schkuhr & Kőszeg & 1938 & Soó Rezső & BPU-03214.JPG \\
\hline 1076 & Polygala comosa Schkuhr & Lábatlan & 1953 & Felföldy Lajos & BPU-03202.JPG \\
\hline 1076 & Polygala comosa Schkuhr & Miskolc & 1937 & Soó Rezső & BPU-03201.JPG \\
\hline 1076 & Polygala comosa Schkuhr & Miskolc & 1950 & Soó Rezső & BPU-03200.JPG \\
\hline 1076 & Polygala comosa Schkuhr & Ócsa & 1955 & Soó Rezső & BPU-03212.JPG \\
\hline 1076 & Polygala comosa Schkuhr & Pécs & 1953 & Soó Rezső, Borsos Olga & BPU-03197.JPG \\
\hline 1076 & Polygala comosa Schkuhr & Szilvásvárad & 1953 & $\begin{array}{l}\text { Felföldy Lajos, Tóth } \\
\text { László }\end{array}$ & BPU-03205.JPG \\
\hline 1076 & Polygala comosa Schkuhr & Tihany & 1958 & Felföldy Lajos & BPU-03218.JPG \\
\hline 1076 & Polygala comosa Schkuhr & Tihany & 1958 & Kozma Erika & BPU-03219.JPG \\
\hline 1076 & Polygala comosa Schkuhr & Vácrátót & 1952 & Felföldy Lajos & BPU-03213.JPG \\
\hline 1076 & Polygala comosa Schkuhr & Várpalota & 1955 & Soó Rezső & BPU-03220.JPG \\
\hline 1078 & Polygala amarella Crantz & Sopron & 1932 & Soó Rezső & BPU-03251.JPG \\
\hline 1078 & Polygala amarella Crantz & Veresegyház & 1949 & Soó Rezső & BPU-03250.JPG \\
\hline 1079 & Polygala amara L. & Bodajk & 1955 & Soó Rezső & BPU-03241.JPG \\
\hline 1079 & Polygala amara L. & Nagykovácsi & 1949 & Soó Rezső & BPU-03243.JPG \\
\hline 1079 & Polygala amara L. & Pilisszentiván & 1953 & Soó Rezső & BPU-03244.JPG \\
\hline 1079 & Polygala amara L. & Sopron & 1960 & Borsos Olga, Simon Tibor & BPU-03252.JPG \\
\hline 1081 & Cotinus coggygria Scop. & Balatonszőlős & 1958 & Felföldy Lajos & BPU-03259.JPG \\
\hline 1081 & Cotinus coggygria Scop. & Bélapátfalva & 1932 & Soó Rezső & BPU-03256.JPG \\
\hline 1081 & Cotinus coggygria Scop. & Budapest & 1952 & Soó Rezső & BPU-15408.JPG \\
\hline 1081 & Cotinus coggygria Scop. & Gyenesdiás & 1960 & Priszter Szaniszló & BPU-03263.JPG \\
\hline 1081 & Cotinus coggygria Scop. & Isztimér & 1949 & Soó Rezső & BPU-03257.JPG \\
\hline 1081 & Cotinus coggygria Scop. & Mosonmagyaróvár & 1962 & Borsos Olga & BPU-03258.JPG \\
\hline 1081 & Cotinus coggygria Scop. & Tihany & 1928 & Soó Rezső & BPU-03261.JPG \\
\hline 1081 & Cotinus coggygria Scop. & Tihany & 1959 & Felföldy Lajos & BPU-03260.JPG \\
\hline 1081 & Cotinus coggygria Scop. & Tihany & 1960 & Priszter Szaniszló & BPU-03262.JPG \\
\hline 1082 & Acer negundo L. & Budapest & 2004 & Felföldy Lajos & BPU-03265.JPG \\
\hline 1083 & Acer tataricum L. & Bisse & 1965 & $\begin{array}{l}\text { Simon Tibor, Priszter } \\
\text { Szaniszló, Borhidi Attila }\end{array}$ & BPU-03270.JPG \\
\hline 1083 & Acer tataricum L. & Budapest & 1942 & Kárpáti Zoltán & BPU-03267.JPG \\
\hline 1083 & Acer tataricum L. & Budapest & 1952 & Dobos L. & BPU-03268.JPG \\
\hline 1083 & Acer tataricum L. & Egyek & 1953 & Borsos Olga & BPU-03272.JPG \\
\hline
\end{tabular}


1. Elektroniukus melléklet - Electronic Appendix 1.

\begin{tabular}{|c|c|c|c|c|c|}
\hline $\begin{array}{l}\text { Sorszám / } \\
\text { Number }\end{array}$ & Taxon-név / Taxon-name & $\begin{array}{l}\text { Település / } \\
\text { Settlement }\end{array}$ & $\begin{array}{l}\text { Év / } \\
\text { Year }\end{array}$ & Gyüjtő / Collector & $\begin{array}{l}\text { Fájlnév / } \\
\text { File-name }\end{array}$ \\
\hline 1083 & Acer tataricum L. & Kerecsend & 1957 & Soó Rezső & BPU-03273.JPG \\
\hline 1083 & Acer tataricum L. & Pécs & 1953 & Soó Rezső, Borsos Olga & BPU-03271.JPG \\
\hline 1083 & Acer tataricum L. & Sima & 1961 & Simon Tibor & BPU-03269.JPG \\
\hline 1083 & Acer tataricum L. & Tarcal & 1947 & Soó Rezső & BPU-03274.JPG \\
\hline 1085 & Acer campestre L. & Ajka & 1954 & Tóth László & BPU-03307.JPG \\
\hline 1085 & Acer campestre L. & Ajka & 1954 & Tóth László & BPU-03308.JPG \\
\hline 1085 & Acer campestre L. & Badacsonytomaj & 1953 & $\begin{array}{l}\text { Felföldy Lajos, Tóth } \\
\text { László }\end{array}$ & BPU-03327.JPG \\
\hline 1085 & Acer campestre L. & Badacsonytördemic & 1953 & $\begin{array}{l}\text { Felföldy Lajos, Tóth } \\
\text { László }\end{array}$ & BPU-03311.JPG \\
\hline 1085 & Acer campestre L. & Badacsonytördemic & 1953 & $\begin{array}{l}\text { Felföldy Lajos, Tóth } \\
\text { László }\end{array}$ & BPU-03312.JPG \\
\hline 1085 & Acer campestre L. & Badacsonytördemic & 1953 & $\begin{array}{l}\text { Felföldy Lajos, Tóth } \\
\text { László }\end{array}$ & BPU-03330.JPG \\
\hline 1085 & Acer campestre L. & Badacsonytördemic & 1953 & $\begin{array}{l}\text { Felföldy Lajos, Tóth } \\
\text { László }\end{array}$ & BPU-03331.JPG \\
\hline 1085 & Acer campestre L. & Balatonszőlős & 1957 & Felföldy Lajos & BPU-03309.JPG \\
\hline 1085 & Acer campestre L. & Budapest & 1954 & Tóth László & BPU-03316.JPG \\
\hline 1085 & Acer campestre L. & Budapest & 1954 & Tóth László & BPU-03317.JPG \\
\hline 1085 & Acer campestre L. & Budapest & 1954 & Tóth László & BPU-03318.JPG \\
\hline 1085 & Acer campestre L. & Debrecen & 1932 & Soó Rezső & BPU-03290.JPG \\
\hline 1085 & Acer campestre L. & Debrecen & 1947 & Soó Rezső & BPU-03320.JPG \\
\hline 1085 & Acer campestre L. & Debrecen & 1953 & Tóth László & BPU-03295.JPG \\
\hline 1085 & Acer campestre L. & Debrecen & 1953 & Tóth László & BPU-03296.JPG \\
\hline 1085 & Acer campestre L. & Debrecen & 1953 & Tóth László & BPU-03297.JPG \\
\hline 1085 & Acer campestre L. & Debrecen & 1953 & Tóth László & BPU-03298.JPG \\
\hline 1085 & Acer campestre L. & Egyek & 1953 & Tóth László & BPU-03299.JPG \\
\hline 1085 & Acer campestre L. & Gyöngyös & 1953 & Tóth László & BPU-03300.JPG \\
\hline 1085 & Acer campestre L. & Gyöngyös & 1953 & Tóth László & BPU-03321.JPG \\
\hline 1085 & Acer campestre L. & Gyöngyös & 1953 & Tóth László & BPU-03322.JPG \\
\hline 1085 & Acer campestre L. & Jósvafő & 1951 & $\begin{array}{l}\text { Soó Rezső, Jakucs Pál, Ér } \\
\text { Lajos }\end{array}$ & BPU-03285.JPG \\
\hline 1085 & Acer campestre L. & Kapolcs & 1959 & $\begin{array}{l}\text { Jávorka Sándor, Felföldy } \\
\text { Lajos, Tóth László }\end{array}$ & BPU-03328.JPG \\
\hline 1085 & Acer campestre L. & Kaposvár & 1954 & Tóth László & BPU-03306.JPG \\
\hline 1085 & Acer campestre L. & Kaposvár & 1954 & Tóth László & BPU-03326.JPG \\
\hline 1085 & Acer campestre L. & Lábatlan & 1953 & Felföldy Lajos & BPU-03303.JPG \\
\hline 1085 & Acer campestre L. & Miskolc & 1955 & Tóth László & BPU-03286.JPG \\
\hline 1085 & Acer campestre L. & Miskolc & 1955 & Tóth László & BPU-03287.JPG \\
\hline 1085 & Acer campestre L. & Miskolc & 1955 & Tóth László & BPU-03288.JPG \\
\hline 1085 & Acer campestre L. & Miskolc & 1955 & Tóth László & BPU-03289.JPG \\
\hline 1085 & Acer campestre L. & Miskolc & 2007 & Simon Tibor & BPU-15506.JPG \\
\hline 1085 & Acer campestre L. & Monostorapáti & 1959 & $\begin{array}{l}\text { Jávorka Sándor, Felföldy } \\
\text { Lajos, Tóth László }\end{array}$ & BPU-03310.JPG \\
\hline 1085 & Acer campestre L. & Monostorapáti & 1959 & $\begin{array}{l}\text { Jávorka Sándor, Felföldy } \\
\text { Lajos, Tóth László }\end{array}$ & BPU-03329.JPG \\
\hline 1085 & Acer campestre L. & Pécsvárad & 1953 & Tóth László & BPU-03294.JPG \\
\hline 1085 & Acer campestre L. & Pécsvárad & 1953 & Tóth László & BPU-03319.JPG \\
\hline 1085 & Acer campestre L. & Szilvásvárad & 1953 & $\begin{array}{l}\text { Felföldy Lajos, Tóth } \\
\text { László }\end{array}$ & BPU-03301.JPG \\
\hline 1085 & Acer campestre L. & Szilvásvárad & 1953 & $\begin{array}{l}\text { Felföldy Lajos, Tóth } \\
\text { László }\end{array}$ & BPU-03302.JPG \\
\hline
\end{tabular}


1. Elektroniukus melléklet - Electronic Appendix 1.

\begin{tabular}{|c|c|c|c|c|c|}
\hline $\begin{array}{l}\text { Sorszám / } \\
\text { Number }\end{array}$ & Taxon-név / Taxon-name & $\begin{array}{l}\text { Település / } \\
\text { Settlement }\end{array}$ & $\begin{array}{l}\text { Év / } \\
\text { Year }\end{array}$ & Gyưjtő / Collector & $\begin{array}{l}\text { Fájlnév / } \\
\text { File-name }\end{array}$ \\
\hline 1085 & Acer campestre L. & Szilvásvárad & 1953 & $\begin{array}{l}\text { Felföldy Lajos, Tóth } \\
\text { László }\end{array}$ & BPU-03323.JPG \\
\hline 1085 & Acer campestre L. & Tihany & 1954 & Tóth László & BPU-03313.JPG \\
\hline 1085 & Acer campestre L. & Tihany & 1954 & Tóth László & BPU-03332.JPG \\
\hline 1085 & Acer campestre L. & Tihany & 1954 & Tóth László & BPU-03336.JPG \\
\hline 1085 & Acer campestre L. & Tihany & 1955 & Tóth László & BPU-03291.JPG \\
\hline 1085 & Acer campestre L. & Tihany & 1955 & Tóth László & BPU-03292.JPG \\
\hline 1085 & Acer campestre L. & Tihany & 1955 & Tóth László & BPU-03293.JPG \\
\hline 1085 & Acer campestre L. & Tihany & 1956 & Felföldy Lajos & BPU-03333.JPG \\
\hline 1085 & Acer campestre L. & Tihany & 1956 & Felföldy Lajos & BPU-03334.JPG \\
\hline 1085 & Acer campestre L. & Tihany & 1956 & Felföldy Lajos & BPU-03335.JPG \\
\hline 1085 & Acer campestre L. & Uzsa & 1959 & $\begin{array}{l}\text { Jávorka Sándor, Felföldy } \\
\text { Lajos }\end{array}$ & BPU-03314.JPG \\
\hline 1085 & Acer campestre L. & Vác & 1953 & Tóth László & BPU-03304.JPG \\
\hline 1085 & Acer campestre L. & Vác & 1953 & Tóth László & BPU-03324.JPG \\
\hline 1085 & Acer campestre L. & Vác & 1953 & Tóth László & BPU-03325.JPG \\
\hline 1085 & Acer campestre L. & Vác & 1956 & Tóth László & BPU-03305.JPG \\
\hline 1085 & Acer campestre L. & Vállus & 1950 & Priszter Szaniszló & BPU-03315.JPG \\
\hline 1086 & Acer pseudoplatanus L. & Barabás & 1951 & Simon Tibor & BPU-03278.JPG \\
\hline 1086 & Acer pseudoplatanus L. & Budapest & 1947 & Soó Rezső & BPU-03277.JPG \\
\hline 1086 & Acer pseudoplatanus L. & Miskolc & 1950 & Soó Rezső & BPU-03279.JPG \\
\hline 1087 & Acer platanoides L. & Abaújalpár & 1948 & Soó Rezső & BPU-03275.JPG \\
\hline 1087 & Acer platanoides L. & Bátorliget & 1934 & Soó Rezső & BPU-03276.JPG \\
\hline 1089 & Impatiens noli-tangere L. & Baktalórántháza & 1948 & Simon Tibor, Jakucs Pál & BPU-03340.JPG \\
\hline 1089 & Impatiens noli-tangere L. & Gyöngyös & 1935 & Soó Rezső & BPU-03337.JPG \\
\hline 1089 & Impatiens noli-tangere L. & Kemence & 1949 & Pólya László & BPU-03338.JPG \\
\hline 1089 & Impatiens noli-tangere L. & Szilvásvárad & 1953 & $\begin{array}{l}\text { Felföldy Lajos, Tóth } \\
\text { László }\end{array}$ & BPU-03342.JPG \\
\hline 1089 & Impatiens noli-tangere L. & Tarpa & 1948 & Simon Tibor, Jakucs Pál & BPU-03341.JPG \\
\hline 1090 & Impatiens parviflora DC. & Bodajk & 1961 & Kuria Kálmán & BPU-03346.JPG \\
\hline 1090 & Impatiens parviflora DC. & Budapest & 1991 & Felföldy Lajos & BPU-03345.JPG \\
\hline 1090 & Impatiens parviflora DC. & Budapest & 1993 & Felföldy Lajos & BPU-03344.JPG \\
\hline 1090 & Impatiens parviflora DC. & Budapest & 2004 & Felföldy Lajos & BPU-03343.JPG \\
\hline 1090 & Impatiens parviflora DC. & Debrecen & 1935 & Soó Rezső & BPU-03347.JPG \\
\hline 1090 & Impatiens parviflora DC. & Debrecen & 1946 & anonim & BPU-03351.JPG \\
\hline 1090 & Impatiens parviflora DC. & Nagykovácsi & 2005 & Felföldy Lajos & BPU-03348.JPG \\
\hline 1090 & Impatiens parviflora DC. & Tihany & 1958 & Felföldy Lajos & BPU-03350.JPG \\
\hline 1090 & Impatiens parviflora DC. & Zákány & 1948 & Károlyi Árpád & BPU-03349.JPG \\
\hline 1095 & Ilex aquifolium L. & Háromhuta & 1955 & Simon Tibor & BPU-03352.JPG \\
\hline 1096 & Euonymus europaeus L. & Balatonszőlős & 1958 & Felföldy Lajos & BPU-03368.JPG \\
\hline 1096 & Euonymus europaeus L. & Budapest & 2005 & Felföldy Lajos & BPU-03363.JPG \\
\hline 1096 & Euonymus europaeus L. & Gánt & 1943 & Soó Rezső & BPU-03365.JPG \\
\hline 1096 & Euonymus europaeus L. & Göd & 1943 & Kárpáti Zoltán & BPU-03369.JPG \\
\hline 1096 & Euonymus europaeus L. & Kaposvár & 1955 & Tóth László & BPU-03366.JPG \\
\hline 1096 & Euonymus europaeus L. & Miskolc & 1950 & Soó Rezső & BPU-03364.JPG \\
\hline 1096 & Euonymus europaeus L. & Sopron & 1944 & Kárpáti Zoltán & BPU-03367.JPG \\
\hline 1097 & Euonymus verrucosus Scop. & Budapest & 1982 & Felföldy Lajos & BPU-03354.JPG \\
\hline 1097 & Euonymus verrucosus Scop. & Budapest & 2004 & Felföldy Lajos & BPU-03353.JPG \\
\hline 1097 & Euonymus verrucosus Scop. & Csesznek & 1963 & Soó Rezső & BPU-03362.JPG \\
\hline
\end{tabular}


1. Elektroniukus melléklet - Electronic Appendix 1.

\begin{tabular}{|c|c|c|c|c|c|}
\hline $\begin{array}{l}\text { Sorszám / } \\
\text { Number }\end{array}$ & Taxon-név / Taxon-name & $\begin{array}{l}\text { Település / } \\
\text { Settlement }\end{array}$ & $\begin{array}{l}\text { Év / } \\
\text { Year }\end{array}$ & Gyüjtő / Collector & $\begin{array}{l}\text { Fájlnév / } \\
\text { File-name }\end{array}$ \\
\hline 1097 & Euonymus verrucosus Scop. & Debrecen & 1931 & Pátkay Imre & BPU-03355.JPG \\
\hline 1097 & Euonymus verrucosus Scop. & Kapolcs & 1959 & $\begin{array}{l}\text { Jávorka Sándor, Felföldy } \\
\text { Lajos, Tóth László }\end{array}$ & BPU-03359.JPG \\
\hline 1097 & Euonymus verrucosus Scop. & Kerecsend & 1957 & Soó Rezső & BPU-03357.JPG \\
\hline 1097 & Euonymus verrucosus Scop. & Miskolc & 1939 & Soó Rezső & BPU-03356.JPG \\
\hline 1097 & Euonymus verrucosus Scop. & Tihany & 1956 & Felföldy Lajos & BPU-03360.JPG \\
\hline 1097 & Euonymus verrucosus Scop. & Tihany & 1958 & Felföldy Lajos & BPU-03361.JPG \\
\hline 1097 & Euonymus verrucosus Scop. & Visegrád & 1964 & Soó Rezső, Borsos Olga & BPU-03358.JPG \\
\hline 1098 & Staphylea pinnata L. & Bátorliget & 1951 & Simon Tibor & BPU-03378.JPG \\
\hline 1098 & Staphylea pinnata L. & Budapest & 1953 & Soó Rezső & BPU-03373.JPG \\
\hline 1098 & Staphylea pinnata L. & Budapest & 1982 & Felföldy Lajos & BPU-03374.JPG \\
\hline 1098 & Staphylea pinnata L. & Debrecen & 1937 & Soó Rezső & BPU-03371.JPG \\
\hline 1098 & Staphylea pinnata L. & Miskolc & 1950 & Soó Rezső, Simon Tibor & BPU-03376.JPG \\
\hline 1098 & Staphylea pinnata L. & Pécs & 1953 & Soó Rezső, Borsos Olga & BPU-03372.JPG \\
\hline 1098 & Staphylea pinnata L. & Sárospatak & 1939 & Soó Rezső & BPU-03377.JPG \\
\hline 1098 & Staphylea pinnata L. & Visegrád & 1964 & Soó Rezső, Borsos Olga & BPU-03375.JPG \\
\hline 1100 & Rhamnus catharticus L. & Debrecen & 1934 & Soó Rezső & BPU-03379.JPG \\
\hline 1100 & Rhamnus catharticus L. & Fót & 1960 & Soó Rezső & BPU-03380.JPG \\
\hline 1100 & Rhamnus catharticus L. & Pécsely & 1950 & Felföldy Lajos & BPU-03382.JPG \\
\hline 1100 & Rhamnus catharticus L. & Tihany & 1956 & Felföldy Lajos & BPU-03383.JPG \\
\hline 1101 & Rhamnus saxatilis Jacq. & Sopron & 1960 & Soó Rezső, Simon Tibor & BPU-03384.JPG \\
\hline $\begin{array}{c}1100 \times \\
1101\end{array}$ & Rhamnus × gayeri Kárpáti & Sopron & 1932 & Soó Rezső & BPU-03386.JPG \\
\hline 1102 & Frangula alnus Mill. & Budapest & 2004 & Felföldy Lajos & BPU-03387.JPG \\
\hline 1102 & Frangula alnus Mill. & Debrecen & 1933 & Soó Rezső & BPU-03389.JPG \\
\hline 1102 & Frangula alnus Mill. & Nagybajcs & 1931 & Soó Rezső & BPU-03388.JPG \\
\hline 1102 & Frangula alnus Mill. & Szentgotthárd & 1955 & Soó Rezső & BPU-03390.JPG \\
\hline 1102 & Frangula alnus Mill. & Zalagyömörő & 1957 & Felföldy Lajos & BPU-03391.JPG \\
\hline 1104 & Vitis rupestris Scheele & Tiszafüred & 1953 & Borsos Olga & BPU-15472.JPG \\
\hline 1107 & Vitis sylvestris C.C. Gmel. & Bátorliget & 1934 & Soó Rezső & BPU-03396.JPG \\
\hline 1107 & Vitis sylvestris C.C. Gmel. & Bátorliget & 1950 & $\begin{array}{l}\text { Simon Tibor, Kárpáti } \\
\text { István }\end{array}$ & BPU-03397.JPG \\
\hline 1107 & Vitis sylvestris C.C. Gmel. & Gyula & 1935 & Soó Rezső & BPU-03394.JPG \\
\hline 1107 & Vitis sylvestris C.C. Gmel. & Jánd & 1951 & Simon Tibor & BPU-03395.JPG \\
\hline 1107 & Vitis sylvestris C.C. Gmel. & Lakitelek & 1961 & Muray Katalin & BPU-03393.JPG \\
\hline 1107 & Vitis sylvestris C.C. Gmel. & Lakitelek & 1961 & Simon Tibor & BPU-03392.JPG \\
\hline 1107 & Vitis sylvestris C.C. Gmel. & Tiszaug & 1953 & Soó Rezső & BPU-03398.JPG \\
\hline 1107 & Vitis sylvestris C.C. Gmel. & Tokaj & 1949 & Soó Rezső & BPU-03399.JPG \\
\hline 1108 & $\begin{array}{l}\text { Parthenocissus tricuspidata Siebold et } \\
\text { Zucc. }\end{array}$ & Debrecen & 1947 & Soó Rezső & BPU-03400.JPG \\
\hline 1110 & $\begin{array}{l}\text { Parthenocissus quinquefolia (L.) } \\
\text { Planch. }\end{array}$ & Tarpa & 1948 & $\begin{array}{l}\text { Pólya László, Simon } \\
\text { Tibor, Jakucs Pál }\end{array}$ & BPU-03401.JPG \\
\hline 1111 & Tilia tomentosa Moench & Bátorliget & 1932 & Soó Rezső & BPU-04410.JPG \\
\hline 1111 & Tilia tomentosa Moench & Bátorliget & 1932 & Soó Rezső & BPU-04411.JPG \\
\hline 1111 & Tilia tomentosa Moench & Bátorliget & 1934 & Soó Rezső & BPU-04409.JPG \\
\hline 1111 & Tilia tomentosa Moench & Bisse & 1965 & $\begin{array}{l}\text { Simon Tibor, Priszter } \\
\text { Szaniszló, Borhidi Attila }\end{array}$ & BPU-04416.JPG \\
\hline 1111 & Tilia tomentosa Moench & Debrecen & 1947 & Soó Rezső & BPU-04407.JPG \\
\hline 1111 & Tilia tomentosa Moench & Debrecen & 1951 & Kulcsár Gábor & BPU-04412.JPG \\
\hline 1111 & Tilia tomentosa Moench & Pécs & 1953 & Soó Rezső, Borsos Olga & BPU-04415.JPG \\
\hline
\end{tabular}


1. Elektroniukus melléklet - Electronic Appendix 1.

\begin{tabular}{|c|c|c|c|c|c|}
\hline $\begin{array}{l}\text { Sorszám / } \\
\text { Number }\end{array}$ & Taxon-név / Taxon-name & $\begin{array}{l}\text { Település / } \\
\text { Settlement }\end{array}$ & $\begin{array}{l}\text { Év / } \\
\text { Year }\end{array}$ & Gyűjtő / Collector & $\begin{array}{l}\text { Fájlnév / } \\
\text { File-name }\end{array}$ \\
\hline 1111 & Tilia tomentosa Moench & Szenna & 1957 & Soó Rezső & BPU-04408.JPG \\
\hline 1111 & Tilia tomentosa Moench & Villány & 1935 & Soó Rezső & BPU-04414.JPG \\
\hline 1112 & Tilia platyphyllos Scop. & $\begin{array}{l}\text { Badacsonytomaj, } \\
\text { Badacsonytördemic }\end{array}$ & 1928 & Soó Rezső & BPU-04433.JPG \\
\hline 1112 & Tilia platyphyllos Scop. & Bélapátfalva & 1953 & Simon Tibor & BPU-04435.JPG \\
\hline 1112 & Tilia platyphyllos Scop. & Budapest & 1947 & Soó Rezső & BPU-04437.JPG \\
\hline 1112 & Tilia platyphyllos Scop. & Eplény & 1932 & Polgár Sándor & BPU-04430.JPG \\
\hline 1112 & Tilia platyphyllos Scop. & Gyöngyös & 1953 & Soó Rezső & BPU-04429.JPG \\
\hline 1112 & Tilia platyphyllos Scop. & Miskolc & 1937 & Soó Rezső & BPU-04436.JPG \\
\hline 1112 & Tilia platyphyllos Scop. & Sarkad & 1935 & Soó Rezső & BPU-04431.JPG \\
\hline 1112 & Tilia platyphyllos Scop. & Sopron & 1937 & Soó Rezső & BPU-04432.JPG \\
\hline 1112 & Tilia platyphyllos Scop. & Szilvásvárad & 1953 & Soó Rezső & BPU-04434.JPG \\
\hline 1112 & Tilia platyphyllos Scop. & Vác & 1949 & $\begin{array}{l}\text { Soó Rezső, Simon Tibor, } \\
\text { Jakucs Pál }\end{array}$ & BPU-04428.JPG \\
\hline 1113 & Tilia cordata Mill. & Bátorliget & 1934 & Soó Rezső & BPU-04423.JPG \\
\hline 1113 & Tilia cordata Mill. & Beregdaróc & 1951 & Simon Tibor & BPU-04420.JPG \\
\hline 1113 & Tilia cordata Mill. & Beregdaróc & 1951 & $\begin{array}{l}\text { Simon Tibor, Vozáry } \\
\text { Elemér }\end{array}$ & BPU-04421.JPG \\
\hline 1113 & Tilia cordata Mill. & Bozsok & 1938 & Soó Rezső & BPU-04424.JPG \\
\hline 1113 & Tilia cordata Mill. & Fony & 1961 & Simon Tibor & BPU-04417.JPG \\
\hline 1113 & Tilia cordata Mill. & Füzér & 1937 & Soó Rezső & BPU-04418.JPG \\
\hline 1113 & Tilia cordata Mill. & Háromhuta & 1961 & Simon Tibor & BPU-04426.JPG \\
\hline 1113 & Tilia cordata Mill. & Kőszeg & 1932 & Soó Rezső & BPU-04425.JPG \\
\hline 1113 & Tilia cordata Mill. & Pálháza & 1952 & Simon Tibor & BPU-04419.JPG \\
\hline 1113 & Tilia cordata Mill. & Pilisszentiván & 1953 & Soó Rezső & BPU-04422.JPG \\
\hline 1113 & Tilia cordata Mill. & Sárospatak & 1938 & Soó Rezső & BPU-04427.JPG \\
\hline 1115 & Malva alcea L. & Lesenceistvánd & 1928 & Soó Rezső & BPU-04460.JPG \\
\hline 1115 & Malva alcea L. & Uzsa & 1952 & Simon Tibor & BPU-04458.JPG \\
\hline 1115 & Malva alcea L. & Uzsa & 1953 & $\begin{array}{l}\text { Felföldy Lajos, Tóth } \\
\text { László }\end{array}$ & BPU-04459.JPG \\
\hline 1117 & Malva sylvestris L. & Debrecen & 1938 & Soó Rezső & BPU-04461.JPG \\
\hline 1119 & Malva neglecta Wallr. & Budapest & 1993 & Felföldy Lajos & BPU-04463.JPG \\
\hline 1119 & Malva neglecta Wallr. & Budapest & 2005 & Felföldy Lajos & BPU-04462.JPG \\
\hline 1119 & Malva neglecta Wallr. & Nyíregyháza & 1947 & Soó Rezső & BPU-04464.JPG \\
\hline 1121 & Lavatera thuringiaca L. & Budaörs & 1982 & Felföldy Lajos & BPU-04443.JPG \\
\hline 1121 & Lavatera thuringiaca L. & Budapest & 1922 & Soó Rezső & BPU-04445.JPG \\
\hline 1121 & Lavatera thuringiaca L. & Szigetújfalu & 1943 & Kárpáti Zoltán & BPU-04442.JPG \\
\hline 1121 & Lavatera thuringiaca L. & Zámoly & 1982 & Felföldy Lajos & BPU-04444.JPG \\
\hline 1123 & Althaea hirsuta L. & Budapest & 1944 & Kárpáti Zoltán & BPU-04446.JPG \\
\hline 1124 & Althaea cannabina L. & Budapest & 1947 & Soó Rezső & BPU-04449.JPG \\
\hline 1124 & Althaea cannabina L. & Nagyharsány & 1965 & $\begin{array}{l}\text { Simon Tibor, Priszter } \\
\text { Szaniszló, Borhidi Attila }\end{array}$ & BPU-04448.JPG \\
\hline 1124 & Althaea cannabina L. & Szentendre & 1943 & Kárpáti Zoltán & BPU-04447.JPG \\
\hline 1125 & Althaea officinalis L. & Gárdony & 1980 & Felföldy Lajos & BPU-04455.JPG \\
\hline 1125 & Althaea officinalis L. & Kiskunhalas & 1964 & Soó Rezső & BPU-04453.JPG \\
\hline 1125 & Althaea officinalis L. & Monoszló & 1982 & Felföldy Lajos & BPU-04452.JPG \\
\hline 1125 & Althaea officinalis L. & Nagybajcs & 1912 & Polgár Sándor & BPU-04450.JPG \\
\hline 1125 & Althaea officinalis L. & Szigetmonostor & 1947 & Priszter Szaniszló & BPU-04451.JPG \\
\hline 1127 & Alcea biennis Winterl & Nyúl & 1913 & Polgár Sándor & BPU-04457.JPG \\
\hline 1129 & Abutilon theophrasti Medik. & Keszthely & 1960 & Csepinszky Béla & BPU-04440.JPG \\
\hline
\end{tabular}


1. Elektroniukus melléklet - Electronic Appendix 1.

\begin{tabular}{|c|c|c|c|c|c|}
\hline $\begin{array}{l}\text { Sorszám / } \\
\text { Number }\end{array}$ & Taxon-név / Taxon-name & $\begin{array}{l}\text { Település / } \\
\text { Settlement }\end{array}$ & $\begin{array}{l}\text { Év / } \\
\text { Year }\end{array}$ & Gyújtő / Collector & $\begin{array}{l}\text { Fájlnév / } \\
\text { File-name }\end{array}$ \\
\hline 1130 & Hibiscus trionum L. & Budapest & 1991 & Felföldy Lajos & BPU-04468.JPG \\
\hline 1130 & Hibiscus trionum L. & Kecskemét & 1926 & Lengyel Géza & BPU-04469.JPG \\
\hline 1130 & Hibiscus trionum L. & Kiskunmajsa & 1964 & Soó Rezső & BPU-04467.JPG \\
\hline 1130 & Hibiscus trionum L. & Nyíracsád & 1932 & Soó Rezső & BPU-04470.JPG \\
\hline 1130 & Hibiscus trionum L. & Tihany & 1961 & $\begin{array}{l}\text { Pankov H., Felföldy Lajos, } \\
\text { Sólymosy G. }\end{array}$ & BPU-04466.JPG \\
\hline 1130 & Hibiscus trionum L. & Tokaj & 1963 & Isépy István & BPU-04465.JPG \\
\hline 1132 & Daphne cneorum L. & Balatongyörök & 1959 & Entz Béla & BPU-02993.JPG \\
\hline 1132 & Daphne cneorum L. & Budaörs & 1944 & Kárpáti Zoltán & BPU-02989.JPG \\
\hline 1132 & Daphne cneorum L. & Győr & 1959 & $\begin{array}{l}\text { Simon Tibor, Borhidi } \\
\text { Attila }\end{array}$ & BPU-02988.JPG \\
\hline 1132 & Daphne cneorum L. & Isztimér & 1932 & Polgár Sándor & BPU-02992.JPG \\
\hline 1132 & Daphne cneorum L. & Pilisszentiván & 1953 & Soó Rezső & BPU-02990.JPG \\
\hline 1132 & Daphne cneorum L. & Szentgotthárd & 1955 & Soó Rezső & BPU-02991.JPG \\
\hline 1133 & Daphne laureola L. & Bakonybél & 1955 & Soó Rezső & BPU-03002.JPG \\
\hline 1133 & Daphne laureola L. & Gánt & 1949 & Soó Rezső & BPU-03003.JPG \\
\hline 1133 & Daphne laureola L. & Gánt & 1949 & Soó Rezső & BPU-03005.JPG \\
\hline 1133 & Daphne laureola L. & Szentgál & 1932 & Boros Ádám & BPU-03004.JPG \\
\hline 1134 & Daphne mezereum L. & Bakonybél & 1955 & Soó Rezső & BPU-03001.JPG \\
\hline 1134 & Daphne mezereum L. & Bélapátfalva & 1948 & $\begin{array}{l}\text { Simon Tibor, Pólya } \\
\text { László, Jakucs Pál }\end{array}$ & BPU-02998.JPG \\
\hline 1134 & Daphne mezereum L. & Gyöngyös & 1964 & Soó Rezső & BPU-02999.JPG \\
\hline 1134 & Daphne mezereum L. & Jósvafő & 1949 & Jakucs Pál & BPU-02996.JPG \\
\hline 1134 & Daphne mezereum L. & Pálháza & 1952 & Simon Tibor & BPU-02997.JPG \\
\hline 1134 & Daphne mezereum L. & Szenna & 1957 & Soó Rezső & BPU-03000.JPG \\
\hline 1135 & $\begin{array}{l}\text { Thymelaea passerina (L.) Coss. et } \\
\text { Germ. }\end{array}$ & Dörgicse & 1957 & Felföldy Lajos & BPU-02986.JPG \\
\hline 1135 & $\begin{array}{l}\text { Thymelaea passerina (L.) Coss. et } \\
\text { Germ. }\end{array}$ & Nyíradony & 1932 & Soó Rezső & BPU-02987.JPG \\
\hline 1135 & $\begin{array}{l}\text { Thymelaea passerina (L.) Coss. et } \\
\text { Germ. }\end{array}$ & Pusztamiske & 1949 & Borsos Olga & BPU-02985.JPG \\
\hline 1139 & Hypericum humifusum L. & Szentgotthárd & 1955 & Soó Rezső & BPU-10948.JPG \\
\hline 1141 & Hypericum perforatum L. & Budapest & 1982 & Felföldy Lajos & BPU-10960.JPG \\
\hline 1141 & Hypericum perforatum L. & Budapest & 2004 & Felföldy Lajos & BPU-10959.JPG \\
\hline 1141 & Hypericum perforatum L. & Budapest & 2005 & Felföldy Lajos & BPU-10958.JPG \\
\hline 1141 & Hypericum perforatum L. & Nagyharsány & 1965 & $\begin{array}{l}\text { Simon Tibor, Priszter } \\
\text { Szaniszló }\end{array}$ & BPU-10961.JPG \\
\hline 1141 & Hypericum perforatum L. & Szentbékkálla & 1957 & Felföldy Lajos & BPU-10962.JPG \\
\hline 1141 & Hypericum perforatum L. & Tihany & 1959 & Felföldy Lajos & BPU-10963.JPG \\
\hline 1142 & Hypericum tetrapterum Fr. & Balatonszepezd & 1956 & $\begin{array}{l}\text { Felföldy Lajos, Tóth } \\
\text { László }\end{array}$ & BPU-10957.JPG \\
\hline 1142 & Hypericum tetrapterum Fr. & Balatonszőlős & 1957 & $\begin{array}{l}\text { Felföldy Lajos, Kovács } \\
\text { Margit, Tóth László }\end{array}$ & BPU-10956.JPG \\
\hline 1142 & Hypericum tetrapterum Fr. & Bátorliget & 1932 & Soó Rezső & BPU-10953.JPG \\
\hline 1142 & Hypericum tetrapterum Fr. & Kaszó & 1957 & Soó Rezső & BPU-10950.JPG \\
\hline 1142 & Hypericum tetrapterum Fr. & Kemence & 1952 & Simon Tibor & BPU-10954.JPG \\
\hline 1142 & Hypericum tetrapterum Fr. & Örvényes & 1958 & Felföldy Lajos & BPU-10955.JPG \\
\hline 1142 & Hypericum tetrapterum Fr. & Uzsa & 1952 & Simon Tibor & BPU-10949.JPG \\
\hline 1142 & Hypericum tetrapterum Fr. & Vászoly & 1958 & Felföldy Lajos & BPU-10952.JPG \\
\hline 1142 & Hypericum tetrapterum Fr. & Veresegyház & 1949 & Soó Rezső & BPU-10951.JPG \\
\hline 1144 & Hypericum dubium Leers & Sopron & 1947 & Kárpáti Zoltán & BPU-10970.JPG \\
\hline
\end{tabular}


1. Elektroniukus melléklet - Electronic Appendix 1.

\begin{tabular}{|c|c|c|c|c|c|}
\hline $\begin{array}{l}\text { Sorszám / } \\
\text { Number }\end{array}$ & Taxon-név / Taxon-name & $\begin{array}{l}\text { Település / } \\
\text { Settlement }\end{array}$ & $\begin{array}{l}\text { Év / } \\
\text { Year }\end{array}$ & Gyűjtő / Collector & $\begin{array}{l}\text { Fájlnév / } \\
\text { File-name }\end{array}$ \\
\hline 1145 & Hypericum hirsutum L. & Balatonszőlős & 1956 & $\begin{array}{l}\text { Felföldy Lajos, Tóth } \\
\text { László }\end{array}$ & BPU-10977.JPG \\
\hline 1145 & Hypericum hirsutum L. & Budapest & 1947 & Soó Rezső & BPU-10971.JPG \\
\hline 1145 & Hypericum hirsutum L. & Gyöngyös & 1952 & Soó Rezső & BPU-10973.JPG \\
\hline 1145 & Hypericum hirsutum L. & Nagybajom & 1955 & Soó Rezső & BPU-10975.JPG \\
\hline 1145 & Hypericum hirsutum L. & Nagykovácsi & 2005 & Felföldy Lajos & BPU-10974.JPG \\
\hline 1145 & Hypericum hirsutum L. & Szilvásvárad & 1953 & $\begin{array}{l}\text { Felföldy Lajos, Tóth } \\
\text { László }\end{array}$ & BPU-10972.JPG \\
\hline 1145 & Hypericum hirsutum L. & Vászoly & 1958 & Felföldy Lajos & BPU-10978.JPG \\
\hline 1147 & Hypericum montanum L. & Alsószölnök & 1955 & Soó Rezső & BPU-10979.JPG \\
\hline 1147 & Hypericum montanum L. & $\begin{array}{l}\text { Badacsonytomaj, } \\
\text { Badacsonytördemic }\end{array}$ & 1928 & Soó Rezső & BPU-10985.JPG \\
\hline 1147 & Hypericum montanum L. & Badacsonytördemic & 1953 & $\begin{array}{l}\text { Felföldy Lajos, Tóth } \\
\text { László }\end{array}$ & BPU-10986.JPG \\
\hline 1147 & Hypericum montanum L. & Kemence & 1952 & Simon Tibor & BPU-10984.JPG \\
\hline 1147 & Hypericum montanum L. & Mátraszentimre & 1961 & Soó Rezső & BPU-10987.JPG \\
\hline 1147 & Hypericum montanum L. & Miskolc & 1950 & Soó Rezső & BPU-10980.JPG \\
\hline 1147 & Hypericum montanum L. & Szögliget & 1951 & $\begin{array}{l}\text { Soó Rezső, Jakucs Pál, Ér } \\
\text { Lajos }\end{array}$ & BPU-10981.JPG \\
\hline 1148 & Hypericum elegans Stephan ex Willd. & Bélapátfalva & 1934 & Boros Ádám & BPU-10988.JPG \\
\hline 1150 & Viola tricolor L. & Bagamér & 1933 & Soó Rezső & BPU-07400.JPG \\
\hline 1150 & Viola tricolor L. & Bélapátfalva & 1948 & $\begin{array}{l}\text { Simon Tibor, Pólya } \\
\text { László, Jakucs Pál }\end{array}$ & BPU-07413.JPG \\
\hline 1150 & Viola tricolor L. & Bélapátfalva & 1948 & $\begin{array}{l}\text { Simon Tibor, Pólya } \\
\text { László, Jakucs Pál }\end{array}$ & BPU-07414.JPG \\
\hline 1150 & Viola tricolor L. & Bélapátfalva & 1948 & $\begin{array}{l}\text { Simon Tibor, Pólya } \\
\text { László, Jakucs Pál }\end{array}$ & BPU-07415.JPG \\
\hline 1150 & Viola tricolor L. & Bélapátfalva & 1953 & Simon Tibor & BPU-07412.JPG \\
\hline 1150 & Viola tricolor L. & Beregsurány & 1951 & $\begin{array}{l}\text { Simon Tibor, Vozáry } \\
\text { Elemér }\end{array}$ & BPU-07419.JPG \\
\hline 1150 & Viola tricolor L. & Csaroda & 1951 & Simon Tibor & BPU-07411.JPG \\
\hline 1150 & Viola tricolor L. & Debrecen & 1933 & Soó Rezső & BPU-07399.JPG \\
\hline 1150 & Viola tricolor L. & Füzér & 1938 & Soó Rezső & BPU-07395.JPG \\
\hline 1150 & Viola tricolor L. & Füzér & 1949 & Simon Tibor, Jakucs Pál & BPU-07416.JPG \\
\hline 1150 & Viola tricolor L. & Füzér & 1949 & Simon Tibor, Jakucs Pál & BPU-07417.JPG \\
\hline 1150 & Viola tricolor L. & Gáborján & 1926 & Kovács L. & BPU-07402.JPG \\
\hline 1150 & Viola tricolor L. & Gáborján & 1939 & Kovács L. & BPU-07398.JPG \\
\hline 1150 & Viola tricolor L. & Gyöngyös & 1953 & Soó Rezső & BPU-07405.JPG \\
\hline 1150 & Viola tricolor L. & Gyöngyös & 1963 & Soó Rezső & BPU-07401.JPG \\
\hline 1150 & Viola tricolor L. & Hajdúbagos & 1933 & Soó Rezső & BPU-07396.JPG \\
\hline 1150 & Viola tricolor L. & Háromhuta & 1960 & Simon Tibor & BPU-07392.JPG \\
\hline 1150 & Viola tricolor L. & Hosszúpályi & 1933 & Soó Rezső & BPU-07404.JPG \\
\hline 1150 & Viola tricolor L. & Miskolc & 1930 & Soó Rezső & BPU-07393.JPG \\
\hline 1150 & Viola tricolor L. & Miskolc & 1937 & Soó Rezső & BPU-07394.JPG \\
\hline 1150 & Viola tricolor L. & Szilvásvárad & 1953 & $\begin{array}{l}\text { Felföldy Lajos, Tóth } \\
\text { László }\end{array}$ & BPU-07423.JPG \\
\hline 1150 & Viola tricolor L. & Szurdokpüspöki & 1937 & Máthé Imre & BPU-07406.JPG \\
\hline 1150 & Viola tricolor L. & Tokaj & 1933 & Soó Rezső & BPU-07443.JPG \\
\hline 1151 & Viola arvensis Murray & Bátorliget & 1928 & Soó Rezső & BPU-07433.JPG \\
\hline 1151 & Viola arvensis Murray & Beregdaróc & 1951 & $\begin{array}{l}\text { Simon Tibor, Vozáry } \\
\text { Elemér }\end{array}$ & BPU-07427.JPG \\
\hline 1151 & Viola arvensis Murray & Budapest & 2005 & Felföldy Lajos & BPU-07424.JPG \\
\hline
\end{tabular}


1. Elektroniukus melléklet - Electronic Appendix 1.

\begin{tabular}{|c|c|c|c|c|c|}
\hline $\begin{array}{l}\text { Sorszám / } \\
\text { Number }\end{array}$ & Taxon-név / Taxon-name & $\begin{array}{l}\text { Település / } \\
\text { Settlement }\end{array}$ & $\begin{array}{l}\text { Év / } \\
\text { Year }\end{array}$ & Gyűjtő / Collector & $\begin{array}{l}\text { Fájlnév / } \\
\text { File-name }\end{array}$ \\
\hline 1151 & Viola arvensis Murray & Csaroda & 1951 & $\begin{array}{l}\text { Simon Tibor, Vozáry } \\
\text { Elemér }\end{array}$ & BPU-07426.JPG \\
\hline 1151 & Viola arvensis Murray & Debrecen & 1943 & Felföldy Lajos & BPU-07429.JPG \\
\hline 1151 & Viola arvensis Murray & Gáborján & 1939 & Kovács L. & BPU-07432.JPG \\
\hline 1151 & Viola arvensis Murray & Mór & 1953 & Felföldy Lajos & BPU-07428.JPG \\
\hline 1151 & Viola arvensis Murray & Regéc & 1963 & Isépy István & BPU-07425.JPG \\
\hline 1151 & Viola arvensis Murray & Sáránd & 1932 & Soó Rezső & BPU-07431.JPG \\
\hline 1151 & Viola arvensis Murray & Tihany & 1942 & Felföldy Lajos & BPU-07430.JPG \\
\hline 1151 & Viola arvensis Murray & Tihany & 1958 & Felföldy Lajos & BPU-07434.JPG \\
\hline 1151 & Viola arvensis Murray & Tihany & 1958 & Felföldy Lajos & BPU-07435.JPG \\
\hline 1151 & Viola arvensis Murray & Uzsa & 1959 & $\begin{array}{l}\text { Jávorka Sándor, Felföldy } \\
\text { Lajos }\end{array}$ & BPU-07436.JPG \\
\hline 1152 & Viola kitaibeliana Roem. et Schult. & Bagamér & 1933 & Soó Rezső & BPU-07444.JPG \\
\hline 1152 & Viola kitaibeliana Roem. et Schult. & Budapest & 1989 & Felföldy Lajos & BPU-07439.JPG \\
\hline 1152 & Viola kitaibeliana Roem. et Schult. & Csévharaszt & 1959 & Simon Tibor, Borsos Olga & BPU-07447.JPG \\
\hline 1152 & Viola kitaibeliana Roem. et Schult. & Debrecen & 1933 & Soó Rezső & BPU-07442.JPG \\
\hline 1152 & Viola kitaibeliana Roem. et Schult. & Hajdúbagos & 1933 & Soó Rezső & BPU-07441.JPG \\
\hline 1152 & Viola kitaibeliana Roem. et Schult. & Miskolc & 1929 & Soó Rezső & BPU-07446.JPG \\
\hline 1152 & Viola kitaibeliana Roem. et Schult. & Nagyharsány & 1965 & $\begin{array}{l}\text { Simon Tibor, Priszter } \\
\text { Szaniszló, Borhidi Attila }\end{array}$ & BPU-07448.JPG \\
\hline 1152 & Viola kitaibeliana Roem. et Schult. & Tihany & 1959 & Felföldy Lajos & BPU-07440.JPG \\
\hline 1152 & Viola kitaibeliana Roem. et Schult. & Tihany & 1982 & Felföldy Lajos & BPU-07445.JPG \\
\hline 1152 & Viola kitaibeliana Roem. et Schult. & Vácrátót & 1953 & Felföldy Lajos & BPU-07449.JPG \\
\hline 1153 & Viola alba Besser & Gyöngyös & 1964 & Soó Rezső & BPU-07274.JPG \\
\hline 1153 & Viola alba Besser & Győr & - & Polgár Sándor & BPU-07278.JPG \\
\hline 1153 & Viola alba Besser & Hidegkút & 1959 & Felföldy Lajos & BPU-07279.JPG \\
\hline 1153 & Viola alba Besser & Makkoshotyka & 1955 & Simon Tibor & BPU-07276.JPG \\
\hline 1153 & Viola alba Besser & Pilisszentiván & 1949 & Soó Rezső & BPU-07275.JPG \\
\hline 1154 & Viola suavis M. Bieb. & Bakonypölöske & 1962 & Simon Tibor, Borsos Olga & BPU-07288.JPG \\
\hline 1154 & Viola suavis M. Bieb. & Balatonfüred & 1959 & Felföldy Lajos & BPU-07289.JPG \\
\hline 1154 & Viola suavis M. Bieb. & Biatorbágy & 1992 & Felföldy Lajos & BPU-07286.JPG \\
\hline 1154 & Viola suavis M. Bieb. & Budaörs & 1982 & Felföldy Lajos & BPU-07287.JPG \\
\hline 1154 & Viola suavis M. Bieb. & Budapest & 1961 & Isépy István & BPU-07280.JPG \\
\hline 1154 & Viola suavis M. Bieb. & Budapest & 1982 & Felföldy Lajos & BPU-07257.JPG \\
\hline 1154 & Viola suavis M. Bieb. & Debrecen & 1934 & Soó Rezső & BPU-07282.JPG \\
\hline 1154 & Viola suavis M. Bieb. & Debrecen & 1934 & Soó Rezső & BPU-07283.JPG \\
\hline 1154 & Viola suavis M. Bieb. & Debrecen & 1943 & Felföldy Lajos & BPU-07466.JPG \\
\hline 1154 & Viola suavis M. Bieb. & Gyöngyös & 1964 & Soó Rezső & BPU-07284.JPG \\
\hline 1154 & Viola suavis M. Bieb. & Gyula & 1935 & Soó Rezső & BPU-07281.JPG \\
\hline 1154 & Viola suavis M. Bieb. & Hidegkút & 1958 & Felföldy Lajos & BPU-07291.JPG \\
\hline 1154 & Viola suavis M. Bieb. & Hidegkút & 1959 & Felföldy Lajos & BPU-07290.JPG \\
\hline 1154 & Viola suavis M. Bieb. & Hidegkút & 1959 & Felföldy Lajos & BPU-07292.JPG \\
\hline 1154 & Viola suavis M. Bieb. & Mosonmagyaróvár & 1962 & Borsos Olga & BPU-07285.JPG \\
\hline 1154 & Viola suavis M. Bieb. & Tihany & 1946 & Felföldy Lajos & BPU-07294.JPG \\
\hline 1154 & Viola suavis M. Bieb. & Tihany & 1958 & Felföldy Lajos & BPU-07293.JPG \\
\hline 1155 & Viola odorata L. & Balatonszőlős & 1959 & Felföldy Lajos & BPU-07265.JPG \\
\hline 1155 & Viola odorata L. & Balatonszőlős & 1959 & Felföldy Lajos & BPU-07266.JPG \\
\hline 1155 & Viola odorata L. & Balatonszőlős & 1959 & Felföldy Lajos & BPU-07267.JPG \\
\hline 1155 & Viola odorata L. & Balatonszőlős & 1959 & Felföldy Lajos & BPU-07268.JPG \\
\hline
\end{tabular}


1. Elektroniukus melléklet - Electronic Appendix 1.

\begin{tabular}{|c|c|c|c|c|c|}
\hline $\begin{array}{l}\text { Sorszám / } \\
\text { Number }\end{array}$ & Taxon-név / Taxon-name & $\begin{array}{l}\text { Település / } \\
\text { Settlement }\end{array}$ & $\begin{array}{l}\text { Év / } \\
\text { Year }\end{array}$ & Gyújtő / Collector & $\begin{array}{l}\text { Fájlnév / } \\
\text { File-name }\end{array}$ \\
\hline 1155 & Viola odorata L. & Budapest & 1992 & Felföldy Lajos & BPU-07256.JPG \\
\hline 1155 & Viola odorata L. & Debrecen & 1939 & Soó Rezső & BPU-07262.JPG \\
\hline 1155 & Viola odorata L. & Gánt & 1949 & Soó Rezső & BPU-07259.JPG \\
\hline 1155 & Viola odorata L. & Gánt & 1949 & Soó Rezső & BPU-07260.JPG \\
\hline 1155 & Viola odorata L. & Gánt & 1949 & Soó Rezső & BPU-07261.JPG \\
\hline 1155 & Viola odorata L. & Hidegkút & 1958 & Felföldy Lajos & BPU-07269.JPG \\
\hline 1155 & Viola odorata L. & Hidegkút & 1959 & Felföldy Lajos & BPU-07270.JPG \\
\hline 1155 & Viola odorata L. & Hidegkút & 1959 & Felföldy Lajos & BPU-07271.JPG \\
\hline 1155 & Viola odorata L. & Jósvafő & 1952 & Kulcsár Gábor & BPU-07258.JPG \\
\hline 1155 & Viola odorata L. & Nagykovácsi & 1949 & Soó Rezső & BPU-07263.JPG \\
\hline 1155 & Viola odorata L. & Pécsely & 1959 & Felföldy Lajos & BPU-07272.JPG \\
\hline 1155 & Viola odorata L. & Szamosszeg & 1948 & $\begin{array}{l}\text { Simon Tibor, Pólya } \\
\text { László, Jakucs Pál }\end{array}$ & BPU-07264.JPG \\
\hline 1155 & Viola odorata L. & Tihany & 1957 & Felföldy Lajos & BPU-07273.JPG \\
\hline 1156 & Viola mirabilis L. & Balatonszőlős & 1958 & Felföldy Lajos & BPU-07322.JPG \\
\hline 1156 & Viola mirabilis L. & Bátorliget & 1932 & Soó Rezső & BPU-07321.JPG \\
\hline 1156 & Viola mirabilis L. & Budapest & 1949 & Soó Rezső & BPU-07315.JPG \\
\hline 1156 & Viola mirabilis L. & Budapest & 1964 & Soó Rezső & BPU-07313.JPG \\
\hline 1156 & Viola mirabilis L. & Budapest & 1982 & Felföldy Lajos & BPU-07314.JPG \\
\hline 1156 & Viola mirabilis L. & Dávod & 1957 & Soó Rezső & BPU-07320.JPG \\
\hline 1156 & Viola mirabilis L. & Debrecen & 1949 & Soó Rezső & BPU-07317.JPG \\
\hline 1156 & Viola mirabilis L. & Gánt & 1949 & Soó Rezső & BPU-07316.JPG \\
\hline 1156 & Viola mirabilis L. & Hidegkút & 1959 & Felföldy Lajos & BPU-07323.JPG \\
\hline 1156 & Viola mirabilis L. & Mosonmagyaróvár & 1965 & Borsos Olga & BPU-07318.JPG \\
\hline 1156 & Viola mirabilis L. & Pécsely & 1959 & Felföldy Lajos & BPU-07324.JPG \\
\hline 1156 & Viola mirabilis L. & Várpalota & 1955 & Soó Rezső & BPU-07325.JPG \\
\hline 1156 & Viola mirabilis L. & Velence & 1992 & Felföldy Lajos & BPU-07319.JPG \\
\hline 1158 & Viola hirta L. & Bagamér & 1949 & Soó Rezső & BPU-07300.JPG \\
\hline 1158 & Viola hirta L. & Bátorliget & 1934 & Soó Rezső & BPU-07306.JPG \\
\hline 1158 & Viola hirta L. & Budapest & 1961 & Isépy István & BPU-07295.JPG \\
\hline 1158 & Viola hirta L. & Budapest & 1982 & Felföldy Lajos & BPU-07298.JPG \\
\hline 1158 & Viola hirta L. & Budapest & 1990 & Felföldy Lajos & BPU-07296.JPG \\
\hline 1158 & Viola hirta L. & Budapest & 1990 & Felföldy Lajos & BPU-07297.JPG \\
\hline 1158 & Viola hirta L. & Debrecen & 1932 & Soó Rezső & BPU-07303.JPG \\
\hline 1158 & Viola hirta L. & Debrecen & 1934 & Soó Rezső & BPU-07302.JPG \\
\hline 1158 & Viola hirta L. & Gyöngyös & 1964 & Soó Rezső & BPU-07304.JPG \\
\hline 1158 & Viola hirta L. & Győr & 1909 & Polgár Sándor & BPU-07301.JPG \\
\hline 1158 & Viola hirta L. & Hidegkút & 1959 & Felföldy Lajos & BPU-07307.JPG \\
\hline 1158 & Viola hirta L. & Hidegkút & 1959 & Felföldy Lajos & BPU-07308.JPG \\
\hline 1158 & Viola hirta L. & Hidegkút & 1959 & Felföldy Lajos & BPU-07309.JPG \\
\hline 1158 & Viola hirta L. & Jósvafő & 1952 & Kulcsár Gábor & BPU-07299.JPG \\
\hline 1158 & Viola hirta L. & Üröm & 1991 & Felföldy Lajos & BPU-07305.JPG \\
\hline 1159 & Viola ambigua Waldst. et Kit. & Budaörs & 1982 & Felföldy Lajos & BPU-07312.JPG \\
\hline 1159 & Viola ambigua Waldst. et Kit. & Budapest & 1943 & Kárpáti Zoltán & BPU-07310.JPG \\
\hline 1159 & Viola ambigua Waldst. et Kit. & Budapest & 1963 & Isépy István & BPU-07311.JPG \\
\hline 1159 & Viola ambigua Waldst. et Kit. & Tihany & 1958 & Felföldy Lajos & BPU-07462.JPG \\
\hline 1160 & Viola rupestris F.W. Schmidt & Bagamér & 1949 & Soó Rezső & BPU-07329.JPG \\
\hline 1160 & Viola rupestris F.W. Schmidt & Pilisszentiván & 1949 & Soó Rezső & BPU-07328.JPG \\
\hline
\end{tabular}


1. Elektroniukus melléklet - Electronic Appendix 1.

\begin{tabular}{|c|c|c|c|c|c|}
\hline $\begin{array}{l}\text { Sorszám / } \\
\text { Number }\end{array}$ & Taxon-név / Taxon-name & $\begin{array}{l}\text { Település / } \\
\text { Settlement }\end{array}$ & $\begin{array}{l}\text { Év / } \\
\text { Year }\end{array}$ & Gyújtő / Collector & $\begin{array}{l}\text { Fájlnév / } \\
\text { File-name }\end{array}$ \\
\hline 1160 & Viola rupestris F.W. Schmidt & Pilisszentiván & 1949 & Soó Rezső & BPU-07330.JPG \\
\hline 1160 & Viola rupestris F.W. Schmidt & Sopron & 1944 & Kárpáti Zoltán & BPU-07333.JPG \\
\hline 1160 & Viola rupestris F.W. Schmidt & Vácrátót & 1950 & Soó Rezső & BPU-07330.JPG \\
\hline 1161 & Viola reichenbachiana Jord. & "Cuha-völgy" & 1954 & Soó Rezső & BPU-07334.JPG \\
\hline 1161 & Viola reichenbachiana Jord. & Balatonszőlős & 1959 & Felföldy Lajos & BPU-07348.JPG \\
\hline 1161 & Viola reichenbachiana Jord. & Bátorliget & 1942 & Máthé Imre & BPU-07339.JPG \\
\hline 1161 & Viola reichenbachiana Jord. & Dömös & 1965 & Borsos Olga & BPU-07358.JPG \\
\hline 1161 & Viola reichenbachiana Jord. & Gyöngyös & 1952 & Soó Rezső & BPU-07341.JPG \\
\hline 1161 & Viola reichenbachiana Jord. & Háromhuta & 1949 & Simon Tibor, Jakucs Pál & BPU-07351.JPG \\
\hline 1161 & Viola reichenbachiana Jord. & Hidegkút & 1958 & Felföldy Lajos & BPU-07344.JPG \\
\hline 1161 & Viola reichenbachiana Jord. & Hidegkút & 1959 & Felföldy Lajos & BPU-07349.JPG \\
\hline 1161 & Viola reichenbachiana Jord. & Hidegkút & 1959 & Felföldy Lajos & BPU-07350.JPG \\
\hline 1161 & Viola reichenbachiana Jord. & Kapolcs & 1959 & $\begin{array}{l}\text { Jávorka Sándor, Felföldy } \\
\text { Lajos, Tóth László }\end{array}$ & BPU-07353.JPG \\
\hline 1161 & Viola reichenbachiana Jord. & Lábatlan & 1953 & Felföldy Lajos & BPU-07342.JPG \\
\hline 1161 & Viola reichenbachiana Jord. & Miskolc & 1937 & Soó Rezső & BPU-07346.JPG \\
\hline 1161 & Viola reichenbachiana Jord. & Pilisszentiván & 1963 & Isépy István & BPU-07340.JPG \\
\hline 1161 & Viola reichenbachiana Jord. & Sáránd & 1932 & Soó Rezső & BPU-07347.JPG \\
\hline 1161 & Viola reichenbachiana Jord. & Szilvásvárad & 1953 & $\begin{array}{l}\text { Felföldy Lajos, Tóth } \\
\text { László }\end{array}$ & BPU-07343.JPG \\
\hline 1161 & Viola reichenbachiana Jord. & Uzsa & 1959 & $\begin{array}{l}\text { Jávorka Sándor, Felföldy } \\
\text { Lajos }\end{array}$ & BPU-07352.JPG \\
\hline 1162 & Viola riviniana Rchb. & Beregdaróc & 1953 & $\begin{array}{l}\text { Simon Tibor, Helmeczy } \\
\text { Balázs }\end{array}$ & BPU-07338.JPG \\
\hline 1162 & Viola riviniana Rchb. & Csesznek & 1953 & $\begin{array}{l}\text { Kulcsár Gábor, Vozáry } \\
\text { Elemér }\end{array}$ & BPU-07359.JPG \\
\hline 1162 & Viola riviniana Rchb. & Debrecen & 1923 & Soó Rezső & BPU-07354.JPG \\
\hline 1162 & Viola riviniana Rchb. & Háromhuta & 1939 & Soó Rezső & BPU-07355.JPG \\
\hline 1162 & Viola riviniana Rchb. & Sopron & 1960 & Borsos Olga, Simon Tibor & BPU-07357.JPG \\
\hline 1163 & Viola elatior Fr. & "Szigetköz" & 1931 & Soó Rezső & BPU-07377.JPG \\
\hline 1163 & Viola elatior Fr. & Bátorliget & 1950 & $\begin{array}{l}\text { Simon Tibor, Kárpáti } \\
\text { István }\end{array}$ & BPU-07381.JPG \\
\hline 1163 & Viola elatior Fr. & Bátorliget & 1951 & Simon Tibor & BPU-07379.JPG \\
\hline 1163 & Viola elatior Fr. & Bátorliget & 1953 & Simon Tibor & BPU-07380.JPG \\
\hline 1163 & Viola elatior Fr. & Devecser & 1957 & Tallós Pál & BPU-07382.JPG \\
\hline 1163 & Viola elatior Fr. & Szigetújfalu & 1943 & Kárpáti Zoltán & BPU-07378.JPG \\
\hline 1163 & Viola elatior Fr. & Tarpa & 1948 & Soó Rezső & BPU-07376.JPG \\
\hline 1164 & Viola canina L. & Bakonyszentlászló & 1929 & Soó Rezső & BPU-07369.JPG \\
\hline 1164 & Viola canina L. & Bozsok & 1938 & Soó Rezső & BPU-07361.JPG \\
\hline 1164 & Viola canina L. & Devecser & 1957 & Tallós Pál & BPU-07375.JPG \\
\hline 1164 & Viola canina L. & Egyek & 1932 & Soó Rezső & BPU-07371.JPG \\
\hline 1164 & Viola canina L. & Kapolcs & 1959 & $\begin{array}{l}\text { Jávorka Sándor, Felföldy } \\
\text { Lajos, Tóth László }\end{array}$ & BPU-07374.JPG \\
\hline 1164 & Viola canina L. & Mátraszentimre & 1961 & Soó Rezső & BPU-07372.JPG \\
\hline 1164 & Viola canina L. & Nyíradony & 1937 & Soó Rezső & BPU-07367.JPG \\
\hline 1164 & Viola canina L. & Nyíradony & 1951 & Simon Tibor & BPU-07373.JPG \\
\hline 1164 & Viola canina L. & Tákos & 1948 & $\begin{array}{l}\text { Pólya László, Simon } \\
\text { Tibor, Jakucs Pál }\end{array}$ & BPU-07360.JPG \\
\hline 1164 & Viola canina L. & Tarpa & 1948 & $\begin{array}{l}\text { Pólya László, Simon } \\
\text { Tibor, Jakucs Pál }\end{array}$ & BPU-07366.JPG \\
\hline 1164 & Viola canina L. & Uzsa & 1959 & $\begin{array}{l}\text { Jávorka Sándor, Felföldy } \\
\text { Lajos }\end{array}$ & BPU-07362.JPG \\
\hline
\end{tabular}


1. Elektroniukus melléklet - Electronic Appendix 1.

\begin{tabular}{|c|c|c|c|c|c|}
\hline $\begin{array}{l}\text { Sorszám / } \\
\text { Number }\end{array}$ & Taxon-név / Taxon-name & $\begin{array}{l}\text { Település / } \\
\text { Settlement }\end{array}$ & $\begin{array}{l}\text { Év / } \\
\text { Year }\end{array}$ & Gyújtő / Collector & $\begin{array}{l}\text { Fájlnév / } \\
\text { File-name }\end{array}$ \\
\hline 1164 & Viola canina L. & Uzsa & 1959 & $\begin{array}{l}\text { Jávorka Sándor, Felföldy } \\
\text { Lajos }\end{array}$ & BPU-07363.JPG \\
\hline 1165 & Viola pumila Chaix & Aszófő & 1950 & Felföldy Lajos & BPU-07387.JPG \\
\hline 1165 & Viola pumila Chaix & Miskolc & 1955 & Tóth László & BPU-07388.JPG \\
\hline 1165 & Viola pumila Chaix & Ócsa & 1959 & Simon Tibor & BPU-07385.JPG \\
\hline 1165 & Viola pumila Chaix & Szarvas & 1949 & Soó Rezső & BPU-07383.JPG \\
\hline 1165 & Viola pumila Chaix & Tákos & 1948 & $\begin{array}{l}\text { Pólya László, Simon } \\
\text { Tibor, Jakucs Pál }\end{array}$ & BPU-07386.JPG \\
\hline 1165 & Viola pumila Chaix & Tarpa & 1949 & $\begin{array}{l}\text { Soó Rezső, Simon Tibor, } \\
\text { Jakucs Pál }\end{array}$ & BPU-07384.JPG \\
\hline 1166 & Viola stagnina Kit. & Beregdaróc & 1951 & Simon Tibor & BPU-07391.JPG \\
\hline 1166 & Viola stagnina Kit. & Csaroda & 1951 & $\begin{array}{l}\text { Simon Tibor, Vozáry } \\
\text { Elemér }\end{array}$ & BPU-07390.JPG \\
\hline 1166 & Viola stagnina Kit. & Penészlek & 1932 & Soó Rezső & BPU-07389.JPG \\
\hline $\begin{array}{c}1153 \times \\
1155\end{array}$ & Viola alba Besser $\times$ Viola odorata L. & Hidegkút & 1959 & Felföldy Lajos & BPU-07464.JPG \\
\hline $\begin{array}{c}1153 \times \\
1155\end{array}$ & Viola alba Besser $\times$ Viola odorata $L$ & Kesztölc & 1943 & Kárpáti Zoltán & BPU-07467.JPG \\
\hline $\begin{array}{c}1153 \times \\
1155\end{array}$ & Viola alba Besser $\times$ Viola odorata L. & Pannonhalma & 1917 & Polgár Sándor & BPU-07471.JPG \\
\hline $\begin{array}{c}1153 \times \\
1158\end{array}$ & Viola alba Besser $\times$ Viola hirta $L$. & Écs & 1931 & Pető & BPU-07470.JPG \\
\hline $\begin{array}{c}1153 \times \\
1158\end{array}$ & Viola alba Besser × Viola hirta L. & Hidegkút & 1959 & Felföldy Lajos & BPU-07465.JPG \\
\hline $\begin{array}{c}1155 \times \\
1158\end{array}$ & Viola $\times$ scabra F. Braun & Hidegkút & 1959 & Felföldy Lajos & BPU-07463.JPG \\
\hline $\begin{array}{c}1162 \times \\
1164\end{array}$ & Viola riviniana Rchb. $\times$ Viola canina $\mathrm{L}$ & Kőszeg & 1895 & Waisbecker Antal & BPU-07469.JPG \\
\hline 1167 & Helianthemum canum (L.) Hornem. & Balatonfüred & 1959 & Felföldy Lajos & BPU-07212.JPG \\
\hline 1167 & Helianthemum canum (L.) Hornem. & Fertőrákos & 1933 & Polgár Sándor & BPU-07211.JPG \\
\hline 1167 & Helianthemum canum (L.) Hornem. & Nagyharsány & 1965 & $\begin{array}{l}\text { Simon Tibor, Priszter } \\
\text { Szaniszló, Borhidi Attila }\end{array}$ & BPU-07208.JPG \\
\hline 1167 & Helianthemum canum (L.) Hornem. & Nagykovácsi & 1963 & Isépy István & BPU-07209.JPG \\
\hline 1167 & Helianthemum canum (L.) Hornem. & Telki & 1947 & Soó Rezső & BPU-07210.JPG \\
\hline 1168 & Helianthemum nummularium (L.) Mill. & Eplény & 1955 & Soó Rezső & BPU-07229.JPG \\
\hline 1168 & Helianthemum nummularium (L.) Mill. & Hajmáskér & 1951 & Felföldy Lajos & BPU-07230.JPG \\
\hline 1168 & Helianthemum nummularium (L.) Mill. & Kiskunhalas & 1964 & Soó Rezső & BPU-07227.JPG \\
\hline 1168 & Helianthemum nummularium (L.) Mill. & Monostorapáti & 1959 & $\begin{array}{l}\text { Jávorka Sándor, Felföldy } \\
\text { Lajos, Tóth László }\end{array}$ & BPU-07231.JPG \\
\hline 1168 & Helianthemum nummularium (L.) Mill. & Nagyharsány & 1956 & Soó Rezső & BPU-07228.JPG \\
\hline 1168 & Helianthemum nummularium (L.) Mill. & Tihany & 1928 & Soó Rezső & BPU-07234.JPG \\
\hline 1168 & Helianthemum nummularium (L.) Mill. & Tihany & 1959 & Felföldy Lajos & BPU-07232.JPG \\
\hline 1168 & Helianthemum nummularium (L.) Mill. & Tihany & 1959 & Felföldy Lajos & BPU-07233.JPG \\
\hline 1168 & Helianthemum nummularium (L.) Mill. & Uzsa & 1952 & Borsos Olga & BPU-07235.JPG \\
\hline 1168 & Helianthemum nummularium (L.) Mill. & Uzsa & 1959 & $\begin{array}{l}\text { Jávorka Sándor, Felföldy } \\
\text { Lajos }\end{array}$ & BPU-07236.JPG \\
\hline 1169 & $\begin{array}{l}\text { Helianthemum ovatum (Viv.) Dunal in } \\
\text { DC. }\end{array}$ & Budaörs & 1982 & Felföldy Lajos & BPU-07221.JPG \\
\hline 1169 & $\begin{array}{l}\text { Helianthemum ovatum (Viv.) Dunal in } \\
\text { DC. }\end{array}$ & Budapest & 2005 & Felföldy Lajos & BPU-07215.JPG \\
\hline 1169 & $\begin{array}{l}\text { Helianthemum ovatum (Viv.) Dunal in } \\
\text { DC. }\end{array}$ & Debrecen & 1934 & Juhász Lajos & BPU-07219.JPG \\
\hline 1169 & $\begin{array}{l}\text { Helianthemum ovatum (Viv.) Dunal in } \\
\text { DC. }\end{array}$ & Eplény & 1955 & Soó Rezső & BPU-07224.JPG \\
\hline
\end{tabular}


1. Elektroniukus melléklet - Electronic Appendix 1.

\begin{tabular}{|c|c|c|c|c|c|}
\hline $\begin{array}{l}\text { Sorszám / } \\
\text { Number }\end{array}$ & Taxon-név / Taxon-name & $\begin{array}{l}\text { Település / } \\
\text { Settlement }\end{array}$ & $\begin{array}{l}\text { Év / } \\
\text { Year }\end{array}$ & Gyűjtő / Collector & $\begin{array}{l}\text { Fájlnév / } \\
\text { File-name }\end{array}$ \\
\hline 1169 & $\begin{array}{l}\text { Helianthemum ovatum (Viv.) Dunal in } \\
\text { DC. }\end{array}$ & Háromhuta & 1949 & Simon Tibor, Jakucs Pál & BPU-07225.JPG \\
\hline 1169 & $\begin{array}{l}\text { Helianthemum ovatum (Viv.) Dunal in } \\
\text { DC. }\end{array}$ & Nagyharsány & 1965 & $\begin{array}{l}\text { Simon Tibor, Priszter } \\
\text { Szaniszló }\end{array}$ & BPU-07217.JPG \\
\hline 1169 & $\begin{array}{l}\text { Helianthemum ovatum (Viv.) Dunal in } \\
\text { DC. }\end{array}$ & Pécs & 1953 & Soó Rezső, Borsos Olga & BPU-07218.JPG \\
\hline 1169 & $\begin{array}{l}\text { Helianthemum ovatum (Viv.) Dunal in } \\
\text { DC. }\end{array}$ & Szigetmonostor & 1947 & Soó Rezső & BPU-07222.JPG \\
\hline 1169 & $\begin{array}{l}\text { Helianthemum ovatum (Viv.) Dunal in } \\
\text { DC. }\end{array}$ & Szilvásvárad & 1953 & $\begin{array}{l}\text { Felföldy Lajos, Tóth } \\
\text { László }\end{array}$ & BPU-07220.JPG \\
\hline 1169 & $\begin{array}{l}\text { Helianthemum ovatum (Viv.) Dunal in } \\
\text { DC. }\end{array}$ & Telkibánya & 1952 & Simon Tibor & BPU-07216.JPG \\
\hline 1169 & $\begin{array}{l}\text { Helianthemum ovatum (Viv.) Dunal in } \\
\text { DC. }\end{array}$ & Vác & 1949 & $\begin{array}{l}\text { Soó Rezső, Simon Tibor, } \\
\text { Jakucs Pál }\end{array}$ & BPU-07223.JPG \\
\hline 1170 & $\begin{array}{l}\text { Fumana procumbens (Dunal) Gren. et } \\
\text { Godr. }\end{array}$ & "Szentendrei-sziget" & 1953 & Borsos Olga & BPU-07247.JPG \\
\hline 1170 & $\begin{array}{l}\text { Fumana procumbens (Dunal) Gren. et } \\
\text { Godr. }\end{array}$ & Budapest & 1947 & Soó Rezső & BPU-07241.JPG \\
\hline 1170 & $\begin{array}{l}\text { Fumana procumbens (Dunal) Gren. et } \\
\text { Godr. }\end{array}$ & Csévharaszt & 1959 & Simon Tibor, Borsos Olga & BPU-07244.JPG \\
\hline 1170 & $\begin{array}{l}\text { Fumana procumbens (Dunal) Gren. et } \\
\text { Godr. }\end{array}$ & Gánt & 1948 & Soó Rezső & BPU-07243.JPG \\
\hline 1170 & $\begin{array}{l}\text { Fumana procumbens (Dunal) Gren. et } \\
\text { Godr. }\end{array}$ & Nagykovácsi & 1962 & Isépy István & BPU-07245.JPG \\
\hline 1170 & $\begin{array}{l}\text { Fumana procumbens (Dunal) Gren. et } \\
\text { Godr. }\end{array}$ & Pécs & 1925 & Soó Rezső & BPU-07242.JPG \\
\hline 1170 & $\begin{array}{l}\text { Fumana procumbens (Dunal) Gren. et } \\
\text { Godr. }\end{array}$ & Szigetmonostor & 1947 & Soó Rezső & BPU-07246.JPG \\
\hline 1175 & Elatine alsinastrum L. & Dörgicse & 1957 & Felföldy Lajos & BPU-07477.JPG \\
\hline 1175 & Elatine alsinastrum L. & Egyek & 1947 & Soó Rezső & BPU-07474.JPG \\
\hline 1175 & Elatine alsinastrum L. & Egyek & 1950 & $\begin{array}{l}\text { Simon Tibor, Borsos Olga, } \\
\text { Kulcsár Gábor }\end{array}$ & BPU-07475.JPG \\
\hline 1175 & Elatine alsinastrum L. & Gyula & 1922 & Boros Ádám & BPU-07478.JPG \\
\hline 1175 & Elatine alsinastrum L. & Hortobágy & 1947 & Soó Rezső & BPU-07473.JPG \\
\hline 1175 & Elatine alsinastrum L. & Pomáz & 1945 & Vajda László & BPU-07479.JPG \\
\hline 1175 & Elatine alsinastrum L. & Tivadar & 1948 & Simon Tibor, Jakucs Pál & BPU-07476.JPG \\
\hline 1176 & Elatine hungarica Moesz & Hortobágy & 1947 & Soó Rezső & BPU-07483.JPG \\
\hline 1176 & Elatine hungarica Moesz & Hortobágy & 1947 & Soó Rezső & BPU-07483.JPG \\
\hline 1176 & Elatine hungarica Moesz & Szarvas & 1947 & Soó Rezső & BPU-07482.JPG \\
\hline 1178 & Elatine triandra Schkuhr & Szarvas & 1947 & Soó Rezső & BPU-07480.JPG \\
\hline 1178 & Elatine triandra Schkuhr & Szarvas & 1947 & Ubrizsy Gábor & BPU-07481.JPG \\
\hline 1178 & Elatine triandra Schkuhr & Szarvas & 1947 & Ubrizsy Gábor & BPU-07481.JPG \\
\hline 1180 & Thladiantha dubia Bunge & Debrecen & 1942 & Felföldy Lajos & BPU-07484.JPG \\
\hline 1180 & Thladiantha dubia Bunge & Nyírpilis & 1951 & Simon Tibor & BPU-07486.JPG \\
\hline 1180 & Thladiantha dubia Bunge & Ócsa & 1955 & Soó Rezső & BPU-07485.JPG \\
\hline 1180 & Thladiantha dubia Bunge & Pápa & 1953 & Czucza E. & BPU-07487.JPG \\
\hline 1182 & Bryonia alba L. & Ócsa & 1955 & Soó Rezső & BPU-07490.JPG \\
\hline 1182 & Bryonia alba L. & Pilisszentiván & 1953 & Soó Rezső & BPU-07488.JPG \\
\hline 1182 & Bryonia alba L. & Téglás & 1937 & Soó Rezső & BPU-07489.JPG \\
\hline 1182 & Bryonia alba L. & Tihany & 1931 & Polgár Sándor & BPU-07491.JPG \\
\hline 1183 & Bryonia dioica Jacq. & Sopron & 1944 & Kárpáti Zoltán & BPU-07492.JPG \\
\hline 1183 & Bryonia dioica Jacq. & Zákány & 1948 & Károlyi Árpád & BPU-07493.JPG \\
\hline 1186 & Cucumis melo L. & Budapest & 1948 & Soó Rezső & BPU-07495.JPG \\
\hline
\end{tabular}


1. Elektroniukus melléklet - Electronic Appendix 1.

\begin{tabular}{|c|c|c|c|c|c|}
\hline $\begin{array}{l}\text { Sorszám / } \\
\text { Number }\end{array}$ & Taxon-név / Taxon-name & $\begin{array}{l}\text { Település / } \\
\text { Settlement }\end{array}$ & $\begin{array}{l}\text { Év / } \\
\text { Year }\end{array}$ & Gyújtő / Collector & $\begin{array}{l}\text { Fájlnév / } \\
\text { File-name }\end{array}$ \\
\hline 1189 & $\begin{array}{l}\text { Echinocystis lobata (Michx.) Torr. et A. } \\
\text { Gray }\end{array}$ & Nagyharsány & 1961 & Simon Tibor & BPU-07498.JPG \\
\hline 1189 & $\begin{array}{l}\text { Echinocystis lobata (Michx.) Torr. et A. } \\
\text { Gray }\end{array}$ & Sarkad & 1948 & Jakucs Pál & BPU-07499.JPG \\
\hline 1190 & Sicyos angulatus L. & Tiszafüred & 1954 & Dobos L. & BPU-07497.JPG \\
\hline 1190 & Sicyos angulatus L. & Tiszaszalka & 1953 & Simon Tibor & BPU-07496.JPG \\
\hline 1194 & Peplis portula L. & Dörgicse & 1957 & Felföldy Lajos & BPU-03012.JPG \\
\hline 1194 & Peplis portula L. & Egyek & 1950 & $\begin{array}{l}\text { Simon Tibor, Borsos Olga, } \\
\text { Kulcsár Gábor }\end{array}$ & BPU-03009.JPG \\
\hline 1194 & Peplis portula L. & Hortobágy & 1947 & Soó Rezső & BPU-03008.JPG \\
\hline 1194 & Peplis portula L. & Nagykanizsa & 1956 & Felföldy Lajos & BPU-03013.JPG \\
\hline 1194 & Peplis portula L. & Regéc & 1953 & Vajda László & BPU-03007.JPG \\
\hline 1194 & Peplis portula L. & Szőce & 1955 & Soó Rezső & BPU-03011.JPG \\
\hline 1194 & Peplis portula L. & Vásárosnamény & 1933 & Soó Rezső & BPU-03010.JPG \\
\hline 1195 & Lythrum virgatum L. & Egyek & 1947 & Soó Rezső & BPU-03023.JPG \\
\hline 1195 & Lythrum virgatum L. & Kerecsend & 1957 & Soó Rezső & BPU-03024.JPG \\
\hline 1195 & Lythrum virgatum L. & Miskolc & 1937 & Soó Rezső & BPU-03022.JPG \\
\hline 1195 & Lythrum virgatum L. & Tiszavasvári & 1949 & Igmándy József & BPU-03026.JPG \\
\hline 1195 & Lythrum virgatum L. & Törökbálint & 1991 & Felföldy Lajos & BPU-03025.JPG \\
\hline 1196 & Lythrum salicaria L. & Debrecen & 1933 & Soó Rezső & BPU-03021.JPG \\
\hline 1196 & Lythrum salicaria L. & Zámoly & 1982 & Felföldy Lajos & BPU-03020.JPG \\
\hline 1197 & Lythrum hyssopifolia L. & Budapest & 1917 & Degen Árpád & BPU-03014.JPG \\
\hline 1197 & Lythrum hyssopifolia L. & Gyöngyös & 1936 & Soó Rezső & BPU-03016.JPG \\
\hline 1197 & Lythrum hyssopifolia L. & Hortobágy & 1947 & Soó Rezső & BPU-03015.JPG \\
\hline 1197 & Lythrum hyssopifolia L. & Tiszafüred & 1990 & Felföldy Lajos & BPU-03018.JPG \\
\hline 1197 & Lythrum hyssopifolia L. & Vácrátót & 1953 & Felföldy Lajos & BPU-03017.JPG \\
\hline $\begin{array}{c}1195 \times \\
1196\end{array}$ & $\begin{array}{l}\text { Lythrum virgatum L. } \times \text { Lythrum } \\
\text { salicaria L. }\end{array}$ & Egyek & 1947 & Soó Rezső & BPU-03027.JPG \\
\hline 1203 & Trapa natans L. & Keszthely & 1958 & Tóth László & BPU-03153.JPG \\
\hline 1203 & Trapa natans L. & Lesenceistvánd & 1951 & Borsos Olga & BPU-03152.JPG \\
\hline 1203 & Trapa natans L. & Nádudvar & 1963 & Simon Tibor & BPU-03150.JPG \\
\hline 1203 & Trapa natans L. & Szamosszeg & 1951 & Simon Tibor & BPU-03151.JPG \\
\hline 1203 & Trapa natans L. & Szarvas & 1947 & Soó Rezső & BPU-03149.JPG \\
\hline 1203 & Trapa natans L. & Vonyarcvashegy & 1958 & Tóth László & BPU-03154.JPG \\
\hline 1204 & Circaea lutetiana L. & Alsószölnök & 1955 & Soó Rezső & BPU-03140.JPG \\
\hline 1204 & Circaea lutetiana L. & Debrecen & 1951 & Kulcsár Gábor & BPU-03135.JPG \\
\hline 1204 & Circaea lutetiana L. & Kapolcs & 1959 & $\begin{array}{l}\text { Jávorka Sándor, Felföldy } \\
\text { Lajos, Tóth László }\end{array}$ & BPU-03142.JPG \\
\hline 1204 & Circaea lutetiana L. & Kemence & 1952 & Simon Tibor & BPU-03136.JPG \\
\hline 1204 & Circaea lutetiana L. & Pusztavacs & 1950 & Soó Rezső & BPU-03137.JPG \\
\hline 1204 & Circaea lutetiana L. & Regéc & 1991 & Simon Tibor & BPU-03132.JPG \\
\hline 1204 & Circaea lutetiana L. & Tarpa & 1948 & Simon Tibor, Jakucs Pál & BPU-03133.JPG \\
\hline 1204 & Circaea lutetiana L. & Tornyospálca & 1933 & Soó Rezső & BPU-03138.JPG \\
\hline 1204 & Circaea lutetiana L. & Vashosszúfalu & 1951 & Felföldy Lajos & BPU-03141.JPG \\
\hline 1204 & Circaea lutetiana L. & Zámoly & 1982 & Felföldy Lajos & BPU-03134.JPG \\
\hline 1205 & Circaea alpina L. & Diósjenő & 1993 & Simon Tibor & BPU-03144.JPG \\
\hline 1206 & Oenothera salicifolia Desf. & Budapest & 1943 & Soó Rezső & BPU-03126.JPG \\
\hline 1206 & Oenothera salicifolia Desf. & Győr & 1929 & Polgár Sándor & BPU-03128.JPG \\
\hline 1206 & Oenothera salicifolia Desf. & Paks & 1971 & Priszter Szaniszló & BPU-03129.JPG \\
\hline 1212 & Oenothera biennis L. & Csesznek & 1963 & Soó Rezső & BPU-03124.JPG \\
\hline
\end{tabular}


1. Elektroniukus melléklet - Electronic Appendix 1.

\begin{tabular}{|c|c|c|c|c|c|}
\hline $\begin{array}{l}\text { Sorszám / } \\
\text { Number }\end{array}$ & Taxon-név / Taxon-name & $\begin{array}{l}\text { Település / } \\
\text { Settlement }\end{array}$ & $\begin{array}{l}\text { Év / } \\
\text { Year }\end{array}$ & Gyújtő / Collector & $\begin{array}{l}\text { Fájlnév / } \\
\text { File-name }\end{array}$ \\
\hline 1212 & Oenothera biennis L. & Debrecen & 1953 & Kulcsár Gábor & BPU-03125.JPG \\
\hline 1212 & Oenothera biennis L. & Keszthely & 1958 & Sebestyén Olga & BPU-03123.JPG \\
\hline 1212 & Oenothera biennis L. & Vásárosnamény & 1951 & Simon Tibor & BPU-03127.JPG \\
\hline $\begin{array}{c}1206- \\
1213\end{array}$ & Oenothera & Jászszentlászló & 1964 & Soó Rezső & BPU-03130.JPG \\
\hline $\begin{array}{c}1206- \\
1213\end{array}$ & Oenothera & Jászszentlászló & 1964 & Soó Rezső & BPU-03131.JPG \\
\hline 1215 & Ludwigia palustris (L.) Elliott & Alsóújlak & 1956 & Jeanplong József & BPU-03030.JPG \\
\hline 1215 & Ludwigia palustris (L.) Elliott & Darány & 1923 & Boros Ádám & BPU-03028.JPG \\
\hline 1215 & Ludwigia palustris (L.) Elliott & Szenta & 1958 & $\begin{array}{l}\text { Járainé Komlódi Magda, } \\
\text { Borhidi Attila }\end{array}$ & BPU-03029.JPG \\
\hline 1216 & Ludwigia repens J.R. Forst. & Miskolc & 1937 & Soó Rezső & BPU-03031.JPG \\
\hline 1217 & Chamaenerion angustifolium (L.) Scop. & Bagamér & 1937 & Soó Rezső & BPU-03118.JPG \\
\hline 1217 & Chamaenerion angustifolium (L.) Scop. & Kállósemjén & 1950 & Simon Tibor & BPU-03119.JPG \\
\hline 1218 & Chamaenerion dodonaei (Vill.) Holub & Ágfalva & 1947 & Kárpáti Zoltán & BPU-03120.JPG \\
\hline 1218 & Chamaenerion dodonaei (Vill.) Holub & Veszprém & 1931 & Soó Rezső & BPU-03122.JPG \\
\hline 1219 & Epilobium hirsutum L. & Bagamér & 1932 & Máthé Imre & BPU-03033.JPG \\
\hline 1219 & Epilobium hirsutum L. & Budapest & 1991 & Felföldy Lajos & BPU-03032.JPG \\
\hline 1219 & Epilobium hirsutum L. & Vászoly & 1958 & Felföldy Lajos & BPU-03035.JPG \\
\hline 1220 & Epilobium parviflorum Schreb. & Balatongyörök & 1961 & Felföldy Lajos & BPU-03044.JPG \\
\hline 1220 & Epilobium parviflorum Schreb. & Balatonszőlős & 1956 & $\begin{array}{l}\text { Felföldy Lajos, Tóth } \\
\text { László }\end{array}$ & BPU-03037.JPG \\
\hline 1220 & Epilobium parviflorum Schreb. & Balatonszőlős & 1957 & $\begin{array}{l}\text { Felföldy Lajos, Kovács } \\
\text { Margit, Tóth László }\end{array}$ & BPU-03042.JPG \\
\hline 1220 & Epilobium parviflorum Schreb. & Gárdony & 1980 & Felföldy Lajos & BPU-03039.JPG \\
\hline 1220 & Epilobium parviflorum Schreb. & Gárdony & 1980 & Felföldy Lajos & BPU-03040.JPG \\
\hline 1220 & Epilobium parviflorum Schreb. & Konyár & 1933 & Soó Rezső & BPU-03036.JPG \\
\hline 1220 & Epilobium parviflorum Schreb. & Örvényes & 1958 & $\begin{array}{l}\text { Felföldy Lajos, Tóth } \\
\text { László }\end{array}$ & BPU-03041.JPG \\
\hline 1220 & Epilobium parviflorum Schreb. & Szögliget & 1951 & $\begin{array}{l}\text { Soó Rezső, Jakucs Pál, Ér } \\
\text { Lajos }\end{array}$ & BPU-03112.JPG \\
\hline 1220 & Epilobium parviflorum Schreb. & Uzsa & 1953 & $\begin{array}{l}\text { Felföldy Lajos, Tóth } \\
\text { László }\end{array}$ & BPU-03043.JPG \\
\hline 1220 & Epilobium parviflorum Schreb. & Zámoly & 1982 & Felföldy Lajos & BPU-03038.JPG \\
\hline 1221 & $\begin{array}{l}\text { Epilobium lanceolatum Sebast. et } \\
\text { Mauri }\end{array}$ & Fót & 1960 & Soó Rezső & BPU-03047.JPG \\
\hline 1221 & $\begin{array}{l}\text { Epilobium lanceolatum Sebast. et } \\
\text { Mauri }\end{array}$ & Háromhuta & 1961 & Simon Tibor & BPU-03050.JPG \\
\hline 1221 & $\begin{array}{l}\text { Epilobium lanceolatum Sebast. et } \\
\text { Mauri }\end{array}$ & Miskolc & 1953 & Soó Rezső & BPU-03049.JPG \\
\hline 1221 & $\begin{array}{l}\text { Epilobium lanceolatum Sebast. et } \\
\text { Mauri }\end{array}$ & Nagyvisnyó & 1953 & Soó Rezső & BPU-03048.JPG \\
\hline 1221 & $\begin{array}{l}\text { Epilobium lanceolatum Sebast. et } \\
\text { Mauri }\end{array}$ & Uzsa & 1931 & Soó Rezső & BPU-03046.JPG \\
\hline 1222 & Epilobium montanum L. & Baktalórántháza & 1933 & Soó Rezső & BPU-03053.JPG \\
\hline 1222 & Epilobium montanum L. & Beregdaróc & 1951 & $\begin{array}{l}\text { Simon Tibor, Vozáry } \\
\text { Elemér }\end{array}$ & BPU-03060.JPG \\
\hline 1222 & Epilobium montanum L. & Debrecen & 1951 & Kulcsár Gábor & BPU-03057.JPG \\
\hline 1222 & Epilobium montanum L. & Debrecen & 1951 & Kulcsár Gábor & BPU-03058.JPG \\
\hline 1222 & Epilobium montanum L. & Füzér & 1937 & Soó Rezső & BPU-03061.JPG \\
\hline 1222 & Epilobium montanum L. & Gyöngyös & 1952 & Soó Rezső & BPU-03056.JPG \\
\hline 1222 & Epilobium montanum L. & Gyöngyös & 1961 & Soó Rezső & BPU-03055.JPG \\
\hline 1222 & Epilobium montanum L. & Hercegkút & 1955 & Simon Tibor & BPU-03051.JPG \\
\hline
\end{tabular}


1. Elektroniukus melléklet - Electronic Appendix 1.

\begin{tabular}{|c|c|c|c|c|c|}
\hline $\begin{array}{l}\text { Sorszám / } \\
\text { Number }\end{array}$ & Taxon-név / Taxon-name & $\begin{array}{l}\text { Település / } \\
\text { Settlement }\end{array}$ & $\begin{array}{l}\text { Év / } \\
\text { Year }\end{array}$ & Gyújtő / Collector & $\begin{array}{l}\text { Fájlnév / } \\
\text { File-name }\end{array}$ \\
\hline 1222 & Epilobium montanum L. & Kőszeg & 1932 & Soó Rezső & BPU-03054.JPG \\
\hline 1222 & Epilobium montanum L. & Miskolc & 1950 & Soó Rezső & BPU-03052.JPG \\
\hline 1222 & Epilobium montanum L. & Miskolc & 1950 & Soó Rezső & BPU-03059.JPG \\
\hline 1223 & Epilobium collinum C.C. Gmel. & Füzérkomlós & 2007 & Simon Tibor & BPU-15504.JPG \\
\hline 1223 & Epilobium collinum C.C. Gmel. & Kőszeg & 1932 & Soó Rezső & BPU-03066.JPG \\
\hline 1223 & Epilobium collinum C.C. Gmel. & Tarpa & 1948 & Simon Tibor, Jakucs Pál & BPU-03063.JPG \\
\hline 1223 & Epilobium collinum C.C. Gmel. & Telkibánya & 1948 & Borsos Olga & BPU-03064.JPG \\
\hline 1223 & Epilobium collinum C.C. Gmel. & Vácrátót & 1953 & Felföldy Lajos & BPU-03065.JPG \\
\hline 1224 & Epilobium palustre L. & Alsószölnök & 1955 & Soó Rezső & BPU-03095.JPG \\
\hline 1224 & Epilobium palustre L. & Kaszó & 1957 & Soó Rezső & BPU-03096.JPG \\
\hline 1224 & Epilobium palustre L. & Nagykanizsa & 1956 & Felföldy Lajos & BPU-03102.JPG \\
\hline 1224 & Epilobium palustre L. & Nyírbéltek & 1932 & Soó Rezső & BPU-03093.JPG \\
\hline 1224 & Epilobium palustre L. & Tákos & 1948 & Simon Tibor, Jakucs Pál & BPU-03100.JPG \\
\hline 1225 & Epilobium roseum Schreb. & Dömös & 1948 & Soó Rezső & BPU-03072.JPG \\
\hline 1225 & Epilobium roseum Schreb. & Gyöngyös & 1952 & Soó Rezső & BPU-03069.JPG \\
\hline 1225 & Epilobium roseum Schreb. & Sátoraljaújhely & 1937 & Soó Rezső & BPU-03070.JPG \\
\hline 1225 & Epilobium roseum Schreb. & Szőce & 1955 & Soó Rezső & BPU-03073.JPG \\
\hline 1225 & Epilobium roseum Schreb. & Velem & 1932 & Soó Rezső & BPU-03071.JPG \\
\hline 1227 & Epilobium obscurum Schreb. & Tákos & 1948 & Simon Tibor, Jakucs Pál & BPU-03087.JPG \\
\hline 1227 & Epilobium obscurum Schreb. & Vácrátót & 1949 & Soó Rezső & BPU-03088.JPG \\
\hline 1227 & Epilobium obscurum Schreb. & Zebegény & 1947 & Soó Rezső & BPU-03103.JPG \\
\hline 1228 & Epilobium tetragonum L. & Budaörs & 1991 & Felföldy Lajos & BPU-03083.JPG \\
\hline 1228 & Epilobium tetragonum L. & Debrecen & 1932 & Soó Rezső & BPU-03076.JPG \\
\hline 1228 & Epilobium tetragonum L. & Debrecen & 1933 & Soó Rezső & BPU-03077.JPG \\
\hline 1228 & Epilobium tetragonum L. & Dörgicse & 1957 & Felföldy Lajos & BPU-03084.JPG \\
\hline 1228 & Epilobium tetragonum L. & Gelénes & 1951 & $\begin{array}{l}\text { Simon Tibor, Vozáry } \\
\text { Elemér }\end{array}$ & BPU-03081.JPG \\
\hline 1228 & Epilobium tetragonum L. & Hegykő & 1960 & Tóth László & BPU-03074.JPG \\
\hline 1228 & Epilobium tetragonum L. & Kaszó & 1957 & Soó Rezső & BPU-03086.JPG \\
\hline 1228 & Epilobium tetragonum L. & Miskolc & 1936 & Soó Rezső & BPU-03079.JPG \\
\hline 1228 & Epilobium tetragonum L. & Miskolc & 1937 & Soó Rezső & BPU-03085.JPG \\
\hline 1228 & Epilobium tetragonum L. & Nagybajom & 1957 & Soó Rezső & BPU-03075.JPG \\
\hline 1228 & Epilobium tetragonum L. & Szarvas & 1947 & Soó Rezső & BPU-03089.JPG \\
\hline 1228 & Epilobium tetragonum L. & Szőce & 1955 & Soó Rezső & BPU-03080.JPG \\
\hline 1228 & Epilobium tetragonum L. & Tihany & 1959 & Felföldy Lajos & BPU-03082.JPG \\
\hline 1228 & Epilobium tetragonum L. & Zámoly & 1982 & Felföldy Lajos & BPU-03078.JPG \\
\hline $\begin{array}{c}1219 \times \\
1220\end{array}$ & $\begin{array}{l}\text { Epilobium hirsutum L. } \times \text { Epilobium } \\
\text { parviflorum Schreb. }\end{array}$ & Csobánka & 1992 & Felföldy Lajos & BPU-03114.JPG \\
\hline $\begin{array}{c}1220 \times \\
1225\end{array}$ & $\begin{array}{l}\text { Epilobium parviflorum Schreb. } \times \\
\text { Epilobium roseum Schreb. }\end{array}$ & Kőszeg & 1932 & Soó Rezső & BPU-03115.JPG \\
\hline $\begin{array}{c}1220 \times \\
1228\end{array}$ & $\begin{array}{l}\text { Epilobium parviflorum Schreb. } \times \\
\text { Epilobium tetragonum L. }\end{array}$ & Miskolc & 1937 & Soó Rezső & BPU-03113.JPG \\
\hline $\begin{array}{c}1222 \times \\
1225\end{array}$ & $\begin{array}{l}\text { Epilobium montanum L. } \times \text { Epilobium } \\
\text { roseum Schreb. }\end{array}$ & Pusztafalu & 1939 & Soó Rezső & BPU-03117.JPG \\
\hline 1229 & Myriophyllum spicatum L. & Budapest & 1909 & Kocsis István & BPU-03165.JPG \\
\hline 1229 & Myriophyllum spicatum L. & Gávavencsellő & 1990 & Felföldy Lajos & BPU-03170.JPG \\
\hline 1229 & Myriophyllum spicatum L. & Gyömrő & 1974 & Tóth László & BPU-03169.JPG \\
\hline 1229 & Myriophyllum spicatum L. & Konyár & 1949 & Soó Rezső & BPU-03168.JPG \\
\hline 1229 & Myriophyllum spicatum L. & Kunmadaras & 1991 & Csányi Béla & BPU-03172.JPG \\
\hline 1229 & Myriophyllum spicatum L. & Olcsvaapáti & 1951 & Simon Tibor & BPU-03171.JPG \\
\hline
\end{tabular}


1. Elektroniukus melléklet - Electronic Appendix 1.

\begin{tabular}{|c|c|c|c|c|c|}
\hline $\begin{array}{l}\text { Sorszám / } \\
\text { Number }\end{array}$ & Taxon-név / Taxon-name & $\begin{array}{l}\text { Település / } \\
\text { Settlement }\end{array}$ & $\begin{array}{l}\text { Év / } \\
\text { Year }\end{array}$ & Gyüjtő / Collector & $\begin{array}{l}\text { Fájlnév / } \\
\text { File-name }\end{array}$ \\
\hline 1229 & Myriophyllum spicatum L. & Soltvadkert & 1992 & Felföldy Lajos & BPU-03166.JPG \\
\hline 1229 & Myriophyllum spicatum L. & Szigliget & 1956 & $\begin{array}{l}\text { Felföldy Lajos, Tóth } \\
\text { László }\end{array}$ & BPU-03173.JPG \\
\hline 1229 & Myriophyllum spicatum L. & Tiszafüred & 1955 & Borsos Olga & BPU-15551.JPG \\
\hline 1229 & Myriophyllum spicatum L. & Tivadar & 1948 & Simon Tibor, Jakucs Pál & BPU-03167.JPG \\
\hline 1229 & Myriophyllum spicatum L. & Zalaapáti & 1958 & Entz Béla & BPU-03174.JPG \\
\hline 1231 & Myriophyllum verticillatum L. & Balatonfüzfő & 1956 & $\begin{array}{l}\text { Felföldy Lajos, Tóth } \\
\text { László }\end{array}$ & BPU-03160.JPG \\
\hline 1231 & Myriophyllum verticillatum L. & Jánd & 1951 & Simon Tibor & BPU-03161.JPG \\
\hline 1231 & Myriophyllum verticillatum L. & Kunmadaras & 1991 & Csányi Béla & BPU-03157.JPG \\
\hline 1231 & Myriophyllum verticillatum L. & Lakitelek & 1961 & Simon Tibor & BPU-03162.JPG \\
\hline 1231 & Myriophyllum verticillatum L. & Révfülöp & 1956 & $\begin{array}{l}\text { Felföldy Lajos, Tóth } \\
\text { László }\end{array}$ & BPU-03159.JPG \\
\hline 1231 & Myriophyllum verticillatum L. & Tiszafüred & 1953 & Borsos Olga & BPU-03155.JPG \\
\hline 1231 & Myriophyllum verticillatum L. & Tiszafüred & 1953 & Borsos Olga & BPU-03156.JPG \\
\hline 1231 & Myriophyllum verticillatum L. & Veresegyház & 1949 & Soó Rezső & BPU-03164.JPG \\
\hline 1231 & Myriophyllum verticillatum L. & Vonyarcvashegy & 1958 & Tóth László & BPU-03158.JPG \\
\hline 1233 & Cornus mas L. & Bátorliget & 1948 & $\begin{array}{l}\text { Simon Tibor, Pólya } \\
\text { László, Jakucs Pál }\end{array}$ & BPU-03412.JPG \\
\hline 1233 & Cornus mas L. & Bódvaszilas & 1950 & Jakucs Pál, Kulcsár Gábor & BPU-03411.JPG \\
\hline 1233 & Cornus mas L. & Nagykovácsi & 1949 & Soó Rezső & BPU-03409.JPG \\
\hline 1233 & Cornus mas L. & Szilvásvárad & 1953 & $\begin{array}{l}\text { Felföldy Lajos, Tóth } \\
\text { László }\end{array}$ & BPU-03414.JPG \\
\hline 1233 & Cornus mas L. & Tihany & 1958 & Felföldy Lajos & BPU-03413.JPG \\
\hline 1233 & Cornus mas L. & Vác & 1949 & $\begin{array}{l}\text { Soó Rezső, Simon Tibor, } \\
\text { Jakucs Pál }\end{array}$ & BPU-03410.JPG \\
\hline 1233 & Cornus mas L. & Zebegény & 1947 & Soó Rezső & BPU-03408.JPG \\
\hline 1234 & Cornus sanguinea L. & Budapest & 1952 & Soó Rezső & BPU-03415.JPG \\
\hline 1234 & Cornus sanguinea L. & Hajdúbagos & 1933 & Soó Rezső & BPU-03416.JPG \\
\hline 1234 & Cornus sanguinea L. & Hidegkút & 1958 & Felföldy Lajos & BPU-03417.JPG \\
\hline 1237 & Hippuris vulgaris L. & Budapest & 1922 & Soó Rezső & BPU-03175.JPG \\
\hline 1237 & Hippuris vulgaris L. & Budapest & 1980 & Csányi Béla & BPU-03178.JPG \\
\hline 1237 & Hippuris vulgaris L. & Dabas & 1951 & Borsos Olga, Simon Tibor & BPU-03176.JPG \\
\hline 1237 & Hippuris vulgaris L. & Dabas & 1951 & Simon Tibor, Borsos Olga & BPU-03177.JPG \\
\hline 1238 & Hedera helix L. & Budapest & 2004 & Felföldy Lajos & BPU-03402.JPG \\
\hline 1238 & Hedera helix L. & Nyíregyháza & 1934 & Soó Rezső & BPU-03404.JPG \\
\hline 1238 & Hedera helix L. & Sárospatak & 1963 & Isépy István & BPU-03407.JPG \\
\hline 1238 & Hedera helix L. & Szigliget & 1962 & Soó Rezső & BPU-03406.JPG \\
\hline 1238 & Hedera helix L. & Tihany & 1958 & Felföldy Lajos & BPU-03405.JPG \\
\hline 1239 & Hydrocotyle vulgaris L. & Darány & 1922 & Boros Ádám & BPU-03418.JPG \\
\hline 1241 & Sanicula europaea L. & "Cuha-völgy" & 1954 & Soó Rezső & BPU-03428.JPG \\
\hline 1241 & Sanicula europaea L. & Beregdaróc & 1952 & $\begin{array}{l}\text { Simon Tibor, Vozáry } \\
\text { Elemér }\end{array}$ & BPU-03423.JPG \\
\hline 1241 & Sanicula europaea L. & Budapest & 1953 & Borsos Olga & BPU-15416.JPG \\
\hline 1241 & Sanicula europaea L. & Isztimér & 1949 & Soó Rezső & BPU-03425.JPG \\
\hline 1241 & Sanicula europaea L. & Kapolcs & 1959 & $\begin{array}{l}\text { Jávorka Sándor, Felföldy } \\
\text { Lajos, Tóth László }\end{array}$ & BPU-03429.JPG \\
\hline 1241 & Sanicula europaea L. & Kemence & 1952 & Simon Tibor & BPU-03426.JPG \\
\hline 1241 & Sanicula europaea L. & Lábatlan & 1953 & Felföldy Lajos & BPU-03424.JPG \\
\hline 1241 & Sanicula europaea L. & Nagybajom & 1955 & Soó Rezső & BPU-03427.JPG \\
\hline 1241 & Sanicula europaea L. & Pécs & 1935 & Soó Rezső & BPU-03421.JPG \\
\hline
\end{tabular}


1. Elektroniukus melléklet - Electronic Appendix 1.

\begin{tabular}{|c|c|c|c|c|c|}
\hline $\begin{array}{l}\text { Sorszám / } \\
\text { Number }\end{array}$ & Taxon-név / Taxon-name & $\begin{array}{l}\text { Település / } \\
\text { Settlement }\end{array}$ & $\begin{array}{l}\text { Év / } \\
\text { Year }\end{array}$ & Gyújtő / Collector & $\begin{array}{l}\text { Fájlnév / } \\
\text { File-name }\end{array}$ \\
\hline 1241 & Sanicula europaea L. & Pécs & 1956 & Soó Rezső & BPU-03422.JPG \\
\hline 1241 & Sanicula europaea L. & Regéc & 1955 & Simon Tibor & BPU-03419.JPG \\
\hline 1241 & Sanicula europaea L. & Telkibánya & 1952 & Simon Tibor & BPU-03420.JPG \\
\hline 1242 & Astrantia major L. & Alsószölnök & 1955 & Soó Rezső & BPU-03435.JPG \\
\hline 1242 & Astrantia major L. & Bózsva & 1950 & Simon Tibor & BPU-03431.JPG \\
\hline 1242 & Astrantia major L. & Jósvafó & 1948 & $\begin{array}{l}\text { Simon Tibor, Pólya } \\
\text { László, Böszörményi Z. }\end{array}$ & BPU-03430.JPG \\
\hline 1242 & Astrantia major L. & Kemence & 1952 & Simon Tibor & BPU-03433.JPG \\
\hline 1242 & Astrantia major L. & Kőszeg & 1963 & Soó Rezső, Borsos Olga & BPU-03436.JPG \\
\hline 1242 & Astrantia major L. & Miskolc & 1950 & Soó Rezső & BPU-03432.JPG \\
\hline 1242 & Astrantia major L. & Sopron & 1948 & Kárpáti Zoltán & BPU-03434.JPG \\
\hline 1243 & Eryngium campestre L. & Nyíracsád & 1947 & Soó Rezső & BPU-03443.JPG \\
\hline 1244 & Eryngium planum L. & Bátorliget & 1953 & Simon Tibor & BPU-03440.JPG \\
\hline 1244 & Eryngium planum L. & Bátorliget & 1953 & Simon Tibor & BPU-03441.JPG \\
\hline 1244 & Eryngium planum L. & Erdőbénye & 1948 & Soó Rezső & BPU-03444.JPG \\
\hline 1244 & Eryngium planum L. & Tarpa & 1948 & Simon Tibor, Jakucs Pál & BPU-03442.JPG \\
\hline 1245 & Physocaulis nodosus (L.) W.D.J. Koch & $\begin{array}{l}\text { Badacsonytomaj, } \\
\text { Badacsonytördemic }\end{array}$ & 1948 & Károlyi Árpád & BPU-03447.JPG \\
\hline 1245 & Physocaulis nodosus (L.) W.D.J. Koch & Tarján & 1932 & Boros Ádám & BPU-03445.JPG \\
\hline 1245 & Physocaulis nodosus (L.) W.D.J. Koch & Tihany & 1959 & Felföldy Lajos & BPU-03446.JPG \\
\hline 1247 & Chaerophyllum aromaticum L. & Kismaros & 1982 & Felföldy Lajos & BPU-03449.JPG \\
\hline 1247 & Chaerophyllum aromaticum L. & Miskolc & 1934 & Soó Rezső & BPU-03448.JPG \\
\hline 1247 & Chaerophyllum aromaticum L. & Sopron & 1937 & Soó Rezső & BPU-03450.JPG \\
\hline 1248 & Chaerophyllum aureum L. & Pécs & 1953 & Soó Rezső, Borsos Olga & BPU-03460.JPG \\
\hline 1249 & Chaerophyllum bulbosum L. & Budaörs & 1960 & Priszter Szaniszló & BPU-03467.JPG \\
\hline 1249 & Chaerophyllum bulbosum L. & Budapest & 1981 & Felföldy Lajos & BPU-03468.JPG \\
\hline 1249 & Chaerophyllum bulbosum L. & Debrecen & 1947 & Soó Rezső & BPU-03469.JPG \\
\hline 1250 & Chaerophyllum temulum L. & Budapest & 1982 & Felföldy Lajos & BPU-03461.JPG \\
\hline 1250 & Chaerophyllum temulum L. & Debrecen & 1931 & Soó Rezső & BPU-03462.JPG \\
\hline 1250 & Chaerophyllum temulum L. & Nagykovácsi & 2005 & Felföldy Lajos & BPU-03463.JPG \\
\hline 1250 & Chaerophyllum temulum L. & Tihany & 1956 & Felföldy Lajos & BPU-03464.JPG \\
\hline 1250 & Chaerophyllum temulum L. & Tihany & 1959 & Felföldy Lajos & BPU-03465.JPG \\
\hline 1251 & Anthriscus caucalis M. Bieb. & Balmazújváros & 1947 & Soó Rezső & BPU-03473.JPG \\
\hline 1251 & Anthriscus caucalis M. Bieb. & Budapest & 1982 & Felföldy Lajos & BPU-03474.JPG \\
\hline 1251 & Anthriscus caucalis M. Bieb. & Dabas & 1951 & Borsos Olga, Simon Tibor & BPU-03472.JPG \\
\hline 1251 & Anthriscus caucalis M. Bieb. & Örvényes & 1959 & Felföldy Lajos & BPU-03471.JPG \\
\hline 1251 & Anthriscus caucalis M. Bieb. & Sormás & 1950 & Károlyi Árpád & BPU-03470.JPG \\
\hline 1251 & Anthriscus caucalis M. Bieb. & Tihany & 1956 & Felföldy Lajos & BPU-03475.JPG \\
\hline 1252 & Anthriscus cerefolium (L.) Hoffm. & Budapest & 1991 & Felföldy Lajos & BPU-03476.JPG \\
\hline 1252 & Anthriscus cerefolium (L.) Hoffm. & Budapest & 1992 & Felföldy Lajos & BPU-03481.JPG \\
\hline 1252 & Anthriscus cerefolium (L.) Hoffm. & Csákberény & 1982 & Felföldy Lajos & BPU-03480.JPG \\
\hline 1252 & Anthriscus cerefolium (L.) Hoffm. & Lábatlan & 1953 & Felföldy Lajos & BPU-03478.JPG \\
\hline 1252 & Anthriscus cerefolium (L.) Hoffm. & Tihany & 1956 & Felföldy Lajos & BPU-03479.JPG \\
\hline 1252 & Anthriscus cerefolium (L.) Hoffm. & Tihany & 1959 & Felföldy Lajos & BPU-03477.JPG \\
\hline 1253 & Anthriscus sylvestris (L.) Hoffm. & Budapest & 2004 & Felföldy Lajos & BPU-03482.JPG \\
\hline 1253 & Anthriscus sylvestris (L.) Hoffm. & Gyöngyös & 1965 & Soó Rezső & BPU-03484.JPG \\
\hline 1253 & Anthriscus sylvestris (L.) Hoffm. & Jósvafó & 1951 & Soó Rezső & BPU-03483.JPG \\
\hline 1253 & Anthriscus sylvestris (L.) Hoffm. & Nyíracsád & 1931 & Soó Rezső & BPU-03485.JPG \\
\hline
\end{tabular}


1. Elektroniukus melléklet - Electronic Appendix 1.

\begin{tabular}{|c|c|c|c|c|c|}
\hline $\begin{array}{l}\text { Sorszám / } \\
\text { Number }\end{array}$ & Taxon-név / Taxon-name & $\begin{array}{l}\text { Település / } \\
\text { Settlement }\end{array}$ & $\begin{array}{l}\text { Év / } \\
\text { Year }\end{array}$ & Gyűjtő / Collector & $\begin{array}{l}\text { Fájlnév / } \\
\text { File-name }\end{array}$ \\
\hline 1254 & Anthriscus nitidus (Wahlenb.) Hazsl. & Bakonybél & 1955 & Soó Rezső & BPU-03488.JPG \\
\hline 1254 & Anthriscus nitidus (Wahlenb.) Hazsl. & Bakonybél & 1955 & Soó Rezső & BPU-03489.JPG \\
\hline 1254 & Anthriscus nitidus (Wahlenb.) Hazsl. & Isztimér & 1949 & Soó Rezső & BPU-03486.JPG \\
\hline 1257 & Bifora radians M. Bieb. & Bélmegyer & 1935 & Soó Rezső & BPU-03526.JPG \\
\hline 1258 & Smyrnium perfoliatum L. & Aszófő & 1956 & $\begin{array}{l}\text { Felföldy Lajos, Tóth } \\
\text { László }\end{array}$ & BPU-03530.JPG \\
\hline 1258 & Smyrnium perfoliatum L. & Badacsonytomaj & 1928 & Soó Rezső & BPU-03531.JPG \\
\hline 1258 & Smyrnium perfoliatum L. & Bodajk & 1955 & Soó Rezső & BPU-03529.JPG \\
\hline 1258 & Smyrnium perfoliatum L. & Isztimér & 1949 & Soó Rezső & BPU-03527.JPG \\
\hline 1258 & Smyrnium perfoliatum L. & Mosonmagyaróvár & 1965 & Borsos Olga & BPU-03528.JPG \\
\hline 1260 & Pimpinella major (L.) Huds. & Galambok & 1950 & Károlyi Árpád & BPU-03624.JPG \\
\hline 1260 & Pimpinella major (L.) Huds. & Hidegkút & 1957 & Felföldy Lajos & BPU-03623.JPG \\
\hline 1260 & Pimpinella major (L.) Huds. & Kőszeg & 1932 & Soó Rezső & BPU-03622.JPG \\
\hline 1260 & Pimpinella major (L.) Huds. & Miskolc & 1950 & Soó Rezső & BPU-03619.JPG \\
\hline 1260 & Pimpinella major (L.) Huds. & Szenna & 1957 & Soó Rezső & BPU-03621.JPG \\
\hline 1260 & Pimpinella major (L.) Huds. & Telkibánya & 1962 & Simon Tibor & BPU-03618.JPG \\
\hline 1261 & Pimpinella saxifraga L. & Budapest & 1982 & Felföldy Lajos & BPU-03627.JPG \\
\hline 1261 & Pimpinella saxifraga L. & Budapest & 1991 & Felföldy Lajos & BPU-03628.JPG \\
\hline 1261 & Pimpinella saxifraga L. & Debrecen & 1933 & Soó Rezső & BPU-03632.JPG \\
\hline 1261 & Pimpinella saxifraga L. & Diósjenő & 1952 & Simon Tibor & BPU-03633.JPG \\
\hline 1261 & Pimpinella saxifraga L. & Erdőbénye & 1948 & Soó Rezső & BPU-03638.JPG \\
\hline 1261 & Pimpinella saxifraga L. & Geszt & 1947 & Soó Rezső & BPU-03629.JPG \\
\hline 1261 & Pimpinella saxifraga L. & Győr & - & Polgár Sándor & BPU-03630.JPG \\
\hline 1261 & Pimpinella saxifraga L. & Hajdúnánás & 1935 & Ujvárosi Miklós & BPU-03631.JPG \\
\hline 1261 & Pimpinella saxifraga L. & Kisújszállás & 1938 & Soó Rezső & BPU-03636.JPG \\
\hline 1261 & Pimpinella saxifraga L. & Nagybajom & 1955 & Soó Rezső & BPU-03634.JPG \\
\hline 1261 & Pimpinella saxifraga L. & Nyírbéltek & 1932 & Soó Rezső & BPU-03635.JPG \\
\hline 1261 & Pimpinella saxifraga L. & Tihany & 1958 & Felföldy Lajos & BPU-03637.JPG \\
\hline 1262 & Aegopodium podagraria L. & Bátorliget & 1928 & Soó Rezső & BPU-03645.JPG \\
\hline 1262 & Aegopodium podagraria L. & Bátorliget & 1950 & $\begin{array}{l}\text { Simon Tibor, Kárpáti } \\
\text { István }\end{array}$ & BPU-03647.JPG \\
\hline 1262 & Aegopodium podagraria L. & Bisse & 1965 & $\begin{array}{l}\text { Simon Tibor, Priszter } \\
\text { Szaniszló, Borhidi Attila }\end{array}$ & BPU-03646.JPG \\
\hline 1262 & Aegopodium podagraria L. & Kishuta & 1950 & Soó Rezső & BPU-03648.JPG \\
\hline 1262 & Aegopodium podagraria L. & Lesenceistvánd & 1952 & Borsos Olga & BPU-03643.JPG \\
\hline 1262 & Aegopodium podagraria L. & Tarpa & 1951 & $\begin{array}{l}\text { Simon Tibor, Vozáry } \\
\text { Elemér }\end{array}$ & BPU-03644.JPG \\
\hline 1263 & Sium latifolium L. & Budapest & 1990 & Felföldy Lajos & BPU-03657.JPG \\
\hline 1263 & Sium latifolium L. & Debrecen & - & Soó Rezső & BPU-03661.JPG \\
\hline 1263 & Sium latifolium L. & Gárdony & 1980 & Felföldy Lajos & BPU-03659.JPG \\
\hline 1265 & Berula erecta (Huds.) Coville & Balatongyörök & 1961 & Felföldy Lajos & BPU-03654.JPG \\
\hline 1265 & Berula erecta (Huds.) Coville & Balatonszőlős & 1957 & $\begin{array}{l}\text { Felföldy Lajos, Kovács } \\
\text { Margit, Tóth László }\end{array}$ & BPU-03655.JPG \\
\hline 1265 & Berula erecta (Huds.) Coville & Budapest & 1947 & Soó Rezső & BPU-03656.JPG \\
\hline 1265 & Berula erecta (Huds.) Coville & Csobánka & 1992 & Felföldy Lajos & BPU-03651.JPG \\
\hline 1265 & Berula erecta (Huds.) Coville & Dávod & 1955 & Soó Rezső & BPU-03649.JPG \\
\hline 1265 & Berula erecta (Huds.) Coville & Gárdony & 1980 & Felföldy Lajos & BPU-03653.JPG \\
\hline 1265 & Berula erecta (Huds.) Coville & Kemence & 1952 & Simon Tibor & BPU-03652.JPG \\
\hline 1265 & Berula erecta (Huds.) Coville & Szenna & 1957 & Soó Rezső & BPU-03650.JPG \\
\hline 1266 & Libanotis pyrenaica (L.) Bourg. & Bélapátfalva & 1930 & Soó Rezső & BPU-03693.JPG \\
\hline
\end{tabular}


1. Elektroniukus melléklet - Electronic Appendix 1.

\begin{tabular}{|c|c|c|c|c|c|}
\hline $\begin{array}{l}\text { Sorszám / } \\
\text { Number }\end{array}$ & Taxon-név / Taxon-name & $\begin{array}{l}\text { Település / } \\
\text { Settlement }\end{array}$ & $\begin{array}{l}\text { Év / } \\
\text { Year }\end{array}$ & Gyújtő / Collector & $\begin{array}{l}\text { Fájlnév / } \\
\text { File-name }\end{array}$ \\
\hline 1266 & Libanotis pyrenaica (L.) Bourg. & Bélapátfalva & 1950 & $\begin{array}{l}\text { Simon Tibor, Jakucs Pál, } \\
\text { Kárpáti István }\end{array}$ & BPU-03692.JPG \\
\hline 1266 & Libanotis pyrenaica (L.) Bourg. & Miskolc & 1950 & Soó Rezső & BPU-03690.JPG \\
\hline 1266 & Libanotis pyrenaica (L.) Bourg. & Piricse & 1932 & Soó Rezső & BPU-03691.JPG \\
\hline 1266 & Libanotis pyrenaica (L.) Bourg. & Tarpa & 1951 & $\begin{array}{l}\text { Simon Tibor, Vozáry } \\
\text { Elemér }\end{array}$ & BPU-03689.JPG \\
\hline 1268 & Seseli hippomarathrum Jacq. & Budapest & 1982 & Felföldy Lajos & BPU-03666.JPG \\
\hline 1268 & Seseli hippomarathrum Jacq. & Fertőrákos & 1937 & Soó Rezső & BPU-03663.JPG \\
\hline 1268 & Seseli hippomarathrum Jacq. & Gánt & 1948 & Soó Rezső & BPU-03662.JPG \\
\hline 1268 & Seseli hippomarathrum Jacq. & Nagyharsány & 1961 & Simon Tibor & BPU-03665.JPG \\
\hline 1268 & Seseli hippomarathrum Jacq. & Tihany & 1949 & Simon Tibor & BPU-03664.JPG \\
\hline 1269 & Seseli annuum L. & Szentbékkálla & 1957 & Felföldy Lajos & BPU-03668.JPG \\
\hline 1269 & Seseli annuum L. & Vászoly & 1958 & Felföldy Lajos & BPU-03667.JPG \\
\hline 1269 & Seseli annuum L. & Zalagyömörő & 1957 & Felföldy Lajos & BPU-03669.JPG \\
\hline 1269 & Seseli annuum L. & Zámoly & 1982 & Felföldy Lajos & BPU-03670.JPG \\
\hline 1270 & Seseli leucospermum Waldst. et Kit. & Bodajk & 1961 & Borsos Olga, Simon Tibor & BPU-03671.JPG \\
\hline 1270 & Seseli leucospermum Waldst. et Kit. & Budapest & - & Steinitz, W. & BPU-03673.JPG \\
\hline 1270 & Seseli leucospermum Waldst. et Kit. & Gyenesdiás & 1928 & Soó Rezső & BPU-03672.JPG \\
\hline 1271 & Seseli varium Trevir. & Abaújszántó & 1948 & Soó Rezső & BPU-03675.JPG \\
\hline 1271 & Seseli varium Trevir. & Budapest & 1982 & Felföldy Lajos & BPU-03679.JPG \\
\hline 1271 & Seseli varium Trevir. & Budapest & 1991 & Felföldy Lajos & BPU-03674.JPG \\
\hline 1271 & Seseli varium Trevir. & Csomád & 1953 & Felföldy Lajos & BPU-03676.JPG \\
\hline 1271 & Seseli varium Trevir. & Egyek & 1947 & Soó Rezső & BPU-03680.JPG \\
\hline 1271 & Seseli varium Trevir. & Egyek & 1960 & Simon Tibor & BPU-03677.JPG \\
\hline 1271 & Seseli varium Trevir. & Göd & 1948 & Soó Rezső & BPU-03678.JPG \\
\hline 1272 & Seseli osseum Crantz em. Simonk. & Bakonyszentlászló & 1963 & Soó Rezső & BPU-03687.JPG \\
\hline 1272 & Seseli osseum Crantz em. Simonk. & Budapest & 1947 & Soó Rezső & BPU-03686.JPG \\
\hline 1272 & Seseli osseum Crantz em. Simonk. & Budapest & 1982 & Felföldy Lajos & BPU-03683.JPG \\
\hline 1272 & Seseli osseum Crantz em. Simonk. & Jósvafő & 1951 & Soó Rezső & BPU-03681.JPG \\
\hline 1272 & Seseli osseum Crantz em. Simonk. & Nagyvisnyó & 1948 & $\begin{array}{l}\text { Simon Tibor, Pólya } \\
\text { László, Jakucs Pál }\end{array}$ & BPU-03685.JPG \\
\hline 1272 & Seseli osseum Crantz em. Simonk. & Tibolddaróc & 1953 & Soó Rezső & BPU-03684.JPG \\
\hline 1272 & Seseli osseum Crantz em. Simonk. & Tihany & 1958 & Felföldy Lajos & BPU-03682.JPG \\
\hline 1273 & Oenanthe aquatica (L.) Poir. & Kapolcs & 1959 & $\begin{array}{l}\text { Jávorka Sándor, Felföldy } \\
\text { Lajos, Tóth László }\end{array}$ & BPU-03700.JPG \\
\hline 1273 & Oenanthe aquatica (L.) Poir. & Tiszakerecseny & 1987 & Felföldy Lajos & BPU-03698.JPG \\
\hline 1273 & Oenanthe aquatica (L.) Poir. & Újfehértó & 1935 & Soó Rezső & BPU-03699.JPG \\
\hline 1274 & Oenanthe fistulosa L. & Budapest & 1946 & Kárpáti Zoltán & BPU-03703.JPG \\
\hline 1274 & Oenanthe fistulosa L. & Hatvan & 1931 & Tuzson János & BPU-03702.JPG \\
\hline 1274 & Oenanthe fistulosa L. & Nagykanizsa & 1949 & Károlyi Árpád & BPU-03704.JPG \\
\hline 1275 & Oenanthe silaifolia M. Bieb. & Beregdaróc & 1951 & $\begin{array}{l}\text { Simon Tibor, Vozáry } \\
\text { Elemér }\end{array}$ & BPU-03705.JPG \\
\hline 1275 & Oenanthe silaifolia M. Bieb. & Hortobágy & 1947 & Soó Rezső & BPU-03707.JPG \\
\hline 1275 & Oenanthe silaifolia M. Bieb. & Szentbékkálla & 1959 & $\begin{array}{l}\text { Jávorka Sándor, Felföldy } \\
\text { Lajos, Tóth László }\end{array}$ & BPU-03708.JPG \\
\hline 1275 & Oenanthe silaifolia M. Bieb. & Tarpa & 1949 & Soó Rezső & BPU-03709.JPG \\
\hline 1275 & Oenanthe silaifolia M. Bieb. & Ukk & 1957 & Felföldy Lajos & BPU-03706.JPG \\
\hline 1276 & Oenanthe banatica Heuff. & Debrecen & 1937 & Soó Rezső & BPU-03711.JPG \\
\hline 1276 & Oenanthe banatica Heuff. & Tákos & 1948 & $\begin{array}{l}\text { Pólya László, Simon } \\
\text { Tibor, Jakucs Pál }\end{array}$ & BPU-03710.JPG \\
\hline
\end{tabular}


1. Elektroniukus melléklet - Electronic Appendix 1.

\begin{tabular}{|c|c|c|c|c|c|}
\hline $\begin{array}{l}\text { Sorszám / } \\
\text { Number }\end{array}$ & Taxon-név / Taxon-name & $\begin{array}{l}\text { Település / } \\
\text { Settlement }\end{array}$ & $\begin{array}{l}\text { Év / } \\
\text { Year }\end{array}$ & Gyűjtő / Collector & $\begin{array}{l}\text { Fájlnév / } \\
\text { File-name }\end{array}$ \\
\hline 1277 & Aethusa cynapium L. & Aszófő & 1957 & $\begin{array}{l}\text { Felföldy Lajos, Kovács } \\
\text { Margit, Tóth László }\end{array}$ & BPU-03717.JPG \\
\hline 1277 & Aethusa cynapium L. & Csesznek & 1963 & Soó Rezső & BPU-03715.JPG \\
\hline 1277 & Aethusa cynapium L. & Csesznek & 1963 & Soó Rezső & BPU-03716.JPG \\
\hline 1277 & Aethusa cynapium L. & Debrecen & 1948 & Soó Rezső & BPU-03724.JPG \\
\hline 1277 & Aethusa cynapium L. & Gyöngyös & 1952 & Soó Rezső & BPU-03723.JPG \\
\hline 1277 & Aethusa cynapium L. & Miskolc & 1934 & Soó Rezső & BPU-03714.JPG \\
\hline 1277 & Aethusa cynapium L. & Örvényes & 1958 & Felföldy Lajos & BPU-03720.JPG \\
\hline 1277 & Aethusa cynapium L. & Szenna & 1957 & Soó Rezső & BPU-03721.JPG \\
\hline 1277 & Aethusa cynapium L. & Szilvásvárad & 1953 & $\begin{array}{l}\text { Felföldy Lajos, Tóth } \\
\text { László }\end{array}$ & BPU-03719.JPG \\
\hline 1277 & Aethusa cynapium L. & Uzsa & 1952 & Simon Tibor & BPU-03722.JPG \\
\hline 1277 & Aethusa cynapium L. & Visegrád & 1949 & Soó Rezső & BPU-03718.JPG \\
\hline 1277 & Aethusa cynapium L. & Zámoly & 1982 & Felföldy Lajos & BPU-03725.JPG \\
\hline 1280 & Silaum silaus (L.) Schinz et Thell. & Miskolc & 1934 & Soó Rezső & BPU-03727.JPG \\
\hline 1280 & Silaum silaus (L.) Schinz et Thell. & Miskolc & 1942 & Soó Rezső & BPU-03729.JPG \\
\hline 1280 & Silaum silaus (L.) Schinz et Thell. & Sopron & 1947 & Kárpáti Zoltán & BPU-03728.JPG \\
\hline 1281 & Physospermum cornubiense (L.) DC. & Dömös & 1948 & Vajda László & BPU-03533.JPG \\
\hline 1282 & Conium maculatum L. & Budapest & 1991 & Felföldy Lajos & BPU-03532.JPG \\
\hline 1283 & $\begin{array}{l}\text { Pleurospermum austriacum (L.) } \\
\text { Hoffm. }\end{array}$ & Ágfalva & 1944 & Kárpáti Zoltán & BPU-03538.JPG \\
\hline 1283 & $\begin{array}{l}\text { Pleurospermum austriacum (L.) } \\
\text { Hoffm. }\end{array}$ & Gyöngyös & 1953 & Soó Rezső & BPU-03537.JPG \\
\hline 1283 & $\begin{array}{l}\text { Pleurospermum austriacum (L.) } \\
\text { Hoffm. }\end{array}$ & Kőszeg & 1932 & Soó Rezső & BPU-03539.JPG \\
\hline 1283 & $\begin{array}{l}\text { Pleurospermum austriacum (L.) } \\
\text { Hoffm. }\end{array}$ & Miskolc & 1950 & Soó Rezső & BPU-03534.JPG \\
\hline 1283 & $\begin{array}{l}\text { Pleurospermum austriacum (L.) } \\
\text { Hoffm. }\end{array}$ & Szilvásvárad & 1953 & Soó Rezső & BPU-03536.JPG \\
\hline 1284 & Bupleurum rotundifolium L. & Arka & 1938 & Soó Rezső & BPU-03540.JPG \\
\hline 1284 & Bupleurum rotundifolium L. & Tihany & 1959 & Felföldy Lajos & BPU-03541.JPG \\
\hline 1286 & Bupleurum longifolium L. & Bélapátfalva & 1932 & Soó Rezső & BPU-03546.JPG \\
\hline 1286 & Bupleurum longifolium L. & Budakeszi & 1947 & Soó Rezső & BPU-03549.JPG \\
\hline 1286 & Bupleurum longifolium L. & Gyöngyös & 1951 & Jakucs Pál, Ér Lajos & BPU-03551.JPG \\
\hline 1286 & Bupleurum longifolium L. & Gyöngyös & 1963 & Soó Rezső & BPU-03547.JPG \\
\hline 1286 & Bupleurum longifolium L. & Isztimér & 1949 & Soó Rezső & BPU-03550.JPG \\
\hline 1286 & Bupleurum longifolium L. & Miskolc & 1953 & Soó Rezső & BPU-03548.JPG \\
\hline 1286 & Bupleurum longifolium L. & Miskolc & 1960 & Simon Tibor & BPU-03542.JPG \\
\hline 1287 & Bupleurum tenuissimum L. & Balatonszőlős & 1957 & Felföldy Lajos & BPU-03554.JPG \\
\hline 1287 & Bupleurum tenuissimum L. & Beregdaróc & 1951 & $\begin{array}{l}\text { Simon Tibor, Vozáry } \\
\text { Elemér }\end{array}$ & BPU-03557.JPG \\
\hline 1287 & Bupleurum tenuissimum L. & Budapest & 1944 & Papp József & BPU-03553.JPG \\
\hline 1287 & Bupleurum tenuissimum L. & Bugyi & 1950 & Soó Rezső & BPU-03552.JPG \\
\hline 1287 & Bupleurum tenuissimum L. & Egyek & 1932 & Soó Rezső & BPU-03558.JPG \\
\hline 1287 & Bupleurum tenuissimum L. & Gelénes & 1951 & $\begin{array}{l}\text { Simon Tibor, Vozáry } \\
\text { Elemér }\end{array}$ & BPU-03556.JPG \\
\hline 1287 & Bupleurum tenuissimum L. & Tihany & 1956 & Felföldy Lajos & BPU-03555.JPG \\
\hline 1288 & Bupleurum falcatum L. & Balatonszőlős & 1957 & Felföldy Lajos & BPU-03559.JPG \\
\hline 1288 & Bupleurum falcatum L. & Bódvaszilas & 1950 & Jakucs Pál, Kulcsár Gábor & BPU-03567.JPG \\
\hline 1288 & Bupleurum falcatum L. & Budapest & 1947 & Soó Rezső & BPU-03563.JPG \\
\hline 1288 & Bupleurum falcatum L. & Budapest & 1982 & Felföldy Lajos & BPU-03562.JPG \\
\hline
\end{tabular}


1. Elektroniukus melléklet - Electronic Appendix 1.

\begin{tabular}{|c|c|c|c|c|c|}
\hline $\begin{array}{l}\text { Sorszám / } \\
\text { Number }\end{array}$ & Taxon-név / Taxon-name & $\begin{array}{l}\text { Település / } \\
\text { Settlement }\end{array}$ & $\begin{array}{l}\text { Év / } \\
\text { Year }\end{array}$ & Gyűjtő / Collector & $\begin{array}{l}\text { Fájlnév / } \\
\text { File-name }\end{array}$ \\
\hline 1288 & Bupleurum falcatum L. & Budapest & 1991 & Felföldy Lajos & BPU-03560.JPG \\
\hline 1288 & Bupleurum falcatum L. & Miskolc & 1952 & Borsos Olga & BPU-03565.JPG \\
\hline 1288 & Bupleurum falcatum L. & Miskolc & 1952 & Borsos Olga & BPU-03568.JPG \\
\hline 1288 & Bupleurum falcatum L. & Tihany & 1959 & Felföldy Lajos & BPU-03561.JPG \\
\hline 1288 & Bupleurum falcatum L. & Uzsa & 1952 & Borsos Olga & BPU-03566.JPG \\
\hline 1289 & Bupleurum praealtum L. & Balatonalmádi & 1934 & Jávorka Sándor & BPU-03575.JPG \\
\hline 1289 & Bupleurum praealtum L. & Bélapátfalva & 1974 & Priszter Szaniszló & BPU-03572.JPG \\
\hline 1289 & Bupleurum praealtum L. & Gyöngyös & 1961 & Soó Rezső & BPU-03571.JPG \\
\hline 1289 & Bupleurum praealtum L. & Nagymaros & 1947 & Soó Rezső & BPU-03574.JPG \\
\hline 1289 & Bupleurum praealtum L. & Szilvásvárad & 1953 & $\begin{array}{l}\text { Felföldy Lajos, Tóth } \\
\text { László }\end{array}$ & BPU-03573.JPG \\
\hline 1291 & Bupleurum affine Sadler & Csarnóta & 1968 & Priszter Szaniszló & BPU-03579.JPG \\
\hline 1291 & Bupleurum affine Sadler & Harka & 1948 & Kárpáti Zoltán & BPU-03583.JPG \\
\hline 1291 & Bupleurum affine Sadler & Nagyharsány & 1961 & Simon Tibor & BPU-03580.JPG \\
\hline 1291 & Bupleurum affine Sadler & Nagyharsány & 1964 & Priszter Szaniszló & BPU-03581.JPG \\
\hline 1291 & Bupleurum affine Sadler & Nagymaros & 1947 & Soó Rezső & BPU-03582.JPG \\
\hline 1291 & Bupleurum affine Sadler & Tihany & 1948 & Károlyi Árpád & BPU-03585.JPG \\
\hline 1291 & Bupleurum affine Sadler & Uzsa & 1952 & Borsos Olga & BPU-03584.JPG \\
\hline 1292 & Bupleurum pachnospermum Pančić & Tihany & 1959 & Felföldy Lajos & BPU-03577.JPG \\
\hline 1292 & Bupleurum pachnospermum Pančić & Tihany & 1961 & Felföldy Lajos & BPU-03576.JPG \\
\hline 1292 & Bupleurum pachnospermum Pančić & Tokaj & 1949 & Soó Rezső & BPU-03578.JPG \\
\hline 1293 & Trinia glauca (L.) Dumort. & Abaújszántó & 1938 & Soó Rezső & BPU-03594.JPG \\
\hline 1293 & Trinia glauca (L.) Dumort. & Budapest & 1947 & Soó Rezső & BPU-03592.JPG \\
\hline 1293 & Trinia glauca (L.) Dumort. & Budapest & 1952 & Soó Rezső & BPU-15423.JPG \\
\hline 1293 & Trinia glauca (L.) Dumort. & Budapest & 1982 & Felföldy Lajos & BPU-03593.JPG \\
\hline 1293 & Trinia glauca (L.) Dumort. & Fót & 1960 & Soó Rezső & BPU-03597.JPG \\
\hline 1293 & Trinia glauca (L.) Dumort. & Pécs & 1936 & Soó Rezső & BPU-03596.JPG \\
\hline 1293 & Trinia glauca (L.) Dumort. & Pécs & 1956 & Soó Rezső & BPU-03595.JPG \\
\hline 1293 & Trinia glauca (L.) Dumort. & Tihany & 1958 & Felföldy Lajos & BPU-03598.JPG \\
\hline 1294 & $\begin{array}{l}\text { Trinia ramosissima (Fisch.) W.D.J. } \\
\text { Koch }\end{array}$ & Pomáz & 1913 & Degen Árpád & BPU-03600.JPG \\
\hline 1295 & Apium repens (Jacq.) Lag. & Budapest & 1943 & Kárpáti Zoltán & BPU-03602.JPG \\
\hline 1295 & Apium repens (Jacq.) Lag. & Dunakeszi & 1948 & Soó Rezső & BPU-03603.JPG \\
\hline 1297 & Petroselinum crispum (Mill.) A.W. Hill & Szilvásvárad & 1952 & Borsos Olga & BPU-03604.JPG \\
\hline 1298 & Cicuta virosa L. & Csaroda & 1952 & Soó Rezső & BPU-03606.JPG \\
\hline 1298 & Cicuta virosa L. & Csaroda & 1952 & Soó Rezső & BPU-03607.JPG \\
\hline 1298 & Cicuta virosa L. & Keszthely & 1982 & Plósz Sándor & BPU-03608.JPG \\
\hline 1298 & Cicuta virosa $L$. & Szőce & 1955 & Soó Rezső & BPU-03605.JPG \\
\hline 1299 & Falcaria vulgaris Bernh. & Budapest & 1982 & Felföldy Lajos & BPU-03610.JPG \\
\hline 1299 & Falcaria vulgaris Bernh. & Budapest & 2004 & Felföldy Lajos & BPU-03609.JPG \\
\hline 1299 & Falcaria vulgaris Bernh. & Tokaj & 1949 & Soó Rezső & BPU-03611.JPG \\
\hline 1300 & Carum carvi L. & Aszófő & 1959 & Felföldy Lajos & BPU-03616.JPG \\
\hline 1300 & Carum carvi L. & Kétegyháza & 1923 & Soó Rezső & BPU-03615.JPG \\
\hline 1300 & Carum carvi L. & Miskolc & 1939 & Soó Rezső & BPU-03614.JPG \\
\hline 1300 & Carum carvi L. & Nagyvisnyó & 1953 & Soó Rezső & BPU-03612.JPG \\
\hline 1300 & Carum carvi L. & Pálháza & 1952 & Simon Tibor & BPU-03613.JPG \\
\hline 1301 & Cnidium dubium (Schkuhr) Thell. & Egyek & 1938 & Soó Rezső & BPU-03733.JPG \\
\hline 1301 & Cnidium dubium (Schkuhr) Thell. & Kiskunhalas & 1964 & Soó Rezső & BPU-03730.JPG \\
\hline
\end{tabular}


1. Elektroniukus melléklet - Electronic Appendix 1.

\begin{tabular}{|c|c|c|c|c|c|}
\hline $\begin{array}{l}\text { Sorszám / } \\
\text { Number }\end{array}$ & Taxon-név / Taxon-name & $\begin{array}{l}\text { Település / } \\
\text { Settlement }\end{array}$ & $\begin{array}{l}\text { Év / } \\
\text { Year }\end{array}$ & Gyújtő / Collector & $\begin{array}{l}\text { Fájlnév / } \\
\text { File-name }\end{array}$ \\
\hline 1301 & Cnidium dubium (Schkuhr) Thell. & Miskolc & 1936 & Soó Rezső & BPU-03732.JPG \\
\hline 1301 & Cnidium dubium (Schkuhr) Thell. & Tatárszentgyörgy & 1950 & Soó Rezső & BPU-03731.JPG \\
\hline 1302 & Selinum carvifolia L. & Debrecen & 1934 & Soó Rezső & BPU-03742.JPG \\
\hline 1302 & Selinum carvifolia L. & Gyöngyös & 1952 & Soó Rezső & BPU-03738.JPG \\
\hline 1302 & Selinum carvifolia L. & Kemence & 1952 & Simon Tibor & BPU-03740.JPG \\
\hline 1302 & Selinum carvifolia L. & Kőszeg & 1963 & Soó Rezső, Borsos Olga & BPU-03737.JPG \\
\hline 1302 & Selinum carvifolia L. & Miskolc & 1934 & Soó Rezső & BPU-03741.JPG \\
\hline 1302 & Selinum carvifolia L. & Szenta & 1955 & Soó Rezső & BPU-03736.JPG \\
\hline 1302 & Selinum carvifolia L. & Tákos & 1948 & Simon Tibor, Jakucs Pál & BPU-03739.JPG \\
\hline 1303 & Angelica palustris (Besser) Hoffm. & Bátorliget & 1932 & Soó Rezső & BPU-03749.JPG \\
\hline 1303 & Angelica palustris (Besser) Hoffm. & Bátorliget & 1950 & Simon Tibor & BPU-03750.JPG \\
\hline 1303 & Angelica palustris (Besser) Hoffm. & Bátorliget & 1950 & $\begin{array}{l}\text { Simon Tibor, Kárpáti } \\
\text { István }\end{array}$ & BPU-03748.JPG \\
\hline 1304 & Angelica sylvestris L. & Alsószölnök & 1955 & Soó Rezső & BPU-03782.JPG \\
\hline 1304 & Angelica sylvestris L. & Alsószölnök & 1955 & Soó Rezső & BPU-03783.JPG \\
\hline 1304 & Angelica sylvestris L. & Bagamér & 1932 & Soó Rezső & BPU-03757.JPG \\
\hline 1304 & Angelica sylvestris L. & Bagamér & 1932 & Soó Rezső & BPU-03758.JPG \\
\hline 1304 & Angelica sylvestris L. & Balatonszőlős & 1957 & Felföldy Lajos & BPU-03762.JPG \\
\hline 1304 & Angelica sylvestris L. & Bátorliget & 1934 & Igmándy József & BPU-03772.JPG \\
\hline 1304 & Angelica sylvestris L. & Bodajk & 1942 & Felföldy Lajos & BPU-03780.JPG \\
\hline 1304 & Angelica sylvestris L. & Budapest & 1916 & Degen Árpád & BPU-03752.JPG \\
\hline 1304 & Angelica sylvestris L. & Budapest & 2004 & Felföldy Lajos & BPU-03751.JPG \\
\hline 1304 & Angelica sylvestris L. & Csorna & 1920 & Polgár Sándor & BPU-03771.JPG \\
\hline 1304 & Angelica sylvestris L. & Debrecen & 1933 & Soó Rezső & BPU-03756.JPG \\
\hline 1304 & Angelica sylvestris L. & Erdőbénye & 1948 & Soó Rezső & BPU-03774.JPG \\
\hline 1304 & Angelica sylvestris L. & Erdőbénye & 1952 & Borsos Olga & BPU-03775.JPG \\
\hline 1304 & Angelica sylvestris L. & Győr & 1908 & Polgár Sándor & BPU-03755.JPG \\
\hline 1304 & Angelica sylvestris L. & Háromhuta & 1937 & Igmándy József & BPU-03754.JPG \\
\hline 1304 & Angelica sylvestris L. & Hosszúpályi & 1932 & Máthé Imre & BPU-03770.JPG \\
\hline 1304 & Angelica sylvestris L. & Mór & 1942 & Felföldy Lajos & BPU-03781.JPG \\
\hline 1304 & Angelica sylvestris L. & Nagykanizsa & 1949 & Károlyi Árpád & BPU-03765.JPG \\
\hline 1304 & Angelica sylvestris L. & Nagyvisnyó & 1928 & Igmándy József & BPU-03767.JPG \\
\hline 1304 & Angelica sylvestris L. & Nyírmártonfalva & 1934 & Máthé Imre & BPU-03760.JPG \\
\hline 1304 & Angelica sylvestris L. & Nyírpilis & 1932 & Soó Rezső & BPU-03761.JPG \\
\hline 1304 & Angelica sylvestris L. & Ócsa & 1950 & Soó Rezső & BPU-03759.JPG \\
\hline 1304 & Angelica sylvestris L. & Örvényes & 1958 & Felföldy Lajos & BPU-03763.JPG \\
\hline 1304 & Angelica sylvestris L. & Pálháza & 1952 & Borsos Olga & BPU-03766.JPG \\
\hline 1304 & Angelica sylvestris L. & Piricse & 1932 & Soó Rezső & BPU-03773.JPG \\
\hline 1304 & Angelica sylvestris L. & Pusztamiske & 1948 & Borsos Olga & BPU-03785.JPG \\
\hline 1304 & Angelica sylvestris L. & Szőce & 1955 & Soó Rezső & BPU-03784.JPG \\
\hline 1304 & Angelica sylvestris L. & Tapolca & 1928 & Soó Rezső & BPU-03764.JPG \\
\hline 1304 & Angelica sylvestris L. & Vének & 1920 & Polgár Sándor & BPU-03769.JPG \\
\hline 1304 & Angelica sylvestris L. & Zámoly & 1982 & Felföldy Lajos & BPU-03768.JPG \\
\hline 1308 & $\begin{array}{l}\text { Peucedanum verticillare (L.) W.D.J. } \\
\text { Koch }\end{array}$ & Őrtilos & 1949 & Károlyi Árpád & BPU-03789.JPG \\
\hline 1309 & Peucedanum carvifolia Vill. & Erdőbénye & 1948 & Soó Rezső & BPU-03793.JPG \\
\hline 1309 & Peucedanum carvifolia Vill. & Gyöngyös & 1953 & Soó Rezső & BPU-03791.JPG \\
\hline 1309 & Peucedanum carvifolia Vill. & Tarpa & 1948 & Simon Tibor, Jakucs Pál & BPU-03792.JPG \\
\hline
\end{tabular}


1. Elektroniukus melléklet - Electronic Appendix 1.

\begin{tabular}{|c|c|c|c|c|c|}
\hline $\begin{array}{l}\text { Sorszám / } \\
\text { Number }\end{array}$ & Taxon-név / Taxon-name & $\begin{array}{l}\text { Település / } \\
\text { Settlement }\end{array}$ & $\begin{array}{l}\text { Év / } \\
\text { Year }\end{array}$ & Gyújtő / Collector & $\begin{array}{l}\text { Fájlnév / } \\
\text { File-name }\end{array}$ \\
\hline 1310 & Peucedanum arenarium Waldst. et Kit. & Bátorliget & 1932 & Soó Rezső & BPU-03796.JPG \\
\hline 1310 & Peucedanum arenarium Waldst. et Kit. & Göd & 1948 & Soó Rezső & BPU-03794.JPG \\
\hline 1310 & Peucedanum arenarium Waldst. et Kit. & Gödöllő & 1949 & Soó Rezső & BPU-03795.JPG \\
\hline 1311 & Peucedanum officinale L. & Hortobágy & 1947 & Soó Rezső & BPU-03799.JPG \\
\hline 1311 & Peucedanum officinale L. & Kerecsend & 1957 & Soó Rezső & BPU-03797.JPG \\
\hline 1311 & Peucedanum officinale L. & Kerecsend & 1957 & Soó Rezső & BPU-03798.JPG \\
\hline 1311 & Peucedanum officinale L. & Tarpa & 1948 & Simon Tibor, Jakucs Pál & BPU-03801.JPG \\
\hline 1312 & Peucedanum alsaticum L. & Budapest & 1947 & Soó Rezső & BPU-03804.JPG \\
\hline 1312 & Peucedanum alsaticum L. & Budapest & 1989 & Felföldy Lajos & BPU-03806.JPG \\
\hline 1312 & Peucedanum alsaticum L. & Csarnóta & 1968 & Priszter Szaniszló & BPU-03805.JPG \\
\hline 1312 & Peucedanum alsaticum L. & Egyek & 1934 & Soó Rezső & BPU-03807.JPG \\
\hline 1312 & Peucedanum alsaticum L. & Erdőbénye & 1948 & Soó Rezső & BPU-03808.JPG \\
\hline 1312 & Peucedanum alsaticum L. & Kerecsend & 1957 & Soó Rezső & BPU-03803.JPG \\
\hline 1313 & Peucedanum palustre (L.) Moench & Csaroda & 1952 & Soó Rezső & BPU-03813.JPG \\
\hline 1313 & Peucedanum palustre (L.) Moench & Nyírpilis & 1932 & Soó Rezső & BPU-03809.JPG \\
\hline 1313 & Peucedanum palustre (L.) Moench & Tatárszentgyörgy & 1950 & Soó Rezső & BPU-03811.JPG \\
\hline 1313 & Peucedanum palustre (L.) Moench & Veresegyház & 1949 & Soó Rezső & BPU-03810.JPG \\
\hline 1314 & Peucedanum cervaria (L.) Lapeyr. & Bátorliget & 1932 & Soó Rezső & BPU-03818.JPG \\
\hline 1314 & Peucedanum cervaria (L.) Lapeyr. & Budapest & 1982 & Felföldy Lajos & BPU-03815.JPG \\
\hline 1314 & Peucedanum cervaria (L.) Lapeyr. & Budapest & 1991 & Felföldy Lajos & BPU-03816.JPG \\
\hline 1314 & Peucedanum cervaria (L.) Lapeyr. & Csákvár & 1948 & Soó Rezső & BPU-03814.JPG \\
\hline 1314 & Peucedanum cervaria (L.) Lapeyr. & Jósvafő & 1951 & Jakucs Pál, Ér Lajos & BPU-03779.JPG \\
\hline 1314 & Peucedanum cervaria (L.) Lapeyr. & Kerecsend & 1957 & Soó Rezső & BPU-03817.JPG \\
\hline 1315 & Peucedanum oreoselinum (L.) Moench & Debrecen & 1989 & Felföldy Lajos & BPU-03822.JPG \\
\hline 1315 & Peucedanum oreoselinum (L.) Moench & Nagybajom & 1957 & Soó Rezső & BPU-03823.JPG \\
\hline 1315 & Peucedanum oreoselinum (L.) Moench & Vállaj & 1934 & Soó Rezső & BPU-03821.JPG \\
\hline 1315 & Peucedanum oreoselinum (L.) Moench & Zalagyömörő & 1957 & Felföldy Lajos & BPU-03820.JPG \\
\hline 1316 & Pastinaca sativa L. & Aszófő & 1957 & $\begin{array}{l}\text { Felföldy Lajos, Kovács } \\
\text { Margit, Tóth László }\end{array}$ & BPU-03826.JPG \\
\hline 1316 & Pastinaca sativa L. & Bátorliget & 1934 & Soó Rezső & BPU-03825.JPG \\
\hline 1316 & Pastinaca sativa L. & Zámoly & 1982 & Felföldy Lajos & BPU-03824.JPG \\
\hline 1317 & Heracleum sphondylium L. & Balatonszőlős & 1957 & $\begin{array}{l}\text { Felföldy Lajos, Kovács } \\
\text { Margit, Tóth László }\end{array}$ & BPU-03827.JPG \\
\hline 1317 & Heracleum sphondylium L. & Csesznek & 1963 & Soó Rezső & BPU-03831.JPG \\
\hline 1317 & Heracleum sphondylium L. & Kismaros & 1982 & Felföldy Lajos & BPU-03832.JPG \\
\hline 1317 & Heracleum sphondylium L. & Kőszeg & 1963 & Soó Rezső, Borsos Olga & BPU-03830.JPG \\
\hline 1317 & Heracleum sphondylium L. & Szilvásvárad & 1953 & $\begin{array}{l}\text { Felföldy Lajos, Tóth } \\
\text { László }\end{array}$ & BPU-03829.JPG \\
\hline 1317 & Heracleum sphondylium L. & Zámoly & 1982 & Felföldy Lajos & BPU-03828.JPG \\
\hline 1320 & Tordylium maximum L. & Gyöngyös & 1935 & Soó Rezső & BPU-03834.JPG \\
\hline 1320 & Tordylium maximum L. & Nagymaros & 1947 & Soó Rezső & BPU-03833.JPG \\
\hline 1321 & Laser trilobum (L.) Borkh. & Budapest & 1947 & Soó Rezső & BPU-03837.JPG \\
\hline 1321 & Laser trilobum (L.) Borkh. & Gyöngyös & 1961 & Soó Rezső & BPU-03839.JPG \\
\hline 1321 & Laser trilobum (L.) Borkh. & Miskolc & 1929 & Soó Rezső & BPU-03836.JPG \\
\hline 1321 & Laser trilobum (L.) Borkh. & Nagyvisnyó & 1953 & Soó Rezső & BPU-03838.JPG \\
\hline 1321 & Laser trilobum (L.) Borkh. & Pécs & 1936 & Soó Rezső & BPU-03835.JPG \\
\hline 1322 & Laserpitium prutenicum L. & Hosszúpályi & 1933 & Soó Rezső & BPU-03841.JPG \\
\hline 1322 & Laserpitium prutenicum L. & Kőszeg & 1932 & Soó Rezső & BPU-03840.JPG \\
\hline 1322 & Laserpitium prutenicum L. & Miskolc & 1929 & Soó Rezső & BPU-03842.JPG \\
\hline
\end{tabular}


1. Elektroniukus melléklet - Electronic Appendix 1.

\begin{tabular}{|c|c|c|c|c|c|}
\hline $\begin{array}{l}\text { Sorszám / } \\
\text { Number }\end{array}$ & Taxon-név / Taxon-name & $\begin{array}{l}\text { Település / } \\
\text { Settlement }\end{array}$ & $\begin{array}{l}\text { Év / } \\
\text { Year }\end{array}$ & Gyüjtő / Collector & $\begin{array}{l}\text { Fájlnév / } \\
\text { File-name }\end{array}$ \\
\hline 1323 & Laserpitium latifolium L. & Balatonszőlős & 1958 & Felföldy Lajos & BPU-03845.JPG \\
\hline 1323 & Laserpitium latifolium L. & Budapest & 1947 & Soó Rezső & BPU-03846.JPG \\
\hline 1323 & Laserpitium latifolium L. & Budapest & 1982 & Felföldy Lajos & BPU-03843.JPG \\
\hline 1323 & Laserpitium latifolium L. & Hidegkút & 1959 & Felföldy Lajos & BPU-03844.JPG \\
\hline 1323 & Laserpitium latifolium L. & Szilvásvárad & 1953 & Soó Rezső & BPU-03847.JPG \\
\hline 1324 & Torilis arvensis (Huds.) Link & Balatonfüred & 1947 & Pólya László & BPU-03495.JPG \\
\hline 1324 & Torilis arvensis (Huds.) Link & Budaörs & 1991 & Felföldy Lajos & BPU-03493.JPG \\
\hline 1324 & Torilis arvensis (Huds.) Link & Budapest & 2004 & Felföldy Lajos & BPU-03491.JPG \\
\hline 1324 & Torilis arvensis (Huds.) Link & Gyöngyös & 1961 & Soó Rezső & BPU-03502.JPG \\
\hline 1324 & Torilis arvensis (Huds.) Link & Hajdúszoboszló & 1937 & Soó Rezső & BPU-03492.JPG \\
\hline 1324 & Torilis arvensis (Huds.) Link & Tihany & 1959 & Felföldy Lajos & BPU-03494.JPG \\
\hline 1325 & Torilis japonica (Houtt.) DC. & Balatonszőlős & 1957 & $\begin{array}{l}\text { Felföldy Lajos, Kovács } \\
\text { Margit, Tóth László }\end{array}$ & BPU-03506.JPG \\
\hline 1325 & Torilis japonica (Houtt.) DC. & Bélapátfalva & 1948 & $\begin{array}{l}\text { Simon Tibor, Pólya } \\
\text { László, Jakucs Pál }\end{array}$ & BPU-03498.JPG \\
\hline 1325 & Torilis japonica (Houtt.) DC. & Budapest & 1982 & Felföldy Lajos & BPU-03497.JPG \\
\hline 1325 & Torilis japonica (Houtt.) DC. & Budapest & 2004 & Felföldy Lajos & BPU-03496.JPG \\
\hline 1325 & Torilis japonica (Houtt.) DC. & Miskolc & 1950 & Soó Rezső & BPU-03499.JPG \\
\hline 1325 & Torilis japonica (Houtt.) DC. & Nagybajom & 1955 & Soó Rezső & BPU-03504.JPG \\
\hline 1325 & Torilis japonica (Houtt.) DC. & Nyíregyháza & 1933 & Soó Rezső & BPU-03505.JPG \\
\hline 1325 & Torilis japonica (Houtt.) DC. & Pécsely & 1990 & Felföldy Lajos & BPU-03507.JPG \\
\hline 1325 & Torilis japonica (Houtt.) DC. & Vászoly & 1958 & $\begin{array}{l}\text { Felföldy Lajos, Kovács } \\
\text { Margit, Tóth László }\end{array}$ & BPU-03508.JPG \\
\hline 1325 & Torilis japonica (Houtt.) DC. & Verőce & 1982 & Felföldy Lajos & BPU-03503.JPG \\
\hline 1325 & Torilis japonica (Houtt.) DC. & Zámoly & 1982 & Felföldy Lajos & BPU-03500.JPG \\
\hline 1325 & Torilis japonica (Houtt.) DC. & Zámoly & 1982 & Felföldy Lajos & BPU-03501.JPG \\
\hline 1326 & Torilis ucranica Spreng. & Tokaj & 1936 & Hulják János & BPU-03509.JPG \\
\hline 1327 & Caucalis platycarpos L. & Budapest & 1946 & Kárpáti Zoltán & BPU-03519.JPG \\
\hline 1327 & Caucalis platycarpos L. & Egyek & 1953 & Borsos Olga & BPU-03518.JPG \\
\hline 1327 & Caucalis platycarpos L. & Hortobágy & 1947 & Soó Rezső & BPU-03517.JPG \\
\hline 1327 & Caucalis platycarpos L. & Nagyharsány & 1965 & $\begin{array}{l}\text { Simon Tibor, Priszter } \\
\text { Szaniszló }\end{array}$ & BPU-03515.JPG \\
\hline 1328 & Turgenia latifolia (L.) Hoffm. & Hortobágy & 1947 & Soó Rezső & BPU-03511.JPG \\
\hline 1328 & Turgenia latifolia (L.) Hoffm. & Szögliget & 1951 & $\begin{array}{l}\text { Soó Rezső, Jakucs Pál, Ér } \\
\text { Lajos }\end{array}$ & BPU-03510.JPG \\
\hline 1328 & Turgenia latifolia (L.) Hoffm. & Tiszacsege & 1950 & $\begin{array}{l}\text { Simon Tibor, Borsos Olga, } \\
\text { Kulcsár Gábor }\end{array}$ & BPU-03512.JPG \\
\hline 1329 & Orlaya grandiflora (L.) Hoffm. & "Szentendrei-sziget" & 1953 & Borsos Olga & BPU-03522.JPG \\
\hline 1329 & Orlaya grandiflora (L.) Hoffm. & Budapest & 1923 & Soó Rezső & BPU-03514.JPG \\
\hline 1329 & Orlaya grandiflora (L.) Hoffm. & Nagyharsány & 1965 & $\begin{array}{l}\text { Simon Tibor, Priszter } \\
\text { Szaniszló }\end{array}$ & BPU-03521.JPG \\
\hline 1329 & Orlaya grandiflora (L.) Hoffm. & Pécs & 1935 & Soó Rezső & BPU-03523.JPG \\
\hline 1329 & Orlaya grandiflora (L.) Hoffm. & Tihany & 1942 & Felföldy Lajos & BPU-03513.JPG \\
\hline 1329 & Orlaya grandiflora (L.) Hoffm. & Tihany & 1959 & Felföldy Lajos & BPU-03516.JPG \\
\hline 1329 & Orlaya grandiflora (L.) Hoffm. & Tihany & 1959 & Felföldy Lajos & BPU-03520.JPG \\
\hline 1329 & Orlaya grandiflora (L.) Hoffm. & Tihany & 1968 & Priszter Szaniszló & BPU-03524.JPG \\
\hline 1330 & Daucus carota L. & Budapest & 2004 & Felföldy Lajos & BPU-03853.JPG \\
\hline 1330 & Daucus carota L. & Vászoly & 1958 & Felföldy Lajos & BPU-03854.JPG \\
\hline 1331 & Pyrola minor L. & Alsószölnök & 1955 & Vajda László & BPU-09031.JPG \\
\hline 1331 & Pyrola minor L. & Arka & 1961 & Simon Tibor & BPU-09034.JPG \\
\hline
\end{tabular}


1. Elektroniukus melléklet - Electronic Appendix 1.

\begin{tabular}{|c|c|c|c|c|c|}
\hline $\begin{array}{l}\text { Sorszám / } \\
\text { Number }\end{array}$ & Taxon-név / Taxon-name & $\begin{array}{l}\text { Település / } \\
\text { Settlement }\end{array}$ & $\begin{array}{l}\text { Év / } \\
\text { Year }\end{array}$ & Gyüjtő / Collector & $\begin{array}{l}\text { Fájlnév / } \\
\text { File-name }\end{array}$ \\
\hline 1331 & Pyrola minor L. & Fenyőfő & 1930 & Soó Rezső & BPU-09033.JPG \\
\hline 1331 & Pyrola minor L. & Nagyvisnyó & 1953 & Soó Rezső & BPU-09029.JPG \\
\hline 1331 & Pyrola minor L. & Szenna & 1957 & Soó Rezső & BPU-09030.JPG \\
\hline 1331 & Pyrola minor L. & Szőce & 1955 & Soó Rezső & BPU-09032.JPG \\
\hline 1332 & Pyrola chlorantha Sw. & Fenyőfő & 1955 & Soó Rezső & BPU-09025.JPG \\
\hline 1332 & Pyrola chlorantha Sw. & Fenyőfő & 1955 & Soó Rezső & BPU-09028.JPG \\
\hline 1332 & Pyrola chlorantha Sw. & Szakonyfalu & 1955 & Soó Rezső & BPU-09027.JPG \\
\hline 1333 & Pyrola rotundifolia L. & Imola & 1936 & Soó Rezső & BPU-09020.JPG \\
\hline 1333 & Pyrola rotundifolia L. & Miskolc & 1950 & Soó Rezső & BPU-09019.JPG \\
\hline 1333 & Pyrola rotundifolia L. & Piricse & 1934 & Soó Rezső & BPU-09021.JPG \\
\hline 1333 & Pyrola rotundifolia L. & Telkibánya & 1962 & Simon Tibor & BPU-09018.JPG \\
\hline 1335 & Orthilia secunda (L.) House & Kishuta & 1950 & Soó Rezső & BPU-09009.JPG \\
\hline 1335 & Orthilia secunda (L.) House & Nagyhuta & 1952 & Simon Tibor & BPU-09010.JPG \\
\hline 1335 & Orthilia secunda (L.) House & Pálháza & 1952 & Simon Tibor & BPU-09007.JPG \\
\hline 1335 & Orthilia secunda (L.) House & Pálháza & 1952 & Simon Tibor & BPU-09008.JPG \\
\hline 1335 & Orthilia secunda (L.) House & Szőce & 1955 & Soó Rezső & BPU-09011.JPG \\
\hline 1336 & Moneses uniflora (L.) A. Gray & Regéc & 1955 & Simon Tibor & BPU-09001.JPG \\
\hline 1337 & $\begin{array}{l}\text { Chimaphila umbellata (L.) W.P.C. } \\
\text { Barton }\end{array}$ & Alsószölnök & 1955 & Vajda László & BPU-08997.JPG \\
\hline 1337 & $\begin{array}{l}\text { Chimaphila umbellata (L.) W.P.C. } \\
\text { Barton }\end{array}$ & Kőszeg & 1932 & Soó Rezső & BPU-08998.JPG \\
\hline 1337 & $\begin{array}{l}\text { Chimaphila umbellata (L.) W.P.C. } \\
\text { Barton }\end{array}$ & Nagyhuta & 1952 & Simon Tibor & BPU-08996.JPG \\
\hline 1337 & $\begin{array}{l}\text { Chimaphila umbellata (L.) W.P.C. } \\
\text { Barton }\end{array}$ & Szakonyfalu & 1955 & Soó Rezső & BPU-08999.JPG \\
\hline 1337 & $\begin{array}{l}\text { Chimaphila umbellata (L.) W.P.C. } \\
\text { Barton }\end{array}$ & Szőce & 1955 & Soó Rezső & BPU-09000.JPG \\
\hline 1338 & Monotropa hypopitys L. & Ágfalva & 1951 & Kárpáti Zoltán & BPU-09036.JPG \\
\hline 1338 & Monotropa hypopitys L. & Alsószölnök & 1955 & Soó Rezső & BPU-09037.JPG \\
\hline 1338 & Monotropa hypopitys L. & Bozsok & 1932 & Kárpáti Zoltán & BPU-09038.JPG \\
\hline 1338 & Monotropa hypopitys L. & Nagyvisnyó & 1953 & Vozáry Elemér & BPU-09035.JPG \\
\hline 1340 & Calluna vulgaris (L.) Hull & Alsószölnök & 1955 & Soó Rezső & BPU-09082.JPG \\
\hline 1340 & Calluna vulgaris (L.) Hull & Kishuta & 1963 & Isépy István & BPU-09080.JPG \\
\hline 1340 & Calluna vulgaris (L.) Hull & Őrimagyarósd & 1968 & Priszter Szaniszló & BPU-09083.JPG \\
\hline 1340 & Calluna vulgaris (L.) Hull & Pálháza & 1938 & Soó Rezső & BPU-09081.JPG \\
\hline 1340 & Calluna vulgaris (L.) Hull & Uzsa & 1952 & Simon Tibor & BPU-09084.JPG \\
\hline 1340 & Calluna vulgaris (L.) Hull & Uzsa & 1953 & Soó Rezső & BPU-09085.JPG \\
\hline 1342 & Vaccinium oxycoccos L. & Csaroda & 1952 & $\begin{array}{l}\text { Simon Tibor, Vozáry } \\
\text { Elemér }\end{array}$ & BPU-09065.JPG \\
\hline 1342 & Vaccinium oxycoccos L. & Csaroda & 1963 & Isépy István & BPU-09067.JPG \\
\hline 1342 & Vaccinium oxycoccos L. & Csaroda & - & $\begin{array}{l}\text { Soó Rezső, Simon Tibor, } \\
\text { Vozáry Elemér }\end{array}$ & BPU-09066.JPG \\
\hline 1343 & Vaccinium vitis-idaea L. & Alsószölnök & 1955 & Soó Rezső & BPU-09042.JPG \\
\hline 1343 & Vaccinium vitis-idaea L. & Háromhuta & 1940 & Hulják János & BPU-09044.JPG \\
\hline 1343 & Vaccinium vitis-idaea L. & Háromhuta & 1955 & Simon Tibor & BPU-09045.JPG \\
\hline 1343 & Vaccinium vitis-idaea L. & Pálháza & 1939 & Soó Rezső & BPU-09041.JPG \\
\hline 1343 & Vaccinium vitis-idaea L. & Szakonyfalu & 1955 & Soó Rezső & BPU-09043.JPG \\
\hline 1344 & Vaccinium myrtillus L. & Alsószölnök & 1955 & Soó Rezső & BPU-09054.JPG \\
\hline 1344 & Vaccinium myrtillus L. & Bátonyterenye & 1937 & Máthé Imre & BPU-09053.JPG \\
\hline 1344 & Vaccinium myrtillus L. & Füzér & 1963 & Isépy István & BPU-09050.JPG \\
\hline
\end{tabular}


1. Elektroniukus melléklet - Electronic Appendix 1.

\begin{tabular}{|c|c|c|c|c|c|}
\hline $\begin{array}{l}\text { Sorszám / } \\
\text { Number }\end{array}$ & Taxon-név / Taxon-name & $\begin{array}{l}\text { Település / } \\
\text { Settlement }\end{array}$ & $\begin{array}{l}\text { Év / } \\
\text { Year }\end{array}$ & Gyújtő / Collector & $\begin{array}{l}\text { Fájlnév / } \\
\text { File-name }\end{array}$ \\
\hline 1344 & Vaccinium myrtillus L. & Gyöngyös & 1951 & Jakucs Pál, Ér Lajos & BPU-09052.JPG \\
\hline 1344 & Vaccinium myrtillus L. & Háromhuta & 1940 & Hulják János & BPU-09058.JPG \\
\hline 1344 & Vaccinium myrtillus L. & Háromhuta & 1949 & Simon Tibor, Jakucs Pál & BPU-09059.JPG \\
\hline 1344 & Vaccinium myrtillus L. & Szakonyfalu & 1955 & Soó Rezső & BPU-09055.JPG \\
\hline 1344 & Vaccinium myrtillus L. & Telkibánya & 1959 & Simon Tibor & BPU-09051.JPG \\
\hline 1344 & Vaccinium myrtillus L. & Uzsa & 1950 & Felföldy Lajos & BPU-09056.JPG \\
\hline 1344 & Vaccinium myrtillus L. & Uzsa & 1959 & $\begin{array}{l}\text { Jávorka Sándor, Felföldy } \\
\text { Lajos }\end{array}$ & BPU-09057.JPG \\
\hline 1345 & Primula auricula L. & Gánt & 1949 & Soó Rezső & BPU-11543.JPG \\
\hline 1345 & Primula auricula L. & Gánt & 1949 & Soó Rezső & BPU-11544.JPG \\
\hline 1345 & Primula auricula L. & Gyenesdiás & 1957 & Entz Béla, Szabó E. & BPU-11542.JPG \\
\hline 1346 & Primula farinosa $\mathrm{L}$. & Lesenceistvánd & 1952 & Simon Tibor & BPU-11550.JPG \\
\hline 1346 & Primula farinosa $\mathrm{L}$. & Lesencetomaj & 1957 & Entz Béla, Szabó E. & BPU-11549.JPG \\
\hline 1346 & Primula farinosa $\mathrm{L}$. & Sopron & 1932 & Soó Rezső & BPU-11551.JPG \\
\hline 1346 & Primula farinosa $\mathrm{L}$. & Tapolca & 1928 & Soó Rezső & BPU-11548.JPG \\
\hline 1346 & Primula farinosa $\mathrm{L}$. & Uzsa & 1951 & Felföldy Lajos & BPU-11547.JPG \\
\hline 1347 & Primula vulgaris Huds. & Bakonybél & 1955 & Soó Rezső & BPU-11536.JPG \\
\hline 1347 & Primula vulgaris Huds. & Fehérgyarmat & 1953 & Simon Tibor & BPU-11535.JPG \\
\hline 1347 & Primula vulgaris Huds. & Nagybajom & 1957 & Soó Rezső & BPU-11533.JPG \\
\hline 1348 & Primula veris $\mathrm{L}$. & Budapest & 1949 & Soó Rezső & BPU-11529.JPG \\
\hline 1348 & Primula veris $\mathrm{L}$. & Gánt & 1949 & Soó Rezső & BPU-11530.JPG \\
\hline 1348 & Primula veris $\mathrm{L}$. & Hidegkút & 1958 & Felföldy Lajos & BPU-11527.JPG \\
\hline 1348 & Primula veris L. & Kapolcs & 1959 & $\begin{array}{l}\text { Jávorka Sándor, Felföldy } \\
\text { Lajos, Tóth László }\end{array}$ & BPU-11526.JPG \\
\hline 1348 & Primula veris $\mathrm{L}$. & Mosonmagyaróvár & 1965 & Borsos Olga & BPU-11531.JPG \\
\hline 1348 & Primula veris $\mathrm{L}$. & Sopron & 1960 & Borsos Olga, Simon Tibor & BPU-11528.JPG \\
\hline 1348 & Primula veris $\mathrm{L}$. & Tihany & 1958 & Felföldy Lajos & BPU-11525.JPG \\
\hline 1348 & Primula veris $\mathrm{L}$. & Visegrád & 1964 & Soó Rezső, Borsos Olga & BPU-11532.JPG \\
\hline 1349 & Primula elatior (L.) Hill & Tibolddaróc & 1953 & Soó Rezső & BPU-11511.JPG \\
\hline $\begin{array}{c}1347 \times \\
1348\end{array}$ & Primula $\times$ brevistyla DC & Csesznek & 1929 & Polgár Sándor & BPU-11506.JPG \\
\hline 1350 & Androsace maxima L. & Budapest & 1927 & Moesz Gusztáv & BPU-11505.JPG \\
\hline 1350 & Androsace maxima L. & Keszthely & 1960 & Priszter Szaniszló & BPU-11500.JPG \\
\hline 1350 & Androsace maxima L. & Nagykovácsi & 1940 & Soó Rezső & BPU-11504.JPG \\
\hline 1350 & Androsace maxima L. & Tarcal & 1951 & Soó Rezső & BPU-11501.JPG \\
\hline 1350 & Androsace maxima L. & Tihany & 1928 & Soó Rezső & BPU-11503.JPG \\
\hline 1350 & Androsace maxima L. & Tihany & 1958 & Felföldy Lajos & BPU-11502.JPG \\
\hline 1351 & Androsace elongata L. & Egyek & 1940 & Soó Rezső & BPU-11497.JPG \\
\hline 1351 & Androsace elongata L. & Pomáz & 1947 & Kárpáti Zoltán & BPU-11498.JPG \\
\hline 1351 & Androsace elongata L. & Tihany & 1929 & Soó Rezső & BPU-11499.JPG \\
\hline 1352 & Hottonia palustris L. & Balatonhenye & 1959 & $\begin{array}{l}\text { Jávorka Sándor, Felföldy } \\
\text { Lajos, Tóth László }\end{array}$ & BPU-11481.JPG \\
\hline 1352 & Hottonia palustris L. & Dabas & 1959 & Simon Tibor & BPU-11486.JPG \\
\hline 1352 & Hottonia palustris L. & Dabas & 1964 & Soó Rezső & BPU-11482.JPG \\
\hline 1352 & Hottonia palustris L. & Debrecen & 1933 & Soó Rezső & BPU-11487.JPG \\
\hline 1352 & Hottonia palustris L. & Nagykanizsa & 1950 & Károlyi Árpád & BPU-11479.JPG \\
\hline 1352 & Hottonia palustris L. & Ócsa & 1953 & Soó Rezső, Borsos Olga & BPU-11485.JPG \\
\hline 1352 & Hottonia palustris L. & Ócsa & 1955 & Soó Rezső & BPU-11484.JPG \\
\hline 1352 & Hottonia palustris L. & Pomáz & 1923 & Boros Ádám & BPU-11483.JPG \\
\hline
\end{tabular}


1. Elektroniukus melléklet - Electronic Appendix 1.

\begin{tabular}{|c|c|c|c|c|c|}
\hline $\begin{array}{l}\text { Sorszám / } \\
\text { Number }\end{array}$ & Taxon-név / Taxon-name & $\begin{array}{l}\text { Település / } \\
\text { Settlement }\end{array}$ & $\begin{array}{l}\text { Év / } \\
\text { Year }\end{array}$ & Gyújtő / Collector & $\begin{array}{l}\text { Fájlnév / } \\
\text { File-name }\end{array}$ \\
\hline 1352 & Hottonia palustris L. & Szentbékkálla & 1959 & $\begin{array}{l}\text { Jávorka Sándor, Felföldy } \\
\text { Lajos, Tóth László }\end{array}$ & BPU-11480.JPG \\
\hline 1353 & Cyclamen purpurascens Mill. & Alsószölnök & 1955 & Soó Rezső & BPU-11437.JPG \\
\hline 1353 & Cyclamen purpurascens Mill. & Pilisszentkereszt & 1923 & Andrasovszky József & BPU-11444.JPG \\
\hline 1353 & Cyclamen purpurascens Mill. & Somogyszob & 1957 & Soó Rezső & BPU-11443.JPG \\
\hline 1353 & Cyclamen purpurascens Mill. & Sopron & 1929 & Soó Rezső & BPU-11442.JPG \\
\hline 1353 & Cyclamen purpurascens Mill. & Sopron & 1970 & Priszter Szaniszló & BPU-11438.JPG \\
\hline 1353 & Cyclamen purpurascens Mill. & Sopron & 1970 & Priszter Szaniszló & BPU-11439.JPG \\
\hline 1353 & Cyclamen purpurascens Mill. & Sopron & 1970 & Priszter Szaniszló & BPU-11440.JPG \\
\hline 1353 & Cyclamen purpurascens Mill. & Sopron & 1970 & Priszter Szaniszló & BPU-11441.JPG \\
\hline 1353 & Cyclamen purpurascens Mill. & Zalakomár & 1954 & Borsos Olga & BPU-11436.JPG \\
\hline 1354 & Lysimachia nummularia L. & Bátorliget & 1932 & Soó Rezső & BPU-11467.JPG \\
\hline 1354 & Lysimachia nummularia L. & Pákozd & 1980 & Felföldy Lajos & BPU-11469.JPG \\
\hline 1354 & Lysimachia nummularia L. & Tihany & 1956 & $\begin{array}{l}\text { Felföldy Lajos, Tóth } \\
\text { László }\end{array}$ & BPU-11468.JPG \\
\hline 1357 & Lysimachia punctata L. & Isztimér & 1949 & Soó Rezső & BPU-11459.JPG \\
\hline 1357 & Lysimachia punctata L. & Szentgotthárd & 1955 & Soó Rezső & BPU-11458.JPG \\
\hline 1358 & Lysimachia vulgaris L. & Bagamér & 1947 & Soó Rezső & BPU-11463.JPG \\
\hline 1358 & Lysimachia vulgaris L. & Balatonszepezd & 1956 & $\begin{array}{l}\text { Felföldy Lajos, Tóth } \\
\text { László }\end{array}$ & BPU-11461.JPG \\
\hline 1358 & Lysimachia vulgaris L. & Balatonszepezd & 1958 & Felföldy Lajos & BPU-11460.JPG \\
\hline 1358 & Lysimachia vulgaris L. & Gárdony & 1980 & Felföldy Lajos & BPU-11462.JPG \\
\hline 1358 & Lysimachia vulgaris L. & Pálháza & 1952 & Simon Tibor & BPU-11464.JPG \\
\hline 1359 & Glaux maritima L. & Sárkeresztúr & 1932 & Boros Ádám & BPU-11456.JPG \\
\hline 1360 & Centunculus minimus L. & Alsószölnök & 1955 & Soó Rezső & BPU-11445.JPG \\
\hline 1360 & Centunculus minimus L. & Darány & 1923 & Boros Ádám & BPU-11448.JPG \\
\hline 1360 & Centunculus minimus L. & Szőce & 1955 & Soó Rezső & BPU-11445.JPG \\
\hline 1360 & Centunculus minimus L. & Tornyospálca & 1967 & Gondola István & BPU-11446.JPG \\
\hline 1360 & Centunculus minimus L. & Tuzsér & 1965 & Gondola István & BPU-11447.JPG \\
\hline 1361 & Anagallis arvensis L. & Budapest & 2005 & Felföldy Lajos & BPU-11453.JPG \\
\hline 1361 & Anagallis arvensis L. & Budapest & 2005 & Felföldy Lajos & BPU-11454.JPG \\
\hline 1361 & Anagallis arvensis L. & Hajdúszoboszló & 1938 & Fäller & BPU-11451.JPG \\
\hline 1361 & Anagallis arvensis L. & Mór & 1942 & Felföldy Lajos & BPU-11452.JPG \\
\hline 1361 & Anagallis arvensis L. & Szenna & 1957 & Soó Rezső & BPU-11450.JPG \\
\hline 1362 & Anagallis foemina Mill. & Debrecen & 1934 & Soó Rezső & BPU-11449.JPG \\
\hline 1363 & Samolus valerandi $\mathrm{L}$. & Balatongyörök & 1961 & Felföldy Lajos & BPU-11472.JPG \\
\hline 1363 & Samolus valerandi L. & Balatongyörök & 1961 & Felföldy Lajos & BPU-11473.JPG \\
\hline 1363 & Samolus valerandi L. & Balatonszárszó & 1960 & Soó Rezső & BPU-11478.JPG \\
\hline 1363 & Samolus valerandi L. & Hévíz & 1961 & Felföldy Lajos & BPU-11476.JPG \\
\hline 1363 & Samolus valerandi L. & Lesenceistvánd & 1952 & Simon Tibor & BPU-11475.JPG \\
\hline 1363 & Samolus valerandi L. & Paloznak & 1956 & Felföldy Lajos & BPU-11474.JPG \\
\hline 1363 & Samolus valerandi L. & Tata & 1944 & Papp József & BPU-11477.JPG \\
\hline 1363 & Samolus valerandi L. & Vácrátót & 1949 & Soó Rezső & BPU-11471.JPG \\
\hline 1366 & Limonium gmelinii (Willd.) Kuntze & Budapest & 1950 & Pócs Tamás & BPU-11405.JPG \\
\hline 1366 & Limonium gmelinii (Willd.) Kuntze & Bugyi & 1950 & Soó Rezső & BPU-11401.JPG \\
\hline 1366 & Limonium gmelinii (Willd.) Kuntze & Egyek & 1960 & Simon Tibor & BPU-11404.JPG \\
\hline 1366 & Limonium gmelinii (Willd.) Kuntze & Kunmadaras & 1991 & Csányi Béla & BPU-11406.JPG \\
\hline 1372 & Fraxinus ornus L. & Budapest & 1953 & Soó Rezső & BPU-04825.JPG \\
\hline 1372 & Fraxinus ornus L. & Budapest & 1953 & Soó Rezső & BPU-15438.JPG \\
\hline
\end{tabular}


1. Elektroniukus melléklet - Electronic Appendix 1.

\begin{tabular}{|c|c|c|c|c|c|}
\hline $\begin{array}{l}\text { Sorszám / } \\
\text { Number }\end{array}$ & Taxon-név / Taxon-name & $\begin{array}{l}\text { Település / } \\
\text { Settlement }\end{array}$ & $\begin{array}{l}\text { Év / } \\
\text { Year }\end{array}$ & Gyűjtő / Collector & $\begin{array}{l}\text { Fájlnév / } \\
\text { File-name }\end{array}$ \\
\hline 1372 & Fraxinus ornus L. & Budapest & 1990 & Felföldy Lajos & BPU-04824.JPG \\
\hline 1372 & Fraxinus ornus L. & Mór & 1953 & Felföldy Lajos & BPU-04831.JPG \\
\hline 1372 & Fraxinus ornus L. & Pécs & 1953 & Soó Rezső, Borsos Olga & BPU-04830.JPG \\
\hline 1372 & Fraxinus ornus L. & Pécs & 1956 & Soó Rezső & BPU-04826.JPG \\
\hline 1372 & Fraxinus ornus L. & Pilisszentiván & 1953 & Soó Rezső & BPU-04827.JPG \\
\hline 1372 & Fraxinus ornus L. & Villány & 1936 & Soó Rezső & BPU-04829.JPG \\
\hline 1372 & Fraxinus ornus L. & Visegrád & 1964 & Soó Rezső, Borsos Olga & BPU-04828.JPG \\
\hline 1373 & Fraxinus pennsylvanica Marshall & Tarpa & 1949 & Soó Rezső & BPU-04834.JPG \\
\hline 1375 & Fraxinus excelsior L. & Aszófó & 1959 & Felföldy Lajos & BPU-04832.JPG \\
\hline 1376 & Fraxinus angustifolia Vahl & Dabas & 1959 & Simon Tibor & BPU-04836.JPG \\
\hline 1376 & Fraxinus angustifolia Vahl & Dabas & 1963 & Isépy István & BPU-04837.JPG \\
\hline 1376 & Fraxinus angustifolia Vahl & Egyek & 1960 & Simon Tibor & BPU-04835.JPG \\
\hline 1377 & Syringa vulgaris L. & Budapest & 1956 & Isépy István & BPU-04838.JPG \\
\hline 1378 & Ligustrum vulgare $\mathrm{L}$. & Nagyharsány & 1967 & Priszter Szaniszló & BPU-04840.JPG \\
\hline 1378 & Ligustrum vulgare $\mathrm{L}$. & Nyíracsád & 1931 & Soó Rezső & BPU-04842.JPG \\
\hline 1380 & $\begin{array}{l}\text { Blackstonia acuminata (W.D.J. Koch et } \\
\text { Ziz) Domin }\end{array}$ & Ásványráró & 1931 & Soó Rezső & BPU-04868.JPG \\
\hline 1381 & Centaurium littorale (Turner) Gilmour & Bugac & 1952 & Vozáry Elemér & BPU-04844.JPG \\
\hline 1381 & Centaurium littorale (Turner) Gilmour & Debrecen & 1952 & Soó Rezső & BPU-04846.JPG \\
\hline 1381 & Centaurium littorale (Turner) Gilmour & Győr & 1948 & Kárpáti Zoltán & BPU-04845.JPG \\
\hline 1381 & Centaurium littorale (Turner) Gilmour & Révfülöp & 1956 & $\begin{array}{l}\text { Felföldy Lajos, Tóth } \\
\text { László }\end{array}$ & BPU-04848.JPG \\
\hline 1381 & Centaurium littorale (Turner) Gilmour & Tapolca & 1928 & Soó Rezső & BPU-04849.JPG \\
\hline 1381 & Centaurium littorale (Turner) Gilmour & Vácrátót & 1953 & Felföldy Lajos & BPU-04847.JPG \\
\hline 1381 & Centaurium littorale (Turner) Gilmour & Zalagyömörő & 1957 & Felföldy Lajos & BPU-04850.JPG \\
\hline 1382 & Centaurium pulchellum (Sw.) Druce & Hortobágy & 1947 & Soó Rezső & BPU-04860.JPG \\
\hline 1382 & Centaurium pulchellum (Sw.) Druce & Kiskunhalas & 1964 & Soó Rezső & BPU-04859.JPG \\
\hline 1382 & Centaurium pulchellum (Sw.) Druce & Kiskunmajsa & 1964 & Soó Rezső & BPU-04867.JPG \\
\hline 1382 & Centaurium pulchellum (Sw.) Druce & Révfülöp & 1956 & $\begin{array}{l}\text { Felföldy Lajos, Tóth } \\
\text { László }\end{array}$ & BPU-04865.JPG \\
\hline 1382 & Centaurium pulchellum (Sw.) Druce & Szilvásvárad & 1953 & $\begin{array}{l}\text { Felföldy Lajos, Tóth } \\
\text { László }\end{array}$ & BPU-04863.JPG \\
\hline 1382 & Centaurium pulchellum (Sw.) Druce & Vácrátót & 1953 & Felföldy Lajos & BPU-04864.JPG \\
\hline 1383 & Centaurium erythraea Raf. & Alsószölnök & 1955 & Soó Rezső & BPU-04855.JPG \\
\hline 1383 & Centaurium erythraea Raf. & Bózsva & 1950 & Simon Tibor & BPU-04851.JPG \\
\hline 1383 & Centaurium erythraea Raf. & Csaroda & 1951 & $\begin{array}{l}\text { Simon Tibor, Vozáry } \\
\text { Elemér }\end{array}$ & BPU-04852.JPG \\
\hline 1383 & Centaurium erythraea Raf. & Debrecen & 1934 & Soó Rezső & BPU-04853.JPG \\
\hline 1383 & Centaurium erythraea Raf. & Szentbékkálla & 1957 & Felföldy Lajos & BPU-04856.JPG \\
\hline 1383 & Centaurium erythraea Raf. & Szentendre & 1963 & Soó Rezső & BPU-04854.JPG \\
\hline 1383 & Centaurium erythraea Raf. & Uzsa & 1953 & $\begin{array}{l}\text { Felföldy Lajos, Tóth } \\
\text { László }\end{array}$ & BPU-04857.JPG \\
\hline 1384 & Gentiana cruciata L. & Mátraszentimre & 1963 & Isépy István & BPU-04891.JPG \\
\hline 1384 & Gentiana cruciata L. & Miskolc & 1938 & Soó Rezső & BPU-04889.JPG \\
\hline 1384 & Gentiana cruciata L. & Miskolc & 1950 & Soó Rezső & BPU-04890.JPG \\
\hline 1384 & Gentiana cruciata L. & Pilisszentiván & 1961 & Borsos Olga, Simon Tibor & BPU-04893.JPG \\
\hline 1384 & Gentiana cruciata L. & Szilvásvárad & 1953 & $\begin{array}{l}\text { Felföldy Lajos, Tóth } \\
\text { László }\end{array}$ & BPU-04892.JPG \\
\hline 1385 & Gentiana pneumonanthe L. & Alsószölnök & 1955 & Soó Rezső & BPU-04904.JPG \\
\hline 1385 & Gentiana pneumonanthe L. & Debrecen & 1937 & Soó Rezső & BPU-04908.JPG \\
\hline
\end{tabular}


1. Elektroniukus melléklet - Electronic Appendix 1.

\begin{tabular}{|c|c|c|c|c|c|}
\hline $\begin{array}{l}\text { Sorszám / } \\
\text { Number }\end{array}$ & Taxon-név / Taxon-name & $\begin{array}{l}\text { Település / } \\
\text { Settlement }\end{array}$ & $\begin{array}{l}\text { Év / } \\
\text { Year }\end{array}$ & Gyújtő / Collector & $\begin{array}{l}\text { Fájlnév / } \\
\text { File-name }\end{array}$ \\
\hline 1385 & Gentiana pneumonanthe L. & Debrecen & 1953 & Kulcsár Gábor & BPU-15442.JPG \\
\hline 1385 & Gentiana pneumonanthe L. & Miskolc & 1929 & Soó Rezső & BPU-04905.JPG \\
\hline 1385 & Gentiana pneumonanthe L. & Szentgotthárd & 1955 & Soó Rezső & BPU-04907.JPG \\
\hline 1385 & Gentiana pneumonanthe L. & Tatárszentgyörgy & 1950 & Soó Rezső & BPU-04909.JPG \\
\hline 1385 & Gentiana pneumonanthe L. & Vámosatya & 1948 & Simon Tibor, Jakucs Pál & BPU-04906.JPG \\
\hline 1387 & Gentianopsis ciliata (L.) Ma & Szilvásvárad & 1953 & $\begin{array}{l}\text { Felföldy Lajos, Tóth } \\
\text { László }\end{array}$ & BPU-04869.JPG \\
\hline 1388 & $\begin{array}{l}\text { Gentianella austriaca (A. Kern. et J. } \\
\text { Kern.) Holub }\end{array}$ & Kőszeg & 1932 & Soó Rezső & BPU-04884.JPG \\
\hline 1388 & $\begin{array}{l}\text { Gentianella austriaca (A. Kern. et J. } \\
\text { Kern.) Holub }\end{array}$ & Nagyhuta & 1948 & Vajda László & BPU-04881.JPG \\
\hline 1388 & $\begin{array}{l}\text { Gentianella austriaca (A. Kern. et J. } \\
\text { Kern.) Holub }\end{array}$ & Nagyvisnyó & 1929 & Soó Rezső & BPU-04882.JPG \\
\hline 1388 & $\begin{array}{l}\text { Gentianella austriaca (A. Kern. et J. } \\
\text { Kern.) Holub }\end{array}$ & Regéc & 1952 & Simon Tibor & BPU-04880.JPG \\
\hline 1388 & $\begin{array}{l}\text { Gentianella austriaca (A. Kern. et J. } \\
\text { Kern.) Holub }\end{array}$ & Szilvásvárad & 1953 & $\begin{array}{l}\text { Felföldy Lajos, Tóth } \\
\text { László }\end{array}$ & BPU-04883.JPG \\
\hline 1389 & Gentianella amarella (L.) Börner & Miskolc & 1929 & Soó Rezső & BPU-04871.JPG \\
\hline 1389 & Gentianella amarella (L.) Börner & Miskolc & 1950 & Soó Rezső, Simon Tibor & BPU-04873.JPG \\
\hline 1389 & Gentianella amarella (L.) Börner & Miskolc & 1960 & Simon Tibor & BPU-04872.JPG \\
\hline 1389 & Gentianella amarella (L.) Börner & Miskolc & 1965 & Soó Rezső, Simon Tibor & BPU-04874.JPG \\
\hline 1390 & Menyanthes trifoliata $\mathrm{L}$. & Bátorliget & 1950 & $\begin{array}{l}\text { Simon Tibor, Kárpáti } \\
\text { István }\end{array}$ & BPU-04934.JPG \\
\hline 1390 & Menyanthes trifoliata L. & Debrecen & 1946 & Soó Rezső & BPU-04935.JPG \\
\hline 1390 & Menyanthes trifoliata L. & Nagykanizsa & 1949 & Soó Rezső & BPU-04938.JPG \\
\hline 1390 & Menyanthes trifoliata L. & Szőce & 1955 & Soó Rezső & BPU-04937.JPG \\
\hline 1390 & Menyanthes trifoliata L. & Veresegyház & 1949 & Soó Rezső & BPU-04936.JPG \\
\hline 1391 & $\begin{array}{l}\text { Nymphoides peltata (S.G. Gmel.) } \\
\text { Kuntze }\end{array}$ & Budapest & 1923 & Soó Rezső & BPU-04941.JPG \\
\hline 1391 & $\begin{array}{l}\text { Nymphoides peltata (S.G. Gmel.) } \\
\text { Kuntze }\end{array}$ & Dunakiliti & 1931 & Soó Rezső & BPU-04942.JPG \\
\hline 1391 & $\begin{array}{l}\text { Nymphoides peltata (S.G. Gmel.) } \\
\text { Kuntze }\end{array}$ & Kunmadaras & 1991 & Csányi Béla & BPU-04939.JPG \\
\hline 1391 & $\begin{array}{l}\text { Nymphoides peltata (S.G. Gmel.) } \\
\text { Kuntze }\end{array}$ & Tiszafüred & 1953 & Borsos Olga & BPU-04943.JPG \\
\hline 1391 & $\begin{array}{l}\text { Nymphoides peltata (S.G. Gmel.) } \\
\text { Kuntze }\end{array}$ & Tiszafüred & 1953 & Borsos Olga & BPU-15471.JPG \\
\hline 1391 & $\begin{array}{l}\text { Nymphoides peltata (S.G. Gmel.) } \\
\text { Kuntze }\end{array}$ & Tiszafüred & 1954 & Dobos L. & BPU-04940.JPG \\
\hline 1392 & Vinca herbacea Waldst. et Kit. & Balatonfüred & 1928 & Soó Rezső & BPU-04981.JPG \\
\hline 1392 & Vinca herbacea Waldst. et Kit. & Balatonfüred & 1959 & Priszter Szaniszló & BPU-04980.JPG \\
\hline 1392 & Vinca herbacea Waldst. et Kit. & Budaörs & 1982 & Felföldy Lajos & BPU-04979.JPG \\
\hline 1392 & Vinca herbacea Waldst. et Kit. & Budapest & 1982 & Felföldy Lajos & BPU-04974.JPG \\
\hline 1392 & Vinca herbacea Waldst. et Kit. & Miskolc & 1939 & Soó Rezső & BPU-04978.JPG \\
\hline 1392 & Vinca herbacea Waldst. et Kit. & Nagyharsány & 1965 & $\begin{array}{l}\text { Simon Tibor, Priszter } \\
\text { Szaniszló, Borhidi Attila }\end{array}$ & BPU-04975.JPG \\
\hline 1392 & Vinca herbacea Waldst. et Kit. & Pécs & 1936 & Soó Rezső & BPU-04976.JPG \\
\hline 1392 & Vinca herbacea Waldst. et Kit. & Pécs & 1953 & Soó Rezső, Borsos Olga & BPU-04977.JPG \\
\hline 1392 & Vinca herbacea Waldst. et Kit. & Tarcal & 1951 & Soó Rezső & BPU-04983.JPG \\
\hline 1392 & Vinca herbacea Waldst. et Kit. & Tihany & 1957 & $\begin{array}{l}\text { Felföldy Lajos, Tóth } \\
\text { László }\end{array}$ & BPU-04982.JPG \\
\hline 1393 & Vinca minor L. & Balatonszőlős & 1959 & Felföldy Lajos & BPU-04972.JPG \\
\hline 1393 & Vinca minor L. & Budapest & 1961 & Isépy István & BPU-04968.JPG \\
\hline 1393 & Vinca minor L. & Ófehértó & 1934 & Máthé Imre & BPU-04971.JPG \\
\hline
\end{tabular}


1. Elektroniukus melléklet - Electronic Appendix 1.

\begin{tabular}{|c|c|c|c|c|c|}
\hline $\begin{array}{l}\text { Sorszám / } \\
\text { Number }\end{array}$ & Taxon-név / Taxon-name & $\begin{array}{l}\text { Település / } \\
\text { Settlement }\end{array}$ & $\begin{array}{l}\text { Év / } \\
\text { Year }\end{array}$ & Gyűjtő / Collector & $\begin{array}{l}\text { Fájlnév / } \\
\text { File-name }\end{array}$ \\
\hline 1393 & Vinca minor L. & Sátoraljaújhely & 1952 & $\begin{array}{l}\text { Kulcsár Gábor, Vozáry } \\
\text { Elemér }\end{array}$ & BPU-15473.JPG \\
\hline 1393 & Vinca minor L. & Szigetmonostor & 1963 & Isépy István & BPU-04969.JPG \\
\hline 1393 & Vinca minor L. & Vácrátót & 1950 & Soó Rezső & BPU-04970.JPG \\
\hline 1394 & Vinca major L. & Nagyharsány & 1965 & $\begin{array}{l}\text { Simon Tibor, Priszter } \\
\text { Szaniszló }\end{array}$ & BPU-04984.JPG \\
\hline 1395 & Asclepias syriaca L. & Debrecen & 1934 & Soó Rezső & BPU-04945.JPG \\
\hline 1395 & Asclepias syriaca L. & Dömös & 1964 & Isépy István & BPU-04944.JPG \\
\hline 1395 & Asclepias syriaca L. & Resznek & 1958 & Cserni J. & BPU-04946.JPG \\
\hline 1396 & Vincetoxicum hirundinaria Medik. & Budapest & 1982 & Felföldy Lajos & BPU-04948.JPG \\
\hline 1396 & Vincetoxicum hirundinaria Medik. & Budapest & 2005 & Felföldy Lajos & BPU-04947.JPG \\
\hline 1396 & Vincetoxicum hirundinaria Medik. & Csévharaszt & 1959 & Simon Tibor, Borsos Olga & BPU-04959.JPG \\
\hline 1396 & Vincetoxicum hirundinaria Medik. & Debrecen & 1931 & Soó Rezső & BPU-04955.JPG \\
\hline 1396 & Vincetoxicum hirundinaria Medik. & Farmos & 1923 & Soó Rezső & BPU-04960.JPG \\
\hline 1396 & Vincetoxicum hirundinaria Medik. & Garadna & 1955 & Tóth László & BPU-04952.JPG \\
\hline 1396 & Vincetoxicum hirundinaria Medik. & Gyöngyös & 1961 & Soó Rezső & BPU-04956.JPG \\
\hline 1396 & Vincetoxicum hirundinaria Medik. & Gyöngyös & 1965 & Soó Rezső & BPU-04957.JPG \\
\hline 1396 & Vincetoxicum hirundinaria Medik. & Lábatlan & 1953 & Felföldy Lajos & BPU-04954.JPG \\
\hline 1396 & Vincetoxicum hirundinaria Medik. & Mosonmagyaróvár & 1962 & Borsos Olga & BPU-04958.JPG \\
\hline 1396 & Vincetoxicum hirundinaria Medik. & Nagyharsány & 1965 & $\begin{array}{l}\text { Simon Tibor, Priszter } \\
\text { Szaniszló }\end{array}$ & BPU-04950.JPG \\
\hline 1396 & Vincetoxicum hirundinaria Medik. & Pécs & 1953 & Soó Rezső, Borsos Olga & BPU-04951.JPG \\
\hline 1396 & Vincetoxicum hirundinaria Medik. & Pusztafalu & 1962 & Simon Tibor & BPU-04949.JPG \\
\hline 1396 & Vincetoxicum hirundinaria Medik. & Szalonna & 1966 & Felföldy Lajos & BPU-04953.JPG \\
\hline 1396 & Vincetoxicum hirundinaria Medik. & Tokaj & 1947 & Soó Rezső & BPU-04963.JPG \\
\hline 1396 & Vincetoxicum hirundinaria Medik. & Uzsa & 1959 & $\begin{array}{l}\text { Jávorka Sándor, Felföldy } \\
\text { Lajos }\end{array}$ & BPU-04962.JPG \\
\hline 1396 & Vincetoxicum hirundinaria Medik. & Zebegény & 1966 & Priszter Szaniszló & BPU-04961.JPG \\
\hline 1397 & $\begin{array}{l}\text { Vincetoxicum pannonicum (Borhidi) } \\
\text { Holub }\end{array}$ & Budaörs & 1966 & $\begin{array}{l}\text { Borhidi Attila, Priszter } \\
\text { Szaniszló }\end{array}$ & BPU-04965.JPG \\
\hline 1397 & $\begin{array}{l}\text { Vincetoxicum pannonicum (Borhidi) } \\
\text { Holub }\end{array}$ & Budaörs & 1966 & Priszter Szaniszló & BPU-04966.JPG \\
\hline 1397 & $\begin{array}{l}\text { Vincetoxicum pannonicum (Borhidi) } \\
\text { Holub }\end{array}$ & Pilisvörösvár & 1917 & Boros Ádám & BPU-04967.JPG \\
\hline 1397 & $\begin{array}{l}\text { Vincetoxicum pannonicum (Borhidi) } \\
\text { Holub }\end{array}$ & Villány & 1966 & $\begin{array}{l}\text { Borhidi Attila, Priszter } \\
\text { Szaniszló, Thury Zs. }\end{array}$ & BPU-04964.JPG \\
\hline 1398 & Calystegia sepium (L.) R. Br. & Debrecen & 1947 & Soó Rezső & BPU-05025.JPG \\
\hline 1399 & Convolvulus arvensis L. & Debrecen & 1945 & Felföldy Lajos & BPU-05010.JPG \\
\hline 1399 & Convolvulus arvensis L. & Debrecen & 1945 & Felföldy Lajos & BPU-05012.JPG \\
\hline 1399 & Convolvulus arvensis L. & Debrecen & 1945 & Felföldy Lajos & BPU-05013.JPG \\
\hline 1399 & Convolvulus arvensis L. & Tihany & 1958 & Felföldy Lajos & BPU-05011.JPG \\
\hline 1400 & Convolvulus cantabrica L. & Budapest & 1952 & Vozáry Elemér & BPU-05014.JPG \\
\hline 1400 & Convolvulus cantabrica L. & Budapest & 1991 & Felföldy Lajos & BPU-05020.JPG \\
\hline 1400 & Convolvulus cantabrica L. & Csákvár & 1955 & Soó Rezső & BPU-05022.JPG \\
\hline 1400 & Convolvulus cantabrica L. & Nagyharsány & 1956 & Soó Rezső & BPU-05021.JPG \\
\hline 1400 & Convolvulus cantabrica L. & Nagyharsány & 1965 & $\begin{array}{l}\text { Simon Tibor, Priszter } \\
\text { Szaniszló }\end{array}$ & BPU-05016.JPG \\
\hline 1400 & Convolvulus cantabrica L. & Pécs & 1925 & Soó Rezső & BPU-05024.JPG \\
\hline 1400 & Convolvulus cantabrica L. & Tihany & 1947 & Pólya László & BPU-05023.JPG \\
\hline 1400 & Convolvulus cantabrica L. & Tihany & 1954 & Felföldy Lajos & BPU-05015.JPG \\
\hline 1400 & Convolvulus cantabrica L. & Tihany & 1956 & $\begin{array}{l}\text { Felföldy Lajos, Tóth } \\
\text { László }\end{array}$ & BPU-05019.JPG \\
\hline
\end{tabular}


1. Elektroniukus melléklet - Electronic Appendix 1.

\begin{tabular}{|c|c|c|c|c|c|}
\hline $\begin{array}{l}\text { Sorszám / } \\
\text { Number }\end{array}$ & Taxon-név / Taxon-name & $\begin{array}{l}\text { Település / } \\
\text { Settlement }\end{array}$ & $\begin{array}{l}\text { Év / } \\
\text { Year }\end{array}$ & Gyújtő / Collector & $\begin{array}{l}\text { Fájlnév / } \\
\text { File-name }\end{array}$ \\
\hline 1400 & Convolvulus cantabrica L. & Tihany & 1959 & Felföldy Lajos & BPU-05017.JPG \\
\hline 1400 & Convolvulus cantabrica L. & Tihany & 1959 & Felföldy Lajos & BPU-05018.JPG \\
\hline 1404 & Cuscuta campestris Yunck. & Budapest & 1982 & Felföldy Lajos & BPU-04985.JPG \\
\hline 1404 & Cuscuta campestris Yunck. & Budapest & 1982 & Felföldy Lajos & BPU-04987.JPG \\
\hline 1404 & Cuscuta campestris Yunck. & Budapest & 1982 & Felföldy Lajos & BPU-04988.JPG \\
\hline 1404 & Cuscuta campestris Yunck. & Budapest & 1982 & Felföldy Lajos & BPU-04989.JPG \\
\hline 1404 & Cuscuta campestris Yunck. & Budapest & 1991 & Felföldy Lajos & BPU-04986.JPG \\
\hline 1407 & Cuscuta epithymum (L.) Nath. & Ábrahámhegy & 1982 & Felföldy Lajos & BPU-05008.JPG \\
\hline 1407 & Cuscuta epithymum (L.) Nath. & Aszófő & 1957 & $\begin{array}{l}\text { Felföldy Lajos, Kovács } \\
\text { Margit, Tóth László }\end{array}$ & BPU-04996.JPG \\
\hline 1407 & Cuscuta epithymum (L.) Nath. & Budapest & 1912 & Degen Árpád & BPU-04992.JPG \\
\hline 1407 & Cuscuta epithymum (L.) Nath. & Budapest & 1982 & Felföldy Lajos & BPU-05000.JPG \\
\hline 1407 & Cuscuta epithymum (L.) Nath. & Budapest & 1982 & Felföldy Lajos & BPU-05001.JPG \\
\hline 1407 & Cuscuta epithymum (L.) Nath. & Budapest & 1982 & Felföldy Lajos & BPU-05002.JPG \\
\hline 1407 & Cuscuta epithymum (L.) Nath. & Budapest & 1982 & Felföldy Lajos & BPU-05003.JPG \\
\hline 1407 & Cuscuta epithymum (L.) Nath. & Budapest & 1982 & Felföldy Lajos & BPU-05006.JPG \\
\hline 1407 & Cuscuta epithymum (L.) Nath. & Budapest & 1982 & Felföldy Lajos & BPU-05007.JPG \\
\hline 1407 & Cuscuta epithymum (L.) Nath. & Budapest & 1991 & Felföldy Lajos & BPU-05005.JPG \\
\hline 1407 & Cuscuta epithymum (L.) Nath. & Egyek & 1933 & Máthé Imre & BPU-04990.JPG \\
\hline 1407 & Cuscuta epithymum (L.) Nath. & Gárdony & 1982 & Felföldy Lajos & BPU-05009.JPG \\
\hline 1407 & Cuscuta epithymum (L.) Nath. & Gelénes & 1951 & $\begin{array}{l}\text { Simon Tibor, Vozáry } \\
\text { Elemér }\end{array}$ & BPU-04993.JPG \\
\hline 1407 & Cuscuta epithymum (L.) Nath. & Jósvafő & 1951 & $\begin{array}{l}\text { Soó Rezső, Jakucs Pál, Ér } \\
\text { Lajos }\end{array}$ & BPU-04991.JPG \\
\hline 1407 & Cuscuta epithymum (L.) Nath. & Nagyharsány & 1965 & $\begin{array}{l}\text { Simon Tibor, Priszter } \\
\text { Szaniszló, Borhidi Attila }\end{array}$ & BPU-04999.JPG \\
\hline 1407 & Cuscuta epithymum (L.) Nath. & Tihany & 1959 & Felföldy Lajos & BPU-05004.JPG \\
\hline 1407 & Cuscuta epithymum (L.) Nath. & Vácrátót & 1953 & Felföldy Lajos & BPU-04997.JPG \\
\hline 1407 & Cuscuta epithymum (L.) Nath. & Vászoly & 1958 & Felföldy Lajos & BPU-04995.JPG \\
\hline 1407 & Cuscuta epithymum (L.) Nath. & Zalagyömörő & 1957 & Felföldy Lajos & BPU-04994.JPG \\
\hline 1409 & Cuscuta europaea L. & Vászoly & 1958 & Felföldy Lajos & BPU-04998.JPG \\
\hline 1410 & Heliotropium europaeum L. & Tihany & 1954 & Felföldy Lajos & BPU-05028.JPG \\
\hline 1410 & Heliotropium europaeum L. & Tihany & 1959 & Felföldy Lajos & BPU-05029.JPG \\
\hline 1410 & Heliotropium europaeum L. & Vácrátót & 1953 & Felföldy Lajos & BPU-05030.JPG \\
\hline 1411 & Heliotropium supinum L. & Szeged & 1950 & Timár Lajos & BPU-05031.JPG \\
\hline 1412 & Lithospermum officinale L. & Balatonszőlős & 1958 & Felföldy Lajos & BPU-05223.JPG \\
\hline 1412 & Lithospermum officinale L. & Budapest & 1953 & Soó Rezső & BPU-05214.JPG \\
\hline 1412 & Lithospermum officinale L. & Kelemér & 1950 & Soó Rezső & BPU-05218.JPG \\
\hline 1412 & Lithospermum officinale L. & Kerecsend & 1963 & Isépy István & BPU-05220.JPG \\
\hline 1412 & Lithospermum officinale L. & Lábatlan & 1953 & Felföldy Lajos & BPU-05216.JPG \\
\hline 1412 & Lithospermum officinale L. & Mór & 1953 & Felföldy Lajos & BPU-05217.JPG \\
\hline 1412 & Lithospermum officinale L. & Mosonmagyaróvár & 1965 & Borsos Olga & BPU-05221.JPG \\
\hline 1412 & Lithospermum officinale L. & Ófehértó & 1934 & Máthé Imre, Soó Rezső & BPU-05213.JPG \\
\hline 1412 & Lithospermum officinale L. & Pécs & 1936 & Soó Rezső & BPU-05215.JPG \\
\hline 1412 & Lithospermum officinale L. & Sáránd & 1934 & Soó Rezső & BPU-05219.JPG \\
\hline 1412 & Lithospermum officinale L. & Szigetmonostor & 1947 & Soó Rezső & BPU-05222.JPG \\
\hline 1412 & Lithospermum officinale L. & Tihany & 1947 & Pólya László & BPU-05212.JPG \\
\hline 1412 & Lithospermum officinale L. & Tihany & 1949 & Simon Tibor & BPU-15522.JPG \\
\hline 1412 & Lithospermum officinale L. & Tihany & 1956 & Felföldy Lajos & BPU-05224.JPG \\
\hline
\end{tabular}


1. Elektroniukus melléklet - Electronic Appendix 1.

\begin{tabular}{|c|c|c|c|c|c|}
\hline $\begin{array}{l}\text { Sorszám / } \\
\text { Number }\end{array}$ & Taxon-név / Taxon-name & $\begin{array}{l}\text { Település / } \\
\text { Settlement }\end{array}$ & $\begin{array}{l}\text { Év / } \\
\text { Year }\end{array}$ & Gyưjtő / Collector & $\begin{array}{l}\text { Fájlnév / } \\
\text { File-name }\end{array}$ \\
\hline 1412 & Lithospermum officinale L. & Tihany & 1959 & Felföldy Lajos & BPU-05211.JPG \\
\hline 1414 & $\begin{array}{l}\text { Buglossoides arvensis (L.) I.M. } \\
\text { Johnston }\end{array}$ & Budapest & 1922 & Soó Rezső & BPU-05228.JPG \\
\hline 1414 & $\begin{array}{l}\text { Buglossoides arvensis (L.) I.M. } \\
\text { Johnston }\end{array}$ & Budapest & 1982 & Felföldy Lajos & BPU-05226.JPG \\
\hline 1414 & $\begin{array}{l}\text { Buglossoides arvensis (L.) I.M. } \\
\text { Johnston }\end{array}$ & Budapest & 1982 & Felföldy Lajos & BPU-05227.JPG \\
\hline 1414 & $\begin{array}{l}\text { Buglossoides arvensis (L.) I.M. } \\
\text { Johnston }\end{array}$ & Budapest & 2004 & Felföldy Lajos & BPU-05225.JPG \\
\hline 1414 & $\begin{array}{l}\text { Buglossoides arvensis (L.) I.M. } \\
\text { Johnston }\end{array}$ & Gárdony & 1963 & Isépy István & BPU-05229.JPG \\
\hline 1414 & $\begin{array}{l}\text { Buglossoides arvensis (L.) I.M. } \\
\text { Johnston }\end{array}$ & Gyöngyös & 1964 & Soó Rezső & BPU-05230.JPG \\
\hline 1414 & $\begin{array}{l}\text { Buglossoides arvensis (L.) I.M. } \\
\text { Johnston }\end{array}$ & Tihany & 1958 & Felföldy Lajos & BPU-05233.JPG \\
\hline 1414 & $\begin{array}{l}\text { Buglossoides arvensis (L.) I.M. } \\
\text { Johnston }\end{array}$ & Vácrátót & 1953 & Felföldy Lajos & BPU-05231.JPG \\
\hline 1414 & $\begin{array}{l}\text { Buglossoides arvensis (L.) I.M. } \\
\text { Johnston }\end{array}$ & Vácrátót & 1953 & Felföldy Lajos & BPU-05232.JPG \\
\hline 1415 & Onosma visianii Clementi & Bélapátfalva & 1932 & Soó Rezső, Máthé Ime & BPU-05235.JPG \\
\hline 1415 & Onosma visianii Clementi & Bélapátfalva & 1950 & $\begin{array}{l}\text { Simon Tibor, Jakucs Pál, } \\
\text { Kárpáti István }\end{array}$ & BPU-05236.JPG \\
\hline 1415 & Onosma visianii Clementi & Budapest & 1947 & Soó Rezső & BPU-05234.JPG \\
\hline 1415 & Onosma visianii Clementi & Budapest & 1952 & Soó Rezső & BPU-15430.JPG \\
\hline 1415 & Onosma visianii Clementi & Fót & 1960 & Soó Rezső & BPU-05237.JPG \\
\hline 1415 & Onosma visianii Clementi & Tihany & 1947 & Pólya László & BPU-05238.JPG \\
\hline 1416 & Onosma arenaria Waldst. et Kit. & Budapest & 1947 & Soó Rezső & BPU-05249.JPG \\
\hline 1416 & Onosma arenaria Waldst. et Kit. & Bugac & 1964 & Soó Rezső & BPU-05247.JPG \\
\hline 1416 & Onosma arenaria Waldst. et Kit. & Bugac & 1964 & Soó Rezső & BPU-05248.JPG \\
\hline 1416 & Onosma arenaria Waldst. et Kit. & Csévharaszt & 1959 & Simon Tibor, Borsos Olga & BPU-05246.JPG \\
\hline 1416 & Onosma arenaria Waldst. et Kit. & Debrecen & 1946 & Soó Rezső & BPU-05242.JPG \\
\hline 1416 & Onosma arenaria Waldst. et Kit. & Kiskunfélegyháza & 1923 & Soó Rezső & BPU-05241.JPG \\
\hline 1416 & Onosma arenaria Waldst. et Kit. & Nagyharsány & 1965 & $\begin{array}{l}\text { Simon Tibor, Priszter } \\
\text { Szaniszló }\end{array}$ & BPU-05245.JPG \\
\hline 1416 & Onosma arenaria Waldst. et Kit. & Pécs & 1938 & Ubrizsy Gábor & BPU-05250.JPG \\
\hline 1416 & Onosma arenaria Waldst. et Kit. & Tihany & 1946 & Felföldy Lajos & BPU-05244.JPG \\
\hline 1416 & Onosma arenaria Waldst. et Kit. & Tihany & 1959 & Felföldy Lajos & BPU-05240.JPG \\
\hline 1416 & Onosma arenaria Waldst. et Kit. & Tokaj & 1947 & Soó Rezső & BPU-05243.JPG \\
\hline 1417 & Onosma tornense Jáv. & Tornanádaska & 1952 & Soó Rezső & BPU-05254.JPG \\
\hline 1418 & Cerinthe minor L. & Debrecen & 1931 & Soó Rezső & BPU-05255.JPG \\
\hline 1418 & Cerinthe minor L. & Jánosháza & 1957 & Szalay József & BPU-05257.JPG \\
\hline 1418 & Cerinthe minor L. & Szigetmonostor & 1963 & Isépy István & BPU-05256.JPG \\
\hline 1418 & Cerinthe minor L. & Tihany & 1958 & Felföldy Lajos & BPU-05258.JPG \\
\hline 1419 & Alkanna tinctoria (L.) Tausch & "Szentendrei-sziget" & 1953 & Borsos Olga & BPU-05110.JPG \\
\hline 1419 & Alkanna tinctoria (L.) Tausch & Bugac & 1962 & Simon Tibor & BPU-05107.JPG \\
\hline 1419 & Alkanna tinctoria (L.) Tausch & Dabas & 1959 & Simon Tibor & BPU-05108.JPG \\
\hline 1419 & Alkanna tinctoria (L.) Tausch & Ócsa & 1923 & Soó Rezső & BPU-05109.JPG \\
\hline 1419 & Alkanna tinctoria (L.) Tausch & Szigetmonostor & 1963 & Isépy István & BPU-05111.JPG \\
\hline 1419 & Alkanna tinctoria (L.) Tausch & Szigetújfalu & 1943 & Kárpáti Zoltán & BPU-05112.JPG \\
\hline 1420 & Echium italicum L. & Bisse & 1965 & $\begin{array}{l}\text { Simon Tibor, Priszter } \\
\text { Szaniszló, Borhidi Attila }\end{array}$ & BPU-05263.JPG \\
\hline 1420 & Echium italicum L. & Budaörs & 1943 & Kárpáti Zoltán & BPU-05261.JPG \\
\hline
\end{tabular}


1. Elektroniukus melléklet - Electronic Appendix 1.

\begin{tabular}{|c|c|c|c|c|c|}
\hline $\begin{array}{l}\text { Sorszám / } \\
\text { Number }\end{array}$ & Taxon-név / Taxon-name & $\begin{array}{l}\text { Település / } \\
\text { Settlement }\end{array}$ & $\begin{array}{l}\text { Év / } \\
\text { Year }\end{array}$ & Gyújtő / Collector & $\begin{array}{l}\text { Fájlnév / } \\
\text { File-name }\end{array}$ \\
\hline 1420 & Echium italicum L. & Nagyvisnyó & 1953 & Soó Rezső & BPU-05262.JPG \\
\hline 1420 & Echium italicum L. & Tihany & 1959 & Felföldy Lajos & BPU-05260.JPG \\
\hline 1421 & Echium vulgare L. & Felsőtelekes & 2005 & Gulyás Pál & BPU-05265.JPG \\
\hline 1421 & Echium vulgare L. & Sáránd & - & anonim & BPU-05264.JPG \\
\hline 1421 & Echium vulgare L. & Tállya & 1958 & Isépy István & BPU-05268.JPG \\
\hline 1421 & Echium vulgare L. & Tihany & 1958 & F. Kalkó Zsuzsa & BPU-05266.JPG \\
\hline 1421 & Echium vulgare L. & Veszprém & 1931 & Soó Rezső & BPU-05267.JPG \\
\hline 1422 & Echium maculatum L. & Budapest & 1947 & Soó Rezső & BPU-05273.JPG \\
\hline 1422 & Echium maculatum L. & Budapest & 1952 & Soó Rezső & BPU-15448.JPG \\
\hline 1422 & Echium maculatum L. & Miskolc & 1937 & Soó Rezső & BPU-05272.JPG \\
\hline 1422 & Echium maculatum L. & Tállya & 1938 & Soó Rezső & BPU-05270.JPG \\
\hline 1424 & Pulmonaria officinalis L. s. l. & Balatonszőlős & 1959 & Felföldy Lajos & BPU-05124.JPG \\
\hline 1424 & Pulmonaria officinalis L. s. l. & Bátorliget & 1934 & Soó Rezső & BPU-05123.JPG \\
\hline 1424 & Pulmonaria officinalis L. s. l. & Beregdaróc & 1952 & Kulcsár Gábor & BPU-05117.JPG \\
\hline 1424 & Pulmonaria officinalis L. s. l. & Budapest & 1961 & Isépy István & BPU-05113.JPG \\
\hline 1424 & Pulmonaria officinalis L. s. l. & Budapest & 1964 & Soó Rezső & BPU-05115.JPG \\
\hline 1424 & Pulmonaria officinalis L. s. l. & Budapest & 2004 & Felföldy Lajos & BPU-05114.JPG \\
\hline 1424 & Pulmonaria officinalis L. s. l. & Gánt & 1949 & Soó Rezső & BPU-05119.JPG \\
\hline 1424 & Pulmonaria officinalis L. s. l. & Gyula & 1935 & Soó Rezső & BPU-05116.JPG \\
\hline 1424 & Pulmonaria officinalis L. s. l. & Miskolc & 1937 & Soó Rezső & BPU-05118.JPG \\
\hline 1424 & Pulmonaria officinalis L. s. l. & Mosonmagyaróvár & 1965 & Borsos Olga & BPU-05120.JPG \\
\hline 1424 & Pulmonaria officinalis L. s. l. & Mosonmagyaróvár & 1965 & Borsos Olga & BPU-05121.JPG \\
\hline 1424 & Pulmonaria officinalis L. s. l. & Visegrád & 1964 & Soó Rezső, Borsos Olga & BPU-05122.JPG \\
\hline 1425 & Pulmonaria angustifolia L. & Devecser & 1959 & Tallós Pál & BPU-05127.JPG \\
\hline 1425 & Pulmonaria angustifolia L. & Sopron & 1960 & Borsos Olga, Simon Tibor & BPU-05128.JPG \\
\hline 1426 & Pulmonaria mollissima A. Kern. & Balatonfüred & 1959 & Felföldy Lajos & BPU-05134.JPG \\
\hline 1426 & Pulmonaria mollissima A. Kern. & Budapest & 1961 & Isépy István & BPU-05129.JPG \\
\hline 1426 & Pulmonaria mollissima A. Kern. & Debrecen & 1934 & Soó Rezső & BPU-05130.JPG \\
\hline 1426 & Pulmonaria mollissima A. Kern. & Gyöngyös & 1964 & Soó Rezső & BPU-05131.JPG \\
\hline 1426 & Pulmonaria mollissima A. Kern. & Hidegkút & 1959 & Felföldy Lajos & BPU-05135.JPG \\
\hline 1426 & Pulmonaria mollissima A. Kern. & Hidegkút & 1959 & Felföldy Lajos & BPU-05136.JPG \\
\hline 1426 & Pulmonaria mollissima A. Kern. & Nagykovácsi & 1949 & Soó Rezső & BPU-05132.JPG \\
\hline 1426 & Pulmonaria mollissima A. Kern. & Nyíregyháza & 1934 & Soó Rezső & BPU-05133.JPG \\
\hline 1426 & Pulmonaria mollissima A. Kern. & Pécsely & 1959 & Felföldy Lajos & BPU-05137.JPG \\
\hline $\begin{array}{c}1424 \times \\
1426\end{array}$ & $\begin{array}{l}\text { Pulmonaria officinalis L. } \times \text { Pulmonaria } \\
\text { mollissima A. Kern. }\end{array}$ & Budapest & 1943 & Kárpáti Zoltán & BPU-05141.JPG \\
\hline 1428 & Nonea pulla (L.) DC. & Egyek & 1938 & Soó Rezső & BPU-05106.JPG \\
\hline 1428 & Nonea pulla (L.) DC. & Hajdúnánás & 1935 & Soó Rezső & BPU-05102.JPG \\
\hline 1428 & Nonea pulla (L.) DC. & Lábatlan & 1953 & Felföldy Lajos & BPU-05104.JPG \\
\hline 1428 & Nonea pulla (L.) DC. & Szokolya & 1922 & Soó Rezső & BPU-05105.JPG \\
\hline 1428 & Nonea pulla (L.) DC. & Tihany & 1958 & Felföldy Lajos & BPU-05103.JPG \\
\hline 1428 & Nonea pulla (L.) DC. & Tihany & 1959 & Felföldy Lajos & BPU-05101.JPG \\
\hline 1429 & Symphytum tuberosum L. & "Cuha-völgy" & 1954 & Soó Rezső & BPU-05059.JPG \\
\hline 1429 & Symphytum tuberosum L. & Bátorliget & 1932 & Soó Rezső & BPU-05062.JPG \\
\hline 1429 & Symphytum tuberosum L. & Budapest & 1953 & Soó Rezső & BPU-05052.JPG \\
\hline 1429 & Symphytum tuberosum L. & Budapest & 1953 & Soó Rezső & BPU-15417.JPG \\
\hline 1429 & Symphytum tuberosum L. & Budapest & 1964 & Soó Rezső & BPU-05058.JPG \\
\hline 1429 & Symphytum tuberosum L. & Gyöngyös & 1965 & Soó Rezső & BPU-05057.JPG \\
\hline
\end{tabular}


1. Elektroniukus melléklet - Electronic Appendix 1.

\begin{tabular}{|c|c|c|c|c|c|}
\hline $\begin{array}{l}\text { Sorszám / } \\
\text { Number }\end{array}$ & Taxon-név / Taxon-name & $\begin{array}{l}\text { Település / } \\
\text { Settlement }\end{array}$ & $\begin{array}{l}\text { Év / } \\
\text { Year }\end{array}$ & Gyüjtő / Collector & $\begin{array}{l}\text { Fájlnév / } \\
\text { File-name }\end{array}$ \\
\hline 1429 & Symphytum tuberosum L. & Hidegkút & 1958 & Felföldy Lajos & BPU-05054.JPG \\
\hline 1429 & Symphytum tuberosum L. & Hidegkút & 1959 & Felföldy Lajos & BPU-05053.JPG \\
\hline 1429 & Symphytum tuberosum L. & Miskolc & 1937 & Soó Rezső & BPU-05056.JPG \\
\hline 1429 & Symphytum tuberosum L. & Pécs & 1935 & Soó Rezső & BPU-05060.JPG \\
\hline 1429 & Symphytum tuberosum L. & Pécs & 1953 & Soó Rezső, Borsos Olga & BPU-05061.JPG \\
\hline 1430 & Symphytum officinale L. & Aszófő & 1959 & Felföldy Lajos & BPU-05067.JPG \\
\hline 1430 & Symphytum officinale L. & Bátorliget & 1932 & Soó Rezső & BPU-05083.JPG \\
\hline 1430 & Symphytum officinale L. & Budapest & 2005 & Felföldy Lajos & BPU-05063.JPG \\
\hline 1430 & Symphytum officinale L. & Csaroda & 1953 & $\begin{array}{l}\text { Simon Tibor, Helmeczy } \\
\text { Balázs }\end{array}$ & BPU-05084.JPG \\
\hline 1430 & Symphytum officinale L. & Dabas & 1951 & Simon Tibor, Borsos Olga & BPU-05074.JPG \\
\hline 1430 & Symphytum officinale L. & Dabas & 1959 & Soó Rezső & BPU-05065.JPG \\
\hline 1430 & Symphytum officinale L. & Dabas & 1959 & Soó Rezső & BPU-05066.JPG \\
\hline 1430 & Symphytum officinale L. & Dabas & 1963 & Isépy István & BPU-05064.JPG \\
\hline 1430 & Symphytum officinale L. & Debrecen & 1931 & Soó Rezső & BPU-05076.JPG \\
\hline 1430 & Symphytum officinale L. & Gyöngyös & 1952 & Soó Rezső & BPU-05070.JPG \\
\hline 1430 & Symphytum officinale L. & Hajdúsámson & 1950 & Soó Rezső & BPU-05078.JPG \\
\hline 1430 & Symphytum officinale L. & Miskolc & 1934 & Soó Rezső & BPU-05069.JPG \\
\hline 1430 & Symphytum officinale L. & Nagyvisnyó & 1936 & Soó Rezső & BPU-05091.JPG \\
\hline 1430 & Symphytum officinale L. & Ócsa & 1953 & Soó Rezső, Borsos Olga & BPU-05081.JPG \\
\hline 1430 & Symphytum officinale L. & Pécs & 1953 & Soó Rezső, Borsos Olga & BPU-05068.JPG \\
\hline 1430 & Symphytum officinale L. & Sárospatak & 1939 & Soó Rezső & BPU-05079.JPG \\
\hline 1430 & Symphytum officinale L. & Sopron & 1937 & Soó Rezső & BPU-05072.JPG \\
\hline 1430 & Symphytum officinale L. & Tákos & 1948 & $\begin{array}{l}\text { Pólya László, Simon } \\
\text { Tibor, Jakucs Pál }\end{array}$ & BPU-05085.JPG \\
\hline 1430 & Symphytum officinale L. & Tákos & 1949 & Soó Rezső & BPU-05080.JPG \\
\hline 1430 & Symphytum officinale L. & Tiszafüred & 1932 & Soó Rezső & BPU-05073.JPG \\
\hline 1430 & Symphytum officinale L. & Ugod & 1955 & Soó Rezső & BPU-05075.JPG \\
\hline 1430 & Symphytum officinale L. & Ugod & 1955 & Soó Rezső & BPU-05077.JPG \\
\hline 1430 & Symphytum officinale L. & Újkígyós & 1923 & Soó Rezső & BPU-05082.JPG \\
\hline 1434 & Lycopsis arvensis L. & Debrecen & 1952 & Dobos L. & BPU-05100.JPG \\
\hline 1435 & Anchusa barrelieri (All.) Vitman & Pécs & 1953 & Soó Rezső, Borsos Olga & BPU-05093.JPG \\
\hline 1436 & Anchusa azurea Mill. & Balatonfüred & 1947 & Pólya László & BPU-05095.JPG \\
\hline 1436 & Anchusa azurea Mill. & Csopak & 1957 & Entz Béla & BPU-05094.JPG \\
\hline 1437 & Anchusa officinalis L. & Budapest & 1961 & Isépy István & BPU-05097.JPG \\
\hline 1437 & Anchusa officinalis L. & Debrecen & 1931 & Soó Rezső & BPU-05096.JPG \\
\hline 1437 & Anchusa officinalis L. & Tállya & 1962 & Isépy István & BPU-05098.JPG \\
\hline 1440 & Asperugo procumbens L. & Debrecen & 1947 & Soó Rezső & BPU-05051.JPG \\
\hline 1440 & Asperugo procumbens L. & Nagyharsány & 1956 & Soó Rezső & BPU-05049.JPG \\
\hline 1440 & Asperugo procumbens L. & Tokaj & 1933 & Soó Rezső & BPU-05050.JPG \\
\hline 1442 & Myosotis caespitosa Schultz & Hortobágy & 1935 & Soó Rezső & BPU-05164.JPG \\
\hline 1443 & Myosotis nemorosa Besser & Balatonszepezd & 1956 & $\begin{array}{l}\text { Felföldy Lajos, Tóth } \\
\text { László }\end{array}$ & BPU-05160.JPG \\
\hline 1443 & Myosotis nemorosa Besser & Bükkszentkereszt & 1938 & Soó Rezső & BPU-05143.JPG \\
\hline 1443 & Myosotis nemorosa Besser & Dabas & 1963 & Isépy István & BPU-05151.JPG \\
\hline 1443 & Myosotis nemorosa Besser & Lesenceistvánd & 1952 & Borsos Olga & BPU-05157.JPG \\
\hline 1443 & Myosotis nemorosa Besser & Örvényes & 1958 & Felföldy Lajos & BPU-05158.JPG \\
\hline 1443 & Myosotis nemorosa Besser & Örvényes & 1958 & Felföldy Lajos & BPU-05159.JPG \\
\hline 1443 & Myosotis nemorosa Besser & Sopron & 1960 & Borsos Olga, Simon Tibor & BPU-05153.JPG \\
\hline
\end{tabular}


1. Elektroniukus melléklet - Electronic Appendix 1.

\begin{tabular}{|c|c|c|c|c|c|}
\hline $\begin{array}{l}\text { Sorszám / } \\
\text { Number }\end{array}$ & Taxon-név / Taxon-name & $\begin{array}{l}\text { Település / } \\
\text { Settlement }\end{array}$ & $\begin{array}{l}\text { Év / } \\
\text { Year }\end{array}$ & Gyüjtő / Collector & $\begin{array}{l}\text { Fájlnév / } \\
\text { File-name }\end{array}$ \\
\hline 1443 & Myosotis nemorosa Besser & Veresegyház & 1949 & Soó Rezső & BPU-05152.JPG \\
\hline 1444 & Myosotis scorpioides L. & Debrecen & 1934 & Soó Rezső & BPU-05144.JPG \\
\hline 1444 & Myosotis scorpioides L. & Debrecen & 1934 & Soó Rezső & BPU-05145.JPG \\
\hline 1444 & Myosotis scorpioides L. & Debrecen & 1989 & Felföldy Lajos & BPU-05146.JPG \\
\hline 1444 & Myosotis scorpioides L. & Debrecen & 1989 & Felföldy Lajos & BPU-05147.JPG \\
\hline 1444 & Myosotis scorpioides L. & Debrecen & 1989 & Felföldy Lajos & BPU-05148.JPG \\
\hline 1444 & Myosotis scorpioides L. & Gávavencsellő & 1990 & Felföldy Lajos & BPU-05154.JPG \\
\hline 1444 & Myosotis scorpioides L. & Hajdúsámson & 1989 & Felföldy Lajos & BPU-05149.JPG \\
\hline 1444 & Myosotis scorpioides L. & Hajdúsámson & 1989 & Felföldy Lajos & BPU-05150.JPG \\
\hline 1444 & Myosotis scorpioides L. & Kömörő & 1948 & $\begin{array}{l}\text { Pólya László, Simon } \\
\text { Tibor, Jakucs Pál }\end{array}$ & BPU-05155.JPG \\
\hline 1444 & Myosotis scorpioides L. & Sáránd & 1934 & Soó Rezső & BPU-05142.JPG \\
\hline 1444 & Myosotis scorpioides L. & Tihany & 1956 & $\begin{array}{l}\text { Felföldy Lajos, Tóth } \\
\text { László }\end{array}$ & BPU-05161.JPG \\
\hline 1444 & Myosotis scorpioides L. & Tiszafüred & 1990 & Felföldy Lajos & BPU-05156.JPG \\
\hline 1444 & Myosotis scorpioides L. & Uzsa & 1950 & $\begin{array}{l}\text { Felföldy Lajos, Tóth } \\
\text { László }\end{array}$ & BPU-05162.JPG \\
\hline 1446 & Myosotis sylvatica (Ehrh.) Hoffm. & "Cuha-völgy" & 1954 & Soó Rezső & BPU-05201.JPG \\
\hline 1446 & Myosotis sylvatica (Ehrh.) Hoffm. & Bozsok & 1938 & Soó Rezső & BPU-05203.JPG \\
\hline 1446 & Myosotis sylvatica (Ehrh.) Hoffm. & Miskolc & 1939 & Soó Rezső & BPU-05200.JPG \\
\hline 1446 & Myosotis sylvatica (Ehrh.) Hoffm. & Pécs & 1925 & Soó Rezső & BPU-05202.JPG \\
\hline 1447 & Myosotis stricta Link & Budapest & 2004 & Felföldy Lajos & BPU-05165.JPG \\
\hline 1447 & Myosotis stricta Link & Budapest & 2004 & Felföldy Lajos & BPU-05166.JPG \\
\hline 1447 & Myosotis stricta Link & Debrecen & 1933 & Soó Rezső & BPU-05168.JPG \\
\hline 1447 & Myosotis stricta Link & Nyíradony & 1951 & Simon Tibor & BPU-05170.JPG \\
\hline 1447 & Myosotis stricta Link & Tihany & 1958 & Felföldy Lajos & BPU-05171.JPG \\
\hline 1447 & Myosotis stricta Link & Vásárosnamény & 1951 & Simon Tibor & BPU-05167.JPG \\
\hline 1447 & Myosotis stricta Link & Visegrád & 1964 & Soó Rezső, Borsos Olga & BPU-05169.JPG \\
\hline 1448 & Myosotis discolor Pers. & Nagykanizsa & 1948 & Károlyi Árpád & BPU-05173.JPG \\
\hline 1449 & Myosotis sparsiflora J.G. Mikan & "Cuha-völgy" & 1954 & Soó Rezső & BPU-05181.JPG \\
\hline 1449 & Myosotis sparsiflora J.G. Mikan & Csaholc & 1993 & Felföldy Lajos & BPU-05180.JPG \\
\hline 1449 & Myosotis sparsiflora J.G. Mikan & Debrecen & 1937 & Felföldy Lajos & BPU-05177.JPG \\
\hline 1449 & Myosotis sparsiflora J.G. Mikan & Pécsvárad & 1936 & Soó Rezső & BPU-05174.JPG \\
\hline 1449 & Myosotis sparsiflora J.G. Mikan & Salgótarján & 1936 & Soó Rezső & BPU-05179.JPG \\
\hline 1449 & Myosotis sparsiflora J.G. Mikan & Téglás & 1937 & Soó Rezső & BPU-05178.JPG \\
\hline 1449 & Myosotis sparsiflora J.G. Mikan & Tihany & 1958 & Felföldy Lajos & BPU-05182.JPG \\
\hline 1449 & Myosotis sparsiflora J.G. Mikan & Tihany & 1958 & Felföldy Lajos & BPU-05183.JPG \\
\hline 1449 & Myosotis sparsiflora J.G. Mikan & Uzsa & 1959 & $\begin{array}{l}\text { Jávorka Sándor, Felföldy } \\
\text { Lajos }\end{array}$ & BPU-05184.JPG \\
\hline 1449 & Myosotis sparsiflora J.G. Mikan & Vászoly & 1955 & $\begin{array}{l}\text { Felföldy Lajos, Tóth } \\
\text { László }\end{array}$ & BPU-05185.JPG \\
\hline 1449 & Myosotis sparsiflora J.G. Mikan & Villány & 1956 & Soó Rezső & BPU-05175.JPG \\
\hline 1449 & Myosotis sparsiflora J.G. Mikan & Villány & 1956 & Soó Rezső & BPU-05176.JPG \\
\hline 1450 & Myosotis arvensis (L.) Hill & Debrecen & 1931 & Soó Rezső & BPU-05193.JPG \\
\hline 1450 & Myosotis arvensis (L.) Hill & Gávavencsellő & 1990 & Felföldy Lajos & BPU-05195.JPG \\
\hline 1450 & Myosotis arvensis (L.) Hill & Nagyharsány & 1965 & $\begin{array}{l}\text { Simon Tibor, Priszter } \\
\text { Szaniszló }\end{array}$ & BPU-05191.JPG \\
\hline 1450 & Myosotis arvensis (L.) Hill & Nagyrada & 1987 & Felföldy Lajos & BPU-05199.JPG \\
\hline 1450 & Myosotis arvensis (L.) Hill & Örvényes & 1959 & Felföldy Lajos & BPU-05196.JPG \\
\hline 1450 & Myosotis arvensis (L.) Hill & Salgótarján & 1936 & Soó Rezső & BPU-05194.JPG \\
\hline
\end{tabular}


1. Elektroniukus melléklet - Electronic Appendix 1.

\begin{tabular}{|c|c|c|c|c|c|}
\hline $\begin{array}{l}\text { Sorszám / } \\
\text { Number }\end{array}$ & Taxon-név / Taxon-name & $\begin{array}{l}\text { Település / } \\
\text { Settlement }\end{array}$ & $\begin{array}{l}\text { Év / } \\
\text { Year }\end{array}$ & Gyújtő / Collector & $\begin{array}{l}\text { Fájlnév / } \\
\text { File-name }\end{array}$ \\
\hline 1450 & Myosotis arvensis (L.) Hill & Tákos & 1993 & Felföldy Lajos & BPU-05192.JPG \\
\hline 1450 & Myosotis arvensis (L.) Hill & Tihany & 1956 & Felföldy Lajos & BPU-05197.JPG \\
\hline 1450 & Myosotis arvensis (L.) Hill & Tihany & 1959 & Felföldy Lajos & BPU-05198.JPG \\
\hline 1451 & Myosotis ramosissima Rochel & Tihany & 1946 & Felföldy Lajos & BPU-05187.JPG \\
\hline 1451 & Myosotis ramosissima Rochel & Tihany & 1958 & Felföldy Lajos & BPU-05188.JPG \\
\hline 1451 & Myosotis ramosissima Rochel & Tihany & 1958 & Felföldy Lajos & BPU-05189.JPG \\
\hline 1451 & Myosotis ramosissima Rochel & Uzsa & 1959 & $\begin{array}{l}\text { Jávorka Sándor, Felföldy } \\
\text { Lajos }\end{array}$ & BPU-05190.JPG \\
\hline 1451 & Myosotis ramosissima Rochel & Zámoly & 1982 & Felföldy Lajos & BPU-05186.JPG \\
\hline---- & $\begin{array}{l}\text { Myosotis suaveolens Waldst. \& Kit. ex } \\
\text { Willd. }\end{array}$ & Pilisszentiván & 1914 & Degen Árpád & BPU-05210.JPG \\
\hline 1455 & Lappula squarrosa (Retz.) Dumort. & Budapest & 1993 & Felföldy Lajos & BPU-05043.JPG \\
\hline 1455 & Lappula squarrosa (Retz.) Dumort. & Konyár & 1949 & Soó Rezső & BPU-05045.JPG \\
\hline 1455 & Lappula squarrosa (Retz.) Dumort. & Szögliget & 1951 & $\begin{array}{l}\text { Soó Rezső, Jakucs Pál, Ér } \\
\text { Lajos }\end{array}$ & BPU-05044.JPG \\
\hline 1455 & Lappula squarrosa (Retz.) Dumort. & Tihany & 1961 & Felföldy Lajos & BPU-05047.JPG \\
\hline 1455 & Lappula squarrosa (Retz.) Dumort. & Vácrátót & 1952 & Felföldy Lajos & BPU-05046.JPG \\
\hline 1456 & Lappula heteracantha (Ledeb.) Borbás & Budapest & 1929 & Zsák Zoltán & BPU-05048.JPG \\
\hline 1457 & $\begin{array}{l}\text { Omphalodes scorpioides (Haenke) } \\
\text { Schrank }\end{array}$ & "Pilis" & 1932 & Soó Rezső & BPU-05035.JPG \\
\hline 1457 & $\begin{array}{l}\text { Omphalodes scorpioides (Haenke) } \\
\text { Schrank }\end{array}$ & Gánt & 1949 & Soó Rezső & BPU-05034.JPG \\
\hline 1457 & $\begin{array}{l}\text { Omphalodes scorpioides (Haenke) } \\
\text { Schrank }\end{array}$ & Gánt & 1949 & Soó Rezső & BPU-15569.JPG \\
\hline 1457 & $\begin{array}{l}\text { Omphalodes scorpioides (Haenke) } \\
\text { Schrank }\end{array}$ & Isztimér & 1959 & Simon Tibor & BPU-05032.JPG \\
\hline 1457 & $\begin{array}{l}\text { Omphalodes scorpioides (Haenke) } \\
\text { Schrank }\end{array}$ & Isztimér & 1959 & Simon Tibor & BPU-05036.JPG \\
\hline 1459 & Cynoglossum officinale L. & Bugac & 1963 & Isépy István & BPU-05037.JPG \\
\hline 1459 & Cynoglossum officinale L. & Debrecen & 1930 & Soó Rezső & BPU-05038.JPG \\
\hline 1460 & Cynoglossum hungaricum Simonk. & Csévharaszt & 1959 & Simon Tibor, Borsos Olga & BPU-05040.JPG \\
\hline 1460 & Cynoglossum hungaricum Simonk. & Debrecen & 1946 & Soó Rezső & BPU-05042.JPG \\
\hline 1460 & Cynoglossum hungaricum Simonk. & Fenyőfő & 1955 & Soó Rezső & BPU-05041.JPG \\
\hline 1460 & Cynoglossum hungaricum Simonk. & Pilisszentiván & 1953 & Soó Rezső & BPU-05039.JPG \\
\hline 1461 & Phacelia tanacetifolia Benth. & Tihany & 1958 & Felföldy Lajos & BPU-05027.JPG \\
\hline 1463 & Verbena officinalis L. & Budapest & 2004 & Felföldy Lajos & BPU-05275.JPG \\
\hline 1463 & Verbena officinalis L. & Budapest & 2005 & Felföldy Lajos & BPU-05276.JPG \\
\hline 1463 & Verbena officinalis L. & Tállya & 1962 & Isépy István & BPU-05278.JPG \\
\hline 1463 & Verbena officinalis L. & Tihany & 1959 & Felföldy Lajos & BPU-05277.JPG \\
\hline 1464 & Verbena supina L. & Szarvas & 1947 & Soó Rezső & BPU-05279.JPG \\
\hline 1464 & Verbena supina L. & Szerep & 1911 & Rácz Béla & BPU-05280.JPG \\
\hline 1466 & Callitriche cophocarpa Sendtn. & Dávod & 1955 & Soó Rezső & BPU-04820.JPG \\
\hline 1466 & Callitriche cophocarpa Sendtn. & Nyírpilis & 1932 & Soó Rezső & BPU-04818.JPG \\
\hline 1466 & Callitriche cophocarpa Sendtn. & Olcsvaapáti & 1951 & Simon Tibor & BPU-04821.JPG \\
\hline 1466 & Callitriche cophocarpa Sendtn. & Putnok & 1950 & Soó Rezső & BPU-04822.JPG \\
\hline 1466 & Callitriche cophocarpa Sendtn. & Szenta & 1955 & Soó Rezső & BPU-04819.JPG \\
\hline 1466 & Callitriche cophocarpa Sendtn. & Szőce & 1955 & Soó Rezső & BPU-04821.JPG \\
\hline 1467 & Callitriche palustris L. & Beregdaróc & 1951 & Simon Tibor, Jakucs Pál & BPU-04816.JPG \\
\hline 1467 & Callitriche palustris L. & Hortobágy & 1947 & Soó Rezső & BPU-04816.JPG \\
\hline 1467 & Callitriche palustris L. & Miskolc & 1950 & Jakucs Pál & BPU-04817.JPG \\
\hline
\end{tabular}


1. Elektroniukus melléklet - Electronic Appendix 1.

\begin{tabular}{|c|c|c|c|c|c|}
\hline $\begin{array}{l}\text { Sorszám / } \\
\text { Number }\end{array}$ & Taxon-név / Taxon-name & $\begin{array}{l}\text { Település / } \\
\text { Settlement }\end{array}$ & $\begin{array}{l}\text { Év / } \\
\text { Year }\end{array}$ & Gyújtő / Collector & $\begin{array}{l}\text { Fájlnév / } \\
\text { File-name }\end{array}$ \\
\hline 1467 & Callitriche palustris L. & Tákos & 1948 & $\begin{array}{l}\text { Pólya László, Simon } \\
\text { Tibor, Jakucs Pál }\end{array}$ & BPU-04815.JPG \\
\hline 1469 & Callitriche platycarpa Kütz. & Kelemér & 2003 & Virók Viktor & BPU-04823.JPG \\
\hline 1470 & Ajuga chamaepitys (L.) Schreb. & Budapest & 1945 & Soó Rezső & BPU-05282.JPG \\
\hline 1470 & Ajuga chamaepitys (L.) Schreb. & Bugac & 1964 & Soó Rezső & BPU-05284.JPG \\
\hline 1470 & Ajuga chamaepitys (L.) Schreb. & Tihany & 1961 & $\begin{array}{l}\text { Pankov H., Felföldy Lajos, } \\
\text { Sólymosy G. }\end{array}$ & BPU-05281.JPG \\
\hline 1470 & Ajuga chamaepitys (L.) Schreb. & Vácrátót & 1952 & Felföldy Lajos & BPU-05283.JPG \\
\hline 1471 & Ajuga laxmannii (L.) Benth. & Ebes & 1965 & Simon Tibor & BPU-05285.JPG \\
\hline 1472 & Ajuga reptans L. & Bátorliget & 1932 & Soó Rezső & BPU-05293.JPG \\
\hline 1472 & Ajuga reptans L. & Biatorbágy & 1992 & Felföldy Lajos & BPU-05291.JPG \\
\hline 1472 & Ajuga reptans L. & Budapest & 1961 & Isépy István & BPU-05287.JPG \\
\hline 1472 & Ajuga reptans L. & Lábatlan & 1953 & Felföldy Lajos & BPU-05289.JPG \\
\hline 1472 & Ajuga reptans L. & Nagykovácsi & 1963 & Isépy István & BPU-05292.JPG \\
\hline 1472 & Ajuga reptans L. & Örvényes & 1959 & Felföldy Lajos & BPU-05294.JPG \\
\hline 1472 & Ajuga reptans L. & Örvényes & 1959 & Felföldy Lajos & BPU-05295.JPG \\
\hline 1472 & Ajuga reptans L. & Pécs & 1935 & Soó Rezső & BPU-05288.JPG \\
\hline 1472 & Ajuga reptans L. & Szokolya & 1922 & Soó Rezső & BPU-05290.JPG \\
\hline 1473 & Ajuga genevensis L. & Nagyharsány & 1967 & Priszter Szaniszló & BPU-05298.JPG \\
\hline 1473 & Ajuga genevensis L. & Sáránd & - & anonim & BPU-05299.JPG \\
\hline 1473 & Ajuga genevensis L. & Siklós & 1967 & Priszter Szaniszló & BPU-05297.JPG \\
\hline 1474 & Teucrium botrys L. & Budapest & 1923 & Soó Rezső & BPU-05312.JPG \\
\hline 1475 & Teucrium montanum L. & Bélapátfalva & 1948 & $\begin{array}{l}\text { Simon Tibor, Pólya } \\
\text { László, Jakucs Pál }\end{array}$ & BPU-05305.JPG \\
\hline 1475 & Teucrium montanum L. & Bélapátfalva & 1950 & $\begin{array}{l}\text { Simon Tibor, Jakucs Pál, } \\
\text { Kárpáti István }\end{array}$ & BPU-05306.JPG \\
\hline 1475 & Teucrium montanum L. & Bodajk & 1961 & Borsos Olga, Simon Tibor & BPU-05304.JPG \\
\hline 1475 & Teucrium montanum L. & Budapest & 1982 & Felföldy Lajos & BPU-05300.JPG \\
\hline 1475 & Teucrium montanum L. & Jósvafő & 1952 & Soó Rezső & BPU-05301.JPG \\
\hline 1475 & Teucrium montanum L. & Jósvafő & 1955 & Jakucs Pál & BPU-05302.JPG \\
\hline 1475 & Teucrium montanum L. & Miskolc & 1950 & Soó Rezső & BPU-05303.JPG \\
\hline 1475 & Teucrium montanum L. & Nagykovácsi & 1962 & Isépy István & BPU-05307.JPG \\
\hline 1477 & Teucrium chamaedrys L. & Bagamér & 1932 & Máthé Imre, Soó Rezső & BPU-05316.JPG \\
\hline 1477 & Teucrium chamaedrys L. & Balatonakali & 1950 & Felföldy Lajos & BPU-05321.JPG \\
\hline 1477 & Teucrium chamaedrys L. & Budapest & 2004 & Felföldy Lajos & BPU-05314.JPG \\
\hline 1477 & Teucrium chamaedrys L. & Csomád & 1953 & Felföldy Lajos & BPU-05318.JPG \\
\hline 1477 & Teucrium chamaedrys L. & Gárdony & 1982 & Felföldy Lajos & BPU-05315.JPG \\
\hline 1477 & Teucrium chamaedrys L. & Nagykovácsi & 1962 & Isépy István & BPU-05319.JPG \\
\hline 1477 & Teucrium chamaedrys L. & Pilisszentiván & 1969 & Priszter Szaniszló & BPU-05320.JPG \\
\hline 1477 & Teucrium chamaedrys L. & Salgótarján & 1936 & Soó Rezső & BPU-05317.JPG \\
\hline 1477 & Teucrium chamaedrys L. & Tihany & 1959 & Felföldy Lajos & BPU-05323.JPG \\
\hline 1477 & Teucrium chamaedrys L. & Tihany & 1959 & Felföldy Lajos & BPU-05324.JPG \\
\hline 1477 & Teucrium chamaedrys L. & Tihany & 1961 & $\begin{array}{l}\text { Pankov H., Felföldy Lajos, } \\
\text { Sólymosy G. }\end{array}$ & BPU-05322.JPG \\
\hline 1478 & Teucrium scordium L. & Aszófő & 1957 & $\begin{array}{l}\text { Felföldy Lajos, Kovács } \\
\text { Margit, Tóth László }\end{array}$ & BPU-05329.JPG \\
\hline 1478 & Teucrium scordium L. & Bagamér & 1932 & Soó Rezső & BPU-05330.JPG \\
\hline 1478 & Teucrium scordium L. & Budapest & 1947 & Soó Rezső & BPU-05327.JPG \\
\hline 1478 & Teucrium scordium L. & Debrecen & 1932 & Soó Rezső & BPU-05331.JPG \\
\hline 1478 & Teucrium scordium L. & Tihany & 1954 & Felföldy Lajos & BPU-05328.JPG \\
\hline
\end{tabular}


1. Elektroniukus melléklet - Electronic Appendix 1.

\begin{tabular}{|c|c|c|c|c|c|}
\hline $\begin{array}{l}\text { Sorszám / } \\
\text { Number }\end{array}$ & Taxon-név / Taxon-name & $\begin{array}{l}\text { Település / } \\
\text { Settlement }\end{array}$ & $\begin{array}{l}\text { Év / } \\
\text { Year }\end{array}$ & Gyűjtő / Collector & $\begin{array}{l}\text { Fájlnév / } \\
\text { File-name }\end{array}$ \\
\hline 1478 & Teucrium scordium L. & Tihany & 1961 & $\begin{array}{l}\text { Pankov H., Felföldy Lajos, } \\
\text { Sólymosy G. }\end{array}$ & BPU-05326.JPG \\
\hline 1479 & Scutellaria hastifolia L. & Budapest & 2005 & Felföldy Lajos & BPU-05341.JPG \\
\hline 1479 & Scutellaria hastifolia L. & Pécsely & 1950 & Felföldy Lajos & BPU-05343.JPG \\
\hline 1479 & Scutellaria hastifolia L. & Tarpa & 1949 & Soó Rezső & BPU-05342.JPG \\
\hline 1480 & Scutellaria galericulata $\mathrm{L}$. & Debrecen & 1989 & Felföldy Lajos & BPU-05336.JPG \\
\hline 1480 & Scutellaria galericulata L. & Debrecen & 1989 & Felföldy Lajos & BPU-05337.JPG \\
\hline 1480 & Scutellaria galericulata L. & Gárdony & 1980 & Felföldy Lajos & BPU-05334.JPG \\
\hline 1480 & Scutellaria galericulata L. & Gárdony & 1982 & Felföldy Lajos & BPU-05333.JPG \\
\hline 1480 & Scutellaria galericulata L. & Kelemér & 1950 & Soó Rezső & BPU-05335.JPG \\
\hline 1480 & Scutellaria galericulata L. & Kimle & 1962 & Borsos Olga & BPU-05338.JPG \\
\hline 1480 & Scutellaria galericulata L. & Szentbékkálla & 1959 & $\begin{array}{l}\text { Jávorka Sándor, Felföldy } \\
\text { Lajos, Tóth László }\end{array}$ & BPU-05339.JPG \\
\hline 1480 & Scutellaria galericulata L. & Tarpa & 1949 & Soó Rezső & BPU-05332.JPG \\
\hline 1480 & Scutellaria galericulata L. & Tihany & 1956 & $\begin{array}{l}\text { Felföldy Lajos, Tóth } \\
\text { László }\end{array}$ & BPU-05340.JPG \\
\hline 1481 & Scutellaria columnae All. & Csesznek & 1929 & Soó Rezső & BPU-05345.JPG \\
\hline 1481 & Scutellaria columnae All. & Pilismarót & 1946 & Vajda László & BPU-05344.JPG \\
\hline 1482 & Scutellaria altissima L. & Bélapátfalva & 1950 & $\begin{array}{l}\text { Simon Tibor, Jakucs Pál, } \\
\text { Kárpáti István }\end{array}$ & BPU-05347.JPG \\
\hline 1482 & Scutellaria altissima L. & Budapest & 1948 & Kárpáti Zoltán & BPU-05349.JPG \\
\hline 1482 & Scutellaria altissima L. & Budapest & 1961 & Soó Rezső & BPU-05346.JPG \\
\hline 1482 & Scutellaria altissima L. & Eger & 1953 & Simon Tibor & BPU-05348.JPG \\
\hline 1483 & Marrubium vulgare L. & Hajdúszoboszló & 1937 & Soó Rezső & BPU-05351.JPG \\
\hline 1484 & Marrubium peregrinum $\mathrm{L}$. & Bugac & 1952 & Vozáry Elemér & BPU-05353.JPG \\
\hline 1484 & Marrubium peregrinum L. & Csákvár & 1948 & Soó Rezső & BPU-05355.JPG \\
\hline 1484 & Marrubium peregrinum L. & Tihany & 1949 & Simon Tibor & BPU-05352.JPG \\
\hline 1484 & Marrubium peregrinum $\mathrm{L}$. & Tihany & 1961 & $\begin{array}{l}\text { Pankov H., Felföldy Lajos, } \\
\text { Sólymosy G. }\end{array}$ & BPU-05354.JPG \\
\hline 1485 & Marrubium $\times$ paniculatum Desr. & Göd & 1936 & Kárpáti Zoltán & BPU-05357.JPG \\
\hline 1485 & Marrubium $\times$ paniculatum Desr. & Pilisszentiván & 1953 & Soó Rezső & BPU-05356.JPG \\
\hline 1485 & Marrubium $\times$ paniculatum Desr. & Újfehértó & 1935 & Soó Rezső & BPU-05359.JPG \\
\hline 1486 & Sideritis montana L. & Bélapátfalva & 1948 & $\begin{array}{l}\text { Simon Tibor, Pólya } \\
\text { László, Jakucs Pál }\end{array}$ & BPU-05361.JPG \\
\hline 1486 & Sideritis montana L. & Budaörs & 1982 & Felföldy Lajos & BPU-05362.JPG \\
\hline 1486 & Sideritis montana L. & Budapest & 1947 & Soó Rezső & BPU-05360.JPG \\
\hline 1486 & Sideritis montana L. & Tihany & 1961 & $\begin{array}{l}\text { Pankov H., Felföldy Lajos, } \\
\text { Sólymosy G. }\end{array}$ & BPU-05363.JPG \\
\hline 1487 & Melittis melissophyllum L. & Balatonszőlős & 1958 & Felföldy Lajos & BPU-05467.JPG \\
\hline 1487 & Melittis melissophyllum L. & Bátorliget & 1941 & Máthé Imre & BPU-05461.JPG \\
\hline 1487 & Melittis melissophyllum L. & Bodajk & 1961 & Borsos Olga & BPU-05452.JPG \\
\hline 1487 & Melittis melissophyllum L. & Bodajk & 1961 & Borsos Olga & BPU-05454.JPG \\
\hline 1487 & Melittis melissophyllum L. & Budaörs & 1922 & Zsák Zoltán & BPU-05456.JPG \\
\hline 1487 & Melittis melissophyllum L. & Budaörs & 1961 & Borsos Olga, Simon Tibor & BPU-05474.JPG \\
\hline 1487 & Melittis melissophyllum L. & Budaörs & 1961 & Borsos Olga, Simon Tibor & BPU-05475.JPG \\
\hline 1487 & Melittis melissophyllum L. & Budaörs & 1961 & Borsos Olga, Simon Tibor & BPU-05476.JPG \\
\hline 1487 & Melittis melissophyllum L. & Budaörs & 1961 & Borsos Olga, Simon Tibor & BPU-05477.JPG \\
\hline 1487 & Melittis melissophyllum L. & Budaörs & 1961 & Borsos Olga, Simon Tibor & BPU-05478.JPG \\
\hline 1487 & Melittis melissophyllum L. & Budaörs & 1961 & Simon Tibor, Borsos Olga & BPU-05464.JPG \\
\hline 1487 & Melittis melissophyllum L. & Budapest & 1947 & Soó Rezső & BPU-05457.JPG \\
\hline
\end{tabular}


1. Elektroniukus melléklet - Electronic Appendix 1.

\begin{tabular}{|c|c|c|c|c|c|}
\hline $\begin{array}{l}\text { Sorszám / } \\
\text { Number }\end{array}$ & Taxon-név / Taxon-name & $\begin{array}{l}\text { Település / } \\
\text { Settlement }\end{array}$ & $\begin{array}{l}\text { Év / } \\
\text { Year }\end{array}$ & Gyújtő / Collector & $\begin{array}{l}\text { Fájlnév / } \\
\text { File-name }\end{array}$ \\
\hline 1487 & Melittis melissophyllum L. & Budapest & 1961 & Isépy István & BPU-05471.JPG \\
\hline 1487 & Melittis melissophyllum L. & Budapest & 1992 & Felföldy Lajos & BPU-05472.JPG \\
\hline 1487 & Melittis melissophyllum L. & Devecser & 1962 & Tallós Pál & BPU-05463.JPG \\
\hline 1487 & Melittis melissophyllum L. & Erdőbénye & 1961 & Borsos Olga & BPU-05440.JPG \\
\hline 1487 & Melittis melissophyllum L. & Erdőbénye & 1961 & Borsos Olga & BPU-05441.JPG \\
\hline 1487 & Melittis melissophyllum L. & Erdőbénye & 1961 & Borsos Olga & BPU-05442.JPG \\
\hline 1487 & Melittis melissophyllum L. & Gyöngyös & 1961 & Soó Rezső & BPU-05455.JPG \\
\hline 1487 & Melittis melissophyllum L. & Gyöngyös & 1961 & Soó Rezső, Borsos Olga & BPU-05443.JPG \\
\hline 1487 & Melittis melissophyllum L. & Gyöngyös & 1961 & Soó Rezső, Borsos Olga & BPU-05444.JPG \\
\hline 1487 & Melittis melissophyllum L. & Gyöngyös & 1965 & Soó Rezső & BPU-05459.JPG \\
\hline 1487 & Melittis melissophyllum L. & Hidegkút & 1958 & Felföldy Lajos & BPU-05468.JPG \\
\hline 1487 & Melittis melissophyllum L. & Hollóháza & 1961 & Simon Tibor & BPU-05473.JPG \\
\hline 1487 & Melittis melissophyllum L. & Kőszeg & 1924 & Boros Ádám & BPU-05465.JPG \\
\hline 1487 & Melittis melissophyllum L. & Lábatlan & 1953 & Felföldy Lajos & BPU-05466.JPG \\
\hline 1487 & Melittis melissophyllum L. & Nagykovácsi & 2005 & Felföldy Lajos & BPU-05470.JPG \\
\hline 1487 & Melittis melissophyllum L. & Pécs & 1953 & Soó Rezső, Borsos Olga & BPU-05458.JPG \\
\hline 1487 & Melittis melissophyllum L. & Pécsely & 1950 & Felföldy Lajos & BPU-05462.JPG \\
\hline 1487 & Melittis melissophyllum L. & Pilisszentiván & 1961 & Borsos Olga & BPU-05448.JPG \\
\hline 1487 & Melittis melissophyllum L. & Pilisszentiván & 1961 & Borsos Olga & BPU-05449.JPG \\
\hline 1487 & Melittis melissophyllum L. & Pilisszentiván & 1961 & Borsos Olga & BPU-05450.JPG \\
\hline 1487 & Melittis melissophyllum L. & Pilisszentiván & 1961 & Borsos Olga & BPU-05451.JPG \\
\hline 1487 & Melittis melissophyllum L. & Tihany & 1956 & Felföldy Lajos & BPU-05469.JPG \\
\hline 1487 & Melittis melissophyllum L. & Zebegény & 1961 & Borsos Olga & BPU-05445.JPG \\
\hline 1487 & Melittis melissophyllum L. & Zebegény & 1961 & Borsos Olga & BPU-05446.JPG \\
\hline 1487 & Melittis melissophyllum L. & Zebegény & 1961 & Borsos Olga & BPU-05447.JPG \\
\hline 1487 & Melittis melissophyllum L. & Zebegény & 1961 & Borsos Olga & BPU-05453.JPG \\
\hline 1488 & Phlomis tuberosa $\mathrm{L}$. & Budapest & 1989 & Felföldy Lajos & BPU-05479.JPG \\
\hline 1488 & Phlomis tuberosa L. & Egyek & 1950 & $\begin{array}{l}\text { Simon Tibor, Borsos Olga, } \\
\text { Kulcsár Gábor }\end{array}$ & BPU-05483.JPG \\
\hline 1488 & Phlomis tuberosa L. & Fót & 1960 & Soó Rezső & BPU-05484.JPG \\
\hline 1488 & Phlomis tuberosa $\mathrm{L}$. & Hortobágy & 1947 & Soó Rezső & BPU-05482.JPG \\
\hline 1488 & Phlomis tuberosa L. & Miskolc & 1940 & Soó Rezső & BPU-05481.JPG \\
\hline 1488 & Phlomis tuberosa L. & Pere & 1961 & Simon Tibor & BPU-05480.JPG \\
\hline 1490 & Galeopsis ladanum L. & Beregdaróc & 1951 & $\begin{array}{l}\text { Simon Tibor, Vozáry } \\
\text { Elemér }\end{array}$ & BPU-05489.JPG \\
\hline 1490 & Galeopsis ladanum L. & Eger & 1948 & Koppányi Tibor & BPU-05487.JPG \\
\hline 1490 & Galeopsis ladanum L. & Tihany & 1959 & Felföldy Lajos & BPU-05485.JPG \\
\hline 1491 & Galeopsis angustifolia (Ehrh.) Hoffm. & Gelénes & 1948 & Simon Tibor, Jakucs Pál & BPU-05486.JPG \\
\hline 1492 & Galeopsis pubescens Besser & Bakonyszentlászló & 1963 & Soó Rezső & BPU-05506.JPG \\
\hline 1492 & Galeopsis pubescens Besser & Csákvár & 1948 & Soó Rezső & BPU-05512.JPG \\
\hline 1492 & Galeopsis pubescens Besser & Diósjenő & 1952 & Simon Tibor & BPU-05508.JPG \\
\hline 1492 & Galeopsis pubescens Besser & Gyöngyös & 1953 & Soó Rezső & BPU-05509.JPG \\
\hline 1492 & Galeopsis pubescens Besser & Mérk & 1948 & Simon Tibor, Jakucs Pál & BPU-05510.JPG \\
\hline 1492 & Galeopsis pubescens Besser & Monoszló & 1982 & Felföldy Lajos & BPU-05511.JPG \\
\hline 1492 & Galeopsis pubescens Besser & Somogyszob & 1957 & Soó Rezső & BPU-05505.JPG \\
\hline $\begin{array}{c}1492 \times \\
1493\end{array}$ & $\begin{array}{l}\text { Galeopsis pubescens Besser } \times \\
\text { Galeopsis speciosa Mill. }\end{array}$ & Gyöngyös & 1953 & Soó Rezső & BPU-05514.JPG \\
\hline $\begin{array}{c}1492 \times \\
1493\end{array}$ & $\begin{array}{l}\text { Galeopsis pubescens Besser × } \\
\text { Galeopsis speciosa Mill. }\end{array}$ & Parád & 1935 & Soó Rezső & BPU-05513.JPG \\
\hline
\end{tabular}


1. Elektroniukus melléklet - Electronic Appendix 1.

\begin{tabular}{|c|c|c|c|c|c|}
\hline $\begin{array}{l}\text { Sorszám / } \\
\text { Number }\end{array}$ & Taxon-név / Taxon-name & $\begin{array}{l}\text { Település / } \\
\text { Settlement }\end{array}$ & $\begin{array}{l}\text { Év / } \\
\text { Year }\end{array}$ & Gyűjtő / Collector & $\begin{array}{l}\text { Fájlnév / } \\
\text { File-name }\end{array}$ \\
\hline 1493 & Galeopsis speciosa Mill. & Beregsurány & 1951 & Simon Tibor & BPU-05501.JPG \\
\hline 1493 & Galeopsis speciosa Mill. & Kaszó & 1957 & Soó Rezső & BPU-05497.JPG \\
\hline 1493 & Galeopsis speciosa Mill. & Nagybajom & 1957 & Soó Rezső & BPU-05496.JPG \\
\hline 1493 & Galeopsis speciosa Mill. & Szilvásvárad & 1953 & $\begin{array}{l}\text { Felföldy Lajos, Tóth } \\
\text { László }\end{array}$ & BPU-05503.JPG \\
\hline 1493 & Galeopsis speciosa Mill. & Szögliget & 1951 & $\begin{array}{l}\text { Soó Rezső, Jakucs Pál, Ér } \\
\text { Lajos }\end{array}$ & BPU-05499.JPG \\
\hline 1493 & Galeopsis speciosa Mill. & Uzsa & 1951 & Borsos Olga & BPU-05500.JPG \\
\hline 1493 & Galeopsis speciosa Mill. & Uzsa & 1953 & $\begin{array}{l}\text { Felföldy Lajos, Tóth } \\
\text { László }\end{array}$ & BPU-05502.JPG \\
\hline 1493 & Galeopsis speciosa Mill. & Zalakomár & 1954 & Borsos Olga & BPU-05498.JPG \\
\hline 1494 & Galeopsis tetrahit L. & Gyöngyös & 1952 & Soó Rezső & BPU-05491.JPG \\
\hline 1494 & Galeopsis tetrahit L. & Miskolc & 1937 & Soó Rezső & BPU-05492.JPG \\
\hline 1494 & Galeopsis tetrahit L. & Tornyospálca & 1933 & Soó Rezső & BPU-05490.JPG \\
\hline 1495 & Galeopsis bifida Boenn. & Ivánc & 1955 & Soó Rezső & BPU-05494.JPG \\
\hline 1496 & Lamium orvala L. & Zákány & 1948 & Károlyi Árpád & BPU-05528.JPG \\
\hline 1497 & Lamium album L. & Debrecen & 1931 & Soó Rezső & BPU-05544.JPG \\
\hline 1497 & Lamium album L. & Felsőtelekes & 2005 & Gulyás Pál & BPU-05542.JPG \\
\hline 1497 & Lamium album L. & Garadna & 1955 & Tóth László & BPU-05543.JPG \\
\hline 1497 & Lamium album L. & Tállya & 1958 & Isépy István & BPU-05545.JPG \\
\hline 1498 & Lamium maculatum L. & Budapest & 1947 & Soó Rezső & BPU-05550.JPG \\
\hline 1498 & Lamium maculatum L. & Isztimér & 1949 & Soó Rezső & BPU-05548.JPG \\
\hline 1498 & Lamium maculatum L. & Mór & 1953 & Felföldy Lajos & BPU-05554.JPG \\
\hline 1498 & Lamium maculatum L. & Pécs & 1953 & Soó Rezső, Borsos Olga & BPU-05547.JPG \\
\hline 1498 & Lamium maculatum L. & Sátoraljaújhely & 1952 & $\begin{array}{l}\text { Kulcsár Gábor, Vozáry } \\
\text { Elemér }\end{array}$ & BPU-15464.JPG \\
\hline 1498 & Lamium maculatum L. & Szentendre & 1963 & Soó Rezső & BPU-05549.JPG \\
\hline 1498 & Lamium maculatum L. & Uzsa & 1959 & $\begin{array}{l}\text { Jávorka Sándor, Felföldy } \\
\text { Lajos }\end{array}$ & BPU-05556.JPG \\
\hline 1498 & Lamium maculatum L. & Visegrád & 1964 & Soó Rezső, Borsos Olga & BPU-05546.JPG \\
\hline 1499 & Lamium amplexicaule L. & Budapest & 1982 & Felföldy Lajos & BPU-05529.JPG \\
\hline 1499 & Lamium amplexicaule L. & Tihany & 1958 & Felföldy Lajos & BPU-05531.JPG \\
\hline 1499 & Lamium amplexicaule L. & Vácrátót & 1953 & Felföldy Lajos & BPU-05530.JPG \\
\hline 1500 & Lamium purpureum L. & Biatorbágy & 1991 & Felföldy Lajos & BPU-05536.JPG \\
\hline 1500 & Lamium purpureum L. & Budapest & 1982 & Felföldy Lajos & BPU-05533.JPG \\
\hline 1500 & Lamium purpureum L. & Budapest & 2005 & Felföldy Lajos & BPU-05532.JPG \\
\hline 1500 & Lamium purpureum L. & Egyek & 1932 & Soó Rezső & BPU-05535.JPG \\
\hline 1500 & Lamium purpureum L. & Hidegkút & 1959 & Felföldy Lajos & BPU-05538.JPG \\
\hline 1500 & Lamium purpureum L. & Nagyharsány & 1965 & $\begin{array}{l}\text { Simon Tibor, Priszter } \\
\text { Szaniszló, Borhidi Attila }\end{array}$ & BPU-05534.JPG \\
\hline 1500 & Lamium purpureum L. & Tihany & 1951 & Felföldy Lajos & BPU-05539.JPG \\
\hline 1500 & Lamium purpureum L. & Tihany & 1958 & Felföldy Lajos & BPU-05540.JPG \\
\hline 1500 & Lamium purpureum L. & Tihany & 1958 & Felföldy Lajos & BPU-05541.JPG \\
\hline 1500 & Lamium purpureum L. & Üröm & 1991 & Felföldy Lajos & BPU-05537.JPG \\
\hline 1502 & Galeobdolon luteum Huds. & "Cuha-völgy" & 1954 & Soó Rezső & BPU-05519.JPG \\
\hline 1502 & Galeobdolon luteum Huds. & Bátorliget & 1934 & Soó Rezső & BPU-05520.JPG \\
\hline 1502 & Galeobdolon luteum Huds. & Budapest & 1952 & Soó Rezső & BPU-05517.JPG \\
\hline 1502 & Galeobdolon luteum Huds. & Budapest & 1964 & Soó Rezső & BPU-05518.JPG \\
\hline 1502 & Galeobdolon luteum Huds. & Dömös & 1918 & Boros Ádám & BPU-05526.JPG \\
\hline
\end{tabular}


1. Elektroniukus melléklet - Electronic Appendix 1.

\begin{tabular}{|c|c|c|c|c|c|}
\hline $\begin{array}{l}\text { Sorszám / } \\
\text { Number }\end{array}$ & Taxon-név / Taxon-name & $\begin{array}{l}\text { Település / } \\
\text { Settlement }\end{array}$ & $\begin{array}{l}\text { Év / } \\
\text { Year }\end{array}$ & Gyűjtő / Collector & $\begin{array}{l}\text { Fájlnév / } \\
\text { File-name }\end{array}$ \\
\hline 1502 & Galeobdolon luteum Huds. & Kapolcs & 1959 & $\begin{array}{l}\text { Jávorka Sándor, Felföldy } \\
\text { Lajos, Tóth László }\end{array}$ & BPU-05516.JPG \\
\hline 1502 & Galeobdolon luteum Huds. & Kőszeg & 1938 & Soó Rezső & BPU-05515.JPG \\
\hline 1502 & Galeobdolon luteum Huds. & Miskolc & 1910 & Hulják János & BPU-05527.JPG \\
\hline 1502 & Galeobdolon luteum Huds. & Mór & 1953 & Felföldy Lajos & BPU-05524.JPG \\
\hline 1502 & Galeobdolon luteum Huds. & Pécs & 1953 & Soó Rezső, Borsos Olga & BPU-05525.JPG \\
\hline 1502 & Galeobdolon luteum Huds. & Sátoraljaújhely & 1952 & $\begin{array}{l}\text { Kulcsár Gábor, Vozáry } \\
\text { Elemér }\end{array}$ & BPU-15538.JPG \\
\hline 1502 & Galeobdolon luteum Huds. & Tarpa & 1951 & Soó Rezső, Jakucs Pál & BPU-05522.JPG \\
\hline 1504 & Leonurus cardiaca L. & Bagamér & 1947 & Soó Rezső & BPU-05558.JPG \\
\hline 1505 & Leonurus marrubiastrum L. & Budakeszi & 1947 & Soó Rezső & BPU-05559.JPG \\
\hline 1505 & Leonurus marrubiastrum L. & Kerecsend & 1957 & Soó Rezső & BPU-05560.JPG \\
\hline 1506 & Ballota nigra L. & Baja & 1968 & Priszter Szaniszló & BPU-05563.JPG \\
\hline 1506 & Ballota nigra L. & Budapest & 1982 & Felföldy Lajos & BPU-05562.JPG \\
\hline 1506 & Ballota nigra L. & Budapest & 2004 & Felföldy Lajos & BPU-05561.JPG \\
\hline 1506 & Ballota nigra L. & Debrecen & 1930 & Soó Rezső & BPU-05564.JPG \\
\hline 1506 & Ballota nigra L. & Tállya & 1958 & Isépy István & BPU-05566.JPG \\
\hline 1506 & Ballota nigra L. & Tihany & 1958 & Felföldy Lajos & BPU-05565.JPG \\
\hline 1507 & Betonica officinalis L. & Budapest & 1961 & Isépy István & BPU-05567.JPG \\
\hline 1507 & Betonica officinalis L. & Debrecen & 1933 & Juhász Lajos & BPU-05569.JPG \\
\hline 1507 & Betonica officinalis L. & Eger & 1948 & Koppányi Tibor & BPU-05570.JPG \\
\hline 1507 & Betonica officinalis L. & Gyöngyös & 1961 & Soó Rezső & BPU-05571.JPG \\
\hline 1507 & Betonica officinalis L. & Szilvásvárad & 1953 & $\begin{array}{l}\text { Felföldy Lajos, Tóth } \\
\text { László }\end{array}$ & BPU-05568.JPG \\
\hline 1508 & Stachys annua L. & Budapest & 2004 & Felföldy Lajos & BPU-05572.JPG \\
\hline 1508 & Stachys annua L. & Tihany & 1947 & Pólya László & BPU-05573.JPG \\
\hline 1508 & Stachys annua L. & Vácrátót & 1952 & Felföldy Lajos & BPU-05574.JPG \\
\hline 1509 & Stachys recta L. & Budapest & 1982 & Felföldy Lajos & BPU-05575.JPG \\
\hline 1509 & Stachys recta L. & Budapest & 1989 & Felföldy Lajos & BPU-05576.JPG \\
\hline 1509 & Stachys recta L. & Göd & 1948 & Soó Rezső & BPU-05581.JPG \\
\hline 1509 & Stachys recta L. & Gyöngyös & 1923 & Soó Rezső & BPU-05580.JPG \\
\hline 1509 & Stachys recta $\mathrm{L}$. & Sáránd & 1934 & Soó Rezső & BPU-05579.JPG \\
\hline 1509 & Stachys recta L. & Szigetmonostor & 1947 & Soó Rezső & BPU-05582.JPG \\
\hline 1509 & Stachys recta L. & Szögliget & 1920 & Jakucs Pál, Kulcsár Gábor & BPU-05577.JPG \\
\hline 1509 & Stachys recta L. & Zámoly & 1982 & Felföldy Lajos & BPU-05578.JPG \\
\hline 1509 & Stachys recta L. & Zebegény & 1966 & Priszter Szaniszló & BPU-05583.JPG \\
\hline 1510 & Stachys sylvatica L. & Aszófő & 1957 & Felföldy Lajos & BPU-05589.JPG \\
\hline 1510 & Stachys sylvatica L. & Bisse & 1965 & $\begin{array}{l}\text { Simon Tibor, Priszter } \\
\text { Szaniszló, Borhidi Attila }\end{array}$ & BPU-05587.JPG \\
\hline 1510 & Stachys sylvatica L. & Budapest & 1982 & Felföldy Lajos & BPU-05586.JPG \\
\hline 1510 & Stachys sylvatica L. & Budapest & 2004 & Felföldy Lajos & BPU-05585.JPG \\
\hline 1510 & Stachys sylvatica L. & Nagyvisnyó & 1953 & Soó Rezső & BPU-05588.JPG \\
\hline 1510 & Stachys sylvatica L. & Tarcal & 1947 & Soó Rezső & BPU-05590.JPG \\
\hline 1511 & Stachys palustris L. & Bagamér & 1947 & Soó Rezső & BPU-05593.JPG \\
\hline 1511 & Stachys palustris L. & Balatonszemes & 1974 & Tóth László & BPU-05595.JPG \\
\hline 1511 & Stachys palustris L. & Balatonszepezd & 1958 & Felföldy Lajos & BPU-05596.JPG \\
\hline 1511 & Stachys palustris L. & Balatonszepezd & 1958 & Felföldy Lajos & BPU-05597.JPG \\
\hline 1511 & Stachys palustris L. & Debrecen & 1989 & Felföldy Lajos & BPU-05592.JPG \\
\hline 1511 & Stachys palustris L. & Gárdony & 1980 & Felföldy Lajos & BPU-05591.JPG \\
\hline
\end{tabular}


1. Elektroniukus melléklet - Electronic Appendix 1.

\begin{tabular}{|c|c|c|c|c|c|}
\hline $\begin{array}{l}\text { Sorszám / } \\
\text { Number }\end{array}$ & Taxon-név / Taxon-name & $\begin{array}{l}\text { Település / } \\
\text { Settlement }\end{array}$ & $\begin{array}{l}\text { Év / } \\
\text { Year }\end{array}$ & Gyüjtő / Collector & $\begin{array}{l}\text { Fájlnév / } \\
\text { File-name }\end{array}$ \\
\hline 1511 & Stachys palustris L. & Keszthely & 1958 & Tóth László & BPU-05599.JPG \\
\hline 1511 & Stachys palustris L. & Szigetmonostor & 1964 & Isépy István & BPU-05594.JPG \\
\hline 1511 & Stachys palustris L. & Tihany & 1947 & Pólya László & BPU-05598.JPG \\
\hline 1512 & Stachys alpina L. & Karancslapujtő & 1964 & Isépy István & BPU-05601.JPG \\
\hline 1512 & Stachys alpina L. & Pécs & 1925 & Soó Rezső & BPU-05600.JPG \\
\hline 1513 & Stachys germanica L. & Aszófő & 1931 & Soó Rezső & BPU-05610.JPG \\
\hline 1513 & Stachys germanica L. & Aszófő & 1931 & Soó Rezső & BPU-05611.JPG \\
\hline 1513 & Stachys germanica L. & Csaroda & 1951 & $\begin{array}{l}\text { Simon Tibor, Vozáry } \\
\text { Elemér }\end{array}$ & BPU-05607.JPG \\
\hline 1513 & Stachys germanica L. & Hajdúhadház & 1933 & Soó Rezső & BPU-05609.JPG \\
\hline 1513 & Stachys germanica L. & Tihany & 1959 & Felföldy Lajos & BPU-05612.JPG \\
\hline 1513 & Stachys germanica L. & Zámoly & 1982 & Felföldy Lajos & BPU-05608.JPG \\
\hline 1514 & Stachys byzantina K. Koch & Gyöngyös & 1961 & Soó Rezső & BPU-05613.JPG \\
\hline 1516 & Nepeta pannonica L. & Budapest & 2005 & Felföldy Lajos & BPU-05365.JPG \\
\hline 1516 & Nepeta pannonica L. & Debrecen & 1951 & Kulcsár Gábor & BPU-05366.JPG \\
\hline 1516 & Nepeta pannonica L. & Egyek & 1950 & $\begin{array}{l}\text { Simon Tibor, Borsos Olga, } \\
\text { Kulcsár Gábor }\end{array}$ & BPU-05367.JPG \\
\hline 1516 & Nepeta pannonica L. & Nyíracsád & 1949 & Soó Rezső & BPU-05368.JPG \\
\hline 1516 & Nepeta pannonica L. & Tokaj & 1947 & Soó Rezső & BPU-05369.JPG \\
\hline 1517 & Nepeta cataria L. & Aszófő & 1957 & Felföldy Lajos & BPU-05371.JPG \\
\hline 1517 & Nepeta cataria L. & Hajdúhadház & 1933 & Soó Rezső & BPU-05370.JPG \\
\hline 1517 & Nepeta cataria L. & Tihany & 1949 & Simon Tibor & BPU-05372.JPG \\
\hline 1517 & Nepeta cataria L. & Tihany & 1959 & Felföldy Lajos & BPU-05373.JPG \\
\hline 1518 & Glechoma hederacea L. & Baktalórántháza & 1934 & Máthé Imre & BPU-05388.JPG \\
\hline 1518 & Glechoma hederacea L. & Bélapátfalva & 1948 & $\begin{array}{l}\text { Simon Tibor, Pólya } \\
\text { László, Jakucs Pál }\end{array}$ & BPU-05387.JPG \\
\hline 1518 & Glechoma hederacea L. & Budapest & 1990 & Felföldy Lajos & BPU-05378.JPG \\
\hline 1518 & Glechoma hederacea L. & Budapest & 1991 & Felföldy Lajos & BPU-05375.JPG \\
\hline 1518 & Glechoma hederacea L. & Budapest & 1991 & Felföldy Lajos & BPU-05376.JPG \\
\hline 1518 & Glechoma hederacea L. & Budapest & 1991 & Felföldy Lajos & BPU-05377.JPG \\
\hline 1518 & Glechoma hederacea L. & Debrecen & 1931 & Soó Rezső & BPU-05383.JPG \\
\hline 1518 & Glechoma hederacea L. & Debrecen & 1934 & Soó Rezső & BPU-05386.JPG \\
\hline 1518 & Glechoma hederacea L. & Dömös & 1963 & Isépy István & BPU-05380.JPG \\
\hline 1518 & Glechoma hederacea L. & Gyöngyös & 1964 & Soó Rezső & BPU-05400.JPG \\
\hline 1518 & Glechoma hederacea L. & Hidegkút & 1958 & Felföldy Lajos & BPU-05390.JPG \\
\hline 1518 & Glechoma hederacea L. & Ófehértó & 1934 & Máthé Imre & BPU-05389.JPG \\
\hline 1518 & Glechoma hederacea L. & Sáránd & 1932 & Soó Rezső & BPU-05399.JPG \\
\hline 1518 & Glechoma hederacea L. & Tass & 1982 & Felföldy Lajos & BPU-05379.JPG \\
\hline 1518 & Glechoma hederacea L. & Tihany & 1959 & Felföldy Lajos & BPU-05391.JPG \\
\hline 1518 & Glechoma hederacea L. & Visegrád & 1964 & Soó Rezső, Borsos Olga & BPU-05401.JPG \\
\hline 1518 & Glechoma hederacea L. & Zámoly & 1982 & Felföldy Lajos & BPU-05381.JPG \\
\hline 1518 & Glechoma hederacea L. & Zámoly & 1982 & Felföldy Lajos & BPU-05382.JPG \\
\hline 1519 & Glechoma hirsuta Waldst. et Kit. & Balatonfüred & 1959 & Felföldy Lajos & BPU-05402.JPG \\
\hline 1519 & Glechoma hirsuta Waldst. et Kit. & Balatonszőlős & 1959 & Felföldy Lajos & BPU-05403.JPG \\
\hline 1519 & Glechoma hirsuta Waldst. et Kit. & Budapest & 1992 & Felföldy Lajos & BPU-05396.JPG \\
\hline 1519 & Glechoma hirsuta Waldst. et Kit. & Debrecen & 1943 & Felföldy Lajos & BPU-05384.JPG \\
\hline 1519 & Glechoma hirsuta Waldst. et Kit. & Debrecen & 1943 & Felföldy Lajos & BPU-05385.JPG \\
\hline 1519 & Glechoma hirsuta Waldst. et Kit. & Hajdúbagos & 1933 & Soó Rezső & BPU-05398.JPG \\
\hline 1519 & Glechoma hirsuta Waldst. et Kit. & Háromhuta & 1961 & Simon Tibor & BPU-05407.JPG \\
\hline
\end{tabular}


1. Elektroniukus melléklet - Electronic Appendix 1.

\begin{tabular}{|c|c|c|c|c|c|}
\hline $\begin{array}{l}\text { Sorszám / } \\
\text { Number }\end{array}$ & Taxon-név / Taxon-name & $\begin{array}{l}\text { Település / } \\
\text { Settlement }\end{array}$ & $\begin{array}{l}\text { Év / } \\
\text { Year }\end{array}$ & Gyűjtő / Collector & $\begin{array}{l}\text { Fájlnév / } \\
\text { File-name }\end{array}$ \\
\hline 1519 & Glechoma hirsuta Waldst. et Kit. & Pécsely & 1959 & Felföldy Lajos & BPU-05404.JPG \\
\hline 1519 & Glechoma hirsuta Waldst. et Kit. & Sima & 1961 & Simon Tibor & BPU-05397.JPG \\
\hline 1519 & Glechoma hirsuta Waldst. et Kit. & Tihany & 1956 & Felföldy Lajos & BPU-05406.JPG \\
\hline 1519 & Glechoma hirsuta Waldst. et Kit. & Tihany & 1958 & Felföldy Lajos & BPU-05392.JPG \\
\hline 1519 & Glechoma hirsuta Waldst. et Kit. & Tihany & 1958 & Felföldy Lajos & BPU-05393.JPG \\
\hline 1519 & Glechoma hirsuta Waldst. et Kit. & Tihany & 1959 & Felföldy Lajos & BPU-05405.JPG \\
\hline 1520 & Dracocephalum ruyschiana L. & Miskolc & 1940 & Hulják János & BPU-05413.JPG \\
\hline 1520 & Dracocephalum ruyschiana L. & Nagyvisnyó & 1953 & Soó Rezső & BPU-05412.JPG \\
\hline 1521 & Dracocephalum austriacum L. & Jósvafő & 1952 & Jakucs Pál, Ér Lajos & BPU-05411.JPG \\
\hline 1521 & Dracocephalum austriacum L. & Jósvafő & 1955 & Jakucs Pál & BPU-05409.JPG \\
\hline 1522 & Prunella laciniata L. & Balatonakali & 1958 & Felföldy Lajos & BPU-05431.JPG \\
\hline 1522 & Prunella laciniata L. & Beregdaróc & 1951 & $\begin{array}{l}\text { Simon Tibor, Vozáry } \\
\text { Elemér }\end{array}$ & BPU-05435.JPG \\
\hline 1522 & Prunella laciniata L. & Budapest & 1982 & Felföldy Lajos & BPU-05424.JPG \\
\hline 1522 & Prunella laciniata L. & Debrecen & 1934 & Juhász Lajos, Soó Rezső & BPU-05426.JPG \\
\hline 1522 & Prunella laciniata L. & Diósjenő & 1952 & Simon Tibor & BPU-05430.JPG \\
\hline 1522 & Prunella laciniata L. & Gyöngyös & 1961 & Soó Rezső & BPU-05425.JPG \\
\hline 1522 & Prunella laciniata L. & Kelemér & 1950 & Soó Rezső & BPU-05428.JPG \\
\hline 1522 & Prunella laciniata L. & Nagyvisnyó & 1953 & Soó Rezső & BPU-05427.JPG \\
\hline 1523 & Prunella vulgaris L. & Bisse & 1965 & $\begin{array}{l}\text { Simon Tibor, Priszter } \\
\text { Szaniszló, Borhidi Attila }\end{array}$ & BPU-05420.JPG \\
\hline 1523 & Prunella vulgaris L. & Budapest & 1961 & Soó Rezső & BPU-05417.JPG \\
\hline 1523 & Prunella vulgaris L. & Budapest & 1982 & Felföldy Lajos & BPU-05419.JPG \\
\hline 1523 & Prunella vulgaris L. & Budapest & 2005 & Felföldy Lajos & BPU-05418.JPG \\
\hline 1523 & Prunella vulgaris L. & Hévíz & 1961 & Felföldy Lajos & BPU-05422.JPG \\
\hline 1523 & Prunella vulgaris L. & Nyíregyháza & 1933 & Soó Rezső & BPU-05421.JPG \\
\hline 1524 & Prunella grandiflora (L.) Scholler & Hollóháza & 2007 & Simon Tibor & BPU-15505.JPG \\
\hline 1524 & Prunella grandiflora (L.) Scholler & Mátraszentimre & 1963 & Isépy István & BPU-05415.JPG \\
\hline 1524 & Prunella grandiflora (L.) Scholler & Miskolc & 1934 & Soó Rezső & BPU-05414.JPG \\
\hline 1524 & Prunella grandiflora (L.) Scholler & Pálháza & 1949 & Simon Tibor, Jakucs Pál & BPU-05439.JPG \\
\hline 1524 & Prunella grandiflora (L.) Scholler & Tokaj & 1947 & Soó Rezső & BPU-05416.JPG \\
\hline $\begin{array}{c}1522 \\
\times 1523\end{array}$ & Prunella $\times$ intermedia Link & Balatonakali & 1958 & Felföldy Lajos & BPU-05437.JPG \\
\hline $\begin{array}{c}1522 \\
\times 1523\end{array}$ & Prunella $\times$ intermedia Link & Balatonszőlős & 1957 & Felföldy Lajos & BPU-05436.JPG \\
\hline $\begin{array}{c}1522 \\
\times 1523\end{array}$ & Prunella $\times$ intermedia Link & Jósvafó & 1951 & $\begin{array}{l}\text { Soó Rezső, Jakucs Pál, Ér } \\
\text { Lajos }\end{array}$ & BPU-05433.JPG \\
\hline $\begin{array}{c}1522 \\
\times 1523\end{array}$ & Prunella $\times$ intermedia Link & Kám & 1929 & Polgár Sándor & BPU-05438.JPG \\
\hline $\begin{array}{c}1522 \\
\times 1523\end{array}$ & Prunella $\times$ intermedia Link & Pomáz & 1945 & Vajda László & BPU-05434.JPG \\
\hline $\begin{array}{c}1524 \\
\times 1522\end{array}$ & Prunella $\times$ dissecta Wender. & Budapest & 1944 & Papp József & BPU-05432.JPG \\
\hline 1525 & Melissa officinalis L. & Budakeszi & 1946 & Kárpáti Zoltán & BPU-05653.JPG \\
\hline 1526 & Satureja hortensis L. & Győr & 1915 & Polgár Sándor & BPU-05683.JPG \\
\hline 1527 & Acinos arvensis (Lam.) Dandy & Bélapátfalva & 1948 & $\begin{array}{l}\text { Simon Tibor, Pólya } \\
\text { László, Jakucs Pál }\end{array}$ & BPU-05661.JPG \\
\hline 1527 & Acinos arvensis (Lam.) Dandy & Budaörs & 1982 & Felföldy Lajos & BPU-05662.JPG \\
\hline 1527 & Acinos arvensis (Lam.) Dandy & Pécs & 1953 & Soó Rezső, Borsos Olga & BPU-05660.JPG \\
\hline 1527 & Acinos arvensis (Lam.) Dandy & Szigetmonostor & 1959 & Soó Rezső & BPU-05663.JPG \\
\hline 1527 & Acinos arvensis (Lam.) Dandy & Uzsa & 1959 & $\begin{array}{l}\text { Jávorka Sándor, Felföldy } \\
\text { Lajos }\end{array}$ & BPU-05664.JPG \\
\hline
\end{tabular}


1. Elektroniukus melléklet - Electronic Appendix 1.

\begin{tabular}{|c|c|c|c|c|c|}
\hline $\begin{array}{l}\text { Sorszám / } \\
\text { Number }\end{array}$ & Taxon-név / Taxon-name & $\begin{array}{l}\text { Település / } \\
\text { Settlement }\end{array}$ & $\begin{array}{l}\text { Év / } \\
\text { Year }\end{array}$ & Gyújtő / Collector & $\begin{array}{l}\text { Fájlnév / } \\
\text { File-name }\end{array}$ \\
\hline 1528 & Calamintha menthifolia Host & Aszófő & 1957 & Felföldy Lajos & BPU-05677.JPG \\
\hline 1528 & Calamintha menthifolia Host & Badacsonytördemic & 1953 & $\begin{array}{l}\text { Felföldy Lajos, Tóth } \\
\text { László }\end{array}$ & BPU-05678.JPG \\
\hline 1528 & Calamintha menthifolia Host & Balatonszőlős & 1957 & Felföldy Lajos & BPU-05679.JPG \\
\hline 1528 & Calamintha menthifolia Host & Diósjenő & 1952 & Simon Tibor & BPU-05675.JPG \\
\hline 1528 & Calamintha menthifolia Host & Dörgicse & 1957 & Felföldy Lajos & BPU-05680.JPG \\
\hline 1528 & Calamintha menthifolia Host & Gyöngyös & 1952 & Soó Rezső & BPU-05674.JPG \\
\hline 1528 & Calamintha menthifolia Host & Harka & 1948 & Kárpáti Zoltán & BPU-05676.JPG \\
\hline 1528 & Calamintha menthifolia Host & Jósvafő & 1951 & Soó Rezső & BPU-05673.JPG \\
\hline 1530 & Clinopodium vulgare L. & Budapest & 1982 & Felföldy Lajos & BPU-05656.JPG \\
\hline 1530 & Clinopodium vulgare L. & Budapest & 1982 & Felföldy Lajos & BPU-05659.JPG \\
\hline 1530 & Clinopodium vulgare L. & Budapest & 2005 & Felföldy Lajos & BPU-05655.JPG \\
\hline 1530 & Clinopodium vulgare L. & Magyarlukafa & 1957 & Soó Rezső, Borhidi Attila & BPU-05657.JPG \\
\hline 1530 & Clinopodium vulgare $\mathrm{L}$. & Miskolc & 1936 & Soó Rezső & BPU-05658.JPG \\
\hline 1531 & Micromeria thymifolia (Scop.) Fritsch & Bélapátfalva & 1930 & Soó Rezső & BPU-05682.JPG \\
\hline 1532 & Hyssopus officinalis L. & Budapest & 1915 & Degen Árpád & BPU-05684.JPG \\
\hline 1532 & Hyssopus officinalis L. & Tihany & 1961 & $\begin{array}{l}\text { Pankov H., Felföldy Lajos, } \\
\text { Sólymosy G. }\end{array}$ & BPU-05685.JPG \\
\hline 1534 & Origanum vulgare $\mathrm{L}$. & Bódvarákó & 1951 & Jakucs Pál, Ér Lajos & BPU-05686.JPG \\
\hline 1534 & Origanum vulgare $\mathrm{L}$. & Gyöngyös & 1953 & Soó Rezső & BPU-05688.JPG \\
\hline 1534 & Origanum vulgare $\mathrm{L}$. & Kőszeg & 1932 & Soó Rezső & BPU-05690.JPG \\
\hline 1534 & Origanum vulgare $\mathrm{L}$. & Nagykovácsi & 1947 & Soó Rezső & BPU-05689.JPG \\
\hline 1534 & Origanum vulgare $\mathrm{L}$. & Uzsa & 1953 & $\begin{array}{l}\text { Felföldy Lajos, Tóth } \\
\text { László }\end{array}$ & BPU-05691.JPG \\
\hline 1536 & Thymus pulegioides L. & Beregdaróc & 1951 & Simon Tibor, Jakucs Pál & BPU-05698.JPG \\
\hline 1536 & Thymus pulegioides L. & Csákvár & 1955 & Soó Rezső & BPU-05702.JPG \\
\hline 1536 & Thymus pulegioides L. & Hajdúnánás & 1935 & Ujvárosi Miklós & BPU-05730.JPG \\
\hline 1536 & Thymus pulegioides L. & Nagyharsány & 1956 & Soó Rezső & BPU-05695.JPG \\
\hline 1536 & Thymus pulegioides L. & Szakonyfalu & 1955 & Soó Rezső & BPU-05694.JPG \\
\hline 1536 & Thymus pulegioides L. & Szakonyfalu & 1955 & Soó Rezső & BPU-05701.JPG \\
\hline 1536 & Thymus pulegioides L. & Szilvásvárad & 1953 & $\begin{array}{l}\text { Felföldy Lajos, Tóth } \\
\text { László }\end{array}$ & BPU-05699.JPG \\
\hline 1537 & Thymus pannonicus All. & Bakonybél & 1955 & Soó Rezső & BPU-05718.JPG \\
\hline 1537 & Thymus pannonicus All. & Balatonszőlős & 1957 & Felföldy Lajos & BPU-05719.JPG \\
\hline 1537 & Thymus pannonicus All. & Ercsi & 1933 & Ötvös László & BPU-05714.JPG \\
\hline 1537 & Thymus pannonicus All. & Hajdúbagos & 1933 & Soó Rezső & BPU-05751.JPG \\
\hline 1537 & Thymus pannonicus All. & Hajdúnánás & 1928 & Igmándy József & BPU-05713.JPG \\
\hline 1537 & Thymus pannonicus All. & Háromhuta & 1943 & Igmándy József & BPU-05715.JPG \\
\hline 1537 & Thymus pannonicus All. & Jászkarajenő & 1928 & Lengyel Géza & BPU-05722.JPG \\
\hline 1537 & Thymus pannonicus All. & Nagykovácsi & 1932 & Soó Rezső & BPU-05712.JPG \\
\hline 1537 & Thymus pannonicus All. & Pilisszentiván & 1917 & Degen Árpád & BPU-05724.JPG \\
\hline 1537 & Thymus pannonicus All. & Szentendre & 1918 & Boros Ádám & BPU-05723.JPG \\
\hline 1537 & Thymus pannonicus All. & Szigetszentmiklós & 1926 & Degen Árpád & BPU-05725.JPG \\
\hline 1537 & Thymus pannonicus All. & Tiszavasvári & 1949 & Igmándy József & BPU-05717.JPG \\
\hline 1537 & Thymus pannonicus All. & Tiszavasvári & 1949 & Igmándy József & BPU-05720.JPG \\
\hline 1537 & Thymus pannonicus All. & Tiszavasvári & 1949 & Igmándy József & BPU-05721.JPG \\
\hline 1537 & Thymus pannonicus All. & Tokaj & 1933 & Igmándy József & BPU-05716.JPG \\
\hline 1538 & Thymus glabrescens Willd. & Baktalórántháza & 1932 & Soó Rezső & BPU-05741.JPG \\
\hline 1538 & Thymus glabrescens Willd. & Bátorliget & 1934 & Soó Rezső & BPU-05711.JPG \\
\hline
\end{tabular}


1. Elektroniukus melléklet - Electronic Appendix 1.

\begin{tabular}{|c|c|c|c|c|c|}
\hline $\begin{array}{l}\text { Sorszám / } \\
\text { Number }\end{array}$ & Taxon-név / Taxon-name & $\begin{array}{l}\text { Település / } \\
\text { Settlement }\end{array}$ & $\begin{array}{l}\text { Év / } \\
\text { Year }\end{array}$ & Gyújtő / Collector & $\begin{array}{l}\text { Fájlnév / } \\
\text { File-name }\end{array}$ \\
\hline 1538 & Thymus glabrescens Willd. & Bátorliget & 1934 & Soó Rezső & BPU-05735.JPG \\
\hline 1538 & Thymus glabrescens Willd. & Budapest & 1920 & Degen Árpád & BPU-05736.JPG \\
\hline 1538 & Thymus glabrescens Willd. & Debrecen & 1931 & Soó Rezső & BPU-05738.JPG \\
\hline 1538 & Thymus glabrescens Willd. & Debrecen & 1931 & Soó Rezső & BPU-05740.JPG \\
\hline 1538 & Thymus glabrescens Willd. & Kaposmérő & 1923 & Jávorka Sándor & BPU-05732.JPG \\
\hline 1538 & Thymus glabrescens Willd. & Nagyharsány & 1965 & $\begin{array}{l}\text { Simon Tibor, Priszter } \\
\text { Szaniszló }\end{array}$ & BPU-05734.JPG \\
\hline 1538 & Thymus glabrescens Willd. & Somoskőújfalu & 1936 & Soó Rezső & BPU-05737.JPG \\
\hline 1538 & Thymus glabrescens Willd. & Tihany & 1959 & Felföldy Lajos & BPU-05731.JPG \\
\hline 1539 & Thymus serpyllum L. & Fenyőfő & 1929 & Soó Rezső & BPU-05761.JPG \\
\hline 1539 & Thymus serpyllum L. & Kaszó & 1957 & Soó Rezső & BPU-05757.JPG \\
\hline 1539 & Thymus serpyllum L. & Kecskemét & 1918 & Boros Ádám & BPU-05739.JPG \\
\hline 1539 & Thymus serpyllum L. & Leányvár & 1920 & Boros Ádám & BPU-05760.JPG \\
\hline 1539 & Thymus serpyllum L. & Leányvár & 1920 & Trautmann Róbert & BPU-05758.JPG \\
\hline 1539 & Thymus serpyllum L. & Tihany & 1959 & Felföldy Lajos & BPU-05755.JPG \\
\hline 1539 & Thymus serpyllum L. & Tihany & 1959 & Felföldy Lajos & BPU-05756.JPG \\
\hline 1539 & Thymus serpyllum L. & Uzsa & 1953 & $\begin{array}{l}\text { Felföldy Lajos, Tóth } \\
\text { László }\end{array}$ & BPU-05700.JPG \\
\hline 1540 & Thymus praecox Opiz & Bélapátfalva & 1948 & $\begin{array}{l}\text { Simon Tibor, Pólya } \\
\text { László, Jakucs Pál }\end{array}$ & BPU-05710.JPG \\
\hline 1540 & Thymus praecox Opiz & Isztimér & 1955 & Soó Rezső & BPU-05705.JPG \\
\hline 1540 & Thymus praecox Opiz & Nagyharsány & 1965 & $\begin{array}{l}\text { Simon Tibor, Priszter } \\
\text { Szaniszló, Borhidi Attila }\end{array}$ & BPU-05707.JPG \\
\hline 1540 & Thymus praecox Opiz & Nagykovácsi & 1963 & Isépy István & BPU-05704.JPG \\
\hline 1540 & Thymus praecox Opiz & Pilisszentiván & 1917 & Degen Árpád & BPU-05708.JPG \\
\hline $\begin{array}{c}1535- \\
1540\end{array}$ & Thymus & Bakonyszentlászló & 1963 & Soó Rezső & BPU-05759.JPG \\
\hline 1541 & Lycopus europaeus L. & Nyírpilis & 1932 & Soó Rezső & BPU-05764.JPG \\
\hline 1541 & Lycopus europaeus L. & Pákozd & 1980 & Felföldy Lajos & BPU-05762.JPG \\
\hline 1541 & Lycopus europaeus L. & Tiszafüred & 1963 & Isépy István & BPU-05765.JPG \\
\hline 1541 & Lycopus europaeus L. & Vácrátót & 1949 & Soó Rezső & BPU-05763.JPG \\
\hline 1542 & Lycopus exaltatus L. f. & Lakitelek & 1961 & Simon Tibor & BPU-05768.JPG \\
\hline 1542 & Lycopus exaltatus L. f. & Szarvas & 1947 & Soó Rezső & BPU-05769.JPG \\
\hline $\begin{array}{c}1541 \times \\
1542\end{array}$ & $\begin{array}{l}\text { Lycopus europaeus L. } \times \text { Lycopus } \\
\text { exaltatus L. f. }\end{array}$ & Vásárosnamény & 1933 & Soó Rezső & BPU-05767.JPG \\
\hline 1543 & Mentha pulegium L. & Baja & 1968 & Priszter Szaniszló & BPU-05770.JPG \\
\hline 1543 & Mentha pulegium L. & Kunmadaras & 1991 & Csányi Béla & BPU-05774.JPG \\
\hline 1543 & Mentha pulegium L. & Szarvas & 1947 & Soó Rezső & BPU-05771.JPG \\
\hline 1543 & Mentha pulegium L. & Szenta & 1955 & Soó Rezső & BPU-05773.JPG \\
\hline 1543 & Mentha pulegium L. & Vácrátót & 1953 & Felföldy Lajos & BPU-05772.JPG \\
\hline 1544 & Mentha longifolia (L.) Nath. & Budapest & 1990 & Felföldy Lajos & BPU-05775.JPG \\
\hline 1544 & Mentha longifolia (L.) Nath. & Debrecen & 1938 & Soó Rezső & BPU-05782.JPG \\
\hline 1544 & Mentha longifolia (L.) Nath. & Miskolc & 1937 & Soó Rezső & BPU-05780.JPG \\
\hline 1544 & Mentha longifolia (L.) Nath. & Szenna & 1957 & Soó Rezső & BPU-05781.JPG \\
\hline 1544 & Mentha longifolia (L.) Nath. & Szögliget & 1951 & $\begin{array}{l}\text { Soó Rezső, Jakucs Pál, Ér } \\
\text { Lajos }\end{array}$ & BPU-05779.JPG \\
\hline 1544 & Mentha longifolia (L.) Nath. & Tihany & 1929 & Soó Rezső & BPU-05777.JPG \\
\hline 1544 & Mentha longifolia (L.) Nath. & Tihany & 1959 & Felföldy Lajos & BPU-05776.JPG \\
\hline 1546 & Mentha aquatica L. & Budapest & 1989 & Felföldy Lajos & BPU-05785.JPG \\
\hline 1546 & Mentha aquatica L. & Budapest & 1989 & Felföldy Lajos & BPU-05786.JPG \\
\hline
\end{tabular}


1. Elektroniukus melléklet - Electronic Appendix 1.

\begin{tabular}{|c|c|c|c|c|c|}
\hline $\begin{array}{l}\text { Sorszám / } \\
\text { Number }\end{array}$ & Taxon-név / Taxon-name & $\begin{array}{l}\text { Település / } \\
\text { Settlement }\end{array}$ & $\begin{array}{l}\text { Év / } \\
\text { Year }\end{array}$ & Gyújtő / Collector & $\begin{array}{l}\text { Fájlnév / } \\
\text { File-name }\end{array}$ \\
\hline 1546 & Mentha aquatica L. & Dávod & 1957 & Soó Rezső & BPU-05792.JPG \\
\hline 1546 & Mentha aquatica L. & Fertőhomok & 1960 & Tóth László & BPU-05793.JPG \\
\hline 1546 & Mentha aquatica L. & Gárdony & 1980 & Felföldy Lajos & BPU-05787.JPG \\
\hline 1546 & Mentha aquatica L. & Hortobágy & 1947 & Soó Rezső & BPU-05789.JPG \\
\hline 1546 & Mentha aquatica L. & Miskolc & 1937 & Soó Rezső & BPU-05794.JPG \\
\hline 1546 & Mentha aquatica L. & Nyírmihálydi & 1934 & Máthé Imre & BPU-05783.JPG \\
\hline 1546 & Mentha aquatica L. & Pákozd & 1980 & Felföldy Lajos & BPU-05788.JPG \\
\hline 1546 & Mentha aquatica $\mathrm{L}$. & Szenta & 1955 & Soó Rezső & BPU-05791.JPG \\
\hline 1546 & Mentha aquatica L. & Vácrátót & 1949 & Soó Rezső & BPU-05790.JPG \\
\hline 1547 & Mentha $\times$ dumetorum Schult. & Tihany & 1946 & Felföldy Lajos & BPU-05784.JPG \\
\hline 1549 & Mentha arvensis L. & Hajdúnánás & 1935 & Ujvárosi Miklós & BPU-05798.JPG \\
\hline 1549 & Mentha arvensis L. & Miskolc & 1937 & Soó Rezső & BPU-05800.JPG \\
\hline 1549 & Mentha arvensis L. & Szarvas & 1947 & Soó Rezső & BPU-05801.JPG \\
\hline 1549 & Mentha arvensis L. & Szenta & 1955 & Soó Rezső & BPU-05796.JPG \\
\hline 1551 & Mentha $\times$ verticillata $L$ & Piricse & 1932 & Soó Rezső & BPU-05795.JPG \\
\hline $\begin{array}{c}1543- \\
1553\end{array}$ & Mentha & Erdőbénye & 1948 & Soó Rezső & BPU-05797.JPG \\
\hline $\begin{array}{c}1543- \\
1553\end{array}$ & Mentha & Tarpa & 1948 & Simon Tibor, Jakucs Pál & BPU-05799.JPG \\
\hline 1556 & Salvia verticillata $\mathrm{L}$. & Balatongyörök & 1961 & Felföldy Lajos & BPU-05621.JPG \\
\hline 1556 & Salvia verticillata $\mathrm{L}$. & Budapest & 2005 & Felföldy Lajos & BPU-05618.JPG \\
\hline 1556 & Salvia verticillata $\mathrm{L}$. & Rudabánya & 1936 & Soó Rezső & BPU-05619.JPG \\
\hline 1556 & Salvia verticillata $\mathrm{L}$. & Tihany & 1959 & Felföldy Lajos & BPU-05620.JPG \\
\hline 1557 & Salvia glutinosa L. & Dávod & 1957 & Soó Rezső & BPU-05616.JPG \\
\hline 1557 & Salvia glutinosa L. & Dömös & 1948 & Soó Rezső & BPU-05614.JPG \\
\hline 1557 & Salvia glutinosa L. & Miskolc & 1952 & Borsos Olga & BPU-05617.JPG \\
\hline 1557 & Salvia glutinosa L. & Pusztavacs & 1950 & Soó Rezső & BPU-05615.JPG \\
\hline 1559 & Salvia aethiopis L. & Balatonszőlős & 1957 & Entz Béla & BPU-05626.JPG \\
\hline 1559 & Salvia aethiopis L. & Budaörs & 1947 & Soó Rezső & BPU-05623.JPG \\
\hline 1559 & Salvia aethiopis L. & Fót & 1960 & Soó Rezső & BPU-05622.JPG \\
\hline 1559 & Salvia aethiopis L. & Hajdúszoboszló & 1937 & Soó Rezső & BPU-05624.JPG \\
\hline 1559 & Salvia aethiopis L. & Hajdúszoboszló & 1937 & Soó Rezső & BPU-05625.JPG \\
\hline 1560 & Salvia austriaca Jacq. & Balmazújváros & 1937 & Soó Rezső & BPU-05628.JPG \\
\hline 1560 & Salvia austriaca Jacq. & Budapest & 2004 & Felföldy Lajos & BPU-05627.JPG \\
\hline 1560 & Salvia austriaca Jacq. & Lábatlan & 1953 & Felföldy Lajos & BPU-05629.JPG \\
\hline 1562 & Salvia nemorosa L. & Budapest & 1991 & Felföldy Lajos & BPU-05632.JPG \\
\hline 1562 & Salvia nemorosa L. & Hortobágy & 1947 & Soó Rezső & BPU-05633.JPG \\
\hline 1562 & Salvia nemorosa L. & Hortobágy & 1947 & Soó Rezső & BPU-05634.JPG \\
\hline 1562 & Salvia nemorosa L. & Nagyharsány & 1965 & $\begin{array}{l}\text { Simon Tibor, Priszter } \\
\text { Szaniszló }\end{array}$ & BPU-05635.JPG \\
\hline 1562 & Salvia nemorosa L. & Tihany & 1959 & Felföldy Lajos & BPU-05631.JPG \\
\hline 1563 & Salvia pratensis L. & Baktalórántháza & 1934 & Máthé Imre & BPU-05644.JPG \\
\hline 1563 & Salvia pratensis L. & Budapest & 1949 & Simon Tibor & BPU-05643.JPG \\
\hline 1563 & Salvia pratensis L. & Budapest & 1969 & Priszter Szaniszló & BPU-05637.JPG \\
\hline 1563 & Salvia pratensis L. & Budapest & 1969 & Priszter Szaniszló & BPU-05638.JPG \\
\hline 1563 & Salvia pratensis L. & Budapest & 2004 & Felföldy Lajos & BPU-05636.JPG \\
\hline 1563 & Salvia pratensis L. & Debrecen & 1932 & Soó Rezső & BPU-05641.JPG \\
\hline 1563 & Salvia pratensis L. & Hajdúbagos & 1937 & Soó Rezső & BPU-05640.JPG \\
\hline 1563 & Salvia pratensis L. & Nagyharsány & 1969 & Priszter Szaniszló & BPU-05639.JPG \\
\hline
\end{tabular}


1. Elektroniukus melléklet - Electronic Appendix 1.

\begin{tabular}{|c|c|c|c|c|c|}
\hline $\begin{array}{l}\text { Sorszám / } \\
\text { Number }\end{array}$ & Taxon-név / Taxon-name & $\begin{array}{l}\text { Település / } \\
\text { Settlement }\end{array}$ & $\begin{array}{l}\text { Év / } \\
\text { Year }\end{array}$ & Gyűjtő / Collector & $\begin{array}{l}\text { Fájlnév / } \\
\text { File-name }\end{array}$ \\
\hline 1563 & Salvia pratensis L. & Szilvásvárad & 1953 & Soó Rezső & BPU-05642.JPG \\
\hline 1563 & Salvia pratensis L. & Tihany & 1959 & Felföldy Lajos & BPU-05645.JPG \\
\hline 1563 & Salvia pratensis L. & Tihany & 1961 & Felföldy Lajos & BPU-05646.JPG \\
\hline $\begin{array}{c}1562 \times \\
1563\end{array}$ & Salvia $\times$ sylvestris L. & Budapest & 1945 & Vajda László & BPU-05649.JPG \\
\hline 1566 & Nicandra physalodes (L.) Gaertn. & Debrecen & 1932 & Máthé Imre & BPU-05804.JPG \\
\hline 1566 & Nicandra physalodes (L.) Gaertn. & Keszthely & 1958 & Kiss Magdolna & BPU-05805.JPG \\
\hline 1567 & Lycium barbarum L. & Budapest & 1948 & Soó Rezső & BPU-05806.JPG \\
\hline 1567 & Lycium barbarum L. & Budapest & 1948 & Soó Rezső & BPU-05807.JPG \\
\hline 1567 & Lycium barbarum L. & Fót & 1963 & Isépy István & BPU-05808.JPG \\
\hline 1568 & Lycium chinense Mill. & Budapest & 1948 & Soó Rezső & BPU-05809.JPG \\
\hline 1568 & Lycium chinense Mill. & Csákvár & 1948 & Soó Rezső & BPU-05810.JPG \\
\hline 1569 & Atropa belladonna L. & Isztimér & 1949 & Soó Rezső & BPU-05812.JPG \\
\hline 1569 & Atropa belladonna L. & Miskolc & 1939 & Soó Rezső & BPU-05811.JPG \\
\hline 1571 & Hyoscyamus niger L. & Budapest & 2005 & Felföldy Lajos & BPU-05815.JPG \\
\hline 1571 & Hyoscyamus niger L. & Debrecen & 1937 & Felföldy Lajos & BPU-05816.JPG \\
\hline 1572 & Physalis alkekengi L. & Budapest & 1982 & Felföldy Lajos & BPU-05817.JPG \\
\hline 1572 & Physalis alkekengi L. & Jánd & 1952 & $\begin{array}{l}\text { Simon Tibor, Vozáry } \\
\text { Elemér }\end{array}$ & BPU-05818.JPG \\
\hline 1572 & Physalis alkekengi L. & Mosonmagyaróvár & 1962 & Borsos Olga & BPU-05820.JPG \\
\hline 1572 & Physalis alkekengi L. & Szigetmonostor & 1947 & Soó Rezső & BPU-05821.JPG \\
\hline 1572 & Physalis alkekengi L. & Tihany & 1959 & Felföldy Lajos & BPU-05823.JPG \\
\hline 1572 & Physalis alkekengi L. & Vác & 1949 & $\begin{array}{l}\text { Soó Rezső, Simon Tibor, } \\
\text { Jakucs Pál }\end{array}$ & BPU-05822.JPG \\
\hline 1572 & Physalis alkekengi L. & Zámoly & 1982 & Felföldy Lajos & BPU-05819.JPG \\
\hline 1576 & Solanum tuberosum L. & Tállya & 1958 & Isépy István & BPU-05842.JPG \\
\hline 1577 & Solanum dulcamara L. & Budaörs & 1967 & $\begin{array}{l}\text { Priszter Szaniszló, } \\
\text { Borhidi attila }\end{array}$ & BPU-05824.JPG \\
\hline 1577 & Solanum dulcamara L. & Dabas & 1965 & Simon Tibor & BPU-05827.JPG \\
\hline 1577 & Solanum dulcamara L. & Pusztavacs & 1963 & Isépy István & BPU-05826.JPG \\
\hline 1577 & Solanum dulcamara L. & Tihany & 1959 & Felföldy Lajos & BPU-05828.JPG \\
\hline 1579 & Solanum nigrum L. & Debrecen & 1942 & Felföldy Lajos & BPU-05836.JPG \\
\hline 1579 & Solanum nigrum L. & Győr & 1926 & Polgár Sándor & BPU-05840.JPG \\
\hline 1579 & Solanum nigrum L. & Győr & 1927 & Polgár Sándor & BPU-05838.JPG \\
\hline 1579 & Solanum nigrum L. & Győr & 1927 & Polgár Sándor & BPU-05839.JPG \\
\hline 1579 & Solanum nigrum L. & Győr & 1929 & Polgár Sándor & BPU-05837.JPG \\
\hline 1579 & Solanum nigrum L. & Nagyharsány & 1965 & $\begin{array}{l}\text { Simon Tibor, Priszter } \\
\text { Szaniszló, Borhidi Attila }\end{array}$ & BPU-05833.JPG \\
\hline 1579 & Solanum nigrum L. & Szalonna & 1926 & Boros Ádám & BPU-05848.JPG \\
\hline 1579 & Solanum nigrum L. & Vácrátót & 1953 & Felföldy Lajos & BPU-05834.JPG \\
\hline 1579 & Solanum nigrum L. & Vácrátót & 1953 & Felföldy Lajos & BPU-05835.JPG \\
\hline 1579 & Solanum nigrum L. & Vácrátót & 1953 & Felföldy Lajos & BPU-05841.JPG \\
\hline 1580 & Solanum alatum Moench & Győr & 1926 & Polgár Sándor & BPU-05830.JPG \\
\hline 1580 & Solanum alatum Moench & Szögliget & 1937 & Boros Ádám & BPU-05832.JPG \\
\hline 1581 & Solanum villosum Mill. & Budapest & 1946 & Boros Ádám & BPU-05829.JPG \\
\hline 1581 & Solanum villosum Mill. & Bugac & 1954 & Dobos L. & BPU-05831.JPG \\
\hline---- & Solanum adventitium Polgár & Győr & 1915 & Polgár Sándor & BPU-05843.JPG \\
\hline---- & Solanum allanii Polgar & Győr & 1938 & Polgár Sándor & BPU-05844.JPG \\
\hline---- & Solanum humile Bernh. ex Willd. & Budapest & 1947 & Priszter Szaniszló & BPU-05849.JPG \\
\hline---- & Solanum probstianum Polgár & Győr & 1939 & Polgár Sándor & BPU-05845.JPG \\
\hline
\end{tabular}


1. Elektroniukus melléklet - Electronic Appendix 1.

\begin{tabular}{|c|c|c|c|c|c|}
\hline $\begin{array}{l}\text { Sorszám / } \\
\text { Number }\end{array}$ & Taxon-név / Taxon-name & $\begin{array}{l}\text { Település / } \\
\text { Settlement }\end{array}$ & $\begin{array}{l}\text { Év / } \\
\text { Year }\end{array}$ & Gyújtő / Collector & $\begin{array}{l}\text { Fájlnév / } \\
\text { File-name }\end{array}$ \\
\hline---- & Solanum sarrachoides Sendth. & Győr & 1926 & Polgár Sándor & BPU-05846.JPG \\
\hline---- & Solanum sisymbriifolium Lam. & Győr & 1931 & Polgár Sándor & BPU-05847.JPG \\
\hline--- & Solanum triflorum Nutt. & Győr & 1918 & Polgár Sándor & BPU-05850.JPG \\
\hline 1583 & Datura stramonium L. & Budapest & 1982 & Felföldy Lajos & BPU-05851.JPG \\
\hline 1590 & Gratiola officinalis L. & Bodajk & 1942 & Felföldy Lajos & BPU-05969.JPG \\
\hline 1590 & Gratiola officinalis L. & Budapest & 1915 & Filarszky Nándor & BPU-05971.JPG \\
\hline 1590 & Gratiola officinalis L. & Debrecen & 1931 & Soó Rezső & BPU-05968.JPG \\
\hline 1590 & Gratiola officinalis L. & Gyöngyös & 1935 & Soó Rezső & BPU-05970.JPG \\
\hline 1590 & Gratiola officinalis L. & Szentbékkálla & 1957 & Felföldy Lajos & BPU-05972.JPG \\
\hline 1591 & $\begin{array}{l}\text { Lindernia procumbens (Krock.) } \\
\text { Philcox }\end{array}$ & Csaroda & 1951 & Simon Tibor & BPU-05985.JPG \\
\hline 1591 & $\begin{array}{l}\text { Lindernia procumbens (Krock.) } \\
\text { Philcox }\end{array}$ & Nagykanizsa & 1956 & Felföldy Lajos & BPU-05984.JPG \\
\hline 1591 & $\begin{array}{l}\text { Lindernia procumbens (Krock.) } \\
\text { Philcox }\end{array}$ & Szarvas & 1947 & Soó Rezső & BPU-05984.JPG \\
\hline 1591 & $\begin{array}{l}\text { Lindernia procumbens (Krock.) } \\
\text { Philcox }\end{array}$ & Vásárosnamény & 1933 & Soó Rezső & BPU-05983.JPG \\
\hline 1593 & Limosella aquatica L. & Csehbánya & 1955 & Tallós Pál & BPU-05979.JPG \\
\hline 1593 & Limosella aquatica L. & Hortobágy & 1947 & Soó Rezső & BPU-05982.JPG \\
\hline 1593 & Limosella aquatica L. & Szolnok & 1948 & Timár Lajos & BPU-05981.JPG \\
\hline 1593 & Limosella aquatica L. & Tivadar & 1948 & Simon Tibor, Jakucs Pál & BPU-05980.JPG \\
\hline 1593 & Limosella aquatica L. & Vásárosnamény & 1933 & Soó Rezső & BPU-05978.JPG \\
\hline 1595 & Verbascum phoeniceum L. & Miskolc & 1948 & $\begin{array}{l}\text { Simon Tibor, Borsos Olga, } \\
\text { Jakucs Pál }\end{array}$ & BPU-05854.JPG \\
\hline 1595 & Verbascum phoeniceum L. & Pécs & 1953 & Soó Rezső, Borsos Olga & BPU-05852.JPG \\
\hline 1595 & Verbascum phoeniceum L. & Pécsely & 1955 & Entz Béla & BPU-05855.JPG \\
\hline 1595 & Verbascum phoeniceum L. & Zebegény & 1963 & Isépy István & BPU-05853.JPG \\
\hline 1596 & Verbascum blattaria L. & Hortobágy & 1947 & Soó Rezső & BPU-05856.JPG \\
\hline 1596 & Verbascum blattaria L. & Tihany & 1947 & Pólya László & BPU-05857.JPG \\
\hline 1597 & Verbascum chaixii Vill. & Budapest & 1982 & Felföldy Lajos & BPU-05880.JPG \\
\hline 1597 & Verbascum chaixii Vill. & Budapest & 2004 & Felföldy Lajos & BPU-05879.JPG \\
\hline 1597 & Verbascum chaixii Vill. & Debrecen & 1934 & Máthé Imre & BPU-05883.JPG \\
\hline 1597 & Verbascum chaixii Vill. & Diósjenő & 1952 & Simon Tibor & BPU-05882.JPG \\
\hline 1597 & Verbascum chaixii Vill. & Nagykovácsi & 1947 & Soó Rezső & BPU-05881.JPG \\
\hline 1597 & Verbascum chaixii Vill. & Putnok & 1950 & Soó Rezső & BPU-05884.JPG \\
\hline 1598 & Verbascum nigrum L. & Budapest & 1947 & Soó Rezső & BPU-05885.JPG \\
\hline 1598 & Verbascum nigrum L. & Debrecen & 1933 & Soó Rezső & BPU-05887.JPG \\
\hline 1598 & Verbascum nigrum L. & Debrecen & 1989 & Felföldy Lajos & BPU-05888.JPG \\
\hline 1598 & Verbascum nigrum L. & Miskolc & 1950 & Soó Rezső & BPU-05886.JPG \\
\hline 1598 & Verbascum nigrum L. & Visegrád & 1947 & Vajda László & BPU-05889.JPG \\
\hline 1599 & Verbascum lychnitis L. & Bélapátfalva & 1950 & $\begin{array}{l}\text { Simon Tibor, Jakucs Pál, } \\
\text { Kárpáti István }\end{array}$ & BPU-05868.JPG \\
\hline 1599 & Verbascum lychnitis L. & Bugac & 1964 & Soó Rezső & BPU-05871.JPG \\
\hline 1599 & Verbascum lychnitis L. & Hosszúpályi & 1933 & Soó Rezső & BPU-05869.JPG \\
\hline 1599 & Verbascum lychnitis L. & Nagyharsány & 1965 & Priszter Szaniszló & BPU-05870.JPG \\
\hline 1599 & Verbascum lychnitis L. & Nyíracsád & 1949 & Soó Rezső & BPU-05872.JPG \\
\hline 1599 & Verbascum lychnitis L. & Pilisszentkereszt & 1948 & Papp József & BPU-05867.JPG \\
\hline 1599 & Verbascum lychnitis L. & Vámospércs & 1934 & Máthé Imre & BPU-05865.JPG \\
\hline 1599 & Verbascum lychnitis L. & Vámospércs & 1934 & Máthé Imre & BPU-05866.JPG \\
\hline 1600 & Verbascum pulverulentum Vill. & Zákány & 1948 & Károlyi Árpád & BPU-05878.JPG \\
\hline
\end{tabular}


1. Elektroniukus melléklet - Electronic Appendix 1.

\begin{tabular}{|c|c|c|c|c|c|}
\hline $\begin{array}{l}\text { Sorszám / } \\
\text { Number }\end{array}$ & Taxon-név / Taxon-name & $\begin{array}{l}\text { Település / } \\
\text { Settlement }\end{array}$ & $\begin{array}{l}\text { Év / } \\
\text { Year }\end{array}$ & Gyüjtő / Collector & $\begin{array}{l}\text { Fájlnév / } \\
\text { File-name }\end{array}$ \\
\hline 1601 & Verbascum speciosum Schrad. & Debrecen & 1934 & Soó Rezső & BPU-05877.JPG \\
\hline 1601 & Verbascum speciosum Schrad. & Pécs & 1938 & Soó Rezső & BPU-05875.JPG \\
\hline 1601 & Verbascum speciosum Schrad. & Pécs & 1938 & Soó Rezső & BPU-05876.JPG \\
\hline 1601 & Verbascum speciosum Schrad. & Pomáz & 1921 & Soó Rezső & BPU-05873.JPG \\
\hline 1601 & Verbascum speciosum Schrad. & Tihany & 1928 & Soó Rezső & BPU-05874.JPG \\
\hline 1602 & Verbascum thapsus L. & Mátraszentimre & 1935 & Soó Rezső & BPU-05860.JPG \\
\hline 1602 & Verbascum thapsus L. & Mátraszentimre & 1961 & Soó Rezső & BPU-05858.JPG \\
\hline 1602 & Verbascum thapsus L. & Miskolc & 1950 & Soó Rezső & BPU-05859.JPG \\
\hline 1602 & Verbascum thapsus L. & Pusztavacs & 1950 & Soó Rezső & BPU-05862.JPG \\
\hline 1603 & Verbascum densiflorum Bertol. & Debrecen & 1933 & Soó Rezső & BPU-05861.JPG \\
\hline 1604 & Verbascum phlomoides L. & Hosszúpályi & 1933 & Soó Rezső & BPU-05864.JPG \\
\hline 1604 & Verbascum phlomoides L. & Tarpa & 1948 & Simon Tibor, Jakucs Pál & BPU-05863.JPG \\
\hline $\begin{array}{c}1596 \times \\
1604\end{array}$ & $\begin{array}{l}\text { Verbascum blattaria L. } \times \text { Verbascum } \\
\text { phlomoides L. }\end{array}$ & Győr & 1896 & Polgár Sándor & BPU-05896.JPG \\
\hline $\begin{array}{c}1596 \times \\
1604\end{array}$ & $\begin{array}{l}\text { Verbascum blattaria L. } \times \text { Verbascum } \\
\text { phlomoides L. }\end{array}$ & Hortobágy & 1947 & Soó Rezső & BPU-05891.JPG \\
\hline $\begin{array}{c}1599 \times \\
1604\end{array}$ & $\begin{array}{l}\text { Verbascum lychnitis L. } \times \text { Verbascum } \\
\text { phlomoides L. }\end{array}$ & Győr & 1912 & Polgár Sándor & BPU-05895.JPG \\
\hline $\begin{array}{c}1599 \times \\
1604\end{array}$ & $\begin{array}{l}\text { Verbascum lychnitis L. } \times \text { Verbascum } \\
\text { phlomoides L. }\end{array}$ & Uppony & 1934 & Boros Ádám & BPU-05892.JPG \\
\hline $\begin{array}{c}1601 \times \\
1604\end{array}$ & $\begin{array}{l}\text { Verbascum speciosum Schrad. } \times \\
\text { Verbascum phlomoides L. }\end{array}$ & Győr & 1936 & Polgár Sándor & BPU-05894.JPG \\
\hline $\begin{array}{c}1601 \times \\
1604\end{array}$ & $\begin{array}{l}\text { Verbascum speciosum Schrad. } \times \\
\text { Verbascum phlomoides L. }\end{array}$ & Szentendre & 1948 & Vajda László & BPU-05893.JPG \\
\hline---- & Verbascum virgatum Stokes & Győr & 1924 & Polgár Sándor & BPU-05890.JPG \\
\hline 1605 & Scrophularia vernalis L. & "Pilis" & 1932 & Soó Rezső & BPU-05954.JPG \\
\hline 1605 & Scrophularia vernalis L. & Miskolc & 1937 & Soó Rezső & BPU-05952.JPG \\
\hline 1605 & Scrophularia vernalis L. & Tibolddaróc & 1953 & Soó Rezső & BPU-05953.JPG \\
\hline 1606 & Scrophularia umbrosa Dumort. & Balatonszepezd & 1958 & Felföldy Lajos & BPU-05966.JPG \\
\hline 1606 & Scrophularia umbrosa Dumort. & Bátorliget & 1934 & Soó Rezső & BPU-05965.JPG \\
\hline 1606 & Scrophularia umbrosa Dumort. & Isaszeg & 1949 & Soó Rezső & BPU-05964.JPG \\
\hline 1606 & Scrophularia umbrosa Dumort. & Jósvafő & 1951 & Soó Rezső, Jakucs Pál & BPU-05961.JPG \\
\hline 1606 & Scrophularia umbrosa Dumort. & Putnok & 1950 & Soó Rezső & BPU-05963.JPG \\
\hline 1606 & Scrophularia umbrosa Dumort. & Vászoly & 1958 & Felföldy Lajos & BPU-05967.JPG \\
\hline 1606 & Scrophularia umbrosa Dumort. & Zámoly & 1982 & Felföldy Lajos & BPU-05962.JPG \\
\hline 1607 & Scrophularia scopolii Hoppe & Pécs & 1956 & Soó Rezső & BPU-05955.JPG \\
\hline 1608 & Scrophularia nodosa L. & Bátorliget & 1948 & $\begin{array}{l}\text { Simon Tibor, Jakucs Pál, } \\
\text { Böszörményi Zoltán }\end{array}$ & BPU-05959.JPG \\
\hline 1608 & Scrophularia nodosa L. & Budapest & 1947 & Soó Rezső & BPU-05958.JPG \\
\hline 1608 & Scrophularia nodosa L. & Budapest & 2004 & Felföldy Lajos & BPU-05957.JPG \\
\hline 1608 & Scrophularia nodosa L. & Kőszeg & 1932 & Soó Rezső & BPU-05960.JPG \\
\hline 1611 & Misopates orontium (L.) Raf. & Kőszeg & 1932 & Soó Rezső & BPU-05944.JPG \\
\hline 1611 & Misopates orontium (L.) Raf. & Nagyharsány & 1965 & $\begin{array}{l}\text { Simon Tibor, Priszter } \\
\text { Szaniszló, Borhidi Attila }\end{array}$ & BPU-05943.JPG \\
\hline 1611 & Misopates orontium (L.) Raf. & Szilvásvárad & 1953 & Soó Rezső & BPU-05942.JPG \\
\hline 1611 & Misopates orontium (L.) Raf. & Tihany & 1959 & Felföldy Lajos & BPU-05946.JPG \\
\hline 1612 & Microrrhinum minus (L.) Fourr. & Budapest & 1921 & Soó Rezső & BPU-05951.JPG \\
\hline 1612 & Microrrhinum minus (L.) Fourr. & Miskolc & 1929 & Soó Rezső & BPU-05950.JPG \\
\hline 1612 & Microrrhinum minus (L.) Fourr. & Pécs & 1938 & Soó Rezső & BPU-05947.JPG \\
\hline 1612 & Microrrhinum minus (L.) Fourr. & Révfülöp & 1956 & $\begin{array}{l}\text { Felföldy Lajos, Tóth } \\
\text { László }\end{array}$ & BPU-05948.JPG \\
\hline
\end{tabular}


1. Elektroniukus melléklet - Electronic Appendix 1.

\begin{tabular}{|c|c|c|c|c|c|}
\hline $\begin{array}{l}\text { Sorszám / } \\
\text { Number }\end{array}$ & Taxon-név / Taxon-name & $\begin{array}{l}\text { Település / } \\
\text { Settlement }\end{array}$ & $\begin{array}{l}\text { Év / } \\
\text { Year }\end{array}$ & Gyújtő / Collector & $\begin{array}{l}\text { Fájlnév / } \\
\text { File-name }\end{array}$ \\
\hline 1612 & Microrrhinum minus (L.) Fourr. & Vácrátót & 1952 & Felföldy Lajos & BPU-05949.JPG \\
\hline 1614 & Linaria genistifolia (L.) Mill. & Abaújszántó & 1948 & Soó Rezső & BPU-05910.JPG \\
\hline 1614 & Linaria genistifolia (L.) Mill. & Bakonyszentlászló & 1963 & Soó Rezső & BPU-05920.JPG \\
\hline 1614 & Linaria genistifolia (L.) Mill. & Budapest & 1982 & Felföldy Lajos & BPU-05907.JPG \\
\hline 1614 & Linaria genistifolia (L.) Mill. & Budapest & 1982 & Felföldy Lajos & BPU-05908.JPG \\
\hline 1614 & Linaria genistifolia (L.) Mill. & Budapest & 1982 & Felföldy Lajos & BPU-05909.JPG \\
\hline 1614 & Linaria genistifolia (L.) Mill. & Budapest & 2004 & Felföldy Lajos & BPU-05905.JPG \\
\hline 1614 & Linaria genistifolia (L.) Mill. & Budapest & 2004 & Felföldy Lajos & BPU-05906.JPG \\
\hline 1614 & Linaria genistifolia (L.) Mill. & Bugac & 1923 & Soó Rezső & BPU-05913.JPG \\
\hline 1614 & Linaria genistifolia (L.) Mill. & Bugac & 1964 & Soó Rezső & BPU-05911.JPG \\
\hline 1614 & Linaria genistifolia (L.) Mill. & Bugac & 1964 & Soó Rezső & BPU-05912.JPG \\
\hline 1614 & Linaria genistifolia (L.) Mill. & Gánt & 1948 & Soó Rezső & BPU-05915.JPG \\
\hline 1614 & Linaria genistifolia (L.) Mill. & Gyöngyös & 1961 & Soó Rezső & BPU-05916.JPG \\
\hline 1614 & Linaria genistifolia (L.) Mill. & Nagyharsány & 1961 & Simon Tibor & BPU-05914.JPG \\
\hline 1614 & Linaria genistifolia (L.) Mill. & Pilisszentiván & 1953 & Soó Rezső & BPU-05917.JPG \\
\hline 1614 & Linaria genistifolia (L.) Mill. & Tihany & 1959 & Felföldy Lajos & BPU-05921.JPG \\
\hline 1614 & Linaria genistifolia (L.) Mill. & Tihany & 1959 & Felföldy Lajos & BPU-05922.JPG \\
\hline 1614 & Linaria genistifolia (L.) Mill. & Tihany & 1959 & Felföldy Lajos & BPU-05923.JPG \\
\hline 1614 & Linaria genistifolia (L.) Mill. & Tihany & 1959 & Felföldy Lajos & BPU-05924.JPG \\
\hline 1614 & Linaria genistifolia (L.) Mill. & Uzsa & 1952 & Simon Tibor & BPU-05925.JPG \\
\hline 1614 & Linaria genistifolia (L.) Mill. & Vácrátót & 1953 & Felföldy Lajos & BPU-05918.JPG \\
\hline 1614 & Linaria genistifolia (L.) Mill. & Visegrád & 1949 & Soó Rezső & BPU-05919.JPG \\
\hline 1615 & Linaria angustissima (Loisel.) Borbás & Budapest & 1991 & Felföldy Lajos & BPU-05927.JPG \\
\hline 1615 & Linaria angustissima (Loisel.) Borbás & Miskolc & 1934 & Soó Rezső & BPU-05931.JPG \\
\hline 1615 & Linaria angustissima (Loisel.) Borbás & Nagybajom & 1957 & Soó Rezső & BPU-05929.JPG \\
\hline 1615 & Linaria angustissima (Loisel.) Borbás & Szarvas & 1949 & Soó Rezső & BPU-05932.JPG \\
\hline 1615 & Linaria angustissima (Loisel.) Borbás & Szilvásvárad & 1953 & Soó Rezső & BPU-05926.JPG \\
\hline 1616 & Linaria vulgaris Mill. & Budapest & 1982 & Felföldy Lajos & BPU-05935.JPG \\
\hline 1616 & Linaria vulgaris Mill. & Debrecen & 1948 & Simon Tibor & BPU-05934.JPG \\
\hline 1616 & Linaria vulgaris Mill. & Hortobágy & 1947 & Soó Rezső & BPU-05933.JPG \\
\hline 1616 & Linaria vulgaris Mill. & Mór & 1946 & Felföldy Lajos & BPU-05936.JPG \\
\hline 1616 & Linaria vulgaris Mill. & Szilvásvárad & 1953 & $\begin{array}{l}\text { Felföldy Lajos, Tóth } \\
\text { László }\end{array}$ & BPU-05938.JPG \\
\hline 1616 & Linaria vulgaris Mill. & Uzsa & 1953 & $\begin{array}{l}\text { Felföldy Lajos, Tóth } \\
\text { László }\end{array}$ & BPU-05937.JPG \\
\hline 1617 & Linaria biebersteinii Besser & Bélapátfalva & 1948 & $\begin{array}{l}\text { Simon Tibor, Pólya } \\
\text { László, Jakucs Pál }\end{array}$ & BPU-05928.JPG \\
\hline 1618 & $\begin{array}{l}\text { Cymbalaria muralis G. Gaertn., B. Mey. } \\
\text { et Scherb. }\end{array}$ & Budapest & 1947 & Soó Rezső & BPU-05898.JPG \\
\hline 1618 & $\begin{array}{l}\text { Cymbalaria muralis G. Gaertn., B. Mey. } \\
\text { et Scherb. }\end{array}$ & Miskolc & 1963 & Isépy István & BPU-05899.JPG \\
\hline 1619 & Kickxia spuria (L.) Dumort. & Jósvafő & 1951 & Soó Rezső, Jakucs Pál & BPU-05900.JPG \\
\hline 1619 & Kickxia spuria (L.) Dumort. & Keszthely & 1958 & Csizmazia István & BPU-05901.JPG \\
\hline 1620 & Kickxia elatine (L.) Dumort. & Debrecen & 1931 & Soó Rezső & BPU-05902.JPG \\
\hline 1620 & Kickxia elatine (L.) Dumort. & Kőszeg & 1932 & Soó Rezső & BPU-05903.JPG \\
\hline 1620 & Kickxia elatine (L.) Dumort. & Kőszeg & 1932 & Soó Rezső & BPU-05904.JPG \\
\hline 1621 & Digitalis purpurea L. & Nagykanizsa & 1949 & Károlyi Árpád & BPU-06275.JPG \\
\hline 1622 & Digitalis grandiflora Mill. & Dömös & 1952 & Soó Rezső & BPU-06268.JPG \\
\hline 1622 & Digitalis grandiflora Mill. & Isztimér & 1949 & Soó Rezső & BPU-06269.JPG \\
\hline 1622 & Digitalis grandiflora Mill. & Mátraszentimre & 1963 & Isépy István & BPU-06270.JPG \\
\hline
\end{tabular}


1. Elektroniukus melléklet - Electronic Appendix 1.

\begin{tabular}{|c|c|c|c|c|c|}
\hline $\begin{array}{l}\text { Sorszám / } \\
\text { Number }\end{array}$ & Taxon-név / Taxon-name & $\begin{array}{l}\text { Település / } \\
\text { Settlement }\end{array}$ & $\begin{array}{l}\text { Év / } \\
\text { Year }\end{array}$ & Gyújtő / Collector & $\begin{array}{l}\text { Fájlnév / } \\
\text { File-name }\end{array}$ \\
\hline 1622 & Digitalis grandiflora Mill. & Nyíracsád & 1947 & Soó Rezső & BPU-06271.JPG \\
\hline 1622 & Digitalis grandiflora Mill. & Szilvásvárad & 1953 & $\begin{array}{l}\text { Felföldy Lajos, Tóth } \\
\text { László }\end{array}$ & BPU-06267.JPG \\
\hline 1623 & Digitalis lanata Ehrh. & Budapest & 1947 & Soó Rezső & BPU-06272.JPG \\
\hline 1624 & Digitalis ferruginea L. & Nagyharsány & 1965 & $\begin{array}{l}\text { Simon Tibor, Priszter } \\
\text { Szaniszló }\end{array}$ & BPU-06274.JPG \\
\hline 1625 & $\begin{array}{l}\text { Pseudolysimachion longifolium (L.) } \\
\text { Opiz }\end{array}$ & Bátorliget & 1922 & Boros Ádám & BPU-06102.JPG \\
\hline 1625 & $\begin{array}{l}\text { Pseudolysimachion longifolium (L.) } \\
\text { Opiz }\end{array}$ & Bátorliget & 1932 & Soó Rezső & BPU-06104.JPG \\
\hline 1625 & $\begin{array}{l}\text { Pseudolysimachion longifolium (L.) } \\
\text { Opiz }\end{array}$ & Dabas & 1928 & Boros Ádám & BPU-06096.JPG \\
\hline 1625 & $\begin{array}{l}\text { Pseudolysimachion longifolium (L.) } \\
\text { Opiz }\end{array}$ & Debrecen & 1932 & Soó Rezső & BPU-06105.JPG \\
\hline 1625 & $\begin{array}{l}\text { Pseudolysimachion longifolium (L.) } \\
\text { Opiz }\end{array}$ & Debrecen & 1952 & Kulcsár Gábor & BPU-06101.JPG \\
\hline 1625 & $\begin{array}{l}\text { Pseudolysimachion longifolium (L.) } \\
\text { Opiz }\end{array}$ & Debrecen & 1952 & Kulcsár Gábor & BPU-15440.JPG \\
\hline 1625 & $\begin{array}{l}\text { Pseudolysimachion longifolium (L.) } \\
\text { Opiz }\end{array}$ & Kiskőrös & 1920 & Boros Ádám & BPU-06097.JPG \\
\hline 1625 & $\begin{array}{l}\text { Pseudolysimachion longifolium (L.) } \\
\text { Opiz }\end{array}$ & Kőszeg & 1932 & Soó Rezső & BPU-06100.JPG \\
\hline 1625 & $\begin{array}{l}\text { Pseudolysimachion longifolium (L.) } \\
\text { Opiz }\end{array}$ & Kunpeszér & 1920 & Boros Ádám & BPU-06099.JPG \\
\hline 1625 & $\begin{array}{l}\text { Pseudolysimachion longifolium (L.) } \\
\text { Opiz }\end{array}$ & Szigetújfalu & 1949 & Boros Ádám & BPU-06103.JPG \\
\hline 1626 & $\begin{array}{l}\text { Pseudolysimachion spurium (L.) } \\
\text { Rauschert }\end{array}$ & Bélapátfalva & 1953 & Simon Tibor & BPU-06110.JPG \\
\hline 1626 & $\begin{array}{l}\text { Pseudolysimachion spurium (L.) } \\
\text { Rauschert }\end{array}$ & Fót & 1960 & Soó Rezső & BPU-06107.JPG \\
\hline 1626 & $\begin{array}{l}\text { Pseudolysimachion spurium (L.) } \\
\text { Rauschert }\end{array}$ & Fót & 1960 & Soó Rezső & BPU-06108.JPG \\
\hline 1626 & $\begin{array}{l}\text { Pseudolysimachion spurium (L.) } \\
\text { Rauschert }\end{array}$ & Gyöngyös & 1961 & Soó Rezső & BPU-06109.JPG \\
\hline 1626 & $\begin{array}{l}\text { Pseudolysimachion spurium (L.) } \\
\text { Rauschert }\end{array}$ & Hajdúböszörmény & 1932 & Soó Rezső & BPU-06106.JPG \\
\hline 1627 & $\begin{array}{l}\text { Pseudolysimachion incanum (L.) } \\
\text { Holub }\end{array}$ & Bagamér & 1932 & Soó Rezső & BPU-06111.JPG \\
\hline 1627 & $\begin{array}{l}\text { Pseudolysimachion incanum (L.) } \\
\text { Holub }\end{array}$ & Bátorliget & 1951 & Simon Tibor & BPU-06112.JPG \\
\hline 1628 & Pseudolysimachion spicatum (L.) Opiz & Bátorliget & 1932 & Soó Rezső & BPU-06126.JPG \\
\hline 1628 & Pseudolysimachion spicatum (L.) Opiz & Bélapátfalva & 1953 & Simon Tibor & BPU-06119.JPG \\
\hline 1628 & Pseudolysimachion spicatum (L.) Opiz & Budapest & 1982 & Felföldy Lajos & BPU-06114.JPG \\
\hline 1628 & Pseudolysimachion spicatum (L.) Opiz & Budapest & 2004 & Felföldy Lajos & BPU-06113.JPG \\
\hline 1628 & Pseudolysimachion spicatum (L.) Opiz & Csomád & 1953 & Felföldy Lajos & BPU-06120.JPG \\
\hline 1628 & Pseudolysimachion spicatum (L.) Opiz & Debrecen & 1951 & Soó Rezső, Simon Tibor & BPU-06118.JPG \\
\hline 1628 & Pseudolysimachion spicatum (L.) Opiz & Hosszúpályi & 1933 & Soó Rezső & BPU-06116.JPG \\
\hline 1628 & Pseudolysimachion spicatum (L.) Opiz & Nagybajom & 1957 & Soó Rezső & BPU-06125.JPG \\
\hline 1628 & Pseudolysimachion spicatum (L.) Opiz & Nagyharsány & 1966 & Priszter Szaniszló & BPU-06115.JPG \\
\hline 1628 & Pseudolysimachion spicatum (L.) Opiz & Piliscsaba & 1916 & Filarszky Nándor & BPU-06121.JPG \\
\hline 1628 & Pseudolysimachion spicatum (L.) Opiz & Pilisszentiván & 1969 & Priszter Szaniszló & BPU-06122.JPG \\
\hline 1628 & Pseudolysimachion spicatum (L.) Opiz & Pusztavacs & 1950 & Soó Rezső & BPU-06123.JPG \\
\hline 1628 & Pseudolysimachion spicatum (L.) Opiz & Szilvásvárad & 1949 & Koppányi Tibor & BPU-06117.JPG \\
\hline 1628 & Pseudolysimachion spicatum (L.) Opiz & Tállya & 1958 & Isépy István & BPU-06129.JPG \\
\hline 1628 & Pseudolysimachion spicatum (L.) Opiz & Uzsa & 1952 & Simon Tibor & BPU-06127.JPG \\
\hline
\end{tabular}


1. Elektroniukus melléklet - Electronic Appendix 1.

\begin{tabular}{|c|c|c|c|c|c|}
\hline $\begin{array}{l}\text { Sorszám / } \\
\text { Number }\end{array}$ & Taxon-név / Taxon-name & $\begin{array}{l}\text { Település / } \\
\text { Settlement }\end{array}$ & $\begin{array}{l}\text { Év / } \\
\text { Year }\end{array}$ & Gyújtő / Collector & $\begin{array}{l}\text { Fájlnév / } \\
\text { File-name }\end{array}$ \\
\hline 1628 & Pseudolysimachion spicatum (L.) Opiz & Uzsa & 1953 & $\begin{array}{l}\text { Felföldy Lajos, Tóth } \\
\text { László }\end{array}$ & BPU-06128.JPG \\
\hline 1628 & Pseudolysimachion spicatum (L.) Opiz & Vácrátót & 1952 & Felföldy Lajos & BPU-06124.JPG \\
\hline 1629 & $\begin{array}{l}\text { Pseudolysimachion orchideum } \\
\text { (Crantz) Wraber }\end{array}$ & Beregdaróc & 1951 & $\begin{array}{l}\text { Simon Tibor, Vozáry } \\
\text { Elemér }\end{array}$ & BPU-06137.JPG \\
\hline 1629 & $\begin{array}{l}\text { Pseudolysimachion orchideum } \\
\text { (Crantz) Wraber }\end{array}$ & Dörgicse & 1957 & Felföldy Lajos & BPU-06140.JPG \\
\hline 1629 & $\begin{array}{l}\text { Pseudolysimachion orchideum } \\
\text { (Crantz) Wraber }\end{array}$ & Egyek & 1947 & Soó Rezső & BPU-06132.JPG \\
\hline 1629 & $\begin{array}{l}\text { Pseudolysimachion orchideum } \\
\text { (Crantz) Wraber }\end{array}$ & Kerecsend & 1957 & Soó Rezső & BPU-06133.JPG \\
\hline 1629 & $\begin{array}{l}\text { Pseudolysimachion orchideum } \\
\text { (Crantz) Wraber }\end{array}$ & Kisharsány & 1961 & Simon Tibor & BPU-06136.JPG \\
\hline 1629 & $\begin{array}{l}\text { Pseudolysimachion orchideum } \\
\text { (Crantz) Wraber }\end{array}$ & Mátraszentimre & 1935 & Soó Rezső & BPU-06134.JPG \\
\hline 1629 & $\begin{array}{l}\text { Pseudolysimachion orchideum } \\
\text { (Crantz) Wraber }\end{array}$ & Tiborszállás & 1948 & Simon Tibor, Jakucs Pál & BPU-06138.JPG \\
\hline 1629 & $\begin{array}{l}\text { Pseudolysimachion orchideum } \\
\text { (Crantz) Wraber }\end{array}$ & Vác & 1949 & Soó Rezső & BPU-06131.JPG \\
\hline 1629 & $\begin{array}{l}\text { Pseudolysimachion orchideum } \\
\text { (Crantz) Wraber }\end{array}$ & Vászoly & 1958 & Felföldy Lajos & BPU-06139.JPG \\
\hline 1630 & Veronica scutellata L. & Balatonszepezd & 1931 & Soó Rezső & BPU-05989.JPG \\
\hline 1630 & Veronica scutellata L. & Debrecen & 1989 & Felföldy Lajos & BPU-05987.JPG \\
\hline 1630 & Veronica scutellata L. & Hortobágy & 1935 & Soó Rezső & BPU-05988.JPG \\
\hline 1630 & Veronica scutellata L. & Lakitelek & 1961 & Simon Tibor & BPU-05986.JPG \\
\hline 1631 & Veronica beccabunga L. & Ásványráró & 1927 & Polgár Sándor & BPU-06029.JPG \\
\hline 1631 & Veronica beccabunga L. & Balatonszőlős & 1956 & $\begin{array}{l}\text { Felföldy Lajos, Tóth } \\
\text { László }\end{array}$ & BPU-06032.JPG \\
\hline 1631 & Veronica beccabunga L. & Bózsva & 1950 & Simon Tibor & BPU-06026.JPG \\
\hline 1631 & Veronica beccabunga L. & Dömös & 1948 & Soó Rezső & BPU-06028.JPG \\
\hline 1631 & Veronica beccabunga L. & Kapolcs & 1959 & $\begin{array}{l}\text { Jávorka Sándor, Felföldy } \\
\text { Lajos, Tóth László }\end{array}$ & BPU-06033.JPG \\
\hline 1631 & Veronica beccabunga L. & Miskolc & 1950 & Jakucs Pál & BPU-06027.JPG \\
\hline 1631 & Veronica beccabunga L. & Olcsvaapáti & 1951 & Simon Tibor & BPU-06030.JPG \\
\hline 1631 & Veronica beccabunga L. & Örvényes & 1959 & Felföldy Lajos & BPU-06034.JPG \\
\hline 1631 & Veronica beccabunga L. & Szőce & 1955 & Soó Rezső & BPU-06031.JPG \\
\hline 1631 & Veronica beccabunga L. & Tállya & 1962 & Isépy István & BPU-06036.JPG \\
\hline 1631 & Veronica beccabunga L. & Uzsa & 1950 & Felföldy Lajos & BPU-06035.JPG \\
\hline 1632 & Veronica scardica Griseb. & Aszófő & 1957 & Felföldy Lajos & BPU-06020.JPG \\
\hline 1632 & Veronica scardica Griseb. & Aszófő & 1959 & Felföldy Lajos & BPU-06019.JPG \\
\hline 1632 & Veronica scardica Griseb. & Balatonfüred & 1946 & Felföldy Lajos & BPU-06025.JPG \\
\hline 1632 & Veronica scardica Griseb. & Balatonszőlős & 1957 & Felföldy Lajos & BPU-06021.JPG \\
\hline 1632 & Veronica scardica Griseb. & Dörgicse & 1957 & Felföldy Lajos & BPU-06022.JPG \\
\hline 1632 & Veronica scardica Griseb. & Kisszékely & 1921 & Boros Ádám & BPU-06018.JPG \\
\hline 1632 & Veronica scardica Griseb. & Makó & 1947 & Timár Lajos & BPU-06013.JPG \\
\hline 1632 & Veronica scardica Griseb. & Örvényes & 1958 & Felföldy Lajos & BPU-06023.JPG \\
\hline 1632 & Veronica scardica Griseb. & Sárbogárd & 1927 & $\begin{array}{l}\text { Moesz Gusztáv, Jávorka } \\
\text { Sándor }\end{array}$ & BPU-06016.JPG \\
\hline 1632 & Veronica scardica Griseb. & Szeged & 1943 & Timár Lajos & BPU-06014.JPG \\
\hline 1632 & Veronica scardica Griseb. & Szeged & 1950 & Timár Lajos & BPU-06015.JPG \\
\hline 1632 & Veronica scardica Griseb. & Szolnok & 1944 & Timár Lajos & BPU-06017.JPG \\
\hline 1632 & Veronica scardica Griseb. & Uzsa & 1953 & $\begin{array}{l}\text { Felföldy Lajos, Tóth } \\
\text { László }\end{array}$ & BPU-06024.JPG \\
\hline
\end{tabular}


1. Elektroniukus melléklet - Electronic Appendix 1.

\begin{tabular}{|c|c|c|c|c|c|}
\hline $\begin{array}{l}\text { Sorszám / } \\
\text { Number }\end{array}$ & Taxon-név / Taxon-name & $\begin{array}{l}\text { Település / } \\
\text { Settlement }\end{array}$ & $\begin{array}{l}\text { Év / } \\
\text { Year }\end{array}$ & Gyűjtő / Collector & $\begin{array}{c}\text { Fájlnév / } \\
\text { File-name }\end{array}$ \\
\hline 1633 & Veronica anagalloides Guss. & Balatonszőlős & 1956 & $\begin{array}{l}\text { Felföldy Lajos, Tóth } \\
\text { László }\end{array}$ & BPU-05998.JPG \\
\hline 1633 & Veronica anagalloides Guss. & Balmazújváros & 1932 & Soó Rezső & BPU-05995.JPG \\
\hline 1633 & Veronica anagalloides Guss. & Debrecen & 1951 & Soó Rezső, Simon Tibor & BPU-05994.JPG \\
\hline 1633 & Veronica anagalloides Guss. & Győrszemere & 1916 & Polgár Sándor & BPU-05993.JPG \\
\hline 1633 & Veronica anagalloides Guss. & Kiskőrös & 1933 & Boros Ádám & BPU-05990.JPG \\
\hline 1633 & Veronica anagalloides Guss. & Kiskunmajsa & 1964 & Soó Rezső & BPU-05991.JPG \\
\hline 1633 & Veronica anagalloides Guss. & Pákozd & 1980 & Felföldy Lajos & BPU-05992.JPG \\
\hline 1633 & Veronica anagalloides Guss. & Tihany & 1929 & Soó Rezső & BPU-05999.JPG \\
\hline 1633 & Veronica anagalloides Guss. & Vácrátót & 1953 & Felföldy Lajos & BPU-05996.JPG \\
\hline 1633 & Veronica anagalloides Guss. & Vácrátót & 1953 & Felföldy Lajos & BPU-05997.JPG \\
\hline 1635 & Veronica anagallis-aquatica L. & Aszófő & 1959 & Felföldy Lajos & BPU-06007.JPG \\
\hline 1635 & Veronica anagallis-aquatica L. & Bagamér & 1947 & Soó Rezső & BPU-06002.JPG \\
\hline 1635 & Veronica anagallis-aquatica L. & Baktalórántháza & 1933 & Soó Rezső & BPU-06005.JPG \\
\hline 1635 & Veronica anagallis-aquatica L. & Balatonakali & 1958 & Felföldy Lajos & BPU-06006.JPG \\
\hline 1635 & Veronica anagallis-aquatica L. & Balatongyörök & 1961 & Felföldy Lajos & BPU-06009.JPG \\
\hline 1635 & Veronica anagallis-aquatica L. & Bisse & 1965 & $\begin{array}{l}\text { Simon Tibor, Priszter } \\
\text { Szaniszló, Borhidi Attila }\end{array}$ & BPU-06001.JPG \\
\hline 1635 & Veronica anagallis-aquatica L. & Bózsva & 1950 & Simon Tibor & BPU-06000.JPG \\
\hline 1635 & Veronica anagallis-aquatica L. & Debrecen & 1937 & Soó Rezső & BPU-06010.JPG \\
\hline 1635 & Veronica anagallis-aquatica L. & Győr & 1911 & Polgár Sándor & BPU-06012.JPG \\
\hline 1635 & Veronica anagallis-aquatica L. & Gyulakeszi & 1938 & Soó Rezső & BPU-06008.JPG \\
\hline 1635 & Veronica anagallis-aquatica L. & Pákozd & 1980 & Felföldy Lajos & BPU-06003.JPG \\
\hline 1635 & Veronica anagallis-aquatica L. & Sávoly/Főnyed/Vörs & 1928 & Soó Rezső & BPU-06011.JPG \\
\hline 1635 & Veronica anagallis-aquatica L. & Vácrátót & 1949 & Soó Rezső & BPU-06004.JPG \\
\hline 1636 & Veronica prostrata L. & Budapest & 2005 & Felföldy Lajos & BPU-06057.JPG \\
\hline 1636 & Veronica prostrata L. & Debrecen & 1939 & Soó Rezső & BPU-06058.JPG \\
\hline 1636 & Veronica prostrata L. & Nógrád & 1922 & Soó Rezső & BPU-06059.JPG \\
\hline 1636 & Veronica prostrata L. & Szigetmonostor & 1963 & Isépy István & BPU-06061.JPG \\
\hline 1636 & Veronica prostrata L. & Szigetszentmiklós & 1947 & Kárpáti Zoltán & BPU-06060.JPG \\
\hline 1636 & Veronica prostrata $\mathrm{L}$. & Tihany & 1958 & Felföldy Lajos & BPU-06062.JPG \\
\hline 1637 & Veronica jacquinii Baumg. & Dabas & 1951 & Borsos Olga, Simon Tibor & BPU-06066.JPG \\
\hline 1637 & Veronica jacquinii Baumg. & Pécs & 1953 & Soó Rezső, Borsos Olga & BPU-06065.JPG \\
\hline 1638 & Veronica austriaca L. & Baktalórántháza & 1934 & Máthé Imre & BPU-06076.JPG \\
\hline 1638 & Veronica austriaca L. & Balatonszőlős & 1958 & Felföldy Lajos & BPU-06078.JPG \\
\hline 1638 & Veronica austriaca L. & Bátorliget & 1934 & Soó Rezső & BPU-06075.JPG \\
\hline 1638 & Veronica austriaca L. & Budapest & 1947 & Soó Rezső & BPU-06069.JPG \\
\hline 1638 & Veronica austriaca L. & Budapest & 1982 & Felföldy Lajos & BPU-06068.JPG \\
\hline 1638 & Veronica austriaca L. & Debrecen & 1933 & Soó Rezső & BPU-06073.JPG \\
\hline 1638 & Veronica austriaca L. & Füzérkomlós & 1948 & Vajda László & BPU-06070.JPG \\
\hline 1638 & Veronica austriaca L. & Lábatlan & 1953 & Felföldy Lajos & BPU-06072.JPG \\
\hline 1638 & Veronica austriaca L. & Nagyharsány & 1956 & Soó Rezső & BPU-06071.JPG \\
\hline 1638 & Veronica austriaca L. & Nagykovácsi & 1963 & Isépy István & BPU-06074.JPG \\
\hline 1638 & Veronica austriaca L. & Tihany & 1956 & Felföldy Lajos & BPU-06079.JPG \\
\hline 1638 & Veronica austriaca L. & Tokaj & 1933 & Soó Rezső & BPU-06081.JPG \\
\hline 1638 & Veronica austriaca L. & Tokaj & 1947 & Soó Rezső & BPU-06082.JPG \\
\hline 1639 & Veronica teucrium L. & Bátorliget & 1934 & Soó Rezső & BPU-06093.JPG \\
\hline 1639 & Veronica teucrium L. & Budapest & 1947 & Soó Rezső & BPU-06092.JPG \\
\hline
\end{tabular}


1. Elektroniukus melléklet - Electronic Appendix 1.

\begin{tabular}{|c|c|c|c|c|c|}
\hline $\begin{array}{l}\text { Sorszám / } \\
\text { Number }\end{array}$ & Taxon-név / Taxon-name & $\begin{array}{l}\text { Település / } \\
\text { Settlement }\end{array}$ & $\begin{array}{l}\text { Év / } \\
\text { Year }\end{array}$ & Gyűjtő / Collector & $\begin{array}{l}\text { Fájlnév / } \\
\text { File-name }\end{array}$ \\
\hline 1639 & Veronica teucrium L. & Budapest & 2005 & Felföldy Lajos & BPU-06084.JPG \\
\hline 1639 & Veronica teucrium L. & Eger & 1936 & Soó Rezső & BPU-06088.JPG \\
\hline 1639 & Veronica teucrium L. & Gyöngyös & 1951 & Jakucs Pál, Ér Lajos & BPU-06090.JPG \\
\hline 1639 & Veronica teucrium L. & Gyöngyös & 1961 & Soó Rezső & BPU-06091.JPG \\
\hline 1639 & Veronica teucrium L. & Isztimér & 1949 & Soó Rezső & BPU-06087.JPG \\
\hline 1639 & Veronica teucrium L. & Mátraszentimre & 1961 & Soó Rezső & BPU-06089.JPG \\
\hline 1639 & Veronica teucrium L. & Miskolc & 1932 & $\begin{array}{l}\text { Zólyomi Bálint, Máthé } \\
\text { Imre }\end{array}$ & BPU-06086.JPG \\
\hline 1639 & Veronica teucrium L. & Nagyharsány & 1967 & Priszter Szaniszló & BPU-06085.JPG \\
\hline 1639 & Veronica teucrium L. & Tihany & 1956 & Felföldy Lajos & BPU-06094.JPG \\
\hline 1640 & $\begin{array}{l}\text { Veronica vindobonensis (M.A. Fisch.) } \\
\text { M.A. Fisch. }\end{array}$ & Budapest & 2005 & Felföldy Lajos & BPU-06037.JPG \\
\hline 1641 & Veronica chamaedrys L. & Budapest & 1961 & Isépy István & BPU-06038.JPG \\
\hline 1641 & Veronica chamaedrys L. & Doboz & 1935 & Máthé Imre & BPU-06040.JPG \\
\hline 1641 & Veronica chamaedrys L. & Felsőtelekes & 2005 & Gulyás Pál & BPU-06042.JPG \\
\hline 1641 & Veronica chamaedrys L. & Hidegkút & 1958 & Felföldy Lajos & BPU-06045.JPG \\
\hline 1641 & Veronica chamaedrys L. & Hidegkút & 1958 & Felföldy Lajos & BPU-06046.JPG \\
\hline 1641 & Veronica chamaedrys L. & Miskolc & 1939 & Soó Rezső & BPU-06043.JPG \\
\hline 1641 & Veronica chamaedrys L. & Téglás & 1937 & Soó Rezső & BPU-06044.JPG \\
\hline 1641 & Veronica chamaedrys L. & Tihany & 1956 & Felföldy Lajos & BPU-06047.JPG \\
\hline 1641 & Veronica chamaedrys L. & Villány & 1935 & Soó Rezső & BPU-06039.JPG \\
\hline 1642 & Veronica officinalis L. & Baktalórántháza & 1932 & Soó Rezső & BPU-06055.JPG \\
\hline 1642 & Veronica officinalis L. & Dömös & 1948 & Soó Rezső & BPU-06054.JPG \\
\hline 1642 & Veronica officinalis L. & Gyöngyös & 1961 & Soó Rezső & BPU-06050.JPG \\
\hline 1642 & Veronica officinalis L. & Kapolcs & 1959 & $\begin{array}{l}\text { Jávorka Sándor, Felföldy } \\
\text { Lajos, Tóth László }\end{array}$ & BPU-06052.JPG \\
\hline 1642 & Veronica officinalis L. & Miskolc & 1936 & Soó Rezső & BPU-06049.JPG \\
\hline 1642 & Veronica officinalis L. & Miskolc & 1953 & Soó Rezső & BPU-06051.JPG \\
\hline 1642 & Veronica officinalis L. & Uzsa & 1959 & $\begin{array}{l}\text { Jávorka Sándor, Felföldy } \\
\text { Lajos }\end{array}$ & BPU-06053.JPG \\
\hline 1643 & Veronica montana L. & Pécs & 1953 & Soó Rezső, Borsos Olga & BPU-06056.JPG \\
\hline 1644 & Veronica triphyllos L. & Aszófő & 1965 & Priszter Szaniszló & BPU-06145.JPG \\
\hline 1644 & Veronica triphyllos L. & Budapest & 1982 & Felföldy Lajos & BPU-06141.JPG \\
\hline 1644 & Veronica triphyllos L. & Budapest & 1989 & Felföldy Lajos & BPU-06142.JPG \\
\hline 1644 & Veronica triphyllos L. & Hajdúsámson & 1932 & Soó Rezső & BPU-06143.JPG \\
\hline 1644 & Veronica triphyllos L. & Tihany & 1951 & Felföldy Lajos & BPU-06146.JPG \\
\hline 1644 & Veronica triphyllos L. & Tihany & 1958 & Felföldy Lajos & BPU-06147.JPG \\
\hline 1644 & Veronica triphyllos L. & Tihany & 1958 & Felföldy Lajos & BPU-06148.JPG \\
\hline 1644 & Veronica triphyllos L. & Vácrátót & 1953 & Felföldy Lajos & BPU-06144.JPG \\
\hline 1645 & Veronica verna L. & Egyek & 1947 & Soó Rezső & BPU-06153.JPG \\
\hline 1645 & Veronica verna L. & Sukoró & 1934 & Boros Ádám & BPU-06149.JPG \\
\hline 1646 & Veronica dillenii Crantz & Hajdúsámson & 1932 & Soó Rezső & BPU-06155.JPG \\
\hline 1646 & Veronica dillenii Crantz & Uzsa & 1950 & Felföldy Lajos & BPU-06154.JPG \\
\hline 1647 & Veronica serpyllifolia L. & Debrecen & 1933 & Soó Rezső & BPU-06175.JPG \\
\hline 1647 & Veronica serpyllifolia L. & Egyek & 1933 & Soó Rezső & BPU-06176.JPG \\
\hline 1647 & Veronica serpyllifolia L. & Gyöngyös & 1965 & Soó Rezső & BPU-06177.JPG \\
\hline 1647 & Veronica serpyllifolia L. & Háromhuta & 1960 & Simon Tibor & BPU-06179.JPG \\
\hline 1647 & Veronica serpyllifolia L. & Hidegkút & 1958 & Felföldy Lajos & BPU-06178.JPG \\
\hline 1647 & Veronica serpyllifolia L. & Miskolc & 1939 & Soó Rezső & BPU-06173.JPG \\
\hline
\end{tabular}


1. Elektroniukus melléklet - Electronic Appendix 1.

\begin{tabular}{|c|c|c|c|c|c|}
\hline $\begin{array}{c}\text { Sorszám / } \\
\text { Number }\end{array}$ & Taxon-név / Taxon-name & $\begin{array}{l}\text { Település / } \\
\text { Settlement }\end{array}$ & $\begin{array}{l}\text { Év / } \\
\text { Year }\end{array}$ & Gyưjtő / Collector & $\begin{array}{l}\text { Fájlnév / } \\
\text { File-name }\end{array}$ \\
\hline 1647 & Veronica serpyllifolia L. & Szeged & 1950 & Timár Lajos & BPU-06174.JPG \\
\hline 1648 & Veronica arvensis L. & Bagamér & 1933 & Soó Rezső & BPU-06162.JPG \\
\hline 1648 & Veronica arvensis L. & Budapest & 1922 & Soó Rezső & BPU-06158.JPG \\
\hline 1648 & Veronica arvensis L. & Budapest & 1982 & Felföldy Lajos & BPU-06157.JPG \\
\hline 1648 & Veronica arvensis L. & Budapest & 2004 & Felföldy Lajos & BPU-06156.JPG \\
\hline 1648 & Veronica arvensis L. & Tass & 1982 & Felföldy Lajos & BPU-06159.JPG \\
\hline 1648 & Veronica arvensis L. & Tass & 1982 & Felföldy Lajos & BPU-06160.JPG \\
\hline 1648 & Veronica arvensis L. & Tihany & 1956 & Felföldy Lajos & BPU-06163.JPG \\
\hline 1648 & Veronica arvensis L. & Tihany & 1958 & Felföldy Lajos & BPU-06164.JPG \\
\hline 1648 & Veronica arvensis L. & Zámoly & 1982 & Felföldy Lajos & BPU-06161.JPG \\
\hline 1650 & Veronica praecox All. & Abaújszántó & 1944 & Siroki Zoltán & BPU-06166.JPG \\
\hline 1650 & Veronica praecox All. & Budapest & 1982 & Felföldy Lajos & BPU-06165.JPG \\
\hline 1650 & Veronica praecox All. & Lábatlan & 1953 & Felföldy Lajos & BPU-06167.JPG \\
\hline 1650 & Veronica praecox All. & Mór & 1953 & Felföldy Lajos & BPU-06168.JPG \\
\hline 1650 & Veronica praecox All. & Nagykovácsi & 1949 & Soó Rezső & BPU-06169.JPG \\
\hline 1650 & Veronica praecox All. & Tihany & 1956 & Felföldy Lajos & BPU-06171.JPG \\
\hline 1650 & Veronica praecox All. & Tihany & 1958 & Felföldy Lajos & BPU-06170.JPG \\
\hline 1650 & Veronica praecox All. & Vászoly & 1951 & Felföldy Lajos & BPU-06172.JPG \\
\hline $\begin{array}{c}1652- \\
1654\end{array}$ & Veronica hederifolia agg. & Budaörs & 1982 & Felföldy Lajos & BPU-06212.JPG \\
\hline $\begin{array}{c}1652- \\
1654\end{array}$ & Veronica hederifolia agg. & Budapest & 1944 & Boros Ádám & BPU-06193.JPG \\
\hline $\begin{array}{c}1652- \\
1654\end{array}$ & Veronica hederifolia agg. & Budapest & 1982 & Felföldy Lajos & BPU-06188.JPG \\
\hline $\begin{array}{c}1652- \\
1654\end{array}$ & Veronica hederifolia agg. & Budapest & 1982 & Felföldy Lajos & BPU-06189.JPG \\
\hline $\begin{array}{c}1652- \\
1654\end{array}$ & Veronica hederifolia agg. & Budapest & 1982 & Felföldy Lajos & BPU-06191.JPG \\
\hline $\begin{array}{c}1652- \\
1654\end{array}$ & Veronica hederifolia agg. & Budapest & 1982 & Felföldy Lajos & BPU-06192.JPG \\
\hline $\begin{array}{c}1652- \\
1654\end{array}$ & Veronica hederifolia agg. & Budapest & 1991 & Felföldy Lajos & BPU-06187.JPG \\
\hline $\begin{array}{c}1652- \\
1654\end{array}$ & Veronica hederifolia agg. & Budapest & 1993 & Felföldy Lajos & BPU-06190.JPG \\
\hline $\begin{array}{c}1652- \\
1654\end{array}$ & Veronica hederifolia agg. & Csákberény & 1982 & Felföldy Lajos & BPU-06197.JPG \\
\hline $\begin{array}{c}1652- \\
1654\end{array}$ & Veronica hederifolia agg. & Csákvár & 1967 & Borsos Olga & BPU-06200.JPG \\
\hline $\begin{array}{c}1652- \\
1654\end{array}$ & Veronica hederifolia agg. & Csákvár & 1967 & Borsos Olga & BPU-06201.JPG \\
\hline $\begin{array}{c}1652- \\
1654\end{array}$ & Veronica hederifolia agg. & Csákvár & 1967 & Borsos Olga & BPU-06202.JPG \\
\hline $\begin{array}{c}1652- \\
1654\end{array}$ & Veronica hederifolia agg. & Csákvár & 1967 & Borsos Olga & BPU-06203.JPG \\
\hline $\begin{array}{c}1652- \\
1654\end{array}$ & Veronica hederifolia agg. & Csákvár & 1967 & Borsos Olga & BPU-06204.JPG \\
\hline $\begin{array}{c}1652- \\
1654\end{array}$ & Veronica hederifolia agg. & Csákvár & 1967 & Borsos Olga & BPU-06205.JPG \\
\hline $\begin{array}{c}1652- \\
1654\end{array}$ & Veronica hederifolia agg. & Csákvár & 1967 & Borsos Olga & BPU-06206.JPG \\
\hline $\begin{array}{c}1652- \\
1654\end{array}$ & Veronica hederifolia agg. & Csobánka & 1963 & Isépy István & BPU-06213.JPG \\
\hline $\begin{array}{c}1652- \\
1654\end{array}$ & Veronica hederifolia agg. & Debrecen & 1931 & Soó Rezső & BPU-06210.JPG \\
\hline
\end{tabular}


1. Elektroniukus melléklet - Electronic Appendix 1.

\begin{tabular}{|c|c|c|c|c|c|}
\hline $\begin{array}{c}\text { Sorszám / } \\
\text { Number }\end{array}$ & Taxon-név / Taxon-name & $\begin{array}{l}\text { Település / } \\
\text { Settlement }\end{array}$ & $\begin{array}{l}\text { Év / } \\
\text { Year }\end{array}$ & Gyưjtő / Collector & $\begin{array}{l}\text { Fájlnév / } \\
\text { File-name }\end{array}$ \\
\hline $\begin{array}{c}1652- \\
1654\end{array}$ & Veronica hederifolia agg. & Debrecen & 1948 & Soó Rezső & BPU-06209.JPG \\
\hline $\begin{array}{c}1652- \\
1654\end{array}$ & Veronica hederifolia agg. & Gárdony & 1966 & Priszter Szaniszló & BPU-06207.JPG \\
\hline $\begin{array}{c}1652- \\
1654\end{array}$ & Veronica hederifolia agg. & Gyenesdiás & 1965 & Priszter Szaniszló & BPU-06223.JPG \\
\hline $\begin{array}{c}1652- \\
1654\end{array}$ & Veronica hederifolia agg. & Gyenesdiás & 1965 & Priszter Szaniszló & BPU-06224.JPG \\
\hline $\begin{array}{c}1652- \\
1654\end{array}$ & Veronica hederifolia agg. & Mezőcsát & 1967 & Borsos Olga & BPU-06199.JPG \\
\hline $\begin{array}{c}1652- \\
1654\end{array}$ & Veronica hederifolia agg. & Mór & 1967 & Borsos Olga & BPU-06198.JPG \\
\hline $\begin{array}{c}1652- \\
1654\end{array}$ & Veronica hederifolia agg. & Nagyharsány & 1967 & Borhidi Attila & BPU-06194.JPG \\
\hline $\begin{array}{c}1652- \\
1654\end{array}$ & Veronica hederifolia agg. & Nagykovácsi & 1949 & Soó Rezső & BPU-06214.JPG \\
\hline $\begin{array}{c}1652- \\
1654\end{array}$ & Veronica hederifolia agg. & Siklós & 1967 & Priszter Szaniszló & BPU-06195.JPG \\
\hline $\begin{array}{c}1652- \\
1654\end{array}$ & Veronica hederifolia agg. & Szalonna & 1966 & Felföldy Lajos & BPU-06196.JPG \\
\hline $\begin{array}{c}1652- \\
1654\end{array}$ & Veronica hederifolia agg. & Szár & 1935 & Boros Ádám & BPU-06208.JPG \\
\hline $\begin{array}{c}1652- \\
1654\end{array}$ & Veronica hederifolia agg. & Tatabánya & 1928 & Boros Ádám & BPU-06211.JPG \\
\hline $\begin{array}{c}1652- \\
1654\end{array}$ & Veronica hederifolia agg. & Tihany & 1958 & Felföldy Lajos & BPU-06218.JPG \\
\hline $\begin{array}{c}1652- \\
1654\end{array}$ & Veronica hederifolia agg. & Tihany & 1958 & Felföldy Lajos & BPU-06219.JPG \\
\hline $\begin{array}{c}1652- \\
1654\end{array}$ & Veronica hederifolia agg. & Tihany & 1958 & Felföldy Lajos & BPU-06220.JPG \\
\hline $\begin{array}{c}1652- \\
1654\end{array}$ & Veronica hederifolia agg. & Tihany & 1958 & Felföldy Lajos & BPU-06221.JPG \\
\hline $\begin{array}{c}1652- \\
1654\end{array}$ & Veronica hederifolia agg. & Tihany & 1958 & Felföldy Lajos & BPU-06222.JPG \\
\hline $\begin{array}{c}1652- \\
1654\end{array}$ & Veronica hederifolia agg. & Üröm & 1991 & Felföldy Lajos & BPU-06215.JPG \\
\hline $\begin{array}{c}1652- \\
1654\end{array}$ & Veronica hederifolia agg. & Vácrátót & 1953 & Felföldy Lajos & BPU-06216.JPG \\
\hline $\begin{array}{c}1652- \\
1654\end{array}$ & Veronica hederifolia agg. & Vácrátót & 1953 & Felföldy Lajos & BPU-06217.JPG \\
\hline 1656 & Veronica persica Poir. & "Szent György-hegy" & 1928 & Soó Rezső & BPU-06230.JPG \\
\hline 1656 & Veronica persica Poir. & Budapest & 1982 & Felföldy Lajos & BPU-06226.JPG \\
\hline 1656 & Veronica persica Poir. & Geszt & 1947 & Soó Rezső & BPU-06229.JPG \\
\hline 1656 & Veronica persica Poir. & Gyenesdiás & 1965 & Priszter Szaniszló & BPU-06231.JPG \\
\hline 1656 & Veronica persica Poir. & Jósvafő & 1967 & Priszter Szaniszló & BPU-06227.JPG \\
\hline 1656 & Veronica persica Poir. & Siklós & 1967 & Priszter Szaniszló & BPU-06228.JPG \\
\hline 1658 & Veronica polita Fr. & Budapest & 1982 & Felföldy Lajos & BPU-06232.JPG \\
\hline 1658 & Veronica polita Fr. & Budapest & 1982 & Felföldy Lajos & BPU-06235.JPG \\
\hline 1658 & Veronica polita Fr. & Budapest & 1989 & Felföldy Lajos & BPU-06234.JPG \\
\hline 1658 & Veronica polita Fr. & Budapest & 1991 & Felföldy Lajos & BPU-06233.JPG \\
\hline 1658 & Veronica polita Fr. & Csákberény & 1982 & Felföldy Lajos & BPU-06239.JPG \\
\hline 1658 & Veronica polita Fr. & Dörgicse & 1961 & Csaby Sándor & BPU-06243.JPG \\
\hline 1658 & Veronica polita Fr. & Hajdúnánás & 1935 & Soó Rezső & BPU-06240.JPG \\
\hline 1658 & Veronica polita Fr. & Jósvafő & 1967 & Priszter Szaniszló & BPU-06236.JPG \\
\hline 1658 & Veronica polita Fr. & Tass & 1982 & Felföldy Lajos & BPU-06237.JPG \\
\hline 1658 & Veronica polita Fr. & Tass & 1982 & Felföldy Lajos & BPU-06238.JPG \\
\hline
\end{tabular}


1. Elektroniukus melléklet - Electronic Appendix 1.

\begin{tabular}{|c|c|c|c|c|c|}
\hline $\begin{array}{l}\text { Sorszám / } \\
\text { Number }\end{array}$ & Taxon-név / Taxon-name & $\begin{array}{l}\text { Település / } \\
\text { Settlement }\end{array}$ & $\begin{array}{l}\text { Év / } \\
\text { Year }\end{array}$ & Gyújtő / Collector & $\begin{array}{l}\text { Fájlnév / } \\
\text { File-name }\end{array}$ \\
\hline 1658 & Veronica polita Fr. & Tihany & 1946 & Felföldy Lajos & BPU-06244.JPG \\
\hline 1658 & Veronica polita Fr. & Tihany & 1958 & Felföldy Lajos & BPU-06245.JPG \\
\hline 1658 & Veronica polita Fr. & Tihany & 1958 & Felföldy Lajos & BPU-06246.JPG \\
\hline 1658 & Veronica polita Fr. & Tihany & 1958 & Felföldy Lajos & BPU-06247.JPG \\
\hline 1658 & Veronica polita Fr. & Vácrátót & 1953 & Felföldy Lajos & BPU-06241.JPG \\
\hline 1658 & Veronica polita Fr. & Vácrátót & 1953 & Felföldy Lajos & BPU-06242.JPG \\
\hline 1659 & Veronica opaca Fr. & Budapest & 1982 & Felföldy Lajos & BPU-06248.JPG \\
\hline 1660 & Melampyrum cristatum L. & Budapest & 1947 & Soó Rezső & BPU-06277.JPG \\
\hline 1660 & Melampyrum cristatum L. & Budapest & 1982 & Felföldy Lajos & BPU-06276.JPG \\
\hline 1660 & Melampyrum cristatum L. & Egyek & 1947 & Soó Rezső & BPU-06281.JPG \\
\hline 1660 & Melampyrum cristatum L. & Fony & 1961 & Simon Tibor & BPU-06278.JPG \\
\hline 1660 & Melampyrum cristatum L. & Fót & 1960 & Soó Rezső & BPU-06286.JPG \\
\hline 1660 & Melampyrum cristatum L. & Gyenesdiás & 1929 & Soó Rezső & BPU-06291.JPG \\
\hline 1660 & Melampyrum cristatum L. & Gyöngyös & 1953 & Soó Rezső & BPU-06283.JPG \\
\hline 1660 & Melampyrum cristatum L. & Gyöngyös & 1961 & Soó Rezső & BPU-06284.JPG \\
\hline 1660 & Melampyrum cristatum L. & Gyöngyös & 1961 & Soó Rezső & BPU-06285.JPG \\
\hline 1660 & Melampyrum cristatum L. & Isztimér & 1949 & Soó Rezső & BPU-06280.JPG \\
\hline 1660 & Melampyrum cristatum L. & Jósvafő & 1951 & $\begin{array}{l}\text { Soó Rezső, Jakucs Pál, Ér } \\
\text { Lajos }\end{array}$ & BPU-06279.JPG \\
\hline 1660 & Melampyrum cristatum L. & Kerecsend & 1957 & Soó Rezső & BPU-06282.JPG \\
\hline 1660 & Melampyrum cristatum L. & Nyíregyháza & 1933 & Soó Rezső & BPU-06289.JPG \\
\hline 1660 & Melampyrum cristatum L. & Ócsa & 1953 & Soó Rezső, Borsos Olga & BPU-06287.JPG \\
\hline 1660 & Melampyrum cristatum L. & Telki & 1947 & Soó Rezső & BPU-06288.JPG \\
\hline 1660 & Melampyrum cristatum L. & Uzsa & 1952 & Borsos Olga & BPU-06290.JPG \\
\hline 1661 & Melampyrum arvense L. & Beregdaróc & 1951 & $\begin{array}{l}\text { Simon Tibor, Vozáry } \\
\text { Elemér }\end{array}$ & BPU-06300.JPG \\
\hline 1661 & Melampyrum arvense L. & Budapest & 1982 & Felföldy Lajos & BPU-06294.JPG \\
\hline 1661 & Melampyrum arvense L. & Jósvafő & 1940 & Soó Rezső & BPU-06296.JPG \\
\hline 1661 & Melampyrum arvense L. & Jósvafő & 1951 & Soó Rezső, Jakucs Pál & BPU-06297.JPG \\
\hline 1661 & Melampyrum arvense L. & Pusztamiske & 1948 & Borsos Olga & BPU-06301.JPG \\
\hline 1661 & Melampyrum arvense L. & Tarpa & 1948 & $\begin{array}{l}\text { Pólya László, Simon } \\
\text { Tibor, Jakucs Pál }\end{array}$ & BPU-06302.JPG \\
\hline 1661 & Melampyrum arvense L. & Tihany & 1961 & $\begin{array}{l}\text { Pankov H., Felföldy Lajos, } \\
\text { Sólymosy G. }\end{array}$ & BPU-06295.JPG \\
\hline 1661 & Melampyrum arvense L. & Vác & 1949 & $\begin{array}{l}\text { Soó Rezső, Simon Tibor, } \\
\text { Jakucs Pál }\end{array}$ & BPU-06298.JPG \\
\hline 1662 & Melampyrum barbatum Waldst. et Kit. & Bőny & 1929 & Polgár Sándor & BPU-06307.JPG \\
\hline 1662 & Melampyrum barbatum Waldst. et Kit. & Budaörs & 1982 & Felföldy Lajos & BPU-06309.JPG \\
\hline 1662 & Melampyrum barbatum Waldst. et Kit. & Budapest & 1947 & Soó Rezső & BPU-06304.JPG \\
\hline 1662 & Melampyrum barbatum Waldst. et Kit. & Budapest & 2004 & Felföldy Lajos & BPU-06303.JPG \\
\hline 1662 & Melampyrum barbatum Waldst. et Kit. & Lábatlan & 1953 & Felföldy Lajos & BPU-06308.JPG \\
\hline 1662 & Melampyrum barbatum Waldst. et Kit. & Miskolc & 1936 & Soó Rezső & BPU-06306.JPG \\
\hline 1662 & Melampyrum barbatum Waldst. et Kit. & Pomáz & 1946 & Boros Ádám & BPU-06310.JPG \\
\hline 1662 & Melampyrum barbatum Waldst. et Kit. & Tarpa & 1949 & Soó Rezső & BPU-06305.JPG \\
\hline 1662 & Melampyrum barbatum Waldst. et Kit. & Tihany & 1928 & Soó Rezső & BPU-06311.JPG \\
\hline 1662 & Melampyrum barbatum Waldst. et Kit. & Tihany & 1959 & Felföldy Lajos & BPU-06312.JPG \\
\hline 1663 & Melampyrum pratense L. & Alsószölnök & 1955 & Soó Rezső & BPU-06351.JPG \\
\hline 1663 & Melampyrum pratense L. & Alsószölnök & 1955 & Soó Rezső & BPU-06356.JPG \\
\hline 1663 & Melampyrum pratense L. & Dávod & 1955 & Soó Rezső & BPU-06354.JPG \\
\hline 1663 & Melampyrum pratense L. & Gyöngyös & 1961 & Soó Rezső & BPU-06350.JPG \\
\hline
\end{tabular}


1. Elektroniukus melléklet - Electronic Appendix 1.

\begin{tabular}{|c|c|c|c|c|c|}
\hline $\begin{array}{l}\text { Sorszám / } \\
\text { Number }\end{array}$ & Taxon-név / Taxon-name & $\begin{array}{l}\text { Település / } \\
\text { Settlement }\end{array}$ & $\begin{array}{l}\text { Év / } \\
\text { Year }\end{array}$ & Gyűjtő / Collector & $\begin{array}{c}\text { Fájlnév / } \\
\text { File-name }\end{array}$ \\
\hline 1663 & Melampyrum pratense L. & Jósvafő & 1951 & $\begin{array}{l}\text { Soó Rezső, Jakucs Pál, Ér } \\
\text { Lajos }\end{array}$ & BPU-06353.JPG \\
\hline 1663 & Melampyrum pratense L. & Kaszó & 1958 & $\begin{array}{l}\text { Járainé Komlódi Magda, } \\
\text { Borhidi Attila }\end{array}$ & BPU-06355.JPG \\
\hline 1663 & Melampyrum pratense L. & Kőszeg & 1924 & Boros Ádám & BPU-06363.JPG \\
\hline 1663 & Melampyrum pratense L. & Kőszeg & 1932 & Soó Rezső & BPU-06361.JPG \\
\hline 1663 & Melampyrum pratense L. & Kőszeg & 1963 & Soó Rezső, Borsos Olga & BPU-06352.JPG \\
\hline 1663 & Melampyrum pratense L. & Pálháza & 1962 & Simon Tibor & BPU-06347.JPG \\
\hline 1663 & Melampyrum pratense L. & Rudabánya & 1936 & Soó Rezső & BPU-06362.JPG \\
\hline 1663 & Melampyrum pratense L. & Tállya & 1958 & Isépy István & BPU-06348.JPG \\
\hline 1663 & Melampyrum pratense L. & Uzsa & 1959 & $\begin{array}{l}\text { Jávorka Sándor, Felföldy } \\
\text { Lajos }\end{array}$ & BPU-06349.JPG \\
\hline 1664 & Melampyrum bihariense A. Kern. & Bátorliget & 1950 & $\begin{array}{l}\text { Simon Tibor, Kárpáti } \\
\text { István }\end{array}$ & BPU-06315.JPG \\
\hline 1664 & Melampyrum bihariense A. Kern. & Bátorliget & 1953 & Soó Rezső & BPU-06314.JPG \\
\hline 1665 & Melampyrum nemorosum L. & Bátorliget & 1928 & Soó Rezső & BPU-06342.JPG \\
\hline 1665 & Melampyrum nemorosum L. & Bátorliget & 1934 & Soó Rezső & BPU-06340.JPG \\
\hline 1665 & Melampyrum nemorosum L. & Bátorliget & 1953 & Simon Tibor & BPU-06339.JPG \\
\hline 1665 & Melampyrum nemorosum L. & Bózsva & 1950 & Simon Tibor & BPU-06322.JPG \\
\hline 1665 & Melampyrum nemorosum L. & Budapest & 1982 & Felföldy Lajos & BPU-06324.JPG \\
\hline 1665 & Melampyrum nemorosum L. & Debrecen & 1930 & Pátkay Imre & BPU-06335.JPG \\
\hline 1665 & Melampyrum nemorosum L. & Debrecen & 1931 & Soó Rezső & BPU-06341.JPG \\
\hline 1665 & Melampyrum nemorosum L. & Debrecen & 1932 & Soó Rezső & BPU-06336.JPG \\
\hline 1665 & Melampyrum nemorosum L. & Dömös & 1948 & Soó Rezső & BPU-06330.JPG \\
\hline 1665 & Melampyrum nemorosum L. & Hajdúbagos & 1933 & Soó Rezső & BPU-06338.JPG \\
\hline 1665 & Melampyrum nemorosum L. & Kőszeg & 1932 & Soó Rezső & BPU-06327.JPG \\
\hline 1665 & Melampyrum nemorosum L. & Kőszeg & 1963 & Soó Rezső, Borsos Olga & BPU-06346.JPG \\
\hline 1665 & Melampyrum nemorosum L. & Miskolc & 1929 & Soó Rezső & BPU-06333.JPG \\
\hline 1665 & Melampyrum nemorosum L. & Nagybajom & 1957 & Soó Rezső & BPU-06329.JPG \\
\hline 1665 & Melampyrum nemorosum L. & Nagyvisnyó & 1953 & Soó Rezső & BPU-06344.JPG \\
\hline 1665 & Melampyrum nemorosum L. & Piricse & 1932 & Soó Rezső & BPU-06337.JPG \\
\hline 1665 & Melampyrum nemorosum L. & Putnok & 1950 & Soó Rezső & BPU-06345.JPG \\
\hline 1665 & Melampyrum nemorosum L. & Sopron & 1937 & Soó Rezső & BPU-06328.JPG \\
\hline 1665 & Melampyrum nemorosum L. & Szamosszeg & 1948 & Simon Tibor, Jakucs Pál & BPU-06332.JPG \\
\hline 1665 & Melampyrum nemorosum L. & Szenna & 1957 & Soó Rezső & BPU-06325.JPG \\
\hline 1665 & Melampyrum nemorosum L. & Tákos & 1948 & Simon Tibor, Jakucs Pál & BPU-06334.JPG \\
\hline 1665 & Melampyrum nemorosum L. & Tállya & 1958 & Isépy István & BPU-06323.JPG \\
\hline 1665 & Melampyrum nemorosum L. & Tarpa & 1949 & Soó Rezső & BPU-06326.JPG \\
\hline 1665 & Melampyrum nemorosum L. & Vác & 1949 & $\begin{array}{l}\text { Soó Rezső, Simon Tibor, } \\
\text { Jakucs Pál }\end{array}$ & BPU-06331.JPG \\
\hline 1666 & Euphrasia rostkoviana Hayne & Alsószölnök & 1955 & Soó Rezső & BPU-06385.JPG \\
\hline 1666 & Euphrasia rostkoviana Hayne & Bak & 1955 & Soó Rezső & BPU-06389.JPG \\
\hline 1666 & Euphrasia rostkoviana Hayne & Balatonakali & 1958 & Felföldy Lajos & BPU-06387.JPG \\
\hline 1666 & Euphrasia rostkoviana Hayne & Kőszeg & 1963 & Soó Rezső, Borsos Olga & BPU-06386.JPG \\
\hline 1666 & Euphrasia rostkoviana Hayne & Nagybajom & 1957 & Soó Rezső & BPU-06384.JPG \\
\hline 1666 & Euphrasia rostkoviana Hayne & Nagykanizsa & 1956 & Felföldy Lajos & BPU-06390.JPG \\
\hline 1666 & Euphrasia rostkoviana Hayne & Szilvásvárad & 1953 & $\begin{array}{l}\text { Felföldy Lajos, Tóth } \\
\text { László }\end{array}$ & BPU-06382.JPG \\
\hline 1666 & Euphrasia rostkoviana Hayne & Uzsa & 1952 & Simon Tibor & BPU-06388.JPG \\
\hline
\end{tabular}


1. Elektroniukus melléklet - Electronic Appendix 1.

\begin{tabular}{|c|c|c|c|c|c|}
\hline $\begin{array}{l}\text { Sorszám / } \\
\text { Number }\end{array}$ & Taxon-név / Taxon-name & $\begin{array}{l}\text { Település / } \\
\text { Settlement }\end{array}$ & $\begin{array}{l}\text { Év / } \\
\text { Year }\end{array}$ & Gyűjtő / Collector & $\begin{array}{l}\text { Fájlnév / } \\
\text { File-name }\end{array}$ \\
\hline 1666 & Euphrasia rostkoviana Hayne & Uzsa & 1953 & $\begin{array}{l}\text { Felföldy Lajos, Tóth } \\
\text { László }\end{array}$ & BPU-06412.JPG \\
\hline 1666 & Euphrasia rostkoviana Hayne & Veresegyház & 1949 & Soó Rezső & BPU-06383.JPG \\
\hline 1667 & Euphrasia kerneri Wettst. & Kimle & 1962 & Borsos Olga & BPU-06398.JPG \\
\hline 1667 & Euphrasia kerneri Wettst. & Miskolc & 1933 & Soó Rezső & BPU-06400.JPG \\
\hline 1667 & Euphrasia kerneri Wettst. & Töltéstava & 1981 & Polgár Sándor & BPU-06399.JPG \\
\hline 1668 & Euphrasia stricta Wolf & Balatongyörök & 1961 & Felföldy Lajos & BPU-06413.JPG \\
\hline 1668 & Euphrasia stricta Wolf & Beregdaróc & 1951 & $\begin{array}{l}\text { Simon Tibor, Vozáry } \\
\text { Elemér }\end{array}$ & BPU-06402.JPG \\
\hline 1668 & Euphrasia stricta Wolf & Csesznek & 1929 & Soó Rezső & BPU-06408.JPG \\
\hline 1668 & Euphrasia stricta Wolf & Debrecen & 1933 & Soó Rezső & BPU-06404.JPG \\
\hline 1668 & Euphrasia stricta Wolf & Gyöngyös & 1952 & Soó Rezső & BPU-06405.JPG \\
\hline 1668 & Euphrasia stricta Wolf & Gyöngyös & 1953 & Soó Rezső & BPU-06406.JPG \\
\hline 1668 & Euphrasia stricta Wolf & Hollóháza & 1961 & Simon Tibor & BPU-06401.JPG \\
\hline 1668 & Euphrasia stricta Wolf & Lesenceistvánd & 1952 & Borsos Olga & BPU-06410.JPG \\
\hline 1668 & Euphrasia stricta Wolf & Miskolc & 1934 & Soó Rezső & BPU-06403.JPG \\
\hline 1668 & Euphrasia stricta Wolf & Uzsa & 1929 & Soó Rezső & BPU-06409.JPG \\
\hline 1668 & Euphrasia stricta Wolf & Vácrátót & 1949 & Soó Rezső & BPU-06407.JPG \\
\hline 1669 & Euphrasia tatarica Fisch. & Budapest & 2004 & Felföldy Lajos & BPU-06418.JPG \\
\hline 1669 & Euphrasia tatarica Fisch. & Mátraszentimre & 1961 & Soó Rezső & BPU-06420.JPG \\
\hline 1669 & Euphrasia tatarica Fisch. & Miskolc & 1929 & Soó Rezső & BPU-06419.JPG \\
\hline 1669 & Euphrasia tatarica Fisch. & Örvényes & 1958 & Felföldy Lajos & BPU-06422.JPG \\
\hline 1669 & Euphrasia tatarica Fisch. & Pomáz & 1948 & Koppányi Tibor & BPU-06421.JPG \\
\hline 1669 & Euphrasia tatarica Fisch. & Tihany & 1954 & Felföldy Lajos & BPU-06411.JPG \\
\hline 1669 & Euphrasia tatarica Fisch. & Uzsa & 1959 & $\begin{array}{l}\text { Jávorka Sándor, Felföldy } \\
\text { Lajos }\end{array}$ & BPU-06423.JPG \\
\hline 1669 & Euphrasia tatarica Fisch. & Zalagyömörő & 1957 & Felföldy Lajos & BPU-06424.JPG \\
\hline 1670 & Odontites lutea (L.) Clairv. & Budapest & 1923 & Soó Rezső & BPU-06440.JPG \\
\hline 1670 & Odontites lutea (L.) Clairv. & Budapest & 1954 & Tóth László & BPU-06439.JPG \\
\hline 1670 & Odontites lutea (L.) Clairv. & Budapest & 1982 & Felföldy Lajos & BPU-06437.JPG \\
\hline 1670 & Odontites lutea (L.) Clairv. & Budapest & 1982 & Felföldy Lajos & BPU-06438.JPG \\
\hline 1670 & Odontites lutea (L.) Clairv. & Csákvár & 1948 & Soó Rezső & BPU-06441.JPG \\
\hline 1670 & Odontites lutea (L.) Clairv. & Egyek & 1947 & Soó Rezső & BPU-06442.JPG \\
\hline 1670 & Odontites lutea (L.) Clairv. & Tállya & 1961 & Simon Tibor & BPU-06443.JPG \\
\hline 1670 & Odontites lutea (L.) Clairv. & Tállya & 1962 & Isépy István & BPU-00001.JPG \\
\hline 1671 & Odontites vernus (Bellardi) Dumort. & Barcs & 1922 & Boros Ádám & BPU-00011.JPG \\
\hline 1671 & Odontites vernus (Bellardi) Dumort. & Bátorliget & 1920 & Boros Ádám & BPU-00013.JPG \\
\hline 1671 & Odontites vernus (Bellardi) Dumort. & Bicske & 1991 & Felföldy Lajos & BPU-00007.JPG \\
\hline 1671 & Odontites vernus (Bellardi) Dumort. & Budapest & 1919 & Boros Ádám & BPU-00004.JPG \\
\hline 1671 & Odontites vernus (Bellardi) Dumort. & Budapest & 2004 & Felföldy Lajos & BPU-00003.JPG \\
\hline 1671 & Odontites vernus (Bellardi) Dumort. & Bugac & 1964 & Soó Rezső & BPU-00005.JPG \\
\hline 1671 & Odontites vernus (Bellardi) Dumort. & Egyek & 1933 & Máthé Imre & BPU-00009.JPG \\
\hline 1671 & Odontites vernus (Bellardi) Dumort. & Gyöngyös & 1953 & Soó Rezső & BPU-00010.JPG \\
\hline 1671 & Odontites vernus (Bellardi) Dumort. & Kőszeg & 1932 & Soó Rezső & BPU-00014.JPG \\
\hline 1671 & Odontites vernus (Bellardi) Dumort. & Nagyharsány & 1965 & $\begin{array}{l}\text { Simon Tibor, Priszter } \\
\text { Szaniszló, Borhidi Attila }\end{array}$ & BPU-00006.JPG \\
\hline 1671 & Odontites vernus (Bellardi) Dumort. & Ócsa & 1919 & Boros Ádám & BPU-00018.JPG \\
\hline 1671 & Odontites vernus (Bellardi) Dumort. & Szentendre & 1963 & Soó Rezső & BPU-00017.JPG \\
\hline 1671 & Odontites vernus (Bellardi) Dumort. & Tihany & 1959 & Felföldy Lajos & BPU-00015.JPG \\
\hline
\end{tabular}


1. Elektroniukus melléklet - Electronic Appendix 1.

\begin{tabular}{|c|c|c|c|c|c|}
\hline $\begin{array}{l}\text { Sorszám / } \\
\text { Number }\end{array}$ & Taxon-név / Taxon-name & $\begin{array}{l}\text { Település / } \\
\text { Settlement }\end{array}$ & $\begin{array}{l}\text { Év / } \\
\text { Year }\end{array}$ & Gyűjtő / Collector & $\begin{array}{l}\text { Fájlnév / } \\
\text { File-name }\end{array}$ \\
\hline 1671 & Odontites vernus (Bellardi) Dumort. & Tihany & 1961 & Sólymossy Gizella & BPU-00016.JPG \\
\hline 1671 & Odontites vernus (Bellardi) Dumort. & Vácrátót & 1953 & Felföldy Lajos & BPU-00019.JPG \\
\hline 1671 & Odontites vernus (Bellardi) Dumort. & Veresegyház & 1919 & Boros Ádám & BPU-00020.JPG \\
\hline 1671 & Odontites vernus (Bellardi) Dumort. & Veresegyház & 1949 & Soó Rezső & BPU-00021.JPG \\
\hline 1671 & Odontites vernus (Bellardi) Dumort. & Zámoly & 1982 & Felföldy Lajos & BPU-00008.JPG \\
\hline 1672 & Pedicularis palustris L. & Lesencetomaj & 1951 & Borsos Olga & BPU-00088.JPG \\
\hline 1672 & Pedicularis palustris L. & Telekes & 1955 & Soó Rezső & BPU-00087.JPG \\
\hline 1672 & Pedicularis palustris L. & Uzsa & 1952 & Simon Tibor & BPU-00091.JPG \\
\hline 1672 & Pedicularis palustris L. & Veresegyház & 1949 & Soó Rezső & BPU-00086.JPG \\
\hline 1673 & Rhinanthus minor L. & Aszófő & 1959 & Felföldy Lajos & BPU-00031.JPG \\
\hline 1673 & Rhinanthus minor L. & Aszófő & 1959 & Felföldy Lajos & BPU-00038.JPG \\
\hline 1673 & Rhinanthus minor L. & Aszófő & 1959 & Felföldy Lajos & BPU-00039.JPG \\
\hline 1673 & Rhinanthus minor L. & Balatonszőlős & 1957 & Felföldy Lajos & BPU-00032.JPG \\
\hline 1673 & Rhinanthus minor L. & Bátorliget & 1934 & Soó Rezső & BPU-00030.JPG \\
\hline 1673 & Rhinanthus minor L. & Dabas & 1963 & Isépy István & BPU-00029.JPG \\
\hline 1673 & Rhinanthus minor L. & Lábatlan & 1953 & Felföldy Lajos & BPU-00027.JPG \\
\hline 1673 & Rhinanthus minor L. & Miskolc & 1939 & Soó Rezső & BPU-00026.JPG \\
\hline 1673 & Rhinanthus minor L. & Nagyvisnyó & 1953 & Soó Rezső & BPU-00028.JPG \\
\hline 1673 & Rhinanthus minor L. & Örvényes & 1959 & Felföldy Lajos & BPU-00033.JPG \\
\hline 1673 & Rhinanthus minor L. & Örvényes & 1959 & Felföldy Lajos & BPU-00034.JPG \\
\hline 1673 & Rhinanthus minor L. & Tihany & 1959 & Felföldy Lajos & BPU-00035.JPG \\
\hline 1674 & $\begin{array}{l}\text { Rhinanthus serotinus (Schönh.) } \\
\text { Oborný }\end{array}$ & Balatonakali & 1958 & Felföldy Lajos & BPU-00042.JPG \\
\hline 1674 & $\begin{array}{l}\text { Rhinanthus serotinus (Schönh.) } \\
\text { Oborný }\end{array}$ & Balatonföldvár & 1928 & Soó Rezső & BPU-00050.JPG \\
\hline 1674 & $\begin{array}{l}\text { Rhinanthus serotinus (Schönh.) } \\
\text { Oborný }\end{array}$ & Balatonszepezd & 1958 & Felföldy Lajos & BPU-00061.JPG \\
\hline 1674 & $\begin{array}{l}\text { Rhinanthus serotinus (Schönh.) } \\
\text { Oborný }\end{array}$ & Balatonszőlős & 1957 & Felföldy Lajos & BPU-00041.JPG \\
\hline 1674 & $\begin{array}{l}\text { Rhinanthus serotinus (Schönh.) } \\
\text { Oborný }\end{array}$ & Balatonszőlős & 1957 & Felföldy Lajos & BPU-00062.JPG \\
\hline 1674 & $\begin{array}{l}\text { Rhinanthus serotinus (Schönh.) } \\
\text { Oborný }\end{array}$ & Bátorliget & 1932 & Soó Rezső & BPU-00065.JPG \\
\hline 1674 & $\begin{array}{l}\text { Rhinanthus serotinus (Schönh.) } \\
\text { Oborný }\end{array}$ & Bátorliget & 1934 & Soó Rezső & BPU-00066.JPG \\
\hline 1674 & $\begin{array}{l}\text { Rhinanthus serotinus (Schönh.) } \\
\text { Oborný }\end{array}$ & Bisse & 1965 & $\begin{array}{l}\text { Simon Tibor, Priszter } \\
\text { Szaniszló, Borhidi Attila }\end{array}$ & BPU-00046.JPG \\
\hline 1674 & $\begin{array}{l}\text { Rhinanthus serotinus (Schönh.) } \\
\text { Oborný }\end{array}$ & Bőny & 1913 & Polgár Sándor & BPU-00058.JPG \\
\hline 1674 & $\begin{array}{l}\text { Rhinanthus serotinus (Schönh.) } \\
\text { Oborný }\end{array}$ & Budapest & 1950 & Timár Lajos & BPU-00059.JPG \\
\hline 1674 & $\begin{array}{l}\text { Rhinanthus serotinus (Schönh.) } \\
\text { Oborný }\end{array}$ & Csévharaszt & 1932 & Boros Ádám & BPU-00063.JPG \\
\hline 1674 & $\begin{array}{l}\text { Rhinanthus serotinus (Schönh.) } \\
\text { Oborný }\end{array}$ & Dabas & 1959 & Soó Rezső & BPU-00051.JPG \\
\hline 1674 & $\begin{array}{l}\text { Rhinanthus serotinus (Schönh.) } \\
\text { Oborný }\end{array}$ & Farmos & 1922 & Soó Rezső & BPU-00064.JPG \\
\hline 1674 & $\begin{array}{l}\text { Rhinanthus serotinus (Schönh.) } \\
\text { Oborný }\end{array}$ & Hajdúsámson & 1951 & Soó Rezső & BPU-00053.JPG \\
\hline 1674 & $\begin{array}{l}\text { Rhinanthus serotinus (Schönh.) } \\
\text { Oborný }\end{array}$ & Kimle & 1962 & Borsos Olga & BPU-00047.JPG \\
\hline 1674 & $\begin{array}{l}\text { Rhinanthus serotinus (Schönh.) } \\
\text { Oborný }\end{array}$ & Lébény & 1927 & Polgár Sándor & BPU-00060.JPG \\
\hline 1674 & $\begin{array}{l}\text { Rhinanthus serotinus (Schönh.) } \\
\text { Oborný }\end{array}$ & Miskolc & 1931 & Hulják János & BPU-00081.JPG \\
\hline
\end{tabular}


1. Elektroniukus melléklet - Electronic Appendix 1.

\begin{tabular}{|c|c|c|c|c|c|}
\hline $\begin{array}{l}\text { Sorszám / } \\
\text { Number }\end{array}$ & Taxon-név / Taxon-name & $\begin{array}{l}\text { Település / } \\
\text { Settlement }\end{array}$ & $\begin{array}{l}\text { Év / } \\
\text { Year }\end{array}$ & Gyűjtő / Collector & $\begin{array}{l}\text { Fájlnév / } \\
\text { File-name }\end{array}$ \\
\hline 1674 & $\begin{array}{l}\text { Rhinanthus serotinus (Schönh.) } \\
\text { Oborný }\end{array}$ & Pákozd & 1980 & Felföldy Lajos & BPU-00056.JPG \\
\hline 1674 & $\begin{array}{l}\text { Rhinanthus serotinus (Schönh.) } \\
\text { Oborný }\end{array}$ & Pákozd & 1980 & Felföldy Lajos & BPU-00057.JPG \\
\hline 1674 & $\begin{array}{l}\text { Rhinanthus serotinus (Schönh.) } \\
\text { Oborný }\end{array}$ & Panyola & 1948 & $\begin{array}{l}\text { Pólya László, Simon } \\
\text { Tibor, Jakucs Pál }\end{array}$ & BPU-00045.JPG \\
\hline 1674 & $\begin{array}{l}\text { Rhinanthus serotinus (Schönh.) } \\
\text { Oborný }\end{array}$ & Tarpa & 1949 & Soó Rezső & BPU-00052.JPG \\
\hline 1674 & $\begin{array}{l}\text { Rhinanthus serotinus (Schönh.) } \\
\text { Oborný }\end{array}$ & Vászoly & 1951 & Felföldy Lajos, Zsolt János & BPU-00043.JPG \\
\hline 1675 & Rhinanthus alectorolophus Pollich & Kőszeg & 1938 & Soó Rezső & BPU-00068.JPG \\
\hline 1675 & Rhinanthus alectorolophus Pollich & Tapolca & 1914 & Lengyel Géza & BPU-00067.JPG \\
\hline 1677 & Rhinanthus rumelicus Velen. & Hortobágy & 1947 & Soó Rezső & BPU-00070.JPG \\
\hline 1677 & Rhinanthus rumelicus Velen. & Kács & 1933 & Boros Ádám & BPU-00084.JPG \\
\hline 1677 & Rhinanthus rumelicus Velen. & Miskolc & 1938 & Soó Rezső & BPU-00085.JPG \\
\hline 1677 & Rhinanthus rumelicus Velen. & Szinpetri & 1928 & Boros Ádám & BPU-00083.JPG \\
\hline 1677 & Rhinanthus rumelicus Velen. & Tarpa & 1948 & $\begin{array}{l}\text { Pólya László, Simon } \\
\text { Tibor, Jakucs Pál }\end{array}$ & BPU-00069.JPG \\
\hline 1678 & Lathraea squamaria L. & "Cuha-völgy" & 1954 & Soó Rezső & BPU-00116.JPG \\
\hline 1678 & Lathraea squamaria L. & Balatonszőlős & 1959 & Felföldy Lajos & BPU-00112.JPG \\
\hline 1678 & Lathraea squamaria L. & Bátorliget & 1932 & Soó Rezső & BPU-00117.JPG \\
\hline 1678 & Lathraea squamaria L. & Gánt & 1949 & Felföldy Lajos & BPU-00113.JPG \\
\hline 1678 & Lathraea squamaria L. & Miskolc & 1949 & Jakucs Pál & BPU-00114.JPG \\
\hline 1681 & Globularia cordifolia L. & Sopron & 1950 & Tallós Pál & BPU-00151.JPG \\
\hline 1682 & Globularia punctata Lapeyr. & Balatonszőlős & 1958 & Felföldy Lajos & BPU-00145.JPG \\
\hline 1682 & Globularia punctata Lapeyr. & Balatonszőlős & 1959 & Felföldy Lajos & BPU-00144.JPG \\
\hline 1682 & Globularia punctata Lapeyr. & Budaörs & 1982 & Felföldy Lajos & BPU-00142.JPG \\
\hline 1682 & Globularia punctata Lapeyr. & Budapest & 1953 & Soó Rezső & BPU-00137.JPG \\
\hline 1682 & Globularia punctata Lapeyr. & Budapest & 1953 & Soó Rezső & BPU-15409.JPG \\
\hline 1682 & Globularia punctata Lapeyr. & Budapest & 1957 & Isépy István & BPU-00139.JPG \\
\hline 1682 & Globularia punctata Lapeyr. & Budapest & 1982 & Felföldy Lajos & BPU-00138.JPG \\
\hline 1682 & Globularia punctata Lapeyr. & Dabas & 1959 & Simon Tibor & BPU-00143.JPG \\
\hline 1682 & Globularia punctata Lapeyr. & Gánt & 1949 & Soó Rezső & BPU-00141.JPG \\
\hline 1682 & Globularia punctata Lapeyr. & Lesenceistvánd & 1952 & Borsos Olga & BPU-00147.JPG \\
\hline 1682 & Globularia punctata Lapeyr. & Pécs & 1953 & Soó Rezső, Borsos Olga & BPU-00140.JPG \\
\hline 1682 & Globularia punctata Lapeyr. & Tihany & 1958 & Felföldy Lajos & BPU-00146.JPG \\
\hline 1682 & Globularia punctata Lapeyr. & Uzsa & 1959 & $\begin{array}{l}\text { Jávorka Sándor, Felföldy } \\
\text { Lajos }\end{array}$ & BPU-00148.JPG \\
\hline 1682 & Globularia punctata Lapeyr. & Vonyarcvashegy & 1930 & Lengyel Géza & BPU-00149.JPG \\
\hline 1685 & Orobanche ramosa L. & Hosszúpályi & 1933 & Soó Rezső & BPU-00152.JPG \\
\hline 1685 & Orobanche ramosa L. & Kiskunhalas & 1964 & Soó Rezső & BPU-00153.JPG \\
\hline 1687 & Orobanche purpurea Jacq. & Debrecen & 1941 & $\begin{array}{l}\text { Igmándy József, Soó } \\
\text { Rezső }\end{array}$ & BPU-00165.JPG \\
\hline 1687 & Orobanche purpurea Jacq. & Zamárdi & 1957 & Entz Béla & BPU-00158.JPG \\
\hline 1688 & Orobanche arenaria Borkh. & Boldogkőváralja & 1938 & Soó Rezső & BPU-00157.JPG \\
\hline 1688 & Orobanche arenaria Borkh. & Debrecen & 1932 & Soó Rezső & BPU-00156.JPG \\
\hline 1688 & Orobanche arenaria Borkh. & Nagyharsány & 1956 & Soó Rezső & BPU-00155.JPG \\
\hline 1692 & Orobanche cumana Wallr. & Kisar & 1948 & Simon Tibor, Jakucs Pál & BPU-00164.JPG \\
\hline 1692 & Orobanche cumana Wallr. & Kistelek & 1953 & Soó Rezső & BPU-00166.JPG \\
\hline 1692 & Orobanche cumana Wallr. & Kunadacs & 1954 & Soó Rezső & BPU-00167.JPG \\
\hline 1694 & Orobanche alba Stephan ex Willd. & Balatonszőlős & 1957 & Felföldy Lajos & BPU-00159.JPG \\
\hline
\end{tabular}


1. Elektroniukus melléklet - Electronic Appendix 1.

\begin{tabular}{|c|c|c|c|c|c|}
\hline $\begin{array}{l}\text { Sorszám / } \\
\text { Number }\end{array}$ & Taxon-név / Taxon-name & $\begin{array}{l}\text { Település / } \\
\text { Settlement }\end{array}$ & $\begin{array}{l}\text { Év / } \\
\text { Year }\end{array}$ & Gyűjtő / Collector & $\begin{array}{l}\text { Fájlnév / } \\
\text { File-name }\end{array}$ \\
\hline 1694 & Orobanche alba Stephan ex Willd. & Isztimér & 1949 & Soó Rezső & BPU-00162.JPG \\
\hline 1694 & Orobanche alba Stephan ex Willd. & Jósvafő & 1952 & Jakucs Pál, Ér Lajos & BPU-00160.JPG \\
\hline 1695 & Orobanche reticulata Wallr. & Tihany & 1959 & Felföldy Lajos & BPU-00163.JPG \\
\hline 1697 & Orobanche minor Sm. & Debrecen & 1947 & Csongor Győző & BPU-00168.JPG \\
\hline 1700 & Orobanche gracilis $\mathrm{Sm}$. & Sopron & 1944 & Kárpáti Zoltán & BPU-00169.JPG \\
\hline 1701 & Orobanche caryophyllacea Sm. & Badacsonytomaj & 1928 & Soó Rezső & BPU-00184.JPG \\
\hline 1701 & Orobanche caryophyllacea Sm. & Gyöngyös & 1961 & Soó Rezső & BPU-00177.JPG \\
\hline 1701 & Orobanche caryophyllacea Sm. & Isztimér & 1955 & Soó Rezső & BPU-00178.JPG \\
\hline 1701 & Orobanche caryophyllacea Sm. & Isztimér & 1955 & Soó Rezső & BPU-00179.JPG \\
\hline 1701 & Orobanche caryophyllacea Sm. & Nagyvisnyó & 1936 & Soó Rezső & BPU-00175.JPG \\
\hline 1701 & Orobanche caryophyllacea Sm. & Nagyvisnyó & 1953 & Soó Rezső & BPU-00176.JPG \\
\hline 1701 & Orobanche caryophyllacea Sm. & Örvényes & 1950 & Felföldy Lajos & BPU-00174.JPG \\
\hline 1701 & Orobanche caryophyllacea Sm. & Szentendre & 1916 & Andrasovszky József & BPU-00182.JPG \\
\hline 1701 & Orobanche caryophyllacea Sm. & Tihany & 1955 & Felföldy Lajos & BPU-00181.JPG \\
\hline 1701 & Orobanche caryophyllacea Sm. & Villány & 1956 & Soó Rezső & BPU-00185.JPG \\
\hline 1703 & Orobanche lutea Baumg. & Lábatlan & 1953 & Felföldy Lajos & BPU-00172.JPG \\
\hline 1703 & Orobanche lutea Baumg. & Nagyharsány & 1936 & Soó Rezső & BPU-00173.JPG \\
\hline 1703 & Orobanche lutea Baumg. & Vászoly & 1951 & Felföldy Lajos, Zsolt János & BPU-00171.JPG \\
\hline 1705 & Orobanche flava Mart. ex F.W. Schultz & Bakonybél & 1955 & Tallós Pál & BPU-00180.JPG \\
\hline $\begin{array}{c}1685- \\
1707\end{array}$ & Orobanche & Arka & 1938 & Soó Rezső & BPU-00188.JPG \\
\hline $\begin{array}{c}1685- \\
1707\end{array}$ & Orobanche & Bélapátfalva & 1953 & Simon Tibor & BPU-00186.JPG \\
\hline $\begin{array}{c}1685- \\
1707\end{array}$ & Orobanche & Budapest & 1947 & Soó Rezső & BPU-00189.JPG \\
\hline $\begin{array}{l}1685- \\
1707\end{array}$ & Orobanche & Komjáti & 1950 & Jakucs Pál, Kulcsár Gábor & BPU-00187.JPG \\
\hline 1708 & Pinguicula vulgaris L. & Sopron & 1932 & Soó Rezső & BPU-00118.JPG \\
\hline 1709 & Pinguicula alpina L. & Lesenceistvánd & 1928 & Soó Rezső & BPU-00122.JPG \\
\hline 1711 & Utricularia minor L. & Uzsa & 1949 & Károlyi Árpád & BPU-00133.JPG \\
\hline 1712 & Utricularia bremii Heer & Isaszeg & 1949 & Soó Rezső & BPU-00134.JPG \\
\hline 1712 & Utricularia bremii Heer & Kállósemjén & 1948 & Simon Tibor & BPU-00135.JPG \\
\hline 1712 & Utricularia bremii Heer & Veresegyház & 1949 & Soó Rezső & BPU-00136.JPG \\
\hline 1713 & Utricularia vulgaris L. & Badacsonytomaj & 1984 & Felföldy Lajos & BPU-00129.JPG \\
\hline 1713 & Utricularia vulgaris L. & Debrecen & 1937 & Soó Rezső & BPU-00127.JPG \\
\hline 1713 & Utricularia vulgaris L. & Hortobágy & 1963 & Isépy István & BPU-00128.JPG \\
\hline 1713 & Utricularia vulgaris L. & Szarvas & 1947 & Soó Rezső & BPU-00126.JPG \\
\hline 1713 & Utricularia vulgaris $\mathrm{L}$. & Tihany & 1956 & $\begin{array}{l}\text { Felföldy Lajos, Tóth } \\
\text { László }\end{array}$ & BPU-00130.JPG \\
\hline 1713 & Utricularia vulgaris L. & Tiszafüred & 1953 & Borsos Olga & BPU-15549.JPG \\
\hline 1713 & Utricularia vulgaris L. & Uzsa & 1952 & Simon Tibor & BPU-00131.JPG \\
\hline 1715 & Sherardia arvensis L. & Borzavár & 1934 & Polgár Sándor & BPU-03859.JPG \\
\hline 1715 & Sherardia arvensis L. & Nagyharsány & 1956 & Soó Rezső & BPU-03857.JPG \\
\hline 1715 & Sherardia arvensis L. & Nagyharsány & 1965 & $\begin{array}{l}\text { Simon Tibor, Priszter } \\
\text { Szaniszló, Borhidi Attila }\end{array}$ & BPU-03858.JPG \\
\hline 1716 & Asperula taurina L. & Pécs & 1953 & Soó Rezső, Borsos Olga & BPU-03860.JPG \\
\hline 1716 & Asperula taurina L. & Pécsvárad & 1936 & Soó Rezső & BPU-03861.JPG \\
\hline 1716 & Asperula taurina L. & Villány & 1956 & Soó Rezső & BPU-03862.JPG \\
\hline 1717 & Asperula tinctoria L. & Isztimér & 1949 & Soó Rezső & BPU-03895.JPG \\
\hline
\end{tabular}


1. Elektroniukus melléklet - Electronic Appendix 1.

\begin{tabular}{|c|c|c|c|c|c|}
\hline $\begin{array}{l}\text { Sorszám / } \\
\text { Number }\end{array}$ & Taxon-név / Taxon-name & $\begin{array}{l}\text { Település / } \\
\text { Settlement }\end{array}$ & $\begin{array}{l}\text { Év / } \\
\text { Year }\end{array}$ & Gyűjtő / Collector & $\begin{array}{c}\text { Fájlnév / } \\
\text { File-name }\end{array}$ \\
\hline 1717 & Asperula tinctoria L. & Uzsa & 1959 & $\begin{array}{l}\text { Jávorka Sándor, Felföldy } \\
\text { Lajos }\end{array}$ & BPU-03896.JPG \\
\hline 1718 & Asperula cynanchica L. & Budapest & 1982 & Felföldy Lajos & BPU-03900.JPG \\
\hline 1718 & Asperula cynanchica L. & Budapest & 1991 & Felföldy Lajos & BPU-03899.JPG \\
\hline 1718 & Asperula cynanchica L. & Mátraszentimre & 1961 & Soó Rezső & BPU-03902.JPG \\
\hline 1718 & Asperula cynanchica L. & Miskolc & 1929 & Soó Rezső & BPU-03901.JPG \\
\hline 1718 & Asperula cynanchica L. & Nagybajom & 1957 & Soó Rezső & BPU-03903.JPG \\
\hline 1718 & Asperula cynanchica L. & Veszprém & 1931 & Soó Rezső & BPU-03904.JPG \\
\hline 1718 & Asperula cynanchica L. & Zámoly & 1982 & Felföldy Lajos & BPU-03898.JPG \\
\hline 1722 & Galium rotundifolium L. & Ágfalva & 1947 & Kárpáti Zoltán & BPU-03937.JPG \\
\hline 1722 & Galium rotundifolium L. & Alsószölnök & 1955 & Soó Rezső & BPU-03940.JPG \\
\hline 1722 & Galium rotundifolium L. & Döbrönte & 1955 & Tallós Pál & BPU-03935.JPG \\
\hline 1722 & Galium rotundifolium L. & Kőszeg & 1938 & Soó Rezső & BPU-03939.JPG \\
\hline 1722 & Galium rotundifolium L. & Sopron & 1960 & Borsos Olga, Simon Tibor & BPU-03936.JPG \\
\hline 1722 & Galium rotundifolium L. & Szakonyfalu & 1955 & Soó Rezső & BPU-03938.JPG \\
\hline 1723 & Galium boreale L. & "Szentendrei-sziget" & 1947 & Kárpáti Zoltán & BPU-03947.JPG \\
\hline 1723 & Galium boreale L. & Aszaló & 1944 & Siroki Zoltán & BPU-03951.JPG \\
\hline 1723 & Galium boreale L. & Bagamér & 1932 & Soó Rezső & BPU-03943.JPG \\
\hline 1723 & Galium boreale L. & Budapest & 2005 & Felföldy Lajos & BPU-03941.JPG \\
\hline 1723 & Galium boreale L. & Debrecen & 1989 & Felföldy Lajos & BPU-03944.JPG \\
\hline 1723 & Galium boreale L. & Gávavencsellő & 1990 & Felföldy Lajos & BPU-03948.JPG \\
\hline 1723 & Galium boreale L. & Kerecsend & 1957 & Soó Rezső & BPU-03945.JPG \\
\hline 1723 & Galium boreale L. & Miskolc & 1934 & Soó Rezső & BPU-03942.JPG \\
\hline 1723 & Galium boreale L. & Nagykanizsa & 1950 & Károlyi Árpád & BPU-03949.JPG \\
\hline 1723 & Galium boreale L. & Szigetmonostor & 1947 & Soó Rezső & BPU-03946.JPG \\
\hline 1723 & Galium boreale L. & Tiszafüred & 1952 & Soó Rezső & BPU-03959.JPG \\
\hline 1723 & Galium boreale L. & Tiszafüred & 1952 & Soó Rezső & BPU-15457.JPG \\
\hline 1724 & Galium rubioides L. & Bátorliget & 1932 & Soó Rezső & BPU-03957.JPG \\
\hline 1724 & Galium rubioides L. & Doboz & 1935 & Soó Rezső & BPU-03952.JPG \\
\hline 1724 & Galium rubioides L. & Erdőbénye & 1948 & Soó Rezső & BPU-03960.JPG \\
\hline 1724 & Galium rubioides L. & Miskolc & 1934 & Soó Rezső & BPU-03954.JPG \\
\hline 1724 & Galium rubioides L. & Mosonmagyaróvár & 1962 & Borsos Olga & BPU-03956.JPG \\
\hline 1724 & Galium rubioides L. & Szilvásvárad & 1953 & $\begin{array}{l}\text { Felföldy Lajos, Tóth } \\
\text { László }\end{array}$ & BPU-03955.JPG \\
\hline 1724 & Galium rubioides L. & Tarpa & 1949 & Soó Rezső & BPU-03953.JPG \\
\hline 1724 & Galium rubioides L. & Tiszafüred & 1953 & Borsos Olga & BPU-15475.JPG \\
\hline 1724 & Galium rubioides L. & Tiszaroff & 1937 & Soó Rezső & BPU-03958.JPG \\
\hline 1725 & Galium rivale (Sibth. et Sm.) Griseb. & Budapest & 1990 & Felföldy Lajos & BPU-03876.JPG \\
\hline 1725 & Galium rivale (Sibth. et Sm.) Griseb. & Göd & 1948 & Soó Rezső & BPU-03881.JPG \\
\hline 1725 & Galium rivale (Sibth. et Sm.) Griseb. & Ipolytarnóc & 1987 & Felföldy Lajos & BPU-03880.JPG \\
\hline 1725 & Galium rivale (Sibth. et Sm.) Griseb. & Miskolc & 1950 & Soó Rezső & BPU-03878.JPG \\
\hline 1726 & Galium odoratum (L.) Scop. & Beregdaróc & 1951 & $\begin{array}{l}\text { Simon Tibor, Jakucs Pál, } \\
\text { Kulcsár Gábor }\end{array}$ & BPU-03865.JPG \\
\hline 1726 & Galium odoratum (L.) Scop. & Budapest & 2004 & Felföldy Lajos & BPU-03863.JPG \\
\hline 1726 & Galium odoratum (L.) Scop. & Gyöngyös & 1961 & Soó Rezső & BPU-03867.JPG \\
\hline 1726 & Galium odoratum (L.) Scop. & Kapolcs & 1959 & $\begin{array}{l}\text { Jávorka Sándor, Felföldy } \\
\text { Lajos, Tóth László }\end{array}$ & BPU-03874.JPG \\
\hline 1726 & Galium odoratum (L.) Scop. & Lábatlan & 1953 & Felföldy Lajos & BPU-03868.JPG \\
\hline 1726 & Galium odoratum (L.) Scop. & Lesenceistvánd & 1952 & Borsos Olga & BPU-03872.JPG \\
\hline
\end{tabular}


1. Elektroniukus melléklet - Electronic Appendix 1.

\begin{tabular}{|c|c|c|c|c|c|}
\hline $\begin{array}{l}\text { Sorszám / } \\
\text { Number }\end{array}$ & Taxon-név / Taxon-name & $\begin{array}{l}\text { Település / } \\
\text { Settlement }\end{array}$ & $\begin{array}{l}\text { Év / } \\
\text { Year }\end{array}$ & Gyújtő / Collector & $\begin{array}{l}\text { Fájlnév / } \\
\text { File-name }\end{array}$ \\
\hline 1726 & Galium odoratum (L.) Scop. & Miskolc & 1939 & Soó Rezső & BPU-03866.JPG \\
\hline 1726 & Galium odoratum (L.) Scop. & Mosonmagyaróvár & 1965 & Borsos Olga & BPU-03869.JPG \\
\hline 1726 & Galium odoratum (L.) Scop. & Pécs & 1935 & Soó Rezső & BPU-03864.JPG \\
\hline 1726 & Galium odoratum (L.) Scop. & Sopron & 1960 & Borsos Olga, Simon Tibor & BPU-03871.JPG \\
\hline 1726 & Galium odoratum (L.) Scop. & Uzsa & 1959 & $\begin{array}{l}\text { Jávorka Sándor, Felföldy } \\
\text { Lajos }\end{array}$ & BPU-03873.JPG \\
\hline 1726 & Galium odoratum (L.) Scop. & Vászoly & 1958 & Felföldy Lajos & BPU-03875.JPG \\
\hline 1727 & Galium humifusum M. Bieb. & Szeged & 1950 & Timár Lajos & BPU-03911.JPG \\
\hline 1728 & Galium glaucum L. & Budapest & 1947 & Soó Rezső & BPU-03883.JPG \\
\hline 1728 & Galium glaucum L. & Budapest & 2005 & Felföldy Lajos & BPU-03884.JPG \\
\hline 1728 & Galium glaucum L. & Fót & 1960 & Soó Rezső & BPU-03890.JPG \\
\hline 1728 & Galium glaucum L. & Gyöngyös & 1965 & Soó Rezső & BPU-03888.JPG \\
\hline 1728 & Galium glaucum L. & Konyár & 1949 & Soó Rezső & BPU-03885.JPG \\
\hline 1728 & Galium glaucum L. & Lábatlan & 1953 & Felföldy Lajos & BPU-03889.JPG \\
\hline 1728 & Galium glaucum L. & Miskolc & 1934 & Soó Rezső & BPU-03887.JPG \\
\hline 1728 & Galium glaucum L. & Miskolc & 1960 & Simon Tibor & BPU-03886.JPG \\
\hline 1728 & Galium glaucum L. & Sárospatak & 1949 & Simon Tibor, Jakucs Pál & BPU-03894.JPG \\
\hline 1728 & Galium glaucum L. & Tihany & 1959 & Felföldy Lajos & BPU-03893.JPG \\
\hline 1728 & Galium glaucum L. & Tihany & 1961 & $\begin{array}{l}\text { Pankov H., Felföldy Lajos, } \\
\text { Sólymosy G. }\end{array}$ & BPU-03892.JPG \\
\hline 1728 & Galium glaucum L. & Visegrád & 1964 & Soó Rezső, Borsos Olga & BPU-03891.JPG \\
\hline 1729 & Galium palustre L. & Aszófő & 1947 & Pólya László & BPU-04013.JPG \\
\hline 1729 & Galium palustre L. & Aszófő & 1959 & Felföldy Lajos & BPU-03998.JPG \\
\hline 1729 & Galium palustre L. & Balatonszepezd & 1958 & Felföldy Lajos & BPU-04003.JPG \\
\hline 1729 & Galium palustre L. & Balatonszőlős & 1956 & $\begin{array}{l}\text { Felföldy Lajos, Tóth } \\
\text { László }\end{array}$ & BPU-04001.JPG \\
\hline 1729 & Galium palustre L. & Balatonszőlős & 1957 & Felföldy Lajos & BPU-03999.JPG \\
\hline 1729 & Galium palustre L. & Balatonszőlős & 1957 & Felföldy Lajos & BPU-04000.JPG \\
\hline 1729 & Galium palustre L. & Bátorliget & 1932 & Soó Rezső & BPU-03997.JPG \\
\hline 1729 & Galium palustre L. & Bátorliget & 1932 & Soó Rezső & BPU-04012.JPG \\
\hline 1729 & Galium palustre L. & Beregdaróc & 1993 & Felföldy Lajos & BPU-03991.JPG \\
\hline 1729 & Galium palustre L. & Budapest & 1991 & Felföldy Lajos & BPU-03985.JPG \\
\hline 1729 & Galium palustre L. & Budapest & 1993 & Felföldy Lajos & BPU-03986.JPG \\
\hline 1729 & Galium palustre L. & Bugac & 1923 & Soó Rezső & BPU-03989.JPG \\
\hline 1729 & Galium palustre L. & Dabas & 1964 & Soó Rezső & BPU-03996.JPG \\
\hline 1729 & Galium palustre L. & Debrecen & 1933 & Soó Rezső & BPU-04011.JPG \\
\hline 1729 & Galium palustre L. & Gárdony & 1980 & Felföldy Lajos & BPU-04010.JPG \\
\hline 1729 & Galium palustre L. & Gyula & 1935 & Soó Rezső & BPU-03990.JPG \\
\hline 1729 & Galium palustre L. & Kapolcs & 1959 & $\begin{array}{l}\text { Jávorka Sándor, Felföldy } \\
\text { Lajos, Tóth László }\end{array}$ & BPU-04002.JPG \\
\hline 1729 & Galium palustre L. & Kishuta & 1952 & Simon Tibor & BPU-03987.JPG \\
\hline 1729 & Galium palustre L. & Lakitelek & 1961 & Simon Tibor & BPU-04006.JPG \\
\hline 1729 & Galium palustre L. & Magyarlukafa & 1957 & Soó Rezső & BPU-04007.JPG \\
\hline 1729 & Galium palustre L. & Miskolc & 1936 & Soó Rezső & BPU-04009.JPG \\
\hline 1729 & Galium palustre L. & Ócsa & 1953 & Soó Rezső, Borsos Olga & BPU-03995.JPG \\
\hline 1729 & Galium palustre L. & Pákozd & 1978 & Felföldy Lajos & BPU-03992.JPG \\
\hline 1729 & Galium palustre L. & Pákozd & 1980 & Felföldy Lajos & BPU-03993.JPG \\
\hline 1729 & Galium palustre L. & Pákozd & 1980 & Felföldy Lajos & BPU-03994.JPG \\
\hline 1729 & Galium palustre L. & Pálháza & 1949 & Simon Tibor, Jakucs Pál & BPU-03988.JPG \\
\hline
\end{tabular}


1. Elektroniukus melléklet - Electronic Appendix 1.

\begin{tabular}{|c|c|c|c|c|c|}
\hline $\begin{array}{l}\text { Sorszám / } \\
\text { Number }\end{array}$ & Taxon-név / Taxon-name & $\begin{array}{l}\text { Település / } \\
\text { Settlement }\end{array}$ & $\begin{array}{l}\text { Év / } \\
\text { Year }\end{array}$ & Gyűjtő / Collector & $\begin{array}{l}\text { Fájlnév / } \\
\text { File-name }\end{array}$ \\
\hline 1729 & Galium palustre L. & Tákos & 1948 & $\begin{array}{l}\text { Pólya László, Simon } \\
\text { Tibor, Jakucs Pál }\end{array}$ & BPU-04008.JPG \\
\hline 1729 & Galium palustre L. & Tihany & 1956 & Felföldy Lajos & BPU-04005.JPG \\
\hline 1729 & Galium palustre L. & Tihany & 1956 & $\begin{array}{l}\text { Felföldy Lajos, Tóth } \\
\text { László }\end{array}$ & BPU-04004.JPG \\
\hline 1729 & Galium palustre L. & Tihany & 1956 & $\begin{array}{l}\text { Felföldy Lajos, Tóth } \\
\text { László }\end{array}$ & BPU-04015.JPG \\
\hline 1729 & Galium palustre L. & Zalakomár & 1949 & Károlyi Árpád & BPU-04017.JPG \\
\hline 1729 & Galium palustre L. & Zánka & 1958 & Felföldy Lajos & BPU-04016.JPG \\
\hline 1730 & $\begin{array}{l}\text { Galium elongatum C. Presl in J. Presl et } \\
\text { C. Presl }\end{array}$ & Paloznak & 1956 & $\begin{array}{l}\text { Felföldy Lajos, Tóth } \\
\text { László }\end{array}$ & BPU-04014.JPG \\
\hline 1731 & Galium tricornutum Dandy & Balatonszőlős & 1956 & $\begin{array}{l}\text { Felföldy Lajos, Tóth } \\
\text { László }\end{array}$ & BPU-03963.JPG \\
\hline 1731 & Galium tricornutum Dandy & Szentendre & 1947 & Vajda László & BPU-03962.JPG \\
\hline 1731 & Galium tricornutum Dandy & Uzsa & 1959 & $\begin{array}{l}\text { Jávorka Sándor, Felföldy } \\
\text { Lajos }\end{array}$ & BPU-03961.JPG \\
\hline 1732 & Galium aparine L. & Budapest & 2004 & Felföldy Lajos & BPU-03964.JPG \\
\hline 1732 & Galium aparine L. & Budapest & 2005 & Felföldy Lajos & BPU-03965.JPG \\
\hline 1732 & Galium aparine L. & Debrecen & 1933 & Soó Rezső & BPU-03879.JPG \\
\hline 1732 & Galium aparine L. & Egyek & 1947 & Soó Rezső & BPU-03967.JPG \\
\hline 1732 & Galium aparine L. & Miskolc & 1934 & Soó Rezső & BPU-03877.JPG \\
\hline 1732 & Galium aparine L. & Tihany & 1957 & Soó Rezső & BPU-03970.JPG \\
\hline 1733 & Galium spurium L. & Budaörs & 1947 & Soó Rezső & BPU-03969.JPG \\
\hline 1733 & Galium spurium L. & Budapest & 1991 & Felföldy Lajos & BPU-03968.JPG \\
\hline 1733 & Galium spurium L. & Vácrátót & 1950 & Soó Rezső & BPU-03966.JPG \\
\hline 1734 & Galium uliginosum L. & Debrecen & 1952 & Kulcsár Gábor & BPU-15450.JPG \\
\hline 1734 & Galium uliginosum L. & Debrecen & 1952 & Kulcsár Gábor & BPU-15451.JPG \\
\hline 1734 & Galium uliginosum L. & Debrecen & 1989 & Felföldy Lajos & BPU-03973.JPG \\
\hline 1734 & Galium uliginosum L. & Isaszeg & 1949 & Soó Rezső & BPU-03974.JPG \\
\hline 1734 & Galium uliginosum L. & Isaszeg & 1949 & Soó Rezső & BPU-15521.JPG \\
\hline 1734 & Galium uliginosum L. & Nyírpilis & 1932 & Soó Rezső & BPU-03975.JPG \\
\hline 1734 & Galium uliginosum L. & Pákozd & 1980 & Felföldy Lajos & BPU-03972.JPG \\
\hline 1734 & Galium uliginosum L. & Pálháza & 1949 & Simon Tibor, Jakucs Pál & BPU-03971.JPG \\
\hline 1734 & Galium uliginosum L. & Tapolca & 1928 & Soó Rezső & BPU-03976.JPG \\
\hline 1735 & Galium tenuissimum M. Bieb. & Pomáz & 1923 & Boros Ádám & BPU-03978.JPG \\
\hline 1735 & Galium tenuissimum M. Bieb. & Uzsa & 1952 & Simon Tibor & BPU-03984.JPG \\
\hline 1736 & Galium parisiense L. & Kőszeg & 1948 & Kárpáti Zoltán & BPU-03979.JPG \\
\hline 1736 & Galium parisiense L. & Zalalövő & 1953 & Pócs Tamás & BPU-03980.JPG \\
\hline 1737 & Galium divaricatum Pourr. ex Lam. & Balatonakali & 1950 & Felföldy Lajos & BPU-03981.JPG \\
\hline 1737 & Galium divaricatum Pourr. ex Lam. & Böhönye & 1923 & Boros Ádám & BPU-03982.JPG \\
\hline 1737 & Galium divaricatum Pourr. ex Lam. & Pogányszentpéter & 1948 & Károlyi Árpád & BPU-03983.JPG \\
\hline 1738 & Galium pumilum Murray & Kőszeg & 1932 & Soó Rezső & BPU-04060.JPG \\
\hline 1738 & Galium pumilum Murray & Sopron & 1946 & Kárpáti Zoltán & BPU-04059.JPG \\
\hline 1738 & Galium pumilum Murray & Vászoly & 1950 & Felföldy Lajos & BPU-04063.JPG \\
\hline 1739 & Galium austriacum Jacq. & Budaörs & 1947 & Soó Rezső & BPU-04061.JPG \\
\hline 1739 & Galium austriacum Jacq. & Gyulakeszi & 1938 & Soó Rezső & BPU-04065.JPG \\
\hline 1739 & Galium austriacum Jacq. & Pilisszentiván & 1932 & Soó Rezső & BPU-04064.JPG \\
\hline 1740 & Galium verum L. & Bagamér & 1934 & Soó Rezső & BPU-04045.JPG \\
\hline 1740 & Galium verum L. & Bátorliget & 1934 & Soó Rezső & BPU-03933.JPG \\
\hline 1740 & Galium verum L. & Bokod & 1938 & Boros Ádám & BPU-04049.JPG \\
\hline
\end{tabular}


1. Elektroniukus melléklet - Electronic Appendix 1.

\begin{tabular}{|c|c|c|c|c|c|}
\hline $\begin{array}{l}\text { Sorszám / } \\
\text { Number }\end{array}$ & Taxon-név / Taxon-name & $\begin{array}{l}\text { Település / } \\
\text { Settlement }\end{array}$ & $\begin{array}{l}\text { Év / } \\
\text { Year }\end{array}$ & Gyűjtő / Collector & $\begin{array}{l}\text { Fájlnév / } \\
\text { File-name }\end{array}$ \\
\hline 1740 & Galium verum $\mathrm{L}$. & Budapest & 1982 & Felföldy Lajos & BPU-04041.JPG \\
\hline 1740 & Galium verum $\mathrm{L}$. & Budapest & 2004 & Felföldy Lajos & BPU-04039.JPG \\
\hline 1740 & Galium verum $\mathrm{L}$. & Budapest & 2004 & Felföldy Lajos & BPU-04040.JPG \\
\hline 1740 & Galium verum L. & Budapest & 2005 & Felföldy Lajos & BPU-04042.JPG \\
\hline 1740 & Galium verum $\mathrm{L}$. & Csévharaszt & 1959 & Simon Tibor, Borsos Olga & BPU-04050.JPG \\
\hline 1740 & Galium verum $\mathrm{L}$. & Debrecen & 1934 & Soó Rezső & BPU-04047.JPG \\
\hline 1740 & Galium verum $\mathrm{L}$. & Fertőhomok & 1960 & Tóth László & BPU-04053.JPG \\
\hline 1740 & Galium verum L. & Hévíz & 1961 & Felföldy Lajos & BPU-04056.JPG \\
\hline 1740 & Galium verum $\mathrm{L}$. & Kálmáncsa & 1922 & Boros Ádám & BPU-04052.JPG \\
\hline 1740 & Galium verum $\mathrm{L}$. & Kapolcs & 1959 & $\begin{array}{l}\text { Jávorka Sándor, Felföldy } \\
\text { Lajos, Tóth László }\end{array}$ & BPU-03927.JPG \\
\hline 1740 & Galium verum L. & Kőszeg & 1932 & Soó Rezső & BPU-04054.JPG \\
\hline 1740 & Galium verum $\mathrm{L}$. & Mezőcsát & 1934 & Soó Rezső & BPU-04046.JPG \\
\hline 1740 & Galium verum $\mathrm{L}$. & Miskolc & 1937 & Soó Rezső & BPU-03934.JPG \\
\hline 1740 & Galium verum L. & Parád & 1923 & Soó Rezső & BPU-04048.JPG \\
\hline 1740 & Galium verum $\mathrm{L}$. & Szarvas & 1949 & Soó Rezső & BPU-04043.JPG \\
\hline 1740 & Galium verum L. & Tarpa & 1949 & Soó Rezső & BPU-04044.JPG \\
\hline 1740 & Galium verum L. & Tihany & 1959 & Felföldy Lajos & BPU-04055.JPG \\
\hline 1740 & Galium verum $\mathrm{L}$. & Vác & 1917 & Boros Ádám & BPU-04051.JPG \\
\hline 1740 & Galium verum $\mathrm{L}$. & Vászoly & 1955 & $\begin{array}{l}\text { Felföldy Lajos, Tóth } \\
\text { László }\end{array}$ & BPU-03928.JPG \\
\hline 1741 & Galium sylvaticum L. & $\begin{array}{l}\text { Badacsonytomaj, } \\
\text { Badacsonytördemic }\end{array}$ & 1928 & Soó Rezső & BPU-04035.JPG \\
\hline 1741 & Galium sylvaticum L. & Csesznek & 1963 & Soó Rezső & BPU-04034.JPG \\
\hline 1741 & Galium sylvaticum L. & Hidegkút & 1957 & Felföldy Lajos & BPU-04028.JPG \\
\hline 1741 & Galium sylvaticum L. & Isztimér & 1949 & Soó Rezső & BPU-04033.JPG \\
\hline 1741 & Galium sylvaticum L. & Ivánc & 1955 & Soó Rezső & BPU-04038.JPG \\
\hline 1741 & Galium sylvaticum L. & Kőszeg & 1963 & Soó Rezső, Borsos Olga & BPU-04037.JPG \\
\hline 1741 & Galium sylvaticum L. & Nagybajom & 1955 & Soó Rezső & BPU-04036.JPG \\
\hline 1741 & Galium sylvaticum L. & Rezi & 1953 & Borsos Olga & BPU-04029.JPG \\
\hline 1741 & Galium sylvaticum L. & Uzsa & 1952 & Borsos Olga & BPU-04032.JPG \\
\hline 1741 & Galium sylvaticum L. & Zalakomár & 1954 & Borsos Olga & BPU-04031.JPG \\
\hline 1742 & Galium schultesii Vest & Budapest & 1982 & Felföldy Lajos & BPU-04018.JPG \\
\hline 1742 & Galium schultesii Vest & Diósjenő & 1949 & Pólya László & BPU-15520.JPG \\
\hline 1742 & Galium schultesii Vest & Dömös & 1948 & Soó Rezső & BPU-04022.JPG \\
\hline 1742 & Galium schultesii Vest & Gyöngyös & 1962 & Soó Rezső, Borsos Olga & BPU-04024.JPG \\
\hline 1742 & Galium schultesii Vest & Gyöngyös & 1963 & Soó Rezső & BPU-04023.JPG \\
\hline 1742 & Galium schultesii Vest & Kemence & 1952 & Simon Tibor & BPU-04025.JPG \\
\hline 1742 & Galium schultesii Vest & Nagykovácsi & 2005 & Felföldy Lajos & BPU-04026.JPG \\
\hline 1742 & Galium schultesii Vest & Nagyvisnyó & 1948 & $\begin{array}{l}\text { Simon Tibor, Pólya } \\
\text { László, Jakucs Pál }\end{array}$ & BPU-04021.JPG \\
\hline 1742 & Galium schultesii Vest & Tákos & 1949 & Soó Rezső & BPU-04020.JPG \\
\hline 1742 & Galium schultesii Vest & Telkibánya & 1950 & Soó Rezső & BPU-04019.JPG \\
\hline 1743 & Galium abaujense Borbás & Baskó & 1937 & Hulják János & BPU-04072.JPG \\
\hline 1743 & Galium abaujense Borbás & Bózsva & 1950 & Simon Tibor & BPU-04071.JPG \\
\hline 1743 & Galium abaujense Borbás & Erdőbénye & 1948 & Soó Rezső & BPU-04077.JPG \\
\hline 1743 & Galium abaujense Borbás & Háromhuta & 1949 & Simon Tibor, Jakucs Pál & BPU-04136.JPG \\
\hline 1743 & Galium abaujense Borbás & Miskolc & 1927 & Hulják János & BPU-04075.JPG \\
\hline 1743 & Galium abaujense Borbás & Miskolc & 1929 & Soó Rezső & BPU-04076.JPG \\
\hline
\end{tabular}


1. Elektroniukus melléklet - Electronic Appendix 1.

\begin{tabular}{|c|c|c|c|c|c|}
\hline $\begin{array}{l}\text { Sorszám / } \\
\text { Number }\end{array}$ & Taxon-név / Taxon-name & $\begin{array}{l}\text { Település / } \\
\text { Settlement }\end{array}$ & $\begin{array}{l}\text { Év / } \\
\text { Year }\end{array}$ & Gyűjtő / Collector & $\begin{array}{l}\text { Fájlnév / } \\
\text { File-name }\end{array}$ \\
\hline 1743 & Galium abaujense Borbás & Nagyvisnyó & 1953 & Vozáry Elemér & BPU-04074.JPG \\
\hline 1743 & Galium abaujense Borbás & Sárospatak & 1920 & Boros Ádám & BPU-04078.JPG \\
\hline 1743 & Galium abaujense Borbás & Telkibánya & 1956 & Soó Rezső & BPU-04073.JPG \\
\hline 1743 & Galium abaujense Borbás & Tokaj & 1935 & Hulják János & BPU-04079.JPG \\
\hline 1744 & Galium lucidum All. & Magyarlukafa & 1957 & Soó Rezső & BPU-04120.JPG \\
\hline 1744 & Galium lucidum All. & Nagyharsány & 1956 & Soó Rezső & BPU-04118.JPG \\
\hline 1744 & Galium lucidum All. & Nagyharsány & 1965 & $\begin{array}{l}\text { Simon Tibor, Priszter } \\
\text { Szaniszló, Borhidi Attila }\end{array}$ & BPU-04121.JPG \\
\hline 1745 & Galium mollugo L. & Aggtelek & 1951 & Soó Rezső & BPU-04108.JPG \\
\hline 1745 & Galium mollugo L. & Bicske & 1991 & Felföldy Lajos & BPU-04093.JPG \\
\hline 1745 & Galium mollugo L. & Bicske & 1991 & Felföldy Lajos & BPU-04094.JPG \\
\hline 1745 & Galium mollugo L. & Bicske & 1991 & Felföldy Lajos & BPU-04095.JPG \\
\hline 1745 & Galium mollugo L. & Budapest & 1947 & Soó Rezső & BPU-04084.JPG \\
\hline 1745 & Galium mollugo L. & Budapest & 1982 & Felföldy Lajos & BPU-04086.JPG \\
\hline 1745 & Galium mollugo L. & Budapest & 1991 & Felföldy Lajos & BPU-04085.JPG \\
\hline 1745 & Galium mollugo L. & Budapest & 2005 & Felföldy Lajos & BPU-04083.JPG \\
\hline 1745 & Galium mollugo L. & Csabrendek & 1988 & Dévai György & BPU-04090.JPG \\
\hline 1745 & Galium mollugo L. & Csákvár & 1937 & Boros Ádám & BPU-04106.JPG \\
\hline 1745 & Galium mollugo L. & Dédestapolcsány & 1932 & $\begin{array}{l}\text { Zólyomi Bálint, Máthé } \\
\text { Imre }\end{array}$ & BPU-04109.JPG \\
\hline 1745 & Galium mollugo L. & Gödöllő & 1922 & Soó Rezső & BPU-04117.JPG \\
\hline 1745 & Galium mollugo L. & Gyöngyös & 1935 & Soó Rezső & BPU-04112.JPG \\
\hline 1745 & Galium mollugo L. & Gyöngyös & 1961 & Soó Rezső & BPU-04116.JPG \\
\hline 1745 & Galium mollugo L. & Hajdúsámson & 1933 & Soó Rezső & BPU-04087.JPG \\
\hline 1745 & Galium mollugo L. & Isztimér & 1949 & Soó Rezső & BPU-04113.JPG \\
\hline 1745 & Galium mollugo L. & Konyár & 1949 & Soó Rezső & BPU-04099.JPG \\
\hline 1745 & Galium mollugo L. & Kőszeg & 1932 & Soó Rezső & BPU-04103.JPG \\
\hline 1745 & Galium mollugo L. & Miskolc & 1929 & Soó Rezső & BPU-04114.JPG \\
\hline 1745 & Galium mollugo L. & Miskolc & 1929 & Soó Rezső & BPU-04115.JPG \\
\hline 1745 & Galium mollugo L. & Miskolc & 1932 & Soó Rezső & BPU-04066.JPG \\
\hline 1745 & Galium mollugo L. & Miskolc & 1934 & Soó Rezső & BPU-04110.JPG \\
\hline 1745 & Galium mollugo L. & Miskolc & 1937 & Soó Rezső & BPU-04100.JPG \\
\hline 1745 & Galium mollugo L. & Pécs & 1953 & Soó Rezső, Borsos Olga & BPU-04097.JPG \\
\hline 1745 & Galium mollugo L. & Somogyszob & 1957 & Soó Rezső & BPU-04102.JPG \\
\hline 1745 & Galium mollugo L. & Tarján & 1935 & Boros Ádám & BPU-04107.JPG \\
\hline 1745 & Galium mollugo L. & Tarpa & 1949 & Soó Rezső & BPU-04098.JPG \\
\hline 1745 & Galium mollugo L. & Telekes & 1955 & Soó Rezső & BPU-04104.JPG \\
\hline 1745 & Galium mollugo L. & Tibolddaróc & 1953 & Soó Rezső & BPU-04111.JPG \\
\hline 1745 & Galium mollugo L. & Tihany & 1949 & Simon Tibor & BPU-04068.JPG \\
\hline 1745 & Galium mollugo L. & Tihany & 1956 & $\begin{array}{l}\text { Felföldy Lajos, Tóth } \\
\text { László }\end{array}$ & BPU-04092.JPG \\
\hline 1745 & Galium mollugo L. & Tihany & 1958 & Felföldy Lajos & BPU-04070.JPG \\
\hline 1745 & Galium mollugo L. & Tihany & 1959 & Felföldy Lajos & BPU-04088.JPG \\
\hline 1745 & Galium mollugo L. & Tihany & 1959 & Felföldy Lajos & BPU-04089.JPG \\
\hline 1745 & Galium mollugo L. & Tihany & 1959 & Felföldy Lajos & BPU-04091.JPG \\
\hline 1745 & Galium mollugo L. & Tokaj & 1933 & Soó Rezső & BPU-04067.JPG \\
\hline 1745 & Galium mollugo L. & Törökbálint & 1991 & Felföldy Lajos & BPU-04096.JPG \\
\hline 1745 & Galium mollugo L. & Vácrátót & 1949 & Soó Rezső & BPU-04101.JPG \\
\hline
\end{tabular}


1. Elektroniukus melléklet - Electronic Appendix 1.

\begin{tabular}{|c|c|c|c|c|c|}
\hline $\begin{array}{l}\text { Sorszám / } \\
\text { Number }\end{array}$ & Taxon-név / Taxon-name & $\begin{array}{l}\text { Település / } \\
\text { Settlement }\end{array}$ & $\begin{array}{l}\text { Év / } \\
\text { Year }\end{array}$ & Gyújtő / Collector & $\begin{array}{l}\text { Fájlnév / } \\
\text { File-name }\end{array}$ \\
\hline 1746 & Galium album Mill. & Balatonszőlős & 1956 & $\begin{array}{l}\text { Felföldy Lajos, Tóth } \\
\text { László }\end{array}$ & BPU-04105.JPG \\
\hline 1746 & Galium album Mill. & Budapest & 1918 & Kocsis István & BPU-04069.JPG \\
\hline $\begin{array}{c}1740 \times \\
1743\end{array}$ & $\begin{array}{l}\text { Galium verum L. × Galium abaujense } \\
\text { Borbás }\end{array}$ & Abaújalpár & 1948 & Soó Rezső & BPU-04134.JPG \\
\hline $\begin{array}{c}1740 \times \\
1743\end{array}$ & $\begin{array}{l}\text { Galium verum L. × Galium abaujense } \\
\text { Borbás }\end{array}$ & Abaújkér & 1938 & Hulják János & BPU-04131.JPG \\
\hline $\begin{array}{c}1740 \times \\
1743\end{array}$ & $\begin{array}{l}\text { Galium verum L. × Galium abaujense } \\
\text { Borbás }\end{array}$ & Abaújkér & 1938 & Hulják János & BPU-04137.JPG \\
\hline $\begin{array}{c}1740 \times \\
1743\end{array}$ & $\begin{array}{l}\text { Galium verum L. × Galium abaujense } \\
\text { Borbás }\end{array}$ & Miskolc & 1929 & Soó Rezső & BPU-04132.JPG \\
\hline $\begin{array}{c}1740 \times \\
1743\end{array}$ & $\begin{array}{l}\text { Galium verum L. × Galium abaujense } \\
\text { Borbás }\end{array}$ & Miskolc & 1929 & Soó Rezső & BPU-04135.JPG \\
\hline $\begin{array}{l}1740 \times \\
1745\end{array}$ & Galium verum L. × Galium mollugo L. & Aszófő & 1956 & $\begin{array}{l}\text { Felföldy Lajos, Tóth } \\
\text { László }\end{array}$ & BPU-04139.JPG \\
\hline $\begin{array}{c}1740 \times \\
1745\end{array}$ & Galium verum L. × Galium mollugo L. & Budapest & 1989 & Felföldy Lajos & BPU-04133.JPG \\
\hline $\begin{array}{c}1740 \times \\
1745\end{array}$ & Galium verum L. × Galium mollugo L. & Encs & 1944 & Siroki Zoltán & BPU-04141.JPG \\
\hline $\begin{array}{c}1740 \times \\
1745\end{array}$ & Galium verum L. × Galium mollugo L. & Szilvásvárad & 1934 & Boros Ádám & BPU-04140.JPG \\
\hline $\begin{array}{c}1740 \times \\
1746\end{array}$ & Galium verum L. × Galium album Mill. & Miskolc & 1929 & Soó Rezső & BPU-04138.JPG \\
\hline $\begin{array}{c}1744- \\
1745\end{array}$ & $\begin{array}{l}\text { Galium lucidum All. - Galium mollugo } \\
\text { L. }\end{array}$ & Pécs & 1935 & Soó Rezső & BPU-04119.JPG \\
\hline 1747 & $\begin{array}{l}\text { Cruciata pedemontana (Bellardi) } \\
\text { Ehrend. }\end{array}$ & Budapest & 2004 & Felföldy Lajos & BPU-03912.JPG \\
\hline 1747 & $\begin{array}{l}\text { Cruciata pedemontana (Bellardi) } \\
\text { Ehrend. }\end{array}$ & Debrecen & 1934 & Soó Rezső & BPU-03914.JPG \\
\hline 1747 & $\begin{array}{l}\text { Cruciata pedemontana (Bellardi) } \\
\text { Ehrend. }\end{array}$ & Egyek & 1947 & Soó Rezső & BPU-03915.JPG \\
\hline 1747 & $\begin{array}{l}\text { Cruciata pedemontana (Bellardi) } \\
\text { Ehrend. }\end{array}$ & Isztimér & 1955 & Soó Rezső & BPU-03916.JPG \\
\hline 1747 & $\begin{array}{l}\text { Cruciata pedemontana (Bellardi) } \\
\text { Ehrend. }\end{array}$ & Nagykanizsa & 1948 & Károlyi Árpád & BPU-03920.JPG \\
\hline 1747 & $\begin{array}{l}\text { Cruciata pedemontana (Bellardi) } \\
\text { Ehrend. }\end{array}$ & Rudabánya & 1936 & Soó Rezső & BPU-03913.JPG \\
\hline 1747 & $\begin{array}{l}\text { Cruciata pedemontana (Bellardi) } \\
\text { Ehrend. }\end{array}$ & Tihany & 1956 & Felföldy Lajos & BPU-03917.JPG \\
\hline 1747 & $\begin{array}{l}\text { Cruciata pedemontana (Bellardi) } \\
\text { Ehrend. }\end{array}$ & Tihany & 1958 & Felföldy Lajos & BPU-03918.JPG \\
\hline 1747 & $\begin{array}{l}\text { Cruciata pedemontana (Bellardi) } \\
\text { Ehrend. }\end{array}$ & Uzsa & 1959 & Felföldy Lajos & BPU-03919.JPG \\
\hline 1748 & Cruciata laevipes Opiz & Balatonszőlős & 1959 & Felföldy Lajos & BPU-03921.JPG \\
\hline 1748 & Cruciata laevipes Opiz & Budapest & 1982 & Felföldy Lajos & BPU-03923.JPG \\
\hline 1748 & Cruciata laevipes Opiz & Hajdúhadház & 1933 & Soó Rezső & BPU-03926.JPG \\
\hline 1748 & Cruciata laevipes Opiz & Hidegkút & 1958 & Felföldy Lajos & BPU-03925.JPG \\
\hline 1748 & Cruciata laevipes Opiz & Pécsely & 1951 & Felföldy Lajos & BPU-03924.JPG \\
\hline 1748 & Cruciata laevipes Opiz & Tihany & 1959 & Felföldy Lajos & BPU-03922.JPG \\
\hline 1750 & Rubia tinctorum L. & Szeged & 1947 & Timár Lajos & BPU-04142.JPG \\
\hline 1751 & Plantago indica L. & Baktalórántháza & 1933 & Soó Rezső & BPU-00211.JPG \\
\hline 1751 & Plantago indica L. & Budapest & 1948 & Soó Rezső & BPU-00214.JPG \\
\hline 1751 & Plantago indica L. & Budapest & 1982 & Felföldy Lajos & BPU-00193.JPG \\
\hline 1751 & Plantago indica L. & Bugac & 1964 & Soó Rezső & BPU-00212.JPG \\
\hline 1751 & Plantago indica L. & Dávod & 1955 & Soó Rezső & BPU-00213.JPG \\
\hline 1751 & Plantago indica L. & Vácrátót & 1953 & Felföldy Lajos & BPU-00210.JPG \\
\hline
\end{tabular}


1. Elektroniukus melléklet - Electronic Appendix 1.

\begin{tabular}{|c|c|c|c|c|c|}
\hline $\begin{array}{l}\text { Sorszám / } \\
\text { Number }\end{array}$ & Taxon-név / Taxon-name & $\begin{array}{l}\text { Település / } \\
\text { Settlement }\end{array}$ & $\begin{array}{l}\text { Év / } \\
\text { Year }\end{array}$ & Gyűjtő / Collector & $\begin{array}{l}\text { Fájlnév / } \\
\text { File-name }\end{array}$ \\
\hline 1752 & Plantago maritima L. & Budapest & 1991 & Felföldy Lajos & BPU-00229.JPG \\
\hline 1752 & Plantago maritima L. & Debrecen & 1989 & Felföldy Lajos & BPU-09223.JPG \\
\hline 1752 & Plantago maritima L. & Egyek & 1947 & Soó Rezső & BPU-09224.JPG \\
\hline 1752 & Plantago maritima L. & Felpéc & 1908 & Polgár Sándor & BPU-09222.JPG \\
\hline 1752 & Plantago maritima L. & Hegykő & 1960 & Felföldy Lajos & BPU-09226.JPG \\
\hline 1752 & Plantago maritima L. & Kiskunhalas & 1964 & Soó Rezső & BPU-09220.JPG \\
\hline 1752 & Plantago maritima L. & Kiskunmajsa & 1964 & Soó Rezső & BPU-09221.JPG \\
\hline 1752 & Plantago maritima L. & Nyíregyháza & 1933 & Soó Rezső & BPU-09227.JPG \\
\hline 1752 & Plantago maritima L. & Tihany & 1959 & Felföldy Lajos & BPU-09228.JPG \\
\hline 1752 & Plantago maritima L. & Vácrátót & 1949 & Soó Rezső & BPU-09225.JPG \\
\hline 1753 & Plantago tenuiflora Waldst. et Kit. & Akasztó & 1961 & Borsos Olga, Simon Tibor & BPU-06452.JPG \\
\hline 1753 & Plantago tenuiflora Waldst. et Kit. & Debrecen & 1947 & Soó Rezső & BPU-06454.JPG \\
\hline 1753 & Plantago tenuiflora Waldst. et Kit. & Farmos & 1923 & Soó Rezső & BPU-06454.JPG \\
\hline 1753 & Plantago tenuiflora Waldst. et Kit. & Hajdúnánás & 1935 & Soó Rezső & BPU-15489.JPG \\
\hline 1753 & Plantago tenuiflora Waldst. et Kit. & Kétegyháza & 1923 & Soó Rezső & BPU-06453.JPG \\
\hline 1753 & Plantago tenuiflora Waldst. et Kit. & Konyár & 1949 & Soó Rezső & BPU-06455.JPG \\
\hline 1754 & Plantago schwarzenbergiana Schur & Kétegyháza & 1923 & Soó Rezső & BPU-09229.JPG \\
\hline 1755 & Plantago argentea Chaix in Vill. & Pécs & 1925 & Soó Rezső & BPU-06445.JPG \\
\hline 1755 & Plantago argentea Chaix in Vill. & Pécs & 1953 & Soó Rezső, Borsos Olga & BPU-09230.JPG \\
\hline 1756 & Plantago lanceolata L. & Budapest & 1982 & Felföldy Lajos & BPU-06446.JPG \\
\hline 1756 & Plantago lanceolata L. & Budapest & 1990 & Felföldy Lajos & BPU-06447.JPG \\
\hline 1756 & Plantago lanceolata L. & Debrecen & 1932 & Soó Rezső & BPU-06451.JPG \\
\hline 1756 & Plantago lanceolata L. & Egyek & 1950 & $\begin{array}{l}\text { Simon Tibor, Borsos Olga, } \\
\text { Kulcsár Gábor }\end{array}$ & BPU-06449.JPG \\
\hline 1756 & Plantago lanceolata L. & Kiskunhalas & 1964 & Soó Rezső & BPU-06450.JPG \\
\hline 1756 & Plantago lanceolata L. & Tihany & 1946 & Udvardy Miklós & BPU-06448.JPG \\
\hline 1757 & Plantago altissima L. & Bicske & 1991 & Felföldy Lajos & BPU-00217.JPG \\
\hline 1757 & Plantago altissima L. & Bugyi & 1959 & Soó Rezső & BPU-00220.JPG \\
\hline 1757 & Plantago altissima L. & Hosszúpereszteg & 1955 & Soó Rezső & BPU-00222.JPG \\
\hline 1757 & Plantago altissima L. & Isaszeg & 1949 & Soó Rezső & BPU-00221.JPG \\
\hline 1757 & Plantago altissima L. & Tiszacsege & 1950 & $\begin{array}{l}\text { Simon Tibor, Borsos Olga, } \\
\text { Kulcsár Gábor }\end{array}$ & BPU-00219.JPG \\
\hline 1757 & Plantago altissima L. & Vámosszabadi & 1931 & Soó Rezső & BPU-00218.JPG \\
\hline 1758 & Plantago media L. & Bagamér & 1949 & Soó Rezső & BPU-00226.JPG \\
\hline 1758 & Plantago media L. & Budapest & 2005 & Felföldy Lajos & BPU-00223.JPG \\
\hline 1758 & Plantago media L. & Budapest & 2005 & Felföldy Lajos & BPU-00224.JPG \\
\hline 1758 & Plantago media L. & Kóspallag & 1962 & Isépy István & BPU-00227.JPG \\
\hline 1758 & Plantago media L. & Révfülöp & 1962 & Priszter Szaniszló & BPU-00228.JPG \\
\hline 1758 & Plantago media L. & Tarpa & 1949 & Soó Rezső & BPU-00225.JPG \\
\hline 1759 & Plantago maxima Juss. ex Jacq. & Bugyi & 1959 & Soó Rezső & BPU-00216.JPG \\
\hline 1759 & Plantago maxima Juss. ex Jacq. & Dabas & 1931 & Pénzes Antal & BPU-00215.JPG \\
\hline 1760 & Plantago major L. & Bicske & 1991 & Felföldy Lajos & BPU-00198.JPG \\
\hline 1760 & Plantago major L. & Budaörs & 1991 & Felföldy Lajos & BPU-00204.JPG \\
\hline 1760 & Plantago major L. & Budaörs & 1991 & Felföldy Lajos & BPU-00205.JPG \\
\hline 1760 & Plantago major L. & Budapest & 1991 & Felföldy Lajos & BPU-00194.JPG \\
\hline 1760 & Plantago major L. & Csaroda & 1948 & Simon Tibor, Jakucs Pál & BPU-00196.JPG \\
\hline 1760 & Plantago major L. & Debrecen & 1945 & Felföldy Lajos & BPU-00201.JPG \\
\hline 1760 & Plantago major L. & Hajdúbagos & 1937 & Soó Rezső & BPU-00197.JPG \\
\hline
\end{tabular}


1. Elektroniukus melléklet - Electronic Appendix 1.

\begin{tabular}{|c|c|c|c|c|c|}
\hline $\begin{array}{l}\text { Sorszám / } \\
\text { Number }\end{array}$ & Taxon-név / Taxon-name & $\begin{array}{l}\text { Település / } \\
\text { Settlement }\end{array}$ & $\begin{array}{l}\text { Év / } \\
\text { Year }\end{array}$ & Gyújtő / Collector & $\begin{array}{l}\text { Fájlnév / } \\
\text { File-name }\end{array}$ \\
\hline 1760 & Plantago major L. & Hortobágy & 1947 & Soó Rezső & BPU-00202.JPG \\
\hline 1760 & Plantago major L. & Hortobágy & 1947 & Soó Rezső & BPU-00203.JPG \\
\hline 1760 & Plantago major L. & Kiskunmajsa & 1964 & Soó Rezső & BPU-00195.JPG \\
\hline 1760 & Plantago major L. & Nagyiván & 1991 & Csányi Béla & BPU-00207.JPG \\
\hline 1760 & Plantago major L. & Szár & 1948 & Soó Rezső & BPU-00199.JPG \\
\hline 1760 & Plantago major L. & Tállya & 1958 & Isépy István & BPU-00209.JPG \\
\hline 1760 & Plantago major L. & Vácrátót & 1953 & Felföldy Lajos & BPU-00206.JPG \\
\hline 1760 & Plantago major L. & Zámoly & 1982 & Felföldy Lajos & BPU-00200.JPG \\
\hline 1761 & Sambucus ebulus L. & Geszt & 1947 & Soó Rezső & BPU-04144.JPG \\
\hline 1761 & Sambucus ebulus L. & Nagyharsány & 1965 & Priszter Szaniszló & BPU-04143.JPG \\
\hline 1761 & Sambucus ebulus L. & Tállya & 1958 & Isépy István & BPU-04145.JPG \\
\hline 1762 & Sambucus nigra L. & Balatonszőlős & 1957 & Felföldy Lajos & BPU-04146.JPG \\
\hline 1762 & Sambucus nigra L. & Debrecen & 1931 & Soó Rezső & BPU-04147.JPG \\
\hline 1763 & Sambucus racemosa L. & Gyöngyös & 1962 & Soó Rezső, Borsos Olga & BPU-04150.JPG \\
\hline 1763 & Sambucus racemosa L. & Kőszeg & 1932 & Soó Rezső & BPU-04151.JPG \\
\hline 1763 & Sambucus racemosa L. & Miskolc & 1937 & Soó Rezső & BPU-04152.JPG \\
\hline 1763 & Sambucus racemosa L. & Miskolc & 1950 & Soó Rezső & BPU-04149.JPG \\
\hline 1763 & Sambucus racemosa L. & Telkibánya & 1948 & Borsos Olga & BPU-04148.JPG \\
\hline 1764 & Viburnum opulus L. & Dabas & 1964 & Soó Rezső & BPU-04154.JPG \\
\hline 1764 & Viburnum opulus L. & Debrecen & 1931 & Soó Rezső & BPU-04155.JPG \\
\hline 1764 & Viburnum opulus L. & Pomáz & 1923 & Soó Rezső & BPU-04153.JPG \\
\hline 1765 & Viburnum lantana L. & Budapest & 1953 & Soó Rezső & BPU-04157.JPG \\
\hline 1765 & Viburnum lantana L. & Budapest & 2005 & Felföldy Lajos & BPU-04158.JPG \\
\hline 1765 & Viburnum lantana L. & Hidegkút & 1958 & Felföldy Lajos & BPU-04165.JPG \\
\hline 1765 & Viburnum lantana L. & Mór & 1953 & Felföldy Lajos & BPU-04161.JPG \\
\hline 1765 & Viburnum lantana L. & Mosonmagyaróvár & 1965 & Borsos Olga & BPU-04162.JPG \\
\hline 1765 & Viburnum lantana L. & Pécs & 1935 & Soó Rezső & BPU-04159.JPG \\
\hline 1765 & Viburnum lantana L. & Pécs & 1953 & Soó Rezső, Borsos Olga & BPU-04160.JPG \\
\hline 1765 & Viburnum lantana L. & Sopron & 1960 & Borsos Olga, Simon Tibor & BPU-04164.JPG \\
\hline 1765 & Viburnum lantana L. & Visegrád & 1964 & Soó Rezső, Borsos Olga & BPU-04163.JPG \\
\hline 1768 & Lonicera caprifolium L. & Bisse & 1965 & $\begin{array}{l}\text { Simon Tibor, Priszter } \\
\text { Szaniszló, Borhidi Attila }\end{array}$ & BPU-04171.JPG \\
\hline 1768 & Lonicera caprifolium L. & Kisharsány & 1961 & Simon Tibor & BPU-04170.JPG \\
\hline 1768 & Lonicera caprifolium L. & Kőszeg & 1932 & Soó Rezső & BPU-04168.JPG \\
\hline 1768 & Lonicera caprifolium L. & Miskolc & 1929 & Soó Rezső & BPU-04169.JPG \\
\hline 1768 & Lonicera caprifolium L. & Pécs & 1950 & Felföldy Lajos & BPU-04172.JPG \\
\hline 1768 & Lonicera caprifolium L. & Pécs & 1956 & Soó Rezső & BPU-04167.JPG \\
\hline 1770 & Lonicera xylosteum L. & Budapest & 1953 & Soó Rezső & BPU-15411.JPG \\
\hline 1770 & Lonicera xylosteum L. & Garadna & 1955 & Tóth László & BPU-04173.JPG \\
\hline 1770 & Lonicera xylosteum L. & Miskolc & 1937 & Soó Rezső & BPU-04174.JPG \\
\hline 1770 & Lonicera xylosteum L. & Nagykovácsi & 2005 & Felföldy Lajos & BPU-04175.JPG \\
\hline 1771 & Lonicera tatarica L. & Debrecen & 1947 & Soó Rezső & BPU-04185.JPG \\
\hline 1771 & Lonicera tatarica L. & Debrecen & - & Soó Rezső & BPU-04184.JPG \\
\hline 1771 & Lonicera tatarica L. & Pécs & 1953 & Soó Rezső, Borsos Olga & BPU-04186.JPG \\
\hline 1773 & Adoxa moschatellina L. & Bakonypölöske & 1962 & Simon Tibor, Borsos Olga & BPU-04401.JPG \\
\hline 1773 & Adoxa moschatellina L. & Balatonszőlős & 1959 & Felföldy Lajos & BPU-04399.JPG \\
\hline 1773 & Adoxa moschatellina L. & Bisse & 1965 & $\begin{array}{l}\text { Simon Tibor, Priszter } \\
\text { Szaniszló, Borhidi Attila }\end{array}$ & BPU-04402.JPG \\
\hline
\end{tabular}


1. Elektroniukus melléklet - Electronic Appendix 1.

\begin{tabular}{|c|c|c|c|c|c|}
\hline $\begin{array}{l}\text { Sorszám / } \\
\text { Number }\end{array}$ & Taxon-név / Taxon-name & $\begin{array}{l}\text { Település / } \\
\text { Settlement }\end{array}$ & $\begin{array}{l}\text { Év / } \\
\text { Year }\end{array}$ & Gyújtő / Collector & $\begin{array}{l}\text { Fájlnév / } \\
\text { File-name }\end{array}$ \\
\hline 1773 & Adoxa moschatellina L. & Pécs & 1956 & Soó Rezső & BPU-04404.JPG \\
\hline 1773 & Adoxa moschatellina L. & Tarcal & 1951 & Soó Rezső & BPU-04403.JPG \\
\hline 1773 & Adoxa moschatellina L. & Uzsa & 1959 & $\begin{array}{l}\text { Jávorka Sándor, Felföldy } \\
\text { Lajos }\end{array}$ & BPU-04398.JPG \\
\hline 1774 & Valerianella coronata (L.) DC. & Nagyharsány & 1936 & Soó Rezső & BPU-04188.JPG \\
\hline 1774 & Valerianella coronata (L.) DC. & Nagyharsány & 1955 & Soó Rezső & BPU-04190.JPG \\
\hline 1774 & Valerianella coronata (L.) DC. & Nagyharsány & 1956 & Soó Rezső & BPU-04199.JPG \\
\hline 1774 & Valerianella coronata (L.) DC. & Tihany & 1928 & Soó Rezső & BPU-04189.JPG \\
\hline 1774 & Valerianella coronata (L.) DC. & Tihany & 1956 & Felföldy Lajos & BPU-04187.JPG \\
\hline 1775 & Valerianella locusta (L.) Laterr. & Balmazújváros & 1932 & Soó Rezső & BPU-04208.JPG \\
\hline 1775 & Valerianella locusta (L.) Laterr. & Budapest & 1922 & Soó Rezső & BPU-04214.JPG \\
\hline 1775 & Valerianella locusta (L.) Laterr. & Budapest & 1991 & Felföldy Lajos & BPU-04213.JPG \\
\hline 1775 & Valerianella locusta (L.) Laterr. & Budapest & 1991 & Felföldy Lajos & BPU-04216.JPG \\
\hline 1775 & Valerianella locusta (L.) Laterr. & Budapest & 1993 & Felföldy Lajos & BPU-04215.JPG \\
\hline 1775 & Valerianella locusta (L.) Laterr. & Budapest & 2004 & Felföldy Lajos & BPU-04217.JPG \\
\hline 1775 & Valerianella locusta (L.) Laterr. & Csévharaszt & 1959 & $\begin{array}{l}\text { Simon Tibor, Horánszky } \\
\text { András }\end{array}$ & BPU-04207.JPG \\
\hline 1775 & Valerianella locusta (L.) Laterr. & Lábatlan & 1953 & Felföldy Lajos & BPU-04211.JPG \\
\hline 1775 & Valerianella locusta (L.) Laterr. & Mór & 1953 & Felföldy Lajos & BPU-04210.JPG \\
\hline 1775 & Valerianella locusta (L.) Laterr. & Tass & 1982 & Felföldy Lajos & BPU-04212.JPG \\
\hline 1775 & Valerianella locusta (L.) Laterr. & Tihany & 1958 & Felföldy Lajos & BPU-04204.JPG \\
\hline 1775 & Valerianella locusta (L.) Laterr. & Tihany & 1958 & Felföldy Lajos & BPU-04205.JPG \\
\hline 1775 & Valerianella locusta (L.) Laterr. & Vácrátót & 1953 & Felföldy Lajos & BPU-04206.JPG \\
\hline 1775 & Valerianella locusta (L.) Laterr. & Zámoly & 1982 & Felföldy Lajos & BPU-04209.JPG \\
\hline 1776 & Valerianella carinata Loisel. & Budapest & 1982 & Felföldy Lajos & BPU-04220.JPG \\
\hline 1776 & Valerianella carinata Loisel. & Kiskunfélegyháza & 1923 & Soó Rezső & BPU-04219.JPG \\
\hline 1776 & Valerianella carinata Loisel. & Lábatlan & 1953 & Felföldy Lajos & BPU-04218.JPG \\
\hline 1776 & Valerianella carinata Loisel. & Tihany & 1946 & Felföldy Lajos & BPU-04222.JPG \\
\hline 1776 & Valerianella carinata Loisel. & Tihany & 1959 & Felföldy Lajos & BPU-04221.JPG \\
\hline 1777 & Valerianella dentata (L.) Pollich & Bélapátfalva & - & Soó Rezső & BPU-04191.JPG \\
\hline 1777 & Valerianella dentata (L.) Pollich & Budapest & 1946 & Kárpáti Zoltán & BPU-04192.JPG \\
\hline 1777 & Valerianella dentata (L.) Pollich & Lábatlan & 1953 & Felföldy Lajos & BPU-04193.JPG \\
\hline 1777 & Valerianella dentata (L.) Pollich & Lábatlan & 1953 & Felföldy Lajos & BPU-04194.JPG \\
\hline 1777 & Valerianella dentata (L.) Pollich & Pécsely & 1951 & Felföldy Lajos & BPU-04195.JPG \\
\hline 1778 & Valerianella rimosa Bastard & Lábatlan & 1953 & Felföldy Lajos & BPU-04197.JPG \\
\hline 1778 & Valerianella rimosa Bastard & Pécsely & 1950 & Felföldy Lajos & BPU-04196.JPG \\
\hline 1779 & Valerianella pumila (L.) DC. & Aszófő & 1959 & Felföldy Lajos & BPU-04200.JPG \\
\hline 1779 & Valerianella pumila (L.) DC. & Nagyharsány & 1956 & Soó Rezső & BPU-04199.JPG \\
\hline 1779 & Valerianella pumila (L.) DC. & Tihany & 1928 & Soó Rezső & BPU-04202.JPG \\
\hline 1779 & Valerianella pumila (L.) DC. & Tihany & 1956 & Felföldy Lajos & BPU-04201.JPG \\
\hline 1780 & Valeriana officinalis L. & Beregdaróc & 1951 & Simon Tibor & BPU-04227.JPG \\
\hline 1780 & Valeriana officinalis L. & Budapest & 1964 & Soó Rezső & BPU-04223.JPG \\
\hline 1780 & Valeriana officinalis L. & Budapest & 1991 & Felföldy Lajos & BPU-04224.JPG \\
\hline 1780 & Valeriana officinalis L. & Budapest & 2005 & Felföldy Lajos & BPU-04225.JPG \\
\hline 1780 & Valeriana officinalis L. & Gyöngyös & 1965 & Soó Rezső & BPU-04229.JPG \\
\hline 1780 & Valeriana officinalis L. & Gyöngyös & 1965 & Soó Rezső & BPU-04230.JPG \\
\hline 1780 & Valeriana officinalis L. & Hajdúsámson & 1950 & Soó Rezső & BPU-04228.JPG \\
\hline 1780 & Valeriana officinalis L. & Magyarlukafa & 1957 & Soó Rezső & BPU-04232.JPG \\
\hline
\end{tabular}


1. Elektroniukus melléklet - Electronic Appendix 1.

\begin{tabular}{|c|c|c|c|c|c|}
\hline $\begin{array}{l}\text { Sorszám / } \\
\text { Number }\end{array}$ & Taxon-név / Taxon-name & $\begin{array}{l}\text { Település / } \\
\text { Settlement }\end{array}$ & $\begin{array}{l}\text { Év / } \\
\text { Year }\end{array}$ & Gyűjtő / Collector & $\begin{array}{l}\text { Fájlnév / } \\
\text { File-name }\end{array}$ \\
\hline 1780 & Valeriana officinalis L. & Pécs & 1925 & Soó Rezső & BPU-04226.JPG \\
\hline 1780 & Valeriana officinalis L. & Tihany & 1959 & Felföldy Lajos & BPU-04231.JPG \\
\hline 1780 & Valeriana officinalis L. & Vászoly & 1950 & Felföldy Lajos & BPU-04233.JPG \\
\hline 1781 & Valeriana tripteris L. & Bélapátfalva & 1953 & Simon Tibor & BPU-04254.JPG \\
\hline 1781 & Valeriana tripteris L. & Miskolc & 1937 & Soó Rezső & BPU-04261.JPG \\
\hline 1781 & Valeriana tripteris $\mathrm{L}$. & Pilisszentiván & 1932 & Soó Rezső & BPU-04260.JPG \\
\hline 1781 & Valeriana tripteris L. & Tibolddaróc & 1953 & Soó Rezső & BPU-04262.JPG \\
\hline 1782 & Valeriana dioica L. & Balatonszőlős & 1957 & Felföldy Lajos & BPU-04243.JPG \\
\hline 1782 & Valeriana dioica L. & Balatonudvari & 1959 & Felföldy Lajos & BPU-04238.JPG \\
\hline 1782 & Valeriana dioica L. & Dabas & 1958 & Simon Tibor & BPU-04239.JPG \\
\hline 1782 & Valeriana dioica L. & Hévíz & 1951 & Felföldy Lajos & BPU-04237.JPG \\
\hline 1782 & Valeriana dioica L. & Nagybajom & 1957 & Soó Rezső & BPU-04241.JPG \\
\hline 1782 & Valeriana dioica L. & Ócsa & 1923 & Soó Rezső & BPU-04242.JPG \\
\hline 1782 & Valeriana dioica $\mathrm{L}$. & Örvényes & 1959 & Felföldy Lajos & BPU-04245.JPG \\
\hline 1782 & Valeriana dioica $\mathrm{L}$. & Örvényes & 1959 & Felföldy Lajos & BPU-04246.JPG \\
\hline 1782 & Valeriana dioica L. & Porva & 1929 & Soó Rezső & BPU-04240.JPG \\
\hline 1782 & Valeriana dioica L. & Tihany & 1956 & Felföldy Lajos & BPU-04244.JPG \\
\hline 1784 & Cephalaria transsylvanica (L.) Schrad. & Budapest & 1943 & Kárpáti Zoltán & BPU-04275.JPG \\
\hline 1784 & Cephalaria transsylvanica (L.) Schrad. & Budapest & 1947 & Soó Rezső & BPU-04276.JPG \\
\hline 1784 & Cephalaria transsylvanica (L.) Schrad. & Debrecen & 1931 & Soó Rezső & BPU-04279.JPG \\
\hline 1784 & Cephalaria transsylvanica (L.) Schrad. & Kerecsend & 1957 & Soó Rezső & BPU-04280.JPG \\
\hline 1784 & Cephalaria transsylvanica (L.) Schrad. & Nagyharsány & 1964 & Priszter Szaniszló & BPU-04277.JPG \\
\hline 1784 & Cephalaria transsylvanica (L.) Schrad. & Nagyharsány & 1965 & $\begin{array}{l}\text { Simon Tibor, Priszter } \\
\text { Szaniszló, Borhidi Attila }\end{array}$ & BPU-04278.JPG \\
\hline 1784 & Cephalaria transsylvanica (L.) Schrad. & Tokaj & 1949 & Soó Rezső & BPU-04281.JPG \\
\hline 1785 & Dipsacus pilosus L. & Debrecen & 1946 & Soó Rezső & BPU-04274.JPG \\
\hline 1785 & Dipsacus pilosus L. & Miskolc & 1937 & Soó Rezső & BPU-04271.JPG \\
\hline 1785 & Dipsacus pilosus L. & Miskolc & 1950 & Soó Rezső & BPU-04273.JPG \\
\hline 1785 & Dipsacus pilosus L. & Szögliget & 1951 & $\begin{array}{l}\text { Soó Rezső, Jakucs Pál, Ér } \\
\text { Lajos }\end{array}$ & BPU-04272.JPG \\
\hline 1786 & Dipsacus laciniatus L. & Bagamér & 1933 & Soó Rezső & BPU-04268.JPG \\
\hline 1786 & Dipsacus laciniatus L. & Csaroda & 1952 & Soó Rezső & BPU-04269.JPG \\
\hline 1787 & Dipsacus fullonum L. & Karcag & 1936 & Soó Rezső & BPU-04270.JPG \\
\hline 1788 & Succisa pratensis Moench & Alsószölnök & 1955 & Soó Rezső & BPU-04291.JPG \\
\hline 1788 & Succisa pratensis Moench & Balatongyörök & 1961 & Felföldy Lajos & BPU-04298.JPG \\
\hline 1788 & Succisa pratensis Moench & Balatongyörök & 1961 & Felföldy Lajos & BPU-04299.JPG \\
\hline 1788 & Succisa pratensis Moench & Balatonszőlős & 1957 & Felföldy Lajos & BPU-04292.JPG \\
\hline 1788 & Succisa pratensis Moench & Bátorliget & 1932 & Soó Rezső & BPU-04290.JPG \\
\hline 1788 & Succisa pratensis Moench & Göd & 1948 & Soó Rezső & BPU-04288.JPG \\
\hline 1788 & Succisa pratensis Moench & Gyöngyös & 1952 & Soó Rezső & BPU-04286.JPG \\
\hline 1788 & Succisa pratensis Moench & Gyöngyös & 1953 & Soó Rezső & BPU-04287.JPG \\
\hline 1788 & Succisa pratensis Moench & Hévíz & 1961 & Felföldy Lajos & BPU-04300.JPG \\
\hline 1788 & Succisa pratensis Moench & Lesencetomaj & 1951 & Borsos Olga & BPU-04293.JPG \\
\hline 1788 & Succisa pratensis Moench & Ócsa & 1950 & Soó Rezső & BPU-04289.JPG \\
\hline 1788 & Succisa pratensis Moench & Örvényes & 1958 & Felföldy Lajos & BPU-04294.JPG \\
\hline 1788 & Succisa pratensis Moench & Örvényes & 1958 & Felföldy Lajos & BPU-04295.JPG \\
\hline 1788 & Succisa pratensis Moench & Pálháza & 1949 & Simon Tibor, Jakucs Pál & BPU-15516.JPG \\
\hline 1788 & Succisa pratensis Moench & Pusztafalu & 1939 & Soó Rezső & BPU-04285.JPG \\
\hline
\end{tabular}


1. Elektroniukus melléklet - Electronic Appendix 1.

\begin{tabular}{|c|c|c|c|c|c|}
\hline $\begin{array}{l}\text { Sorszám / } \\
\text { Number }\end{array}$ & Taxon-név / Taxon-name & $\begin{array}{l}\text { Település / } \\
\text { Settlement }\end{array}$ & $\begin{array}{l}\text { Év / } \\
\text { Year }\end{array}$ & Gyújtő / Collector & $\begin{array}{l}\text { Fájlnév / } \\
\text { File-name }\end{array}$ \\
\hline 1788 & Succisa pratensis Moench & Uzsa & 1952 & Simon Tibor & BPU-04296.JPG \\
\hline 1788 & Succisa pratensis Moench & Zalagyömörő & 1957 & Felföldy Lajos & BPU-04297.JPG \\
\hline 1789 & Succisella inflexa (Kluk.) Beck & Alsószölnök & 1955 & Soó Rezső & BPU-04304.JPG \\
\hline 1789 & Succisella inflexa (Kluk.) Beck & Alsószölnök & 1955 & Soó Rezső & BPU-04311.JPG \\
\hline 1789 & Succisella inflexa (Kluk.) Beck & Bak & 1955 & Soó Rezső & BPU-04310.JPG \\
\hline 1789 & Succisella inflexa (Kluk.) Beck & Beregdaróc & 1951 & $\begin{array}{l}\text { Simon Tibor, Vozáry } \\
\text { Elemér }\end{array}$ & BPU-04307.JPG \\
\hline 1789 & Succisella inflexa (Kluk.) Beck & Kőszeg & 1932 & Soó Rezső & BPU-04312.JPG \\
\hline 1789 & Succisella inflexa (Kluk.) Beck & Miskolc & 1934 & Soó Rezső & BPU-04308.JPG \\
\hline 1789 & Succisella inflexa (Kluk.) Beck & Somogyszob & 1957 & Soó Rezső & BPU-04309.JPG \\
\hline 1789 & Succisella inflexa (Kluk.) Beck & Tákos & 1948 & Simon Tibor, Jakucs Pál & BPU-04306.JPG \\
\hline 1789 & Succisella inflexa (Kluk.) Beck & Zalagyömörő & 1957 & Felföldy Lajos & BPU-04305.JPG \\
\hline 1790 & Knautia arvensis (L.) Coult. & Bagamér & 1934 & Soó Rezső & BPU-04322.JPG \\
\hline 1790 & Knautia arvensis (L.) Coult. & Baktalórántháza & 1933 & Soó Rezső & BPU-04323.JPG \\
\hline 1790 & Knautia arvensis (L.) Coult. & Bátorliget & 1948 & $\begin{array}{l}\text { Simon Tibor, Jakucs Pál, } \\
\text { Böszörményi Zoltán }\end{array}$ & BPU-04324.JPG \\
\hline 1790 & Knautia arvensis (L.) Coult. & Bodajk & 1955 & Soó Rezső & BPU-04317.JPG \\
\hline 1790 & Knautia arvensis (L.) Coult. & Bodajk & 1955 & Soó Rezső & BPU-04318.JPG \\
\hline 1790 & Knautia arvensis (L.) Coult. & Budapest & 1923 & Soó Rezső & BPU-04315.JPG \\
\hline 1790 & Knautia arvensis (L.) Coult. & Budapest & 1991 & Felföldy Lajos & BPU-04313.JPG \\
\hline 1790 & Knautia arvensis (L.) Coult. & Budapest & 2005 & Felföldy Lajos & BPU-04314.JPG \\
\hline 1790 & Knautia arvensis (L.) Coult. & Debrecen & 1937 & Soó Rezső & BPU-04320.JPG \\
\hline 1790 & Knautia arvensis (L.) Coult. & Debrecen & 1945 & Felföldy Lajos & BPU-04321.JPG \\
\hline 1790 & Knautia arvensis (L.) Coult. & Hajdúbagos & 1934 & Soó Rezső & BPU-04316.JPG \\
\hline 1790 & Knautia arvensis (L.) Coult. & Mór & 1936 & Felföldy Lajos & BPU-04319.JPG \\
\hline 1791 & Knautia drymeia Heuff. & Bisse & 1965 & $\begin{array}{l}\text { Simon Tibor, Priszter } \\
\text { Szaniszló, Borhidi Attila }\end{array}$ & BPU-04353.JPG \\
\hline 1791 & Knautia drymeia Heuff. & Csepreg & 1947 & Papp József & BPU-04349.JPG \\
\hline 1791 & Knautia drymeia Heuff. & Csesznek & 1963 & Soó Rezső & BPU-04347.JPG \\
\hline 1791 & Knautia drymeia Heuff. & Hidegkút & 1958 & Felföldy Lajos & BPU-04346.JPG \\
\hline 1791 & Knautia drymeia Heuff. & Isztimér & 1949 & Soó Rezső & BPU-15515.JPG \\
\hline 1791 & Knautia drymeia Heuff. & Kőszeg & 1963 & Soó Rezső, Borsos Olga & BPU-04348.JPG \\
\hline 1791 & Knautia drymeia Heuff. & Köveskál & 1959 & $\begin{array}{l}\text { Jávorka Sándor, Felföldy } \\
\text { Lajos, Tóth László }\end{array}$ & BPU-04345.JPG \\
\hline 1791 & Knautia drymeia Heuff. & Nagybajom & 1955 & Soó Rezső & BPU-04350.JPG \\
\hline 1791 & Knautia drymeia Heuff. & Pécs & 1925 & Soó Rezső & BPU-04352.JPG \\
\hline 1791 & Knautia drymeia Heuff. & Pécs & 1953 & Soó Rezső, Borsos Olga & BPU-04351.JPG \\
\hline 1791 & Knautia drymeia Heuff. & Uzsa & 1950 & Felföldy Lajos & BPU-04344.JPG \\
\hline 1791 & Knautia drymeia Heuff. & Uzsa & 1952 & Simon Tibor & BPU-04343.JPG \\
\hline 1791 & Knautia drymeia Heuff. & Vászoly & 1958 & Felföldy Lajos & BPU-04342.JPG \\
\hline 1792 & Knautia dipsacifolia Kreutzer & Csaroda & 1984 & Simon Tibor & BPU-04333.JPG \\
\hline 1792 & Knautia dipsacifolia Kreutzer & Szentendre & 1946 & Papp József & BPU-04335.JPG \\
\hline $\begin{array}{c}1790 \times \\
1791\end{array}$ & Knautia dipsacifolia Kreutzer & Csákánydoroszló & 1955 & Soó Rezső & BPU-04341.JPG \\
\hline $\begin{array}{c}1790 \times \\
1791\end{array}$ & $\begin{array}{l}\text { Knautia arvensis (L.) Coult. × Knautia } \\
\text { drymeia Heuff. }\end{array}$ & Pusztamiske & 1948 & Borsos Olga & BPU-04364.JPG \\
\hline 1793 & Scabiosa canescens Waldst. et Kit. & Abaújszántó & 1948 & Soó Rezső & BPU-04367.JPG \\
\hline 1793 & Scabiosa canescens Waldst. et Kit. & Bátorliget & 1932 & Soó Rezső & BPU-04366.JPG \\
\hline 1793 & Scabiosa canescens Waldst. et Kit. & Budapest & 1954 & Tóth László & BPU-04368.JPG \\
\hline 1793 & Scabiosa canescens Waldst. et Kit. & Csákvár & 1948 & Soó Rezső & BPU-04365.JPG \\
\hline
\end{tabular}


1. Elektroniukus melléklet - Electronic Appendix 1.

\begin{tabular}{|c|c|c|c|c|c|}
\hline $\begin{array}{l}\text { Sorszám / } \\
\text { Number }\end{array}$ & Taxon-név / Taxon-name & $\begin{array}{l}\text { Település / } \\
\text { Settlement }\end{array}$ & $\begin{array}{l}\text { Év / } \\
\text { Year }\end{array}$ & Gyűjtő / Collector & $\begin{array}{l}\text { Fájlnév / } \\
\text { File-name }\end{array}$ \\
\hline 1794 & Scabiosa ochroleuca L. & Budapest & 1982 & Felföldy Lajos & BPU-04370.JPG \\
\hline 1794 & Scabiosa ochroleuca L. & Budapest & 1993 & Felföldy Lajos & BPU-04369.JPG \\
\hline 1794 & Scabiosa ochroleuca L. & Hortobágy & 1947 & Soó Rezső & BPU-04371.JPG \\
\hline 1794 & Scabiosa ochroleuca L. & Tihany & 1947 & Pólya László & BPU-04372.JPG \\
\hline 1795 & Scabiosa columbaria L. & Alsószölnök & 1955 & Soó Rezső & BPU-04377.JPG \\
\hline 1795 & Scabiosa columbaria L. & Bélapátfalva & 1930 & Soó Rezső & BPU-04389.JPG \\
\hline 1795 & Scabiosa columbaria L. & Bélapátfalva & 1950 & $\begin{array}{l}\text { Simon Tibor, Jakucs Pál, } \\
\text { Kárpáti István }\end{array}$ & BPU-04385.JPG \\
\hline 1795 & Scabiosa columbaria L. & Gyöngyös & 1952 & Soó Rezső & BPU-04378.JPG \\
\hline 1795 & Scabiosa columbaria L. & Gyöngyös & 1953 & Soó Rezső & BPU-04379.JPG \\
\hline 1795 & Scabiosa columbaria L. & Nagykanizsa & 1950 & Károlyi Árpád & BPU-04381.JPG \\
\hline 1795 & Scabiosa columbaria L. & Nagyvisnyó & 1953 & Vozáry Elemér & BPU-04382.JPG \\
\hline 1797 & Campanula glomerata L. & Abaújszántó & 1948 & Soó Rezső & BPU-07506.JPG \\
\hline 1797 & Campanula glomerata L. & Abaújszántó & 1962 & Isépy István & BPU-07505.JPG \\
\hline 1797 & Campanula glomerata L. & Bátorliget & 1932 & Soó Rezső & BPU-07507.JPG \\
\hline 1797 & Campanula glomerata L. & Beregdaróc & 1951 & $\begin{array}{l}\text { Simon Tibor, Vozáry } \\
\text { Elemér }\end{array}$ & BPU-07503.JPG \\
\hline 1797 & Campanula glomerata L. & Budapest & 1947 & Soó Rezső & BPU-07500.JPG \\
\hline 1797 & Campanula glomerata L. & Budapest & 1982 & Felföldy Lajos & BPU-07501.JPG \\
\hline 1797 & Campanula glomerata L. & Miskolc & 1937 & Soó Rezső & BPU-07504.JPG \\
\hline 1797 & Campanula glomerata L. & Nagymaros & 1947 & Soó Rezső & BPU-07502.JPG \\
\hline 1797 & Campanula glomerata L. & Pusztamiske & 1948 & Borsos Olga & BPU-07508.JPG \\
\hline 1798 & Campanula macrostachya Kit. & Miskolc & 1938 & Soó Rezső & BPU-07517.JPG \\
\hline 1799 & Campanula cervicaria L. & Alsószölnök & 1955 & Soó Rezső & BPU-07526.JPG \\
\hline 1799 & Campanula cervicaria L. & Bak & 1955 & Soó Rezső & BPU-07528.JPG \\
\hline 1799 & Campanula cervicaria L. & Bátorliget & 1948 & $\begin{array}{l}\text { Simon Tibor, Jakucs Pál, } \\
\text { Böszörményi Zoltán }\end{array}$ & BPU-07524.JPG \\
\hline 1799 & Campanula cervicaria L. & Bátorliget & 1950 & $\begin{array}{l}\text { Simon Tibor, Kárpáti } \\
\text { István }\end{array}$ & BPU-07525.JPG \\
\hline 1799 & Campanula cervicaria L. & Debrecen & 1933 & Soó Rezső & BPU-07519.JPG \\
\hline 1799 & Campanula cervicaria L. & Gyöngyös & 1961 & Soó Rezső & BPU-07520.JPG \\
\hline 1799 & Campanula cervicaria L. & Háromhuta & 1949 & Simon Tibor, Jakucs Pál & BPU-15510.JPG \\
\hline 1799 & Campanula cervicaria L. & Nagybajom & 1957 & Soó Rezső & BPU-07523.JPG \\
\hline 1799 & Campanula cervicaria L. & Nagykovácsi & 2005 & Felföldy Lajos & BPU-07521.JPG \\
\hline 1799 & Campanula cervicaria L. & Ócsa & 1953 & Soó Rezső, Borsos Olga & BPU-07522.JPG \\
\hline 1799 & Campanula cervicaria L. & Pécs & 1925 & Soó Rezső & BPU-07518.JPG \\
\hline 1799 & Campanula cervicaria L. & Szentbékkálla & 1957 & Felföldy Lajos & BPU-07527.JPG \\
\hline 1800 & Campanula sibirica L. & Abaújszántó & 1938 & Soó Rezső & BPU-07539.JPG \\
\hline 1800 & Campanula sibirica L. & Bugyi & 1959 & Soó Rezső & BPU-07537.JPG \\
\hline 1800 & Campanula sibirica L. & Dabas & 1951 & Simon Tibor, Borsos Olga & BPU-07532.JPG \\
\hline 1800 & Campanula sibirica L. & Debrecen & 1934 & Soó Rezső & BPU-07531.JPG \\
\hline 1800 & Campanula sibirica L. & Jósvafő & 1952 & Jakucs Pál, Ér Lajos & BPU-07540.JPG \\
\hline 1800 & Campanula sibirica L. & Lábatlan & 1953 & Felföldy Lajos & BPU-07533.JPG \\
\hline 1800 & Campanula sibirica L. & Miskolc & 1939 & Soó Rezső & BPU-07541.JPG \\
\hline 1800 & Campanula sibirica L. & Nagyharsány & 1956 & Soó Rezső & BPU-07538.JPG \\
\hline 1800 & Campanula sibirica L. & Nagyharsány & 1967 & Priszter Szaniszló & BPU-07536.JPG \\
\hline 1800 & Campanula sibirica L. & Nagykovácsi & 1932 & Soó Rezső & BPU-07530.JPG \\
\hline 1800 & Campanula sibirica L. & Uzsa & 1959 & $\begin{array}{l}\text { Jávorka Sándor, Felföldy } \\
\text { Lajos }\end{array}$ & BPU-07535.JPG \\
\hline 1801 & Campanula bononiensis L. & Aggtelek & 1951 & Soó Rezső & BPU-07569.JPG \\
\hline
\end{tabular}


1. Elektroniukus melléklet - Electronic Appendix 1.

\begin{tabular}{|c|c|c|c|c|c|}
\hline $\begin{array}{l}\text { Sorszám / } \\
\text { Number }\end{array}$ & Taxon-név / Taxon-name & $\begin{array}{l}\text { Település / } \\
\text { Settlement }\end{array}$ & $\begin{array}{l}\text { Év / } \\
\text { Year }\end{array}$ & Gyűjtő / Collector & $\begin{array}{l}\text { Fájlnév / } \\
\text { File-name }\end{array}$ \\
\hline 1801 & Campanula bononiensis L. & Budapest & 1982 & Felföldy Lajos & BPU-07568.JPG \\
\hline 1801 & Campanula bononiensis L. & Budapest & 2004 & Felföldy Lajos & BPU-07566.JPG \\
\hline 1801 & Campanula bononiensis L. & Budapest & 2005 & Felföldy Lajos & BPU-07567.JPG \\
\hline 1801 & Campanula bononiensis L. & Csákvár & 1948 & Soó Rezső & BPU-07572.JPG \\
\hline 1801 & Campanula bononiensis L. & Füzér & 1949 & Simon Tibor, Jakucs Pál & BPU-15513.JPG \\
\hline 1801 & Campanula bononiensis L. & Gyöngyös & 1961 & Soó Rezső & BPU-07573.JPG \\
\hline 1801 & Campanula bononiensis L. & Mezőcsát & 1934 & Soó Rezső & BPU-07571.JPG \\
\hline 1801 & Campanula bononiensis L. & Nyíracsád & 1947 & Soó Rezső & BPU-07574.JPG \\
\hline 1801 & Campanula bononiensis L. & Pécs & 1938 & Soó Rezső & BPU-07570.JPG \\
\hline 1802 & Campanula rapunculoides L. & Bélapátfalva & 1974 & Priszter Szaniszló & BPU-07547.JPG \\
\hline 1802 & Campanula rapunculoides L. & Bélapátfalva & 1974 & Priszter Szaniszló & BPU-07548.JPG \\
\hline 1802 & Campanula rapunculoides L. & Budapest & 1947 & Soó Rezső & BPU-07545.JPG \\
\hline 1802 & Campanula rapunculoides L. & Eger & 1948 & Koppányi Tibor & BPU-07565.JPG \\
\hline 1802 & Campanula rapunculoides L. & Gyöngyös & 1961 & Soó Rezső & BPU-07549.JPG \\
\hline 1802 & Campanula rapunculoides L. & Nagykovácsi & 2005 & Felföldy Lajos & BPU-07550.JPG \\
\hline 1802 & Campanula rapunculoides L. & Nagyvisnyó & 1953 & Soó Rezső & BPU-07546.JPG \\
\hline 1802 & Campanula rapunculoides L. & Solymár & 2005 & Felföldy Lajos & BPU-07551.JPG \\
\hline 1802 & Campanula rapunculoides L. & Tállya & 1958 & Isépy István & BPU-07554.JPG \\
\hline 1802 & Campanula rapunculoides L. & Vászoly & 1950 & Felföldy Lajos & BPU-07552.JPG \\
\hline 1802 & Campanula rapunculoides L. & Vászoly & 1958 & Felföldy Lajos & BPU-07553.JPG \\
\hline 1804 & Campanula trachelium L. & Bátorliget & 1934 & Soó Rezső & BPU-07563.JPG \\
\hline 1804 & Campanula trachelium L. & Budapest & 1947 & Soó Rezső & BPU-07559.JPG \\
\hline 1804 & Campanula trachelium L. & Eger & 1948 & Koppányi Tibor & BPU-07565.JPG \\
\hline 1804 & Campanula trachelium L. & Miskolc & 1950 & Soó Rezső & BPU-07560.JPG \\
\hline 1804 & Campanula trachelium L. & Nagykovácsi & 2005 & Felföldy Lajos & BPU-07562.JPG \\
\hline 1804 & Campanula trachelium L. & Rezi & 1953 & Borsos Olga & BPU-07564.JPG \\
\hline 1805 & Campanula persicifolia L. & Bátorliget & 1934 & Soó Rezső & BPU-07588.JPG \\
\hline 1805 & Campanula persicifolia L. & Bátorliget & 1951 & Simon Tibor & BPU-07587.JPG \\
\hline 1805 & Campanula persicifolia L. & Budapest & 2004 & Felföldy Lajos & BPU-07593.JPG \\
\hline 1805 & Campanula persicifolia L. & Dömös & 1948 & Soó Rezső & BPU-07591.JPG \\
\hline 1805 & Campanula persicifolia L. & Salgótarján & 1936 & Soó Rezső & BPU-07589.JPG \\
\hline 1805 & Campanula persicifolia L. & Tihany & 1947 & Pólya László & BPU-07592.JPG \\
\hline 1806 & Campanula rapunculus L. & Debrecen & 1951 & Kulcsár Gábor & BPU-07599.JPG \\
\hline 1806 & Campanula rapunculus L. & Egyek & 1932 & Soó Rezső & BPU-07598.JPG \\
\hline 1806 & Campanula rapunculus L. & Egyek & 1950 & $\begin{array}{l}\text { Simon Tibor, Borsos Olga, } \\
\text { Kulcsár Gábor }\end{array}$ & BPU-07594.JPG \\
\hline 1806 & Campanula rapunculus $\mathrm{L}$. & Fenyőfő & 1919 & Polgár Sándor & BPU-07595.JPG \\
\hline 1806 & Campanula rapunculus L. & Gödöllő & 1922 & Soó Rezső & BPU-07596.JPG \\
\hline 1806 & Campanula rapunculus L. & Hajdúbagos & 1933 & Soó Rezső & BPU-07597.JPG \\
\hline 1807 & Campanula patula L. & Bátorliget & 1934 & Soó Rezső & BPU-07602.JPG \\
\hline 1807 & Campanula patula L. & Beregdaróc & 1951 & $\begin{array}{l}\text { Simon Tibor, Vozáry } \\
\text { Elemér }\end{array}$ & BPU-07606.JPG \\
\hline 1807 & Campanula patula L. & Bisse & 1965 & $\begin{array}{l}\text { Simon Tibor, Priszter } \\
\text { Szaniszló, Borhidi Attila }\end{array}$ & BPU-07608.JPG \\
\hline 1807 & Campanula patula L. & Debrecen & 1933 & Soó Rezső & BPU-07605.JPG \\
\hline 1807 & Campanula patula $\mathrm{L}$. & Nagyvisnyó & 1948 & $\begin{array}{l}\text { Simon Tibor, Borsos Olga, } \\
\text { Jakucs Pál }\end{array}$ & BPU-07601.JPG \\
\hline 1807 & Campanula patula L. & Szilvásvárad & 1953 & $\begin{array}{l}\text { Felföldy Lajos, Tóth } \\
\text { László }\end{array}$ & BPU-07607.JPG \\
\hline
\end{tabular}


1. Elektroniukus melléklet - Electronic Appendix 1.

\begin{tabular}{|c|c|c|c|c|c|}
\hline $\begin{array}{l}\text { Sorszám / } \\
\text { Number }\end{array}$ & Taxon-név / Taxon-name & $\begin{array}{l}\text { Település / } \\
\text { Settlement }\end{array}$ & $\begin{array}{l}\text { Év / } \\
\text { Year }\end{array}$ & Gyüjtő / Collector & $\begin{array}{l}\text { Fájlnév / } \\
\text { File-name }\end{array}$ \\
\hline 1807 & Campanula patula L. & Uzsa & 1959 & $\begin{array}{l}\text { Jávorka Sándor, Felföldy } \\
\text { Lajos }\end{array}$ & BPU-07600.JPG \\
\hline 1808 & Campanula rotundifolia L. & Baktalórántháza & 1933 & Soó Rezső & BPU-07580.JPG \\
\hline 1808 & Campanula rotundifolia L. & Bódvarákó & 1951 & Soó Rezső, Jakucs Pál & BPU-07581.JPG \\
\hline 1808 & Campanula rotundifolia L. & Debrecen & 1931 & Soó Rezső & BPU-07579.JPG \\
\hline 1808 & Campanula rotundifolia L. & Debrecen & 1953 & Kulcsár Gábor & BPU-07576.JPG \\
\hline 1808 & Campanula rotundifolia L. & Debrecen & 1953 & Kulcsár Gábor & BPU-15441.JPG \\
\hline 1808 & Campanula rotundifolia L. & Gyöngyös & 1953 & Soó Rezső & BPU-07575.JPG \\
\hline 1808 & Campanula rotundifolia L. & Nagyvisnyó & 1953 & Soó Rezső & BPU-07578.JPG \\
\hline 1808 & Campanula rotundifolia L. & Sümeg & 1953 & Borsos Olga & BPU-07582.JPG \\
\hline 1808 & Campanula rotundifolia L. & Sümeg & 1953 & Borsos Olga & BPU-07583.JPG \\
\hline 1808 & Campanula rotundifolia L. & Tapolca & 1953 & Borsos Olga & BPU-07577.JPG \\
\hline 1808 & Campanula rotundifolia L. & Uzsa & 1953 & Borsos Olga & BPU-07584.JPG \\
\hline 1808 & Campanula rotundifolia $\mathrm{L}$. & Vácrátót & 1952 & Felföldy Lajos & BPU-07586.JPG \\
\hline 1811 & $\begin{array}{l}\text { Adenophora liliifolia (L.) Ledeb. ex A. } \\
\text { DC. }\end{array}$ & Aggtelek & 1953 & Jakucs Pál & BPU-07667.JPG \\
\hline 1811 & $\begin{array}{l}\text { Adenophora liliifolia (L.) Ledeb. ex A. } \\
\text { DC. }\end{array}$ & Bátorliget & 1932 & Soó Rezső & BPU-07668.JPG \\
\hline 1811 & $\begin{array}{l}\text { Adenophora liliifolia (L.) Ledeb. ex A. } \\
\text { DC. }\end{array}$ & Dabas & 1951 & Pócs Tamás & BPU-07666.JPG \\
\hline 1811 & $\begin{array}{l}\text { Adenophora liliifolia (L.) Ledeb. ex A. } \\
\text { DC. }\end{array}$ & Kiskőrös & 1921 & Boros Ádám & BPU-07669.JPG \\
\hline 1811 & $\begin{array}{l}\text { Adenophora liliifolia (L.) Ledeb. ex A. } \\
\text { DC. }\end{array}$ & Telkibánya & 1952 & Simon Tibor & BPU-07670.JPG \\
\hline 1812 & Legousia speculum-veneris (L.) Chaix & Hetvehely & 1950 & Felföldy Lajos & BPU-07672.JPG \\
\hline 1812 & Legousia speculum-veneris (L.) Chaix & Nagyharsány & 1965 & $\begin{array}{l}\text { Simon Tibor, Priszter } \\
\text { Szaniszló, Borhidi Attila }\end{array}$ & BPU-07671.JPG \\
\hline 1813 & $\begin{array}{l}\text { Asyneuma canescens (Waldst. et Kit.) } \\
\text { Griseb. et Schenk }\end{array}$ & Abaújszántó & 1962 & Isépy István & BPU-07675.JPG \\
\hline 1813 & $\begin{array}{l}\text { Asyneuma canescens (Waldst. et Kit.) } \\
\text { Griseb. et Schenk }\end{array}$ & Budapest & 1947 & Soó Rezső & BPU-07674.JPG \\
\hline 1814 & Phyteuma spicatum L. & Kőszeg & 1932 & Soó Rezső & BPU-07681.JPG \\
\hline 1814 & Phyteuma spicatum L. & Pálháza & 1938 & Soó Rezső & BPU-07682.JPG \\
\hline 1815 & Phyteuma orbiculare L. & Bélapátfalva & 1953 & Simon Tibor & BPU-07692.JPG \\
\hline 1815 & Phyteuma orbiculare L. & Bodajk & 1955 & Soó Rezső & BPU-07693.JPG \\
\hline 1815 & Phyteuma orbiculare L. & Budapest & 1947 & Soó Rezső & BPU-07690.JPG \\
\hline 1815 & Phyteuma orbiculare L. & Isztimér & 1949 & Soó Rezső & BPU-07691.JPG \\
\hline 1815 & Phyteuma orbiculare L. & Isztimér & 1949 & Soó Rezső & BPU-15514.JPG \\
\hline 1816 & Jasione montana L. & Bakonyszentlászló & 1963 & Soó Rezső & BPU-07710.JPG \\
\hline 1816 & Jasione montana L. & Bátorliget & 1948 & $\begin{array}{l}\text { Simon Tibor, Jakucs Pál, } \\
\text { Böszörményi Zoltán }\end{array}$ & BPU-07708.JPG \\
\hline 1816 & Jasione montana L. & Debrecen & 1934 & Soó Rezső & BPU-07706.JPG \\
\hline 1816 & Jasione montana L. & Kaszó & 1957 & Soó Rezső & BPU-07709.JPG \\
\hline 1816 & Jasione montana L. & Nagykőrös & 1920 & Boros Ádám & BPU-07711.JPG \\
\hline 1816 & Jasione montana L. & Vácrátót & 1923 & Soó Rezső & BPU-07707.JPG \\
\hline 1817 & Eupatorium cannabinum L. & Erdőbénye & 1952 & Borsos Olga & BPU-07713.JPG \\
\hline 1817 & Eupatorium cannabinum L. & Gárdony & 1980 & Felföldy Lajos & BPU-07712.JPG \\
\hline 1817 & Eupatorium cannabinum L. & Örvényes & 1958 & Felföldy Lajos & BPU-07715.JPG \\
\hline 1817 & Eupatorium cannabinum L. & Uzsa & 1953 & $\begin{array}{l}\text { Felföldy Lajos, Tóth } \\
\text { László }\end{array}$ & BPU-07714.JPG \\
\hline 1821 & Solidago virgaurea L. & Balatonrendes & 1956 & $\begin{array}{l}\text { Felföldy Lajos, Tóth } \\
\text { László }\end{array}$ & BPU-07722.JPG \\
\hline
\end{tabular}


1. Elektroniukus melléklet - Electronic Appendix 1.

\begin{tabular}{|c|c|c|c|c|c|}
\hline $\begin{array}{l}\text { Sorszám / } \\
\text { Number }\end{array}$ & Taxon-név / Taxon-name & $\begin{array}{l}\text { Település / } \\
\text { Settlement }\end{array}$ & $\begin{array}{l}\text { Év / } \\
\text { Year }\end{array}$ & Gyűjtő / Collector & $\begin{array}{l}\text { Fájlnév / } \\
\text { File-name }\end{array}$ \\
\hline 1821 & Solidago virgaurea L. & Hidegkút & 1957 & Felföldy Lajos & BPU-07723.JPG \\
\hline 1821 & Solidago virgaurea L. & Tapolca & 1953 & Borsos Olga & BPU-07721.JPG \\
\hline 1823 & Solidago gigantea Aiton & Debrecen & 1948 & Soó Rezső & BPU-07728.JPG \\
\hline 1823 & Solidago gigantea Aiton & Gárdony & 1980 & Felföldy Lajos & BPU-07727.JPG \\
\hline 1823 & Solidago gigantea Aiton & Göd & 1948 & Soó Rezső & BPU-07729.JPG \\
\hline 1823 & Solidago gigantea Aiton & Pécs & 1965 & Priszter Szaniszló & BPU-07726.JPG \\
\hline 1824 & Solidago canadensis L. & Budapest & 2004 & Felföldy Lajos & BPU-07730.JPG \\
\hline 1824 & Solidago canadensis L. & Budapest & 2004 & Felföldy Lajos & BPU-07731.JPG \\
\hline 1824 & Solidago canadensis L. & Debrecen & 1945 & Soó Rezső & BPU-07733.JPG \\
\hline 1824 & Solidago canadensis L. & Pere & 1961 & Simon Tibor & BPU-07732.JPG \\
\hline 1825 & Bellis perennis L. & Hévíz & 1951 & Felföldy Lajos & BPU-07739.JPG \\
\hline 1825 & Bellis perennis L. & Mogyoróska & 1938 & Soó Rezső & BPU-07735.JPG \\
\hline 1825 & Bellis perennis L. & Pécs & 1936 & Soó Rezső & BPU-07737.JPG \\
\hline 1825 & Bellis perennis L. & Téglás & 1948 & Soó Rezső & BPU-07734.JPG \\
\hline 1825 & Bellis perennis $\mathrm{L}$. & Tihany & 1958 & Felföldy Lajos & BPU-07736.JPG \\
\hline 1825 & Bellis perennis L. & Tihany & 1958 & Felföldy Lajos & BPU-07738.JPG \\
\hline 1828 & Aster linosyris (L.) Bernh. & Badacsonytördemic & 1953 & $\begin{array}{l}\text { Felföldy Lajos, Tóth } \\
\text { László }\end{array}$ & BPU-07747.JPG \\
\hline 1828 & Aster linosyris (L.) Bernh. & Budapest & 1982 & Felföldy Lajos & BPU-07742.JPG \\
\hline 1828 & Aster linosyris (L.) Bernh. & Budapest & 1991 & Felföldy Lajos & BPU-07741.JPG \\
\hline 1828 & Aster linosyris (L.) Bernh. & Csákvár & 1948 & Soó Rezső & BPU-07745.JPG \\
\hline 1828 & Aster linosyris (L.) Bernh. & Csarnóta & 1968 & Priszter Szaniszló & BPU-07743.JPG \\
\hline 1828 & Aster linosyris (L.) Bernh. & Kerecsend & 1957 & Soó Rezső & BPU-07746.JPG \\
\hline 1828 & Aster linosyris (L.) Bernh. & Miskolc & 1936 & Soó Rezső & BPU-07744.JPG \\
\hline 1828 & Aster linosyris (L.) Bernh. & Tihany & 1954 & Felföldy Lajos & BPU-07748.JPG \\
\hline 1828 & Aster linosyris (L.) Bernh. & Tihany & 1958 & Felföldy Lajos & BPU-07749.JPG \\
\hline 1829 & Aster sedifolius L. & Budaörs & 1920 & Wagner János & BPU-07755.JPG \\
\hline 1829 & Aster sedifolius L. & Hortobágy & 1947 & Soó Rezső & BPU-07751.JPG \\
\hline 1829 & Aster sedifolius L. & Újszentmargita & 1971 & Priszter Szaniszló & BPU-07752.JPG \\
\hline 1829 & Aster sedifolius L. & Újszentmargita & 1971 & Priszter Szaniszló & BPU-07753.JPG \\
\hline 1829 & Aster sedifolius L. & Újszentmargita & 1971 & Priszter Szaniszló & BPU-07754.JPG \\
\hline 1829 & Aster sedifolius L. & Vásárosnamény & 1952 & $\begin{array}{l}\text { Simon Tibor, Vozáry } \\
\text { Elemér }\end{array}$ & BPU-07750.JPG \\
\hline 1830 & Aster tripolium L. & Bugac & 1952 & Vozáry Elemér & BPU-07763.JPG \\
\hline 1830 & Aster tripolium L. & Debrecen & 1947 & Soó Rezső & BPU-07768.JPG \\
\hline 1830 & Aster tripolium L. & Debrecen & 1952 & Kulcsár Gábor & BPU-15432.JPG \\
\hline 1830 & Aster tripolium L. & Egyek & 1947 & Soó Rezső & BPU-07769.JPG \\
\hline 1830 & Aster tripolium L. & Hajdúbagos & 1934 & Soó Rezső & BPU-07766.JPG \\
\hline 1830 & Aster tripolium L. & Kiskunmajsa & 1964 & Soó Rezső & BPU-07767.JPG \\
\hline 1830 & Aster tripolium L. & Tihany & 1956 & Felföldy Lajos & BPU-07764.JPG \\
\hline 1830 & Aster tripolium L. & Tihany & 1961 & Sólymossy Gizella & BPU-07765.JPG \\
\hline 1831 & Aster amellus L. & Abaújszántó & 1948 & Soó Rezső & BPU-07760.JPG \\
\hline 1831 & Aster amellus L. & Budapest & 1958 & Simon Tibor & BPU-07762.JPG \\
\hline 1831 & Aster amellus L. & Budapest & 1982 & Felföldy Lajos & BPU-07757.JPG \\
\hline 1831 & Aster amellus L. & Pécs & 1925 & Soó Rezső & BPU-07758.JPG \\
\hline 1831 & Aster amellus L. & Somoskőúffalu & 1937 & Máthé Imre & BPU-07759.JPG \\
\hline 1833 & Aster novi-belgii L. & Debrecen & 1945 & Soó Rezső & BPU-07771.JPG \\
\hline 1835 & Aster $\times$ salignus Willd. & Baracska & 1922 & Trautmann Róbert & BPU-07777.JPG \\
\hline
\end{tabular}


1. Elektroniukus melléklet - Electronic Appendix 1.

\begin{tabular}{|c|c|c|c|c|c|}
\hline $\begin{array}{l}\text { Sorszám / } \\
\text { Number }\end{array}$ & Taxon-név / Taxon-name & $\begin{array}{l}\text { Település / } \\
\text { Settlement }\end{array}$ & $\begin{array}{l}\text { Év / } \\
\text { Year }\end{array}$ & Gyüjtő / Collector & $\begin{array}{l}\text { Fájlnév / } \\
\text { File-name }\end{array}$ \\
\hline 1836 & Aster lanceolatus Willd. & Balassagyarmat & 1971 & Priszter Szaniszló & BPU-07773.JPG \\
\hline 1836 & Aster lanceolatus Willd. & Debrecen & 1945 & Soó Rezső & BPU-07774.JPG \\
\hline 1837 & Aster tradescantii L. & Dabas & 1965 & Priszter Szaniszló & BPU-07775.JPG \\
\hline 1837 & Aster tradescantii L. & Göd & 1948 & Soó Rezső & BPU-07776.JPG \\
\hline $\begin{array}{c}1833 \times \\
1834\end{array}$ & $\begin{array}{l}\text { Aster novi-belgii L. } \times \text { Aster laevis } \\
\text { Willd. }\end{array}$ & Debrecen & 1945 & Soó Rezső & BPU-07772.JPG \\
\hline 1838 & Erigeron annuus (L.) Pers. & Bisse & 1965 & $\begin{array}{l}\text { Simon Tibor, Priszter } \\
\text { Szaniszló, Borhidi Attila }\end{array}$ & BPU-07792.JPG \\
\hline 1838 & Erigeron annuus (L.) Pers. & Budapest & 2004 & Felföldy Lajos & BPU-07788.JPG \\
\hline 1838 & Erigeron annuus (L.) Pers. & Budapest & 2004 & Felföldy Lajos & BPU-07797.JPG \\
\hline 1838 & Erigeron annuus (L.) Pers. & Budapest & 2005 & Felföldy Lajos & BPU-07798.JPG \\
\hline 1838 & Erigeron annuus (L.) Pers. & Debrecen & 1935 & Soó Rezső & BPU-07802.JPG \\
\hline 1838 & Erigeron annuus (L.) Pers. & Doboz & 1934 & Máthé Imre & BPU-07790.JPG \\
\hline 1838 & Erigeron annuus (L.) Pers. & Jánd & 1951 & Simon Tibor & BPU-07791.JPG \\
\hline 1838 & Erigeron annuus (L.) Pers. & Magyarlukafa & 1957 & Soó Rezső & BPU-07794.JPG \\
\hline 1838 & Erigeron annuus (L.) Pers. & Nagybajom & 1957 & Soó Rezső & BPU-07795.JPG \\
\hline 1838 & Erigeron annuus (L.) Pers. & Nagyharsány & 1965 & $\begin{array}{l}\text { Simon Tibor, Priszter } \\
\text { Szaniszló, Borhidi Attila }\end{array}$ & BPU-07793.JPG \\
\hline 1838 & Erigeron annuus (L.) Pers. & Pécs & 1925 & Soó Rezső & BPU-07799.JPG \\
\hline 1838 & Erigeron annuus (L.) Pers. & Szigliget & 1962 & Soó Rezső & BPU-07796.JPG \\
\hline 1838 & Erigeron annuus (L.) Pers. & Tarpa & 1949 & Simon Tibor & BPU-15566.JPG \\
\hline 1838 & Erigeron annuus (L.) Pers. & Tarpa & 1949 & Soó Rezső & BPU-07800.JPG \\
\hline 1838 & Erigeron annuus (L.) Pers. & Vácrátót & 1949 & Soó Rezső & BPU-07801.JPG \\
\hline 1838 & Erigeron annuus (L.) Pers. & Vácrátót & 1949 & Soó Rezső & BPU-15565.JPG \\
\hline 1839 & Erigeron acris L. & Budapest & 1947 & Soó Rezső & BPU-07807.JPG \\
\hline 1839 & Erigeron acris L. & Budapest & 1982 & Felföldy Lajos & BPU-07808.JPG \\
\hline 1839 & Erigeron acris L. & Budapest & 2004 & Felföldy Lajos & BPU-07806.JPG \\
\hline 1839 & Erigeron acris L. & Dédestapolcsány & 1928 & Hulják János & BPU-07819.JPG \\
\hline 1839 & Erigeron acris L. & Dörgicse & 1957 & Felföldy Lajos & BPU-07812.JPG \\
\hline 1839 & Erigeron acris L. & Kemence & 1952 & Simon Tibor & BPU-07811.JPG \\
\hline 1839 & Erigeron acris L. & Miskolc & 1950 & Soó Rezső & BPU-07818.JPG \\
\hline 1839 & Erigeron acris L. & Miskolc & 1950 & Soó Rezső, Simon Tibor & BPU-07810.JPG \\
\hline 1839 & Erigeron acris L. & Regéc & 1952 & Simon Tibor & BPU-07809.JPG \\
\hline 1839 & Erigeron acris L. & Vászoly & 1958 & Felföldy Lajos & BPU-07813.JPG \\
\hline 1840 & Conyza canadensis (L.) Cronquist & Budapest & 2004 & Felföldy Lajos & BPU-07803.JPG \\
\hline 1840 & Conyza canadensis (L.) Cronquist & Füzér & 1949 & Simon Tibor, Jakucs Pál & BPU-07804.JPG \\
\hline 1840 & Conyza canadensis (L.) Cronquist & Szilvásvárad & 1953 & $\begin{array}{l}\text { Felföldy Lajos, Tóth } \\
\text { László }\end{array}$ & BPU-07805.JPG \\
\hline 1842 & Filago vulgaris L. & Pécs & 1925 & Soó Rezső & BPU-07829.JPG \\
\hline 1842 & Filago vulgaris L. & Tihany & 1954 & Felföldy Lajos & BPU-07827.JPG \\
\hline 1843 & Filago arvensis L. & Badacsonytomaj & 1953 & Felföldy Lajos & BPU-07832.JPG \\
\hline 1843 & Filago arvensis L. & Beregdaróc & 1951 & $\begin{array}{l}\text { Simon Tibor, Vozáry } \\
\text { Elemér }\end{array}$ & BPU-07834.JPG \\
\hline 1843 & Filago arvensis L. & Dömös & 1948 & Soó Rezső & BPU-07831.JPG \\
\hline 1843 & Filago arvensis L. & Miskolc & 1936 & Soó Rezső & BPU-07837.JPG \\
\hline 1843 & Filago arvensis L. & Tihany & 1954 & Felföldy Lajos & BPU-07833.JPG \\
\hline 1843 & Filago arvensis L. & Tihany & 1959 & Felföldy Lajos & BPU-07836.JPG \\
\hline 1843 & Filago arvensis L. & Uzsa & 1952 & Simon Tibor & BPU-07835.JPG \\
\hline 1844 & Filago minima (Sm.) Pers. & Balatonalmádi & 1913 & Lyka Károly & BPU-07843.JPG \\
\hline 1844 & Filago minima (Sm.) Pers. & Budapest & 2004 & Felföldy Lajos & BPU-07838.JPG \\
\hline
\end{tabular}


1. Elektroniukus melléklet - Electronic Appendix 1.

\begin{tabular}{|c|c|c|c|c|c|}
\hline $\begin{array}{l}\text { Sorszám / } \\
\text { Number }\end{array}$ & Taxon-név / Taxon-name & $\begin{array}{l}\text { Település / } \\
\text { Settlement }\end{array}$ & $\begin{array}{l}\text { Év / } \\
\text { Year }\end{array}$ & Gyưjtő / Collector & $\begin{array}{l}\text { Fájlnév / } \\
\text { File-name }\end{array}$ \\
\hline 1844 & Filago minima (Sm.) Pers. & Sopron & 1948 & Kárpáti Zoltán & BPU-07841.JPG \\
\hline 1844 & Filago minima (Sm.) Pers. & Szenta & 1957 & Soó Rezső & BPU-07840.JPG \\
\hline 1844 & Filago minima (Sm.) Pers. & Szőce & 1955 & Soó Rezső & BPU-07842.JPG \\
\hline 1844 & Filago minima (Sm.) Pers. & Vác & 1923 & Soó Rezső & BPU-07839.JPG \\
\hline 1845 & Micropus erectus L. & Pilisszentiván & 1953 & Soó Rezső & BPU-07823.JPG \\
\hline 1845 & Micropus erectus L. & Tihany & 1928 & Soó Rezső & BPU-07824.JPG \\
\hline 1845 & Micropus erectus L. & Tihany & 1929 & Soó Rezső & BPU-07825.JPG \\
\hline 1845 & Micropus erectus L. & Tihany & 1956 & $\begin{array}{l}\text { Felföldy Lajos, Tóth } \\
\text { László }\end{array}$ & BPU-07826.JPG \\
\hline 1846 & Gnaphalium sylvaticum L. & Bakonyszombathely & 1942 & Felföldy Lajos & BPU-07862.JPG \\
\hline 1846 & Gnaphalium sylvaticum L. & Csaroda & 1952 & Soó Rezső & BPU-07858.JPG \\
\hline 1846 & Gnaphalium sylvaticum L. & Diósjenő & 1952 & Simon Tibor & BPU-07861.JPG \\
\hline 1846 & Gnaphalium sylvaticum L. & Gyöngyös & 1952 & Soó Rezső & BPU-07860.JPG \\
\hline 1846 & Gnaphalium sylvaticum L. & Hidegkút & 1957 & Felföldy Lajos & BPU-07863.JPG \\
\hline 1846 & Gnaphalium sylvaticum L. & Miskolc & 1934 & Soó Rezső & BPU-07859.JPG \\
\hline 1847 & Gnaphalium uliginosum L. & Budapest & - & Soó Rezső & BPU-07868.JPG \\
\hline 1847 & Gnaphalium uliginosum L. & Dörgicse & 1957 & Felföldy Lajos & BPU-07875.JPG \\
\hline 1847 & Gnaphalium uliginosum L. & Egyek & 1950 & $\begin{array}{l}\text { Simon Tibor, Borsos Olga, } \\
\text { Kulcsár Gábor }\end{array}$ & BPU-07869.JPG \\
\hline 1847 & Gnaphalium uliginosum L. & Gyöngyös & 1953 & Soó Rezső & BPU-07870.JPG \\
\hline 1847 & Gnaphalium uliginosum L. & Kaszó & 1957 & Soó Rezső & BPU-07871.JPG \\
\hline 1847 & Gnaphalium uliginosum L. & Kőszeg & 1932 & Soó Rezső & BPU-07872.JPG \\
\hline 1847 & Gnaphalium uliginosum L. & Sárospatak & 1939 & Soó Rezső & BPU-07876.JPG \\
\hline 1847 & Gnaphalium uliginosum L. & Szentgotthárd & 1955 & Soó Rezső & BPU-07873.JPG \\
\hline 1847 & Gnaphalium uliginosum L. & Szombathely & 1964 & Simon Tibor & BPU-07874.JPG \\
\hline 1848 & Gnaphalium luteoalbum L. & Bisse & 1965 & $\begin{array}{l}\text { Simon Tibor, Priszter } \\
\text { Szaniszló, Borhidi Attila }\end{array}$ & BPU-07878.JPG \\
\hline 1848 & Gnaphalium luteoalbum L. & Bugac & 1952 & Vozáry Elemér & BPU-15445.JPG \\
\hline 1848 & Gnaphalium luteoalbum L. & Dávod & 1955 & Soó Rezső & BPU-07882.JPG \\
\hline 1848 & Gnaphalium luteoalbum L. & Debrecen & 1951 & Kulcsár Gábor & BPU-07880.JPG \\
\hline 1848 & Gnaphalium luteoalbum L. & Debrecen & - & Soó Rezső & BPU-07881.JPG \\
\hline 1848 & Gnaphalium luteoalbum L. & Dorog & 1965 & Priszter Szaniszló & BPU-07879.JPG \\
\hline 1848 & Gnaphalium luteoalbum L. & Kaszó & 1957 & Soó Rezső & BPU-07883.JPG \\
\hline 1848 & Gnaphalium luteoalbum L. & Pusztamiske & 1948 & Borsos Olga & BPU-07884.JPG \\
\hline 1849 & Helichrysum arenarium (L.) Moench & "Szentendrei-sziget" & 1952 & Vozáry Elemér & BPU-07896.JPG \\
\hline 1849 & Helichrysum arenarium (L.) Moench & "Szentendrei-sziget" & 1953 & Borsos Olga & BPU-07895.JPG \\
\hline 1849 & Helichrysum arenarium (L.) Moench & Bakonyszentlászló & 1963 & Soó Rezső & BPU-07893.JPG \\
\hline 1849 & Helichrysum arenarium (L.) Moench & Baktalórántháza & 1933 & Soó Rezső & BPU-07894.JPG \\
\hline 1849 & Helichrysum arenarium (L.) Moench & Uzsa & 1953 & Borsos Olga & BPU-07892.JPG \\
\hline 1849 & Helichrysum arenarium (L.) Moench & Uzsa & 1953 & $\begin{array}{l}\text { Felföldy Lajos, Tóth } \\
\text { László }\end{array}$ & BPU-07897.JPG \\
\hline 1850 & Antennaria dioica (L.) Gaertn. & Bükkszentkereszt & 1938 & Soó Rezső & BPU-07848.JPG \\
\hline 1851 & Inula helenium L. & Regéc & 1951 & Simon Tibor & BPU-07899.JPG \\
\hline 1851 & Inula helenium L. & Szenna & 1957 & Soó Rezső & BPU-07900.JPG \\
\hline 1852 & Inula conyza DC. & Badacsonytördemic & 1953 & $\begin{array}{l}\text { Felföldy Lajos, Tóth } \\
\text { László }\end{array}$ & BPU-07908.JPG \\
\hline 1852 & Inula conyza DC. & Budapest & 1947 & Soó Rezső & BPU-07905.JPG \\
\hline 1852 & Inula conyza DC. & Budapest & 1982 & Felföldy Lajos & BPU-07907.JPG \\
\hline 1852 & Inula conyza DC. & Gyöngyös & 1952 & Soó Rezső & BPU-07904.JPG \\
\hline 1852 & Inula conyza DC. & Kemence & 1952 & Simon Tibor & BPU-07903.JPG \\
\hline
\end{tabular}


1. Elektroniukus melléklet - Electronic Appendix 1.

\begin{tabular}{|c|c|c|c|c|c|}
\hline $\begin{array}{l}\text { Sorszám / } \\
\text { Number }\end{array}$ & Taxon-név / Taxon-name & $\begin{array}{l}\text { Település / } \\
\text { Settlement }\end{array}$ & $\begin{array}{l}\text { Év / } \\
\text { Year }\end{array}$ & Gyűjtő / Collector & $\begin{array}{l}\text { Fájlnév / } \\
\text { File-name }\end{array}$ \\
\hline 1852 & Inula conyza DC. & Miskolc & 1937 & Soó Rezső & BPU-07906.JPG \\
\hline 1852 & Inula conyza DC. & Tihany & 1949 & Simon Tibor & BPU-07902.JPG \\
\hline 1853 & Inula hirta L. & Badacsonytördemic & 1953 & $\begin{array}{l}\text { Felföldy Lajos, Tóth } \\
\text { László }\end{array}$ & BPU-07948.JPG \\
\hline 1853 & Inula hirta L. & Bagamér & 1933 & Máthé Imre & BPU-07944.JPG \\
\hline 1853 & Inula hirta L. & Bátorliget & 1951 & Simon Tibor & BPU-07947.JPG \\
\hline 1853 & Inula hirta L. & Budapest & 1991 & Felföldy Lajos & BPU-07942.JPG \\
\hline 1853 & Inula hirta L. & Dömös & 1952 & Soó Rezső & BPU-07943.JPG \\
\hline 1853 & Inula hirta L. & Gyöngyös & 1961 & Soó Rezső & BPU-07945.JPG \\
\hline 1853 & Inula hirta L. & Nagykovácsi & 1968 & Priszter Szaniszló & BPU-07946.JPG \\
\hline 1853 & Inula hirta L. & Tihany & 1961 & Sólymossy Gizella & BPU-07949.JPG \\
\hline 1853 & Inula hirta L. & Vászoly & 1958 & Felföldy Lajos & BPU-07950.JPG \\
\hline 1854 & Inula britannica L. & Balatonszőlős & 1957 & $\begin{array}{l}\text { Felföldy Lajos, Kovács } \\
\text { Margit, Tóth László }\end{array}$ & BPU-07965.JPG \\
\hline 1854 & Inula britannica L. & Bódvarákó & 1951 & $\begin{array}{l}\text { Soó Rezső, Jakucs Pál, Ér } \\
\text { Lajos }\end{array}$ & BPU-07960.JPG \\
\hline 1854 & Inula britannica L. & Egyek & 1934 & Soó Rezső & BPU-07961.JPG \\
\hline 1854 & Inula britannica L. & Fertőhomok & 1960 & Tóth László & BPU-07959.JPG \\
\hline 1854 & Inula britannica L. & Gelénes & 1951 & $\begin{array}{l}\text { Simon Tibor, Vozáry } \\
\text { Elemér }\end{array}$ & BPU-07963.JPG \\
\hline 1854 & Inula britannica L. & Rácalmás & 1932 & Pátkay Imre & BPU-07962.JPG \\
\hline 1854 & Inula britannica L. & Szilvásvárad & 1953 & $\begin{array}{l}\text { Felföldy Lajos, Tóth } \\
\text { László }\end{array}$ & BPU-07966.JPG \\
\hline 1854 & Inula britannica L. & Vácrátót & 1953 & Felföldy Lajos & BPU-07964.JPG \\
\hline 1855 & Inula oculus-christi L. & Budapest & 1947 & Soó Rezső & BPU-07967.JPG \\
\hline 1855 & Inula oculus-christi L. & Csákvár & 1948 & Soó Rezső & BPU-07968.JPG \\
\hline 1855 & Inula oculus-christi L. & Tihany & 1959 & Felföldy Lajos & BPU-07969.JPG \\
\hline 1856 & Inula germanica L. & Balatonfüred & 1947 & Pólya László & BPU-07957.JPG \\
\hline 1856 & Inula germanica L. & Budapest & 1953 & Vajda László & BPU-07952.JPG \\
\hline 1856 & Inula germanica L. & Budapest & 1964 & Priszter Szaniszló & BPU-07951.JPG \\
\hline 1856 & Inula germanica L. & Debrecen & 1948 & Simon Tibor & BPU-07953.JPG \\
\hline 1856 & Inula germanica L. & Egyek & 1960 & Simon Tibor & BPU-07954.JPG \\
\hline 1856 & Inula germanica L. & Kerecsend & 1957 & Soó Rezső & BPU-07955.JPG \\
\hline 1856 & Inula germanica L. & Nagymaros & 1947 & Soó Rezső & BPU-07956.JPG \\
\hline 1856 & Inula germanica L. & Tihany & 1961 & $\begin{array}{l}\text { Pankov H., Felföldy Lajos, } \\
\text { Sólymosy G. }\end{array}$ & BPU-07958.JPG \\
\hline 1857 & Inula salicina L. & "Csepel-sziget" & 1909 & Kocsis István & BPU-07926.JPG \\
\hline 1857 & Inula salicina L. & Bagamér & 1932 & Soó Rezső & BPU-07930.JPG \\
\hline 1857 & Inula salicina L. & Budaörs & 1982 & Felföldy Lajos & BPU-07936.JPG \\
\hline 1857 & Inula salicina L. & Budapest & 1982 & Felföldy Lajos & BPU-07924.JPG \\
\hline 1857 & Inula salicina L. & Budapest & 1982 & Felföldy Lajos & BPU-07925.JPG \\
\hline 1857 & Inula salicina L. & Budapest & 1992 & Felföldy Lajos & BPU-07921.JPG \\
\hline 1857 & Inula salicina L. & Budapest & 2005 & Felföldy Lajos & BPU-07922.JPG \\
\hline 1857 & Inula salicina L. & Budapest & 2005 & Felföldy Lajos & BPU-07923.JPG \\
\hline 1857 & Inula salicina L. & Diósjenő & 1952 & Simon Tibor & BPU-07935.JPG \\
\hline 1857 & Inula salicina L. & Egyek & 1932 & Soó Rezső & BPU-07929.JPG \\
\hline 1857 & Inula salicina L. & Gyöngyös & 1961 & Soó Rezső & BPU-07933.JPG \\
\hline 1857 & Inula salicina L. & Hévíz & 1961 & Felföldy Lajos & BPU-07939.JPG \\
\hline 1857 & Inula salicina L. & Hosszúpályi & 1933 & Soó Rezső & BPU-07927.JPG \\
\hline 1857 & Inula salicina L. & Kaszó & 1957 & Soó Rezső & BPU-07937.JPG \\
\hline
\end{tabular}


1. Elektroniukus melléklet - Electronic Appendix 1.

\begin{tabular}{|c|c|c|c|c|c|}
\hline $\begin{array}{l}\text { Sorszám / } \\
\text { Number }\end{array}$ & Taxon-név / Taxon-name & $\begin{array}{l}\text { Település / } \\
\text { Settlement }\end{array}$ & $\begin{array}{l}\text { Év / } \\
\text { Year }\end{array}$ & Gyűjtő / Collector & $\begin{array}{l}\text { Fájlnév / } \\
\text { File-name }\end{array}$ \\
\hline 1857 & Inula salicina $\mathrm{L}$. & Kerecsend & 1957 & Soó Rezső & BPU-07931.JPG \\
\hline 1857 & Inula salicina $\mathrm{L}$. & Kerecsend & 1957 & Soó Rezső & BPU-07932.JPG \\
\hline 1857 & Inula salicina L. & Kimle & 1962 & Borsos Olga & BPU-07934.JPG \\
\hline 1857 & Inula salicina L. & Kőszeg & 1932 & Soó Rezső & BPU-07938.JPG \\
\hline 1857 & Inula salicina L. & Miskolc & 1929 & Soó Rezső & BPU-07928.JPG \\
\hline 1857 & Inula salicina L. & Tarpa & 1949 & Simon Tibor & BPU-15564.JPG \\
\hline 1857 & Inula salicina L. & Tokaj & 1947 & Soó Rezső & BPU-07940.JPG \\
\hline 1858 & Inula ensifolia L. & Badacsonytördemic & 1953 & $\begin{array}{l}\text { Felföldy Lajos, Tóth } \\
\text { László }\end{array}$ & BPU-07918.JPG \\
\hline 1858 & Inula ensifolia L. & Bodajk & 1961 & Borsos Olga, Simon Tibor & BPU-07914.JPG \\
\hline 1858 & Inula ensifolia L. & Budaörs & 1982 & Felföldy Lajos & BPU-07916.JPG \\
\hline 1858 & Inula ensifolia L. & Budapest & 1947 & Soó Rezső & BPU-07912.JPG \\
\hline 1858 & Inula ensifolia L. & Budapest & 1982 & Felföldy Lajos & BPU-07910.JPG \\
\hline 1858 & Inula ensifolia L. & Budapest & 1982 & Felföldy Lajos & BPU-07911.JPG \\
\hline 1858 & Inula ensifolia L. & Budapest & 1991 & Felföldy Lajos & BPU-07909.JPG \\
\hline 1858 & Inula ensifolia L. & Eger & 1953 & Simon Tibor & BPU-07915.JPG \\
\hline 1858 & Inula ensifolia L. & Nagykovácsi & 1968 & Priszter Szaniszló & BPU-07917.JPG \\
\hline 1858 & Inula ensifolia L. & Pécs & 1938 & Soó Rezső & BPU-07913.JPG \\
\hline 1858 & Inula ensifolia L. & Sátoraljaújhely & 1937 & Soó Rezső & BPU-07919.JPG \\
\hline 1859 & Inula spiraeifolia L. & Pécs & 1935 & Soó Rezső & BPU-07941.JPG \\
\hline $\begin{array}{c}1853 \times \\
1857\end{array}$ & Inula $\times$ rigida Döll & Miskolc & 1938 & Soó Rezső & BPU-07975.JPG \\
\hline $\begin{array}{c}1856 \times \\
1857\end{array}$ & Inula germanica L. $\times$ Inula salicina L. & Budapest & 1946 & Vajda László & BPU-07973.JPG \\
\hline $\begin{array}{c}1856 \times \\
1857 \times \\
1858\end{array}$ & $\begin{array}{l}\text { Inula germanica L. } \times \text { Inula salicina L. } \times \\
\text { Inula ensifolia L. }\end{array}$ & Budapest & 1945 & Vajda László & BPU-07976.JPG \\
\hline $\begin{array}{c}1856 \times \\
1858\end{array}$ & Inula × hybrida Baumg. & Budapest & 1953 & Vajda László & BPU-07972.JPG \\
\hline $\begin{array}{c}1856 \times \\
1858\end{array}$ & Inula $\times$ hybrida Baumg & Nagymaros & 1949 & Vajda László & BPU-07971.JPG \\
\hline $\begin{array}{c}1857 \times \\
1858\end{array}$ & Inula $\times$ stricta Tausch & Kisgyőr & 1932 & Hulják János & BPU-07977.JPG \\
\hline $\begin{array}{c}1857 \times \\
1859\end{array}$ & Inula salicina L. $\times$ Inula spiraeifolia L. & Pécs & 1938 & Soó Rezső & BPU-07974.JPG \\
\hline--- & Inula vrabelyiana Kern. & Aszófő & 1931 & Soó Rezső & BPU-07978.JPG \\
\hline 1860 & Pulicaria vulgaris Gaertn. & Balmazújváros & 1932 & Soó Rezső & BPU-07980.JPG \\
\hline 1860 & Pulicaria vulgaris Gaertn. & Csaroda & 1948 & Simon Tibor, Jakucs Pál & BPU-07982.JPG \\
\hline 1860 & Pulicaria vulgaris Gaertn. & Gelénes & 1951 & $\begin{array}{l}\text { Simon Tibor, Vozáry } \\
\text { Elemér }\end{array}$ & BPU-07981.JPG \\
\hline 1860 & Pulicaria vulgaris Gaertn. & Vácrátót & 1953 & Felföldy Lajos & BPU-07983.JPG \\
\hline 1861 & Pulicaria dysenterica (L.) Bernh. & Balatongyörök & 1961 & Felföldy Lajos & BPU-07988.JPG \\
\hline 1861 & Pulicaria dysenterica (L.) Bernh. & Budapest & 1911 & Kocsis István & BPU-07987.JPG \\
\hline 1861 & Pulicaria dysenterica (L.) Bernh. & Gárdony & 1980 & Felföldy Lajos & BPU-07989.JPG \\
\hline 1861 & Pulicaria dysenterica (L.) Bernh. & Hencida & 1939 & Kovács L. & BPU-07985.JPG \\
\hline 1861 & Pulicaria dysenterica (L.) Bernh. & Siófok & 1928 & Soó Rezső & BPU-07984.JPG \\
\hline 1861 & Pulicaria dysenterica (L.) Bernh. & Uzsa & 1952 & Simon Tibor & BPU-07986.JPG \\
\hline 1864 & Buphthalmum salicifolium L. & Devecser & 1957 & Tallós Pál & BPU-07991.JPG \\
\hline 1864 & Buphthalmum salicifolium L. & Sopron & 1932 & Soó Rezső & BPU-07990.JPG \\
\hline 1865 & Telekia speciosa (Schreb.) Baumg. & Miskolc & 1950 & Soó Rezső & BPU-07993.JPG \\
\hline 1865 & Telekia speciosa (Schreb.) Baumg. & Miskolc & 1950 & Soó Rezső, Simon Tibor & BPU-07992.JPG \\
\hline
\end{tabular}


1. Elektroniukus melléklet - Electronic Appendix 1.

\begin{tabular}{|c|c|c|c|c|c|}
\hline $\begin{array}{l}\text { Sorszám / } \\
\text { Number }\end{array}$ & Taxon-név / Taxon-name & $\begin{array}{l}\text { Település / } \\
\text { Settlement }\end{array}$ & $\begin{array}{l}\text { Év / } \\
\text { Year }\end{array}$ & Gyújtő / Collector & $\begin{array}{l}\text { Fájlnév / } \\
\text { File-name }\end{array}$ \\
\hline 1866 & Bidens cernua L. & Egyek & 1934 & Soó Rezső & BPU-08053.JPG \\
\hline 1866 & Bidens cernua L. & Isaszeg & 1949 & Soó Rezső & BPU-08052.JPG \\
\hline 1866 & Bidens cernua L. & Örvényes & 1958 & Felföldy Lajos & BPU-08058.JPG \\
\hline 1866 & Bidens cernua L. & Ukk & 1957 & Felföldy Lajos & BPU-08057.JPG \\
\hline 1866 & Bidens cernua L. & Vácrátót & 1953 & Felföldy Lajos & BPU-08056.JPG \\
\hline 1866 & Bidens cernua L. & Veresegyház & 1944 & Kárpáti Zoltán & BPU-08055.JPG \\
\hline 1867 & Bidens tripartita L. & Adony & 1971 & Priszter Szaniszló & BPU-08040.JPG \\
\hline 1867 & Bidens tripartita L. & Balassagyarmat & 1971 & Priszter Szaniszló & BPU-08044.JPG \\
\hline 1867 & Bidens tripartita L. & Balassagyarmat & 1971 & Priszter Szaniszló & BPU-08045.JPG \\
\hline 1867 & Bidens tripartita L. & Balatongyörök & 1961 & Felföldy Lajos & BPU-08048.JPG \\
\hline 1867 & Bidens tripartita L. & Dörgicse & 1957 & Felföldy Lajos & BPU-08046.JPG \\
\hline 1867 & Bidens tripartita L. & Hajdúbagos & 1937 & Soó Rezső & BPU-08039.JPG \\
\hline 1867 & Bidens tripartita L. & Monoszló & 1982 & Felföldy Lajos & BPU-08047.JPG \\
\hline 1867 & Bidens tripartita L. & Pellérd & 1968 & Priszter Szaniszló & BPU-08037.JPG \\
\hline 1867 & Bidens tripartita L. & Pellérd & 1968 & Priszter Szaniszló & BPU-09231.JPG \\
\hline 1867 & Bidens tripartita L. & Szarvas & 1947 & Soó Rezső & BPU-08038.JPG \\
\hline 1867 & Bidens tripartita L. & Szilvásvárad & 1953 & $\begin{array}{l}\text { Felföldy Lajos, Tóth } \\
\text { László }\end{array}$ & BPU-08042.JPG \\
\hline 1867 & Bidens tripartita L. & Tiszafüred & 1952 & Soó Rezső & BPU-08043.JPG \\
\hline 1867 & Bidens tripartita L. & Újszentmargita & 1961 & Priszter Szaniszló & BPU-08041.JPG \\
\hline 1868 & Bidens frondosa L. & Budapest & 1994 & Felföldy Lajos & BPU-08049.JPG \\
\hline 1868 & Bidens frondosa L. & Szigetmonostor & 1964 & Isépy István & BPU-08050.JPG \\
\hline 1868 & Bidens frondosa L. & Visegrád & 1967 & Priszter Szaniszló & BPU-08051.JPG \\
\hline 1869 & Rudbeckia laciniata L. & Szigetújfalu & 1949 & Vajda László & BPU-08026.JPG \\
\hline 1869 & Rudbeckia laciniata L. & Tarpa & 1948 & Simon Tibor, Jakucs Pál & BPU-08025.JPG \\
\hline 1871 & Helianthus annuus L. & Balatonalmádi & 1963 & Soó Rezső & BPU-08028.JPG \\
\hline 1872 & Helianthus tuberosus L. & Győr & 1930 & Polgár Sándor & BPU-08034.JPG \\
\hline 1872 & Helianthus tuberosus L. & Keszthely & 1959 & Priszter Szaniszló & BPU-08029.JPG \\
\hline 1872 & Helianthus tuberosus L. & Keszthely & 1959 & Priszter Szaniszló & BPU-08030.JPG \\
\hline 1872 & Helianthus tuberosus L. & Keszthely & 1959 & Priszter Szaniszló & BPU-08032.JPG \\
\hline 1872 & Helianthus tuberosus L. & Nőtincs & 1974 & Priszter Szaniszló & BPU-08035.JPG \\
\hline 1872 & Helianthus tuberosus L. & Nőtincs & 1974 & Priszter Szaniszló & BPU-08036.JPG \\
\hline 1873 & Helianthus pauciflorus Nutt. & Budapest & 1958 & Priszter Szaniszló & BPU-08031.JPG \\
\hline 1875 & Iva xanthiifolia Nutt. & Tiszaug & 1953 & Jávorka Sándor & BPU-08016.JPG \\
\hline 1876 & Ambrosia artemisiifolia L. & Bakonyszentlászló & 1963 & Soó Rezső & BPU-08012.JPG \\
\hline 1876 & Ambrosia artemisiifolia L. & Balatongyörök & 1961 & Felföldy Lajos & BPU-08014.JPG \\
\hline 1876 & Ambrosia artemisiifolia L. & Balatonkenese & 1982 & Felföldy Lajos & BPU-08011.JPG \\
\hline 1876 & Ambrosia artemisiifolia L. & Bárdudvarnok & 1966 & Priszter Szaniszló & BPU-08010.JPG \\
\hline 1876 & Ambrosia artemisiifolia L. & Budapest & 1982 & Felföldy Lajos & BPU-07998.JPG \\
\hline 1876 & Ambrosia artemisiifolia L. & Budapest & 2004 & Felföldy Lajos & BPU-07997.JPG \\
\hline 1876 & Ambrosia artemisiifolia L. & Bugac & 1964 & Soó Rezső & BPU-07999.JPG \\
\hline 1876 & Ambrosia artemisiifolia L. & Csányoszró & 1968 & Priszter Szaniszló & BPU-08000.JPG \\
\hline 1876 & Ambrosia artemisiifolia L. & Dabas & 1964 & Soó Rezső & BPU-08009.JPG \\
\hline 1876 & Ambrosia artemisiifolia L. & Drávaszerdahely & 1968 & Priszter Szaniszló & BPU-08001.JPG \\
\hline 1876 & Ambrosia artemisiifolia L. & Mohács & 1966 & Priszter Szaniszló & BPU-08002.JPG \\
\hline 1876 & Ambrosia artemisiifolia L. & Nagyharsány & 1965 & Priszter Szaniszló & BPU-08003.JPG \\
\hline 1876 & Ambrosia artemisiifolia L. & Nagykanizsa & 1956 & Felföldy Lajos & BPU-08015.JPG \\
\hline 1876 & Ambrosia artemisiifolia L. & Ócsa & 1965 & Priszter Szaniszló & BPU-08006.JPG \\
\hline
\end{tabular}


1. Elektroniukus melléklet - Electronic Appendix 1.

\begin{tabular}{|c|c|c|c|c|c|}
\hline $\begin{array}{l}\text { Sorszám / } \\
\text { Number }\end{array}$ & Taxon-név / Taxon-name & $\begin{array}{l}\text { Település / } \\
\text { Settlement }\end{array}$ & $\begin{array}{l}\text { Év / } \\
\text { Year }\end{array}$ & Gyújtő / Collector & $\begin{array}{l}\text { Fájlnév / } \\
\text { File-name }\end{array}$ \\
\hline 1876 & Ambrosia artemisiifolia L. & Ócsa & 1965 & Priszter Szaniszló & BPU-08007.JPG \\
\hline 1876 & Ambrosia artemisiifolia L. & Örkény & 1964 & Soó Rezső & BPU-08008.JPG \\
\hline 1876 & Ambrosia artemisiifolia L. & Pécs & 1965 & Priszter Szaniszló & BPU-08004.JPG \\
\hline 1876 & Ambrosia artemisiifolia L. & Uzsa & 1952 & Simon Tibor & BPU-08013.JPG \\
\hline 1876 & Ambrosia artemisiifolia L. & Villány & 1962 & Beremendi Ferenc & BPU-08005.JPG \\
\hline 1878 & Xanthium spinosum L. & Komjáti & 1950 & Jakucs Pál, Kulcsár Gábor & BPU-15478.JPG \\
\hline 1879 & Xanthium strumarium L. & Göd & 1947 & Kárpáti Zoltán & BPU-08024.JPG \\
\hline 1881 & Xanthium italicum Moretti & Lakitelek & 1968 & Priszter Szaniszló & BPU-08019.JPG \\
\hline 1881 & Xanthium italicum Moretti & Nagyrév & 1968 & Priszter Szaniszló & BPU-08020.JPG \\
\hline 1881 & Xanthium italicum Moretti & Szolnok & 1968 & Priszter Szaniszló & BPU-08021.JPG \\
\hline 1881 & Xanthium italicum Moretti & Szolnok & 1968 & Priszter Szaniszló & BPU-08022.JPG \\
\hline 1881 & Xanthium italicum Moretti & Szolnok & 1968 & Priszter Szaniszló & BPU-08023.JPG \\
\hline 1882 & Xanthium saccharatum Wallr. & Lakitelek & 1968 & Priszter Szaniszló & BPU-08017.JPG \\
\hline 1882 & Xanthium saccharatum Wallr. & Szolnok & 1968 & Priszter Szaniszló & BPU-08018.JPG \\
\hline 1885 & Galinsoga ciliata (Raf.) S.F. Blake & Budapest & 1946 & Kárpáti Zoltán & BPU-08061.JPG \\
\hline 1885 & Galinsoga ciliata (Raf.) S.F. Blake & Szarvas & 1949 & Soó Rezső & BPU-08059.JPG \\
\hline 1885 & Galinsoga ciliata (Raf.) S.F. Blake & Szarvas & 1949 & Soó Rezső & BPU-08060.JPG \\
\hline 1886 & Galinsoga parviflora Cav. & Tihany & 1959 & Felföldy Lajos & BPU-08062.JPG \\
\hline 1890 & Anthemis cotula L. & Gelénes & 1951 & $\begin{array}{l}\text { Simon Tibor, Vozáry } \\
\text { Elemér }\end{array}$ & BPU-08064.JPG \\
\hline 1891 & Anthemis arvensis L. & Hollóháza & 1940 & Siroki Zoltán & BPU-08076.JPG \\
\hline 1891 & Anthemis arvensis L. & Tarpa & 1949 & Soó Rezső & BPU-08077.JPG \\
\hline 1892 & Anthemis ruthenica L. & Debrecen & 1931 & Soó Rezső & BPU-08079.JPG \\
\hline 1892 & Anthemis ruthenica L. & Tihany & 1942 & Felföldy Lajos & BPU-08078.JPG \\
\hline 1893 & Anthemis austriaca Jacq. & Debrecen & 1949 & Soó Rezső & BPU-08066.JPG \\
\hline 1893 & Anthemis austriaca Jacq. & Egyek & 1950 & $\begin{array}{l}\text { Simon Tibor, Borsos Olga, } \\
\text { Kulcsár Gábor }\end{array}$ & BPU-08067.JPG \\
\hline 1893 & Anthemis austriaca Jacq. & Gyöngyös & 1923 & Soó Rezső & BPU-08065.JPG \\
\hline 1894 & Anthemis tinctoria L. & Balatonszőlős & 1957 & Felföldy Lajos & BPU-08073.JPG \\
\hline 1894 & Anthemis tinctoria L. & Bátorliget & 1950 & $\begin{array}{l}\text { Simon Tibor, Kárpáti } \\
\text { István }\end{array}$ & BPU-08072.JPG \\
\hline 1894 & Anthemis tinctoria L. & Budapest & 2004 & Felföldy Lajos & BPU-08068.JPG \\
\hline 1894 & Anthemis tinctoria L. & Hajdúszoboszló & 1937 & Soó Rezső & BPU-08070.JPG \\
\hline 1894 & Anthemis tinctoria L. & Nagyvisnyó & 1948 & $\begin{array}{l}\text { Simon Tibor, Pólya } \\
\text { László, Jakucs Pál }\end{array}$ & BPU-08069.JPG \\
\hline 1894 & Anthemis tinctoria L. & Vác & 1923 & Soó Rezső & BPU-08071.JPG \\
\hline 1895 & Achillea ptarmica L. & Aggtelek & 1953 & Jakucs Pál & BPU-08091.JPG \\
\hline 1895 & Achillea ptarmica L. & Alsószölnök & 1955 & Soó Rezső & BPU-08087.JPG \\
\hline 1895 & Achillea ptarmica L. & Hollóháza & 2007 & Simon Tibor & BPU-15509.JPG \\
\hline 1895 & Achillea ptarmica L. & Somogyszob & 1953 & Tallós Pál & BPU-08090.JPG \\
\hline 1895 & Achillea ptarmica L. & Túrricse & 1953 & Simon Tibor & BPU-08089.JPG \\
\hline 1896 & Achillea ochroleuca Ehrh. & Csévharaszt & 1959 & Simon Tibor, Borsos Olga & BPU-08093.JPG \\
\hline 1896 & Achillea ochroleuca Ehrh. & Szigetmonostor & 1947 & Soó Rezső & BPU-08092.JPG \\
\hline 1897 & Achillea crithmifolia Waldst. et Kit. & Gyöngyös & 1923 & Soó Rezső & BPU-08119.JPG \\
\hline 1897 & Achillea crithmifolia Waldst. et Kit. & Miskolc & 1938 & Soó Rezső & BPU-08118.JPG \\
\hline 1899 & Achillea nobilis L. & Budapest & 1991 & Felföldy Lajos & BPU-08095.JPG \\
\hline 1899 & Achillea nobilis L. & Budapest & 2004 & Felföldy Lajos & BPU-08094.JPG \\
\hline 1899 & Achillea nobilis L. & Debrecen & 1926 & Máthé Imre & BPU-08101.JPG \\
\hline 1899 & Achillea nobilis L. & Debrecen & 1947 & Soó Rezső & BPU-08099.JPG \\
\hline
\end{tabular}


1. Elektroniukus melléklet - Electronic Appendix 1.

\begin{tabular}{|c|c|c|c|c|c|}
\hline $\begin{array}{l}\text { Sorszám / } \\
\text { Number }\end{array}$ & Taxon-név / Taxon-name & $\begin{array}{l}\text { Település / } \\
\text { Settlement }\end{array}$ & $\begin{array}{l}\text { Év / } \\
\text { Year }\end{array}$ & Gyújtő / Collector & $\begin{array}{l}\text { Fájlnév / } \\
\text { File-name }\end{array}$ \\
\hline 1899 & Achillea nobilis L. & Miskolc & 1934 & Soó Rezső & BPU-08098.JPG \\
\hline 1899 & Achillea nobilis L. & Tihany & 1947 & Pólya László & BPU-08100.JPG \\
\hline 1899 & Achillea nobilis L. & Tihany & 1959 & Felföldy Lajos & BPU-08096.JPG \\
\hline 1899 & Achillea nobilis L. & Tihany & 1961 & $\begin{array}{l}\text { Pankov H., Felföldy Lajos, } \\
\text { Sólymosy G. }\end{array}$ & BPU-08097.JPG \\
\hline 1901 & $\begin{array}{l}\text { Achillea distans Waldst. et Kit. ex } \\
\text { Willd. }\end{array}$ & Bélapátfalva & 1950 & $\begin{array}{l}\text { Simon Tibor, Jakucs Pál, } \\
\text { Kárpáti István }\end{array}$ & BPU-08103.JPG \\
\hline 1901 & $\begin{array}{l}\text { Achillea distans Waldst. et Kit. ex } \\
\text { Willd. }\end{array}$ & Budapest & 1961 & Soó Rezső & BPU-08102.JPG \\
\hline 1901 & $\begin{array}{l}\text { Achillea distans Waldst. et Kit. ex } \\
\text { Willd. }\end{array}$ & Dömös & 1948 & Soó Rezső & BPU-08105.JPG \\
\hline 1901 & $\begin{array}{l}\text { Achillea distans Waldst. et Kit. ex } \\
\text { Willd. }\end{array}$ & Gánt & 1953 & Vajda László & BPU-08107.JPG \\
\hline 1901 & $\begin{array}{l}\text { Achillea distans Waldst. et Kit. ex } \\
\text { Willd. }\end{array}$ & Isztimér & 1949 & Soó Rezső & BPU-08106.JPG \\
\hline 1901 & $\begin{array}{l}\text { Achillea distans Waldst. et Kit. ex } \\
\text { Willd. }\end{array}$ & Kisgyőr & 1938 & Soó Rezső & BPU-08104.JPG \\
\hline 1901 & $\begin{array}{l}\text { Achillea distans Waldst. et Kit. ex } \\
\text { Willd. }\end{array}$ & Pilisszentkereszt & 1905 & Filarszky Nándor & BPU-08110.JPG \\
\hline 1901 & $\begin{array}{l}\text { Achillea distans Waldst. et Kit. ex } \\
\text { Willd. }\end{array}$ & Szilvásvárad & 1953 & $\begin{array}{l}\text { Felföldy Lajos, Tóth } \\
\text { László }\end{array}$ & BPU-08108.JPG \\
\hline 1901 & $\begin{array}{l}\text { Achillea distans Waldst. et Kit. ex } \\
\text { Willd. }\end{array}$ & Szilvásvárad & 1974 & Priszter Szaniszló & BPU-08109.JPG \\
\hline 1903 & Achillea setacea Waldst. et Kit. & Abaújszántó & 1944 & Siroki Zoltán & BPU-08124.JPG \\
\hline 1903 & Achillea setacea Waldst. et Kit. & Akasztó & 1961 & Borsos Olga, Simon Tibor & BPU-08123.JPG \\
\hline 1903 & Achillea setacea Waldst. et Kit. & Egyek & 1947 & Soó Rezső & BPU-08122.JPG \\
\hline 1903 & Achillea setacea Waldst. et Kit. & Pécsely & 1950 & Felföldy Lajos & BPU-08126.JPG \\
\hline 1903 & Achillea setacea Waldst. et Kit. & Tihany & 1959 & Felföldy Lajos & BPU-08120.JPG \\
\hline 1903 & Achillea setacea Waldst. et Kit. & Tihany & 1959 & Felföldy Lajos & BPU-08121.JPG \\
\hline 1903 & Achillea setacea Waldst. et Kit. & Tihany & 1959 & Felföldy Lajos & BPU-08125.JPG \\
\hline 1904 & Achillea asplenifolia Vent. & Balatonszőlős & 1956 & $\begin{array}{l}\text { Felföldy Lajos, Tóth } \\
\text { László }\end{array}$ & BPU-08133.JPG \\
\hline 1904 & Achillea asplenifolia Vent. & Balatonszőlős & 1957 & $\begin{array}{l}\text { Felföldy Lajos, Tóth } \\
\text { László }\end{array}$ & BPU-08134.JPG \\
\hline 1904 & Achillea asplenifolia Vent. & Bátorliget & 1953 & Soó Rezső & BPU-08132.JPG \\
\hline 1904 & Achillea asplenifolia Vent. & Budapest & 2005 & Felföldy Lajos & BPU-08127.JPG \\
\hline 1904 & Achillea asplenifolia Vent. & Bugac & 1964 & Soó Rezső & BPU-08128.JPG \\
\hline 1904 & Achillea asplenifolia Vent. & Ócsa & 1923 & Soó Rezső & BPU-08129.JPG \\
\hline 1904 & Achillea asplenifolia Vent. & Ócsa & 1953 & Soó Rezső, Borsos Olga & BPU-08130.JPG \\
\hline 1904 & Achillea asplenifolia Vent. & Somogyszob & 1957 & Soó Rezső & BPU-08131.JPG \\
\hline 1905 & Achillea pannonica Scheele & Bodajk & 1955 & Soó Rezső & BPU-08146.JPG \\
\hline 1905 & Achillea pannonica Scheele & Budaörs & 1947 & Soó Rezső & BPU-08147.JPG \\
\hline 1905 & Achillea pannonica Scheele & Budaörs & 1982 & Felföldy Lajos & BPU-08145.JPG \\
\hline 1905 & Achillea pannonica Scheele & Budapest & 1982 & Felföldy Lajos & BPU-08151.JPG \\
\hline 1905 & Achillea pannonica Scheele & Budapest & 2004 & Felföldy Lajos & BPU-08155.JPG \\
\hline 1905 & Achillea pannonica Scheele & Budapest & 2005 & Felföldy Lajos & BPU-08154.JPG \\
\hline 1905 & Achillea pannonica Scheele & Fót & 1960 & Soó Rezső & BPU-08152.JPG \\
\hline 1905 & Achillea pannonica Scheele & Tihany & 1956 & $\begin{array}{l}\text { Felföldy Lajos, Tóth } \\
\text { László }\end{array}$ & BPU-08150.JPG \\
\hline 1905 & Achillea pannonica Scheele & Tokaj & 1947 & Soó Rezső & BPU-08149.JPG \\
\hline 1905 & Achillea pannonica Scheele & Uzsa & 1959 & $\begin{array}{l}\text { Jávorka Sándor, Felföldy } \\
\text { Lajos }\end{array}$ & BPU-08158.JPG \\
\hline 1906 & Achillea collina Becker ex Rchb. & Budaörs & 1982 & Felföldy Lajos & BPU-08157.JPG \\
\hline
\end{tabular}


1. Elektroniukus melléklet - Electronic Appendix 1.

\begin{tabular}{|c|c|c|c|c|c|}
\hline $\begin{array}{l}\text { Sorszám / } \\
\text { Number }\end{array}$ & Taxon-név / Taxon-name & $\begin{array}{l}\text { Település / } \\
\text { Settlement }\end{array}$ & $\begin{array}{l}\text { Év / } \\
\text { Year }\end{array}$ & Gyüjtő / Collector & $\begin{array}{l}\text { Fájlnév / } \\
\text { File-name }\end{array}$ \\
\hline 1906 & Achillea collina Becker ex Rchb. & Budapest & 2004 & Felföldy Lajos & BPU-08156.JPG \\
\hline 1906 & Achillea collina Becker ex Rchb. & Hortobágy & 1931 & Soó Rezső & BPU-08159.JPG \\
\hline 1906 & Achillea collina Becker ex Rchb. & Nyíregyháza & 1948 & Soó Rezső & BPU-08160.JPG \\
\hline 1907 & Achillea millefolium L. & Bátorliget & 1948 & $\begin{array}{l}\text { Simon Tibor, Jakucs Pál, } \\
\text { Böszörményi Zoltán }\end{array}$ & BPU-08141.JPG \\
\hline 1907 & Achillea millefolium L. & Beregsurány & 1951 & $\begin{array}{l}\text { Simon Tibor, Vozáry } \\
\text { Elemér }\end{array}$ & BPU-08142.JPG \\
\hline 1907 & Achillea millefolium L. & Budapest & 1991 & Felföldy Lajos & BPU-08135.JPG \\
\hline 1907 & Achillea millefolium L. & Debrecen & 1946 & Soó Rezső & BPU-08138.JPG \\
\hline 1907 & Achillea millefolium L. & Gyöngyös & 1961 & Soó Rezső & BPU-08136.JPG \\
\hline 1907 & Achillea millefolium L. & Kaposvár & 1955 & Tóth László & BPU-08144.JPG \\
\hline 1907 & Achillea millefolium L. & Nagybajom & 1957 & Soó Rezső & BPU-08139.JPG \\
\hline 1907 & Achillea millefolium L. & Tihany & 1946 & Soó Rezső & BPU-08153.JPG \\
\hline 1907 & Achillea millefolium L. & Vácrátót & 1953 & Felföldy Lajos & BPU-08143.JPG \\
\hline 1910 & $\begin{array}{l}\text { Tripleurospermum perforatum } \\
\text { (Mérat) M. Laínz }\end{array}$ & Budapest & 1982 & Felföldy Lajos & BPU-08176.JPG \\
\hline 1910 & $\begin{array}{l}\text { Tripleurospermum perforatum } \\
\text { (Mérat) M. Laínz }\end{array}$ & Budapest & 1982 & Felföldy Lajos & BPU-08177.JPG \\
\hline 1910 & $\begin{array}{l}\text { Tripleurospermum perforatum } \\
\text { (Mérat) M. Laínz }\end{array}$ & Gelénes & 1948 & Simon Tibor, Jakucs Pál & BPU-08180.JPG \\
\hline 1910 & $\begin{array}{l}\text { Tripleurospermum perforatum } \\
\text { (Mérat) M. Laínz }\end{array}$ & Miskolc & 1937 & Soó Rezső & BPU-08178.JPG \\
\hline 1910 & $\begin{array}{l}\text { Tripleurospermum perforatum } \\
\text { (Mérat) M. Laínz }\end{array}$ & Szentendre & 1963 & Soó Rezső & BPU-08179.JPG \\
\hline 1911 & Matricaria recutita L. & Hortobágy & 1934 & Soó Rezső & BPU-08174.JPG \\
\hline 1911 & Matricaria recutita L. & Szarvas & 1949 & Soó Rezső & BPU-08175.JPG \\
\hline 1912 & Matricaria discoidea DC. & Budapest & 1923 & Soó Rezső & BPU-08172.JPG \\
\hline 1913 & Tanacetum vulgare L. & Budapest & 1982 & Felföldy Lajos & BPU-08181.JPG \\
\hline 1913 & Tanacetum vulgare L. & Hajdúbagos & 1933 & Soó Rezső & BPU-08182.JPG \\
\hline 1913 & Tanacetum vulgare L. & Kaposvár & 1955 & Tóth László & BPU-08183.JPG \\
\hline 1914 & Tanacetum corymbosum (L.) Sch. Bip. & Budapest & 1947 & Soó Rezső & BPU-08185.JPG \\
\hline 1914 & Tanacetum corymbosum (L.) Sch. Bip. & Budapest & 1982 & Felföldy Lajos & BPU-08186.JPG \\
\hline 1914 & Tanacetum corymbosum (L.) Sch. Bip. & Budapest & 2005 & Felföldy Lajos & BPU-08184.JPG \\
\hline 1914 & Tanacetum corymbosum (L.) Sch. Bip. & Miskolc & 1929 & Soó Rezső & BPU-08188.JPG \\
\hline 1914 & Tanacetum corymbosum (L.) Sch. Bip. & Pécs & 1935 & Soó Rezső & BPU-08187.JPG \\
\hline 1914 & Tanacetum corymbosum (L.) Sch. Bip. & Solymár & 2005 & Felföldy Lajos & BPU-08189.JPG \\
\hline 1914 & Tanacetum corymbosum (L.) Sch. Bip. & Uzsa & 1959 & $\begin{array}{l}\text { Jávorka Sándor, Felföldy } \\
\text { Lajos }\end{array}$ & BPU-08190.JPG \\
\hline 1915 & Tanacetum parthenium (L.) Sch. Bip. & Sopron & 1946 & Kárpáti Zoltán & BPU-08195.JPG \\
\hline 1916 & Leucanthemella serotina (L.) Tzvelev & Dabas & 1932 & Boros Ádám & BPU-08200.JPG \\
\hline 1916 & Leucanthemella serotina (L.) Tzvelev & Debrecen & 1931 & Soó Rezső & BPU-08197.JPG \\
\hline 1916 & Leucanthemella serotina (L.) Tzvelev & Debrecen & 1953 & Kulcsár Gábor & BPU-15443.JPG \\
\hline 1916 & Leucanthemella serotina (L.) Tzvelev & Sárospatak & 1939 & Soó Rezső & BPU-08196.JPG \\
\hline 1916 & Leucanthemella serotina (L.) Tzvelev & Tákos & 1948 & Simon Tibor, Jakucs Pál & BPU-08199.JPG \\
\hline 1916 & Leucanthemella serotina (L.) Tzvelev & Tiszafüred & 1952 & Soó Rezső & BPU-08198.JPG \\
\hline 1918 & Leucanthemum vulgare Lam. & Aszófő & 1959 & Felföldy Lajos & BPU-08212.JPG \\
\hline 1918 & Leucanthemum vulgare Lam. & Bátorliget & 1932 & Soó Rezső & BPU-08211.JPG \\
\hline 1918 & Leucanthemum vulgare Lam. & Budapest & 1952 & Soó Rezső & BPU-08203.JPG \\
\hline 1918 & Leucanthemum vulgare Lam. & Budapest & 2005 & Felföldy Lajos & BPU-08201.JPG \\
\hline 1918 & Leucanthemum vulgare Lam. & Budapest & 2005 & Felföldy Lajos & BPU-08202.JPG \\
\hline 1918 & Leucanthemum vulgare Lam. & Eger & 1948 & Koppányi Tibor & BPU-08206.JPG \\
\hline
\end{tabular}


1. Elektroniukus melléklet - Electronic Appendix 1.

\begin{tabular}{|c|c|c|c|c|c|}
\hline $\begin{array}{l}\text { Sorszám / } \\
\text { Number }\end{array}$ & Taxon-név / Taxon-name & $\begin{array}{l}\text { Település / } \\
\text { Settlement }\end{array}$ & $\begin{array}{l}\text { Év / } \\
\text { Year }\end{array}$ & Gyújtő / Collector & $\begin{array}{l}\text { Fájlnév / } \\
\text { File-name }\end{array}$ \\
\hline 1918 & Leucanthemum vulgare Lam. & Gyöngyös & 1962 & Soó Rezső, Borsos Olga & BPU-08207.JPG \\
\hline 1918 & Leucanthemum vulgare Lam. & Gyöngyös & 1962 & Soó Rezső, Borsos Olga & BPU-08208.JPG \\
\hline 1918 & Leucanthemum vulgare Lam. & Kapolcs & 1959 & $\begin{array}{l}\text { Jávorka Sándor, Felföldy } \\
\text { Lajos, Tóth László }\end{array}$ & BPU-08213.JPG \\
\hline 1918 & Leucanthemum vulgare Lam. & Miskolc & 1939 & Soó Rezső & BPU-08204.JPG \\
\hline 1918 & Leucanthemum vulgare Lam. & Miskolc & 1953 & Soó Rezső & BPU-08205.JPG \\
\hline 1918 & Leucanthemum vulgare Lam. & Örvényes & 1959 & Felföldy Lajos & BPU-08214.JPG \\
\hline 1918 & Leucanthemum vulgare Lam. & Pomáz & 1957 & Isépy István & BPU-08210.JPG \\
\hline 1918 & Leucanthemum vulgare Lam. & Szilvásvárad & 1953 & $\begin{array}{l}\text { Felföldy Lajos, Tóth } \\
\text { László }\end{array}$ & BPU-08209.JPG \\
\hline 1918 & Leucanthemum vulgare Lam. & Tihany & 1956 & Felföldy Lajos & BPU-08215.JPG \\
\hline 1918 & Leucanthemum vulgare Lam. & Vászoly & 1951 & Felföldy Lajos, Zsolt János & BPU-08216.JPG \\
\hline 1921 & Artemisia scoparia Waldst. et Kit. & Balmazújváros & 1932 & Soó Rezső & BPU-08277.JPG \\
\hline 1921 & Artemisia scoparia Waldst. et Kit. & Csókakő & 1948 & Vajda László & BPU-08278.JPG \\
\hline 1921 & Artemisia scoparia Waldst. et Kit. & Veresegyház & 1949 & Soó Rezső & BPU-08276.JPG \\
\hline 1922 & Artemisia annua L. & Budapest & 1982 & Felföldy Lajos & BPU-08280.JPG \\
\hline 1922 & Artemisia annua L. & Budapest & 1991 & Felföldy Lajos & BPU-08279.JPG \\
\hline 1922 & Artemisia annua L. & Dabas & 1964 & Soó Rezső & BPU-08281.JPG \\
\hline 1922 & Artemisia annua L. & Debrecen & 1931 & Soó Rezső & BPU-08282.JPG \\
\hline 1923 & Artemisia santonicum L. & Bugyi & 1950 & Soó Rezső & BPU-08245.JPG \\
\hline 1923 & Artemisia santonicum L. & Gyomaendrőd & 1947 & Soó Rezső & BPU-08242.JPG \\
\hline 1923 & Artemisia santonicum L. & Győr & 1921 & Polgár Sándor & BPU-08243.JPG \\
\hline 1923 & Artemisia santonicum L. & Hortobágy & 1930 & Soó Rezső & BPU-08244.JPG \\
\hline 1924 & Artemisia campestris L. & Bélapátfalva & 1948 & $\begin{array}{l}\text { Simon Tibor, Pólya } \\
\text { László, Jakucs Pál }\end{array}$ & BPU-08252.JPG \\
\hline 1924 & Artemisia campestris L. & Budapest & 1921 & Soó Rezső & BPU-08250.JPG \\
\hline 1924 & Artemisia campestris L. & Budapest & 1982 & Felföldy Lajos & BPU-08251.JPG \\
\hline 1924 & Artemisia campestris L. & Debrecen & 1942 & Felföldy Lajos & BPU-08255.JPG \\
\hline 1924 & Artemisia campestris L. & Tihany & 1958 & Felföldy Lajos & BPU-08254.JPG \\
\hline 1925 & Artemisia absinthium L. & Bajna & 1961 & Priszter Szaniszló & BPU-08247.JPG \\
\hline 1925 & Artemisia absinthium L. & Budapest & 1982 & Felföldy Lajos & BPU-08246.JPG \\
\hline 1927 & Artemisia vulgaris L. & Budapest & 2004 & Felföldy Lajos & BPU-08248.JPG \\
\hline 1927 & Artemisia vulgaris L. & Váncsod & 1927 & Kovács L. & BPU-08249.JPG \\
\hline 1928 & Artemisia alba Turra & Bodajk & 1926 & Polgár Sándor & BPU-08275.JPG \\
\hline 1928 & Artemisia alba Turra & Csákvár & 1955 & Soó Rezső & BPU-08272.JPG \\
\hline 1928 & Artemisia alba Turra & Csókakő & 1948 & Papp József & BPU-08271.JPG \\
\hline 1928 & Artemisia alba Turra & Csókakő & 1948 & Papp József & BPU-08274.JPG \\
\hline 1928 & Artemisia alba Turra & Nagyharsány & 1965 & $\begin{array}{l}\text { Simon Tibor, Priszter } \\
\text { Szaniszló, Borhidi Attila }\end{array}$ & BPU-08273.JPG \\
\hline 1929 & Artemisia pontica L. & Arka & 1961 & Simon Tibor & BPU-08262.JPG \\
\hline 1929 & Artemisia pontica L. & Bodrogkeresztúr & 1963 & Isépy István & BPU-08270.JPG \\
\hline 1929 & Artemisia pontica L. & Egyek & 1938 & Soó Rezső & BPU-08266.JPG \\
\hline 1929 & Artemisia pontica L. & Gyomaendrőd & 1947 & Soó Rezső & BPU-08264.JPG \\
\hline 1929 & Artemisia pontica L. & Hortobágy & 1947 & Soó Rezső & BPU-08265.JPG \\
\hline 1929 & Artemisia pontica L. & Kerecsend & 1957 & Soó Rezső & BPU-08267.JPG \\
\hline 1929 & Artemisia pontica L. & Pilisborosjenő & 1953 & Vajda László & BPU-08268.JPG \\
\hline 1929 & Artemisia pontica L. & Tornanádaska & 1951 & $\begin{array}{l}\text { Soó Rezső, Jakucs Pál, Ér } \\
\text { Lajos }\end{array}$ & BPU-08263.JPG \\
\hline 1929 & Artemisia pontica L. & Vácrátót & 1949 & Soó Rezső & BPU-08269.JPG \\
\hline 1930 & Artemisia austriaca Jacq. & Budapest & 1947 & Soó Rezső & BPU-08256.JPG \\
\hline
\end{tabular}


1. Elektroniukus melléklet - Electronic Appendix 1.

\begin{tabular}{|c|c|c|c|c|c|}
\hline $\begin{array}{l}\text { Sorszám / } \\
\text { Number }\end{array}$ & Taxon-név / Taxon-name & $\begin{array}{l}\text { Település / } \\
\text { Settlement }\end{array}$ & $\begin{array}{l}\text { Év / } \\
\text { Year }\end{array}$ & Gyüjtő / Collector & $\begin{array}{l}\text { Fájlnév / } \\
\text { File-name }\end{array}$ \\
\hline 1930 & Artemisia austriaca Jacq. & Csákvár & 1948 & Soó Rezső & BPU-08257.JPG \\
\hline 1930 & Artemisia austriaca Jacq. & Csákvár & 1966 & Priszter Szaniszló & BPU-08258.JPG \\
\hline 1930 & Artemisia austriaca Jacq. & Tihany & 1949 & Simon Tibor & BPU-08260.JPG \\
\hline 1930 & Artemisia austriaca Jacq. & Tihany & 1954 & Felföldy Lajos & BPU-08259.JPG \\
\hline 1930 & Artemisia austriaca Jacq. & Tihany & 1954 & Felföldy Lajos & BPU-08261.JPG \\
\hline $\begin{array}{c}1925 \times \\
1929\end{array}$ & $\begin{array}{l}\text { Artemisia absinthium L. } \times \text { Artemisia } \\
\text { pontica L. }\end{array}$ & Sopron & 1947 & Kárpáti Zoltán & BPU-08289.JPG \\
\hline 1931 & Tussilago farfara L. & Balatonszepezd & 1958 & Felföldy Lajos & BPU-08298.JPG \\
\hline 1931 & Tussilago farfara L. & Balatonszőlős & 1957 & Felföldy Lajos & BPU-08297.JPG \\
\hline 1931 & Tussilago farfara L. & Bátorliget & 1934 & Soó Rezső & BPU-08296.JPG \\
\hline 1931 & Tussilago farfara L. & Budapest & 2004 & Felföldy Lajos & BPU-08294.JPG \\
\hline 1931 & Tussilago farfara L. & Solymár & 2005 & Felföldy Lajos & BPU-08295.JPG \\
\hline 1932 & Petasites albus (L.) Gaertn. & Háromhuta & 1938 & Soó Rezső & BPU-08308.JPG \\
\hline 1933 & $\begin{array}{l}\text { Petasites hybridus (L.) G. Gaertn., B. } \\
\text { Mey. et Scherb. }\end{array}$ & Budapest & 1989 & Felföldy Lajos & BPU-08307.JPG \\
\hline 1933 & $\begin{array}{l}\text { Petasites hybridus (L.) G. Gaertn., B. } \\
\text { Mey. et Scherb. }\end{array}$ & Jósvafő & 1949 & Jakucs Pál & BPU-08299.JPG \\
\hline 1933 & $\begin{array}{l}\text { Petasites hybridus (L.) G. Gaertn., B. } \\
\text { Mey. et Scherb. }\end{array}$ & Örvényes & 1959 & Felföldy Lajos & BPU-08306.JPG \\
\hline 1933 & $\begin{array}{l}\text { Petasites hybridus (L.) G. Gaertn., B. } \\
\text { Mey. et Scherb. }\end{array}$ & Pécs & 1936 & Soó Rezső & BPU-08302.JPG \\
\hline 1933 & $\begin{array}{l}\text { Petasites hybridus (L.) G. Gaertn., B. } \\
\text { Mey. et Scherb. }\end{array}$ & Sopron & 1960 & Borsos Olga, Simon Tibor & BPU-08303.JPG \\
\hline 1933 & $\begin{array}{l}\text { Petasites hybridus (L.) G. Gaertn., B. } \\
\text { Mey. et Scherb. }\end{array}$ & Sopron & 1960 & Borsos Olga, Simon Tibor & BPU-08304.JPG \\
\hline 1933 & $\begin{array}{l}\text { Petasites hybridus (L.) G. Gaertn., B. } \\
\text { Mey. et Scherb. }\end{array}$ & Tihany & 1958 & Felföldy Lajos & BPU-08305.JPG \\
\hline 1933 & $\begin{array}{l}\text { Petasites hybridus (L.) G. Gaertn., B. } \\
\text { Mey. et Scherb. }\end{array}$ & Vácrátót & 1950 & Soó Rezső & BPU-08301.JPG \\
\hline 1934 & Arnica montana L. & Alsószölnök & 1955 & Soó Rezső & BPU-08322.JPG \\
\hline 1934 & Arnica montana L. & Sopron & 1950 & Csapody István & BPU-08321.JPG \\
\hline 1935 & Doronicum hungaricum (Sadler) Rchb. & Budapest & 1948 & Papp József & BPU-08328.JPG \\
\hline 1935 & Doronicum hungaricum (Sadler) Rchb. & Csévharaszt & 1959 & $\begin{array}{l}\text { Simon Tibor, Horánszky } \\
\text { András }\end{array}$ & BPU-08326.JPG \\
\hline 1935 & Doronicum hungaricum (Sadler) Rchb. & Egyek & 1947 & Soó Rezső & BPU-08324.JPG \\
\hline 1935 & Doronicum hungaricum (Sadler) Rchb. & Lábatlan & 1953 & Felföldy Lajos & BPU-08327.JPG \\
\hline 1935 & Doronicum hungaricum (Sadler) Rchb. & Pécs & 1936 & Soó Rezső & BPU-08329.JPG \\
\hline 1935 & Doronicum hungaricum (Sadler) Rchb. & Várpalota & 1955 & Soó Rezső & BPU-08325.JPG \\
\hline 1936 & Doronicum austriacum Jacq. & Kőszeg & 1948 & Kárpáti Zoltán & BPU-08337.JPG \\
\hline 1936 & Doronicum austriacum Jacq. & Szőce & 1955 & Soó Rezső & BPU-08338.JPG \\
\hline 1937 & Doronicum orientale Hoffm. & Pécs & 1946 & Papp József & BPU-08341.JPG \\
\hline 1937 & Doronicum orientale Hoffm. & Pécs & 1953 & Vajda László & BPU-08342.JPG \\
\hline 1938 & Erechtites hieracifolia (L.) Raf. ex DC. & Alsószölnök & 1955 & Soó Rezső & BPU-08320.JPG \\
\hline 1938 & Erechtites hieracifolia (L.) Raf. ex DC. & Csaroda & 1951 & $\begin{array}{l}\text { Simon Tibor, Vozáry } \\
\text { Elemér }\end{array}$ & BPU-08316.JPG \\
\hline 1938 & Erechtites hieracifolia (L.) Raf. ex DC. & Csaroda & 1951 & $\begin{array}{l}\text { Simon Tibor, Vozáry } \\
\text { Elemér }\end{array}$ & BPU-08317.JPG \\
\hline 1938 & Erechtites hieracifolia (L.) Raf. ex DC. & Csaroda & 1952 & $\begin{array}{l}\text { Simon Tibor, Vozáry } \\
\text { Elemér }\end{array}$ & BPU-08315.JPG \\
\hline 1938 & Erechtites hieracifolia (L.) Raf. ex DC. & Pálháza & 1939 & Soó Rezső & BPU-08318.JPG \\
\hline 1938 & Erechtites hieracifolia (L.) Raf. ex DC. & Somogyszob & 1957 & Soó Rezső & BPU-08319.JPG \\
\hline 1939 & Tephroseris crispa (Jacq.) Rchb. & Miskolc & 1939 & Soó Rezső & BPU-08358.JPG \\
\hline
\end{tabular}


1. Elektroniukus melléklet - Electronic Appendix 1.

\begin{tabular}{|c|c|c|c|c|c|}
\hline $\begin{array}{l}\text { Sorszám / } \\
\text { Number }\end{array}$ & Taxon-név / Taxon-name & $\begin{array}{l}\text { Település / } \\
\text { Settlement }\end{array}$ & $\begin{array}{l}\text { Év / } \\
\text { Year }\end{array}$ & Gyűjtő / Collector & $\begin{array}{l}\text { Fájlnév / } \\
\text { File-name }\end{array}$ \\
\hline 1940 & $\begin{array}{l}\text { Tephroseris longifolia (Jacq.) Griseb. } \\
\text { et Schenk }\end{array}$ & Devecser & 1959 & Tallós Pál & BPU-08359.JPG \\
\hline 1941 & Tephroseris integrifolia (L.) Holub & Csévharaszt & 1959 & $\begin{array}{l}\text { Simon Tibor, Horánszky } \\
\text { András }\end{array}$ & BPU-08353.JPG \\
\hline 1941 & Tephroseris integrifolia (L.) Holub & Füzér & 1938 & Soó Rezső & BPU-08352.JPG \\
\hline 1941 & Tephroseris integrifolia (L.) Holub & Füzér & 1960 & Simon Tibor & BPU-08350.JPG \\
\hline 1941 & Tephroseris integrifolia (L.) Holub & Kisgyőr & 1938 & Soó Rezső & BPU-08351.JPG \\
\hline 1941 & Tephroseris integrifolia (L.) Holub & Sopron & 1933 & Kárpáti Zoltán & BPU-08354.JPG \\
\hline 1943 & Senecio vulgaris L. & Tihany & 1958 & Felföldy Lajos & BPU-08360.JPG \\
\hline 1944 & Senecio sylvaticus L. & Alsószölnök & 1955 & Soó Rezső & BPU-08363.JPG \\
\hline 1944 & Senecio sylvaticus L. & Gyöngyös & 1953 & Soó Rezső & BPU-08362.JPG \\
\hline 1944 & Senecio sylvaticus L. & Pálháza & 1952 & Simon Tibor & BPU-08361.JPG \\
\hline 1945 & Senecio viscosus L. & Bélapátfalva & 1930 & Soó Rezső & BPU-08367.JPG \\
\hline 1945 & Senecio viscosus L. & Beregdaróc & 1951 & Simon Tibor, Jakucs Pál & BPU-08366.JPG \\
\hline 1945 & Senecio viscosus L. & Pálháza & 1952 & Simon Tibor & BPU-08365.JPG \\
\hline 1945 & Senecio viscosus L. & Szilvásvárad & 1953 & $\begin{array}{l}\text { Felföldy Lajos, Tóth } \\
\text { László }\end{array}$ & BPU-08368.JPG \\
\hline 1946 & Senecio vernalis Waldst. et Kit. & Budapest & 1991 & Felföldy Lajos & BPU-08370.JPG \\
\hline 1947 & Senecio rupestris Waldst. et Kit. & Ágfalva & 1947 & Kárpáti Zoltán & BPU-08371.JPG \\
\hline 1948 & Senecio erucifolius L. & Bátorliget & 1932 & Soó Rezső & BPU-08379.JPG \\
\hline 1948 & Senecio erucifolius L. & Csarnóta & 1968 & Priszter Szaniszló & BPU-08375.JPG \\
\hline 1948 & Senecio erucifolius L. & Sellye & 1968 & Priszter Szaniszló & BPU-08376.JPG \\
\hline 1948 & Senecio erucifolius L. & Tákos & 1948 & Simon Tibor, Jakucs Pál & BPU-08378.JPG \\
\hline 1948 & Senecio erucifolius L. & Villány & 1968 & Priszter Szaniszló & BPU-08377.JPG \\
\hline 1949 & Senecio jacobaea L. & Budapest & 2004 & Felföldy Lajos & BPU-08381.JPG \\
\hline 1949 & Senecio jacobaea L. & Debrecen & 1934 & Soó Rezső & BPU-08384.JPG \\
\hline 1949 & Senecio jacobaea L. & Miskolc & 1929 & Soó Rezső & BPU-08382.JPG \\
\hline 1949 & Senecio jacobaea L. & Nagybajom & 1957 & Soó Rezső & BPU-08386.JPG \\
\hline 1949 & Senecio jacobaea L. & Nagykovácsi & 1947 & Soó Rezső & BPU-08385.JPG \\
\hline 1949 & Senecio jacobaea L. & Szilvásvárad & 1953 & $\begin{array}{l}\text { Felföldy Lajos, Tóth } \\
\text { László }\end{array}$ & BPU-08383.JPG \\
\hline 1949 & Senecio jacobaea L. & Tihany & 1954 & Felföldy Lajos & BPU-08390.JPG \\
\hline 1949 & Senecio jacobaea L. & Tihany & 1959 & Felföldy Lajos & BPU-08387.JPG \\
\hline 1949 & Senecio jacobaea L. & Tihany & 1959 & Felföldy Lajos & BPU-08389.JPG \\
\hline 1949 & Senecio jacobaea L. & Tihany & 1961 & Felföldy Lajos & BPU-08388.JPG \\
\hline 1951 & Senecio erraticus Bertol. & Budapest & 1990 & Felföldy Lajos & BPU-08393.JPG \\
\hline 1951 & Senecio erraticus Bertol. & Budapest & 1991 & Felföldy Lajos & BPU-08394.JPG \\
\hline 1951 & Senecio erraticus Bertol. & Debrecen & 1933 & Soó Rezső & BPU-08396.JPG \\
\hline 1951 & Senecio erraticus Bertol. & Debrecen & 1987 & Felföldy Lajos & BPU-08397.JPG \\
\hline 1951 & Senecio erraticus Bertol. & Dörgicse & 1957 & Felföldy Lajos & BPU-08400.JPG \\
\hline 1951 & Senecio erraticus Bertol. & Gyomaendrőd & 1947 & Soó Rezső & BPU-08395.JPG \\
\hline 1951 & Senecio erraticus Bertol. & Szenta & 1957 & Soó Rezső & BPU-08399.JPG \\
\hline 1951 & Senecio erraticus Bertol. & Vácrátót & 1953 & Felföldy Lajos & BPU-08398.JPG \\
\hline 1953 & Senecio paludosus L. & Bagamér & 1947 & Soó Rezső & BPU-08401.JPG \\
\hline 1953 & Senecio paludosus L. & Egyek & 1932 & Máthé Imre & BPU-08404.JPG \\
\hline 1953 & Senecio paludosus L. & Győr & 1917 & Polgár Sándor & BPU-08406.JPG \\
\hline 1953 & Senecio paludosus L. & Kimle & 1962 & Borsos Olga & BPU-08405.JPG \\
\hline 1953 & Senecio paludosus L. & Ócsa & 1953 & Soó Rezső, Borsos Olga & BPU-08402.JPG \\
\hline 1953 & Senecio paludosus L. & Tiszafüred & 1952 & Soó Rezső & BPU-15546.JPG \\
\hline
\end{tabular}


1. Elektroniukus melléklet - Electronic Appendix 1.

\begin{tabular}{|c|c|c|c|c|c|}
\hline $\begin{array}{l}\text { Sorszám / } \\
\text { Number }\end{array}$ & Taxon-név / Taxon-name & $\begin{array}{l}\text { Település / } \\
\text { Settlement }\end{array}$ & $\begin{array}{l}\text { Év / } \\
\text { Year }\end{array}$ & Gyüjtő / Collector & $\begin{array}{l}\text { Fájlnév / } \\
\text { File-name }\end{array}$ \\
\hline 1953 & Senecio paludosus L. & Tiszafüred & 1953 & Borsos Olga & BPU-15469.JPG \\
\hline 1954 & Senecio sarracenicus L. & Ásványráró & 1910 & Polgár Sándor & BPU-08408.JPG \\
\hline 1955 & Senecio germanicus Wallr. & Gyöngyös & 1952 & Soó Rezső & BPU-08412.JPG \\
\hline 1955 & Senecio germanicus Wallr. & Gyöngyös & 1953 & Soó Rezső & BPU-08422.JPG \\
\hline 1955 & Senecio germanicus Wallr. & Jósvafő & 1960 & Priszter Szaniszló & BPU-08409.JPG \\
\hline 1955 & Senecio germanicus Wallr. & Kemence & 1952 & Simon Tibor & BPU-08413.JPG \\
\hline 1955 & Senecio germanicus Wallr. & Miskolc & 1937 & Soó Rezső & BPU-08410.JPG \\
\hline 1955 & Senecio germanicus Wallr. & Miskolc & 1950 & Soó Rezső & BPU-08424.JPG \\
\hline 1955 & Senecio germanicus Wallr. & Miskolc & 1952 & Borsos Olga & BPU-08411.JPG \\
\hline 1955 & Senecio germanicus Wallr. & Nyíregyháza & 1935 & Soó Rezső & BPU-08414.JPG \\
\hline 1955 & Senecio germanicus Wallr. & Szilvásvárad & 1953 & $\begin{array}{l}\text { Felföldy Lajos, Tóth } \\
\text { László }\end{array}$ & BPU-08431.JPG \\
\hline 1955 & Senecio germanicus Wallr. & Szőce & 1955 & Soó Rezső & BPU-08421.JPG \\
\hline 1955 & Senecio germanicus Wallr. & Taksony & 1946 & Vajda László & BPU-08407.JPG \\
\hline 1956 & $\begin{array}{l}\text { Senecio ovatus (G. Gaertn., B. Mey. et } \\
\text { Scherb.) Willd. }\end{array}$ & Gyöngyös & 1952 & Soó Rezső & BPU-08423.JPG \\
\hline 1956 & $\begin{array}{l}\text { Senecio ovatus (G. Gaertn., B. Mey. et } \\
\text { Scherb.) Willd. }\end{array}$ & Miskolc & 1937 & Soó Rezső & BPU-08425.JPG \\
\hline 1956 & $\begin{array}{l}\text { Senecio ovatus (G. Gaertn., B. Mey. et } \\
\text { Scherb.) Willd. }\end{array}$ & Sopron & 1948 & Kárpáti Zoltán & BPU-08432.JPG \\
\hline 1957 & Senecio doria Nath. & Debrecen & 1932 & Soó Rezső & BPU-08433.JPG \\
\hline 1958 & Senecio umbrosus Waldst. et Kit. & Tata & 1925 & Boros Ádám & BPU-08435.JPG \\
\hline 1959 & Ligularia sibirica (L.) Cass. & Bátorliget & 1950 & $\begin{array}{l}\text { Simon Tibor, Kárpáti } \\
\text { István }\end{array}$ & BPU-08458.JPG \\
\hline 1959 & Ligularia sibirica (L.) Cass. & Bátorliget & 1951 & Simon Tibor & BPU-08459.JPG \\
\hline 1959 & Ligularia sibirica (L.) Cass. & Nyírpilis & 1932 & Soó Rezső & BPU-08457.JPG \\
\hline 1961 & Carlina acaulis L. & Sopron & 1932 & Soó Rezső & BPU-08487.JPG \\
\hline 1961 & Carlina acaulis L. & Telkibánya & 1952 & Simon Tibor & BPU-08486.JPG \\
\hline 1962 & Carlina vulgaris L. & Bagamér & 1932 & Soó Rezső & BPU-08502.JPG \\
\hline 1962 & Carlina vulgaris L. & Budapest & 1947 & Soó Rezső & BPU-08498.JPG \\
\hline 1962 & Carlina vulgaris L. & Budapest & 1982 & Felföldy Lajos & BPU-08499.JPG \\
\hline 1962 & Carlina vulgaris L. & Csákvár & 1948 & Soó Rezső & BPU-08491.JPG \\
\hline 1962 & Carlina vulgaris L. & Csákvár & 1966 & Priszter Szaniszló & BPU-08492.JPG \\
\hline 1962 & Carlina vulgaris L. & Diósjenő & 1952 & Simon Tibor & BPU-08504.JPG \\
\hline 1962 & Carlina vulgaris L. & Dörgicse & 1957 & Felföldy Lajos & BPU-08494.JPG \\
\hline 1962 & Carlina vulgaris L. & Erdőbénye & 1948 & Soó Rezső & BPU-08507.JPG \\
\hline 1962 & Carlina vulgaris L. & Miskolc & 1934 & Soó Rezső & BPU-08501.JPG \\
\hline 1962 & Carlina vulgaris L. & Miskolc & 1950 & Soó Rezső & BPU-08500.JPG \\
\hline 1962 & Carlina vulgaris L. & Nyíracsád & 1949 & Soó Rezső & BPU-15563.JPG \\
\hline 1962 & Carlina vulgaris L. & Pusztamiske & 1948 & Borsos Olga & BPU-08495.JPG \\
\hline 1962 & Carlina vulgaris L. & Szentgotthárd & 1955 & Soó Rezső & BPU-08505.JPG \\
\hline 1962 & Carlina vulgaris L. & Szilvásvárad & 1953 & $\begin{array}{l}\text { Felföldy Lajos, Tóth } \\
\text { László }\end{array}$ & BPU-08503.JPG \\
\hline 1962 & Carlina vulgaris L. & Szőce & 1955 & Soó Rezső & BPU-08493.JPG \\
\hline 1962 & Carlina vulgaris L. & Uzsa & 1953 & $\begin{array}{l}\text { Felföldy Lajos, Tóth } \\
\text { László }\end{array}$ & BPU-08496.JPG \\
\hline 1962 & Carlina vulgaris L. & Vászoly & 1958 & Felföldy Lajos & BPU-08497.JPG \\
\hline 1962 & Carlina vulgaris L. & Velem & 1932 & Soó Rezső & BPU-08506.JPG \\
\hline 1963 & $\begin{array}{l}\text { Xeranthemum cylindraceum Sibth. et } \\
\text { Sm. }\end{array}$ & Pomáz & 1948 & Koppányi Tibor & BPU-08484.JPG \\
\hline
\end{tabular}


1. Elektroniukus melléklet - Electronic Appendix 1.

\begin{tabular}{|c|c|c|c|c|c|}
\hline $\begin{array}{l}\text { Sorszám / } \\
\text { Number }\end{array}$ & Taxon-név / Taxon-name & $\begin{array}{l}\text { Település / } \\
\text { Settlement }\end{array}$ & $\begin{array}{l}\text { Év / } \\
\text { Year }\end{array}$ & Gyújtő / Collector & $\begin{array}{l}\text { Fájlnév / } \\
\text { File-name }\end{array}$ \\
\hline 1963 & $\begin{array}{l}\text { Xeranthemum cylindraceum Sibth. et } \\
\text { Sm. }\end{array}$ & Szentendre & 1943 & Papp József & BPU-08485.JPG \\
\hline 1964 & Xeranthemum annuum L. & Abaújszántó & 1948 & Soó Rezső & BPU-08482.JPG \\
\hline 1964 & Xeranthemum annuum L. & Budapest & 1947 & Soó Rezső & BPU-08479.JPG \\
\hline 1964 & Xeranthemum annuum L. & Budapest & 1947 & Soó Rezső & BPU-08481.JPG \\
\hline 1964 & Xeranthemum annuum L. & Sümeg & 1953 & Borsos Olga & BPU-08483.JPG \\
\hline 1964 & Xeranthemum annuum L. & Tihany & 1961 & $\begin{array}{l}\text { Pankov H., Felföldy Lajos, } \\
\text { Sólymosy G. }\end{array}$ & BPU-08478.JPG \\
\hline 1964 & Xeranthemum annuum L. & Villány & 1935 & Soó Rezső & BPU-08480.JPG \\
\hline 1965 & Echinops sphaerocephalus L. & Budapest & 1947 & Soó Rezső & BPU-08466.JPG \\
\hline 1965 & Echinops sphaerocephalus L. & Budapest & 2004 & Felföldy Lajos & BPU-08465.JPG \\
\hline 1965 & Echinops sphaerocephalus L. & Debrecen & 1932 & Soó Rezső & BPU-08470.JPG \\
\hline 1965 & Echinops sphaerocephalus L. & Komjáti & 1950 & Jakucs Pál, Kulcsár Gábor & BPU-08467.JPG \\
\hline 1965 & Echinops sphaerocephalus L. & Miskolc & 1937 & Soó Rezső & BPU-08469.JPG \\
\hline 1965 & Echinops sphaerocephalus L. & Pécs & 1938 & Soó Rezső & BPU-08468.JPG \\
\hline 1965 & Echinops sphaerocephalus L. & Tihany & 1961 & $\begin{array}{l}\text { Pankov H., Felföldy Lajos, } \\
\text { Sólymosy G. }\end{array}$ & BPU-08471.JPG \\
\hline 1966 & Echinops ruthenicus (Fisch.) M. Bieb. & Balatonszabadi & 1947 & Priszter Szaniszló & BPU-08477.JPG \\
\hline 1966 & Echinops ruthenicus (Fisch.) M. Bieb. & Budapest & 1947 & Soó Rezső & BPU-08473.JPG \\
\hline 1966 & Echinops ruthenicus (Fisch.) M. Bieb. & Bugac & 1964 & Soó Rezső & BPU-08472.JPG \\
\hline 1966 & Echinops ruthenicus (Fisch.) M. Bieb. & Bugac & 1964 & Soó Rezső & BPU-08475.JPG \\
\hline 1966 & Echinops ruthenicus (Fisch.) M. Bieb. & Siófok & 1929 & Soó Rezső & BPU-08474.JPG \\
\hline 1966 & Echinops ruthenicus (Fisch.) M. Bieb. & Vácrátót & 1950 & Soó Rezső & BPU-08476.JPG \\
\hline 1969 & Arctium minus (Hill) Bernh. & Budapest & 1991 & Felföldy Lajos & BPU-08565.JPG \\
\hline 1969 & Arctium minus (Hill) Bernh. & Miskolc & 1937 & Soó Rezső & BPU-08567.JPG \\
\hline 1969 & Arctium minus (Hill) Bernh. & Tihany & 1961 & Sólymossy Gizella & BPU-08566.JPG \\
\hline 1969 & Arctium minus (Hill) Bernh. & Tokaj & 1933 & Soó Rezső & BPU-08568.JPG \\
\hline 1970 & Arctium nemorosum Lej. & Budakeszi & 1947 & Soó Rezső & BPU-08573.JPG \\
\hline 1970 & Arctium nemorosum Lej. & Debrecen & 1933 & Soó Rezső & BPU-08571.JPG \\
\hline 1970 & Arctium nemorosum Lej. & Miskolc & 1937 & Soó Rezső & BPU-08570.JPG \\
\hline 1970 & Arctium nemorosum Lej. & Szilvásvárad & 1953 & $\begin{array}{l}\text { Felföldy Lajos, Tóth } \\
\text { László }\end{array}$ & BPU-08569.JPG \\
\hline 1970 & Arctium nemorosum Lej. & Zebegény & 1947 & Soó Rezső & BPU-08572.JPG \\
\hline $\begin{array}{c}1967 \times \\
1968\end{array}$ & Arctium $\times$ ambiguum (Čelak.) Nyman & Eger & 1936 & Soó Rezső & BPU-08575.JPG \\
\hline $\begin{array}{c}1967 \times \\
1969\end{array}$ & Arctium $\times$ mixtum (Simonk.) Nyman & Pomáz & 1945 & Vajda László & BPU-08577.JPG \\
\hline $\begin{array}{c}1967 \times \\
1969\end{array}$ & Arctium $\times$ mixtum (Simonk.) Nyman & Tihany & 1942 & Felföldy Lajos & BPU-08576.JPG \\
\hline 1971 & Jurinea mollis (L.) Rchb. & Aszófő & 1951 & Felföldy Lajos & BPU-08548.JPG \\
\hline 1971 & Jurinea mollis (L.) Rchb. & Bélapátfalva & 1948 & $\begin{array}{l}\text { Simon Tibor, Pólya } \\
\text { László, Jakucs Pál }\end{array}$ & BPU-08545.JPG \\
\hline 1971 & Jurinea mollis (L.) Rchb. & Budapest & 1948 & Soó Rezső & BPU-08540.JPG \\
\hline 1971 & Jurinea mollis (L.) Rchb. & Budapest & 1952 & Soó Rezső & BPU-15431.JPG \\
\hline 1971 & Jurinea mollis (L.) Rchb. & Budapest & 1992 & Felföldy Lajos & BPU-08541.JPG \\
\hline 1971 & Jurinea mollis (L.) Rchb. & Csákvár & 1955 & Soó Rezső & BPU-08544.JPG \\
\hline 1971 & Jurinea mollis (L.) Rchb. & Eplény & 1955 & Soó Rezső & BPU-08549.JPG \\
\hline 1971 & Jurinea mollis (L.) Rchb. & Jósvafő & 1953 & Jakucs Pál & BPU-08542.JPG \\
\hline 1971 & Jurinea mollis (L.) Rchb. & Pilisszentiván & 1953 & Soó Rezső & BPU-08546.JPG \\
\hline 1971 & Jurinea mollis (L.) Rchb. & Pilisszentiván & 1955 & Jakucs Pál & BPU-08547.JPG \\
\hline 1971 & Jurinea mollis (L.) Rchb. & Siklós & 1967 & Priszter Szaniszló & BPU-08543.JPG \\
\hline
\end{tabular}


1. Elektroniukus melléklet - Electronic Appendix 1.

\begin{tabular}{|c|c|c|c|c|c|}
\hline $\begin{array}{l}\text { Sorszám / } \\
\text { Number }\end{array}$ & Taxon-név / Taxon-name & $\begin{array}{l}\text { Település / } \\
\text { Settlement }\end{array}$ & $\begin{array}{l}\text { Év / } \\
\text { Year }\end{array}$ & Gyűjtő / Collector & $\begin{array}{l}\text { Fájlnév / } \\
\text { File-name }\end{array}$ \\
\hline 1971 & Jurinea mollis (L.) Rchb. & Tihany & 1959 & Felföldy Lajos & BPU-08550.JPG \\
\hline 1971 & Jurinea mollis (L.) Rchb. & Tokaj & 1937 & Soó Rezső & BPU-08551.JPG \\
\hline 1973 & Carduus nutans L. & Budapest & 1952 & Soó Rezső & BPU-08553.JPG \\
\hline 1973 & Carduus nutans L. & Tata & 1968 & Priszter Szaniszló & BPU-08554.JPG \\
\hline 1974 & Carduus acanthoides L. & Debrecen & 1951 & Soó Rezső, Simon Tibor & BPU-08531.JPG \\
\hline 1974 & Carduus acanthoides L. & Gelénes & 1951 & $\begin{array}{l}\text { Simon Tibor, Vozáry } \\
\text { Elemér }\end{array}$ & BPU-08532.JPG \\
\hline 1974 & Carduus acanthoides L. & Visegrád & 1949 & Soó Rezső & BPU-08530.JPG \\
\hline 1975 & Carduus crispus L. & Ásványráró & 1931 & Soó Rezső & BPU-08535.JPG \\
\hline 1975 & Carduus crispus L. & Lábatlan & 1953 & Felföldy Lajos & BPU-08539.JPG \\
\hline 1975 & Carduus crispus L. & Miskolc & 1934 & Soó Rezső & BPU-08533.JPG \\
\hline 1975 & Carduus crispus L. & Sáránd & 1933 & Soó Rezső & BPU-08538.JPG \\
\hline 1975 & Carduus crispus L. & Szilvásvárad & 1953 & Soó Rezső & BPU-08536.JPG \\
\hline 1976 & Carduus crassifolius Willd. & Pilisszentiván & 1953 & Soó Rezső & BPU-08557.JPG \\
\hline 1976 & Carduus crassifolius Willd. & Pilisszentiván & 1953 & Soó Rezső & BPU-08558.JPG \\
\hline 1976 & Carduus crassifolius Willd. & Várpalota & 1928 & Jávorka Sándor & BPU-08564.JPG \\
\hline 1977 & Carduus hamulosus Ehrh. & Budapest & 1947 & Soó Rezső & BPU-08555.JPG \\
\hline 1978 & Carduus collinus Waldst. et Kit. & Bélapátfalva & 1953 & Simon Tibor & BPU-08529.JPG \\
\hline 1978 & Carduus collinus Waldst. et Kit. & Budapest & 1947 & Soó Rezső & BPU-08526.JPG \\
\hline 1978 & Carduus collinus Waldst. et Kit. & Dömös & 1948 & Soó Rezső & BPU-08524.JPG \\
\hline 1978 & Carduus collinus Waldst. et Kit. & Gyöngyös & 1961 & Soó Rezső & BPU-08527.JPG \\
\hline 1978 & Carduus collinus Waldst. et Kit. & Jósvafő & 1951 & Soó Rezső & BPU-08528.JPG \\
\hline 1978 & Carduus collinus Waldst. et Kit. & Pilisszentkereszt & 1955 & Soó Rezső & BPU-08525.JPG \\
\hline 1978 & Carduus collinus Waldst. et Kit. & Tarcal & 1933 & Soó Rezső & BPU-08519.JPG \\
\hline $\begin{array}{c}1973 \times \\
1974\end{array}$ & Carduus $\times$ ortocephalus Wallr. & Debrecen & 1929 & Kovács L. & BPU-08520.JPG \\
\hline $\begin{array}{c}1973 \times \\
1978\end{array}$ & $\begin{array}{l}\text { Carduus nutans L. } \times \text { Carduus collinus } \\
\text { Waldst. et Kit. }\end{array}$ & Budapest & 1947 & Soó Rezső & BPU-08518.JPG \\
\hline $\begin{array}{c}1973 \times \\
1978\end{array}$ & $\begin{array}{l}\text { Carduus nutans L. } \times \text { Carduus collinus } \\
\text { Waldst. et Kit. }\end{array}$ & Miskolc & 1929 & Soó Rezső & BPU-08521.JPG \\
\hline 1979 & Cirsium vulgare (Savi) Ten. & Debrecen & 1936 & Soó Rezső & BPU-08522.JPG \\
\hline 1979 & Cirsium vulgare (Savi) Ten. & Gáborján & 1936 & Kovács L. & BPU-08523.JPG \\
\hline 1980 & Cirsium eriophorum (L.) Scop. & Budapest & 2004 & Felföldy Lajos & BPU-08583.JPG \\
\hline 1980 & Cirsium eriophorum (L.) Scop. & Füzér & 1949 & Simon Tibor, Jakucs Pál & BPU-08584.JPG \\
\hline 1980 & Cirsium eriophorum (L.) Scop. & Kimle & 1924 & Polgár Sándor & BPU-08585.JPG \\
\hline 1980 & Cirsium eriophorum (L.) Scop. & Pomáz & 1921 & Soó Rezső & BPU-08586.JPG \\
\hline 1981 & Cirsium furiens Griseb. et Schenk & Debrecen & 1932 & Soó Rezső & BPU-08579.JPG \\
\hline 1983 & Cirsium arvense (L.) Scop. & Budapest & 2004 & Felföldy Lajos & BPU-08588.JPG \\
\hline 1983 & Cirsium arvense (L.) Scop. & Debrecen & 1937 & Felföldy Lajos & BPU-08591.JPG \\
\hline 1983 & Cirsium arvense (L.) Scop. & Erdőbénye & 1948 & Soó Rezső & BPU-08594.JPG \\
\hline 1983 & Cirsium arvense (L.) Scop. & Gelénes & 1951 & $\begin{array}{l}\text { Simon Tibor, Vozáry } \\
\text { Elemér }\end{array}$ & BPU-08589.JPG \\
\hline 1983 & Cirsium arvense (L.) Scop. & Győr & 1910 & Polgár Sándor & BPU-08590.JPG \\
\hline 1983 & Cirsium arvense (L.) Scop. & Nagykanizsa & 1949 & Károlyi Árpád & BPU-08593.JPG \\
\hline 1983 & Cirsium arvense (L.) Scop. & Pusztamiske & 1948 & Borsos Olga & BPU-08592.JPG \\
\hline 1984 & Cirsium brachycephalum Jur. & Bátorliget & 1953 & Soó Rezső & BPU-08602.JPG \\
\hline 1984 & Cirsium brachycephalum Jur. & Bátorliget & 1953 & Soó Rezső & BPU-08603.JPG \\
\hline 1984 & Cirsium brachycephalum Jur. & Csaroda & 1948 & Simon Tibor, Jakucs Pál & BPU-08595.JPG \\
\hline 1984 & Cirsium brachycephalum Jur. & Dabas & 1963 & Isépy István & BPU-08601.JPG \\
\hline 1984 & Cirsium brachycephalum Jur. & Debrecen & 1949 & Soó Rezső & BPU-08598.JPG \\
\hline
\end{tabular}


1. Elektroniukus melléklet - Electronic Appendix 1.

\begin{tabular}{|c|c|c|c|c|c|}
\hline $\begin{array}{l}\text { Sorszám / } \\
\text { Number }\end{array}$ & Taxon-név / Taxon-name & $\begin{array}{l}\text { Település / } \\
\text { Settlement }\end{array}$ & $\begin{array}{l}\text { Év / } \\
\text { Year }\end{array}$ & Gyüjtő / Collector & $\begin{array}{l}\text { Fájlnév / } \\
\text { File-name }\end{array}$ \\
\hline 1984 & Cirsium brachycephalum Jur. & Debrecen & 1951 & Kulcsár Gábor & BPU-08599.JPG \\
\hline 1984 & Cirsium brachycephalum Jur. & Kimle & 1962 & Borsos Olga & BPU-08600.JPG \\
\hline 1984 & Cirsium brachycephalum Jur. & Pákozd & 1980 & Felföldy Lajos & BPU-08596.JPG \\
\hline 1984 & Cirsium brachycephalum Jur. & Pákozd & 1980 & Felföldy Lajos & BPU-08597.JPG \\
\hline 1984 & Cirsium brachycephalum Jur. & Tihany & 1928 & Soó Rezső & BPU-08605.JPG \\
\hline 1984 & Cirsium brachycephalum Jur. & Tihany & 1956 & $\begin{array}{l}\text { Felföldy Lajos, Tóth } \\
\text { László }\end{array}$ & BPU-08604.JPG \\
\hline 1984 & Cirsium brachycephalum Jur. & Tihany & 1959 & Felföldy Lajos & BPU-08606.JPG \\
\hline 1985 & Cirsium palustre (L.) Scop. & Bátorliget & 1950 & $\begin{array}{l}\text { Simon Tibor, Kárpáti } \\
\text { István }\end{array}$ & BPU-08614.JPG \\
\hline 1985 & Cirsium palustre (L.) Scop. & Hévíz & 1928 & Soó Rezső & BPU-08608.JPG \\
\hline 1985 & Cirsium palustre (L.) Scop. & Isaszeg & 1949 & Soó Rezső & BPU-08611.JPG \\
\hline 1985 & Cirsium palustre (L.) Scop. & Kaszó & 1957 & Soó Rezső & BPU-08610.JPG \\
\hline 1985 & Cirsium palustre (L.) Scop. & Ócsa & 1953 & Soó Rezső, Borsos Olga & BPU-08609.JPG \\
\hline 1985 & Cirsium palustre (L.) Scop. & Szőce & 1955 & Soó Rezső & BPU-08607.JPG \\
\hline 1985 & Cirsium palustre (L.) Scop. & Szögliget & 1951 & $\begin{array}{l}\text { Soó Rezső, Jakucs Pál, Ér } \\
\text { Lajos }\end{array}$ & BPU-08676.JPG \\
\hline 1986 & Cirsium canum (L.) All. & Balatongyörök & 1961 & Felföldy Lajos & BPU-08621.JPG \\
\hline 1986 & Cirsium canum (L.) All. & Budapest & 2004 & Felföldy Lajos & BPU-08615.JPG \\
\hline 1986 & Cirsium canum (L.) All. & Debrecen & 1932 & Soó Rezső & BPU-08617.JPG \\
\hline 1986 & Cirsium canum (L.) All. & Erdőbénye & 1948 & Soó Rezső & BPU-08622.JPG \\
\hline 1986 & Cirsium canum (L.) All. & Kőszeg & 1963 & Soó Rezső, Borsos Olga & BPU-08619.JPG \\
\hline 1986 & Cirsium canum (L.) All. & Lesenceistvánd & 1952 & Borsos Olga & BPU-08620.JPG \\
\hline 1986 & Cirsium canum (L.) All. & Mór & 1942 & Felföldy Lajos & BPU-08616.JPG \\
\hline 1986 & Cirsium canum (L.) All. & Ócsa & 1950 & Soó Rezső & BPU-08618.JPG \\
\hline 1987 & Cirsium pannonicum (L. f.) Link & Bátorliget & 1948 & $\begin{array}{l}\text { Simon Tibor, Jakucs Pál, } \\
\text { Böszörményi Zoltán }\end{array}$ & BPU-08629.JPG \\
\hline 1987 & Cirsium pannonicum (L.f.) Link & Budapest & 1947 & Soó Rezső & BPU-08625.JPG \\
\hline 1987 & Cirsium pannonicum (L. f.) Link & Budapest & 1963 & Borsos Olga & BPU-08624.JPG \\
\hline 1987 & Cirsium pannonicum (L.f.) Link & Gyöngyös & 1961 & Soó Rezső & BPU-08628.JPG \\
\hline 1987 & Cirsium pannonicum (L.f.) Link & Miskolc & 1934 & Soó Rezső & BPU-08627.JPG \\
\hline 1987 & Cirsium pannonicum (L. f.) Link & Miskolc & 1960 & Simon Tibor & BPU-08626.JPG \\
\hline 1988 & Cirsium rivulare (Jacq.) All. & Balatonszőlős & 1957 & Felföldy Lajos & BPU-08641.JPG \\
\hline 1988 & Cirsium rivulare (Jacq.) All. & Bátorliget & 1932 & Soó Rezső & BPU-08633.JPG \\
\hline 1988 & Cirsium rivulare (Jacq.) All. & Debrecen & 1931 & Soó Rezső & BPU-08632.JPG \\
\hline 1988 & Cirsium rivulare (Jacq.) All. & Kimle & 1962 & Borsos Olga & BPU-08638.JPG \\
\hline 1988 & Cirsium rivulare (Jacq.) All. & Nagybajom & 1957 & Soó Rezső & BPU-08634.JPG \\
\hline 1988 & Cirsium rivulare (Jacq.) All. & Tihany & 1956 & Felföldy Lajos & BPU-08642.JPG \\
\hline 1988 & Cirsium rivulare (Jacq.) All. & Tihany & 1957 & Entz Béla & BPU-08640.JPG \\
\hline 1988 & Cirsium rivulare (Jacq.) All. & Ugod & 1955 & Soó Rezső & BPU-08631.JPG \\
\hline 1988 & Cirsium rivulare (Jacq.) All. & Ugod & 1955 & Soó Rezső & BPU-08635.JPG \\
\hline 1989 & Cirsium oleraceum (L.) Scop. & Bak & 1955 & Soó Rezső & BPU-08646.JPG \\
\hline 1989 & Cirsium oleraceum (L.) Scop. & Kőszeg & 1963 & Soó Rezső, Borsos Olga & BPU-08647.JPG \\
\hline 1989 & Cirsium oleraceum (L.) Scop. & Miskolc & 1952 & Borsos Olga & BPU-08651.JPG \\
\hline 1989 & Cirsium oleraceum (L.) Scop. & Ócsa & 1950 & Soó Rezső & BPU-08643.JPG \\
\hline 1989 & Cirsium oleraceum (L.) Scop. & Putnok & 1953 & Dobos L. & BPU-08644.JPG \\
\hline 1989 & Cirsium oleraceum (L.) Scop. & Sopron & 1934 & Soó Rezső & BPU-08645.JPG \\
\hline 1989 & Cirsium oleraceum (L.) Scop. & Szenta & 1957 & Soó Rezső & BPU-08648.JPG \\
\hline 1989 & Cirsium oleraceum (L.) Scop. & Uzsa & 1951 & Borsos Olga & BPU-08650.JPG \\
\hline
\end{tabular}


1. Elektroniukus melléklet - Electronic Appendix 1.

\begin{tabular}{|c|c|c|c|c|c|}
\hline $\begin{array}{l}\text { Sorszám / } \\
\text { Number }\end{array}$ & Taxon-név / Taxon-name & $\begin{array}{l}\text { Település / } \\
\text { Settlement }\end{array}$ & $\begin{array}{l}\text { Év / } \\
\text { Year }\end{array}$ & Gyújtő / Collector & $\begin{array}{l}\text { Fájlnév / } \\
\text { File-name }\end{array}$ \\
\hline 1989 & Cirsium oleraceum (L.) Scop. & Uzsa & 1953 & $\begin{array}{l}\text { Felföldy Lajos, Tóth } \\
\text { László }\end{array}$ & BPU-08652.JPG \\
\hline 1990 & Cirsium erisithales (Jacq.) Scop. & Kőszeg & 1932 & Soó Rezső & BPU-08655.JPG \\
\hline 1990 & Cirsium erisithales (Jacq.) Scop. & Miskolc & 1932 & Soó Rezső & BPU-08660.JPG \\
\hline 1990 & Cirsium erisithales (Jacq.) Scop. & Miskolc & 1950 & Soó Rezső & BPU-08656.JPG \\
\hline 1990 & Cirsium erisithales (Jacq.) Scop. & Tibolddaróc & 1953 & Soó Rezső & BPU-08654.JPG \\
\hline $\begin{array}{c}1979- \\
1990\end{array}$ & Cirsium & Ócsa & 1955 & Soó Rezső & BPU-08665.JPG \\
\hline $\begin{array}{c}1984 \times \\
1986\end{array}$ & $\begin{array}{l}\text { Cirsium brachycephalum Jur. } \times \\
\text { Cirsium canum (L.) All. }\end{array}$ & Demecser & 1911 & Radó E. & BPU-08675.JPG \\
\hline $\begin{array}{c}1985 \times \\
1989\end{array}$ & $\begin{array}{l}\text { Cirsium palustre (L.) Scop. } \times \text { Cirsium } \\
\text { oleraceum (L.) Scop. }\end{array}$ & Szögliget & 1951 & $\begin{array}{l}\text { Soó Rezső, Jakucs Pál, Ér } \\
\text { Lajos }\end{array}$ & BPU-08677.JPG \\
\hline $\begin{array}{c}1986 \times \\
1988\end{array}$ & Cirsium $\times$ siegertii Sch. Bip. & Debrecen & 1947 & Soó Rezső & BPU-08669.JPG \\
\hline $\begin{array}{c}1986 \times \\
1988\end{array}$ & Cirsium $\times$ siegertii Sch. Bip. & Hévíz & 1928 & Soó Rezső & BPU-08672.JPG \\
\hline $\begin{array}{c}1986 \times \\
1989\end{array}$ & Cirsium $\times$ tataricum All & Jósvafő & 1951 & Jakucs Pál, Ér Lajos & BPU-08680.JPG \\
\hline $\begin{array}{c}1986 \times \\
1989\end{array}$ & Cirsium $\times$ tataricum All & Kőszeg & 1932 & Soó Rezső & BPU-08670.JPG \\
\hline $\begin{array}{c}1986 \times \\
1989\end{array}$ & Cirsium $\times$ tataricum All & Szilvásvárad & 1953 & $\begin{array}{l}\text { Felföldy Lajos, Tóth } \\
\text { László }\end{array}$ & BPU-08681.JPG \\
\hline $\begin{array}{c}1986 \times \\
1989\end{array}$ & Cirsium $\times$ tataricum All. & Szögliget & 1951 & $\begin{array}{l}\text { Soó Rezső, Jakucs Pál, Ér } \\
\text { Lajos }\end{array}$ & BPU-08679.JPG \\
\hline $\begin{array}{c}1987 \times \\
1990\end{array}$ & $\begin{array}{l}\text { Cirsium pannonicum (L.f.) Link } \times \\
\text { Cirsium erisithales (Jacq.) Scop. }\end{array}$ & Miskolc & 1929 & Soó Rezső & BPU-08674.JPG \\
\hline $\begin{array}{c}1988 \times \\
1989\end{array}$ & $\begin{array}{l}\text { Cirsium } \times \text { erucagineum DC. in Lam. et } \\
\text { DC. }\end{array}$ & Sopron & 1932 & Soó Rezső & BPU-08671.JPG \\
\hline $\begin{array}{c}1988 \times \\
1989\end{array}$ & $\begin{array}{l}\text { Cirsium } \times \text { erucagineum DC. in Lam. et } \\
\text { DC. }\end{array}$ & Szögliget & 1951 & $\begin{array}{l}\text { Soó Rezső, Jakucs Pál, Ér } \\
\text { Lajos }\end{array}$ & BPU-08678.JPG \\
\hline 1991 & Onopordum acanthium L. & Debrecen & 1938 & Soó Rezső & BPU-08682.JPG \\
\hline 1993 & Serratula tinctoria L. & Balatonszőlős & 1957 & Felföldy Lajos & BPU-08699.JPG \\
\hline 1993 & Serratula tinctoria L. & Budapest & 1947 & Soó Rezső & BPU-08691.JPG \\
\hline 1993 & Serratula tinctoria L. & Budapest & 1991 & Felföldy Lajos & BPU-08693.JPG \\
\hline 1993 & Serratula tinctoria L. & Budapest & 1992 & Felföldy Lajos & BPU-08692.JPG \\
\hline 1993 & Serratula tinctoria L. & Gelénes & 1951 & $\begin{array}{l}\text { Simon Tibor, Vozáry } \\
\text { Elemér }\end{array}$ & BPU-08694.JPG \\
\hline 1993 & Serratula tinctoria L. & Gyöngyös & 1952 & Soó Rezső & BPU-08697.JPG \\
\hline 1993 & Serratula tinctoria L. & Hévíz & 1961 & Felföldy Lajos & BPU-08701.JPG \\
\hline 1993 & Serratula tinctoria L. & Kerecsend & 1957 & Soó Rezső & BPU-08696.JPG \\
\hline 1993 & Serratula tinctoria L. & Kőszeg & 1963 & Soó Rezső, Borsos Olga & BPU-08698.JPG \\
\hline 1993 & Serratula tinctoria L. & Vámosatya & 1952 & $\begin{array}{l}\text { Simon Tibor, Vozáry } \\
\text { Elemér }\end{array}$ & BPU-08695.JPG \\
\hline 1993 & Serratula tinctoria L. & Zalagyömörő & 1957 & Felföldy Lajos & BPU-08700.JPG \\
\hline 1994 & Serratula lycopifolia (Vill.) A. Kern. & Gánt & 1934 & Boros Ádám & BPU-08705.JPG \\
\hline 1995 & $\begin{array}{l}\text { Serratula radiata (Waldst. et Kit.) M. } \\
\text { Bieb. }\end{array}$ & Budaörs & 1922 & Soó Rezső & BPU-08707.JPG \\
\hline 1995 & $\begin{array}{l}\text { Serratula radiata (Waldst. et Kit.) M. } \\
\text { Bieb. }\end{array}$ & Budaörs & 1961 & Borsos Olga, Simon Tibor & BPU-08709.JPG \\
\hline 1996 & Centaurea solstitialis L. & Budapest & 1947 & Papp József & BPU-08711.JPG \\
\hline 1996 & Centaurea solstitialis L. & Gyomaendrőd & 1947 & Soó Rezső & BPU-08713.JPG \\
\hline 1996 & Centaurea solstitialis L. & Nádudvar & 1963 & Simon Tibor & BPU-08714.JPG \\
\hline 1996 & Centaurea solstitialis L. & Nagyharsány & 1965 & $\begin{array}{l}\text { Simon Tibor, Priszter } \\
\text { Szaniszló, Borhidi Attila }\end{array}$ & BPU-08712.JPG \\
\hline 1996 & Centaurea solstitialis L. & Szentendre & 1947 & Papp József & BPU-08715.JPG \\
\hline
\end{tabular}


1. Elektroniukus melléklet - Electronic Appendix 1.

\begin{tabular}{|c|c|c|c|c|c|}
\hline $\begin{array}{l}\text { Sorszám / } \\
\text { Number }\end{array}$ & Taxon-név / Taxon-name & $\begin{array}{l}\text { Település / } \\
\text { Settlement }\end{array}$ & $\begin{array}{l}\text { Év / } \\
\text { Year }\end{array}$ & Gyújtő / Collector & $\begin{array}{l}\text { Fájlnév / } \\
\text { File-name }\end{array}$ \\
\hline 1996 & Centaurea solstitialis L. & Törökbálint & 1991 & Felföldy Lajos & BPU-08716.JPG \\
\hline 1998 & Centaurea nigrescens Willd. & Alsószölnök & 1955 & Soó Rezső & BPU-08752.JPG \\
\hline 1998 & Centaurea nigrescens Willd. & Bak & 1955 & Soó Rezső & BPU-08761.JPG \\
\hline 1998 & Centaurea nigrescens Willd. & Bisse & 1965 & $\begin{array}{l}\text { Simon Tibor, Priszter } \\
\text { Szaniszló }\end{array}$ & BPU-08757.JPG \\
\hline 1998 & Centaurea nigrescens Willd. & Budapest & 1947 & Soó Rezső & BPU-08759.JPG \\
\hline 1998 & Centaurea nigrescens Willd. & Hetvehely & 1950 & Felföldy Lajos & BPU-08758.JPG \\
\hline 1998 & Centaurea nigrescens Willd. & Kaszó & 1953 & Tallós Pál & BPU-08762.JPG \\
\hline 1998 & Centaurea nigrescens Willd. & Kaszó & 1957 & Soó Rezső & BPU-08751.JPG \\
\hline 1998 & Centaurea nigrescens Willd. & Kaszó & 1957 & Soó Rezső & BPU-08753.JPG \\
\hline 1998 & Centaurea nigrescens Willd. & Magyarlukafa & 1957 & Soó Rezső & BPU-08755.JPG \\
\hline 1998 & Centaurea nigrescens Willd. & Pécs & 1938 & Soó Rezső & BPU-08756.JPG \\
\hline 1998 & Centaurea nigrescens Willd. & Szécsisziget & 1949 & Papp József & BPU-08760.JPG \\
\hline 1998 & Centaurea nigrescens Willd. & Szenna & 1957 & Soó Rezső & BPU-08754.JPG \\
\hline 1999 & Centaurea jacea L. s. l. & Aszófő & 1958 & Felföldy Lajos & BPU-08742.JPG \\
\hline 1999 & Centaurea jacea L. s. l. & Balatonakali & 1958 & Felföldy Lajos & BPU-08741.JPG \\
\hline 1999 & Centaurea jacea L. s. l. & Balatonfüred & 1946 & Felföldy Lajos & BPU-08743.JPG \\
\hline 1999 & Centaurea jacea L. s. l. & Balatongyörök & 1961 & Felföldy Lajos & BPU-08749.JPG \\
\hline 1999 & Centaurea jacea L. s. l. & Budapest & 1990 & Felföldy Lajos & BPU-08727.JPG \\
\hline 1999 & Centaurea jacea L. s. l. & Budapest & 1992 & Felföldy Lajos & BPU-08728.JPG \\
\hline 1999 & Centaurea jacea L. s. l. & Budapest & 2004 & Felföldy Lajos & BPU-08726.JPG \\
\hline 1999 & Centaurea jacea L. s. l. & Csaroda & 1952 & Soó Rezső & BPU-08720.JPG \\
\hline 1999 & Centaurea jacea L. s. l. & Debrecen & 1931 & Soó Rezső & BPU-08735.JPG \\
\hline 1999 & Centaurea jacea L. s. l. & Debrecen & 1946 & Soó Rezső & BPU-08734.JPG \\
\hline 1999 & Centaurea jacea L. s. l. & Dömös & 1948 & Soó Rezső & BPU-08730.JPG \\
\hline 1999 & Centaurea jacea L. s. l. & Dömös & 1948 & Soó Rezső & BPU-08731.JPG \\
\hline 1999 & Centaurea jacea L. s. l. & Dörgicse & 1957 & Felföldy Lajos & BPU-08744.JPG \\
\hline 1999 & Centaurea jacea L. s. l. & Eger & 1948 & Koppányi Tibor & BPU-08719.JPG \\
\hline 1999 & Centaurea jacea L. s. l. & Erdőbénye & 1948 & Soó Rezső & BPU-08721.JPG \\
\hline 1999 & Centaurea jacea L. s. l. & Erdőbénye & 1948 & Soó Rezső & BPU-08750.JPG \\
\hline 1999 & Centaurea jacea L. s. l. & Fertőhomok & 1960 & Tóth László & BPU-08725.JPG \\
\hline 1999 & Centaurea jacea L. s. l. & Gelénes & 1951 & $\begin{array}{l}\text { Simon Tibor, Vozáry } \\
\text { Elemér }\end{array}$ & BPU-08729.JPG \\
\hline 1999 & Centaurea jacea L. s. l. & Gödöllő & 1949 & Soó Rezső & BPU-08736.JPG \\
\hline 1999 & Centaurea jacea L. s. l. & Gyöngyös & 1952 & Soó Rezső & BPU-08723.JPG \\
\hline 1999 & Centaurea jacea L. s. l. & Győr & 1912 & Polgár Sándor & BPU-08902.JPG \\
\hline 1999 & Centaurea jacea L. s. l. & Jósvafó & 1948 & $\begin{array}{l}\text { Simon Tibor, Pólya } \\
\text { László, Böszörményi } \\
\text { Zoltán }\end{array}$ & BPU-08717.JPG \\
\hline 1999 & Centaurea jacea L. s. l. & Kaposvár & 1955 & Tóth László & BPU-08739.JPG \\
\hline 1999 & Centaurea jacea L. s. l. & Karcag & 1935 & Oláh J. & BPU-08740.JPG \\
\hline 1999 & Centaurea jacea L. s. l. & Mátraszentimre & 1933 & Jávorka Sándor & BPU-08912.JPG \\
\hline 1999 & Centaurea jacea L. s. l. & Miskolc & 1950 & Soó Rezső & BPU-08724.JPG \\
\hline 1999 & Centaurea jacea L. s. l. & Révfülöp & 1956 & $\begin{array}{l}\text { Felföldy Lajos, Tóth } \\
\text { László }\end{array}$ & BPU-08745.JPG \\
\hline 1999 & Centaurea jacea L. s. l. & Salgótarján & 1936 & Soó Rezső & BPU-08763.JPG \\
\hline 1999 & Centaurea jacea L. s. l. & Szentbékkálla & 1957 & Felföldy Lajos & BPU-08746.JPG \\
\hline 1999 & Centaurea jacea L. s. l. & Szilvásvárad & 1953 & $\begin{array}{l}\text { Felföldy Lajos, Tóth } \\
\text { László }\end{array}$ & BPU-08764.JPG \\
\hline 1999 & Centaurea jacea L. s. l. & Tarpa & 1948 & Simon Tibor, Jakucs Pál & BPU-08718.JPG \\
\hline
\end{tabular}


1. Elektroniukus melléklet - Electronic Appendix 1.

\begin{tabular}{|c|c|c|c|c|c|}
\hline $\begin{array}{l}\text { Sorszám / } \\
\text { Number }\end{array}$ & Taxon-név / Taxon-name & $\begin{array}{l}\text { Település / } \\
\text { Settlement }\end{array}$ & $\begin{array}{l}\text { Év / } \\
\text { Year }\end{array}$ & Gyújtő / Collector & $\begin{array}{l}\text { Fájlnév / } \\
\text { File-name }\end{array}$ \\
\hline 1999 & Centaurea jacea L. s. l. & Tatabánya & 1925 & Boros Ádám & BPU-08916.JPG \\
\hline 1999 & Centaurea jacea L. s. l. & Tihany & 1959 & Felföldy Lajos & BPU-08747.JPG \\
\hline 1999 & Centaurea jacea L. s. l. & Uzsa & 1952 & Borsos Olga & BPU-08748.JPG \\
\hline 1999 & Centaurea jacea L. s. l. & Vácrátót & 1949 & Soó Rezső & BPU-08722.JPG \\
\hline 1999 & Centaurea jacea L. s. l. & Veresegyház & 1949 & Soó Rezső & BPU-08737.JPG \\
\hline 1999 & Centaurea jacea L. s. l. & Veresegyház & 1949 & Soó Rezső & BPU-08738.JPG \\
\hline 1999 & Centaurea jacea L. s. l. & Zámoly & 1982 & Felföldy Lajos & BPU-08732.JPG \\
\hline 1999 & Centaurea jacea L. s. l. & Zámoly & 1982 & Felföldy Lajos & BPU-08733.JPG \\
\hline 2000 & Centaurea indurata Janka & Beregdaróc & 1951 & $\begin{array}{l}\text { Simon Tibor, Vozáry } \\
\text { Elemér }\end{array}$ & BPU-08774.JPG \\
\hline 2000 & Centaurea indurata Janka & Diósjenő & 1952 & Simon Tibor & BPU-08773.JPG \\
\hline 2000 & Centaurea indurata Janka & Hencida & 1939 & Kovács L. & BPU-08771.JPG \\
\hline 2000 & Centaurea indurata Janka & Jánd & 1951 & Simon Tibor & BPU-08775.JPG \\
\hline 2000 & Centaurea indurata Janka & Piricse & 1932 & Soó Rezső & BPU-08772.JPG \\
\hline 2002 & Centaurea stenolepis A. Kern. & Budapest & 1909 & Kocsis István & BPU-08769.JPG \\
\hline 2002 & Centaurea stenolepis A. Kern. & Gánt & 1948 & Soó Rezső & BPU-08767.JPG \\
\hline 2003 & Centaurea cyanus L. & Beregdaróc & 1951 & Simon Tibor & BPU-08845.JPG \\
\hline 2003 & Centaurea cyanus L. & Egyek & 1947 & Soó Rezső & BPU-08847.JPG \\
\hline 2003 & Centaurea cyanus L. & Felsőtelekes & 2005 & Gulyás Pál & BPU-08846.JPG \\
\hline 2004 & Centaurea mollis Waldst. et Kit. & Gyöngyös & 1953 & Soó Rezső & BPU-08781.JPG \\
\hline 2004 & Centaurea mollis Waldst. et Kit. & Miskolc & 1929 & Soó Rezső & BPU-08779.JPG \\
\hline 2004 & Centaurea mollis Waldst. et Kit. & Tibolddaróc & 1953 & Soó Rezső & BPU-08780.JPG \\
\hline 2005 & Centaurea triumfettii All. & Bagamér & 1933 & Soó Rezső & BPU-08785.JPG \\
\hline 2005 & Centaurea triumfettii All. & Bélapátfalva & 1950 & $\begin{array}{l}\text { Simon Tibor, Jakucs Pál, } \\
\text { Kárpáti István }\end{array}$ & BPU-08798.JPG \\
\hline 2005 & Centaurea triumfettii All. & Budapest & 1947 & Soó Rezső & BPU-08784.JPG \\
\hline 2005 & Centaurea triumfettii All. & Budapest & 1982 & Felföldy Lajos & BPU-08791.JPG \\
\hline 2005 & Centaurea triumfettii All. & Budapest & 1982 & Felföldy Lajos & BPU-08792.JPG \\
\hline 2005 & Centaurea triumfettii All. & Budapest & 1982 & Felföldy Lajos & BPU-08793.JPG \\
\hline 2005 & Centaurea triumfettii All. & Csákvár & 1955 & Soó Rezső & BPU-08786.JPG \\
\hline 2005 & Centaurea triumfettii All. & Csákvár & 1955 & Soó Rezső & BPU-08787.JPG \\
\hline 2005 & Centaurea triumfettii All. & Gyöngyös & 1953 & Soó Rezső & BPU-08790.JPG \\
\hline 2005 & Centaurea triumfettii All. & Gyöngyös & 1961 & Soó Rezső & BPU-08788.JPG \\
\hline 2005 & Centaurea triumfettii All. & Isztimér & 1949 & Soó Rezső & BPU-08783.JPG \\
\hline 2005 & Centaurea triumfettii All. & Miskolc & 1934 & Soó Rezső & BPU-08794.JPG \\
\hline 2005 & Centaurea triumfettii All. & Pécs & 1953 & Soó Rezső, Borsos Olga & BPU-08789.JPG \\
\hline 2005 & Centaurea triumfettii All. & Tokaj & 1933 & Soó Rezső & BPU-08796.JPG \\
\hline 2006 & Centaurea salonitana Vis. & Budapest & 1948 & Soó Rezső & BPU-08848.JPG \\
\hline 2007 & Centaurea diffusa Lam. & Budapest & 1948 & Soó Rezső & BPU-08803.JPG \\
\hline 2008 & Centaurea scabiosa L. & Aszófő & 1957 & $\begin{array}{l}\text { Felföldy Lajos, Kovács } \\
\text { Margit, Tóth László }\end{array}$ & BPU-08869.JPG \\
\hline 2008 & Centaurea scabiosa L. & Balatonfüred & 1947 & Pólya László & BPU-08870.JPG \\
\hline 2008 & Centaurea scabiosa L. & Barabás & 1948 & Simon Tibor, Jakucs Pál & BPU-08857.JPG \\
\hline 2008 & Centaurea scabiosa L. & Budaörs & 1982 & Felföldy Lajos & BPU-08850.JPG \\
\hline 2008 & Centaurea scabiosa L. & Budapest & 1947 & Soó Rezső & BPU-08854.JPG \\
\hline 2008 & Centaurea scabiosa L. & Budapest & 1982 & Felföldy Lajos & BPU-08849.JPG \\
\hline 2008 & Centaurea scabiosa L. & Budapest & 1982 & Felföldy Lajos & BPU-08851.JPG \\
\hline 2008 & Centaurea scabiosa L. & Budapest & 2004 & Felföldy Lajos & BPU-08855.JPG \\
\hline 2008 & Centaurea scabiosa L. & Isztimér & 1949 & Soó Rezső & BPU-08867.JPG \\
\hline
\end{tabular}


1. Elektroniukus melléklet - Electronic Appendix 1.

\begin{tabular}{|c|c|c|c|c|c|}
\hline $\begin{array}{l}\text { Sorszám / } \\
\text { Number }\end{array}$ & Taxon-név / Taxon-name & $\begin{array}{l}\text { Település / } \\
\text { Settlement }\end{array}$ & $\begin{array}{l}\text { Év / } \\
\text { Year }\end{array}$ & Gyűjtő / Collector & $\begin{array}{l}\text { Fájlnév / } \\
\text { File-name }\end{array}$ \\
\hline 2008 & Centaurea scabiosa L. & Kesztölc & 1949 & Jakucs Pál & BPU-08852.JPG \\
\hline 2008 & Centaurea scabiosa L. & Kőszeg & 1932 & Soó Rezső & BPU-08863.JPG \\
\hline 2008 & Centaurea scabiosa L. & Miskolc & 1950 & Soó Rezső & BPU-08866.JPG \\
\hline 2008 & Centaurea scabiosa L. & Nagyharsány & 1961 & Simon Tibor & BPU-08856.JPG \\
\hline 2008 & Centaurea scabiosa L. & Nyíracsád & 1932 & Soó Rezső & BPU-08859.JPG \\
\hline 2008 & Centaurea scabiosa L. & Sárospatak & 1932 & Soó Rezső & BPU-08917.JPG \\
\hline 2008 & Centaurea scabiosa L. & Sümeg & 1953 & Borsos Olga & BPU-08865.JPG \\
\hline 2008 & Centaurea scabiosa L. & Szeged & 1950 & Timár Lajos & BPU-08889.JPG \\
\hline 2008 & Centaurea scabiosa L. & Szilvásvárad & 1949 & Koppányi Tibor & BPU-08919.JPG \\
\hline 2008 & Centaurea scabiosa L. & Tarpa & 1949 & Soó Rezső & BPU-08858.JPG \\
\hline 2008 & Centaurea scabiosa L. & Tihany & 1959 & Felföldy Lajos & BPU-08871.JPG \\
\hline 2008 & Centaurea scabiosa L. & Tihany & 1959 & Felföldy Lajos & BPU-08872.JPG \\
\hline 2008 & Centaurea scabiosa L. & Tokaj & 1933 & Soó Rezső & BPU-08860.JPG \\
\hline 2008 & Centaurea scabiosa L. & Uzsa & 1952 & Simon Tibor & BPU-08864.JPG \\
\hline 2008 & Centaurea scabiosa L. & Vácrátót & 1939 & Soó Rezső & BPU-08853.JPG \\
\hline 2008 & Centaurea scabiosa L. & Zalagyömörő & 1957 & Felföldy Lajos & BPU-08873.JPG \\
\hline 2008 & Centaurea scabiosa L. & Zámoly & 1982 & Felföldy Lajos & BPU-08868.JPG \\
\hline 2009 & Centaurea arenaria M. Bieb. ex Willd. & Baktalórántháza & 1948 & Simon Tibor, Jakucs Pál & BPU-08806.JPG \\
\hline 2009 & Centaurea arenaria M. Bieb. ex Willd. & Budapest & 1948 & Soó Rezső & BPU-08811.JPG \\
\hline 2009 & Centaurea arenaria M. Bieb. ex Willd. & Budapest & 1982 & Felföldy Lajos & BPU-08808.JPG \\
\hline 2009 & Centaurea arenaria M. Bieb. ex Willd. & Budapest & 1982 & Felföldy Lajos & BPU-08809.JPG \\
\hline 2009 & Centaurea arenaria M. Bieb. ex Willd. & Dunakeszi & 1948 & Soó Rezső & BPU-08805.JPG \\
\hline 2009 & Centaurea arenaria M. Bieb. ex Willd. & Kecskemét & 1931 & Degen Árpád & BPU-08807.JPG \\
\hline 2009 & Centaurea arenaria M. Bieb. ex Willd. & Nyíradony & 1932 & Soó Rezső & BPU-08804.JPG \\
\hline 2009 & Centaurea arenaria M. Bieb. ex Willd. & Tihany & 1961 & $\begin{array}{l}\text { Pankov H., Felföldy Lajos, } \\
\text { Sólymosy G. }\end{array}$ & BPU-08810.JPG \\
\hline 2010 & Centaurea stoebe L. & Abaújszántó & 1948 & Soó Rezső & BPU-08816.JPG \\
\hline 2010 & Centaurea stoebe L. & Baktalórántháza & 1933 & Soó Rezső & BPU-08814.JPG \\
\hline 2010 & Centaurea stoebe L. & Budapest & 1947 & Soó Rezső & BPU-08827.JPG \\
\hline 2010 & Centaurea stoebe L. & Budapest & 1947 & Soó Rezső & BPU-08828.JPG \\
\hline 2010 & Centaurea stoebe L. & Budapest & 1948 & Soó Rezső & BPU-08813.JPG \\
\hline 2010 & Centaurea stoebe L. & Budapest & 1982 & Felföldy Lajos & BPU-08824.JPG \\
\hline 2010 & Centaurea stoebe L. & Budapest & 1982 & Felföldy Lajos & BPU-08825.JPG \\
\hline 2010 & Centaurea stoebe L. & Budapest & 1982 & Felföldy Lajos & BPU-08826.JPG \\
\hline 2010 & Centaurea stoebe L. & Budapest & 2004 & Felföldy Lajos & BPU-08823.JPG \\
\hline 2010 & Centaurea stoebe L. & Csákvár & 1948 & Soó Rezső & BPU-08812.JPG \\
\hline 2010 & Centaurea stoebe L. & Csomád & 1953 & Felföldy Lajos & BPU-08817.JPG \\
\hline 2010 & Centaurea stoebe L. & Gánt & 1948 & Soó Rezső & BPU-08815.JPG \\
\hline 2010 & Centaurea stoebe L. & Nagyharsány & 1961 & Simon Tibor & BPU-08829.JPG \\
\hline 2010 & Centaurea stoebe L. & Solymár & 1992 & Felföldy Lajos & BPU-08834.JPG \\
\hline 2010 & Centaurea stoebe L. & Tihany & 1959 & Felföldy Lajos & BPU-08819.JPG \\
\hline 2010 & Centaurea stoebe L. & Tihany & 1959 & Felföldy Lajos & BPU-08820.JPG \\
\hline 2010 & Centaurea stoebe L. & Tihany & 1959 & Felföldy Lajos & BPU-08821.JPG \\
\hline 2010 & Centaurea stoebe L. & Tihany & 1959 & Felföldy Lajos & BPU-08822.JPG \\
\hline 2010 & Centaurea stoebe L. & Tihany & 1959 & Felföldy Lajos & BPU-08837.JPG \\
\hline 2010 & Centaurea stoebe L. & Tihany & 1959 & Felföldy Lajos & BPU-08838.JPG \\
\hline 2010 & Centaurea stoebe L. & Tihany & 1959 & Felföldy Lajos & BPU-08839.JPG \\
\hline 2010 & Centaurea stoebe L. & Tihany & 1961 & Felföldy Lajos & BPU-08835.JPG \\
\hline
\end{tabular}


1. Elektroniukus melléklet - Electronic Appendix 1.

\begin{tabular}{|c|c|c|c|c|c|}
\hline $\begin{array}{l}\text { Sorszám / } \\
\text { Number }\end{array}$ & Taxon-név / Taxon-name & $\begin{array}{l}\text { Település / } \\
\text { Settlement }\end{array}$ & $\begin{array}{l}\text { Év / } \\
\text { Year }\end{array}$ & Gyújtő / Collector & $\begin{array}{l}\text { Fájlnév / } \\
\text { File-name }\end{array}$ \\
\hline 2010 & Centaurea stoebe L. & Tihany & 1982 & Felföldy Lajos & BPU-08836.JPG \\
\hline 2010 & Centaurea stoebe L. & Vácrátót & 1952 & Felföldy Lajos & BPU-08818.JPG \\
\hline 2010 & Centaurea stoebe L. & Zámoly & 1982 & Felföldy Lajos & BPU-08830.JPG \\
\hline 2010 & Centaurea stoebe L. & Zámoly & 1982 & Felföldy Lajos & BPU-08831.JPG \\
\hline 2010 & Centaurea stoebe L. & Zámoly & 1982 & Felföldy Lajos & BPU-08832.JPG \\
\hline 2010 & Centaurea stoebe L. & Zámoly & 1982 & Felföldy Lajos & BPU-08833.JPG \\
\hline $\begin{array}{c}1998 \times \\
1999\end{array}$ & $\begin{array}{l}\text { Centaurea nigrescens Willd. } \times \\
\text { Centaurea jacea L. }\end{array}$ & Gánt & 1943 & Kárpáti Zoltán & BPU-08897.JPG \\
\hline $\begin{array}{c}1998 \times \\
1999\end{array}$ & $\begin{array}{l}\text { Centaurea nigrescens Willd. } \times \\
\text { Centaurea jacea L. }\end{array}$ & Sajólád & 1909 & Budai József & BPU-08896.JPG \\
\hline $\begin{array}{c}1998 \times \\
1999\end{array}$ & $\begin{array}{l}\text { Centaurea nigrescens Willd. } \times \\
\text { Centaurea jacea L. }\end{array}$ & Sopron & 1940 & Kárpáti Zoltán & BPU-08900.JPG \\
\hline $\begin{array}{c}1999 \times \\
2000\end{array}$ & $\begin{array}{l}\text { Centaurea jacea L. s. l. × Centaurea } \\
\text { indurata Janka }\end{array}$ & Bátorliget & 1932 & Soó Rezső & BPU-08904.JPG \\
\hline $\begin{array}{c}1999 \times \\
2000\end{array}$ & $\begin{array}{l}\text { Centaurea jacea L. s. l. × Centaurea } \\
\text { indurata Janka }\end{array}$ & Hortobágy & 1947 & Siroki Zoltán & BPU-08903.JPG \\
\hline $\begin{array}{c}1999 \times \\
2002\end{array}$ & $\begin{array}{l}\text { Centaurea stenolepis A. Kern. × } \\
\text { Centaurea jacea L. }\end{array}$ & Gánt & 1948 & Soó Rezső & BPU-08894.JPG \\
\hline $\begin{array}{c}1999 \times \\
2010\end{array}$ & $\begin{array}{l}\text { Centaurea jacea L. } \times \text { Centaurea stoebe } \\
\text { L. }\end{array}$ & Budapest & 1943 & Kárpáti Zoltán & BPU-08914.JPG \\
\hline $\begin{array}{c}1999 \times \\
2010\end{array}$ & $\begin{array}{l}\text { Centaurea jacea L. } \times \text { Centaurea stoebe } \\
\text { L. }\end{array}$ & Tihany & 1961 & Sólymossy Gizella & BPU-08898.JPG \\
\hline $\begin{array}{c}1999 \times \\
2010\end{array}$ & $\begin{array}{l}\text { Centaurea jacea L. × Centaurea stoebe } \\
\text { L. }\end{array}$ & Tihany & 1961 & Sólymossy Gizella & BPU-08899.JPG \\
\hline $\begin{array}{l}2001 \times \\
2002\end{array}$ & $\begin{array}{l}\text { Centaurea } \times \text { castriferrei Borbás et } \\
\text { Wagner }\end{array}$ & Kőszeg & 1932 & Soó Rezső & BPU-08768.JPG \\
\hline $\begin{array}{c}2007 \times \\
2010\end{array}$ & $\begin{array}{l}\text { Centaurea stoebe L. } \times \text { Centaurea } \\
\text { diffusa Lam. }\end{array}$ & Győr & 1933 & Polgár Sándor & BPU-08915.JPG \\
\hline $\begin{array}{c}2008 \times \\
2010\end{array}$ & $\begin{array}{l}\text { Centaurea stoebe L. } \times \text { Centaurea } \\
\text { scabiosa L. }\end{array}$ & Budapest & 1947 & Soó Rezső & BPU-08913.JPG \\
\hline 2011 & Crupina vulgaris Cass. & Budapest & 1947 & Soó Rezső & BPU-08688.JPG \\
\hline 2011 & Crupina vulgaris Cass. & Budapest & 1982 & Felföldy Lajos & BPU-08683.JPG \\
\hline 2011 & Crupina vulgaris Cass. & Budapest & 1991 & Felföldy Lajos & BPU-08684.JPG \\
\hline 2011 & Crupina vulgaris Cass. & Csákvár & 1955 & Soó Rezső & BPU-08686.JPG \\
\hline 2011 & Crupina vulgaris Cass. & Nagyharsány & 1956 & Soó Rezső & BPU-08687.JPG \\
\hline 2011 & Crupina vulgaris Cass. & Tarcal & 1943 & Felföldy Lajos & BPU-08690.JPG \\
\hline 2011 & Crupina vulgaris Cass. & Tihany & 1947 & Pólya László & BPU-08689.JPG \\
\hline 2011 & Crupina vulgaris Cass. & Tihany & 1959 & Felföldy Lajos & BPU-08685.JPG \\
\hline 2013 & Carthamus lanatus L. & Budapest & 1947 & Soó Rezső & BPU-08920.JPG \\
\hline 2013 & Carthamus lanatus L. & Nagyharsány & 1961 & Simon Tibor & BPU-08921.JPG \\
\hline 2015 & Cichorium intybus L. & Sáránd & 1933 & Juhász Lajos & BPU-08923.JPG \\
\hline 2015 & Cichorium intybus L. & Tokaj & 1949 & Soó Rezső & BPU-08922.JPG \\
\hline 2016 & Hypochoeris maculata L. & "Szentendrei-sziget" & 1953 & Borsos Olga & BPU-08938.JPG \\
\hline 2016 & Hypochoeris maculata L. & Budapest & 1947 & Soó Rezső & BPU-08935.JPG \\
\hline 2016 & Hypochoeris maculata L. & Fót & 1960 & Soó Rezső & BPU-08934.JPG \\
\hline 2016 & Hypochoeris maculata L. & Gyöngyös & 1961 & Soó Rezső & BPU-08940.JPG \\
\hline 2016 & Hypochoeris maculata L. & Nagyvisnyó & 1948 & $\begin{array}{l}\text { Simon Tibor, Pólya } \\
\text { László, Jakucs Pál }\end{array}$ & BPU-08937.JPG \\
\hline 2016 & Hypochoeris maculata L. & Nyíracsád & 1931 & Soó Rezső & BPU-08936.JPG \\
\hline 2016 & Hypochoeris maculata L. & Uzsa & 1959 & $\begin{array}{l}\text { Jávorka Sándor, Felföldy } \\
\text { Lajos }\end{array}$ & BPU-08939.JPG \\
\hline 2017 & Hypochoeris radicata L. & Debrecen & 1932 & Soó Rezső & BPU-08942.JPG \\
\hline 2017 & Hypochoeris radicata L. & Pálháza & 1938 & Soó Rezső & BPU-08945.JPG \\
\hline
\end{tabular}


1. Elektroniukus melléklet - Electronic Appendix 1.

\begin{tabular}{|c|c|c|c|c|c|}
\hline $\begin{array}{l}\text { Sorszám / } \\
\text { Number }\end{array}$ & Taxon-név / Taxon-name & $\begin{array}{l}\text { Település / } \\
\text { Settlement }\end{array}$ & $\begin{array}{l}\text { Év / } \\
\text { Year }\end{array}$ & Gyűjtő / Collector & $\begin{array}{l}\text { Fájlnév / } \\
\text { File-name }\end{array}$ \\
\hline 2017 & Hypochoeris radicata L. & Pálháza & 1949 & Simon Tibor, Jakucs Pál & BPU-08941.JPG \\
\hline 2017 & Hypochoeris radicata L. & Uzsa & 1950 & Felföldy Lajos & BPU-08943.JPG \\
\hline 2018 & Thrincia nudicaulis (L.) Dostál & Balatonakali & 1958 & Felföldy Lajos & BPU-08965.JPG \\
\hline 2018 & Thrincia nudicaulis (L.) Dostál & Balatongyörök & 1961 & Felföldy Lajos & BPU-08968.JPG \\
\hline 2018 & Thrincia nudicaulis (L.) Dostál & Balatonszemes & 1982 & Felföldy Lajos & BPU-08967.JPG \\
\hline 2018 & Thrincia nudicaulis (L.) Dostál & Balatonszepezd & 1958 & Felföldy Lajos & BPU-08960.JPG \\
\hline 2018 & Thrincia nudicaulis (L.) Dostál & Balatonszepezd & 1958 & Felföldy Lajos & BPU-08962.JPG \\
\hline 2018 & Thrincia nudicaulis (L.) Dostál & Budapest & 1922 & Koszilkov János & BPU-08957.JPG \\
\hline 2018 & Thrincia nudicaulis (L.) Dostál & Fertőhomok & 1960 & Tóth László & BPU-08971.JPG \\
\hline 2018 & Thrincia nudicaulis (L.) Dostál & Hévíz & 1928 & Soó Rezső & BPU-08958.JPG \\
\hline 2018 & Thrincia nudicaulis (L.) Dostál & Hévíz & 1961 & Felföldy Lajos & BPU-08969.JPG \\
\hline 2018 & Thrincia nudicaulis (L.) Dostál & Hévíz & 1961 & Felföldy Lajos & BPU-08970.JPG \\
\hline 2018 & Thrincia nudicaulis (L.) Dostál & Hévíz & 1961 & Felföldy Lajos & BPU-08972.JPG \\
\hline 2018 & Thrincia nudicaulis (L.) Dostál & Révfülöp & 1956 & $\begin{array}{l}\text { Felföldy Lajos, Tóth } \\
\text { László }\end{array}$ & BPU-08963.JPG \\
\hline 2018 & Thrincia nudicaulis (L.) Dostál & Tihany & 1957 & Felföldy Lajos & BPU-08961.JPG \\
\hline 2018 & Thrincia nudicaulis (L.) Dostál & Ukk & 1957 & Felföldy Lajos & BPU-08964.JPG \\
\hline 2018 & Thrincia nudicaulis (L.) Dostál & Uzsa & 1950 & Felföldy Lajos & BPU-08959.JPG \\
\hline 2018 & Thrincia nudicaulis (L.) Dostál & Uzsa & 1952 & Simon Tibor & BPU-08966.JPG \\
\hline 2019 & Leontodon incanus (L.) Schrank & Gyenesdiás & 1928 & Soó Rezső & BPU-08975.JPG \\
\hline 2019 & Leontodon incanus (L.) Schrank & Uzsa & 1959 & $\begin{array}{l}\text { Jávorka Sándor, Felföldy } \\
\text { Lajos }\end{array}$ & BPU-08976.JPG \\
\hline 2020 & Leontodon autumnalis L. & Balatongyörök & 1961 & Felföldy Lajos & BPU-08991.JPG \\
\hline 2020 & Leontodon autumnalis L. & Debrecen & 1932 & Máthé Imre & BPU-08989.JPG \\
\hline 2020 & Leontodon autumnalis L. & Erdőbénye & 1948 & Soó Rezső & BPU-08980.JPG \\
\hline 2020 & Leontodon autumnalis L. & Farmos & 1928 & Soó Rezső & BPU-08995.JPG \\
\hline 2020 & Leontodon autumnalis L. & Gelénes & 1951 & $\begin{array}{l}\text { Simon Tibor, Vozáry } \\
\text { Elemér }\end{array}$ & BPU-08979.JPG \\
\hline 2020 & Leontodon autumnalis L. & Hévíz & 1961 & Felföldy Lajos & BPU-08992.JPG \\
\hline 2020 & Leontodon autumnalis L. & Mátraszentimre & 1935 & Soó Rezső & BPU-08985.JPG \\
\hline 2020 & Leontodon autumnalis L. & Monoszló & 1982 & Felföldy Lajos & BPU-08990.JPG \\
\hline 2020 & Leontodon autumnalis L. & Somogyszob & 1957 & Soó Rezső & BPU-08988.JPG \\
\hline 2020 & Leontodon autumnalis L. & Tákos & 1948 & Simon Tibor, Jakucs Pál & BPU-08982.JPG \\
\hline 2021 & Leontodon hispidus L. & Bakonybél & 1955 & Soó Rezső & BPU-14014.JPG \\
\hline 2021 & Leontodon hispidus L. & Baktalórántháza & 1932 & Soó Rezső & BPU-14015.JPG \\
\hline 2021 & Leontodon hispidus L. & Balatongyörök & 1961 & Felföldy Lajos & BPU-14005.JPG \\
\hline 2021 & Leontodon hispidus L. & Balatongyörök & 1961 & Felföldy Lajos & BPU-14006.JPG \\
\hline 2021 & Leontodon hispidus L. & Balatongyörök & 1961 & Felföldy Lajos & BPU-14007.JPG \\
\hline 2021 & Leontodon hispidus L. & Balatongyörök & 1961 & Felföldy Lajos & BPU-14008.JPG \\
\hline 2021 & Leontodon hispidus L. & Bisse & 1965 & $\begin{array}{l}\text { Simon Tibor, Priszter } \\
\text { Szaniszló }\end{array}$ & BPU-14025.JPG \\
\hline 2021 & Leontodon hispidus L. & Budaörs & 1967 & $\begin{array}{l}\text { Priszter Szaniszló, } \\
\text { Borhidi Attila }\end{array}$ & BPU-14017.JPG \\
\hline 2021 & Leontodon hispidus L. & Budapest & 1923 & Soó Rezső & BPU-14026.JPG \\
\hline 2021 & Leontodon hispidus L. & Budapest & 1991 & Felföldy Lajos & BPU-14027.JPG \\
\hline 2021 & Leontodon hispidus L. & Budapest & 2004 & Felföldy Lajos & BPU-14028.JPG \\
\hline 2021 & Leontodon hispidus L. & Cák & 1932 & Soó Rezső & BPU-13995.JPG \\
\hline 2021 & Leontodon hispidus L. & Cák & 1941 & Soó Rezső & BPU-13994.JPG \\
\hline 2021 & Leontodon hispidus L. & Debrecen & 1933 & Soó Rezső & BPU-14019.JPG \\
\hline 2021 & Leontodon hispidus L. & Debrecen & 1933 & Soó Rezső & BPU-14022.JPG \\
\hline
\end{tabular}


1. Elektroniukus melléklet - Electronic Appendix 1.

\begin{tabular}{|c|c|c|c|c|c|}
\hline $\begin{array}{l}\text { Sorszám / } \\
\text { Number }\end{array}$ & Taxon-név / Taxon-name & $\begin{array}{l}\text { Település / } \\
\text { Settlement }\end{array}$ & $\begin{array}{l}\text { Év / } \\
\text { Year }\end{array}$ & Gyújtő / Collector & $\begin{array}{l}\text { Fájlnév / } \\
\text { File-name }\end{array}$ \\
\hline 2021 & Leontodon hispidus L. & Debrecen & 1938 & Soó Rezső & BPU-14020.JPG \\
\hline 2021 & Leontodon hispidus L. & Miskolc & 1937 & Soó Rezső & BPU-14024.JPG \\
\hline 2021 & Leontodon hispidus L. & Monostorapáti & 1959 & $\begin{array}{l}\text { Jávorka Sándor, Felföldy } \\
\text { Lajos, Tóth László }\end{array}$ & BPU-14013.JPG \\
\hline 2021 & Leontodon hispidus L. & Örvényes & 1959 & Felföldy Lajos & BPU-14012.JPG \\
\hline 2021 & Leontodon hispidus L. & Solymár & 1992 & Felföldy Lajos & BPU-14016.JPG \\
\hline 2021 & Leontodon hispidus L. & Szomor & 1938 & Boros Ádám & BPU-14018.JPG \\
\hline 2021 & Leontodon hispidus L. & Uzsa & 1959 & $\begin{array}{l}\text { Jávorka Sándor, Felföldy } \\
\text { Lajos }\end{array}$ & BPU-14009.JPG \\
\hline 2021 & Leontodon hispidus L. & Uzsa & 1959 & $\begin{array}{l}\text { Jávorka Sándor, Felföldy } \\
\text { Lajos }\end{array}$ & BPU-14010.JPG \\
\hline 2021 & Leontodon hispidus L. & Uzsa & 1959 & $\begin{array}{l}\text { Jávorka Sándor, Felföldy } \\
\text { Lajos }\end{array}$ & BPU-14011.JPG \\
\hline 2022 & Helminthia echioides (L.) Gaertn. & Budapest & 1943 & Kárpáti Zoltán & BPU-13979.JPG \\
\hline 2023 & Picris hieracioides L. & Balatongyörök & 1961 & Felföldy Lajos & BPU-13971.JPG \\
\hline 2023 & Picris hieracioides L. & Budapest & 1982 & Felföldy Lajos & BPU-13975.JPG \\
\hline 2023 & Picris hieracioides L. & Budapest & 1982 & Felföldy Lajos & BPU-13976.JPG \\
\hline 2023 & Picris hieracioides L. & Budapest & 1982 & Felföldy Lajos & BPU-13977.JPG \\
\hline 2023 & Picris hieracioides L. & Budapest & 1991 & Felföldy Lajos & BPU-13974.JPG \\
\hline 2023 & Picris hieracioides L. & Budapest & 2004 & Felföldy Lajos & BPU-13978.JPG \\
\hline 2023 & Picris hieracioides L. & Debrecen & 1931 & Soó Rezső & BPU-13973.JPG \\
\hline 2023 & Picris hieracioides L. & Tihany & 1959 & Felföldy Lajos & BPU-13972.JPG \\
\hline 2024 & $\begin{array}{l}\text { Podospermum canum (C.A. Mey.) } \\
\text { Griseb. }\end{array}$ & Bodajk & 1955 & Soó Rezső & BPU-13923.JPG \\
\hline 2024 & $\begin{array}{l}\text { Podospermum canum (C.A. Mey.) } \\
\text { Griseb. }\end{array}$ & Budapest & 1916 & Andrasovszky József & BPU-13925.JPG \\
\hline 2024 & $\begin{array}{l}\text { Podospermum canum (C.A. Mey.) } \\
\text { Griseb. }\end{array}$ & Dabas & 1968 & Priszter Szaniszló & BPU-13921.JPG \\
\hline 2024 & $\begin{array}{l}\text { Podospermum canum (C.A. Mey.) } \\
\text { Griseb. }\end{array}$ & Debrecen & 1931 & Soó Rezső & BPU-13922.JPG \\
\hline 2024 & $\begin{array}{l}\text { Podospermum canum (C.A. Mey.) } \\
\text { Griseb. }\end{array}$ & Gelénes & 1951 & $\begin{array}{l}\text { Simon Tibor, Vozáry } \\
\text { Elemér }\end{array}$ & BPU-13924.JPG \\
\hline 2026 & Scorzonera purpurea L. & Abaújszántó & 1938 & Soó Rezső & BPU-13957.JPG \\
\hline 2026 & Scorzonera purpurea L. & Budapest & 1952 & Soó Rezső & BPU-13956.JPG \\
\hline 2026 & Scorzonera purpurea L. & Debrecen & 1953 & Soó Rezső & BPU-13955.JPG \\
\hline 2026 & Scorzonera purpurea L. & Fenyőfö & 1955 & Soó Rezső & BPU-13954.JPG \\
\hline 2026 & Scorzonera purpurea L. & Uzsa & 1959 & $\begin{array}{l}\text { Jávorka Sándor, Felföldy } \\
\text { Lajos }\end{array}$ & BPU-13953.JPG \\
\hline 2027 & Scorzonera hispanica L. & Budapest & 1947 & Soó Rezső & BPU-13952.JPG \\
\hline 2027 & Scorzonera hispanica L. & Budapest & 1982 & Felföldy Lajos & BPU-13945.JPG \\
\hline 2027 & Scorzonera hispanica L. & Budapest & 1982 & Felföldy Lajos & BPU-13946.JPG \\
\hline 2027 & Scorzonera hispanica L. & Budapest & 1991 & Felföldy Lajos & BPU-13948.JPG \\
\hline 2027 & Scorzonera hispanica L. & Szalonna & 1966 & Felföldy Lajos & BPU-13947.JPG \\
\hline 2027 & Scorzonera hispanica L. & Tállya & 1938 & Soó Rezső & BPU-13951.JPG \\
\hline 2027 & Scorzonera hispanica L. & Tihany & 1959 & Felföldy Lajos & BPU-13950.JPG \\
\hline 2027 & Scorzonera hispanica L. & Üröm & 1991 & Felföldy Lajos & BPU-13949.JPG \\
\hline 2028 & Scorzonera austriaca L. & Abaújszántó & 1938 & Soó Rezső & BPU-13940.JPG \\
\hline 2028 & Scorzonera austriaca L. & Budaörs & 1982 & Felföldy Lajos & BPU-13941.JPG \\
\hline 2028 & Scorzonera austriaca L. & Budapest & 1958 & Isépy István & BPU-13943.JPG \\
\hline 2028 & Scorzonera austriaca L. & Budapest & 1982 & Felföldy Lajos & BPU-13944.JPG \\
\hline 2028 & Scorzonera austriaca L. & Gánt & 1949 & Soó Rezső & BPU-13942.JPG \\
\hline 2029 & Scorzonera humilis L. & Bugyi & 1959 & Soó Rezső & BPU-13934.JPG \\
\hline
\end{tabular}


1. Elektroniukus melléklet - Electronic Appendix 1.

\begin{tabular}{|c|c|c|c|c|c|}
\hline $\begin{array}{l}\text { Sorszám / } \\
\text { Number }\end{array}$ & Taxon-név / Taxon-name & $\begin{array}{l}\text { Település / } \\
\text { Settlement }\end{array}$ & $\begin{array}{l}\text { Év / } \\
\text { Year }\end{array}$ & Gyűjtő / Collector & $\begin{array}{l}\text { Fájlnév / } \\
\text { File-name }\end{array}$ \\
\hline 2029 & Scorzonera humilis L. & Dabas & 1928 & Palik Piroska & BPU-13933.JPG \\
\hline 2029 & Scorzonera humilis L. & Gyöngyös & 1965 & Soó Rezső & BPU-13935.JPG \\
\hline 2029 & Scorzonera humilis L. & Ócsa & 1959 & Simon Tibor & BPU-13937.JPG \\
\hline 2029 & Scorzonera humilis L. & Sopron & 1944 & Kárpáti Zoltán & BPU-13939.JPG \\
\hline 2029 & Scorzonera humilis L. & Uzsa & 1950 & Felföldy Lajos & BPU-13938.JPG \\
\hline 2030 & Scorzonera parviflora Jacq. & Bugyi & 1959 & Soó Rezső & BPU-13926.JPG \\
\hline 2030 & Scorzonera parviflora Jacq. & Dabas & 1951 & Borsos Olga, Simon Tibor & BPU-13929.JPG \\
\hline 2030 & Scorzonera parviflora Jacq. & Dabas & 1951 & Simon Tibor, Borsos Olga & BPU-13930.JPG \\
\hline 2030 & Scorzonera parviflora Jacq. & Dabas & 1959 & Soó Rezső & BPU-13927.JPG \\
\hline 2030 & Scorzonera parviflora Jacq. & Győr & 1927 & Polgár Sándor & BPU-13931.JPG \\
\hline 2030 & Scorzonera parviflora Jacq. & Pákozd & 1980 & Felföldy Lajos & BPU-13932.JPG \\
\hline 2032 & Tragopogon dubius Scop. & Egyek & 1932 & Soó Rezső & BPU-13966.JPG \\
\hline 2032 & Tragopogon dubius Scop. & Nagyharsány & 1965 & $\begin{array}{l}\text { Simon Tibor, Priszter } \\
\text { Szaniszló }\end{array}$ & BPU-13968.JPG \\
\hline 2032 & Tragopogon dubius Scop. & Nagyharsány & 1967 & Priszter Szaniszló & BPU-13967.JPG \\
\hline 2033 & Tragopogon orientalis L. & Miskolc & 1934 & Soó Rezső & BPU-13962.JPG \\
\hline 2033 & Tragopogon orientalis L. & Szigetmonostor & 1947 & Soó Rezső & BPU-13961.JPG \\
\hline 2034 & Tragopogon floccosus Waldst. et Kit. & Budapest & 1923 & Soó Rezső & BPU-13963.JPG \\
\hline 2034 & Tragopogon floccosus Waldst. et Kit. & Bugac & 1964 & Soó Rezső & BPU-13964.JPG \\
\hline 2034 & Tragopogon floccosus Waldst. et Kit. & Csévharaszt & 1959 & Simon Tibor, Borsos Olga & BPU-13965.JPG \\
\hline 2035 & Sonchus asper (L.) Hill & Budaörs & 1991 & Felföldy Lajos & BPU-13846.JPG \\
\hline 2035 & Sonchus asper (L.) Hill & Szarvas & 1947 & Soó Rezső & BPU-13847.JPG \\
\hline 2036 & Sonchus oleraceus L. & Debrecen & 1938 & Soó Rezső & BPU-13851.JPG \\
\hline 2036 & Sonchus oleraceus L. & Tákos & 1948 & Soó Rezső & BPU-13852.JPG \\
\hline 2036 & Sonchus oleraceus L. & Tihany & 1961 & Sólymossy Gizella & BPU-13848.JPG \\
\hline 2036 & Sonchus oleraceus L. & Tihany & 1961 & Sólymossy Gizella & BPU-13849.JPG \\
\hline 2037 & Sonchus palustris L. & Budapest & 2004 & Felföldy Lajos & BPU-13866.JPG \\
\hline 2037 & Sonchus palustris L. & Göd & 1938 & Kárpáti Zoltán & BPU-13865.JPG \\
\hline 2038 & Sonchus arvensis L. & Balatongyörök & 1961 & Felföldy Lajos & BPU-13853.JPG \\
\hline 2038 & Sonchus arvensis L. & Budapest & 1990 & Felföldy Lajos & BPU-13864.JPG \\
\hline 2038 & Sonchus arvensis L. & Diósjenő & 1952 & Simon Tibor & BPU-13860.JPG \\
\hline 2038 & Sonchus arvensis L. & Kiskunmajsa & 1954 & Soó Rezső & BPU-13863.JPG \\
\hline 2038 & Sonchus arvensis L. & Mosonmagyaróvár & 1939 & Siroki Zoltán & BPU-13861.JPG \\
\hline 2038 & Sonchus arvensis L. & Nyírbéltek & 1948 & Simon Tibor, Jakucs Pál & BPU-13857.JPG \\
\hline 2038 & Sonchus arvensis L. & Pákozd & 1980 & Felföldy Lajos & BPU-13862.JPG \\
\hline 2038 & Sonchus arvensis L. & Solymár & 1992 & Felföldy Lajos & BPU-13859.JPG \\
\hline 2038 & Sonchus arvensis L. & Tihany & 1959 & Felföldy Lajos & BPU-13854.JPG \\
\hline 2038 & Sonchus arvensis L. & Tihany & 1959 & Felföldy Lajos & BPU-13855.JPG \\
\hline 2038 & Sonchus arvensis L. & Tihany & 1959 & Felföldy Lajos & BPU-13856.JPG \\
\hline 2038 & Sonchus arvensis L. & Vácrátót & 1949 & Soó Rezső & BPU-13858.JPG \\
\hline 2039 & Lactuca perennis L. & Bélapátfalva & 1948 & $\begin{array}{l}\text { Pólya László, Simon } \\
\text { Tibor, Jakucs Pál }\end{array}$ & BPU-13844.JPG \\
\hline 2039 & Lactuca perennis L. & Budapest & 1947 & Soó Rezső & BPU-13842.JPG \\
\hline 2039 & Lactuca perennis L. & Miskolc & 1929 & Soó Rezső & BPU-13843.JPG \\
\hline 2039 & Lactuca perennis L. & Szin & 1951 & Jakucs Pál, Ér Lajos & BPU-13845.JPG \\
\hline 2040 & Lactuca viminea (L.) J. Presl et C. Presl & Budapest & 1947 & Soó Rezső & BPU-13839.JPG \\
\hline 2040 & Lactuca viminea (L.) J. Presl et C. Presl & Budapest & 1982 & Felföldy Lajos & BPU-13841.JPG \\
\hline 2040 & Lactuca viminea (L.) J. Presl et C. Presl & Csákvár & 1948 & Soó Rezső & BPU-13838.JPG \\
\hline
\end{tabular}


1. Elektroniukus melléklet - Electronic Appendix 1.

\begin{tabular}{|c|c|c|c|c|c|}
\hline $\begin{array}{l}\text { Sorszám / } \\
\text { Number }\end{array}$ & Taxon-név / Taxon-name & $\begin{array}{l}\text { Település / } \\
\text { Settlement }\end{array}$ & $\begin{array}{l}\text { Év / } \\
\text { Year }\end{array}$ & Gyűjtő / Collector & $\begin{array}{l}\text { Fájlnév / } \\
\text { File-name }\end{array}$ \\
\hline 2040 & Lactuca viminea (L.) J. Presl et C. Presl & Tihany & 1959 & Felföldy Lajos & BPU-13840.JPG \\
\hline 2041 & Lactuca quercina $\mathrm{L}$. & Bagamér & 1947 & Soó Rezső & BPU-13837.JPG \\
\hline 2041 & Lactuca quercina $\mathrm{L}$. & Budapest & 1947 & Soó Rezső & BPU-13834.JPG \\
\hline 2041 & Lactuca quercina $\mathrm{L}$. & Budapest & 1982 & Felföldy Lajos & BPU-13832.JPG \\
\hline 2041 & Lactuca quercina L. & Egyek & 1947 & Soó Rezső & BPU-13833.JPG \\
\hline 2041 & Lactuca quercina L. & Gyöngyös & 1961 & Soó Rezső & BPU-13830.JPG \\
\hline 2041 & Lactuca quercina $\mathrm{L}$. & Gyöngyös & 1961 & Soó Rezső & BPU-13831.JPG \\
\hline 2041 & Lactuca quercina $\mathrm{L}$. & Miskolc & 1937 & Soó Rezső & BPU-13835.JPG \\
\hline 2041 & Lactuca quercina $\mathrm{L}$. & Zebegény & 1947 & Soó Rezső & BPU-13836.JPG \\
\hline 2042 & Lactuca saligna L. & Szarvas & 1947 & Soó Rezső & BPU-13828.JPG \\
\hline 2043 & Lactuca serriola L. & Budapest & 1982 & Felföldy Lajos & BPU-13826.JPG \\
\hline 2043 & Lactuca serriola L. & Budapest & 1982 & Felföldy Lajos & BPU-13827.JPG \\
\hline 2043 & Lactuca serriola L. & Nyíregyháza & 1935 & Soó Rezső & BPU-13824.JPG \\
\hline 2043 & Lactuca serriola L. & Szarvas & 1947 & Soó Rezső & BPU-13823.JPG \\
\hline 2043 & Lactuca serriola L. & Tihany & 1959 & Felföldy Lajos & BPU-13825.JPG \\
\hline $\begin{array}{c}2042 \times \\
2043\end{array}$ & Lactuca saligna L. × Lactuca serriola L. & Budapest & 1944 & Kárpáti Zoltán & BPU-13822.JPG \\
\hline 2045 & Prenanthes purpurea L. & Alsószölnök & 1955 & Soó Rezső & BPU-13749.JPG \\
\hline 2045 & Prenanthes purpurea L. & Kemence & 1952 & Simon Tibor & BPU-13756.JPG \\
\hline 2045 & Prenanthes purpurea L. & Kőszeg & 1963 & Soó Rezső, Borsos Olga & BPU-13748.JPG \\
\hline 2045 & Prenanthes purpurea L. & Miskolc & 1937 & Soó Rezső & BPU-13751.JPG \\
\hline 2045 & Prenanthes purpurea L. & Szögliget & 1951 & Soó Rezső, Jakucs Pál & BPU-13752.JPG \\
\hline 2045 & Prenanthes purpurea L. & Tibolddaróc & 1953 & Soó Rezső & BPU-13750.JPG \\
\hline 2046 & Mycelis muralis (L.) Dumort. & Bélapátfalva & 1943 & $\begin{array}{l}\text { Simon Tibor, Pólya } \\
\text { László, Jakucs Pál }\end{array}$ & BPU-13875.JPG \\
\hline 2046 & Mycelis muralis (L.) Dumort. & Budapest & 1947 & Soó Rezső & BPU-13876.JPG \\
\hline 2046 & Mycelis muralis (L.) Dumort. & Budapest & 2004 & Felföldy Lajos & BPU-13877.JPG \\
\hline 2046 & Mycelis muralis (L.) Dumort. & Hosszúpályi & 1933 & Soó Rezső & BPU-13874.JPG \\
\hline 2046 & Mycelis muralis (L.) Dumort. & Nagykovácsi & 2005 & Felföldy Lajos & BPU-13873.JPG \\
\hline 2047 & $\begin{array}{l}\text { Taraxacum bessarabicum (Hornem.) } \\
\text { Hand.-Mazz. }\end{array}$ & Budapest & 1922 & Magyar Pál & BPU-13905.JPG \\
\hline 2047 & $\begin{array}{l}\text { Taraxacum bessarabicum (Hornem.) } \\
\text { Hand.-Mazz. }\end{array}$ & Győr & 1918 & Polgár Sándor & BPU-13908.JPG \\
\hline 2047 & $\begin{array}{l}\text { Taraxacum bessarabicum (Hornem.) } \\
\text { Hand.-Mazz. }\end{array}$ & Hajdúnánás & 1935 & Ujvárosi Miklós & BPU-13907.JPG \\
\hline 2047 & $\begin{array}{l}\text { Taraxacum bessarabicum (Hornem.) } \\
\text { Hand.-Mazz. }\end{array}$ & Lakitelek & 1961 & Simon Tibor & BPU-13906.JPG \\
\hline 2047 & $\begin{array}{l}\text { Taraxacum bessarabicum (Hornem.) } \\
\text { Hand.-Mazz. }\end{array}$ & Nyíradony & 1932 & Soó Rezső & BPU-13904.JPG \\
\hline 2048 & $\begin{array}{l}\text { Taraxacum serotinum (Waldst. et Kit.) } \\
\text { Poir. }\end{array}$ & Göd & 1948 & Soó Rezső & BPU-13910.JPG \\
\hline 2048 & $\begin{array}{l}\text { Taraxacum serotinum (Waldst. et Kit.) } \\
\text { Poir. }\end{array}$ & Pere & 1961 & Simon Tibor & BPU-13912.JPG \\
\hline 2048 & $\begin{array}{l}\text { Taraxacum serotinum (Waldst. et Kit.) } \\
\text { Poir. }\end{array}$ & Szár & 1943 & Kárpáti Zoltán & BPU-13911.JPG \\
\hline 2048 & $\begin{array}{l}\text { Taraxacum serotinum (Waldst. et Kit.) } \\
\text { Poir. }\end{array}$ & Tihany & 1959 & Felföldy Lajos & BPU-13909.JPG \\
\hline 2049 & Taraxacum laevigatum agg. & Balatonszőlős & 1959 & Felföldy Lajos & BPU-13901.JPG \\
\hline 2049 & Taraxacum laevigatum agg. & Balmazújváros & 1932 & Soó Rezső & BPU-13898.JPG \\
\hline 2049 & Taraxacum laevigatum agg. & Bisse & 1965 & $\begin{array}{l}\text { Simon Tibor, Priszter } \\
\text { Szaniszló, Borhidi Attila }\end{array}$ & BPU-13900.JPG \\
\hline 2049 & Taraxacum laevigatum agg. & Hidegkút & 1959 & Felföldy Lajos & BPU-13902.JPG \\
\hline
\end{tabular}


1. Elektroniukus melléklet - Electronic Appendix 1.

\begin{tabular}{|c|c|c|c|c|c|}
\hline $\begin{array}{l}\text { Sorszám / } \\
\text { Number }\end{array}$ & Taxon-név / Taxon-name & $\begin{array}{l}\text { Település / } \\
\text { Settlement }\end{array}$ & $\begin{array}{l}\text { Év / } \\
\text { Year }\end{array}$ & Gyüjtő / Collector & $\begin{array}{l}\text { Fájlnév / } \\
\text { File-name }\end{array}$ \\
\hline 2049 & Taraxacum laevigatum agg. & Nagykovácsi & 1949 & Soó Rezső & BPU-13896.JPG \\
\hline 2049 & Taraxacum laevigatum agg. & Tarcal & 1951 & Soó Rezső & BPU-13903.JPG \\
\hline 2049 & Taraxacum laevigatum agg. & Tihany & 1951 & Felföldy Lajos & BPU-13897.JPG \\
\hline 2050 & Taraxacum officinale agg. & Bagamér & 1949 & Soó Rezső & BPU-13893.JPG \\
\hline 2050 & Taraxacum officinale agg. & Pécs & 1953 & Soó Rezső, Borsos Olga & BPU-13892.JPG \\
\hline 2051 & Taraxacum palustre agg. & Bagamér & 1949 & Soó Rezső & BPU-13884.JPG \\
\hline 2051 & Taraxacum palustre agg. & Debrecen & 1932 & Soó Rezső & BPU-13883.JPG \\
\hline 2051 & Taraxacum palustre agg. & Kimle & 1962 & Borsos Olga & BPU-13887.JPG \\
\hline 2051 & Taraxacum palustre agg. & Örvényes & 1959 & Felföldy Lajos & BPU-13889.JPG \\
\hline 2051 & Taraxacum palustre agg. & Szalonna & 1966 & Felföldy Lajos & BPU-13888.JPG \\
\hline 2051 & Taraxacum palustre agg. & Tihany & 1946 & Felföldy Lajos & BPU-13890.JPG \\
\hline 2051 & Taraxacum palustre agg. & Vácrátót & 1950 & Soó Rezső & BPU-13885.JPG \\
\hline 2052 & Chondrilla juncea L. & Bagamér & 1932 & Soó Rezső & BPU-13915.JPG \\
\hline 2052 & Chondrilla juncea L. & Budapest & 1982 & Felföldy Lajos & BPU-13916.JPG \\
\hline 2052 & Chondrilla juncea L. & Kaszó & 1957 & Soó Rezső & BPU-13914.JPG \\
\hline 2053 & Lapsana communis L. & Budapest & 1982 & Felföldy Lajos & BPU-08926.JPG \\
\hline 2053 & Lapsana communis L. & Budapest & 1991 & Felföldy Lajos & BPU-08925.JPG \\
\hline 2053 & Lapsana communis L. & Csévharaszt & 1959 & Simon Tibor, Borsos Olga & BPU-08927.JPG \\
\hline 2053 & Lapsana communis L. & Hajdúhadház & 1933 & Soó Rezső & BPU-08931.JPG \\
\hline 2053 & Lapsana communis L. & Nagykovácsi & 2005 & Felföldy Lajos & BPU-08928.JPG \\
\hline 2053 & Lapsana communis L. & Szentendre & 1947 & Soó Rezső & BPU-08929.JPG \\
\hline 2053 & Lapsana communis L. & Vászoly & 1950 & Felföldy Lajos & BPU-08930.JPG \\
\hline 2054 & Crepis rhoeadifolia M. Bieb. & Budapest & 1982 & Felföldy Lajos & BPU-13821.JPG \\
\hline 2054 & Crepis rhoeadifolia M. Bieb. & Debrecen & 1931 & Soó Rezső & BPU-13819.JPG \\
\hline 2054 & Crepis rhoeadifolia M. Bieb. & Zámoly & 1982 & Felföldy Lajos & BPU-13820.JPG \\
\hline 2055 & Crepis taraxacifolia Thuill. & Nagykanizsa & 1949 & Károlyi Árpád & BPU-13769.JPG \\
\hline 2056 & Crepis setosa Haller & Aszaló & 1944 & Siroki Zoltán & BPU-13816.JPG \\
\hline 2056 & Crepis setosa Haller & Gelénes & 1951 & $\begin{array}{l}\text { Simon Tibor, Vozáry } \\
\text { Elemér }\end{array}$ & BPU-13817.JPG \\
\hline 2056 & Crepis setosa Haller & Szőce & 1955 & Soó Rezső & BPU-13818.JPG \\
\hline 2057 & Crepis paludosa (L.) Moench & Uzsa & 1950 & Felföldy Lajos & BPU-13801.JPG \\
\hline 2057 & Crepis paludosa (L.) Moench & Uzsa & 1959 & $\begin{array}{l}\text { Jávorka Sándor, Felföldy } \\
\text { Lajos }\end{array}$ & BPU-13802.JPG \\
\hline 2058 & Crepis praemorsa (L.) Walther & Bátorliget & 1932 & Soó Rezső & BPU-13815.JPG \\
\hline 2058 & Crepis praemorsa (L.) Walther & Budapest & 1947 & Soó Rezső & BPU-13811.JPG \\
\hline 2058 & Crepis praemorsa (L.) Walther & Gyöngyös & 1965 & Soó Rezső & BPU-13813.JPG \\
\hline 2058 & Crepis praemorsa (L.) Walther & Márianosztra & 1949 & Papp József & BPU-13812.JPG \\
\hline 2059 & Crepis pulchra L. & Budapest & 1922 & Soó Rezső & BPU-13773.JPG \\
\hline 2059 & Crepis pulchra L. & Miskolc & 1929 & Soó Rezső & BPU-13775.JPG \\
\hline 2059 & Crepis pulchra L. & Miskolc & 1937 & Soó Rezső & BPU-13775.JPG \\
\hline 2059 & Crepis pulchra L. & Nagyharsány & 1965 & Simon Tibor & BPU-13774.JPG \\
\hline 2060 & Crepis pannonica (Jacq.) K. Koch & Budapest & 1947 & Soó Rezső & BPU-13799.JPG \\
\hline 2060 & Crepis pannonica (Jacq.) K. Koch & Tokaj & 1938 & Soó Rezső & BPU-13800.JPG \\
\hline 2061 & Crepis tectorum $\mathrm{L}$. & Budapest & 1982 & Felföldy Lajos & BPU-13797.JPG \\
\hline 2061 & Crepis tectorum L. & Budapest & 1982 & Felföldy Lajos & BPU-13798.JPG \\
\hline 2061 & Crepis tectorum L. & Budapest & 2005 & Felföldy Lajos & BPU-13796.JPG \\
\hline 2061 & Crepis tectorum L. & Debrecen & 1931 & Soó Rezső & BPU-13794.JPG \\
\hline 2061 & Crepis tectorum L. & Kapolcs & 1959 & $\begin{array}{l}\text { Jávorka Sándor, Felföldy } \\
\text { Lajos, Tóth László }\end{array}$ & BPU-13792.JPG \\
\hline
\end{tabular}


1. Elektroniukus melléklet - Electronic Appendix 1.

\begin{tabular}{|c|c|c|c|c|c|}
\hline $\begin{array}{l}\text { Sorszám / } \\
\text { Number }\end{array}$ & Taxon-név / Taxon-name & $\begin{array}{l}\text { Település / } \\
\text { Settlement }\end{array}$ & $\begin{array}{l}\text { Év / } \\
\text { Year }\end{array}$ & Gyüjtő / Collector & $\begin{array}{l}\text { Fájlnév / } \\
\text { File-name }\end{array}$ \\
\hline 2061 & Crepis tectorum L. & Kapolcs & 1959 & $\begin{array}{l}\text { Jávorka Sándor, Felföldy } \\
\text { Lajos, Tóth László }\end{array}$ & BPU-13793.JPG \\
\hline 2061 & Crepis tectorum L. & Paks & 1961 & Simon Tibor & BPU-13795.JPG \\
\hline 2061 & Crepis tectorum L. & Tihany & 1942 & Felföldy Lajos & BPU-13791.JPG \\
\hline 2061 & Crepis tectorum L. & Uzsa & 1959 & $\begin{array}{l}\text { Jávorka Sándor, Felföldy } \\
\text { Lajos }\end{array}$ & BPU-13790.JPG \\
\hline 2062 & Crepis biennis L. & Aszófő & 1959 & Felföldy Lajos & BPU-13785.JPG \\
\hline 2062 & Crepis biennis L. & Balatongyörök & 1961 & Felföldy Lajos & BPU-13788.JPG \\
\hline 2062 & Crepis biennis L. & Bátorliget & 1934 & Soó Rezső & BPU-13784.JPG \\
\hline 2062 & Crepis biennis L. & Bicske & 1991 & Felföldy Lajos & BPU-13782.JPG \\
\hline 2062 & Crepis biennis L. & Bisse & 1965 & $\begin{array}{l}\text { Simon Tibor, Priszter } \\
\text { Szaniszló, Borhidi Attila }\end{array}$ & BPU-13780.JPG \\
\hline 2062 & Crepis biennis L. & Budapest & 1991 & Felföldy Lajos & BPU-13779.JPG \\
\hline 2062 & Crepis biennis L. & Budapest & 2005 & Felföldy Lajos & BPU-13778.JPG \\
\hline 2062 & Crepis biennis L. & Miskolc & 1948 & Simon Tibor & BPU-13781.JPG \\
\hline 2062 & Crepis biennis L. & Tihany & 1949 & Simon Tibor & BPU-13786.JPG \\
\hline 2062 & Crepis biennis L. & Tihany & 1959 & Felföldy Lajos & BPU-13787.JPG \\
\hline 2062 & Crepis biennis L. & Zámoly & 1982 & Felföldy Lajos & BPU-13783.JPG \\
\hline 2063 & Crepis nicaeensis Balb. & Balatonfüred & 1928 & Soó Rezső & BPU-13777.JPG \\
\hline 2063 & Crepis nicaeensis Balb. & Budapest & 1947 & Soó Rezső & BPU-13776.JPG \\
\hline 2064 & Crepis capillaris (L.) Wallr. & Kőszeg & 1932 & Soó Rezső & BPU-13771.JPG \\
\hline 2064 & Crepis capillaris (L.) Wallr. & Nagykanizsa & 1955 & Soó Rezső & BPU-13770.JPG \\
\hline 2064 & Crepis capillaris (L.) Wallr. & Sopron & 1948 & Kárpáti Zoltán & BPU-13772.JPG \\
\hline 2065 & Hieracium pilosella L. & Balatonboglár & 1941 & Kováts Ferenc & BPU-13705.JPG \\
\hline 2065 & Hieracium pilosella L. & Budapest & 1925 & Kováts Ferenc & BPU-13739.JPG \\
\hline 2065 & Hieracium pilosella L. & Budapest & 1934 & Kováts Ferenc & BPU-13704.JPG \\
\hline 2065 & Hieracium pilosella L. & Budapest & 1935 & Kováts Ferenc & BPU-13710.JPG \\
\hline 2065 & Hieracium pilosella L. & Budapest & 1935 & Kováts Ferenc & BPU-13738.JPG \\
\hline 2065 & Hieracium pilosella L. & Budapest & 1935 & Kováts Ferenc & BPU-13740.JPG \\
\hline 2065 & Hieracium pilosella L. & Budapest & 1937 & Kováts Ferenc & BPU-13702.JPG \\
\hline 2065 & Hieracium pilosella L. & Budapest & 1938 & Kováts Ferenc & BPU-13701.JPG \\
\hline 2065 & Hieracium pilosella L. & Budapest & 1938 & Kováts Ferenc & BPU-13708.JPG \\
\hline 2065 & Hieracium pilosella L. & Budapest & 1940 & Kováts Ferenc & BPU-13703.JPG \\
\hline 2065 & Hieracium pilosella L. & Budapest & 1943 & Kováts Ferenc & BPU-13700.JPG \\
\hline 2065 & Hieracium pilosella L. & Debrecen & 1931 & Soó Rezső & BPU-13728.JPG \\
\hline 2065 & Hieracium pilosella L. & Debrecen & 1933 & Juhász Lajos & BPU-13731.JPG \\
\hline 2065 & Hieracium pilosella L. & Debrecen & 1935 & Soó Rezső & BPU-13726.JPG \\
\hline 2065 & Hieracium pilosella L. & Debrecen & 1937 & Felföldy Lajos & BPU-13689.JPG \\
\hline 2065 & Hieracium pilosella L. & Debrecen & 1946 & Soó Rezső & BPU-13727.JPG \\
\hline 2065 & Hieracium pilosella L. & Gánt & 1948 & Soó Rezső & BPU-13735.JPG \\
\hline 2065 & Hieracium pilosella L. & Hajdúböszörmény & 1930 & Igmándy József & BPU-13711.JPG \\
\hline 2065 & Hieracium pilosella L. & Hajdúnánás & 1936 & Ujvárosi Miklós & BPU-13692.JPG \\
\hline 2065 & Hieracium pilosella L. & Hajdúnánás & 1948 & Igmándy József & BPU-13714.JPG \\
\hline 2065 & Hieracium pilosella L. & Hajdúnánás & 1948 & Igmándy József & BPU-13716.JPG \\
\hline 2065 & Hieracium pilosella L. & Háromhuta & 1937 & Igmándy József & BPU-13713.JPG \\
\hline 2065 & Hieracium pilosella L. & Jósvafő & 1948 & $\begin{array}{l}\text { Simon Tibor, Pólya } \\
\text { László, Böszörményi } \\
\text { Zoltán }\end{array}$ & BPU-13693.JPG \\
\hline 2065 & Hieracium pilosella L. & Karcag & 1936 & Oláh J. & BPU-13734.JPG \\
\hline
\end{tabular}


1. Elektroniukus melléklet - Electronic Appendix 1.

\begin{tabular}{|c|c|c|c|c|c|}
\hline $\begin{array}{l}\text { Sorszám / } \\
\text { Number }\end{array}$ & Taxon-név / Taxon-name & $\begin{array}{l}\text { Település / } \\
\text { Settlement }\end{array}$ & $\begin{array}{l}\text { Év / } \\
\text { Year }\end{array}$ & Gyưjtő / Collector & $\begin{array}{l}\text { Fájlnév / } \\
\text { File-name }\end{array}$ \\
\hline 2065 & Hieracium pilosella L. & Konyár & 1949 & Soó Rezső & BPU-13730.JPG \\
\hline 2065 & Hieracium pilosella L. & Kömörő & 1948 & $\begin{array}{l}\text { Pólya László, Simon } \\
\text { Tibor, Jakucs Pál }\end{array}$ & BPU-13694.JPG \\
\hline 2065 & Hieracium pilosella L. & Kömörő & 1948 & $\begin{array}{l}\text { Pólya László, Simon } \\
\text { Tibor, Jakucs Pál }\end{array}$ & BPU-13695.JPG \\
\hline 2065 & Hieracium pilosella L. & Mike & 1926 & Kováts Ferenc & BPU-13707.JPG \\
\hline 2065 & Hieracium pilosella L. & Misefa & 1935 & Kováts Ferenc & BPU-13706.JPG \\
\hline 2065 & Hieracium pilosella L. & Misefa & 1935 & Kováts Ferenc & BPU-13709.JPG \\
\hline 2065 & Hieracium pilosella L. & Mór & 1942 & Felföldy Lajos & BPU-13691.JPG \\
\hline 2065 & Hieracium pilosella L. & Nagyvisnyó & 1928 & Igmándy József & BPU-13712.JPG \\
\hline 2065 & Hieracium pilosella L. & Nyíracsád & 1931 & Soó Rezső & BPU-13732.JPG \\
\hline 2065 & Hieracium pilosella L. & Pécs & 1935 & Soó Rezső & BPU-13725.JPG \\
\hline 2065 & Hieracium pilosella L. & Pécs & 1935 & Soó Rezső & BPU-13745.JPG \\
\hline 2065 & Hieracium pilosella L. & Pusztamiske & 1948 & Borsos Olga & BPU-13697.JPG \\
\hline 2065 & Hieracium pilosella L. & Sárospatak & 1943 & Felföldy Lajos & BPU-13688.JPG \\
\hline 2065 & Hieracium pilosella L. & Szilvásvárad & 1949 & Koppányi Tibor & BPU-13699.JPG \\
\hline 2065 & Hieracium pilosella L. & Téglás & 1948 & Soó Rezső & BPU-13729.JPG \\
\hline 2065 & Hieracium pilosella L. & Tiborszállás & 1948 & Simon Tibor, Jakucs Pál & BPU-13696.JPG \\
\hline 2065 & Hieracium pilosella L. & Tihany & 1947 & Pólya László & BPU-13743.JPG \\
\hline 2065 & Hieracium pilosella L. & Tiszavasvári & 1948 & Igmándy József & BPU-13715.JPG \\
\hline 2066 & Hieracium macranthum Ten. & Budapest & 1934 & Kováts Ferenc & BPU-13747.JPG \\
\hline 2066 & Hieracium macranthum Ten. & Budapest & 1937 & Kováts Ferenc & BPU-13746.JPG \\
\hline 2066 & Hieracium macranthum Ten. & Budapest & 1992 & Felföldy Lajos & BPU-13744.JPG \\
\hline 2066 & Hieracium macranthum Ten. & Tihany & 1959 & Felföldy Lajos & BPU-13742.JPG \\
\hline 2066 & Hieracium macranthum Ten. & Tokaj & 1937 & Soó Rezső & BPU-13741.JPG \\
\hline 2068 & Hieracium lactucella Wallr. & Vámosatya & 1951 & Simon Tibor & BPU-13664.JPG \\
\hline 2069 & Hieracium echioides Lumn. & Bakonyszentlászló & 1963 & Soó Rezső & BPU-13505.JPG \\
\hline 2069 & Hieracium echioides Lumn. & Boldogkőváralja & 1938 & Soó Rezső & BPU-13512.JPG \\
\hline 2069 & Hieracium echioides Lumn. & Domony & 1992 & Felföldy Lajos & BPU-13508.JPG \\
\hline 2069 & Hieracium echioides Lumn. & Hajdúbagos & 1933 & Soó Rezső & BPU-13510.JPG \\
\hline 2069 & Hieracium echioides Lumn. & Lesenceistvánd & 1931 & Soó Rezső & BPU-13504.JPG \\
\hline 2069 & Hieracium echioides Lumn. & Nagyharsány & 1961 & Simon Tibor & BPU-13511.JPG \\
\hline 2069 & Hieracium echioides Lumn. & Nagykovácsi & 1933 & Kováts Ferenc & BPU-13507.JPG \\
\hline 2069 & Hieracium echioides Lumn. & Pilisvörösvár & 1933 & Kováts Ferenc & BPU-13506.JPG \\
\hline 2069 & Hieracium echioides Lumn. & Szarvaskő & 1948 & $\begin{array}{l}\text { Simon Tibor, Pólya } \\
\text { László, Jakucs Pál }\end{array}$ & BPU-13509.JPG \\
\hline 2069 & Hieracium echioides Lumn. & Tihany & 1943 & Felföldy Lajos & BPU-13503.JPG \\
\hline 2070 & Hieracium cymosum L. & Bátorliget & 1934 & Soó Rezső & BPU-13636.JPG \\
\hline 2070 & Hieracium cymosum L. & Budapest & 1935 & Kováts Ferenc & BPU-13534.JPG \\
\hline 2070 & Hieracium cymosum L. & Budapest & 1937 & Kováts Ferenc & BPU-13535.JPG \\
\hline 2070 & Hieracium cymosum L. & Hajdúszoboszló & 1947 & Simon Tibor & BPU-13530.JPG \\
\hline 2070 & Hieracium cymosum L. & Magyarlukafa & 1957 & Soó Rezső & BPU-13526.JPG \\
\hline 2070 & Hieracium cymosum L. & Nagyharsány & 1956 & Soó Rezső & BPU-13523.JPG \\
\hline 2070 & Hieracium cymosum L. & Nyíracsád & 1937 & Soó Rezső & BPU-13522.JPG \\
\hline 2070 & Hieracium cymosum L. & Pécs & 1935 & Soó Rezső & BPU-13525.JPG \\
\hline 2070 & Hieracium cymosum L. & Pécs & 1939 & Kováts Ferenc & BPU-13532.JPG \\
\hline 2070 & Hieracium cymosum L. & Pécs & 1939 & Kováts Ferenc & BPU-13533.JPG \\
\hline 2070 & Hieracium cymosum L. & Tákos & 1949 & Soó Rezső & BPU-13524.JPG \\
\hline 2070 & Hieracium cymosum L. & Tarcal & 1936 & Hulják János & BPU-13536.JPG \\
\hline
\end{tabular}


1. Elektroniukus melléklet - Electronic Appendix 1.

\begin{tabular}{|c|c|c|c|c|c|}
\hline $\begin{array}{l}\text { Sorszám / } \\
\text { Number }\end{array}$ & Taxon-név / Taxon-name & $\begin{array}{l}\text { Település / } \\
\text { Settlement }\end{array}$ & $\begin{array}{l}\text { Év / } \\
\text { Year }\end{array}$ & Gyújtő / Collector & $\begin{array}{l}\text { Fájlnév / } \\
\text { File-name }\end{array}$ \\
\hline 2070 & Hieracium cymosum L. & Tokaj & 1933 & Soó Rezső & BPU-13528.JPG \\
\hline 2071 & Hieracium caespitosum Dumort. & Bugyi & 1959 & Soó Rezső & BPU-13635.JPG \\
\hline 2071 & Hieracium caespitosum Dumort. & Nagyvisnyó & 1948 & $\begin{array}{l}\text { Simon Tibor, Borsos Olga, } \\
\text { Jakucs Pál }\end{array}$ & BPU-13639.JPG \\
\hline 2071 & Hieracium caespitosum Dumort. & Penészlek & 1932 & Soó Rezső & BPU-13637.JPG \\
\hline 2071 & Hieracium caespitosum Dumort. & Tarpa & 1948 & $\begin{array}{l}\text { Pólya László, Simon } \\
\text { Tibor, Jakucs Pál }\end{array}$ & BPU-13638.JPG \\
\hline 2072 & Hieracium piloselloides Vill. & Balatonboglár & 1940 & Kováts Ferenc & BPU-13516.JPG \\
\hline 2072 & Hieracium piloselloides Vill. & Balatonboglár & 1940 & Kováts Ferenc & BPU-13517.JPG \\
\hline 2072 & Hieracium piloselloides Vill. & Dömös & 1948 & Soó Rezső & BPU-13519.JPG \\
\hline 2073 & Hieracium bauhinii Schult. ex Besser & Balatonfüred & 1928 & Soó Rezső & BPU-13604.JPG \\
\hline 2073 & Hieracium bauhinii Schult. ex Besser & Bátorliget & 1934 & Soó Rezső & BPU-13619.JPG \\
\hline 2073 & Hieracium bauhinii Schult. ex Besser & Budapest & 1925 & Soó Rezső & BPU-13620.JPG \\
\hline 2073 & Hieracium bauhinii Schult. ex Besser & Budapest & 1929 & Kováts Ferenc & BPU-13593.JPG \\
\hline 2073 & Hieracium bauhinii Schult. ex Besser & Budapest & 1936 & Kováts Ferenc & BPU-13591.JPG \\
\hline 2073 & Hieracium bauhinii Schult. ex Besser & Budapest & 1947 & Soó Rezső & BPU-13607.JPG \\
\hline 2073 & Hieracium bauhinii Schult. ex Besser & Budapest & 1991 & Felföldy Lajos & BPU-13602.JPG \\
\hline 2073 & Hieracium bauhinii Schult. ex Besser & Budapest & - & Kováts Ferenc & BPU-13595.JPG \\
\hline 2073 & Hieracium bauhinii Schult. ex Besser & Csákvár & 1948 & Soó Rezső & BPU-13606.JPG \\
\hline 2073 & Hieracium bauhinii Schult. ex Besser & Csaroda & 1948 & $\begin{array}{l}\text { Pólya László, Simon } \\
\text { Tibor, Jakucs Pál }\end{array}$ & BPU-13577.JPG \\
\hline 2073 & Hieracium bauhinii Schult. ex Besser & Csobánka & 1938 & Kováts Ferenc & BPU-13594.JPG \\
\hline 2073 & Hieracium bauhinii Schult. ex Besser & Hajdúnánás & 1938 & Igmándy József & BPU-13600.JPG \\
\hline 2073 & Hieracium bauhinii Schult. ex Besser & Hajdúnánás & 1948 & Igmándy József & BPU-13597.JPG \\
\hline 2073 & Hieracium bauhinii Schult. ex Besser & Hajdúnánás & 1948 & Igmándy József & BPU-13601.JPG \\
\hline 2073 & Hieracium bauhinii Schult. ex Besser & Isztimér & 1949 & Soó Rezső & BPU-13605.JPG \\
\hline 2073 & Hieracium bauhinii Schult. ex Besser & Konyár & 1949 & Soó Rezső & BPU-13624.JPG \\
\hline 2073 & Hieracium bauhinii Schult. ex Besser & Misefa & 1934 & Kováts Ferenc & BPU-13585.JPG \\
\hline 2073 & Hieracium bauhinii Schult. ex Besser & Miskolc & 1938 & Soó Rezső & BPU-13612.JPG \\
\hline 2073 & Hieracium bauhinii Schult. ex Besser & Miskolc & 1939 & Soó Rezső & BPU-13611.JPG \\
\hline 2073 & Hieracium bauhinii Schult. ex Besser & Nagykanizsa & 1948 & Károlyi Árpád & BPU-13583.JPG \\
\hline 2073 & Hieracium bauhinii Schult. ex Besser & Nagykovácsi & 1932 & Soó Rezső & BPU-13608.JPG \\
\hline 2073 & Hieracium bauhinii Schult. ex Besser & Nyíregyháza & 1938 & Ubrizsy Gábor & BPU-13617.JPG \\
\hline 2073 & Hieracium bauhinii Schult. ex Besser & Orbányosfa & 1935 & Kováts Ferenc & BPU-13587.JPG \\
\hline 2073 & Hieracium bauhinii Schult. ex Besser & Pécs & 1935 & Soó Rezső & BPU-13603.JPG \\
\hline 2073 & Hieracium bauhinii Schult. ex Besser & Sáránd & - & anonim & BPU-13623.JPG \\
\hline 2073 & Hieracium bauhinii Schult. ex Besser & Sárospatak & 1940 & $\begin{array}{l}\text { Ubrizsy Gábor, Felföldy } \\
\text { Lajos, Szű́cs Lajos }\end{array}$ & BPU-13579.JPG \\
\hline 2073 & Hieracium bauhinii Schult. ex Besser & Sárospatak & 1943 & Felföldy Lajos & BPU-13582.JPG \\
\hline 2073 & Hieracium bauhinii Schult. ex Besser & Sárospatak & 1943 & Felföldy Lajos & BPU-13621.JPG \\
\hline 2073 & Hieracium bauhinii Schult. ex Besser & Somlóvásárhely & 1953 & $\begin{array}{l}\text { Kulcsár Gábor, Vozáry } \\
\text { Elemér }\end{array}$ & BPU-13618.JPG \\
\hline 2073 & Hieracium bauhinii Schult. ex Besser & Szarvas & 1949 & Soó Rezső & BPU-13625.JPG \\
\hline 2073 & Hieracium bauhinii Schult. ex Besser & Tákos & 1948 & $\begin{array}{l}\text { Pólya László, Simon } \\
\text { Tibor, Jakucs Pál }\end{array}$ & BPU-13559.JPG \\
\hline 2073 & Hieracium bauhinii Schult. ex Besser & Tákos & 1948 & $\begin{array}{l}\text { Pólya László, Simon } \\
\text { Tibor, Jakucs Pál }\end{array}$ & BPU-13576.JPG \\
\hline 2073 & Hieracium bauhinii Schult. ex Besser & Tállya & 1938 & Soó Rezső & BPU-13614.JPG \\
\hline 2073 & Hieracium bauhinii Schult. ex Besser & Tarcal & 1933 & Soó Rezső & BPU-13613.JPG \\
\hline 2073 & Hieracium bauhinii Schult. ex Besser & Tarcal & 1937 & Hulják János & BPU-13584.JPG \\
\hline
\end{tabular}


1. Elektroniukus melléklet - Electronic Appendix 1.

\begin{tabular}{|c|c|c|c|c|c|}
\hline $\begin{array}{l}\text { Sorszám / } \\
\text { Number }\end{array}$ & Taxon-név / Taxon-name & $\begin{array}{l}\text { Település / } \\
\text { Settlement }\end{array}$ & $\begin{array}{l}\text { Év / } \\
\text { Year }\end{array}$ & Gyújtő / Collector & $\begin{array}{l}\text { Fájlnév / } \\
\text { File-name }\end{array}$ \\
\hline 2073 & Hieracium bauhinii Schult. ex Besser & Tarcal & 1937 & Hulják János & BPU-13586.JPG \\
\hline 2073 & Hieracium bauhinii Schult. ex Besser & Tarcal & 1937 & Hulják János & BPU-13588.JPG \\
\hline 2073 & Hieracium bauhinii Schult. ex Besser & Tarcal & 1937 & Hulják János & BPU-13590.JPG \\
\hline 2073 & Hieracium bauhinii Schult. ex Besser & Tarcal & 1937 & Hulják János & BPU-13592.JPG \\
\hline 2073 & Hieracium bauhinii Schult. ex Besser & Tarpa & 1948 & $\begin{array}{l}\text { Pólya László, Simon } \\
\text { Tibor, Jakucs Pál }\end{array}$ & BPU-13575.JPG \\
\hline 2073 & Hieracium bauhinii Schult. ex Besser & Tarpa & 1948 & Simon Tibor, Jakucs Pál & BPU-13574.JPG \\
\hline 2073 & Hieracium bauhinii Schult. ex Besser & Tarpa & 1948 & Simon Tibor, Jakucs Pál & BPU-13578.JPG \\
\hline 2073 & Hieracium bauhinii Schult. ex Besser & Tarpa & 1949 & Soó Rezső & BPU-13622.JPG \\
\hline 2073 & Hieracium bauhinii Schult. ex Besser & Tarpa & 1949 & Soó Rezső & BPU-13626.JPG \\
\hline 2073 & Hieracium bauhinii Schult. ex Besser & Telkibánya & 1937 & Hulják János & BPU-13589.JPG \\
\hline 2073 & Hieracium bauhinii Schult. ex Besser & Tihany & 1942 & Felföldy Lajos & BPU-13581.JPG \\
\hline 2073 & Hieracium bauhinii Schult. ex Besser & Tiszavasvári & 1949 & Igmándy József & BPU-13598.JPG \\
\hline 2073 & Hieracium bauhinii Schult. ex Besser & Tiszavasvári & 1949 & Igmándy József & BPU-13599.JPG \\
\hline 2073 & Hieracium bauhinii Schult. ex Besser & Vámosatya & 1951 & Simon Tibor & BPU-13573.JPG \\
\hline 2075 & Hieracium bupleuroides C.C. Gmel. & Bélapátfalva & 1953 & Simon Tibor & BPU-13493.JPG \\
\hline 2075 & Hieracium bupleuroides C.C. Gmel. & Miskolc & 1950 & Soó Rezső, Simon Tibor & BPU-13492.JPG \\
\hline 2076 & Hieracium schmidtii Tausch & Fenyőfö & 1955 & Soó Rezső & BPU-13490.JPG \\
\hline 2077 & Hieracium bifidum Kit. & Budapest & 1936 & Kováts Ferenc & BPU-10835.JPG \\
\hline 2077 & Hieracium bifidum Kit. & Budapest & 1937 & Kováts Ferenc & BPU-10834.JPG \\
\hline 2077 & Hieracium bifidum Kit. & Budapest & 1940 & Kováts Ferenc & BPU-10833.JPG \\
\hline 2077 & Hieracium bifidum Kit. & Budapest & 1941 & Kováts Ferenc & BPU-10832.JPG \\
\hline 2077 & Hieracium bifidum Kit. & Budapest & 1947 & Soó Rezső & BPU-10831.JPG \\
\hline 2077 & Hieracium bifidum Kit. & Erdőbénye & 1948 & Soó Rezső & BPU-10841.JPG \\
\hline 2077 & Hieracium bifidum Kit. & Szilvásvárad & 1949 & Koppányi Tibor & BPU-10838.JPG \\
\hline 2078 & Hieracium murorum L. & Balatonfüred & 1942 & Felföldy Lajos & BPU-10780.JPG \\
\hline 2078 & Hieracium murorum L. & Balatonfüred & 1942 & Felföldy Lajos & BPU-10781.JPG \\
\hline 2078 & Hieracium murorum L. & Budakeszi & 1940 & Kováts Ferenc & BPU-13454.JPG \\
\hline 2078 & Hieracium murorum L. & Budakeszi & 1942 & Kováts Ferenc & BPU-13461.JPG \\
\hline 2078 & Hieracium murorum L. & Budapest & 1936 & Kováts Ferenc & BPU-13455.JPG \\
\hline 2078 & Hieracium murorum L. & Budapest & 1937 & Kováts Ferenc & BPU-13456.JPG \\
\hline 2078 & Hieracium murorum L. & Budapest & 1937 & Kováts Ferenc & BPU-13462.JPG \\
\hline 2078 & Hieracium murorum L. & Budapest & 1938 & Kováts Ferenc & BPU-13463.JPG \\
\hline 2078 & Hieracium murorum L. & Budapest & 1940 & Kováts Ferenc & BPU-10768.JPG \\
\hline 2078 & Hieracium murorum L. & Budapest & 1940 & Kováts Ferenc & BPU-13457.JPG \\
\hline 2078 & Hieracium murorum L. & Budapest & 1940 & Kováts Ferenc & BPU-13464.JPG \\
\hline 2078 & Hieracium murorum L. & Budapest & 1942 & Kováts Ferenc & BPU-10769.JPG \\
\hline 2078 & Hieracium murorum L. & Budapest & 1947 & Kováts Ferenc & BPU-13453.JPG \\
\hline 2078 & Hieracium murorum L. & Budapest & 1947 & Soó Rezső & BPU-13473.JPG \\
\hline 2078 & Hieracium murorum L. & Dömös & 1948 & Soó Rezső & BPU-13489.JPG \\
\hline 2078 & Hieracium murorum L. & Dömös & 1952 & Soó Rezső & BPU-13467.JPG \\
\hline 2078 & Hieracium murorum L. & Dömös & 1952 & Soó Rezső & BPU-15419.JPG \\
\hline 2078 & Hieracium murorum L. & Dömös & 1952 & Soó Rezső & BPU-15420.JPG \\
\hline 2078 & Hieracium murorum L. & Erdőbénye & 1948 & Soó Rezső & BPU-13478.JPG \\
\hline 2078 & Hieracium murorum L. & Kemence & 1952 & Simon Tibor & BPU-10778.JPG \\
\hline 2078 & Hieracium murorum L. & Kőszeg & 1938 & Soó Rezső & BPU-13471.JPG \\
\hline 2078 & Hieracium murorum L. & Misefa & 1934 & Kováts Ferenc & BPU-10774.JPG \\
\hline 2078 & Hieracium murorum L. & Misefa & 1935 & Kováts Ferenc & BPU-10773.JPG \\
\hline
\end{tabular}


1. Elektroniukus melléklet - Electronic Appendix 1.

\begin{tabular}{|c|c|c|c|c|c|}
\hline $\begin{array}{l}\text { Sorszám / } \\
\text { Number }\end{array}$ & Taxon-név / Taxon-name & $\begin{array}{l}\text { Település / } \\
\text { Settlement }\end{array}$ & $\begin{array}{l}\text { Év / } \\
\text { Year }\end{array}$ & Gyüjtő / Collector & $\begin{array}{l}\text { Fájlnév / } \\
\text { File-name }\end{array}$ \\
\hline 2078 & Hieracium murorum L. & Misefa & 1935 & Kováts Ferenc & BPU-13458.JPG \\
\hline 2078 & Hieracium murorum L. & Misefa & 1935 & Kováts Ferenc & BPU-13459.JPG \\
\hline 2078 & Hieracium murorum L. & Miskolc & 1936 & Soó Rezső & BPU-13484.JPG \\
\hline 2078 & Hieracium murorum L. & Miskolc & 1938 & Soó Rezső & BPU-13476.JPG \\
\hline 2078 & Hieracium murorum L. & Miskolc & 1939 & Soó Rezső & BPU-13475.JPG \\
\hline 2078 & Hieracium murorum L. & Monoszló & 1938 & Ubrizsy Gábor & BPU-10775.JPG \\
\hline 2078 & Hieracium murorum L. & Nagykapornak & 1938 & Kováts Ferenc & BPU-10772.JPG \\
\hline 2078 & Hieracium murorum L. & Nagykapornak & 1942 & Kováts Ferenc & BPU-13460.JPG \\
\hline 2078 & Hieracium murorum L. & Nagyvisnyó & 1953 & Vozáry Elemér & BPU-10776.JPG \\
\hline 2078 & Hieracium murorum L. & Orbányosfa & 1935 & Kováts Ferenc & BPU-10771.JPG \\
\hline 2078 & Hieracium murorum L. & Pécs & 1936 & Soó Rezső & BPU-13472.JPG \\
\hline 2078 & Hieracium murorum L. & Pécsvárad & 1936 & Soó Rezső & BPU-10785.JPG \\
\hline 2078 & Hieracium murorum L. & Pilisszentkereszt & 1938 & Kováts Ferenc & BPU-10770.JPG \\
\hline 2078 & Hieracium murorum L. & Sárospatak & 1943 & Felföldy Lajos & BPU-10779.JPG \\
\hline 2078 & Hieracium murorum L. & Sopron & 1937 & Soó Rezső & BPU-13469.JPG \\
\hline 2078 & Hieracium murorum L. & Sopron & 1938 & Soó Rezső & BPU-13468.JPG \\
\hline 2078 & Hieracium murorum L. & Szakonyfalu & 1955 & Soó Rezső & BPU-10786.JPG \\
\hline 2078 & Hieracium murorum L. & Szakonyfalu & 1955 & Soó Rezső & BPU-13470.JPG \\
\hline 2078 & Hieracium murorum L. & Tállya & 1936 & Hulják János & BPU-13465.JPG \\
\hline 2078 & Hieracium murorum L. & Vácrátót & 1949 & Soó Rezső & BPU-13466.JPG \\
\hline 2078 & Hieracium murorum L. & Zebegény & 1947 & Soó Rezső & BPU-13474.JPG \\
\hline 2078 & Hieracium murorum L. & Zebegény & 1950 & Simon Tibor, Borsos Olga & BPU-10777.JPG \\
\hline 2079 & Hieracium lachenalii C.C. Gmel. & Bakonybél & 1955 & Soó Rezső & BPU-10803.JPG \\
\hline 2079 & Hieracium lachenalii C.C. Gmel. & Balatonfüred & 1947 & Felföldy Lajos & BPU-10819.JPG \\
\hline 2079 & Hieracium lachenalii C.C. Gmel. & Budapest & 1936 & Kováts Ferenc & BPU-10815.JPG \\
\hline 2079 & Hieracium lachenalii C.C. Gmel. & Budapest & 1937 & Kováts Ferenc & BPU-10809.JPG \\
\hline 2079 & Hieracium lachenalii C.C. Gmel. & Budapest & 1937 & Kováts Ferenc & BPU-10813.JPG \\
\hline 2079 & Hieracium lachenalii C.C. Gmel. & Budapest & 1947 & Kováts Ferenc & BPU-10812.JPG \\
\hline 2079 & Hieracium lachenalii C.C. Gmel. & Budapest & 1947 & Soó Rezső & BPU-10802.JPG \\
\hline 2079 & Hieracium lachenalii C.C. Gmel. & Bükkszentkereszt & 1937 & Soó Rezső & BPU-10825.JPG \\
\hline 2079 & Hieracium lachenalii C.C. Gmel. & Fót & 1960 & Soó Rezső & BPU-10801.JPG \\
\hline 2079 & Hieracium lachenalii C.C. Gmel. & Gyöngyös & 1961 & Soó Rezső & BPU-10800.JPG \\
\hline 2079 & Hieracium lachenalii C.C. Gmel. & Gyöngyös & 1961 & Soó Rezső & BPU-10806.JPG \\
\hline 2079 & Hieracium lachenalii C.C. Gmel. & Gyöngyös & 1962 & Soó Rezső, Borsos Olga & BPU-10799.JPG \\
\hline 2079 & Hieracium lachenalii C.C. Gmel. & Hajmáskér & 1932 & Kováts Ferenc & BPU-10816.JPG \\
\hline 2079 & Hieracium lachenalii C.C. Gmel. & Jósvafó & 1948 & $\begin{array}{l}\text { Simon Tibor, Pólya } \\
\text { László, Böszörményi } \\
\text { Zoltán }\end{array}$ & BPU-10824.JPG \\
\hline 2079 & Hieracium lachenalii C.C. Gmel. & Jósvafő & 1951 & Soó Rezső & BPU-10796.JPG \\
\hline 2079 & Hieracium lachenalii C.C. Gmel. & Misefa & 1935 & Kováts Ferenc & BPU-10808.JPG \\
\hline 2079 & Hieracium lachenalii C.C. Gmel. & Misefa & 1935 & Kováts Ferenc & BPU-10817.JPG \\
\hline 2079 & Hieracium lachenalii C.C. Gmel. & Misefa & 1937 & Kováts Ferenc & BPU-10811.JPG \\
\hline 2079 & Hieracium lachenalii C.C. Gmel. & Misefa & 1937 & Kováts Ferenc & BPU-10814.JPG \\
\hline 2079 & Hieracium lachenalii C.C. Gmel. & Misefa & 1938 & Kováts Ferenc & BPU-10810.JPG \\
\hline 2079 & Hieracium lachenalii C.C. Gmel. & Miskolc & 1929 & Soó Rezső & BPU-10798.JPG \\
\hline 2079 & Hieracium lachenalii C.C. Gmel. & Nagykovácsi & 1946 & Vajda László & BPU-10818.JPG \\
\hline 2079 & Hieracium lachenalii C.C. Gmel. & Nagyvisnyó & 1948 & $\begin{array}{l}\text { Simon Tibor, Pólya } \\
\text { László, Jakucs Pál }\end{array}$ & BPU-10823.JPG \\
\hline
\end{tabular}


1. Elektroniukus melléklet - Electronic Appendix 1.

\begin{tabular}{|c|c|c|c|c|c|}
\hline $\begin{array}{l}\text { Sorszám / } \\
\text { Number }\end{array}$ & Taxon-név / Taxon-name & $\begin{array}{l}\text { Település / } \\
\text { Settlement }\end{array}$ & $\begin{array}{l}\text { Év / } \\
\text { Year }\end{array}$ & Gyüjtő / Collector & $\begin{array}{l}\text { Fájlnév / } \\
\text { File-name }\end{array}$ \\
\hline 2079 & Hieracium lachenalii C.C. Gmel. & Pécsely & 1950 & Felföldy Lajos & BPU-10820.JPG \\
\hline 2079 & Hieracium lachenalii C.C. Gmel. & Pécsely & 1950 & Felföldy Lajos & BPU-10821.JPG \\
\hline 2079 & Hieracium lachenalii C.C. Gmel. & Szalonna & 1948 & $\begin{array}{l}\text { Böszörményi, Simon } \\
\text { Tibor, Jakucs Pál }\end{array}$ & BPU-10822.JPG \\
\hline 2079 & Hieracium lachenalii C.C. Gmel. & Szőce & 1955 & Soó Rezső & BPU-10807.JPG \\
\hline 2079 & Hieracium lachenalii C.C. Gmel. & Szögliget & 1951 & $\begin{array}{l}\text { Soó Rezső, Jakucs Pál, Ér } \\
\text { Lajos }\end{array}$ & BPU-10797.JPG \\
\hline 2079 & Hieracium lachenalii C.C. Gmel. & Telkibánya & 1950 & Soó Rezső & BPU-10794.JPG \\
\hline 2080 & Hieracium laevigatum Willd. & Jósvafő & 1951 & $\begin{array}{l}\text { Soó Rezső, Jakucs Pál, Ér } \\
\text { Lajos }\end{array}$ & BPU-10854.JPG \\
\hline 2080 & Hieracium laevigatum Willd. & Szakonyfalu & 1955 & Soó Rezső & BPU-10855.JPG \\
\hline 2080 & Hieracium laevigatum Willd. & Szakonyfalu & 1955 & Soó Rezső & BPU-10856.JPG \\
\hline 2081 & Hieracium umbellatum L. & Beregdaróc & 1951 & $\begin{array}{l}\text { Simon Tibor, Vozáry } \\
\text { Elemér }\end{array}$ & BPU-10870.JPG \\
\hline 2081 & Hieracium umbellatum L. & Budapest & 1947 & Soó Rezső & BPU-10860.JPG \\
\hline 2081 & Hieracium umbellatum L. & Debrecen & 1932 & Máthé Imre & BPU-10862.JPG \\
\hline 2081 & Hieracium umbellatum L. & Erdőbénye & 1948 & Soó Rezső & BPU-10858.JPG \\
\hline 2081 & Hieracium umbellatum L. & Göd & 1948 & Soó Rezső & BPU-10864.JPG \\
\hline 2081 & Hieracium umbellatum L. & Hajdúhadház & 1933 & Soó Rezső & BPU-10863.JPG \\
\hline 2081 & Hieracium umbellatum L. & Kerecsend & 1957 & Soó Rezső & BPU-10859.JPG \\
\hline 2081 & Hieracium umbellatum L. & Mátraszentimre & 1943 & Kováts Ferenc & BPU-10865.JPG \\
\hline 2081 & Hieracium umbellatum L. & Sáránd & 1942 & Soó Rezső & BPU-10861.JPG \\
\hline 2081 & Hieracium umbellatum L. & Sáránd & 1947 & Simon Tibor & BPU-10868.JPG \\
\hline 2081 & Hieracium umbellatum L. & Tarpa & 1948 & Simon Tibor, Jakucs Pál & BPU-10867.JPG \\
\hline 2081 & Hieracium umbellatum L. & Újszentmargita & 1942 & Máthé Imre & BPU-10869.JPG \\
\hline 2082 & Hieracium sabaudum L. & Budakeszi & 1947 & Soó Rezső & BPU-10879.JPG \\
\hline 2082 & Hieracium sabaudum L. & Budapest & 1934 & Kováts Ferenc & BPU-10880.JPG \\
\hline 2082 & Hieracium sabaudum L. & Budapest & 1934 & Kováts Ferenc & BPU-10881.JPG \\
\hline 2082 & Hieracium sabaudum L. & Budapest & 1934 & Kováts Ferenc & BPU-10883.JPG \\
\hline 2082 & Hieracium sabaudum L. & Budapest & 1934 & Kováts Ferenc & BPU-10884.JPG \\
\hline 2082 & Hieracium sabaudum L. & Budapest & 1940 & Kováts Ferenc & BPU-10882.JPG \\
\hline 2082 & Hieracium sabaudum L. & Budapest & 1940 & Kováts Ferenc & BPU-10885.JPG \\
\hline 2082 & Hieracium sabaudum L. & Budapest & 1940 & Kováts Ferenc & BPU-10886.JPG \\
\hline 2082 & Hieracium sabaudum L. & Budapest & 1940 & Kováts Ferenc & BPU-10887.JPG \\
\hline 2082 & Hieracium sabaudum L. & Eger & 1948 & Koppányi Tibor & BPU-10888.JPG \\
\hline 2082 & Hieracium sabaudum L. & Magyarlukafa & 1957 & Soó Rezső & BPU-10874.JPG \\
\hline 2082 & Hieracium sabaudum L. & Somoskőújfalu & 1937 & Máthé Imre & BPU-10891.JPG \\
\hline 2082 & Hieracium sabaudum L. & Szögliget & 1951 & $\begin{array}{l}\text { Soó Rezső, Jakucs Pál, Ér } \\
\text { Lajos }\end{array}$ & BPU-10877.JPG \\
\hline 2082 & Hieracium sabaudum L. & Vácrátót & 1949 & Soó Rezső & BPU-10875.JPG \\
\hline 2082 & Hieracium sabaudum L. & Zebegény & 1947 & Soó Rezső & BPU-10876.JPG \\
\hline 2082 & Hieracium sabaudum L. & Zebegény & 1950 & Simon Tibor, Borsos Olga & BPU-10889.JPG \\
\hline 2083 & Hieracium racemosum Waldst. et Kit. & Budapest & 1992 & Felföldy Lajos & BPU-10896.JPG \\
\hline 2083 & Hieracium racemosum Waldst. et Kit. & Csákvár & 1948 & Soó Rezső & BPU-10898.JPG \\
\hline 2083 & Hieracium racemosum Waldst. et Kit. & Diósjenő & 1949 & Pólya László & BPU-10902.JPG \\
\hline 2083 & Hieracium racemosum Waldst. et Kit. & Dömös & 1948 & Soó Rezső & BPU-10894.JPG \\
\hline 2083 & Hieracium racemosum Waldst. et Kit. & Dömös & 1948 & Soó Rezső & BPU-10897.JPG \\
\hline 2083 & Hieracium racemosum Waldst. et Kit. & Gánt & 1948 & Soó Rezső & BPU-10899.JPG \\
\hline 2083 & Hieracium racemosum Waldst. et Kit. & Mátraszentimre & 1943 & Kováts Ferenc & BPU-10900.JPG \\
\hline 2083 & Hieracium racemosum Waldst. et Kit. & Mátraszentimre & 1943 & Kováts Ferenc & BPU-10901.JPG \\
\hline
\end{tabular}


1. Elektroniukus melléklet - Electronic Appendix 1.

\begin{tabular}{|c|c|c|c|c|c|}
\hline $\begin{array}{l}\text { Sorszám / } \\
\text { Number }\end{array}$ & Taxon-név / Taxon-name & $\begin{array}{l}\text { Település / } \\
\text { Settlement }\end{array}$ & $\begin{array}{l}\text { Év / } \\
\text { Year }\end{array}$ & Gyújtő / Collector & $\begin{array}{l}\text { Fájlnév / } \\
\text { File-name }\end{array}$ \\
\hline 2083 & Hieracium racemosum Waldst. et Kit. & Nagybajom & 1955 & Soó Rezső & BPU-10905.JPG \\
\hline 2083 & Hieracium racemosum Waldst. et Kit. & Nagykapornak & 1942 & Kováts Ferenc & BPU-10906.JPG \\
\hline 2083 & Hieracium racemosum Waldst. et Kit. & Sárospatak & 1949 & Simon Tibor, Jakucs Pál & BPU-10907.JPG \\
\hline 2083 & Hieracium racemosum Waldst. et Kit. & Szenna & 1957 & Soó Rezső & BPU-10904.JPG \\
\hline 2083 & Hieracium racemosum Waldst. et Kit. & Szentendre & 1963 & Soó Rezső & BPU-10903.JPG \\
\hline 2084 & $\begin{array}{l}\text { Hieracium } \times \text { hypereuryum Nägeli et } \\
\text { Peter }\end{array}$ & Szarvas & 1949 & Soó Rezső & BPU-13669.JPG \\
\hline 2086 & Hieracium $\times$ schultesii F.W. Schultz & Misefa & 1938 & Kováts Ferenc & BPU-13662.JPG \\
\hline 2086 & Hieracium $\times$ schultesii F.W. Schultz & Pálháza & 1948 & Vajda László & BPU-13661.JPG \\
\hline 2087 & Hieracium $\times$ bifurcum M. Bieb. & Budapest & 1936 & Kováts Ferenc & BPU-13660.JPG \\
\hline 2087 & Hieracium $\times$ bifurcum M. Bieb. & Budapest & 1945 & Kováts Ferenc & BPU-13659.JPG \\
\hline 2087 & Hieracium $\times$ bifurcum M. Bieb. & Hajdúnánás & 1935 & Ujvárosi Miklós & BPU-13658.JPG \\
\hline 2089 & Hieracium $\times$ laschii Zahn & Budapest & 1937 & Kováts Ferenc & BPU-13656.JPG \\
\hline 2089 & Hieracium $\times$ laschii Zahn & Budapest & 1943 & Kováts Ferenc & BPU-13657.JPG \\
\hline 2090 & Hieracium $\times$ brachiatum Bertol. ex DC. & Budapest & 1935 & Kováts Ferenc & BPU-13651.JPG \\
\hline 2090 & Hieracium $\times$ brachiatum Bertol. ex DC. & Budapest & 1935 & Kováts Ferenc & BPU-13721.JPG \\
\hline 2090 & Hieracium $\times$ brachiatum Bertol. ex DC. & Budapest & 1937 & Kováts Ferenc & BPU-13648.JPG \\
\hline 2090 & Hieracium $\times$ brachiatum Bertol. ex DC. & Budapest & 1937 & Kováts Ferenc & BPU-13654.JPG \\
\hline 2090 & Hieracium $\times$ brachiatum Bertol. ex DC. & Budapest & 1937 & Kováts Ferenc & BPU-13655.JPG \\
\hline 2090 & Hieracium $\times$ brachiatum Bertol. ex DC. & Budapest & 1937 & Kováts Ferenc & BPU-13723.JPG \\
\hline 2090 & Hieracium $\times$ brachiatum Bertol. ex DC. & Budapest & 1938 & Kováts Ferenc & BPU-13646.JPG \\
\hline 2090 & Hieracium $\times$ brachiatum Bertol. ex DC. & Budapest & 1940 & Kováts Ferenc & BPU-13647.JPG \\
\hline 2090 & Hieracium $\times$ brachiatum Bertol. ex DC. & Budapest & 1940 & Kováts Ferenc & BPU-13650.JPG \\
\hline 2090 & Hieracium $\times$ brachiatum Bertol. ex DC. & Budapest & 1942 & Kováts Ferenc & BPU-13652.JPG \\
\hline 2090 & Hieracium $\times$ brachiatum Bertol. ex DC. & Budapest & 1958 & Kováts Ferenc & BPU-13653.JPG \\
\hline 2090 & Hieracium $\times$ brachiatum Bertol. ex DC. & Misefa & 1935 & Kováts Ferenc & BPU-13724.JPG \\
\hline 2090 & Hieracium $\times$ brachiatum Bertol. ex DC. & Misefa & 1937 & Kováts Ferenc & BPU-13722.JPG \\
\hline 2090 & Hieracium $\times$ brachiatum Bertol. ex DC. & Miskolc & 1911 & Budai József & BPU-13645.JPG \\
\hline 2090 & Hieracium $\times$ brachiatum Bertol. ex DC. & Nagykapornak & 1940 & Kováts Ferenc & BPU-13720.JPG \\
\hline 2090 & Hieracium $\times$ brachiatum Bertol. ex DC. & Nagykapornak & 1941 & Kováts Ferenc & BPU-13649.JPG \\
\hline 2090 & Hieracium $\times$ brachiatum Bertol. ex DC. & Tákos & 1948 & $\begin{array}{l}\text { Pólya László, Jakucs Pál, } \\
\text { Simon Tibor }\end{array}$ & BPU-13642.JPG \\
\hline 2090 & Hieracium $\times$ brachiatum Bertol. ex DC. & Tarcal & 1937 & Hulják János & BPU-13643.JPG \\
\hline 2090 & Hieracium $\times$ brachiatum Bertol. ex DC. & Tihany & 1946 & Felföldy Lajos & BPU-13644.JPG \\
\hline 2090 & Hieracium $\times$ brachiatum Bertol. ex DC. & Tiszavasvári & 1949 & Igmándy József & BPU-13717.JPG \\
\hline 2090 & Hieracium $\times$ brachiatum Bertol. ex DC. & Tiszavasvári & 1949 & Igmándy József & BPU-13718.JPG \\
\hline 2090 & Hieracium $\times$ brachiatum Bertol. ex DC. & Tiszavasvári & 1949 & Igmándy József & BPU-13719.JPG \\
\hline 2091 & $\begin{array}{l}\text { Hieracium } \times \text { euchaetium Nägeli et } \\
\text { Peter }\end{array}$ & Budapest & 1938 & Kováts Ferenc & BPU-13498.JPG \\
\hline 2091 & $\begin{array}{l}\text { Hieracium } \times \text { euchaetium Nägeli et } \\
\text { Peter }\end{array}$ & Budapest & 1947 & Kováts Ferenc & BPU-13497.JPG \\
\hline 2092 & Hieracium $\times$ rothianum Wallr. & Budaörs & 1948 & Kováts Ferenc & BPU-13501.JPG \\
\hline 2092 & Hieracium $\times$ rothianum Wallr. & Ordacsehi & 1936 & Kováts Ferenc & BPU-13499.JPG \\
\hline 2092 & Hieracium $\times$ rothianum Wallr. & Pilisvörösvár & 1933 & Kováts Ferenc & BPU-13500.JPG \\
\hline 2093 & Hieracium $\times$ fallacinum F.W. Schultz & Budapest & 1936 & Kováts Ferenc & BPU-13521.JPG \\
\hline 2093 & Hieracium $\times$ fallacinum F.W. Schultz & Budapest & 1941 & Kováts Ferenc & BPU-13520.JPG \\
\hline 2094 & Hieracium $\times$ flagellare Willd. & Mátraszentimre & 1942 & Kováts Ferenc & BPU-13633.JPG \\
\hline 2094 & Hieracium $\times$ flagellare Willd. & Pilisszentkereszt & 1938 & Kováts Ferenc & BPU-13632.JPG \\
\hline 2095 & Hieracium $\times$ prussicum Nägeli et Peter & Tokaj & 1935 & Hulják János & BPU-13634.JPG \\
\hline
\end{tabular}


1. Elektroniukus melléklet - Electronic Appendix 1.

\begin{tabular}{|c|c|c|c|c|c|}
\hline $\begin{array}{l}\text { Sorszám / } \\
\text { Number }\end{array}$ & Taxon-név / Taxon-name & $\begin{array}{l}\text { Település / } \\
\text { Settlement }\end{array}$ & $\begin{array}{l}\text { Év / } \\
\text { Year }\end{array}$ & Gyűjtő / Collector & $\begin{array}{l}\text { Fájlnév / } \\
\text { File-name }\end{array}$ \\
\hline 2096 & $\begin{array}{l}\text { Hieracium } \times \text { leptophyton Nägeli et } \\
\text { Peter }\end{array}$ & Budapest & 1935 & Kováts Ferenc & BPU-13629.JPG \\
\hline 2096 & $\begin{array}{l}\text { Hieracium } \times \text { leptophyton Nägeli et } \\
\text { Peter }\end{array}$ & Misefa & 1935 & Kováts Ferenc & BPU-13628.JPG \\
\hline 2096 & $\begin{array}{l}\text { Hieracium } \times \text { leptophyton Nägeli et } \\
\text { Peter }\end{array}$ & Nemesrádó & 1935 & Kováts Ferenc & BPU-13627.JPG \\
\hline 2098 & Hieracium fallax Willd. & Budapest & 1948 & Kováts Ferenc & BPU-13496.JPG \\
\hline 2099 & Hieracium auriculoides Láng & Budapest & 1922 & Soó Rezső & BPU-13541.JPG \\
\hline 2099 & Hieracium auriculoides Láng & Budapest & 1932 & Kováts Ferenc & BPU-13558.JPG \\
\hline 2099 & Hieracium auriculoides Láng & Budapest & 1935 & Kováts Ferenc & BPU-13554.JPG \\
\hline 2099 & Hieracium auriculoides Láng & Budapest & 1935 & Kováts Ferenc & BPU-13555.JPG \\
\hline 2099 & Hieracium auriculoides Láng & Budapest & 1935 & Kováts Ferenc & BPU-13556.JPG \\
\hline 2099 & Hieracium auriculoides Láng & Budapest & 1935 & Kováts Ferenc & BPU-13557.JPG \\
\hline 2099 & Hieracium auriculoides Láng & Budapest & 1936 & Kováts Ferenc & BPU-13553.JPG \\
\hline 2099 & Hieracium auriculoides Láng & Budapest & 1941 & Kováts Ferenc & BPU-13543.JPG \\
\hline 2099 & Hieracium auriculoides Láng & Budapest & 1947 & Soó Rezső & BPU-13539.JPG \\
\hline 2099 & Hieracium auriculoides Láng & Budapest & 1947 & Soó Rezső & BPU-13540.JPG \\
\hline 2099 & Hieracium auriculoides Láng & Budapest & 1947 & Soó Rezső & BPU-13609.JPG \\
\hline 2099 & Hieracium auriculoides Láng & Bükkszentkereszt & 1925 & Budai József & BPU-13668.JPG \\
\hline 2099 & Hieracium auriculoides Láng & Egyek & 1935 & Soó Rezső & BPU-13537.JPG \\
\hline 2099 & Hieracium auriculoides Láng & Füzér & 1938 & Soó Rezső & BPU-13665.JPG \\
\hline 2099 & Hieracium auriculoides Láng & Hajdúbagos & 1937 & Soó Rezső & BPU-13538.JPG \\
\hline 2099 & Hieracium auriculoides Láng & Hajdúnánás & 1938 & Igmándy József & BPU-13552.JPG \\
\hline 2099 & Hieracium auriculoides Láng & Parád & 1939 & Igmándy József & BPU-13551.JPG \\
\hline 2099 & Hieracium auriculoides Láng & Somlóvásárhely & 1953 & $\begin{array}{l}\text { Kulcsár Gábor, Vozáry } \\
\text { Elemér }\end{array}$ & BPU-13542.JPG \\
\hline 2099 & Hieracium auriculoides Láng & Tarcal & 1936 & Hulják János & BPU-13546.JPG \\
\hline 2099 & Hieracium auriculoides Láng & Tarcal & 1936 & Hulják János & BPU-13550.JPG \\
\hline 2099 & Hieracium auriculoides Láng & Tarcal & 1937 & Hulják János & BPU-13544.JPG \\
\hline 2099 & Hieracium auriculoides Láng & Tarcal & 1937 & Hulják János & BPU-13545.JPG \\
\hline 2099 & Hieracium auriculoides Láng & Tarcal & 1937 & Hulják János & BPU-13547.JPG \\
\hline 2099 & Hieracium auriculoides Láng & Tarcal & 1937 & Hulják János & BPU-13548.JPG \\
\hline 2099 & Hieracium auriculoides Láng & Tarcal & 1937 & Hulják János & BPU-13549.JPG \\
\hline 2099 & Hieracium auriculoides Láng & Vác & 1938 & Balázs Ferenc & BPU-13610.JPG \\
\hline 2104 & Hieracium densiflorum Tausch & Abaújszántó & 1936 & Hulják János & BPU-13570.JPG \\
\hline 2104 & Hieracium densiflorum Tausch & Balatonalmádi & 1938 & Soó Rezső & BPU-13564.JPG \\
\hline 2104 & Hieracium densiflorum Tausch & Budapest & 1942 & Kováts Ferenc & BPU-13567.JPG \\
\hline 2104 & Hieracium densiflorum Tausch & Nyíregyháza & 1938 & Soó Rezső & BPU-13563.JPG \\
\hline 2104 & Hieracium densiflorum Tausch & Pilisszentiván & 1926 & Kováts Ferenc & BPU-13566.JPG \\
\hline 2104 & Hieracium densiflorum Tausch & Pilisszentiván & 1945 & Vajda László & BPU-13572.JPG \\
\hline 2104 & Hieracium densiflorum Tausch & Tarcal & 1937 & Hulják János & BPU-13568.JPG \\
\hline 2104 & Hieracium densiflorum Tausch & Tarcal & 1937 & Hulják János & BPU-13569.JPG \\
\hline 2104 & Hieracium densiflorum Tausch & Tarcal & 1937 & Hulják János & BPU-13571.JPG \\
\hline 2104 & Hieracium densiflorum Tausch & Tarpa & 1949 & Soó Rezső & BPU-13562.JPG \\
\hline 2104 & Hieracium densiflorum Tausch & Tarpa & 1949 & Soó Rezső & BPU-13565.JPG \\
\hline 2105 & Hieracium zizianum Tausch & Tarpa & 1949 & Soó Rezső & BPU-13513.JPG \\
\hline 2108 & Hieracium $\times$ polymastix Peter & Debrecen & 1947 & Soó Rezső & BPU-13560.JPG \\
\hline 2109 & Hieracium wiesbaurianum Uechtr. & Pilisszentiván & 1949 & Vajda László & BPU-13481.JPG \\
\hline 2109 & Hieracium wiesbaurianum Uechtr. & Tapolca & 1949 & Vajda László & BPU-13482.JPG \\
\hline
\end{tabular}


1. Elektroniukus melléklet - Electronic Appendix 1.

\begin{tabular}{|c|c|c|c|c|c|}
\hline $\begin{array}{l}\text { Sorszám / } \\
\text { Number }\end{array}$ & Taxon-név / Taxon-name & $\begin{array}{l}\text { Település / } \\
\text { Settlement }\end{array}$ & $\begin{array}{l}\text { Év / } \\
\text { Year }\end{array}$ & Gyűjtő / Collector & $\begin{array}{l}\text { Fájlnév / } \\
\text { File-name }\end{array}$ \\
\hline 2110 & Hieracium glaucinum Jord. & Erdőbénye & 1948 & Soó Rezső & BPU-13485.JPG \\
\hline 2110 & Hieracium glaucinum Jord. & Erdőbénye & 1948 & Soó Rezső & BPU-13488.JPG \\
\hline 2111 & Hieracium praecurrens Vuk. & Bakonybél & 1955 & Soó Rezső & BPU-10792.JPG \\
\hline 2111 & Hieracium praecurrens Vuk. & Bélapátfalva & 1936 & Igmándy József & BPU-10787.JPG \\
\hline 2112 & Hieracium kossuthianum Soó & Budapest & 1947 & Soó Rezső & BPU-13491.JPG \\
\hline 2113 & Hieracium maculatum Schrank & Csákvár & 1948 & Soó Rezső & BPU-13483.JPG \\
\hline 2113 & Hieracium maculatum Schrank & Fenyőfő & 1930 & Soó Rezső & BPU-10830.JPG \\
\hline 2114 & Hieracium diaphanoides Lindeb. & Budapest & 1936 & Kováts Ferenc & BPU-10827.JPG \\
\hline 2114 & Hieracium diaphanoides Lindeb. & Budapest & 1947 & Soó Rezső & BPU-10829.JPG \\
\hline 2114 & Hieracium diaphanoides Lindeb. & Fertőrákos & 1935 & Kováts Ferenc & BPU-10828.JPG \\
\hline 2115 & Hieracium caesium Fr. & Eger & 1928 & Koppányi Tibor & BPU-10842.JPG \\
\hline 2115 & Hieracium caesium Fr. & Erdőbénye & 1951 & Kováts L. & BPU-10853.JPG \\
\hline 2115 & Hieracium caesium Fr. & Pálháza & 1939 & Soó Rezső & BPU-10844.JPG \\
\hline 2117 & Hieracium ramosum Waldst. et Kit. & Gyöngyös & 1935 & Soó Rezső & BPU-10848.JPG \\
\hline 2117 & Hieracium ramosum Waldst. et Kit. & Gyöngyössolymos & 1942 & Kováts Ferenc & BPU-10847.JPG \\
\hline 2117 & Hieracium ramosum Waldst. et Kit. & Háromhuta & 1937 & Hulják János & BPU-10846.JPG \\
\hline 2117 & Hieracium ramosum Waldst. et Kit. & Parádsasvár & 1942 & Kováts Ferenc & BPU-10849.JPG \\
\hline 2076 & Hieracium laevicaule Jord. & Budapest & 1936 & Kováts Ferenc & BPU-10851.JPG \\
\hline 2076 & Hieracium laevicaule Jord. & Háromhuta & 1935 & Igmándy József & BPU-10850.JPG \\
\hline 2076 & Hieracium laevicaule Jord. & Parádsasvár & 1942 & Kováts Ferenc & BPU-10852.JPG \\
\hline 2119 & Hieracium laurinum Arv.-Touv. & Budapest & 2004 & Felföldy Lajos & BPU-10892.JPG \\
\hline 2121 & Hieracium latifolium Spreng. & Budaörs & 1948 & Kováts Ferenc & BPU-10871.JPG \\
\hline 2121 & Hieracium latifolium Spreng. & Budapest & 1937 & Soó Rezső & BPU-10872.JPG \\
\hline 2121 & Hieracium latifolium Spreng. & Dömös & 1948 & Soó Rezső & BPU-10878.JPG \\
\hline---- & Hieracium erythrodontum Zahn & Balatonboglár & 1941 & Kováts Ferenc & BPU-13502.JPG \\
\hline $\begin{array}{c}2065- \\
2121\end{array}$ & Hieracium & Gyöngyös & 1965 & Soó Rezső & BPU-10935.JPG \\
\hline $\begin{array}{c}2065- \\
2121\end{array}$ & Hieracium & Gyöngyös & 1965 & Soó Rezső & BPU-13527.JPG \\
\hline $\begin{array}{c}2065- \\
2121\end{array}$ & Hieracium & Nagybajom & 1957 & Soó Rezső & BPU-10936.JPG \\
\hline $\begin{array}{c}2065- \\
2121\end{array}$ & Hieracium & Nagybajom & 1957 & Soó Rezső & BPU-10937.JPG \\
\hline $\begin{array}{c}2065- \\
2121\end{array}$ & Hieracium & Nagybajom & 1957 & Soó Rezső & BPU-10938.JPG \\
\hline $\begin{array}{c}2065- \\
2121\end{array}$ & Hieracium & Nagybajom & 1957 & Soó Rezső & BPU-10939.JPG \\
\hline $\begin{array}{c}2065- \\
2121\end{array}$ & Hieracium & Pilisszentiván & 1953 & Soó Rezső & BPU-10940.JPG \\
\hline $\begin{array}{c}2065- \\
2121\end{array}$ & Hieracium & Pilisszentiván & 1953 & Soó Rezső & BPU-10942.JPG \\
\hline $\begin{array}{c}2065- \\
2121\end{array}$ & Hieracium & Pilisszentiván & 1953 & Soó Rezső & BPU-10945.JPG \\
\hline $\begin{array}{c}2065- \\
2121\end{array}$ & Hieracium & Szarvas & 1949 & Soó Rezső & BPU-13733.JPG \\
\hline $\begin{array}{c}2065- \\
2121\end{array}$ & Hieracium & Tapolca & 1953 & Borsos Olga & BPU-10941.JPG \\
\hline $\begin{array}{c}2065- \\
2121\end{array}$ & Hieracium & Uzsa & 1953 & Soó Rezső & BPU-10943.JPG \\
\hline $\begin{array}{c}2065- \\
2121\end{array}$ & Hieracium & Zebegény & 1950 & Simon Tibor, Borsos Olga & BPU-10944.JPG \\
\hline---- & Heliopsis helianthoides (L.) Sweet & Debrecen & 1945 & Soó Rezső & BPU-10946.JPG \\
\hline---- & Heliopsis helianthoides (L.) Sweet & Debrecen & 1945 & Soó Rezső & BPU-10947.JPG \\
\hline
\end{tabular}


1. Elektroniukus melléklet - Electronic Appendix 1.

\begin{tabular}{|c|c|c|c|c|c|}
\hline $\begin{array}{l}\text { Sorszám / } \\
\text { Number }\end{array}$ & Taxon-név / Taxon-name & $\begin{array}{l}\text { Település / } \\
\text { Settlement }\end{array}$ & $\begin{array}{l}\text { Év / } \\
\text { Year }\end{array}$ & Gyüjtő / Collector & $\begin{array}{l}\text { Fájlnév / } \\
\text { File-name }\end{array}$ \\
\hline---- & Rudbeckia bicolor Nutt. & Debrecen & 1934 & Soó Rezső & BPU-08027.JPG \\
\hline 2122 & Sagittaria sagittifolia L. & Budapest & 1913 & $\begin{array}{l}\text { Filarszky Nándor, Szurák } \\
\text { János }\end{array}$ & BPU-13382.JPG \\
\hline 2122 & Sagittaria sagittifolia L. & Budapest & 1913 & $\begin{array}{l}\text { Filarszky Nándor, Szurák } \\
\text { János }\end{array}$ & BPU-13382.JPG \\
\hline 2122 & Sagittaria sagittifolia L. & Budapest & 1947 & Priszter Szaniszló & BPU-13381.JPG \\
\hline 2122 & Sagittaria sagittifolia L. & Keszthely & 1958 & Tóth László & BPU-13378.JPG \\
\hline 2122 & Sagittaria sagittifolia L. & Sávoly/Főnyed/Vörs & 1928 & Soó Rezső & BPU-13379.JPG \\
\hline 2122 & Sagittaria sagittifolia L. & Tivadar & 1948 & Simon Tibor, Jakucs Pál & BPU-13380.JPG \\
\hline 2123 & Alisma gramineum Lej. & Budapest & 1946 & Priszter Szaniszló & BPU-13394.JPG \\
\hline 2123 & Alisma gramineum Lej. & Debrecen & 1989 & Felföldy Lajos & BPU-13392.JPG \\
\hline 2123 & Alisma gramineum Lej. & Fülöpszállás & 1925 & Boros Ádám & BPU-13393.JPG \\
\hline 2123 & Alisma gramineum Lej. & Nyírmada & 1933 & Soó Rezső & BPU-13395.JPG \\
\hline 2123 & Alisma gramineum Lej. & Ráckeve & 1919 & Boros Ádám & BPU-13396.JPG \\
\hline 2123 & Alisma gramineum Lej. & Tihany & 1963 & Felföldy Lajos & BPU-13397.JPG \\
\hline 2123 & Alisma gramineum Lej. & Tihany & 1963 & Felföldy Lajos & BPU-13398.JPG \\
\hline 2124 & Alisma plantago-aquatica L. & Aranyosapáti & 1933 & Soó Rezső & BPU-13390.JPG \\
\hline 2124 & Alisma plantago-aquatica L. & Budaörs & 1991 & Felföldy Lajos & BPU-13391.JPG \\
\hline 2124 & Alisma plantago-aquatica L. & Vásárosnamény & 1933 & Soó Rezső & BPU-13389.JPG \\
\hline 2125 & Alisma lanceolatum With. & Hortobágy & 1947 & Soó Rezső & BPU-13388.JPG \\
\hline 2125 & Alisma lanceolatum With. & Nagykanizsa & 1956 & Felföldy Lajos & BPU-13399.JPG \\
\hline 2126 & Caldesia parnassifolia (L.) Parl. & Kállósemjén & 1926 & Boros Ádám & BPU-13387.JPG \\
\hline 2126 & Caldesia parnassifolia (L.) Parl. & Nyírmada & 1933 & Soó Rezső & BPU-13383.JPG \\
\hline 2126 & Caldesia parnassifolia (L.) Parl. & Nyírmada & 1933 & Soó Rezső & BPU-13384.JPG \\
\hline 2126 & Caldesia parnassifolia (L.) Parl. & Szenta & 1923 & Boros Ádám & BPU-13386.JPG \\
\hline 2126 & Caldesia parnassifolia (L.) Parl. & Szenta & 1958 & $\begin{array}{l}\text { Borhidi Attila, Járainé } \\
\text { Komlódi Magda }\end{array}$ & BPU-13385.JPG \\
\hline 2127 & Butomus umbellatus L. & Budapest & 1913 & $\begin{array}{l}\text { Filarszky Nándor, } \\
\text { Kümmerle Jenő Béla }\end{array}$ & BPU-13377.JPG \\
\hline 2127 & Butomus umbellatus L. & Budapest & 1947 & Priszter Szaniszló & BPU-13376.JPG \\
\hline 2127 & Butomus umbellatus L. & Konyár & 1949 & Soó Rezső & BPU-13375.JPG \\
\hline 2128 & Hydrocharis morsus-ranae L. & Badacsonytomaj & 1942 & Priszter Szaniszló & BPU-13345.JPG \\
\hline 2128 & Hydrocharis morsus-ranae L. & Balatonalmádi & 1952 & Soó Rezső & BPU-13344.JPG \\
\hline 2128 & Hydrocharis morsus-ranae L. & Budapest & 1947 & Priszter Szaniszló & BPU-13352.JPG \\
\hline 2128 & Hydrocharis morsus-ranae L. & Budapest & 1947 & Priszter Szaniszló & BPU-13353.JPG \\
\hline 2128 & Hydrocharis morsus-ranae L. & Debrecen & 1989 & Felföldy Lajos & BPU-13349.JPG \\
\hline 2128 & Hydrocharis morsus-ranae L. & Dunaharaszti & 1946 & Priszter Szaniszló & BPU-13346.JPG \\
\hline 2128 & Hydrocharis morsus-ranae L. & Gelénes & 1951 & $\begin{array}{l}\text { Simon Tibor, Vozáry } \\
\text { Elemér }\end{array}$ & BPU-13350.JPG \\
\hline 2128 & Hydrocharis morsus-ranae L. & Göd & 1948 & Soó Rezső & BPU-13347.JPG \\
\hline 2128 & Hydrocharis morsus-ranae L. & Szarvas & 1991 & Felföldy Lajos & BPU-13351.JPG \\
\hline 2128 & Hydrocharis morsus-ranae L. & Tiszafüred & 1953 & Borsos Olga & BPU-13348.JPG \\
\hline 2128 & Hydrocharis morsus-ranae L. & Veresegyház & 1949 & Soó Rezső & BPU-15532.JPG \\
\hline 2129 & Stratiotes aloides L. & Badacsonytomaj & 1935 & Priszter Szaniszló & BPU-13354.JPG \\
\hline 2129 & Stratiotes aloides L. & Debrecen & 1934 & Soó Rezső & BPU-13357.JPG \\
\hline 2129 & Stratiotes aloides L. & Debrecen & 1989 & Felföldy Lajos & BPU-13356.JPG \\
\hline 2129 & Stratiotes aloides L. & Gávavencsellő & 1990 & Felföldy Lajos & BPU-13355.JPG \\
\hline 2129 & Stratiotes aloides L. & Kecel & - & Haynald Lajos & BPU-13359.JPG \\
\hline 2129 & Stratiotes aloides L. & Tákos & 1948 & Simon Tibor, Jakucs Pál & BPU-13358.JPG \\
\hline
\end{tabular}


1. Elektroniukus melléklet - Electronic Appendix 1.

\begin{tabular}{|c|c|c|c|c|c|}
\hline $\begin{array}{l}\text { Sorszám / } \\
\text { Number }\end{array}$ & Taxon-név / Taxon-name & $\begin{array}{l}\text { Település / } \\
\text { Settlement }\end{array}$ & $\begin{array}{l}\text { Év / } \\
\text { Year }\end{array}$ & Gyújtő / Collector & $\begin{array}{l}\text { Fájlnév / } \\
\text { File-name }\end{array}$ \\
\hline 2131 & Elodea canadensis Michx. & Balatongyörök & 1956 & $\begin{array}{l}\text { Felföldy Lajos, Tóth } \\
\text { László }\end{array}$ & BPU-13368.JPG \\
\hline 2131 & Elodea canadensis Michx. & Balatongyörök & 1958 & Iharos Gyula & BPU-13367.JPG \\
\hline 2131 & Elodea canadensis Michx. & Balatonszemes & 1974 & Tóth László & BPU-13369.JPG \\
\hline 2131 & Elodea canadensis Michx. & Budapest & 1909 & Degen Árpád & BPU-13374.JPG \\
\hline 2131 & Elodea canadensis Michx. & Budapest & 1916 & Degen Árpád & BPU-13374.JPG \\
\hline 2131 & Elodea canadensis Michx. & Budapest & 1946 & Kárpáti Zoltán & BPU-13372.JPG \\
\hline 2131 & Elodea canadensis Michx. & Budapest & 1946 & Priszter Szaniszló & BPU-13371.JPG \\
\hline 2131 & Elodea canadensis Michx. & Gyenesdiás & 1957 & Gizella Tamás & BPU-13365.JPG \\
\hline 2131 & Elodea canadensis Michx. & Keszthely & 1958 & Tóth László & BPU-13364.JPG \\
\hline 2131 & Elodea canadensis Michx. & Keszthely & 1960 & Sebestyén Olga & BPU-13363.JPG \\
\hline 2131 & Elodea canadensis Michx. & Keszthely & 1960 & Sebestyén Olga & BPU-13366.JPG \\
\hline 2131 & Elodea canadensis Michx. & Szigetcsép & 1992 & Felföldy Lajos & BPU-13370.JPG \\
\hline 2131 & Elodea canadensis Michx. & Vámosújfalu & 1993 & Felföldy Lajos & BPU-13362.JPG \\
\hline 2136 & Vallisneria spiralis L. & Budapest & 1905 & Lengyel Géza & BPU-13360.JPG \\
\hline 2136 & Vallisneria spiralis $\mathrm{L}$. & Budapest & 1910 & $\begin{array}{l}\text { Kümmerle Jenő Béla, } \\
\text { Jávorka Sándor, Szurák } \\
\text { János }\end{array}$ & BPU-13361.JPG \\
\hline 2138 & Triglochin palustre L. & Balatonszőlős & 1957 & $\begin{array}{l}\text { Felföldy Lajos, Kovács } \\
\text { Margit, Tóth László }\end{array}$ & BPU-13208.JPG \\
\hline 2138 & Triglochin palustre L. & Debrecen & 1952 & Kulcsár Gábor & BPU-15433.JPG \\
\hline 2138 & Triglochin palustre L. & Fertőhomok & 1960 & Tóth László & BPU-13211.JPG \\
\hline 2138 & Triglochin palustre L. & Kállósemjén & 1934 & Soó Rezső & BPU-13209.JPG \\
\hline 2138 & Triglochin palustre L. & Kemence & 1952 & Simon Tibor & BPU-13217.JPG \\
\hline 2138 & Triglochin palustre L. & Kiskunhalas & 1964 & Soó Rezső & BPU-13218.JPG \\
\hline 2138 & Triglochin palustre L. & Mór & 1942 & Priszter Szaniszló & BPU-13216.JPG \\
\hline 2138 & Triglochin palustre L. & Sopron & 1942 & Priszter Szaniszló & BPU-13210.JPG \\
\hline 2138 & Triglochin palustre L. & Vácrátót & 1953 & Felföldy Lajos & BPU-13215.JPG \\
\hline 2138 & Triglochin palustre L. & Veresegyház & 1947 & Papp József & BPU-13214.JPG \\
\hline 2138 & Triglochin palustre L. & Veresegyház & 1949 & Soó Rezső & BPU-13213.JPG \\
\hline 2138 & Triglochin palustre L. & Veresegyház & 1949 & Soó Rezső & BPU-15533.JPG \\
\hline 2138 & Triglochin palustre L. & Veresegyház & 1950 & Soó Rezső & BPU-13212.JPG \\
\hline 2140 & Veratrum nigrum L. & Balatonszőlős & 1958 & Felföldy Lajos & BPU-13181.JPG \\
\hline 2140 & Veratrum nigrum L. & Csákvár & 1948 & Soó Rezső & BPU-13182.JPG \\
\hline 2141 & Veratrum album L. & Bátorliget & 1950 & Simon Tibor & BPU-13176.JPG \\
\hline 2141 & Veratrum album L. & Dabas & 1951 & Borsos Olga, Simon Tibor & BPU-13179.JPG \\
\hline 2141 & Veratrum album L. & Debrecen & 1933 & Soó Rezső & BPU-13180.JPG \\
\hline 2141 & Veratrum album L. & Kiskőrös & 1950 & Papp József & BPU-13178.JPG \\
\hline 2141 & Veratrum album L. & Ócsa & 1953 & Soó Rezső, Borsos Olga & BPU-13177.JPG \\
\hline 2142 & Asphodelus albus Mill. & Gyenesdiás & 1928 & Soó Rezső & BPU-09800.JPG \\
\hline 2142 & Asphodelus albus Mill. & Uzsa & 1959 & $\begin{array}{l}\text { Jávorka Sándor, Felföldy } \\
\text { Lajos }\end{array}$ & BPU-09799.JPG \\
\hline 2143 & Anthericum liliago L. & Budapest & 1947 & Soó Rezső & BPU-09801.JPG \\
\hline 2143 & Anthericum liliago L. & Villány & 1956 & Soó Rezső & BPU-09802.JPG \\
\hline 2144 & Anthericum ramosum L. & Bátorliget & 1948 & Soó Rezső & BPU-09811.JPG \\
\hline 2144 & Anthericum ramosum L. & Bátorliget & 1953 & Simon Tibor & BPU-09812.JPG \\
\hline 2144 & Anthericum ramosum L. & Bodajk & 1961 & Borsos Olga, Simon Tibor & BPU-09807.JPG \\
\hline 2144 & Anthericum ramosum L. & Budapest & 1947 & Soó Rezső & BPU-09805.JPG \\
\hline 2144 & Anthericum ramosum L. & Budapest & 1982 & Felföldy Lajos & BPU-09804.JPG \\
\hline
\end{tabular}


1. Elektroniukus melléklet - Electronic Appendix 1.

\begin{tabular}{|c|c|c|c|c|c|}
\hline $\begin{array}{l}\text { Sorszám / } \\
\text { Number }\end{array}$ & Taxon-név / Taxon-name & $\begin{array}{l}\text { Település / } \\
\text { Settlement }\end{array}$ & $\begin{array}{l}\text { Év / } \\
\text { Year }\end{array}$ & Gyüjtő / Collector & $\begin{array}{l}\text { Fájlnév / } \\
\text { File-name }\end{array}$ \\
\hline 2144 & Anthericum ramosum L. & Budapest & 2004 & Felföldy Lajos & BPU-09803.JPG \\
\hline 2144 & Anthericum ramosum L. & Fót & 1960 & Soó Rezső & BPU-09810.JPG \\
\hline 2144 & Anthericum ramosum L. & Gyöngyös & 1961 & Soó Rezső & BPU-09808.JPG \\
\hline 2144 & Anthericum ramosum L. & Gyöngyös & 1961 & Soó Rezső & BPU-09809.JPG \\
\hline 2144 & Anthericum ramosum L. & Tibolddaróc & 1953 & Soó Rezső & BPU-09806.JPG \\
\hline 2144 & Anthericum ramosum L. & Uzsa & 1952 & Simon Tibor & BPU-09813.JPG \\
\hline 2145 & Hemerocallis lilio-asphodelus L. & Szentgotthárd & 1955 & Soó Rezső & BPU-09814.JPG \\
\hline 2146 & Hemerocallis fulva L. & Nagyvázsony & 1933 & Jávorka Sándor & BPU-09815.JPG \\
\hline 2147 & Colchicum hungaricum Janka & Nagyharsány & 1925 & Boros Ádám & BPU-13169.JPG \\
\hline 2147 & Colchicum hungaricum Janka & Nagyharsány & 1956 & Soó Rezső & BPU-13168.JPG \\
\hline 2147 & Colchicum hungaricum Janka & Nagyharsány & 1967 & Priszter Szaniszló & BPU-09781.JPG \\
\hline 2147 & Colchicum hungaricum Janka & Nagyharsány & 1967 & Priszter Szaniszló & BPU-13166.JPG \\
\hline 2147 & Colchicum hungaricum Janka & Nagyharsány & 1967 & Priszter Szaniszló & BPU-13167.JPG \\
\hline 2148 & Colchicum arenarium Waldst. et Kit. & Bugac & 1962 & Simon Tibor & BPU-09782.JPG \\
\hline 2148 & Colchicum arenarium Waldst. et Kit. & Dunakeszi & 1943 & Kárpáti Zoltán & BPU-09783.JPG \\
\hline 2148 & Colchicum arenarium Waldst. et Kit. & Dunakeszi & 1948 & Soó Rezső & BPU-09784.JPG \\
\hline 2148 & Colchicum arenarium Waldst. et Kit. & Fót & 1963 & Isépy István & BPU-09785.JPG \\
\hline 2148 & Colchicum arenarium Waldst. et Kit. & Szigetcsép & 1948 & Priszter Szaniszló & BPU-09786.JPG \\
\hline 2149 & Colchicum autumnale L. & Balatongyörök & 1961 & Felföldy Lajos & BPU-09797.JPG \\
\hline 2149 & Colchicum autumnale L. & Drávafok & 1968 & Priszter Szaniszló & BPU-09787.JPG \\
\hline 2149 & Colchicum autumnale L. & Hévíz & 1961 & Felföldy Lajos & BPU-09798.JPG \\
\hline 2149 & Colchicum autumnale L. & Hidegkút & 1957 & Felföldy Lajos & BPU-09795.JPG \\
\hline 2149 & Colchicum autumnale L. & Kerecsend & 1957 & Soó Rezső & BPU-09789.JPG \\
\hline 2149 & Colchicum autumnale L. & Miskolc & 1937 & Soó Rezső & BPU-09788.JPG \\
\hline 2149 & Colchicum autumnale L. & Ócsa & 1965 & Priszter Szaniszló & BPU-09792.JPG \\
\hline 2149 & Colchicum autumnale L. & Ócsa & 1965 & Priszter Szaniszló & BPU-09793.JPG \\
\hline 2149 & Colchicum autumnale L. & Sopron & 1969 & Priszter Szaniszló & BPU-09794.JPG \\
\hline 2149 & Colchicum autumnale L. & Szilvásvárad & 1949 & Koppányi Tibor & BPU-09790.JPG \\
\hline 2149 & Colchicum autumnale L. & Szilvásvárad & 1953 & $\begin{array}{l}\text { Felföldy Lajos, Tóth } \\
\text { László }\end{array}$ & BPU-09791.JPG \\
\hline 2149 & Colchicum autumnale L. & Vászoly & 1958 & Felföldy Lajos & BPU-09796.JPG \\
\hline 2150 & Bulbocodium vernum L. & Hosszúpályi & 1955 & Soó Rezső & BPU-13172.JPG \\
\hline 2150 & Bulbocodium vernum L. & Sáránd & 1932 & Soó Rezső & BPU-13171.JPG \\
\hline 2152 & Ornithogalum $\times$ degenianum Polgár & Pápakovácsi & 1930 & $\begin{array}{l}\text { Polgár Sándor, Jávorka } \\
\text { Sándor }\end{array}$ & BPU-13102.JPG \\
\hline 2152 & Ornithogalum $\times$ degenianum Polgár & Pápakovácsi & 1933 & Polgár Sándor & BPU-13101.JPG \\
\hline 2153 & $\begin{array}{l}\text { Ornithogalum boucheanum (Kunth) } \\
\text { Asch. }\end{array}$ & Debrecen & 1931 & Soó Rezső & BPU-13140.JPG \\
\hline 2153 & $\begin{array}{l}\text { Ornithogalum boucheanum (Kunth) } \\
\text { Asch. }\end{array}$ & Solymár & 1947 & Priszter Szaniszló & BPU-13139.JPG \\
\hline 2153 & $\begin{array}{l}\text { Ornithogalum boucheanum (Kunth) } \\
\text { Asch. }\end{array}$ & Szigetújfalu & 1948 & Priszter Szaniszló & BPU-13138.JPG \\
\hline 2153 & $\begin{array}{l}\text { Ornithogalum boucheanum (Kunth) } \\
\text { Asch. }\end{array}$ & Tihany & 1958 & Felföldy Lajos & BPU-13136.JPG \\
\hline 2153 & $\begin{array}{l}\text { Ornithogalum boucheanum (Kunth) } \\
\text { Asch. }\end{array}$ & Visegrád & 1941 & Priszter Szaniszló & BPU-13137.JPG \\
\hline 2154 & Ornithogalum sphaerocarpum A. Kern. & Badacsonytomaj & 1928 & Soó Rezső & BPU-13134.JPG \\
\hline 2154 & Ornithogalum sphaerocarpum A. Kern. & Nagykanizsa & 1948 & Károlyi Árpád & BPU-13133.JPG \\
\hline 2154 & Ornithogalum sphaerocarpum A. Kern. & Sopron & 1948 & Kárpáti Zoltán & BPU-13135.JPG \\
\hline 2155 & Ornithogalum brevistylum Wolfner & Budapest & 1947 & Priszter Szaniszló & BPU-13131.JPG \\
\hline
\end{tabular}


1. Elektroniukus melléklet - Electronic Appendix 1.

\begin{tabular}{|c|c|c|c|c|c|}
\hline $\begin{array}{l}\text { Sorszám / } \\
\text { Number }\end{array}$ & Taxon-név / Taxon-name & $\begin{array}{l}\text { Település / } \\
\text { Settlement }\end{array}$ & $\begin{array}{l}\text { Év / } \\
\text { Year }\end{array}$ & Gyújtő / Collector & $\begin{array}{l}\text { Fájlnév / } \\
\text { File-name }\end{array}$ \\
\hline 2155 & Ornithogalum brevistylum Wolfner & Budapest & 1982 & Felföldy Lajos & BPU-13130.JPG \\
\hline 2155 & Ornithogalum brevistylum Wolfner & Mezőtúr & 1969 & Priszter Szaniszló & BPU-13123.JPG \\
\hline 2155 & Ornithogalum brevistylum Wolfner & Pécsely & 1950 & Felföldy Lajos & BPU-13122.JPG \\
\hline 2155 & Ornithogalum brevistylum Wolfner & Pilisszentkereszt & 1940 & Kárpáti Zoltán & BPU-13127.JPG \\
\hline 2155 & Ornithogalum brevistylum Wolfner & Pomáz & 1942 & Priszter Szaniszló & BPU-13125.JPG \\
\hline 2155 & Ornithogalum brevistylum Wolfner & Szentendre & 1941 & Priszter Szaniszló & BPU-13126.JPG \\
\hline 2155 & Ornithogalum brevistylum Wolfner & Szentendre & 1947 & Priszter Szaniszló & BPU-13124.JPG \\
\hline 2155 & Ornithogalum brevistylum Wolfner & Tiszacsege & 1951 & Borsos Olga, Simon Tibor & BPU-13128.JPG \\
\hline 2155 & Ornithogalum brevistylum Wolfner & Tiszacsege & 1951 & Borsos Olga, Simon Tibor & BPU-13129.JPG \\
\hline 2156 & Ornithogalum pannonicum Chaix & Budapest & 1947 & Soó Rezső & BPU-13120.JPG \\
\hline 2156 & Ornithogalum pannonicum Chaix & Csákvár & 1955 & Soó Rezső & BPU-13119.JPG \\
\hline 2157 & Ornithogalum kochii Parl. & Hajdúnánás & 1935 & Soó Rezső & BPU-13117.JPG \\
\hline 2157 & Ornithogalum kochii Parl. & Hidegkút & 1958 & Felföldy Lajos & BPU-13115.JPG \\
\hline 2157 & Ornithogalum kochii Parl. & Villány & 1956 & Soó Rezső & BPU-13118.JPG \\
\hline 2158 & Ornithogalum refractum Kit. in Willd. & Budapest & 2005 & Felföldy Lajos & BPU-13113.JPG \\
\hline 2158 & Ornithogalum refractum Kit. in Willd. & Solymár & 1947 & Kárpáti Zoltán & BPU-13112.JPG \\
\hline 2158 & Ornithogalum refractum Kit. in Willd. & Tihany & 1956 & Felföldy Lajos & BPU-13111.JPG \\
\hline 2159 & Ornithogalum umbellatum L. & "Cuha-völgy" & 1954 & Soó Rezső & BPU-13104.JPG \\
\hline 2159 & Ornithogalum umbellatum L. & Balatonszőlős & 1958 & Felföldy Lajos & BPU-13116.JPG \\
\hline 2159 & Ornithogalum umbellatum L. & Budapest & 1939 & Priszter Szaniszló & BPU-13109.JPG \\
\hline 2159 & Ornithogalum umbellatum L. & Budapest & 2004 & Felföldy Lajos & BPU-13110.JPG \\
\hline 2159 & Ornithogalum umbellatum L. & Debrecen & 1932 & Soó Rezső & BPU-13106.JPG \\
\hline 2159 & Ornithogalum umbellatum L. & Szigetújfalu & 1948 & Priszter Szaniszló & BPU-13105.JPG \\
\hline 2159 & Ornithogalum umbellatum L. & Tihany & 1958 & Felföldy Lajos & BPU-13114.JPG \\
\hline 2159 & Ornithogalum umbellatum L. & Villány & 1941 & Priszter Szaniszló & BPU-13108.JPG \\
\hline 2159 & Ornithogalum umbellatum L. & Zámoly & 1982 & Felföldy Lajos & BPU-13107.JPG \\
\hline 2161 & Scilla autumnalis L. & Tihany & 1911 & $\begin{array}{l}\text { Filarszky Nándor, Jávorka } \\
\text { Sándor }\end{array}$ & BPU-13150.JPG \\
\hline 2161 & Scilla autumnalis L. & Tihany & 1949 & Simon Tibor & BPU-13149.JPG \\
\hline $\begin{array}{l}2162- \\
2165\end{array}$ & Scilla bifolia agg. & Budapest & 1937 & Priszter Szaniszló & BPU-13148.JPG \\
\hline $\begin{array}{l}2162- \\
2165\end{array}$ & Scilla bifolia agg. & Budapest & 1948 & Priszter Szaniszló & BPU-13147.JPG \\
\hline $\begin{array}{l}2162- \\
2165\end{array}$ & Scilla bifolia agg. & Debrecen & 1932 & Soó Rezső & BPU-13144.JPG \\
\hline $\begin{array}{c}2162- \\
2165\end{array}$ & Scilla bifolia agg. & Debrecen & 1932 & Soó Rezső & BPU-13145.JPG \\
\hline $\begin{array}{l}2162- \\
2165\end{array}$ & Scilla bifolia agg. & Érd & 1947 & Papp József & BPU-13143.JPG \\
\hline $\begin{array}{l}2162- \\
2165\end{array}$ & Scilla bifolia agg. & Miskolc & 1949 & Jakucs Pál & BPU-13146.JPG \\
\hline $\begin{array}{l}2162- \\
2165\end{array}$ & Scilla bifolia agg. & Perőcsény & 1948 & Priszter Szaniszló & BPU-13142.JPG \\
\hline 2167 & Muscari tenuiflorum Tausch & Csarnóta & 1965 & Simon Tibor & BPU-13092.JPG \\
\hline 2167 & Muscari tenuiflorum Tausch & Nagyharsány & 1965 & $\begin{array}{l}\text { Simon Tibor, Priszter } \\
\text { Szaniszló }\end{array}$ & BPU-13091.JPG \\
\hline 2167 & Muscari tenuiflorum Tausch & Nagyharsány & 1968 & Priszter Szaniszló & BPU-13090.JPG \\
\hline 2167 & Muscari tenuiflorum Tausch & Százhalombatta & 1969 & Priszter Szaniszló & BPU-13089.JPG \\
\hline 2168 & Muscari comosum (L.) Mill. & Budapest & 1923 & Soó Rezső & BPU-13098.JPG \\
\hline 2168 & Muscari comosum (L.) Mill. & Budapest & 2005 & Felföldy Lajos & BPU-13099.JPG \\
\hline 2168 & Muscari comosum (L.) Mill. & Budapest & 2005 & Felföldy Lajos & BPU-13100.JPG \\
\hline
\end{tabular}


1. Elektroniukus melléklet - Electronic Appendix 1.

\begin{tabular}{|c|c|c|c|c|c|}
\hline $\begin{array}{l}\text { Sorszám / } \\
\text { Number }\end{array}$ & Taxon-név / Taxon-name & $\begin{array}{l}\text { Település / } \\
\text { Settlement }\end{array}$ & $\begin{array}{l}\text { Év / } \\
\text { Year }\end{array}$ & Gyüjtő / Collector & $\begin{array}{l}\text { Fájlnév / } \\
\text { File-name }\end{array}$ \\
\hline 2168 & Muscari comosum (L.) Mill. & Nagykovácsi & 1939 & Priszter Szaniszló & BPU-13095.JPG \\
\hline 2168 & Muscari comosum (L.) Mill. & Parád & 1940 & Priszter Szaniszló & BPU-13096.JPG \\
\hline 2168 & Muscari comosum (L.) Mill. & Pomáz & 1948 & Priszter Szaniszló & BPU-13094.JPG \\
\hline 2168 & Muscari comosum (L.) Mill. & Százhalombatta & 1969 & Priszter Szaniszló & BPU-13093.JPG \\
\hline 2168 & Muscari comosum (L.) Mill. & Téglás & 1948 & Soó Rezső & BPU-13097.JPG \\
\hline 2169 & Muscari neglectum Guss. ex Ten. & Balatonfüred & 1928 & Soó Rezső & BPU-13081.JPG \\
\hline 2169 & Muscari neglectum Guss. ex Ten. & Bodajk & 1955 & Soó Rezső & BPU-13087.JPG \\
\hline 2169 & Muscari neglectum Guss. ex Ten. & Gyöngyös & 1964 & Soó Rezső & BPU-13085.JPG \\
\hline 2169 & Muscari neglectum Guss. ex Ten. & Hajdúszoboszló & 1938 & Fäller & BPU-13086.JPG \\
\hline 2169 & Muscari neglectum Guss. ex Ten. & Nagykovácsi & 1938 & Priszter Szaniszló & BPU-13084.JPG \\
\hline 2169 & Muscari neglectum Guss. ex Ten. & Tihany & 1958 & Felföldy Lajos & BPU-13079.JPG \\
\hline 2169 & Muscari neglectum Guss. ex Ten. & Tihany & 1958 & Felföldy Lajos & BPU-13080.JPG \\
\hline 2169 & Muscari neglectum Guss. ex Ten. & Vácrátót & 1953 & Felföldy Lajos & BPU-13083.JPG \\
\hline 2169 & Muscari neglectum Guss. ex Ten. & Visegrád & 1964 & Soó Rezső, Borsos Olga & BPU-13082.JPG \\
\hline 2170 & Muscari botryoides (L.) Mill. & Nagykőrös & 1935 & Hargitai Zoltán & BPU-13078.JPG \\
\hline 2170 & Muscari botryoides (L.) Mill. & Rezi & 1962 & Priszter Szaniszló & BPU-13077.JPG \\
\hline 2171 & Allium vineale $\mathrm{L}$. & Hajdúsámson & 1933 & Soó Rezső & BPU-09841.JPG \\
\hline 2172 & Allium sphaerocephalon L. & Budapest & 1949 & Jakucs Pál & BPU-09844.JPG \\
\hline 2172 & Allium sphaerocephalon L. & Debrecen & 1952 & Kulcsár Gábor & BPU-09846.JPG \\
\hline 2172 & Allium sphaerocephalon L. & Nagyharsány & 1961 & Simon Tibor & BPU-09845.JPG \\
\hline 2172 & Allium sphaerocephalon L. & Pilisborosjenő & 1942 & Priszter Szaniszló & BPU-09848.JPG \\
\hline 2172 & Allium sphaerocephalon L. & Piliscsaba & 1916 & Filarszky Nándor & BPU-09843.JPG \\
\hline 2172 & Allium sphaerocephalon L. & Tihany & 1959 & Felföldy Lajos & BPU-09850.JPG \\
\hline 2172 & Allium sphaerocephalon L. & Tihany & 1959 & Felföldy Lajos & BPU-09851.JPG \\
\hline 2172 & Allium sphaerocephalon L. & Tihany & 1961 & Felföldy Lajos & BPU-09852.JPG \\
\hline 2172 & Allium sphaerocephalon L. & Uzsa & 1952 & Simon Tibor & BPU-09853.JPG \\
\hline 2172 & Allium sphaerocephalon L. & Vácrátót & 1953 & Felföldy Lajos & BPU-09849.JPG \\
\hline 2172 & Allium sphaerocephalon L. & Zebegény & 1947 & Soó Rezső & BPU-09847.JPG \\
\hline 2174 & Allium scorodoprasum L. & Beregdaróc & 1951 & $\begin{array}{l}\text { Simon Tibor, Vozáry } \\
\text { Elemér }\end{array}$ & BPU-09860.JPG \\
\hline 2174 & Allium scorodoprasum L. & Bisse & 1965 & $\begin{array}{l}\text { Simon Tibor, Priszter } \\
\text { Szaniszló, Borhidi Attila }\end{array}$ & BPU-09859.JPG \\
\hline 2174 & Allium scorodoprasum L. & Budapest & 1921 & Filarszky Nándor & BPU-09855.JPG \\
\hline 2174 & Allium scorodoprasum L. & Budapest & 1942 & Priszter Szaniszló & BPU-09856.JPG \\
\hline 2174 & Allium scorodoprasum L. & Budapest & 1947 & Priszter Szaniszló & BPU-09857.JPG \\
\hline 2174 & Allium scorodoprasum L. & Debrecen & 1930 & Pátkay Imre & BPU-09862.JPG \\
\hline 2174 & Allium scorodoprasum L. & Diósjenő & 1947 & Priszter Szaniszló & BPU-09863.JPG \\
\hline 2174 & Allium scorodoprasum L. & Dörgicse & 1958 & Felföldy Lajos & BPU-09865.JPG \\
\hline 2174 & Allium scorodoprasum L. & Pákozd & 1980 & Felföldy Lajos & BPU-09861.JPG \\
\hline 2174 & Allium scorodoprasum L. & Szentendre & 1947 & Priszter Szaniszló & BPU-09864.JPG \\
\hline 2174 & Allium scorodoprasum L. & Szögliget & 1951 & $\begin{array}{l}\text { Soó Rezső, Jakucs Pál, Ér } \\
\text { Lajos }\end{array}$ & BPU-09858.JPG \\
\hline 2175 & Allium rotundum L. & Csarnóta & 1965 & Simon Tibor & BPU-09866.JPG \\
\hline 2175 & Allium rotundum L. & Nagyharsány & 1965 & $\begin{array}{l}\text { Simon Tibor, Priszter } \\
\text { Szaniszló }\end{array}$ & BPU-09867.JPG \\
\hline 2175 & Allium rotundum L. & Nagykovácsi & 1939 & Priszter Szaniszló & BPU-09869.JPG \\
\hline 2175 & Allium rotundum L. & Pilisszántó & 1942 & Priszter Szaniszló & BPU-09870.JPG \\
\hline 2175 & Allium rotundum L. & Szentendre & 1947 & Priszter Szaniszló & BPU-09871.JPG \\
\hline 2175 & Allium rotundum L. & Tibolddaróc & 1953 & Soó Rezső & BPU-09868.JPG \\
\hline
\end{tabular}


1. Elektroniukus melléklet - Electronic Appendix 1.

\begin{tabular}{|c|c|c|c|c|c|}
\hline $\begin{array}{l}\text { Sorszám / } \\
\text { Number }\end{array}$ & Taxon-név / Taxon-name & $\begin{array}{l}\text { Település / } \\
\text { Settlement }\end{array}$ & $\begin{array}{l}\text { Év / } \\
\text { Year }\end{array}$ & Gyújtő / Collector & $\begin{array}{l}\text { Fájlnév / } \\
\text { File-name }\end{array}$ \\
\hline 2175 & Allium rotundum L. & Tihany & 1947 & Pólya László & BPU-09872.JPG \\
\hline 2175 & Allium rotundum L. & Tokaj & 1938 & Soó Rezső & BPU-09873.JPG \\
\hline 2176 & Allium atroviolaceum Boiss. & Budapest & 1911 & Zsák Zoltán & BPU-09874.JPG \\
\hline 2176 & Allium atroviolaceum Boiss. & Budapest & 1947 & Priszter Szaniszló & BPU-09875.JPG \\
\hline 2178 & Allium moschatum L. & Budapest & 1914 & $\begin{array}{l}\text { Filarszky Nándor, Moesz } \\
\text { Gusztáv }\end{array}$ & BPU-09876.JPG \\
\hline 2178 & Allium moschatum L. & Budapest & 1982 & Felföldy Lajos & BPU-09877.JPG \\
\hline 2178 & Allium moschatum L. & Szigetmonostor & 1947 & Priszter Szaniszló & BPU-09878.JPG \\
\hline 2182 & Allium ursinum L. & Bátorliget & 1935 & Soó Rezső & BPU-09886.JPG \\
\hline 2182 & Allium ursinum L. & Gyenesdiás & 1957 & Entz Béla, Szabó E. & BPU-09887.JPG \\
\hline 2182 & Allium ursinum L. & Mosonmagyaróvár & 1962 & Borsos Olga & BPU-09885.JPG \\
\hline 2182 & Allium ursinum L. & Nagyharsány & 1948 & Priszter Szaniszló & BPU-09881.JPG \\
\hline 2182 & Allium ursinum L. & Pécs & 1935 & Soó Rezső & BPU-09882.JPG \\
\hline 2182 & Allium ursinum L. & Pécs & 1953 & Soó Rezső, Borsos Olga & BPU-09884.JPG \\
\hline 2182 & Allium ursinum L. & Pécs & 1956 & Soó Rezső & BPU-09883.JPG \\
\hline 2182 & Allium ursinum L. & Várpalota & 1955 & Soó Rezső & BPU-09888.JPG \\
\hline 2183 & Allium victorialis L. & Isztimér & 1949 & Soó Rezső & BPU-09890.JPG \\
\hline 2183 & Allium victorialis L. & Tibolddaróc & 1953 & Soó Rezső & BPU-09889.JPG \\
\hline 2183 & Allium victorialis L. & Várpalota & 1955 & Soó Rezső & BPU-09891.JPG \\
\hline 2183 & Allium victorialis L. & Várpalota & 1955 & Soó Rezső & BPU-09892.JPG \\
\hline 2184 & Allium atropurpureum Waldst. et Kit. & Pécsely & 1950 & Felföldy Lajos & BPU-09896.JPG \\
\hline 2184 & Allium atropurpureum Waldst. et Kit. & Tihany & 1958 & Kozma Erika & BPU-09897.JPG \\
\hline 2185 & Allium suaveolens Jacq. & Devecser & 1957 & Tallós Pál & BPU-09900.JPG \\
\hline 2185 & Allium suaveolens Jacq. & Lébény & 1927 & Tuzson János & BPU-09898.JPG \\
\hline 2185 & Allium suaveolens Jacq. & Lesencetomaj & 1964 & Isépy István & BPU-09901.JPG \\
\hline 2185 & Allium suaveolens Jacq. & Sopron & 1947 & Kárpáti L. & BPU-09899.JPG \\
\hline 2186 & Allium angulosum L. & Budapest & 1990 & Felföldy Lajos & BPU-09905.JPG \\
\hline 2186 & Allium angulosum L. & Debrecen & 1936 & Soó Rezső & BPU-09903.JPG \\
\hline 2186 & Allium angulosum L. & Gelénes & 1951 & $\begin{array}{l}\text { Simon Tibor, Vozáry } \\
\text { Elemér }\end{array}$ & BPU-09904.JPG \\
\hline 2186 & Allium angulosum L. & Miskolc & 1936 & Soó Rezső & BPU-09902.JPG \\
\hline 2186 & Allium angulosum L. & Tarpa & 1948 & Simon Tibor, Jakucs Pál & BPU-09906.JPG \\
\hline 2187 & Allium lusitanicum Lam. & Bélapátfalva & 1974 & Priszter Szaniszló & BPU-09909.JPG \\
\hline 2187 & Allium lusitanicum Lam. & Gánt & 1948 & Soó Rezső & BPU-09907.JPG \\
\hline 2187 & Allium lusitanicum Lam. & Gyöngyös & 1952 & Soó Rezső & BPU-09908.JPG \\
\hline 2187 & Allium lusitanicum Lam. & Gyöngyös & 1953 & Soó Rezső & BPU-09910.JPG \\
\hline 2187 & Allium lusitanicum Lam. & Pomáz & 1960 & Isépy István & BPU-09911.JPG \\
\hline 2187 & Allium lusitanicum Lam. & Tapolca & 1953 & Borsos Olga & BPU-09913.JPG \\
\hline 2187 & Allium lusitanicum Lam. & Uzsa & 1952 & Borsos Olga & BPU-09914.JPG \\
\hline 2187 & Allium lusitanicum Lam. & Vác & 1949 & $\begin{array}{l}\text { Soó Rezső, Simon Tibor, } \\
\text { Jakucs Pál }\end{array}$ & BPU-09912.JPG \\
\hline 2188 & Allium oleraceum L. & Bagamér & 1934 & Soó Rezső & BPU-09923.JPG \\
\hline 2188 & Allium oleraceum L. & Budapest & 2004 & Felföldy Lajos & BPU-09918.JPG \\
\hline 2188 & Allium oleraceum L. & Gelénes & 1951 & Simon Tibor & BPU-09919.JPG \\
\hline 2188 & Allium oleraceum L. & Miskolc & 1930 & Soó Rezső & BPU-09923.JPG \\
\hline 2188 & Allium oleraceum L. & Pilisszentkereszt & 1942 & Priszter Szaniszló & BPU-09920.JPG \\
\hline 2188 & Allium oleraceum L. & Telki & 1947 & Soó Rezső & BPU-09921.JPG \\
\hline 2188 & Allium oleraceum L. & Uzsa & 1952 & Simon Tibor & BPU-09922.JPG \\
\hline
\end{tabular}


1. Elektroniukus melléklet - Electronic Appendix 1.

\begin{tabular}{|c|c|c|c|c|c|}
\hline $\begin{array}{l}\text { Sorszám / } \\
\text { Number }\end{array}$ & Taxon-név / Taxon-name & $\begin{array}{l}\text { Település / } \\
\text { Settlement }\end{array}$ & $\begin{array}{l}\text { Év / } \\
\text { Year }\end{array}$ & Gyűjtő / Collector & $\begin{array}{l}\text { Fájlnév / } \\
\text { File-name }\end{array}$ \\
\hline 2189 & Allium carinatum L. & Aszófó & 1957 & $\begin{array}{l}\text { Felföldy Lajos, Kovács } \\
\text { Margit, Tóth László }\end{array}$ & BPU-09925.JPG \\
\hline 2189 & Allium carinatum L. & Fertőrákos & 1946 & Kárpáti Zoltán & BPU-09924.JPG \\
\hline 2190 & Allium flavum L. & Budapest & 1982 & Felföldy Lajos & BPU-09928.JPG \\
\hline 2190 & Allium flavum L. & Csákvár & 1948 & Soó Rezső & BPU-09931.JPG \\
\hline 2190 & Allium flavum L. & Miskolc & 1937 & Soó Rezső & BPU-09926.JPG \\
\hline 2190 & Allium flavum L. & Nagyharsány & 1961 & Simon Tibor & BPU-09933.JPG \\
\hline 2190 & Allium flavum L. & Tihany & 1959 & $\begin{array}{l}\text { Felföldy Lajos, Kovács } \\
\text { Margit, Tóth László }\end{array}$ & BPU-09929.JPG \\
\hline 2190 & Allium flavum L. & Tihany & 1959 & $\begin{array}{l}\text { Felföldy Lajos, Kovács } \\
\text { Margit, Tóth László }\end{array}$ & BPU-09930.JPG \\
\hline 2190 & Allium flavum L. & Tihany & 1961 & Sólymossy Gizella & BPU-09927.JPG \\
\hline 2190 & Allium flavum L. & Uzsa & 1952 & Borsos Olga & BPU-09934.JPG \\
\hline 2191 & Allium paniculatum L. & Nagykőrös & 1913 & Degen Árpád & BPU-09936.JPG \\
\hline 2192 & Convallaria majalis L. & Budapest & 2004 & Felföldy Lajos & BPU-13008.JPG \\
\hline 2192 & Convallaria majalis L. & Csévharaszt & 1959 & Simon Tibor & BPU-13005.JPG \\
\hline 2192 & Convallaria majalis L. & Debrecen & 1931 & Soó Rezső & BPU-13007.JPG \\
\hline 2192 & Convallaria majalis L. & Hidegkút & 1959 & Felföldy Lajos & BPU-13003.JPG \\
\hline 2192 & Convallaria majalis L. & Mosonmagyaróvár & 1965 & Borsos Olga & BPU-13006.JPG \\
\hline 2192 & Convallaria majalis L. & Nagykovácsi & 2005 & Felföldy Lajos & BPU-13004.JPG \\
\hline 2193 & $\begin{array}{l}\text { Maianthemum bifolium (L.) F.W. } \\
\text { Schmidt }\end{array}$ & Bakonybél & 1935 & Soó Rezső & BPU-13044.JPG \\
\hline 2193 & $\begin{array}{l}\text { Maianthemum bifolium (L.) F.W. } \\
\text { Schmidt }\end{array}$ & Bátorliget & 1932 & Soó Rezső & BPU-13043.JPG \\
\hline 2193 & $\begin{array}{l}\text { Maianthemum bifolium (L.) F.W. } \\
\text { Schmidt }\end{array}$ & Tarpa & 1948 & $\begin{array}{l}\text { Pólya László, Simon } \\
\text { Tibor, Jakucs Pál }\end{array}$ & BPU-13045.JPG \\
\hline 2194 & Polygonatum verticillatum (L.) All. & Gyöngyös & 1953 & Soó Rezső & BPU-13030.JPG \\
\hline 2194 & Polygonatum verticillatum (L.) All. & Mátraszentimre & 1961 & Soó Rezső & BPU-13035.JPG \\
\hline 2194 & Polygonatum verticillatum (L.) All. & Miskolc & 1950 & Soó Rezső & BPU-13031.JPG \\
\hline 2195 & Polygonatum latifolium (Jacq.) Desf. & Beregdaróc & 1962 & Simon Tibor & BPU-13028.JPG \\
\hline 2195 & Polygonatum latifolium (Jacq.) Desf. & Biatorbágy & 1992 & Felföldy Lajos & BPU-13025.JPG \\
\hline 2195 & Polygonatum latifolium (Jacq.) Desf. & Debrecen & 1931 & Soó Rezső & BPU-13026.JPG \\
\hline 2195 & Polygonatum latifolium (Jacq.) Desf. & Kiskunhalas & 1964 & Soó Rezső & BPU-13029.JPG \\
\hline 2195 & Polygonatum latifolium (Jacq.) Desf. & Pécsely & 1950 & Felföldy Lajos & BPU-13023.JPG \\
\hline 2195 & Polygonatum latifolium (Jacq.) Desf. & Szigetszentmiklós & 1947 & Kárpáti Zoltán & BPU-13024.JPG \\
\hline 2195 & Polygonatum latifolium (Jacq.) Desf. & Tihany & 1956 & Felföldy Lajos & BPU-13022.JPG \\
\hline 2195 & Polygonatum latifolium (Jacq.) Desf. & Zámoly & 1982 & Felföldy Lajos & BPU-13027.JPG \\
\hline 2196 & Polygonatum odoratum (Mill.) Druce & Balatonszőlős & 1958 & Felföldy Lajos & BPU-13016.JPG \\
\hline 2196 & Polygonatum odoratum (Mill.) Druce & Budapest & 1953 & Soó Rezső & BPU-13021.JPG \\
\hline 2196 & Polygonatum odoratum (Mill.) Druce & Budapest & 1953 & Soó Rezső & BPU-15414.JPG \\
\hline 2196 & Polygonatum odoratum (Mill.) Druce & Budapest & 1982 & Felföldy Lajos & BPU-13019.JPG \\
\hline 2196 & Polygonatum odoratum (Mill.) Druce & Budapest & 2005 & Felföldy Lajos & BPU-13020.JPG \\
\hline 2196 & Polygonatum odoratum (Mill.) Druce & Kovácsvágás & 1962 & Simon Tibor & BPU-13018.JPG \\
\hline 2196 & Polygonatum odoratum (Mill.) Druce & Tihany & 1956 & Felföldy Lajos & BPU-13015.JPG \\
\hline 2196 & Polygonatum odoratum (Mill.) Druce & Visegrád & 1964 & Soó Rezső, Borsos Olga & BPU-13017.JPG \\
\hline 2197 & Polygonatum multiflorum (L.) All. & Budapest & 1964 & Soó Rezső & BPU-13013.JPG \\
\hline 2197 & Polygonatum multiflorum (L.) All. & Budapest & 2004 & Felföldy Lajos & BPU-13014.JPG \\
\hline 2197 & Polygonatum multiflorum (L.) All. & Hajdúsámson & 1932 & Soó Rezső & BPU-13010.JPG \\
\hline 2197 & Polygonatum multiflorum (L.) All. & Pécs & 1953 & Soó Rezső, Borsos Olga & BPU-13011.JPG \\
\hline 2197 & Polygonatum multiflorum (L.) All. & Pécs & 1956 & Soó Rezső & BPU-13012.JPG \\
\hline
\end{tabular}


1. Elektroniukus melléklet - Electronic Appendix 1.

\begin{tabular}{|c|c|c|c|c|c|}
\hline $\begin{array}{l}\text { Sorszám / } \\
\text { Number }\end{array}$ & Taxon-név / Taxon-name & $\begin{array}{l}\text { Település / } \\
\text { Settlement }\end{array}$ & $\begin{array}{l}\text { Év / } \\
\text { Year }\end{array}$ & Gyüjtő / Collector & $\begin{array}{l}\text { Fájlnév / } \\
\text { File-name }\end{array}$ \\
\hline 2198 & Asparagus officinalis L. & Csákvár & 1955 & Soó Rezső & BPU-13073.JPG \\
\hline 2198 & Asparagus officinalis L. & Csókakő & 1948 & Priszter Szaniszló & BPU-13072.JPG \\
\hline 2198 & Asparagus officinalis L. & Debrecen & 1934 & Soó Rezső & BPU-13071.JPG \\
\hline 2198 & Asparagus officinalis L. & Lábatlan & 1953 & Felföldy Lajos & BPU-13074.JPG \\
\hline 2198 & Asparagus officinalis L. & Lesenceistvánd & 1928 & Soó Rezső & BPU-13067.JPG \\
\hline 2198 & Asparagus officinalis L. & Mosonmagyaróvár & 1962 & Borsos Olga & BPU-13070.JPG \\
\hline 2198 & Asparagus officinalis L. & Nagyharsány & 1965 & $\begin{array}{l}\text { Simon Tibor, Priszter } \\
\text { Szaniszló }\end{array}$ & BPU-13076.JPG \\
\hline 2198 & Asparagus officinalis L. & Nagyharsány & 1966 & Priszter Szaniszló & BPU-13075.JPG \\
\hline 2198 & Asparagus officinalis L. & Ócsa & 1923 & Soó Rezső & BPU-13069.JPG \\
\hline 2198 & Asparagus officinalis L. & Tököl & 1942 & Priszter Szaniszló & BPU-13068.JPG \\
\hline 2199 & Paris quadrifolia $\mathrm{L}$. & "Cuha-völgy" & 1954 & Soó Rezső & BPU-12995.JPG \\
\hline 2199 & Paris quadrifolia L. & Bátorliget & 1932 & Soó Rezső & BPU-12997.JPG \\
\hline 2199 & Paris quadrifolia L. & Bátorliget & 1950 & $\begin{array}{l}\text { Simon Tibor, Kárpáti } \\
\text { István }\end{array}$ & BPU-12996.JPG \\
\hline 2199 & Paris quadrifolia L. & Isztimér & 1959 & Simon Tibor & BPU-13000.JPG \\
\hline 2199 & Paris quadrifolia L. & Miskolc & 1950 & Soó Rezső & BPU-13001.JPG \\
\hline 2199 & Paris quadrifolia L. & Mosonmagyaróvár & 1962 & Borsos Olga & BPU-12999.JPG \\
\hline 2199 & Paris quadrifolia L. & Ócsa & 1953 & Soó Rezső, Borsos Olga & BPU-12998.JPG \\
\hline 2199 & Paris quadrifolia $\mathrm{L}$. & Pécs & 1925 & Soó Rezső & BPU-13002.JPG \\
\hline 2199 & Paris quadrifolia L. & Uzsa & 1950 & Felföldy Lajos & BPU-12994.JPG \\
\hline 2199 & Paris quadrifolia L. & Uzsa & 1959 & $\begin{array}{l}\text { Jávorka Sándor, Felföldy } \\
\text { Lajos }\end{array}$ & BPU-12993.JPG \\
\hline 2200 & Ruscus aculeatus L. & Bisse & 1965 & Priszter Szaniszló & BPU-13055.JPG \\
\hline 2200 & Ruscus aculeatus L. & Bisse & 1965 & Priszter Szaniszló & BPU-13056.JPG \\
\hline 2200 & Ruscus aculeatus L. & Bisse & 1965 & Priszter Szaniszló & BPU-13057.JPG \\
\hline 2200 & Ruscus aculeatus L. & Bisse & 1965 & $\begin{array}{l}\text { Simon Tibor, Priszter } \\
\text { Szaniszló, Borhidi Attila }\end{array}$ & BPU-13054.JPG \\
\hline 2200 & Ruscus aculeatus L. & Kaszó & 1957 & Soó Rezső & BPU-13047.JPG \\
\hline 2200 & Ruscus aculeatus L. & Nagyharsány & 1966 & Priszter Szaniszló & BPU-13053.JPG \\
\hline 2200 & Ruscus aculeatus L. & Pécs & 1936 & Soó Rezső & BPU-13052.JPG \\
\hline 2200 & Ruscus aculeatus L. & Pécs & 1956 & Soó Rezső & BPU-13051.JPG \\
\hline 2200 & Ruscus aculeatus L. & Pécs & 1966 & Priszter Szaniszló & BPU-13050.JPG \\
\hline 2200 & Ruscus aculeatus L. & Rezi & 1953 & Borsos Olga & BPU-13046.JPG \\
\hline 2200 & Ruscus aculeatus L. & Szenna & 1957 & Soó Rezső & BPU-13048.JPG \\
\hline 2200 & Ruscus aculeatus L. & Villány & 1956 & Soó Rezső & BPU-13049.JPG \\
\hline 2201 & Ruscus hypoglossum L. & Abaliget & 1938 & Soó Rezső & BPU-13064.JPG \\
\hline 2201 & Ruscus hypoglossum L. & Bárdudvarnok & 1966 & Priszter Szaniszló & BPU-13060.JPG \\
\hline 2201 & Ruscus hypoglossum L. & Nagykanizsa & 1950 & Károlyi Árpád & BPU-13059.JPG \\
\hline 2201 & Ruscus hypoglossum L. & Pécs & 1953 & Soó Rezső, Borsos Olga & BPU-13063.JPG \\
\hline 2201 & Ruscus hypoglossum L. & Szenna & 1957 & Soó Rezső & BPU-13061.JPG \\
\hline 2201 & Ruscus hypoglossum L. & Villány & 1936 & Soó Rezső & BPU-13062.JPG \\
\hline 2202 & Potamogeton pectinatus L. & Algyő & 1947 & Timár Lajos & BPU-13269.JPG \\
\hline 2202 & Potamogeton pectinatus L. & Balatonalmádi & 1948 & Borsos Olga & BPU-13252.JPG \\
\hline 2202 & Potamogeton pectinatus L. & Balatonberény & 1958 & Tóth László & BPU-13258.JPG \\
\hline 2202 & Potamogeton pectinatus L. & Balatonfüzfő & 1956 & Felföldy Lajos & BPU-13250.JPG \\
\hline 2202 & Potamogeton pectinatus L. & Balatonkenese & 1956 & Felföldy Lajos & BPU-13251.JPG \\
\hline 2202 & Potamogeton pectinatus L. & Debrecen & 1989 & Felföldy Lajos & BPU-13263.JPG \\
\hline 2202 & Potamogeton pectinatus L. & Debrecen & 1989 & Felföldy Lajos & BPU-13264.JPG \\
\hline
\end{tabular}


1. Elektroniukus melléklet - Electronic Appendix 1.

\begin{tabular}{|c|c|c|c|c|c|}
\hline $\begin{array}{l}\text { Sorszám / } \\
\text { Number }\end{array}$ & Taxon-név / Taxon-name & $\begin{array}{l}\text { Település / } \\
\text { Settlement }\end{array}$ & $\begin{array}{l}\text { Év / } \\
\text { Year }\end{array}$ & Gyújtő / Collector & $\begin{array}{l}\text { Fájlnév / } \\
\text { File-name }\end{array}$ \\
\hline 2202 & Potamogeton pectinatus L. & Esztergom & 1947 & Priszter Szaniszló & BPU-13268.JPG \\
\hline 2202 & Potamogeton pectinatus L. & Fonyód & 1954 & Kovács Margit & BPU-13257.JPG \\
\hline 2202 & Potamogeton pectinatus L. & Gárdony & 1966 & Priszter Szaniszló & BPU-13267.JPG \\
\hline 2202 & Potamogeton pectinatus L. & Gávavencsellő & 1990 & Felföldy Lajos & BPU-13256.JPG \\
\hline 2202 & Potamogeton pectinatus L. & Hortobágy & 1947 & Soó Rezső & BPU-13261.JPG \\
\hline 2202 & Potamogeton pectinatus L. & Hortobágy & 1953 & Borsos Olga & BPU-13260.JPG \\
\hline 2202 & Potamogeton pectinatus L. & Jánoshida & 1992 & Csányi Béla & BPU-13254.JPG \\
\hline 2202 & Potamogeton pectinatus L. & Kunmadaras & 1991 & Csányi Béla & BPU-13253.JPG \\
\hline 2202 & Potamogeton pectinatus L. & Pákozd & 1939 & Boros Ádám & BPU-13265.JPG \\
\hline 2202 & Potamogeton pectinatus L. & Szeged & 1947 & Timár Lajos & BPU-13270.JPG \\
\hline 2202 & Potamogeton pectinatus L. & Szigliget & 1956 & $\begin{array}{l}\text { Felföldy Lajos, Tóth } \\
\text { László }\end{array}$ & BPU-13249.JPG \\
\hline 2202 & Potamogeton pectinatus L. & Tata & 1922 & Moesz Gusztáv & BPU-13259.JPG \\
\hline 2202 & Potamogeton pectinatus L. & Tihany & 1924 & Soó Rezső & BPU-13248.JPG \\
\hline 2202 & Potamogeton pectinatus L. & Tihany & 1928 & Soó Rezső & BPU-13247.JPG \\
\hline 2202 & Potamogeton pectinatus L. & Tihany & 1956 & Felföldy Lajos & BPU-13246.JPG \\
\hline 2202 & Potamogeton pectinatus L. & Tiszabercel & 1990 & Felföldy Lajos & BPU-13255.JPG \\
\hline 2202 & Potamogeton pectinatus L. & Velence & 1930 & Boros Ádám & BPU-13266.JPG \\
\hline 2202 & Potamogeton pectinatus L. & Zalaapáti & 1958 & Entz Béla & BPU-13244.JPG \\
\hline 2202 & Potamogeton pectinatus L. & Zánka & 1958 & Felföldy Lajos & BPU-13245.JPG \\
\hline 2204 & Potamogeton acutifolius Link & Devecser & 1957 & Tallós Pál & BPU-13242.JPG \\
\hline 2204 & Potamogeton acutifolius Link & Nyírmada & 1933 & Soó Rezső & BPU-13243.JPG \\
\hline 2204 & Potamogeton acutifolius Link & Szentbékkálla & 1959 & $\begin{array}{l}\text { Jávorka Sándor, Felföldy } \\
\text { Lajos, Tóth László }\end{array}$ & BPU-13241.JPG \\
\hline 2204 & Potamogeton acutifolius Link & Vámosszabadi & 1991 & Felföldy Lajos & BPU-13237.JPG \\
\hline 2204 & Potamogeton acutifolius Link & Vámosszabadi & 1992 & Csányi Béla & BPU-13236.JPG \\
\hline 2206 & $\begin{array}{l}\text { Potamogeton trichoides Cham. et } \\
\text { Schltdl. }\end{array}$ & Debrecen & 1989 & Felföldy Lajos & BPU-13240.JPG \\
\hline 2206 & $\begin{array}{l}\text { Potamogeton trichoides Cham. et } \\
\text { Schltdl. }\end{array}$ & Gávavencsellő & 1990 & Felföldy Lajos & BPU-13239.JPG \\
\hline 2207 & Potamogeton pusillus L. em. Fieber & Algyő & 1947 & Timár Lajos & BPU-13229.JPG \\
\hline 2207 & Potamogeton pusillus L. em. Fieber & Budapest & 1947 & Pénzes Antal & BPU-13234.JPG \\
\hline 2207 & Potamogeton pusillus L. em. Fieber & Budapest & 1989 & Felföldy Lajos & BPU-13233.JPG \\
\hline 2207 & Potamogeton pusillus L. em. Fieber & Budapest & 1992 & Felföldy Lajos & BPU-13232.JPG \\
\hline 2207 & Potamogeton pusillus L. em. Fieber & Gávavencsellő & 1990 & Felföldy Lajos & BPU-13223.JPG \\
\hline 2207 & Potamogeton pusillus L. em. Fieber & Gávavencsellő & 1990 & Felföldy Lajos & BPU-13224.JPG \\
\hline 2207 & Potamogeton pusillus L. em. Fieber & Gödöllő & 1949 & Soó Rezső & BPU-13226.JPG \\
\hline 2207 & Potamogeton pusillus L. em. Fieber & Gödöllő & 1949 & Soó Rezső & BPU-13227.JPG \\
\hline 2207 & Potamogeton pusillus L. em. Fieber & Szentendre & 1942 & Priszter Szaniszló & BPU-13225.JPG \\
\hline 2207 & Potamogeton pusillus L. em. Fieber & Tiszaújváros & 1982 & Felföldy Lajos & BPU-13230.JPG \\
\hline 2208 & Potamogeton berchtoldii Fieber & Budapest & 1949 & Priszter Szaniszló & BPU-13235.JPG \\
\hline 2208 & Potamogeton berchtoldii Fieber & Budapest & 1989 & Felföldy Lajos & BPU-13231.JPG \\
\hline 2208 & Potamogeton berchtoldii Fieber & Dunakeszi & 1947 & Priszter Szaniszló & BPU-13228.JPG \\
\hline 2209 & Potamogeton crispus L. & Alcsútdoboz & 1992 & Felföldy Lajos & BPU-13303.JPG \\
\hline 2209 & Potamogeton crispus L. & Balatonszemes & 1974 & Tóth László & BPU-13296.JPG \\
\hline 2209 & Potamogeton crispus L. & Budapest & 1942 & Priszter Szaniszló & BPU-13305.JPG \\
\hline 2209 & Potamogeton crispus L. & Budapest & 1943 & Priszter Szaniszló & BPU-13304.JPG \\
\hline 2209 & Potamogeton crispus L. & Debrecen & 1989 & Felföldy Lajos & BPU-13301.JPG \\
\hline 2209 & Potamogeton crispus L. & Debrecen & 1989 & Felföldy Lajos & BPU-13302.JPG \\
\hline
\end{tabular}


1. Elektroniukus melléklet - Electronic Appendix 1.

\begin{tabular}{|c|c|c|c|c|c|}
\hline $\begin{array}{l}\text { Sorszám / } \\
\text { Number }\end{array}$ & Taxon-név / Taxon-name & $\begin{array}{l}\text { Település / } \\
\text { Settlement }\end{array}$ & $\begin{array}{l}\text { Év / } \\
\text { Year }\end{array}$ & Gyưjtő / Collector & $\begin{array}{l}\text { Fájlnév / } \\
\text { File-name }\end{array}$ \\
\hline 2209 & Potamogeton crispus L. & Gyömrő & 1974 & Tóth László & BPU-13298.JPG \\
\hline 2209 & Potamogeton crispus L. & Hortobágy & 1947 & Soó Rezső & BPU-13300.JPG \\
\hline 2209 & Potamogeton crispus L. & Hortobágy & 1953 & Borsos Olga & BPU-13299.JPG \\
\hline 2209 & Potamogeton crispus L. & Ócsa & 1955 & Soó Rezső & BPU-13297.JPG \\
\hline 2209 & Potamogeton crispus L. & Tiszabercel & 1990 & Felföldy Lajos & BPU-13295.JPG \\
\hline 2209 & Potamogeton crispus L. & Zalaapáti & 1956 & Entz Béla & BPU-13294.JPG \\
\hline 2210 & Potamogeton perfoliatus L. & Abádszalók & 1989 & $\begin{array}{l}\text { Felföldy Lajos, Hamar } \\
\text { József }\end{array}$ & BPU-13309.JPG \\
\hline 2210 & Potamogeton perfoliatus L. & Balatonalmádi & 1928 & Soó Rezső & BPU-13308.JPG \\
\hline 2210 & Potamogeton perfoliatus L. & Balatonszabadi & 1940 & Priszter Szaniszló & BPU-13311.JPG \\
\hline 2210 & Potamogeton perfoliatus L. & Budapest & 1947 & Priszter Szaniszló & BPU-13315.JPG \\
\hline 2210 & Potamogeton perfoliatus L. & Bugyi & 1959 & Soó Rezső & BPU-13312.JPG \\
\hline 2210 & Potamogeton perfoliatus L. & Esztergom & 1947 & Priszter Szaniszló & BPU-13313.JPG \\
\hline 2210 & Potamogeton perfoliatus L. & Kömörő & 1948 & $\begin{array}{l}\text { Pólya László, Simon } \\
\text { Tibor, Jakucs Pál }\end{array}$ & BPU-13310.JPG \\
\hline 2210 & Potamogeton perfoliatus L. & Paloznak & 1956 & Felföldy Lajos & BPU-13307.JPG \\
\hline 2210 & Potamogeton perfoliatus L. & Szeged & 1943 & Timár Lajos & BPU-13314.JPG \\
\hline 2210 & Potamogeton perfoliatus L. & Tihany & 1928 & Soó Rezső & BPU-13306.JPG \\
\hline 2211 & Potamogeton gramineus L. & Abádszalók & 1989 & $\begin{array}{l}\text { Felföldy Lajos, Hamar } \\
\text { József }\end{array}$ & BPU-13284.JPG \\
\hline 2211 & Potamogeton gramineus L. & Csévharaszt & 1948 & Priszter Szaniszló & BPU-13287.JPG \\
\hline 2211 & Potamogeton gramineus L. & Kisoroszi & 1985 & Ráth Borbála & BPU-13288.JPG \\
\hline 2211 & Potamogeton gramineus L. & Nyirád & 1963 & Tallós Pál & BPU-13281.JPG \\
\hline 2211 & Potamogeton gramineus L. & Nyirád & 1963 & Tallós Pál & BPU-13282.JPG \\
\hline 2211 & Potamogeton gramineus L. & Nyírbéltek & 1932 & Soó Rezső & BPU-13286.JPG \\
\hline 2211 & Potamogeton gramineus L. & Szeged & 1947 & Timár Lajos & BPU-13289.JPG \\
\hline 2211 & Potamogeton gramineus L. & Szeged & 1947 & Timár Lajos & BPU-13290.JPG \\
\hline 2211 & Potamogeton gramineus L. & Szolnok & 1937 & Soó Rezső & BPU-13283.JPG \\
\hline 2211 & Potamogeton gramineus L. & Tiszabercel & 1990 & Felföldy Lajos & BPU-13285.JPG \\
\hline 2211 & Potamogeton gramineus L. & Ukk & 1957 & Felföldy Lajos & BPU-13279.JPG \\
\hline 2211 & Potamogeton gramineus L. & Ukk & 1957 & Felföldy Lajos & BPU-13280.JPG \\
\hline 2212 & Potamogeton lucens L. & Gávavencsellő & 1990 & Felföldy Lajos & BPU-13274.JPG \\
\hline 2212 & Potamogeton lucens L. & Kunmadaras & 1991 & Csányi Béla & BPU-13272.JPG \\
\hline 2212 & Potamogeton lucens L. & Miskolc & 1929 & Soó Rezső & BPU-13278.JPG \\
\hline 2212 & Potamogeton lucens L. & Nagykanizsa & 1956 & Felföldy Lajos & BPU-13271.JPG \\
\hline 2212 & Potamogeton lucens L. & Nagykáta & 1990 & Felföldy Lajos & BPU-13276.JPG \\
\hline 2212 & Potamogeton lucens L. & Nyírmada & 1933 & Soó Rezső & BPU-13273.JPG \\
\hline 2212 & Potamogeton lucens L. & Tiszafüred & 1952 & Soó Rezső & BPU-15543.JPG \\
\hline 2212 & Potamogeton lucens L. & Vámosszabadi & 1991 & Felföldy Lajos & BPU-13277.JPG \\
\hline 2212 & Potamogeton lucens L. & Veresegyház & 1949 & Soó Rezső & BPU-15531.JPG \\
\hline 2212 & Potamogeton lucens L. & Veresegyház & 1950 & Soó Rezső & BPU-13275.JPG \\
\hline 2214 & Potamogeton natans L. & Csévharaszt & 1942 & Priszter Szaniszló & BPU-13335.JPG \\
\hline 2214 & Potamogeton natans L. & Debrecen & 1935 & Soó Rezső & BPU-13339.JPG \\
\hline 2214 & Potamogeton natans L. & Debrecen & 1989 & Felföldy Lajos & BPU-13336.JPG \\
\hline 2214 & Potamogeton natans L. & Debrecen & 1989 & Felföldy Lajos & BPU-13337.JPG \\
\hline 2214 & Potamogeton natans L. & Debrecen & 1989 & Felföldy Lajos & BPU-13338.JPG \\
\hline 2214 & Potamogeton natans L. & Keszthely & 1958 & Tóth László & BPU-13327.JPG \\
\hline 2214 & Potamogeton natans L. & Kőszeg & 1932 & Soó Rezső & BPU-13333.JPG \\
\hline 2214 & Potamogeton natans L. & Pusztamiske & 1948 & Borsos Olga & BPU-13320.JPG \\
\hline
\end{tabular}


1. Elektroniukus melléklet - Electronic Appendix 1.

\begin{tabular}{|c|c|c|c|c|c|}
\hline $\begin{array}{l}\text { Sorszám / } \\
\text { Number }\end{array}$ & Taxon-név / Taxon-name & $\begin{array}{l}\text { Település / } \\
\text { Settlement }\end{array}$ & $\begin{array}{l}\text { Év / } \\
\text { Year }\end{array}$ & Gyűjtő / Collector & $\begin{array}{l}\text { Fájlnév / } \\
\text { File-name }\end{array}$ \\
\hline 2214 & Potamogeton natans L. & Szentbékkálla & 1959 & $\begin{array}{l}\text { Jávorka Sándor, Felföldy } \\
\text { Lajos, Tóth László }\end{array}$ & BPU-13331.JPG \\
\hline 2214 & Potamogeton natans L. & Szentbékkálla & 1959 & $\begin{array}{l}\text { Jávorka Sándor, Felföldy } \\
\text { Lajos, Tóth László }\end{array}$ & BPU-13332.JPG \\
\hline 2214 & Potamogeton natans L. & Tiszafüred & 1987 & Felföldy Lajos & BPU-13334.JPG \\
\hline 2214 & Potamogeton natans L. & Vonyarcvashegy & 1958 & Tóth László & BPU-13328.JPG \\
\hline 2215 & Potamogeton coloratus Hornem. & Budapest & 1942 & Priszter Szaniszló & BPU-13341.JPG \\
\hline 2215 & Potamogeton coloratus Hornem. & Budapest & - & Staub Móric & BPU-13342.JPG \\
\hline 2215 & Potamogeton coloratus Hornem. & Lébény & 1931 & Zólyomi Bálint & BPU-13340.JPG \\
\hline 2216 & Potamogeton nodosus Poir. & Hortobágy & 1947 & Soó Rezső & BPU-13325.JPG \\
\hline 2216 & Potamogeton nodosus Poir. & Hortobágy & 1953 & Borsos Olga & BPU-13322.JPG \\
\hline 2216 & Potamogeton nodosus Poir. & Hortobágy & 1953 & Borsos Olga & BPU-13323.JPG \\
\hline 2216 & Potamogeton nodosus Poir. & Kaszó & 1957 & Soó Rezső & BPU-13324.JPG \\
\hline 2216 & Potamogeton nodosus Poir. & Kömörő & 1948 & $\begin{array}{l}\text { Pólya László, Simon } \\
\text { Tibor, Jakucs Pál }\end{array}$ & BPU-13321.JPG \\
\hline 2216 & Potamogeton nodosus Poir. & Kömörő & 1948 & $\begin{array}{l}\text { Pólya László, Simon } \\
\text { Tibor, Jakucs Pál }\end{array}$ & BPU-13329.JPG \\
\hline 2216 & Potamogeton nodosus Poir. & Szigliget & 1956 & $\begin{array}{l}\text { Felföldy Lajos, Tóth } \\
\text { László }\end{array}$ & BPU-13318.JPG \\
\hline 2216 & Potamogeton nodosus Poir. & Tiszafüred & 1990 & Felföldy Lajos & BPU-13326.JPG \\
\hline 2216 & Potamogeton nodosus Poir. & Zalaapáti & 1958 & Entz Béla & BPU-13317.JPG \\
\hline 2217 & Groenlandia densa (L.) Fourr. & Tatabánya & 1934 & Boros Ádám & BPU-13316.JPG \\
\hline 2218 & Zannichellia palustris L. & Debrecen & 1949 & Soó Rezső & BPU-13189.JPG \\
\hline 2218 & Zannichellia palustris L. & Dunakeszi & 1947 & Priszter Szaniszló & BPU-13187.JPG \\
\hline 2218 & Zannichellia palustris L. & Pázmánd & 1976 & Felföldy Lajos & BPU-13190.JPG \\
\hline 2218 & Zannichellia palustris L. & Szeged & 1947 & Timár Lajos & BPU-13191.JPG \\
\hline 2218 & Zannichellia palustris L. & Tapolca & 1931 & Soó Rezső & BPU-13188.JPG \\
\hline 2219 & Najas marina L. & Balatonalmádi & 1952 & Soó Rezső & BPU-13200.JPG \\
\hline 2219 & Najas marina L. & Balatonfüzfő & 1956 & $\begin{array}{l}\text { Felföldy Lajos, Tóth } \\
\text { László }\end{array}$ & BPU-13199.JPG \\
\hline 2219 & Najas marina L. & Balatonszemes & 1974 & Tóth László & BPU-13201.JPG \\
\hline 2219 & Najas marina L. & Budapest & 1946 & Priszter Szaniszló & BPU-13202.JPG \\
\hline 2219 & Najas marina L. & Budapest & 1947 & Priszter Szaniszló & BPU-13203.JPG \\
\hline 2219 & Najas marina L. & Tihany & 1961 & Sólymossy Gizella & BPU-13198.JPG \\
\hline 2219 & Najas marina L. & Vonyarcvashegy & 1958 & Tóth László & BPU-13197.JPG \\
\hline 2220 & Najas minor All. & Budapest & 1946 & Priszter Szaniszló & BPU-13196.JPG \\
\hline 2220 & Najas minor All. & Debrecen & 1987 & Felföldy Lajos & BPU-13195.JPG \\
\hline 2220 & Najas minor All. & Kállósemjén & 1948 & Soó Rezső & BPU-13194.JPG \\
\hline 2220 & Najas minor All. & Nagykanizsa & 1956 & Felföldy Lajos & BPU-13192.JPG \\
\hline 2220 & Najas minor All. & Paks & 1974 & Felföldy Lajos & BPU-13193.JPG \\
\hline 2222 & Gagea pratensis (Pers.) Dumort. & Budapest & 1990 & Felföldy Lajos & BPU-09816.JPG \\
\hline 2222 & Gagea pratensis (Pers.) Dumort. & Gyöngyös & 1933 & Soó Rezső & BPU-09817.JPG \\
\hline 2222 & Gagea pratensis (Pers.) Dumort. & Gyöngyös & 1964 & Soó Rezső & BPU-09818.JPG \\
\hline 2222 & Gagea pratensis (Pers.) Dumort. & Nyíregyháza & 1934 & Soó Rezső & BPU-09819.JPG \\
\hline 2222 & Gagea pratensis (Pers.) Dumort. & Tihany & 1958 & Felföldy Lajos & BPU-09820.JPG \\
\hline 2222 & Gagea pratensis (Pers.) Dumort. & Tihany & 1958 & Felföldy Lajos & BPU-09821.JPG \\
\hline 2223 & Gagea lutea (L.) Ker Gawl. & Gánt & 1949 & Soó Rezső & BPU-09829.JPG \\
\hline 2223 & Gagea lutea (L.) Ker Gawl. & Szamosszeg & 1948 & $\begin{array}{l}\text { Simon Tibor, Pólya } \\
\text { László, Jakucs Pál }\end{array}$ & BPU-09830.JPG \\
\hline 2223 & Gagea lutea (L.) Ker Gawl. & Tihany & 1958 & Felföldy Lajos & BPU-09831.JPG \\
\hline
\end{tabular}


1. Elektroniukus melléklet - Electronic Appendix 1.

\begin{tabular}{|c|c|c|c|c|c|}
\hline $\begin{array}{l}\text { Sorszám / } \\
\text { Number }\end{array}$ & Taxon-név / Taxon-name & $\begin{array}{l}\text { Település / } \\
\text { Settlement }\end{array}$ & $\begin{array}{l}\text { Év / } \\
\text { Year }\end{array}$ & Gyüjtő / Collector & $\begin{array}{l}\text { Fájlnév / } \\
\text { File-name }\end{array}$ \\
\hline 2224 & Gagea minima (L.) Ker Gawl. & Gánt & 1949 & Soó Rezső & BPU-09839.JPG \\
\hline 2224 & Gagea minima (L.) Ker Gawl. & Tarcal & 1951 & Soó Rezső & BPU-09840.JPG \\
\hline 2225 & $\begin{array}{l}\text { Gagea pusilla (F.W. Schmidt) Schult. et } \\
\text { Schult. f. }\end{array}$ & Bagamér & 1933 & Soó Rezső & BPU-09834.JPG \\
\hline 2225 & $\begin{array}{l}\text { Gagea pusilla (F.W. Schmidt) Schult. et } \\
\text { Schult. f. }\end{array}$ & Nagykovácsi & 1949 & Soó Rezső & BPU-09835.JPG \\
\hline 2225 & $\begin{array}{l}\text { Gagea pusilla (F.W. Schmidt) Schult. et } \\
\text { Schult. f. }\end{array}$ & Tihany & 1958 & Felföldy Lajos & BPU-09836.JPG \\
\hline 2225 & $\begin{array}{l}\text { Gagea pusilla (F.W. Schmidt) Schult. et } \\
\text { Schult. f. }\end{array}$ & Tihany & 1958 & Felföldy Lajos & BPU-09837.JPG \\
\hline 2225 & $\begin{array}{l}\text { Gagea pusilla (F.W. Schmidt) Schult. et } \\
\text { Schult. f. }\end{array}$ & Tihany & 1958 & Felföldy Lajos & BPU-09838.JPG \\
\hline 2226 & Gagea spathacea (Hayne) Salisb. & Tarpa & 1948 & $\begin{array}{l}\text { Simon Tibor, Pólya } \\
\text { László, Jakucs Pál }\end{array}$ & BPU-09832.JPG \\
\hline 2226 & Gagea spathacea (Hayne) Salisb. & Tarpa & 1952 & Kulcsár Gábor & BPU-09833.JPG \\
\hline 2227 & Gagea villosa (M. Bieb.) Duby & Hencida & 1926 & Kovács L. & BPU-09823.JPG \\
\hline 2227 & Gagea villosa (M. Bieb.) Duby & Hidegkút & 1959 & Felföldy Lajos & BPU-09822.JPG \\
\hline 2227 & Gagea villosa (M. Bieb.) Duby & Tihany & 1958 & Felföldy Lajos & BPU-09824.JPG \\
\hline 2227 & Gagea villosa (M. Bieb.) Duby & Tihany & 1958 & Felföldy Lajos & BPU-09825.JPG \\
\hline 2227 & Gagea villosa (M. Bieb.) Duby & Tihany & 1958 & Felföldy Lajos & BPU-09826.JPG \\
\hline 2228 & $\begin{array}{l}\text { Gagea bohemica (Zauschn.) Schult. et } \\
\text { Schult. f. }\end{array}$ & Budapest & 2005 & Felföldy Lajos & BPU-09827.JPG \\
\hline 2228 & $\begin{array}{l}\text { Gagea bohemica (Zauschn.) Schult. et } \\
\text { Schult. f. }\end{array}$ & Pomáz & 1946 & Vajda László & BPU-09828.JPG \\
\hline 2230 & Erythronium dens-canis L. & Jósvafő & 1951 & Jakucs Pál, Kulcsár Gábor & BPU-15480.JPG \\
\hline 2230 & Erythronium dens-canis L. & Lispeszentadorján & 1948 & Károlyi Árpád & BPU-13153.JPG \\
\hline 2230 & Erythronium dens-canis L. & Szentgotthárd & 1927 & Gáyer Gyula & BPU-13155.JPG \\
\hline 2230 & Erythronium dens-canis L. & Szentgotthárd & 1947 & anonim & BPU-13154.JPG \\
\hline 2233 & Fritillaria meleagris L. & Ikervár & 1923 & Gáyer Gyula, Mályusz E. & BPU-13161.JPG \\
\hline 2233 & Fritillaria meleagris L. & Lenti & 1948 & Károlyi Árpád & BPU-13158.JPG \\
\hline 2233 & Fritillaria meleagris L. & Lenti & 1948 & Károlyi Árpád & BPU-13159.JPG \\
\hline 2233 & Fritillaria meleagris L. & Szászfa & 1953 & Jakucs Pál & BPU-13160.JPG \\
\hline 2235 & Lilium martagon L. & Balatonszőlős & 1958 & Felföldy Lajos & BPU-09958.JPG \\
\hline 2235 & Lilium martagon L. & Bátorliget & 1948 & $\begin{array}{l}\text { Simon Tibor, Jakucs Pál, } \\
\text { Böszörményi Zoltán }\end{array}$ & BPU-09955.JPG \\
\hline 2235 & Lilium martagon L. & Bátorliget & 1950 & Simon Tibor & BPU-09956.JPG \\
\hline 2235 & Lilium martagon L. & Bátorliget & 1950 & $\begin{array}{l}\text { Simon Tibor, Kárpáti } \\
\text { István }\end{array}$ & BPU-09957.JPG \\
\hline 2235 & Lilium martagon L. & Bélapátfalva & 1948 & $\begin{array}{l}\text { Simon Tibor, Pólya } \\
\text { László, Jakucs Pál }\end{array}$ & BPU-09948.JPG \\
\hline 2235 & Lilium martagon L. & Budapest & 1913 & $\begin{array}{l}\text { Filarszky Nándor, Timkó } \\
\text { György }\end{array}$ & BPU-09945.JPG \\
\hline 2235 & Lilium martagon L. & Budapest & 2004 & Felföldy Lajos & BPU-09946.JPG \\
\hline 2235 & Lilium martagon L. & Gyöngyös & 1961 & Soó Rezső & BPU-09952.JPG \\
\hline 2235 & Lilium martagon L. & Gyöngyös & 1962 & Soó Rezső & BPU-09953.JPG \\
\hline 2235 & Lilium martagon L. & Isztimér & 1949 & Soó Rezső & BPU-09951.JPG \\
\hline 2235 & Lilium martagon L. & Kisharsány & 1967 & Priszter Szaniszló & BPU-09947.JPG \\
\hline 2235 & Lilium martagon L. & Miskolc & 1953 & Soó Rezső & BPU-09949.JPG \\
\hline 2235 & Lilium martagon L. & Pilisszentiván & 1947 & Priszter Szaniszló & BPU-09954.JPG \\
\hline 2235 & Lilium martagon L. & Tibolddaróc & 1953 & Soó Rezső & BPU-09950.JPG \\
\hline 2236 & Lilium bulbiferum L. & Miskolc & 1950 & Soó Rezső & BPU-13164.JPG \\
\hline 2236 & Lilium bulbiferum L. & Miskolc & 1950 & Soó Rezső, Simon Tibor & BPU-13165.JPG \\
\hline
\end{tabular}


1. Elektroniukus melléklet - Electronic Appendix 1.

\begin{tabular}{|c|c|c|c|c|c|}
\hline $\begin{array}{l}\text { Sorszám / } \\
\text { Number }\end{array}$ & Taxon-név / Taxon-name & $\begin{array}{l}\text { Település / } \\
\text { Settlement }\end{array}$ & $\begin{array}{l}\text { Év / } \\
\text { Year }\end{array}$ & Gyújtő / Collector & $\begin{array}{l}\text { Fájlnév / } \\
\text { File-name }\end{array}$ \\
\hline 2238 & $\begin{array}{l}\text { Sternbergia colchiciflora Waldst. et } \\
\text { Kit. }\end{array}$ & Budapest & 1921 & Soó Rezső & BPU-12980.JPG \\
\hline 2239 & Leucojum vernum L. & Bakonybél & 1954 & Tallós Pál & BPU-12988.JPG \\
\hline 2239 & Leucojum vernum $\mathrm{L}$. & Beregdaróc & 1952 & Kulcsár Gábor & BPU-12987.JPG \\
\hline 2239 & Leucojum vernum L. & Garbolc & 1953 & $\begin{array}{l}\text { Simon Tibor, Kulcsár } \\
\text { Gábor }\end{array}$ & BPU-12989.JPG \\
\hline 2240 & Leucojum aestivum L. & Barabás & 1956 & Simon Tibor & BPU-12986.JPG \\
\hline 2240 & Leucojum aestivum L. & Bátorliget & 1928 & Soó Rezső & BPU-12981.JPG \\
\hline 2240 & Leucojum aestivum L. & Csaroda & 1951 & $\begin{array}{l}\text { Simon Tibor, Jakucs Pál, } \\
\text { Kulcsár Gábor }\end{array}$ & BPU-12985.JPG \\
\hline 2240 & Leucojum aestivum L. & Szigetújfalu & 1948 & Kárpáti Zoltán & BPU-12983.JPG \\
\hline 2240 & Leucojum aestivum L. & Szigetújfalu & 1948 & Priszter Szaniszló & BPU-12982.JPG \\
\hline 2240 & Leucojum aestivum L. & Tiszacsege & 1932 & Soó Rezső & BPU-12984.JPG \\
\hline 2241 & Galanthus nivalis L. & Balatonszőlős & 1959 & Felföldy Lajos & BPU-12990.JPG \\
\hline 2241 & Galanthus nivalis L. & Beregdaróc & 1953 & $\begin{array}{l}\text { Simon Tibor, Kulcsár } \\
\text { Gábor }\end{array}$ & BPU-12991.JPG \\
\hline 2244 & Narcissus poëticus L. & Babócsa & 1923 & Boros Ádám & BPU-12979.JPG \\
\hline 2246 & Tamus communis L. & Badacsonytomaj & 1928 & Soó Rezső & BPU-12969.JPG \\
\hline 2246 & Tamus communis L. & Bakonyjákó & 1954 & Tallós Pál & BPU-12967.JPG \\
\hline 2246 & Tamus communis L. & Balatonederics & 1960 & Simon Tibor & BPU-12968.JPG \\
\hline 2246 & Tamus communis L. & Bisse & 1965 & $\begin{array}{l}\text { Simon Tibor, Priszter } \\
\text { Szaniszló, Borhidi Attila }\end{array}$ & BPU-12977.JPG \\
\hline 2246 & Tamus communis L. & Eszteregnye & 1948 & Károlyi Árpád & BPU-12964.JPG \\
\hline 2246 & Tamus communis L. & Kisharsány & 1961 & Simon Tibor & BPU-12976.JPG \\
\hline 2246 & Tamus communis L. & Nagybajom & 1957 & Soó Rezső & BPU-12970.JPG \\
\hline 2246 & Tamus communis L. & Nagyharsány & 1948 & Priszter Szaniszló & BPU-12975.JPG \\
\hline 2246 & Tamus communis L. & Pécs & 1936 & Soó Rezső & BPU-12974.JPG \\
\hline 2246 & Tamus communis L. & Pécs & 1956 & Soó Rezső & BPU-12973.JPG \\
\hline 2246 & Tamus communis L. & Rezi & 1953 & Borsos Olga & BPU-12963.JPG \\
\hline 2246 & Tamus communis L. & Szenna & 1957 & Soó Rezső & BPU-12971.JPG \\
\hline 2246 & Tamus communis L. & Uzsa & 1959 & $\begin{array}{l}\text { Jávorka Sándor, Felföldy } \\
\text { Lajos }\end{array}$ & BPU-12966.JPG \\
\hline 2246 & Tamus communis L. & Villány & 1941 & Priszter Szaniszló & BPU-12972.JPG \\
\hline 2246 & Tamus communis L. & Zalakomár & 1954 & Borsos Olga & BPU-12965.JPG \\
\hline 2251 & Iris pseudacorus L. & Budapest & 1942 & Priszter Szaniszló & BPU-12923.JPG \\
\hline 2252 & Iris graminea $\mathrm{L}$. & Balatonszőlős & 1951 & Felföldy Lajos & BPU-12916.JPG \\
\hline 2252 & Iris graminea L. & Csengersima & 1953 & Simon Tibor & BPU-12917.JPG \\
\hline 2252 & Iris graminea $\mathrm{L}$. & Miskolc & 1939 & Soó Rezső & BPU-12920.JPG \\
\hline 2252 & Iris graminea $\mathrm{L}$. & Pécs & 1953 & Soó Rezső, Borsos Olga & BPU-12921.JPG \\
\hline 2252 & Iris graminea $\mathrm{L}$. & Pilisszentkereszt & 1932 & Soó Rezső & BPU-12919.JPG \\
\hline 2252 & Iris graminea L. & Sopron & 1947 & Priszter Szaniszló & BPU-12918.JPG \\
\hline 2252 & Iris graminea $\mathrm{L}$. & Szentbékkálla & 1959 & $\begin{array}{l}\text { Jávorka Sándor, Felföldy } \\
\text { Lajos, Tóth László }\end{array}$ & BPU-12915.JPG \\
\hline 2253 & Iris spuria L. & Dabas & 1951 & Simon Tibor, Borsos Olga & BPU-12902.JPG \\
\hline 2253 & Iris spuria L. & Dabas & 1958 & Soó Rezső & BPU-12903.JPG \\
\hline 2254 & Iris sibirica L. & Balatonfüred & 1954 & Entz Béla & BPU-12905.JPG \\
\hline 2254 & Iris sibirica L. & Barabás & 1956 & Simon Tibor & BPU-12913.JPG \\
\hline 2254 & Iris sibirica L. & Bátorliget & 1950 & Simon Tibor & BPU-12908.JPG \\
\hline 2254 & Iris sibirica L. & Bugyi & 1959 & Soó Rezső & BPU-12911.JPG \\
\hline 2254 & Iris sibirica L. & Dabas & 1952 & Borsos Olga, Simon Tibor & BPU-12910.JPG \\
\hline
\end{tabular}


1. Elektroniukus melléklet - Electronic Appendix 1.

\begin{tabular}{|c|c|c|c|c|c|}
\hline $\begin{array}{l}\text { Sorszám / } \\
\text { Number }\end{array}$ & Taxon-név / Taxon-name & $\begin{array}{l}\text { Település / } \\
\text { Settlement }\end{array}$ & $\begin{array}{l}\text { Év / } \\
\text { Year }\end{array}$ & Gyújtő / Collector & $\begin{array}{l}\text { Fájlnév / } \\
\text { File-name }\end{array}$ \\
\hline 2254 & Iris sibirica L. & Felsőtárkány & 1954 & Soó Rezső & BPU-12912.JPG \\
\hline 2254 & Iris sibirica L. & Nyírpilis & 1932 & Soó Rezső & BPU-12907.JPG \\
\hline 2254 & Iris sibirica L. & Ócsa & 1953 & Soó Rezső, Borsos Olga & BPU-12909.JPG \\
\hline 2254 & Iris sibirica L. & Telkibánya & 1955 & Simon Tibor & BPU-12914.JPG \\
\hline 2255 & Iris arenaria Waldst. et Kit. & Dabas & 1959 & Simon Tibor & BPU-12939.JPG \\
\hline 2255 & Iris arenaria Waldst. et Kit. & Dunaharaszti & 1948 & Priszter Szaniszló & BPU-12938.JPG \\
\hline 2255 & Iris arenaria Waldst. et Kit. & Győr & 1959 & $\begin{array}{l}\text { Simon Tibor, Borhidi } \\
\text { Attila }\end{array}$ & BPU-12940.JPG \\
\hline 2255 & Iris arenaria Waldst. et Kit. & Szigetszentmárton & 1948 & Priszter Szaniszló & BPU-12937.JPG \\
\hline 2255 & Iris arenaria Waldst. et Kit. & Szigetújfalu & 1948 & Papp József & BPU-12936.JPG \\
\hline 2256 & Iris pumila $\mathrm{L}$. & Budapest & 1913 & $\begin{array}{l}\text { Kümmerle Jenő Béla, } \\
\text { Timkó György }\end{array}$ & BPU-12950.JPG \\
\hline 2256 & Iris pumila L. & Budapest & 1938 & Priszter Szaniszló & BPU-12948.JPG \\
\hline 2256 & Iris pumila L. & Budapest & 1948 & Priszter Szaniszló & BPU-12949.JPG \\
\hline 2256 & Iris pumila L. & Budapest & - & Priszter Szaniszló & BPU-12947.JPG \\
\hline 2256 & Iris pumila L. & Egyek & 1948 & Soó Rezső & BPU-12944.JPG \\
\hline 2256 & Iris pumila $\mathrm{L}$. & Gánt & 1949 & Soó Rezső & BPU-12945.JPG \\
\hline 2256 & Iris pumila L. & Gánt & 1949 & Soó Rezső & BPU-12946.JPG \\
\hline 2256 & Iris pumila L. & Sopron & 1951 & Tallós Pál & BPU-12943.JPG \\
\hline 2256 & Iris pumila $\mathrm{L}$. & Tarcal & 1951 & Soó Rezső & BPU-12942.JPG \\
\hline 2259 & Iris aphylla L. & Penészlek & 1932 & Soó Rezső & BPU-12934.JPG \\
\hline 2259 & Iris aphylla L. & Téglás & 1937 & Soó Rezső & BPU-12935.JPG \\
\hline 2260 & Iris variegata $\mathrm{L}$. & Budapest & 1953 & Borsos Olga & BPU-12931.JPG \\
\hline 2260 & Iris variegata $L$. & Csévharaszt & 1959 & Simon Tibor, Borsos Olga & BPU-12930.JPG \\
\hline 2260 & Iris variegata $\mathrm{L}$. & Hidegkút & 1958 & Felföldy Lajos & BPU-12927.JPG \\
\hline 2260 & Iris variegata L. & Kapolcs & 1959 & $\begin{array}{l}\text { Jávorka Sándor, Felföldy } \\
\text { Lajos, Tóth László }\end{array}$ & BPU-12926.JPG \\
\hline 2260 & Iris variegata $L$. & Nagykovácsi & 1947 & Priszter Szaniszló & BPU-12929.JPG \\
\hline 2260 & Iris variegata $\mathrm{L}$. & Pilisszentkereszt & 1942 & Priszter Szaniszló & BPU-12928.JPG \\
\hline 2260 & Iris variegata $\mathrm{L}$. & Szentbékkálla & 1959 & $\begin{array}{l}\text { Jávorka Sándor, Felföldy } \\
\text { Lajos, Tóth László }\end{array}$ & BPU-12925.JPG \\
\hline 2260 & Iris variegata $\mathrm{L}$. & Uzsa & 1959 & $\begin{array}{l}\text { Jávorka Sándor, Felföldy } \\
\text { Lajos }\end{array}$ & BPU-12924.JPG \\
\hline 2261 & Crocus reticulatus Steven & Szeged & 1928 & Györffy István & BPU-12961.JPG \\
\hline 2261 & Crocus reticulatus Steven & Szeged & 1929 & Györffy István & BPU-12962.JPG \\
\hline 2261 & Crocus reticulatus Steven & Sződ & 1943 & Kárpáti Zoltán & BPU-12960.JPG \\
\hline 2264 & Crocus heuffelianus Herb. & Pápasalamon & 1955 & Tallós Pál & BPU-12959.JPG \\
\hline 2266 & Gladiolus imbricatus L. & Bátorliget & 1951 & Simon Tibor & BPU-12893.JPG \\
\hline 2266 & Gladiolus imbricatus L. & Miskolc & 1940 & Hulják János & BPU-12894.JPG \\
\hline 2266 & Gladiolus imbricatus L. & Miskolc & 1940 & Hulják János & BPU-12895.JPG \\
\hline 2266 & Gladiolus imbricatus L. & Nyíregyháza & 1939 & Ubrizsy Gábor & BPU-12892.JPG \\
\hline 2266 & Gladiolus imbricatus L. & Regéc & 1957 & Hulják János & BPU-12896.JPG \\
\hline 2267 & Gladiolus palustris Gaudin & Bugyi & 1928 & Zsák Zoltán & BPU-12897.JPG \\
\hline 2268 & Juncus maritimus Lam. & Balatonszabadi & 1936 & Priszter Szaniszló & BPU-12872.JPG \\
\hline 2268 & Juncus maritimus Lam. & Sarród & 1923 & Polgár Sándor & BPU-12876.JPG \\
\hline 2268 & Juncus maritimus Lam. & Zamárdi & 1926 & Szartorisz Béla & BPU-12873.JPG \\
\hline 2268 & Juncus maritimus Lam. & Zamárdi & 1928 & Soó Rezső & BPU-12871.JPG \\
\hline 2269 & Juncus inflexus L. & Balatonfüred & 1931 & Soó Rezső & BPU-12809.JPG \\
\hline 2269 & Juncus inflexus L. & Balatonszepezd & 1958 & Felföldy Lajos & BPU-12810.JPG \\
\hline
\end{tabular}


1. Elektroniukus melléklet - Electronic Appendix 1.

\begin{tabular}{|c|c|c|c|c|c|}
\hline $\begin{array}{l}\text { Sorszám / } \\
\text { Number }\end{array}$ & Taxon-név / Taxon-name & $\begin{array}{l}\text { Település / } \\
\text { Settlement }\end{array}$ & $\begin{array}{l}\text { Év / } \\
\text { Year }\end{array}$ & Gyüjtő / Collector & $\begin{array}{l}\text { Fájlnév / } \\
\text { File-name }\end{array}$ \\
\hline 2269 & Juncus inflexus L. & Balatonszőlős & 1956 & $\begin{array}{l}\text { Felföldy Lajos, Tóth } \\
\text { László }\end{array}$ & BPU-12811.JPG \\
\hline 2269 & Juncus inflexus L. & Balatonszőlős & 1956 & $\begin{array}{l}\text { Felföldy Lajos, Tóth } \\
\text { László }\end{array}$ & BPU-12812.JPG \\
\hline 2269 & Juncus inflexus L. & Budapest & 1909 & Degen Árpád & BPU-12818.JPG \\
\hline 2269 & Juncus inflexus L. & Debrecen & 1931 & Soó Rezső & BPU-12814.JPG \\
\hline 2269 & Juncus inflexus L. & Győr & 1916 & Polgár Sándor & BPU-12816.JPG \\
\hline 2269 & Juncus inflexus L. & Kimle & 1962 & Borsos Olga & BPU-12813.JPG \\
\hline 2269 & Juncus inflexus L. & Nagyrada & 1987 & Felföldy Lajos & BPU-12808.JPG \\
\hline 2270 & Juncus effusus L. & Badacsonytomaj & 1984 & Felföldy Lajos & BPU-12801.JPG \\
\hline 2270 & Juncus effusus L. & Budapest & 1909 & Degen Árpád & BPU-12805.JPG \\
\hline 2270 & Juncus effusus L. & Debrecen & 1916 & Rapaics Rajmund & BPU-12806.JPG \\
\hline 2270 & Juncus effusus L. & Debrecen & 1934 & Soó Rezső & BPU-12803.JPG \\
\hline 2270 & Juncus effusus L. & Debrecen & 1947 & Felföldy Lajos & BPU-12804.JPG \\
\hline 2270 & Juncus effusus L. & Szentbékkálla & 1957 & Felföldy Lajos & BPU-12800.JPG \\
\hline 2270 & Juncus effusus L. & Szentendre & 1942 & Priszter Szaniszló & BPU-12802.JPG \\
\hline 2271 & Juncus conglomeratus L. & Bátorliget & 1932 & Soó Rezső & BPU-12793.JPG \\
\hline 2271 & Juncus conglomeratus L. & Miskolc & 1934 & Soó Rezső & BPU-12794.JPG \\
\hline 2271 & Juncus conglomeratus L. & Nagyvisnyó & 1928 & Igmándy József & BPU-12815.JPG \\
\hline 2273 & Juncus sphaerocarpus Nees & Balatonszőlős & 1956 & $\begin{array}{l}\text { Felföldy Lajos, Tóth } \\
\text { László }\end{array}$ & BPU-12746.JPG \\
\hline 2273 & Juncus sphaerocarpus Nees & Budapest & 1915 & Degen Árpád & BPU-12748.JPG \\
\hline 2273 & Juncus sphaerocarpus Nees & Töltéstava & 1927 & Polgár Sándor & BPU-12749.JPG \\
\hline 2274 & $\begin{array}{l}\text { Juncus ranarius Songeon et E.P. } \\
\text { Perrier }\end{array}$ & Budaörs & 1915 & Degen Árpád, Zsák Zoltán & BPU-12744.JPG \\
\hline 2274 & $\begin{array}{l}\text { Juncus ranarius Songeon et E.P. } \\
\text { Perrier }\end{array}$ & Budaörs & 1915 & Degen Árpád, Zsák Zoltán & BPU-12744.JPG \\
\hline 2274 & $\begin{array}{l}\text { Juncus ranarius Songeon et E.P. } \\
\text { Perrier }\end{array}$ & Budapest & 1916 & Degen Árpád, Zsák Zoltán & BPU-12742.JPG \\
\hline 2274 & $\begin{array}{l}\text { Juncus ranarius Songeon et E.P. } \\
\text { Perrier }\end{array}$ & Bugyi & 1927 & Lengyel Géza & BPU-12743.JPG \\
\hline 2275 & Juncus bufonius L. & Balatonszőlős & 1957 & Felföldy Lajos & BPU-12733.JPG \\
\hline 2275 & Juncus bufonius L. & Bisse & 1965 & $\begin{array}{l}\text { Simon Tibor, Priszter } \\
\text { Szaniszló, Borhidi Attila }\end{array}$ & BPU-12741.JPG \\
\hline 2275 & Juncus bufonius L. & Budaörs & 1991 & Felföldy Lajos & BPU-12737.JPG \\
\hline 2275 & Juncus bufonius L. & Budapest & 1909 & Degen Árpád & BPU-12745.JPG \\
\hline 2275 & Juncus bufonius L. & Gávavencsellő & 1990 & Felföldy Lajos & BPU-12735.JPG \\
\hline 2275 & Juncus bufonius L. & Gyöngyös & 1940 & Priszter Szaniszló & BPU-12738.JPG \\
\hline 2275 & Juncus bufonius L. & Kőszeg & 1941 & Priszter Szaniszló & BPU-12734.JPG \\
\hline 2275 & Juncus bufonius L. & Pákozd & 1980 & Felföldy Lajos & BPU-12727.JPG \\
\hline 2275 & Juncus bufonius L. & Pákozd & 1980 & Felföldy Lajos & BPU-12728.JPG \\
\hline 2275 & Juncus bufonius L. & Pákozd & 1980 & Felföldy Lajos & BPU-12729.JPG \\
\hline 2275 & Juncus bufonius L. & Pákozd & 1982 & Felföldy Lajos & BPU-12726.JPG \\
\hline 2275 & Juncus bufonius L. & Szilvásvárad & 1953 & $\begin{array}{l}\text { Felföldy Lajos, Tóth } \\
\text { László }\end{array}$ & BPU-12740.JPG \\
\hline 2275 & Juncus bufonius L. & Szőce & 1955 & Soó Rezső & BPU-12734.JPG \\
\hline 2275 & Juncus bufonius L. & Tényő & - & Polgár Sándor & BPU-12739.JPG \\
\hline 2275 & Juncus bufonius L. & Uzsa & 1953 & $\begin{array}{l}\text { Felföldy Lajos, Tóth } \\
\text { László }\end{array}$ & BPU-12732.JPG \\
\hline 2275 & Juncus bufonius L. & Vácrátót & 1953 & Felföldy Lajos & BPU-12736.JPG \\
\hline 2276 & Juncus tenuis Willd. & Alsószölnök & 1955 & Soó Rezső & BPU-12783.JPG \\
\hline 2276 & Juncus tenuis Willd. & Göd & 1948 & Soó Rezső & BPU-12786.JPG \\
\hline
\end{tabular}


1. Elektroniukus melléklet - Electronic Appendix 1.

\begin{tabular}{|c|c|c|c|c|c|}
\hline $\begin{array}{l}\text { Sorszám / } \\
\text { Number }\end{array}$ & Taxon-név / Taxon-name & $\begin{array}{l}\text { Település / } \\
\text { Settlement }\end{array}$ & $\begin{array}{l}\text { Év / } \\
\text { Year }\end{array}$ & Gyűjtő / Collector & $\begin{array}{l}\text { Fájlnév / } \\
\text { File-name }\end{array}$ \\
\hline 2276 & Juncus tenuis Willd. & Nagykanizsa & 1950 & Károlyi Árpád & BPU-12781.JPG \\
\hline 2276 & Juncus tenuis Willd. & Nagykanizsa & 1956 & Felföldy Lajos & BPU-12782.JPG \\
\hline 2276 & Juncus tenuis Willd. & Pálháza & 1960 & Simon Tibor & BPU-12788.JPG \\
\hline 2276 & Juncus tenuis Willd. & Somogyszob & 1957 & Soó Rezső & BPU-12785.JPG \\
\hline 2276 & Juncus tenuis Willd. & Sopron & 1947 & Priszter Szaniszló & BPU-12784.JPG \\
\hline 2276 & Juncus tenuis Willd. & Tarpa & 1949 & Soó Rezső & BPU-12787.JPG \\
\hline 2277 & Juncus compressus Jacq. & Balatonalmádi & 1924 & Polgár Sándor & BPU-12765.JPG \\
\hline 2277 & Juncus compressus Jacq. & Balatonszőlős & 1956 & $\begin{array}{l}\text { Felföldy Lajos, Tóth } \\
\text { László }\end{array}$ & BPU-12764.JPG \\
\hline 2277 & Juncus compressus Jacq. & Budapest & 1909 & Degen Árpád & BPU-12778.JPG \\
\hline 2277 & Juncus compressus Jacq. & Budapest & 1989 & Felföldy Lajos & BPU-12773.JPG \\
\hline 2277 & Juncus compressus Jacq. & Budapest & 1990 & Felföldy Lajos & BPU-12774.JPG \\
\hline 2277 & Juncus compressus Jacq. & Budapest & 1992 & Felföldy Lajos & BPU-12775.JPG \\
\hline 2277 & Juncus compressus Jacq. & Debrecen & 1939 & Felföldy Lajos & BPU-12770.JPG \\
\hline 2277 & Juncus compressus Jacq. & Debrecen & 1988 & Sümegi Gy., Turi B. A. & BPU-12771.JPG \\
\hline 2277 & Juncus compressus Jacq. & Hajdúszoboszló & 1937 & Soó Rezső & BPU-12769.JPG \\
\hline 2277 & Juncus compressus Jacq. & Ipolytarnóc & 1987 & Felföldy Lajos & BPU-12768.JPG \\
\hline 2277 & Juncus compressus Jacq. & Nagykanizsa & 1956 & Felföldy Lajos & BPU-12762.JPG \\
\hline 2277 & Juncus compressus Jacq. & Nagykanizsa & 1956 & Felföldy Lajos & BPU-12763.JPG \\
\hline 2277 & Juncus compressus Jacq. & Nagyrada & 1987 & Felföldy Lajos & BPU-12761.JPG \\
\hline 2277 & Juncus compressus Jacq. & Rábatamási & 1989 & Tóth László & BPU-12766.JPG \\
\hline 2277 & Juncus compressus Jacq. & Szegvár & 1942 & Timár Lajos & BPU-12772.JPG \\
\hline 2277 & Juncus compressus Jacq. & Törökbálint & 1991 & Felföldy Lajos & BPU-12767.JPG \\
\hline 2277 & Juncus compressus Jacq. & Újfehértó & 1910 & Zsák Zoltán & BPU-12776.JPG \\
\hline 2278 & Juncus gerardii Loisel. & Aszófó & 1956 & $\begin{array}{l}\text { Felföldy Lajos, Tóth } \\
\text { László }\end{array}$ & BPU-12711.JPG \\
\hline 2278 & Juncus gerardii Loisel. & Balatonszőlős & 1956 & $\begin{array}{l}\text { Felföldy Lajos, Tóth } \\
\text { László }\end{array}$ & BPU-12712.JPG \\
\hline 2278 & Juncus gerardii Loisel. & Balatonudvari & 1959 & Felföldy Lajos & BPU-12713.JPG \\
\hline 2278 & Juncus gerardii Loisel. & Budapest & 1947 & Pénzes Antal & BPU-12716.JPG \\
\hline 2278 & Juncus gerardii Loisel. & Budapest & 1947 & Pénzes Antal & BPU-12758.JPG \\
\hline 2278 & Juncus gerardii Loisel. & Dunaharaszti & 1948 & Priszter Szaniszló & BPU-12709.JPG \\
\hline 2278 & Juncus gerardii Loisel. & Gárdony & 1982 & Felföldy Lajos & BPU-12708.JPG \\
\hline 2278 & Juncus gerardii Loisel. & Nyíregyháza & 1933 & Soó Rezső & BPU-12710.JPG \\
\hline 2278 & Juncus gerardii Loisel. & Tihany & 1947 & Pólya László & BPU-12714.JPG \\
\hline 2278 & Juncus gerardii Loisel. & Tihany & 1959 & Felföldy Lajos & BPU-12715.JPG \\
\hline 2279 & Juncus bulbosus L. & Tihany & 1929 & Soó Rezső & BPU-12877.JPG \\
\hline 2280 & Juncus capitatus Weigel & Hajdúsámson & 1967 & Gondola István & BPU-12878.JPG \\
\hline 2281 & Juncus alpinoarticulatus Chaix in Vill. & Budapest & 1913 & Degen Árpád & BPU-12824.JPG \\
\hline 2281 & Juncus alpinoarticulatus Chaix in Vill. & Budapest & 1913 & Zsák Zoltán & BPU-12825.JPG \\
\hline 2281 & Juncus alpinoarticulatus Chaix in Vill. & Budapest & 1913 & Zsák Zoltán & BPU-12826.JPG \\
\hline 2282 & Juncus subnodulosus Schrank & Bak & 1955 & Soó Rezső & BPU-12860.JPG \\
\hline 2282 & Juncus subnodulosus Schrank & Balatonszepezd & 1958 & Felföldy Lajos & BPU-12862.JPG \\
\hline 2282 & Juncus subnodulosus Schrank & Balatonszőlős & 1956 & $\begin{array}{l}\text { Felföldy Lajos, Tóth } \\
\text { László }\end{array}$ & BPU-12865.JPG \\
\hline 2282 & Juncus subnodulosus Schrank & Balatonszőlős & 1990 & Felföldy Lajos & BPU-12864.JPG \\
\hline 2282 & Juncus subnodulosus Schrank & Budapest & 1909 & Degen Árpád & BPU-12869.JPG \\
\hline 2282 & Juncus subnodulosus Schrank & Budapest & 1990 & Felföldy Lajos & BPU-12867.JPG \\
\hline 2282 & Juncus subnodulosus Schrank & Budapest & 1991 & Felföldy Lajos & BPU-12868.JPG \\
\hline
\end{tabular}


1. Elektroniukus melléklet - Electronic Appendix 1.

\begin{tabular}{|c|c|c|c|c|c|}
\hline $\begin{array}{l}\text { Sorszám / } \\
\text { Number }\end{array}$ & Taxon-név / Taxon-name & $\begin{array}{l}\text { Település / } \\
\text { Settlement }\end{array}$ & $\begin{array}{l}\text { Év / } \\
\text { Year }\end{array}$ & Gyüjtő / Collector & $\begin{array}{l}\text { Fájlnév / } \\
\text { File-name }\end{array}$ \\
\hline 2282 & Juncus subnodulosus Schrank & Dunakeszi & 1917 & Boros Ádám & BPU-12870.JPG \\
\hline 2282 & Juncus subnodulosus Schrank & Lesenceistvánd & 1928 & Soó Rezső & BPU-12863.JPG \\
\hline 2282 & Juncus subnodulosus Schrank & Sopron & 1938 & Soó Rezső & BPU-12866.JPG \\
\hline 2282 & Juncus subnodulosus Schrank & Tihany & 1956 & Felföldy Lajos & BPU-12861.JPG \\
\hline 2283 & Juncus atratus Krock. & Bajánsenye & 1956 & Kovács Margit & BPU-12850.JPG \\
\hline 2283 & Juncus atratus Krock. & Bátorliget & 1932 & Soó Rezső & BPU-12853.JPG \\
\hline 2283 & Juncus atratus Krock. & Dabas & 1951 & Simon Tibor, Borsos Olga & BPU-12854.JPG \\
\hline 2283 & Juncus atratus Krock. & Egyek & 1950 & $\begin{array}{l}\text { Simon Tibor, Borsos Olga, } \\
\text { Kulcsár Gábor }\end{array}$ & BPU-12855.JPG \\
\hline 2283 & Juncus atratus Krock. & Gávavencsellő & 1988 & Felföldy Lajos & BPU-12852.JPG \\
\hline 2283 & Juncus atratus Krock. & Gávavencsellő & 1990 & Felföldy Lajos & BPU-12851.JPG \\
\hline 2283 & Juncus atratus Krock. & Mezőcsát & 1915 & Budai József & BPU-12857.JPG \\
\hline 2283 & Juncus atratus Krock. & Pákozd & 1980 & Felföldy Lajos & BPU-12856.JPG \\
\hline 2283 & Juncus atratus Krock. & Szentbékkálla & 1957 & Felföldy Lajos & BPU-12849.JPG \\
\hline 2284 & Juncus articulatus L. & Alsószölnök & 1955 & Soó Rezső & BPU-12833.JPG \\
\hline 2284 & Juncus articulatus L. & Balatongyörök & 1961 & Felföldy Lajos & BPU-12829.JPG \\
\hline 2284 & Juncus articulatus L. & Balatonszepezd & 1958 & Felföldy Lajos & BPU-12830.JPG \\
\hline 2284 & Juncus articulatus L. & Balatonszőlős & 1956 & $\begin{array}{l}\text { Felföldy Lajos, Tóth } \\
\text { László }\end{array}$ & BPU-12832.JPG \\
\hline 2284 & Juncus articulatus L. & Budapest & 1906 & Lengyel Géza & BPU-12840.JPG \\
\hline 2284 & Juncus articulatus L. & Budapest & 1909 & Degen Árpád & BPU-12844.JPG \\
\hline 2284 & Juncus articulatus L. & Budapest & 1942 & Priszter Szaniszló & BPU-12842.JPG \\
\hline 2284 & Juncus articulatus L. & Budapest & 1942 & Priszter Szaniszló & BPU-12843.JPG \\
\hline 2284 & Juncus articulatus L. & Budapest & 1947 & Priszter Szaniszló & BPU-12839.JPG \\
\hline 2284 & Juncus articulatus L. & Budapest & 1992 & Felföldy Lajos & BPU-12841.JPG \\
\hline 2284 & Juncus articulatus L. & Gyöngyös & 1953 & Soó Rezső & BPU-12836.JPG \\
\hline 2284 & Juncus articulatus L. & Kiskunmajsa & 1964 & Soó Rezső & BPU-12838.JPG \\
\hline 2284 & Juncus articulatus L. & Lesenceistvánd & 1952 & Borsos Olga & BPU-12831.JPG \\
\hline 2284 & Juncus articulatus L. & Monor & 1942 & Priszter Szaniszló & BPU-12835.JPG \\
\hline 2284 & Juncus articulatus L. & Nagykanizsa & 1956 & Felföldy Lajos & BPU-12828.JPG \\
\hline 2284 & Juncus articulatus L. & Nyírmada & 1933 & Soó Rezső & BPU-12834.JPG \\
\hline 2284 & Juncus articulatus L. & Vilmány & 1910 & Thaisz Lajos & BPU-12846.JPG \\
\hline $\begin{array}{l}2281 \times \\
2284\end{array}$ & $\begin{array}{l}\text { Juncus alpinoarticulatus Chaix in Vill. } \\
\times \text { Juncus articulatus L. }\end{array}$ & Budapest & 1916 & Zsák Zoltán & BPU-12725.JPG \\
\hline $\begin{array}{c}2281 \times \\
2284\end{array}$ & $\begin{array}{l}\text { Juncus alpinoarticulatus Chaix in Vill. } \\
\times \text { Juncus articulatus L. }\end{array}$ & Veresegyház & 1949 & Soó Rezső & BPU-12723.JPG \\
\hline 2285 & Luzula forsteri (Sm.) DC. & "Cuha-völgy" & 1954 & Soó Rezső & BPU-12658.JPG \\
\hline 2285 & Luzula forsteri (Sm.) DC. & Bakonybél & 1955 & Soó Rezső & BPU-12661.JPG \\
\hline 2285 & Luzula forsteri (Sm.) DC. & Balatonszepezd & 1952 & Szabados A. & BPU-12660.JPG \\
\hline 2285 & Luzula forsteri (Sm.) DC. & Csesznek & 1929 & Soó Rezső & BPU-12659.JPG \\
\hline 2285 & Luzula forsteri (Sm.) DC. & Hidegkút & 1958 & Felföldy Lajos & BPU-12657.JPG \\
\hline 2285 & Luzula forsteri (Sm.) DC. & Kapolcs & 1959 & $\begin{array}{l}\text { Jávorka Sándor, Felföldy } \\
\text { Lajos, Tóth László }\end{array}$ & BPU-12656.JPG \\
\hline 2285 & Luzula forsteri (Sm.) DC. & Pécs & 1925 & Soó Rezső & BPU-12663.JPG \\
\hline 2285 & Luzula forsteri (Sm.) DC. & Pécs & 1953 & Soó Rezső, Borsos Olga & BPU-12662.JPG \\
\hline 2286 & Luzula pilosa (L.) Willd. & Beregdaróc & 1951 & Simon Tibor, Jakucs Pál & BPU-12653.JPG \\
\hline 2286 & Luzula pilosa (L.) Willd. & Nagykovácsi & 1914 & $\begin{array}{l}\text { Degen Árpád, Lengyel } \\
\text { Géza }\end{array}$ & BPU-12655.JPG \\
\hline 2286 & Luzula pilosa (L.) Willd. & Sopron & 1938 & Soó Rezső & BPU-12651.JPG \\
\hline 2286 & Luzula pilosa (L.) Willd. & Sopron & 1942 & Priszter Szaniszló & BPU-12652.JPG \\
\hline
\end{tabular}


1. Elektroniukus melléklet - Electronic Appendix 1.

\begin{tabular}{|c|c|c|c|c|c|}
\hline $\begin{array}{l}\text { Sorszám / } \\
\text { Number }\end{array}$ & Taxon-név / Taxon-name & $\begin{array}{l}\text { Település / } \\
\text { Settlement }\end{array}$ & $\begin{array}{l}\text { Év / } \\
\text { Year }\end{array}$ & Gyújtő / Collector & $\begin{array}{l}\text { Fájlnév / } \\
\text { File-name }\end{array}$ \\
\hline 2287 & $\begin{array}{l}\text { Luzula luzuloides (Lam.) Dandy et } \\
\text { Wilmott }\end{array}$ & Alsószölnök & 1955 & Soó Rezső & BPU-12675.JPG \\
\hline 2287 & $\begin{array}{l}\text { Luzula luzuloides (Lam.) Dandy et } \\
\text { Wilmott }\end{array}$ & Bakonybél & 1955 & Soó Rezső & BPU-12673.JPG \\
\hline 2287 & $\begin{array}{l}\text { Luzula luzuloides (Lam.) Dandy et } \\
\text { Wilmott }\end{array}$ & Dömös & 1952 & Soó Rezső & BPU-15422.JPG \\
\hline 2287 & $\begin{array}{l}\text { Luzula luzuloides (Lam.) Dandy et } \\
\text { Wilmott }\end{array}$ & Fenyőfő & 1955 & Soó Rezső & BPU-12672.JPG \\
\hline 2287 & $\begin{array}{l}\text { Luzula luzuloides (Lam.) Dandy et } \\
\text { Wilmott }\end{array}$ & Gyöngyös & 1965 & Soó Rezső & BPU-12677.JPG \\
\hline 2287 & $\begin{array}{l}\text { Luzula luzuloides (Lam.) Dandy et } \\
\text { Wilmott }\end{array}$ & Isztimér & 1949 & Soó Rezső & BPU-12679.JPG \\
\hline 2287 & $\begin{array}{l}\text { Luzula luzuloides (Lam.) Dandy et } \\
\text { Wilmott }\end{array}$ & Komló & 1941 & Priszter Szaniszló & BPU-12683.JPG \\
\hline 2287 & $\begin{array}{l}\text { Luzula luzuloides (Lam.) Dandy et } \\
\text { Wilmott }\end{array}$ & Kőszeg & 1941 & Priszter Szaniszló & BPU-12674.JPG \\
\hline 2287 & $\begin{array}{l}\text { Luzula luzuloides (Lam.) Dandy et } \\
\text { Wilmott }\end{array}$ & Köveskál & 1959 & $\begin{array}{l}\text { Jávorka Sándor, Felföldy } \\
\text { Lajos, Tóth László }\end{array}$ & BPU-12671.JPG \\
\hline 2287 & $\begin{array}{l}\text { Luzula luzuloides (Lam.) Dandy et } \\
\text { Wilmott }\end{array}$ & Miskolc & 1963 & Isépy István & BPU-12680.JPG \\
\hline 2287 & $\begin{array}{l}\text { Luzula luzuloides (Lam.) Dandy et } \\
\text { Wilmott }\end{array}$ & Pécs & 1948 & Priszter Szaniszló & BPU-12682.JPG \\
\hline 2287 & $\begin{array}{l}\text { Luzula luzuloides (Lam.) Dandy et } \\
\text { Wilmott }\end{array}$ & Pécs & 1953 & Soó Rezső, Borsos Olga & BPU-12681.JPG \\
\hline 2287 & $\begin{array}{l}\text { Luzula luzuloides (Lam.) Dandy et } \\
\text { Wilmott }\end{array}$ & Pilisborosjenő & 1947 & Priszter Szaniszló & BPU-12676.JPG \\
\hline 2287 & $\begin{array}{l}\text { Luzula luzuloides (Lam.) Dandy et } \\
\text { Wilmott }\end{array}$ & Putnok & 1950 & Soó Rezső & BPU-12678.JPG \\
\hline 2287 & $\begin{array}{l}\text { Luzula luzuloides (Lam.) Dandy et } \\
\text { Wilmott }\end{array}$ & Uzsa & 1959 & $\begin{array}{l}\text { Jávorka Sándor, Felföldy } \\
\text { Lajos }\end{array}$ & BPU-12670.JPG \\
\hline 2288 & Luzula pallidula Kirschner & Háromhuta & 1955 & Simon Tibor & BPU-12619.JPG \\
\hline 2288 & Luzula pallidula Kirschner & Kimle & 1962 & Borsos Olga & BPU-12624.JPG \\
\hline 2288 & Luzula pallidula Kirschner & Lovászi & 1950 & Károlyi Árpád & BPU-12620.JPG \\
\hline 2288 & Luzula pallidula Kirschner & Mezőcsát & 1912 & Budai József & BPU-12621.JPG \\
\hline 2289 & Luzula campestris (L.) DC. & Budapest & 1916 & Zsák Zoltán & BPU-12648.JPG \\
\hline 2289 & Luzula campestris (L.) DC. & Budapest & 1938 & Priszter Szaniszló & BPU-12645.JPG \\
\hline 2289 & Luzula campestris (L.) DC. & Budapest & 1941 & Priszter Szaniszló & BPU-12646.JPG \\
\hline 2289 & Luzula campestris (L.) DC. & Budapest & 2005 & Felföldy Lajos & BPU-12644.JPG \\
\hline 2289 & Luzula campestris (L.) DC. & Dömös & 1964 & Isépy István & BPU-12642.JPG \\
\hline 2289 & Luzula campestris (L.) DC. & Gyöngyös & 1964 & Soó Rezső & BPU-12641.JPG \\
\hline 2289 & Luzula campestris (L.) DC. & Hidegkút & 1958 & Felföldy Lajos & BPU-12636.JPG \\
\hline 2289 & Luzula campestris (L.) DC. & Hidegkút & 1959 & Felföldy Lajos & BPU-12635.JPG \\
\hline 2289 & Luzula campestris (L.) DC. & Komló & 1941 & Priszter Szaniszló & BPU-12626.JPG \\
\hline 2289 & Luzula campestris (L.) DC. & Nagyvisnyó & 1953 & Soó Rezső & BPU-12625.JPG \\
\hline 2289 & Luzula campestris (L.) DC. & Penészlek & 1932 & Soó Rezső & BPU-12622.JPG \\
\hline 2289 & Luzula campestris (L.) DC. & Sopron & 1938 & Soó Rezső & BPU-12623.JPG \\
\hline 2289 & Luzula campestris (L.) DC. & Sopron & 1942 & Priszter Szaniszló & BPU-12637.JPG \\
\hline 2289 & Luzula campestris (L.) DC. & Szentbékkálla & 1959 & $\begin{array}{l}\text { Jávorka Sándor, Felföldy } \\
\text { Lajos, Tóth László }\end{array}$ & BPU-12634.JPG \\
\hline 2289 & Luzula campestris (L.) DC. & Tatabánya & 1942 & Priszter Szaniszló & BPU-12640.JPG \\
\hline 2289 & Luzula campestris (L.) DC. & Tihany & 1958 & Felföldy Lajos & BPU-12632.JPG \\
\hline 2289 & Luzula campestris (L.) DC. & Tihany & 1958 & Felföldy Lajos & BPU-12633.JPG \\
\hline 2289 & Luzula campestris (L.) DC. & Uzsa & 1959 & $\begin{array}{l}\text { Jávorka Sándor, Felföldy } \\
\text { Lajos }\end{array}$ & BPU-12631.JPG \\
\hline 2289 & Luzula campestris (L.) DC. & Vác & 1942 & Priszter Szaniszló & BPU-12639.JPG \\
\hline
\end{tabular}


1. Elektroniukus melléklet - Electronic Appendix 1.

\begin{tabular}{|c|c|c|c|c|c|}
\hline $\begin{array}{l}\text { Sorszám / } \\
\text { Number }\end{array}$ & Taxon-név / Taxon-name & $\begin{array}{l}\text { Település / } \\
\text { Settlement }\end{array}$ & $\begin{array}{l}\text { Év / } \\
\text { Year }\end{array}$ & Gyüjtő / Collector & $\begin{array}{l}\text { Fájlnév / } \\
\text { File-name }\end{array}$ \\
\hline 2289 & Luzula campestris (L.) DC. & Vácrátót & 1953 & Felföldy Lajos & BPU-12638.JPG \\
\hline 2290 & Luzula multiflora (Ehrh.) Lej. & Nagykőrös & 1916 & Zsák Zoltán & BPU-12627.JPG \\
\hline 2291 & Luzula divulgata Kirschner & Budapest & 2005 & Felföldy Lajos & BPU-12643.JPG \\
\hline 2293 & Commelina communis L. & Balatongyörök & 1960 & Priszter Szaniszló & BPU-12370.JPG \\
\hline 2294 & Festuca altissima All. & Bakonybél & 1955 & Soó Rezső & BPU-12141.JPG \\
\hline 2294 & Festuca altissima All. & Bakonybél & 1955 & Soó Rezső & BPU-12142.JPG \\
\hline 2294 & Festuca altissima All. & Bükkszentkereszt & 1938 & Soó Rezső & BPU-12144.JPG \\
\hline 2294 & Festuca altissima All. & Gyöngyös & 1953 & Soó Rezső & BPU-12143.JPG \\
\hline 2294 & Festuca altissima All. & Tibolddaróc & 1960 & Simon Tibor & BPU-12145.JPG \\
\hline 2295 & Festuca drymeja Mert. et W.D.J. Koch & Pécs & 1925 & Soó Rezső & BPU-12137.JPG \\
\hline 2295 & Festuca drymeja Mert. et W.D.J. Koch & Regéc & 1991 & Simon Tibor & BPU-12134.JPG \\
\hline 2295 & Festuca drymeja Mert. et W.D.J. Koch & Szenna & 1957 & Soó Rezső & BPU-12139.JPG \\
\hline 2296 & Festuca gigantea (L.) Vill. & Alsószölnök & 1955 & Soó Rezső & BPU-12161.JPG \\
\hline 2296 & Festuca gigantea (L.) Vill. & Budakeszi & 1947 & Soó Rezső & BPU-12162.JPG \\
\hline 2296 & Festuca gigantea (L.) Vill. & Debrecen & 1933 & Soó Rezső & BPU-12163.JPG \\
\hline 2296 & Festuca gigantea (L.) Vill. & Miskolc & 1950 & Soó Rezső & BPU-12164.JPG \\
\hline 2296 & Festuca gigantea (L.) Vill. & Sátoraljaújhely & 1993 & Felföldy Lajos & BPU-12160.JPG \\
\hline 2297 & Festuca arundinacea Schreb. & Budapest & 2005 & Felföldy Lajos & BPU-12157.JPG \\
\hline 2297 & Festuca arundinacea Schreb. & Budapest & 2005 & Felföldy Lajos & BPU-12158.JPG \\
\hline 2297 & Festuca arundinacea Schreb. & Dabas & 1951 & Simon Tibor, Borsos Olga & BPU-12154.JPG \\
\hline 2297 & Festuca arundinacea Schreb. & Miskolc & 1950 & Soó Rezső, Simon Tibor & BPU-12156.JPG \\
\hline 2297 & Festuca arundinacea Schreb. & Nyíregyháza & 1948 & Soó Rezső & BPU-12153.JPG \\
\hline 2297 & Festuca arundinacea Schreb. & Vámospércs & 1932 & Soó Rezső & BPU-12155.JPG \\
\hline 2298 & Festuca pratensis Huds. & Akasztó & 1968 & Priszter Szaniszló & BPU-12151.JPG \\
\hline 2298 & Festuca pratensis Huds. & Hajdúbagos & 1933 & Soó Rezső & BPU-12150.JPG \\
\hline 2298 & Festuca pratensis Huds. & Pannonhalma & 1917 & Polgár Sándor & BPU-12152.JPG \\
\hline 2298 & Festuca pratensis Huds. & Pécsely & 1950 & Felföldy Lajos & BPU-12149.JPG \\
\hline 2298 & Festuca pratensis Huds. & Tihany & 1943 & Felföldy Lajos & BPU-12148.JPG \\
\hline 2299 & Festuca heterophylla Lam. & Balatonfüred & 1942 & Felföldy Lajos & BPU-12180.JPG \\
\hline 2299 & Festuca heterophylla Lam. & Bátorliget & 1953 & Simon Tibor & BPU-12182.JPG \\
\hline 2299 & Festuca heterophylla Lam. & Bélapátfalva & 1948 & $\begin{array}{l}\text { Simon Tibor, Pólya } \\
\text { László, Jakucs Pál }\end{array}$ & BPU-12179.JPG \\
\hline 2299 & Festuca heterophylla Lam. & Gyöngyös & 1953 & Soó Rezső & BPU-12183.JPG \\
\hline 2299 & Festuca heterophylla Lam. & Gyöngyös & 1961 & Soó Rezső & BPU-12184.JPG \\
\hline 2299 & Festuca heterophylla Lam. & Isztimér & 1949 & Soó Rezső & BPU-15526.JPG \\
\hline 2299 & Festuca heterophylla Lam. & Köveskál & 1959 & $\begin{array}{l}\text { Jávorka Sándor, Felföldy } \\
\text { Lajos, Tóth László }\end{array}$ & BPU-12181.JPG \\
\hline 2301 & Festuca rubra L. & Budapest & 1991 & Felföldy Lajos & BPU-12175.JPG \\
\hline 2301 & Festuca rubra L. & Debrecen & 1945 & Felföldy Lajos & BPU-12172.JPG \\
\hline 2301 & Festuca rubra L. & Nyíracsád & 1949 & Soó Rezső & BPU-12171.JPG \\
\hline 2301 & Festuca rubra L. & Sárospatak & 1938 & Soó Rezső, Hargitai Zoltán & BPU-12174.JPG \\
\hline 2301 & Festuca rubra L. & Vámospércs & 1932 & Soó Rezső & BPU-12173.JPG \\
\hline 2301 & Festuca rubra L. & Vitnyéd & 1928 & Rigler József & BPU-12177.JPG \\
\hline 2302 & Festuca amethystina L. & Pilisszentiván & 1953 & Soó Rezső & BPU-12269.JPG \\
\hline 2305 & $\begin{array}{l}\text { Festuca vaginata Waldst. et Kit. ex } \\
\text { Willd. }\end{array}$ & Debrecen & 1934 & Soó Rezső & BPU-12246.JPG \\
\hline 2305 & $\begin{array}{l}\text { Festuca vaginata Waldst. et Kit. ex } \\
\text { Willd. }\end{array}$ & Fenyőfő & 1955 & Soó Rezső & BPU-12241.JPG \\
\hline 2305 & $\begin{array}{l}\text { Festuca vaginata Waldst. et Kit. ex } \\
\text { Willd. }\end{array}$ & Fenyőfő & 1955 & Soó Rezső & BPU-12242.JPG \\
\hline
\end{tabular}


1. Elektroniukus melléklet - Electronic Appendix 1.

\begin{tabular}{|c|c|c|c|c|c|}
\hline $\begin{array}{l}\text { Sorszám / } \\
\text { Number }\end{array}$ & Taxon-név / Taxon-name & $\begin{array}{l}\text { Település / } \\
\text { Settlement }\end{array}$ & $\begin{array}{l}\text { Év / } \\
\text { Year }\end{array}$ & Gyújtő / Collector & $\begin{array}{l}\text { Fájlnév / } \\
\text { File-name }\end{array}$ \\
\hline 2305 & $\begin{array}{l}\text { Festuca vaginata Waldst. et Kit. ex } \\
\text { Willd. }\end{array}$ & Nagykanizsa & 1953 & Pócs Tamás & BPU-12240.JPG \\
\hline 2305 & $\begin{array}{l}\text { Festuca vaginata Waldst. et Kit. ex } \\
\text { Willd. }\end{array}$ & Pilisszentiván & 1953 & Soó Rezső & BPU-12244.JPG \\
\hline 2305 & $\begin{array}{l}\text { Festuca vaginata Waldst. et Kit. ex } \\
\text { Willd. }\end{array}$ & Pilisszentiván & 1953 & Soó Rezső & BPU-12245.JPG \\
\hline 2305 & $\begin{array}{l}\text { Festuca vaginata Waldst. et Kit. ex } \\
\text { Willd. }\end{array}$ & Szigetmonostor & 1963 & Isépy István & BPU-12243.JPG \\
\hline 2307 & Festuca pallens Host & Budapest & 1947 & Soó Rezső & BPU-12254.JPG \\
\hline 2307 & Festuca pallens Host & Miskolc & 1939 & Soó Rezső & BPU-12253.JPG \\
\hline 2307 & Festuca pallens Host & Tihany & 1950 & Felföldy Lajos & BPU-12252.JPG \\
\hline 2309 & Festuca dalmatica (Hack.) K. Richt. & Nagyharsány & 1961 & Simon Tibor & BPU-12186.JPG \\
\hline 2309 & Festuca dalmatica (Hack.) K. Richt. & Nagyharsány & 1963 & Simon Tibor & BPU-12185.JPG \\
\hline 2309 & Festuca dalmatica (Hack.) K. Richt. & Nagyharsány & 1963 & Simon Tibor & BPU-12188.JPG \\
\hline 2310 & Festuca rupicola Heuff. & Abaújszántó & 1938 & Soó Rezső & BPU-12227.JPG \\
\hline 2310 & Festuca rupicola Heuff. & Balatonfüred & 1942 & Felföldy Lajos & BPU-12191.JPG \\
\hline 2310 & Festuca rupicola Heuff. & Bátorliget & 1932 & Soó Rezső & BPU-12222.JPG \\
\hline 2310 & Festuca rupicola Heuff. & Bicske & 1992 & Felföldy Lajos & BPU-12225.JPG \\
\hline 2310 & Festuca rupicola Heuff. & Budapest & 1905 & Kocsis István & BPU-12228.JPG \\
\hline 2310 & Festuca rupicola Heuff. & Budapest & 2004 & Felföldy Lajos & BPU-12229.JPG \\
\hline 2310 & Festuca rupicola Heuff. & Debrecen & 1931 & Soó Rezső & BPU-12224.JPG \\
\hline 2310 & Festuca rupicola Heuff. & Hajdúsámson & 1932 & Soó Rezső & BPU-12223.JPG \\
\hline 2310 & Festuca rupicola Heuff. & Hidegkút & 1958 & Felföldy Lajos & BPU-12220.JPG \\
\hline 2310 & Festuca rupicola Heuff. & Pécs & 1936 & Soó Rezső & BPU-12226.JPG \\
\hline 2310 & Festuca rupicola Heuff. & Tihany & 1942 & Felföldy Lajos & BPU-12218.JPG \\
\hline 2310 & Festuca rupicola Heuff. & Tihany & 1943 & Felföldy Lajos & BPU-12217.JPG \\
\hline 2310 & Festuca rupicola Heuff. & Tihany & 1946 & Felföldy Lajos & BPU-12219.JPG \\
\hline 2310 & Festuca rupicola Heuff. & Tiszabercel & 1990 & Felföldy Lajos & BPU-12221.JPG \\
\hline 2310 & Festuca rupicola Heuff. & Tokaj & 1943 & Felföldy Lajos & BPU-12214.JPG \\
\hline 2310 & Festuca rupicola Heuff. & Uzsa & 1959 & $\begin{array}{l}\text { Jávorka Sándor, Felföldy } \\
\text { Lajos }\end{array}$ & BPU-12215.JPG \\
\hline 2310 & Festuca rupicola Heuff. & Uzsa & 1959 & $\begin{array}{l}\text { Jávorka Sándor, Felföldy } \\
\text { Lajos }\end{array}$ & BPU-12216.JPG \\
\hline 2312 & Festuca filiformis Pourr. & ??? & 1931 & Thaisz Lajos & BPU-12261.JPG \\
\hline 2312 & Festuca filiformis Pourr. & Alsószölnök & 1955 & Soó Rezső & BPU-12258.JPG \\
\hline 2312 & Festuca filiformis Pourr. & Debrecen & 1948 & Siroki Zoltán & BPU-12259.JPG \\
\hline 2312 & Festuca filiformis Pourr. & Szakonyfalu & 1955 & Soó Rezső & BPU-12257.JPG \\
\hline 2312 & Festuca filiformis Pourr. & Szőce & 1955 & Soó Rezső & BPU-12260.JPG \\
\hline 2313 & Festuca ovina L. & Hernádpetri & 1952 & Jakucs Pál, Ér Lajos & BPU-12263.JPG \\
\hline 2313 & Festuca ovina L. & Kishuta & 1952 & Simon Tibor & BPU-12264.JPG \\
\hline 2313 & Festuca ovina L. & Miskolc & 1950 & Soó Rezső, Simon Tibor & BPU-12262.JPG \\
\hline 2314 & $\begin{array}{l}\text { Festuca pseudodalmatica Krajina ex } \\
\text { Domin }\end{array}$ & Bükkszentkereszt & 1938 & Soó Rezső & BPU-12197.JPG \\
\hline 2314 & $\begin{array}{l}\text { Festuca pseudodalmatica Krajina ex } \\
\text { Domin }\end{array}$ & Füzér & 1938 & Soó Rezső & BPU-12200.JPG \\
\hline 2314 & $\begin{array}{l}\text { Festuca pseudodalmatica Krajina ex } \\
\text { Domin }\end{array}$ & Gyöngyös & 1963 & Simon Tibor & BPU-12196.JPG \\
\hline 2314 & $\begin{array}{l}\text { Festuca pseudodalmatica Krajina ex } \\
\text { Domin }\end{array}$ & Miskolc & 1937 & Soó Rezső & BPU-12199.JPG \\
\hline 2314 & $\begin{array}{l}\text { Festuca pseudodalmatica Krajina ex } \\
\text { Domin }\end{array}$ & Nagyvisnyó & 1953 & Soó Rezső & BPU-12194.JPG \\
\hline
\end{tabular}


1. Elektroniukus melléklet - Electronic Appendix 1.

\begin{tabular}{|c|c|c|c|c|c|}
\hline $\begin{array}{l}\text { Sorszám / } \\
\text { Number }\end{array}$ & Taxon-név / Taxon-name & $\begin{array}{l}\text { Település / } \\
\text { Settlement }\end{array}$ & $\begin{array}{l}\text { Év / } \\
\text { Year }\end{array}$ & Gyűjtő / Collector & $\begin{array}{l}\text { Fájlnév / } \\
\text { File-name }\end{array}$ \\
\hline 2314 & $\begin{array}{l}\text { Festuca pseudodalmatica Krajina ex } \\
\text { Domin }\end{array}$ & Pécsely & 1950 & Felföldy Lajos & BPU-12190.JPG \\
\hline 2314 & $\begin{array}{l}\text { Festuca pseudodalmatica Krajina ex } \\
\text { Domin }\end{array}$ & Pusztafalu & 1939 & Soó Rezső & BPU-12198.JPG \\
\hline 2314 & $\begin{array}{l}\text { Festuca pseudodalmatica Krajina ex } \\
\text { Domin }\end{array}$ & Salgótarján & 1936 & Soó Rezső & BPU-12193.JPG \\
\hline 2314 & $\begin{array}{l}\text { Festuca pseudodalmatica Krajina ex } \\
\text { Domin }\end{array}$ & Salgótarján & 1963 & Simon Tibor & BPU-12192.JPG \\
\hline 2315 & Festuca valesiaca Schleich. ex Gaudin & Bátonyterenye & 1937 & Máthé Imre & BPU-12195.JPG \\
\hline 2315 & Festuca valesiaca Schleich. ex Gaudin & Budapest & 1989 & Felföldy Lajos & BPU-12208.JPG \\
\hline 2315 & Festuca valesiaca Schleich. ex Gaudin & Budapest & 1991 & Felföldy Lajos & BPU-12209.JPG \\
\hline 2315 & Festuca valesiaca Schleich. ex Gaudin & Budapest & 2004 & Felföldy Lajos & BPU-12210.JPG \\
\hline 2315 & Festuca valesiaca Schleich. ex Gaudin & Egyek & 1932 & Soó Rezső & BPU-12206.JPG \\
\hline 2315 & Festuca valesiaca Schleich. ex Gaudin & Gyöngyös & 1965 & Soó Rezső & BPU-12205.JPG \\
\hline 2315 & Festuca valesiaca Schleich. ex Gaudin & Háromhuta & 1960 & Simon Tibor & BPU-12201.JPG \\
\hline 2315 & Festuca valesiaca Schleich. ex Gaudin & Soltvadkert & 1992 & Felföldy Lajos & BPU-12207.JPG \\
\hline 2315 & Festuca valesiaca Schleich. ex Gaudin & Tarcal & 1933 & Soó Rezső & BPU-12202.JPG \\
\hline 2315 & Festuca valesiaca Schleich. ex Gaudin & Tihany & 1943 & Felföldy Lajos & BPU-12204.JPG \\
\hline 2315 & Festuca valesiaca Schleich. ex Gaudin & Uzsa & 1950 & Felföldy Lajos & BPU-12203.JPG \\
\hline 2315 & Festuca valesiaca Schleich. ex Gaudin & Villány & 1907 & Degen Árpád & BPU-12212.JPG \\
\hline 2316 & Festuca pseudovina Hack. & Balatonszőlős & 1957 & Felföldy Lajos & BPU-12234.JPG \\
\hline 2316 & Festuca pseudovina Hack. & Debrecen & 1933 & Soó Rezső & BPU-12236.JPG \\
\hline 2316 & Festuca pseudovina Hack. & Regéc & 1939 & Soó Rezső & BPU-12237.JPG \\
\hline 2316 & Festuca pseudovina Hack. & Szolnok & 1902 & Degen Árpád & BPU-12239.JPG \\
\hline 2316 & Festuca pseudovina Hack. & Újfehértó & 1936 & Soó Rezső & BPU-12235.JPG \\
\hline $\begin{array}{l}2294- \\
2316\end{array}$ & Festuca & Sopron & 1938 & Soó Rezső & BPU-12089.JPG \\
\hline $\begin{array}{c}2294- \\
2316\end{array}$ & Festuca & Szarvas & 1949 & Soó Rezső & BPU-12090.JPG \\
\hline $\begin{array}{l}2294- \\
2316\end{array}$ & Festuca & Szarvas & 1949 & Soó Rezső & BPU-12095.JPG \\
\hline $\begin{array}{c}2294- \\
2316\end{array}$ & Festuca & Szolnok & 1937 & Soó Rezső & BPU-12094.JPG \\
\hline $2300-2470$ & Festuca & Budapest & 1952 & Pócs Tamás & BPU-12256.JPG \\
\hline 2317 & Lolium perenne L. & Budapest & 1947 & Priszter Szaniszló & BPU-11826.JPG \\
\hline 2317 & Lolium perenne L. & Budapest & 1948 & Priszter Szaniszló & BPU-11825.JPG \\
\hline 2317 & Lolium perenne L. & Budapest & 1948 & Priszter Szaniszló & BPU-11828.JPG \\
\hline 2317 & Lolium perenne L. & Budapest & 1948 & Priszter Szaniszló & BPU-11829.JPG \\
\hline 2317 & Lolium perenne L. & Budapest & 1948 & Priszter Szaniszló & BPU-11830.JPG \\
\hline 2317 & Lolium perenne L. & Budapest & 2005 & Felföldy Lajos & BPU-11827.JPG \\
\hline 2317 & Lolium perenne L. & Debrecen & 1941 & Soó Rezső & BPU-11822.JPG \\
\hline 2317 & Lolium perenne L. & Egyek & 1947 & Soó Rezső & BPU-11823.JPG \\
\hline 2317 & Lolium perenne L. & Keszthely & 1960 & Ördög Gizella & BPU-11821.JPG \\
\hline 2317 & Lolium perenne L. & Miskolc & 1938 & Soó Rezső & BPU-11824.JPG \\
\hline 2318 & Lolium multiflorum Lam. & Keszthely & 1960 & Sándor Béla & BPU-11819.JPG \\
\hline 2318 & Lolium multiflorum Lam. & Keszthely & 1963 & Péter István & BPU-11818.JPG \\
\hline 2318 & Lolium multiflorum Lam. & Tokaj & 1948 & Soó Rezső & BPU-11820.JPG \\
\hline 2319 & Lolium temulentum L. & Dorog & 1948 & Priszter Szaniszló & BPU-11816.JPG \\
\hline 2319 & Lolium temulentum L. & Nagykanizsa & 1950 & Károlyi Árpád & BPU-11815.JPG \\
\hline 2319 & Lolium temulentum L. & Szeged & 1957 & Timár Lajos & BPU-11817.JPG \\
\hline 2322 & Vulpia myuros (L.) C.C. Gmel. & Badacsonytomaj & 1984 & Felföldy Lajos & BPU-12077.JPG \\
\hline
\end{tabular}


1. Elektroniukus melléklet - Electronic Appendix 1.

\begin{tabular}{|c|c|c|c|c|c|}
\hline $\begin{array}{l}\text { Sorszám / } \\
\text { Number }\end{array}$ & Taxon-név / Taxon-name & $\begin{array}{l}\text { Település / } \\
\text { Settlement }\end{array}$ & $\begin{array}{l}\text { Év / } \\
\text { Year }\end{array}$ & Gyújtő / Collector & $\begin{array}{l}\text { Fájlnév / } \\
\text { File-name }\end{array}$ \\
\hline 2322 & Vulpia myuros (L.) C.C. Gmel. & Bodajk & 1938 & Boros Ádám & BPU-12080.JPG \\
\hline 2322 & Vulpia myuros (L.) C.C. Gmel. & Budapest & 2004 & Felföldy Lajos & BPU-12083.JPG \\
\hline 2322 & Vulpia myuros (L.) C.C. Gmel. & Monostorapáti & 1959 & $\begin{array}{l}\text { Jávorka Sándor, Felföldy } \\
\text { Lajos, Tóth László }\end{array}$ & BPU-12076.JPG \\
\hline 2322 & Vulpia myuros (L.) C.C. Gmel. & Nagyharsány & 1961 & Simon Tibor & BPU-12081.JPG \\
\hline 2322 & Vulpia myuros (L.) C.C. Gmel. & Pilisszentiván & 1953 & Soó Rezső & BPU-12079.JPG \\
\hline 2322 & Vulpia myuros (L.) C.C. Gmel. & Szentgotthárd & 1955 & Soó Rezső & BPU-12078.JPG \\
\hline 2322 & Vulpia myuros (L.) C.C. Gmel. & Tihany & 1942 & Felföldy Lajos & BPU-12075.JPG \\
\hline 2322 & Vulpia myuros (L.) C.C. Gmel. & Törökbálint & 1915 & Jávorka Sándor & BPU-12084.JPG \\
\hline 2322 & Vulpia myuros (L.) C.C. Gmel. & Uzsa & 1959 & $\begin{array}{l}\text { Jávorka Sándor, Felföldy } \\
\text { Lajos }\end{array}$ & BPU-12074.JPG \\
\hline 2323 & Vulpia bromoides (L.) Gray & Debrecen & 1916 & Rapaics Rajmund & BPU-12073.JPG \\
\hline 2323 & Vulpia bromoides (L.) Gray & Kőszeg & 1948 & Kárpáti Zoltán & BPU-12071.JPG \\
\hline 2323 & Vulpia bromoides (L.) Gray & Vitnyéd & 1928 & Rigler József & BPU-12072.JPG \\
\hline 2324 & Poa bulbosa L. & Budakeszi & 1991 & Felföldy Lajos & BPU-09990.JPG \\
\hline 2324 & Poa bulbosa L. & Budaörs & 1942 & Priszter Szaniszló & BPU-09991.JPG \\
\hline 2324 & Poa bulbosa L. & Budapest & 1941 & Priszter Szaniszló & BPU-09985.JPG \\
\hline 2324 & Poa bulbosa L. & Budapest & 1948 & Priszter Szaniszló & BPU-09983.JPG \\
\hline 2324 & Poa bulbosa L. & Budapest & 1948 & Priszter Szaniszló & BPU-09984.JPG \\
\hline 2324 & Poa bulbosa L. & Budapest & 1951 & Priszter Szaniszló & BPU-09982.JPG \\
\hline 2324 & Poa bulbosa L. & Budapest & 1982 & Felföldy Lajos & BPU-09981.JPG \\
\hline 2324 & Poa bulbosa L. & Budapest & 2004 & Felföldy Lajos & BPU-09979.JPG \\
\hline 2324 & Poa bulbosa L. & Budapest & 2004 & Felföldy Lajos & BPU-09980.JPG \\
\hline 2324 & Poa bulbosa L. & Debrecen & 1935 & Máthé Imre & BPU-09988.JPG \\
\hline 2324 & Poa bulbosa L. & Diósjenő & 1951 & Priszter Szaniszló & BPU-09989.JPG \\
\hline 2324 & Poa bulbosa L. & Mór & 1953 & Felföldy Lajos & BPU-09987.JPG \\
\hline 2324 & Poa bulbosa L. & Pilisszentiván & 1947 & Priszter Szaniszló & BPU-09992.JPG \\
\hline 2324 & Poa bulbosa L. & Sáránd & 1933 & Soó Rezső & BPU-09986.JPG \\
\hline 2324 & Poa bulbosa L. & Szigetszentmárton & 1948 & Priszter Szaniszló & BPU-09993.JPG \\
\hline 2324 & Poa bulbosa L. & Tihany & 1958 & Felföldy Lajos & BPU-09978.JPG \\
\hline 2324 & Poa bulbosa L. & Tihany & 1959 & Felföldy Lajos & BPU-09995.JPG \\
\hline 2324 & Poa bulbosa L. & Vácrátót & 1953 & Felföldy Lajos & BPU-09994.JPG \\
\hline 2325 & Poa badensis Haenke ex Willd. & Budapest & 1947 & Priszter Szaniszló & BPU-09997.JPG \\
\hline 2325 & Poa badensis Haenke ex Willd. & Budapest & 1947 & Soó Rezső & BPU-09996.JPG \\
\hline 2325 & Poa badensis Haenke ex Willd. & Budapest & 1989 & Felföldy Lajos & BPU-09998.JPG \\
\hline 2325 & Poa badensis Haenke ex Willd. & Csákvár & 1955 & Soó Rezső & BPU-09999.JPG \\
\hline 2325 & Poa badensis Haenke ex Willd. & Fertőrákos & 1938 & Soó Rezső & BPU-10002.JPG \\
\hline 2325 & Poa badensis Haenke ex Willd. & Isztimér & 1955 & Soó Rezső & BPU-10003.JPG \\
\hline 2325 & Poa badensis Haenke ex Willd. & Pilisborosjenő & 1942 & Priszter Szaniszló & BPU-10000.JPG \\
\hline 2325 & Poa badensis Haenke ex Willd. & Pilisszentiván & 1942 & Priszter Szaniszló & BPU-10001.JPG \\
\hline 2327 & Poa compressa L. & Ágfalva & 1947 & Kárpáti Zoltán & BPU-09972.JPG \\
\hline 2327 & Poa compressa L. & Balatonhenye & 1959 & $\begin{array}{l}\text { Jávorka Sándor, Felföldy } \\
\text { Lajos, Tóth László }\end{array}$ & BPU-09973.JPG \\
\hline 2327 & Poa compressa L. & Balatonszepezd & 1958 & Felföldy Lajos & BPU-09974.JPG \\
\hline 2327 & Poa compressa L. & Bélapátfalva & 1951 & Priszter Szaniszló & BPU-09966.JPG \\
\hline 2327 & Poa compressa L. & Budaörs & 1946 & Kárpáti Zoltán & BPU-09969.JPG \\
\hline 2327 & Poa compressa L. & Budapest & 1942 & Priszter Szaniszló & BPU-09963.JPG \\
\hline 2327 & Poa compressa L. & Budapest & 1943 & Priszter Szaniszló & BPU-11935.JPG \\
\hline 2327 & Poa compressa L. & Budapest & 1948 & Priszter Szaniszló & BPU-11934.JPG \\
\hline
\end{tabular}


1. Elektroniukus melléklet - Electronic Appendix 1.

\begin{tabular}{|c|c|c|c|c|c|}
\hline $\begin{array}{l}\text { Sorszám / } \\
\text { Number }\end{array}$ & Taxon-név / Taxon-name & $\begin{array}{l}\text { Település / } \\
\text { Settlement }\end{array}$ & $\begin{array}{l}\text { Év / } \\
\text { Year }\end{array}$ & Gyújtő / Collector & $\begin{array}{l}\text { Fájlnév / } \\
\text { File-name }\end{array}$ \\
\hline 2327 & Poa compressa L. & Budapest & 1949 & Priszter Szaniszló & BPU-09961.JPG \\
\hline 2327 & Poa compressa L. & Budapest & 1949 & Priszter Szaniszló & BPU-09962.JPG \\
\hline 2327 & Poa compressa L. & Budapest & 1949 & Priszter Szaniszló & BPU-11933.JPG \\
\hline 2327 & Poa compressa L. & Budapest & 1982 & Felföldy Lajos & BPU-11925.JPG \\
\hline 2327 & Poa compressa L. & Budapest & 1982 & Felföldy Lajos & BPU-11927.JPG \\
\hline 2327 & Poa compressa L. & Budapest & 1991 & Felföldy Lajos & BPU-11928.JPG \\
\hline 2327 & Poa compressa L. & Budapest & 1991 & Felföldy Lajos & BPU-11929.JPG \\
\hline 2327 & Poa compressa L. & Budapest & 1991 & Felföldy Lajos & BPU-11930.JPG \\
\hline 2327 & Poa compressa L. & Budapest & 1992 & Felföldy Lajos & BPU-11926.JPG \\
\hline 2327 & Poa compressa L. & Budapest & 2004 & Felföldy Lajos & BPU-11931.JPG \\
\hline 2327 & Poa compressa L. & Budapest & 2005 & Felföldy Lajos & BPU-09960.JPG \\
\hline 2327 & Poa compressa L. & Budapest & 2005 & Felföldy Lajos & BPU-11932.JPG \\
\hline 2327 & Poa compressa L. & Gyöngyös & 1961 & Soó Rezső & BPU-09967.JPG \\
\hline 2327 & Poa compressa L. & Litke & 1987 & Felföldy Lajos & BPU-09968.JPG \\
\hline 2327 & Poa compressa L. & Szentendre & 1947 & Priszter Szaniszló & BPU-09970.JPG \\
\hline 2327 & Poa compressa L. & Tahitótfalu & 1950 & Priszter Szaniszló & BPU-09971.JPG \\
\hline 2327 & Poa compressa L. & Tihany & 1946 & Felföldy Lajos & BPU-09977.JPG \\
\hline 2327 & Poa compressa L. & Tihany & 1959 & Felföldy Lajos & BPU-09975.JPG \\
\hline 2327 & Poa compressa L. & Tihany & 1959 & Felföldy Lajos & BPU-09976.JPG \\
\hline 2327 & Poa compressa L. & Zámoly & 1982 & Felföldy Lajos & BPU-09965.JPG \\
\hline 2328 & Poa annua L. & Badacsonytomaj & 1953 & Priszter Szaniszló & BPU-11939.JPG \\
\hline 2328 & Poa annua L. & Budapest & 1947 & Priszter Szaniszló & BPU-11943.JPG \\
\hline 2328 & Poa annua L. & Debrecen & 1945 & Felföldy Lajos & BPU-11941.JPG \\
\hline 2328 & Poa annua L. & Debrecen & 1948 & Borsos Olga & BPU-11942.JPG \\
\hline 2328 & Poa annua L. & Kemence & 1952 & Simon Tibor & BPU-11940.JPG \\
\hline 2331 & Poa trivialis L. & Alcsútdoboz & 1992 & Felföldy Lajos & BPU-11958.JPG \\
\hline 2331 & Poa trivialis L. & Aszófő & 1957 & Felföldy Lajos & BPU-11949.JPG \\
\hline 2331 & Poa trivialis L. & Balatonakali & 1958 & Felföldy Lajos & BPU-11950.JPG \\
\hline 2331 & Poa trivialis L. & Bátorliget & 1932 & Soó Rezső & BPU-11951.JPG \\
\hline 2331 & Poa trivialis L. & Budapest & 1951 & Priszter Szaniszló & BPU-11959.JPG \\
\hline 2331 & Poa trivialis L. & Budapest & 1982 & Felföldy Lajos & BPU-11960.JPG \\
\hline 2331 & Poa trivialis L. & Debrecen & 1934 & Soó Rezső & BPU-11954.JPG \\
\hline 2331 & Poa trivialis L. & Gödöllő & 1951 & Priszter Szaniszló & BPU-11952.JPG \\
\hline 2331 & Poa trivialis L. & Háromhuta & 1960 & Simon Tibor & BPU-11945.JPG \\
\hline 2331 & Poa trivialis L. & Ipolytarnóc & 1987 & Felföldy Lajos & BPU-11953.JPG \\
\hline 2331 & Poa trivialis L. & Pákozd & 1980 & Felföldy Lajos & BPU-11955.JPG \\
\hline 2331 & Poa trivialis L. & Pákozd & 1980 & Felföldy Lajos & BPU-11956.JPG \\
\hline 2331 & Poa trivialis L. & Pákozd & 1980 & Felföldy Lajos & BPU-11957.JPG \\
\hline 2331 & Poa trivialis L. & Tihany & 1947 & Felföldy Lajos & BPU-11948.JPG \\
\hline 2331 & Poa trivialis L. & Tihany & 1952 & Priszter Szaniszló & BPU-11946.JPG \\
\hline 2331 & Poa trivialis L. & Uzsa & 1959 & $\begin{array}{l}\text { Jávorka Sándor, Felföldy } \\
\text { Lajos }\end{array}$ & BPU-11947.JPG \\
\hline 2332 & Poa scabra Kit. & Dunabogdány & 1921 & Jávorka Sándor & BPU-10051.JPG \\
\hline 2332 & Poa scabra Kit. & Gyöngyös & 1922 & Soó Rezső & BPU-10053.JPG \\
\hline 2332 & Poa scabra Kit. & Szarvaskő & 1932 & Zólyomi Bálint & BPU-10052.JPG \\
\hline 2333 & Poa nemoralis L. & Budapest & 1982 & Felföldy Lajos & BPU-10009.JPG \\
\hline 2333 & Poa nemoralis L. & Budapest & 2004 & Felföldy Lajos & BPU-10008.JPG \\
\hline 2333 & Poa nemoralis L. & Debrecen & 1934 & Soó Rezső & BPU-10013.JPG \\
\hline
\end{tabular}


1. Elektroniukus melléklet - Electronic Appendix 1.

\begin{tabular}{|c|c|c|c|c|c|}
\hline $\begin{array}{l}\text { Sorszám / } \\
\text { Number }\end{array}$ & Taxon-név / Taxon-name & $\begin{array}{l}\text { Település / } \\
\text { Settlement }\end{array}$ & $\begin{array}{l}\text { Év / } \\
\text { Year }\end{array}$ & Gyűjtő / Collector & $\begin{array}{c}\text { Fájlnév / } \\
\text { File-name }\end{array}$ \\
\hline 2333 & Poa nemoralis L. & Gelénes & 1953 & $\begin{array}{l}\text { Simon Tibor, Vozáry } \\
\text { Elemér }\end{array}$ & BPU-10010.JPG \\
\hline 2333 & Poa nemoralis L. & Gyöngyös & 1952 & Soó Rezső & BPU-10014.JPG \\
\hline 2333 & Poa nemoralis L. & Háromhuta & 1955 & Simon Tibor & BPU-10021.JPG \\
\hline 2333 & Poa nemoralis L. & Kapolcs & 1959 & $\begin{array}{l}\text { Jávorka Sándor, Felföldy } \\
\text { Lajos, Tóth László }\end{array}$ & BPU-10019.JPG \\
\hline 2333 & Poa nemoralis L. & Miskolc & 1951 & Priszter Szaniszló & BPU-10011.JPG \\
\hline 2333 & Poa nemoralis L. & Miskolc & 1951 & Priszter Szaniszló & BPU-10012.JPG \\
\hline 2333 & Poa nemoralis L. & Nyíregyháza & 1933 & Soó Rezső & BPU-10017.JPG \\
\hline 2333 & Poa nemoralis L. & Penészlek & 1932 & Soó Rezső & BPU-10018.JPG \\
\hline 2333 & Poa nemoralis L. & Pilisszentiván & 1942 & Priszter Szaniszló & BPU-10015.JPG \\
\hline 2333 & Poa nemoralis L. & Szigetújfalu & 1951 & Priszter Szaniszló & BPU-10016.JPG \\
\hline 2333 & Poa nemoralis L. & Uzsa & 1952 & Borsos Olga & BPU-10020.JPG \\
\hline 2334 & Poa palustris L. & Balmazújváros & 1937 & Soó Rezső & BPU-10042.JPG \\
\hline 2334 & Poa palustris L. & Bicske & 1992 & Felföldy Lajos & BPU-10039.JPG \\
\hline 2334 & Poa palustris L. & Budapest & 1992 & Felföldy Lajos & BPU-10036.JPG \\
\hline 2334 & Poa palustris L. & Dunaújváros & 1973 & $\begin{array}{l}\text { Felföldy Lajos, Tóth } \\
\text { László }\end{array}$ & BPU-10040.JPG \\
\hline 2334 & Poa palustris L. & Gávavencsellő & 1990 & Felföldy Lajos & BPU-10050.JPG \\
\hline 2334 & Poa palustris L. & Ipolytarnóc & 1987 & Felföldy Lajos & BPU-10043.JPG \\
\hline 2334 & Poa palustris L. & Ipolytarnóc & 1987 & Felföldy Lajos & BPU-10044.JPG \\
\hline 2334 & Poa palustris L. & Ipolytarnóc & 1987 & Felföldy Lajos & BPU-10045.JPG \\
\hline 2334 & Poa palustris L. & Ipolytarnóc & 1987 & Felföldy Lajos & BPU-10046.JPG \\
\hline 2334 & Poa palustris L. & Nagybajom & 1957 & Soó Rezső & BPU-10049.JPG \\
\hline 2334 & Poa palustris L. & Pákozd & 1978 & $\begin{array}{l}\text { Felföldy Lajos, Tóth } \\
\text { László }\end{array}$ & BPU-10041.JPG \\
\hline 2334 & Poa palustris L. & Pomáz & 1949 & Priszter Szaniszló & BPU-10047.JPG \\
\hline 2334 & Poa palustris L. & Szenta & 1955 & Soó Rezső & BPU-10048.JPG \\
\hline 2334 & Poa palustris L. & Tarpa & 1949 & Soó Rezső & BPU-10037.JPG \\
\hline 2334 & Poa palustris L. & Tarpa & 1949 & Soó Rezső & BPU-10038.JPG \\
\hline 2336 & Poa pratensis L. & Balatonhenye & 1959 & $\begin{array}{l}\text { Jávorka Sándor, Felföldy } \\
\text { Lajos, Tóth László }\end{array}$ & BPU-11965.JPG \\
\hline 2336 & Poa pratensis L. & Budapest & 1941 & Priszter Szaniszló & BPU-11976.JPG \\
\hline 2336 & Poa pratensis L. & Budapest & 1982 & Felföldy Lajos & BPU-11977.JPG \\
\hline 2336 & Poa pratensis L. & Budapest & 1982 & Felföldy Lajos & BPU-11979.JPG \\
\hline 2336 & Poa pratensis L. & Budapest & 1982 & Felföldy Lajos & BPU-11980.JPG \\
\hline 2336 & Poa pratensis L. & Debrecen & 1945 & Felföldy Lajos & BPU-11973.JPG \\
\hline 2336 & Poa pratensis L. & Debrecen & 1947 & Soó Rezső & BPU-11972.JPG \\
\hline 2336 & Poa pratensis L. & Debrecen & 1988 & Sümegi Gy., Turi B. A. & BPU-11969.JPG \\
\hline 2336 & Poa pratensis L. & Gödöllő & 1951 & Priszter Szaniszló & BPU-11968.JPG \\
\hline 2336 & Poa pratensis L. & Hortobágy & 1931 & Soó Rezső & BPU-11971.JPG \\
\hline 2336 & Poa pratensis L. & Pomáz & 1951 & Priszter Szaniszló & BPU-11966.JPG \\
\hline 2336 & Poa pratensis L. & Tihany & 1946 & Felföldy Lajos & BPU-11963.JPG \\
\hline 2336 & Poa pratensis L. & Tihany & 1959 & Felföldy Lajos & BPU-11964.JPG \\
\hline 2337 & Poa angustifolia L. & Budapest & 1947 & Pénzes Antal & BPU-11978.JPG \\
\hline 2337 & Poa angustifolia L. & Gödöllő & 1951 & Priszter Szaniszló & BPU-11967.JPG \\
\hline 2337 & Poa angustifolia L. & Hortobágy & 1931 & Soó Rezső & BPU-11970.JPG \\
\hline 2337 & Poa angustifolia L. & Tihany & 1956 & $\begin{array}{l}\text { Felföldy Lajos, Tóth } \\
\text { László }\end{array}$ & BPU-11962.JPG \\
\hline 2337 & Poa angustifolia L. & Villány & 1941 & Priszter Szaniszló & BPU-11974.JPG \\
\hline
\end{tabular}


1. Elektroniukus melléklet - Electronic Appendix 1.

\begin{tabular}{|c|c|c|c|c|c|}
\hline $\begin{array}{l}\text { Sorszám / } \\
\text { Number }\end{array}$ & Taxon-név / Taxon-name & $\begin{array}{l}\text { Település / } \\
\text { Settlement }\end{array}$ & $\begin{array}{l}\text { Év / } \\
\text { Year }\end{array}$ & Gyújtő / Collector & $\begin{array}{l}\text { Fájlnév / } \\
\text { File-name }\end{array}$ \\
\hline 2337 & Poa angustifolia L. & Villány & 1956 & Soó Rezső & BPU-11975.JPG \\
\hline 2338 & Puccinellia distans (Jacq.) Parl. & - & 1954 & anonim & BPU-11987.JPG \\
\hline 2338 & Puccinellia distans (Jacq.) Parl. & Balmazújváros & 1953 & anonim & BPU-11988.JPG \\
\hline 2338 & Puccinellia distans (Jacq.) Parl. & Budapest & 1948 & Priszter Szaniszló & BPU-12012.JPG \\
\hline 2338 & Puccinellia distans (Jacq.) Parl. & Budapest & 1991 & Felföldy Lajos & BPU-12010.JPG \\
\hline 2338 & Puccinellia distans (Jacq.) Parl. & Budapest & 2004 & Felföldy Lajos & BPU-12011.JPG \\
\hline 2338 & Puccinellia distans (Jacq.) Parl. & Debrecen & 1947 & Soó Rezső & BPU-12008.JPG \\
\hline 2338 & Puccinellia distans (Jacq.) Parl. & Hajdúbagos & 1934 & Soó Rezső & BPU-12007.JPG \\
\hline 2338 & Puccinellia distans (Jacq.) Parl. & Szeged & 1948 & Timár Lajos & BPU-12009.JPG \\
\hline 2338 & Puccinellia distans (Jacq.) Parl. & Vácrátót & 1953 & Felföldy Lajos & BPU-12006.JPG \\
\hline 2339 & Puccinellia limosa (Schur) Holmb. & - & 1953 & anonim & BPU-11995.JPG \\
\hline 2339 & Puccinellia limosa (Schur) Holmb. & - & 1953 & anonim & BPU-11996.JPG \\
\hline 2339 & Puccinellia limosa (Schur) Holmb. & - & 1953 & anonim & BPU-11997.JPG \\
\hline 2339 & Puccinellia limosa (Schur) Holmb. & - & 1953 & Borsos Olga & BPU-11994.JPG \\
\hline 2339 & Puccinellia limosa (Schur) Holmb. & Egyek & 1947 & Soó Rezső & BPU-12000.JPG \\
\hline 2339 & Puccinellia limosa (Schur) Holmb. & Hajdúsámson & 1951 & Soó Rezső & BPU-12001.JPG \\
\hline 2339 & Puccinellia limosa (Schur) Holmb. & Kiskunmajsa & 1964 & Soó Rezső & BPU-12002.JPG \\
\hline 2339 & Puccinellia limosa (Schur) Holmb. & Kiskunmajsa & 1964 & Soó Rezső & BPU-12003.JPG \\
\hline 2339 & Puccinellia limosa (Schur) Holmb. & Kunszentmiklós & 1905 & Degen Árpád & BPU-12004.JPG \\
\hline 2339 & Puccinellia limosa (Schur) Holmb. & Nyíregyháza & 1948 & Soó Rezső & BPU-11998.JPG \\
\hline 2339 & Puccinellia limosa (Schur) Holmb. & Tiszavasvári & 1949 & Igmándy József & BPU-11999.JPG \\
\hline 2340 & Puccinellia peisonis (Beck) Jáv. & Pákozd & 1980 & Felföldy Lajos & BPU-11984.JPG \\
\hline---- & Puccinellia pannonica (Hack.) Holmb. & Budapest & 1901 & Flatt Károly & BPU-11983.JPG \\
\hline---- & Puccinellia pannonica (Hack.) Holmb. & Budapest & - & Flatt Károly & BPU-11982.JPG \\
\hline 2341 & Sclerochloa dura (L.) P. Beauv. & Alcsútdoboz & 1992 & Felföldy Lajos & BPU-12022.JPG \\
\hline 2341 & Sclerochloa dura (L.) P. Beauv. & Budapest & 1948 & Priszter Szaniszló & BPU-12025.JPG \\
\hline 2341 & Sclerochloa dura (L.) P. Beauv. & Hajdúnánás & 1935 & Soó Rezső & BPU-12020.JPG \\
\hline 2341 & Sclerochloa dura (L.) P. Beauv. & Pilisszentiván & 1942 & Priszter Szaniszló & BPU-12018.JPG \\
\hline 2341 & Sclerochloa dura (L.) P. Beauv. & Szigetszentmárton & 1948 & Priszter Szaniszló & BPU-12017.JPG \\
\hline 2341 & Sclerochloa dura (L.) P. Beauv. & Tihany & 1959 & Felföldy Lajos & BPU-12014.JPG \\
\hline 2341 & Sclerochloa dura (L.) P. Beauv. & Tiszacsege & 1950 & $\begin{array}{l}\text { Simon Tibor, Borsos Olga, } \\
\text { Kulcsár Gábor }\end{array}$ & BPU-12019.JPG \\
\hline 2341 & Sclerochloa dura (L.) P. Beauv. & Vácrátót & 1953 & Felföldy Lajos & BPU-12015.JPG \\
\hline 2341 & Sclerochloa dura (L.) P. Beauv. & Vácrátót & 1953 & Felföldy Lajos & BPU-12016.JPG \\
\hline 2341 & Sclerochloa dura (L.) P. Beauv. & Vásárosnamény & 1951 & $\begin{array}{l}\text { Simon Tibor, Jakucs Pál, } \\
\text { Kulcsár Gábor }\end{array}$ & BPU-12023.JPG \\
\hline 2341 & Sclerochloa dura (L.) P. Beauv. & Villány & 1948 & Priszter Szaniszló & BPU-12024.JPG \\
\hline 2341 & Sclerochloa dura (L.) P. Beauv. & Zámoly & 1982 & Felföldy Lajos & BPU-12021.JPG \\
\hline 2342 & Dactylis glomerata L. & Abaliget & 1938 & Soó Rezső & BPU-10124.JPG \\
\hline 2342 & Dactylis glomerata L. & Bakonyszentlászló & 1929 & Soó Rezső & BPU-10129.JPG \\
\hline 2342 & Dactylis glomerata L. & Balatonfüred & 1942 & Felföldy Lajos & BPU-10130.JPG \\
\hline 2342 & Dactylis glomerata L. & Balatongyörök & 1961 & Felföldy Lajos & BPU-10119.JPG \\
\hline 2342 & Dactylis glomerata L. & Budapest & 2004 & Felföldy Lajos & BPU-10111.JPG \\
\hline 2342 & Dactylis glomerata L. & Debrecen & 1932 & Soó Rezső & BPU-10127.JPG \\
\hline 2342 & Dactylis glomerata L. & Debrecen & 1938 & Soó Rezső & BPU-10113.JPG \\
\hline 2342 & Dactylis glomerata L. & Pákozd & 1980 & Felföldy Lajos & BPU-10112.JPG \\
\hline 2342 & Dactylis glomerata L. & Szabadszállás & 1914 & $\begin{array}{l}\text { Degen Árpád, Wagner } \\
\text { János, Lengyel Géza }\end{array}$ & BPU-10109.JPG \\
\hline 2342 & Dactylis glomerata L. & Szabadszállás & 1918 & Degen Árpád & BPU-10110.JPG \\
\hline
\end{tabular}


1. Elektroniukus melléklet - Electronic Appendix 1.

\begin{tabular}{|c|c|c|c|c|c|}
\hline $\begin{array}{l}\text { Sorszám / } \\
\text { Number }\end{array}$ & Taxon-név / Taxon-name & $\begin{array}{l}\text { Település / } \\
\text { Settlement }\end{array}$ & $\begin{array}{l}\text { Év / } \\
\text { Year }\end{array}$ & Gyűjtő / Collector & $\begin{array}{l}\text { Fájlnév / } \\
\text { File-name }\end{array}$ \\
\hline 2342 & Dactylis glomerata L. & Szilvásvárad & 1953 & $\begin{array}{l}\text { Felföldy Lajos, Tóth } \\
\text { László }\end{array}$ & BPU-10114.JPG \\
\hline 2342 & Dactylis glomerata L. & Tákos & 1948 & $\begin{array}{l}\text { Pólya László, Simon } \\
\text { Tibor, Jakucs Pál }\end{array}$ & BPU-10126.JPG \\
\hline 2342 & Dactylis glomerata L. & Tihany & 1928 & Soó Rezső & BPU-10117.JPG \\
\hline 2342 & Dactylis glomerata L. & Tihany & 1946 & Felföldy Lajos & BPU-10115.JPG \\
\hline 2342 & Dactylis glomerata L. & Tihany & 1947 & Pólya László & BPU-10131.JPG \\
\hline 2342 & Dactylis glomerata L. & Tihany & 1956 & Felföldy Lajos & BPU-10116.JPG \\
\hline 2342 & Dactylis glomerata L. & Uzsa & 1959 & $\begin{array}{l}\text { Jávorka Sándor, Felföldy } \\
\text { Lajos }\end{array}$ & BPU-10118.JPG \\
\hline 2342 & Dactylis glomerata L. & Vászoly & 1950 & Felföldy Lajos & BPU-10133.JPG \\
\hline 2342 & Dactylis glomerata L. & Villány & 1956 & Soó Rezső & BPU-10125.JPG \\
\hline 2343 & Dactylis polygama Horv. & Budapest & 1982 & Felföldy Lajos & BPU-10122.JPG \\
\hline 2343 & Dactylis polygama Horv. & Budapest & 2005 & Felföldy Lajos & BPU-10121.JPG \\
\hline 2343 & Dactylis polygama Horv. & Pusztafalu & 1962 & Simon Tibor & BPU-10123.JPG \\
\hline 2343 & Dactylis polygama Horv. & Solymár & 2005 & Felföldy Lajos & BPU-10128.JPG \\
\hline 2343 & Dactylis polygama Horv. & Tihany & 1959 & Felföldy Lajos & BPU-10132.JPG \\
\hline 2344 & Beckmannia eruciformis (L.) Host & Budapest & 1993 & Felföldy Lajos & BPU-11653.JPG \\
\hline 2344 & Beckmannia eruciformis (L.) Host & Ecsegfalva & 1969 & Priszter Szaniszló & BPU-11652.JPG \\
\hline 2344 & Beckmannia eruciformis (L.) Host & Egyek & 1950 & $\begin{array}{l}\text { Simon Tibor, Borsos Olga, } \\
\text { Kulcsár Gábor }\end{array}$ & BPU-10138.JPG \\
\hline 2344 & Beckmannia eruciformis (L.) Host & Hortobágy & 1947 & Soó Rezső & BPU-15988.JPG \\
\hline 2345 & Cynosurus cristatus L. & Bátorliget & 1932 & Soó Rezső & BPU-10135.JPG \\
\hline 2345 & Cynosurus cristatus L. & Bátorliget & 1953 & Simon Tibor & BPU-10136.JPG \\
\hline 2345 & Cynosurus cristatus L. & Debrecen & 1934 & Soó Rezső & BPU-10134.JPG \\
\hline 2345 & Cynosurus cristatus L. & Kapolcs & 1959 & $\begin{array}{l}\text { Jávorka Sándor, Felföldy } \\
\text { Lajos, Tóth László }\end{array}$ & BPU-10137.JPG \\
\hline 2346 & Catabrosa aquatica (L.) P. Beauv. & Aszófő & 1959 & Felföldy Lajos & BPU-10105.JPG \\
\hline 2346 & Catabrosa aquatica (L.) P. Beauv. & Balatonszőlős & 1957 & Felföldy Lajos & BPU-10104.JPG \\
\hline 2346 & Catabrosa aquatica (L.) P. Beauv. & Budapest & 1913 & $\begin{array}{l}\text { Filarszky Nándor, } \\
\text { Kümmerle Jenő Béla }\end{array}$ & BPU-12051.JPG \\
\hline 2346 & Catabrosa aquatica (L.) P. Beauv. & Komlóska & 1955 & Simon Tibor & BPU-10107.JPG \\
\hline 2346 & Catabrosa aquatica (L.) P. Beauv. & Miskolc & 1908 & Hulják János & BPU-12052.JPG \\
\hline 2346 & Catabrosa aquatica (L.) P. Beauv. & Pátka & 1987 & Felföldy Lajos & BPU-10103.JPG \\
\hline 2346 & Catabrosa aquatica (L.) P. Beauv. & Uzsa & 1950 & Felföldy Lajos & BPU-10106.JPG \\
\hline 2347 & Apera spica-venti (L.) P. Beauv. & Budapest & 2005 & Felföldy Lajos & BPU-15896.JPG \\
\hline 2347 & Apera spica-venti (L.) P. Beauv. & Debrecen & 1934 & Soó Rezső & BPU-15895.JPG \\
\hline 2347 & Apera spica-venti (L.) P. Beauv. & Vácrátót & 1953 & Felföldy Lajos & BPU-15894.JPG \\
\hline 2349 & Briza media L. & Balatonszepezd & 1958 & Felföldy Lajos & BPU-10102.JPG \\
\hline 2349 & Briza media L. & Bicske & 1992 & Felföldy Lajos & BPU-10099.JPG \\
\hline 2349 & Briza media L. & Budapest & 1982 & Felföldy Lajos & BPU-10097.JPG \\
\hline 2349 & Briza media L. & Budapest & 2004 & Felföldy Lajos & BPU-10096.JPG \\
\hline 2349 & Briza media L. & Debrecen & 1934 & Soó Rezső & BPU-10100.JPG \\
\hline 2349 & Briza media L. & Lábatlan & 1953 & Felföldy Lajos & BPU-10101.JPG \\
\hline 2349 & Briza media L. & Miskolc & 1948 & $\begin{array}{l}\text { Simon Tibor, Borsos Olga, } \\
\text { Jakucs Pál }\end{array}$ & BPU-10098.JPG \\
\hline 2349 & Briza media L. & Pilisszentkereszt & 1904 & Degen Árpád & BPU-10095.JPG \\
\hline 2350 & Sesleria heufleriana Schur & Bélapátfalva & 1950 & $\begin{array}{l}\text { Simon Tibor, Jakucs Pál, } \\
\text { Kárpáti István }\end{array}$ & BPU-11874.JPG \\
\hline 2350 & Sesleria heufleriana Schur & Miskolc & 1911 & Hulják János & BPU-11880.JPG \\
\hline 2350 & Sesleria heufleriana Schur & Miskolc & 1911 & Hulják János & BPU-11880.JPG \\
\hline
\end{tabular}


1. Elektroniukus melléklet - Electronic Appendix 1.

\begin{tabular}{|c|c|c|c|c|c|}
\hline $\begin{array}{l}\text { Sorszám / } \\
\text { Number }\end{array}$ & Taxon-név / Taxon-name & $\begin{array}{l}\text { Település / } \\
\text { Settlement }\end{array}$ & $\begin{array}{l}\text { Év / } \\
\text { Year }\end{array}$ & Gyújtő / Collector & $\begin{array}{l}\text { Fájlnév / } \\
\text { File-name }\end{array}$ \\
\hline 2350 & Sesleria heufleriana Schur & Miskolc & 1916 & Hulják János & BPU-11879.JPG \\
\hline 2350 & Sesleria heufleriana Schur & Miskolc & 1947 & Papp József & BPU-11877.JPG \\
\hline 2350 & Sesleria heufleriana Schur & Miskolc & 1949 & Priszter Szaniszló & BPU-11876.JPG \\
\hline 2350 & Sesleria heufleriana Schur & Miskolc & 1952 & Priszter Szaniszló & BPU-11875.JPG \\
\hline 2350 & Sesleria heufleriana Schur & Szilvásvárad & 1916 & Hulják János & BPU-11881.JPG \\
\hline 2350 & Sesleria heufleriana Schur & Szögliget & 1951 & Jakucs Pál, Ér Lajos & BPU-11878.JPG \\
\hline 2351 & Sesleria hungarica Ujhelyi & Tibolddaróc & 1953 & Soó Rezső & BPU-11872.JPG \\
\hline 2351 & Sesleria hungarica Ujhelyi & Tibolddaróc & 1960 & Simon Tibor & BPU-11871.JPG \\
\hline 2352 & Sesleria uliginosa Opiz & Bozsok & 1938 & Soó Rezső & BPU-11855.JPG \\
\hline 2352 & Sesleria uliginosa Opiz & Bozsok & 1938 & Soó Rezső & BPU-11855.JPG \\
\hline 2353 & Sesleria albicans Kit. ex Schult. & Sopron & 1942 & Priszter Szaniszló & BPU-11856.JPG \\
\hline 2354 & Sesleria sadleriana Janka & Budapest & 1912 & Jávorka Sándor & BPU-11870.JPG \\
\hline 2354 & Sesleria sadleriana Janka & Budapest & 1922 & Soó Rezső & BPU-11868.JPG \\
\hline 2354 & Sesleria sadleriana Janka & Budapest & 1949 & Soó Rezső & BPU-15528.JPG \\
\hline 2354 & Sesleria sadleriana Janka & Budapest & 1958 & Isépy István & BPU-11867.JPG \\
\hline 2354 & Sesleria sadleriana Janka & Pilisszentiván & 1919 & Boros Ádám & BPU-11869.JPG \\
\hline 2354 & Sesleria sadleriana Janka & Pilisszentiván & 1932 & Soó Rezső & BPU-11865.JPG \\
\hline 2354 & Sesleria sadleriana Janka & Szentendre & 1963 & Soó Rezső & BPU-11866.JPG \\
\hline 2354 & Sesleria sadleriana Janka & Vác & 1942 & Priszter Szaniszló & BPU-11864.JPG \\
\hline 2355 & Melica ciliata L. & Bélapátfalva & 1930 & Soó Rezső & BPU-11916.JPG \\
\hline 2355 & Melica ciliata L. & Budaörs & 1982 & Felföldy Lajos & BPU-11913.JPG \\
\hline 2355 & Melica ciliata L. & Budapest & 1942 & Priszter Szaniszló & BPU-11923.JPG \\
\hline 2355 & Melica ciliata L. & Budapest & 1947 & Priszter Szaniszló & BPU-11921.JPG \\
\hline 2355 & Melica ciliata L. & Budapest & 1947 & Soó Rezső & BPU-11922.JPG \\
\hline 2355 & Melica ciliata L. & Mályinka & 1929 & Soó Rezső & BPU-11915.JPG \\
\hline 2355 & Melica ciliata L. & Nagyharsány & 1948 & Priszter Szaniszló & BPU-11920.JPG \\
\hline 2355 & Melica ciliata L. & Nagyharsány & 1956 & Soó Rezső & BPU-11919.JPG \\
\hline 2355 & Melica ciliata L. & Nagyharsány & 1965 & $\begin{array}{l}\text { Simon Tibor, Priszter } \\
\text { Szaniszló, Borhidi Attila }\end{array}$ & BPU-11917.JPG \\
\hline 2355 & Melica ciliata L. & Pásztó & 1936 & Soó Rezső & BPU-11914.JPG \\
\hline 2355 & Melica ciliata L. & Szigetmonostor & 1948 & Priszter Szaniszló & BPU-11912.JPG \\
\hline 2355 & Melica ciliata L. & Tihany & 1956 & Felföldy Lajos & BPU-11911.JPG \\
\hline 2356 & Melica transsilvanica Schur & Budapest & 1923 & Soó Rezső & BPU-11907.JPG \\
\hline 2356 & Melica transsilvanica Schur & Budapest & 1991 & Felföldy Lajos & BPU-11909.JPG \\
\hline 2356 & Melica transsilvanica Schur & Budapest & 2005 & Felföldy Lajos & BPU-11908.JPG \\
\hline 2356 & Melica transsilvanica Schur & Debrecen & 1937 & Soó Rezső & BPU-11905.JPG \\
\hline 2356 & Melica transsilvanica Schur & Miskolc & 1950 & Soó Rezső & BPU-11906.JPG \\
\hline 2356 & Melica transsilvanica Schur & Szentendre & 1952 & Vozáry Elemér & BPU-15449.JPG \\
\hline 2357 & Melica altissima L. & Bélapátfalva & 1932 & Soó Rezső & BPU-11903.JPG \\
\hline 2357 & Melica altissima L. & Egyek & 1932 & Soó Rezső & BPU-11901.JPG \\
\hline 2357 & Melica altissima L. & Kerecsend & 1957 & Soó Rezső & BPU-11900.JPG \\
\hline 2357 & Melica altissima L. & Miskolc & 1940 & Priszter Szaniszló & BPU-11902.JPG \\
\hline 2358 & Melica uniflora L. & Budapest & 1953 & Soó Rezső & BPU-11898.JPG \\
\hline 2358 & Melica uniflora L. & Budapest & 1989 & Felföldy Lajos & BPU-11896.JPG \\
\hline 2358 & Melica uniflora L. & Budapest & 2004 & Felföldy Lajos & BPU-11897.JPG \\
\hline 2358 & Melica uniflora L. & Garadna & 1955 & Tóth László & BPU-11895.JPG \\
\hline 2358 & Melica uniflora L. & Kapolcs & 1959 & Felföldy Lajos & BPU-11894.JPG \\
\hline 2358 & Melica uniflora L. & Tihany & 1956 & Felföldy Lajos & BPU-11893.JPG \\
\hline
\end{tabular}


1. Elektroniukus melléklet - Electronic Appendix 1.

\begin{tabular}{|c|c|c|c|c|c|}
\hline $\begin{array}{l}\text { Sorszám / } \\
\text { Number }\end{array}$ & Taxon-név / Taxon-name & $\begin{array}{l}\text { Település / } \\
\text { Settlement }\end{array}$ & $\begin{array}{l}\text { Év / } \\
\text { Year }\end{array}$ & Gyüjtő / Collector & $\begin{array}{l}\text { Fájlnév / } \\
\text { File-name }\end{array}$ \\
\hline 2358 & Melica uniflora L. & Tihany & 1958 & Felföldy Lajos & BPU-11892.JPG \\
\hline 2358 & Melica uniflora L. & Tihany & 1959 & Felföldy Lajos & BPU-11891.JPG \\
\hline 2359 & Melica nutans L. & Aszófő & 1956 & $\begin{array}{l}\text { Felföldy Lajos, Tóth } \\
\text { László }\end{array}$ & BPU-11886.JPG \\
\hline 2359 & Melica nutans L. & Kapolcs & 1959 & $\begin{array}{l}\text { Jávorka Sándor, Felföldy } \\
\text { Lajos, Tóth László }\end{array}$ & BPU-11885.JPG \\
\hline 2359 & Melica nutans L. & Miskolc & 1929 & Soó Rezső & BPU-11889.JPG \\
\hline 2359 & Melica nutans L. & Miskolc & 1949 & Priszter Szaniszló & BPU-11888.JPG \\
\hline 2359 & Melica nutans L. & Mosonmagyaróvár & 1965 & Borsos Olga & BPU-11887.JPG \\
\hline 2360 & Melica picta K. Koch & Bátorliget & 1935 & Soó Rezső & BPU-11883.JPG \\
\hline 2361 & Glyceria maxima (Hartm.) Holmb. & Bodrogolaszi & 1993 & Felföldy Lajos & BPU-12045.JPG \\
\hline 2361 & Glyceria maxima (Hartm.) Holmb. & Budapest & 1947 & Priszter Szaniszló & BPU-12050.JPG \\
\hline 2361 & Glyceria maxima (Hartm.) Holmb. & Egyek & 1947 & Soó Rezső & BPU-12048.JPG \\
\hline 2361 & Glyceria maxima (Hartm.) Holmb. & Gárdony & 1982 & Felföldy Lajos & BPU-12049.JPG \\
\hline 2361 & Glyceria maxima (Hartm.) Holmb. & Pomáz & 1941 & Priszter Szaniszló & BPU-12047.JPG \\
\hline 2361 & Glyceria maxima (Hartm.) Holmb. & Sárospatak & 1993 & Felföldy Lajos & BPU-12044.JPG \\
\hline 2361 & Glyceria maxima (Hartm.) Holmb. & Tihany & 1956 & $\begin{array}{l}\text { Felföldy Lajos, Tóth } \\
\text { László }\end{array}$ & BPU-12046.JPG \\
\hline 2363 & Glyceria fluitans (L.) R. Br. & Balatonszepezd & 1958 & Felföldy Lajos & BPU-12039.JPG \\
\hline 2363 & Glyceria fluitans (L.) R. Br. & Egyek & 1947 & Soó Rezső & BPU-12042.JPG \\
\hline 2363 & Glyceria fluitans (L.) R. Br. & Miskolc & 1934 & Soó Rezső & BPU-12043.JPG \\
\hline 2363 & Glyceria fluitans (L.) R. Br. & Szécsény & 1953 & Priszter Szaniszló & BPU-12041.JPG \\
\hline 2363 & Glyceria fluitans (L.) R. Br. & Szenta & 1957 & Soó Rezső & BPU-12040.JPG \\
\hline 2363 & Glyceria fluitans (L.) R. Br. & Ukk & 1957 & Felföldy Lajos & BPU-12038.JPG \\
\hline 2365 & Glyceria notata Chevall. & Balatonszőlős & 1956 & $\begin{array}{l}\text { Felföldy Lajos, Tóth } \\
\text { László }\end{array}$ & BPU-12031.JPG \\
\hline 2365 & Glyceria notata Chevall. & Budaörs & 1992 & Felföldy Lajos & BPU-12033.JPG \\
\hline 2365 & Glyceria notata Chevall. & Budapest & 1913 & Jávorka Sándor & BPU-12037.JPG \\
\hline 2365 & Glyceria notata Chevall. & Csesznek & 1929 & Soó Rezső & BPU-12030.JPG \\
\hline 2365 & Glyceria notata Chevall. & Debrecen & 1934 & Soó Rezső & BPU-12036.JPG \\
\hline 2365 & Glyceria notata Chevall. & Kapolcs & 1959 & $\begin{array}{l}\text { Jávorka Sándor, Felföldy } \\
\text { Lajos, Tóth László }\end{array}$ & BPU-12029.JPG \\
\hline 2365 & Glyceria notata Chevall. & Pomáz & 1941 & Priszter Szaniszló & BPU-12032.JPG \\
\hline 2365 & Glyceria notata Chevall. & Szilvásvárad & 1951 & Priszter Szaniszló & BPU-12035.JPG \\
\hline 2366 & $\begin{array}{l}\text { Glyceria nemoralis (Uechtr.) Uechtr. et } \\
\text { Körn. }\end{array}$ & Szilvásvárad & 1953 & Soó Rezső & BPU-12028.JPG \\
\hline 2367 & Bromus catharticus Vahl & Győr & 1916 & Polgár Sándor & BPU-12271.JPG \\
\hline 2370 & Bromus hordeaceus L. & Budapest & 2004 & Felföldy Lajos & BPU-12354.JPG \\
\hline 2370 & Bromus hordeaceus L. & Debrecen & 1945 & Felföldy Lajos & BPU-12351.JPG \\
\hline 2370 & Bromus hordeaceus L. & Debrecen & 1947 & Soó Rezső & BPU-12352.JPG \\
\hline 2370 & Bromus hordeaceus L. & Tihany & 1958 & Felföldy Lajos & BPU-12349.JPG \\
\hline 2370 & Bromus hordeaceus L. & Vácrátót & 1953 & Felföldy Lajos & BPU-12350.JPG \\
\hline 2370 & Bromus hordeaceus L. & Zámoly & 1982 & Felföldy Lajos & BPU-12353.JPG \\
\hline 2371 & Bromus lepidus Holmb. & Budapest & 1931 & Pénzes Antal & BPU-12355.JPG \\
\hline 2373 & Bromus secalinus L. & Bagamér & 1947 & Soó Rezső & BPU-12368.JPG \\
\hline 2373 & Bromus secalinus L. & Nagymaros & 1950 & Priszter Szaniszló & BPU-12367.JPG \\
\hline 2373 & Bromus secalinus L. & Szolnok & 1944 & Timár Lajos & BPU-12366.JPG \\
\hline 2374 & Bromus squarrosus L. & Aszófo" & 1957 & $\begin{array}{l}\text { Felföldy Lajos, Kovács } \\
\text { Margit, Tóth László }\end{array}$ & BPU-12333.JPG \\
\hline 2374 & Bromus squarrosus L. & Budapest & 1991 & Felföldy Lajos & BPU-12331.JPG \\
\hline
\end{tabular}


1. Elektroniukus melléklet - Electronic Appendix 1.

\begin{tabular}{|c|c|c|c|c|c|}
\hline $\begin{array}{l}\text { Sorszám / } \\
\text { Number }\end{array}$ & Taxon-név / Taxon-name & $\begin{array}{l}\text { Település / } \\
\text { Settlement }\end{array}$ & $\begin{array}{l}\text { Év / } \\
\text { Year }\end{array}$ & Gyújtő / Collector & $\begin{array}{l}\text { Fájlnév / } \\
\text { File-name }\end{array}$ \\
\hline 2374 & Bromus squarrosus L. & Budapest & 2004 & Felföldy Lajos & BPU-12340.JPG \\
\hline 2374 & Bromus squarrosus L. & Csévharaszt & 1959 & Simon Tibor, Borsos Olga & BPU-12338.JPG \\
\hline 2374 & Bromus squarrosus L. & Dabas & 1951 & Borsos Olga, Simon Tibor & BPU-12336.JPG \\
\hline 2374 & Bromus squarrosus L. & Kisharsány & 1961 & Simon Tibor & BPU-12337.JPG \\
\hline 2374 & Bromus squarrosus L. & Nagyharsány & 1956 & Soó Rezső & BPU-12335.JPG \\
\hline 2374 & Bromus squarrosus L. & Nagyharsány & 1968 & Priszter Szaniszló & BPU-12339.JPG \\
\hline 2374 & Bromus squarrosus L. & Szigetmonostor & 1947 & Soó Rezső & BPU-12328.JPG \\
\hline 2374 & Bromus squarrosus L. & Tihany & 1942 & Felföldy Lajos & BPU-12332.JPG \\
\hline 2374 & Bromus squarrosus L. & Tihany & 1956 & Felföldy Lajos & BPU-12334.JPG \\
\hline 2374 & Bromus squarrosus L. & Tihany & 1959 & Felföldy Lajos & BPU-12329.JPG \\
\hline 2374 & Bromus squarrosus L. & Tihany & 1959 & Felföldy Lajos & BPU-12330.JPG \\
\hline 2375 & Bromus arvensis L. & Budapest & 2004 & Felföldy Lajos & BPU-12362.JPG \\
\hline 2375 & Bromus arvensis L. & Budapest & 2004 & Felföldy Lajos & BPU-12363.JPG \\
\hline 2375 & Bromus arvensis L. & Budapest & 2004 & Felföldy Lajos & BPU-12364.JPG \\
\hline 2375 & Bromus arvensis L. & Debrecen & 1942 & Felföldy Lajos & BPU-12361.JPG \\
\hline 2375 & Bromus arvensis L. & Egyek & 1947 & Siroki Zoltán & BPU-12360.JPG \\
\hline 2375 & Bromus arvensis L. & Egyek & 1950 & $\begin{array}{l}\text { Simon Tibor, Borsos Olga, } \\
\text { Kulcsár Gábor }\end{array}$ & BPU-12359.JPG \\
\hline 2375 & Bromus arvensis L. & Szentendre & 1918 & Degen Árpád, Zsák Zoltán & BPU-12365.JPG \\
\hline 2376 & Bromus japonicus Thunb. & Balmazújváros & 1937 & Soó Rezső & BPU-12323.JPG \\
\hline 2376 & Bromus japonicus Thunb. & Budapest & 1913 & Szartorisz Béla & BPU-12327.JPG \\
\hline 2376 & Bromus japonicus Thunb. & Budapest & 1982 & Felföldy Lajos & BPU-12324.JPG \\
\hline 2376 & Bromus japonicus Thunb. & Budapest & 1982 & Felföldy Lajos & BPU-12325.JPG \\
\hline 2376 & Bromus japonicus Thunb. & Budapest & 2004 & Felföldy Lajos & BPU-12326.JPG \\
\hline 2376 & Bromus japonicus Thunb. & Köveskál & 1959 & $\begin{array}{l}\text { Jávorka Sándor, Felföldy } \\
\text { Lajos, Tóth László }\end{array}$ & BPU-12322.JPG \\
\hline 2377 & Bromus racemosus L. & Táp & 1912 & Polgár Sándor & BPU-12357.JPG \\
\hline 2378 & Bromus commutatus Schrad. & Bakonybél & 1955 & Soó Rezső & BPU-12341.JPG \\
\hline 2378 & Bromus commutatus Schrad. & Bátorliget & 1934 & Soó Rezső & BPU-12345.JPG \\
\hline 2378 & Bromus commutatus Schrad. & Budapest & 2005 & Felföldy Lajos & BPU-12346.JPG \\
\hline 2378 & Bromus commutatus Schrad. & Hatvan & 1913 & Degen Árpád & BPU-12348.JPG \\
\hline 2378 & Bromus commutatus Schrad. & Monostorapáti & 1959 & $\begin{array}{l}\text { Jávorka Sándor, Felföldy } \\
\text { Lajos, Tóth László }\end{array}$ & BPU-12344.JPG \\
\hline 2378 & Bromus commutatus Schrad. & Tihany & 1959 & Felföldy Lajos & BPU-12342.JPG \\
\hline 2378 & Bromus commutatus Schrad. & Tihany & 1959 & Felföldy Lajos & BPU-12343.JPG \\
\hline 2379 & Bromus inermis Leyss. & Budapest & 1982 & Felföldy Lajos & BPU-12312.JPG \\
\hline 2379 & Bromus inermis Leyss. & Budapest & 2004 & Felföldy Lajos & BPU-12313.JPG \\
\hline 2379 & Bromus inermis Leyss. & Budapest & 2005 & Felföldy Lajos & BPU-12310.JPG \\
\hline 2379 & Bromus inermis Leyss. & Budapest & 2005 & Felföldy Lajos & BPU-12311.JPG \\
\hline 2379 & Bromus inermis Leyss. & Debrecen & 1935 & Soó Rezső & BPU-12308.JPG \\
\hline 2379 & Bromus inermis Leyss. & Debrecen & 1945 & Felföldy Lajos & BPU-12306.JPG \\
\hline 2379 & Bromus inermis Leyss. & Dédestapolcsány & 1932 & $\begin{array}{l}\text { Zólyomi Bálint, Máthé } \\
\text { Imre }\end{array}$ & BPU-12309.JPG \\
\hline 2379 & Bromus inermis Leyss. & Szigetmonostor & 1947 & Soó Rezső & BPU-12307.JPG \\
\hline 2380 & Bromus erectus Huds. & Bakonybél & 1955 & Soó Rezső & BPU-12296.JPG \\
\hline 2380 & Bromus erectus Huds. & Budapest & 1929 & Schermann Szilád & BPU-12303.JPG \\
\hline 2380 & Bromus erectus Huds. & Budapest & 1982 & Felföldy Lajos & BPU-12301.JPG \\
\hline 2380 & Bromus erectus Huds. & Budapest & 1991 & Felföldy Lajos & BPU-12302.JPG \\
\hline 2380 & Bromus erectus Huds. & Háromhuta & 1960 & Simon Tibor & BPU-12294.JPG \\
\hline
\end{tabular}


1. Elektroniukus melléklet - Electronic Appendix 1.

\begin{tabular}{|c|c|c|c|c|c|}
\hline $\begin{array}{l}\text { Sorszám / } \\
\text { Number }\end{array}$ & Taxon-név / Taxon-name & $\begin{array}{l}\text { Település / } \\
\text { Settlement }\end{array}$ & $\begin{array}{l}\text { Év / } \\
\text { Year }\end{array}$ & Gyưjtő / Collector & $\begin{array}{l}\text { Fájlnév / } \\
\text { File-name }\end{array}$ \\
\hline 2380 & Bromus erectus Huds. & Lábatlan & 1953 & Felföldy Lajos & BPU-12298.JPG \\
\hline 2380 & Bromus erectus Huds. & Nagyharsány & 1956 & Soó Rezső & BPU-12292.JPG \\
\hline 2380 & Bromus erectus Huds. & Nyíracsád & 1949 & Soó Rezső & BPU-15529.JPG \\
\hline 2380 & Bromus erectus Huds. & Örvényes & 1959 & Felföldy Lajos & BPU-12295.JPG \\
\hline 2380 & Bromus erectus Huds. & Pécs & 1925 & Soó Rezső & BPU-12291.JPG \\
\hline 2380 & Bromus erectus Huds. & Pilisszentkereszt & 1932 & Soó Rezső & BPU-12297.JPG \\
\hline 2380 & Bromus erectus Huds. & Szarvas & 1940 & Soó Rezső & BPU-12299.JPG \\
\hline 2380 & Bromus erectus Huds. & Szarvas & 1949 & Soó Rezső & BPU-15530.JPG \\
\hline 2381 & Bromus pannonicus Kumm. et Sendtn. & Pécs & 1935 & Soó Rezső & BPU-12293.JPG \\
\hline 2381 & Bromus pannonicus Kumm. et Sendtn. & Pécs & 1953 & Soó Rezső, Borsos Olga & BPU-12300.JPG \\
\hline 2383 & Bromus ramosus Huds. & Bátorliget & 1932 & Soó Rezső & BPU-12320.JPG \\
\hline 2383 & Bromus ramosus Huds. & Budapest & 1992 & Felföldy Lajos & BPU-12318.JPG \\
\hline 2383 & Bromus ramosus Huds. & Budapest & 2004 & Felföldy Lajos & BPU-12321.JPG \\
\hline 2383 & Bromus ramosus Huds. & Csesznek & 1929 & Soó Rezső & BPU-12314.JPG \\
\hline 2383 & Bromus ramosus Huds. & Egyek & 1947 & Soó Rezső & BPU-12317.JPG \\
\hline 2383 & Bromus ramosus Huds. & Szilvásvárad & 1953 & Soó Rezső & BPU-12316.JPG \\
\hline 2384 & Bromus benekenii (Lange) Trimen & Gyöngyös & 1961 & Soó Rezső & BPU-12315.JPG \\
\hline 2386 & Bromus madritensis L. & Budapest & 1931 & Pénzes Antal & BPU-12272.JPG \\
\hline 2386 & Bromus madritensis L. & Budapest & 1931 & Pénzes Antal & BPU-12288.JPG \\
\hline 2387 & Bromus sterilis L. & Budapest & 1918 & Zsák Zoltán & BPU-12286.JPG \\
\hline 2387 & Bromus sterilis L. & Budapest & 1951 & Priszter Szaniszló & BPU-12282.JPG \\
\hline 2387 & Bromus sterilis L. & Budapest & 2004 & Felföldy Lajos & BPU-12284.JPG \\
\hline 2387 & Bromus sterilis L. & Budapest & 2004 & Felföldy Lajos & BPU-12285.JPG \\
\hline 2387 & Bromus sterilis L. & Budapest & 2005 & Felföldy Lajos & BPU-12283.JPG \\
\hline 2387 & Bromus sterilis L. & Debrecen & 1931 & Soó Rezső & BPU-12281.JPG \\
\hline 2387 & Bromus sterilis L. & Murarátka & 1956 & Károlyi Árpád & BPU-12287.JPG \\
\hline 2387 & Bromus sterilis L. & Nagyharsány & 1936 & Soó Rezső & BPU-12289.JPG \\
\hline 2388 & Bromus tectorum L. & Budapest & 1924 & $\begin{array}{l}\text { Degen Árpád, Schermann } \\
\text { Szilárd }\end{array}$ & BPU-12279.JPG \\
\hline 2388 & Bromus tectorum L. & Budapest & 2004 & Felföldy Lajos & BPU-12277.JPG \\
\hline 2388 & Bromus tectorum L. & Budapest & 2004 & Felföldy Lajos & BPU-12278.JPG \\
\hline 2388 & Bromus tectorum L. & Gárdony & 1966 & Priszter Szaniszló & BPU-12275.JPG \\
\hline 2388 & Bromus tectorum L. & Monostorapáti & 1959 & $\begin{array}{l}\text { Jávorka Sándor, Felföldy } \\
\text { Lajos, Tóth László }\end{array}$ & BPU-12273.JPG \\
\hline 2388 & Bromus tectorum L. & Nagyharsány & 1956 & Soó Rezső & BPU-12276.JPG \\
\hline 2388 & Bromus tectorum L. & Szigetcsép & 1919 & Degen Árpád & BPU-12280.JPG \\
\hline 2388 & Bromus tectorum L. & Szigetmonostor & 1947 & Soó Rezső & BPU-12274.JPG \\
\hline 2389 & $\begin{array}{l}\text { Brachypodium sylvaticum (Huds.) P. } \\
\text { Beauv. }\end{array}$ & Budapest & 1982 & Felföldy Lajos & BPU-12064.JPG \\
\hline 2389 & $\begin{array}{l}\text { Brachypodium sylvaticum (Huds.) P. } \\
\text { Beauv. }\end{array}$ & Egyek & 1947 & Soó Rezső & BPU-12068.JPG \\
\hline 2389 & $\begin{array}{l}\text { Brachypodium sylvaticum (Huds.) P. } \\
\text { Beauv. }\end{array}$ & Hajdúhadház & 1934 & Soó Rezső & BPU-12069.JPG \\
\hline 2389 & $\begin{array}{l}\text { Brachypodium sylvaticum (Huds.) P. } \\
\text { Beauv. }\end{array}$ & Miskolc & 1934 & Soó Rezső & BPU-12070.JPG \\
\hline 2389 & $\begin{array}{l}\text { Brachypodium sylvaticum (Huds.) P. } \\
\text { Beauv. }\end{array}$ & Solymár & 2005 & Felföldy Lajos & BPU-12067.JPG \\
\hline 2389 & $\begin{array}{l}\text { Brachypodium sylvaticum (Huds.) P. } \\
\text { Beauv. }\end{array}$ & Tihany & 1959 & Felföldy Lajos & BPU-12066.JPG \\
\hline 2390 & Brachypodium pinnatum (L.) P. Beauv. & Budapest & 1909 & Flatt Károly, Thaisz Lajos & BPU-12061.JPG \\
\hline 2390 & Brachypodium pinnatum (L.) P. Beauv. & Fenyőfő & 1908 & Polgár Sándor & BPU-12057.JPG \\
\hline
\end{tabular}


1. Elektroniukus melléklet - Electronic Appendix 1.

\begin{tabular}{|c|c|c|c|c|c|}
\hline $\begin{array}{l}\text { Sorszám / } \\
\text { Number }\end{array}$ & Taxon-név / Taxon-name & $\begin{array}{l}\text { Település / } \\
\text { Settlement }\end{array}$ & $\begin{array}{l}\text { Év / } \\
\text { Year }\end{array}$ & Gyújtő / Collector & $\begin{array}{l}\text { Fájlnév / } \\
\text { File-name }\end{array}$ \\
\hline 2390 & Brachypodium pinnatum (L.) P. Beauv. & Győr & 1907 & Polgár Sándor & BPU-12055.JPG \\
\hline 2390 & Brachypodium pinnatum (L.) P. Beauv. & Győr & 1912 & Polgár Sándor & BPU-12056.JPG \\
\hline 2390 & Brachypodium pinnatum (L.) P. Beauv. & Győrszemere & 1928 & Polgár Sándor & BPU-12054.JPG \\
\hline 2390 & Brachypodium pinnatum (L.) P. Beauv. & Jósvafő & 1948 & $\begin{array}{l}\text { Simon Tibor, Pólya } \\
\text { László, Böszörményi } \\
\text { Zoltán }\end{array}$ & BPU-12059.JPG \\
\hline 2390 & Brachypodium pinnatum (L.) P. Beauv. & Kőszeg & 1932 & Soó Rezső & BPU-12060.JPG \\
\hline 2390 & Brachypodium pinnatum (L.) P. Beauv. & Nyíradony & 1920 & Ujvárosi Miklós & BPU-12062.JPG \\
\hline 2390 & Brachypodium pinnatum (L.) P. Beauv. & Zákány & 1949 & Károlyi Árpád & BPU-12053.JPG \\
\hline 2391 & $\begin{array}{l}\text { Brachypodium rupestre (Host) Roem. } \\
\text { et Schult. }\end{array}$ & Budapest & 1991 & Felföldy Lajos & BPU-12063.JPG \\
\hline 2393 & Elymus caninus (L.) L. & Debrecen & 1935 & Soó Rezső & BPU-11759.JPG \\
\hline 2393 & Elymus caninus (L.) L. & Tihany & 1959 & Felföldy Lajos & BPU-11758.JPG \\
\hline 2395 & Elymus repens (L.) Gould & Budaörs & 1913 & Zsák Zoltán & BPU-11770.JPG \\
\hline 2395 & Elymus repens (L.) Gould & Budapest & 1993 & Felföldy Lajos & BPU-11767.JPG \\
\hline 2395 & Elymus repens (L.) Gould & Budapest & 2004 & Felföldy Lajos & BPU-11768.JPG \\
\hline 2395 & Elymus repens (L.) Gould & Budapest & 2004 & Felföldy Lajos & BPU-11769.JPG \\
\hline 2395 & Elymus repens (L.) Gould & Debrecen & 1933 & Soó Rezső & BPU-11766.JPG \\
\hline 2395 & Elymus repens (L.) Gould & Debrecen & 1942 & Felföldy Lajos & BPU-11762.JPG \\
\hline 2395 & Elymus repens (L.) Gould & Debrecen & 1943 & Felföldy Lajos & BPU-11763.JPG \\
\hline 2395 & Elymus repens (L.) Gould & Debrecen & 1943 & Felföldy Lajos & BPU-11764.JPG \\
\hline 2395 & Elymus repens (L.) Gould & Debrecen & 1943 & Felföldy Lajos & BPU-11765.JPG \\
\hline 2396 & Elymus hispidus (Opiz) Melderis & Budaörs & 1982 & Felföldy Lajos & BPU-11777.JPG \\
\hline 2396 & Elymus hispidus (Opiz) Melderis & Budapest & 1947 & Soó Rezső & BPU-11789.JPG \\
\hline 2396 & Elymus hispidus (Opiz) Melderis & Budapest & 1982 & Felföldy Lajos & BPU-11784.JPG \\
\hline 2396 & Elymus hispidus (Opiz) Melderis & Budapest & 1991 & Felföldy Lajos & BPU-11788.JPG \\
\hline 2396 & Elymus hispidus (Opiz) Melderis & Budapest & 2004 & Felföldy Lajos & BPU-11787.JPG \\
\hline 2396 & Elymus hispidus (Opiz) Melderis & Budapest & 2005 & Felföldy Lajos & BPU-11785.JPG \\
\hline 2396 & Elymus hispidus (Opiz) Melderis & Budapest & 2005 & Felföldy Lajos & BPU-11786.JPG \\
\hline 2396 & Elymus hispidus (Opiz) Melderis & Debrecen & 1937 & Felföldy Lajos & BPU-11780.JPG \\
\hline 2396 & Elymus hispidus (Opiz) Melderis & Debrecen & 1943 & Felföldy Lajos & BPU-11779.JPG \\
\hline 2396 & Elymus hispidus (Opiz) Melderis & Hajdúhadház & 1933 & Soó Rezső & BPU-11778.JPG \\
\hline 2396 & Elymus hispidus (Opiz) Melderis & Iregszemcse & 1962 & Varga Lajos & BPU-11761.JPG \\
\hline 2396 & Elymus hispidus (Opiz) Melderis & Nagyharsány & 1965 & $\begin{array}{l}\text { Simon Tibor, Priszter } \\
\text { Szaniszló, Borhidi Attila }\end{array}$ & BPU-11781.JPG \\
\hline 2396 & Elymus hispidus (Opiz) Melderis & Pere & 1961 & Simon Tibor & BPU-11783.JPG \\
\hline 2396 & Elymus hispidus (Opiz) Melderis & Siklós & 1967 & Priszter Szaniszló & BPU-11782.JPG \\
\hline 2396 & Elymus hispidus (Opiz) Melderis & Tihany & 1943 & Felföldy Lajos & BPU-11774.JPG \\
\hline 2396 & Elymus hispidus (Opiz) Melderis & Tihany & 1943 & Felföldy Lajos & BPU-11775.JPG \\
\hline 2396 & Elymus hispidus (Opiz) Melderis & Tihany & 1943 & Felföldy Lajos & BPU-11776.JPG \\
\hline 2396 & Elymus hispidus (Opiz) Melderis & Tihany & 1959 & Felföldy Lajos & BPU-11773.JPG \\
\hline 2396 & Elymus hispidus (Opiz) Melderis & Törökbálint & 1932 & Schermann Szilád & BPU-11790.JPG \\
\hline 2397 & Agropyron cristatum (L.) Gaertn. & Budapest & 1947 & Soó Rezső & BPU-11794.JPG \\
\hline 2397 & Agropyron cristatum (L.) Gaertn. & Budapest & 1950 & Priszter Szaniszló & BPU-11793.JPG \\
\hline 2397 & Agropyron cristatum (L.) Gaertn. & Tarcal & 1937 & Soó Rezső & BPU-11792.JPG \\
\hline 2398 & Aegilops cylindrica Host & Budapest & 2005 & Felföldy Lajos & BPU-11720.JPG \\
\hline 2398 & Aegilops cylindrica Host & Hortobágy & 1947 & Soó Rezső & BPU-11719.JPG \\
\hline 2398 & Aegilops cylindrica Host & Köveskál & 1959 & $\begin{array}{l}\text { Jávorka Sándor, Felföldy } \\
\text { Lajos, Tóth László }\end{array}$ & BPU-11718.JPG \\
\hline
\end{tabular}


1. Elektroniukus melléklet - Electronic Appendix 1.

\begin{tabular}{|c|c|c|c|c|c|}
\hline $\begin{array}{l}\text { Sorszám / } \\
\text { Number }\end{array}$ & Taxon-név / Taxon-name & $\begin{array}{l}\text { Település / } \\
\text { Settlement }\end{array}$ & $\begin{array}{l}\text { Év / } \\
\text { Year }\end{array}$ & Gyüjtő / Collector & $\begin{array}{l}\text { Fájlnév / } \\
\text { File-name }\end{array}$ \\
\hline 2398 & Aegilops cylindrica Host & Tihany & 1947 & Pólya László & BPU-11717.JPG \\
\hline 2401 & Triticum aestivum L. & Budapest & 1947 & Priszter Szaniszló & BPU-11715.JPG \\
\hline 2401 & Triticum aestivum L. & Budapest & 1948 & Priszter Szaniszló & BPU-11714.JPG \\
\hline 2401 & Triticum aestivum L. & Budapest & 1950 & Priszter Szaniszló & BPU-11713.JPG \\
\hline 2403 & Secale cereale L. & Budapest & 1947 & Priszter Szaniszló & BPU-11723.JPG \\
\hline 2403 & Secale cereale L. & Budapest & 1948 & Priszter Szaniszló & BPU-11722.JPG \\
\hline 2403 & Secale cereale L. & Debrecen & 1947 & Soó Rezső & BPU-11721.JPG \\
\hline 2404 & Secale sylvestre Host & "Szentendrei-sziget" & 1953 & Borsos Olga & BPU-11724.JPG \\
\hline 2404 & Secale sylvestre Host & Budapest & 1913 & Moesz Gusztáv & BPU-11733.JPG \\
\hline 2404 & Secale sylvestre Host & Budapest & 1943 & Kárpáti Zoltán & BPU-11731.JPG \\
\hline 2404 & Secale sylvestre Host & Bugac & 1962 & Simon Tibor & BPU-11730.JPG \\
\hline 2404 & Secale sylvestre Host & Csévharaszt & 1959 & Simon Tibor, Borsos Olga & BPU-11726.JPG \\
\hline 2404 & Secale sylvestre Host & Dabas & 1947 & Papp József & BPU-11728.JPG \\
\hline 2404 & Secale sylvestre Host & Dabas & 1951 & Borsos Olga, Simon Tibor & BPU-11727.JPG \\
\hline 2404 & Secale sylvestre Host & Szeged & 1941 & Timár Lajos & BPU-11729.JPG \\
\hline 2404 & Secale sylvestre Host & Szigetmonostor & 1947 & Soó Rezső & BPU-11725.JPG \\
\hline 2405 & Hordeum jubatum L. & Debrecen & 1954 & Simon Tibor & BPU-11685.JPG \\
\hline 2407 & Hordeum vulgare L. & Budapest & 1948 & Priszter Szaniszló & BPU-11693.JPG \\
\hline 2408 & Hordeum murinum L. & Budapest & 1948 & Priszter Szaniszló & BPU-11710.JPG \\
\hline 2408 & Hordeum murinum L. & Budapest & 1948 & Priszter Szaniszló & BPU-11711.JPG \\
\hline 2408 & Hordeum murinum L. & Kunszentmiklós & 1968 & Priszter Szaniszló & BPU-11709.JPG \\
\hline 2408 & Hordeum murinum L. & Szentendre & 1941 & Priszter Szaniszló & BPU-11708.JPG \\
\hline 2408 & Hordeum murinum L. & Tihany & 1951 & Priszter Szaniszló & BPU-11707.JPG \\
\hline 2409 & Hordeum secalinum Schreb. & Budapest & 1911 & Degen Árpád & BPU-11712.JPG \\
\hline 2410 & Hordeum hystrix Roth & Akasztó & 1961 & Borsos Olga, Simon Tibor & BPU-11701.JPG \\
\hline 2410 & Hordeum hystrix Roth & Budapest & 1942 & Priszter Szaniszló & BPU-11702.JPG \\
\hline 2410 & Hordeum hystrix Roth & Budapest & 1947 & Priszter Szaniszló & BPU-11703.JPG \\
\hline 2410 & Hordeum hystrix Roth & Budapest & 1948 & Priszter Szaniszló & BPU-11704.JPG \\
\hline 2410 & Hordeum hystrix Roth & Budapest & 1948 & Priszter Szaniszló & BPU-11705.JPG \\
\hline 2410 & Hordeum hystrix Roth & Ecsegfalva & 1969 & Priszter Szaniszló & BPU-11700.JPG \\
\hline 2410 & Hordeum hystrix Roth & Gelénes & 1951 & Simon Tibor & BPU-11698.JPG \\
\hline 2410 & Hordeum hystrix Roth & Hortobágy & 1947 & Soó Rezső & BPU-11697.JPG \\
\hline 2410 & Hordeum hystrix Roth & Kunszentmiklós & 1918 & $\begin{array}{l}\text { Filarszky Nándor, Jávorka } \\
\text { Sándor }\end{array}$ & BPU-11706.JPG \\
\hline 2410 & Hordeum hystrix Roth & Szarvas & 1940 & Soó Rezső & BPU-11699.JPG \\
\hline 2410 & Hordeum hystrix Roth & Szentendre & 1943 & Kárpáti Zoltán & BPU-11695.JPG \\
\hline 2410 & Hordeum hystrix Roth & Szentendre & 1947 & Priszter Szaniszló & BPU-11696.JPG \\
\hline 2412 & $\begin{array}{l}\text { Hordelymus europaeus (L.) Less. ex } \\
\text { Harz }\end{array}$ & Budapest & 1947 & Soó Rezső & BPU-11688.JPG \\
\hline 2412 & $\begin{array}{l}\text { Hordelymus europaeus (L.) Less. ex } \\
\text { Harz }\end{array}$ & Gyöngyös & 1952 & Soó Rezső & BPU-11689.JPG \\
\hline 2412 & $\begin{array}{l}\text { Hordelymus europaeus (L.) Less. ex } \\
\text { Harz }\end{array}$ & Miskolc & 1937 & Soó Rezső & BPU-11692.JPG \\
\hline 2412 & $\begin{array}{l}\text { Hordelymus europaeus (L.) Less. ex } \\
\text { Harz }\end{array}$ & Miskolc & 1950 & Soó Rezső & BPU-11687.JPG \\
\hline 2412 & $\begin{array}{l}\text { Hordelymus europaeus (L.) Less. ex } \\
\text { Harz }\end{array}$ & Miskolc & 1953 & Soó Rezső & BPU-11690.JPG \\
\hline 2413 & $\begin{array}{l}\text { Taeniatherum caput-medusae (L.) } \\
\text { Nevski }\end{array}$ & Hortobágy & 1989 & Tóth Albert & BPU-11684.JPG \\
\hline 2415 & Avena sativa L. & Debrecen & 1935 & Juhász Lajos & BPU-10239.JPG \\
\hline
\end{tabular}


1. Elektroniukus melléklet - Electronic Appendix 1.

\begin{tabular}{|c|c|c|c|c|c|}
\hline $\begin{array}{l}\text { Sorszám / } \\
\text { Number }\end{array}$ & Taxon-név / Taxon-name & $\begin{array}{l}\text { Település / } \\
\text { Settlement }\end{array}$ & $\begin{array}{l}\text { Év / } \\
\text { Year }\end{array}$ & Gyűjtő / Collector & $\begin{array}{l}\text { Fájlnév / } \\
\text { File-name }\end{array}$ \\
\hline 2415 & Avena sativa L. & Nyalka & 1913 & Polgár Sándor & BPU-10237.JPG \\
\hline 2420 & Helictotrichon pubescens (Huds.) Pilg. & Bakonybél & 1955 & Soó Rezső & BPU-10249.JPG \\
\hline 2420 & Helictotrichon pubescens (Huds.) Pilg. & Balatonszőlős & 1958 & Felföldy Lajos & BPU-10250.JPG \\
\hline 2420 & Helictotrichon pubescens (Huds.) Pilg. & Bátorliget & 1932 & Soó Rezső & BPU-10248.JPG \\
\hline 2420 & Helictotrichon pubescens (Huds.) Pilg. & Budapest & 1991 & Felföldy Lajos & BPU-10241.JPG \\
\hline 2420 & Helictotrichon pubescens (Huds.) Pilg. & Budapest & 1993 & Felföldy Lajos & BPU-10242.JPG \\
\hline 2420 & Helictotrichon pubescens (Huds.) Pilg. & Csákvár & 1955 & Soó Rezső & BPU-10245.JPG \\
\hline 2420 & Helictotrichon pubescens (Huds.) Pilg. & Csévharaszt & 1959 & $\begin{array}{l}\text { Simon Tibor, Horánszky } \\
\text { András }\end{array}$ & BPU-10247.JPG \\
\hline 2420 & Helictotrichon pubescens (Huds.) Pilg. & Debrecen & 1933 & Soó Rezső & BPU-10246.JPG \\
\hline 2420 & Helictotrichon pubescens (Huds.) Pilg. & Hidegkút & 1958 & Felföldy Lajos & BPU-10251.JPG \\
\hline 2420 & Helictotrichon pubescens (Huds.) Pilg. & Lábatlan & 1953 & Felföldy Lajos & BPU-10244.JPG \\
\hline 2420 & Helictotrichon pubescens (Huds.) Pilg. & Monostorapáti & 1959 & $\begin{array}{l}\text { Jávorka Sándor, Felföldy } \\
\text { Lajos, Tóth László }\end{array}$ & BPU-10252.JPG \\
\hline 2420 & Helictotrichon pubescens (Huds.) Pilg. & Nagyvisnyó & 1953 & Soó Rezső & BPU-10243.JPG \\
\hline 2421 & $\begin{array}{l}\text { Helictotrichon compressum (Heuff.) } \\
\text { Henrard }\end{array}$ & Miskolc & 1938 & Soó Rezső & BPU-10256.JPG \\
\hline 2421 & $\begin{array}{l}\text { Helictotrichon compressum (Heuff.) } \\
\text { Henrard }\end{array}$ & Szentendre & 1916 & Degen Árpád, Zsák Zoltán & BPU-10255.JPG \\
\hline 2422 & $\begin{array}{l}\text { Helictotrichon adsurgens (Schur ex } \\
\text { Simonk.) Conert }\end{array}$ & Budapest & 2005 & Felföldy Lajos & BPU-10258.JPG \\
\hline 2423 & Helictotrichon pratense (L.) Besser & Uzsa & 1959 & $\begin{array}{l}\text { Jávorka Sándor, Felföldy } \\
\text { Lajos }\end{array}$ & BPU-10261.JPG \\
\hline 2424 & $\begin{array}{l}\text { Arrhenatherum elatius (L.) P. Beauv. } \\
\text { ex J. Presl et C. Presl }\end{array}$ & Bózsva & 1950 & Simon Tibor & BPU-10203.JPG \\
\hline 2424 & $\begin{array}{l}\text { Arrhenatherum elatius (L.) P. Beauv. } \\
\text { ex J. Presl et C. Presl }\end{array}$ & Budapest & 1982 & Felföldy Lajos & BPU-10202.JPG \\
\hline 2424 & $\begin{array}{l}\text { Arrhenatherum elatius (L.) P. Beauv. } \\
\text { ex J. Presl et C. Presl }\end{array}$ & Budapest & 2004 & Felföldy Lajos & BPU-10200.JPG \\
\hline 2424 & $\begin{array}{l}\text { Arrhenatherum elatius (L.) P. Beauv. } \\
\text { ex J. Presl et C. Presl }\end{array}$ & Budapest & 2004 & Felföldy Lajos & BPU-10201.JPG \\
\hline 2424 & $\begin{array}{l}\text { Arrhenatherum elatius (L.) P. Beauv. } \\
\text { ex J. Presl et C. Presl }\end{array}$ & Miskolc & 1937 & Soó Rezső & BPU-10205.JPG \\
\hline 2424 & $\begin{array}{l}\text { Arrhenatherum elatius (L.) P. Beauv. } \\
\text { ex J. Presl et C. Presl }\end{array}$ & Monostorapáti & 1959 & $\begin{array}{l}\text { Jávorka Sándor, Felföldy } \\
\text { Lajos, Tóth László }\end{array}$ & BPU-10207.JPG \\
\hline 2424 & $\begin{array}{l}\text { Arrhenatherum elatius (L.) P. Beauv. } \\
\text { ex J. Presl et C. Presl }\end{array}$ & Nagyharsány & 1956 & Soó Rezső & BPU-10204.JPG \\
\hline 2424 & $\begin{array}{l}\text { Arrhenatherum elatius (L.) P. Beauv. } \\
\text { ex J. Presl et C. Presl }\end{array}$ & Nyíregyháza & 1938 & Soó Rezső & BPU-10206.JPG \\
\hline 2425 & Gaudinia fragilis (L.) P. Beauv. & Bejcgyertyános & 1956 & Jeanplong József & BPU-10276.JPG \\
\hline 2426 & Ventenata dubia (Leers) Coss. & Eszteregnye & 1950 & Károlyi Árpád & BPU-10235.JPG \\
\hline 2426 & Ventenata dubia (Leers) Coss. & Gelénes & 1951 & $\begin{array}{l}\text { Simon Tibor, Vozáry } \\
\text { Elemér }\end{array}$ & BPU-10229.JPG \\
\hline 2426 & Ventenata dubia (Leers) Coss. & Kapolcs & 1959 & $\begin{array}{l}\text { Jávorka Sándor, Felföldy } \\
\text { Lajos, Tóth László }\end{array}$ & BPU-10234.JPG \\
\hline 2426 & Ventenata dubia (Leers) Coss. & Pécs & 1948 & Priszter Szaniszló & BPU-10228.JPG \\
\hline 2426 & Ventenata dubia (Leers) Coss. & Pomáz & 1948 & Priszter Szaniszló & BPU-10231.JPG \\
\hline 2426 & Ventenata dubia (Leers) Coss. & Rudabánya & 1936 & Soó Rezső & BPU-10230.JPG \\
\hline 2426 & Ventenata dubia (Leers) Coss. & Szentendre & 1947 & Priszter Szaniszló & BPU-10233.JPG \\
\hline 2426 & Ventenata dubia (Leers) Coss. & Szentendre & 1948 & Priszter Szaniszló & BPU-10232.JPG \\
\hline 2427 & Koeleria glauca (Spreng.) DC. & Bagamér & 1933 & Soó Rezső & BPU-15977.JPG \\
\hline 2427 & Koeleria glauca (Spreng.) DC. & Dabas & 1951 & Borsos Olga, Simon Tibor & BPU-15976.JPG \\
\hline 2427 & Koeleria glauca (Spreng.) DC. & Füzér & 1938 & Soó Rezső & BPU-15978.JPG \\
\hline 2427 & Koeleria glauca (Spreng.) DC. & Szigetmonostor & 1947 & Soó Rezső & BPU-15974.JPG \\
\hline
\end{tabular}


1. Elektroniukus melléklet - Electronic Appendix 1.

\begin{tabular}{|c|c|c|c|c|c|}
\hline $\begin{array}{l}\text { Sorszám / } \\
\text { Number }\end{array}$ & Taxon-név / Taxon-name & $\begin{array}{l}\text { Település / } \\
\text { Settlement }\end{array}$ & $\begin{array}{l}\text { Év / } \\
\text { Year }\end{array}$ & Gyưjtő / Collector & $\begin{array}{l}\text { Fájlnév / } \\
\text { File-name }\end{array}$ \\
\hline 2427 & Koeleria glauca (Spreng.) DC. & Szigetmonostor & 1947 & Soó Rezső & BPU-15975.JPG \\
\hline 2430 & $\begin{array}{l}\text { Koeleria cristata (L.) Pers. em. Borbás } \\
\text { ex Domin }\end{array}$ & "Szentendrei-sziget" & 1953 & Borsos Olga & BPU-15959.JPG \\
\hline 2430 & $\begin{array}{l}\text { Koeleria cristata (L.) Pers. em. Borbás } \\
\text { ex Domin }\end{array}$ & Budapest & 1951 & Priszter Szaniszló & BPU-15965.JPG \\
\hline 2430 & $\begin{array}{l}\text { Koeleria cristata (L.) Pers. em. Borbás } \\
\text { ex Domin }\end{array}$ & Budapest & 2004 & Felföldy Lajos & BPU-15966.JPG \\
\hline 2430 & $\begin{array}{l}\text { Koeleria cristata (L.) Pers. em. Borbás } \\
\text { ex Domin }\end{array}$ & Fenyőfő & 1955 & Soó Rezső & BPU-15951.JPG \\
\hline 2430 & $\begin{array}{l}\text { Koeleria cristata (L.) Pers. em. Borbás } \\
\text { ex Domin }\end{array}$ & Hortobágy & 1947 & Soó Rezső & BPU-15961.JPG \\
\hline 2430 & $\begin{array}{l}\text { Koeleria cristata (L.) Pers. em. Borbás } \\
\text { ex Domin }\end{array}$ & Isztimér & 1955 & Soó Rezső & BPU-15957.JPG \\
\hline 2430 & $\begin{array}{l}\text { Koeleria cristata (L.) Pers. em. Borbás } \\
\text { ex Domin }\end{array}$ & Lábatlan & 1953 & Felföldy Lajos & BPU-15962.JPG \\
\hline 2430 & $\begin{array}{l}\text { Koeleria cristata (L.) Pers. em. Borbás } \\
\text { ex Domin }\end{array}$ & Nagyharsány & 1956 & Soó Rezső & BPU-15963.JPG \\
\hline 2430 & $\begin{array}{l}\text { Koeleria cristata (L.) Pers. em. Borbás } \\
\text { ex Domin }\end{array}$ & Nagyharsány & 1956 & Soó Rezső & BPU-15964.JPG \\
\hline 2430 & $\begin{array}{l}\text { Koeleria cristata (L.) Pers. em. Borbás } \\
\text { ex Domin }\end{array}$ & Pilisszentiván & 1918 & Degen Árpád & BPU-15969.JPG \\
\hline 2430 & $\begin{array}{l}\text { Koeleria cristata (L.) Pers. em. Borbás } \\
\text { ex Domin }\end{array}$ & Tihany & 1959 & Felföldy Lajos & BPU-15958.JPG \\
\hline 2430 & $\begin{array}{l}\text { Koeleria cristata (L.) Pers. em. Borbás } \\
\text { ex Domin }\end{array}$ & Vác & 1923 & Soó Rezső & BPU-15960.JPG \\
\hline 2431 & $\begin{array}{l}\text { Koeleria majoriflora (Borbás) Borbás } \\
\text { ex Domin }\end{array}$ & Bátorliget & 1934 & Soó Rezső & BPU-15950.JPG \\
\hline 2431 & $\begin{array}{l}\text { Koeleria majoriflora (Borbás) Borbás } \\
\text { ex Domin }\end{array}$ & Kecskemét & 1914 & $\begin{array}{l}\text { Degen Árpád, Lengyel } \\
\text { Géza }\end{array}$ & BPU-15953.JPG \\
\hline 2432 & Koeleria javorkae Ujhelyi & Budapest & 1989 & Hegedűs Á. & BPU-15955.JPG \\
\hline 2432 & Koeleria javorkae Ujhelyi & Budapest & 2005 & Felföldy Lajos & BPU-15954.JPG \\
\hline 2434 & Trisetum flavescens (L.) P. Beauv. & Bakonybél & 1955 & Soó Rezső & BPU-10213.JPG \\
\hline 2434 & Trisetum flavescens (L.) P. Beauv. & Bakonybél & 1955 & Soó Rezső & BPU-10214.JPG \\
\hline 2434 & Trisetum flavescens (L.) P. Beauv. & Bisse & 1965 & $\begin{array}{l}\text { Simon Tibor, Priszter } \\
\text { Szaniszló }\end{array}$ & BPU-10209.JPG \\
\hline 2434 & Trisetum flavescens (L.) P. Beauv. & Miskolc & 1948 & $\begin{array}{l}\text { Simon Tibor, Pólya } \\
\text { László, Borsos Olga }\end{array}$ & BPU-10210.JPG \\
\hline 2434 & Trisetum flavescens (L.) P. Beauv. & Nagyvisnyó & 1953 & Soó Rezső & BPU-10211.JPG \\
\hline 2434 & Trisetum flavescens (L.) P. Beauv. & Szilvásvárad & 1953 & $\begin{array}{l}\text { Felföldy Lajos, Tóth } \\
\text { László }\end{array}$ & BPU-10212.JPG \\
\hline 2434 & Trisetum flavescens (L.) P. Beauv. & Tihany & 1959 & Felföldy Lajos & BPU-10215.JPG \\
\hline 2435 & Deschampsia caespitosa (L.) P. Beauv. & Bicske & 1991 & Felföldy Lajos & BPU-10173.JPG \\
\hline 2435 & Deschampsia caespitosa (L.) P. Beauv. & Budapest & 1993 & Felföldy Lajos & BPU-10170.JPG \\
\hline 2435 & Deschampsia caespitosa (L.) P. Beauv. & Budapest & 2005 & Felföldy Lajos & BPU-10171.JPG \\
\hline 2435 & Deschampsia caespitosa (L.) P. Beauv. & Debrecen & 1934 & Soó Rezső & BPU-10174.JPG \\
\hline 2435 & Deschampsia caespitosa (L.) P. Beauv. & Dunasziget & 1987 & Gulyás Pál & BPU-10178.JPG \\
\hline 2435 & Deschampsia caespitosa (L.) P. Beauv. & Gyöngyös & 1953 & Soó Rezső & BPU-10176.JPG \\
\hline 2435 & Deschampsia caespitosa (L.) P. Beauv. & Gyöngyös & 1953 & Soó Rezső & BPU-10177.JPG \\
\hline 2435 & Deschampsia caespitosa (L.) P. Beauv. & Hévíz & 1952 & Simon Tibor & BPU-10181.JPG \\
\hline 2435 & Deschampsia caespitosa (L.) P. Beauv. & Mátraszentimre & 1935 & Soó Rezső & BPU-10175.JPG \\
\hline 2435 & Deschampsia caespitosa (L.) P. Beauv. & Pálháza & 1949 & Simon Tibor, Jakucs Pál & BPU-10172.JPG \\
\hline 2435 & Deschampsia caespitosa (L.) P. Beauv. & Újhartyán & 1948 & Priszter Szaniszló & BPU-10179.JPG \\
\hline 2435 & Deschampsia caespitosa (L.) P. Beauv. & Vashosszúfalu & 1951 & Felföldy Lajos & BPU-10180.JPG \\
\hline 2436 & Deschampsia flexuosa (L.) Trin. & Gyöngyös & 1953 & Soó Rezső & BPU-10167.JPG \\
\hline 2436 & Deschampsia flexuosa (L.) Trin. & Kőszeg & 1938 & Soó Rezső & BPU-10165.JPG \\
\hline
\end{tabular}


1. Elektroniukus melléklet - Electronic Appendix 1.

\begin{tabular}{|c|c|c|c|c|c|}
\hline $\begin{array}{l}\text { Sorszám / } \\
\text { Number }\end{array}$ & Taxon-név / Taxon-name & $\begin{array}{l}\text { Település / } \\
\text { Settlement }\end{array}$ & $\begin{array}{l}\text { Év / } \\
\text { Year }\end{array}$ & Gyüjtő / Collector & $\begin{array}{l}\text { Fájlnév / } \\
\text { File-name }\end{array}$ \\
\hline 2437 & Aira elegantissima Schur & Budapest & 2004 & Felföldy Lajos & BPU-10158.JPG \\
\hline 2437 & Aira elegantissima Schur & Diósjenő & 1952 & Simon Tibor & BPU-10161.JPG \\
\hline 2437 & Aira elegantissima Schur & Pécs & 1938 & Soó Rezső & BPU-10159.JPG \\
\hline 2437 & Aira elegantissima Schur & Rudabánya & 1936 & Soó Rezső & BPU-10160.JPG \\
\hline 2437 & Aira elegantissima Schur & Salgótarján & 1940 & Hulják János & BPU-10162.JPG \\
\hline 2437 & Aira elegantissima Schur & Uzsa & 1959 & $\begin{array}{l}\text { Jávorka Sándor, Felföldy } \\
\text { Lajos }\end{array}$ & BPU-10163.JPG \\
\hline 2438 & Aira caryophyllea L. & Alsószölnök & 1955 & Soó Rezső & BPU-10157.JPG \\
\hline 2438 & Aira caryophyllea L. & Kőszeg & 1932 & Soó Rezső & BPU-10155.JPG \\
\hline 2438 & Aira caryophyllea L. & Uzsa & 1952 & Simon Tibor & BPU-10156.JPG \\
\hline 2439 & $\begin{array}{l}\text { Hierochloë australis (Schrad.) Roem. } \\
\text { et Schult. }\end{array}$ & Gyenesdiás & 1928 & Soó Rezső & BPU-15717.JPG \\
\hline 2439 & $\begin{array}{l}\text { Hierochloë australis (Schrad.) Roem. } \\
\text { et Schult. }\end{array}$ & Hidegkút & 1959 & Felföldy Lajos & BPU-15718.JPG \\
\hline 2439 & $\begin{array}{l}\text { Hierochloë australis (Schrad.) Roem. } \\
\text { et Schult. }\end{array}$ & Hidegkút & 1959 & Felföldy Lajos & BPU-15719.JPG \\
\hline 2439 & $\begin{array}{l}\text { Hierochloë australis (Schrad.) Roem. } \\
\text { et Schult. }\end{array}$ & Sopron & 1944 & Kárpáti Zoltán & BPU-15720.JPG \\
\hline 2440 & Hierochloë repens (Host) P. Beauv. & Balatonszőlős & 1958 & Felföldy Lajos & BPU-15724.JPG \\
\hline 2440 & Hierochloë repens (Host) P. Beauv. & Budapest & 1942 & Priszter Szaniszló & BPU-15728.JPG \\
\hline 2440 & Hierochloë repens (Host) P. Beauv. & Debrecen & 1934 & Soó Rezső & BPU-15726.JPG \\
\hline 2440 & Hierochloë repens (Host) P. Beauv. & Debrecen & 1949 & Soó Rezső & BPU-15727.JPG \\
\hline 2440 & Hierochloë repens (Host) P. Beauv. & Hidegkút & 1955 & Felföldy Lajos & BPU-15723.JPG \\
\hline 2440 & Hierochloë repens (Host) P. Beauv. & Szigetszentmiklós & 1947 & Kárpáti Zoltán & BPU-15725.JPG \\
\hline 2441 & Anthoxanthum odoratum L. & Beregdaróc & 1951 & $\begin{array}{l}\text { Simon Tibor, Vozáry } \\
\text { Elemér }\end{array}$ & BPU-15713.JPG \\
\hline 2441 & Anthoxanthum odoratum L. & Budapest & 1922 & Soó Rezső & BPU-15715.JPG \\
\hline 2441 & Anthoxanthum odoratum L. & Budapest & 2005 & Felföldy Lajos & BPU-15716.JPG \\
\hline 2441 & Anthoxanthum odoratum L. & Csákberény & 1982 & Felföldy Lajos & BPU-15712.JPG \\
\hline 2441 & Anthoxanthum odoratum L. & Debrecen & 1931 & Soó Rezső & BPU-15711.JPG \\
\hline 2441 & Anthoxanthum odoratum L. & Debrecen & 1988 & Turi B. A., Sümegi Gy. & BPU-15710.JPG \\
\hline 2441 & Anthoxanthum odoratum L. & Diósjenő & 1952 & Simon Tibor & BPU-15709.JPG \\
\hline 2441 & Anthoxanthum odoratum L. & Pálháza & 1991 & Simon Tibor & BPU-15714.JPG \\
\hline 2443 & Holcus lanatus L. & Balatonszepezd & 1958 & Felföldy Lajos & BPU-10196.JPG \\
\hline 2443 & Holcus lanatus L. & Budapest & 1948 & Priszter Szaniszló & BPU-10187.JPG \\
\hline 2443 & Holcus lanatus L. & Budapest & 1991 & Felföldy Lajos & BPU-10188.JPG \\
\hline 2443 & Holcus lanatus L. & Dabas & 1948 & Priszter Szaniszló & BPU-10193.JPG \\
\hline 2443 & Holcus lanatus L. & Debrecen & 1933 & Soó Rezső & BPU-10190.JPG \\
\hline 2443 & Holcus lanatus L. & Debrecen & 1989 & Felföldy Lajos & BPU-10191.JPG \\
\hline 2443 & Holcus lanatus L. & Felsőtelekes & 2005 & Gulyás Pál & BPU-10189.JPG \\
\hline 2443 & Holcus lanatus L. & Kőszeg & 1941 & Priszter Szaniszló & BPU-10194.JPG \\
\hline 2443 & Holcus lanatus L. & Örvényes & 1959 & Felföldy Lajos & BPU-10195.JPG \\
\hline 2443 & Holcus lanatus L. & Pilisszentiván & 1951 & Priszter Szaniszló & BPU-10192.JPG \\
\hline 2443 & Holcus lanatus L. & Szentendre & 1952 & Vozáry Elemér & BPU-15405.JPG \\
\hline 2443 & Holcus lanatus L. & Tihany & 1958 & Felföldy Lajos & BPU-10197.JPG \\
\hline 2444 & Holcus mollis L. & Alsószölnök & 1955 & Soó Rezső & BPU-10185.JPG \\
\hline 2444 & Holcus mollis L. & Szalafő & 1953 & Pócs Tamás & BPU-10186.JPG \\
\hline 2445 & Corynephorus canescens (L.) P. Beauv. & Baktalórántháza & 1934 & Máthé Imre & BPU-10279.JPG \\
\hline 2445 & Corynephorus canescens (L.) P. Beauv. & Fenyőfő & 1955 & Soó Rezső & BPU-10278.JPG \\
\hline 2446 & Agrostis capillaris L. & Dörgicse & 1957 & Felföldy Lajos & BPU-15930.JPG \\
\hline
\end{tabular}


1. Elektroniukus melléklet - Electronic Appendix 1.

\begin{tabular}{|c|c|c|c|c|c|}
\hline $\begin{array}{l}\text { Sorszám / } \\
\text { Number }\end{array}$ & Taxon-név / Taxon-name & $\begin{array}{l}\text { Település / } \\
\text { Settlement }\end{array}$ & $\begin{array}{l}\text { Év / } \\
\text { Year }\end{array}$ & Gyűjtő / Collector & $\begin{array}{l}\text { Fájlnév / } \\
\text { File-name }\end{array}$ \\
\hline 2446 & Agrostis capillaris L. & Felsőtelekes & 2005 & Gulyás Pál & BPU-15935.JPG \\
\hline 2446 & Agrostis capillaris L. & Gyöngyös & 1953 & Soó Rezső & BPU-15933.JPG \\
\hline 2446 & Agrostis capillaris L. & Mátraszentimre & 1961 & Soó Rezső & BPU-15934.JPG \\
\hline 2446 & Agrostis capillaris L. & Nyíracsád & 1949 & Soó Rezső & BPU-15931.JPG \\
\hline 2446 & Agrostis capillaris L. & Nyíracsád & 1949 & Soó Rezső & BPU-15932.JPG \\
\hline 2447 & Agrostis stolonifera L. & Balatonszemes & 1974 & Tóth László & BPU-15918.JPG \\
\hline 2447 & Agrostis stolonifera L. & Balatonszepezd & 1958 & Felföldy Lajos & BPU-15912.JPG \\
\hline 2447 & Agrostis stolonifera L. & Balatonszepezd & 1958 & Felföldy Lajos & BPU-15913.JPG \\
\hline 2447 & Agrostis stolonifera L. & Balatonszőlős & 1957 & $\begin{array}{l}\text { Felföldy Lajos, Kovács } \\
\text { Margit, Tóth László }\end{array}$ & BPU-15914.JPG \\
\hline 2447 & Agrostis stolonifera L. & Bózsva & 1950 & Simon Tibor & BPU-15908.JPG \\
\hline 2447 & Agrostis stolonifera L. & Budapest & 1991 & Felföldy Lajos & BPU-15910.JPG \\
\hline 2447 & Agrostis stolonifera L. & Budapest & 1991 & Felföldy Lajos & BPU-15925.JPG \\
\hline 2447 & Agrostis stolonifera L. & Budapest & 1991 & Felföldy Lajos & BPU-15926.JPG \\
\hline 2447 & Agrostis stolonifera L. & Budapest & 1993 & Felföldy Lajos & BPU-15924.JPG \\
\hline 2447 & Agrostis stolonifera L. & Debrecen & 1935 & Soó Rezső & BPU-15920.JPG \\
\hline 2447 & Agrostis stolonifera L. & Debrecen & 1951 & Soó Rezső & BPU-15919.JPG \\
\hline 2447 & Agrostis stolonifera L. & Gárdony & 1980 & Felföldy Lajos & BPU-15923.JPG \\
\hline 2447 & Agrostis stolonifera L. & Hegykő & 1960 & Tóth László & BPU-15917.JPG \\
\hline 2447 & Agrostis stolonifera L. & Nagycserkesz & 1933 & Soó Rezső & BPU-15916.JPG \\
\hline 2447 & Agrostis stolonifera L. & Pákozd & 1978 & Felföldy Lajos & BPU-15921.JPG \\
\hline 2447 & Agrostis stolonifera L. & Pákozd & 1980 & Felföldy Lajos & BPU-15922.JPG \\
\hline 2447 & Agrostis stolonifera L. & Szombathely & 1964 & Simon Tibor & BPU-15915.JPG \\
\hline 2447 & Agrostis stolonifera L. & Tihany & 1942 & Felföldy Lajos & BPU-15911.JPG \\
\hline 2447 & Agrostis stolonifera L. & Tihany & 1946 & Felföldy Lajos & BPU-15927.JPG \\
\hline 2448 & Agrostis gigantea Roth & Budapest & 1993 & Felföldy Lajos & BPU-15909.JPG \\
\hline 2448 & Agrostis gigantea Roth & Dunasziget & 1987 & Gulyás Pál & BPU-15906.JPG \\
\hline 2448 & Agrostis gigantea Roth & Gelénes & 1951 & Simon Tibor & BPU-15907.JPG \\
\hline 2448 & Agrostis gigantea Roth & Tihany & 1946 & Felföldy Lajos & BPU-15905.JPG \\
\hline 2449 & Agrostis canina L. & Bakonyszentlászló & 1930 & Polgár Sándor & BPU-15937.JPG \\
\hline 2449 & Agrostis canina L. & Szőce & 1955 & Soó Rezső & BPU-15938.JPG \\
\hline 2449 & Agrostis canina L. & Uzsa & 1959 & $\begin{array}{l}\text { Jávorka Sándor, Felföldy } \\
\text { Lajos }\end{array}$ & BPU-15936.JPG \\
\hline 2449 & Agrostis canina L. & Zámoly & 1982 & Felföldy Lajos & BPU-15939.JPG \\
\hline 2451 & Calamagrostis stricta (Timm) Koeler & Bátorliget & 1914 & $\begin{array}{l}\text { Degen Árpád, Lengyel } \\
\text { Géza }\end{array}$ & BPU-15885.JPG \\
\hline 2451 & Calamagrostis stricta (Timm) Koeler & Debrecen & 1989 & Felföldy Lajos & BPU-15884.JPG \\
\hline 2451 & Calamagrostis stricta (Timm) Koeler & Nyírpilis & 1932 & Soó Rezső & BPU-15883.JPG \\
\hline 2451 & Calamagrostis stricta (Timm) Koeler & Nyírpilis & 1951 & Simon Tibor & BPU-15855.JPG \\
\hline 2452 & Calamagrostis arundinacea (L.) Roth & Jósvafő & 1951 & $\begin{array}{l}\text { Soó Rezső, Jakucs Pál, Ér } \\
\text { Lajos }\end{array}$ & BPU-15892.JPG \\
\hline 2452 & Calamagrostis arundinacea (L.) Roth & Pálháza & 1952 & Simon Tibor & BPU-15891.JPG \\
\hline 2452 & Calamagrostis arundinacea (L.) Roth & Szilvásvárad & 1974 & Priszter Szaniszló & BPU-15889.JPG \\
\hline 2452 & Calamagrostis arundinacea (L.) Roth & Tahitótfalu & 1950 & Priszter Szaniszló & BPU-15888.JPG \\
\hline 2452 & Calamagrostis arundinacea (L.) Roth & Tibolddaróc & 1953 & Soó Rezső & BPU-15890.JPG \\
\hline 2453 & Calamagrostis varia (Schrad.) Host & Hollóháza & 2007 & Simon Tibor & BPU-15503.JPG \\
\hline 2453 & Calamagrostis varia (Schrad.) Host & Isztimér & 1949 & Soó Rezső & BPU-15879.JPG \\
\hline 2453 & Calamagrostis varia (Schrad.) Host & Nagyvisnyó & 1953 & Vozáry Elemér & BPU-15880.JPG \\
\hline 2455 & Calamagrostis phragmitoides Hartm. & Szokolya & 1994 & Simon Tibor, Tatár Dóra & BPU-15848.JPG \\
\hline
\end{tabular}


1. Elektroniukus melléklet - Electronic Appendix 1.

\begin{tabular}{|c|c|c|c|c|c|}
\hline $\begin{array}{l}\text { Sorszám / } \\
\text { Number }\end{array}$ & Taxon-név / Taxon-name & $\begin{array}{l}\text { Település / } \\
\text { Settlement }\end{array}$ & $\begin{array}{l}\text { Év / } \\
\text { Year }\end{array}$ & Gyűjtő / Collector & $\begin{array}{l}\text { Fájlnév / } \\
\text { File-name }\end{array}$ \\
\hline 2456 & $\begin{array}{l}\text { Calamagrostis canescens (Weber) } \\
\text { Roth em. Druce }\end{array}$ & Bátorliget & 1934 & Soó Rezső & BPU-15856.JPG \\
\hline 2456 & $\begin{array}{l}\text { Calamagrostis canescens (Weber) } \\
\text { Roth em. Druce }\end{array}$ & Debrecen & 1932 & Soó Rezső & BPU-15857.JPG \\
\hline 2456 & $\begin{array}{l}\text { Calamagrostis canescens (Weber) } \\
\text { Roth em. Druce }\end{array}$ & Gelénes & 1951 & $\begin{array}{l}\text { Simon Tibor, Vozáry } \\
\text { Elemér }\end{array}$ & BPU-15859.JPG \\
\hline 2456 & $\begin{array}{l}\text { Calamagrostis canescens (Weber) } \\
\text { Roth em. Druce }\end{array}$ & Kelemér & 1950 & Soó Rezső & BPU-15858.JPG \\
\hline 2457 & $\begin{array}{l}\text { Calamagrostis pseudophragmites } \\
\text { (Haller f.) Koeler }\end{array}$ & Szenta & 1958 & $\begin{array}{l}\text { Járainé Komlódi Magda, } \\
\text { Borhidi Attila }\end{array}$ & BPU-15866.JPG \\
\hline 2458 & Calamagrostis epigeios (L.) Roth & Badacsonytomaj & 1984 & Felföldy Lajos & BPU-15863.JPG \\
\hline 2458 & Calamagrostis epigeios (L.) Roth & Baktalórántháza & 1933 & Soó Rezső & BPU-15865.JPG \\
\hline 2458 & Calamagrostis epigeios (L.) Roth & Balatonakali & 1958 & Felföldy Lajos & BPU-15864.JPG \\
\hline 2458 & Calamagrostis epigeios (L.) Roth & Balatonmagyaród & 1987 & Mándoki Mónika & BPU-15861.JPG \\
\hline 2458 & Calamagrostis epigeios (L.) Roth & Balatonszemes & 1974 & Tóth László & BPU-15867.JPG \\
\hline 2458 & Calamagrostis epigeios (L.) Roth & Balatonszőlős & 1990 & Felföldy Lajos & BPU-15862.JPG \\
\hline 2458 & Calamagrostis epigeios (L.) Roth & Budapest & 1900 & Flatt Károly & BPU-15876.JPG \\
\hline 2458 & Calamagrostis epigeios (L.) Roth & Budapest & 1903 & Kocsis István & BPU-15847.JPG \\
\hline 2458 & Calamagrostis epigeios (L.) Roth & Budapest & 2005 & Felföldy Lajos & BPU-15875.JPG \\
\hline 2458 & Calamagrostis epigeios (L.) Roth & Debrecen & 1934 & Soó Rezső & BPU-15868.JPG \\
\hline 2458 & Calamagrostis epigeios (L.) Roth & Dunasziget & 1987 & Gulyás Pál & BPU-15870.JPG \\
\hline 2458 & Calamagrostis epigeios (L.) Roth & Gárdony & 1980 & Felföldy Lajos & BPU-15871.JPG \\
\hline 2458 & Calamagrostis epigeios (L.) Roth & Gárdony & 1980 & Felföldy Lajos & BPU-15872.JPG \\
\hline 2458 & Calamagrostis epigeios (L.) Roth & Gárdony & 1980 & Felföldy Lajos & BPU-15873.JPG \\
\hline 2458 & Calamagrostis epigeios (L.) Roth & Szeged & 1953 & Priszter Szaniszló & BPU-15874.JPG \\
\hline 2458 & Calamagrostis epigeios (L.) Roth & Szilvásvárad & 1953 & $\begin{array}{l}\text { Felföldy Lajos, Tóth } \\
\text { László }\end{array}$ & BPU-15869.JPG \\
\hline 2460 & Phleum phleoides (L.) H. Karst. & Budapest & 1942 & Priszter Szaniszló & BPU-15835.JPG \\
\hline 2460 & Phleum phleoides (L.) H. Karst. & Budapest & 1991 & Felföldy Lajos & BPU-15836.JPG \\
\hline 2460 & Phleum phleoides (L.) H. Karst. & Budapest & 2005 & Felföldy Lajos & BPU-15837.JPG \\
\hline 2460 & Phleum phleoides (L.) H. Karst. & Debrecen & 1934 & Soó Rezső & BPU-15834.JPG \\
\hline 2460 & Phleum phleoides (L.) H. Karst. & Dunaharaszti & 1948 & Priszter Szaniszló & BPU-15833.JPG \\
\hline 2460 & Phleum phleoides (L.) H. Karst. & Pilisszentiván & 1941 & Priszter Szaniszló & BPU-15832.JPG \\
\hline 2460 & Phleum phleoides (L.) H. Karst. & Szigetszentmiklós & 1942 & Priszter Szaniszló & BPU-15831.JPG \\
\hline 2460 & Phleum phleoides (L.) H. Karst. & Tihany & 1959 & Felföldy Lajos & BPU-15829.JPG \\
\hline 2460 & Phleum phleoides (L.) H. Karst. & Tihany & 1959 & Felföldy Lajos & BPU-15830.JPG \\
\hline 2460 & Phleum phleoides (L.) H. Karst. & Uzsa & 1959 & $\begin{array}{l}\text { Jávorka Sándor, Felföldy } \\
\text { Lajos }\end{array}$ & BPU-15828.JPG \\
\hline 2461 & Phleum pratense L. & Ipolytarnóc & 1987 & Felföldy Lajos & BPU-15845.JPG \\
\hline 2461 & Phleum pratense L. & Kishartyán & 1953 & Priszter Szaniszló & BPU-15844.JPG \\
\hline 2461 & Phleum pratense L. & Nyíregyháza & 1942 & Soó Rezső & BPU-15840.JPG \\
\hline 2461 & Phleum pratense L. & Pomáz & 1942 & Priszter Szaniszló & BPU-15843.JPG \\
\hline 2461 & Phleum pratense L. & Pomáz & 1948 & Priszter Szaniszló & BPU-15842.JPG \\
\hline 2461 & Phleum pratense L. & Szentendre & 1947 & Priszter Szaniszló & BPU-15841.JPG \\
\hline 2461 & Phleum pratense L. & Tihany & 1928 & Soó Rezső & BPU-15839.JPG \\
\hline 2463 & Alopecurus pratensis L. & Debrecen & 1934 & Soó Rezső & BPU-15814.JPG \\
\hline 2463 & Alopecurus pratensis L. & Pátka & 1987 & Felföldy Lajos & BPU-15815.JPG \\
\hline 2464 & Alopecurus myosuroides Huds. & Győr & 1928 & Polgár Sándor & BPU-15816.JPG \\
\hline 2465 & Alopecurus geniculatus L. & Debrecen & 1933 & Soó Rezső & BPU-15813.JPG \\
\hline 2465 & Alopecurus geniculatus L. & Kapolcs & 1959 & $\begin{array}{l}\text { Jávorka Sándor, Felföldy } \\
\text { Lajos, Tóth László }\end{array}$ & BPU-15811.JPG \\
\hline
\end{tabular}


1. Elektroniukus melléklet - Electronic Appendix 1.

\begin{tabular}{|c|c|c|c|c|c|}
\hline $\begin{array}{l}\text { Sorszám / } \\
\text { Number }\end{array}$ & Taxon-név / Taxon-name & $\begin{array}{l}\text { Település / } \\
\text { Settlement }\end{array}$ & $\begin{array}{l}\text { Év / } \\
\text { Year }\end{array}$ & Gyújtő / Collector & $\begin{array}{l}\text { Fájlnév / } \\
\text { File-name }\end{array}$ \\
\hline 2465 & Alopecurus geniculatus L. & Tiszabercel & 1990 & Felföldy Lajos & BPU-15812.JPG \\
\hline 2467 & Pholiurus pannonicus Trin. & Akasztó & 1961 & Borsos Olga, Simon Tibor & BPU-10153.JPG \\
\hline 2467 & Pholiurus pannonicus Trin. & Debrecen & 1948 & Jakobinyi A. & BPU-10152.JPG \\
\hline 2467 & Pholiurus pannonicus Trin. & Debrecen & 1951 & Soó Rezső & BPU-10149.JPG \\
\hline 2467 & Pholiurus pannonicus Trin. & Egyek & 1950 & $\begin{array}{l}\text { Simon Tibor, Borsos Olga, } \\
\text { Kulcsár Gábor }\end{array}$ & BPU-10150.JPG \\
\hline 2467 & Pholiurus pannonicus Trin. & Egyek & 1950 & $\begin{array}{l}\text { Simon Tibor, Borsos Olga, } \\
\text { Kulcsár Gábor }\end{array}$ & BPU-10151.JPG \\
\hline 2467 & Pholiurus pannonicus Trin. & Hortobágy & 1947 & Soó Rezső & BPU-10148.JPG \\
\hline 2467 & Pholiurus pannonicus Trin. & Szarvas & 1949 & Soó Rezső & BPU-10146.JPG \\
\hline 2467 & Pholiurus pannonicus Trin. & Szeged & 1951 & Timár Lajos & BPU-10147.JPG \\
\hline 2467 & Pholiurus pannonicus Trin. & Szolnok & 1944 & Timár Lajos & BPU-10154.JPG \\
\hline 2469 & Phalaris arundinacea L. & Balatonakali & 1958 & Felföldy Lajos & BPU-15701.JPG \\
\hline 2469 & Phalaris arundinacea L. & Budapest & 2005 & Felföldy Lajos & BPU-15706.JPG \\
\hline 2469 & Phalaris arundinacea L. & Debrecen & 1931 & Soó Rezső & BPU-15704.JPG \\
\hline 2469 & Phalaris arundinacea L. & Nagyrada & 1987 & Felföldy Lajos & BPU-15699.JPG \\
\hline 2469 & Phalaris arundinacea L. & Pákozd & 1980 & Felföldy Lajos & BPU-15705.JPG \\
\hline 2469 & Phalaris arundinacea L. & Szentendre & 1949 & Soó Rezső & BPU-15703.JPG \\
\hline 2469 & Phalaris arundinacea L. & Tihany & 1956 & $\begin{array}{l}\text { Felföldy Lajos, Tóth } \\
\text { László }\end{array}$ & BPU-15700.JPG \\
\hline 2469 & Phalaris arundinacea L. & Tiszaroff & 1937 & Soó Rezső & BPU-15702.JPG \\
\hline 2471 & Milium effusum L. & Debrecen & 1934 & Soó Rezső & BPU-15743.JPG \\
\hline 2471 & Milium effusum L. & Kisunyom & 1954 & $\begin{array}{l}\text { Máthé Imre, Jeanplon } \\
\text { József, Kovács Margit }\end{array}$ & BPU-15740.JPG \\
\hline 2471 & Milium effusum L. & Mátraszentimre & 1961 & Soó Rezső & BPU-15742.JPG \\
\hline 2471 & Milium effusum L. & Pécs & 1953 & Soó Rezső, Borsos Olga & BPU-15746.JPG \\
\hline 2471 & Milium effusum L. & Pécs & 1956 & Soó Rezső & BPU-15745.JPG \\
\hline 2471 & Milium effusum L. & Sopron & 1942 & Priszter Szaniszló & BPU-15741.JPG \\
\hline 2471 & Milium effusum L. & Tákos & 1949 & Soó Rezső & BPU-15744.JPG \\
\hline 2472 & Piptatherum virescens (Trin.) Boiss. & Balatonszőlős & 1958 & Felföldy Lajos & BPU-15749.JPG \\
\hline 2472 & Piptatherum virescens (Trin.) Boiss. & Bélapátfalva & 1948 & $\begin{array}{l}\text { Simon Tibor, Pólya } \\
\text { László, Jakucs Pál }\end{array}$ & BPU-15753.JPG \\
\hline 2472 & Piptatherum virescens (Trin.) Boiss. & Budapest & 1947 & Priszter Szaniszló & BPU-15755.JPG \\
\hline 2472 & Piptatherum virescens (Trin.) Boiss. & Budapest & 1982 & Felföldy Lajos & BPU-15754.JPG \\
\hline 2472 & Piptatherum virescens (Trin.) Boiss. & Csákberény & 1948 & Priszter Szaniszló & BPU-15752.JPG \\
\hline 2472 & Piptatherum virescens (Trin.) Boiss. & Csákvár & 1955 & Soó Rezső & BPU-15750.JPG \\
\hline 2472 & Piptatherum virescens (Trin.) Boiss. & Csákvár & 1955 & Soó Rezső & BPU-15751.JPG \\
\hline 2473 & Stipa capillata L. & Bugac & 1964 & Soó Rezső & BPU-15795.JPG \\
\hline 2473 & Stipa capillata L. & Csókakő & 1948 & Priszter Szaniszló & BPU-15793.JPG \\
\hline 2473 & Stipa capillata L. & Debrecen & 1936 & Juhász Lajos & BPU-15794.JPG \\
\hline 2473 & Stipa capillata L. & Pilisborosjenő & 1941 & Priszter Szaniszló & BPU-15791.JPG \\
\hline 2473 & Stipa capillata L. & Tatárszentgyörgy & 1950 & Soó Rezső & BPU-15792.JPG \\
\hline 2473 & Stipa capillata L. & Tihany & 1959 & Felföldy Lajos & BPU-15790.JPG \\
\hline 2473 & Stipa capillata L. & Tokaj & 1949 & Soó Rezső & BPU-15788.JPG \\
\hline 2473 & Stipa capillata L. & Uzsa & 1952 & Borsos Olga & BPU-15789.JPG \\
\hline 2475 & Stipa tirsa Steven em. Čelak. & Budapest & 1904 & Degen Árpád & BPU-15787.JPG \\
\hline 2475 & Stipa tirsa Steven em. Čelak. & Pomáz & 1942 & Priszter Szaniszló & BPU-15785.JPG \\
\hline 2475 & Stipa tirsa Steven em. Čelak. & Szentendre & 1942 & Priszter Szaniszló & BPU-15786.JPG \\
\hline 2475 & Stipa tirsa Steven em. Čelak. & Tihany & 1927 & Soó Rezső & BPU-15784.JPG \\
\hline
\end{tabular}


1. Elektroniukus melléklet - Electronic Appendix 1.

\begin{tabular}{|c|c|c|c|c|c|}
\hline $\begin{array}{l}\text { Sorszám / } \\
\text { Number }\end{array}$ & Taxon-név / Taxon-name & $\begin{array}{l}\text { Település / } \\
\text { Settlement }\end{array}$ & $\begin{array}{l}\text { Év / } \\
\text { Year }\end{array}$ & Gyújtő / Collector & $\begin{array}{l}\text { Fájlnév / } \\
\text { File-name }\end{array}$ \\
\hline 2476 & $\begin{array}{l}\text { Stipa dasyphylla (Czern. ex Lindem.) } \\
\text { Trautv. }\end{array}$ & Budapest & 1939 & Soó Rezső & BPU-15782.JPG \\
\hline 2476 & $\begin{array}{l}\text { Stipa dasyphylla (Czern. ex Lindem.) } \\
\text { Trautv. }\end{array}$ & Budapest & 1947 & Priszter Szaniszló & BPU-15781.JPG \\
\hline 2476 & $\begin{array}{l}\text { Stipa dasyphylla (Czern. ex Lindem.) } \\
\text { Trautv. }\end{array}$ & Budapest & 1947 & Soó Rezső & BPU-15779.JPG \\
\hline 2476 & $\begin{array}{l}\text { Stipa dasyphylla (Czern. ex Lindem.) } \\
\text { Trautv. }\end{array}$ & Budapest & 1947 & Soó Rezső & BPU-15780.JPG \\
\hline 2477 & Stipa pennata $\mathrm{L}$. & Budaörs & 1947 & Soó Rezső & BPU-15769.JPG \\
\hline 2477 & Stipa pennata L. & Budapest & 2004 & Felföldy Lajos & BPU-15773.JPG \\
\hline 2477 & Stipa pennata L. & Csákvár & 1955 & Soó Rezső & BPU-15766.JPG \\
\hline 2477 & Stipa pennata L. & Csákvár & 1955 & Soó Rezső & BPU-15772.JPG \\
\hline 2477 & Stipa pennata L. & Csévharaszt & 1959 & Simon Tibor, Borsos Olga & BPU-15760.JPG \\
\hline 2477 & Stipa pennata L. & Dabas & 1951 & Borsos Olga, Simon Tibor & BPU-15761.JPG \\
\hline 2477 & Stipa pennata L. & Debrecen & 1938 & Soó Rezső & BPU-15767.JPG \\
\hline 2477 & Stipa pennata L. & Fenyőfő & 1955 & Soó Rezső & BPU-15765.JPG \\
\hline 2477 & Stipa pennata L. & Győr & 1959 & $\begin{array}{l}\text { Simon Tibor, Borhidi } \\
\text { Attila }\end{array}$ & BPU-15762.JPG \\
\hline 2477 & Stipa pennata L. & Lábatlan & 1953 & Felföldy Lajos & BPU-15768.JPG \\
\hline 2477 & Stipa pennata L. & Szalonna & 1966 & Felföldy Lajos & BPU-15771.JPG \\
\hline 2477 & Stipa pennata L. & Szigetmonostor & 1947 & Soó Rezső & BPU-15759.JPG \\
\hline 2477 & Stipa pennata L. & Tállya & 1938 & Soó Rezső & BPU-15763.JPG \\
\hline 2477 & Stipa pennata L. & Telki & 1947 & Soó Rezső & BPU-15770.JPG \\
\hline 2477 & Stipa pennata L. & Tihany & 1959 & Felföldy Lajos & BPU-15764.JPG \\
\hline 2479 & Stipa pulcherrima K. Koch & Budapest & 1947 & Priszter Szaniszló & BPU-15777.JPG \\
\hline 2479 & Stipa pulcherrima K. Koch & Budapest & 1947 & Soó Rezső & BPU-15778.JPG \\
\hline 2479 & Stipa pulcherrima K. Koch & Budapest & 1982 & Felföldy Lajos & BPU-15776.JPG \\
\hline 2479 & Stipa pulcherrima K. Koch & Pilisborosjenő & 1942 & Priszter Szaniszló & BPU-15775.JPG \\
\hline 2479 & Stipa pulcherrima K. Koch & Pilisszentiván & 1942 & Priszter Szaniszló & BPU-15774.JPG \\
\hline 2481 & Phragmites australis (Cav.) Steud. & Balatonberény & 1958 & Tóth László & BPU-11679.JPG \\
\hline 2481 & Phragmites australis (Cav.) Steud. & Csókakő & 1948 & Priszter Szaniszló & BPU-11681.JPG \\
\hline 2481 & Phragmites australis (Cav.) Steud. & Esztergom & 1947 & Priszter Szaniszló & BPU-11682.JPG \\
\hline 2481 & Phragmites australis (Cav.) Steud. & Keszthely & 1958 & Tóth László & BPU-11678.JPG \\
\hline 2481 & Phragmites australis (Cav.) Steud. & Sarród & 1923 & Polgár Sándor & BPU-11680.JPG \\
\hline 2481 & Phragmites australis (Cav.) Steud. & Vonyarcvashegy & 1958 & Tóth László & BPU-11677.JPG \\
\hline 2482 & Danthonia alpina Vest & Gyöngyös & 1961 & Soó Rezső & BPU-10273.JPG \\
\hline 2482 & Danthonia alpina Vest & Miskolc & 1938 & Soó Rezső & BPU-10272.JPG \\
\hline 2482 & Danthonia alpina Vest & Uzsa & 1959 & $\begin{array}{l}\text { Jávorka Sándor, Felföldy } \\
\text { Lajos }\end{array}$ & BPU-10274.JPG \\
\hline 2483 & Danthonia decumbens (L.) DC. & Alsószölnök & 1955 & Soó Rezső & BPU-15982.JPG \\
\hline 2483 & Danthonia decumbens (L.) DC. & Miskolc & 1929 & Soó Rezső & BPU-15986.JPG \\
\hline 2483 & Danthonia decumbens (L.) DC. & Pilisszentlászló & - & $\begin{array}{l}\text { Degen Árpád, Trautmann } \\
\text { Róbert }\end{array}$ & BPU-15987.JPG \\
\hline 2483 & Danthonia decumbens (L.) DC. & Szakonyfalu & 1955 & Soó Rezső & BPU-15983.JPG \\
\hline 2483 & Danthonia decumbens (L.) DC. & Szentendre & 1942 & Priszter Szaniszló & BPU-15985.JPG \\
\hline 2483 & Danthonia decumbens (L.) DC. & Szentendre & 1947 & Priszter Szaniszló & BPU-15984.JPG \\
\hline 2483 & Danthonia decumbens (L.) DC. & Uzsa & 1952 & Simon Tibor & BPU-15981.JPG \\
\hline 2483 & Danthonia decumbens (L.) DC. & Uzsa & 1959 & $\begin{array}{l}\text { Jávorka Sándor, Felföldy } \\
\text { Lajos }\end{array}$ & BPU-15980.JPG \\
\hline 2484 & Molinia caerulea (L.) Moench & Balatonszőlős & 1957 & $\begin{array}{l}\text { Felföldy Lajos, Kovács } \\
\text { Margit, Tóth László }\end{array}$ & BPU-11797.JPG \\
\hline
\end{tabular}


1. Elektroniukus melléklet - Electronic Appendix 1.

\begin{tabular}{|c|c|c|c|c|c|}
\hline $\begin{array}{l}\text { Sorszám / } \\
\text { Number }\end{array}$ & Taxon-név / Taxon-name & $\begin{array}{l}\text { Település / } \\
\text { Settlement }\end{array}$ & $\begin{array}{l}\text { Év / } \\
\text { Year }\end{array}$ & Gyüjtő / Collector & $\begin{array}{l}\text { Fájlnév / } \\
\text { File-name }\end{array}$ \\
\hline 2484 & Molinia caerulea (L.) Moench & Balatonszőlős & 1990 & Felföldy Lajos & BPU-11796.JPG \\
\hline 2484 & Molinia caerulea (L.) Moench & Budapest & 1989 & Felföldy Lajos & BPU-11814.JPG \\
\hline 2484 & Molinia caerulea (L.) Moench & Budapest & 1991 & Felföldy Lajos & BPU-11813.JPG \\
\hline 2484 & Molinia caerulea (L.) Moench & Budapest & 1993 & Felföldy Lajos & BPU-11812.JPG \\
\hline 2484 & Molinia caerulea (L.) Moench & Eszteregnye & 1950 & Károlyi Árpád & BPU-11795.JPG \\
\hline 2484 & Molinia caerulea (L.) Moench & Esztergom & 1947 & Priszter Szaniszló & BPU-11811.JPG \\
\hline 2484 & Molinia caerulea (L.) Moench & Hegykő & 1960 & Tóth László & BPU-11809.JPG \\
\hline 2484 & Molinia caerulea (L.) Moench & Hévíz & 1961 & Felföldy Lajos & BPU-11803.JPG \\
\hline 2484 & Molinia caerulea (L.) Moench & Kaszó & 1957 & Soó Rezső & BPU-11802.JPG \\
\hline 2484 & Molinia caerulea (L.) Moench & Kőszeg & 1932 & Soó Rezső & BPU-11799.JPG \\
\hline 2484 & Molinia caerulea (L.) Moench & Örvényes & 1958 & Felföldy Lajos & BPU-11804.JPG \\
\hline 2484 & Molinia caerulea (L.) Moench & Örvényes & 1958 & Felföldy Lajos & BPU-11805.JPG \\
\hline 2484 & Molinia caerulea (L.) Moench & Piricse & 1932 & Soó Rezső & BPU-11801.JPG \\
\hline 2484 & Molinia caerulea (L.) Moench & Sopron & 1942 & Priszter Szaniszló & BPU-11806.JPG \\
\hline 2484 & Molinia caerulea (L.) Moench & Sopron & 1942 & Priszter Szaniszló & BPU-11808.JPG \\
\hline 2484 & Molinia caerulea (L.) Moench & Sopron & 1947 & Priszter Szaniszló & BPU-11807.JPG \\
\hline 2484 & Molinia caerulea (L.) Moench & Szakonyfalu & 1955 & Soó Rezső & BPU-11798.JPG \\
\hline 2484 & Molinia caerulea (L.) Moench & Szentgotthárd & 1955 & Soó Rezső & BPU-11800.JPG \\
\hline 2484 & Molinia caerulea (L.) Moench & Veresegyház & 1949 & Soó Rezső & BPU-11810.JPG \\
\hline 2486 & Nardus stricta L. & Nagyvisnyó & 1953 & Soó Rezső & BPU-10141.JPG \\
\hline 2486 & Nardus stricta L. & Pálháza & 1960 & Simon Tibor & BPU-10140.JPG \\
\hline 2486 & Nardus stricta L. & Pilisszentlászló & 1917 & $\begin{array}{l}\text { Degen Árpád, Trautmann } \\
\text { Róbert }\end{array}$ & BPU-10139.JPG \\
\hline 2486 & Nardus stricta L. & Pomáz & 1948 & Priszter Szaniszló & BPU-10142.JPG \\
\hline 2487 & Cleistogenes serotina (L.) Keng & Budapest & 1958 & Simon Tibor & BPU-11662.JPG \\
\hline 2487 & Cleistogenes serotina (L.) Keng & Nagymaros & 1947 & Soó Rezső & BPU-11661.JPG \\
\hline 2488 & Eragrostis minor Host & Budapest & 1991 & Felföldy Lajos & BPU-11673.JPG \\
\hline 2488 & Eragrostis minor Host & Budapest & 1993 & Felföldy Lajos & BPU-11672.JPG \\
\hline 2488 & Eragrostis minor Host & Csarnóta & 1968 & Priszter Szaniszló & BPU-11670.JPG \\
\hline 2488 & Eragrostis minor Host & Debrecen & 1931 & Soó Rezső & BPU-11667.JPG \\
\hline 2488 & Eragrostis minor Host & Debrecen & - & Soó Rezső & BPU-11668.JPG \\
\hline 2488 & Eragrostis minor Host & Kemence & 1952 & Simon Tibor & BPU-11666.JPG \\
\hline 2488 & Eragrostis minor Host & Kiskunmajsa & 1954 & Soó Rezső & BPU-11671.JPG \\
\hline 2488 & Eragrostis minor Host & Orosháza & 1974 & Priszter Szaniszló & BPU-11669.JPG \\
\hline 2488 & Eragrostis minor Host & Törökbálint & 1991 & Felföldy Lajos & BPU-11665.JPG \\
\hline 2489 & $\begin{array}{l}\text { Eragrostis cilianensis (All.) Vignolo ex } \\
\text { Janch. }\end{array}$ & Nagyharsány & 1964 & Priszter Szaniszló & BPU-11664.JPG \\
\hline 2489 & $\begin{array}{l}\text { Eragrostis cilianensis (All.) Vignolo ex } \\
\text { Janch. }\end{array}$ & Szeged & 1947 & Timár Lajos & BPU-11663.JPG \\
\hline 2490 & Eragrostis pilosa (L.) P. Beauv. & Egyek & 1947 & Soó Rezső & BPU-11674.JPG \\
\hline 2490 & Eragrostis pilosa (L.) P. Beauv. & Szigetszentmiklós & 1900 & Degen Árpád & BPU-11675.JPG \\
\hline 2490 & Eragrostis pilosa (L.) P. Beauv. & Szigetszentmiklós & 1909 & Degen Árpád & BPU-11675.JPG \\
\hline 2494 & Crypsis aculeata (L.) Aiton & Gárdony & 1959 & Kovács Miklós & BPU-15807.JPG \\
\hline 2494 & Crypsis aculeata (L.) Aiton & Hajdúbagos & 1937 & Soó Rezső & BPU-15809.JPG \\
\hline 2494 & Crypsis aculeata (L.) Aiton & Kiskunmajsa & 1964 & Soó Rezső & BPU-15810.JPG \\
\hline 2494 & Crypsis aculeata (L.) Aiton & Konyár & 1933 & Soó Rezső & BPU-15808.JPG \\
\hline 2495 & Crypsis schoenoides (L.) Lam. & Budapest & 1913 & Moesz Gusztáv & BPU-15801.JPG \\
\hline 2495 & Crypsis schoenoides (L.) Lam. & Budapest & 1941 & Priszter Szaniszló & BPU-15800.JPG \\
\hline 2495 & Crypsis schoenoides (L.) Lam. & Budapest & 1947 & Priszter Szaniszló & BPU-15799.JPG \\
\hline
\end{tabular}


1. Elektroniukus melléklet - Electronic Appendix 1.

\begin{tabular}{|c|c|c|c|c|c|}
\hline $\begin{array}{l}\text { Sorszám / } \\
\text { Number }\end{array}$ & Taxon-név / Taxon-name & $\begin{array}{l}\text { Település / } \\
\text { Settlement }\end{array}$ & $\begin{array}{l}\text { Év / } \\
\text { Year }\end{array}$ & Gyújtő / Collector & $\begin{array}{l}\text { Fájlnév / } \\
\text { File-name }\end{array}$ \\
\hline 2495 & Crypsis schoenoides (L.) Lam. & Egyek & 1947 & Soó Rezső & BPU-15796.JPG \\
\hline 2495 & Crypsis schoenoides (L.) Lam. & Kiskunmajsa & 1964 & Soó Rezső & BPU-15798.JPG \\
\hline 2495 & Crypsis schoenoides (L.) Lam. & Szeged & 1949 & Timár Lajos & BPU-15797.JPG \\
\hline 2496 & $\begin{array}{l}\text { Crypsis alopecuroides (Piller et } \\
\text { Mitterp.) Schrad. }\end{array}$ & Budapest & 1948 & Priszter Szaniszló & BPU-15804.JPG \\
\hline 2496 & $\begin{array}{l}\text { Crypsis alopecuroides (Piller et } \\
\text { Mitterp.) Schrad. }\end{array}$ & Budapest & 1948 & Priszter Szaniszló & BPU-15805.JPG \\
\hline 2496 & $\begin{array}{l}\text { Crypsis alopecuroides (Piller et } \\
\text { Mitterp.) Schrad. }\end{array}$ & Ecsegfalva & 1901 & Degen Árpád & BPU-15806.JPG \\
\hline 2496 & $\begin{array}{l}\text { Crypsis alopecuroides (Piller et } \\
\text { Mitterp.) Schrad. }\end{array}$ & Egyek & 1947 & Soó Rezső & BPU-15802.JPG \\
\hline 2496 & $\begin{array}{l}\text { Crypsis alopecuroides (Piller et } \\
\text { Mitterp.) Schrad. }\end{array}$ & Szeged & 1947 & Timár Lajos & BPU-15803.JPG \\
\hline 2497 & Eleusine indica (L.) Gaertn. & Budapest & 1947 & Soó Rezső & BPU-11654.JPG \\
\hline 2497 & Eleusine indica (L.) Gaertn. & Budapest & 2004 & Felföldy Lajos & BPU-11655.JPG \\
\hline 2497 & Eleusine indica (L.) Gaertn. & Budapest & 2004 & Felföldy Lajos & BPU-11656.JPG \\
\hline 2497 & Eleusine indica (L.) Gaertn. & Budapest & $?$ & Kárpáti Zoltán & BPU-11657.JPG \\
\hline 2498 & Cynodon dactylon (L.) Pers. & Baja & 1965 & Priszter Szaniszló & BPU-11660.JPG \\
\hline 2498 & Cynodon dactylon (L.) Pers. & Bicske & 1991 & Felföldy Lajos & BPU-11659.JPG \\
\hline 2498 & Cynodon dactylon (L.) Pers. & Debrecen & 1947 & Soó Rezső & BPU-11658.JPG \\
\hline 2499 & Tragus racemosus (L.) All. & Budapest & 1947 & Priszter Szaniszló & BPU-15737.JPG \\
\hline 2499 & Tragus racemosus (L.) All. & Budapest & 1948 & Priszter Szaniszló & BPU-15739.JPG \\
\hline 2499 & Tragus racemosus (L.) All. & Budapest & 1982 & Felföldy Lajos & BPU-15738.JPG \\
\hline 2499 & Tragus racemosus (L.) All. & Csarnóta & 1968 & Priszter Szaniszló & BPU-15736.JPG \\
\hline 2499 & Tragus racemosus (L.) All. & Szigetcsép & 1966 & Priszter Szaniszló & BPU-15735.JPG \\
\hline 2499 & Tragus racemosus (L.) All. & Tihany & 1954 & Felföldy Lajos & BPU-15733.JPG \\
\hline 2499 & Tragus racemosus (L.) All. & Tihany & 1959 & Felföldy Lajos & BPU-15732.JPG \\
\hline 2499 & Tragus racemosus (L.) All. & Vácrátót & 1949 & Soó Rezső & BPU-15734.JPG \\
\hline 2501 & Leersia oryzoides (L.) Sw. & Budapest & 1947 & Priszter Szaniszló & BPU-15698.JPG \\
\hline 2501 & Leersia oryzoides (L.) Sw. & Dávod & 1955 & Soó Rezső & BPU-15695.JPG \\
\hline 2501 & Leersia oryzoides (L.) Sw. & Gyomaendrőd & 1947 & Soó Rezső & BPU-15697.JPG \\
\hline 2501 & Leersia oryzoides (L.) Sw. & Szeged & 1947 & Timár Lajos & BPU-15696.JPG \\
\hline 2504 & Panicum capillare L. & Bátorliget & 1926 & Boros Ádám & BPU-15681.JPG \\
\hline 2504 & Panicum capillare L. & Sellye & 1968 & Priszter Szaniszló & BPU-15682.JPG \\
\hline 2504 & Panicum capillare L. & Vácrátót & 1949 & Priszter Szaniszló & BPU-15683.JPG \\
\hline 2504 & Panicum capillare L. & Vácrátót & 1949 & Soó Rezső & BPU-15684.JPG \\
\hline 2504 & Panicum capillare L. & Vácrátót & 1949 & Soó Rezső & BPU-15685.JPG \\
\hline 2505 & Panicum miliaceum L. & Budapest & 1942 & Soó Rezső & BPU-15688.JPG \\
\hline 2505 & Panicum miliaceum L. & Budapest & 1947 & Priszter Szaniszló & BPU-15686.JPG \\
\hline 2505 & Panicum miliaceum L. & Budapest & 1947 & Priszter Szaniszló & BPU-15687.JPG \\
\hline 2505 & Panicum miliaceum L. & Budapest & 1947 & Priszter Szaniszló & BPU-15689.JPG \\
\hline 2507 & Echinochloa crus-galli (L.) P. Beauv. & Bugyi & 1950 & Soó Rezső & BPU-15676.JPG \\
\hline 2507 & Echinochloa crus-galli (L.) P. Beauv. & Zámoly & 1982 & Felföldy Lajos & BPU-15678.JPG \\
\hline 2507 & Echinochloa crus-galli (L.) P. Beauv. & Zámoly & 1982 & Felföldy Lajos & BPU-15679.JPG \\
\hline 2508 & Echinochloa oryzoides (Ard.) Fritsch & Nádudvar & 1963 & Simon Tibor & BPU-15675.JPG \\
\hline 2510 & Digitaria sanguinalis (L.) Scop. & Bátorliget & 1932 & Soó Rezső & BPU-15690.JPG \\
\hline 2511 & Digitaria ciliaris (Retz.) Koeler & Budapest & 1901 & Degen Árpád & BPU-15692.JPG \\
\hline 2511 & Digitaria ciliaris (Retz.) Koeler & Budapest & 1902 & Flatt Károly & BPU-15691.JPG \\
\hline 2512 & Setaria pumila (Poir.) Schult. & Baja & 1965 & Priszter Szaniszló & BPU-15667.JPG \\
\hline 2512 & Setaria pumila (Poir.) Schult. & Debrecen & 1934 & Soó Rezső & BPU-15666.JPG \\
\hline
\end{tabular}


1. Elektroniukus melléklet - Electronic Appendix 1.

\begin{tabular}{|c|c|c|c|c|c|}
\hline $\begin{array}{l}\text { Sorszám / } \\
\text { Number }\end{array}$ & Taxon-név / Taxon-name & $\begin{array}{l}\text { Település / } \\
\text { Settlement }\end{array}$ & $\begin{array}{l}\text { Év / } \\
\text { Year }\end{array}$ & Gyüjtő / Collector & $\begin{array}{l}\text { Fájlnév / } \\
\text { File-name }\end{array}$ \\
\hline 2513 & Setaria italica (L.) P. Beauv. & Budapest & 1946 & Priszter Szaniszló & BPU-15660.JPG \\
\hline 2513 & Setaria italica (L.) P. Beauv. & Budapest & 1947 & Priszter Szaniszló & BPU-15661.JPG \\
\hline 2513 & Setaria italica (L.) P. Beauv. & Budapest & 1947 & Priszter Szaniszló & BPU-15662.JPG \\
\hline 2513 & Setaria italica (L.) P. Beauv. & Budapest & 1947 & Priszter Szaniszló & BPU-15663.JPG \\
\hline 2513 & Setaria italica (L.) P. Beauv. & Budapest & 1948 & Priszter Szaniszló & BPU-15664.JPG \\
\hline 2514 & Setaria verticillata (L.) P. Beauv. & Budapest & 1947 & Priszter Szaniszló & BPU-15671.JPG \\
\hline 2514 & Setaria verticillata (L.) P. Beauv. & Budapest & 1948 & Priszter Szaniszló & BPU-15672.JPG \\
\hline 2514 & Setaria verticillata (L.) P. Beauv. & Budapest & 1948 & Priszter Szaniszló & BPU-15673.JPG \\
\hline 2514 & Setaria verticillata (L.) P. Beauv. & Hajdúnánás & 1935 & Ujvárosi Miklós & BPU-15669.JPG \\
\hline 2514 & Setaria verticillata (L.) P. Beauv. & Miskolc & 1960 & Priszter Szaniszló & BPU-15670.JPG \\
\hline 2514 & Setaria verticillata (L.) P. Beauv. & Tihany & 1961 & Sólymossy Gizella & BPU-15668.JPG \\
\hline 2515 & Setaria viridis (L.) P. Beauv. & Budapest & 1947 & Priszter Szaniszló & BPU-15655.JPG \\
\hline 2515 & Setaria viridis (L.) P. Beauv. & Budapest & 1947 & Priszter Szaniszló & BPU-15659.JPG \\
\hline 2515 & Setaria viridis (L.) P. Beauv. & Budapest & 1948 & Priszter Szaniszló & BPU-15656.JPG \\
\hline 2515 & Setaria viridis (L.) P. Beauv. & Budapest & 1948 & Priszter Szaniszló & BPU-15657.JPG \\
\hline 2515 & Setaria viridis (L.) P. Beauv. & Budapest & 1948 & Priszter Szaniszló & BPU-15658.JPG \\
\hline 2515 & Setaria viridis (L.) P. Beauv. & Debrecen & 1936 & Soó Rezső & BPU-15652.JPG \\
\hline 2515 & Setaria viridis (L.) P. Beauv. & Göd & 1938 & Kárpáti Zoltán & BPU-15654.JPG \\
\hline 2515 & Setaria viridis (L.) P. Beauv. & Szentendre & 1948 & Priszter Szaniszló & BPU-15653.JPG \\
\hline 2517 & Cenchrus incertus M.A. Curtis & Budapest & 1982 & Felföldy Lajos & BPU-15731.JPG \\
\hline 2517 & Cenchrus incertus M.A. Curtis & Kunadacs & 1964 & Soó Rezső & BPU-15729.JPG \\
\hline 2517 & Cenchrus incertus M.A. Curtis & Tatárszentgyörgy & 1950 & Soó Rezső & BPU-15730.JPG \\
\hline 2518 & Sorghum halepense (L.) Pers. & Budapest & 1949 & Priszter Szaniszló & BPU-15636.JPG \\
\hline 2518 & Sorghum halepense (L.) Pers. & Budapest & 1949 & Priszter Szaniszló & BPU-15637.JPG \\
\hline 2518 & Sorghum halepense (L.) Pers. & Leányfalu & 1948 & Priszter Szaniszló & BPU-15633.JPG \\
\hline 2518 & Sorghum halepense (L.) Pers. & Nagyharsány & 1968 & Priszter Szaniszló & BPU-15635.JPG \\
\hline 2518 & Sorghum halepense (L.) Pers. & Tihany & 1959 & Felföldy Lajos & BPU-15640.JPG \\
\hline 2519 & Sorghum bicolor (L.) Moench & Budapest & 1948 & Priszter Szaniszló & BPU-15632.JPG \\
\hline 2521 & Chrysopogon gryllus (L.) Trin. & Budaörs & 1982 & Felföldy Lajos & BPU-15642.JPG \\
\hline 2521 & Chrysopogon gryllus (L.) Trin. & Budapest & 1947 & Soó Rezső & BPU-15645.JPG \\
\hline 2521 & Chrysopogon gryllus (L.) Trin. & Csomád & 1953 & Felföldy Lajos & BPU-15643.JPG \\
\hline 2521 & Chrysopogon gryllus (L.) Trin. & Hosszúpályi & 1942 & Soó Rezső & BPU-15646.JPG \\
\hline 2521 & Chrysopogon gryllus (L.) Trin. & Uzsa & 1952 & Simon Tibor & BPU-15644.JPG \\
\hline 2522 & Bothriochloa ischaemum (L.) Keng & Bátorliget & 1932 & Soó Rezső & BPU-15650.JPG \\
\hline 2522 & Bothriochloa ischaemum (L.) Keng & Budapest & 1982 & Felföldy Lajos & BPU-15649.JPG \\
\hline 2522 & Bothriochloa ischaemum (L.) Keng & Tihany & 1958 & Felföldy Lajos & BPU-15647.JPG \\
\hline 2522 & Bothriochloa ischaemum (L.) Keng & Tihany & 1961 & Sólymossy Gizella & BPU-15648.JPG \\
\hline 2524 & Acorus calamus L. & Murarátka & 1948 & Károlyi Árpád & BPU-15627.JPG \\
\hline 2524 & Acorus calamus L. & Nagykanizsa & 1950 & Károlyi Árpád & BPU-15628.JPG \\
\hline 2524 & Acorus calamus L. & Tormafölde & 1949 & Károlyi Árpád & BPU-15626.JPG \\
\hline 2524 & Acorus calamus L. & Veresegyház & 1949 & Soó Rezső & BPU-15629.JPG \\
\hline 2526 & Arum maculatum L. & Budakeszi & 1937 & Priszter Szaniszló & BPU-15619.JPG \\
\hline 2526 & Arum maculatum L. & Budapest & 1913 & Filarszky Nándor & BPU-15624.JPG \\
\hline 2526 & Arum maculatum L. & Budapest & 1948 & Priszter Szaniszló & BPU-15621.JPG \\
\hline 2526 & Arum maculatum L. & Budapest & 1948 & Priszter Szaniszló & BPU-15622.JPG \\
\hline 2526 & Arum maculatum L. & Budapest & 1948 & Priszter Szaniszló & BPU-15623.JPG \\
\hline 2526 & Arum maculatum L. & Budapest & 1964 & Soó Rezső & BPU-15620.JPG \\
\hline
\end{tabular}


1. Elektroniukus melléklet - Electronic Appendix 1.

\begin{tabular}{|c|c|c|c|c|c|}
\hline $\begin{array}{l}\text { Sorszám / } \\
\text { Number }\end{array}$ & Taxon-név / Taxon-name & $\begin{array}{l}\text { Település / } \\
\text { Settlement }\end{array}$ & $\begin{array}{l}\text { Év / } \\
\text { Year }\end{array}$ & Gyújtő / Collector & $\begin{array}{l}\text { Fájlnév / } \\
\text { File-name }\end{array}$ \\
\hline 2526 & Arum maculatum L. & Nagyharsány & 1948 & Priszter Szaniszló & BPU-15615.JPG \\
\hline 2526 & Arum maculatum L. & Pécs & 1937 & Priszter Szaniszló & BPU-15617.JPG \\
\hline 2526 & Arum maculatum L. & Pécs & 1948 & Priszter Szaniszló & BPU-15616.JPG \\
\hline 2526 & Arum maculatum L. & Pécs & 1953 & Soó Rezső, Borsos Olga & BPU-15612.JPG \\
\hline 2526 & Arum maculatum L. & Pécs & 1953 & Soó Rezső, Borsos Olga & BPU-15614.JPG \\
\hline 2526 & Arum maculatum L. & Pécsvárad & 1936 & Soó Rezső & BPU-15613.JPG \\
\hline 2526 & Arum maculatum L. & Somlóvásárhely & 1953 & $\begin{array}{l}\text { Kulcsár Gábor, Vozáry } \\
\text { Elemér }\end{array}$ & BPU-15618.JPG \\
\hline 2529 & Wolffia arrhiza (L.) Horkel ex Wimm. & Debrecen & 1989 & Dévai György & BPU-15601.JPG \\
\hline 2529 & Wolffia arrhiza (L.) Horkel ex Wimm. & Dunaharaszti & 1946 & Kárpáti Zoltán & BPU-15600.JPG \\
\hline 2529 & Wolffia arrhiza (L.) Horkel ex Wimm. & Dunaharaszti & 1946 & Papp József & BPU-15601.JPG \\
\hline 2530 & Lemna trisulca L. & Badacsonytomaj & 1984 & Felföldy Lajos & BPU-15607.JPG \\
\hline 2530 & Lemna trisulca L. & Budapest & 1913 & $\begin{array}{l}\text { Filarszky Nándor, } \\
\text { Kümmerle Jenő Béla }\end{array}$ & BPU-15608.JPG \\
\hline 2530 & Lemna trisulca L. & Budapest & 1946 & Kárpáti Zoltán & BPU-15610.JPG \\
\hline 2530 & Lemna trisulca L. & Budapest & 1947 & Priszter Szaniszló & BPU-15608.JPG \\
\hline 2530 & Lemna trisulca L. & Debrecen & 1989 & Felföldy Lajos & BPU-15607.JPG \\
\hline 2530 & Lemna trisulca L. & Debrecen & 1989 & Felföldy Lajos & BPU-15607.JPG \\
\hline 2530 & Lemna trisulca L. & Debrecen & 1989 & Felföldy Lajos & BPU-15608.JPG \\
\hline 2530 & Lemna trisulca L. & Debrecen & 1989 & Felföldy Lajos & BPU-15608.JPG \\
\hline 2530 & Lemna trisulca L. & Gávavencsellő & 1984 & Felföldy Lajos & BPU-15607.JPG \\
\hline 2530 & Lemna trisulca L. & Gávavencsellő & 1989 & Felföldy Lajos & BPU-15607.JPG \\
\hline 2530 & Lemna trisulca L. & Gávavencsellő & 1989 & Felföldy Lajos & BPU-15607.JPG \\
\hline 2530 & Lemna trisulca L. & Gávavencsellő & 1990 & Felföldy Lajos & BPU-15607.JPG \\
\hline 2530 & Lemna trisulca L. & Nyírmada & 1933 & Soó Rezső & BPU-15609.JPG \\
\hline 2531 & Lemna gibba L. & Bicske & 1991 & Felföldy Lajos & BPU-15603.JPG \\
\hline 2531 & Lemna gibba L. & Budapest & 1915 & Degen Árpád & BPU-15603.JPG \\
\hline 2531 & Lemna gibba L. & Sopron & 1947 & Kárpáti Zoltán & BPU-15604.JPG \\
\hline 2531 & Lemna gibba L. & Tiszafüred & 1953 & Borsos Olga & BPU-15604.JPG \\
\hline 2531 & Lemna gibba L. & Velence & 1988 & Tóth László & BPU-15603.JPG \\
\hline 2534 & Lemna minor L. & Badacsonytomaj & 1942 & Priszter Szaniszló & BPU-15605.JPG \\
\hline 2534 & Lemna minor L. & Badacsonytomaj & 1984 & Felföldy Lajos & BPU-15605.JPG \\
\hline 2534 & Lemna minor L. & Bátorliget & 1928 & Soó Rezső & BPU-15604.JPG \\
\hline 2534 & Lemna minor L. & Bátorliget & 1934 & Soó Rezső & BPU-15606.JPG \\
\hline 2534 & Lemna minor L. & Budapest & 1989 & Felföldy Lajos & BPU-15605.JPG \\
\hline 2534 & Lemna minor L. & Egyek & 1947 & Soó Rezső & BPU-15606.JPG \\
\hline 2534 & Lemna minor L. & Törökbálint & 1989 & Felföldy Lajos & BPU-15605.JPG \\
\hline 2536 & Spirodela polyrhiza (L.) Schleid. & Budapest & 1916 & Jávorka Sándor & BPU-15602.JPG \\
\hline 2536 & Spirodela polyrhiza (L.) Schleid. & Budapest & 1947 & Priszter Szaniszló & BPU-15602.JPG \\
\hline 2536 & Spirodela polyrhiza (L.) Schleid. & Debrecen & 1969 & Felföldy Lajos & BPU-15602.JPG \\
\hline 2536 & Spirodela polyrhiza (L.) Schleid. & Szarvas & 1991 & Felföldy Lajos & BPU-15602.JPG \\
\hline 2537 & Sparganium erectum L. & Budapest & 1910 & Földváry Dezső & BPU-15590.JPG \\
\hline 2537 & Sparganium erectum L. & Budapest & 1942 & Priszter Szaniszló & BPU-15589.JPG \\
\hline 2537 & Sparganium erectum L. & Kállósemjén & 1932 & Soó Rezső & BPU-15587.JPG \\
\hline 2537 & Sparganium erectum L. & Kapolcs & 1959 & $\begin{array}{l}\text { Jávorka Sándor, Felföldy } \\
\text { Lajos, Tóth László }\end{array}$ & BPU-15586.JPG \\
\hline 2537 & Sparganium erectum L. & Tarpa & 1949 & Soó Rezső & BPU-15588.JPG \\
\hline 2537 & Sparganium erectum L. & Tihany & 1954 & Felföldy Lajos & BPU-15585.JPG \\
\hline 2538 & Sparganium emersum Rehmann & Győr & 1940 & Polgár Sándor & BPU-15592.JPG \\
\hline
\end{tabular}


1. Elektroniukus melléklet - Electronic Appendix 1.

\begin{tabular}{|c|c|c|c|c|c|}
\hline $\begin{array}{l}\text { Sorszám / } \\
\text { Number }\end{array}$ & Taxon-név / Taxon-name & $\begin{array}{l}\text { Település / } \\
\text { Settlement }\end{array}$ & $\begin{array}{l}\text { Év / } \\
\text { Year }\end{array}$ & Gyűjtő / Collector & $\begin{array}{l}\text { Fájlnév / } \\
\text { File-name }\end{array}$ \\
\hline 2538 & Sparganium emersum Rehmann & Kőszeg & 1932 & Soó Rezső & BPU-15591.JPG \\
\hline 2539 & Sparganium natans L. & Csévharaszt & 1942 & Priszter Szaniszló & BPU-15596.JPG \\
\hline 2539 & Sparganium natans L. & Kiskőrös & 1932 & Boros Ádám & BPU-15597.JPG \\
\hline 2539 & Sparganium natans L. & Kiskőrös & 1932 & Boros Ádám & BPU-15598.JPG \\
\hline 2539 & Sparganium natans L. & Nyírbéltek & 1932 & Soó Rezső & BPU-15594.JPG \\
\hline 2539 & Sparganium natans L. & Ócsa & 1929 & Boros Ádám & BPU-15595.JPG \\
\hline 2539 & Sparganium natans L. & Szentgotthárd & 1955 & Soó Rezső & BPU-15593.JPG \\
\hline 2541 & Typha laxmannii Lepech. & Budapest & 1993 & Felföldy Lajos & BPU-15573.JPG \\
\hline 2541 & Typha laxmannii Lepech. & Szolnok & 1956 & Jeanplong József & BPU-15572.JPG \\
\hline 2542 & Typha angustifolia L. & Balatonszepezd & 1958 & Felföldy Lajos & BPU-15578.JPG \\
\hline 2542 & Typha angustifolia L. & Budapest & 1906 & Degen Árpád & BPU-15582.JPG \\
\hline 2542 & Typha angustifolia L. & Debrecen & 1935 & Soó Rezső & BPU-15581.JPG \\
\hline 2542 & Typha angustifolia L. & Hajdúnánás & 1943 & Igmándy József & BPU-15580.JPG \\
\hline 2542 & Typha angustifolia L. & Nyírpilis & 1951 & Simon Tibor & BPU-15579.JPG \\
\hline 2544 & Typha latifolia L. & Budapest & 1908 & Degen Árpád & BPU-15577.JPG \\
\hline 2544 & Typha latifolia L. & Csévharaszt & 1942 & Priszter Szaniszló & BPU-15575.JPG \\
\hline 2544 & Typha latifolia L. & Debrecen & 1948 & Soó Rezső & BPU-15576.JPG \\
\hline 2547 & Scirpoides holoschoenus (L.) Soják & Bagamér & 1937 & Soó Rezső & BPU-10693.JPG \\
\hline 2547 & Scirpoides holoschoenus (L.) Soják & Balatonszabadi & 1942 & Priszter Szaniszló & BPU-10697.JPG \\
\hline 2547 & Scirpoides holoschoenus (L.) Soják & Balatonszemes & 1974 & Tóth László & BPU-10698.JPG \\
\hline 2547 & Scirpoides holoschoenus (L.) Soják & Budapest & 1909 & Lengyel Géza & BPU-10689.JPG \\
\hline 2547 & Scirpoides holoschoenus (L.) Soják & Budapest & 1923 & Soó Rezső & BPU-10690.JPG \\
\hline 2547 & Scirpoides holoschoenus (L.) Soják & Budapest & 1942 & Priszter Szaniszló & BPU-10691.JPG \\
\hline 2547 & Scirpoides holoschoenus (L.) Soják & Budapest & 1992 & Felföldy Lajos & BPU-10692.JPG \\
\hline 2547 & Scirpoides holoschoenus (L.) Soják & Dabas & 1914 & Tuzson János & BPU-10688.JPG \\
\hline 2547 & Scirpoides holoschoenus (L.) Soják & Debrecen & 1932 & Soó Rezső & BPU-10694.JPG \\
\hline 2547 & Scirpoides holoschoenus (L.) Soják & Dunaharaszti & 1948 & Priszter Szaniszló & BPU-10695.JPG \\
\hline 2547 & Scirpoides holoschoenus (L.) Soják & Ócsa & 1953 & Soó Rezső, Borsos Olga & BPU-10696.JPG \\
\hline 2547 & Scirpoides holoschoenus (L.) Soják & Uzsa & 1952 & Borsos Olga & BPU-10699.JPG \\
\hline 2548 & Schoenoplectus setaceus (L.) Palla & Hosszúvölgy & 1948 & Károlyi Árpád & BPU-10636.JPG \\
\hline 2548 & Schoenoplectus setaceus (L.) Palla & Nagykanizsa & 1948 & Károlyi Árpád & BPU-10638.JPG \\
\hline 2548 & Schoenoplectus setaceus (L.) Palla & Nagykanizsa & 1949 & Károlyi Árpád & BPU-10639.JPG \\
\hline 2548 & Schoenoplectus setaceus (L.) Palla & Szepetnek & 1949 & Károlyi Árpád & BPU-10637.JPG \\
\hline 2549 & Schoenoplectus supinus (L.) Palla & Hortobágy & 1947 & Simon Tibor & BPU-10644.JPG \\
\hline 2549 & Schoenoplectus supinus (L.) Palla & Hortobágy & 1947 & Soó Rezső & BPU-10643.JPG \\
\hline 2549 & Schoenoplectus supinus (L.) Palla & Szenta & 1958 & $\begin{array}{l}\text { Járainé Komlódi Magda, } \\
\text { Borhidi Attila }\end{array}$ & BPU-10646.JPG \\
\hline 2549 & Schoenoplectus supinus (L.) Palla & Tököl & 1917 & Degen Árpád & BPU-10645.JPG \\
\hline 2549 & Schoenoplectus supinus (L.) Palla & Tököl & 1917 & Degen Árpád, Zsák Zoltán & BPU-10642.JPG \\
\hline 2549 & Schoenoplectus supinus (L.) Palla & Tököl & 1917 & $\begin{array}{l}\text { Jávorka Sándor, } \\
\text { Trautmann Róbert }\end{array}$ & BPU-10640.JPG \\
\hline 2549 & Schoenoplectus supinus (L.) Palla & Vámosszabadi & 1912 & Polgár Sándor & BPU-10641.JPG \\
\hline 2549 & Schoenoplectus supinus (L.) Palla & Vámosszabadi & 1912 & Polgár Sándor & BPU-10641.JPG \\
\hline 2549 & Schoenoplectus supinus (L.) Palla & Vásárosnamény & 1933 & Soó Rezső & BPU-10647.JPG \\
\hline 2550 & Schoenoplectus lacustris (L.) Palla & Balatonakali & 1958 & Felföldy Lajos & BPU-10655.JPG \\
\hline 2550 & Schoenoplectus lacustris (L.) Palla & Balatonszemes & 1974 & Tóth László & BPU-10654.JPG \\
\hline 2550 & Schoenoplectus lacustris (L.) Palla & Budapest & 1909 & Degen Árpád & BPU-10648.JPG \\
\hline 2550 & Schoenoplectus lacustris (L.) Palla & Debrecen & 1934 & Soó Rezső & BPU-10652.JPG \\
\hline
\end{tabular}


1. Elektroniukus melléklet - Electronic Appendix 1.

\begin{tabular}{|c|c|c|c|c|c|}
\hline $\begin{array}{l}\text { Sorszám / } \\
\text { Number }\end{array}$ & Taxon-név / Taxon-name & $\begin{array}{l}\text { Település / } \\
\text { Settlement }\end{array}$ & $\begin{array}{l}\text { Év / } \\
\text { Year }\end{array}$ & Gyújtő / Collector & $\begin{array}{l}\text { Fájlnév / } \\
\text { File-name }\end{array}$ \\
\hline 2550 & Schoenoplectus lacustris (L.) Palla & Gárdony & 1980 & Felföldy Lajos & BPU-10650.JPG \\
\hline 2550 & Schoenoplectus lacustris (L.) Palla & Kiskunmajsa & 1938 & Priszter Szaniszló & BPU-10649.JPG \\
\hline 2550 & Schoenoplectus lacustris (L.) Palla & Litke & 1987 & Felföldy Lajos & BPU-10653.JPG \\
\hline 2550 & Schoenoplectus lacustris (L.) Palla & Pátka & 1987 & Felföldy Lajos & BPU-10651.JPG \\
\hline 2550 & Schoenoplectus lacustris (L.) Palla & Tihany & 1961 & $\begin{array}{l}\text { Pankov H., Felföldy Lajos, } \\
\text { Sólymosy G. }\end{array}$ & BPU-10656.JPG \\
\hline 2551 & $\begin{array}{l}\text { Schoenoplectus tabernaemontani (C.C. } \\
\text { Gmel.) Palla }\end{array}$ & Aszófő & 1956 & $\begin{array}{l}\text { Felföldy Lajos, Tóth } \\
\text { László }\end{array}$ & BPU-10667.JPG \\
\hline 2551 & $\begin{array}{l}\text { Schoenoplectus tabernaemontani (C.C. } \\
\text { Gmel.) Palla }\end{array}$ & Budapest & 1909 & Degen Árpád & BPU-10658.JPG \\
\hline 2551 & $\begin{array}{l}\text { Schoenoplectus tabernaemontani (C.C. } \\
\text { Gmel.) Palla }\end{array}$ & Budapest & 1914 & Szurák János & BPU-10657.JPG \\
\hline 2551 & $\begin{array}{l}\text { Schoenoplectus tabernaemontani (C.C. } \\
\text { Gmel.) Palla }\end{array}$ & Budapest & 1991 & Felföldy Lajos & BPU-10660.JPG \\
\hline 2551 & $\begin{array}{l}\text { Schoenoplectus tabernaemontani (C.C. } \\
\text { Gmel.) Palla }\end{array}$ & Csaroda & 1951 & $\begin{array}{l}\text { Simon Tibor, Vozáry } \\
\text { Elemér }\end{array}$ & BPU-10661.JPG \\
\hline 2551 & $\begin{array}{l}\text { Schoenoplectus tabernaemontani (C.C. } \\
\text { Gmel.) Palla }\end{array}$ & Debrecen & 1931 & Soó Rezső & BPU-10663.JPG \\
\hline 2551 & $\begin{array}{l}\text { Schoenoplectus tabernaemontani (C.C. } \\
\text { Gmel.) Palla }\end{array}$ & Debrecen & 1989 & Felföldy Lajos & BPU-10664.JPG \\
\hline 2551 & $\begin{array}{l}\text { Schoenoplectus tabernaemontani (C.C. } \\
\text { Gmel.) Palla }\end{array}$ & Debrecen & 1989 & Felföldy Lajos & BPU-10665.JPG \\
\hline 2551 & $\begin{array}{l}\text { Schoenoplectus tabernaemontani (C.C. } \\
\text { Gmel.) Palla }\end{array}$ & Gelénes & 1951 & $\begin{array}{l}\text { Simon Tibor, Vozáry } \\
\text { Elemér }\end{array}$ & BPU-10662.JPG \\
\hline 2551 & $\begin{array}{l}\text { Schoenoplectus tabernaemontani (C.C. } \\
\text { Gmel.) Palla }\end{array}$ & Tihany & 1956 & Felföldy Lajos & BPU-10668.JPG \\
\hline 2551 & $\begin{array}{l}\text { Schoenoplectus tabernaemontani (C.C. } \\
\text { Gmel.) Palla }\end{array}$ & Törökbálint & 1991 & Felföldy Lajos & BPU-10666.JPG \\
\hline 2552 & Schoenoplectus mucronatus (L.) Palla & Mátészalka & 1926 & Boros Ádám & BPU-10670.JPG \\
\hline 2552 & Schoenoplectus mucronatus (L.) Palla & Nagykanizsa & 1956 & Felföldy Lajos & BPU-10673.JPG \\
\hline 2552 & Schoenoplectus mucronatus (L.) Palla & Nyírmada & 1927 & Boros Ádám & BPU-10669.JPG \\
\hline 2552 & Schoenoplectus mucronatus (L.) Palla & Nyírmada & 1927 & Boros Ádám & BPU-10672.JPG \\
\hline 2552 & Schoenoplectus mucronatus (L.) Palla & Szenta & 1957 & Soó Rezső & BPU-10671.JPG \\
\hline 2553 & Schoenoplectus pungens (Vahl) Palla & Balatonberény & 1928 & Papp József & BPU-10684.JPG \\
\hline 2553 & Schoenoplectus pungens (Vahl) Palla & Balatonszemes & 1910 & Paikert, Alois & BPU-10683.JPG \\
\hline 2553 & Schoenoplectus pungens (Vahl) Palla & Siófok & 1928 & Soó Rezső & BPU-10685.JPG \\
\hline 2553 & Schoenoplectus pungens (Vahl) Palla & Zamárdi & 1930 & Jávorka Sándor & BPU-10682.JPG \\
\hline 2554 & $\begin{array}{l}\text { Schoenoplectus litoralis (Schrad.) } \\
\text { Palla }\end{array}$ & Fertőrákos & 1982 & Tóth László & BPU-10679.JPG \\
\hline 2554 & $\begin{array}{l}\text { Schoenoplectus litoralis (Schrad.) } \\
\text { Palla }\end{array}$ & Hévíz & 1916 & Lovassy Alex & BPU-10675.JPG \\
\hline 2554 & $\begin{array}{l}\text { Schoenoplectus litoralis (Schrad.) } \\
\text { Palla }\end{array}$ & Hévíz & 1926 & Jávorka Sándor & BPU-10674.JPG \\
\hline 2554 & $\begin{array}{l}\text { Schoenoplectus litoralis (Schrad.) } \\
\text { Palla }\end{array}$ & Hévíz & 1952 & Simon Tibor & BPU-10677.JPG \\
\hline 2554 & $\begin{array}{l}\text { Schoenoplectus litoralis (Schrad.) } \\
\text { Palla }\end{array}$ & Hévíz & 1961 & Felföldy Lajos & BPU-10678.JPG \\
\hline 2554 & $\begin{array}{l}\text { Schoenoplectus litoralis (Schrad.) } \\
\text { Palla }\end{array}$ & Keszthely & 1916 & Lovassy Alex & BPU-10676.JPG \\
\hline 2555 & Schoenoplectus triqueter L. & Budapest & 1909 & Degen Árpád & BPU-10681.JPG \\
\hline 2555 & Schoenoplectus triqueter L. & Budapest & 1913 & $\begin{array}{l}\text { Filarszky Nándor, } \\
\text { Kümmerle Jenő Béla }\end{array}$ & BPU-10680.JPG \\
\hline $\begin{array}{c}2551 \\
\times 2555\end{array}$ & $\begin{array}{l}\text { Schoenoplectus tabernaemontani (C.C. } \\
\text { Gmel.) Palla × Schoenoplectus } \\
\text { triqueter L. }\end{array}$ & Győr & 1912 & Polgár Sándor & BPU-10686.JPG \\
\hline
\end{tabular}


1. Elektroniukus melléklet - Electronic Appendix 1.

\begin{tabular}{|c|c|c|c|c|c|}
\hline $\begin{array}{l}\text { Sorszám / } \\
\text { Number }\end{array}$ & Taxon-név / Taxon-name & $\begin{array}{l}\text { Település / } \\
\text { Settlement }\end{array}$ & $\begin{array}{l}\text { Év / } \\
\text { Year }\end{array}$ & Gyưjtő / Collector & $\begin{array}{l}\text { Fájlnév / } \\
\text { File-name }\end{array}$ \\
\hline $\begin{array}{c}2551 \\
\times 2555\end{array}$ & $\begin{array}{l}\text { Schoenoplectus tabernaemontani (C.C. } \\
\text { Gmel.) Palla } \times \text { Schoenoplectus } \\
\text { triqueter L. }\end{array}$ & Győr & 1912 & Polgár Sándor & BPU-10686.JPG \\
\hline $\begin{array}{r}2551 \\
\times 2555\end{array}$ & $\begin{array}{l}\text { Schoenoplectus tabernaemontani (C.C. } \\
\text { Gmel.) Palla } \times \text { Schoenoplectus } \\
\text { triqueter L. }\end{array}$ & Győr & 1912 & Polgár Sándor & BPU-10687.JPG \\
\hline $\begin{array}{c}2551 \\
\times 2555\end{array}$ & $\begin{array}{l}\text { Schoenoplectus tabernaemontani (C.C. } \\
\text { Gmel.) Palla } \times \text { Schoenoplectus } \\
\text { triqueter L. }\end{array}$ & Győr & 1912 & Polgár Sándor & BPU-10687.JPG \\
\hline $\begin{array}{l}2556- \\
2560\end{array}$ & Bolboschoenus maritimus agg. & Abádszalók & 1989 & $\begin{array}{l}\text { Felföldy Lajos, Hamar } \\
\text { József }\end{array}$ & BPU-10747.JPG \\
\hline $\begin{array}{c}2556- \\
2560\end{array}$ & Bolboschoenus maritimus agg. & Budaörs & 1991 & Felföldy Lajos & BPU-10741.JPG \\
\hline $\begin{array}{c}2556- \\
2560\end{array}$ & Bolboschoenus maritimus agg. & Budapest & 1909 & Degen Árpád & BPU-10730.JPG \\
\hline $\begin{array}{c}2556- \\
2560\end{array}$ & Bolboschoenus maritimus agg. & Budapest & 1913 & Kümmerle Jenő Béla & BPU-10729.JPG \\
\hline $\begin{array}{c}2556- \\
2560\end{array}$ & Bolboschoenus maritimus agg. & Budapest & 1942 & Priszter Szaniszló & BPU-10735.JPG \\
\hline $\begin{array}{c}2556- \\
2560\end{array}$ & Bolboschoenus maritimus agg. & Budapest & 1947 & Soó Rezső & BPU-10734.JPG \\
\hline $\begin{array}{l}2556- \\
2560\end{array}$ & Bolboschoenus maritimus agg. & Budapest & 1991 & Felföldy Lajos & BPU-10733.JPG \\
\hline $\begin{array}{c}2556- \\
2560\end{array}$ & Bolboschoenus maritimus agg. & Budapest & 1993 & Felföldy Lajos & BPU-10736.JPG \\
\hline $\begin{array}{c}2556- \\
2560\end{array}$ & Bolboschoenus maritimus agg. & Dabas & 1963 & Isépy István & BPU-10742.JPG \\
\hline $\begin{array}{c}2556- \\
2560\end{array}$ & Bolboschoenus maritimus agg. & Debrecen & 1946 & Soó Rezső & BPU-10740.JPG \\
\hline $\begin{array}{c}2556- \\
2560\end{array}$ & Bolboschoenus maritimus agg. & Dunaharaszti & 1948 & Priszter Szaniszló & BPU-10743.JPG \\
\hline $\begin{array}{c}2556- \\
2560\end{array}$ & Bolboschoenus maritimus agg. & Gávavencsellő & 1987 & Felföldy Lajos & BPU-10745.JPG \\
\hline $\begin{array}{c}2556- \\
2560\end{array}$ & Bolboschoenus maritimus agg. & Gávavencsellő & 1987 & Felföldy Lajos & BPU-10746.JPG \\
\hline $\begin{array}{c}2556- \\
2560\end{array}$ & Bolboschoenus maritimus agg. & Gávavencsellő & 1990 & Felföldy Lajos & BPU-10744.JPG \\
\hline $\begin{array}{c}2556- \\
2560\end{array}$ & Bolboschoenus maritimus agg. & Kesztölc & 1942 & Priszter Szaniszló & BPU-10737.JPG \\
\hline $\begin{array}{c}2556- \\
2560\end{array}$ & Bolboschoenus maritimus agg. & Pákozd & 1980 & Felföldy Lajos & BPU-10738.JPG \\
\hline $\begin{array}{c}2556- \\
2560\end{array}$ & Bolboschoenus maritimus agg. & Pákozd & 1980 & Felföldy Lajos & BPU-10739.JPG \\
\hline $\begin{array}{l}2556- \\
2560\end{array}$ & Bolboschoenus maritimus agg. & Szikszó & 1909 & Thaisz Lajos & BPU-10731.JPG \\
\hline $\begin{array}{l}2556- \\
2560\end{array}$ & Bolboschoenus maritimus agg. & Tihany & 1961 & $\begin{array}{l}\text { Pankov H., Felföldy Lajos, } \\
\text { Sólymosy G. }\end{array}$ & BPU-10748.JPG \\
\hline 2561 & Scirpus sylvaticus L. & Budapest & 1909 & Degen Árpád & BPU-10721.JPG \\
\hline 2561 & Scirpus sylvaticus L. & Miskolc & 1932 & $\begin{array}{l}\text { Zólyomi Bálint, Máthé } \\
\text { Imre }\end{array}$ & BPU-10723.JPG \\
\hline 2561 & Scirpus sylvaticus L. & Nagyrada & 1987 & Felföldy Lajos & BPU-10727.JPG \\
\hline 2561 & Scirpus sylvaticus L. & Pálháza & 1952 & Borsos Olga & BPU-15476.JPG \\
\hline 2561 & Scirpus sylvaticus L. & Pomáz & 1942 & Priszter Szaniszló & BPU-10725.JPG \\
\hline 2561 & Scirpus sylvaticus L. & Sopron & 1942 & Priszter Szaniszló & BPU-10726.JPG \\
\hline 2561 & Scirpus sylvaticus L. & Szilvásvárad & 1953 & Soó Rezső & BPU-10724.JPG \\
\hline 2562 & Scirpus radicans Schkuhr & Budapest & 1909 & Degen Árpád & BPU-10717.JPG \\
\hline 2562 & Scirpus radicans Schkuhr & Budapest & 1909 & Kocsis István & BPU-10719.JPG \\
\hline 2562 & Scirpus radicans Schkuhr & Budapest & 1910 & Degen Árpád & BPU-10718.JPG \\
\hline
\end{tabular}


1. Elektroniukus melléklet - Electronic Appendix 1.

\begin{tabular}{|c|c|c|c|c|c|}
\hline $\begin{array}{l}\text { Sorszám / } \\
\text { Number }\end{array}$ & Taxon-név / Taxon-name & $\begin{array}{l}\text { Település / } \\
\text { Settlement }\end{array}$ & $\begin{array}{l}\text { Év / } \\
\text { Year }\end{array}$ & Gyűjtő / Collector & $\begin{array}{l}\text { Fájlnév / } \\
\text { File-name }\end{array}$ \\
\hline 2562 & Scirpus radicans Schkuhr & Vasvár & 1950 & Károlyi Árpád & BPU-10720.JPG \\
\hline 2563 & Blysmus compressus (L.) Panz. & Kemence & 1952 & Simon Tibor & BPU-10716.JPG \\
\hline 2563 & Blysmus compressus (L.) Panz. & Miskolc & 1948 & $\begin{array}{l}\text { Simon Tibor, Borsos Olga, } \\
\text { Jakucs Pál }\end{array}$ & BPU-10713.JPG \\
\hline 2563 & Blysmus compressus (L.) Panz. & Miskolc & 1950 & Soó Rezső & BPU-10715.JPG \\
\hline 2563 & Blysmus compressus (L.) Panz. & Miskolc & 1950 & Soó Rezső, Simon Tibor & BPU-10714.JPG \\
\hline 2564 & Eriophorum vaginatum L. & Csaroda & 1953 & $\begin{array}{l}\text { Vozáry Elemér, Kulcsár } \\
\text { Gábor }\end{array}$ & BPU-10586.JPG \\
\hline 2564 & Eriophorum vaginatum L. & Csaroda & 1954 & $\begin{array}{l}\text { Máthé Imre, Jeanplon } \\
\text { József, Kovács Margit }\end{array}$ & BPU-10585.JPG \\
\hline 2564 & Eriophorum vaginatum L. & Kelemér & 1950 & Soó Rezső & BPU-10587.JPG \\
\hline 2564 & Eriophorum vaginatum L. & Kelemér & 1950 & Soó Rezső & BPU-10588.JPG \\
\hline 2565 & Eriophorum angustifolium Honck. & Aszófő & 1959 & Felföldy Lajos & BPU-10624.JPG \\
\hline 2565 & Eriophorum angustifolium Honck. & Aszófő & 1959 & Felföldy Lajos & BPU-10627.JPG \\
\hline 2565 & Eriophorum angustifolium Honck. & Balatonakali & 1958 & Felföldy Lajos & BPU-10623.JPG \\
\hline 2565 & Eriophorum angustifolium Honck. & Balatonszőlős & 1957 & Felföldy Lajos & BPU-10625.JPG \\
\hline 2565 & Eriophorum angustifolium Honck. & Balatonszőlős & 1957 & $\begin{array}{l}\text { Felföldy Lajos, Kovács } \\
\text { Margit, Tóth László }\end{array}$ & BPU-10626.JPG \\
\hline 2565 & Eriophorum angustifolium Honck. & Budapest & 1914 & Koszilkov János & BPU-10612.JPG \\
\hline 2565 & Eriophorum angustifolium Honck. & Dabas & 1961 & Borsos Olga, Simon Tibor & BPU-10618.JPG \\
\hline 2565 & Eriophorum angustifolium Honck. & Debrecen & 1932 & Soó Rezső & BPU-10615.JPG \\
\hline 2565 & Eriophorum angustifolium Honck. & Debrecen & 1934 & Soó Rezső & BPU-10616.JPG \\
\hline 2565 & Eriophorum angustifolium Honck. & Hajdúsámson & 1950 & Soó Rezső & BPU-10617.JPG \\
\hline 2565 & Eriophorum angustifolium Honck. & Sopron & 1942 & Priszter Szaniszló & BPU-10621.JPG \\
\hline 2565 & Eriophorum angustifolium Honck. & Szalonna & 1966 & Felföldy Lajos & BPU-10614.JPG \\
\hline 2565 & Eriophorum angustifolium Honck. & Szentgotthárd & 1955 & Soó Rezső & BPU-10622.JPG \\
\hline 2565 & Eriophorum angustifolium Honck. & Tihany & 1956 & Felföldy Lajos & BPU-10628.JPG \\
\hline 2565 & Eriophorum angustifolium Honck. & Vácrátót & 1950 & Soó Rezső & BPU-10619.JPG \\
\hline 2565 & Eriophorum angustifolium Honck. & Veresegyház & 1949 & Soó Rezső & BPU-10620.JPG \\
\hline 2566 & Eriophorum latifolium Hoppe & Aszófő & 1959 & Felföldy Lajos & BPU-10603.JPG \\
\hline 2566 & Eriophorum latifolium Hoppe & Balatonszőlős & 1956 & Felföldy Lajos & BPU-10604.JPG \\
\hline 2566 & Eriophorum latifolium Hoppe & Balatonszőlős & 1957 & Felföldy Lajos & BPU-10605.JPG \\
\hline 2566 & Eriophorum latifolium Hoppe & Bozsok & 1938 & Soó Rezső & BPU-10602.JPG \\
\hline 2566 & Eriophorum latifolium Hoppe & Bükkszentkereszt & 1938 & Soó Rezső & BPU-10599.JPG \\
\hline 2566 & Eriophorum latifolium Hoppe & Felsőtárkány & 1953 & Soó Rezső & BPU-10600.JPG \\
\hline 2566 & Eriophorum latifolium Hoppe & Miskolc & 1950 & Soó Rezső & BPU-10597.JPG \\
\hline 2566 & Eriophorum latifolium Hoppe & Örvényes & 1959 & Felföldy Lajos & BPU-10606.JPG \\
\hline 2566 & Eriophorum latifolium Hoppe & Örvényes & 1959 & Felföldy Lajos & BPU-10607.JPG \\
\hline 2566 & Eriophorum latifolium Hoppe & Szemere & 1966 & Felföldy Lajos & BPU-10598.JPG \\
\hline 2566 & Eriophorum latifolium Hoppe & Szilvásvárad & 1947 & Papp József & BPU-10601.JPG \\
\hline 2566 & Eriophorum latifolium Hoppe & Szögliget & 1951 & Jakucs Pál, Ér Lajos & BPU-15482.JPG \\
\hline 2566 & Eriophorum latifolium Hoppe & Ugod & 1955 & Soó Rezső & BPU-10608.JPG \\
\hline 2566 & Eriophorum latifolium Hoppe & Uzsa & 1959 & $\begin{array}{l}\text { Jávorka Sándor, Felföldy } \\
\text { Lajos }\end{array}$ & BPU-10609.JPG \\
\hline 2568 & $\begin{array}{l}\text { Eleocharis acicularis (L.) Roem. et } \\
\text { Schult. }\end{array}$ & Balatonszőlős & 1957 & Felföldy Lajos & BPU-10760.JPG \\
\hline 2568 & $\begin{array}{l}\text { Eleocharis acicularis (L.) Roem. et } \\
\text { Schult. }\end{array}$ & Budapest & 1909 & Degen Árpád & BPU-10753.JPG \\
\hline 2568 & $\begin{array}{l}\text { Eleocharis acicularis (L.) Roem. et } \\
\text { Schult. }\end{array}$ & Budapest & 1947 & Priszter Szaniszló & BPU-10754.JPG \\
\hline
\end{tabular}


1. Elektroniukus melléklet - Electronic Appendix 1.

\begin{tabular}{|c|c|c|c|c|c|}
\hline $\begin{array}{l}\text { Sorszám / } \\
\text { Number }\end{array}$ & Taxon-név / Taxon-name & $\begin{array}{l}\text { Település / } \\
\text { Settlement }\end{array}$ & $\begin{array}{l}\text { Év / } \\
\text { Year }\end{array}$ & Gyújtő / Collector & $\begin{array}{l}\text { Fájlnév / } \\
\text { File-name }\end{array}$ \\
\hline 2568 & $\begin{array}{l}\text { Eleocharis acicularis (L.) Roem. et } \\
\text { Schult. }\end{array}$ & Budapest & 1947 & Priszter Szaniszló & BPU-10755.JPG \\
\hline 2568 & $\begin{array}{l}\text { Eleocharis acicularis (L.) Roem. et } \\
\text { Schult. }\end{array}$ & Dömös & 1947 & Priszter Szaniszló & BPU-10757.JPG \\
\hline 2568 & $\begin{array}{l}\text { Eleocharis acicularis (L.) Roem. et } \\
\text { Schult. }\end{array}$ & Mindszent & 1947 & Timár Lajos & BPU-10756.JPG \\
\hline 2568 & $\begin{array}{l}\text { Eleocharis acicularis (L.) Roem. et } \\
\text { Schult. }\end{array}$ & Nagykanizsa & 1956 & Felföldy Lajos & BPU-10762.JPG \\
\hline 2568 & $\begin{array}{l}\text { Eleocharis acicularis (L.) Roem. et } \\
\text { Schult. }\end{array}$ & Révfülöp & 1956 & $\begin{array}{l}\text { Felföldy Lajos, Tóth } \\
\text { László }\end{array}$ & BPU-10761.JPG \\
\hline 2568 & $\begin{array}{l}\text { Eleocharis acicularis (L.) Roem. et } \\
\text { Schult. }\end{array}$ & Sárospatak & 1939 & Soó Rezső & BPU-10763.JPG \\
\hline 2568 & $\begin{array}{l}\text { Eleocharis acicularis (L.) Roem. et } \\
\text { Schult. }\end{array}$ & Szigetszentmiklós & 1915 & $\begin{array}{l}\text { Kümmerle Jenő Béla, } \\
\text { Jávorka Sándor }\end{array}$ & BPU-10752.JPG \\
\hline 2568 & $\begin{array}{l}\text { Eleocharis acicularis (L.) Roem. et } \\
\text { Schult. }\end{array}$ & Tiszafüred & 1932 & Soó Rezső & BPU-10758.JPG \\
\hline 2568 & $\begin{array}{l}\text { Eleocharis acicularis (L.) Roem. et } \\
\text { Schult. }\end{array}$ & Vásárosnamény & 1933 & Soó Rezső & BPU-10759.JPG \\
\hline 2569 & $\begin{array}{l}\text { Eleocharis quinqueflora (Hartmann) } \\
\text { O. Schwarz }\end{array}$ & Balatonboglár & 1911 & Polgár Sándor & BPU-10766.JPG \\
\hline 2569 & $\begin{array}{l}\text { Eleocharis quinqueflora (Hartmann) } \\
\text { O. Schwarz }\end{array}$ & Sopron & 1944 & Kárpáti Zoltán & BPU-10767.JPG \\
\hline 2570 & Eleocharis carniolica W.D.J. Koch & Beregdaróc & 1951 & $\begin{array}{l}\text { Simon Tibor, Vozáry } \\
\text { Elemér }\end{array}$ & BPU-14776.JPG \\
\hline 2570 & Eleocharis carniolica W.D.J. Koch & Beregsurány & 1951 & $\begin{array}{l}\text { Simon Tibor, Vozáry } \\
\text { Elemér }\end{array}$ & BPU-14777.JPG \\
\hline 2570 & Eleocharis carniolica W.D.J. Koch & Gelénes & 1951 & $\begin{array}{l}\text { Simon Tibor, Vozáry } \\
\text { Elemér }\end{array}$ & BPU-14778.JPG \\
\hline 2570 & Eleocharis carniolica W.D.J. Koch & Szakonyfalu & 1955 & Soó Rezső & BPU-14775.JPG \\
\hline 2571 & $\begin{array}{l}\text { Eleocharis ovata (Roth) Roem. et } \\
\text { Schult. }\end{array}$ & Jánd & 1951 & Simon Tibor & BPU-14770.JPG \\
\hline 2571 & $\begin{array}{l}\text { Eleocharis ovata (Roth) Roem. et } \\
\text { Schult. }\end{array}$ & Rinyabesenyő & 1942 & Boros Ádám & BPU-14769.JPG \\
\hline 2572 & Eleocharis uniglumis (Link) Schult. & Balatonszőlős & 1957 & Felföldy Lajos & BPU-14753.JPG \\
\hline 2572 & Eleocharis uniglumis (Link) Schult. & Budapest & 1916 & Degen Árpád & BPU-14756.JPG \\
\hline 2572 & Eleocharis uniglumis (Link) Schult. & Egyek & 1947 & Soó Rezső & BPU-14754.JPG \\
\hline 2572 & Eleocharis uniglumis (Link) Schult. & Tihany & 1956 & Felföldy Lajos & BPU-14752.JPG \\
\hline 2572 & Eleocharis uniglumis (Link) Schult. & Zámoly & 1982 & Felföldy Lajos & BPU-14755.JPG \\
\hline 2573 & $\begin{array}{l}\text { Eleocharis palustris (L.) Roem. et } \\
\text { Schult. }\end{array}$ & Bélapátfalva & 1953 & Simon Tibor & BPU-14764.JPG \\
\hline 2573 & $\begin{array}{l}\text { Eleocharis palustris (L.) Roem. et } \\
\text { Schult. }\end{array}$ & Budapest & 1909 & Degen Árpád & BPU-14766.JPG \\
\hline 2573 & $\begin{array}{l}\text { Eleocharis palustris (L.) Roem. et } \\
\text { Schult. }\end{array}$ & Hortobágy & 1930 & Soó Rezső & BPU-14762.JPG \\
\hline 2573 & $\begin{array}{l}\text { Eleocharis palustris (L.) Roem. et } \\
\text { Schult. }\end{array}$ & Kőszeg & 1941 & Priszter Szaniszló & BPU-14759.JPG \\
\hline 2573 & $\begin{array}{l}\text { Eleocharis palustris (L.) Roem. et } \\
\text { Schult. }\end{array}$ & Pomáz & 1942 & Priszter Szaniszló & BPU-14760.JPG \\
\hline 2573 & $\begin{array}{l}\text { Eleocharis palustris (L.) Roem. et } \\
\text { Schult. }\end{array}$ & Szentendre & 1942 & Priszter Szaniszló & BPU-14761.JPG \\
\hline 2575 & Eleocharis mamillata H. Lindb. & Debrecen & 1987 & Felföldy Lajos & BPU-14763.JPG \\
\hline 2575 & Eleocharis mamillata H. Lindb. & Monostorapáti & 1959 & $\begin{array}{l}\text { Jávorka Sándor, Felföldy } \\
\text { Lajos, Tóth László }\end{array}$ & BPU-14758.JPG \\
\hline 2576 & Dichostylis micheliana (L.) Nees & Baja & 1968 & Priszter Szaniszló & BPU-10705.JPG \\
\hline 2576 & Dichostylis micheliana (L.) Nees & Budapest & 1947 & Priszter Szaniszló & BPU-10703.JPG \\
\hline 2576 & Dichostylis micheliana (L.) Nees & Budapest & 1947 & Priszter Szaniszló & BPU-10704.JPG \\
\hline 2576 & Dichostylis micheliana (L.) Nees & Dömös & 1947 & Priszter Szaniszló & BPU-10707.JPG \\
\hline
\end{tabular}


1. Elektroniukus melléklet - Electronic Appendix 1.

\begin{tabular}{|c|c|c|c|c|c|}
\hline $\begin{array}{l}\text { Sorszám / } \\
\text { Number }\end{array}$ & Taxon-név / Taxon-name & $\begin{array}{l}\text { Település / } \\
\text { Settlement }\end{array}$ & $\begin{array}{l}\text { Év / } \\
\text { Year }\end{array}$ & Gyújtő / Collector & $\begin{array}{l}\text { Fájlnév / } \\
\text { File-name }\end{array}$ \\
\hline 2576 & Dichostylis micheliana (L.) Nees & Szolnok & 1943 & anonim & BPU-10709.JPG \\
\hline 2576 & Dichostylis micheliana (L.) Nees & Tiszafüred & 1952 & Soó Rezső & BPU-10708.JPG \\
\hline 2576 & Dichostylis micheliana (L.) Nees & Tivadar & 1948 & Simon Tibor, Jakucs Pál & BPU-10706.JPG \\
\hline 2576 & Dichostylis micheliana (L.) Nees & Tokaj & 1946 & Priszter Szaniszló & BPU-10710.JPG \\
\hline 2577 & Cyperus pannonicus Jacq. & Hajdúbagos & 1933 & Soó Rezső & BPU-14822.JPG \\
\hline 2577 & Cyperus pannonicus Jacq. & Kiskunhalas & 1964 & Soó Rezső & BPU-14825.JPG \\
\hline 2577 & Cyperus pannonicus Jacq. & Konyár & 1933 & Soó Rezső & BPU-14821.JPG \\
\hline 2577 & Cyperus pannonicus Jacq. & Páhi & 1968 & Priszter Szaniszló & BPU-14824.JPG \\
\hline 2577 & Cyperus pannonicus Jacq. & Pálmonostora & 1926 & Lányi Béla & BPU-14823.JPG \\
\hline 2577 & Cyperus pannonicus Jacq. & Pálmonostora & 1926 & Lengyel Géza, Pinkert S. & BPU-14826.JPG \\
\hline 2577 & Cyperus pannonicus Jacq. & Téglás & 1947 & Simon Tibor & BPU-14820.JPG \\
\hline 2578 & Cyperus flavescens L. & Balatongyörök & 1961 & Felföldy Lajos & BPU-14837.JPG \\
\hline 2578 & Cyperus flavescens L. & Balatonszepezd & 1957 & Entz Béla & BPU-14840.JPG \\
\hline 2578 & Cyperus flavescens L. & Budapest & 1909 & Degen Árpád & BPU-14843.JPG \\
\hline 2578 & Cyperus flavescens L. & Budapest & 1947 & Priszter Szaniszló & BPU-14841.JPG \\
\hline 2578 & Cyperus flavescens L. & Budapest & 1947 & Priszter Szaniszló & BPU-14842.JPG \\
\hline 2578 & Cyperus flavescens L. & Debrecen & 1916 & Rapaics Rajmund & BPU-14844.JPG \\
\hline 2578 & Cyperus flavescens L. & Debrecen & 1922 & Bakos Gy. & BPU-14831.JPG \\
\hline 2578 & Cyperus flavescens L. & Debrecen & 1938 & Soó Rezső & BPU-14830.JPG \\
\hline 2578 & Cyperus flavescens L. & Kállósemjén & 1937 & Soó Rezső & BPU-14833.JPG \\
\hline 2578 & Cyperus flavescens L. & Lesenceistvánd & 1952 & Simon Tibor & BPU-14839.JPG \\
\hline 2578 & Cyperus flavescens L. & Nagykanizsa & 1956 & Felföldy Lajos & BPU-14836.JPG \\
\hline 2578 & Cyperus flavescens L. & Nyírlugos & 1932 & Soó Rezső & BPU-14832.JPG \\
\hline 2578 & Cyperus flavescens L. & Örvényes & 1958 & Felföldy Lajos & BPU-14838.JPG \\
\hline 2578 & Cyperus flavescens L. & Tokaj & 1946 & Priszter Szaniszló & BPU-14835.JPG \\
\hline 2579 & Cyperus fuscus L. & Balatonberény & 1958 & Tóth László & BPU-14809.JPG \\
\hline 2579 & Cyperus fuscus L. & Budapest & 1912 & Degen Árpád & BPU-14818.JPG \\
\hline 2579 & Cyperus fuscus L. & Budapest & 1992 & Felföldy Lajos & BPU-14816.JPG \\
\hline 2579 & Cyperus fuscus L. & Budapest & 1992 & Felföldy Lajos & BPU-14817.JPG \\
\hline 2579 & Cyperus fuscus L. & Budapest & - & Degen Árpád & BPU-14819.JPG \\
\hline 2579 & Cyperus fuscus L. & Dávod & 1955 & Soó Rezső & BPU-14808.JPG \\
\hline 2579 & Cyperus fuscus L. & Debrecen & 1953 & Kulcsár Gábor & BPU-15453.JPG \\
\hline 2579 & Cyperus fuscus L. & Debrecen & 1968 & Felföldy Lajos & BPU-14813.JPG \\
\hline 2579 & Cyperus fuscus L. & Dömös & 1947 & Priszter Szaniszló & BPU-14814.JPG \\
\hline 2579 & Cyperus fuscus L. & Dörgicse & 1957 & Felföldy Lajos & BPU-14805.JPG \\
\hline 2579 & Cyperus fuscus L. & Dunakeszi & 1942 & Priszter Szaniszló & BPU-14811.JPG \\
\hline 2579 & Cyperus fuscus L. & Gávavencsellő & 1990 & Felföldy Lajos & BPU-14807.JPG \\
\hline 2579 & Cyperus fuscus L. & Hortobágy & 1947 & Soó Rezső & BPU-14812.JPG \\
\hline 2579 & Cyperus fuscus L. & Kiskunmajsa & 1964 & Soó Rezső & BPU-14815.JPG \\
\hline 2579 & Cyperus fuscus L. & Nyírpilis & 1932 & Soó Rezső & BPU-14806.JPG \\
\hline 2579 & Cyperus fuscus L. & Révfülöp & 1956 & $\begin{array}{l}\text { Felföldy Lajos, Tóth } \\
\text { László }\end{array}$ & BPU-14804.JPG \\
\hline 2579 & Cyperus fuscus L. & Vácrátót & 1953 & Felföldy Lajos & BPU-14810.JPG \\
\hline 2580 & Cyperus difformis L. & Hortobágy & 1958 & Siroki Zoltán & BPU-14801.JPG \\
\hline 2580 & Cyperus difformis L. & Szarvas & 1951 & Csapody Vera & BPU-14802.JPG \\
\hline 2581 & Cyperus glomeratus L. & Debrecen & 1943 & Felföldy Lajos & BPU-14794.JPG \\
\hline 2581 & Cyperus glomeratus L. & Debrecen & 1988 & Felföldy Lajos & BPU-14793.JPG \\
\hline 2581 & Cyperus glomeratus L. & Dömös & 1947 & Priszter Szaniszló & BPU-14795.JPG \\
\hline
\end{tabular}


1. Elektroniukus melléklet - Electronic Appendix 1.

\begin{tabular}{|c|c|c|c|c|c|}
\hline $\begin{array}{l}\text { Sorszám / } \\
\text { Number }\end{array}$ & Taxon-név / Taxon-name & $\begin{array}{l}\text { Település / } \\
\text { Settlement }\end{array}$ & $\begin{array}{l}\text { Év / } \\
\text { Year }\end{array}$ & Gyújtő / Collector & $\begin{array}{l}\text { Fájlnév / } \\
\text { File-name }\end{array}$ \\
\hline 2581 & Cyperus glomeratus L. & Göd & 1942 & Priszter Szaniszló & BPU-14789.JPG \\
\hline 2581 & Cyperus glomeratus L. & Harkány & 1927 & Boros Ádám & BPU-14796.JPG \\
\hline 2581 & Cyperus glomeratus L. & Lakitelek & 1961 & Simon Tibor & BPU-14797.JPG \\
\hline 2581 & Cyperus glomeratus L. & Tiszafüred & 1952 & Soó Rezső & BPU-14790.JPG \\
\hline 2581 & Cyperus glomeratus L. & Tiszafüred & 1952 & Soó Rezső & BPU-14791.JPG \\
\hline 2582 & Cyperus glaber L. & Budapest & 1909 & Degen Árpád & BPU-14829.JPG \\
\hline 2582 & Cyperus glaber L. & Budapest & 1909 & Kocsis István & BPU-14828.JPG \\
\hline 2584 & Cyperus longus L. & Szigliget & 1956 & $\begin{array}{l}\text { Felföldy Lajos, Tóth } \\
\text { László }\end{array}$ & BPU-14783.JPG \\
\hline 2584 & Cyperus longus L. & Tata & 1911 & Degen Árpád & BPU-14787.JPG \\
\hline 2584 & Cyperus longus L. & Tata & 1911 & Degen Árpád & BPU-14787.JPG \\
\hline 2584 & Cyperus longus L. & Tata & 1911 & Degen Árpád & BPU-14788.JPG \\
\hline 2584 & Cyperus longus L. & Tata & 1911 & Degen Árpád & BPU-14788.JPG \\
\hline 2584 & Cyperus longus L. & Tata & 1926 & Boros Ádám & BPU-14785.JPG \\
\hline 2584 & Cyperus longus L. & Tata & 1926 & Degen Árpád & BPU-14786.JPG \\
\hline 2584 & Cyperus longus L. & Tata & 1942 & Priszter Szaniszló & BPU-14784.JPG \\
\hline 2585 & Cladium mariscus (L.) Pohl & Balatonfenyves & 1928 & Rigler József & BPU-14733.JPG \\
\hline 2585 & Cladium mariscus (L.) Pohl & Budapest & 1909 & Degen Árpád & BPU-14732.JPG \\
\hline 2585 & Cladium mariscus (L.) Pohl & Budapest & 1909 & Degen Árpád & BPU-14732.JPG \\
\hline 2585 & Cladium mariscus (L.) Pohl & Budapest & 1911 & Degen Árpád & BPU-14732.JPG \\
\hline 2585 & Cladium mariscus (L.) Pohl & Budapest & 1917 & Jávorka Sándor & BPU-14734.JPG \\
\hline 2585 & Cladium mariscus (L.) Pohl & Budapest & 1942 & Priszter Szaniszló & BPU-14731.JPG \\
\hline 2585 & Cladium mariscus (L.) Pohl & Kimle & 1962 & Borsos Olga & BPU-14730.JPG \\
\hline 2585 & Cladium mariscus (L.) Pohl & Lesenceistvánd & 1952 & Simon Tibor & BPU-14728.JPG \\
\hline 2585 & Cladium mariscus (L.) Pohl & Siófok & 1928 & Soó Rezső & BPU-14729.JPG \\
\hline 2586 & Rhynchospora alba (L.) Vahl & Szőce & 1955 & Soó Rezső & BPU-14722.JPG \\
\hline 2586 & Rhynchospora alba (L.) Vahl & Szőce & 1965 & $\begin{array}{l}\text { Máthé Imre, Kovács } \\
\text { Margit }\end{array}$ & BPU-14723.JPG \\
\hline 2587 & Schoenus nigricans L. & Balatonszőlős & 1956 & $\begin{array}{l}\text { Felföldy Lajos, Tóth } \\
\text { László }\end{array}$ & BPU-14746.JPG \\
\hline 2587 & Schoenus nigricans L. & Budapest & 1909 & Degen Árpád & BPU-14751.JPG \\
\hline 2587 & Schoenus nigricans L. & Budapest & 1923 & Soó Rezső & BPU-14750.JPG \\
\hline 2587 & Schoenus nigricans L. & Budapest & 1942 & Priszter Szaniszló & BPU-14749.JPG \\
\hline 2587 & Schoenus nigricans L. & Bugyi & 1959 & Soó Rezső & BPU-14742.JPG \\
\hline 2587 & Schoenus nigricans L. & Dunaharaszti & 1948 & Priszter Szaniszló & BPU-14741.JPG \\
\hline 2587 & Schoenus nigricans L. & Fertőhomok & 1960 & Tóth László & BPU-14748.JPG \\
\hline 2587 & Schoenus nigricans L. & Kimle & 1962 & Borsos Olga & BPU-14739.JPG \\
\hline 2587 & Schoenus nigricans L. & Kunpeszér & 1951 & Simon Tibor, Borsos Olga & BPU-14740.JPG \\
\hline 2587 & Schoenus nigricans L. & Lesenceistvánd & 1952 & Simon Tibor & BPU-14745.JPG \\
\hline 2587 & Schoenus nigricans L. & Örvényes & 1959 & Felföldy Lajos & BPU-14743.JPG \\
\hline 2587 & Schoenus nigricans L. & Örvényes & 1959 & Felföldy Lajos & BPU-14744.JPG \\
\hline 2587 & Schoenus nigricans L. & Sopron & 1944 & Kárpáti Zoltán & BPU-14747.JPG \\
\hline 2589 & Carex davalliana Sm. & Aszófő & 1957 & Felföldy Lajos & BPU-14621.JPG \\
\hline 2589 & Carex davalliana Sm. & Aszófő & 1959 & Felföldy Lajos & BPU-14619.JPG \\
\hline 2589 & Carex davalliana Sm. & Balatonszőlős & 1956 & $\begin{array}{l}\text { Felföldy Lajos, Tóth } \\
\text { László }\end{array}$ & BPU-14620.JPG \\
\hline 2589 & Carex davalliana Sm. & Balatonszőlős & 1957 & Felföldy Lajos & BPU-14625.JPG \\
\hline 2589 & Carex davalliana Sm. & Budapest & 1909 & Degen Árpád & BPU-14626.JPG \\
\hline 2589 & Carex davalliana Sm. & Örvényes & 1959 & Felföldy Lajos & BPU-14618.JPG \\
\hline
\end{tabular}


1. Elektroniukus melléklet - Electronic Appendix 1.

\begin{tabular}{|c|c|c|c|c|c|}
\hline $\begin{array}{l}\text { Sorszám / } \\
\text { Number }\end{array}$ & Taxon-név / Taxon-name & $\begin{array}{l}\text { Település / } \\
\text { Settlement }\end{array}$ & $\begin{array}{l}\text { Év / } \\
\text { Year }\end{array}$ & Gyűjtő / Collector & $\begin{array}{l}\text { Fájlnév / } \\
\text { File-name }\end{array}$ \\
\hline 2589 & Carex davalliana Sm. & Sopron & 1938 & Soó Rezső & BPU-14622.JPG \\
\hline 2589 & Carex davalliana Sm. & Sopron & 1960 & Borsos Olga, Simon Tibor & BPU-14623.JPG \\
\hline 2589 & Carex davalliana Sm. & Tapolca & 1928 & Soó Rezső & BPU-14617.JPG \\
\hline 2589 & Carex davalliana Sm. & Vácrátót & 1950 & Soó Rezső & BPU-14624.JPG \\
\hline 2590 & Carex bohemica Schreb. & Vásárosnamény & 1951 & Simon Tibor & BPU-14612.JPG \\
\hline 2591 & Carex remota L. & Bátorliget & 1934 & Soó Rezső & BPU-14407.JPG \\
\hline 2591 & Carex remota L. & Bátorliget & 1950 & $\begin{array}{l}\text { Simon Tibor, Kárpáti } \\
\text { István }\end{array}$ & BPU-14406.JPG \\
\hline 2591 & Carex remota $\mathrm{L}$. & Debrecen & 1989 & Felföldy Lajos & BPU-14413.JPG \\
\hline 2591 & Carex remota L. & Kapolcs & 1959 & $\begin{array}{l}\text { Jávorka Sándor, Felföldy } \\
\text { Lajos, Tóth László }\end{array}$ & BPU-14403.JPG \\
\hline 2591 & Carex remota L. & Kapolcs & 1959 & $\begin{array}{l}\text { Jávorka Sándor, Felföldy } \\
\text { Lajos, Tóth László }\end{array}$ & BPU-14404.JPG \\
\hline 2591 & Carex remota L. & Kaszó & 1957 & Soó Rezső & BPU-14408.JPG \\
\hline 2591 & Carex remota $\mathrm{L}$. & Kőszeg & 1932 & Soó Rezső & BPU-14405.JPG \\
\hline 2591 & Carex remota L. & Miskolc & 1950 & Soó Rezső & BPU-14414.JPG \\
\hline 2591 & Carex remota L. & Nagyvisnyó & 1953 & Soó Rezső & BPU-14412.JPG \\
\hline 2591 & Carex remota L. & Pálháza & 1960 & Simon Tibor & BPU-14416.JPG \\
\hline 2591 & Carex remota L. & Pécs & 1953 & Soó Rezső, Borsos Olga & BPU-14415.JPG \\
\hline 2591 & Carex remota L. & Pilisszentiván & 1941 & Priszter Szaniszló & BPU-14411.JPG \\
\hline 2591 & Carex remota L. & Pomáz & 1909 & Degen Árpád & BPU-14417.JPG \\
\hline 2591 & Carex remota L. & Pomáz & 1909 & Lengyel Géza & BPU-14410.JPG \\
\hline 2591 & Carex remota L. & Sárospatak & 1939 & Soó Rezső & BPU-14402.JPG \\
\hline 2591 & Carex remota L. & Szenna & 1957 & Soó Rezső & BPU-14409.JPG \\
\hline 2592 & Carex ovalis Gooden. & Debrecen & 1934 & Soó Rezső & BPU-14433.JPG \\
\hline 2592 & Carex ovalis Gooden. & Debrecen & 1950 & Soó Rezső & BPU-14432.JPG \\
\hline 2592 & Carex ovalis Gooden. & Debrecen & 1951 & Soó Rezső & BPU-14434.JPG \\
\hline 2592 & Carex ovalis Gooden. & Debrecen & 1988 & Sümegi Gy., Turi B. A. & BPU-14431.JPG \\
\hline 2592 & Carex ovalis Gooden. & Felsőtárkány & 1953 & Soó Rezső & BPU-14429.JPG \\
\hline 2592 & Carex ovalis Gooden. & Gyöngyös & 1965 & Soó Rezső & BPU-14428.JPG \\
\hline 2592 & Carex ovalis Gooden. & Kapolcs & 1959 & $\begin{array}{l}\text { Jávorka Sándor, Felföldy } \\
\text { Lajos, Tóth László }\end{array}$ & BPU-14427.JPG \\
\hline 2592 & Carex ovalis Gooden. & Monostorapáti & 1959 & $\begin{array}{l}\text { Jávorka Sándor, Felföldy } \\
\text { Lajos, Tóth László }\end{array}$ & BPU-14426.JPG \\
\hline 2592 & Carex ovalis Gooden. & Pomáz & 1910 & Degen Árpád & BPU-14436.JPG \\
\hline 2592 & Carex ovalis Gooden. & Szentbékkálla & 1959 & $\begin{array}{l}\text { Jávorka Sándor, Felföldy } \\
\text { Lajos, Tóth László }\end{array}$ & BPU-14425.JPG \\
\hline 2592 & Carex ovalis Gooden. & Vámospércs & 1932 & Soó Rezső & BPU-14430.JPG \\
\hline 2593 & Carex praecox Schreb. & Badacsonytomaj & 1951 & Priszter Szaniszló & BPU-14469.JPG \\
\hline 2593 & Carex praecox Schreb. & Balatonszőlős & 1957 & Felföldy Lajos & BPU-14468.JPG \\
\hline 2593 & Carex praecox Schreb. & Budakeszi & 1991 & Felföldy Lajos & BPU-14471.JPG \\
\hline 2593 & Carex praecox Schreb. & Budapest & 1909 & Degen Árpád & BPU-14477.JPG \\
\hline 2593 & Carex praecox Schreb. & Budapest & 1909 & Degen Árpád & BPU-14478.JPG \\
\hline 2593 & Carex praecox Schreb. & Debrecen & 1949 & Soó Rezső & BPU-14472.JPG \\
\hline 2593 & Carex praecox Schreb. & Gávavencsellő & 1990 & Felföldy Lajos & BPU-14470.JPG \\
\hline 2593 & Carex praecox Schreb. & Háromhuta & 1960 & Simon Tibor & BPU-14462.JPG \\
\hline 2593 & Carex praecox Schreb. & Kétegyháza & 1938 & Boros Ádám & BPU-14473.JPG \\
\hline 2593 & Carex praecox Schreb. & Kovácsvágás & 1962 & Simon Tibor & BPU-14476.JPG \\
\hline 2593 & Carex praecox Schreb. & Monostorapáti & 1959 & $\begin{array}{l}\text { Jávorka Sándor, Felföldy } \\
\text { Lajos, Tóth László }\end{array}$ & BPU-14467.JPG \\
\hline
\end{tabular}


1. Elektroniukus melléklet - Electronic Appendix 1.

\begin{tabular}{|c|c|c|c|c|c|}
\hline $\begin{array}{l}\text { Sorszám / } \\
\text { Number }\end{array}$ & Taxon-név / Taxon-name & $\begin{array}{l}\text { Település / } \\
\text { Settlement }\end{array}$ & $\begin{array}{l}\text { Év / } \\
\text { Year }\end{array}$ & Gyújtő / Collector & $\begin{array}{l}\text { Fájlnév / } \\
\text { File-name }\end{array}$ \\
\hline 2593 & Carex praecox Schreb. & Nagyharsány & 1956 & Soó Rezső & BPU-14474.JPG \\
\hline 2593 & Carex praecox Schreb. & Tass & 1982 & Felföldy Lajos & BPU-14475.JPG \\
\hline 2593 & Carex praecox Schreb. & Tihany & 1928 & Soó Rezső & BPU-14465.JPG \\
\hline 2593 & Carex praecox Schreb. & Tihany & 1956 & Felföldy Lajos & BPU-14466.JPG \\
\hline 2593 & Carex praecox Schreb. & Tihany & 1958 & Felföldy Lajos & BPU-14464.JPG \\
\hline 2593 & Carex praecox Schreb. & Tihany & 1959 & Felföldy Lajos & BPU-14463.JPG \\
\hline 2594 & Carex brizoides L. & Bátorliget & 1934 & Soó Rezső & BPU-14456.JPG \\
\hline 2594 & Carex brizoides L. & Kisunyom & 1954 & $\begin{array}{l}\text { Máthé Imre, Jeanplon } \\
\text { József, Kovács Margit }\end{array}$ & BPU-14455.JPG \\
\hline 2594 & Carex brizoides L. & Kőszeg & 1938 & Soó Rezső & BPU-14454.JPG \\
\hline 2594 & Carex brizoides L. & Regéc & 1960 & Simon Tibor & BPU-14461.JPG \\
\hline 2594 & Carex brizoides L. & Sátoraljaújhely & 1954 & $\begin{array}{l}\text { Máthé Imre, Jeanplon } \\
\text { József, Kovács Margit }\end{array}$ & BPU-14458.JPG \\
\hline 2594 & Carex brizoides L. & Surd & 1949 & Károlyi Árpád & BPU-14457.JPG \\
\hline 2595 & Carex echinata Murray & Debrecen & 1950 & Soó Rezső & BPU-14395.JPG \\
\hline 2595 & Carex echinata Murray & Debrecen & 1950 & Soó Rezső & BPU-14396.JPG \\
\hline 2595 & Carex echinata Murray & Pálháza & 1949 & Simon Tibor, Jakucs Pál & BPU-14398.JPG \\
\hline 2595 & Carex echinata Murray & Sátoraljaújhely & 1954 & $\begin{array}{l}\text { Máthé Imre, Jeanplon } \\
\text { József, Kovács Margit }\end{array}$ & BPU-14397.JPG \\
\hline 2595 & Carex echinata Murray & Szentgotthárd & 1955 & Soó Rezső & BPU-14394.JPG \\
\hline 2596 & Carex elongata L. & Bátorliget & 1935 & Soó Rezső & BPU-14442.JPG \\
\hline 2596 & Carex elongata L. & Dömös & 1917 & Boros Ádám & BPU-14451.JPG \\
\hline 2596 & Carex elongata L. & Füzér & 1938 & Soó Rezső & BPU-14445.JPG \\
\hline 2596 & Carex elongata L. & Kaszó & 1957 & Soó Rezső & BPU-14444.JPG \\
\hline 2596 & Carex elongata L. & Pálháza & 1960 & Simon Tibor & BPU-14447.JPG \\
\hline 2596 & Carex elongata L. & Pálháza & 1960 & Simon Tibor & BPU-14448.JPG \\
\hline 2596 & Carex elongata L. & Sátoraljaújhely & 1954 & $\begin{array}{l}\text { Máthé Imre, Jeanplon } \\
\text { József, Kovács Margit }\end{array}$ & BPU-14446.JPG \\
\hline 2596 & Carex elongata L. & Somogyszob & 1957 & Soó Rezső & BPU-14443.JPG \\
\hline 2596 & Carex elongata L. & Szentbékkálla & 1959 & $\begin{array}{l}\text { Jávorka Sándor, Felföldy } \\
\text { Lajos, Tóth László }\end{array}$ & BPU-14440.JPG \\
\hline 2596 & Carex elongata L. & Szőce & 1955 & Soó Rezső & BPU-14441.JPG \\
\hline 2596 & Carex elongata L. & Tarpa & 1949 & Soó Rezső & BPU-15525.JPG \\
\hline 2597 & Carex canescens L. & Kétvölgy & 1957 & Pócs Tamás, Tallós Pál & BPU-14383.JPG \\
\hline 2597 & Carex canescens L. & Szemere & 1966 & Felföldy Lajos & BPU-14384.JPG \\
\hline 2598 & Carex divulsa Stokes & Badacsonytomaj & 1953 & Priszter Szaniszló & BPU-14484.JPG \\
\hline 2598 & Carex divulsa Stokes & Balatonfüred & 1942 & Felföldy Lajos & BPU-14483.JPG \\
\hline 2598 & Carex divulsa Stokes & Balatonszepezd & 1953 & Priszter Szaniszló & BPU-14482.JPG \\
\hline 2598 & Carex divulsa Stokes & Bisse & 1965 & $\begin{array}{l}\text { Simon Tibor, Priszter } \\
\text { Szaniszló, Borhidi Attila }\end{array}$ & BPU-14491.JPG \\
\hline 2598 & Carex divulsa Stokes & Debrecen & 1934 & Soó Rezső & BPU-14487.JPG \\
\hline 2598 & Carex divulsa Stokes & Debrecen & 1937 & Máthé Imre & BPU-14485.JPG \\
\hline 2598 & Carex divulsa Stokes & Háromhuta & 1960 & Simon Tibor & BPU-14480.JPG \\
\hline 2598 & Carex divulsa Stokes & Kapolcs & 1959 & $\begin{array}{l}\text { Jávorka Sándor, Felföldy } \\
\text { Lajos, Tóth László }\end{array}$ & BPU-14481.JPG \\
\hline 2598 & Carex divulsa Stokes & Kelemér & 1950 & Soó Rezső & BPU-14488.JPG \\
\hline 2598 & Carex divulsa Stokes & Komló & 1948 & Priszter Szaniszló & BPU-14490.JPG \\
\hline 2598 & Carex divulsa Stokes & Pécs & 1915 & Andrasovszky József & BPU-14504.JPG \\
\hline 2598 & Carex divulsa Stokes & Pécs & 1915 & Andrasovszky József & BPU-14505.JPG \\
\hline 2598 & Carex divulsa Stokes & Pécs & 1936 & Soó Rezső & BPU-14489.JPG \\
\hline
\end{tabular}


1. Elektroniukus melléklet - Electronic Appendix 1.

\begin{tabular}{|c|c|c|c|c|c|}
\hline $\begin{array}{l}\text { Sorszám / } \\
\text { Number }\end{array}$ & Taxon-név / Taxon-name & $\begin{array}{l}\text { Település / } \\
\text { Settlement }\end{array}$ & $\begin{array}{l}\text { Év / } \\
\text { Year }\end{array}$ & Gyújtő / Collector & $\begin{array}{l}\text { Fájlnév / } \\
\text { File-name }\end{array}$ \\
\hline 2598 & Carex divulsa Stokes & Sáránd & 1932 & Soó Rezső & BPU-14486.JPG \\
\hline 2598 & Carex divulsa Stokes & Vác & 1923 & Soó Rezső & BPU-14500.JPG \\
\hline 2599 & Carex spicata Huds. & Balatonfüred & 1942 & Felföldy Lajos & BPU-14511.JPG \\
\hline 2599 & Carex spicata Huds. & Bátorliget & 1934 & Soó Rezső & BPU-14512.JPG \\
\hline 2599 & Carex spicata Huds. & Budapest & 1989 & Felföldy Lajos & BPU-14517.JPG \\
\hline 2599 & Carex spicata Huds. & Budapest & 2004 & Felföldy Lajos & BPU-14518.JPG \\
\hline 2599 & Carex spicata Huds. & Debrecen & 1935 & Soó Rezső & BPU-14514.JPG \\
\hline 2599 & Carex spicata Huds. & Kapolcs & 1959 & $\begin{array}{l}\text { Jávorka Sándor, Felföldy } \\
\text { Lajos, Tóth László }\end{array}$ & BPU-14510.JPG \\
\hline 2599 & Carex spicata Huds. & Litke & 1987 & Felföldy Lajos & BPU-14513.JPG \\
\hline 2599 & Carex spicata Huds. & Nagyrada & 1987 & Felföldy Lajos & BPU-14508.JPG \\
\hline 2599 & Carex spicata Huds. & Pilisszentiván & 1909 & Degen Árpád & BPU-14521.JPG \\
\hline 2599 & Carex spicata Huds. & Pilisszentiván & 1915 & $\begin{array}{l}\text { Degen Árpád, Szártorisz } \\
\text { Béla, Földvári Dezső }\end{array}$ & BPU-14520.JPG \\
\hline 2599 & Carex spicata Huds. & Szár & 1943 & Kárpáti Zoltán & BPU-14515.JPG \\
\hline 2599 & Carex spicata Huds. & Szigetújfalu & 1906 & Degen Árpád & BPU-14519.JPG \\
\hline 2600 & Carex pairaei F.W. Schultz & Aszófő & 1957 & Felföldy Lajos & BPU-14499.JPG \\
\hline 2600 & Carex pairaei F.W. Schultz & Bakonybél & 1955 & Soó Rezső & BPU-14498.JPG \\
\hline 2600 & Carex pairaei F.W. Schultz & Bélapátfalva & 1932 & Soó Rezső & BPU-14501.JPG \\
\hline 2600 & Carex pairaei F.W. Schultz & Budapest & 1947 & Pénzes Antal & BPU-14502.JPG \\
\hline 2600 & Carex pairaei F.W. Schultz & Budapest & 2004 & Felföldy Lajos & BPU-14503.JPG \\
\hline 2600 & Carex pairaei F.W. Schultz & Kapolcs & 1959 & $\begin{array}{l}\text { Jávorka Sándor, Felföldy } \\
\text { Lajos, Tóth László }\end{array}$ & BPU-14497.JPG \\
\hline 2600 & Carex pairaei F.W. Schultz & Miskolc & 1915 & Budai József, Hulják János & BPU-14506.JPG \\
\hline 2600 & Carex pairaei F.W. Schultz & Tákos & 1948 & $\begin{array}{l}\text { Pólya László, Simon } \\
\text { Tibor, Jakucs Pál }\end{array}$ & BPU-14516.JPG \\
\hline 2600 & Carex pairaei F.W. Schultz & Tihany & 1942 & Felföldy Lajos & BPU-14494.JPG \\
\hline 2600 & Carex pairaei F.W. Schultz & Tihany & 1956 & Felföldy Lajos & BPU-14496.JPG \\
\hline 2600 & Carex pairaei F.W. Schultz & Tihany & 1959 & Felföldy Lajos & BPU-14493.JPG \\
\hline 2600 & Carex pairaei F.W. Schultz & Tihany & 1959 & Felföldy Lajos & BPU-14495.JPG \\
\hline 2600 & Carex pairaei F.W. Schultz & Uzsa & 1959 & $\begin{array}{l}\text { Jávorka Sándor, Felföldy } \\
\text { Lajos }\end{array}$ & BPU-14492.JPG \\
\hline 2600 & Carex pairaei F.W. Schultz & Vászoly & 1958 & Felföldy Lajos & BPU-14509.JPG \\
\hline 2601 & Carex vulpina $\mathrm{L}$. & Bátorliget & 1932 & Soó Rezső & BPU-14541.JPG \\
\hline 2601 & Carex vulpina L. & Beregdaróc & 1951 & $\begin{array}{l}\text { Simon Tibor, Vozáry } \\
\text { Elemér }\end{array}$ & BPU-14544.JPG \\
\hline 2601 & Carex vulpina $\mathrm{L}$. & Beregdaróc & 1951 & $\begin{array}{l}\text { Simon Tibor, Vozáry } \\
\text { Elemér }\end{array}$ & BPU-14545.JPG \\
\hline 2601 & Carex vulpina $\mathrm{L}$. & Budapest & 1909 & $\begin{array}{l}\text { Degen Árpád, Földváry } \\
\text { Dezső }\end{array}$ & BPU-14548.JPG \\
\hline 2601 & Carex vulpina L. & Budapest & - & $\begin{array}{l}\text { Degen Árpád, Földváry } \\
\text { Dezső }\end{array}$ & BPU-14547.JPG \\
\hline 2601 & Carex vulpina L. & Debrecen & 1935 & Soó Rezső & BPU-14542.JPG \\
\hline 2601 & Carex vulpina $\mathrm{L}$. & Debrecen & 1952 & Kulcsár Gábor & BPU-15434.JPG \\
\hline 2601 & Carex vulpina $\mathrm{L}$. & Gelénes & 1951 & $\begin{array}{l}\text { Simon Tibor, Vozáry } \\
\text { Elemér }\end{array}$ & BPU-14543.JPG \\
\hline 2601 & Carex vulpina L. & Monostorapáti & 1959 & $\begin{array}{l}\text { Jávorka Sándor, Felföldy } \\
\text { Lajos, Tóth László }\end{array}$ & BPU-14540.JPG \\
\hline 2601 & Carex vulpina L. & Tihany & 1925 & Soó Rezső & BPU-14539.JPG \\
\hline 2601 & Carex vulpina L. & Tihany & 1951 & Priszter Szaniszló & BPU-14538.JPG \\
\hline 2602 & Carex otrubae Podp. & Balatonszepezd & 1958 & Felföldy Lajos & BPU-14524.JPG \\
\hline 2602 & Carex otrubae Podp. & Balatonszőlős & 1957 & Felföldy Lajos & BPU-14525.JPG \\
\hline
\end{tabular}


1. Elektroniukus melléklet - Electronic Appendix 1.

\begin{tabular}{|c|c|c|c|c|c|}
\hline $\begin{array}{l}\text { Sorszám / } \\
\text { Number }\end{array}$ & Taxon-név / Taxon-name & $\begin{array}{l}\text { Település / } \\
\text { Settlement }\end{array}$ & $\begin{array}{l}\text { Év / } \\
\text { Year }\end{array}$ & Gyűjtő / Collector & $\begin{array}{l}\text { Fájlnév / } \\
\text { File-name }\end{array}$ \\
\hline 2602 & Carex otrubae Podp. & Budaörs & 1991 & Felföldy Lajos & BPU-14528.JPG \\
\hline 2602 & Carex otrubae Podp. & Budapest & 1991 & Felföldy Lajos & BPU-14537.JPG \\
\hline 2602 & Carex otrubae Podp. & Debrecen & 1935 & Soó Rezső & BPU-14529.JPG \\
\hline 2602 & Carex otrubae Podp. & Dunaharaszti & 1948 & Priszter Szaniszló & BPU-14527.JPG \\
\hline 2602 & Carex otrubae Podp. & Fonyód & 1953 & Priszter Szaniszló & BPU-14526.JPG \\
\hline 2602 & Carex otrubae Podp. & Gárdony & 1980 & Felföldy Lajos & BPU-14535.JPG \\
\hline 2602 & Carex otrubae Podp. & Kunszentmiklós & 1968 & Priszter Szaniszló & BPU-14536.JPG \\
\hline 2602 & Carex otrubae Podp. & Pákozd & 1980 & Felföldy Lajos & BPU-14530.JPG \\
\hline 2602 & Carex otrubae Podp. & Pákozd & 1980 & Felföldy Lajos & BPU-14531.JPG \\
\hline 2602 & Carex otrubae Podp. & Pákozd & 1980 & Felföldy Lajos & BPU-14532.JPG \\
\hline 2602 & Carex otrubae Podp. & Pákozd & 1980 & Felföldy Lajos & BPU-14533.JPG \\
\hline 2602 & Carex otrubae Podp. & Pákozd & 1980 & Felföldy Lajos & BPU-14534.JPG \\
\hline 2602 & Carex otrubae Podp. & Tihany & 1951 & Priszter Szaniszló & BPU-14523.JPG \\
\hline 2603 & Carex paniculata L. & Aszófő & 1931 & Soó Rezső & BPU-14558.JPG \\
\hline 2603 & Carex paniculata L. & Aszófő & 1957 & Felföldy Lajos & BPU-14557.JPG \\
\hline 2603 & Carex paniculata L. & Aszófő & 1959 & Felföldy Lajos & BPU-14556.JPG \\
\hline 2603 & Carex paniculata L. & Budapest & 1917 & Degen Árpád & BPU-14565.JPG \\
\hline 2603 & Carex paniculata L. & Budapest & 1917 & Degen Árpád & BPU-14565.JPG \\
\hline 2603 & Carex paniculata L. & Dabas & 1959 & Simon Tibor & BPU-14561.JPG \\
\hline 2603 & Carex paniculata L. & Gödöllő & 1952 & Priszter Szaniszló & BPU-14560.JPG \\
\hline 2603 & Carex paniculata L. & Komlóska & 1955 & Simon Tibor & BPU-14552.JPG \\
\hline 2603 & Carex paniculata L. & Ugod & 1955 & Soó Rezső & BPU-14555.JPG \\
\hline 2603 & Carex paniculata L. & Uzsa & 1949 & Károlyi Árpád & BPU-14554.JPG \\
\hline 2603 & Carex paniculata L. & Uzsa & 1959 & $\begin{array}{l}\text { Jávorka Sándor, Felföldy } \\
\text { Lajos }\end{array}$ & BPU-14553.JPG \\
\hline 2603 & Carex paniculata L. & Vasvár & 1950 & Károlyi Árpád & BPU-14559.JPG \\
\hline 2604 & Carex appropinquata Schumach. & Badacsonytomaj & 1984 & Felföldy Lajos & BPU-14570.JPG \\
\hline 2604 & Carex appropinquata Schumach. & Balatonszőlős & 1956 & $\begin{array}{l}\text { Felföldy Lajos, Tóth } \\
\text { László }\end{array}$ & BPU-14569.JPG \\
\hline 2604 & Carex appropinquata Schumach. & Gárdony & 1978 & Felföldy Lajos & BPU-14573.JPG \\
\hline 2604 & Carex appropinquata Schumach. & Gárdony & 1978 & Felföldy Lajos & BPU-14575.JPG \\
\hline 2604 & Carex appropinquata Schumach. & Gárdony & 1978 & Felföldy Lajos & BPU-14576.JPG \\
\hline 2604 & Carex appropinquata Schumach. & Gödöllő & 1951 & Priszter Szaniszló & BPU-14571.JPG \\
\hline 2604 & Carex appropinquata Schumach. & Nagykanizsa & 1948 & Károlyi Árpád & BPU-14567.JPG \\
\hline 2604 & Carex appropinquata Schumach. & Nagykanizsa & 1949 & Károlyi Árpád & BPU-14566.JPG \\
\hline 2604 & Carex appropinquata Schumach. & Örvényes & 1959 & Felföldy Lajos & BPU-14568.JPG \\
\hline 2604 & Carex appropinquata Schumach. & Pákozd & 1978 & Felföldy Lajos & BPU-14574.JPG \\
\hline 2605 & Carex diandra Schrank & Háromhuta & 1961 & Siroki Zoltán & BPU-14580.JPG \\
\hline 2605 & Carex diandra Schrank & Kelemér & 1950 & Soó Rezső & BPU-14584.JPG \\
\hline 2605 & Carex diandra Schrank & Kelemér & 1950 & Soó Rezső & BPU-14585.JPG \\
\hline 2605 & Carex diandra Schrank & Ozmánbük & 1950 & Károlyi Árpád & BPU-14581.JPG \\
\hline 2605 & Carex diandra Schrank & Ozmánbük & 1950 & Károlyi Árpád & BPU-14582.JPG \\
\hline 2605 & Carex diandra Schrank & Örvényes & 1959 & Felföldy Lajos & BPU-14583.JPG \\
\hline 2606 & Carex disticha Huds. & Aszófo" & 1959 & Felföldy Lajos & BPU-14366.JPG \\
\hline 2606 & Carex disticha Huds. & Budaörs & 1992 & Felföldy Lajos & BPU-14367.JPG \\
\hline 2606 & Carex disticha Huds. & Budapest & 1909 & Degen Árpád & BPU-14375.JPG \\
\hline 2606 & Carex disticha Huds. & Dabas & 1951 & Borsos Olga, Simon Tibor & BPU-14368.JPG \\
\hline 2606 & Carex disticha Huds. & Debrecen & 1934 & Soó Rezső & BPU-14371.JPG \\
\hline
\end{tabular}


1. Elektroniukus melléklet - Electronic Appendix 1.

\begin{tabular}{|c|c|c|c|c|c|}
\hline $\begin{array}{l}\text { Sorszám / } \\
\text { Number }\end{array}$ & Taxon-név / Taxon-name & $\begin{array}{l}\text { Település / } \\
\text { Settlement }\end{array}$ & $\begin{array}{l}\text { Év / } \\
\text { Year }\end{array}$ & Gyüjtő / Collector & $\begin{array}{l}\text { Fájlnév / } \\
\text { File-name }\end{array}$ \\
\hline 2606 & Carex disticha Huds. & Gárdony & 1979 & Felföldy Lajos & BPU-14374.JPG \\
\hline 2606 & Carex disticha Huds. & Gárdony & 1980 & Felföldy Lajos & BPU-14373.JPG \\
\hline 2606 & Carex disticha Huds. & Hajdúsámson & 1950 & Soó Rezső & BPU-14370.JPG \\
\hline 2606 & Carex disticha Huds. & Lábatlan & 1953 & Felföldy Lajos & BPU-14369.JPG \\
\hline 2606 & Carex disticha Huds. & Pátka & 1987 & Felföldy Lajos & BPU-14372.JPG \\
\hline 2608 & Carex stenophylla Wahlenb. & Bátorliget & 1934 & Soó Rezső & BPU-14598.JPG \\
\hline 2608 & Carex stenophylla Wahlenb. & Budapest & 1909 & Degen Árpád & BPU-14609.JPG \\
\hline 2608 & Carex stenophylla Wahlenb. & Budapest & 1909 & Degen Árpád & BPU-14611.JPG \\
\hline 2608 & Carex stenophylla Wahlenb. & Budapest & 1941 & Priszter Szaniszló & BPU-14607.JPG \\
\hline 2608 & Carex stenophylla Wahlenb. & Budapest & 1941 & Priszter Szaniszló & BPU-14608.JPG \\
\hline 2608 & Carex stenophylla Wahlenb. & Debrecen & 1932 & Soó Rezső & BPU-14604.JPG \\
\hline 2608 & Carex stenophylla Wahlenb. & Gödöllő & 1954 & Priszter Szaniszló & BPU-14603.JPG \\
\hline 2608 & Carex stenophylla Wahlenb. & Hortobágy & 1934 & Zólyomi Bálint, Soó Rezső & BPU-14605.JPG \\
\hline 2608 & Carex stenophylla Wahlenb. & Nadap & 1951 & Priszter Szaniszló & BPU-14606.JPG \\
\hline 2608 & Carex stenophylla Wahlenb. & Nagykovácsi & 1949 & Soó Rezső & BPU-14602.JPG \\
\hline 2608 & Carex stenophylla Wahlenb. & Pilisvörösvár & 1915 & $\begin{array}{l}\text { Degen Árpád, Szártorisz } \\
\text { Béla, Földvári Dezső }\end{array}$ & BPU-14610.JPG \\
\hline 2608 & Carex stenophylla Wahlenb. & Pilisszentiván & 1942 & Priszter Szaniszló & BPU-14601.JPG \\
\hline 2608 & Carex stenophylla Wahlenb. & Pilisszentiván & 1947 & Priszter Szaniszló & BPU-14600.JPG \\
\hline 2608 & Carex stenophylla Wahlenb. & Solymár & 1951 & Priszter Szaniszló & BPU-14599.JPG \\
\hline 2609 & Carex divisa Huds. & Budapest & 1909 & Degen Árpád & BPU-14597.JPG \\
\hline 2609 & Carex divisa Huds. & Budapest & 1947 & Pénzes Antal & BPU-14596.JPG \\
\hline 2609 & Carex divisa Huds. & Dabas & 1951 & Borsos Olga, Simon Tibor & BPU-14590.JPG \\
\hline 2609 & Carex divisa Huds. & Debrecen & 1947 & Soó Rezső & BPU-14591.JPG \\
\hline 2609 & Carex divisa Huds. & Gárdony & 1982 & Felföldy Lajos & BPU-14594.JPG \\
\hline 2609 & Carex divisa Huds. & Kecel & 1954 & Priszter Szaniszló & BPU-14595.JPG \\
\hline 2609 & Carex divisa Huds. & Örvényes & 1959 & Felföldy Lajos & BPU-14588.JPG \\
\hline 2609 & Carex divisa Huds. & Pákozd & 1980 & Felföldy Lajos & BPU-14592.JPG \\
\hline 2609 & Carex divisa Huds. & Pákozd & 1980 & Felföldy Lajos & BPU-14593.JPG \\
\hline 2609 & Carex divisa Huds. & Siófok & 1928 & Soó Rezső & BPU-14589.JPG \\
\hline 2610 & Carex elata All. & Bagamér & 1949 & Soó Rezső & BPU-11740.JPG \\
\hline 2610 & Carex elata All. & Balatonszőlős & 1957 & Felföldy Lajos & BPU-11736.JPG \\
\hline 2610 & Carex elata All. & Budapest & 1916 & Degen Árpád & BPU-11746.JPG \\
\hline 2610 & Carex elata All. & Budapest & 1947 & Pénzes Antal & BPU-11745.JPG \\
\hline 2610 & Carex elata All. & Budapest & 1989 & Felföldy Lajos & BPU-11744.JPG \\
\hline 2610 & Carex elata All. & Csaroda & 1953 & $\begin{array}{l}\text { Simon Tibor, Helmeczy } \\
\text { Balázs }\end{array}$ & BPU-11743.JPG \\
\hline 2610 & Carex elata All. & Csévharaszt & 1959 & $\begin{array}{l}\text { Simon Tibor, Horánszky } \\
\text { András }\end{array}$ & BPU-11738.JPG \\
\hline 2610 & Carex elata All. & Debrecen & 1934 & Soó Rezső & BPU-11741.JPG \\
\hline 2610 & Carex elata All. & Kelemér & 1950 & Soó Rezső & BPU-11742.JPG \\
\hline 2610 & Carex elata All. & Kőszeg & 1932 & Soó Rezső & BPU-11737.JPG \\
\hline 2610 & Carex elata All. & Szécsény & 1953 & Priszter Szaniszló & BPU-11739.JPG \\
\hline 2610 & Carex elata All. & Tihany & 1956 & Felföldy Lajos & BPU-11734.JPG \\
\hline 2610 & Carex elata All. & Tihany & 1959 & Felföldy Lajos & BPU-11735.JPG \\
\hline 2611 & Carex acuta L. & Bozsok & 1938 & Soó Rezső & BPU-14347.JPG \\
\hline 2611 & Carex acuta L. & Budapest & 1909 & Degen Árpád & BPU-14356.JPG \\
\hline 2611 & Carex acuta L. & Budapest & 1909 & Degen Árpád & BPU-14357.JPG \\
\hline 2611 & Carex acuta L. & Budapest & 1915 & Degen Árpád & BPU-14355.JPG \\
\hline
\end{tabular}


1. Elektroniukus melléklet - Electronic Appendix 1.

\begin{tabular}{|c|c|c|c|c|c|}
\hline $\begin{array}{l}\text { Sorszám / } \\
\text { Number }\end{array}$ & Taxon-név / Taxon-name & $\begin{array}{l}\text { Település / } \\
\text { Settlement }\end{array}$ & $\begin{array}{l}\text { Év / } \\
\text { Year }\end{array}$ & Gyújtő / Collector & $\begin{array}{l}\text { Fájlnév / } \\
\text { File-name }\end{array}$ \\
\hline 2611 & Carex acuta L. & Budapest & 1916 & Degen Árpád & BPU-14355.JPG \\
\hline 2611 & Carex acuta L. & Csaroda & 1948 & $\begin{array}{l}\text { Pólya László, Simon } \\
\text { Tibor, Jakucs Pál }\end{array}$ & BPU-14354.JPG \\
\hline 2611 & Carex acuta L. & Gávavencsellő & 1990 & Felföldy Lajos & BPU-14348.JPG \\
\hline 2611 & Carex acuta L. & Gávavencsellő & 1990 & Felföldy Lajos & BPU-14349.JPG \\
\hline 2611 & Carex acuta L. & Ipolytarnóc & 1987 & Felföldy Lajos & BPU-14352.JPG \\
\hline 2611 & Carex acuta L. & Litke & 1987 & Felföldy Lajos & BPU-14351.JPG \\
\hline 2611 & Carex acuta L. & Szécsény & 1953 & Priszter Szaniszló & BPU-14350.JPG \\
\hline 2611 & Carex acuta L. & Tiszafüred & 1932 & Soó Rezső & BPU-14353.JPG \\
\hline 2612 & Carex nigra (L.) Reichard & Aszófő & 1959 & Felföldy Lajos & BPU-14331.JPG \\
\hline 2612 & Carex nigra (L.) Reichard & Bátorliget & 1934 & Soó Rezső & BPU-14334.JPG \\
\hline 2612 & Carex nigra (L.) Reichard & Budapest & 1915 & $\begin{array}{l}\text { Degen Árpád, Földváry } \\
\text { Dezső }\end{array}$ & BPU-14342.JPG \\
\hline 2612 & Carex nigra (L.) Reichard & Debrecen & 1934 & Soó Rezső & BPU-14336.JPG \\
\hline 2612 & Carex nigra (L.) Reichard & Debrecen & 1950 & Soó Rezső & BPU-14335.JPG \\
\hline 2612 & Carex nigra (L.) Reichard & Kőszeg & 1954 & $\begin{array}{l}\text { Máthé Imre, Jeanplon } \\
\text { József, Kovács Margit }\end{array}$ & BPU-14333.JPG \\
\hline 2612 & Carex nigra (L.) Reichard & Pálháza & 1991 & Simon Tibor & BPU-14337.JPG \\
\hline 2612 & Carex nigra (L.) Reichard & Sátoraljaújhely & 1954 & $\begin{array}{l}\text { Máthé Imre, Jeanplon } \\
\text { József, Kovács Margit }\end{array}$ & BPU-14338.JPG \\
\hline 2613 & Carex buekii Wimm. & Zebegény & 2007 & Nagy József & BPU-11755.JPG \\
\hline 2614 & Carex cespitosa L. & Kőszeg & 1896 & Waisbecker Antal & BPU-11751.JPG \\
\hline 2614 & Carex cespitosa L. & Szentgyörgyvölgy & 1963 & $\begin{array}{l}\text { Szodfridt István, Tallós } \\
\text { Pál }\end{array}$ & BPU-11750.JPG \\
\hline 2615 & Carex hartmanii Cajander & Nyirád & 1963 & Tallós Pál & BPU-14359.JPG \\
\hline 2617 & Carex lasiocarpa Ehrh. & Csaroda & 1955 & Soó Rezső & BPU-12480.JPG \\
\hline 2617 & Carex lasiocarpa Ehrh. & Nyirád & 1963 & Tallós Pál & BPU-12479.JPG \\
\hline 2618 & Carex hirta L. & Aszófő & 1951 & Priszter Szaniszló & BPU-12489.JPG \\
\hline 2618 & Carex hirta L. & Balatonberény & 1953 & Priszter Szaniszló & BPU-12492.JPG \\
\hline 2618 & Carex hirta L. & Balatonfenyves & 1953 & Priszter Szaniszló & BPU-12491.JPG \\
\hline 2618 & Carex hirta L. & Balatonszepezd & 1953 & Priszter Szaniszló & BPU-12488.JPG \\
\hline 2618 & Carex hirta L. & Balatonszepezd & 1958 & Felföldy Lajos & BPU-12486.JPG \\
\hline 2618 & Carex hirta L. & Bátorliget & 1932 & Soó Rezső & BPU-12490.JPG \\
\hline 2618 & Carex hirta L. & Budapest & 1909 & Degen Árpád & BPU-12511.JPG \\
\hline 2618 & Carex hirta L. & Budapest & 1909 & Degen Árpád & BPU-12512.JPG \\
\hline 2618 & Carex hirta L. & Budapest & 1916 & Degen Árpád & BPU-12508.JPG \\
\hline 2618 & Carex hirta L. & Budapest & 1991 & Felföldy Lajos & BPU-12505.JPG \\
\hline 2618 & Carex hirta L. & Budapest & 1991 & Felföldy Lajos & BPU-12506.JPG \\
\hline 2618 & Carex hirta L. & Budapest & 1991 & Felföldy Lajos & BPU-12507.JPG \\
\hline 2618 & Carex hirta L. & Budapest & 2005 & Felföldy Lajos & BPU-12504.JPG \\
\hline 2618 & Carex hirta L. & Debrecen & 1988 & Sümegi Gy., Turi B. A. & BPU-12498.JPG \\
\hline 2618 & Carex hirta L. & Debrecen & 1988 & Turi B. A., Sümegi Gy. & BPU-12499.JPG \\
\hline 2618 & Carex hirta L. & Dunaharaszti & 1916 & Zsák Zoltán & BPU-12509.JPG \\
\hline 2618 & Carex hirta L. & Dunaharaszti & 1916 & Zsák Zoltán & BPU-12510.JPG \\
\hline 2618 & Carex hirta L. & Gyöngyös & 1940 & Priszter Szaniszló & BPU-12496.JPG \\
\hline 2618 & Carex hirta L. & Lábatlan & 1953 & Felföldy Lajos & BPU-12495.JPG \\
\hline 2618 & Carex hirta L. & Nagyrada & 1987 & Felföldy Lajos & BPU-12483.JPG \\
\hline 2618 & Carex hirta L. & Örvényes & 1959 & Felföldy Lajos & BPU-12487.JPG \\
\hline 2618 & Carex hirta L. & Pákozd & 1980 & Felföldy Lajos & BPU-12500.JPG \\
\hline 2618 & Carex hirta L. & Pákozd & 1980 & Felföldy Lajos & BPU-12501.JPG \\
\hline
\end{tabular}


1. Elektroniukus melléklet - Electronic Appendix 1.

\begin{tabular}{|c|c|c|c|c|c|}
\hline $\begin{array}{l}\text { Sorszám / } \\
\text { Number }\end{array}$ & Taxon-név / Taxon-name & $\begin{array}{l}\text { Település / } \\
\text { Settlement }\end{array}$ & $\begin{array}{l}\text { Év / } \\
\text { Year }\end{array}$ & Gyújtő / Collector & $\begin{array}{l}\text { Fájlnév / } \\
\text { File-name }\end{array}$ \\
\hline 2618 & Carex hirta L. & Pálháza & 1960 & Simon Tibor & BPU-12502.JPG \\
\hline 2618 & Carex hirta L. & Pálháza & 1960 & Simon Tibor & BPU-12503.JPG \\
\hline 2618 & Carex hirta L. & Szécsény & 1953 & Priszter Szaniszló & BPU-12494.JPG \\
\hline 2618 & Carex hirta L. & Téglás & 1937 & Soó Rezső & BPU-12497.JPG \\
\hline 2618 & Carex hirta L. & Tihany & 1951 & Priszter Szaniszló & BPU-12485.JPG \\
\hline 2618 & Carex hirta L. & Tihany & 1959 & Felföldy Lajos & BPU-12484.JPG \\
\hline 2618 & Carex hirta L. & Törökbálint & 1992 & Felföldy Lajos & BPU-12493.JPG \\
\hline 2619 & Carex halleriana Asso & Balatonfüred & 1928 & Soó Rezső & BPU-14176.JPG \\
\hline 2619 & Carex halleriana Asso & Balatonszőlős & 1959 & Felföldy Lajos & BPU-14175.JPG \\
\hline 2619 & Carex halleriana Asso & Budapest & 1909 & Degen Árpád & BPU-14182.JPG \\
\hline 2619 & Carex halleriana Asso & Budapest & 1947 & Soó Rezső & BPU-14181.JPG \\
\hline 2619 & Carex halleriana Asso & Budapest & 1953 & Soó Rezső & BPU-15446.JPG \\
\hline 2619 & Carex halleriana Asso & Budapest & 1982 & Felföldy Lajos & BPU-14179.JPG \\
\hline 2619 & Carex halleriana Asso & Budapest & 1992 & Felföldy Lajos & BPU-14180.JPG \\
\hline 2619 & Carex halleriana Asso & Pécsely & 1951 & Priszter Szaniszló & BPU-14174.JPG \\
\hline 2619 & Carex halleriana Asso & Pilisborosjenő & 1940 & Kárpáti Zoltán & BPU-14178.JPG \\
\hline 2619 & Carex halleriana Asso & Pilisborosjenő & 1942 & Priszter Szaniszló & BPU-14177.JPG \\
\hline 2619 & Carex halleriana Asso & Szentgál & 1940 & Boros Ádám & BPU-14173.JPG \\
\hline 2619 & Carex halleriana Asso & Tihany & 1957 & $\begin{array}{l}\text { Felföldy Lajos, Tóth } \\
\text { László }\end{array}$ & BPU-14171.JPG \\
\hline 2619 & Carex halleriana Asso & Tihany & 1958 & Felföldy Lajos & BPU-14170.JPG \\
\hline 2619 & Carex halleriana Asso & Tihany & 1959 & Felföldy Lajos & BPU-14172.JPG \\
\hline 2621 & Carex flacca Schreb. & Apc & 1954 & Priszter Szaniszló & BPU-14312.JPG \\
\hline 2621 & Carex flacca Schreb. & Aszófő & 1959 & Felföldy Lajos & BPU-14299.JPG \\
\hline 2621 & Carex flacca Schreb. & Aszófó & 1959 & Felföldy Lajos & BPU-14306.JPG \\
\hline 2621 & Carex flacca Schreb. & Balatonakali & 1958 & Felföldy Lajos & BPU-14307.JPG \\
\hline 2621 & Carex flacca Schreb. & Balatonboglár & 1950 & Priszter Szaniszló & BPU-14310.JPG \\
\hline 2621 & Carex flacca Schreb. & Balatonszemes & 1915 & Paikert, Alois & BPU-14322.JPG \\
\hline 2621 & Carex flacca Schreb. & Balatonszemes & 1917 & Paikert, Alois & BPU-14322.JPG \\
\hline 2621 & Carex flacca Schreb. & Balatonszemes & 1917 & Paikert, Alois & BPU-14322.JPG \\
\hline 2621 & Carex flacca Schreb. & Balatonszepezd & 1958 & Felföldy Lajos & BPU-14296.JPG \\
\hline 2621 & Carex flacca Schreb. & Balatonszepezd & 1958 & Felföldy Lajos & BPU-14297.JPG \\
\hline 2621 & Carex flacca Schreb. & Balatonszőlős & 1956 & $\begin{array}{l}\text { Felföldy Lajos, Tóth } \\
\text { László }\end{array}$ & BPU-14300.JPG \\
\hline 2621 & Carex flacca Schreb. & Balatonszőlős & 1956 & $\begin{array}{l}\text { Felföldy Lajos, Tóth } \\
\text { László }\end{array}$ & BPU-14302.JPG \\
\hline 2621 & Carex flacca Schreb. & Balatonszőlős & 1956 & $\begin{array}{l}\text { Felföldy Lajos, Tóth } \\
\text { László }\end{array}$ & BPU-14303.JPG \\
\hline 2621 & Carex flacca Schreb. & Balatonszőlős & 1956 & $\begin{array}{l}\text { Felföldy Lajos, Tóth } \\
\text { László }\end{array}$ & BPU-14304.JPG \\
\hline 2621 & Carex flacca Schreb. & Balatonszőlős & 1956 & $\begin{array}{l}\text { Felföldy Lajos, Tóth } \\
\text { László }\end{array}$ & BPU-14305.JPG \\
\hline 2621 & Carex flacca Schreb. & Balatonszőlős & 1957 & Felföldy Lajos & BPU-14301.JPG \\
\hline 2621 & Carex flacca Schreb. & Bisse & 1965 & $\begin{array}{l}\text { Simon Tibor, Priszter } \\
\text { Szaniszló, Borhidi Attila }\end{array}$ & BPU-14316.JPG \\
\hline 2621 & Carex flacca Schreb. & Budapest & 1915 & $\begin{array}{l}\text { Degen Árpád, Földváry } \\
\text { Dezső }\end{array}$ & BPU-14321.JPG \\
\hline 2621 & Carex flacca Schreb. & Budapest & 1992 & Felföldy Lajos & BPU-14318.JPG \\
\hline 2621 & Carex flacca Schreb. & Budapest & 2005 & Felföldy Lajos & BPU-14317.JPG \\
\hline 2621 & Carex flacca Schreb. & Fonyód & 1953 & Priszter Szaniszló & BPU-14309.JPG \\
\hline 2621 & Carex flacca Schreb. & Gyöngyös & 1923 & Soó Rezső & BPU-14311.JPG \\
\hline
\end{tabular}


1. Elektroniukus melléklet - Electronic Appendix 1.

\begin{tabular}{|c|c|c|c|c|c|}
\hline $\begin{array}{l}\text { Sorszám / } \\
\text { Number }\end{array}$ & Taxon-név / Taxon-name & $\begin{array}{l}\text { Település / } \\
\text { Settlement }\end{array}$ & $\begin{array}{l}\text { Év / } \\
\text { Year }\end{array}$ & Gyűjtő / Collector & $\begin{array}{l}\text { Fájlnév / } \\
\text { File-name }\end{array}$ \\
\hline 2621 & Carex flacca Schreb. & Pákozd & 1980 & Felföldy Lajos & BPU-14313.JPG \\
\hline 2621 & Carex flacca Schreb. & Pécs & 1911 & Lengyel Géza & BPU-14324.JPG \\
\hline 2621 & Carex flacca Schreb. & Pécs & 1951 & Priszter Szaniszló & BPU-14315.JPG \\
\hline 2621 & Carex flacca Schreb. & Penészlek & 1932 & Soó Rezső & BPU-14308.JPG \\
\hline 2621 & Carex flacca Schreb. & Szalonna & 1966 & Felföldy Lajos & BPU-14314.JPG \\
\hline 2621 & Carex flacca Schreb. & Tihany & 1946 & Felföldy Lajos & BPU-14295.JPG \\
\hline 2621 & Carex flacca Schreb. & Tihany & 1959 & Felföldy Lajos & BPU-14294.JPG \\
\hline 2621 & Carex flacca Schreb. & Ugod & 1955 & Soó Rezső & BPU-14298.JPG \\
\hline 2621 & Carex flacca Schreb. & Uzsa & 1959 & $\begin{array}{l}\text { Jávorka Sándor, Felföldy } \\
\text { Lajos }\end{array}$ & BPU-14293.JPG \\
\hline 2622 & Carex humilis Leyss. & Abaújszántó & 1938 & Soó Rezső & BPU-14167.JPG \\
\hline 2622 & Carex humilis Leyss. & Bátorliget & 1948 & Simon Tibor & BPU-14162.JPG \\
\hline 2622 & Carex humilis Leyss. & Budapest & 1982 & Felföldy Lajos & BPU-14168.JPG \\
\hline 2622 & Carex humilis Leyss. & Csákberény & 1949 & Priszter Szaniszló & BPU-14165.JPG \\
\hline 2622 & Carex humilis Leyss. & Diósjenő & 1952 & Simon Tibor & BPU-14164.JPG \\
\hline 2622 & Carex humilis Leyss. & Fertőrákos & 1938 & Soó Rezső & BPU-14163.JPG \\
\hline 2622 & Carex humilis Leyss. & Jósvafő & 1953 & Priszter Szaniszló & BPU-14166.JPG \\
\hline 2623 & Carex digitata L. & Balatonszőlős & 1958 & Felföldy Lajos & BPU-14146.JPG \\
\hline 2623 & Carex digitata L. & Balatonszőlős & 1959 & Felföldy Lajos & BPU-14147.JPG \\
\hline 2623 & Carex digitata L. & Bátorliget & 1934 & Soó Rezső & BPU-14148.JPG \\
\hline 2623 & Carex digitata L. & Biatorbágy & 1992 & Felföldy Lajos & BPU-14149.JPG \\
\hline 2623 & Carex digitata L. & Bisse & 1965 & $\begin{array}{l}\text { Simon Tibor, Priszter } \\
\text { Szaniszló, Borhidi Attila }\end{array}$ & BPU-14153.JPG \\
\hline 2623 & Carex digitata L. & Budapest & 1939 & Priszter Szaniszló & BPU-14156.JPG \\
\hline 2623 & Carex digitata L. & Budapest & 1949 & Simon Tibor & BPU-14157.JPG \\
\hline 2623 & Carex digitata L. & Csákberény & 1949 & Priszter Szaniszló & BPU-14151.JPG \\
\hline 2623 & Carex digitata L. & Gánt & 1949 & Soó Rezső & BPU-14150.JPG \\
\hline 2623 & Carex digitata L. & Háromhuta & 1949 & Simon Tibor, Jakucs Pál & BPU-14144.JPG \\
\hline 2623 & Carex digitata L. & Hidegkút & 1958 & Felföldy Lajos & BPU-14145.JPG \\
\hline 2623 & Carex digitata L. & Pálháza & 1960 & Simon Tibor & BPU-14154.JPG \\
\hline 2623 & Carex digitata L. & Pécs & 1953 & Soó Rezső, Borsos Olga & BPU-14152.JPG \\
\hline 2624 & Carex umbrosa Host & Bátorliget & 1991 & Simon Tibor & BPU-14191.JPG \\
\hline 2624 & Carex umbrosa Host & Kőszeg & 1896 & Waisbecker Antal & BPU-14190.JPG \\
\hline 2624 & Carex umbrosa Host & Szőce & 1955 & Soó Rezső & BPU-14189.JPG \\
\hline 2624 & Carex umbrosa Host & Uzsa & 1950 & Felföldy Lajos & BPU-14188.JPG \\
\hline 2624 & Carex umbrosa Host & Uzsa & 1959 & $\begin{array}{l}\text { Jávorka Sándor, Felföldy } \\
\text { Lajos }\end{array}$ & BPU-14187.JPG \\
\hline 2625 & Carex caryophyllea Latourr. & Fertőrákos & 1938 & Soó Rezső & BPU-14199.JPG \\
\hline 2625 & Carex caryophyllea Latourr. & Hidegkút & 1958 & Felföldy Lajos & BPU-14198.JPG \\
\hline 2625 & Carex caryophyllea Latourr. & Isztimér & 1955 & Soó Rezső & BPU-14196.JPG \\
\hline 2625 & Carex caryophyllea Latourr. & Isztimér & 1955 & Soó Rezső & BPU-14197.JPG \\
\hline 2625 & Carex caryophyllea Latourr. & Pécs & 1953 & Soó Rezső, Borsos Olga & BPU-14200.JPG \\
\hline 2625 & Carex caryophyllea Latourr. & Pilisszentiván & 1914 & $\begin{array}{l}\text { Degen Árpád, Lengyel } \\
\text { Géza }\end{array}$ & BPU-14204.JPG \\
\hline 2625 & Carex caryophyllea Latourr. & Pilisszentiván & 1916 & Zsák Zoltán & BPU-14201.JPG \\
\hline 2625 & Carex caryophyllea Latourr. & Tihany & 1958 & Felföldy Lajos & BPU-14195.JPG \\
\hline 2626 & Carex tomentosa L. & "Csepel-sziget" & 1909 & Degen Árpád & BPU-14278.JPG \\
\hline 2626 & Carex tomentosa L. & Apc & 1954 & Priszter Szaniszló & BPU-14274.JPG \\
\hline 2626 & Carex tomentosa L. & Aszófő & 1956 & $\begin{array}{l}\text { Felföldy Lajos, Tóth } \\
\text { László }\end{array}$ & BPU-14270.JPG \\
\hline
\end{tabular}


1. Elektroniukus melléklet - Electronic Appendix 1.

\begin{tabular}{|c|c|c|c|c|c|}
\hline $\begin{array}{l}\text { Sorszám / } \\
\text { Number }\end{array}$ & Taxon-név / Taxon-name & $\begin{array}{l}\text { Település / } \\
\text { Settlement }\end{array}$ & $\begin{array}{l}\text { Év / } \\
\text { Year }\end{array}$ & Gyüjtő / Collector & $\begin{array}{l}\text { Fájlnév / } \\
\text { File-name }\end{array}$ \\
\hline 2626 & Carex tomentosa L. & Balatonszőlős & 1957 & Felföldy Lajos & BPU-14269.JPG \\
\hline 2626 & Carex tomentosa L. & Bátorliget & 1932 & Soó Rezső & BPU-14271.JPG \\
\hline 2626 & Carex tomentosa L. & Budapest & 1991 & Felföldy Lajos & BPU-14277.JPG \\
\hline 2626 & Carex tomentosa L. & Háromhuta & 1960 & Simon Tibor & BPU-14265.JPG \\
\hline 2626 & Carex tomentosa L. & Háromhuta & 1960 & Simon Tibor & BPU-14266.JPG \\
\hline 2626 & Carex tomentosa L. & Hidegkút & 1958 & Felföldy Lajos & BPU-14268.JPG \\
\hline 2626 & Carex tomentosa L. & Pákozd & 1980 & Felföldy Lajos & BPU-14275.JPG \\
\hline 2626 & Carex tomentosa L. & Szentendre & 1947 & Pénzes Antal & BPU-14273.JPG \\
\hline 2626 & Carex tomentosa L. & Szigetújfalu & 1943 & Kárpáti Zoltán & BPU-14272.JPG \\
\hline 2626 & Carex tomentosa L. & Tákos & 1949 & Soó Rezső & BPU-14276.JPG \\
\hline 2626 & Carex tomentosa L. & Tihany & 1928 & Soó Rezső & BPU-14267.JPG \\
\hline 2627 & Carex fritschii Waisb. & Kőszeg & 1909 & Waisbecker Antal & BPU-14242.JPG \\
\hline 2627 & Carex fritschii Waisb. & Kőszeg & 1909 & Waisbecker Antal & BPU-14242.JPG \\
\hline 2627 & Carex fritschii Waisb. & Kőszeg & - & Waisbecker Antal & BPU-14243.JPG \\
\hline 2627 & Carex fritschii Waisb. & Uzsa & 1949 & Vajda László & BPU-14241.JPG \\
\hline 2627 & Carex fritschii Waisb. & Uzsa & 1952 & Simon Tibor & BPU-14240.JPG \\
\hline 2627 & Carex fritschii Waisb. & Uzsa & 1953 & Soó Rezső & BPU-14239.JPG \\
\hline 2627 & Carex fritschii Waisb. & Uzsa & 1959 & $\begin{array}{l}\text { Jávorka Sándor, Felföldy } \\
\text { Lajos }\end{array}$ & BPU-14238.JPG \\
\hline 2628 & Carex montana L. & Balatonfüred & 1959 & Felföldy Lajos & BPU-14247.JPG \\
\hline 2628 & Carex montana L. & Bátorliget & 1935 & Soó Rezső & BPU-14251.JPG \\
\hline 2628 & Carex montana L. & Budapest & 1909 & Degen Árpád & BPU-14253.JPG \\
\hline 2628 & Carex montana L. & Budapest & 1909 & Degen Árpád & BPU-14254.JPG \\
\hline 2628 & Carex montana L. & Budapest & 1949 & Simon Tibor & BPU-14255.JPG \\
\hline 2628 & Carex montana L. & Csákberény & 1949 & Priszter Szaniszló & BPU-14252.JPG \\
\hline 2628 & Carex montana L. & Csesznek & 1925 & Polgár Sándor & BPU-14246.JPG \\
\hline 2628 & Carex montana L. & Hidegkút & 1958 & Felföldy Lajos & BPU-14245.JPG \\
\hline 2628 & Carex montana L. & Kőszeg & 1892 & Waisbecker Antal & BPU-14248.JPG \\
\hline 2628 & Carex montana L. & Kőszeg & 1896 & Waisbecker Antal & BPU-14249.JPG \\
\hline 2628 & Carex montana L. & Kőszeg & 1896 & Waisbecker Antal & BPU-14250.JPG \\
\hline 2628 & Carex montana L. & Pécs & 1953 & Soó Rezső, Borsos Olga & BPU-14256.JPG \\
\hline 2629 & Carex ericetorum Pollich & Budapest & 1909 & Degen Árpád & BPU-14577.JPG \\
\hline 2629 & Carex ericetorum Pollich & Budapest & 1911 & Degen Árpád & BPU-14578.JPG \\
\hline 2629 & Carex ericetorum Pollich & Debrecen & 1933 & Soó Rezső & BPU-14572.JPG \\
\hline 2629 & Carex ericetorum Pollich & Debrecen & 1934 & Soó Rezső & BPU-14262.JPG \\
\hline 2629 & Carex ericetorum Pollich & Debrecen & 1934 & Soó Rezső & BPU-14263.JPG \\
\hline 2629 & Carex ericetorum Pollich & Debrecen & 1949 & Soó Rezső & BPU-14261.JPG \\
\hline 2629 & Carex ericetorum Pollich & Fenyőfő & 1955 & Soó Rezső & BPU-14258.JPG \\
\hline 2629 & Carex ericetorum Pollich & Fenyőfö & 1955 & Soó Rezső & BPU-14259.JPG \\
\hline 2629 & Carex ericetorum Pollich & Pilisszentiván & 1914 & $\begin{array}{l}\text { Degen Árpád, Lengyel } \\
\text { Géza }\end{array}$ & BPU-14264.JPG \\
\hline 2629 & Carex ericetorum Pollich & Pilisszentiván & 1944 & Kárpáti Zoltán & BPU-14260.JPG \\
\hline 2631 & Carex pendula Huds. & Dömös & 1948 & Soó Rezső & BPU-14208.JPG \\
\hline 2631 & Carex pendula Huds. & Dömös & 1952 & Soó Rezső & BPU-15404.JPG \\
\hline 2631 & Carex pendula Huds. & Pécs & 1935 & Soó Rezső & BPU-14211.JPG \\
\hline 2631 & Carex pendula Huds. & Pécs & 1953 & Soó Rezső, Borsos Olga & BPU-14210.JPG \\
\hline 2631 & Carex pendula Huds. & Pécs & 1956 & Soó Rezső & BPU-14209.JPG \\
\hline 2631 & Carex pendula Huds. & Pomáz & 1910 & $\begin{array}{l}\text { Degen Árpád, Lengyel } \\
\text { Géza, Zsák Zoltán }\end{array}$ & BPU-14212.JPG \\
\hline
\end{tabular}


1. Elektroniukus melléklet - Electronic Appendix 1.

\begin{tabular}{|c|c|c|c|c|c|}
\hline $\begin{array}{l}\text { Sorszám / } \\
\text { Number }\end{array}$ & Taxon-név / Taxon-name & $\begin{array}{l}\text { Település / } \\
\text { Settlement }\end{array}$ & $\begin{array}{l}\text { Év / } \\
\text { Year }\end{array}$ & Gyűjtő / Collector & $\begin{array}{l}\text { Fájlnév / } \\
\text { File-name }\end{array}$ \\
\hline 2631 & Carex pendula Huds. & Pomáz & 1911 & $\begin{array}{l}\text { Degen Árpád, Lengyel } \\
\text { Géza, Zsák Zoltán }\end{array}$ & BPU-14212.JPG \\
\hline 2631 & Carex pendula Huds. & Sopron & 1942 & Priszter Szaniszló & BPU-14206.JPG \\
\hline 2631 & Carex pendula Huds. & Sopron & 1947 & Kárpáti Zoltán & BPU-14205.JPG \\
\hline 2631 & Carex pendula Huds. & Szenna & 1957 & Soó Rezső & BPU-14207.JPG \\
\hline 2633 & Carex pseudocyperus L. & Balatonfüred & 1982 & $\begin{array}{l}\text { Felföldy Lajos, Tóth } \\
\text { László }\end{array}$ & BPU-12409.JPG \\
\hline 2633 & Carex pseudocyperus L. & Budapest & 1900 & Degen Árpád & BPU-12420.JPG \\
\hline 2633 & Carex pseudocyperus L. & Budapest & 1915 & Degen Árpád & BPU-12413.JPG \\
\hline 2633 & Carex pseudocyperus L. & Debrecen & 1934 & Soó Rezső & BPU-12418.JPG \\
\hline 2633 & Carex pseudocyperus L. & Hajdúsámson & 1989 & Felföldy Lajos & BPU-12417.JPG \\
\hline 2633 & Carex pseudocyperus L. & Kállósemjén & 1950 & Simon Tibor & BPU-12411.JPG \\
\hline 2633 & Carex pseudocyperus L. & Kelemér & 1950 & Soó Rezső & BPU-12419.JPG \\
\hline 2633 & Carex pseudocyperus L. & Kimle & 1962 & Borsos Olga & BPU-12416.JPG \\
\hline 2633 & Carex pseudocyperus L. & Nagykanizsa & 1949 & Károlyi Árpád & BPU-12408.JPG \\
\hline 2633 & Carex pseudocyperus L. & Nyírpilis & 1951 & Simon Tibor & BPU-12410.JPG \\
\hline 2633 & Carex pseudocyperus L. & Ócsa & 1955 & Soó Rezső & BPU-12415.JPG \\
\hline 2633 & Carex pseudocyperus L. & Pilisszentiván & 1951 & Priszter Szaniszló & BPU-12414.JPG \\
\hline 2633 & Carex pseudocyperus L. & Veresegyház & 1949 & Soó Rezső & BPU-12412.JPG \\
\hline 2634 & Carex sylvatica Huds. & Budapest & 2004 & Felföldy Lajos & BPU-14110.JPG \\
\hline 2634 & Carex sylvatica Huds. & Debrecen & 1933 & Máthé Imre & BPU-14101.JPG \\
\hline 2634 & Carex sylvatica Huds. & Debrecen & 1934 & Soó Rezső & BPU-14102.JPG \\
\hline 2634 & Carex sylvatica Huds. & Dömös & 1948 & Priszter Szaniszló & BPU-14104.JPG \\
\hline 2634 & Carex sylvatica Huds. & Isztimér & 1949 & Soó Rezső & BPU-14103.JPG \\
\hline 2634 & Carex sylvatica Huds. & Kapolcs & 1959 & $\begin{array}{l}\text { Jávorka Sándor, Felföldy } \\
\text { Lajos, Tóth László }\end{array}$ & BPU-14095.JPG \\
\hline 2634 & Carex sylvatica Huds. & Kisunyom & 1954 & $\begin{array}{l}\text { Máthé Imre, Jeanplon } \\
\text { József, Kovács Margit }\end{array}$ & BPU-14096.JPG \\
\hline 2634 & Carex sylvatica Huds. & Kővágószőlős & 1938 & Soó Rezső & BPU-14108.JPG \\
\hline 2634 & Carex sylvatica Huds. & Lábatlan & 1953 & Felföldy Lajos & BPU-14100.JPG \\
\hline 2634 & Carex sylvatica Huds. & Lesenceistvánd & 1952 & Borsos Olga & BPU-14094.JPG \\
\hline 2634 & Carex sylvatica Huds. & Miskolc & 1950 & Soó Rezső & BPU-14105.JPG \\
\hline 2634 & Carex sylvatica Huds. & Pécs & 1907 & Kocsis István & BPU-14109.JPG \\
\hline 2634 & Carex sylvatica Huds. & Pécs & 1911 & Lengyel Géza & BPU-14111.JPG \\
\hline 2634 & Carex sylvatica Huds. & Pécs & 1953 & Soó Rezső, Borsos Olga & BPU-14106.JPG \\
\hline 2634 & Carex sylvatica Huds. & Pécs & 1956 & Soó Rezső & BPU-14107.JPG \\
\hline 2634 & Carex sylvatica Huds. & Pilisszentkereszt & 1963 & Isépy István & BPU-14099.JPG \\
\hline 2634 & Carex sylvatica Huds. & Szenta & 1955 & Soó Rezső & BPU-14098.JPG \\
\hline 2634 & Carex sylvatica Huds. & Szentgotthárd & 1955 & Soó Rezső & BPU-14097.JPG \\
\hline 2635 & Carex pilosa Scop. & Bátorliget & 1932 & Soó Rezső & BPU-14058.JPG \\
\hline 2635 & Carex pilosa Scop. & Budapest & 1939 & Priszter Szaniszló & BPU-14063.JPG \\
\hline 2635 & Carex pilosa Scop. & Csákberény & 1949 & Priszter Szaniszló & BPU-14062.JPG \\
\hline 2635 & Carex pilosa Scop. & Gánt & 1949 & Soó Rezső & BPU-14061.JPG \\
\hline 2635 & Carex pilosa Scop. & Gyöngyös & 1964 & Soó Rezső & BPU-14060.JPG \\
\hline 2635 & Carex pilosa Scop. & Háromhuta & 1960 & Sebestyén Olga & BPU-14057.JPG \\
\hline 2635 & Carex pilosa Scop. & Háromhuta & 1960 & Simon Tibor & BPU-14056.JPG \\
\hline 2635 & Carex pilosa Scop. & Piliscsaba & 1911 & Lengyel Géza & BPU-14064.JPG \\
\hline 2635 & Carex pilosa Scop. & Sopron & 1960 & Borsos Olga, Simon Tibor & BPU-14059.JPG \\
\hline 2636 & Carex pallescens L. & Debrecen & 1989 & Felföldy Lajos & BPU-14286.JPG \\
\hline
\end{tabular}


1. Elektroniukus melléklet - Electronic Appendix 1.

\begin{tabular}{|c|c|c|c|c|c|}
\hline $\begin{array}{l}\text { Sorszám / } \\
\text { Number }\end{array}$ & Taxon-név / Taxon-name & $\begin{array}{l}\text { Település / } \\
\text { Settlement }\end{array}$ & $\begin{array}{l}\text { Év / } \\
\text { Year }\end{array}$ & Gyüjtő / Collector & $\begin{array}{l}\text { Fájlnév / } \\
\text { File-name }\end{array}$ \\
\hline 2636 & Carex pallescens L. & Hajdúbagos & 1933 & Soó Rezső & BPU-14287.JPG \\
\hline 2636 & Carex pallescens L. & Háromhuta & 1960 & Simon Tibor & BPU-14281.JPG \\
\hline 2636 & Carex pallescens L. & Kőszeg & 1938 & Soó Rezső & BPU-14283.JPG \\
\hline 2636 & Carex pallescens L. & Nárai & 1954 & $\begin{array}{l}\text { Máthé Imre, Jeanplon } \\
\text { József, Kovács Margit }\end{array}$ & BPU-14282.JPG \\
\hline 2636 & Carex pallescens L. & Pálháza & 1960 & Simon Tibor & BPU-14288.JPG \\
\hline 2636 & Carex pallescens L. & Piliscsaba & 1909 & Lengyel Géza & BPU-14291.JPG \\
\hline 2636 & Carex pallescens L. & Piliscsaba & 1911 & Lengyel Géza & BPU-14291.JPG \\
\hline 2636 & Carex pallescens L. & Pilisszentlászló & 1918 & Degen Árpád & BPU-14290.JPG \\
\hline 2636 & Carex pallescens L. & Sátoraljaújhely & 1954 & $\begin{array}{l}\text { Máthé Imre, Jeanplon } \\
\text { József, Kovács Margit }\end{array}$ & BPU-14289.JPG \\
\hline 2636 & Carex pallescens L. & Szentendre & 1947 & Pénzes Antal & BPU-14285.JPG \\
\hline 2636 & Carex pallescens L. & Szentendre & 1947 & Priszter Szaniszló & BPU-14284.JPG \\
\hline 2637 & Carex supina Wahlenb. & Boldogkőváralja & 1939 & Soó Rezső & BPU-14233.JPG \\
\hline 2637 & Carex supina Wahlenb. & Debrecen & 1947 & Soó Rezső & BPU-14232.JPG \\
\hline 2637 & Carex supina Wahlenb. & Debrecen & 1952 & Kulcsár Gábor & BPU-15452.JPG \\
\hline 2637 & Carex supina Wahlenb. & Mezőcsát & 1911 & Budai József & BPU-14234.JPG \\
\hline 2637 & Carex supina Wahlenb. & Mezőcsát & 1911 & Budai József & BPU-14234.JPG \\
\hline 2637 & Carex supina Wahlenb. & Pilisszentiván & 1909 & $\begin{array}{l}\text { Degen Árpád, Kümmerle } \\
\text { Jenő Béla, Lengyel Géza }\end{array}$ & BPU-14235.JPG \\
\hline 2637 & Carex supina Wahlenb. & Pilisszentiván & 1946 & Boros Ádám & BPU-14231.JPG \\
\hline 2637 & Carex supina Wahlenb. & Vácrátót & 1953 & Felföldy Lajos & BPU-14230.JPG \\
\hline 2638 & Carex acutiformis Ehrh. & Aszófő & 1959 & Felföldy Lajos & BPU-12454.JPG \\
\hline 2638 & Carex acutiformis Ehrh. & Balatonszőlős & 1956 & Felföldy Lajos & BPU-12449.JPG \\
\hline 2638 & Carex acutiformis Ehrh. & Balatonszőlős & 1956 & $\begin{array}{l}\text { Felföldy Lajos, Tóth } \\
\text { László }\end{array}$ & BPU-12451.JPG \\
\hline 2638 & Carex acutiformis Ehrh. & Balatonszőlős & 1956 & $\begin{array}{l}\text { Felföldy Lajos, Tóth } \\
\text { László }\end{array}$ & BPU-12452.JPG \\
\hline 2638 & Carex acutiformis Ehrh. & Balatonszőlős & 1956 & $\begin{array}{l}\text { Felföldy Lajos, Tóth } \\
\text { László }\end{array}$ & BPU-12453.JPG \\
\hline 2638 & Carex acutiformis Ehrh. & Balatonszőlős & 1957 & Felföldy Lajos & BPU-12448.JPG \\
\hline 2638 & Carex acutiformis Ehrh. & Balatonszőlős & 1957 & Felföldy Lajos & BPU-12450.JPG \\
\hline 2638 & Carex acutiformis Ehrh. & Balatonudvari & 1959 & Felföldy Lajos & BPU-12447.JPG \\
\hline 2638 & Carex acutiformis Ehrh. & Bátorliget & 1932 & Soó Rezső & BPU-12455.JPG \\
\hline 2638 & Carex acutiformis Ehrh. & Bátorliget & 1932 & Soó Rezső & BPU-12456.JPG \\
\hline 2638 & Carex acutiformis Ehrh. & Budapest & 1909 & Degen Árpád & BPU-12469.JPG \\
\hline 2638 & Carex acutiformis Ehrh. & Budapest & 1914 & Degen Árpád & BPU-12466.JPG \\
\hline 2638 & Carex acutiformis Ehrh. & Budapest & 1915 & Degen Árpád & BPU-12467.JPG \\
\hline 2638 & Carex acutiformis Ehrh. & Budapest & 1915 & Degen Árpád & BPU-12468.JPG \\
\hline 2638 & Carex acutiformis Ehrh. & Budapest & 1989 & Felföldy Lajos & BPU-12464.JPG \\
\hline 2638 & Carex acutiformis Ehrh. & Budapest & 1989 & Felföldy Lajos & BPU-12465.JPG \\
\hline 2638 & Carex acutiformis Ehrh. & Debrecen & 1932 & Soó Rezső & BPU-12461.JPG \\
\hline 2638 & Carex acutiformis Ehrh. & Debrecen & 1988 & Turi B. A., Sümegi Gy. & BPU-12460.JPG \\
\hline 2638 & Carex acutiformis Ehrh. & Gárdony & 1980 & Felföldy Lajos & BPU-12462.JPG \\
\hline 2638 & Carex acutiformis Ehrh. & Gödöllő & 1951 & Priszter Szaniszló & BPU-12458.JPG \\
\hline 2638 & Carex acutiformis Ehrh. & Gödöllő & 1952 & Priszter Szaniszló & BPU-12457.JPG \\
\hline 2638 & Carex acutiformis Ehrh. & Hajdúhadház & 1932 & Soó Rezső & BPU-12459.JPG \\
\hline 2638 & Carex acutiformis Ehrh. & Konyár & 1949 & Soó Rezső & BPU-15523.JPG \\
\hline 2638 & Carex acutiformis Ehrh. & Nagyrada & 1987 & Felföldy Lajos & BPU-12440.JPG \\
\hline 2638 & Carex acutiformis Ehrh. & Örvényes & 1959 & Felföldy Lajos & BPU-12446.JPG \\
\hline
\end{tabular}


1. Elektroniukus melléklet - Electronic Appendix 1.

\begin{tabular}{|c|c|c|c|c|c|}
\hline $\begin{array}{l}\text { Sorszám / } \\
\text { Number }\end{array}$ & Taxon-név / Taxon-name & $\begin{array}{l}\text { Település / } \\
\text { Settlement }\end{array}$ & $\begin{array}{l}\text { Év / } \\
\text { Year }\end{array}$ & Gyüjtő / Collector & $\begin{array}{l}\text { Fájlnév / } \\
\text { File-name }\end{array}$ \\
\hline 2638 & Carex acutiformis Ehrh. & Pákozd & 1978 & Felföldy Lajos & BPU-12463.JPG \\
\hline 2638 & Carex acutiformis Ehrh. & Tihany & 1956 & Felföldy Lajos & BPU-12442.JPG \\
\hline 2638 & Carex acutiformis Ehrh. & Tihany & 1956 & Felföldy Lajos & BPU-12443.JPG \\
\hline 2638 & Carex acutiformis Ehrh. & Tihany & 1959 & Felföldy Lajos & BPU-12441.JPG \\
\hline 2638 & Carex acutiformis Ehrh. & Ugod & 1955 & Soó Rezső & BPU-12444.JPG \\
\hline 2638 & Carex acutiformis Ehrh. & Ugod & 1955 & Soó Rezső & BPU-12445.JPG \\
\hline 2639 & Carex riparia Curtis & Aszófő & 1957 & Felföldy Lajos & BPU-12430.JPG \\
\hline 2639 & Carex riparia Curtis & Balatonszőlős & 1956 & $\begin{array}{l}\text { Felföldy Lajos, Tóth } \\
\text { László }\end{array}$ & BPU-12428.JPG \\
\hline 2639 & Carex riparia Curtis & Balatonszőlős & 1956 & $\begin{array}{l}\text { Felföldy Lajos, Tóth } \\
\text { László }\end{array}$ & BPU-12429.JPG \\
\hline 2639 & Carex riparia Curtis & Balatonudvari & 1959 & Felföldy Lajos & BPU-12427.JPG \\
\hline 2639 & Carex riparia Curtis & Budapest & 1909 & Degen Árpád & BPU-12437.JPG \\
\hline 2639 & Carex riparia Curtis & Budapest & 1915 & $\begin{array}{l}\text { Degen Árpád, Földváry } \\
\text { Dezső }\end{array}$ & BPU-12438.JPG \\
\hline 2639 & Carex riparia Curtis & Debrecen & 1938 & Soó Rezső & BPU-12432.JPG \\
\hline 2639 & Carex riparia Curtis & Konyár & 1949 & Soó Rezső & BPU-15524.JPG \\
\hline 2639 & Carex riparia Curtis & Leányfalu & 1950 & Priszter Szaniszló & BPU-12431.JPG \\
\hline 2639 & Carex riparia Curtis & Pákozd & 1980 & Felföldy Lajos & BPU-12433.JPG \\
\hline 2639 & Carex riparia Curtis & Pákozd & 1980 & Felföldy Lajos & BPU-12434.JPG \\
\hline 2639 & Carex riparia Curtis & Pákozd & 1980 & Felföldy Lajos & BPU-12435.JPG \\
\hline 2639 & Carex riparia Curtis & Szentbékkálla & 1959 & $\begin{array}{l}\text { Jávorka Sándor, Felföldy } \\
\text { Lajos, Tóth László }\end{array}$ & BPU-12426.JPG \\
\hline 2639 & Carex riparia Curtis & Tarpa & 1948 & $\begin{array}{l}\text { Pólya László, Simon } \\
\text { Tibor, Jakucs Pál }\end{array}$ & BPU-12436.JPG \\
\hline 2639 & Carex riparia Curtis & Tihany & 1951 & Priszter Szaniszló & BPU-12425.JPG \\
\hline 2639 & Carex riparia Curtis & Tihany & 1956 & $\begin{array}{l}\text { Felföldy Lajos, Tóth } \\
\text { László }\end{array}$ & BPU-12422.JPG \\
\hline 2639 & Carex riparia Curtis & Tihany & 1956 & $\begin{array}{l}\text { Felföldy Lajos, Tóth } \\
\text { László }\end{array}$ & BPU-12423.JPG \\
\hline 2639 & Carex riparia Curtis & Tihany & 1961 & $\begin{array}{l}\text { Pankov H., Felföldy Lajos, } \\
\text { Sólymosy G. }\end{array}$ & BPU-12424.JPG \\
\hline 2640 & Carex rostrata Stokes & Bátorliget & 1932 & Soó Rezső & BPU-12393.JPG \\
\hline 2640 & Carex rostrata Stokes & Budapest & 1915 & Degen Árpád & BPU-12405.JPG \\
\hline 2640 & Carex rostrata Stokes & Kállósemjén & 1932 & Soó Rezső & BPU-12404.JPG \\
\hline 2641 & Carex vesicaria L. & Bátorliget & 1950 & $\begin{array}{l}\text { Simon Tibor, Kárpáti } \\
\text { István }\end{array}$ & BPU-12392.JPG \\
\hline 2641 & Carex vesicaria L. & Bátorliget & 1950 & $\begin{array}{l}\text { Simon Tibor, Kárpáti } \\
\text { István }\end{array}$ & BPU-15477.JPG \\
\hline 2641 & Carex vesicaria L. & Dabas & 1957 & Soó Rezső & BPU-12394.JPG \\
\hline 2641 & Carex vesicaria L. & Gávavencsellő & 1990 & Felföldy Lajos & BPU-12391.JPG \\
\hline 2641 & Carex vesicaria L. & Ipolytarnóc & 1987 & Felföldy Lajos & BPU-12396.JPG \\
\hline 2641 & Carex vesicaria L. & Kapolcs & 1959 & $\begin{array}{l}\text { Jávorka Sándor, Felföldy } \\
\text { Lajos, Tóth László }\end{array}$ & BPU-12389.JPG \\
\hline 2641 & Carex vesicaria L. & Kelemér & 1950 & Soó Rezső & BPU-12397.JPG \\
\hline 2641 & Carex vesicaria L. & Monostorapáti & 1959 & $\begin{array}{l}\text { Jávorka Sándor, Felföldy } \\
\text { Lajos, Tóth László }\end{array}$ & BPU-12388.JPG \\
\hline 2641 & Carex vesicaria L. & Nagykanizsa & 1949 & Károlyi Árpád & BPU-12385.JPG \\
\hline 2641 & Carex vesicaria L. & Nyírpilis & 1932 & Soó Rezső & BPU-12390.JPG \\
\hline 2641 & Carex vesicaria L. & Szécsény & 1953 & Priszter Szaniszló & BPU-12395.JPG \\
\hline 2641 & Carex vesicaria L. & Szentbékkálla & 1959 & $\begin{array}{l}\text { Jávorka Sándor, Felföldy } \\
\text { Lajos, Tóth László }\end{array}$ & BPU-12387.JPG \\
\hline 2641 & Carex vesicaria L. & Zánka & 1958 & Felföldy Lajos & BPU-12386.JPG \\
\hline
\end{tabular}


1. Elektroniukus melléklet - Electronic Appendix 1.

\begin{tabular}{|c|c|c|c|c|c|}
\hline $\begin{array}{l}\text { Sorszám / } \\
\text { Number }\end{array}$ & Taxon-név / Taxon-name & $\begin{array}{l}\text { Település / } \\
\text { Settlement }\end{array}$ & $\begin{array}{l}\text { Év / } \\
\text { Year }\end{array}$ & Gyűjtő / Collector & $\begin{array}{l}\text { Fájlnév / } \\
\text { File-name }\end{array}$ \\
\hline 2642 & Carex melanostachya Willd. & Egyek & 1950 & $\begin{array}{l}\text { Simon Tibor, Borsos Olga, } \\
\text { Kulcsár Gábor }\end{array}$ & BPU-12475.JPG \\
\hline 2642 & Carex melanostachya Willd. & Gávavencsellő & 1990 & Felföldy Lajos & BPU-12470.JPG \\
\hline 2642 & Carex melanostachya Willd. & Gávavencsellő & 1990 & Felföldy Lajos & BPU-12471.JPG \\
\hline 2642 & Carex melanostachya Willd. & Gávavencsellő & 1990 & Felföldy Lajos & BPU-12472.JPG \\
\hline 2642 & Carex melanostachya Willd. & Pomáz & 1910 & Degen Árpád & BPU-12477.JPG \\
\hline 2642 & Carex melanostachya Willd. & Szécsény & 1953 & Priszter Szaniszló & BPU-12473.JPG \\
\hline 2642 & Carex melanostachya Willd. & Szekszárd & 1914 & Hollós László & BPU-14323.JPG \\
\hline 2642 & Carex melanostachya Willd. & Tarpa & 1949 & Soó Rezső & BPU-12476.JPG \\
\hline 2642 & Carex melanostachya Willd. & Tiszafüred & 1932 & Soó Rezső & BPU-12474.JPG \\
\hline 2643 & Carex alba Scop. & Aszófő & 1957 & $\begin{array}{l}\text { Felföldy Lajos, Kovács } \\
\text { Margit, Tóth László }\end{array}$ & BPU-14134.JPG \\
\hline 2643 & Carex alba Scop. & Budapest & 1947 & Priszter Szaniszló & BPU-14139.JPG \\
\hline 2643 & Carex alba Scop. & Csákberény & 1948 & Priszter Szaniszló & BPU-14138.JPG \\
\hline 2643 & Carex alba Scop. & Csákberény & 1949 & Priszter Szaniszló & BPU-14137.JPG \\
\hline 2643 & Carex alba Scop. & Gánt & 1949 & Soó Rezső & BPU-15527.JPG \\
\hline 2643 & Carex alba Scop. & Isztimér & 1949 & Soó Rezső & BPU-14136.JPG \\
\hline 2643 & Carex alba Scop. & Várpalota & 1955 & Soó Rezső & BPU-14135.JPG \\
\hline 2644 & Carex liparicarpos Gaudin & Balatonboglár & 1950 & Priszter Szaniszló & BPU-14218.JPG \\
\hline 2644 & Carex liparicarpos Gaudin & Budapest & 1909 & $\begin{array}{l}\text { Degen Árpád, Lengyel } \\
\text { Géza }\end{array}$ & BPU-14229.JPG \\
\hline 2644 & Carex liparicarpos Gaudin & Budapest & 1923 & Soó Rezső & BPU-14225.JPG \\
\hline 2644 & Carex liparicarpos Gaudin & Budapest & 1941 & Priszter Szaniszló & BPU-14227.JPG \\
\hline 2644 & Carex liparicarpos Gaudin & Budapest & 1989 & Felföldy Lajos & BPU-14226.JPG \\
\hline 2644 & Carex liparicarpos Gaudin & Csévharaszt & 1959 & Simon Tibor, Borsos Olga & BPU-14224.JPG \\
\hline 2644 & Carex liparicarpos Gaudin & Dabas & 1951 & Borsos Olga, Simon Tibor & BPU-14223.JPG \\
\hline 2644 & Carex liparicarpos Gaudin & Fonyód & 1953 & Priszter Szaniszló & BPU-14217.JPG \\
\hline 2644 & Carex liparicarpos Gaudin & Pilisborosjenő & 1942 & Priszter Szaniszló & BPU-14222.JPG \\
\hline 2644 & Carex liparicarpos Gaudin & Pilisvörösvár & 1916 & $\begin{array}{l}\text { Degen Árpád, Zsák Z., } \\
\text { Andrasovszky J. }\end{array}$ & BPU-14228.JPG \\
\hline 2644 & Carex liparicarpos Gaudin & Pilisszentiván & 1941 & Kárpáti Zoltán & BPU-14220.JPG \\
\hline 2644 & Carex liparicarpos Gaudin & Pilisszentiván & 1950 & Priszter Szaniszló & BPU-14221.JPG \\
\hline 2644 & Carex liparicarpos Gaudin & Tihany & 1928 & Soó Rezső & BPU-14214.JPG \\
\hline 2644 & Carex liparicarpos Gaudin & Tihany & 1958 & Felföldy Lajos & BPU-14215.JPG \\
\hline 2644 & Carex liparicarpos Gaudin & Tihany & 1959 & Felföldy Lajos & BPU-14216.JPG \\
\hline 2644 & Carex liparicarpos Gaudin & Vácrátót & 1953 & Felföldy Lajos & BPU-14219.JPG \\
\hline 2645 & Carex panicea L. & Aszófő & 1959 & Felföldy Lajos & BPU-14116.JPG \\
\hline 2645 & Carex panicea L. & Aszófő & 1959 & Felföldy Lajos & BPU-14118.JPG \\
\hline 2645 & Carex panicea L. & Balatonszőlős & 1957 & Felföldy Lajos & BPU-14117.JPG \\
\hline 2645 & Carex panicea L. & Budapest & 1909 & Degen Árpád & BPU-14129.JPG \\
\hline 2645 & Carex panicea L. & Debrecen & 1933 & Soó Rezső & BPU-14124.JPG \\
\hline 2645 & Carex panicea L. & Fonyód & 1953 & Priszter Szaniszló & BPU-14121.JPG \\
\hline 2645 & Carex panicea L. & Gödöllő & 1951 & Priszter Szaniszló & BPU-14123.JPG \\
\hline 2645 & Carex panicea L. & Háromhuta & 1950 & Simon Tibor & BPU-14112.JPG \\
\hline 2645 & Carex panicea L. & Jósvafő & 1953 & Priszter Szaniszló & BPU-14127.JPG \\
\hline 2645 & Carex panicea L. & Monor & 1952 & Csapody Vera & BPU-14122.JPG \\
\hline 2645 & Carex panicea L. & Örvényes & 1959 & Felföldy Lajos & BPU-14114.JPG \\
\hline 2645 & Carex panicea L. & Örvényes & 1959 & Felföldy Lajos & BPU-14115.JPG \\
\hline 2645 & Carex panicea L. & Sopron & 1928 & Soó Rezső & BPU-14120.JPG \\
\hline
\end{tabular}


1. Elektroniukus melléklet - Electronic Appendix 1.

\begin{tabular}{|c|c|c|c|c|c|}
\hline $\begin{array}{l}\text { Sorszám / } \\
\text { Number }\end{array}$ & Taxon-név / Taxon-name & $\begin{array}{l}\text { Település / } \\
\text { Settlement }\end{array}$ & $\begin{array}{l}\text { Év / } \\
\text { Year }\end{array}$ & Gyújtő / Collector & $\begin{array}{l}\text { Fájlnév / } \\
\text { File-name }\end{array}$ \\
\hline 2645 & Carex panicea L. & Sopron & 1942 & Priszter Szaniszló & BPU-14119.JPG \\
\hline 2645 & Carex panicea L. & Szalonna & 1966 & Felföldy Lajos & BPU-14126.JPG \\
\hline 2645 & Carex panicea L. & Szemere & 1966 & Felföldy Lajos & BPU-14125.JPG \\
\hline 2645 & Carex panicea L. & Szögliget & 1951 & Jakucs Pál, Ér Lajos & BPU-15468.JPG \\
\hline 2645 & Carex panicea L. & Tihany & 1956 & Felföldy Lajos & BPU-14113.JPG \\
\hline 2646 & Carex brevicollis DC. & Jósvafő & 1953 & Jakucs Pál & BPU-14083.JPG \\
\hline 2646 & Carex brevicollis DC. & Jósvafő & 1953 & Priszter Szaniszló & BPU-14082.JPG \\
\hline 2646 & Carex brevicollis DC. & Miskolc & 1911 & Budai József & BPU-14084.JPG \\
\hline 2646 & Carex brevicollis DC. & Miskolc & 1911 & Budai József & BPU-14084.JPG \\
\hline 2646 & Carex brevicollis DC. & Miskolc & 1911 & Budai József & BPU-14085.JPG \\
\hline 2646 & Carex brevicollis DC. & Miskolc & 1911 & Budai József & BPU-14085.JPG \\
\hline 2646 & Carex brevicollis DC. & Miskolc & 1913 & Budai József & BPU-14087.JPG \\
\hline 2646 & Carex brevicollis DC. & Miskolc & 1939 & Soó Rezső & BPU-14081.JPG \\
\hline 2646 & Carex brevicollis DC. & Miskolc & 1952 & Priszter Szaniszló & BPU-14080.JPG \\
\hline 2646 & Carex brevicollis DC. & Miskolc & 1953 & Soó Rezső & BPU-14079.JPG \\
\hline 2647 & Carex michelii Host & Budapest & 1949 & Simon Tibor & BPU-14076.JPG \\
\hline 2647 & Carex michelii Host & Budapest & 2004 & Felföldy Lajos & BPU-14077.JPG \\
\hline 2647 & Carex michelii Host & Csákberény & 1949 & Priszter Szaniszló & BPU-14074.JPG \\
\hline 2647 & Carex michelii Host & Debrecen & 1934 & Soó Rezső & BPU-14073.JPG \\
\hline 2647 & Carex michelii Host & Hidegkút & 1958 & Felföldy Lajos & BPU-14069.JPG \\
\hline 2647 & Carex michelii Host & Mosonmagyaróvár & 1965 & Borsos Olga & BPU-14071.JPG \\
\hline 2647 & Carex michelii Host & Nagyharsány & 1948 & Priszter Szaniszló & BPU-14075.JPG \\
\hline 2647 & Carex michelii Host & Pilisborosjenő & 1942 & Priszter Szaniszló & BPU-14070.JPG \\
\hline 2647 & Carex michelii Host & Sáránd & 1932 & Soó Rezső & BPU-14072.JPG \\
\hline 2647 & Carex michelii Host & Tata & 1909 & Zsák Zoltán & BPU-14078.JPG \\
\hline 2647 & Carex michelii Host & Tihany & 1956 & Felföldy Lajos & BPU-14067.JPG \\
\hline 2647 & Carex michelii Host & Tihany & 1958 & Felföldy Lajos & BPU-14066.JPG \\
\hline 2647 & Carex michelii Host & Tihany & 1958 & Felföldy Lajos & BPU-14068.JPG \\
\hline 2648 & Carex strigosa Huds. & Baja & 1956 & Tóth & BPU-14089.JPG \\
\hline 2648 & Carex strigosa Huds. & Nagybajom & 1957 & Soó Rezső & BPU-14090.JPG \\
\hline 2649 & Carex hordeistichos Vill. & Aszófő & 1957 & Felföldy Lajos & BPU-12524.JPG \\
\hline 2649 & Carex hordeistichos Vill. & Dunaharaszti & 1948 & Kárpáti Zoltán & BPU-12527.JPG \\
\hline 2649 & Carex hordeistichos Vill. & Dunaharaszti & 1948 & Priszter Szaniszló & BPU-12526.JPG \\
\hline 2649 & Carex hordeistichos Vill. & Monor & 1942 & Priszter Szaniszló & BPU-12525.JPG \\
\hline 2649 & Carex hordeistichos Vill. & Noszlop & 1957 & Pócs Tamás, Tallós Pál & BPU-12523.JPG \\
\hline 2649 & Carex hordeistichos Vill. & Szikszó & 1910 & Thaisz Lajos & BPU-12528.JPG \\
\hline 2650 & Carex secalina Wahlenb. & Alcsútdoboz & 1992 & Felföldy Lajos & BPU-12519.JPG \\
\hline 2650 & Carex secalina Wahlenb. & Balatonberény & 1953 & Priszter Szaniszló & BPU-12515.JPG \\
\hline 2650 & Carex secalina Wahlenb. & Balatonberény & 1953 & Priszter Szaniszló & BPU-12516.JPG \\
\hline 2650 & Carex secalina Wahlenb. & Budapest & 1909 & Degen Árpád & BPU-12521.JPG \\
\hline 2650 & Carex secalina Wahlenb. & Budapest & 1909 & Degen Árpád & BPU-12522.JPG \\
\hline 2650 & Carex secalina Wahlenb. & Budapest & 1932 & Kárpáti Zoltán & BPU-12520.JPG \\
\hline 2650 & Carex secalina Wahlenb. & Debrecen & 1951 & Soó Rezső, Simon Tibor & BPU-12518.JPG \\
\hline 2650 & Carex secalina Wahlenb. & Hortobágy & 1930 & Soó Rezső & BPU-12517.JPG \\
\hline 2650 & Carex secalina Wahlenb. & Tihany & 1951 & Priszter Szaniszló & BPU-12514.JPG \\
\hline 2651 & Carex flava L. & Balatonszőlős & 1956 & $\begin{array}{l}\text { Felföldy Lajos, Tóth } \\
\text { László }\end{array}$ & BPU-12551.JPG \\
\hline 2651 & Carex flava L. & Budapest & 1915 & Degen Árpád & BPU-12554.JPG \\
\hline
\end{tabular}


1. Elektroniukus melléklet - Electronic Appendix 1.

\begin{tabular}{|c|c|c|c|c|c|}
\hline $\begin{array}{l}\text { Sorszám / } \\
\text { Number }\end{array}$ & Taxon-név / Taxon-name & $\begin{array}{l}\text { Település / } \\
\text { Settlement }\end{array}$ & $\begin{array}{l}\text { Év / } \\
\text { Year }\end{array}$ & Gyűjtő / Collector & $\begin{array}{l}\text { Fájlnév / } \\
\text { File-name }\end{array}$ \\
\hline 2651 & Carex flava L. & Budapest & 1915 & Degen Árpád & BPU-12554.JPG \\
\hline 2651 & Carex flava L. & Budapest & 1916 & Degen Árpád & BPU-12554.JPG \\
\hline 2651 & Carex flava L. & Uzsa & 1959 & $\begin{array}{l}\text { Jávorka Sándor, Felföldy } \\
\text { Lajos }\end{array}$ & BPU-12549.JPG \\
\hline 2651 & Carex flava L. & Veresegyház & 1950 & Csapody Vera & BPU-12552.JPG \\
\hline 2651 & Carex flava L. & Zalagyömörő & 1957 & Felföldy Lajos & BPU-12548.JPG \\
\hline 2652 & Carex lepidocarpa Tausch & Bak & 1955 & Soó Rezső & BPU-12532.JPG \\
\hline 2652 & Carex lepidocarpa Tausch & Balatonszőlős & 1957 & Felföldy Lajos & BPU-12535.JPG \\
\hline 2652 & Carex lepidocarpa Tausch & Dunaharaszti & 1915 & Degen Árpád & BPU-12543.JPG \\
\hline 2652 & Carex lepidocarpa Tausch & Dunaharaszti & 1915 & Degen Árpád & BPU-12543.JPG \\
\hline 2652 & Carex lepidocarpa Tausch & Dunaharaszti & 1916 & Degen Árpád & BPU-12543.JPG \\
\hline 2652 & Carex lepidocarpa Tausch & Felsőtárkány & 1953 & Soó Rezső & BPU-12539.JPG \\
\hline 2652 & Carex lepidocarpa Tausch & Miskolc & 1950 & Soó Rezső & BPU-12540.JPG \\
\hline 2652 & Carex lepidocarpa Tausch & Örvényes & 1959 & Felföldy Lajos & BPU-12534.JPG \\
\hline 2652 & Carex lepidocarpa Tausch & Pálháza & 1960 & Simon Tibor & BPU-12542.JPG \\
\hline 2652 & Carex lepidocarpa Tausch & Pálháza & 1991 & Simon Tibor & BPU-12541.JPG \\
\hline 2652 & Carex lepidocarpa Tausch & Somogyszob & 1957 & Soó Rezső & BPU-12537.JPG \\
\hline 2652 & Carex lepidocarpa Tausch & Somoskőújfalu & 1936 & Soó Rezső & BPU-12538.JPG \\
\hline 2652 & Carex lepidocarpa Tausch & Szakonyfalu & 1955 & Soó Rezső & BPU-12536.JPG \\
\hline 2652 & Carex lepidocarpa Tausch & Ugod & 1955 & Soó Rezső & BPU-12533.JPG \\
\hline 2652 & Carex lepidocarpa Tausch & Uzsa & 1950 & Felföldy Lajos & BPU-12550.JPG \\
\hline 2653 & Carex viridula Michx. & Balatonboglár & 1950 & Priszter Szaniszló & BPU-12563.JPG \\
\hline 2653 & Carex viridula Michx. & Balatonfenyves & 1953 & Priszter Szaniszló & BPU-12562.JPG \\
\hline 2653 & Carex viridula Michx. & Balatonszepezd & 1953 & Priszter Szaniszló & BPU-12560.JPG \\
\hline 2653 & Carex viridula Michx. & Balatonszepezd & 1956 & $\begin{array}{l}\text { Felföldy Lajos, Tóth } \\
\text { László }\end{array}$ & BPU-12559.JPG \\
\hline 2653 & Carex viridula Michx. & Balatonszepezd & 1958 & Felföldy Lajos & BPU-12558.JPG \\
\hline 2653 & Carex viridula Michx. & Budapest & 1909 & Degen Árpád & BPU-12574.JPG \\
\hline 2653 & Carex viridula Michx. & Budapest & 1914 & Zsák Zoltán & BPU-12571.JPG \\
\hline 2653 & Carex viridula Michx. & Budapest & 1942 & Priszter Szaniszló & BPU-12572.JPG \\
\hline 2653 & Carex viridula Michx. & Budapest & - & Pénzes Antal & BPU-12573.JPG \\
\hline 2653 & Carex viridula Michx. & Debrecen & 1951 & Kulcsár Gábor & BPU-12569.JPG \\
\hline 2653 & Carex viridula Michx. & Dunaharaszti & 1949 & Priszter Szaniszló & BPU-12568.JPG \\
\hline 2653 & Carex viridula Michx. & Hajdúbagos & 1934 & Soó Rezső & BPU-12570.JPG \\
\hline 2653 & Carex viridula Michx. & Hévíz & 1961 & Felföldy Lajos & BPU-12557.JPG \\
\hline 2653 & Carex viridula Michx. & Paloznak & 1956 & $\begin{array}{l}\text { Felföldy Lajos, Tóth } \\
\text { László }\end{array}$ & BPU-12561.JPG \\
\hline 2653 & Carex viridula Michx. & Pusztamiske & 1948 & Borsos Olga & BPU-12556.JPG \\
\hline 2653 & Carex viridula Michx. & Révfülöp & 1956 & $\begin{array}{l}\text { Felföldy Lajos, Tóth } \\
\text { László }\end{array}$ & BPU-12560.JPG \\
\hline 2653 & Carex viridula Michx. & Vácrátót & 1953 & Felföldy Lajos & BPU-12566.JPG \\
\hline 2653 & Carex viridula Michx. & Vácrátót & 1953 & Felföldy Lajos & BPU-12567.JPG \\
\hline 2653 & Carex viridula Michx. & Veresegyház & 1949 & Soó Rezső & BPU-12565.JPG \\
\hline 2653 & Carex viridula Michx. & Veresegyház & 1950 & Soó Rezső & BPU-12564.JPG \\
\hline 2655 & Carex hostiana DC. & Aszófő & 1959 & Felföldy Lajos & BPU-12582.JPG \\
\hline 2655 & Carex hostiana DC. & Balatonszőlős & 1957 & Felföldy Lajos & BPU-12581.JPG \\
\hline 2655 & Carex hostiana DC. & Balatonudvari & 1959 & Felföldy Lajos & BPU-12580.JPG \\
\hline 2655 & Carex hostiana DC. & Budapest & 1914 & Zsák Zoltán & BPU-12584.JPG \\
\hline 2655 & Carex hostiana DC. & Kimle & 1962 & Borsos Olga & BPU-12583.JPG \\
\hline
\end{tabular}


1. Elektroniukus melléklet - Electronic Appendix 1.

\begin{tabular}{|c|c|c|c|c|c|}
\hline $\begin{array}{l}\text { Sorszám / } \\
\text { Number }\end{array}$ & Taxon-név / Taxon-name & $\begin{array}{l}\text { Település / } \\
\text { Settlement }\end{array}$ & $\begin{array}{l}\text { Év / } \\
\text { Year }\end{array}$ & Gyűjtő / Collector & $\begin{array}{l}\text { Fájlnév / } \\
\text { File-name }\end{array}$ \\
\hline 2655 & Carex hostiana DC. & Örvényes & 1959 & Felföldy Lajos & BPU-12578.JPG \\
\hline 2655 & Carex hostiana DC. & Örvényes & 1959 & Felföldy Lajos & BPU-12579.JPG \\
\hline 2655 & Carex hostiana DC. & Szigliget & 1928 & Soó Rezső & BPU-12575.JPG \\
\hline 2655 & Carex hostiana DC. & Ugod & 1955 & Soó Rezső & BPU-12576.JPG \\
\hline 2655 & Carex hostiana DC. & Ugod & 1955 & Soó Rezső & BPU-12577.JPG \\
\hline 2656 & Carex distans L. & Balatonfenyves & 1953 & Priszter Szaniszló & BPU-14036.JPG \\
\hline 2656 & Carex distans L. & Balatonfenyves & 1953 & Priszter Szaniszló & BPU-14037.JPG \\
\hline 2656 & Carex distans L. & Balatonszőlős & 1956 & $\begin{array}{l}\text { Felföldy Lajos, Tóth } \\
\text { László }\end{array}$ & BPU-14035.JPG \\
\hline 2656 & Carex distans L. & Balatonszőlős & 1957 & $\begin{array}{l}\text { Felföldy Lajos, Kovács } \\
\text { Margit, Tóth László }\end{array}$ & BPU-14034.JPG \\
\hline 2656 & Carex distans L. & Bicske & 1992 & Felföldy Lajos & BPU-14048.JPG \\
\hline 2656 & Carex distans L. & Budapest & 1909 & Degen Árpád & BPU-14055.JPG \\
\hline 2656 & Carex distans L. & Budapest & 1947 & Pénzes Antal & BPU-14054.JPG \\
\hline 2656 & Carex distans L. & Budapest & 1991 & Felföldy Lajos & BPU-14052.JPG \\
\hline 2656 & Carex distans L. & Budapest & 1991 & Felföldy Lajos & BPU-14053.JPG \\
\hline 2656 & Carex distans L. & Budapest & 2005 & Felföldy Lajos & BPU-14051.JPG \\
\hline 2656 & Carex distans L. & Bugac & 1952 & Vozáry Elemér & BPU-14050.JPG \\
\hline 2656 & Carex distans L. & Dabas & 1959 & Simon Tibor & BPU-14040.JPG \\
\hline 2656 & Carex distans L. & Debrecen & 1934 & Soó Rezső & BPU-14041.JPG \\
\hline 2656 & Carex distans L. & Debrecen & 1989 & Felföldy Lajos & BPU-14042.JPG \\
\hline 2656 & Carex distans L. & Győr & 1926 & Polgár Sándor & BPU-14043.JPG \\
\hline 2656 & Carex distans L. & Hidas & 1965 & Simon Tibor & BPU-14049.JPG \\
\hline 2656 & Carex distans L. & Örvényes & 1959 & Felföldy Lajos & BPU-14033.JPG \\
\hline 2656 & Carex distans L. & Pákozd & 1978 & Felföldy Lajos & BPU-14045.JPG \\
\hline 2656 & Carex distans L. & Pákozd & 1980 & Felföldy Lajos & BPU-14044.JPG \\
\hline 2656 & Carex distans L. & Pákozd & 1980 & Felföldy Lajos & BPU-14046.JPG \\
\hline 2656 & Carex distans L. & Pátka & 1987 & Felföldy Lajos & BPU-14047.JPG \\
\hline 2656 & Carex distans L. & Tihany & 1926 & Soó Rezső & BPU-14031.JPG \\
\hline 2656 & Carex distans L. & Tihany & 1951 & Priszter Szaniszló & BPU-14032.JPG \\
\hline 2656 & Carex distans L. & Tihany & 1956 & Felföldy Lajos & BPU-14030.JPG \\
\hline 2656 & Carex distans L. & Törökbálint & 1992 & Felföldy Lajos & BPU-14039.JPG \\
\hline 2656 & Carex distans L. & Vácrátót & 1953 & Felföldy Lajos & BPU-14038.JPG \\
\hline $\begin{array}{l}2610- \\
2656\end{array}$ & Carex subg. Carex & Szentendre & 1917 & Degen Árpád & BPU-12377.JPG \\
\hline $\begin{array}{c}2603 \times \\
2604\end{array}$ & $\begin{array}{l}\text { Carex paniculata L. } \times \text { Carex } \\
\text { appropinquata Schumach. }\end{array}$ & Budapest & 1917 & Degen Árpád & BPU-12371.JPG \\
\hline $\begin{array}{c}2603 \times \\
2604\end{array}$ & $\begin{array}{l}\text { Carex paniculata L. } \times \text { Carex } \\
\text { appropinquata Schumach. }\end{array}$ & Budapest & 1917 & Degen Árpád & BPU-12371.JPG \\
\hline $\begin{array}{c}2610 \times \\
2612\end{array}$ & $\begin{array}{l}\text { Carex elata All. × Carex nigra (L.) } \\
\text { Reichard }\end{array}$ & Bátorliget & 1932 & Soó Rezső & BPU-12380.JPG \\
\hline $\begin{array}{c}2624 \times \\
2625\end{array}$ & $\begin{array}{l}\text { Carex umbrosa Host } \times \text { Carex } \\
\text { caryophyllea Latourr. }\end{array}$ & Kőszeg & 1896 & Waisbecker Antal & BPU-12379.JPG \\
\hline $\begin{array}{c}2651 \times \\
2652\end{array}$ & $\begin{array}{l}\text { Carex flava L. } \times \text { Carex lepidocarpa } \\
\text { Tausch }\end{array}$ & Budapest & 1915 & Degen Árpád & BPU-12373.JPG \\
\hline $\begin{array}{c}2651 \times \\
2652\end{array}$ & $\begin{array}{l}\text { Carex flava L. } \times \text { Carex lepidocarpa } \\
\text { Tausch }\end{array}$ & Budapest & 1915 & Degen Árpád & BPU-12373.JPG \\
\hline $\begin{array}{c}2651 \times \\
2652\end{array}$ & $\begin{array}{l}\text { Carex flava L. } \times \text { Carex lepidocarpa } \\
\text { Tausch }\end{array}$ & Budapest & 1916 & Degen Árpád & BPU-12373.JPG \\
\hline $\begin{array}{c}2651 \times \\
2653\end{array}$ & Carex flava L. $\times$ Carex viridula Michx. & Budapest & 1916 & Degen Árpád & BPU-12375.JPG \\
\hline
\end{tabular}


1. Elektroniukus melléklet - Electronic Appendix 1.

\begin{tabular}{|c|c|c|c|c|c|}
\hline $\begin{array}{l}\text { Sorszám / } \\
\text { Number }\end{array}$ & Taxon-név / Taxon-name & $\begin{array}{l}\text { Település / } \\
\text { Settlement }\end{array}$ & $\begin{array}{l}\text { Év / } \\
\text { Year }\end{array}$ & Gyűjtő / Collector & $\begin{array}{l}\text { Fájlnév / } \\
\text { File-name }\end{array}$ \\
\hline $\begin{array}{c}2651 \times \\
2653\end{array}$ & Carex flava L. × Carex viridula Michx. & Budapest & 1916 & Degen Árpád & BPU-12381.JPG \\
\hline $\begin{array}{c}2651 \times \\
2656\end{array}$ & Carex flava L. $\times$ Carex distans L. & Budapest & 1917 & Degen Árpád & BPU-12374.JPG \\
\hline 2657 & Cypripedium calceolus L. & Felsőtárkány & 1941 & Boros Ádám & BPU-0333.JPG \\
\hline 2657 & Cypripedium calceolus L. & Sopron & 1950 & Tallós Pál & BPU-0338.JPG \\
\hline 2657 & Cypripedium calceolus L. & Sopron & 1952 & Csapody István & BPU-0334.JPG \\
\hline 2657 & Cypripedium calceolus L. & Sopron & 1960 & Borsos Olga, Simon Tibor & BPU-0331.JPG \\
\hline 2657 & Cypripedium calceolus L. & Sopron & 1960 & Borsos Olga, Simon Tibor & BPU-0337.JPG \\
\hline 2658 & Epipactis palustris (L.) Crantz & Balatonszőlős & 1956 & Felföldy Lajos & BPU-0398.JPG \\
\hline 2658 & Epipactis palustris (L.) Crantz & Balatonszőlős & 1990 & Felföldy Lajos & BPU-0399.JPG \\
\hline 2658 & Epipactis palustris (L.) Crantz & Dabas & 1970 & Borsos Olga & BPU-0392.JPG \\
\hline 2658 & Epipactis palustris (L.) Crantz & Devecser & 1957 & Pócs Tamás, Tallós Pál & BPU-0400.JPG \\
\hline 2658 & Epipactis palustris (L.) Crantz & Kecskemét & 1914 & Szepesfalvy János & BPU-0391.JPG \\
\hline 2658 & Epipactis palustris (L.) Crantz & Nyírpilis & 1932 & Soó Rezső & BPU-0397.JPG \\
\hline 2658 & Epipactis palustris (L.) Crantz & Sopron & 1947 & Priszter Szaniszló & BPU-0395.JPG \\
\hline 2658 & Epipactis palustris (L.) Crantz & Sopron & 1952 & Csapody István & BPU-0396.JPG \\
\hline 2658 & Epipactis palustris (L.) Crantz & Szigetcsép & 1948 & Csapody Vera & BPU-0393.JPG \\
\hline 2658 & Epipactis palustris (L.) Crantz & Szigetcsép & 1992 & Felföldy Lajos & BPU-0394.JPG \\
\hline 2659 & Epipactis microphylla (Ehrh.) Sw. & Budapest & 1937 & Priszter Szaniszló & BPU-0404.JPG \\
\hline 2659 & Epipactis microphylla (Ehrh.) Sw. & Budapest & 1938 & Priszter Szaniszló & BPU-0405.JPG \\
\hline 2659 & Epipactis microphylla (Ehrh.) Sw. & Budapest & 1947 & Priszter Szaniszló & BPU-0406.JPG \\
\hline 2659 & Epipactis microphylla (Ehrh.) Sw. & Kács & 1926 & Boros Ádám & BPU-0408.JPG \\
\hline 2659 & Epipactis microphylla (Ehrh.) Sw. & Nagyvisnyó & 1955 & Soó Rezső & BPU-0409.JPG \\
\hline 2659 & Epipactis microphylla (Ehrh.) Sw. & Tihany & 1929 & Soó Rezső & BPU-0410.JPG \\
\hline 2659 & Epipactis microphylla (Ehrh.) Sw. & Vésztő & - & Soó Rezső & BPU-0407.JPG \\
\hline 2661 & Epipactis voethii Robatsch & Bodajk & 1961 & Borsos Olga, Simon Tibor & BPU-0439.JPG \\
\hline 2665 & Epipactis nordeniorum Robatsch & Hidegkút & 1957 & Felföldy Lajos & BPU-0453.JPG \\
\hline 2672 & Epipactis atrorubens Hoffm. ex Besser & Bugac & 1923 & Soó Rezső & BPU-0412.JPG \\
\hline 2672 & Epipactis atrorubens Hoffm. ex Besser & Csévharaszt & 1959 & Simon Tibor, Borsos Olga & BPU-0419.JPG \\
\hline 2672 & Epipactis atrorubens Hoffm. ex Besser & Miskolc & 1940 & Priszter Szaniszló & BPU-0416.JPG \\
\hline 2672 & Epipactis atrorubens Hoffm. ex Besser & Miskolc & 1949 & Priszter Szaniszló & BPU-0417.JPG \\
\hline 2672 & Epipactis atrorubens Hoffm. ex Besser & Nagyvisnyó & 1953 & Soó Rezső & BPU-0414.JPG \\
\hline 2672 & Epipactis atrorubens Hoffm. ex Besser & Nagyvisnyó & 1960 & Simon Tibor & BPU-0415.JPG \\
\hline 2672 & Epipactis atrorubens Hoffm. ex Besser & Szilvásvárad & 1952 & Borsos Olga & BPU-0418.JPG \\
\hline 2673 & Epipactis helleborine (L.) Crantz & Bátorliget & 1950 & $\begin{array}{l}\text { Simon Tibor, Kárpáti } \\
\text { István }\end{array}$ & BPU-0450.JPG \\
\hline 2673 & Epipactis helleborine (L.) Crantz & Bátorliget & 1953 & Simon Tibor & BPU-0449.JPG \\
\hline 2673 & Epipactis helleborine (L.) Crantz & Beregdaróc & 1951 & $\begin{array}{l}\text { Simon Tibor, Vozáry } \\
\text { Elemér }\end{array}$ & BPU-0432.JPG \\
\hline 2673 & Epipactis helleborine (L.) Crantz & Budapest & 1937 & Priszter Szaniszló & BPU-0431.JPG \\
\hline 2673 & Epipactis helleborine (L.) Crantz & Csesznek & 1929 & Soó Rezső & BPU-0452.JPG \\
\hline 2673 & Epipactis helleborine (L.) Crantz & Gyöngyös & 1953 & Soó Rezső & BPU-0442.JPG \\
\hline 2673 & Epipactis helleborine (L.) Crantz & Miskolc & 1929 & Soó Rezső & BPU-0438.JPG \\
\hline 2673 & Epipactis helleborine (L.) Crantz & Miskolc & 1939 & Soó Rezső & BPU-0436.JPG \\
\hline 2673 & Epipactis helleborine (L.) Crantz & Miskolc & 1950 & Soó Rezső & BPU-0434.JPG \\
\hline 2673 & Epipactis helleborine (L.) Crantz & Miskolc & 1950 & Soó Rezső & BPU-0437.JPG \\
\hline 2673 & Epipactis helleborine (L.) Crantz & Nagykovácsi & 1947 & Soó Rezső & BPU-0445.JPG \\
\hline 2673 & Epipactis helleborine (L.) Crantz & Nagyvisnyó & 1953 & Soó Rezső & BPU-0433.JPG \\
\hline
\end{tabular}


1. Elektroniukus melléklet - Electronic Appendix 1.

\begin{tabular}{|c|c|c|c|c|c|}
\hline $\begin{array}{l}\text { Sorszám / } \\
\text { Number }\end{array}$ & Taxon-név / Taxon-name & $\begin{array}{l}\text { Település / } \\
\text { Settlement }\end{array}$ & $\begin{array}{l}\text { Év / } \\
\text { Year }\end{array}$ & Gyűjtő / Collector & $\begin{array}{l}\text { Fájlnév / } \\
\text { File-name }\end{array}$ \\
\hline 2673 & Epipactis helleborine (L.) Crantz & Pilisszentiván & 1961 & Borsos Olga, Simon Tibor & BPU-0446.JPG \\
\hline 2673 & Epipactis helleborine (L.) Crantz & Szilvásvárad & 1947 & Papp József & BPU-0435.JPG \\
\hline $\begin{array}{c}2658- \\
2674\end{array}$ & Epipactis & Bátorliget & 1950 & $\begin{array}{l}\text { Simon Tibor, Kárpáti } \\
\text { István }\end{array}$ & BPU-0448.JPG \\
\hline $\begin{array}{c}2658- \\
2674\end{array}$ & Epipactis & Diósjenő & 1952 & Simon Tibor & BPU-0443.JPG \\
\hline $\begin{array}{c}2658- \\
2674\end{array}$ & Epipactis & Gyöngyös & 1952 & Soó Rezső & BPU-0441.JPG \\
\hline $\begin{array}{c}2658- \\
2674\end{array}$ & Epipactis & Miskolc & 1950 & Soó Rezső & BPU-0413.JPG \\
\hline $\begin{array}{c}2658- \\
2674\end{array}$ & Epipactis & Nyíracsád & 1955 & Jeney Endre & BPU-0440.JPG \\
\hline $\begin{array}{c}2658- \\
2674\end{array}$ & Epipactis & Szenna & 1957 & Soó Rezső & BPU-0447.JPG \\
\hline $\begin{array}{c}2658- \\
2674\end{array}$ & Epipactis & Szentgotthárd & 1955 & Soó Rezső & BPU-0451.JPG \\
\hline $\begin{array}{l}2658- \\
2674\end{array}$ & Epipactis & Szigetmonostor & 1941 & Priszter Szaniszló & BPU-0444.JPG \\
\hline 2675 & Cephalanthera rubra (L.) Rich. & Bélapátfalva & 1953 & Simon Tibor & BPU-0343.JPG \\
\hline 2675 & Cephalanthera rubra (L.) Rich. & Budapest & 1938 & Priszter Szaniszló & BPU-0339.JPG \\
\hline 2675 & Cephalanthera rubra (L.) Rich. & Miskolc & 1929 & Soó Rezső & BPU-0341.JPG \\
\hline 2675 & Cephalanthera rubra (L.) Rich. & Nagyvisnyó & 1953 & Soó Rezső & BPU-0340.JPG \\
\hline 2675 & Cephalanthera rubra (L.) Rich. & Paloznak & 1957 & Entz Béla & BPU-0345.JPG \\
\hline 2675 & Cephalanthera rubra (L.) Rich. & Sopron & 1937 & Soó Rezső & BPU-0344.JPG \\
\hline 2675 & Cephalanthera rubra (L.) Rich. & Süttő & 1938 & Boros Ádám & BPU-0342.JPG \\
\hline 2675 & Cephalanthera rubra (L.) Rich. & Uzsa & 1931 & Soó Rezső & BPU-0347.JPG \\
\hline 2676 & $\begin{array}{l}\text { Cephalanthera damasonium (Mill.) } \\
\text { Druce }\end{array}$ & Bátorliget & 1955 & Soó Rezső & BPU-0370.JPG \\
\hline 2676 & $\begin{array}{l}\text { Cephalanthera damasonium (Mill.) } \\
\text { Druce }\end{array}$ & Budapest & 1937 & Priszter Szaniszló & BPU-0357.JPG \\
\hline 2676 & $\begin{array}{l}\text { Cephalanthera damasonium (Mill.) } \\
\text { Druce }\end{array}$ & Budapest & 1937 & Priszter Szaniszló & BPU-0358.JPG \\
\hline 2676 & $\begin{array}{l}\text { Cephalanthera damasonium (Mill.) } \\
\text { Druce }\end{array}$ & Budapest & 1992 & Felföldy Lajos & BPU-0356.JPG \\
\hline 2676 & $\begin{array}{l}\text { Cephalanthera damasonium (Mill.) } \\
\text { Druce }\end{array}$ & Budapest & 2004 & Felföldy Lajos & BPU-0355.JPG \\
\hline 2676 & $\begin{array}{l}\text { Cephalanthera damasonium (Mill.) } \\
\text { Druce }\end{array}$ & Csákberény & 1948 & Priszter Szaniszló & BPU-0365.JPG \\
\hline 2676 & $\begin{array}{l}\text { Cephalanthera damasonium (Mill.) } \\
\text { Druce }\end{array}$ & Csókakő & 1948 & Priszter Szaniszló & BPU-0366.JPG \\
\hline 2676 & $\begin{array}{l}\text { Cephalanthera damasonium (Mill.) } \\
\text { Druce }\end{array}$ & Isztimér & 1949 & Soó Rezső & BPU-0367.JPG \\
\hline 2676 & $\begin{array}{l}\text { Cephalanthera damasonium (Mill.) } \\
\text { Druce }\end{array}$ & Kapolcs & 1959 & $\begin{array}{l}\text { Jávorka Sándor, Felföldy } \\
\text { Lajos, Tóth László }\end{array}$ & BPU-0371.JPG \\
\hline 2676 & $\begin{array}{l}\text { Cephalanthera damasonium (Mill.) } \\
\text { Druce }\end{array}$ & Köveskál & 1959 & $\begin{array}{l}\text { Jávorka Sándor, Felföldy } \\
\text { Lajos, Tóth László }\end{array}$ & BPU-0372.JPG \\
\hline 2676 & $\begin{array}{l}\text { Cephalanthera damasonium (Mill.) } \\
\text { Druce }\end{array}$ & Miskolc & 1939 & Soó Rezső & BPU-0363.JPG \\
\hline 2676 & $\begin{array}{l}\text { Cephalanthera damasonium (Mill.) } \\
\text { Druce }\end{array}$ & Pécs & 1925 & Soó Rezső & BPU-0361.JPG \\
\hline 2676 & $\begin{array}{l}\text { Cephalanthera damasonium (Mill.) } \\
\text { Druce }\end{array}$ & Pécs & 1935 & Soó Rezső & BPU-0359.JPG \\
\hline 2676 & $\begin{array}{l}\text { Cephalanthera damasonium (Mill.) } \\
\text { Druce }\end{array}$ & Pécs & 1948 & Priszter Szaniszló & BPU-0360.JPG \\
\hline 2676 & $\begin{array}{l}\text { Cephalanthera damasonium (Mill.) } \\
\text { Druce }\end{array}$ & Pilisszentiván & 1953 & Soó Rezső & BPU-0369.JPG \\
\hline 2676 & $\begin{array}{l}\text { Cephalanthera damasonium (Mill.) } \\
\text { Druce }\end{array}$ & Répáshuta & 1953 & Soó Rezső & BPU-0364.JPG \\
\hline
\end{tabular}


1. Elektroniukus melléklet - Electronic Appendix 1.

\begin{tabular}{|c|c|c|c|c|c|}
\hline $\begin{array}{l}\text { Sorszám / } \\
\text { Number }\end{array}$ & Taxon-név / Taxon-name & $\begin{array}{l}\text { Település / } \\
\text { Settlement }\end{array}$ & $\begin{array}{l}\text { Év / } \\
\text { Year }\end{array}$ & Gyűjtő / Collector & $\begin{array}{l}\text { Fájlnév / } \\
\text { File-name }\end{array}$ \\
\hline 2676 & $\begin{array}{l}\text { Cephalanthera damasonium (Mill.) } \\
\text { Druce }\end{array}$ & Szarvas & 1949 & Soó Rezső & BPU-0362.JPG \\
\hline 2676 & $\begin{array}{l}\text { Cephalanthera damasonium (Mill.) } \\
\text { Druce }\end{array}$ & Szilvásvárad & 1953 & $\begin{array}{l}\text { Felföldy Lajos, Tóth } \\
\text { László }\end{array}$ & BPU-0368.JPG \\
\hline 2676 & $\begin{array}{l}\text { Cephalanthera damasonium (Mill.) } \\
\text { Druce }\end{array}$ & Tihany & 1957 & Entz Béla & BPU-0373.JPG \\
\hline 2677 & Cephalanthera longifolia (L.) Fritsch & Beregdaróc & 1951 & Simon Tibor, Jakucs Pál & BPU-0380.JPG \\
\hline 2677 & Cephalanthera longifolia (L.) Fritsch & Dömös & 1948 & Priszter Szaniszló & BPU-0383.JPG \\
\hline 2677 & Cephalanthera longifolia (L.) Fritsch & Dömös & 1952 & Soó Rezső & BPU-15424.JPG \\
\hline 2677 & Cephalanthera longifolia (L.) Fritsch & Fenyőfő & 1955 & Soó Rezső & BPU-0346.JPG \\
\hline 2677 & Cephalanthera longifolia (L.) Fritsch & Isztimér & 1955 & Soó Rezső & BPU-0348.JPG \\
\hline 2677 & Cephalanthera longifolia (L.) Fritsch & Komló & 1941 & Priszter Szaniszló & BPU-0377.JPG \\
\hline 2677 & Cephalanthera longifolia (L.) Fritsch & Kőszeg & 1938 & Soó Rezső & BPU-0387.JPG \\
\hline 2677 & Cephalanthera longifolia (L.) Fritsch & Kőszeg & 1941 & Priszter Szaniszló & BPU-0388.JPG \\
\hline 2677 & Cephalanthera longifolia (L.) Fritsch & Lábatlan & 1953 & Felföldy Lajos & BPU-0384.JPG \\
\hline 2677 & Cephalanthera longifolia (L.) Fritsch & Miskolc & 1939 & Soó Rezső & BPU-0381.JPG \\
\hline 2677 & Cephalanthera longifolia (L.) Fritsch & Miskolc & 1949 & Priszter Szaniszló & BPU-0382.JPG \\
\hline 2677 & Cephalanthera longifolia (L.) Fritsch & Pécs & 1956 & Soó Rezső & BPU-0378.JPG \\
\hline 2677 & Cephalanthera longifolia (L.) Fritsch & Pécs & 1956 & Soó Rezső & BPU-0379.JPG \\
\hline 2677 & Cephalanthera longifolia (L.) Fritsch & Somogyszob & 1957 & Soó Rezső & BPU-0385.JPG \\
\hline 2677 & Cephalanthera longifolia (L.) Fritsch & Telkibánya & 1955 & Simon Tibor & BPU-0376.JPG \\
\hline 2677 & Cephalanthera longifolia (L.) Fritsch & Vállaj & 1934 & Soó Rezső & BPU-0386.JPG \\
\hline 2678 & Limodorum abortivum (L.) Sw. & Gyöngyös & 1961 & Soó Rezső & BPU-0465.JPG \\
\hline 2678 & Limodorum abortivum (L.) Sw. & Paloznak & 1957 & Entz Béla & BPU-0468.JPG \\
\hline 2678 & Limodorum abortivum (L.) Sw. & Pécs & 1935 & Soó Rezső & BPU-0464.JPG \\
\hline 2678 & Limodorum abortivum (L.) Sw. & Pilisszentlászló & 1932 & Soó Rezső & BPU-0466.JPG \\
\hline 2678 & Limodorum abortivum (L.) Sw. & Vác & 1944 & Kárpáti Zoltán & BPU-0467.JPG \\
\hline 2679 & Epipogium aphyllum Sw. & Csesznek & 1924 & Polgár Sándor & BPU-0516.JPG \\
\hline 2680 & Neottia nidus-avis (L.) Rich. & Budapest & 1938 & Priszter Szaniszló & BPU-0487.JPG \\
\hline 2680 & Neottia nidus-avis (L.) Rich. & Budapest & 1938 & Priszter Szaniszló & BPU-0489.JPG \\
\hline 2680 & Neottia nidus-avis (L.) Rich. & Budapest & 1982 & Felföldy Lajos & BPU-0488.JPG \\
\hline 2680 & Neottia nidus-avis (L.) Rich. & Gyöngyös & 1965 & Soó Rezső & BPU-0496.JPG \\
\hline 2680 & Neottia nidus-avis (L.) Rich. & Hajdúsámson & 1932 & Soó Rezső & BPU-0495.JPG \\
\hline 2680 & Neottia nidus-avis (L.) Rich. & Isztimér & 1949 & Soó Rezső & BPU-0494.JPG \\
\hline 2680 & Neottia nidus-avis (L.) Rich. & Lábatlan & 1953 & Felföldy Lajos & BPU-0493.JPG \\
\hline 2680 & Neottia nidus-avis (L.) Rich. & Miskolc & 1950 & Soó Rezső & BPU-0490.JPG \\
\hline 2680 & Neottia nidus-avis (L.) Rich. & Répáshuta & 1953 & Soó Rezső & BPU-0491.JPG \\
\hline 2680 & Neottia nidus-avis (L.) Rich. & Sopron & 1960 & Borsos Olga, Simon Tibor & BPU-0497.JPG \\
\hline 2680 & Neottia nidus-avis (L.) Rich. & Szalonna & 1966 & Felföldy Lajos & BPU-0492.JPG \\
\hline 2680 & Neottia nidus-avis (L.) Rich. & Szentbékkálla & 1959 & $\begin{array}{l}\text { Jávorka Sándor, Felföldy } \\
\text { Lajos, Tóth László }\end{array}$ & BPU-0498.JPG \\
\hline 2680 & Neottia nidus-avis (L.) Rich. & Uzsa & 1952 & Simon Tibor & BPU-0499.JPG \\
\hline 2680 & Neottia nidus-avis (L.) Rich. & Uzsa & 1959 & $\begin{array}{l}\text { Jávorka Sándor, Felföldy } \\
\text { Lajos }\end{array}$ & BPU-0500.JPG \\
\hline 2681 & Listera ovata (L.) R. Br. & Bátorliget & 1932 & Soó Rezső & BPU-0476.JPG \\
\hline 2681 & Listera ovata (L.) R. Br. & Bátorliget & 1951 & Simon Tibor & BPU-0477.JPG \\
\hline 2681 & Listera ovata (L.) R. Br. & Bátorliget & 1953 & Simon Tibor & BPU-0478.JPG \\
\hline 2681 & Listera ovata (L.) R. Br. & Budapest & 1989 & Felföldy Lajos & BPU-0469.JPG \\
\hline 2681 & Listera ovata (L.) R. Br. & Debrecen & 1932 & Soó Rezső & BPU-0471.JPG \\
\hline 2681 & Listera ovata (L.) R. Br. & Komló & 1941 & Priszter Szaniszló & BPU-0470.JPG \\
\hline
\end{tabular}


1. Elektroniukus melléklet - Electronic Appendix 1.

\begin{tabular}{|c|c|c|c|c|c|}
\hline $\begin{array}{l}\text { Sorszám / } \\
\text { Number }\end{array}$ & Taxon-név / Taxon-name & $\begin{array}{l}\text { Település / } \\
\text { Settlement }\end{array}$ & $\begin{array}{l}\text { Év / } \\
\text { Year }\end{array}$ & Gyújtő / Collector & $\begin{array}{l}\text { Fájlnév / } \\
\text { File-name }\end{array}$ \\
\hline 2681 & Listera ovata (L.) R. Br. & Mosonmagyaróvár & 1962 & Borsos Olga & BPU-0472.JPG \\
\hline 2681 & Listera ovata (L.) R. Br. & Perőcsény & 1947 & Priszter Szaniszló & BPU-0473.JPG \\
\hline 2681 & Listera ovata (L.) R. Br. & Sopron & 1952 & Csapody István & BPU-0474.JPG \\
\hline 2681 & Listera ovata (L.) R. Br. & Sopron & 1960 & Borsos Olga, Simon Tibor & BPU-0475.JPG \\
\hline 2682 & Spiranthes aestivalis (Poir.) Rich. & Sormás & 1948 & Károlyi Árpád & BPU-0506.JPG \\
\hline 2683 & Spiranthes spiralis (L.) Chevall. & Pápa & 1952 & Tallós Pál & BPU-0504.JPG \\
\hline 2684 & Goodyera repens (L.) R. Br. & Csákvár & 1937 & Boros Ádám & BPU-0509.JPG \\
\hline 2684 & Goodyera repens (L.) R. Br. & Sopron & 1944 & Kárpáti Zoltán & BPU-0510.JPG \\
\hline 2684 & Goodyera repens (L.) R. Br. & Szőce & 1955 & Soó Rezső & BPU-0511.JPG \\
\hline 2686 & Platanthera bifolia (L.) Rchb. & Gyöngyös & 1940 & Priszter Szaniszló & BPU-0521.JPG \\
\hline 2686 & Platanthera bifolia (L.) Rchb. & Gyöngyös & 1965 & Soó Rezső & BPU-0522.JPG \\
\hline 2686 & Platanthera bifolia (L.) Rchb. & Isztimér & 1949 & Soó Rezső & BPU-0520.JPG \\
\hline 2686 & Platanthera bifolia (L.) Rchb. & Mosonmagyaróvár & 1962 & Borsos Olga & BPU-0523.JPG \\
\hline 2686 & Platanthera bifolia (L.) Rchb. & Nagyvisnyó & 1953 & Soó Rezső & BPU-0519.JPG \\
\hline 2686 & Platanthera bifolia (L.) Rchb. & Nyírlugos & 1951 & Soó Rezső & BPU-0524.JPG \\
\hline 2686 & Platanthera bifolia (L.) Rchb. & Sopron & 1960 & Borsos Olga, Simon Tibor & BPU-0475.JPG \\
\hline 2686 & Platanthera bifolia (L.) Rchb. & Szakonyfalu & 1955 & Soó Rezső & BPU-0525.JPG \\
\hline 2686 & Platanthera bifolia (L.) Rchb. & Uzsa & 1959 & $\begin{array}{l}\text { Jávorka Sándor, Felföldy } \\
\text { Lajos }\end{array}$ & BPU-0526.JPG \\
\hline 2686 & Platanthera bifolia (L.) Rchb. & Uzsa & 1959 & $\begin{array}{l}\text { Jávorka Sándor, Felföldy } \\
\text { Lajos }\end{array}$ & BPU-0527.JPG \\
\hline 2687 & Platanthera chlorantha (Custer) Rchb. & Szentendre & 1916 & Andrasovszky József & BPU-0533.JPG \\
\hline 2687 & Platanthera chlorantha (Custer) Rchb. & Tarpa & 1949 & Soó Rezső & BPU-0518.JPG \\
\hline 2688 & Gymnadenia conopsea (L.) R. Br. & Bátorliget & 1922 & Boros Ádám & BPU-0181.JPG \\
\hline 2688 & Gymnadenia conopsea (L.) R. Br. & Budapest & 1917 & Boros Ádám & BPU-0147.JPG \\
\hline 2688 & Gymnadenia conopsea (L.) R. Br. & Budapest & 1937 & Vajda László & BPU-0179.JPG \\
\hline 2688 & Gymnadenia conopsea (L.) R. Br. & Budapest & 1942 & Priszter Szaniszló & BPU-0180.JPG \\
\hline 2688 & Gymnadenia conopsea (L.) R. Br. & Dabas & 1951 & Borsos Olga, Simon Tibor & BPU-0156.JPG \\
\hline 2688 & Gymnadenia conopsea (L.) R. Br. & Dabas & 1951 & Simon Tibor, Borsos Olga & BPU-0155.JPG \\
\hline 2688 & Gymnadenia conopsea (L.) R. Br. & Gyöngyös & 1961 & Soó Rezső & BPU-0153.JPG \\
\hline 2688 & Gymnadenia conopsea (L.) R. Br. & Kimle & 1962 & Borsos Olga & BPU-0154.JPG \\
\hline 2688 & Gymnadenia conopsea (L.) R. Br. & Kiskőrös & 1920 & Boros Ádám & BPU-0157.JPG \\
\hline 2688 & Gymnadenia conopsea (L.) R. Br. & Mátraszentimre & 1942 & Priszter Szaniszló & BPU-0152.JPG \\
\hline 2688 & Gymnadenia conopsea (L.) R. Br. & Miskolc & 1948 & $\begin{array}{l}\text { Simon Tibor, Borsos Olga, } \\
\text { Jakucs Pál }\end{array}$ & BPU-0149.JPG \\
\hline 2688 & Gymnadenia conopsea (L.) R. Br. & Miskolc & 1950 & Soó Rezső & BPU-0150.JPG \\
\hline 2688 & Gymnadenia conopsea (L.) R. Br. & Nagyvisnyó & 1953 & Soó Rezső & BPU-0151.JPG \\
\hline 2688 & Gymnadenia conopsea (L.) R. Br. & Telkibánya & 1955 & Simon Tibor & BPU-0148.JPG \\
\hline 2690 & Coeloglossum viride (L.) Hartm. & Mályinka & 1950 & Soó Rezső & BPU-0133.JPG \\
\hline 2690 & Coeloglossum viride (L.) Hartm. & Miskolc & 1922 & Boros Ádám & BPU-0132.JPG \\
\hline 2690 & Coeloglossum viride (L.) Hartm. & Telkibánya & 1955 & Simon Tibor & BPU-0131.JPG \\
\hline 2691 & Dactylorhiza sambucina (L.) Soó & Budakeszi & 1937 & Priszter Szaniszló & BPU-0053.JPG \\
\hline 2691 & Dactylorhiza sambucina (L.) Soó & Gánt & 1943 & Kárpáti Zoltán & BPU-0051.JPG \\
\hline 2691 & Dactylorhiza sambucina (L.) Soó & Gánt & 1949 & Soó Rezső & BPU-0052.JPG \\
\hline 2691 & Dactylorhiza sambucina (L.) Soó & Telkibánya & 1955 & Simon Tibor & BPU-0049.JPG \\
\hline 2691 & Dactylorhiza sambucina (L.) Soó & Telkibánya & 1955 & Simon Tibor & BPU-0050.JPG \\
\hline 2693 & Dactylorhiza incarnata (L.) Soó & Aszófő & 1957 & Entz Béla & BPU-0045.JPG \\
\hline 2693 & Dactylorhiza incarnata (L.) Soó & Aszófő & 1957 & Felföldy Lajos & BPU-0044.JPG \\
\hline 2693 & Dactylorhiza incarnata (L.) Soó & Balatonszőlős & 1957 & Felföldy Lajos & BPU-0046.JPG \\
\hline
\end{tabular}


1. Elektroniukus melléklet - Electronic Appendix 1.

\begin{tabular}{|c|c|c|c|c|c|}
\hline $\begin{array}{l}\text { Sorszám / } \\
\text { Number }\end{array}$ & Taxon-név / Taxon-name & $\begin{array}{l}\text { Település / } \\
\text { Settlement }\end{array}$ & $\begin{array}{l}\text { Év / } \\
\text { Year }\end{array}$ & Gyújtő / Collector & $\begin{array}{l}\text { Fájlnév / } \\
\text { File-name }\end{array}$ \\
\hline 2693 & Dactylorhiza incarnata (L.) Soó & Bátorliget & 1932 & Soó Rezső & BPU-0042.JPG \\
\hline 2693 & Dactylorhiza incarnata (L.) Soó & Dabas & 1948 & Priszter Szaniszló & BPU-0041.JPG \\
\hline 2693 & Dactylorhiza incarnata (L.) Soó & Dabas & 1951 & Borsos Olga, Simon Tibor & BPU-0037.JPG \\
\hline 2693 & Dactylorhiza incarnata (L.) Soó & Dabas & 1951 & Borsos Olga, Simon Tibor & BPU-0038.JPG \\
\hline 2693 & Dactylorhiza incarnata (L.) Soó & Dabas & 1951 & Soó Rezső & BPU-0036.JPG \\
\hline 2693 & Dactylorhiza incarnata (L.) Soó & Debrecen & 1931 & Soó Rezső & BPU-0035.JPG \\
\hline 2693 & Dactylorhiza incarnata (L.) Soó & Debrecen & 1952 & Kulcsár Gábor & BPU-15439.JPG \\
\hline 2693 & Dactylorhiza incarnata (L.) Soó & Dunaszeg & 1921 & Polgár Sándor & BPU-0034.JPG \\
\hline 2693 & Dactylorhiza incarnata (L.) Soó & Nyírpilis & 1932 & Soó Rezső & BPU-0043.JPG \\
\hline 2693 & Dactylorhiza incarnata (L.) Soó & Ócsa & 1953 & Soó Rezső, Borsos Olga & BPU-0039.JPG \\
\hline 2693 & Dactylorhiza incarnata (L.) Soó & Ócsa & 1955 & Soó Rezső & BPU-0040.JPG \\
\hline 2693 & Dactylorhiza incarnata (L.) Soó & Szalonna & 1966 & Felföldy Lajos & BPU-0032.JPG \\
\hline 2693 & Dactylorhiza incarnata (L.) Soó & Telkibánya & 1955 & Simon Tibor & BPU-0031.JPG \\
\hline 2694 & $\begin{array}{l}\text { Dactylorhiza majalis (Rchb.) P.F. Hunt } \\
\text { et Summerh. }\end{array}$ & Harkakötöny & 1942 & Priszter Szaniszló & BPU-0086.JPG \\
\hline 2694 & $\begin{array}{l}\text { Dactylorhiza majalis (Rchb.) P.F. Hunt } \\
\text { et Summerh. }\end{array}$ & Háromhuta & 1960 & Simon Tibor & BPU-0095.JPG \\
\hline 2694 & $\begin{array}{l}\text { Dactylorhiza majalis (Rchb.) P.F. Hunt } \\
\text { et Summerh. }\end{array}$ & Kimle & 1962 & Borsos Olga & BPU-0090.JPG \\
\hline 2694 & $\begin{array}{l}\text { Dactylorhiza majalis (Rchb.) P.F. Hunt } \\
\text { et Summerh. }\end{array}$ & Kőszeg & 1938 & Soó Rezső & BPU-0094.JPG \\
\hline 2694 & $\begin{array}{l}\text { Dactylorhiza majalis (Rchb.) P.F. Hunt } \\
\text { et Summerh. }\end{array}$ & Miskolc & 1950 & Soó Rezső & BPU-0089.JPG \\
\hline 2694 & $\begin{array}{l}\text { Dactylorhiza majalis (Rchb.) P.F. Hunt } \\
\text { et Summerh. }\end{array}$ & Sopron & 1937 & Kárpáti Zoltán & BPU-0093.JPG \\
\hline 2694 & $\begin{array}{l}\text { Dactylorhiza majalis (Rchb.) P.F. Hunt } \\
\text { et Summerh. }\end{array}$ & Sopron & 1960 & Borsos Olga, Simon Tibor & BPU-0091.JPG \\
\hline 2694 & $\begin{array}{l}\text { Dactylorhiza majalis (Rchb.) P.F. Hunt } \\
\text { et Summerh. }\end{array}$ & Sopron & 1960 & Borsos Olga, Simon Tibor & BPU-0092.JPG \\
\hline 2694 & $\begin{array}{l}\text { Dactylorhiza majalis (Rchb.) P.F. Hunt } \\
\text { et Summerh. }\end{array}$ & Szemere & 1966 & Felföldy Lajos & BPU-0033.JPG \\
\hline 2694 & $\begin{array}{l}\text { Dactylorhiza majalis (Rchb.) P.F. Hunt } \\
\text { et Summerh. }\end{array}$ & Telkibánya & 1955 & Simon Tibor & BPU-0087.JPG \\
\hline 2694 & $\begin{array}{l}\text { Dactylorhiza majalis (Rchb.) P.F. Hunt } \\
\text { et Summerh. }\end{array}$ & Telkibánya & 1955 & Simon Tibor & BPU-0088.JPG \\
\hline 2697 & Dactylorhiza fuchsii (Druce) Soó & Cserépfalu & 1933 & Boros Ádám & BPU-0057.JPG \\
\hline 2697 & Dactylorhiza fuchsii (Druce) Soó & Gyöngyös & 1922 & Soó Rezső & BPU-0058.JPG \\
\hline 2697 & Dactylorhiza fuchsii (Druce) Soó & Gyöngyös & 1940 & Priszter Szaniszló & BPU-0059.JPG \\
\hline 2697 & Dactylorhiza fuchsii (Druce) Soó & Gyöngyös & 1961 & Soó Rezső & BPU-0060.JPG \\
\hline 2697 & Dactylorhiza fuchsii (Druce) Soó & Gyöngyös & 1961 & Soó Rezső & BPU-0061.JPG \\
\hline 2697 & Dactylorhiza fuchsii (Druce) Soó & Háromhuta & 1960 & Simon Tibor & BPU-0062.JPG \\
\hline 2697 & Dactylorhiza fuchsii (Druce) Soó & Mogyoróska & 1955 & Simon Tibor & BPU-0056.JPG \\
\hline 2698 & Traunsteinera globosa (L.) Rchb. & Mátraszentimre & 1930 & Kárpáti Zoltán & BPU-0309.JPG \\
\hline 2698 & Traunsteinera globosa (L.) Rchb. & Mátraszentimre & 1942 & Felföldy Lajos & BPU-0310.JPG \\
\hline 2698 & Traunsteinera globosa (L.) Rchb. & Mátraszentimre & 1961 & Soó Rezső & BPU-0311.JPG \\
\hline 2698 & Traunsteinera globosa (L.) Rchb. & Telkibánya & 1955 & Simon Tibor & BPU-0308.JPG \\
\hline 2699 & Orchis simia Lam. & Pécs & 1933 & Soó Rezső & BPU-0282.JPG \\
\hline 2699 & Orchis simia Lam. & Pécs & 1953 & Soó Rezső, Borsos Olga & BPU-0283.JPG \\
\hline 2699 & Orchis simia Lam. & Pécsvárad & 1941 & Priszter Szaniszló & BPU-0285.JPG \\
\hline 2700 & Orchis morio L. & Balatonfüred & 1929 & Soó Rezső & BPU-0245.JPG \\
\hline 2700 & Orchis morio L. & Budapest & 1918 & Boros Ádám & BPU-0231.JPG \\
\hline 2700 & Orchis morio L. & Csesznek & 1954 & Soó Rezső & BPU-0246.JPG \\
\hline
\end{tabular}


1. Elektroniukus melléklet - Electronic Appendix 1.

\begin{tabular}{|c|c|c|c|c|c|}
\hline $\begin{array}{l}\text { Sorszám / } \\
\text { Number }\end{array}$ & Taxon-név / Taxon-name & $\begin{array}{l}\text { Település / } \\
\text { Settlement }\end{array}$ & $\begin{array}{l}\text { Év / } \\
\text { Year }\end{array}$ & Gyújtő / Collector & $\begin{array}{l}\text { Fájlnév / } \\
\text { File-name }\end{array}$ \\
\hline 2700 & Orchis morio L. & Egyek & 1932 & Soó Rezső & BPU-0236.JPG \\
\hline 2700 & Orchis morio L. & Felsőtelekes & 2005 & Gulyás Pál & BPU-0232.JPG \\
\hline 2700 & Orchis morio L. & Hajdúsámson & 1989 & Felföldy Lajos & BPU-0235.JPG \\
\hline 2700 & Orchis morio L. & Isztimér & 1959 & Simon Tibor & BPU-0237.JPG \\
\hline 2700 & Orchis morio L. & Lábatlan & 1953 & Felföldy Lajos & BPU-0234.JPG \\
\hline 2700 & Orchis morio L. & Lenti & 1948 & Károlyi Árpád & BPU-0247.JPG \\
\hline 2700 & Orchis morio L. & Miskolc & 1937 & Balázs Ferenc & BPU-0233.JPG \\
\hline 2700 & Orchis morio L. & Nyírlugos & 1951 & Simon Tibor & BPU-0244.JPG \\
\hline 2700 & Orchis morio L. & Pilisszentlászló & 1941 & Priszter Szaniszló & BPU-0238.JPG \\
\hline 2700 & Orchis morio L. & Pomáz & 1942 & Priszter Szaniszló & BPU-0239.JPG \\
\hline 2700 & Orchis morio L. & Sárospatak & - & Soó Rezső & BPU-0248.JPG \\
\hline 2700 & Orchis morio L. & Sopron & 1960 & Borsos Olga, Simon Tibor & BPU-0243.JPG \\
\hline 2700 & Orchis morio L. & Szántód & 1957 & Entz Béla & BPU-0242.JPG \\
\hline 2700 & Orchis morio L. & Visegrád & 1948 & Priszter Szaniszló & BPU-0240.JPG \\
\hline 2700 & Orchis morio L. & Zebegény & 1962 & Simon Tibor & BPU-0241.JPG \\
\hline 2701 & Orchis coriophora L. & Budapest & 1913 & Koszilkov János & BPU-0252.JPG \\
\hline 2701 & Orchis coriophora L. & Dabas & 1948 & Priszter Szaniszló & BPU-0257.JPG \\
\hline 2701 & Orchis coriophora L. & Dabas & 1951 & Borsos Olga, Simon Tibor & BPU-0253.JPG \\
\hline 2701 & Orchis coriophora L. & Dabas & 1959 & Soó Rezső & BPU-0254.JPG \\
\hline 2701 & Orchis coriophora L. & Kunpeszér & 1951 & Borsos Olga, Simon Tibor & BPU-0255.JPG \\
\hline 2701 & Orchis coriophora L. & Pápateszér & 1962 & Tallós Pál & BPU-0258.JPG \\
\hline 2701 & Orchis coriophora L. & Pápateszér & 1962 & Tallós Pál & BPU-0259.JPG \\
\hline 2701 & Orchis coriophora L. & Pápateszér & 1962 & Tallós Pál & BPU-0260.JPG \\
\hline 2701 & Orchis coriophora L. & Pomáz & 1948 & Priszter Szaniszló & BPU-0256.JPG \\
\hline 2701 & Orchis coriophora L. & Sormás & 1950 & Károlyi Árpád & BPU-0261.JPG \\
\hline 2702 & Orchis tridentata Scop. & Budakeszi & 1937 & Priszter Szaniszló & BPU-0271.JPG \\
\hline 2702 & Orchis tridentata Scop. & Budapest & 1931 & Kárpáti Zoltán & BPU-0268.JPG \\
\hline 2702 & Orchis tridentata Scop. & Budapest & 1938 & Priszter Szaniszló & BPU-0267.JPG \\
\hline 2702 & Orchis tridentata Scop. & Budapest & 1948 & Priszter Szaniszló & BPU-0266.JPG \\
\hline 2702 & Orchis tridentata Scop. & Budapest & 2004 & Felföldy Lajos & BPU-0265.JPG \\
\hline 2702 & Orchis tridentata Scop. & Csákberény & 1934 & Boros Ádám & BPU-0270.JPG \\
\hline 2702 & Orchis tridentata Scop. & Gyenesdiás & 1928 & Soó Rezső & BPU-0273.JPG \\
\hline 2702 & Orchis tridentata Scop. & Kecskemét & 1919 & Boros Ádám & BPU-0107.JPG \\
\hline 2702 & Orchis tridentata Scop. & Kecskemét & 1919 & Boros Ádám & BPU-0269.JPG \\
\hline 2702 & Orchis tridentata Scop. & Visegrád & 1941 & Priszter Szaniszló & BPU-0272.JPG \\
\hline 2703 & Orchis ustulata L. & Bozsok & 1938 & Soó Rezső & BPU-0279.JPG \\
\hline 2703 & Orchis ustulata L. & Kecskemét & 1919 & Boros Ádám & BPU-0275.JPG \\
\hline 2703 & Orchis ustulata L. & Miskolc & 1937 & Soó Rezső & BPU-0277.JPG \\
\hline 2703 & Orchis ustulata L. & Miskolc & 1953 & Soó Rezső & BPU-0276.JPG \\
\hline 2703 & Orchis ustulata L. & Sopron & 1960 & Borsos Olga, Simon Tibor & BPU-0278.JPG \\
\hline 2704 & Orchis purpurea Huds. & Aszófő & 1951 & Felföldy Lajos & BPU-0304.JPG \\
\hline 2704 & Orchis purpurea Huds. & Balatonszőlős & 1958 & Felföldy Lajos & BPU-0305.JPG \\
\hline 2704 & Orchis purpurea Huds. & Budakeszi & 1937 & Priszter Szaniszló & BPU-0299.JPG \\
\hline 2704 & Orchis purpurea Huds. & Hidegkút & 1958 & Felföldy Lajos & BPU-0306.JPG \\
\hline 2704 & Orchis purpurea Huds. & Isztimér & 1955 & Soó Rezső & BPU-0307.JPG \\
\hline 2704 & Orchis purpurea Huds. & Mór & 1953 & Felföldy Lajos & BPU-0297.JPG \\
\hline 2704 & Orchis purpurea Huds. & Pécs & 1953 & Soó Rezső, Borsos Olga & BPU-0295.JPG \\
\hline
\end{tabular}


1. Elektroniukus melléklet - Electronic Appendix 1.

\begin{tabular}{|c|c|c|c|c|c|}
\hline $\begin{array}{l}\text { Sorszám / } \\
\text { Number }\end{array}$ & Taxon-név / Taxon-name & $\begin{array}{l}\text { Település / } \\
\text { Settlement }\end{array}$ & $\begin{array}{l}\text { Év / } \\
\text { Year }\end{array}$ & Gyújtő / Collector & $\begin{array}{l}\text { Fájlnév / } \\
\text { File-name }\end{array}$ \\
\hline 2704 & Orchis purpurea Huds. & Pécsvárad & 1936 & Soó Rezső & BPU-0296.JPG \\
\hline 2704 & Orchis purpurea Huds. & Pilisborosjenő & 1942 & Priszter Szaniszló & BPU-0300.JPG \\
\hline 2704 & Orchis purpurea Huds. & Sopron & 1937 & Kárpáti Zoltán & BPU-0302.JPG \\
\hline 2704 & Orchis purpurea Huds. & Sopron & 1960 & Borsos Olga, Simon Tibor & BPU-0303.JPG \\
\hline 2704 & Orchis purpurea Huds. & Vác & 1962 & Pátkai Tamás & BPU-0298.JPG \\
\hline 2704 & Orchis purpurea Huds. & Zebegény & 1962 & Simon Tibor & BPU-0301.JPG \\
\hline 2705 & Orchis militaris L. & Alsónémedi & 1958 & Simon Tibor & BPU-0288.JPG \\
\hline 2705 & Orchis militaris L. & Bátorliget & 1942 & Máthé Imre & BPU-0293.JPG \\
\hline 2705 & Orchis militaris L. & Budapest & 1923 & Soó Rezső & BPU-0286.JPG \\
\hline 2705 & Orchis militaris L. & Bugyi & 1959 & Soó Rezső & BPU-0289.JPG \\
\hline 2705 & Orchis militaris L. & Dabas & 1959 & Soó Rezső & BPU-0290.JPG \\
\hline 2705 & Orchis militaris L. & Szántód & 1957 & Entz Béla & BPU-0292.JPG \\
\hline 2705 & Orchis militaris L. & Szigetszentmiklós & 1947 & Kárpáti Zoltán & BPU-0291.JPG \\
\hline 2705 & Orchis militaris L. & Telkibánya & 1955 & Simon Tibor & BPU-0287.JPG \\
\hline 2706 & Orchis pallens L. & Pécs & 1925 & Soó Rezső & BPU-0327.JPG \\
\hline 2706 & Orchis pallens L. & Pilisszentkereszt & 1918 & Boros Ádám & BPU-0328.JPG \\
\hline 2706 & Orchis pallens L. & Pilisszentkereszt & 1965 & Borsos Olga & BPU-0329.JPG \\
\hline 2706 & Orchis pallens L. & Vác & 1942 & Priszter Szaniszló & BPU-0330.JPG \\
\hline 2707 & Orchis mascula (L.) L. & Bakonybél & 1955 & Soó Rezső & BPU-0322.JPG \\
\hline 2707 & Orchis mascula (L.) L. & Dömös & 1952 & Soó Rezső & BPU-0320.JPG \\
\hline 2707 & Orchis mascula (L.) L. & Dömös & 1952 & Soó Rezső & BPU-0321.JPG \\
\hline 2707 & Orchis mascula (L.) L. & Dömös & 1952 & Soó Rezső & BPU-15418.JPG \\
\hline 2707 & Orchis mascula (L.) L. & Telkibánya & 1955 & Simon Tibor & BPU-0318.JPG \\
\hline 2707 & Orchis mascula (L.) L. & Telkibánya & 1955 & Simon Tibor & BPU-0319.JPG \\
\hline 2708 & Orchis elegans Heuff. & Bátorliget & 1934 & Soó Rezső & BPU-0029.JPG \\
\hline 2708 & Orchis elegans Heuff. & Beregdaróc & 1951 & Simon Tibor & BPU-0024.JPG \\
\hline 2708 & Orchis elegans Heuff. & Debrecen & 1934 & Soó Rezső & BPU-0026.JPG \\
\hline 2708 & Orchis elegans Heuff. & Debrecen & 1947 & Simon Tibor & BPU-0027.JPG \\
\hline 2708 & Orchis elegans Heuff. & Debrecen & 1953 & Jeney Endre & BPU-15437.JPG \\
\hline 2708 & Orchis elegans Heuff. & Hajdúsámson & 1989 & Felföldy Lajos & BPU-0028.JPG \\
\hline 2708 & Orchis elegans Heuff. & Tarpa & 1951 & Simon Tibor, Jakucs Pál & BPU-0025.JPG \\
\hline 2709 & Orchis palustris Jacq. & Aszófő & 1957 & Entz Béla & BPU-0019.JPG \\
\hline 2709 & Orchis palustris Jacq. & Aszófó & 1959 & Felföldy Lajos & BPU-0020.JPG \\
\hline 2709 & Orchis palustris Jacq. & Balatonszőlős & 1956 & $\begin{array}{l}\text { Felföldy Lajos, Tóth } \\
\text { László }\end{array}$ & BPU-0021.JPG \\
\hline 2709 & Orchis palustris Jacq. & Balatonszőlős & 1957 & Felföldy Lajos & BPU-0022.JPG \\
\hline 2709 & Orchis palustris Jacq. & Balatonszőlős & 1957 & Felföldy Lajos & BPU-0109.JPG \\
\hline 2709 & Orchis palustris Jacq. & Budapest & 1913 & Koszilkov János & BPU-0009.JPG \\
\hline 2709 & Orchis palustris Jacq. & Bugyi & 1959 & Soó Rezső & BPU-0013.JPG \\
\hline 2709 & Orchis palustris Jacq. & Dabas & 1951 & Borsos Olga, Simon Tibor & BPU-0015.JPG \\
\hline 2709 & Orchis palustris Jacq. & Dabas & 1961 & Borsos Olga, Simon Tibor & BPU-0016.JPG \\
\hline 2709 & Orchis palustris Jacq. & Dunaharaszti & 1946 & Priszter Szaniszló & BPU-0014.JPG \\
\hline 2709 & Orchis palustris Jacq. & Kimle & 1962 & Borsos Olga & BPU-0012.JPG \\
\hline 2709 & Orchis palustris Jacq. & Ócsa & 1955 & Soó Rezső & BPU-0017.JPG \\
\hline 2709 & Orchis palustris Jacq. & Pákozd & 1939 & Boros Ádám & BPU-0011.JPG \\
\hline 2709 & Orchis palustris Jacq. & Szalonna & 1966 & Felföldy Lajos & BPU-0010.JPG \\
\hline 2709 & Orchis palustris Jacq. & Szántód & 1957 & Entz Béla & BPU-0018.JPG \\
\hline 2709 & Orchis palustris Jacq. & Tihany & 1956 & Felföldy Lajos & BPU-0023.JPG \\
\hline
\end{tabular}


1. Elektroniukus melléklet - Electronic Appendix 1.

\begin{tabular}{|c|c|c|c|c|c|}
\hline $\begin{array}{l}\text { Sorszám / } \\
\text { Number }\end{array}$ & Taxon-név / Taxon-name & $\begin{array}{l}\text { Település / } \\
\text { Settlement }\end{array}$ & $\begin{array}{l}\text { Év / } \\
\text { Year }\end{array}$ & Gyújtő / Collector & $\begin{array}{l}\text { Fájlnév / } \\
\text { File-name }\end{array}$ \\
\hline $\begin{array}{c}2699 \times \\
2704\end{array}$ & Orchis $\times$ angusticruris Franch & Pécsvárad & 1953 & Soó Rezső, Borsos Olga & BPU-0284.JPG \\
\hline 2710 & $\begin{array}{l}\text { Himantoglossum adriaticum } \mathrm{H} \text {. } \\
\text { Baumann }\end{array}$ & Kőszeg & 1991 & Waisbecker Antal & BPU-0123.JPG \\
\hline 2711 & $\begin{array}{l}\text { Himantoglossum jankae Somlyay, } \\
\text { Kreutz \& Óvári }\end{array}$ & Solymár & 1918 & Trautmann Róbert & BPU-0122.JPG \\
\hline 2712 & Anacamptis pyramidalis (L.) Rich. & Bátorliget & 1928 & Soó Rezső & BPU-0117.JPG \\
\hline 2712 & Anacamptis pyramidalis (L.) Rich. & Bátorliget & 1950 & Simon Tibor & BPU-0118.JPG \\
\hline 2712 & Anacamptis pyramidalis (L.) Rich. & Bátorliget & 1950 & $\begin{array}{l}\text { Simon Tibor, Kárpáti } \\
\text { István }\end{array}$ & BPU-0119.JPG \\
\hline 2712 & Anacamptis pyramidalis (L.) Rich. & Budapest & 1913 & Koszilkov János & BPU-0110.JPG \\
\hline 2712 & Anacamptis pyramidalis (L.) Rich. & Budapest & 1913 & Koszilkov János & BPU-0252.JPG \\
\hline 2712 & Anacamptis pyramidalis (L.) Rich. & Csákvár & 1937 & Boros Ádám & BPU-0112.JPG \\
\hline 2712 & Anacamptis pyramidalis (L.) Rich. & Dabas & 1959 & Soó Rezső & BPU-0113.JPG \\
\hline 2712 & Anacamptis pyramidalis (L.) Rich. & Dabas & 1961 & Borsos Olga, Simon Tibor & BPU-0114.JPG \\
\hline 2712 & Anacamptis pyramidalis (L.) Rich. & Gyenesdiás & 1929 & Soó Rezső & BPU-0120.JPG \\
\hline 2712 & Anacamptis pyramidalis (L.) Rich. & Kecskemét & 1920 & Boros Ádám & BPU-0111.JPG \\
\hline 2712 & Anacamptis pyramidalis (L.) Rich. & Pilisszentiván & 1942 & Priszter Szaniszló & BPU-0115.JPG \\
\hline 2712 & Anacamptis pyramidalis (L.) Rich. & Szántód & 1957 & Entz Béla & BPU-0116.JPG \\
\hline 2713 & Ophrys insectifera L. & Dabas & 1959 & Simon Tibor & BPU-0218.JPG \\
\hline 2713 & Ophrys insectifera L. & Dabas & 1959 & Soó Rezső & BPU-0214.JPG \\
\hline 2713 & Ophrys insectifera L. & Gyenesdiás & 1928 & Soó Rezső & BPU-0215.JPG \\
\hline 2713 & Ophrys insectifera L. & Sopron & 1939 & Kárpáti Zoltán & BPU-0219.JPG \\
\hline 2713 & Ophrys insectifera L. & Sopron & 1960 & Borsos Olga, Simon Tibor & BPU-0216.JPG \\
\hline 2714 & Ophrys apifera Huds. & Balatonszőlős & 1956 & $\begin{array}{l}\text { Felföldy Lajos, Tóth } \\
\text { László }\end{array}$ & BPU-0226.JPG \\
\hline 2714 & Ophrys apifera Huds. & Budapest & 1873 & Simonkai Lajos & BPU-0224.JPG \\
\hline 2714 & Ophrys apifera Huds. & Budapest & 1962 & Pátkai Tamás & BPU-0225.JPG \\
\hline 2715 & Ophrys sphegodes Mill. & Balatonberény & 1949 & Károlyi Árpád & BPU-0212.JPG \\
\hline 2715 & Ophrys sphegodes Mill. & Cegléd & 1995 & Borsos Olga, Király J. & BPU-0208.JPG \\
\hline 2715 & Ophrys sphegodes Mill. & Dabas & 1951 & Borsos Olga, Simon Tibor & BPU-0209.JPG \\
\hline 2715 & Ophrys sphegodes Mill. & Dabas & 1959 & Simon Tibor & BPU-0210.JPG \\
\hline 2715 & Ophrys sphegodes Mill. & Devecser & 1957 & Tallós Pál & BPU-0221.JPG \\
\hline 2715 & Ophrys sphegodes Mill. & Felpéc & 1937 & Polgár Sándor & BPU-0205.JPG \\
\hline 2715 & Ophrys sphegodes Mill. & Győr & 1920 & Boros Ádám & BPU-0206.JPG \\
\hline 2715 & Ophrys sphegodes Mill. & Kunpeszér & 1951 & Borsos Olga, Simon Tibor & BPU-0211.JPG \\
\hline 2715 & Ophrys sphegodes Mill. & Nagyszentjános & 1906 & Polgár Sándor & BPU-0207.JPG \\
\hline 2715 & Ophrys sphegodes Mill. & Sopron & 1937 & Kárpáti Zoltán & BPU-0213.JPG \\
\hline 2716 & Ophrys oestrifera M. Bieb. & Budapest & 1943 & Kárpáti Zoltán & BPU-0228.JPG \\
\hline 2716 & Ophrys oestrifera M. Bieb. & Kunpeszér & 1931 & Tuzson János & BPU-0229.JPG \\
\hline 2716 & Ophrys oestrifera M. Bieb. & Kunpeszér & 1933 & Kárpáti Zoltán & BPU-0230.JPG \\
\hline 2718 & Corallorhiza trifida Châtel. & Gánt & 1966 & Borhidi Attila & BPU-0126.JPG \\
\hline 2719 & Liparis loeselii (L.) Rich. & Sopron & 1936 & Kárpáti Zoltán & BPU-0125.JPG \\
\hline
\end{tabular}

